\begin{tabular}{|c|c|}
\hline 2. To: (Receiving Organization) & 3. From: (Originating Organization) \\
\hline 300 Area Stabilization & 300 Area Stabilization Projec \\
\hline 5. Proj./Prog./Dept./Div.: & $\begin{array}{l}\text { 6. Design Authority/Design Agent/Cog. Engr.: } \\
\text { J. L. Shelor }\end{array}$ \\
\hline \multicolumn{2}{|l|}{ 8. Originator Remarks: } \\
\hline $\begin{array}{l}\text { Release of HNE-4799, Rev. 0, "R } \\
\text { Cesium-137 Content of HN-200 G }\end{array}$ & $\begin{array}{l}\text { ed Methodology for Determin } \\
\text { Containers" }\end{array}$ \\
\hline
\end{tabular}

11. Receiver Remarks:

11A. Design Baseline Document? $\mathrm{Yes}$

No

\begin{tabular}{|c|c|c|c|}
\hline \multicolumn{4}{|c|}{$\begin{array}{l}\text { 4. Related EDT No.: } \\
\text { NA }\end{array}$} \\
\hline \multicolumn{4}{|c|}{$\begin{array}{l}\text { 7. Purchase Order No.: } \\
\text { NA }\end{array}$} \\
\hline \multicolumn{4}{|c|}{$\begin{array}{l}\text { 9. Equip./Component No.: } \\
\text { NA }\end{array}$} \\
\hline \multicolumn{4}{|c|}{$\begin{array}{l}\text { 10. System/Bldg./Facility: } \\
324 \text { Building }\end{array}$} \\
\hline \multicolumn{4}{|c|}{$\begin{array}{l}\text { 12. Major Assm. Dwg. No.: } \\
\text { NA }\end{array}$} \\
\hline \multicolumn{4}{|c|}{$\begin{array}{l}\text { 13. PermitPermit Application No:: } \\
\text { NA }\end{array}$} \\
\hline \multicolumn{4}{|c|}{$\begin{array}{l}\text { 14. Required Response Date: } \\
\text { NA }\end{array}$} \\
\hline (F) & (G) & $(H)$ & (i) \\
\hline $\begin{array}{l}\text { Approval } \\
\text { Desig- } \\
\text { nator }\end{array}$ & $\begin{array}{l}\text { Reason } \\
\text { for Trans- } \\
\text { mittal }\end{array}$ & $\begin{array}{l}\text { Origi- } \\
\text { nator } \\
\text { Dispo- } \\
\text { stion }\end{array}$ & $\begin{array}{l}\text { Receiv- } \\
\text { er } \\
\text { Dispo- } \\
\text { sition }\end{array}$ \\
\hline$E, Q$ & 2 & & \\
\hline & & & \\
\hline & & & \\
\hline & & & \\
\hline & & & \\
\hline & & & \\
\hline & & & \\
\hline
\end{tabular}

16.

\begin{tabular}{|c|}
\hline Approval Designator $(F)$ \\
\hline E, S, O, D OR N/A \\
(See WHC.CM-3-5, \\
Sec. 12.7) \\
\hline
\end{tabular}

17
DATA TRANSMITTED (C) Sheet (D) Rev.

KEY

(G) Title or Description of Data Transmitted

HNF-4799, Rev. 0, "Revised

Methodology for

Determining Cesium-137

content of $\mathrm{HN}-200$ Grout Containers"

Disposition (H) \& (l)

Reason for Transmittal (G)

1. Approved

2. Approved w/comment

3. Disapproved w/comment
4. Reviewed no/comment

5. Reviewed w/comment 6. Receipt acknowledged SIGNATURE/DISTRIBUTION (See Approval Designator for required signatures)

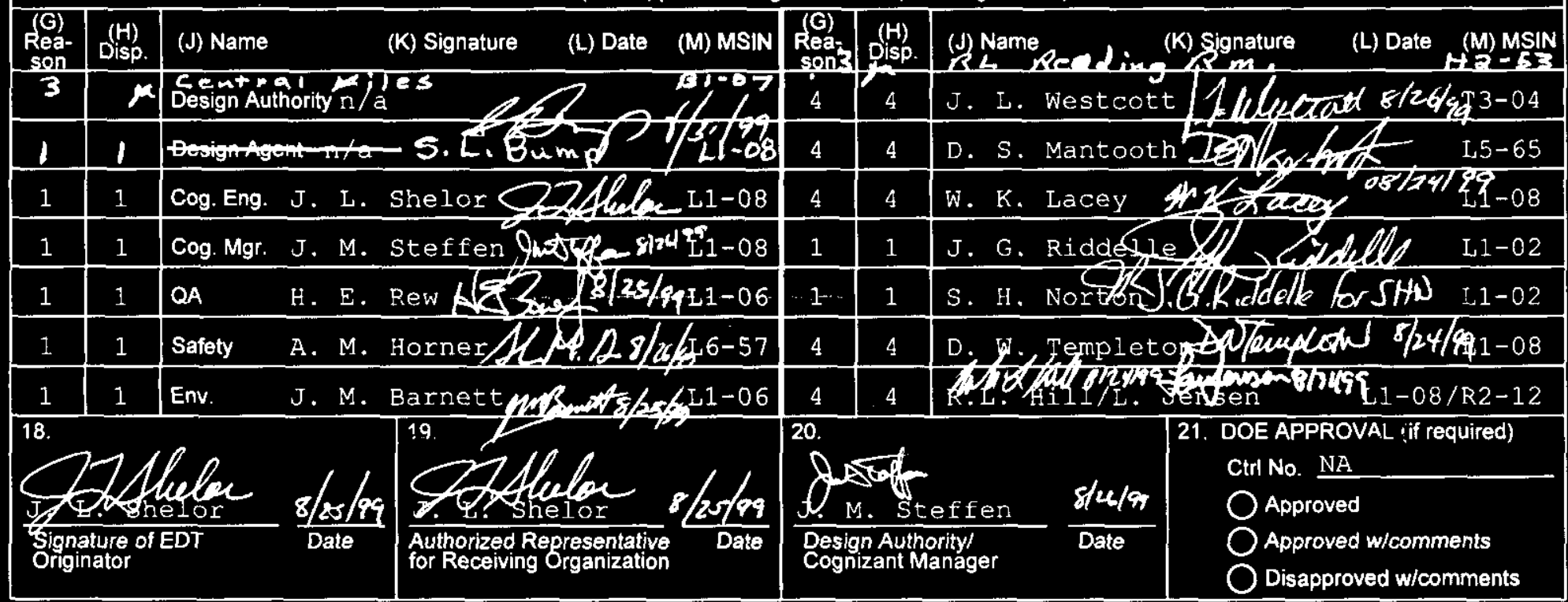




\title{
Revised Methodology for Determining Cesium-137 Content of HN-200 Grout Containers
}

\author{
J. I. Shelor \\ B\&W Hanford Company \\ Richland, WA 99352 \\ U.S. Department of Energy Contract DE-AC06-96RL13200

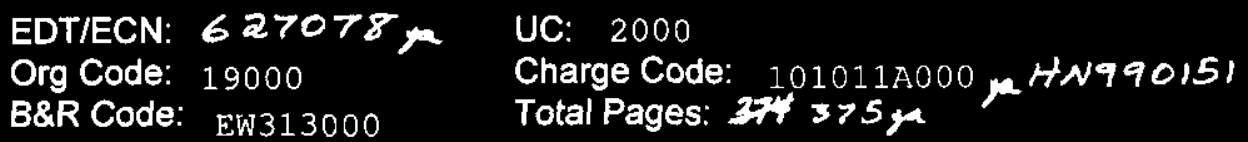 \\ Key Words: 324 Building, grout containers, B-Cell, isotope, sifted \\ debris, cesium, strontium

\section{Abstract:} \\ This paper examines the accuracy of the current method of determining \\ the cesium-137 content of the HN-200 grout containers filled with 324 \\ Building B-Cell equipment dunnage and sifted debxis.
}

TRADEMARK DISCLAIMER. Reference herein to any specific commercial product, process, or service by trade name, trademark, manufacturer, or otherwise, does not necessarily constitute or imply its endorsement, recommendation, or favoring by the United States Government or any agency thereof or its contractors or subcontractors.

Printed in the United States of America. To obtain copies of this document, contact: Document Control Services, P.O. Box 950, Mailstop H6-08, Richland WA 99352, Phone (509) 372-2420; Fax (509) 376-4989.

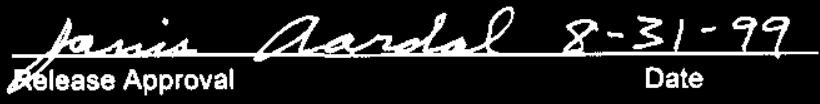

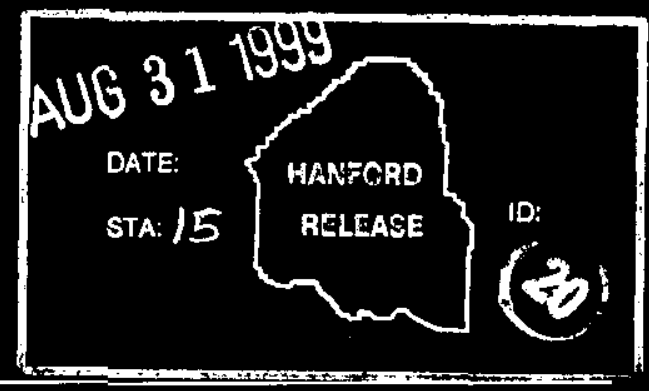

Release Stamp 


\section{RFLEASE AUTHORIZATION}

Document HNF-4799, Rev. 0

Number:

Document

Title:
Revised Methodology for Determining Cesium-137

Content of HN-200 Grout Containers

This document, reviewed in accordance with DOE Order 241.1, "Scientific and Technical Information Management," and 241.1-1, "Guide to the Management of Scientific and Technical Information," does not contain classified or sensitive unclassified information and is:

\section{APPROVFD FOR PUBLIC RELEASE}

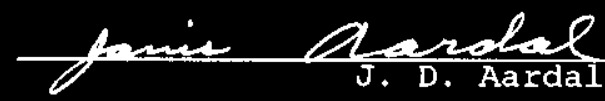

\section{$8-31-99$}

Lockheed Martin Services, Inc.

Document Control/Information Clearance heviewed for Applied Technology, Business Sensitive, Clasgified, Copyrighted, Export Cont rolled, Patent, Personal/Private,
Proprietary, Protected CADA, Trademark, Unclassified Control led Nuclear Informat ion.

Trademark Dlsclaimer. Reference herein to any spectf1c commercial product, process, or service by trade name, trademark, manufacturer, or othorwise, does not necessarlily consticute or impiy its enoorsement, recomuendation, or favoring by the expressed herein do not necessarily state or reflect those of the United States Government or any agency thereof. Th1s report has been reproduced from the best available copy.

Printed in the United States of America. 


\section{REVISED METHODOLOGY FOR DETERMINING CESIUM-137 CONTENT OF HN-200 GROUT CONTAINERS.}

\section{TABLE OF CONTENTS}

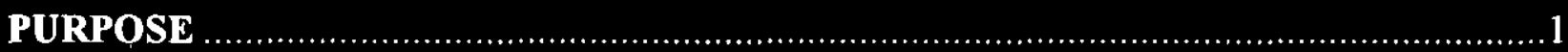

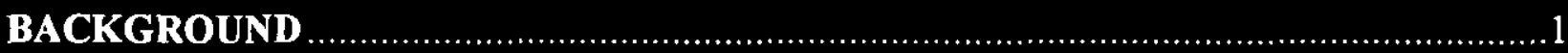

ASSUMPTIONS AND CONSTRAINTS OF THIS ANALYSIS ...................................

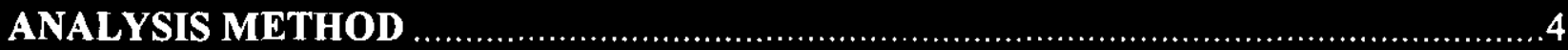

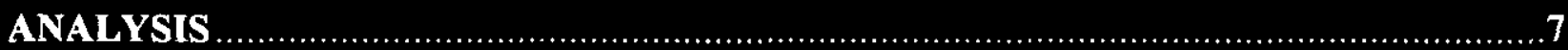

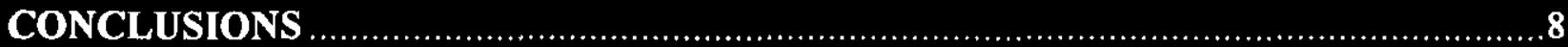

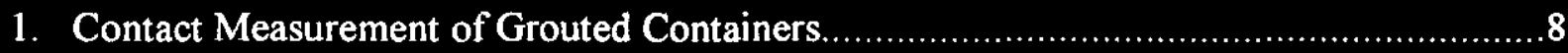

2. Remote Measurement of HN-200 Grout Containers......................................................

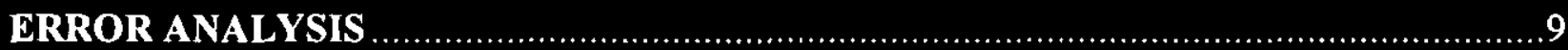

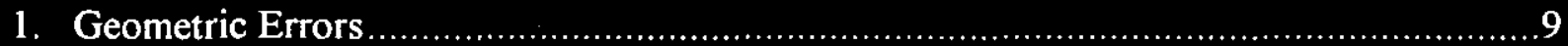

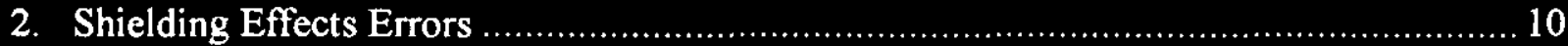

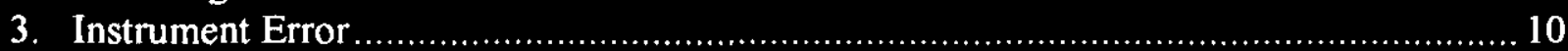

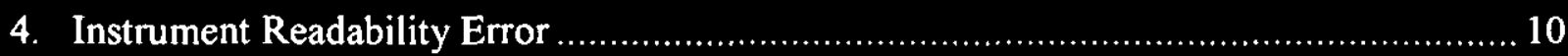

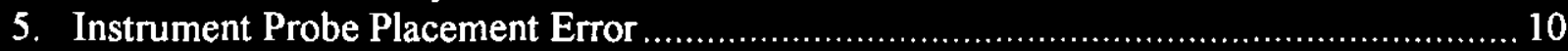

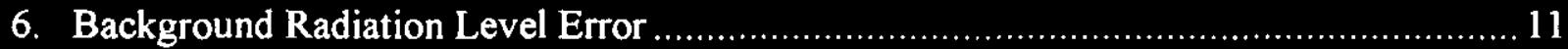

7. Gamma Scatter (Compton Scatter) during Measurements ............................................ 11

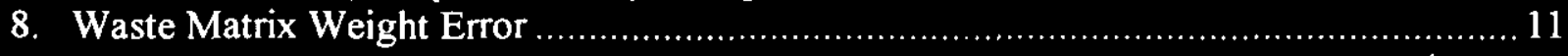

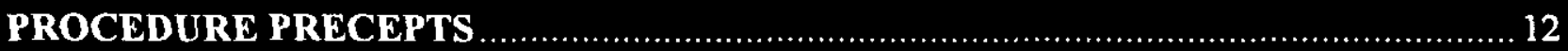

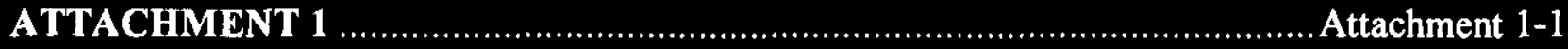

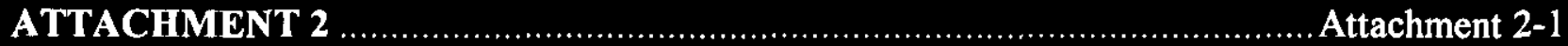

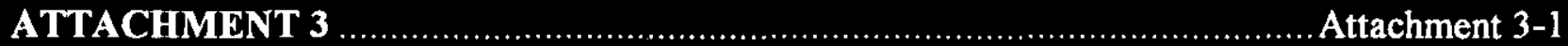

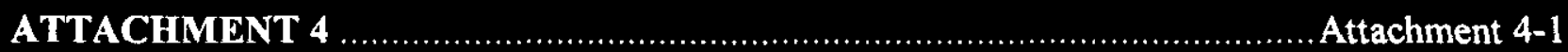

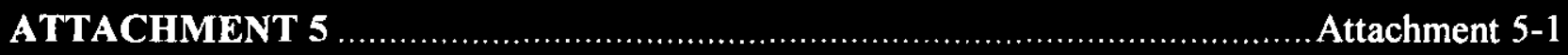

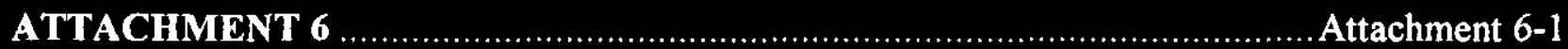

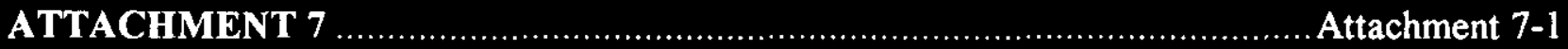




\section{REVISED METHODOLOGY FOR DETERMINING CESIUM-137 CONTENT OF HN-200 GROUT CONTAINERS.}

\section{PURPOSE.}

The purpose of this technical paper is to examine the accuracy of the existing method of determining the Cs-137 content of HN-200 grout containers and compare that accuracy to the accuracy attainable by other methods of measurement.

The methods of measurement to be compared include:

C Contact measurements on a grouted container (existing method)

$\therefore$ Measurements at 5 feet from the surface of a grouted container.

. Measurements at $\mathbf{1 0}$ feet from a grouted container.

$\checkmark$ Measurements on contact with the surface of an ungrouted container.

- Measurements at 5 feet from the surface of an ungrouted container.

- Measurements at $\mathbf{1 0}$ feet from the surface of an ungrouted container.

Once the most accurate and useable method is determined, the precepts for an operating procedure will be provided for determining the Cs- 137 content of newly generated and future HN-200 grout containers as well as the grouted legacy containers currently stored in B Cell.

\section{BACKGROUND.}

HN-200 grout containers are used to dispose of waste from the cleanup of B Cell in the 324 Building. The waste stream placed in the $\mathrm{HN}-200$ grout containers consists of equipment dunnage and sifted debris generated during the dismantling of equipment racks and process equipment in B Cell. This equipment dunnage and sifted debris is coated with a strongly adherent film of contamination consisting primarily of Cs-137 and Sr-90 along with several other isotopes including transuranic isotopes.

Radioisotopic analysis of the film on the equipment dunnage and sifted debris is conducted to determine the amounts of each isotope present and the ratios between the isotopes. Since analysis of these isotopes has determined that greater than $99 \%$ of the gamma energy emitted by this waste is generated by the Cs-137 (and it's daughter products) in the waste, Cs-137 is used as the target isotope for measurement and characterization of the waste in a given container. The characterization of the waste, including the transuranic (TRU) content of the waste, is dependent on obtaining as accurate a Cs-137 measurement as possible.

Obtaining an accurate Cs-137 measurement is the subject of this paper. Once an accurate Cs-137 measurement has been obtained, the ratios of the Cs- 137 to the other isotopes present can be used to determine the characterization of the waste in the container. The process for characterizing the waste once an accurate Cs- 137 measurement has been attained will be the subject of a separate technical paper. 


\section{REVISED METHODOLOGY FOR DETERMINING CESIUM-137 CONTENT OF HN-200 GROUT CONTAINERS.}

The existing method for characterizing these waste containers consists of:

Fill the container with equipment dunnage and sifted debris.

Conduct a pre-grout gamma radiation profile of the container. For this profile, four sets of six readings are obtained using an $\mathrm{RO-7}$ radiation survey instrument in contact with the container. The four sets of readings are taken at the $0^{\circ}, 90^{\circ}, 180^{\circ}$, and $270^{\circ}$ positions around the circumference of the container with six readings, evenly spaced vertically, being taken along the vertical axis of the container at each of these four positions. If all 24 readings are less than a preset maximum value and the average of the 24 readings is less than a preset maximum average value, the waste is grouted. If any reading exceeds the preset maximum or the average exceeds the preset maximum, the container is repackaged to produce a more homogeneous profile with all readings below the preset maximum values and the waste is grouted.

Once the grouting operation is completed, a post-grout gamma radiation profile is conducted on the container. The post-grout gamma radiation profile is conducted in the identical manner as the pre-grout profile with six vertically evenly spaced readings taken at four positions around the circumference of the container $\left(0^{\circ}, 90^{\circ}, 180^{\circ}\right.$, and $\left.270^{\circ}\right)$. The post-grout gamma radiation profile is considered the official profile for the container and is used to characterize the container for waste purposes. The average of the 24 readings taken on contact with the surface of the grouted container is used to calculate the Cs-137 content of the container by using a conversion factor determined assuming the container to be filled with a homogeneous mixture of grout and Cs- 137 activity. Once this Cs- 137 activity determination is made, all other isotopes in the container are determined by ratio to this Cs137 value.

The reason for performing this analysis is that questions have been raised regarding optimization of the current method for determining the Cs-137 content of HN-200 grout containers from two perspectives.

1. Can an accurate determination of Cs-137 content be made in a container that is approximately 4 feet in diameter and approximately 4.5 feet tall using contact readings?

2. Can an accurate determination of Cs-137 content be made for a container under the following conditions?

Approximately $10 \%$ of the volume of the container is filled with material coated with activity.

Approximately $90 \%$ of the volume of the container is filled with essentially uncontaminated grout. 


\section{REVISED METHODOLOGY FOR DETERMINING CESIUM-137 CONTENT OF HN-200 GROUT CONTAINERS.}

ר The assumption used to model the container is that the container is filled with a homogeneous mixture of activity and grout.

\section{ASSUMPTIONS AND CONSTRAINTS OF THIS ANALYSIS.}

1. The HN-200 grout container being measured is filled with equipment dunnage and sifted debris as defined by HNF-2570, Rev 1, Dated 06/25/98, "324 Facility Special Case Waste Analysis in support of 324 Closure (TPA Milestone M-89-05)". Use of this methodology and modeling with any other waste matrix may result in substantial error if the matrix is substantially different and should be proceeded by a specific analysis of that waste matrix.

2. An HN-200 grout container filled with B Cell equipment dunnage and sifted debris can be simulated using a series of 35 randomly generated point sources in a mathematically constructed (computer generated) simulation to accurately assess the effects of geometry (i.e., the placement of the measuring device with respect to the actual position of the source) and the effects of distributed shielding (i.e., the grout) in the container. Thirty-five sources were used to attempt to make the container appear homogeneous. Statistically, seven sources should make the container start to appear homogeneous.

3. Errors that are common to all methods of measurement need not be included in the initial analysis of the effects of geometry and distributed shielding. Errors that are common to all methods of measurement will be examined after the proper measurement technique(s) are determined and these errors will be propagated through the measurement process using standard statistical techniques.

4. The errors common to all measurement techniques include:

c The measurement error of the instrument used to conduct the measurements.

c The measurement error caused by measurement probe placement error.

c The error caused by the shielding effects of the waste matrix of the equipment dunnage and sifted debris loaded into the container. This assumption is true because the only change made in the analysis between the grouted case and the ungrouted case is to remove the grout. The positions and shielding characteristics of the equipment dunnage and sifted debris are maintained constant throughout the analysis.

c The error caused by background radiation levels. For the comparison analysis conducted in this technical paper, the assumption will be that the measurements are being taken in a background radiation free environment.

c The error caused by Compton Scatter of the gamma radiation during the measuring process.

c The error caused by errors in weighing the waste matrix. 


\section{REVISED METHODOLOGY FOR DETERMINING CESIUM-137 CONTENT OF HN-200 GROUT CONTAINERS.}

5. Using different combinations of one source set to obtain multiple source sets for analysis is equivalent to providing multiple sets of random sources. In this analysis, one set of 35 random sources is generated and different combinations of these 35 random sources varying from a single source per container to 35 sources per container are analyzed. Source sets per container analyzed include single sources, 2 sources, 3 sources, 5 sources, 7 sources, 10 sources, 11 sources, 12 sources, 17 sources, 18 sources, 20 sources, 25 sources, 30 sources, and 35 sources. Each multiple source set is a different combination of the same set of 35 random sources. The individualism of the multiple source sets is obtained by randomly varying the source numbers used to comprise the source sets. In addition, individual source strength was restricted to be between 10 and 30 curies per source to prevent a single source from dominating the gamma radiation profile of the container and causing a multiple source container to appear to be a single source container.

6. Standard statistical analysis techniques using linear regression analysis and data correlation analysis provides an accurate and usable basis for using the gamma radiation profile of the container to determine the $95 \%$ assurance bounding value of the Cesium-137 (Cs-137) content of an HN-200 grout container. Waste Management Hanford has imposed the criteria that the TRU content of these containers must be known with $85 \%$ assurance to be less than 100 nanoCuries/gm for the containers to be accepted as low level waste. Use of the $95 \%$ bounding value (i.e., the value for which the measurement has $95 \%$ assurance that the actual content of the container is equal to or less than the calculated $95 \%$ bounding value) is necessary so that after chemical analysis errors and statistical assurance calculations for the isotopic results are propagated through the process in follow on technical papers, the requirement that the TRU characterization of the container be known with $85 \%$ assurance ( $85 \%$ assurance that the TRU content of the container is less than 100 nanoCuries $/ \mathrm{gm}$ ) can be met.

\section{ANALYSIS METHOD.}

The method of the analysis for this technical paper consists of:

1. Mathematically constructing an $\mathrm{HN}-200$ grout container in an Excelه spread sheet, placing 35 randomly generated point sources inside the container using the random number generator included in the spread sheet, designating the $\mathrm{X}, \mathrm{Z}, \mathrm{Y}$ coordinates of contact and remote (10 Feet) measuring points to the intended measuring positions, and calculating Microshield@ 2 version 5.03 input data sheets for the entry of the data into Microshield@. Attachment 1 contains these calculations and ends with the Microshield@ data sheets. Attachment 3 provides the same calculations for the remote measurement at 5 feet cases but the intermediate calculations are not shown, only the initial input data and the calculated Microshield@ input data sheets are displayed. The calculations consist of:

c Calculating the straight-line distance from the random source to the measuring point.

\footnotetext{
Excel is a trademark of the Microsoft Corp.
}

Page 4 of 15

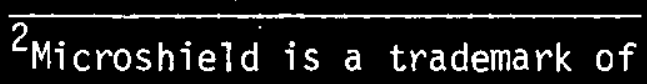
Grove Engineering, Inc. 


\section{REVISED METHODOLOGY FOR DETERMINING CESIUM-137 CONTENT OF HN-200 GROUT CONTAINERS.}

Calculating the distance of travel from the source to the point where the inner wall of the container is encountered along the line of travel to the measuring point. This produces the travel distance inside the container and the shield thickness for the grout during the grouted case analysis.

Calculating the distance from the point where the inner wall of the container is encountered to the point where the outer wall of the container is penetrated along the line of travel to the measuring point. This produces the effective shielding distance for the wall of the container along the path of travel from the source to the measuring point. Calculating the distance from the exit point of the outer wall of the container to the measuring point along the line of travel to the measuring point. This produces the air gap distance from the outer wall of the container to the measuring point. Since Microshield@ also automatically calculates the air gap distance after Shield 1, Shield 2, and Total Distance are entered, the Air Gap distance serves as a check number to ensure the data from the input data sheet has been entered correctly.

2. Calculating the radiation level produced by each source in the container at each measuring point. Manually entering the data calculated in Attachment 1 into Microshield@ and manually entering the output of Microshield@ into Attachment 2 accomplish this calculation. The Microshield@ version 5.03 files used to accomplish this calculation can be provided on request, however, approximately 4100 files are included in this group.

3. Calculating the Conversion Factors for converting the gamma radiation profile exhibited by the container to curies of Cs-137. This is accomplished by generating Microshield\& calculations for a HN-200 grout container filled with a homogeneous mixture of 1 curie of Cs-137 and grout to obtain the grouted container conversion factor. This model was used because the current method for determining the Cs-137 content of the container assumes this model for the container. The ungrouted container conversion factors were obtained by removing the grout from the above model and using a homogeneous mixture of 1 curie of Cs137 and air to simulate a material in the container with little or no shielding value. Using air as the internal source mixture was necessary to obtain a direct correlation with the grouted case since the distributed shielding effect of the waste matrix is common to both measurements and is not being considered in this comparison analysis. Conversion factors are calculated for the contact measurement method, the measurement at 5 feet from the surface of the container method, and the measurement at 10 feet from the surface of the container method. The Microshield@ files used to calculate these conversion factors are available on request.

4. Calculate the total radiation level measured at each measuring point as the contribution from each set of sources to be analyzed by summing the contributions of the sources included in each source set to be analyzed. Then using the conversion factors calculated for the homogeneous containers (grouted and ungrouted) calculate the estimated Cs-137 content that would have been attained using the current method. In addition to the current method of 


\section{REVISED METHODOLOGY FOR DETERMINING CESIUM-137 CONTENT OF HN-200 GROUT CONTAINERS.}

contact readings on a grouted container, the following other methods of calculating the curie content of the Cs-137 in the container are analyzed:

- Measurements taken at 5 feet from the surface of a grouted container.

- Measurements taken at 10 feet from the surface of a grouted container.

- Measurements taken on contact with the surface of an ungrouted container.

- Measurements taken at 5 feet from the surface of an ungrouted container.

r Measurements taken at 10 feet from the surface of an ungrouted container.

Attachment 2 displays these calculations and results for the contact measurement cases and the measurement at 10 feet cases. Attachment 4 displays these calculations for the measurement at 5 feet case.

5. Conduct linear regression analysis on each method of measurement for each of five source combinations with respect to the homogeneity of the container. These combinations are:

Heterogeneous Cases - Cases in which the container is filled in a manner resulting in a single dominating source that drives the gamma radiation profile.

Mostly Heterogeneous Cases - Cases in which the container is filled in a manner resulting in between 2 and 5 sources per container dominating the gamma radiation profile of the container.

Slightly Homogeneous Cases - Cases in which the container is filled in a manner resulting in between 7 an 10 sources per container dominating the gamma radiation profile of the container.

Homogeneous Cases - Cases in which the container is filled in a manner resulting in between 11 and 35 sources per container dominating the gamma radiation profile of the container.

All Data Analysis - In this case, all available data for all cases from 1 source per container to 35 sources per container was analyzed for the contact measurement on a grouted container method (the current method) and the contact method on an ungrouted container. As will be discussed later, the data correlation and state of homogeneity for this case made the use of all data necessary to arrive at a reasonably accurate, useable method of estimation for this case.

Figures 1 through 26 of Attachment 5 show the results of the linear regression analysis for all cases analyzed. Summary regression statistics are available on request. These statistics are included on separate sheets of Attachment 5 but are not printed in this analysis.

6. Conduct modeling to arrive at a Microshield $囚$ model that is representative of the shielding effect of the equipment dunnage and sifted debris waste matrix being placed into $\mathrm{HN}-200$ grout containers. After lengthy discussions of this area with Fluor Daniel Hanford Project 


\section{REVISED METHODOLOGY FOR DETERMINING CESIUM-137 CONTENT OF HN-200 GROUT CONTAINERS.}

Direction and Dr. Joseph Alvarez, Auxier and Associates, a model consisting of 8 cylindrical tubes evenly spaced concentrically in the container from the center of the container outward with the wall thickness of the tubes varied to account for the varying net weight of the waste was considered the most representative model. The surfaces of the 8 cylinders are evenly coated with contamination totaling 1 curie of Cs-137. The modeling is conducted to determine conversion factors for these container models on contact with the surface of the container, at 5 feet from the surface of the container, and at 10 feet from the surface of the container. The modeling is conducted assuming $2500 \mathrm{lb}$., $2200 \mathrm{lb}$., and $1900 \mathrm{lb}$. net weight of the waste. Curves are then plotted for the variance of the conversion factors with net weight of the waste over the 1900 to $2500 \mathrm{lb}$. ranges for all three methods of measurement and for both the grouted and ungrouted cases. Attachment 6 presents the calculations and the curves derived from this analysis. Microshield (8) files used to calculate the conversion factors are available on request.

7. Analyze the results of the regression analyses shown in Attachment 5 and determine the most effective, useable ways to conduct the measurement of Cs-137 for $\mathrm{HN}-200$ grout containers in both the grouted and ungrouted condition. Attachment 7 provides the summary results of the correlation analysis performed for each homogeneity condition and each measurement mode. Attachment 7 also provides recommended data sheets for use in determining the curie content of Cs-137 in HN-200 grout containers in both the grouted and ungrouted condition and for all three measurement modes (contact, 5 feet from the surface, and 10 feet from the surface). The reasons for the choices of measurement methods and homogeneity selections are present in the analysis section below.

\section{ANALYSIS.}

Attachments 5 and 7 provide the summary of the results for all calculations and the data correlation analysis for the relationship between the calculated (estimated) result and the actual curie content of the container being measured.

Attachment 5 Tables 1 and 2 provide the summary of the estimated (calculated) results for each source combination in each grout condition and each measurement mode. Attachment 5 provides the linear regression analysis in Figures 1 through 26 . Figures 1 through 24 provide the linear regression analysis for each homogeneity condition while Figures 25 and 26 provide an analysis of the contact measurement mode for all source combinations regardless of homogeneity condition. These analyses provide best-fit lines describing the relationship between the estimated (calculated) curie content for each homogeneity condition and each measurement mode. The analyses also provide equations for the best-fit lines and the $\mathrm{R}^{2}$ value for the data described by the equation. The $R^{2}$ value is a statistical function that provides an indicator of the percentage of the data analyzed that can be explained by the best-fit line plotted and the equation presented on the graph. An $\mathbf{R}^{2}$ value of 1 is considered a perfect fit and the equation is expected to produce a reliable value. The data correlation coefficient is a second indicator of the 


\section{REVISED METHODOLOGY FOR DETERMINING CESIUM-137 CONTENT OF HN-200 GROUT CONTAINERS.}

reliability of the results attainable from the linear regression equations. By limiting the Cs-137 values used to those attained from a linear regression analysis with a data correlation coefficient of 0.9 or greater and by using the $95 \%$ assurance value from the linear regression, reliable values for the amount of Cs-137 can be attained.

\section{CONCLUSIONS.}

When combined with the correlation analysis conducted in Attachment 7 , the linear regression analyses conducted in Attachment 5 leads to the following conclusions:

\section{Contact Measurement of Grouted Containers.}

The method of estimating the Cs- 137 content of a grouted HN-200 grout container by taking contact radiation level measurements on the container results in answers that are subject to large errors, especially when the container does not exhibit a homogeneous character. In the worst case, the heterogeneous case when the container is primarily dominated by a single strong source, Figure 1 demonstrates there is no consistent relationship between the calculated curie content and the actual curie content of the container. The equation defining the best-fit line through the heterogeneous data describes only $0.06 \%$ of the data, the data correlation coefficient is only 0.024 indicating the estimated (calculated), and actual data do not correlate. Further, an examination of the estimated (calculated) curie content of heterogeneous containers compared to the actual content of the container, Attachments 2 and 4 , indicates that 33 of 35 individual source calculations $(94 \%)$ underestimated the curie content of the container by an average of $70 \%$ (i.e., the answer was only $30 \%$ of the actual answer), although 2 of the 35 sources did show as having been overestimated. Additionally, when the multiple source containers are examined, the results indicate that $100 \%$ ( 39 of 39 cases) are underestimated by an average of $73 \%$, a factor of 3.5 . When the data correlation is examined, it is clear that the best-fit line answer has a high probability of being unreliable and may not bound the content of the container. By using the statistical equation for the upper limit of the linear regression to produce a $95 \%$ probability that the bounding value is equal to or greater than the actual curie content of the container, a result of 10 times the original estimated (calculated) value is not uncommon in the lower curie content cases. This lack of data correlation remains a problem throughout all homogeneity conditions making the task of providing a reasonable estimate for the curie content of a grouted container extremely difficult. However, by taking all the data available from all multiple and single source analyses, a reasonable regression analysis with reasonable data correlation is possible. Attachment 5, Figure 25 displays this "all data" correlation and best-fit equation. By using this best-fit equation and the statistical calculation for a $95 \%$ assurance bounding value, a reasonably accurate estimate of the bounding value for the Cs-137 content of a grouted HN200 grout container is possible. Accordingly, the data sheets provided in Attachment 7 for calculating the content of HN-200 grout container using the contact method use the "All 


\section{REVISED METHODOLOGY FOR DETERMINING CESIUM-137 CONTENT OF HN-200 GROUT CONTAINERS.}

Data" analysis and are provided as Attachment 7 Tables 4 and 7. These data sheets correspond to the regression analyses conducted in Attachment 5 Figures 25 and 26.

\section{Remote Measurement of HN-200 Grout Containers.}

In general, the further away from the surface of the container the gamma radiation profile is measured, the more accurate the answer for curie content will be. However, the data correlation for the grouted container cases is always lower, generally by a substantial margin, that the data correlation for ungrouted containers at the same distance under the same homogeneity conditions. Balancing the accuracy attainable by moving away from the container with the other errors introduced by moving away from the container is the key to success in this operation. The errors present and the precepts of the procedures for performing this operation will be discussed in the next section. For the reasons discussed above, the following data sheets have been provided in Attachment 7 :

- Remote measurement at 10 feet of an ungrouted container using the heterogeneous case model, Attachment 5 Figure 6.

$\checkmark$ Remote measurement at 5 feet of an ungrouted container using the heterogeneous case model, Attachment 5 Figure 4.

c Contact measurement of an ungrouted container using the all data model, Attachment 5 Figure 26.

c Remote measurement at 10 feet of a grouted container using the homogeneous case model, Attachment 5 Figure 23.

c Remote measurement at 5 feet of a grouted container using the homogeneous case model, Attachment 5 Figure 21.

c Contact measurement of a grouted container using the all data model, Attachment 5 Figure 25.

\section{ERROR ANALYSIS.}

1. Geometric Errors. Geometric errors are errors caused by the assumption that the source is a homogeneous mixture of activity rather than the discrete sources of activity that exist within the container. These sources of error have been accounted for and compensated for by calculating a $95 \%$ assurance bounding value for the Cs- 137 content of the container. Since the analyses presented in Attachments 1 through 5 contained only two sources of error, geometric error and distributed shielding error, by using only linear regression equations with a data correlation coefficient of 0.9 or better and by using the $95 \%$ upper bound as the calculated Cs- 137 content, geometric errors are compensated for (bounded) by the calculated result and need not be further considered here. Geometric errors are also referred to as nonhomogeneity errors; accordingly, non-homogeneity errors are accounted for in the linear regression and data correlation analyses. 


\section{REVISED METHODOLOGY FOR DETERMINING CESIUM-137 CONTENT OF HN-200 GROUT CONTAINERS.}

2. Shielding Effects Errors. Shielding effects errors are errors caused by two sources. The shielding of the grout in the container which is compensated for by using linear regressions with a data correlation coefficient of 0.9 or better and using the $95 \%$ assurance bounding value calculated, as explained in item 1 above, and the shielding effect of the waste matrix (equipment dunnage and sifted debris). The effect of the equipment dunnage and sifted debris was accounted for in the construction of the model used to calculate the conversion factors in Attachment 6 . The 8 concentric cylinders evenly spaced through the container and evenly contaminated with Cs-137 is the model chosen to best represent this waste matrix. Accordingly, no shielding effect errors need be reconsidered in this section.

3. Instrument Error. Instrument error is the error introduced by the radiation level measurement instrument used to conduct the measurements. The RO-7 radiation survey instrument is the instrument that will be used for these measurements. The normal instrument error associated with the RO-7 radiation survey instrument is $\pm 20 \%$. By using a special source check procedure and calculating a correction factor and performing this check on the same day when the measurements are taken, the instrument error can be reduced to as little as $3 \%$ depending on the calibration accuracy of the source used for the special source check. For purposes of this analysis, a $4 \%$ error will be assumed.

4. Instrument Readability Error. Instrument readability error is introduced by the smallest graduation on the meter face that can be accurately discerned by the human eye. Since the RO-7 radiation survey instrument is a digital readout instrument with a 3-digit capability, a $1 \%$ instrument readability error is assigned for the $1 \%$ of the reading that cannot be discerned between the third digits of the readout.

5. Instrument Probe Placement Error. This error is introduced by uncertainties in the exact distance from the center of the active volume of the instrument probe to the surface of the container being measured. This error is introduced by two mechanisms.

The container is to be placed on a turntable and turned the prescribed number of degrees for each reading while the measuring probe is maintained in a fixed position. This technique may introduce error if the rotational center of the container is not aligned with the rotational center of the turntable. Techniques for reducing this error will be introduced in the procedure precept section of this paper.

The second error associated with probe placement is the actual position of the probe with respect to the desired position. Care must be taken to ensure the center of the active volume of the probe is the correct distance from the surface of the container.

For purposes of this analysis, these two errors will be assumed to combine to cause a 6-inch error in the distance to the surface of the container for remote measurements at 10 feet and remote measurements at 5 feet. For contact measurements, since these measuring operations 


\section{REVISED METHODOLOGY FOR DETERMINING CESIUM-137 CONTENT OF HN-200 GROUT CONTAINERS.}

will be conducted remotely using manipulators, a distance error of 2.5 " will be assumed for this analysis. This contact measurement error distance may be reduced to 1" if separate correction factors for high, medium, and low range R0-7 probes are developed for contact measurements. The following errors are therefore assigned for probe placement error:

- Measurements conducted at 10 feet from the surface of the container: $\mathbf{8 . 9 7 \%}$.

- Measurements conducted at 5 feet from the surface of the container: $15.82 \%$.

- Measurements conducted on contact with the surface of the container: $20.49 \%$.

6. Background Radiation Level Error. Errors introduced by conducting readings in high background radiation level areas are primarily statistical assurance errors in the reading caused by background fluctuation and by accepting readings too close to the background radiation level to be considered statistically accurate. The errors introduced by these issues will be eliminated by reducing the background in the area where the measurements will be taken by as much as possible and by accepting no raw data readings (reading before subtracting background) that are less than twice the value of the background measured prior to starting the measurement process. Accordingly, no error percentage is assigned to this error.

7. Gamma Scatter (Compton Scatter) during Measurements. Errors are introduced by Compton Scattering of the gamma energy off walls, floors, and ceilings during the measurement process. These errors will always result in the reading at the probe being higher than expected because Microshield® does not account for this scatter in the calculation. This scatter produces gammas of lower energy than the energy of the Cs-137 gamma which results in the R0-7 probe overresponding to this scatter radiation by as much as $60 \%$. Discussions with experts in this field indicate that the scatter gamma from this configuration would not be expected to exceed $7 \%$ of the total and would occur in the $125-250 \mathrm{Kev}$ range of energies. Accordingly, accounting for the overresponse of the RO-7 to these gamma energies, the total response to this scatter mechanism would be less than $10 \%$ of the total radiation level measured. Additionally, since this scatter will always result in the radiation level measurement being higher than expected, the effect of this scatter is to make the curie content calculation more conservative. Therefore, this scatter will not be accounted for in curie content calculations for $\mathrm{HN}-200$ grout containers and no error percentage will be assigned.

8. Waste Matrix Weight Error. Two steps are required to determine the weight of the waste matrix in a HN-200 grout container. The container is weighed while empty and then again after filling. The device used to obtain these weights is a dynamometer with a design (and calibrated) accuracy of $0.5 \%$ of full scale. Full scale on the dynamometer is $10,000 \mathrm{lbs}$. In the worst case, one dynamometer reading $50 \mathrm{lbs}$ low would be used for one step of the operation and a different dynamometer reading $50 \mathrm{lbs}$ high would be used for the second step. This situation would result in an error of $100 \mathrm{lbs}$ in the weight of the waste matrix. 


\section{REVISED METHODOLOGY FOR DETERMINING CESIUM-137 CONTENT OF HN-200 GROUT CONTAINERS.}

Using Attachment 6 to establish the conversion factors for the different weights shows that for grouted containers this $100 \mathrm{lb}$ error results in a $1 \%$ error in the Cs- 137 calculation while for a ungrouted container the $100 \mathrm{lb}$ error results in a $2 \%$ error in the Cs- 137 calculation. Accordingly, $2 \%$ is assigned as the correction factor necessary for weight uncertainty.

By employing the square root of the sum of the squares, the total error for each type of measurement technique can be assigned. The following total errors are assigned for this process:

Measurements taken at 10 feet from the surface of the container: $10.07 \%$.

Measurements taken at 5 feet from the surface of the container: $16.47 \%$.

Measurements taken on contact with the surface of the container: $21.00 \%$.

\section{PROCEDURE PRECEPTS.}

The purpose of this section of this technical paper is to provide the concepts and recommendations that should be incorporated into operating procedures for performing these operations. This section of this technical paper does not provide a checklist or a step by step operating procedure for performing these measurements and calculations. Other methods of meeting these principles may be used when adequate technical bases for those methods have been established.

1. The REC airlock should be free of grout containers and other sources of high radiation prior to the start of this procedure.

2. The turntable should be marked on the base with an index mark to allow aligning the turntable at specific points. The turntable should be marked with markers at the $0,22.5,45$, $67.5,90,112.5,135,157.5,180,202.5,225,247.5,270,292.5,315$, and 337.5 degree points on the turntable in a manner to allow these markers to be aligned to the base index marker to determine the position of the turntable. The turntable should also be marked in a manner than will align a grout container placed on the turntable as close to the center of rotation of the turntable as possible.

3. Set up the REC airlock with the turntable in a predetermined location designated by the BCell Project Manager.

4. Conduct the special source check of the RO-7 and log the calculated correction factor on a datasheet. This special source check should be conducted on a new probe each day measurements are taken and the probe located in the airlock should be replaced by the new probe after the source check is completed. Additionally, the zero adjust of the RO-7 and probe should be conducted in a low background area outside the airlock before the probe is transferred into the airlock. 


\section{REVISED METHODOLOGY FOR DETERMINING CESIUM-137 CONTENT OF HN-200 GROUT CONTAINERS.}

5. Position an RO-7 probe at a location determined by the B-Cell Project Manager that will place the probe 10 feet from the surface of a grout container when the container is placed on the center of the turntable. The probe should also be positioned such that a horizontal line drawn from the probe to a grout container placed on the center of the turntable will intersect the $y$ axis of the container at the vertical centerline of the container (i.e., the container is 54 inches tall, the probe should be positioned so that a horizontal line from the probe to the container intersects the container at the 27 inch point along the vertical axis).

6. Measure the radiation background at the $\mathrm{RO}-7$ probe and log the background on a datasheet. This background should be less than $0.2 \mathrm{R} / \mathrm{hr}$ for conducting these measurements. If the background is greater than $0.2 \mathrm{R} / \mathrm{hr}$, take action to reduce the background to less than or equal to $0.2 \mathrm{R} / \mathrm{hr}$.

7. Repeat steps 5 and 6 with the RO-7 probe positioned such that the distance from the probe to the surface of a container placed on the turntable would be 5 feet and for taking contact readings on a container. Then return the R0-7 probe to the original 10-foot position.

8. Select a grout container to be measured.

9. Determine the gross and net weights of the waste matrix in the grout container and log the gross and net weights in pounds on a datasheet.

10. Move the grout container to be measured to the turntable and place the container on the center of rotation of the turntable.

11. Using the RO-7 probe placement jig, measure the distance from the RO-7 probe to the surface of the container. If the distance is not 10 feet, check the container placement to ensure it is in the center of the turntable by rotating the container in 45 degree increments and measuring the distance from the RO-7 probe to the surface of the container at each $\mathbf{4 5}$ degree increment. If the distances at each 45-degree increment are not equal, the container is not on the center of rotation of the turntable. Adjust the container and repeat this step until the container is on the center of rotation of the turntable within $a \pm 1$ inch tolerance (e.g., the difference between the smallest reading and the largest reading of the 8 reading set is less than or equal to $1 \mathrm{inch}$ ). After assuring the container is on the center of rotation of the turntable \pm 1 inch, measure the distance from the RO-7 probe to the surface of the container and adjust the position of the RO-7 probe as necessary to obtain a distance of 10 feet from the surface of the container.

12. Position the turntable so that the 0 -degree marker for the turntable is aligned with the index marker on the turntable base. Record the RO-7 reading for the 0-degree position on a datasheet in the first reading column. If the reading obtained is less than twice the background recorded for 10 feet, move the RO-7 probe to a distance of 5 feet from the 


\section{REVISED METHODOLOGY FOR DETERMINING CESIUM-137 CONTENT OF HN-200 GROUT CONTAINERS.}

container and repeat step 12 with the probe at 5 feet, then continue the procedure with the RO-7 probe at 5 feet from the container. If readings obtained at 5 feet are less than twice background recorded for the probe at 5 feet, report the condition to management for evaluation. It may be necessary to take actions to reduce the background in the area or necessary to measure the container with the probe on contact with the surface of the container. In either case, special attention to the measurement of this container is warranted.

13. Position the turntable so that the 22.5-degree marker for the turntable is aligned with the index marker on the base. Record the R0-7 reading for the 22.5-degree position on a datasheet in the first reading column.

14. Repeat step 13 for the $45,67.5,90,112.5,135,157.5,180,202.5,225,247.5,270,292.5$, $315,337.5$-degree markers on the turntable until all 16 readings are obtained. If any reading obtained during this process is less than twice background, consult management for evaluation. It may be necessary to adjust the probe to 5 feet from the surface of the container, repeat step 12 , and restart the procedure from step 12 with the probe at the 5-foot position.

15. Repeat steps 12 through 14 for the second reading and third reading columns of a datasheet. If any reading obtained during this process is less than twice background, consult management for evaluation. It may be necessary to adjust the probe to 5 feet from the surface of the container, repeat step 12, and restart the procedure from step 12 with the probe at the 5-foot position.

16. Average the 16 first, second, and third reading columns and log the averages of the first 16 , the second 16 , and the third 16 readings in the space provided under the reading columns on a datasheet.

17. Multiply each of the three readings by the correction factor determined in step 4 and log the corrected value in the corrected average reading block of the datasheet.

18. Subtract the background determined in step six from each of the three averages determined in step 17 and $\log$ the result in the above background block on the datasheet.

19. Determine the correction factor to be used for the measurement method used and multiply the corrected "above background" reading determined in step 18 by this correction factor.

20. Determine the conversion factor to be used for the net weight and type of container measured and divide the result of step 19 by this number. This is the measured Cs-137 result.

21. Determine the linear regression equation for the type of container and measurement method used and calculate the predicted value of Cs-137 from the result in step 20 . 


\section{REVISED METHODOLOGY FOR DETERMINING CESIUM-137 CONTENT OF HN-200 GROUT CONTAINERS.}

22. Determine the equation for the $95 \%$ bounding value for the type of container and measurement method used and multiply the result of step 21 by the result of this equation. This produces a $95 \%$ Assurance Bounding Value for the Cs- 137 which states with $95 \%$ assurance that the Cs-137 is equal to or less than this value.

The above calculations (steps 16 through 22) can be done automatically by using the spreadsheets provided in Attachment 7 Tables 2 through 7. The only data necessary from the operators for these spreadsheets to be used is:

Type of container and measurement method used.

r. RO-7 Special Source Check Correction Factor.

C Net weight of the waste in lbs.

B Background reading in the position the container readings were taken.

r. The RO-7 digital readout (uncorrected) in each measurement position.

After the above data is provided, all the calculations for the container are performed automatically. 


\section{REVISED METHODOLOGY FOR DETERMINING CESIUM-137 CONTENT OF HN-200 GROUT CONTAINERS.}

\section{TABLE 1 \\ DATA FOR HN-200 GROUT CONTAINER LINER \\ DRAWING NUMBER H-3-49415}

$\begin{array}{rcccc}\text { Outer Height } & 54.0000 & \text { in } & 137.1600 & \mathrm{~cm} \\ \text { Inner Height (note 1) } & 53.5000 & \text { in } & 135.8900 & \mathrm{~cm} \\ \text { Outer Radius } & 22.9375 & \text { in } & 58.2613 & \mathrm{~cm} \\ \text { Inner Radius } & 22.7993 & \text { in } & 57.9102 & \mathrm{~cm} \\ \text { Wall Thickness } & 0.1382 & \text { in } & 0.3510 & \mathrm{~cm} \\ & & & & \\ \text { Volume (inner) } & 87366.8507 & \text { in }^{3} & 1431686.17 & \mathrm{~cm}^{3} \\ & 50.5595201 & \mathrm{ft}^{3} & 1.43168617 & \mathrm{~m}^{3}\end{array}$

Note 1 - The top and bottom closure plates for the container are constructed of $0.125^{\prime \prime}$ steel. A bottom stiffening plate, constructed of $0.25^{\prime \prime}$ steel, is inserted into the container. This yields an internal height of $53.5^{\prime \prime}$ for the container.

Note 2 - For purposes of the coordinates and calculations presented in this attachment, the cylinder of interest is placed in an $\mathrm{X}, \mathrm{Z}, \mathrm{Y}$ coordinate frame to match the coordinate frame use in Microshield version 5.03. The circular portion of the cylinder is centered at $X=0, Z=0$. The bottom of the inner height of the cylinder is placed at $Y=0$. This placement matches the orientation used in Microshield for a cylindrical source. 


\section{REVISED METHODOLOGY FOR DETERMINING CESIUM-137 CONTENT OF HN-200 GROUT CONTAINERS.}

TABLE 2

Coordinates of current contact measuring points.

$\begin{array}{lccc}\text { Point } & \mathbf{X} & \mathbf{Z} & \mathbf{Y} \\ \text { C01 } & 23.9375 & 0.0000 & 49.5000 \\ \text { C02 } & 23.9375 & 0.0000 & 40.5000 \\ \text { C03 } & 23.9375 & 0.0000 & 31.5000 \\ \text { C04 } & 23.9375 & 0.0000 & 22.5000 \\ \text { C05 } & 23.9375 & 0.0000 & 13.5000 \\ \text { C06 } & 23.9375 & 0.0000 & 4.5000 \\ \text { C07 } & 0.0000 & 23.9375 & 49.5000 \\ \text { C08 } & 0.0000 & 23.9375 & 40.5000 \\ \text { C09 } & 0.0000 & 23.9375 & 31.5000 \\ \text { C10 } & 0.0000 & 23.9375 & 22.5000 \\ \text { C11 } & 0.0000 & 23.9375 & 13.5000 \\ \text { C12 } & 0.0000 & 23.9375 & 4.5000 \\ \text { C13 } & -23.9375 & 0.0000 & 49.5000 \\ \text { C14 } & -23.9375 & 0.0000 & 40.5000 \\ \text { C15 } & -23.9375 & 0.0000 & 31.5000 \\ \text { C16 } & -23.9375 & 0.0000 & 22.5000 \\ \text { C17 } & -23.9375 & 0.0000 & 13.5000 \\ \text { C18 } & -23.9375 & 0.0000 & 4.5000 \\ \text { C19 } & 0.0000 & -23.9375 & 49.5000 \\ \text { C20 } & 0.0000 & -23.9375 & 40.5000 \\ \text { C21 } & 0.0000 & -23.9375 & 31.5000 \\ \text { C22 } & 0.0000 & -23.9375 & 22.5000 \\ \text { C23 } & 0.0000 & -23.9375 & 13.5000 \\ \text { C24 } & 0.0000 & -23.9375 & 4.5000\end{array}$

Note 1 - The radiation readings taken for this container are normally taken with an RO-7 radiation survey instrument. The coordinates for the contact readings are set at the center of the active portion of the RO-7 probe when the probe face is on contact with the surface of the container.

Note 2 - The center of the active portion of the RO-7 radiation survey instrument probe is one inch from the face of the probe. 
TABLE 3

Coordinates of Remote Measuring Points.

$\begin{array}{cccc}\text { Point } & \mathbf{X} & \mathbf{Z} & \mathbf{Y} \\ \text { R01 } & 142.9375 & 0.0000 & 26.7500 \\ \text { R02 } & 132.0570 & 54.6998 & 26.7500 \\ \text { R03 } & 101.0721 & 101.0721 & 26.7500 \\ \text { R04 } & 54.6998 & 132.0570 & 26.7500 \\ \text { R05 } & 0.0000 & 142.9375 & 26.7500 \\ \text { R06 } & -54.6998 & 132.0570 & 26.7500 \\ \text { R07 } & -101.0721 & 101.0721 & 26.7500 \\ \text { R08 } & -132.0570 & .54 .6998 & 26.7500 \\ \text { R09 } & -142.9375 & 0.0000 & 26.7500 \\ \text { R10 } & -132.0570 & -54.6998 & 26.7500 \\ \text { R11 } & -101.0721 & -101.0721 & 26.7500 \\ \text { R12 } & -54.6998 & -132.0570 & 26.7500 \\ \text { R13 } & 0.0000 & -142.9375 & 26.7500 \\ \text { R14 } & 54.6998 & -132.0570 & 26.7500 \\ \text { R15 } & 101.0721 & -101.0721 & 26.7500 \\ \text { R16 } & 132.0570 & -54.6998 & 26.7500\end{array}$

Note 1 - Remote Measuring Points $(\mathrm{R})$ are 120 inches (10 feet) from the surface of the container. 
REVISED METHODOLOGY FOR DETERMINING CESIUM-137 CONTENT OF HN-200 GROUT CONTAINERS.

TABLE 4

Coordinates and source strengths for random sources.

$\begin{array}{lccclc}\text { Source } & \mathbf{X} & \mathbf{Z} & \mathbf{Y} & \text { Validity } & \text { Strength } \\ \text { S01 } & 19.3677 & 0.5818 & 25.9034 & \text { Valid } & 29.4975 \\ \text { S02 } & -10.6176 & -19.5689 & 18.8660 & \text { Valid } & 13.8502 \\ \text { S03 } & 1.7704 & -7.2590 & 41.8874 & \text { Valid } & 23.5916 \\ \text { S04 } & 5.5262 & -19.2179 & 28.6756 & \text { Valid } & 20.6982 \\ \text { S05 } & -12.5171 & 2.4304 & 36.3581 & \text { Valid } & 10.9510 \\ \text { S06 } & 10.1481 & -6.8169 & 29.6428 & \text { Valid } & 16.3182 \\ \text { S07 } & -3.0040 & -18.4824 & 1.5181 & \text { Valid } & 25.9358 \\ \text { S08 } & -1.4468 & -9.3997 & 20.8621 & \text { Valid } & 25.1678 \\ \text { S09 } & -11.3240 & -1.2877 & 45.4810 & \text { Valid } & 24.9347 \\ \text { S10 } & -8.1594 & 10.5475 & 46.4380 & \text { Valid } & 26.8139 \\ \text { S11 } & 13.0540 & 6.4320 & 29.1917 & \text { Valid } & 12.5933 \\ \text { S12 } & 17.0889 & 8.7507 & 21.2880 & \text { Valid } & 17.5307 \\ \text { S13 } & 3.5123 & 8.3040 & 41.4473 & \text { Valid } & 17.9331 \\ \text { S14 } & -18.2417 & 6.1323 & 39.9213 & \text { Valid } & 26.2138 \\ \text { S15 } & 21.0026 & 5.4076 & 10.7056 & \text { Valid } & 22.7469 \\ \text { S16 } & 12.7799 & 10.3319 & 39.3081 & \text { Valid } & 27.6556 \\ \text { S17 } & -14.4903 & 3.0983 & 8.6941 & \text { Valid } & 28.5227 \\ \text { S18 } & 18.7646 & -12.0033 & 10.5209 & \text { Valid } & 23.2528 \\ \text { S19 } & -18.2584 & 0.2785 & 21.1001 & \text { Valid } & 15.3416 \\ \text { S20 } & 2.8644 & 9.2261 & 44.4497 & \text { Valid } & 16.4988 \\ \text { S21 } & 3.0621 & -7.9137 & 19.8848 & \text { Valid } & 28.6466 \\ \text { S22 } & -17.9782 & 12.8570 & 50.0431 & \text { Valid } & 20.3724 \\ \text { S23 } & 5.0992 & 14.6169 & 52.0038 & \text { Valid } & 13.0146 \\ \text { S24 } & 7.9617 & 19.2122 & 33.0332 & \text { Valid } & 12.5419 \\ \text { S25 } & 8.3975 & 9.5559 & 39.8544 & \text { Valid } & 19.7625 \\ \text { S26 } & 4.1090 & 16.2164 & 34.1935 & \text { Valid } & 24.8512 \\ \text { S27 } & 2.2698 & -21.1016 & 13.9713 & \text { Valid } & 10.2138 \\ \text { S28 } & 18.4153 & -5.6775 & 6.4850 & \text { Valid } & 15.5133 \\ \text { S29 } & -0.0904 & 3.6427 & 38.3454 & \text { Valid } & 18.0653 \\ \text { S30 } & 7.3731 & -19.7854 & 12.4003 & \text { Valid } & 15.0401 \\ \text { S31 } & 5.5574 & -17.7939 & 21.2754 & \text { Valid } & 22.3550 \\ \text { S32 } & -0.9171 & 17.9652 & 26.2433 & \text { Valid } & 21.6866 \\ \text { S33 } & -2.7939 & -22.1819 & 29.8247 & \text { Valid } & 17.8281 \\ \text { S34 } & -14.1407 & 16.9694 & 5.4489 & \text { Valid } & 14.6776 \\ \text { S35 } & 17.6331 & 12.8346 & 30.1155 & \text { Valid } & 28.0146 \\ & & & & & \\ & & & & \end{array}$

TABLE 5 


\section{REVISED METHODOLOGY FOR DETERMINING CESIUM-137 CONTENT OF HN-200 GROUT CONTAINERS.}

Write the equation for the line projection in the $X Z$ plane for the line from each source to each measuring point in the format $\mathrm{Z}=\mathrm{ZO}+\mathrm{SX}$.

\section{SOURCE S01}

$\begin{array}{lcc}\text { Line } & \text { Z0 } & \text { Slope } \\ \text { S01C01 } & 3.04767698 & -0.1273181 \\ \text { S01C02 } & 3.04767698 & -0.1273181 \\ \text { S01C03 } & 3.04767698 & -0.1273181 \\ \text { S01C04 } & 3.04767698 & -0.1273181 \\ \text { S01C05 } & 3.04767698 & -0.1273181 \\ \text { S01C06 } & 3.04767698 & -0.1273181 \\ \text { S01C07 } & 23.9375 & -1.2059087 \\ \text { S01C08 } & 23.9375 & -1.2059087 \\ \text { S01C09 } & 23.9375 & -1.2059087 \\ \text { S01C10 } & 23.9375 & -1.2059087 \\ \text { S01C11 } & 23.9375 & -1.2059087 \\ \text { S01C12 } & 23.9375 & -1.2059087 \\ \text { S01C13 } & 0.32160713 & 0.01343528 \\ \text { S01C14 } & 0.32160713 & 0.01343528 \\ \text { S01C15 } & 0.32160713 & 0.01343528 \\ \text { S01C16 } & 0.32160713 & 0.01343528 \\ \text { S01C17 } & 0.32160713 & 0.01343528 \\ \text { S01C18 } & 0.32160713 & 0.01343528 \\ \text { S01C19 } & -23.9375 & 1.26598991 \\ \text { S01C20 } & -23.9375 & 1.26598991 \\ \text { S01C21 } & -23.9375 & 1.26598991 \\ \text { S01C22 } & -23.9375 & 1.26598991 \\ \text { S01C23 } & -23.9375 & 1.26598991 \\ \text { S01C24 } & -23.9375 & 1.26598991 \\ \text { S01R01 } & 0.67300891 & -0.0047084 \\ \text { S01R02 } & -8.7193412 & 0.48024066 \\ \text { S01R03 } & -23.239006 & 1.22992504 \\ \text { S01R04 } & -71.487849 & 3.72112603 \\ \text { S01R05 } & 142.9375 & -7.3501579 \\ \text { S01R06 } & 34.9608945 & -1.7750724 \\ \text { S01R07 } & 16.7414757 & -0.8343611 \\ \text { S01R08 } & 7.50368076 & -0.357392 \\ \text { S01R09 } & 0.51239007 & 0.00358471 \\ \text { S01R10 } & -6.488878 & 0.36507661 \\ \text { S01R11 } & -15.764962 & 0.84402261 \\ \text { S01R12 } & -34.101535 & 1.79078288 \\ \text { S01R13 } & -142.9375 & 7.41023914 \\ \text { S01R14 } & 73.2893454 & -3.7540603 \\ \text { S01R15 } & 24.6784769 & -1.2441671 \\ \text { S01R16 } & 10.0829686 & -0.4905667\end{array}$




\section{REVISED METHODOLOGY FOR DETERMINING CESIUM-137 CONTENT} OF HN-200 GROUT CONTAINERS.

\begin{tabular}{ccc} 
& \multicolumn{2}{c}{ SOURCE S02 } \\
Line & Z0 & Slope \\
S02C01 & -13.556063 & 0.56631072 \\
S02C02 & -13.556063 & 0.56631072 \\
S02C03 & -13.556063 & 0.56631072 \\
S02C04 & -13.556063 & 0.56631072 \\
S02C05 & -13.556063 & 0.56631072 \\
S02C06 & -13.556063 & 0.56631072 \\
S02C07 & 23.9375 & 4.0975702 \\
S02C08 & 23.9375 & 4.0975702 \\
S02C09 & 23.9375 & 4.0975702 \\
S02C10 & 23.9375 & 4.0975702 \\
S02C11 & 23.9375 & 4.0975702 \\
S02C12 & 23.9375 & 4.0975702 \\
SO2C13 & -35.167829 & -1.4691521 \\
S02C14 & -35.167829 & -1.4691521 \\
S02C15 & -35.167829 & -1.4691521 \\
S02C16 & -35.167829 & -1.4691521 \\
S02C17 & -35.167829 & -1.4691521 \\
S02C18 & -35.167829 & -1.4691521 \\
S02C19 & -23.9375 & -0.4114451 \\
S02C20 & -23.9375 & -0.4114451 \\
S02C21 & -23.9375 & -0.4114451 \\
S02C22 & -23.9375 & -0.4114451 \\
S02C23 & -23.9375 & -0.4114451 \\
S02C24 & -23.9375 & -0.4114451 \\
S02R01 & -18.215833 & 0.12743915 \\
S02R02 & -14.041973 & 0.52054622 \\
S02R03 & -8.1003723 & 1.08014449 \\
S02R04 & 5.07849196 & 2.32137061 \\
S02R05 & 142.9375 & 15.3053575 \\
S02R06 & -56.089498 & -3.4396195 \\
S02R07 & -33.729872 & -1.3337209 \\
S02R08 & -26.062354 & -0.6115704 \\
S02R09 & -21.139185 & -0.1478911 \\
S02R10 & -16.497392 & 0.28928726 \\
S02R11 & -10.002028 & 0.90104066 \\
S02R12 & 7.52489923 & 2.5517808 \\
S02R13 & -142.9375 & -11.619232 \\
S02R14 & -37.854336 & -1.7221757 \\
SO2R15 & -27.316911 & -0.7297285 \\
S02R16 & -22.183317 & -0.2462307 \\
& & \\
\hline
\end{tabular}


REVISED METHODOLOGY FOR DETERMINING CESIUM-137 CONTENT OF HN-200 GROUT CONTAINERS.

\begin{tabular}{ccc} 
& \multicolumn{2}{c}{ SOURCE S03 } \\
Line & Z0 & Slope \\
S03C01 & -7.8387813 & 0.32746867 \\
S03C02 & -7.8387813 & 0.32746867 \\
S03C03 & -7.8387813 & 0.32746867 \\
S03C04 & -7.8387813 & 0.32746867 \\
S03C05 & -7.8387813 & 0.32746867 \\
S03C06 & -7.8387813 & 0.32746867 \\
S03C07 & 23.9375 & -17.620682 \\
S03C08 & 23.9375 & -17.620682 \\
S03C09 & 23.9375 & -17.620682 \\
S03C10 & 23.9375 & -17.620682 \\
S03C11 & 23.9375 & -17.620682 \\
S03C12 & 23.9375 & -17.620682 \\
S03C13 & -6.7591027 & -0.2823646 \\
S03C14 & -6.7591027 & -0.2823646 \\
S03C15 & -6.7591027 & -0.2823646 \\
S03C16 & -6.7591027 & -0.2823646 \\
S03C17 & -6.7591027 & -0.2823646 \\
S03C18 & -6.7591027 & -0.2823646 \\
S03C19 & -23.9375 & 9.42048451 \\
S03C20 & -23.9375 & 9.42048451 \\
S03C21 & -23.9375 & 9.42048451 \\
S03C22 & -23.9375 & 9.42048451 \\
S03C23 & -23.9375 & 9.42048451 \\
S03C24 & -23.9375 & 9.42048451 \\
S03R01 & -7.3500538 & 0.05142145 \\
S03R02 & -8.1009659 & 0.47555802 \\
S03R03 & -9.1904498 & 1.09092964 \\
S03R04 & -11.919035 & 2.63211265 \\
S03R05 & 142.9375 & -84.835279 \\
S03R06 & -2.891195 & -2.4670693 \\
S03R07 & -5.3940796 & -1.0533686 \\
S03R08 & -6.4393406 & -0.4629754 \\
S03R09 & -7.1702034 & -0.0501632 \\
S03R10 & -7.886625 & 0.35449219 \\
S03R11 & -8.8740201 & 0.91220109 \\
S03R12 & -11.171667 & 2.20997761 \\
S03R13 & -142.9375 & 76.6350811 \\
S03R14 & -3.0846118 & -2.3578219 \\
S03R15 & -5.5864216 & -0.9447284 \\
S03R16 & -6.6143475 & -0.3641265 \\
& & \\
\hline
\end{tabular}

SOURCE S04 
REVISED METHODOLOGY FOR DETERMINING CESIUM-137 CONTENT OF HN-200 GROUT CONTAINERS.

$\begin{array}{lcc}\text { Line } & \text { Z0 } & \text { Slope } \\ \text { S04C01 } & -24.986185 & 1.04380929 \\ \text { SO4C02 } & -24.986185 & 1.04380929 \\ \text { S04C03 } & -24.986185 & 1.04380929 \\ \text { S04C04 } & -24.986185 & 1.04380929 \\ \text { S04C05 } & -24.986185 & 1.04380929 \\ \text { S04C06 } & -24.986185 & 1.04380929 \\ \text { S04C07 } & 23.9375 & -7.8092893 \\ \text { S04C08 } & 23.9375 & -7.8092893 \\ \text { S04C09 } & 23.9375 & -7.8092893 \\ \text { S04C10 } & 23.9375 & -7.8092893 \\ \text { S04C11 } & 23.9375 & -7.8092893 \\ \text { S04C12 } & 23.9375 & -7.8092893 \\ \text { S04C13 } & -15.613435 & -0.6522584 \\ \text { S04C14 } & -15.613435 & -0.6522584 \\ \text { S04C15 } & -15.613435 & -0.6522584 \\ \text { S04C16 } & -15.613435 & -0.6522584 \\ \text { S04C17 } & -15.613435 & -0.6522584 \\ \text { S04C18 } & -15.613435 & -0.6522584 \\ \text { S04C19 } & -23.9375 & 0.85404208 \\ \text { S04C20 } & -23.9375 & 0.85404208 \\ \text { S04C21 } & -23.9375 & 0.85404208 \\ \text { S04C22 } & -23.9375 & 0.85404208 \\ \text { S04C23 } & -23.9375 & 0.85404208 \\ \text { S04C24 } & -23.9375 & 0.85404208 \\ \text { S04R01 } & -19.990795 & 0.13985689 \\ \text { S04R02 } & -22.446239 & 0.58418742 \\ \text { S04R03 } & -26.175231 & 1.25897583 \\ \text { S04R04 } & -36.218297 & 3.07634209 \\ \text { S04R05 } & 142.9375 & -29.34321 \\ \text { S04R06 } & -5.3373611 & -2.5117891 \\ \text { S04R07 } & -12.981962 & -1.1284426 \\ \text { S04R08 } & -16.248943 & -0.5372585 \\ \text { S04R09 } & -18.502586 & -0.1294453 \\ \text { S04R10 } & -20.643087 & 0.25789403 \\ \text { S04R11 } & -23.461328 & 0.76787533 \\ \text { S04R12 } & -29.571719 & 1.87359517 \\ \text { S04R13 } & -142.9375 & 22.3879624 \\ \text { S04R14 } & -6.5369933 & -2.2947069 \\ \text { S04R15 } & -14.483658 & -0.85669997 \\ \text { S04R16 } & -17.668271 & -0.2804208 \\ & & \end{array}$

SOURCE S05

Line $\quad$ ZO Slope 
REVISED METHODOLOGY FOR DETERMINING CESIUM-137 CONTENT OF HN-200 GROUT CONTAINERS.

\begin{tabular}{lcl} 
S05C01 & 1.59588055 & -0.0666686 \\
S05C02 & 1.59588055 & -0.0666686 \\
S05C03 & 1.59588055 & -0.0666686 \\
S05C04 & 1.59588055 & -0.0666686 \\
S05C05 & 1.59588055 & -0.0666686 \\
S05C06 & 1.59588055 & -0.0666686 \\
S05C07 & 23.9375 & 1.71822452 \\
S05C08 & 23.9375 & 1.71822452 \\
S05C09 & 23.9375 & 1.71822452 \\
S05C10 & 23.9375 & 1.71822452 \\
S05C11 & 23.9375 & 1.71822452 \\
S05C12 & 23.9375 & 1.71822452 \\
S05C13 & 5.09412429 & 0.21280937 \\
S05C14 & 5.09412429 & 0.21280937 \\
S05C15 & 5.09412429 & 0.21280937 \\
S05C16 & 5.09412429 & 0.21280937 \\
S05C17 & 5.09412429 & 0.21280937 \\
S05C18 & 5.09412429 & 0.21280937 \\
S05C19 & -23.9375 & -2.1065546 \\
S05C20 & -23.9375 & -2.1065546 \\
S05C21 & -23.9375 & -2.1065546 \\
S05C22 & -23.9375 & -2.1065546 \\
S05C23 & -23.9375 & -2.1065546 \\
S05C24 & -23.9375 & -2.1065546 \\
S05R01 & 2.23468437 & -0.015634 \\
S05R02 & 6.95580506 & 0.36154081 \\
S05R03 & 13.3002919 & 0.86840788 \\
S05R04 & 26.5693273 & 1.9284837 \\
S05R05 & 142.9375 & 11.2252473 \\
S05R06 & -36.034282 & -3.072978 \\
S05R07 & -11.512418 & -1.113903 \\
S05R08 & -3.0427709 & -0.4372549 \\
S05R09 & 2.6636307 & 0.01863493 \\
S05R10 & 8.41249392 & 0.47791707 \\
S05R11 & 17.0602268 & 1.16879264 \\
S05R12 & 42.3373858 & 3.18820884 \\
S05R13 & -142.9375 & -11.613577 \\
S05R14 & -22.61374 & -2.0007982 \\
S05R15 & -8.9751748 & -0.9112003 \\
S05R16 & -2.5158915 & -0.395162 \\
& & \\
\hline
\end{tabular}

SOURCE S06

Line $\quad$ Z0 Slope

S06C01 $\quad-11.833666 \quad 0.4943568$ 
REVISED METHODOLOGY FOR DETERMINING CESIUM-137 CONTENT OF HN-200 GROUT CONTAINERS.

\begin{tabular}{|c|c|c|}
\hline & -11.833666 & \\
\hline $06 \mathrm{C} 03$ & -11.833666 & \\
\hline $06 \mathrm{C} 04$ & -11.833666 & \\
\hline $06 \mathrm{CO5}$ & 666 & 0.494356 \\
\hline $6 \mathrm{C} 06$ & & 56 \\
\hline $6 \mathrm{C} 07$ & & 5554 \\
\hline $506 \mathrm{C} 08$ & & \\
\hline $806 C 09$ & 23.9375 & -3.030554 \\
\hline $06 \mathrm{C} 10$ & & -3.030554 \\
\hline $06 \mathrm{C} 11$ & 75 & 0554 \\
\hline $06 \mathrm{C} 12$ & & \\
\hline $06 \mathrm{C} 13$ & & 993 \\
\hline $06 \mathrm{C} 14$ & -4.7 & \\
\hline $\mathrm{S} 06 \mathrm{C} 15$ & -4.7 & \\
\hline SO6C16 & -4.7 & \\
\hline $06 \mathrm{C} 17$ & -4.7 & \\
\hline $06 \mathrm{C} 18$ & -4 & 993 \\
\hline $6 C 19$ & & 1.6 \\
\hline 20 & & 7754 \\
\hline 21 & & 7754 \\
\hline 22 & & 754 \\
\hline & & 0754 \\
\hline 66 & & 0754 \\
\hline $66 R$ & -7.3 & 0.0 \\
\hline $6 \mathrm{R} 02$ & -11. & 0.5 \\
\hline $6 \mathrm{R} 03$ & -18 & 1.18 \\
\hline $6 R 04$ & -38 & 4026 \\
\hline $6 R 05$ & & 6881 \\
\hline & & 15323 \\
\hline 06R07 & & -0.9 \\
\hline 6R08 & -2.42 & -0.4 \\
\hline 66R09 & -6.36 & 45299 \\
\hline $6 \mathrm{R} 10$ & -10.2 & 57173 \\
\hline 6R 11 & -15 & 0.84 \\
\hline & & 1.93129012 \\
\hline & & 13.4134022 \\
\hline 6R 14 & 21.7106516 & -2.8111191 \\
\hline 06R15 & 3.70302511 & -1.0366375 \\
\hline 06R 16 & -2.8309462 & -0.3927763 \\
\hline
\end{tabular}

\begin{tabular}{ccc} 
& \multicolumn{2}{c}{ SOURCE S07 } \\
Line & Z0 & Slope \\
S07C01 & -16.421572 & 0.68601869 \\
S07C02 & -16.421572 & 0.68601869
\end{tabular}

10 of 210 
HNF-4799, Rev 0

August 25, 1999

REVISED METHODOLOGY FOR DETERMINING CESIUM-137 CONTENT OF HN-200 GROUT CONTAINERS.

\begin{tabular}{lcc} 
S07C03 & -16.421572 & 0.68601869 \\
S07C04 & -16.421572 & 0.68601869 \\
S07C05 & -16.421572 & 0.68601869 \\
S07C06 & -16.421572 & 0.68601869 \\
S07C07 & 23.9375 & 14.1211651 \\
S07C08 & 23.9375 & 14.1211651 \\
S07C09 & 23.9375 & 14.1211651 \\
S07C10 & 23.9375 & 14.1211651 \\
S07C11 & 23.9375 & 14.1211651 \\
S07C12 & 23.9375 & 14.1211651 \\
S07C13 & -21.134616 & -0.8829083 \\
S07C14 & -21.134616 & -0.8829083 \\
S07C15 & -21.134616 & -0.8829083 \\
S07C16 & -21.134616 & -0.8829083 \\
S07C17 & -21.134616 & -0.8829083 \\
S07C18 & -21.134616 & -0.8829083 \\
S07C19 & -23.9375 & -1.8159612 \\
S07C20 & -23.9375 & -1.8159612 \\
S07C21 & -23.9375 & -1.8159612 \\
S07C22 & -23.9375 & -1.8159612 \\
S07C23 & -23.9375 & -1.8159612 \\
S07C24 & -23.9375 & -1.8159612 \\
S07R01 & -18.101935 & 0.12664231 \\
S07R02 & -16.854668 & 0.54184532 \\
S07R03 & -15.031616 & 1.14872172 \\
S07R04 & -10.645463 & 2.60882971 \\
S07R05 & 142.9375 & 53.7351188 \\
S07R06 & -27.23006 & -2.9120227 \\
S07R07 & -22.144523 & -1.2190963 \\
S07R08 & -20.185843 & -0.5670706 \\
S07R09 & -18.879133 & -0.1320796 \\
S07R10 & -17.639327 & 0.28063998 \\
S07R11 & -15.952504 & 0.84216709 \\
S07R12 & -11.882658 & 2.19697955 \\
S07R13 & -142.9375 & -41.429915 \\
S07R14 & -24.39493 & -1.9682352 \\
S07R15 & -20.866189 & -0.7935514 \\
S07R16 & -19.287906 & -0.2681561 \\
& & \\
\hline
\end{tabular}

\begin{tabular}{ccc} 
& \multicolumn{2}{c}{ SOURCE S08 } \\
Line & Z0 & Slope \\
S08C01 & -8.8639963 & 0.3702975 \\
S08C02 & -8.8639963 & 0.3702975 \\
S08C03 & -8.8639963 & 0.3702975
\end{tabular}


HNF-4799, Rev 0 August 25, 1999

\section{REVISED METHODOLOGY FOR DETERMINING CESIUM-137 CONTENT} OF HN-200 GROUT CONTAINERS.

$\begin{array}{lll}\text { S08C04 } & -8.8639963 & 0.3702975 \\ \text { S08C05 } & -8.8639963 & 0.3702975 \\ \text { S08C06 } & -8.8639963 & 0.3702975 \\ \text { S08C07 } & 23.9375 & 23.0427577 \\ \text { S08C08 } & 23.9375 & 23.0427577 \\ \text { S08C09 } & 23.9375 & 23.0427577 \\ \text { S08C10 } & 23.9375 & 23.0427577 \\ \text { S08C11 } & 23.9375 & 23.0427577 \\ \text { S08C12 } & 23.9375 & 23.0427577 \\ \text { S08C13 } & -10.004379 & -0.4179375 \\ \text { S08C14 } & -10.004379 & -0.4179375 \\ \text { S08C15 } & -10.004379 & -0.4179375 \\ \text { S08C16 } & -10.004379 & -0.4179375 \\ \text { S08C17 } & -10.004379 & -0.4179375 \\ \text { S08C18 } & -10.004379 & -0.4179375 \\ \text { S08C19 } & -23.9375 & -10.048539 \\ \text { S08C20 } & -23.9375 & -10.048539 \\ \text { S08C21 } & -23.9375 & -10.048539 \\ \text { S08C22 } & -23.9375 & -10.048539 \\ \text { S08C23 } & -23.9375 & -10.048539 \\ \text { S08C24 } & -23.9375 & -10.048539 \\ \text { S08R01 } & -9.3055392 & 0.06510215 \\ \text { S08R02 } & -8.7050916 & 0.48013276 \\ \text { S08R03 } & -7.840738 & 1.07757569 \\ \text { S08R04 } & -5.7547432 & 2.51941951 \\ \text { S08R05 } & 142.9375 & 105.295797 \\ \text { S08R06 } & -13.24276 & -2.6563124 \\ \text { S08R07 } & -11.003993 & -1.1088727 \\ \text { S08R08 } & -10.109749 & -0.4907695 \\ \text { S08R09 } & -9.4958391 & -0.0664335 \\ \text { S08R10 } & -8.8979422 & 0.346834 \\ \text { S08R11 } & -8.0684637 & 0.92017121 \\ \text { S08R12 } & -6.0674278 & 2.30329128 \\ \text { S08R13 } & -142.9375 & -92.301578 \\ \text { S08R14 } & -12.560295 & -2.1845913 \\ \text { S08R15 } & -10.693415 & -0.8942001 \\ \text { S08R16 } & -9.8906344 & -0.3393169\end{array}$

\begin{tabular}{ccc} 
& \multicolumn{2}{c}{ SOURCE S09 } \\
Line & Z0 & Slope \\
S09C01 & -0.8741865 & 0.03651954 \\
S09C02 & -0.8741865 & 0.03651954 \\
S09C03 & -0.8741865 & 0.03651954 \\
S09C04 & -0.8741865 & 0.03651954
\end{tabular}


REVISED METHODOLOGY FOR DETERMINING CESIUM-137 CONTENT OF HN-200 GROUT CONTAINERS.

$\begin{array}{lcc}\text { SO9C05 } & -0.8741865 & 0.03651954 \\ \text { S09C06 } & -0.8741865 & 0.03651954 \\ \text { S09C07 } & 23.9375 & 2.22758955 \\ \text { S09C08 } & 23.9375 & 2.22758955 \\ \text { S09C09 } & 23.9375 & 2.22758955 \\ \text { S09C10 } & 23.9375 & 2.22758955 \\ \text { S09C11 } & 23.9375 & 2.22758955 \\ \text { S09C12 } & 23.9375 & 2.22758955 \\ \text { S09C13 } & -2.4438214 & -0.1020918 \\ \text { S09C14 } & -2.4438214 & -0.1020918 \\ \text { S09C15 } & -2.4438214 & -0.1020918 \\ \text { S09C16 } & -2.4438214 & -0.1020918 \\ \text { S09C17 } & -2.4438214 & -0.1020918 \\ \text { S09C18 } & -2.4438214 & -0.1020918 \\ \text { S09C19 } & -23.9375 & -2.0001552 \\ \text { S09C20 } & -23.9375 & -2.0001552 \\ \text { S09C21 } & -23.9375 & -2.0001552 \\ \text { S09C22 } & -23.9375 & -2.0001552 \\ \text { S09C23 } & -23.9375 & -2.0001552 \\ \text { S09C24 } & -23.9375 & -2.0001552 \\ \text { S09R01 } & -1.1932041 & 0.00834773 \\ \text { S09R02 } & 3.13407278 & 0.39048083 \\ \text { S09R03 } & 9.02510763 & 0.91070624 \\ \text { S09R04 } & 21.58275 & 2.01964632 \\ \text { S09R05 } & 142.9375 & 12.7362396 \\ \text { S09R06 } & -36.099692 & -3.0741738 \\ \text { S09R07 } & -14.20303 & -1.1405237 \\ \text { S09R08 } & -6.5390165 & -0.4637302 \\ \text { S09R09 } & -1.3985303 & -0.0097842 \\ \text { S09R10 } & 3.72198586 & 0.44239825 \\ \text { S09R11 } & 11.3026013 & 1.11182711 \\ \text { S09R12 } & 32.8518532 & 3.0147981 \\ \text { S09R13 } & -142.9375 & -12.508805 \\ \text { S09R14 } & -23.716489 & -1.9806382 \\ \text { S09R15 } & -11.341095 & -0.887792 \\ \text { S09R16 } & -5.5061342 & -0.3725184\end{array}$

\begin{tabular}{ccc} 
& \multicolumn{2}{c}{ SOURCE S10 } \\
Line & Z0 & Slope \\
S10C01 & 7.86617835 & -0.3286132 \\
S10C02 & 7.86617835 & -0.3286132 \\
S10C03 & 7.86617835 & -0.3286132 \\
S10C04 & 7.86617835 & -0.3286132 \\
S10C05 & 7.86617835 & -0.3286132
\end{tabular}


REVISED METHODOLOGY FOR DETERMINING CESIUM-137 CONTENT OF HN-200 GROUT CONTAINERS.

\begin{tabular}{lcc} 
S10C06 & 7.86617835 & -0.3286132 \\
S10C07 & 23.9375 & 1.64104785 \\
S10C08 & 23.9375 & 1.64104785 \\
S10C09 & 23.9375 & 1.64104785 \\
S10C10 & 23.9375 & 1.64104785 \\
S10C11 & 23.9375 & 1.64104785 \\
S10C12 & 23.9375 & 1.64104785 \\
S10C13 & 16.0019762 & 0.66848987 \\
S10C14 & 16.0019762 & 0.66848987 \\
S10C15 & 16.0019762 & 0.66848987 \\
S10C16 & 16.0019762 & 0.66848987 \\
S10C17 & 16.0019762 & 0.66848987 \\
S10C18 & 16.0019762 & 0.66848987 \\
S10C19 & -23.9375 & -4.2263926 \\
S10C20 & -23.9375 & -4.2263926 \\
S10C21 & -23.9375 & -4.2263926 \\
S10C22 & -23.9375 & -4.2263926 \\
S10C23 & -23.9375 & -4.2263926 \\
S10C24 & -23.9375 & -4.2263926 \\
S10R01 & 9.97789869 & -0.069806 \\
S10R02 & 13.116776 & 0.31488694 \\
S10R03 & 17.3095328 & 0.82874074 \\
S10R04 & 26.320005 & 1.93304171 \\
S10R05 & 142.9375 & 16.2253906 \\
S10R06 & -10.755517 & -2.6108417 \\
S10R07 & 2.59775502 & -0.974298 \\
S10R08 & 7.63976775 & -0.3563615 \\
S10R09 & 11.1860184 & 0.07825811 \\
S10R10 & 14.8444206 & 0.52662275 \\
S10R11 & 20.3497214 & 1.20133866 \\
S10R12 & 35.5488272 & 3.06410311 \\
S10R13 & -142.9375 & -18.810735 \\
S10R14 & -7.9632799 & -2.2686321 \\
S10R15 & 2.20965805 & -1.0218622 \\
S10R16 & 6.75062534 & -0.4653326 \\
& & \\
\hline
\end{tabular}

\begin{tabular}{ccc} 
& \multicolumn{2}{c}{ SOURCE S11 } \\
Line & Z0 & Slope \\
S11C01 & 14.1466954 & -0.5909847 \\
S11C02 & 14.1466954 & -0.5909847 \\
S11C03 & 14.1466954 & -0.5909847 \\
S11C04 & 14.1466954 & -0.5909847 \\
S11C05 & 14.1466954 & -0.5909847 \\
S11C06 & 14.1466954 & -0.5909847
\end{tabular}


REVISED METHODOLOGY FOR DETERMINING CESIUM-137 CONTENT OF HN-200 GROUT CONTAINERS.

\begin{tabular}{lcc} 
S11C07 & 23.9375 & -1.3410067 \\
S11C08 & 23.9375 & -1.3410067 \\
S11C09 & 23.9375 & -1.3410067 \\
S11C10 & 23.9375 & -1.3410067 \\
S11C11 & 23.9375 & -1.3410067 \\
S11C12 & 23.9375 & -1.3410067 \\
S11C13 & 4.16217637 & 0.17387682 \\
S11C14 & 4.16217637 & 0.17387682 \\
S11C15 & 4.16217637 & 0.17387682 \\
S11C16 & 4.16217637 & 0.17387682 \\
S11C17 & 4.16217637 & 0.17387682 \\
S11C18 & 4.16217637 & 0.17387682 \\
S11C19 & -23.9375 & 2.32644522 \\
S11C20 & -23.9375 & 2.32644522 \\
S11C21 & -23.9375 & 2.32644522 \\
S11C22 & -23.9375 & 2.32644522 \\
S11C23 & -23.9375 & 2.32644522 \\
S11C24 & -23.9375 & 2.32644522 \\
S11R01 & 7.0784174 & -0.0495211 \\
S11R02 & 1.13723219 & 0.40560188 \\
S11R03 & -7.6041757 & 1.07523516 \\
S11R04 & -32.945661 & 3.01651306 \\
S11R05 & 142.9375 & -10.456971 \\
S11R06 & 30.6359457 & -1.8541394 \\
S11R07 & 17.2571359 & -0.8292592 \\
S11R08 & 10.7740875 & -0.3326269 \\
S11R09 & 5.8937143 & 0.04123281 \\
S11R10 & 0.93262396 & 0.42127584 \\
S11R11 & -5.8646076 & 0.941976 \\
S11R12 & -20.250484 & 2.04400229 \\
S11R13 & -142.9375 & 11.4424093 \\
S11R14 & 49.841846 & -3.3254024 \\
S11R15 & 22.3759717 & -1.2213862 \\
S11R16 & 13.1378129 & -0.5136995 \\
& & \\
\hline
\end{tabular}

\begin{tabular}{ccc} 
& \multicolumn{2}{c}{ SOURCE S12 } \\
Line & $\mathbf{Z 0}$ & Slope \\
$\mathrm{S} 12 \mathrm{C} 01$ & 30.5856043 & -1.2777276 \\
$\mathrm{~S} 12 \mathrm{C} 02$ & 30.5856043 & -1.2777276 \\
$\mathrm{~S} 12 \mathrm{C} 03$ & 30.5856043 & -1.2777276 \\
$\mathrm{~S} 12 \mathrm{C} 04$ & 30.5856043 & -1.2777276 \\
$\mathrm{~S} 12 \mathrm{C} 05$ & 30.5856043 & -1.2777276 \\
$\mathrm{~S} 12 \mathrm{C} 06$ & 30.5856043 & -1.2777276 \\
$\mathrm{~S} 12 \mathrm{C} 07$ & 23.9375 & -0.8886963
\end{tabular}


REVISED METHODOLOGY FOR DETERMINING CESIUM-137 CONTENT

OF HN-200 GROUT CONTAINERS.

$\begin{array}{lcc}\text { S12C08 } & 23.9375 & -0.8886963 \\ \text { S12C09 } & 23.9375 & -0.8886963 \\ \text { S12C10 } & 23.9375 & -0.8886963 \\ \text { S12C11 } & 23.9375 & -0.8886963 \\ \text { S12C12 } & 23.9375 & -0.8886963 \\ \text { S12C13 } & 5.10573009 & 0.21329421 \\ \text { S12C14 } & 5.10573009 & 0.21329421 \\ \text { S12C15 } & 5.10573009 & 0.21329421 \\ \text { S12C16 } & 5.10573009 & 0.21329421 \\ \text { S12C17 } & 5.10573009 & 0.21329421 \\ \text { S12C18 } & 5.10573009 & 0.21329421 \\ \text { S12C19 } & -23.9375 & 1.91283514 \\ \text { S12C20 } & -23.9375 & 1.91283514 \\ \text { S12C21 } & -23.9375 & 1.91283514 \\ \text { S12C22 } & -23.9375 & 1.91283514 \\ \text { S12C23 } & -23.9375 & 1.91283514 \\ \text { S12C24 } & -23.9375 & 1.91283514 \\ \text { S12R01 } & 9.93893408 & -0.0695334 \\ \text { S12R02 } & 1.92080858 & 0.39966826 \\ \text { S12R03 } & -10.03483 & 1.09928388 \\ \text { S12R04 } & -47.274653 & 3.27847 \\ \text { S12R05 } & 142.9375 & -7.852294 \\ \text { S12R06 } & 38.103025 & -1.7176292 \\ \text { S12R07 } & 22.1025442 & -0.781319 \\ \text { S12R08 } & 14.0154541 & -0.3080817 \\ \text { S12R09 } & 7.81621979 & 0.05468278 \\ \text { S12R10 } & 1.48064293 & 0.42542571 \\ \text { S12R11 } & -7.1322831 & 0.92943371 \\ \text { S12R12 } & -24.76775 & 1.96141942 \\ \text { S12R13 } & -142.9375 & 8.87643289 \\ \text { S12R14 } & 72.7279337 & -3.7437968 \\ \text { S12R15 } & 31.0973742 & -1.3076752 \\ \text { S12R16 } & 18.1819693 & -0.5518963 \\ & & \end{array}$

SOURCE S13

\begin{tabular}{|c|c|c|}
\hline Line & $\mathbf{Z O}$ & Slope \\
\hline $\mathrm{S} 13 \mathrm{C} 01$ & 9.73196761 & -0.4065574 \\
\hline $\mathrm{S} 13 \mathrm{CO} 2$ & 9.73196761 & -0.4065574 \\
\hline $\mathrm{S} 13 \mathrm{CO}$ & 9.73196761 & -0.4065574 \\
\hline S13C04 & 9.73196761 & -0.4065574 \\
\hline $\mathrm{S} 13 \mathrm{C} 05$ & 9.73196761 & -0.4065574 \\
\hline S13C06 & 9.73196761 & -0.4065574 \\
\hline $\mathrm{S} 13 \mathrm{C} 07$ & 23.9375 & -4.4511199 \\
\hline$\$ 13 C 08$ & 23.9375 & -4.4511199 \\
\hline
\end{tabular}




\section{REVISED METHODOLOGY FOR DETERMINING CESIUM-137 CONTENT} OF HN-200 GROUT CONTAINERS.

$\begin{array}{lcc}\text { S13C09 } & 23.9375 & -4.4511199 \\ \text { S13C10 } & 23.9375 & -4.4511199 \\ \text { S13C11 } & 23.9375 & -4.4511199 \\ \text { S13C12 } & 23.9375 & -4.4511199 \\ \text { S13C13 } & 7.24151573 & 0.30251763 \\ \text { S13C14 } & 7.24151573 & 0.30251763 \\ \text { S13C15 } & 7.24151573 & 0.30251763 \\ \text { S13C16 } & 7.24151573 & 0.30251763 \\ \text { S13C17 } & 7.24151573 & 0.30251763 \\ \text { S13C18 } & 7.24151573 & 0.30251763 \\ \text { S13C19 } & -23.9375 & 9.17972639 \\ \text { S13C20 } & -23.9375 & 9.17972639 \\ \text { S13C21 } & -23.9375 & 9.17972639 \\ \text { S13C22 } & -23.9375 & 9.17972639 \\ \text { S13C23 } & -23.9375 & 9.17972639 \\ \text { S13C24 } & -23.9375 & 9.17972639 \\ \text { S13R01 } & 8.51322113 & -0.059559 \\ \text { S13R02 } & 7.0363536 & 0.36093086 \\ \text { S13R03 } & 4.96428918 & 0.95088368 \\ \text { S13R04 } & -0.1873258 & 2.4176382 \\ \text { S13R05 } & 142.9375 & -38.332492 \\ \text { S13R06 } & 15.7707338 & -2.1258993 \\ \text { S13R07 } & 11.4194628 & -0.8870167 \\ \text { S13R08 } & 9.50603084 & -0.3422293 \\ \text { S13R09 } & 8.10488178 & 0.05670228 \\ \text { S13R10 } & 6.67176557 & 0.46473542 \\ \text { S13R11 } & 4.63085792 & 1.04581737 \\ \text { S13R12 } & -0.164721 & 2.41120222 \\ \text { S13R13 } & -142.9375 & 43.0610988 \\ \text { S13R14 } & 17.9349645 & -2.7420935 \\ \text { S13R15 } & 12.2416875 & -1.1211184 \\ \text { S13R16 } & 10.0255013 & -0.4901315 \\ & & \end{array}$

\begin{tabular}{ccc} 
& \multicolumn{2}{c}{ SOURCE S14 } \\
Line & $Z \mathbf{Z 0}$ & Slope \\
S14C01 & 3.48019744 & -0.1453868 \\
S14C02 & 3.48019744 & -0.1453868 \\
S14C03 & 3.48019744 & -0.1453868 \\
S14C04 & 3.48019744 & -0.1453868 \\
S14C05 & 3.48019744 & -0.1453868 \\
S14C06 & 3.48019744 & -0.1453868 \\
$S 14 C 07$ & 23.9375 & 0.97606906 \\
$S 14 C 08$ & 23.9375 & 0.97606906 \\
$S 14 C 09$ & 23.9375 & 0.97606906
\end{tabular}


REVISED METHODOLOGY FOR DETERMINING CESIUM-137 CONTENT OF HN-200 GROUT CONTAINERS.

$\begin{array}{lcl}\text { S14C10 } & 23.9375 & 0.97606906 \\ \text { S14C11 } & 23.9375 & 0.97606906 \\ \text { S14C12 } & 23.9375 & 0.97606906 \\ \text { S14C13 } & 25.7721479 & 1.07664326 \\ \text { S14C14 } & 25.7721479 & 1.07664326 \\ \text { S14C15 } & 25.7721479 & 1.07664326 \\ \text { S14C16 } & 25.7721479 & 1.07664326 \\ \text { S14C17 } & 25.7721479 & 1.07664326 \\ \text { S14C18 } & 25.7721479 & 1.07664326 \\ \text { S14C19 } & -23.9375 & -1.648407 \\ \text { S14C20 } & -23.9375 & -1.648407 \\ \text { S14C21 } & -23.9375 & -1.648407 \\ \text { S14C22 } & -23.9375 & -1.648407 \\ \text { S14C23 } & -23.9375 & -1.648407 \\ \text { S14C24 } & -23.9375 & -1.648407 \\ \text { S14R01 } & 5.43827156 & -0.0380465 \\ \text { S14R02 } & 12.0269356 & 0.32313974 \\ \text { S14R03 } & 20.6475265 & 0.79571488 \\ \text { S14R04 } & 37.6244454 & 1.72637842 \\ \text { S14R05 } & 142.9375 & 7.49957095 \\ \text { S14R06 } & -56.873921 & -3.45396 \\ \text { S14R07 } & -14.776291 & -1.1461955 \\ \text { S14R08 } & -1.6518471 & -0.4267222 \\ \text { S14R09 } & 7.02940043 & 0.04917814 \\ \text { S14R10 } & 15.8821687 & 0.53448109 \\ \text { S14R11 } & 29.7419386 & 1.29426458 \\ \text { S14R12 } & 75.2751121 & 3.79036326 \\ \text { S14R13 } & -142.9375 & -8.1719089 \\ \text { S14R14 } & -28.427055 & -1.8945215 \\ \text { S14R15 } & -10.258035 & -0.8985077 \\ \text { S14R16 } & -1.2508782 & -0.4047413 \\ & & \end{array}$

\begin{tabular}{ccc} 
& \multicolumn{2}{c}{ SOURCE S15 } \\
Line & Z0 & Slope \\
S15C01 & 44.1049227 & -1.8425033 \\
S15C02 & 44.1049227 & -1.8425033 \\
S15C03 & 44.1049227 & -1.8425033 \\
S15C04 & 44.1049227 & -1.8425033 \\
S15C05 & 44.1049227 & -1.8425033 \\
S15C06 & 44.1049227 & -1.8425033 \\
S15C07 & 23.9375 & -0.882267 \\
S15C08 & 23.9375 & -0.882267 \\
S15C09 & 23.9375 & -0.882267 \\
S15C10 & 23.9375 & -0.882267
\end{tabular}


REVISED METHODOLOGY FOR DETERMINING CESIUM-137 CONTENT OF HN-200 GROUT CONTAINERS.

$\begin{array}{lcl}\text { S15C11 } & 23.9375 & -0.882267 \\ \text { S15C12 } & 23.9375 & -0.882267 \\ \text { S15C13 } & 2.88039592 & 0.12032986 \\ \text { S15C14 } & 2.88039592 & 0.12032986 \\ \text { S15C15 } & 2.88039592 & 0.12032986 \\ \text { S15C16 } & 2.88039592 & 0.12032986 \\ \text { S15C17 } & 2.88039592 & 0.12032986 \\ \text { S15C18 } & 2.88039592 & 0.12032986 \\ \text { S15C19 } & -23.9375 & 1.39721668 \\ \text { S15C20 } & -23.9375 & 1.39721668 \\ \text { S15C21 } & -23.9375 & 1.39721668 \\ \text { S15C22 } & -23.9375 & 1.39721668 \\ \text { S15C23 } & -23.9375 & 1.39721668 \\ \text { S15C24 } & -23.9375 & 1.39721668 \\ \text { S15R01 } & 6.33906339 & -0.0443485 \\ \text { S15R02 } & -3.9144805 & 0.44385591 \\ \text { S15R03 } & -19.685545 & 1.19476735 \\ \text { S15R04 } & -73.529444 & 3.75844964 \\ \text { S15R05 } & 142.9375 & -6.5482422 \\ \text { S15R06 } & 40.5447291 & -1.672991 \\ \text { S15R07 } & 21.8664031 & -0.7836554 \\ \text { S15R08 } & 12.1714156 & -0.3220457 \\ \text { S15R09 } & 4.71485299 & 0.03298542 \\ \text { S15R10 } & -2.8402043 & 0.39270615 \\ \text { S15R11 } & -12.911873 & 0.87225087 \\ \text { S15R12 } & -32.73001 & 1.81585655 \\ \text { S15R13 } & -142.9375 & 7.06319186 \\ \text { S15R14 } & 91.0855626 & -4.0794036 \\ \text { S15R15 } & 33.3376948 & -1.3298407 \\ \text { S15R16 } & 16.7751204 & -0.541243\end{array}$

\begin{tabular}{ccc} 
& \multicolumn{2}{c}{ SOURCE S16 } \\
Line & Z0 & Slope \\
S16C01 & 22.1660637 & -0.9259974 \\
S16C02 & 22.1660637 & -0.9259974 \\
S16C03 & 22.1660637 & -0.9259974 \\
S16C04 & 22.1660637 & -0.9259974 \\
S16C05 & 22.1660637 & -0.9259974 \\
S16C06 & 22.1660637 & -0.9259974 \\
S16C07 & 23.9375 & -1.0646083 \\
S16C08 & 23.9375 & -1.0646083 \\
S16C09 & 23.9375 & -1.0646083 \\
S16C10 & 23.9375 & -1.0646083 \\
S16C11 & 23.9375 & -1.0646083
\end{tabular}


REVISED METHODOLOGY FOR DETERMINING CESIUM-137 CONTENT OF HN-200 GROUT CONTAINERS.

$\begin{array}{lcc}\text { S16C12 } & 23.9375 & -1.0646083 \\ \text { S16C13 } & 6.735754 & 0.2813892 \\ \text { S16C14 } & 6.735754 & 0.2813892 \\ \text { S16C15 } & 6.735754 & 0.2813892 \\ \text { S16C16 } & 6.735754 & 0.2813892 \\ \text { S16C17 } & 6.735754 & 0.2813892 \\ \text { S16C18 } & 6.735754 & 0.2813892 \\ \text { S16C19 } & -23.9375 & 2.68150156 \\ \text { S16C20 } & -23.9375 & 2.68150156 \\ \text { S16C21 } & -23.9375 & 2.68150156 \\ \text { S16C22 } & -23.9375 & 2.68150156 \\ \text { S16C23 } & -23.9375 & 2.68150156 \\ \text { S16C24 } & -23.9375 & 2.68150156 \\ \text { S16R01 } & 11.3463549 & -0.0793798 \\ \text { S16R02 } & 5.57809331 & 0.37197352 \\ \text { S16R03 } & -2.8023808 & 1.02772655 \\ \text { S16R04 } & -26.777902 & 2.90375653 \\ \text { S16R05 } & 142.9375 & -10.376088 \\ \text { S16R06 } & 33.3852962 & -1.8038769 \\ \text { S16R07 } & 20.5175031 & -0.7970013 \\ \text { S16R08 } & 14.2467624 & -0.3063301 \\ \text { S16R09 } & 9.48393576 & 0.06635023 \\ \text { S16R10 } & 4.59370884 & 0.44899936 \\ \text { S16R11 } & -2.1732446 & 0.97849808 \\ \text { S16R12 } & -16.635016 & 2.11009882 \\ \text { S16R13 } & -142.9375 & 11.9929809 \\ \text { S16R14 } & 53.7413467 & -3.3966915 \\ \text { S16R15 } & 26.457149 & -1.2617651 \\ \text { S16R16 } & 17.2996968 & -0.5452153\end{array}$

\begin{tabular}{|c|c|c|}
\hline \multirow[b]{2}{*}{ Line } & \multicolumn{2}{|c|}{ SOURCE S17 } \\
\hline & $\mathbf{Z} 0$ & Slope \\
\hline $\mathbf{S} 17 \mathrm{C} 01$ & 1.92999286 & -0.0806263 \\
\hline $\mathrm{S} 17 \mathrm{C} 02$ & 1.92999286 & -0.0806263 \\
\hline $\mathrm{S} 17 \mathrm{C} 03$ & 1.92999286 & -0.0806263 \\
\hline S17C04 & 1.92999286 & -0.0806263 \\
\hline S17C05 & 1.92999286 & -0.0806263 \\
\hline S17C06 & 1.92999286 & -0.0806263 \\
\hline $\mathrm{S} 17 \mathrm{C} 07$ & 23.9375 & 1.43815174 \\
\hline $\mathrm{S} 17 \mathrm{C} 08$ & 23.9375 & 1.43815174 \\
\hline S17C09 & 23.9375 & 1.43815174 \\
\hline $\mathbf{S 1 7 C 1 0}$ & 23.9375 & 1.43815174 \\
\hline S17C11 & 23.9375 & 1.43815174 \\
\hline $\mathrm{S} 17 \mathrm{C} 12$ & 23.9375 & 1.43815174 \\
\hline
\end{tabular}


REVISED METHODOLOGY FOR DETERMINING CESIUM-137 CONTENT

OF HN-200 GROUT CONTAINERS.

$\begin{array}{lcl}\text { S17C13 } & 7.85048526 & 0.32795761 \\ \text { S17C14 } & 7.85048526 & 0.32795761 \\ \text { S17C15 } & 7.85048526 & 0.32795761 \\ \text { S17C16 } & 7.85048526 & 0.32795761 \\ \text { S17C17 } & 7.85048526 & 0.32795761 \\ \text { S17C18 } & 7.85048526 & 0.32795761 \\ \text { S17C19 } & -23.9375 & -1.865789 \\ \text { S17C20 } & -23.9375 & -1.865789 \\ \text { S17C21 } & -23.9375 & -1.865789 \\ \text { S17C22 } & -23.9375 & -1.865789 \\ \text { S17C23 } & -23.9375 & -1.865789 \\ \text { S17C24 } & -23.9375 & -1.865789 \\ \text { S17R01 } & 2.81311152 & -0.0196807 \\ \text { S17R02 } & 8.20053411 & 0.35211512 \\ \text { S17R03 } & 15.3831467 & 0.84780027 \\ \text { S17R04 } & 30.105731 & 1.86383257 \\ \text { S17R05 } & 142.9375 & 9.65055805 \\ \text { S17R06 } & -43.374445 & -3.2071679 \\ \text { S17R07 } & -13.298535 & -1.1315747 \\ \text { S17R08 } & -3.2616715 & -0.4389125 \\ \text { S17R09 } & 3.44781187 & 0.02412111 \\ \text { S17R10 } & 10.2219898 & 0.49161945 \\ \text { S17R11 } & 20.5321712 & 1.20314381 \\ \text { S17R12 } & 51.804082 & 3.36127521 \\ \text { S17R13 } & -142.9375 & -10.078195 \\ \text { S17R14 } & -25.206882 & -1.9533914 \\ \text { S17R15 } & -9.9635502 & -0.9014214 \\ \text { S17R16 } & -2.6166577 & -0.394399\end{array}$

SOURCE S18

$\begin{array}{lcc}\text { Line } & \mathbf{Z 0} & \text { Slope } \\ \text { S18C01 } & -55.544832 & 2.32041076 \\ \text { S18C02 } & -55.544832 & 2.32041076 \\ \text { S18C03 } & -\mathbf{5 5 . 5 4 4 8 3 2} & 2.32041076 \\ \text { S18C04 } & -55.544832 & 2.32041076 \\ \text { S18C05 } & -55.544832 & 2.32041076 \\ \text { S18C06 } & -55.544832 & 2.32041076 \\ \text { S18C07 } & 23.9375 & -1.9153509 \\ \text { S18C08 } & 23.9375 & -1.9153509 \\ \text { S18C09 } & 23.9375 & -1.9153509 \\ \text { S18C10 } & 23.9375 & -1.9153509 \\ \text { S18C11 } & 23.9375 & -1.9153509 \\ \text { S18C12 } & 23.9375 & -1.9153509 \\ \text { S18C13 } & -6.7286728 & -0.2810934 \\ & & \end{array}$


REVISED METHODOLOGY FOR DETERMINING CESIUM-137 CONTENT OF HN-200 GROUT CONTAINERS.

$\begin{array}{lcc}\text { S18C14 } & -6.7286728 & -0.2810934 \\ \text { S18C15 } & -6.7286728 & -0.2810934 \\ \text { S18C16 } & -6.7286728 & -0.2810934 \\ \text { S18C17 } & -6.7286728 & -0.2810934 \\ \text { S18C18 } & -6.7286728 & -0.2810934 \\ \text { S18C19 } & -23.9375 & 0.63599709 \\ \text { S18C20 } & -23.9375 & 0.63599709 \\ \text { S18C21 } & -23.9375 & 0.63599709 \\ \text { S18C22 } & -23.9375 & 0.63599709 \\ \text { S18C23 } & -23.9375 & 0.63599709 \\ \text { S18C24 } & -23.9375 & 0.63599709 \\ \text { S18R01 } & -13.817169 & 0.09666581 \\ \text { S18R02 } & -23.051287 & 0.58876914 \\ \text { S18R03 } & -37.78237 & 1.37381602 \\ \text { S18R04 } & -87.22844 & 4.00888924 \\ \text { S18R05 } & 142.9375 & -8.2570827 \\ \text { S18R06 } & 24.7932197 & -1.9609538 \\ \text { S18R07 } & 5.70259696 & -0.9435789 \\ \text { S18R08 } & -3.7043579 & -0.4422648 \\ \text { S18R09 } & -10.610365 & -0.0742308 \\ \text { S18R10 } & -17.315398 & 0.28309292 \\ \text { S18R11 } & -25.950089 & 0.74325171 \\ \text { S18R12 } & -42.667914 & 1.63417575 \\ \text { S18R13 } & -142.9375 & 6.9777289 \\ \text { S18R14 } & 50.6861876 & -3.3408383 \\ \text { S18R15 } & 8.3027703 & -1.082147 \\ \text { S18R16 } & -4.9314614 & -0.3768701\end{array}$

\begin{tabular}{ccc} 
& \multicolumn{2}{c}{ SOURCE S19 } \\
Line & Z0 & Slope \\
S19C01 & 0.15798385 & -0.0065998 \\
S19C02 & 0.15798385 & -0.0065998 \\
S19C03 & 0.15798385 & -0.0065998 \\
S19C04 & 0.15798385 & -0.0065998 \\
S19C05 & 0.15798385 & -0.0065998 \\
S19C06 & 0.15798385 & -0.0065998 \\
S19C07 & 23.9375 & 1.29578803 \\
S19C08 & 23.9375 & 1.29578803 \\
S19C09 & 23.9375 & 1.29578803 \\
S19C10 & 23.9375 & 1.29578803 \\
S19C11 & 23.9375 & 1.29578803 \\
S19C12 & 23.9375 & 1.29578803 \\
S19C13 & 1.17382469 & 0.04903706 \\
S19C14 & 1.17382469 & 0.04903706
\end{tabular}


REVISED METHODOLOGY FOR DETERMINING CESIUM-137 CONTENT

OF HN-200 GROUT CONTAINERS.

$\begin{array}{lcc}\text { S19C15 } & 1.17382469 & 0.04903706 \\ \text { S19C16 } & 1.17382469 & 0.04903706 \\ \text { S19C17 } & 1.17382469 & 0.04903706 \\ \text { S19C18 } & 1.17382469 & 0.04903706 \\ \text { S19C19 } & -23.9375 & -1.3262931 \\ \text { S19C20 } & -23.9375 & -1.3262931 \\ \text { S19C21 } & -23.9375 & -1.3262931 \\ \text { S19C22 } & -23.9375 & -1.3262931 \\ \text { S19C23 } & -23.9375 & -1.3262931 \\ \text { S19C24 } & -23.9375 & -1.3262931 \\ \text { S19R01 } & 0.24694277 & -0.0017276 \\ \text { S19R02 } & 6.88889375 & 0.3620475 \\ \text { S19R03 } & 15.7006119 & 0.84465929 \\ \text { S19R04 } & 33.2571593 & 1.80621941 \\ \text { S19R05 } & 142.9375 & 7.81333685 \\ \text { S19R06 } & -65.747089 & -3.6161757 \\ \text { S19R07 } & -21.944043 & -1.2171128 \\ \text { S19R08 } & -8.4531322 & -0.4782248 \\ \text { S19R09 } & 0.31926892 & 0.00223363 \\ \text { S19R10 } & 9.0994686 & 0.48311917 \\ \text { S19R11 } & 22.6238145 & 1.22383837 \\ \text { S19R12 } & 66.5831246 & 3.6314598 \\ \text { S19R13 } & -142.9375 & -7.8438419 \\ \text { S19R14 } & -32.839573 & -1.8138536 \\ \text { S19R15 } & -15.22886 & -0.8493268 \\ \text { S19R16 } & -6.3995748 & -0.3657529\end{array}$

SOURCE S20

$\begin{array}{ccc}\text { Line } & \mathbf{Z 0} & \text { Slope } \\ \mathrm{S} 20 \mathrm{C} 01 & 10.4801379 & -0.4378125 \\ \mathrm{~S} 20 \mathrm{C} 02 & 10.4801379 & -0.4378125 \\ \mathrm{~S} 20 \mathrm{C} 03 & 10.4801379 & -0.4378125 \\ \mathrm{~S} 20 \mathrm{C} 04 & 10.4801379 & -0.4378125 \\ \mathrm{~S} 20 \mathrm{C} 05 & 10.4801379 & -0.4378125 \\ \mathrm{~S} 20 \mathrm{C} 06 & 10.4801379 & -0.4378125 \\ \mathrm{~S} 20 \mathrm{C} 07 & 23.9375 & -5.1360239 \\ \mathrm{~S} 20 \mathrm{C} 08 & 23.9375 & -5.1360239 \\ \mathrm{~S} 20 \mathrm{C} 09 & 23.9375 & -5.1360239 \\ \mathrm{~S} 20 \mathrm{C} 10 & 23.9375 & -5.1360239 \\ \mathrm{~S} 20 \mathrm{C} 11 & 23.9375 & -5.1360239 \\ \mathrm{~S} 20 \mathrm{C} 12 & 23.9375 & -5.1360239 \\ \mathrm{~S} 20 \mathrm{C} 13 & 8.24007883 & 0.34423306 \\ \mathrm{~S} 20 \mathrm{C} 14 & 8.24007883 & 0.34423306 \\ \mathrm{~S} 20 \mathrm{C} 15 & \mathbf{8 . 2 4 0 0 7 8 8 3} & 0.34423306\end{array}$


REVISED METHODOLOGY FOR DETERMINING CESIUM-137 CONTENT OF HN-200 GROUT CONTAINERS.

$\begin{array}{lcl}\text { S20C16 } & 8.24007883 & 0.34423306 \\ \text { S20C17 } & 8.24007883 & 0.34423306 \\ \text { S20C18 } & 8.24007883 & 0.34423306 \\ \text { S20C19 } & -23.9375 & 11.5780146 \\ \text { S20C20 } & -23.9375 & 11.5780146 \\ \text { S20C21 } & -23.9375 & 11.5780146 \\ \text { S20C22 } & -23.9375 & 11.5780146 \\ \text { S20C23 } & -23.9375 & 11.5780146 \\ \text { S20C24 } & -23.9375 & 11.5780146 \\ \text { S20R01 } & 9.41475018 & -0.0658662 \\ \text { S20R02 } & 8.21787798 & 0.35198378 \\ \text { S20R03 } & 6.54727532 & 0.93522173 \\ \text { S20R04 } & 2.43861027 & 2.36963188 \\ \text { S20R05 } & 142.9375 & -46.681101 \\ \text { S20R06 } & 15.3380797 & -2.1338089 \\ \text { S20R07 } & 11.7572469 & -0.8836747 \\ \text { S20R08 } & 10.1914854 & -0.3370387 \\ \text { S20R09 } & 9.04483417 & 0.06327825 \\ \text { S20R10 } & 7.86894954 & 0.47380108 \\ \text { S20R11 } & 6.18640591 & 1.06120785 \\ \text { S20R12 } & 2.19592265 & 2.45435857 \\ \text { S20R13 } & -142.9375 & 53.1230918 \\ \text { S20R14 } & 17.0332041 & -2.7256078 \\ \text { S20R15 } & 12.4430782 & -1.1231109 \\ \text { S20R16 } & 10.6434007 & -0.4948106\end{array}$

\begin{tabular}{ccc} 
& \multicolumn{2}{c}{ SOURCE S21 } \\
Line & Z0 & Slope \\
S21C01 & -9.0745578 & 0.3790938 \\
S21C02 & -9.0745578 & 0.3790938 \\
S21C03 & -9.0745578 & 0.3790938 \\
S21C04 & -9.0745578 & 0.3790938 \\
S21C05 & -9.0745578 & 0.3790938 \\
S21C06 & -9.0745578 & 0.3790938 \\
S21C07 & 23.9375 & -10.401774 \\
S21C08 & 23.9375 & -10.401774 \\
S21C09 & 23.9375 & -10.401774 \\
S21C10 & 23.9375 & -10.401774 \\
S21C11 & 23.9375 & -10.401774 \\
S21C12 & 23.9375 & -10.401774 \\
S21C13 & -7.0162181 & -0.2931057 \\
S21C14 & -7.0162181 & -0.2931057 \\
S21C15 & -7.0162181 & -0.2931057 \\
S21C16 & -7.0162181 & -0.2931057
\end{tabular}




\section{REVISED METHODOLOGY FOR DETERMINING CESIUM-137 CONTENT OF HN-200 GROUT CONTAINERS.}

$\begin{array}{lcc}\text { S21C17 } & -7.0162181 & -0.2931057 \\ \text { S21C18 } & -7.0162181 & -0.2931057 \\ \text { S21C19 } & -23.9375 & 5.23293891 \\ \text { S21C20 } & -23.9375 & 5.23293891 \\ \text { S21C21 } & -23.9375 & 5.23293891 \\ \text { S21C22 } & -23.9375 & 5.23293891 \\ \text { S21C23 } & -23.9375 & 5.23293891 \\ \text { S21C24 } & -23.9375 & 5.23293891 \\ \text { S21R01 } & -8.0869804 & 0.05657704 \\ \text { S21R02 } & -9.4000637 & 0.48539543 \\ \text { S21R03 } & -11.318747 & 1.11198686 \\ \text { S21R04 } & -16.213948 & 2.71063054 \\ \text { S21R05 } & 142.9375 & -49.264037 \\ \text { S21R06 } & -0.493552 & -2.4232365 \\ \text { S21R07 } & -4.7089757 & -1.0465903 \\ \text { S21R08 } & -6.4947753 & -0.4633952 \\ \text { S21R09 } & -7.7477587 & -0.0542038 \\ \text { S21R10 } & -8.9740114 & 0.34625797 \\ \text { S21R11 } & -10.653085 & 0.89459915 \\ \text { S21R12 } & -14.494868 & 2.14922417 \\ \text { S21R13 } & -142.9375 & 44.095202 \\ \text { S21R14 } & -0.5520869 & -2.4041205 \\ \text { S21R15 } & -5.0032178 & -0.9504985 \\ \text { S21R16 } & -6.8031228 & -0.362697\end{array}$

\begin{tabular}{lcc} 
& \multicolumn{2}{c}{ SOURCE S22 } \\
Line & Z0 & Slope \\
S22C01 & 7.34245033 & -0.3067342 \\
$\mathrm{~S} 22 \mathrm{C} 02$ & 7.34245033 & -0.3067342 \\
$\mathrm{~S} 22 \mathrm{C} 03$ & 7.34245033 & -0.3067342 \\
$\mathrm{~S} 22 \mathrm{C} 04$ & 7.34245033 & -0.3067342 \\
$\mathrm{~S} 22 \mathrm{C} 05$ & 7.34245033 & -0.3067342 \\
$\mathrm{~S} 22 \mathrm{C} 06$ & 7.34245033 & -0.3067342 \\
$\mathrm{~S} 22 \mathrm{C} 07$ & 23.9375 & 0.61633122 \\
$\mathrm{~S} 22 \mathrm{C} 08$ & 23.9375 & 0.61633122 \\
$\mathrm{~S} 22 \mathrm{C} 09$ & 23.9375 & 0.61633122 \\
$\mathrm{~S} 22 \mathrm{C} 10$ & 23.9375 & 0.61633122 \\
$\mathrm{~S} 22 \mathrm{C} 11$ & 23.9375 & 0.61633122 \\
$\mathrm{~S} 22 \mathrm{C} 12$ & 23.9375 & 0.61633122 \\
$\mathrm{~S} 22 \mathrm{C} 13$ & 51.6442541 & 2.15746231 \\
$\mathrm{~S} 22 \mathrm{C} 14$ & 51.6442541 & 2.15746231 \\
$\mathrm{~S} 22 \mathrm{C} 15$ & 51.6442541 & 2.15746231 \\
$\mathrm{~S} 22 \mathrm{C} 16$ & 51.6442541 & 2.15746231 \\
$\mathrm{~S} 22 \mathrm{C} 17$ & 51.6442541 & 2.15746231
\end{tabular}


REVISED METHODOLOGY FOR DETERMINING CESIUM-137 CONTENT OF HN-200 GROUT CONTAINERS.

$\begin{array}{lcc}\text { S22C18 } & 51.6442541 & 2.15746231 \\ \text { S22C19 } & -23.9375 & -2.046617 \\ \text { S22C20 } & -23.9375 & -2.046617 \\ \text { S22C21 } & -23.9375 & -2.046617 \\ \text { S22C22 } & -23.9375 & -2.046617 \\ \text { S22C23 } & -23.9375 & -2.046617 \\ \text { S22C24 } & -23.9375 & -2.046617 \\ \text { S22R01 } & 11.4205408 & -0.0798988 \\ \text { S22R02 } & 17.8708572 & 0.27888671 \\ \text { S22R03 } & 26.1786466 & 0.74099038 \\ \text { S22R04 } & 42.3432228 & 1.64011161 \\ \text { S22R05 } & 142.9375 & 7.23546096 \\ \text { S22R06 } & -45.501068 & -3.246046 \\ \text { S22R07 } & -6.2292427 & -1.0616317 \\ \text { S22R08 } & 6.26277833 & -0.3667887 \\ \text { S22R09 } & 14.7067418 & 0.10288932 \\ \text { S22R10 } & 23.5035554 & 0.59219394 \\ \text { S22R11 } & 37.5066684 & 1.37108825 \\ \text { S22R12 } & 83.804083 & 3.94628651 \\ \text { S22R13 } & -142.9375 & -8.6657467 \\ \text { S22R14 } & -22.990072 & -1.9939182 \\ \text { S22R15 } & -4.347844 & -0.9569827 \\ \text { S22R16 } & 4.76188454 & -0.4502729\end{array}$

\begin{tabular}{ccc} 
& \multicolumn{2}{c}{ SOURCE S23 } \\
Line & $\mathbf{Z 0}$ & Slope \\
S23C01 & 18.573468 & -0.7759151 \\
S23C02 & 18.573468 & -0.7759151 \\
S23C03 & 18.573468 & -0.7759151 \\
S23C04 & 18.573468 & -0.7759151 \\
S23C05 & 18.573468 & -0.7759151 \\
S23C06 & 18.573468 & -0.7759151 \\
S23C07 & 23.9375 & -1.8278555 \\
S23C08 & 23.9375 & -1.8278555 \\
S23C09 & 23.9375 & -1.8278555 \\
S23C10 & 23.9375 & -1.8278555 \\
S23C11 & 23.9375 & -1.8278555 \\
S23C12 & 23.9375 & -1.8278555 \\
S23C13 & 12.0500337 & 0.50339566 \\
S23C14 & 12.0500337 & 0.50339566 \\
S23C15 & 12.0500337 & 0.50339566 \\
S23C16 & 12.0500337 & 0.50339566 \\
S23C17 & 12.0500337 & 0.50339566 \\
S23C18 & 12.0500337 & 0.50339566
\end{tabular}


REVISED METHODOLOGY FOR DETERMINING CESIUM-137 CONTENT OF HN-200 GROUT CONTAINERS.

$\begin{array}{lcc}\text { S23C19 } & -23.9375 & 7.5609114 \\ \text { S23C20 } & -23.9375 & 7.5609114 \\ \text { S23C21 } & -23.9375 & 7.5609114 \\ \text { S23C22 } & -23.9375 & 7.5609114 \\ \text { S23C23 } & -23.9375 & 7.5609114 \\ \text { S23C24 } & -23.9375 & 7.5609114 \\ \text { S23R01 } & 15.1576759 & -0.1060441 \\ \text { S23R02 } & 13.007036 & 0.31571794 \\ \text { S23R03 } & 10.0234517 & 0.9008287 \\ \text { S23R04 } & 2.54354387 & 2.36771352 \\ \text { S23R05 } & 142.9375 & -25.164947 \\ \text { S23R06 } & 24.6312874 & -1.9639142 \\ \text { S23R07 } & 18.7691941 & -0.814299 \\ \text { S23R08 } & 16.1071348 & -0.2922425 \\ \text { S23R09 } & 14.1134523 & 0.09873863 \\ \text { S23R10 } & 12.0398874 & 0.50538546 \\ \text { S23R11 } & 9.06064193 & 1.08964533 \\ \text { S23R12 } & 2.10975771 & 2.45278333 \\ \text { S23R13 } & -142.9375 & 30.898003 \\ \text { S23R14 } & 29.6957133 & -2.9570988 \\ \text { S23R15 } & 20.7636624 & -1.2054342 \\ \text { S23R16 } & 17.4010001 & -0.5459824\end{array}$

SOURCE S24

$\begin{array}{ccc}\text { Line } & \mathbf{Z 0} & \text { Slope } \\ \text { S24C01 } & 28.786894 & -1.2025857 \\ \text { S24C02 } & 28.786894 & -1.2025857 \\ \text { S24C03 } & 28.786894 & -1.2025857 \\ \text { S24C04 } & 28.786894 & -1.2025857 \\ \text { S24C05 } & 28.786894 & -1.2025857 \\ \text { S24C06 } & 28.786894 & -1.2025857 \\ \text { S24C07 } & 23.9375 & -0.5934991 \\ \text { S24C08 } & 23.9375 & -0.5934991 \\ \text { S24C09 } & 23.9375 & -0.5934991 \\ \text { S24C10 } & 23.9375 & -0.5934991 \\ \text { S24C11 } & 23.9375 & -0.5934991 \\ \text { S24C12 } & 23.9375 & -0.5934991 \\ \text { S24C13 } & 14.4170254 & 0.60227782 \\ \text { S24C14 } & 14.4170254 & 0.60227782 \\ \text { S24C15 } & 14.4170254 & 0.60227782 \\ \text { S24C16 } & 14.4170254 & 0.60227782 \\ \text { S24C17 } & 14.4170254 & 0.60227782 \\ \text { S24C18 } & 14.4170254 & 0.60227782 \\ \text { S24C19 } & -23.9375 & 5.41962749\end{array}$


REVISED METHODOLOGY FOR DETERMINING CESIUM-137 CONTENT OF HN-200 GROUT CONTAINERS.

$\begin{array}{lcc}\text { S24C20 } & -23.9375 & 5.41962749 \\ \text { S24C21 } & -23.9375 & 5.41962749 \\ \text { S24C22 } & -23.9375 & 5.41962749 \\ \text { S24C23 } & -23.9375 & 5.41962749 \\ \text { S24C24 } & -23.9375 & 5.41962749 \\ \text { S24R01 } & 20.345471 & -0.1423382 \\ \text { S24R02 } & 16.9353841 & 0.28597057 \\ \text { S24R03 } & 12.2124742 & 0.87917067 \\ \text { S24R04 } & -0.0107099 & 2.41440937 \\ \text { S24R05 } & 142.9375 & -15.539965 \\ \text { S24R06 } & 33.5502183 & -1.8008618 \\ \text { S24R07 } & 25.1896916 & -0.750775 \\ \text { S24R08 } & 21.2301057 & -0.2534488 \\ \text { S24R09 } & 18.1985349 & 0.12731813 \\ \text { S24R10 } & 15.0094239 & 0.52787224 \\ \text { S24R11 } & 10.4289428 & 1.1031832 \\ \text { S24R12 } & -0.0079883 & 2.41406754 \\ \text { S24R13 } & -142.9375 & 20.3660935 \\ \text { S24R14 } & 44.9806645 & -3.2365322 \\ \text { S24R15 } & 29.4975688 & -1.2918468 \\ \text { S24R16 } & 23.9542834 & -0.5956071\end{array}$

\begin{tabular}{ccc} 
& \multicolumn{2}{c}{ SOURCE S25 } \\
Line & Z0 & Slope \\
S25C01 & 14.7196853 & -0.6149216 \\
S25C02 & 14.7196853 & -0.6149216 \\
S25C03 & 14.7196853 & -0.6149216 \\
S25C04 & 14.7196853 & -0.6149216 \\
S25C05 & 14.7196853 & -0.6149216 \\
S25C06 & 14.7196853 & -0.6149216 \\
S25C07 & 23.9375 & -1.7126132 \\
S25C08 & 23.9375 & -1.7126132 \\
S25C09 & 23.9375 & -1.7126132 \\
S25C10 & 23.9375 & -1.7126132 \\
S25C11 & 23.9375 & -1.7126132 \\
S25C12 & 23.9375 & -1.7126132 \\
S25C13 & 7.07422045 & 0.29552879 \\
S25C14 & 7.07422045 & 0.29552879 \\
S25C15 & 7.07422045 & 0.29552879 \\
S25C16 & 7.07422045 & 0.29552879 \\
S25C17 & 7.07422045 & 0.29552879 \\
S25C18 & 7.07422045 & 0.29552879 \\
S25C19 & -23.9375 & 3.98851973 \\
S25C20 & -23.9375 & 3.98851973
\end{tabular}


REVISED METHODOLOGY FOR DETERMINING CESIUM-137 CONTENT OF HN-200 GROUT CONTAINERS.

$\begin{array}{lcc}\text { S25C21 } & -23.9375 & 3.98851973 \\ \text { S25C22 } & -23.9375 & 3.98851973 \\ \text { S25C23 } & -23.9375 & 3.98851973 \\ \text { S25C24 } & -23.9375 & 3.98851973 \\ \text { S25R01 } & 10.1523516 & -0.0710265 \\ \text { S25R02 } & 6.49028535 & 0.36506595 \\ \text { S25R03 } & 1.26342634 & 0.98749975 \\ \text { S25R04 } & -12.661047 & 2.64567781 \\ \text { S25R05 } & 142.9375 & -15.883576 \\ \text { S25R06 } & 25.8592697 & -1.9414647 \\ \text { S25R07 } & 16.5761537 & -0.8359967 \\ \text { S25R08 } & 12.2549608 & -0.321413 \\ \text { S25R09 } & 9.02566012 & 0.0631441 \\ \text { S25R10 } & 5.71420642 & 0.45748432 \\ \text { S25R11 } & 1.06959045 & 1.01058245 \\ \text { S25R12 } & -9.2909937 & 2.24435933 \\ \text { S25R13 } & -142.9375 & 18.159482 \\ \text { S25R14 } & 35.2390112 & -3.0584392 \\ \text { S25R15 } & 19.5801573 & -1.1937247 \\ \text { S25R16 } & 13.9193768 & -0.5196179\end{array}$

\begin{tabular}{lcc} 
& \multicolumn{2}{c}{ SOURCE S26 } \\
Line & Z0 & Slope \\
S26C01 & 19.5768637 & -0.8178324 \\
S26C02 & 19.5768637 & -0.8178324 \\
S26C03 & 19.5768637 & -0.8178324 \\
S26C04 & 19.5768637 & -0.8178324 \\
S26C05 & 19.5768637 & -0.8178324 \\
S26C06 & 19.5768637 & -0.8178324 \\
S26C07 & 23.9375 & -1.8790741 \\
S26C08 & 23.9375 & -1.8790741 \\
S26C09 & 23.9375 & -1.8790741 \\
S26C10 & 23.9375 & -1.8790741 \\
S26C11 & 23.9375 & -1.8790741 \\
S26C12 & 23.9375 & -1.8790741 \\
S26C13 & 13.840587 & 0.57819685 \\
S26C14 & 13.840587 & 0.57819685 \\
S26C15 & 13.840587 & 0.57819685 \\
S26C16 & 13.840587 & 0.57819685 \\
S26C17 & 13.840587 & 0.57819685 \\
S26C18 & 13.840587 & 0.57819685 \\
S26C19 & -23.9375 & 9.77219464 \\
S26C20 & -23.9375 & 9.77219464 \\
S26C21 & -23.9375 & 9.77219464
\end{tabular}


HNF-4799, Rev 0

August 25, 1999

REVISED METHODOLOGY FOR DETERMINING CESIUM-137 CONTENT

OF HN-200 GROUT CONTAINERS.

$\begin{array}{lcc}\text { S26C22 } & -23.9375 & 9.77219464 \\ \text { S26C23 } & -23.9375 & 9.77219464 \\ \text { S26C24 } & -23.9375 & 9.77219464 \\ \text { S26R01 } & 16.6963616 & -0.1168088 \\ \text { S26R02 } & 14.9805169 & 0.30077378 \\ \text { S26R03 } & 12.6204741 & 0.87513395 \\ \text { S26R04 } & 6.80779935 & 2.2897561 \\ \text { S26R05 } & 142.9375 & -30.83993 \\ \text { S26R06 } & 24.3102253 & -1.9697837 \\ \text { S26R07 } & 19.5313596 & -0.8067582 \\ \text { S26R08 } & 17.377684 & -0.2826213 \\ \text { S26R09 } & 15.7632518 & 0.11028073 \\ \text { S26R10 } & 14.0764018 & 0.52080694 \\ \text { S26R11 } & 11.6344136 & 1.11511004 \\ \text { S26R12 } & 5.85647189 & 2.52127927 \\ \text { S26R13 } & -142.9375 & 38.7330506 \\ \text { S26R14 } & 28.2591873 & -2.9308368 \\ \text { S26R15 } & 21.186716 & -1.2096198 \\ \text { S26R16 } & 18.4938375 & -0.5542579\end{array}$

SOURCE S27

$\begin{array}{lcc}\text { Line } & \mathbf{Z 1} & \text { Slope } \\ \text { S27C01 } & -23.312077 & 0.97387266 \\ \text { S27C02 } & -23.312077 & 0.97387266 \\ \text { S27C03 } & -23.312077 & 0.97387266 \\ \text { S27C04 } & -23.312077 & 0.97387266 \\ \text { S27C05 } & -23.312077 & 0.97387266 \\ \text { S27C06 } & -23.312077 & 0.97387266 \\ \text { S27C07 } & 23.9375 & -19.842767 \\ \text { S27C08 } & 23.9375 & -19.842767 \\ \text { S27C09 } & 23.9375 & -19.842767 \\ \text { S27C10 } & 23.9375 & -19.842767 \\ \text { S27C11 } & 23.9375 & -19.842767 \\ \text { S27C12 } & 23.9375 & -19.842767 \\ \text { S27C13 } & -19.273987 & -0.8051796 \\ \text { S27C14 } & -19.273987 & -0.8051796 \\ \text { S27C15 } & -19.273987 & -0.8051796 \\ \text { S27C16 } & -19.273987 & -0.8051796 \\ \text { S27C17 } & -19.273987 & -0.8051796 \\ \text { S27C18 } & -19.273987 & -0.8051796 \\ \text { S27C19 } & -23.9375 & 1.24941394 \\ \text { S27C20 } & -23.9375 & 1.24941394 \\ \text { S27C21 } & -23.9375 & 1.24941394 \\ \text { S27C22 } & -23.9375 & 1.24941394\end{array}$

30 of 210 
REVISED METHODOLOGY FOR DETERMINING CESIUM-137 CONTENT OF HN-200 GROUT CONTAINERS.

$\begin{array}{lll}\text { S27C23 } & -23.9375 & 1.24941394 \\ \text { S27C24 } & -23.9375 & 1.24941394 \\ \text { S27R01 } & -21.442075 & 0.15001014 \\ \text { S27R02 } & -22.427243 & 0.58404358 \\ \text { S27R03 } & -23.908294 & 1.23654693 \\ \text { S27R04 } & -27.73212 & 2.92120118 \\ \text { S27R05 } & 142.9375 & -72.270328 \\ \text { S27R06 } & -14.999396 & -2.6884266 \\ \text { S27R07 } & -18.418163 & -1.182228 \\ \text { S27R08 } & -19.820722 & -0.5643057 \\ \text { S27R09 } & -20.771734 & -0.1453204 \\ \text { S27R10 } & -21.669311 & 0.25012297 \\ \text { S27R11 } & -22.858052 & 0.77384409 \\ \text { S27R12 } & -25.522299 & 1.94762506 \\ \text { S27R13 } & -142.9375 & 53.6769752 \\ \text { S27R14 } & -16.298103 & -2.1162581 \\ \text { S27R15 } & -19.264409 & -0.8093993 \\ \text { S27R16 } & -20.513996 & -0.2588716\end{array}$

\begin{tabular}{lcc} 
& \multicolumn{2}{c}{ SOURCE S28 } \\
Line & Z0 & Slope \\
S28C01 & -24.610722 & 1.02812415 \\
S28C02 & -24.610722 & 1.02812415 \\
S28C03 & -24.610722 & 1.02812415 \\
S28C04 & -24.610722 & 1.02812415 \\
S28C05 & -24.610722 & 1.02812415 \\
S28C06 & -24.610722 & 1.02812415 \\
S28C07 & 23.9375 & -1.6081701 \\
S28C08 & 23.9375 & -1.6081701 \\
S28C09 & 23.9375 & -1.6081701 \\
S28C10 & 23.9375 & -1.6081701 \\
S28C11 & 23.9375 & -1.6081701 \\
S28C12 & 23.9375 & -1.6081701 \\
S28C13 & -3.2088683 & -0.1340519 \\
S28C14 & -3.2088683 & -0.1340519 \\
S28C15 & -3.2088683 & -0.1340519 \\
S28C16 & -3.2088683 & -0.1340519 \\
S28C17 & -3.2088683 & -0.1340519 \\
S28C18 & -3.2088683 & -0.1340519 \\
S28C19 & -23.9375 & 0.99156646 \\
S28C20 & -23.9375 & 0.99156646 \\
S28C21 & -23.9375 & 0.99156646 \\
S28C22 & -23.9375 & 0.99156646 \\
S28C23 & -23.9375 & 0.99156646
\end{tabular}


REVISED METHODOLOGY FOR DETERMINING CESIUM-137 CONTENT OF HN-200 GROUT CONTAINERS.

$\begin{array}{lcc}\text { S28C24 } & -23.9375 & 0.99156646 \\ \text { S28R01 } & -6.5171093 & 0.04559412 \\ \text { S28R02 } & -15.461455 & 0.53129523 \\ \text { S28R03 } & -29.460508 & 1.29148013 \\ \text { S28R04 } & -75.581363 & 3.79596202 \\ \text { S28R05 } & 142.9375 & -8.0701783 \\ \text { S28R06 } & 29.0133663 & -1.8838028 \\ \text { S28R07 } & 10.7746997 & -0.8933959 \\ \text { S28R08 } & 1.71170304 & -0.4012517 \\ \text { S28R09 } & -5.0295035 & -0.0351867 \\ \text { S28R10 } & -11.677001 & 0.32578961 \\ \text { S28R11 } & -20.379638 & 0.79836534 \\ \text { S28R12 } & -37.508378 & 1.72850032 \\ \text { S28R13 } & -142.9375 & 7.45357473 \\ \text { S28R14 } & 58.4634652 & -3.4830194 \\ \text { S28R15 } & 15.5757492 & -1.1541053 \\ \text { S28R16 } & 2.2664568 & -0.4313763\end{array}$

\begin{tabular}{lcc} 
& \multicolumn{2}{c}{ SOURCE S29 } \\
Line & Z0 & Slope \\
S29C01 & 3.62896645 & -0.1516017 \\
S29C02 & 3.62896645 & -0.1516017 \\
S29C03 & 3.62896645 & -0.1516017 \\
S29C04 & 3.62896645 & -0.1516017 \\
S29C05 & 3.62896645 & -0.1516017 \\
S29C06 & 3.62896645 & -0.1516017 \\
S29C07 & 23.9375 & 224.60776 \\
S29C08 & 23.9375 & 224.60776 \\
S29C09 & 23.9375 & 224.60776 \\
S29C10 & 23.9375 & 224.60776 \\
S29C11 & 23.9375 & 224.60776 \\
S29C12 & 23.9375 & 224.60776 \\
S29C13 & 3.65646675 & 0.15275057 \\
S29C14 & 3.65646675 & 0.15275057 \\
S29C15 & 3.65646675 & 0.15275057 \\
S29C16 & 3.65646675 & 0.15275057 \\
S29C17 & 3.65646675 & 0.15275057 \\
S29C18 & 3.65646675 & 0.15275057 \\
S29C19 & -23.9375 & -305.23623 \\
S29C20 & -23.9375 & -305.23623 \\
S29C21 & -23.9375 & -305.23623 \\
S29C22 & -23.9375 & -305.23623 \\
S29C23 & -23.9375 & -305.23623 \\
S29C24 & -23.9375 & -305.23623
\end{tabular}


REVISED METHODOLOGY FOR DETERMINING CESIUM-137 CONTENT OF HN-200 GROUT CONTAINERS.

$\begin{array}{lcc}\text { S29R01 } & 3.64036347 & -0.0254682 \\ \text { S29R02 } & 3.67757541 & 0.38636516 \\ \text { S29R03 } & 3.7296872 & 0.96309875 \\ \text { S29R04 } & 3.85443826 & 2.34374827 \\ \text { S29R05 } & 142.9375 & 1541.60901 \\ \text { S29R06 } & 3.43019033 & -2.3515042 \\ \text { S29R07 } & 3.55548645 & -0.9648223 \\ \text { S29R08 } & 3.60770617 & -0.3868942 \\ \text { S29R09 } & 3.64496883 & 0.02550044 \\ \text { S29R10 } & 3.68261144 & 0.44210009 \\ \text { S29R11 } & 3.73636173 & 1.03696729 \\ \text { S29R12 } & 3.86719336 & 2.48491207 \\ \text { S29R13 } & -142.9375 & -1622.2375 \\ \text { S29R14 } & 3.41887659 & -2.4767161 \\ \text { S29R15 } & 3.54913504 & -1.0351149 \\ \text { S29R16 } & 3.60277258 & -0.4414955 \\ & & \end{array}$

\begin{tabular}{lcc} 
& \multicolumn{2}{c}{ SOURCE S30 } \\
Line & Z0 & Slope \\
S30C01 & -28.592121 & 1.19444893 \\
S30C02 & -28.592121 & 1.19444893 \\
S30C03 & -28.592121 & 1.19444893 \\
S30C04 & -28.592121 & 1.19444893 \\
S30C05 & -28.592121 & 1.19444893 \\
S30C06 & -28.592121 & 1.19444893 \\
S30C07 & 23.9375 & -5.9300759 \\
S30C08 & 23.9375 & -5.9300759 \\
S30C09 & 23.9375 & -5.9300759 \\
S30C10 & 23.9375 & -5.9300759 \\
S30C11 & 23.9375 & -5.9300759 \\
S30C12 & 23.9375 & -5.9300759 \\
S30C13 & -15.126272 & -0.6319069 \\
S30C14 & -15.126272 & -0.6319069 \\
S30C15 & -15.126272 & -0.6319069 \\
S30C16 & -15.126272 & -0.6319069 \\
S30C17 & -15.126272 & -0.6319069 \\
S30C18 & -15.126272 & -0.6319069 \\
S30C19 & -23.9375 & 0.56314864 \\
S30C20 & -23.9375 & 0.56314864 \\
S30C21 & -23.9375 & 0.56314864 \\
S30C22 & -23.9375 & 0.56314864 \\
S30C23 & -23.9375 & 0.56314864 \\
S30C24 & -23.9375 & 0.56314864 \\
S30R01 & -20.861451 & 0.14594806 \\
& & \\
\hline & &
\end{tabular}


REVISED METHODOLOGY FOR DETERMINING CESIUM-137 CONTENT OF HN-200 GROUT CONTAINERS.

$\begin{array}{lcl}\text { S30R02 } & -24.189978 & 0.59739187 \\ \text { S30R03 } & -29.295502 & 1.28984757 \\ \text { S30R04 } & -43.441012 & 3.2083849 \\ \text { S30R05 } & 142.9375 & -22.069893 \\ \text { S30R06 } & -1.7493958 & -2.4461953 \\ \text { S30R07 } & -11.568397 & -1.1144569 \\ \text { S30R08 } & -15.846586 & -0.5342116 \\ \text { S30R09 } & -18.814849 & -0.1316299 \\ \text { S30R10 } & -21.631643 & 0.25040821 \\ \text { S30R11 } & -25.311963 & 0.74956528 \\ \text { S30R12 } & -33.121089 & 1.808707 \\ \text { S30R13 } & -142.9375 & 16.7029653 \\ \text { S30R14 } & -2.2944754 & -2.3722669 \\ \text { S30R15 } & -13.389005 & -0.8675302 \\ \text { S30R16 } & -17.720732 & -0.2800235\end{array}$

\begin{tabular}{ccc} 
& \multicolumn{2}{c}{ SOURCE S31 } \\
Line & Z0 & Slope \\
S31C01 & -23.174045 & 0.96810631 \\
S31C02 & -23.174045 & 0.96810631 \\
S31C03 & -23.174045 & 0.96810631 \\
S31C04 & -23.174045 & 0.96810631 \\
S31C05 & -23.174045 & 0.96810631 \\
S31C06 & -23.174045 & 0.96810631 \\
S31C07 & 23.9375 & -7.5092297 \\
S31C08 & 23.9375 & -7.5092297 \\
S31C09 & 23.9375 & -7.5092297 \\
S31C10 & 23.9375 & -7.5092297 \\
S31C11 & 23.9375 & -7.5092297 \\
S31C12 & 23.9375 & -7.5092297 \\
S31C13 & -14.441244 & -0.6032896 \\
S31C14 & -14.441244 & -0.6032896 \\
S31C15 & -14.441244 & -0.6032896 \\
S31C16 & -14.441244 & -0.6032896 \\
S31C17 & -14.441244 & -0.6032896 \\
S31C18 & -14.441244 & -0.6032896 \\
S31C19 & -23.9375 & 1.1054838 \\
S31C20 & -23.9375 & 1.1054838 \\
S31C21 & -23.9375 & 1.1054838 \\
S31C22 & -23.9375 & 1.1054838 \\
S31C23 & -23.9375 & 1.1054838 \\
S31C24 & -23.9375 & 1.1054838 \\
S31R01 & -18.513744 & 0.12952335 \\
S31R02 & -20.978714 & 0.57307462
\end{tabular}


REVISED METHODOLOGY FOR DETERMINING CESIUM-137 CONTENT OF HN-200 GROUT CONTAINERS.

$\begin{array}{lcl}\text { S31R03 } & -24.709942 & 1.24447837 \\ \text { S31R04 } & -34.74007 & 3.04931774 \\ \text { S31R05 } & 142.9375 & -28.922304 \\ \text { S31R06 } & -3.9735944 & -2.4868573 \\ \text { S31R07 } & -11.598834 & -1.114758 \\ \text { S31R08 } & -14.866384 & -0.5267891 \\ \text { S31R09 } & -17.128007 & -0.1198286 \\ \text { S31R10 } & -19.284326 & 0.26818324 \\ \text { S31R11 } & -22.134258 & 0.78100526 \\ \text { S31R12 } & -28.332107 & 1.89625726 \\ \text { S31R13 } & -142.9375 & 22.5185577 \\ \text { S31R14 } & -4.872315 & -2.3251399 \\ \text { S31R15 } & -12.948548 & -0.871888 \\ \text { S31R16 } & -16.172597 & -0.2917468\end{array}$

\begin{tabular}{lcc} 
& \multicolumn{2}{c}{ SOURCE S32 } \\
Line & Z0 & Slope \\
S32C01 & 17.3023328 & -0.7228129 \\
S32C02 & 17.3023328 & -0.7228129 \\
S32C03 & 17.3023328 & -0.7228129 \\
S32C04 & 17.3023328 & -0.7228129 \\
S32C05 & 17.3023328 & -0.7228129 \\
S32C06 & 17.3023328 & -0.7228129 \\
S32C07 & 23.9375 & 6.51236306 \\
S32C08 & 23.9375 & 6.51236306 \\
S32C09 & 23.9375 & 6.51236306 \\
S32C10 & 23.9375 & 6.51236306 \\
S32C11 & 23.9375 & 6.51236306 \\
S32C12 & 23.9375 & 6.51236306 \\
S32C13 & 18.6808875 & 0.78040261 \\
S32C14 & 18.6808875 & 0.78040261 \\
S32C15 & 18.6808875 & 0.78040261 \\
S32C16 & 18.6808875 & 0.78040261 \\
S32C17 & 18.6808875 & 0.78040261 \\
S32C18 & 18.6808875 & 0.78040261 \\
S32C19 & -23.9375 & -45.691905 \\
S32C20 & -23.9375 & -45.691905 \\
S32C21 & -23.9375 & -45.691905 \\
S32C22 & -23.9375 & -45.691905 \\
S32C23 & -23.9375 & -45.691905 \\
S32C24 & -23.9375 & -45.691905 \\
S32R01 & 17.8506753 & -0.1248845 \\
S32R02 & 18.2185475 & 0.27625383 \\
S32R03 & 18.7124874 & 0.81486001 \\
& &
\end{tabular}


REVISED METHODOLOGY FOR DETERMINING CESIUM-137 CONTENT OF HN-200 GROUT CONTAINERS.

$\begin{array}{lcc}\text { S32R04 } & 19.8464711 & 2.05138828 \\ \text { S32R05 } & 142.9375 & 136.273363 \\ \text { S32R06 } & 16.0197788 & -2.1213464 \\ \text { S32R07 } & 17.204234 & -0.8297826 \\ \text { S32R08 } & 17.7083156 & -0.2801176 \\ \text { S32R09 } & 18.0812102 & 0.12649732 \\ \text { S32R10 } & 18.4733546 & 0.55410281 \\ \text { S32R11 } & 19.0551696 & 1.18853046 \\ \text { S32R12 } & 20.5232912 & 2.78941223 \\ \text { S32R13 } & -142.9375 & -175.4529 \\ \text { S32R14 } & 15.4914762 & -2.6974226 \\ \text { S32R15 } & 16.8948385 & -1.1671563 \\ \text { S32R16 } & 17.4640608 & -0.5464599\end{array}$

\begin{tabular}{lcc} 
& \multicolumn{2}{c}{ SOURCE S33 } \\
Line & Z0 & Slope \\
S33C01 & -19.863487 & 0.82980623 \\
S33C02 & -19.863487 & 0.82980623 \\
S33C03 & -19.863487 & 0.82980623 \\
S33C04 & -19.863487 & 0.82980623 \\
S33C05 & -19.863487 & 0.82980623 \\
S33C06 & -19.863487 & 0.82980623 \\
S33C07 & 23.9375 & 16.5071074 \\
S33C08 & 23.9375 & 16.5071074 \\
S33C09 & 23.9375 & 16.5071074 \\
S33C10 & 23.9375 & 16.5071074 \\
S33C11 & 23.9375 & 16.5071074 \\
S33C12 & 23.9375 & 16.5071074 \\
S33C13 & -25.113004 & -1.0491072 \\
S33C14 & -25.113004 & -1.0491072 \\
S33C15 & -25.113004 & -1.0491072 \\
S33C16 & -25.113004 & -1.0491072 \\
S33C17 & -25.113004 & -1.0491072 \\
S33C18 & -25.113004 & -1.0491072 \\
S33C19 & -23.9375 & -0.6283694 \\
S33C20 & -23.9375 & -0.6283694 \\
S33C21 & -23.9375 & -0.6283694 \\
S33C22 & -23.9375 & -0.6283694 \\
S33C23 & -23.9375 & -0.6283694 \\
S33C24 & -23.9375 & -0.6283694 \\
S33R01 & -21.756628 & 0.15221078 \\
S33R02 & -20.589017 & 0.57012363 \\
S33R03 & -18.86646 & 1.18666338 \\
S33R04 & -14.686641 & 2.68270892 \\
& & \\
\hline & &
\end{tabular}


HNF-4799, Rev 0

August 25, 1999

REVISED METHODOLOGY FOR DETERMINING CESIUM-137 CONTENT

OF HN-200 GROUT CONTAINERS.

$\begin{array}{lll}\text { S33R05 } & 142.9375 & 59.0997288 \\ \text { S33R06 } & -30.484028 & -2.9715105 \\ \text { S33R07 } & -25.68583 & -1.2541337 \\ \text { S33R08 } & -23.843624 & -0.5947691 \\ \text { S33R09 } & -22.624111 & -0.1582797 \\ \text { S33R10 } & -21.479045 & 0.25156376 \\ \text { S33R11 } & -19.939154 & 0.80272347 \\ \text { S33R12 } & -16.267702 & 2.11681393 \\ \text { S33R13 } & -142.9375 & -43.220991 \\ \text { S33R14 } & -27.521281 & -1.9110805 \\ \text { S33R15 } & -24.303974 & -0.7595383 \\ \text { S33R16 } & -22.855614 & -0.2411397\end{array}$

\begin{tabular}{lcc} 
& \multicolumn{2}{c}{ SOURCE S34 } \\
Line & Z0 & Slope \\
S34C01 & 10.6676482 & -0.4456459 \\
S34C02 & 10.6676482 & -0.4456459 \\
S34C03 & 10.6676482 & -0.4456459 \\
S34C04 & 10.6676482 & -0.4456459 \\
S34C05 & 10.6676482 & -0.4456459 \\
S34C06 & 10.6676482 & -0.4456459 \\
S34C07 & 23.9375 & 0.49277287 \\
S34C08 & 23.9375 & 0.49277287 \\
S34C09 & 23.9375 & 0.49277287 \\
S34C10 & 23.9375 & 0.49277287 \\
S34C11 & 23.9375 & 0.49277287 \\
S34C12 & 23.9375 & 0.49277287 \\
S34C13 & 41.4627507 & 1.73212535 \\
S34C14 & 41.4627507 & 1.73212535 \\
S34C15 & 41.4627507 & 1.73212535 \\
S34C16 & 41.4627507 & 1.73212535 \\
S34C17 & 41.4627507 & 1.73212535 \\
S34C18 & 41.4627507 & 1.73212535 \\
S34C19 & -23.9375 & -2.8928563 \\
S34C20 & -23.9375 & -2.8928563 \\
S34C21 & -23.9375 & -2.8928563 \\
S34C22 & -23.9375 & -2.8928563 \\
S34C23 & -23.9375 & -2.8928563 \\
S34C24 & -23.9375 & -2.8928563 \\
S34R01 & 15.4417368 & -0.1080314 \\
S34R02 & 20.6187649 & 0.25807822 \\
S34R03 & 27.2917295 & 0.72997762 \\
S34R04 & 40.6097445 & 1.67180237 \\
S34R05 & 142.9375 & 8.90822716
\end{tabular}


REVISED METHODOLOGY FOR DETERMINING CESIUM-137 CONTENT OF HN-200 GROUT CONTAINERS.

$\begin{array}{lcc}\text { S34R06 } & -23.155089 & -2.8375257 \\ \text { S34R07 } & 3.28885227 & -0.9674603 \\ \text { S34R08 } & 12.4446989 & -0.3199762 \\ \text { S34R09 } & 18.8324441 & 0.131753 \\ \text { S34R10 } & 25.5640122 & 0.60779673 \\ \text { S34R11 } & 36.1705143 & 1.35786844 \\ \text { S34R12 } & 68.9263253 & 3.67429726 \\ \text { S34R13 } & -142.9375 & -11.308311 \\ \text { S34R14 } & -13.642425 & -2.1648082 \\ \text { S34R15 } & 2.48153692 & -1.0245521 \\ \text { S34R16 } & 10.0373258 & -0.4902211\end{array}$

\begin{tabular}{lcc} 
& \multicolumn{2}{c}{ SOURCE S35 } \\
Line & Z0 & Slope \\
S35C01 & 48.7324743 & -2.0358214 \\
S35C02 & 48.7324743 & -2.0358214 \\
S35C03 & 48.7324743 & -2.0358214 \\
S35C04 & 48.7324743 & -2.0358214 \\
S35C05 & 48.7324743 & -2.0358214 \\
S35C06 & 48.7324743 & -2.0358214 \\
S35C07 & 23.9375 & -0.6296619 \\
S35C08 & 23.9375 & -0.6296619 \\
S35C09 & 23.9375 & -0.6296619 \\
S35C10 & 23.9375 & -0.6296619 \\
S35C11 & 23.9375 & -0.6296619 \\
S35C12 & 23.9375 & -0.6296619 \\
S35C13 & 7.39051263 & 0.30874204 \\
S35C14 & 7.39051263 & 0.30874204 \\
S35C15 & 7.39051263 & 0.30874204 \\
S35C16 & 7.39051263 & 0.30874204 \\
S35C17 & 7.39051263 & 0.30874204 \\
S35C18 & 7.39051263 & 0.30874204 \\
S35C19 & -23.9375 & 2.08539969 \\
S35C20 & -23.9375 & 2.08539969 \\
S35C21 & -23.9375 & 2.08539969 \\
S35C22 & -23.9375 & 2.08539969 \\
S35C23 & -23.9375 & 2.08539969 \\
S35C24 & -23.9375 & 2.08539969 \\
S35R01 & 14.6407109 & -0.1024274 \\
S35R02 & 6.3830243 & 0.36587819 \\
S35R03 & -5.8125887 & 1.05750933 \\
S35R04 & -43.881096 & 3.21643033 \\
S35R05 & 142.9375 & -7.3783269 \\
S35R06 & 41.8983027 & -1.6482455 \\
& & \\
\hline & &
\end{tabular}




\section{REVISED METHODOLOGY FOR DETERMINING CESIUM-137 CONTENT OF HN-200 GROUT CONTAINERS.}

$\begin{array}{lll}\text { S35R07 } & 25.9418748 & -0.743333 \\ \text { S35R08 } & 17.7662123 & -0.2796791 \\ \text { S35R09 } & 11.4251616 & 0.07993117 \\ \text { S35R10 } & 4.87921606 & 0.45116136 \\ \text { S35R11 } & -4.0857218 & 0.95957617 \\ \text { S35R12 } & -22.486673 & 2.00312116 \\ \text { S35R13 } & -142.9375 & 8.83406464 \\ \text { S35R14 } & 81.7614698 & -3.9089443 \\ \text { S35R15 } & 36.9064409 & -1.3651496 \\ \text { S35R16 } & 23.2418821 & -0.5902124\end{array}$

TABLE 6 - Determine the coefficients of the quadradic equation $(a, b$, and $c)$ for the intersection of each line in Table 5 to the inner surface (ai, bi, ci) and outer surface (ao, bo, co) of the cylinder wall.

\begin{tabular}{cccc} 
Line & ai & bi & $\begin{array}{c}\text { Source S01 } \\
\text { ci }\end{array}$ \\
S01C01 & 1.0162099 & -0.7760489 & -510.51975 \\
S01C02 & 1.0162099 & -0.7760489 & -510.51975 \\
S01C03 & 1.0162099 & -0.7760489 & -510.51975 \\
S01C04 & 1.0162099 & -0.7760489 & -510.51975 \\
S01C05 & 1.0162099 & -0.7760489 & -510.51975 \\
S01C06 & 1.0162099 & -0.7760489 & -510.51975 \\
S01C07 & 2.45421574 & -57.732878 & 53.1958258 \\
S01C08 & 2.45421574 & -57.732878 & 53.1958258 \\
S01C09 & 2.45421574 & -57.732878 & 53.1958258 \\
S01C10 & 2.45421574 & -57.732878 & 53.1958258 \\
S01C11 & 2.45421574 & -57.732878 & 53.1958258 \\
S01C12 & 2.45421574 & -57.732878 & 53.1958258 \\
S01C13 & 1.00018051 & 0.00864177 & -519.70465 \\
S01C14 & 1.00018051 & 0.00864177 & -519.70465 \\
S01C15 & 1.00018051 & 0.00864177 & -519.70465 \\
S01C16 & 1.00018051 & 0.00864177 & -519.70465 \\
S01C17 & 1.00018051 & 0.00864177 & -519.70465 \\
S01C18 & 1.00018051 & 0.00864177 & -519.70465 \\
S01C19 & 2.60273045 & -60.609267 & 53.1958258 \\
S01C20 & 2.60273045 & -60.609267 & 53.1958258 \\
S01C21 & 2.60273045 & -60.609267 & 53.1958258 \\
S01C22 & 2.60273045 & -60.609267 & 53.1958258 \\
S01C23 & 2.60273045 & -60.609267 & 53.1958258 \\
S01C24 & 2.60273045 & -60.609267 & 53.1958258 \\
S01R01 & 1.00002217 & -0.0063376 & -519.35514 \\
S01R02 & 1.23063109 & -8.3747644 & -443.78117 \\
S01R03 & 2.51271559 & -57.164471 & 20.2433286 \\
S01R04 & 14.8467789 & -532.03059 & 4590.70453 \\
S01R05 & 55.0248213 & -2101.2264 & 19911.3208 \\
S01R06 & 4.15088204 & -124.11624 & 702.456065 \\
S01R07 & 1.69615838 & -27.936871 & -239.53107 \\
& & & \\
\hline
\end{tabular}

$\begin{array}{ccc}\text { ao } & \text { bo } & \text { co } \\ 1.0162099 & -0.7760489 & -516.84057 \\ 1.0162099 & -0.7760489 & -516.84057 \\ 1.0162099 & -0.7760489 & -516.84057 \\ 1.0162099 & -0.7760489 & -516.84057 \\ 1.0162099 & -0.7760489 & -516.84057 \\ 1.0162099 & -0.7760489 & -516.84057 \\ 2.45421574 & -57.732878 & 46.875 \\ 2.45421574 & -57.732878 & 46.875 \\ 2.45421574 & -57.732878 & 46.875 \\ 2.45421574 & -57.732878 & 46.875 \\ 2.45421574 & -57.732878 & 46.875 \\ 2.45421574 & -57.732878 & 46.875 \\ 1.00018051 & 0.00864177 & -526.02548 \\ 1.00018051 & 0.00864177 & -526.02548 \\ 1.00018051 & 0.00864177 & -526.02548 \\ 1.00018051 & 0.00864177 & -526.02548 \\ 1.00018051 & 0.00864177 & -526.02548 \\ 1.00018051 & 0.00864177 & -526.02548 \\ 2.60273045 & -60.609267 & 46.875 \\ 2.60273045 & -60.609267 & 46.875 \\ 2.60273045 & -60.609267 & 46.875 \\ 2.60273045 & -60.609267 & 46.875 \\ 2.60273045 & -60.609267 & 46.875 \\ 2.60273045 & -60.609267 & 46.875 \\ 1.00002217 & -0.0063376 & -525.67597 \\ 1.23063109 & -8.3747644 & -450.10199 \\ 2.51271559 & -57.164471 & 13.9225028 \\ 14.8467789 & -532.03059 & 4584.38371 \\ 55.0248213 & -2101.2264 & 19905 \\ 4.15088204 & -124.11624 & 696.135239 \\ 1.69615838 & -27.936871 & -245.8519\end{array}$




\section{REVISED METHODOLOGY FOR DETERMINING CESIUM-137 CONTENT OF HN-200 GROUT CONTAINERS.}

$\begin{array}{llll}\text { S01R08 } & 1.12772906 & -5.3635114 & -463.50286 \\ \text { S01R09 } & 1.00001285 & 0.00367354 & -519.54554 \\ \text { S01R10 } & 1.13328093 & -4.7378752 & -477.70254 \\ \text { S01R11 } & 1.71237417 & -26.611969 & -271.27404 \\ \text { S01R12 } & 4.20690331 & -122.13689 & 643.106596 \\ \text { S01R13 } & 55.9116442 & -2118.4021 & 19911.3208 \\ \text { S01R14 } & 15.0929684 & -550.26524 & 4851.52007 \\ \text { S01R15 } & 2.54795166 & -61.408296 & 89.2191407 \\ \text { S01R16 } & 1.2406557 & -9.8927376 & -418.14182\end{array}$

\begin{tabular}{lccc} 
Line & ai & bi & ci \\
S02C01 & 1.32070783 & -15.353887 & -336.04124 \\
S02C02 & 1.32070783 & -15.353887 & -336.04124 \\
S02C03 & 1.32070783 & -15.353887 & -336.04124 \\
S02C04 & 1.32070783 & -15.353887 & -336.04124 \\
S02C05 & 1.32070783 & -15.353887 & -336.04124 \\
S02C06 & 1.32070783 & -15.353887 & -336.04124 \\
S02C07 & 17.7900815 & 196.171173 & 53.1958258 \\
S02C08 & 17.7900815 & 196.171173 & 53.1958258 \\
S02C09 & 17.7900815 & 196.171173 & 53.1958258 \\
S02C10 & 17.7900815 & 196.171173 & 53.1958258 \\
S02C11 & 17.7900815 & 196.171173 & 53.1958258 \\
S02C12 & 17.7900815 & 196.171173 & 53.1958258 \\
S02C13 & 3.15840797 & 103.333781 & 716.968116 \\
S02C14 & 3.15840797 & 103.333781 & 716.968116 \\
S02C15 & 3.15840797 & 103.333781 & 716.968116 \\
S02C16 & 3.15840797 & 103.333781 & 716.968116 \\
S02C17 & 3.15840797 & 103.333781 & 716.968116 \\
S02C18 & 3.15840797 & 103.333781 & 716.968116 \\
S02C19 & 1.16928705 & 19.697933 & 53.1958258 \\
S02C20 & 1.16928705 & 19.697933 & 53.1958258 \\
S02C21 & 1.16928705 & 19.697933 & 53.1958258 \\
S02C22 & 1.16928705 & 19.697933 & 53.1958258 \\
S02C23 & 1.16928705 & 19.697933 & 53.1958258 \\
S02C24 & 1.16928705 & 19.697933 & 53.1958258 \\
S02R01 & 1.01624074 & -4.6428206 & -187.9915 \\
S02R02 & 1.27096837 & -14.618992 & -322.63108 \\
S02R03 & 2.16671213 & -17.499145 & -454.19205 \\
S02R04 & 6.3887615 & 23.578124 & -494.017 \\
S02R05 & 235.253968 & 4375.41908 & 19911.3208 \\
S02R06 & 12.8309822 & 385.85306 & 2626.2237 \\
S02R07 & 2.77881143 & 89.9724704 & 617.896186 \\
S02R08 & 1.37401837 & 31.8779297 & 159.438232 \\
& & & \\
\hline
\end{tabular}

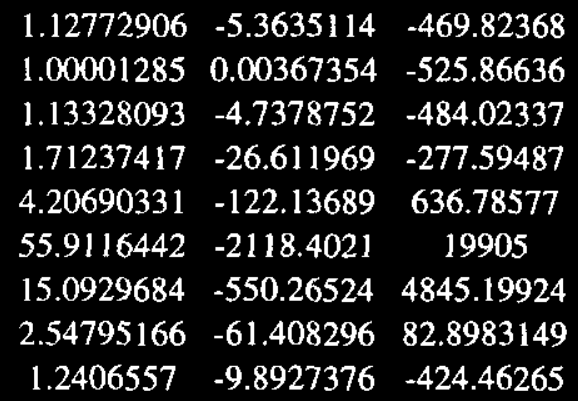




\section{REVISED METHODOLOGY FOR DETERMINING CESIUM-137 CONTENT OF HN-200 GROUT CONTAINERS.}

$\begin{array}{llll}\text { S02R09 } & 1.02187178 & 6.25259473 & -72.942951 \\ \text { S02R10 } & 1.08368712 & -9.5449707 & -247.64414 \\ \text { S02R11 } & 1.81187427 & -18.024468 & -419.76751 \\ \text { S02R12 } & 7.51158524 & 38.4037867 & -463.18397 \\ \text { S02R13 } & 136.006561 & 3321.64806 & 19911.3208 \\ \text { S02R14 } & 3.96588899 & 130.383632 & 913.14268 \\ \text { S02R15 } & 1.53250364 & 39.8678557 & 226.405565 \\ \text { S02R16 } & 1.06062954 & 10.9244258 & -27.708545\end{array}$

\author{
$\begin{array}{llll}1.02187178 & 6.25259473 & -79.263776\end{array}$ \\ $\begin{array}{llll}1.08368712 & -9.5449707 & -253.96496\end{array}$ \\ $\begin{array}{llll}1.81187427 & -18.024468 & -426.08834\end{array}$ \\ $\begin{array}{llll}7.51158524 & 38.4037867 & -469.5048\end{array}$ \\ $136.006561 \quad 3321.64806 \quad 19905$ \\ $3.96588899 \quad 130.383632 \quad 906.821854$ \\ $\begin{array}{lll}1.53250364 & 39.8678557 & 220.084739\end{array}$ \\ $\begin{array}{lll}1.06062954 & 10.9244258 & -34.029371\end{array}$
}

\begin{tabular}{|c|c|c|c|c|c|c|}
\hline \multicolumn{7}{|c|}{ Source S03 } \\
\hline Line & ai & bi & ci & ao & bo & co \\
\hline $\mathrm{S} 03 \mathrm{CO} 1$ & 1.10723573 & -5.1339105 & -458.36159 & 1.10723573 & -5.1339105 & -464.68241 \\
\hline $\mathrm{S} 03 \mathrm{C} 02$ & 1.10723573 & -5.1339105 & -458.36159 & 1.10723573 & -5.1339105 & -464.68241 \\
\hline $\mathrm{S} 03 \mathrm{CO} 3$ & 1.10723573 & -5.1339105 & -458.36159 & 1.10723573 & -5.1339105 & -464.68241 \\
\hline S03C04 & 1.10723573 & -5.1339105 & -458.36159 & 1.10723573 & -5.1339105 & -464.68241 \\
\hline $\mathrm{S} 03 \mathrm{C} 05$ & 1.10723573 & -5.1339105 & -458.36159 & 1.10723573 & -5.1339105 & -464.68241 \\
\hline S03C06 & 1.10723573 & -5.1339105 & -458.36159 & 1.10723573 & -5.1339105 & -464.68241 \\
\hline S03C07 & 311.488433 & -843.59015 & 53.1958258 & 311.488433 & -843.59015 & 46.875 \\
\hline $\mathrm{S} 03 \mathrm{C} 08$ & 311.488433 & -843.59015 & 53.1958258 & 311.488433 & -843.59015 & 46.875 \\
\hline S03C09 & 311.488433 & -843.59015 & 53.1958258 & 311.488433 & -843.59015 & 46.875 \\
\hline $\mathrm{S} 03 \mathrm{C} 10$ & 311.488433 & -843.59015 & 53.1958258 & 311.488433 & -843.59015 & 46.875 \\
\hline $\mathrm{S} 03 \mathrm{C} 11$ & 311.488433 & -843.59015 & 53.1958258 & 311.488433 & -843.59015 & 46.875 \\
\hline $\mathrm{S} 03 \mathrm{C} 12$ & 311.488433 & -843.59015 & 53.1958258 & 311.488433 & -843.59015 & 46.875 \\
\hline $\mathrm{S} 03 \mathrm{C} 13$ & 1.07972977 & 3.8170627 & -474.12261 & 1.07972977 & 3.8170627 & -480.44344 \\
\hline $\mathrm{S} 03 \mathrm{C} 14$ & 1.07972977 & 3.8170627 & -474.12261 & 1.07972977 & 3.8170627 & -480.44344 \\
\hline S03C15 & 1.07972977 & 3.8170627 & -474.12261 & 1.07972977 & 3.8170627 & -480.44344 \\
\hline S03C16 & 1.07972977 & 3.8170627 & -474.12261 & 1.07972977 & 3.8170627 & -480.44344 \\
\hline $\mathrm{S} 03 \mathrm{C} 17$ & 1.07972977 & 3.8170627 & -474.12261 & 1.07972977 & 3.8170627 & -480.44344 \\
\hline S03C18 & 1.07972977 & 3.8170627 & -474.12261 & 1.07972977 & 3.8170627 & -480.44344 \\
\hline SO3C19 & 89.7455285 & -451.0057 & 53.1958258 & 89.7455285 & -451.0057 & 46.875 \\
\hline $\mathrm{S} 03 \mathrm{C} 20$ & 89.7455285 & -451.0057 & 53.1958258 & 89.7455285 & -451.0057 & 46.875 \\
\hline $\mathrm{S} 03 \mathrm{C} 21$ & 89.7455285 & -451.0057 & 53.1958258 & 89.7455285 & -451.0057 & 46.875 \\
\hline $\mathrm{S} 03 \mathrm{C} 22$ & 89.7455285 & -451.0057 & 53.1958258 & 89.7455285 & -451.0057 & 46.875 \\
\hline $\mathrm{S} 03 \mathrm{C} 23$ & 89.7455285 & -451.0057 & 53.1958258 & 89.7455285 & -451.0057 & 46.875 \\
\hline $\mathrm{S} 03 \mathrm{C} 24$ & 89.7455285 & -451.0057 & 53.1958258 & 89.7455285 & -451.0057 & 46.875 \\
\hline S03R01 & 1.00264417 & -0.7559009 & -465.78479 & 1.00264417 & -0.7559009 & -472.10562 \\
\hline S03R02 & 1.22615543 & -7.7049586 & -454.18243 & 1.22615543 & -7.7049586 & -460.50326 \\
\hline SO3R03 & 2.19012748 & -20.052268 & -435.34371 & 2.19012748 & -20.052268 & -441.66454 \\
\hline S03R04 & 7.92801699 & -62.744487 & -377.74468 & 7.92801699 & -62.744487 & -384.0655 \\
\hline S03R05 & 7198.02448 & -24252.285 & 19911.3208 & 7198.02448 & -24252.285 & 19905 \\
\hline S03R06 & 7.08643073 & 14.2655567 & -511.44907 & 7.08643073 & 14.2655567 & -517.7699 \\
\hline S03R07 & 2.10958547 & 11.3639084 & -490.71199 & 2.10958547 & 11.3639084 & -497.03281 \\
\hline S03R08 & 1.21434621 & 5.96251236 & -478.34297 & 1.21434621 & 5.96251236 & -484.6638 \\
\hline S03R09 & 1.00251635 & 0.71936079 & -468.39626 & 1.00251635 & 0.71936079 & -474.71709 \\
\hline
\end{tabular}


REVISED METHODOLOGY FOR DETERMINING CESIUM-137 CONTENT OF HN-200 GROUT CONTAINERS.

$\begin{array}{lccc}\text { S03R10 } & 1.12566471 & -5.5914939 & -457.60923 \\ \text { S03R11 } & 1.83211083 & -16.189782 & -441.05985 \\ \text { S03R12 } & 5.88400102 & -49.378267 & -395.00194 \\ \text { S03R13 } & 5873.93565 & -21908.054 & 19911.3208 \\ \text { S03R14 } & 6.55932427 & 14.5459308 & -510.29325 \\ \text { S03R15 } & 1.89251166 & 10.5553017 & -488.59997 \\ \text { S03R16 } & 1.1325881 & 4.81691831 & -476.05849\end{array}$

\author{
$1.12566471-5.5914939-463.93005$ \\ $\begin{array}{lll}1.83211083 & -16.189782 & -447.38067\end{array}$ \\ $\begin{array}{llll}5.88400102 & -49.378267 & -401.32276\end{array}$ \\ $\begin{array}{lll}5873.93565 & -21908.054 & 19905\end{array}$ \\ $\begin{array}{llll}6.55932427 & 14.5459308 & -516.61408\end{array}$ \\ $\begin{array}{llll}1.89251166 & 10.5553017 & -494.9208\end{array}$ \\ $\begin{array}{llll}1.1325881 & 4.81691831 & -482.37931\end{array}$
}

\begin{tabular}{|c|c|c|c|c|c|c|}
\hline \multicolumn{7}{|c|}{ Source S04 } \\
\hline Line & ai & bi & ci & ao & bo & co \\
\hline SO4C01 & 2.08953784 & -52.161624 & 104.501358 & 2.08953784 & -52.161624 & 98.1805327 \\
\hline $\mathrm{S} 04 \mathrm{C} 02$ & 2.08953784 & -52.161624 & 104.501358 & 2.08953784 & -52.161624 & 98.1805327 \\
\hline SO4C03 & 2.08953784 & -52.161624 & 104.501358 & 2.08953784 & -52.161624 & 98.1805327 \\
\hline S04C04 & 2.08953784 & -52.161624 & 104.501358 & 2.08953784 & -52.161624 & 98.1805327 \\
\hline S04C05 & 2.08953784 & -52.161624 & 104.501358 & 2.08953784 & -52.161624 & 98.1805327 \\
\hline S04C06 & 2.08953784 & -52.161624 & 104.501358 & 2.08953784 & -52.161624 & 98.1805327 \\
\hline S04C07 & 61.9849997 & -373.86973 & 53.1958258 & 61.9849997 & -373.86973 & 46.875 \\
\hline S04C08 & 61.9849997 & -373.86973 & 53.1958258 & 61.9849997 & -373.86973 & 46.875 \\
\hline S04C09 & 61.9849997 & -373.86973 & 53.1958258 & 61.9849997 & -373.86973 & 46.875 \\
\hline SO4C10 & 61.9849997 & -373.86973 & 53.1958258 & 61.9849997 & -373.86973 & 46.875 \\
\hline S04C11 & 61.9849997 & -373.86973 & 53.1958258 & 61.9849997 & -373.86973 & 46.875 \\
\hline $\mathrm{S} 04 \mathrm{Cl} 2$ & 61.9849997 & -373.86973 & 53.1958258 & 61.9849997 & -373.86973 & 46.875 \\
\hline S04C13 & 1.42544099 & 20.3679875 & -276.02873 & 1.42544099 & 20.3679875 & -282.34956 \\
\hline S04C14 & 1.42544099 & 20.3679875 & -276.02873 & 1.42544099 & 20.3679875 & -282.34956 \\
\hline S04C15 & 1.42544099 & 20.3679875 & -276.02873 & 1.42544099 & 20.3679875 & -282.34956 \\
\hline $504 \mathrm{C} 16$ & 1.42544099 & 20.3679875 & -276.02873 & 1.42544099 & 20.3679875 & -282.34956 \\
\hline S04C17 & 1.42544099 & 20.3679875 & -276.02873 & 1.42544099 & 20.3679875 & -282.34956 \\
\hline S04C18 & 1.42544099 & 20.3679875 & -276.02873 & 1.42544099 & 20.3679875 & -282.34956 \\
\hline S04C19 & 1.72938787 & -40.887265 & 53.1958258 & 1.72938787 & -40.887265 & 46.875 \\
\hline $\mathrm{S} 04 \mathrm{C} 20$ & 1.72938787 & -40.887265 & 53.1958258 & 1.72938787 & -40.887265 & 46.875 \\
\hline S04C21 & 1.72938787 & -40.887265 & 53.1958258 & 1.72938787 & -40.887265 & 46.875 \\
\hline $\mathrm{S} 04 \mathrm{C} 22$ & 1.72938787 & -40.887265 & 53.1958258 & 1.72938787 & -40.887265 & 46.875 \\
\hline S04C23 & 1.72938787 & -40.887265 & 53.1958258 & 1.72938787 & -40.887265 & 46.875 \\
\hline S04C24 & 1.72938787 & -40.887265 & 53.1958258 & 1.72938787 & -40.887265 & 46.875 \\
\hline S04R01 & 1.01955995 & -5.5917009 & -120.17621 & 1.01955995 & -5.5917009 & -126.49703 \\
\hline SO4R02 & 1.34127495 & -26.225621 & -15.974453 & 1.34127495 & -26.225621 & -22.295279 \\
\hline S04R03 & 2.58502013 & -65.907966 & 165.334625 & 2.58502013 & -65.907966 & 159.013799 \\
\hline S04R04 & 10.4638806 & -222.83974 & 791.956945 & 10.4638806 & -222.83974 & 785.636119 \\
\hline S04R05 & 862.023954 & -8388.4901 & 19911.3208 & 862.023954 & -8388.4901 & 19905 \\
\hline S04R06 & 7.30908445 & 26.8126508 & -491.32066 & 7.30908445 & 26.8126508 & -497.64148 \\
\hline S04R07 & 2.27338268 & 29.2987984 & -351.27673 & 2.27338268 & 29.2987984 & -357.59756 \\
\hline S04R08 & 1.28864668 & 17.4597653 & -255.77992 & 1.28864668 & 17.4597653 & -262.10075 \\
\hline S04R09 & 1.01675608 & 4.79014538 & -177.46238 & 1.01675608 & 4.79014538 & -183.7832 \\
\hline SO4R 10 & 1.06650933 & -10.647458 & -93.671021 & 1.06650933 & -10.647458 & -99.991847 \\
\hline
\end{tabular}


REVISED METHODOLOGY FOR DETERMINING CESIUM-137 CONTENT OF HN-200 GROUT CONTAINERS.

$\begin{array}{lccc}\text { S04R11 } & 1.58963252 & -36.03075 & 30.6258446 \\ \text { S04R12 } & 4.51035887 & -110.81086 & 354.67847 \\ \text { S04R13 } & 502.220862 & -6400.1588 & 19911.3208 \\ \text { SO4R14 } & 6.2656796 & 30.000967 & -477.0758 \\ \text { S04R15 } & 1.73393444 & 24.8162924 & -310.03173 \\ \text { S04R16 } & 1.07863582 & 9.90910116 & -207.64029\end{array}$

\begin{tabular}{|c|c|c|c|}
\hline & & & Source S05 \\
\hline Line & ai & bi & ci \\
\hline S05C01 & 1.00444471 & -0.2127904 & -517.26125 \\
\hline $\mathrm{S} 05 \mathrm{C} 02$ & 1.00444471 & -0.2127904 & -517.26125 \\
\hline S05C03 & 1.00444471 & -0.2127904 & -517.26125 \\
\hline $\mathrm{S} 05 \mathrm{CO} 4$ & 1.00444471 & -0.2127904 & -517.26125 \\
\hline $\mathrm{S} 05 \mathrm{C} 05$ & 1.00444471 & -0.2127904 & -517.26125 \\
\hline S05C06 & 1.00444471 & -0.2127904 & -517.26125 \\
\hline S05C07 & 3.9522955 & 82.2599989 & 53.1958258 \\
\hline S05C08 & 3.9522955 & 82.2599989 & 53.1958258 \\
\hline S05C09 & 3.9522955 & 82.2599989 & 53.1958258 \\
\hline S05C10 & 3.9522955 & 82.2599989 & 53.1958258 \\
\hline S05C11 & 3.9522955 & 82.2599989 & 53.1958258 \\
\hline $\mathrm{S} 05 \mathrm{C} 12$ & 3.9522955 & 82.2599989 & 53.1958258 \\
\hline S05C13 & 1.04528783 & 2.16815476 & -493.85798 \\
\hline $\mathrm{S} 05 \mathrm{C} 14$ & 1.04528783 & 2.16815476 & -493.85798 \\
\hline S05C15 & 1.04528783 & 2.16815476 & -493.85798 \\
\hline S05C16 & 1.04528783 & 2.16815476 & -493.85798 \\
\hline S05C17 & 1.04528783 & 2.16815476 & -493.85798 \\
\hline S05C18 & 1.04528783 & 2.16815476 & -493.85798 \\
\hline S05C 19 & 5.43757236 & 100.851302 & 53.1958258 \\
\hline $\mathrm{S} 05 \mathrm{C} 20$ & 5.43757236 & 100.851302 & 53.1958258 \\
\hline $\mathrm{S} 05 \mathrm{C} 21$ & 5.43757236 & 100.851302 & 53.1958258 \\
\hline $\mathrm{S} 05 \mathrm{C} 22$ & 5.43757236 & 100.851302 & 53.1958258 \\
\hline S05C23 & 5.43757236 & 100.851302 & 53.1958258 \\
\hline S05C24 & 5.43757236 & 100.851302 & 53.1958258 \\
\hline S05R01 & 1.00024442 & -0.0698741 & -514.81427 \\
\hline S05R02 & 1.13071176 & 5.02961481 & -471.42486 \\
\hline S05R03 & 1.75413225 & 23.1001566 & -342.91032 \\
\hline S05R04 & 4.71904938 & 102.477029 & 186.121074 \\
\hline S05R05 & 127.006178 & 3209.01758 & 19911.3208 \\
\hline S05R06 & 10.4431938 & 221.465112 & 778.661398 \\
\hline S05R07 & 2.24077995 & 25.6474341 & -387.27232 \\
\hline S05R08 & 1.19119185 & 2.66093304 & -510.54963 \\
\hline S05R09 & 1.00034726 & 0.09927316 & -512.71315 \\
\hline S05R 10 & 1.22840472 & 8.04094881 & -449.03803 \\
\hline S05R 11 & 2.36607624 & 39.879735 & -228.75674 \\
\hline
\end{tabular}

1.58963252

$-36.03075$

$502.220862-6400.1588$

$6.2656796 \quad 30.000967$

1.7339344424 .8162924

1.078635829 .90910116
24.3050188

348.357644

19905

$-483.39662$

$-316.35255$

$-213.96111$

\begin{tabular}{ccc} 
ao & bo & co \\
1.00444471 & -0.2127904 & -523.58207 \\
1.00444471 & -0.2127904 & -523.58207 \\
1.00444471 & -0.2127904 & -523.58207 \\
1.00444471 & -0.2127904 & -523.58207 \\
1.00444471 & -0.2127904 & -523.58207 \\
1.00444471 & -0.2127904 & -523.58207 \\
3.9522955 & 82.2599989 & 46.875 \\
3.9522955 & 82.2599989 & 46.875 \\
3.9522955 & 82.2599989 & 46.875 \\
3.9522955 & 82.2599989 & 46.875 \\
3.9522955 & 82.2599989 & 46.875 \\
3.9522955 & 82.2599989 & 46.875 \\
1.04528783 & 2.16815476 & -500.1788 \\
1.04528783 & 2.16815476 & -500.1788 \\
1.04528783 & 2.16815476 & -500.1788 \\
1.04528783 & 2.16815476 & -500.1788 \\
1.04528783 & 2.16815476 & -500.1788 \\
1.04528783 & 2.16815476 & -500.1788 \\
5.43757236 & 100.851302 & 46.875 \\
5.43757236 & 100.851302 & 46.875 \\
5.43757236 & 100.851302 & 46.875 \\
5.43757236 & 100.851302 & 46.875 \\
5.43757236 & 100.851302 & 46.875 \\
5.43757236 & 100.851302 & 46.875 \\
1.00024442 & -0.0698741 & -521.13509 \\
1.13071176 & 5.02961481 & -477.74568 \\
1.75413225 & 23.1001566 & -349.23114 \\
4.71904938 & 102.477029 & 179.800248 \\
127.006178 & 3209.01758 & 19905 \\
10.4431938 & 221.465112 & 772.340572 \\
2.24077995 & 25.6474341 & -393.59314 \\
1.19119185 & 2.66093304 & -516.87045 \\
1.00034726 & 0.09927316 & -519.03398 \\
1.22840472 & 8.04094881 & -455.35885 \\
2.36607624 & 39.879735 & -235.07757 \\
& & \\
\hline & &
\end{tabular}




\section{REVISED METHODOLOGY FOR DETERMINING CESIUM-137 CONTENT OF HN-200 GROUT CONTAINERS.}

$\begin{array}{llll}\text { S05R12 } & 11.1646756 & 269.960855 & 1272.64615 \\ \text { S05R13 } & 135.875181 & 3320.03145 & 19911.3208 \\ \text { S05R14 } & 5.00319334 & 90.4910594 & -8.4268446 \\ \text { S05R15 } & 1.83028594 & 16.3563635 & -439.25432 \\ \text { S05R16 } & 1.15615301 & 1.98836941 & -513.47837\end{array}$

$\begin{array}{lccc}\text { Line } & \text { ai } & \text { bi } & \text { ci } \\ \text { S06C01 } & 1.24438865 & -11.700107 & -379.77243 \\ \text { S06C02 } & 1.24438865 & -11.700107 & -379.77243 \\ \text { S06C03 } & 1.24438865 & -11.700107 & -379.77243 \\ \text { S06C04 } & 1.24438865 & -11.700107 & -379.77243 \\ \text { S06C05 } & 1.24438865 & -11.700107 & -379.77243 \\ \text { S06C06 } & 1.24438865 & -11.700107 & -379.77243 \\ \text { S06C07 } & 10.1842574 & -145.08777 & 53.1958258 \\ \text { S06C08 } & 10.1842574 & -145.08777 & 53.1958258 \\ \text { S06C09 } & 10.1842574 & -145.08777 & 53.1958258 \\ \text { S06C10 } & 10.1842574 & -145.08777 & 53.1958258 \\ \text { S06C11 } & 10.1842574 & -145.08777 & 53.1958258 \\ \text { S06C12 } & 10.1842574 & -145.08777 & 53.1958258 \\ \text { S06C13 } & 1.03999718 & 1.91486516 & -496.88954 \\ \text { S06C14 } & 1.03999718 & 1.91486516 & -496.88954 \\ \text { S06C15 } & 1.03999718 & 1.91486516 & -496.88954 \\ \text { S06C16 } & 1.03999718 & 1.91486516 & -496.88954 \\ \text { S06C17 } & 1.03999718 & 1.91486516 & -496.88954 \\ \text { S06C18 } & 1.03999718 & 1.91486516 & -496.88954 \\ \text { S06C19 } & 3.84622342 & -80.768735 & 53.1958258 \\ \text { S06C20 } & 3.84622342 & -80.768735 & 53.1958258 \\ \text { S06C21 } & 3.84622342 & -80.768735 & 53.1958258 \\ \text { S06C22 } & 3.84622342 & -80.768735 & 53.1958258 \\ \text { S06C23 } & 3.84622342 & -80.768735 & 53.1958258 \\ \text { S06C24 } & 3.84622342 & -80.768735 & 53.1958258 \\ \text { S06R01 } & 1.00263539 & -0.7533917 & -465.96412 \\ \text { S06R02 } & 1.25463319 & -12.047848 & -377.29854 \\ \text { S06R03 } & 2.40798221 & -44.754311 & -164.16645 \\ \text { S06R04 } & 10.7165634 & -239.70777 & 958.590484 \\ \text { S06R05 } & 218.765531 & -4218.6233 & 19911.3208 \\ \text { S06R06 } & 5.5861604 & -63.884537 & -297.33254 \\ \text { S06R07 } & 1.94099388 & -5.873201 & -510.6437 \\ \text { S06R08 } & 1.18713517 & 2.09971104 & -513.91824 \\ \text { S06R09 } & 1.00198291 & 0.56686403 & -479.29502 \\ \text { S06R10 } & 1.11337854 & -6.8918783 & -415.07489 \\ \text { S06R11 } & 1.71819674 & -26.130807 & -282.12283 \\ \text { S06R12 } & 4.72988153 & -102.03321 & 177.98729 \\ & & & \end{array}$

$11.1646756 \quad 269.960855 \quad 1266.32533$

$135.875181 \quad 3320.03145 \quad 19905$

$\begin{array}{lll}5.00319334 & 90.4910594 & -14.74767\end{array}$

$\begin{array}{lll}1.83028594 & 16.3563635 & -445.57514\end{array}$

$\begin{array}{llll}1.15615301 & 1.98836941 & -519.7992\end{array}$

\begin{tabular}{ccc} 
ao & bo & co \\
1.24438865 & -11.700107 & -386.09326 \\
1.24438865 & -11.700107 & -386.09326 \\
1.24438865 & -11.700107 & -386.09326 \\
1.24438865 & -11.700107 & -386.09326 \\
1.24438865 & -11.700107 & -386.09326 \\
1.24438865 & -11.700107 & -386.09326 \\
10.1842574 & -145.08777 & 46.875 \\
10.1842574 & -145.08777 & 46.875 \\
10.1842574 & -145.08777 & 46.875 \\
10.1842574 & -145.08777 & 46.875 \\
10.1842574 & -145.08777 & 46.875 \\
10.1842574 & -145.08777 & 46.875 \\
1.03999718 & 1.91486516 & -503.21036 \\
1.03999718 & 1.91486516 & -503.21036 \\
1.03999718 & 1.91486516 & -503.21036 \\
1.03999718 & 1.91486516 & -503.21036 \\
1.03999718 & 1.91486516 & -503.21036 \\
1.03999718 & 1.91486516 & -503.21036 \\
3.84622342 & -80.768735 & 46.875 \\
3.84622342 & -80.768735 & 46.875 \\
3.84622342 & -80.768735 & 46.875 \\
3.84622342 & -80.768735 & 46.875 \\
3.84622342 & -80.768735 & 46.875 \\
3.84622342 & -80.768735 & 46.875 \\
1.00263539 & -0.7533917 & -472.28494 \\
1.25463319 & -12.047848 & -383.61937 \\
2.40798221 & -44.754311 & -170.48727 \\
10.7165634 & -239.70777 & 952.269658 \\
218.765531 & -4218.6233 & 19905 \\
5.5861604 & -63.884537 & -303.65337 \\
1.94099388 & -5.873201 & -516.96453 \\
1.18713517 & 2.09971104 & -520.23906 \\
1.00198291 & 0.56686403 & -485.61584 \\
1.11337854 & -6.8918783 & -421.39571 \\
1.71819674 & -26.130807 & -288.44366 \\
4.72988153 & -102.03321 & 171.666464 \\
& & \\
\hline
\end{tabular}




\section{REVISED METHODOLOGY FOR DETERMINING CESIUM-137 CONTENT OF HN-200 GROUT CONTAINERS.}

\author{
SO6R 13 \\ S06R14 \\ S06R 15 \\ SO6R16 \\ $\begin{array}{lll}180.919359 & -3834.5564 & 19911.3208\end{array}$ \\ $8.90239049-122.06245 \quad-48.45569$ \\ $\begin{array}{llll}2.07461723 & -7.6773891 & -506.09569\end{array}$ \\ $\begin{array}{lll}1.15427319 & 2.22385692 & -511.79382\end{array}$
}

\author{
$180.919359-3834.5564 \quad 19905$ \\ $\begin{array}{llll}8.90239049 & -122.06245 & -54.776515\end{array}$ \\ $\begin{array}{llll}2.07461723 & -7.6773891 & -512.41651\end{array}$ \\ $\begin{array}{llll}1.15427319 & 2.22385692 & -518.11465\end{array}$
}

\begin{tabular}{|c|c|c|c|c|c|c|}
\hline \multicolumn{7}{|c|}{ Source S07 } \\
\hline Line & ai & bi & ci & ao & bo & co \\
\hline $\mathrm{S} 07 \mathrm{C} 01$ & 1.47062164 & -22.531011 & -250.14004 & 1.47062164 & -22.531011 & -256.46087 \\
\hline $\mathrm{S} 07 \mathrm{C} 02$ & 1.47062164 & -22.531011 & -250.14004 & 1.47062164 & -22.531011 & -256.46087 \\
\hline $\mathrm{S} 07 \mathrm{C} 03$ & 1.47062164 & -22.531011 & -250.14004 & 1.47062164 & -22.531011 & -256.46087 \\
\hline $\mathrm{S} 07 \mathrm{C} 04$ & 1.47062164 & -22.531011 & -250.14004 & 1.47062164 & -22.531011 & -256.46087 \\
\hline S07C05 & 1.47062164 & -22.531011 & -250.14004 & 1.47062164 & -22.531011 & -256.46087 \\
\hline S07C06 & 1.47062164 & -22.531011 & -250.14004 & 1.47062164 & -22.531011 & -256.46087 \\
\hline S07C07 & 200.407304 & 676.05078 & 53.1958258 & 200.407304 & 676.05078 & 46.875 \\
\hline S07C08 & 200.407304 & 676.05078 & 53.1958258 & 200.407304 & 676.05078 & 46.875 \\
\hline S07C09 & 200.407304 & 676.05078 & 53.1958258 & 200.407304 & 676.05078 & 46.875 \\
\hline S07C10 & 200.407304 & 676.05078 & 53.1958258 & 200.407304 & 676.05078 & 46.875 \\
\hline S07C11 & 200.407304 & 676.05078 & 53.1958258 & 200.407304 & 676.05078 & 46.875 \\
\hline S07C12 & 200.407304 & 676.05078 & 53.1958258 & 200.407304 & 676.05078 & 46.875 \\
\hline $\mathrm{S} 07 \mathrm{C} 13$ & 1.77952699 & 37.3198546 & -73.136071 & 1.77952699 & 37.3198546 & -79.456896 \\
\hline S07C14 & 1.77952699 & 37.3198546 & -73.136071 & 1.77952699 & 37.3198546 & -79.456896 \\
\hline S07C15 & 1.77952699 & 37.3198546 & -73.136071 & 1.77952699 & 37.3198546 & -79.456896 \\
\hline S07C16 & 1.77952699 & 37.3198546 & -73.136071 & 1.77952699 & 37.3198546 & -79.456896 \\
\hline S07C17 & 1.77952699 & 37.3198546 & -73.136071 & 1.77952699 & 37.3198546 & -79.456896 \\
\hline S07C18 & 1.77952699 & 37.3198546 & -73.136071 & 1.77952699 & 37.3198546 & -79.456896 \\
\hline S07C19 & 4.29771512 & 86.939143 & 53.1958258 & 4.29771512 & 86.939143 & 46.875 \\
\hline $\mathrm{S} 07 \mathrm{C} 20$ & 4.29771512 & 86.939143 & 53.1958258 & 4.29771512 & 86.939143 & 46.875 \\
\hline $\mathrm{S} 07 \mathrm{C} 21$ & 4.29771512 & 86.939143 & 53.1958258 & 4.29771512 & 86.939143 & 46.875 \\
\hline $\mathrm{S} 07 \mathrm{C} 22$ & 4.29771512 & 86.939143 & 53.1958258 & 4.29771512 & 86.939143 & 46.875 \\
\hline $\mathrm{S} 07 \mathrm{C} 23$ & 4.29771512 & 86.939143 & 53.1958258 & 4.29771512 & 86.939143 & 46.875 \\
\hline S07C24 & 4.29771512 & 86.939143 & 53.1958258 & 4.29771512 & 86.939143 & 46.875 \\
\hline S07R01 & 1.01603827 & -4.5849415 & -192.12805 & 1.01603827 & -4.5849415 & -198.44887 \\
\hline S07R02 & 1.29359636 & -18.265246 & -235.72825 & 1.29359636 & -18.265246 & -242.04907 \\
\hline S07R03 & 2.31956158 & -34.534288 & -293.85859 & 2.31956158 & -34.534288 & -300.17942 \\
\hline S07R04 & 7.80599247 & -55.544403 & -406.48219 & 7.80599247 & -55.544403 & -412.80301 \\
\hline S07R05 & 2888.463 & 15361.5271 & 19911.3208 & 2888.463 & 15361.5271 & 19905 \\
\hline S07R06 & 9.47987628 & 158.589106 & 221.668086 & 9.47987628 & 158.589106 & 215.34726 \\
\hline S07R07 & 2.48619577 & 53.9926108 & -29.428201 & 2.48619577 & 53.9926108 & -35.749027 \\
\hline S07R08 & 1.32156907 & 22.8935959 & -112.33984 & 1.32156907 & 22.8935959 & -118.66066 \\
\hline S07R09 & 1.01744503 & 4.98709816 & -163.38641 & 1.01744503 & 4.98709816 & -169.70723 \\
\hline S07R10 & 1.0787588 & -9.9006005 & -208.66223 & 1.0787588 & -9.9006005 & -214.98306 \\
\hline S07R11 & 1.7092454 & -26.869347 & -265.3257 & 1.7092454 & -26.869347 & -271.64653 \\
\hline S07R12 & 5.82671914 & -52.211914 & -378.61052 & 5.82671914 & -52.211914 & -384.93134 \\
\hline S07R 13 & 1717.43785 & 11843.7769 & 19911.3208 & 1717.43785 & 11843.7769 & 19905 \\
\hline
\end{tabular}




\section{REVISED METHODOLOGY FOR DETERMINING CESIUM-137 CONTENT OF HN-200 GROUT CONTAINERS.}

$\begin{array}{lllll}\text { S07R14 } & 4.87394969 & 96.0299175 & 75.3045175\end{array}$

$\begin{array}{lllll}\text { S07R15 } & 1.62972389 & 33.1167891 & -84.410227\end{array}$

$\begin{array}{lllll}\text { S07R } 16 & 1.07190771 & 10.3443404 & -147.78477\end{array}$
$4.8739496996 .0299175 \quad 68.9836917$

$\begin{array}{llll}1.62972389 & 33.1167891 & -90.731053\end{array}$

$1.07190771 \quad 10.3443404-154.10559$

\begin{tabular}{|c|c|c|c|c|c|c|}
\hline \multicolumn{7}{|c|}{ Source S08 } \\
\hline Line & ai & bi & ci & ao & bo & co \\
\hline S08C01 & 1.13712023 & -6.5646312 & -441.23765 & 1.13712023 & -6.5646312 & -447.55848 \\
\hline $\mathrm{S} 08 \mathrm{C} 02$ & 1.13712023 & -6.5646312 & -441.23765 & 1.13712023 & -6.5646312 & -447.55848 \\
\hline S08C03 & 1.13712023 & -6.5646312 & -441.23765 & 1.13712023 & -6.5646312 & -447.55848 \\
\hline S08C04 & 1.13712023 & -6.5646312 & -441.23765 & 1.13712023 & -6.5646312 & -447.55848 \\
\hline S08C05 & 1.13712023 & -6.5646312 & -441.23765 & 1.13712023 & -6.5646312 & -447.55848 \\
\hline S08C06 & 1.13712023 & -6.5646312 & -441.23765 & 1.13712023 & -6.5646312 & -447.55848 \\
\hline S08C07 & 531.968681 & 1103.17202 & 53.1958258 & 531.968681 & 1103.17202 & 46.875 \\
\hline S08C08 & 531.968681 & 1103.17202 & 53.1958258 & 531.968681 & 1103.17202 & 46.875 \\
\hline S08C09 & 531.968681 & 1103.17202 & 53.1958258 & 531.968681 & 1103.17202 & 46.875 \\
\hline $\mathrm{S} 08 \mathrm{C} 10$ & 531.968681 & 1103.17202 & 53.1958258 & 531.968681 & 1103.17202 & 46.875 \\
\hline S08C11 & 531.968681 & 1103.17202 & 53.1958258 & 531.968681 & 1103.17202 & 46.875 \\
\hline S08C12 & 531.968681 & 1103.17202 & 53.1958258 & 531.968681 & 1103.17202 & 46.875 \\
\hline $\mathrm{S} 08 \mathrm{C} 13$ & 1.17467177 & 8.36241083 & -419.72048 & 1.17467177 & 8.36241083 & -426.0413 \\
\hline S08C14 & 1.17467177 & 8.36241083 & -419.72048 & 1.17467177 & 8.36241083 & -426.0413 \\
\hline S08C15 & 1.17467177 & 8.36241083 & -419.72048 & 1.17467177 & 8.36241083 & -426.0413 \\
\hline S08C16 & 1.17467177 & 8.36241083 & -419.72048 & 1.17467177 & 8.36241083 & -426.0413 \\
\hline S08C17 & 1.17467177 & 8.36241083 & -419.72048 & 1.17467177 & 8.36241083 & -426.0413 \\
\hline S08C18 & 1.17467177 & 8.36241083 & -419.72048 & 1.17467177 & 8.36241083 & -426.0413 \\
\hline S08C19 & 101.973127 & 481.073784 & 53.1958258 & 101.973127 & 481.073784 & 46.875 \\
\hline $\mathrm{S} 08 \mathrm{C} 20$ & 101.973127 & 481.073784 & 53.1958258 & 101.973127 & 481.073784 & 46.875 \\
\hline S08C21 & 101.973127 & 481.073784 & 53.1958258 & 101.973127 & 481.073784 & 46.875 \\
\hline S08C22 & 101.973127 & 481.073784 & 53.1958258 & 101.973127 & 481.073784 & 46.875 \\
\hline S08C23 & 101.973127 & 481.073784 & 53.1958258 & 101.973127 & 481.073784 & 46.875 \\
\hline S08C24 & 101.973127 & 481.073784 & 53.1958258 & 101.973127 & 481.073784 & 46.875 \\
\hline S08R01 & 1.00423829 & -1.2116213 & -433.21502 & 1.00423829 & -1.2116213 & -439.53585 \\
\hline S08R02 & 1.23052746 & -8.3591992 & -444.02946 & 1.23052746 & -8.3591992 & -450.35029 \\
\hline S08R03 & 2.16116937 & -16.897977 & -458.33091 & 2.16116937 & -16.897977 & -464.65173 \\
\hline S08R04 & 7.34747465 & -28.997224 & -486.69101 & 7.34747465 & -28.997224 & -493.01184 \\
\hline S08R05 & 11088.2048 & 30101.4359 & 19911.3208 & 11088.2048 & 30101.4359 & 19905 \\
\hline S08R06 & 8.05599581 & 70.3538138 & -344.4374 & 8.05599581 & 70.3538138 & -350.75823 \\
\hline S08R07 & 2.22959868 & 24.4040556 & -398.72021 & 2.22959868 & 24.4040556 & -405.04104 \\
\hline S08R08 & 1.24085471 & 9.92311347 & -417.60105 & 1.24085471 & 9.92311347 & -423.92187 \\
\hline S08R09 & 1.00441341 & 1.26168374 & -429.63712 & 1.00441341 & 1.26168374 & -435.95795 \\
\hline S08R10 & 1.12029382 & -6.1722178 & -440.6347 & 1.12029382 & -6.1722178 & -446.95553 \\
\hline S08R11 & 1.84671505 & -14.848736 & -454.70797 & 1.84671505 & -14.848736 & -461.0288 \\
\hline S08R12 & 6.30515071 & -27.950107 & -482.9944 & 6.30515071 & -27.950107 & -489.31523 \\
\hline S08R13 & 8520.58126 & 26386.7135 & 19911.3208 & 8520.58126 & 26386.7135 & 19905 \\
\hline S08R 14 & 5.77243901 & 54.8782195 & -362.04708 & 5.77243901 & 54.8782195 & -368.36791 \\
\hline
\end{tabular}


REVISED METHODOLOGY FOR DETERMINING CESIUM-137 CONTENT OF HN-200 GROUT CONTAINERS.

S08R15

S08R16
1.79959388

1.11513593
$19.1241056-405.45896$

$6.71211789-421.98343$
$1.79959388 \quad 19.1241056-411.77979$

$\begin{array}{llll}1.11513593 & 6.71211789 & -428.30426\end{array}$
Source S09

\begin{tabular}{lccc} 
Line & ai & bi & ci \\
S09C01 & 1.00133368 & -0.0638498 & -519.04388 \\
S09C02 & 1.00133368 & -0.0638498 & -519.04388 \\
S09C03 & 1.00133368 & -0.0638498 & -519.04388 \\
S09C04 & 1.00133368 & -0.0638498 & -519.04388 \\
S09C05 & 1.00133368 & -0.0638498 & -519.04388 \\
S09C06 & 1.00133368 & -0.0638498 & -519.04388 \\
S09C07 & 5.96215519 & 106.64585 & 53.1958258 \\
S09C08 & 5.96215519 & 106.64585 & 53.1958258 \\
S09C09 & 5.96215519 & 106.64585 & 53.1958258 \\
S09C10 & 5.96215519 & 106.64585 & 53.1958258 \\
S09C11 & 5.96215519 & 106.64585 & 53.1958258 \\
S09C12 & 5.96215519 & 106.64585 & 53.1958258 \\
S09C13 & 1.01042273 & 0.49898802 & -513.83582 \\
S09C14 & 1.01042273 & 0.49898802 & -513.83582 \\
S09C15 & 1.01042273 & 0.49898802 & -513.83582 \\
S09C16 & 1.01042273 & 0.49898802 & -513.83582 \\
S09C17 & 1.01042273 & 0.49898802 & -513.83582 \\
S09C18 & 1.01042273 & 0.49898802 & -513.83582 \\
S09C19 & 5.00062074 & 95.7574292 & 53.1958258 \\
S09C20 & 5.00062074 & 95.7574292 & 53.1958258 \\
S09C21 & 5.00062074 & 95.7574292 & 53.1958258 \\
S09C22 & 5.00062074 & 95.7574292 & 53.1958258 \\
S09C23 & 5.00062074 & 95.7574292 & 53.1958258 \\
S09C24 & 5.00062074 & 95.7574292 & 53.1958258 \\
S09R01 & 1.00006968 & -0.0199211 & -518.38434 \\
S09R02 & 1.15247528 & 2.44759069 & -509.98567 \\
S09R03 & 1.82938586 & 16.4384437 & -438.35551 \\
S09R04 & 5.07897127 & 87.1790435 & -53.992982 \\
S09R05 & 163.2118 & 3640.9725 & 19911.3208 \\
S09R06 & 10.4505445 & 221.953451 & 783.379647 \\
S09R07 & 2.30079441 & 32.3977854 & -318.08202 \\
S09R08 & 1.21504568 & 6.06467863 & -477.04934 \\
S09R09 & 1.00009573 & 0.02736702 & -517.85219 \\
S09R10 & 1.19571621 & 3.29320007 & -505.9549 \\
S09R11 & 2.23615953 & 25.1330771 & -392.05928 \\
S09R12 & 10.0890076 & 198.083409 & 559.436175 \\
S09R13 & 157.470209 & 3575.9547 & 19911.3208 \\
S09R14 & 4.9229275 & 93.9475664 & 42.6637756 \\
S09R15 & 1.78817469 & 20.1370673 & -391.18765 \\
& & & \\
\hline
\end{tabular}

$\begin{array}{ccc}\text { ao } & \text { bo } & \text { co } \\ 1.00133368 & -0.0638498 & -525.3647 \\ 1.00133368 & -0.0638498 & -525.3647 \\ 1.00133368 & -0.0638498 & -525.3647 \\ 1.00133368 & -0.0638498 & -525.3647 \\ 1.00133368 & -0.0638498 & -525.3647 \\ 1.00133368 & -0.0638498 & -525.3647 \\ 5.96215519 & 106.64585 & 46.875 \\ 5.96215519 & 106.64585 & 46.875 \\ 5.96215519 & 106.64585 & 46.875 \\ 5.96215519 & 106.64585 & 46.875 \\ 5.96215519 & 106.64585 & 46.875 \\ 5.96215519 & 106.64585 & 46.875 \\ 1.01042273 & 0.49898802 & -520.15664 \\ 1.01042273 & 0.49898802 & -520.15664 \\ 1.01042273 & 0.49898802 & -520.15664 \\ 1.01042273 & 0.49898802 & -520.15664 \\ 1.01042273 & 0.49898802 & -520.15664 \\ 1.01042273 & 0.49898802 & -520.15664 \\ 5.00062074 & 95.7574292 & 46.875 \\ 5.00062074 & 95.7574292 & 46.875 \\ 5.00062074 & 95.7574292 & 46.875 \\ 5.00062074 & 95.7574292 & 46.875 \\ 5.00062074 & 95.7574292 & 46.875 \\ 5.00062074 & 95.7574292 & 46.875 \\ 1.00006968 & -0.0199211 & -524.70517 \\ 1.15247528 & 2.44759069 & -516.30649 \\ 1.82938586 & 16.4384437 & -444.67634 \\ 5.07897127 & 87.1790435 & -60.313808 \\ 163.2118 & 3640.9725 & 19905 \\ 10.4505445 & 221.953451 & 777.058821 \\ 2.30079441 & 32.3977854 & -324.40285 \\ 1.21504568 & 6.06467863 & -483.37017 \\ 1.00009573 & 0.02736702 & -524.17302 \\ 1.19571621 & 3.29320007 & -512.27573 \\ 2.23615953 & 25.1330771 & -398.38011 \\ 10.0890076 & 198.083409 & 553.115349 \\ 157.470209 & 3575.9547 & 19905 \\ 4.9229275 & 93.9475664 & 36.3429499 \\ 1.78817469 & 20.1370673 & -397.50847\end{array}$




\section{REVISED METHODOLOGY FOR DETERMINING CESIUM-137 CONTENT OF HN-200 GROUT CONTAINERS.}

$\begin{array}{lllll}\text { S09R16 } & 1.13876998 & 4.10227289 & -489.49057\end{array}$

$1.13876998 \quad 4.10227289 \quad-495.81139$

\begin{tabular}{|c|c|c|c|c|c|c|}
\hline \multicolumn{7}{|c|}{ Source \$10 } \\
\hline Line & ai & bi & ci & ao & bo & co \\
\hline$S 10 C 01$ & 1.10798663 & -5.16986 & -457.93132 & 1.10798663 & -5.16986 & -464.25214 \\
\hline S10C02 & 1.10798663 & -5.16986 & -457.93132 & 1.10798663 & -5.16986 & -464.25214 \\
\hline $\mathrm{S} 10 \mathrm{C} 03$ & 1.10798663 & -5.16986 & -457.93132 & 1.10798663 & -5.16986 & -464.25214 \\
\hline S10C04 & 1.10798663 & -5.16986 & -457.93132 & 1.10798663 & -5.16986 & -464.25214 \\
\hline S10C05 & 1.10798663 & -5.16986 & -457.93132 & 1.10798663 & -5.16986 & -464.25214 \\
\hline S10C06 & 1.10798663 & -5.16986 & -457.93132 & 1.10798663 & -5.16986 & -464.25214 \\
\hline S10C07 & 3.69303804 & 78.5651657 & 53.1958258 & 3.69303804 & 78.5651657 & 46.875 \\
\hline S10C08 & 3.69303804 & 78.5651657 & 53.1958258 & 3.69303804 & 78.5651657 & 46.875 \\
\hline S10C09 & 3.69303804 & 78.5651657 & 53.1958258 & 3.69303804 & 78.5651657 & 46.875 \\
\hline$\$ 10 C 10$ & 3.69303804 & 78.5651657 & 53.1958258 & 3.69303804 & 78.5651657 & 46.875 \\
\hline S10C11 & 3.69303804 & 78.5651657 & 53.1958258 & 3.69303804 & 78.5651657 & 46.875 \\
\hline$S 10 C 12$ & 3.69303804 & 78.5651657 & 53.1958258 & 3.69303804 & 78.5651657 & 46.875 \\
\hline$S 10 C 13$ & 1.4468787 & 21.3943178 & -263.74484 & 1.4468787 & 21.3943178 & -270.06567 \\
\hline S10C14 & 1.4468787 & 21.3943178 & -263.74484 & 1.4468787 & 21.3943178 & -270.06567 \\
\hline S10C15 & 1.4468787 & 21.3943178 & -263.74484 & 1.4468787 & 21.3943178 & -270.06567 \\
\hline S10C16 & 1.4468787 & 21.3943178 & -263.74484 & 1.4468787 & 21.3943178 & -270.06567 \\
\hline S10C17 & 1.4468787 & 21.3943178 & -263.74484 & 1.4468787 & 21.3943178 & -270.06567 \\
\hline S10C18 & 1.4468787 & 21.3943178 & -263.74484 & 1.4468787 & 21.3943178 & -270.06567 \\
\hline S10C19 & 18.8623943 & 202.338545 & 53.1958258 & 18.8623943 & 202.338545 & 46.875 \\
\hline S10C20 & 18.8623943 & 202.338545 & 53.1958258 & 18.8623943 & 202.338545 & 46.875 \\
\hline S10C21 & 18.8623943 & 202.338545 & 53.1958258 & 18.8623943 & 202.338545 & 46.875 \\
\hline $\mathrm{S} 10 \mathrm{C} 22$ & 18.8623943 & 202.338545 & 53.1958258 & 18.8623943 & 202.338545 & 46.875 \\
\hline S10C23 & 18.8623943 & 202.338545 & 53.1958258 & 18.8623943 & 202.338545 & 46.875 \\
\hline S10C24 & 18.8623943 & 202.338545 & 53.1958258 & 18.8623943 & 202.338545 & 46.875 \\
\hline S10R01 & 1.00487288 & -1.3930349 & -420.24962 & 1.00487288 & -1.3930349 & -426.57044 \\
\hline S10R02 & 1.09915378 & 8.26060277 & -347.75827 & 1.09915378 & 8.26060277 & -354.07909 \\
\hline S10R03 & 1.68681122 & 28.6902301 & -220.18815 & 1.68681122 & 28.6902301 & -226.50898 \\
\hline S10R04 & 4.73665026 & 101.755335 & 172.934583 & 4.73665026 & 101.755335 & 166.613757 \\
\hline S10R05 & 264.263302 & 4638.43355 & 19911.3208 & 264.263302 & 4638.43355 & 19905 \\
\hline S10R06 & 7.81649421 & 56.1619037 & -404.12694 & 7.81649421 & 56.1619037 & -410.44776 \\
\hline S10R07 & 1.94925659 & -5.061975 & -513.05975 & 1.94925659 & -5.061975 & -519.38058 \\
\hline S10R08 & 1.12699353 & -5.4450384 & -461.44203 & 1.12699353 & -5.4450384 & -467.76285 \\
\hline S10R09 & 1.00612433 & 1.75079328 & -394.68107 & 1.00612433 & 1.75079328 & -401.0019 \\
\hline S10R10 & 1.27733152 & 15.6348193 & -299.45126 & 1.27733152 & 15.6348193 & -305.77208 \\
\hline S10R11 & 2.44321458 & 48.8938141 & -105.69692 & 2.44321458 & 48.8938141 & -112.01775 \\
\hline S10R12 & 10.3887279 & 217.850544 & 743.911033 & 10.3887279 & 217.850544 & 737.590207 \\
\hline S10R13 & 354.843766 & 5377.51898 & 19911.3208 & 354.843766 & 5377.51898 & 19905 \\
\hline S10R14 & 6.14669144 & 36.1315041 & -456.39425 & 6.14669144 & 36.1315041 & -462.71508 \\
\hline S10R15 & 2.04420235 & -4.5159321 & -514.92549 & 2.04420235 & -4.5159321 & -521.24632 \\
\hline S10R16 & 1.21653442 & -6.2825719 & -474.23714 & 1.21653442 & -6.2825719 & -480.55796 \\
\hline
\end{tabular}




\section{REVISED METHODOLOGY FOR DETERMINING CESIUM-137 CONTENT OF HN-200 GROUT CONTAINERS.}

\begin{tabular}{|c|c|c|c|c|c|c|}
\hline \multicolumn{7}{|c|}{ Source S11 } \\
\hline Line & ai & bi & ci & ao & bo & co \\
\hline S11C01 & 1.34926287 & -16.72096 & -319.67909 & 1.34926287 & -16.72096 & -325.99992 \\
\hline $\mathrm{S} 11 \mathrm{C} 02$ & 1.34926287 & -16.72096 & -319.67909 & 1.34926287 & -16.72096 & -325.99992 \\
\hline S11C03 & 1.34926287 & -16.72096 & -319.67909 & 1.34926287 & -16.72096 & -325.99992 \\
\hline S11C04 & 1.34926287 & -16.72096 & -319.67909 & 1.34926287 & -16.72096 & -325.99992 \\
\hline S11C05 & 1.34926287 & -16.72096 & -319.67909 & 1.34926287 & -16.72096 & -325.99992 \\
\hline S1IC06 & 1.34926287 & -16.72096 & -319.67909 & 1.34926287 & -16.72096 & -325.99992 \\
\hline S11C07 & 2.79829899 & -64.200696 & 53.1958258 & 2.79829899 & -64.200696 & 46.875 \\
\hline S11C08 & 2.79829899 & -64.200696 & 53.1958258 & 2.79829899 & -64.200696 & 46.875 \\
\hline S11C09 & 2.79829899 & -64.200696 & 53.1958258 & 2.79829899 & -64.200696 & 46.875 \\
\hline S11C10 & 2.79829899 & -64.200696 & 53.1958258 & 2.79829899 & -64.200696 & 46.875 \\
\hline S11C11 & 2.79829899 & -64.200696 & 53.1958258 & 2.79829899 & -64.200696 & 46.875 \\
\hline $\mathrm{S} 11 \mathrm{C} 12$ & 2.79829899 & -64.200696 & 53.1958258 & 2.79829899 & -64.200696 & 46.875 \\
\hline S11C13 & 1.03023315 & 1.44741198 & -502.48437 & 1.03023315 & 1.44741198 & -508.80519 \\
\hline S11C14 & 1.03023315 & 1.44741198 & -502.48437 & 1.03023315 & 1.44741198 & -508.80519 \\
\hline S11C15 & 1.03023315 & 1.44741198 & -502.48437 & 1.03023315 & 1.44741198 & -508.80519 \\
\hline S11C16 & 1.03023315 & 1.44741198 & -502.48437 & 1.03023315 & 1.44741198 & -508.80519 \\
\hline S11C17 & 1.03023315 & 1.44741198 & -502.48437 & 1.03023315 & 1.44741198 & -508.80519 \\
\hline S11C18 & 1.03023315 & 1.44741198 & -502.48437 & 1.03023315 & 1.44741198 & -508.80519 \\
\hline S11C19 & 6.41234738 & -111.37857 & 53.1958258 & 6.41234738 & -111.37857 & 46.875 \\
\hline $\mathrm{S} 11 \mathrm{C} 20$ & 6.41234738 & -111.37857 & 53.1958258 & 6.41234738 & -111.37857 & 46.875 \\
\hline S11C21 & 6.41234738 & -111.37857 & 53.1958258 & 6.41234738 & -111.37857 & 46.875 \\
\hline S11C22 & 6.41234738 & -111.37857 & 53.1958258 & 6.41234738 & -111.37857 & 46.875 \\
\hline S11C23 & 6.41234738 & -111.37857 & 53.1958258 & 6.41234738 & -111.37857 & 46.875 \\
\hline S11C24 & 6.41234738 & -111.37857 & 53.1958258 & 6.41234738 & -111.37857 & 46.875 \\
\hline S11R01 & 1.00245234 & -0.7010616 & -469.70409 & 1.00245234 & -0.7010616 & -476.02491 \\
\hline S11R02 & 1.16451289 & 0.92252703 & -518.51478 & 1.16451289 & 0.92252703 & -524.83561 \\
\hline S11R03 & 2.15613065 & -16.352554 & -461.98459 & 2.15613065 & -16.352554 & -468.30542 \\
\hline S11R04 & 10.099351 & -198.76203 & 565.608501 & 10.099351 & -198.76203 & 559.287676 \\
\hline S11R05 & 110.348238 & -2989.3865 & 19911.3208 & 110.348238 & -2989.3865 & 19905 \\
\hline S11R06 & 4.43783291 & -113.60663 & 418.753089 & 4.43783291 & -113.60663 & 412.432263 \\
\hline S11R07 & 1.68767074 & -28.621276 & -221.99934 & 1.68767074 & -28.621276 & -228.32017 \\
\hline S1IR08 & 1.11064066 & -7.167503 & -403.72712 & 1.11064066 & -7.167503 & -410.04794 \\
\hline S11R09 & 1.00170014 & 0.48602876 & -485.07221 & 1.00170014 & 0.48602876 & -491.39304 \\
\hline S11R10 & 1.17747334 & 0.78578389 & -518.93829 & 1.17747334 & 0.78578389 & -525.25912 \\
\hline S11R11 & 1.88731878 & -11.048639 & -485.41446 & 1.88731878 & -11.048639 & -491.73528 \\
\hline S11R12 & 5.17794536 & -82.78407 & -109.726 & 5.17794536 & -82.78407 & -116.04682 \\
\hline S11R13 & 131.92873 & -3271.0988 & 19911.3208 & 131.92873 & -3271.0988 & 19905 \\
\hline S11R14 & 12.0583011 & -331.48839 & 1964.40153 & 12.0583011 & -331.48839 & 1958.0807 \\
\hline S11R15 & 2.49178433 & -54.659408 & -19.123972 & 2.49178433 & -54.659408 & -25.444798 \\
\hline S11R16 & 1.26388716 & -13.497775 & -347.20595 & 1.26388716 & -13.497775 & -353.52678 \\
\hline
\end{tabular}




\section{REVISED METHODOLOGY FOR DETERMINING CESIUM-137 CONTENT OF HN-200 GROUT CONTAINERS.}

\begin{tabular}{|c|c|c|c|}
\hline & & & Source \$12 \\
\hline Line & ai & bi & ci \\
\hline S12C01 & 2.63258781 & -78.160141 & 415.671109 \\
\hline $\mathrm{S} 12 \mathrm{CO} 2$ & 2.63258781 & -78.160141 & 415.671109 \\
\hline $\mathrm{S} 12 \mathrm{CO} 3$ & 2.63258781 & -78.160141 & 415.671109 \\
\hline S12C04 & 2.63258781 & -78.160141 & 415.671109 \\
\hline S12C05 & 2.63258781 & -78.160141 & 415.671109 \\
\hline S12C06 & 2.63258781 & -78.160141 & 415.671109 \\
\hline $\mathrm{S} 12 \mathrm{CO} 7$ & 1.78978111 & -42.546335 & 53.1958258 \\
\hline $\mathrm{S} 12 \mathrm{CO} 8$ & 1.78978111 & -42.546335 & 53.1958258 \\
\hline S12C09 & 1.78978111 & -42.546335 & 53.1958258 \\
\hline $\mathrm{S} 12 \mathrm{C} 10$ & 1.78978111 & -42.546335 & 53.1958258 \\
\hline $\mathrm{S} 12 \mathrm{C} 11$ & 1.78978111 & -42.546335 & 53.1958258 \\
\hline $\mathrm{S} 12 \mathrm{C} 12$ & 1.78978111 & -42.546335 & 53.1958258 \\
\hline $\mathrm{S} 12 \mathrm{C} 13$ & 1.04549442 & 2.17804531 & -493.7396 \\
\hline S12C14 & 1.04549442 & 2.17804531 & -493.7396 \\
\hline $\mathrm{S} 12 \mathrm{C} 15$ & 1.04549442 & 2.17804531 & -493.7396 \\
\hline $\mathrm{S} 12 \mathrm{C} 16$ & 1.04549442 & 2.17804531 & -493.7396 \\
\hline$S 12 C 17$ & 1.04549442 & 2.17804531 & -493.7396 \\
\hline $\mathrm{S} 12 \mathrm{C} 18$ & 1.04549442 & 2.17804531 & -493.7396 \\
\hline $\mathrm{S} 12 \mathrm{C} 19$ & 4.65893829 & -91.576983 & 53.1958258 \\
\hline $\mathrm{S} 12 \mathrm{C} 20$ & 4.65893829 & -91.576983 & 53.1958258 \\
\hline S12C21 & 4.65893829 & -91.576983 & 53.1958258 \\
\hline $\mathrm{S} 12 \mathrm{C} 22$ & 4.65893829 & -91.576983 & 53.1958258 \\
\hline S12C23 & 4.65893829 & -91.576983 & 53.1958258 \\
\hline $\mathrm{S} 12 \mathrm{C} 24$ & 4.65893829 & -91.576983 & 53.1958258 \\
\hline S12R01 & 1.0048349 & -1.3821763 & -421.02567 \\
\hline S12R02 & 1.15973472 & 1.53537244 & -516.11857 \\
\hline S12R03 & 2.20842505 & -22.062254 & -419.11027 \\
\hline S12R04 & 11.7483655 & -309.97706 & 1715.08476 \\
\hline S12R05 & 62.6585217 & -2244.7746 & 19911.3208 \\
\hline S12R06 & 3.95025015 & -130.89374 & 932.032434 \\
\hline S12R07 & 1.61045944 & -34.538277 & -31.285622 \\
\hline S12R08 & 1.09491434 & -8.63581 & -323.37513 \\
\hline S12R09 & 1.00299021 & 0.85482525 & -458.71479 \\
\hline S12R 10 & 1.18098703 & 1.25980714 & -517.61578 \\
\hline S12R11 & 1.86384702 & -13.257969 & -468.93862 \\
\hline S12R12 & 4.84716613 & -97.159892 & 93.6333694 \\
\hline S12R13 & 79.7910608 & -2537.5503 & 19911.3208 \\
\hline SI2RI4 & 15.0160141 & -544.5572 & 4769.54426 \\
\hline S12R15 & 2.71001432 & -81.330527 & 447.238603 \\
\hline S12R16 & 1.30458952 & -20.069123 & -189.22407 \\
\hline
\end{tabular}

\begin{tabular}{ccc} 
ao & bo & co \\
2.63258781 & -78.160141 & 409.350283 \\
2.63258781 & -78.160141 & 409.350283 \\
2.63258781 & -78.160141 & 409.350283 \\
2.63258781 & -78.160141 & 409.350283 \\
2.63258781 & -78.160141 & 409.350283 \\
2.63258781 & -78.160141 & 409.350283 \\
1.78978111 & -42.546335 & 46.875 \\
1.78978111 & -42.546335 & 46.875 \\
1.78978111 & -42.546335 & 46.875 \\
1.78978111 & -42.546335 & 46.875 \\
1.78978111 & -42.546335 & 46.875 \\
1.78978111 & -42.546335 & 46.875 \\
1.04549442 & 2.17804531 & -500.06043 \\
1.04549442 & 2.17804531 & -500.06043 \\
1.04549442 & 2.17804531 & -500.06043 \\
1.04549442 & 2.17804531 & -500.06043 \\
1.04549442 & 2.17804531 & -500.06043 \\
1.04549442 & 2.17804531 & -500.06043 \\
4.65893829 & -91.576983 & 46.875 \\
4.65893829 & -91.576983 & 46.875 \\
\hline 4.65893829 & -91.576983 & 46.875 \\
4.65893829 & -91.576983 & 46.875 \\
4.65893829 & -91.576983 & 46.875 \\
4.65893829 & -91.576983 & 46.875 \\
1.0048349 & -1.3821763 & -427.3465 \\
1.15973472 & 1.53537244 & -522.4394 \\
2.20842505 & -22.062254 & -425.43109 \\
11.7483655 & -309.97706 & 1708.76393 \\
62.6585217 & -2244.7746 & 19905 \\
3.95025015 & -130.89374 & 925.711608 \\
1.61045944 & -34.538277 & -37.606448 \\
1.09491434 & -8.63581 & -329.69595 \\
1.00299021 & 0.85482525 & -465.03561 \\
1.18098703 & 1.25980714 & -523.9366 \\
1.86384702 & -13.257969 & -475.25944 \\
4.84716613 & -97.159892 & 87.3125436 \\
79.7910608 & -2537.5503 & 19905 \\
15.0160141 & -544.5572 & 4763.22344 \\
2.71001432 & -81.330527 & 440.917777 \\
1.30458952 & -20.069123 & -195.5449
\end{tabular}




\section{REVISED METHODOLOGY FOR DETERMINING CESIUM-137 CONTENT OF HN-200 GROUT CONTAINERS.}

\begin{tabular}{|c|c|c|c|c|c|c|}
\hline \multicolumn{7}{|c|}{ Source S13 } \\
\hline Line & ai & bi & ci & ao & bo & co \\
\hline $\mathrm{S} 13 \mathrm{C} 01$ & 1.16528891 & -7.9132068 & -425.09689 & 1.16528891 & -7.9132068 & -431.41771 \\
\hline $\mathrm{S} 13 \mathrm{C} 02$ & 1.16528891 & -7.9132068 & -425.09689 & 1.16528891 & -7.9132068 & -431.4177 \\
\hline $\mathrm{S} 13 \mathrm{C} 03$ & 1.16528891 & -7.9132068 & -425.09689 & 1.16528891 & -7.9132068 & -431.4177 \\
\hline S13C04 & 1.16528891 & -7.9132068 & -425.09689 & 1.16528891 & -7.9132068 & -431.41771 \\
\hline $\mathrm{S} 13 \mathrm{C} 05$ & 1.16528891 & -7.9132068 & -425.09689 & 1.16528891 & -7.9132068 & -431.41771 \\
\hline S13C06 & 1.16528891 & -7.9132068 & -425.09689 & 1.16528891 & -7.9132068 & -431.41771 \\
\hline S13C07 & 20.8124679 & -213.09736 & 53.1958258 & 20.8124679 & -213.09736 & 46.875 \\
\hline S13C08 & 20.8124679 & -213.09736 & 53.1958258 & 20.8124679 & -213.09736 & 46.875 \\
\hline $\mathrm{S} 13 \mathrm{C} 09$ & 20.8124679 & -213.09736 & 53.1958258 & 20.8124679 & -213.09736 & 46.875 \\
\hline $\mathrm{S} 13 \mathrm{C} 10$ & 20.8124679 & -213.09736 & 53.1958258 & 20.8124679 & -213.09736 & 46.875 \\
\hline S13C11 & 20.8124679 & -213.09736 & 53.1958258 & 20.8124679 & -213.09736 & 46.875 \\
\hline $\mathrm{S} 13 \mathrm{C} 12$ & 20.8124679 & -213.09736 & 53.1958258 & 20.8124679 & -213.09736 & 46.875 \\
\hline $\mathrm{S} 13 \mathrm{C} 13$ & 1.09151692 & 4.38137233 & -467.36853 & 1.09151692 & 4.38137233 & $-473.6893 t$ \\
\hline $\mathrm{S} 13 \mathrm{C} 14$ & 1.09151692 & 4.38137233 & -467.36853 & 1.09151692 & 4.38137233 & $-473.6893 t$ \\
\hline $\mathrm{S} 13 \mathrm{C} 15$ & 1.09151692 & 4.38137233 & -467.36853 & 1.09151692 & 4.38137233 & -473.68936 \\
\hline $\mathrm{S} 13 \mathrm{C} 16$ & 1.09151692 & 4.38137233 & -467.36853 & 1,09151692 & 4.38137233 & -473.68936 \\
\hline $\mathrm{S} 13 \mathrm{C} 17$ & 1.09151692 & 4.38137233 & -467.36853 & 1.09151692 & 4.38137233 & -473.68936 \\
\hline S13C18 & 1.09151692 & 4.38137233 & -467.36853 & 1.09151692 & 4.38137233 & -473.68936 \\
\hline S13C19 & 85.2673766 & -439.4794 & 53.1958258 & 85.2673766 & -439.4794 & 46.875 \\
\hline S13C20 & 85.2673766 & -439.4794 & 53.1958258 & 85.2673766 & -439.4794 & 46.875 \\
\hline S13C21 & 85.2673766 & -439.4794 & 53.1958258 & 85.2673766 & -439.4794 & 46.875 \\
\hline$S 13 C 22$ & 85.2673766 & -439.4794 & 53.1958258 & 85.2673766 & -439.4794 & 46.875 \\
\hline $\mathrm{S} 13 \mathrm{C} 23$ & 85.2673766 & -439.4794 & 53.1958258 & 85.2673766 & -439.4794 & 46.875 \\
\hline S13C24 & 85.2673766 & -439.4794 & 53.1958258 & 85.2673766 & -439.4794 & 46.875 \\
\hline S13R01 & 1.00354728 & -1.0140787 & -447.33315 & 1.00354728 & -1.0140787 & -453.65397 \\
\hline S13R02 & 1.13027108 & 5.0792743 & -470.29781 & 1.13027108 & 5.0792743 & $-476.6186 ?$ \\
\hline S13R03 & 1.90417978 & 9.44092318 & -495.16391 & 1.90417978 & 9.44092318 & -501.4847 \\
\hline S13R04 & 6.84497445 & -0.9057722 & -519.77299 & 6.84497445 & -0.9057722 & -526.09382 \\
\hline S13R05 & 1470.37996 & -10958.301 & 19911.3208 & 1470.37996 & -10958.301 & 19905 \\
\hline S13R06 & 5.51944781 & -67.053984 & -271.09204 & 5.51944781 & -67.053984 & -277.41286 \\
\hline S13R07 & 1.78679857 & -20.258508 & -389.40395 & 1.78679857 & -20.258508 & -395.72478 \\
\hline S13R08 & 1.11712087 & -6.5064838 & -429.44346 & 1.11712087 & -6.5064838 & -435.76428 \\
\hline S13R09 & 1.00321515 & 0.91913051 & -454.11897 & 1.00321515 & 0.91913051 & -460.4398 \\
\hline S13R10 & 1.21597901 & 6.20121157 & -475.29562 & 1.21597901 & 6.20121157 & -481.61645 \\
\hline S13R11 & 2.09373397 & 9.68606332 & -498.36324 & 2.09373397 & 9.68606332 & -504.68406 \\
\hline S13R12 & 6.81389613 & -0.7943515 & -519.78095 & 6.81389613 & -0.7943515 & -526.10177 \\
\hline S13R13 & 1855.25823 & -12310.092 & 19911.3208 & 1855.25823 & -12310.092 & 19905 \\
\hline S13R14 & 8.51907661 & -98.358698 & -198.14513 & 8.51907661 & -98.358698 & -204.46595 \\
\hline S13R15 & 2.25690639 & -27.448761 & -369.94917 & 2.25690639 & -27.448761 & -376.26999 \\
\hline S13R 16 & 1.24022893 & -9.8276289 & -419.2974 & 1.24022893 & -9.8276289 & -425.61823 \\
\hline
\end{tabular}




\section{REVISED METHODOLOGY FOR DETERMINING CESIUM-137 CONTENT OF HN-200 GROUT CONTAINERS.}

$\begin{array}{lccc}\text { Line } & \text { ai } & \text { bi } & \text { ci } \\ \text { S14C01 } & 1.02113733 & -1.0119498 & -507.69631 \\ \text { S14C02 } & 1.02113733 & -1.0119498 & -507.69631 \\ \text { S14C03 } & 1.02113733 & -1.0119498 & -507.69631 \\ \text { S14C04 } & 1.02113733 & -1.0119498 & -507.69631 \\ \text { S14C05 } & 1.02113733 & -1.0119498 & -507.69631 \\ \text { S14C06 } & 1.02113733 & -1.0119498 & -507.69631 \\ \text { S14C07 } & 1.95271081 & 46.7293063 & 53.1958258 \\ \text { S14C08 } & 1.95271081 & 46.7293063 & 53.1958258 \\ \text { S14C09 } & 1.95271081 & 46.7293063 & 53.1958258 \\ \text { S14C10 } & 1.95271081 & 46.7293063 & 53.1958258 \\ \text { S14C11 } & 1.95271081 & 46.7293063 & 53.1958258 \\ \text { S14C12 } & 1.95271081 & 46.7293063 & 53.1958258 \\ \text { S14C13 } & 2.1591607 & 55.4948185 & 144.395528 \\ \text { S14C14 } & 2.1591607 & 55.4948185 & 144.395528 \\ \text { S14C15 } & 2.1591607 & 55.4948185 & 144.395528 \\ \text { S14C16 } & 2.1591607 & 55.4948185 & 144.395528 \\ \text { S14C17 } & 2.1591607 & 55.4948185 & 144.395528 \\ \text { S14C18 } & 2.1591607 & 55.4948185 & 144.395528 \\ \text { S14C19 } & 3.71724569 & 78.9174858 & 53.1958258 \\ \text { S14C20 } & 3.71724569 & 78.9174858 & 53.1958258 \\ \text { S14C21 } & 3.71724569 & 78.9174858 & 53.1958258 \\ \text { S14C22 } & 3.71724569 & 78.9174858 & 53.1958258 \\ \text { S14C23 } & 3.71724569 & 78.9174858 & 53.1958258 \\ \text { S14C24 } & 3.71724569 & 78.9174858 & 53.1958258 \\ \text { S14R01 } & 1.00144754 & -0.4138144 & -490.23328 \\ \text { S14R02 } & 1.10441929 & 7.77276164 & -375.1609 \\ \text { S14R03 } & 1.63316216 & 32.859088 & -93.487728 \\ \text { S14R04 } & 3.98038247 & 129.908062 & 895.790814 \\ \text { S14R05 } & 57.2435644 & 2143.93985 & 19911.3208 \\ \text { S14R06 } & 12.9298397 & 392.880496 & 2714.83481 \\ \text { S14R07 } & 2.31376423 & 33.8730377 & -301.46931 \\ \text { S14R08 } & 1.18209179 & 1.4097595 & -517.07948 \\ \text { S14R09 } & 1.00241849 & 0.69138568 & -470.39561 \\ \text { S14R10 } & 1.28567003 & 16.9774375 & -267.5648 \\ \text { S14R11 } & 2.67512079 & 76.9878751 & 364.774833 \\ \text { S14R12 } & 15.3668536 & 570.640038 & 5146.53442 \\ \text { S14R13 } & 67.7800951 & 2336.14446 & 19911.3208 \\ \text { S14R14 } & 4.58921158 & 107.711331 & 288.289366 \\ \text { S14R15 } & 1.80731617 & 18.4338485 & -414.58079 \\ \text { S14R16 } & 1.16381552 & 1.01256414 & -518.24338 \\ & & & \end{array}$

$\begin{array}{ccc}\text { ao } & \text { bo } & \text { co } \\ 1.02113733 & -1.0119498 & -514.01713 \\ 1.02113733 & -1.0119498 & -514.01713 \\ 1.02113733 & -1.0119498 & -514.01713 \\ 1.02113733 & -1.0119498 & -514.01713 \\ 1.02113733 & -1.0119498 & -514.01713 \\ 1.02113733 & -1.0119498 & -514.01713 \\ 1.95271081 & 46.7293063 & 46.875 \\ 1.95271081 & 46.7293063 & 46.875 \\ 1.95271081 & 46.7293063 & 46.875 \\ 1.95271081 & 46.7293063 & 46.875 \\ 1.95271081 & 46.7293063 & 46.875 \\ 1.95271081 & 46.7293063 & 46.875 \\ 2.1591607 & 55.4948185 & 138.074702 \\ 2.1591607 & 55.4948185 & 138.074702 \\ 2.1591607 & 55.4948185 & 138.074702 \\ 2.1591607 & 55.4948185 & 138.074702 \\ 2.1591607 & 55.4948185 & 138.074702 \\ 2.1591607 & 55.4948185 & 138.074702 \\ 3.71724569 & 78.9174858 & 46.875 \\ 3.71724569 & 78.9174858 & 46.875 \\ 3.71724569 & 78.9174858 & 46.875 \\ 3.71724569 & 78.9174858 & 46.875 \\ 3.71724569 & 78.9174858 & 46.875 \\ 3.71724569 & 78.9174858 & 46.875 \\ 1.00144754 & -0.4138144 & -496.55411 \\ 1.10441929 & 7.77276164 & -381.48173 \\ 1.63316216 & 32.859088 & -99.808554 \\ 3.98038247 & 129.908062 & 889.469988 \\ 57.2435644 & 2143.93985 & 19905 \\ 12.9298397 & 392.880496 & 2708.51399 \\ 2.31376423 & 33.8730377 & -307.79013 \\ 1.18209179 & 1.4097595 & -523.40031 \\ 1.00241849 & 0.69138568 & -476.71644 \\ 1.28567003 & 16.9774375 & -273.88562 \\ 2.67512079 & 76.9878751 & 358.454007 \\ 15.3668536 & 570.640038 & 5140.21359 \\ 67.7800951 & 2336.14446 & 19905 \\ 4.58921158 & 107.711331 & 281.96854 \\ 1.80731617 & 18.4338485 & -420.90162 \\ 1.16381552 & 1.01256414 & -524.56421\end{array}$




\section{REVISED METHODOLOGY FOR DETERMINING CESIUM-137 CONTENT OF HN-200 GROUT CONTAINERS.}

\begin{tabular}{|c|c|c|c|c|c|c|}
\hline \multicolumn{7}{|c|}{ Source S15 } \\
\hline Line & ai & bi & ci & $\mathbf{2 0}$ & bo & co \\
\hline $\mathrm{S} 15 \mathrm{C} 01$ & 4.39481841 & -162.52693 & 1425.43613 & 4.39481841 & -162.52693 & 1419.1153 \\
\hline $\mathrm{S} 15 \mathrm{C} 02$ & 4.39481841 & -162.52693 & 1425.43613 & 4.39481841 & -162.52693 & 1419.1153 \\
\hline S15C03 & 4.39481841 & -162.52693 & 1425.43613 & 4.39481841 & -162.52693 & 1419.1153 \\
\hline S15C04 & 4.39481841 & -162.52693 & 1425.43613 & 4.39481841 & -162.52693 & 1419.1153 \\
\hline S15C05 & 4.39481841 & -162.52693 & 1425.43613 & 4.39481841 & -162.52693 & 1419.1153 \\
\hline S15C06 & 4.39481841 & -162.52693 & 1425.43613 & 4.39481841 & -162.52693 & 1419.1153 \\
\hline S15C07 & 1.77839511 & -42.238534 & 53.1958258 & 1.77839511 & -42.238534 & 46.875 \\
\hline $\mathrm{S} 15 \mathrm{C} 08$ & 1.77839511 & -42.238534 & 53.1958258 & 1.77839511 & -42.238534 & 46.875 \\
\hline S15C09 & 1.77839511 & -42.238534 & 53.1958258 & 1.77839511 & -42.238534 & 46.875 \\
\hline S15C10 & 1.77839511 & -42.238534 & 53.1958258 & 1.77839511 & -42.238534 & 46.875 \\
\hline S15C11 & 1.77839511 & -42.238534 & 53.1958258 & 1.77839511 & -42.238534 & 46.875 \\
\hline $\mathrm{S} 15 \mathrm{C} 12$ & 1.77839511 & -42.238534 & 53.1958258 & 1.77839511 & -42.238534 & 46.875 \\
\hline $\mathrm{S} 15 \mathrm{C} 13$ & 1.01447927 & 0.69319525 & -511.5114 & 1.01447927 & 0.69319525 & -517.83223 \\
\hline S15C14 & 1.01447927 & 0.69319525 & -511.5114 & 1.01447927 & 0.69319525 & -517.83223 \\
\hline$S 15 C 15$ & 1.01447927 & 0.69319525 & -511.5114 & 1.01447927 & 0.69319525 & -517.83223 \\
\hline S15C16 & 1.01447927 & 0.69319525 & -511.5114 & 1.01447927 & 0.69319525 & -517.83223 \\
\hline $\mathrm{S} 15 \mathrm{C} 17$ & 1.01447927 & 0.69319525 & -511.5114 & 1.01447927 & 0.69319525 & -517.83223 \\
\hline S15C18 & 1.01447927 & 0.69319525 & -511.5114 & 1.01447927 & 0.69319525 & -517.83223 \\
\hline S15C19 & 2.95221446 & -66.891749 & 53.1958258 & 2.95221446 & -66.891749 & 46.875 \\
\hline $\mathrm{S} 15 \mathrm{C} 20$ & 2.95221446 & -66.891749 & 53.1958258 & 2.95221446 & -66.891749 & 46.875 \\
\hline$S 15 C 21$ & 2.95221446 & -66.891749 & 53.1958258 & 2.95221446 & -66.891749 & 46.875 \\
\hline S15C22 & 2.95221446 & -66.891749 & 53.1958258 & 2.95221446 & -66.891749 & 46.875 \\
\hline$S 15 C 23$ & 2.95221446 & -66.891749 & 53.1958258 & 2.95221446 & -66.891749 & 46.875 \\
\hline S15C24 & 2.95221446 & -66.891749 & 53.1958258 & 2.95221446 & -66.891749 & 46.875 \\
\hline S15R01 & 1.00196679 & -0.5622559 & -479.62436 & 1.00196679 & -0.5622559 & -485.94518 \\
\hline S15R02 & 1.19700807 & -3.4749306 & -504.48492 & 1.19700807 & -3.4749306 & -510.80575 \\
\hline S15R03 & 2.42746902 & -47.039293 & -132.28739 & 2.42746902 & -47.039293 & -138.60822 \\
\hline S15R04 & 15.1259437 & -552.71342 & 4886.77102 & 15.1259437 & -552.71342 & 4880.4502 \\
\hline S15R05 & 43.8794759 & -1871.9787 & 19911.3208 & 43.8794759 & -1871.9787 & 19905 \\
\hline S15R06 & 3.79889875 & -135.66193 & 1124.06698 & 3.79889875 & -135.66193 & 1117.74615 \\
\hline S15R07 & 1.61411579 & -34.27145 & -41.668496 & 1.61411579 & -34.27145 & -47.989322 \\
\hline S15R08 & 1.10371341 & -7.8395033 & -371.66472 & 1.10371341 & -7.8395033 & -377.98555 \\
\hline S15R09 & 1.00108804 & 0.31104278 & -497.57824 & 1.00108804 & 0.31104278 & -503.89907 \\
\hline S15R10 & 1.15421812 & -2.2307314 & -511.74132 & 1.15421812 & -2.2307314 & -518.06215 \\
\hline S15R11 & 1.76082159 & -22.524784 & -353.09163 & 1.76082159 & -22.524784 & -359.41245 \\
\hline S15R 12 & 4.297335 & -118.86601 & 551.445479 & 4.297335 & -118.86601 & 545.124653 \\
\hline S15R13 & 50.8886792 & -2019.19 & 19911.3208 & 50.8886792 & -2019.19 & 19905 \\
\hline S15R14 & 17.641534 & -743.14955 & 7776.77164 & 17.641534 & -743.14955 & 7770.45081 \\
\hline S15R15 & 2.76847635 & -88.667648 & 591.593812 & 2.76847635 & -88.667648 & 585.272986 \\
\hline S15R16 & 1.29294393 & -18.158831 & -238.40342 & 1.29294393 & -18.158831 & -244.72424 \\
\hline
\end{tabular}




\section{REVISED METHODOLOGY FOR DETERMINING CESIUM-137 CONTENT OF HN-200 GROUT CONTAINERS.}

\begin{tabular}{|c|c|c|c|c|c|c|}
\hline \multicolumn{7}{|c|}{ Source S16 } \\
\hline Line & ai & bi & ci & ao & bo & co \\
\hline $\mathrm{S} 16 \mathrm{C} 01$ & 1.85747126 & -41.051437 & -28.4737 & 1.85747126 & -41.051437 & -34.794525 \\
\hline $\mathrm{S} 16 \mathrm{C} 02$ & 1.85747126 & -41.051437 & -28.4737 & 1.85747126 & -41.051437 & -34.794525 \\
\hline $\mathrm{S} 16 \mathrm{C} 03$ & 1.85747126 & -41.051437 & -28.4737 & 1.85747126 & -41.051437 & -34.794525 \\
\hline S16C04 & 1.85747126 & -41.051437 & -28.4737 & 1.85747126 & -41.051437 & -34.794525 \\
\hline S16C05 & 1.85747126 & -41.051437 & -28.4737 & 1.85747126 & -41.051437 & -34.794525 \\
\hline S16C06 & 1.85747126 & -41.051437 & -28.4737 & 1.85747126 & -41.051437 & -34.794525 \\
\hline S16C07 & 2.13339083 & -50.968122 & 53.1958258 & 2.13339083 & -50.968122 & 46.875 \\
\hline S16C08 & 2.13339083 & -50.968122 & 53.1958258 & 2.13339083 & -50.968122 & 46.875 \\
\hline S16C09 & 2.13339083 & -50.968122 & 53.1958258 & 2.13339083 & -50.968122 & 46.875 \\
\hline S16C10 & 2.13339083 & -50.968122 & 53.1958258 & 2.13339083 & -50.968122 & 46.875 \\
\hline S16C11 & 2.13339083 & -50.968122 & 53.1958258 & 2.13339083 & -50.968122 & 46.875 \\
\hline S16C12 & 2.13339083 & -50.968122 & 53.1958258 & 2.13339083 & -50.968122 & 46.875 \\
\hline S16C13 & 1.07917988 & 3.79073687 & -474.4377 & 1.07917988 & 3.79073687 & -480.75852 \\
\hline S16C14 & 1.07917988 & 3.79073687 & -474.4377 & 1.07917988 & 3.79073687 & -480.75852 \\
\hline S16C15 & 1.07917988 & 3.79073687 & -474.4377 & 1.07917988 & 3.79073687 & -480.75852 \\
\hline S16C16 & 1.07917988 & 3.79073687 & -474.4377 & 1.07917988 & 3.79073687 & -480.75852 \\
\hline S16C17 & 1.07917988 & 3.79073687 & -474.4377 & 1.07917988 & 3.79073687 & -480.75852 \\
\hline S16C18 & 1.07917988 & 3.79073687 & -474.4377 & 1.07917988 & 3.79073687 & -480.75852 \\
\hline S16C19 & 8.19045063 & -128.37689 & 53.1958258 & 8.19045063 & -128.37689 & 46.875 \\
\hline S16C20 & 8.19045063 & -128.37689 & 53.1958258 & 8.19045063 & -128.37689 & 46.875 \\
\hline $\mathrm{S} 16 \mathrm{C} 21$ & 8.19045063 & -128.37689 & 53.1958258 & 8.19045063 & -128.37689 & 46.875 \\
\hline $\mathrm{S} 16 \mathrm{C} 22$ & 8.19045063 & -128.37689 & 53.1958258 & 8.19045063 & -128.37689 & 46.875 \\
\hline S16C23 & 8.19045063 & -128.37689 & 53.1958258 & 8.19045063 & -128.37689 & 46.875 \\
\hline S16C24 & 8.19045063 & -128.37689 & 53.1958258 & 8.19045063 & -128.37689 & 46.875 \\
\hline S16R01 & 1.00630116 & -1.8013435 & -391.06831 & 1.00630116 & -1.8013435 & -397.38914 \\
\hline S16R02 & 1.1383643 & 4.14980597 & -488.69296 & 1.1383643 & 4.14980597 & -495.01378 \\
\hline S16R03 & 2.05622186 & -5.7601622 & -511.95474 & 2.05622186 & -5.7601622 & -518.27557 \\
\hline S16R04 & 9.43180201 & -155.51301 & 197.247939 & 9.43180201 & -155.51301 & 190.927113 \\
\hline S16R05 & 108.663195 & -2966.2641 & 19911.3208 & 108.663195 & -2966.2641 & 19905 \\
\hline S16R06 & 4.25397175 & -120.44593 & 594.769923 & 4.25397175 & -120.44593 & 588.449097 \\
\hline S16R07 & 1.6352111 & -32.704954 & -98.840148 & 1.6352111 & -32.704954 & -105.16097 \\
\hline S16R08 & 1.09383815 & -8.7284251 & -316.83784 & 1.09383815 & -8.7284251 & -323.15867 \\
\hline S16R09 & 1.00440235 & 1.25852261 & -429.86304 & 1.00440235 & 1.25852261 & -436.18387 \\
\hline S16R10 & 1.20160043 & 4.12514468 & -498.70592 & 1.20160043 & 4.12514468 & -505.02675 \\
\hline S16R11 & 1.95745849 & -4.2530313 & -515.08509 & 1.95745849 & -4.2530313 & -521.40591 \\
\hline S16R12 & 5.45251705 & -70.203057 & -243.08431 & 5.45251705 & -70.203057 & -249.40514 \\
\hline S16R13 & 144.831591 & -3428.4934 & 19911.3208 & 144.831591 & -3428.4934 & 19905 \\
\hline S16R14 & 12.5375133 & -365.08555 & 2368.32427 & 12.5375133 & -365.08555 & 2362.00344 \\
\hline S16R15 & 2.59205118 & -66.765415 & 180.172655 & 2.59205118 & -66.765415 & 173.851829 \\
\hline S16R16 & 1.29725972 & -18.864119 & -220.52857 & 1.29725972 & $-18.864 ! 19$ & -226.8494 \\
\hline
\end{tabular}

Source S17 


\section{REVISED METHODOLOGY FOR DETERMINING CESIUM-137 CONTENT OF HN-200 GROUT CONTAINERS.}

\begin{tabular}{|c|c|c|c|c|c|c|}
\hline Line & ai & bi & ci & ao & bo & co \\
\hline $\mathrm{S} 17 \mathrm{C} 01$ & 1.00650061 & -0.3112165 & -516.08321 & 1.00650061 & -0.3112165 & -522.40403 \\
\hline $\mathrm{S} 17 \mathrm{C} 02$ & 1.00650061 & -0.3112165 & -516.08321 & 1.00650061 & -0.3112165 & -522.40403 \\
\hline S17C03 & 1.00650061 & -0.3112165 & -516.08321 & 1.00650061 & -0.3112165 & -522.40403 \\
\hline S17C04 & 1.00650061 & -0.3112165 & -516.08321 & 1.00650061 & -0.3112165 & -522.40403 \\
\hline S17C05 & 1.00650061 & -0.3112165 & -516.08321 & 1.00650061 & -0.3112165 & -522.40403 \\
\hline S17C06 & 1.00650061 & -0.3112165 & -516.08321 & 1.00650061 & -0.3112165 & -522.40403 \\
\hline S17C07 & 3.06828042 & 68.8515145 & 53.1958258 & 3.06828042 & 68.8515145 & 46.875 \\
\hline S17C08 & 3.06828042 & 68.8515145 & 53.1958258 & 3.06828042 & 68.8515145 & 46.875 \\
\hline S17C09 & 3.06828042 & 68.8515145 & 53.1958258 & 3.06828042 & 68.8515145 & 46.875 \\
\hline S17C10 & 3.06828042 & 68.8515145 & 53.1958258 & 3.06828042 & 68.8515145 & 46.875 \\
\hline S17C11 & 3.06828042 & 68.8515145 & 53.1958258 & 3.06828042 & 68.8515145 & 46.875 \\
\hline S17C12 & 3.06828042 & 68.8515145 & 53.1958258 & 3.06828042 & 68.8515145 & 46.875 \\
\hline S17C13 & 1.10755619 & 5.14925275 & -458.17796 & 1.10755619 & 5.14925275 & -464.49879 \\
\hline S17C14 & 1.10755619 & 5.14925275 & -458.17796 & 1.10755619 & 5.14925275 & -464.49879 \\
\hline S17C15 & 1.10755619 & 5.14925275 & -458.17796 & 1.10755619 & 5.14925275 & -464.49879 \\
\hline S17C16 & 1.10755619 & 5.14925275 & -458.17796 & 1.10755619 & 5.14925275 & -464.49879 \\
\hline S17C17 & 1.10755619 & 5.14925275 & -458.17796 & 1.10755619 & 5.14925275 & -464.49879 \\
\hline S17C18 & 1.10755619 & 5.14925275 & -458.17796 & 1.10755619 & 5.14925275 & -464.49879 \\
\hline S17C19 & 4.48116873 & 89.3246501 & 53.1958258 & 4.48116873 & 89.3246501 & 46.875 \\
\hline S17C20 & 4.48116873 & 89.3246501 & 53.1958258 & 4.48116873 & 89.3246501 & 46.875 \\
\hline S17C21 & 4.48116873 & 89.3246501 & 53.1958258 & 4.48116873 & 89.3246501 & 46.875 \\
\hline S17C22 & 4.48116873 & 89.3246501 & 53.1958258 & 4.48116873 & 89.3246501 & 46.875 \\
\hline S17C23 & 4.48116873 & 89.3246501 & 53.1958258 & 4.48116873 & 89.3246501 & 46.875 \\
\hline S17C24 & 4.48116873 & 89.3246501 & 53.1958258 & 4.48116873 & 89.3246501 & 46.875 \\
\hline S17R01 & 1.00038733 & -0.1107281 & -511.89448 & 1.00038733 & -0.1107281 & -518.21531 \\
\hline S17R02 & 1.12398505 & 5.77506404 & -452.55932 & 1.12398505 & 5.77506404 & -458.88015 \\
\hline S17R03 & 1.71876529 & 26.0836717 & -283.16688 & 1.71876529 & 26.0836717 & -289.4877 \\
\hline S17R04 & 4.47387186 & 112.224084 & 386.54696 & 4.47387186 & 112.224084 & 380.226134 \\
\hline S17R05 & 94.1332707 & 2758.85328 & 19911.3208 & 94.1332707 & 2758.85328 & 19905 \\
\hline S17R06 & 11.2859262 & 278.218259 & 1361.5344 & 11.2859262 & 278.218259 & 1355.21357 \\
\hline S17R07 & 2.28046139 & 30.0965731 & -342.95704 & 2.28046139 & 30.0965731 & -349.27787 \\
\hline S17R08 & 1.19264421 & 2.86317692 & -509.16958 & 1.19264421 & 2.86317692 & -515.49041 \\
\hline S17R09 & 1.00058183 & 0.16633013 & -507.92067 & 1.00058183 & 0.16633013 & -514.2415 \\
\hline S17R10 & 1.24168968 & 10.0506581 & -415.319 & 1.24168968 & 10.0506581 & -421.63983 \\
\hline S17R11 & 2.44755502 & 49.4063092 & -98.238027 & 2.44755502 & 49.4063092 & -104.55885 \\
\hline S17R12 & 12.2981711 & 348.255553 & 2163.85483 & 12.2981711 & 348.255553 & 2157.534 \\
\hline S17R13 & 102.570022 & 2881.1041 & 19911.3208 & 102.570022 & 2881.1041 & 19905 \\
\hline S17R14 & 4.81573791 & 98.4778118 & 115.578812 & 4.81573791 & 98.4778118 & 109.257987 \\
\hline S17R15 & 1.81256047 & 17.9627139 & -420.53575 & 1.81256047 & 17.9627139 & -426.85657 \\
\hline S17R16 & 1.15555053 & 2.06401408 & -512.96118 & 1.15555053 & 2.06401408 & -519.28201 \\
\hline
\end{tabular}

Source S18

Line

bi

ci

ao

bo

co 
REVISED METHODOLOGY FOR DETERMINING CESIUM-137 CONTENT OF HN-200 GROUT CONTAINERS.

$\begin{array}{lccc}\text { S18C01 } & 6.38430607 & -257.77365 & 2565.42033 \\ \text { S18C02 } & 6.38430607 & -257.77365 & 2565.42033 \\ \text { S18C03 } & 6.38430607 & -257.77365 & 2565.42033 \\ \text { S18C04 } & 6.38430607 & -257.77365 & 2565.42033 \\ \text { S18C05 } & 6.38430607 & -257.77365 & 2565.42033 \\ \text { S18C06 } & 6.38430607 & -257.77365 & 2565.42033 \\ \text { S18C07 } & 4.66856904 & -91.697424 & 53.1958258 \\ \text { S18C08 } & 4.66856904 & -91.697424 & 53.1958258 \\ \text { S18C09 } & 4.66856904 & -91.697424 & 53.1958258 \\ \text { S18C10 } & 4.66856904 & -91.697424 & 53.1958258 \\ \text { S18C11 } & 4.66856904 & -91.697424 & 53.1958258 \\ \text { S18C12 } & 4.66856904 & -91.697424 & 53.1958258 \\ \text { S18C13 } & 1.07901349 & 3.78277081 & -474.53304 \\ \text { S18C14 } & 1.07901349 & 3.78277081 & -474.53304 \\ \text { S18C15 } & 1.07901349 & 3.78277081 & -474.53304 \\ \text { S18C16 } & 1.07901349 & 3.78277081 & -474.53304 \\ \text { S18C17 } & 1.07901349 & 3.78277081 & -474.53304 \\ \text { S18C18 } & 1.07901349 & 3.78277081 & -474.53304 \\ \text { S18C19 } & 1.4044923 & -30.448361 & 53.1958258 \\ \text { S18C20 } & 1.4044923 & -30.448361 & 53.1958258 \\ \text { S18C21 } & 1.4044923 & -30.448361 & 53.1958258 \\ \text { S18C22 } & 1.4044923 & -30.448361 & 53.1958258 \\ \text { S18C23 } & 1.4044923 & -30.448361 & 53.1958258 \\ \text { S18C24 } & 1.4044923 & -30.448361 & 53.1958258 \\ \text { S18R01 } & 1.00934428 & -2.6712958 & -328.89391 \\ \text { S18R02 } & 1.34664911 & -27.143773 & 11.5537456 \\ \text { S18R03 } & 2.88737045 & -103.81205 & 907.699395 \\ \text { S18R04 } & 17.0711929 & -699.37831 & 7088.99261 \\ \text { S18R05 } & 69.1794148 & -2360.4935 & 19911.3208 \\ \text { S18R06 } & 4.84533978 & -97.236717 & 94.8956643 \\ \text { S18R07 } & 1.89034118 & -10.761701 & -487.28847 \\ \text { S18R08 } & 1.19559812 & 3.27661392 & -506.08581 \\ \text { S18R09 } & 1.00551021 & 1.57523163 & -407.22825 \\ \text { S18R10 } & 1.0801416 & -9.8037332 & -219.98508 \\ \text { S18R11 } & 1.5524231 & -38.574896 & 153.599035 \\ \text { S18R12 } & 3.67053037 & -139.45374 & 1300.74277 \\ \text { S18R13 } & 49.6887007 & -1994.7583 & 19911.3208 \\ \text { S18R14 } & 12.1612006 & -338.66872 & 2049.28153 \\ \text { S18R15 } & 2.17104214 & -17.969636 & -450.87209 \\ \text { S18R16 } & 1.14203109 & 3.717041 & -495.48877\end{array}$

$\begin{array}{ccc}6.38430607 & -257.77365 & 2559.09951 \\ 6.38430607 & -257.77365 & 2559.09951 \\ 6.38430607 & -257.77365 & 2559.09951 \\ 6.38430607 & -257.77365 & 2559.09951 \\ 6.38430607 & -257.77365 & 2559.09951 \\ 6.38430607 & -257.77365 & 2559.09951 \\ 4.66856904 & -91.697424 & 46.875 \\ 4.66856904 & -91.697424 & 46.875 \\ 4.66856904 & -91.697424 & 46.875 \\ 4.66856904 & -91.697424 & 46.875 \\ 4.66856904 & -91.697424 & 46.875 \\ 4.66856904 & -91.697424 & 46.875 \\ 1.07901349 & 3.78277081 & -480.85387 \\ 1.07901349 & 3.78277081 & -480.85387 \\ 1.07901349 & 3.78277081 & -480.85387 \\ 1.07901349 & 3.78277081 & -480.85387 \\ 1.07901349 & 3.78277081 & -480.85387 \\ 1.07901349 & 3.78277081 & -480.85387 \\ 1.4044923 & -30.448361 & 46.875 \\ 1.4044923 & -30.448361 & 46.875 \\ 1.4044923 & -30.448361 & 46.875 \\ 1.4044923 & -30.448361 & 46.875 \\ 1.4044923 & -30.448361 & 46.875 \\ 1.4044923 & -30.448361 & 46.875 \\ 1.00934428 & -2.6712958 & -335.21474 \\ 1.34664911 & -27.143773 & 5.23291985 \\ 2.88737045 & -103.81205 & 901.378569 \\ 17.0711929 & -699.37831 & 7082.67178 \\ 69.1794148 & -2360.4935 & 19905 \\ 4.84533978 & -97.236717 & 88.5748385 \\ 1.89034118 & -10.761701 & -493.60929 \\ 1.19559812 & 3.27661392 & -512.40664 \\ 1.00551021 & 1.57523163 & -413.54907 \\ 1.0801416 & -9.8037332 & -226.30591 \\ 1.5524231 & -38.574896 & 147.278209 \\ 3.67053037 & -139.45374 & 1294.42194 \\ 49.6887007 & -1994.7583 & 19905 \\ 12.1612006 & -338.66872 & 2042.96071 \\ 2.17104214 & -17.969636 & -457.19291 \\ 1.14203109 & 3.717041 & -501.80959\end{array}$

\begin{tabular}{cccc} 
& & & \multicolumn{1}{r}{ Source S19 } \\
Line & ai & bi & ci \\
S19C01 & 1.00004356 & -0.0020853 & -519.78312
\end{tabular}

$\begin{array}{ccc}\text { ao } & \text { bo } & \text { co } \\ 1.00004356 & -0.0020853 & -526.10395\end{array}$




\section{REVISED METHODOLOGY FOR DETERMINING CESIUM-137 CONTENT} OF HN-200 GROUT CONTAINERS.

$\begin{array}{llll}\text { S19C02 } & 1.00004356 & -0.0020853 & -519.78312 \\ \text { S19C03 } & 1.00004356 & -0.0020853 & -519.78312 \\ \text { S19C04 } & 1.00004356 & -0.0020853 & -519.78312 \\ \text { S19C05 } & 1.00004356 & -0.0020853 & -519.78312 \\ \text { S19C06 } & 1.00004356 & -0.0020853 & -519.78312 \\ \text { S19C07 } & 2.67906662 & 62.035852 & 53.1958258 \\ \text { S19C08 } & 2.67906662 & 62.035852 & 53.1958258 \\ \text { S19C09 } & 2.67906662 & 62.035852 & 53.1958258 \\ \text { S19C10 } & 2.67906662 & 62.035852 & 53.1958258 \\ \text { S19C11 } & 2.67906662 & 62.035852 & 53.1958258 \\ \text { S19C12 } & 2.67906662 & 62.035852 & 53.1958258 \\ \text { S19C13 } & 1.00240463 & 0.11512183 & -518.43022 \\ \text { S19C14 } & 1.00240463 & 0.11512183 & -518.43022 \\ \text { S19C15 } & 1.00240463 & 0.11512183 & -518.43022 \\ \text { S19C16 } & 1.00240463 & 0.11512183 & -518.43022 \\ \text { S19C17 } & 1.00240463 & 0.11512183 & -518.43022 \\ \text { S19C18 } & 1.00240463 & 0.11512183 & -518.43022 \\ \text { S19C19 } & 2.75905328 & 63.4962802 & 53.1958258 \\ \text { S19C20 } & 2.75905328 & 63.4962802 & 53.1958258 \\ \text { S19C21 } & 2.75905328 & 63.4962802 & 53.1958258 \\ \text { S19C22 } & 2.75905328 & 63.4962802 & 53.1958258 \\ \text { S19C23 } & 2.75905328 & 63.4962802 & 53.1958258 \\ \text { S19C24 } & 2.75905328 & 63.4962802 & 53.1958258 \\ \text { S19R01 } & 1.00000298 & -0.0008533 & -519.7471 \\ \text { S19R02 } & 1.13107839 & 4.98821347 & -472.35122 \\ \text { S19R03 } & 1.71344931 & 26.5233354 & -273.29887 \\ \text { S19R04 } & 4.26242857 & 120.139454 & 586.230565 \\ \text { S19R05 } & 62.0482327 & 2233.63767 & 19911.3208 \\ \text { S19R06 } & 14.0767269 & 475.506054 & 3802.87163 \\ \text { S19R07 } & 2.48136347 & 53.4167486 & -38.267073 \\ \text { S19R08 } & 1.22869896 & 8.08499488 & -448.35264 \\ \text { S19R09 } & 1.00000499 & 0.00142625 & -519.70615 \\ \text { S19R10 } & 1.23340414 & 8.79225548 & -437.00775 \\ \text { S19R11 } & 2.49778037 & 55.3757848 & -7.9710959 \\ \text { S19R12 } & 14.1875003 & 483.58788 & 3913.5044 \\ \text { S19R13 } & 62.5258554 & 2242.3583 & 19911.3208 \\ \text { S19R14 } & 4.29006471 & 119.132354 & 558.629506 \\ \text { S19R15 } & 1.72135596 & 25.8685564 & -287.88991 \\ \text { S19R16 } & 1.13377515 & 4.68132552 & -478.85352 \\ & & & \end{array}$

$\begin{array}{llll}1.00004356 & -0.0020853 & -526.10395\end{array}$

$\begin{array}{llll}1.00004356 & -0.0020853 & -526.10395\end{array}$

$\begin{array}{llll}1.00004356 & -0.0020853 & -526.10395\end{array}$

$\begin{array}{lll}1.00004356 & -0.0020853 & -526.10395\end{array}$

$\begin{array}{llll}1.00004356 & -0.0020853 & -526.10395\end{array}$

$\begin{array}{lll}2.67906662 & 62.035852 & 46.875\end{array}$

$\begin{array}{lll}2.67906662 & 62.035852 & 46.875\end{array}$

$\begin{array}{lll}2.67906662 & 62.035852 & 46.875\end{array}$

$\begin{array}{lll}2.67906662 & 62.035852 & 46.875\end{array}$

$\begin{array}{lll}2.67906662 & 62.035852 & 46.875\end{array}$

$\begin{array}{lll}2.67906662 & 62.035852 & 46.875\end{array}$

$\begin{array}{lll}1.00240463 & 0.11512183 & -524.75104\end{array}$

$\begin{array}{llll}1.00240463 & 0.11512183 & -524.75104\end{array}$

$\begin{array}{llll}1.00240463 & 0.11512183 & -524.75104\end{array}$

$\begin{array}{lll}1.00240463 & 0.11512183 & -524.75104\end{array}$

$\begin{array}{llll}1.00240463 & 0.11512183 & -524.75104\end{array}$

$\begin{array}{llll}1.00240463 & 0.11512183 & -524.75104\end{array}$

$2.75905328 \quad 63.4962802 \quad 46.875$

$2.75905328 \quad 63.4962802 \quad 46.875$

$2.75905328 \quad 63.4962802 \quad 46.875$

$\begin{array}{lll}2.75905328 & 63.4962802 & 46.875\end{array}$

$\begin{array}{llll}2.75905328 & 63.4962802 & 46.875\end{array}$

$\begin{array}{lll}2.75905328 & 63.4962802 & 46.875\end{array}$

$\begin{array}{llll}1.00000298 & -0.0008533 & -526.06793\end{array}$

$\begin{array}{llll}1.13107839 & 4.98821347 & -478.67205\end{array}$

$\begin{array}{llll}1.71344931 & 26.5233354 & -279.61969\end{array}$

$\begin{array}{llll}4.26242857 & 120.139454 & 579.90974\end{array}$

$\begin{array}{lll}62.0482327 & 2233.63767 \quad 19905\end{array}$

$\begin{array}{llll}14.0767269 & 475.506054 & 3796.5508\end{array}$

$\begin{array}{llll}2.48136347 & 53.4167486 & -44.587899\end{array}$

$\begin{array}{llll}1.22869896 & 8.08499488 & -454.67346\end{array}$

$\begin{array}{llll}1.00000499 & 0.00142625 & -526.02697\end{array}$

$\begin{array}{llll}1.23340414 & 8.79225548 & -443.32858\end{array}$

$\begin{array}{llll}2.49778037 & 55.3757848 & -14.291922\end{array}$

$\begin{array}{llll}14.1875003 & 483.58788 & 3907.18357\end{array}$

$\begin{array}{lll}62.5258554 & 2242.3583 & 19905\end{array}$

$\begin{array}{llll}4.29006471 & 119.132354 & 552.30868\end{array}$

$\begin{array}{llll}1.721355596 & 25.8685564 & -294.21074\end{array}$

$\begin{array}{llll}1.13377515 & 4.68132552 & -485.17435\end{array}$

Source S20

\author{
Line \\ $\mathrm{S} 20 \mathrm{COI}$ \\ ai \\ bi \\ ci \\ $\begin{array}{lll}1.19167983 & -9.1766718 & -409.97479\end{array}$ \\ $\mathrm{S} 20 \mathrm{CO} 2$
}
ao
bo
$\begin{array}{lll}1.19167983 & -9.1766718 & -416.29562\end{array}$
$\begin{array}{llll}1.19167983 & -9.1766718 & -416.29562\end{array}$ 


\section{REVISED METHODOLOGY FOR DETERMINING CESIUM-137 CONTENT OF HN-200 GROUT CONTAINERS.}

\begin{tabular}{|c|c|c|c|c|c|c|}
\hline $\mathbf{S} 20 \mathrm{C} 03$ & 1.19167983 & -9.1766718 & -409.97479 & 1.19167983 & -9.1766718 & $-416,29562$ \\
\hline S20C04 & 1.19167983 & -9.1766718 & -409.97479 & 1.19167983 & -9.1766718 & -416.29562 \\
\hline $\mathrm{S} 20 \mathrm{C} 05$ & 1.19167983 & -9.1766718 & -409.97479 & 1.19167983 & -9.1766718 & -416.29562 \\
\hline S20C06 & 1.19167983 & -9.1766718 & -409.97479 & 1.19167983 & -9.1766718 & -416.29562 \\
\hline S20C07 & 27.3787413 & -245.88714 & 53.1958258 & 27.3787413 & -245.88714 & 46.875 \\
\hline S20C08 & 27.3787413 & -245.88714 & 53.1958258 & 27.3787413 & -245.88714 & 46.875 \\
\hline$\$ 20 C 09$ & 27.3787413 & -245.88714 & 53.1958258 & 27.3787413 & -245.88714 & 46.875 \\
\hline $\mathbf{S} 20 \mathrm{C} 10$ & 27.3787413 & -245.88714 & 53.1958258 & 27.3787413 & -245.88714 & 46.875 \\
\hline $\mathrm{S} 20 \mathrm{C} 11$ & 27.3787413 & -245.88714 & 53.1958258 & 27.3787413 & -245.88714 & 46.875 \\
\hline $\mathrm{S} 20 \mathrm{C} 12$ & 27.3787413 & -245.88714 & 53.1958258 & 27.3787413 & -245.88714 & 46.875 \\
\hline $\mathrm{S} 20 \mathrm{C} 13$ & 1.1184964 & 5.67301507 & -451.90918 & 1.1184964 & 5.67301507 & -458.23001 \\
\hline S20C14 & 1.1184964 & 5.67301507 & -451.90918 & 1.1184964 & 5.67301507 & -458.23001 \\
\hline S20C15 & 1.1184964 & 5.67301507 & -451.90918 & 1.1184964 & 5.67301507 & -458.23001 \\
\hline S20C16 & 1.1184964 & 5.67301507 & -451.90918 & 1.1184964 & 5.67301507 & -458.23001 \\
\hline S20C17 & 1.1184964 & 5.67301507 & -451.90918 & 1.1184964 & 5.67301507 & -458.23001 \\
\hline$S 20 C 18$ & 1.1184964 & 5.67301507 & -451.90918 & 1.1184964 & 5.67301507 & -458.23001 \\
\hline S20C19 & 135.050421 & -554.29745 & 53.1958258 & 135.050421 & -554.29745 & 46.875 \\
\hline$S 20 C 20$ & 135.050421 & -554.29745 & 53.1958258 & 135.050421 & -554.29745 & 46.875 \\
\hline$S 20 C 21$ & 135.050421 & -554.29745 & 53.1958258 & 135.050421 & -554.29745 & 46.875 \\
\hline $\mathbf{S} 20 \mathrm{C} 22$ & 135.050421 & -554.29745 & 53.1958258 & 135.050421 & -554.29745 & 46.875 \\
\hline S20C23 & 135.050421 & -554.29745 & 53.1958258 & 135.050421 & -554.29745 & 46.875 \\
\hline $520 \mathrm{C} 24$ & 135.050421 & -554.29745 & 53.1958258 & 135.050421 & -554.29745 & 46.875 \\
\hline S20R01 & 1.00433836 & -1.2402277 & -431.17056 & 1.00433836 & -1.2402277 & -437.49139 \\
\hline S20R02 & 1.12389258 & 5.78511951 & -452.27456 & 1.12389258 & 5.78511951 & -458.59539 \\
\hline S20R03 & 1.87463969 & 12.2463084 & -476.94127 & 1.87463969 & 12.2463084 & -483.26209 \\
\hline S20R04 & 6.61515523 & 11.5572173 & -513.86126 & 6.61515523 & 11.5572173 & -520.18209 \\
\hline S20R05 & 2180.1252 & -13344.96 & 19911.3208 & 2180.1252 & -13344.96 & 19905 \\
\hline S20R06 & 5.55314044 & -65.457062 & -284.55139 & 5.55314044 & -65.457062 & -290.87222 \\
\hline S20R07 & 1.7808809 & -20.779162 & -381.57523 & 1.7808809 & -20.779162 & -387.89605 \\
\hline S20R08 & 1.11359506 & -6.8698492 & -415.94171 & 1.11359506 & -6.8698492 & -422.26253 \\
\hline S20R09 & 1.00400414 & 1.14468247 & -437.99906 & 1.00400414 & 1.14468247 & -444.31988 \\
\hline S20R10 & 1.22448747 & 7.45663362 & -457.88771 & 1.22448747 & 7.45663362 & -464.20854 \\
\hline S20R11 & 2.1261621 & 13.130125 & -481.53646 & 2.1261621 & 13.130125 & -487.85729 \\
\hline S20R 12 & 7.02387597 & 10.7791631 & -514.986 & 7.02387597 & 10.7791631 & -521.30683 \\
\hline S20R13 & 2823.06289 & -15186.564 & 19911.3208 & 2823.06289 & -15186.564 & 19905 \\
\hline S20R 14 & 8.42893813 & -92.851669 & -229.67804 & 8.42893813 & -92.851669 & -235.99887. \\
\hline S20R15 & 2.26137812 & -27.949914 & -364.97789 & 2.26137812 & -27.949914 & -371.29871 \\
\hline S20R16 & 1.24483751 & -10.532935 & -406.5261 & 1.24483751 & -10.532935 & -412.84693 \\
\hline
\end{tabular}

$\begin{array}{cccc}\text { Line } & \text { ai } & \text { bi } & \text { ci } \\ \text { S21C01 } & 1.14371211 & -6.8802172 & -437.46048 \\ \text { S21C02 } & 1.14371211 & -6.8802172 & -437.46048 \\ \text { S21C03 } & 1.14371211 & -6.8802172 & -437.46048\end{array}$

$\begin{array}{ccc}\text { ao } & \text { bo } & \text { co } \\ 1.14371211 & -6.8802172 & -443.78131 \\ 1.14371211 & -6.8802172 & -443.78131 \\ 1.14371211 & -6.8802172 & -443.78131\end{array}$




\section{REVISED METHODOLOGY FOR DETERMINING CESIUM-137 CONTENT OF HN-200 GROUT CONTAINERS.}

$\begin{array}{llll}\text { S21C04 } & 1.14371211 & -6.8802172 & -437.46048 \\ \text { S21C05 } & 1.14371211 & -6.8802172 & -437.46048 \\ \text { S21C06 } & 1.14371211 & -6.8802172 & -437.46048 \\ \text { S21C07 } & 109.196904 & -497.98493 & 53.1958258 \\ \text { S21C08 } & 109.196904 & -497.98493 & 53.1958258 \\ \text { S21C09 } & 109.196904 & -497.98493 & 53.1958258 \\ \text { S21C10 } & 109.196904 & -497.98493 & 53.1958258 \\ \text { S21C11 } & 109.196904 & -497.98493 & 53.1958258 \\ \text { S21C12 } & 109.196904 & -497.98493 & 53.1958258 \\ \text { S21C13 } & 1.08591096 & 4.11298722 & -470.58076 \\ \text { S21C14 } & 1.08591096 & 4.11298722 & -470.58076 \\ \text { S21C15 } & 1.08591096 & 4.11298722 & -470.58076 \\ \text { S21C16 } & 1.08591096 & 4.11298722 & -470.58076 \\ \text { S21C17 } & 1.08591096 & 4.11298722 & -470.58076 \\ \text { S21C18 } & 1.08591096 & 4.11298722 & -470.58076 \\ \text { S21C19 } & 28.3836496 & -250.52695 & 53.1958258 \\ \text { S21C20 } & 28.3836496 & -250.52695 & 53.1958258 \\ \text { S21C21 } & 28.3836496 & -250.52695 & 53.1958258 \\ \text { S21C22 } & 28.3836496 & -250.52695 & 53.1958258 \\ \text { S21C23 } & 28.3836496 & -250.52695 & 53.1958258 \\ \text { S21C24 } & 28.3836496 & -250.52695 & 53.1958258 \\ \text { S21R01 } & 1.00320096 & -0.9150748 & -454.40883 \\ \text { S21R02 } & 1.23560872 & -9.1254958 & -431.44688 \\ \text { S21R03 } & 2.23651478 & -25.172596 & -391.69404 \\ \text { S21R04 } & 8.34751792 & -87.900047 & -256.91596 \\ \text { S21R05 } & 2427.94535 & -14083.357 & 19911.3208 \\ \text { S21R06 } & 6.87207514 & 2.39198648 & -519.56449 \\ \text { S21R07 } & 2.09535118 & 9.85673612 & -497.63363 \\ \text { S21R08 } & 1.21473508 & 6.01929496 & -477.62597 \\ \text { S21R09 } & 1.00293805 & 0.83991626 & -459.78032 \\ \text { S21R10 } & 1.11989458 & -6.2146459 & -439.2752 \\ \text { S21R11 } & 1.80030764 & -19.060482 & -406.31986 \\ \text { S21R12 } & 5.61916452 & -62.305441 & -309.70689 \\ \text { S21R13 } & 1945.38684 & -12605.716 & 19911.3208 \\ \text { S21R14 } & 6.7797956 & 2.65456695 & -519.50328 \\ \text { S21R15 } & 1.90344745 & 9.51110233 & -494.77589 \\ \text { S21R16 } & 1.13154911 & 4.93494443 & -473.5256 \\ & & & \end{array}$

\author{
$\begin{array}{lll}1.14371211 & -6.8802172 & -443.78131\end{array}$
}

$\begin{array}{llll}1.14371211 & -6.8802172 & -443.78131\end{array}$

$\begin{array}{lll}1.14371211 & -6.8802172 & -443.78131\end{array}$

$\begin{array}{lll}109.196904 & -497.98493 & 46.875\end{array}$

$\begin{array}{lll}109.196904 & -497.98493 & 46.875\end{array}$

$109.196904-497.98493 \quad 46.875$

$109.196904 \quad-497.98493 \quad 46.875$

$109.196904 \quad-497.98493 \quad 46.875$

$\begin{array}{lll}109.196904 & -497.98493 \quad 46.875\end{array}$

$\begin{array}{llll}1.08591096 & 4.11298722 & -476.90159\end{array}$

$\begin{array}{llll}1.08591096 & 4.11298722 & -476.90159\end{array}$

$\begin{array}{llll}1.08591096 & 4.11298722 & -476.90159\end{array}$

$\begin{array}{llll}1.08591096 & 4.11298722 & -476.90159\end{array}$

$\begin{array}{llll}1.08591096 & 4.11298722 & -476.90159\end{array}$

$\begin{array}{llll}1.08591096 & 4.11298722 & -476.90159\end{array}$

$\begin{array}{lll}28.3836496 & -250.52695 & 46.875\end{array}$

$\begin{array}{lll}28.3836496 & -250.52695 & 46.875\end{array}$

$\begin{array}{lll}28.3836496 & -250.52695 & 46.875\end{array}$

$28.3836496 \quad-250.52695 \quad 46.875$

$\begin{array}{lll}28.3836496 & -250.52695 & 46.875\end{array}$

$\begin{array}{lll}28.3836496 & -250.52695 & 46.875\end{array}$

$\begin{array}{llll}1.00320096 & -0.9150748 & -460.72965\end{array}$

$\begin{array}{llll}1.23560872 & -9.1254958 & -437.76771\end{array}$

$\begin{array}{llll}2.23651478 & -25.172596 & -398.01487\end{array}$

$\begin{array}{llll}8.34751792 & -87.900047 & -263.23679\end{array}$

$\begin{array}{lllr}2427.94535 & -14083.357 & 19905\end{array}$

$\begin{array}{llll}6.87207514 & 2.39198648 & -525.88531\end{array}$

$\begin{array}{llll}2.09535118 & 9.85673612 & -503.95445\end{array}$

$\begin{array}{llll}1.21473508 & 6.01929496 & -483.9468\end{array}$

$\begin{array}{llll}1.00293805 & 0.83991626 & -466.10114\end{array}$

$\begin{array}{llll}1.11989458 & -6.2146459 & -445.59603\end{array}$

$1.80030764-19.060482 \quad-412.64069$

$\begin{array}{llll}5.61916452 & -62.305441 & -316.02771\end{array}$

$\begin{array}{lll}1945.38684 & -12605.716 & 19905\end{array}$

$\begin{array}{llll}6.7797956 & 2.65456695 & -525.82411\end{array}$

$\begin{array}{llll}1.90344745 & 9.51110233 & -501.09672\end{array}$

$\begin{array}{llll}1.13154911 & 4.93494443 & -479.84643\end{array}$

$\begin{array}{cccc}\text { Line } & \text { ai } & \text { bi } & \text { ci } \\ \text { S22C01 } & 1.09408588 & -4.5043615 & -465.8965 \\ \mathrm{~S} 22 \mathrm{C02} & 1.09408588 & -4.5043615 & -465.8965 \\ \mathrm{~S} 22 \mathrm{C} 03 & 1.09408588 & -4.5043615 & -465.8965 \\ \mathrm{~S} 22 \mathrm{C} 04 & 1.09408588 & -4.5043615 & -465.8965\end{array}$

$\begin{array}{ccc}\text { ao } & \text { bo } & \text { co } \\ 1.09408588 & -4.5043615 & -472.21733 \\ 1.09408588 & -4.5043615 & -472.21733 \\ 1.09408588 & -4.5043615 & -472.21733 \\ 1.09408588 & -4.5043615 & -472.21733\end{array}$




\section{REVISED METHODOLOGY FOR DETERMINING CESIUM-137 CONTENT OF HN-200 GROUT CONTAINERS.}

\begin{tabular}{lccc} 
S22C05 & 1.09408588 & -4.5043615 & -465.8965 \\
S22C06 & 1.09408588 & -4.5043615 & -465.8965 \\
S22C07 & 1.37986417 & 29.5068571 & 53.1958258 \\
S22C08 & 1.37986417 & 29.5068571 & 53.1958258 \\
S22C09 & 1.37986417 & 29.5068571 & 53.1958258 \\
S22C10 & 1.37986417 & 29.5068571 & 53.1958258 \\
S22C11 & 1.37986417 & 29.5068571 & 53.1958258 \\
S22C12 & 1.37986417 & 29.5068571 & 53.1958258 \\
S22C13 & 5.65464363 & 222.841064 & 2147.3209 \\
S22C14 & 5.65464363 & 222.841064 & 2147.3209 \\
S22C15 & 5.65464363 & 222.841064 & 2147.3209 \\
S22C16 & 5.65464363 & 222.841064 & 2147.3209 \\
S22C17 & 5.65464363 & 222.841064 & 2147.3209 \\
S22C18 & 5.65464363 & 222.841064 & 2147.3209 \\
S22C19 & 5.18864109 & 97.9817883 & 53.1958258 \\
S22C20 & 5.18864109 & 97.9817883 & 53.1958258 \\
S22C21 & 5.18864109 & 97.9817883 & 53.1958258 \\
S22C22 & 5.18864109 & 97.9817883 & 53.1958258 \\
S22C23 & 5.18864109 & 97.9817883 & 53.1958258 \\
S22C24 & 5.18864109 & 97.9817883 & 53.1958258 \\
S22R01 & 1.00638382 & -1.824976 & -389.37933 \\
S22R02 & 1.0777778 & 9.96788928 & -200.44054 \\
S22R03 & 1.54906674 & 38.7962503 & 165.513455 \\
S22R04 & 3.6899661 & 138.895223 & 1273.14043 \\
S22R05 & 53.3518953 & 2068.4374 & 19911.3208 \\
S22R06 & 11.5368148 & 295.397122 & 1550.53912 \\
S22R07 & 2.12706181 & 13.2263228 & -481.00462 \\
S22R08 & 1.13453399 & -4.5942332 & -480.58569 \\
S22R09 & 1.01058621 & 3.02633325 & -303.51983 \\
S22R10 & 1.35069366 & 27.8373262 & 32.6090339 \\
S22R11 & 2.87988298 & 102.849905 & 886.942098 \\
S22R12 & 16.5731772 & 661.429845 & 6503.31624 \\
S22R13 & 76.0951664 & 2477.32035 & 19911.3208 \\
S22R14 & 4.97570991 & 91.6806455 & 8.73530826 \\
S22R15 & 1.91581598 & 8.32162344 & -500.90433 \\
S22R16 & 1.20274566 & -4.2882949 & -497.13254 \\
& & & \\
\hline
\end{tabular}

\begin{tabular}{ccc}
1.09408588 & -4.5043615 & -472.21733 \\
1.09408588 & -4.5043615 & -472.21733 \\
1.37986417 & 29.5068571 & 46.875 \\
1.37986417 & 29.5068571 & 46.875 \\
1.37986417 & 29.5068571 & 46.875 \\
1.37986417 & 29.5068571 & 46.875 \\
1.37986417 & 29.5068571 & 46.875 \\
1.37986417 & 29.5068571 & 46.875 \\
5.65464363 & 222.841064 & 2141.00008 \\
5.65464363 & 222.841064 & 2141.00008 \\
5.65464363 & 222.841064 & 2141.00008 \\
5.65464363 & 222.841064 & 2141.00008 \\
5.65464363 & 222.841064 & 2141.00008 \\
5.65464363 & 222.841064 & 2141.00008 \\
5.18864109 & 97.9817883 & 46.875 \\
5.18864109 & 97.9817883 & 46.875 \\
5.18864109 & 97.9817883 & 46.875 \\
5.18864109 & 97.9817883 & 46.875 \\
5.18864109 & 97.9817883 & 46.875 \\
5.18864109 & 97.9817883 & 46.875 \\
1.00638382 & -1.824976 & -395.70015 \\
1.0777778 & 9.96788928 & -206.76137 \\
1.54906674 & 38.7962503 & 159.192629 \\
3.6899661 & 138.895223 & 1266.81961 \\
53.3518953 & 2068.4374 & 19905 \\
11.5368148 & 295.397122 & 1544.21829 \\
2.12706181 & 13.2263228 & -487.32544 \\
1.13453399 & -4.5942332 & -486.90651 \\
1.01058621 & 3.02633325 & -309.84065 \\
1.35069366 & 27.8373262 & 26.2882081 \\
2.87988298 & 102.849905 & 880.621272 \\
16.5731772 & 661.429845 & 6496.99542 \\
76.0951664 & 2477.32035 & 19905 \\
4.97570991 & 91.6806455 & 2.4144825 \\
1.91581598 & 8.32162344 & -507.22516 \\
1.20274566 & -4.2882949 & -503.45336 \\
& & \\
\hline
\end{tabular}

$\begin{array}{cccc}\text { Line } & \text { ai } & \text { bi } & \text { Source S23 } \\ \text { S23C01 } & 1.60204426 & -28.822869 & -174.83437 \\ \text { S23C02 } & 1.60204426 & -28.822869 & -174.83437 \\ \text { S23C03 } & 1.60204426 & -28.822869 & -174.83437 \\ \text { S23C04 } & 1.60204426 & -28.822869 & -174.83437 \\ \text { S23C05 } & 1.60204426 & -28.822869 & -174.83437\end{array}$

$\begin{array}{ccc}\text { ao } & \text { bo } & \text { co } \\ 1.60204426 & -28.822869 & -181.15519 \\ 1.60204426 & -28.822869 & -181.15519 \\ 1.60204426 & -28.822869 & -181.15519 \\ 1.60204426 & -28.822869 & -181.15519 \\ 1.60204426 & -28.822869 & -181.15519\end{array}$


REVISED METHODOLOGY FOR DETERMINING CESIUM-137 CONTENT OF HN-200 GROUT CONTAINERS.

\begin{tabular}{lccc} 
S23C06 & 1.60204426 & -28.822869 & -174.83437 \\
S23C07 & 4.34105576 & -87.508582 & 53.1958258 \\
S23C08 & 4.34105576 & -87.508582 & 53.1958258 \\
S23C09 & 4.34105576 & -87.508582 & 53.1958258 \\
S23C10 & 4.34105576 & -87.508582 & 53.1958258 \\
S23C11 & 4.34105576 & -87.508582 & 53.1958258 \\
S23C12 & 4.34105576 & -87.508582 & 53.1958258 \\
S23C13 & 1.2534072 & 12.1318695 & -374.60477 \\
S23C14 & 1.2534072 & 12.1318695 & -374.60477 \\
S23C15 & 1.2534072 & 12.1318695 & -374.60477 \\
S23C16 & 1.2534072 & 12.1318695 & -374.60477 \\
S23C17 & 1.2534072 & 12.1318695 & -374.60477 \\
S23C18 & 1.2534072 & 12.1318695 & -374.60477 \\
S23C19 & 58.1673812 & -361.97863 & 53.1958258 \\
S23C20 & 58.1673812 & -361.97863 & 53.1958258 \\
S23C21 & 58.1673812 & -361.97863 & 53.1958258 \\
S23C22 & 58.1673812 & -361.97863 & 53.1958258 \\
S23C23 & 58.1673812 & -361.97863 & 53.1958258 \\
S23C24 & 58.1673812 & -361.97863 & 53.1958258 \\
S23R01 & 1.01124535 & -3.2147636 & -290.05294 \\
S23R02 & 1.09967782 & 8.21310921 & -350.6251 \\
S23R03 & 1.81149234 & 18.0588259 & -419.3385 \\
S23R04 & 6.60606732 & 12.0447664 & -513.33847 \\
S23R05 & 634.274565 & -7194.0293 & 19911.3208 \\
S23R06 & 4.85695888 & -96.747469 & 86.8922408 \\
S23R07 & 1.6630828 & -30.56747 & -167.52543 \\
S23R08 & 1.08540567 & -9.414378 & -260.36829 \\
S23R09 & 1.00974932 & 2.78708576 & -320.61855 \\
S23R10 & 1.25541446 & 12.169568 & -374.84919 \\
S23R11 & 2.18732695 & 19.7457724 & -437.71285 \\
S23R12 & 7.01614607 & 10.3495571 & -515.357 \\
S23R13 & 955.686591 & -8832.9666 & 19911.3208 \\
S23R14 & 9.74443336 & -175.62632 & 362.027311 \\
S23R15 & 2.45307152 & -50.058456 & -88.678406 \\
S23R16 & 1.2980968 & -19.00128 & -217.01328 \\
& & & \\
\hline
\end{tabular}

\begin{tabular}{ccc}
1.60204426 & -28.822869 & -181.15519 \\
4.34105576 & -87.508582 & 46.875 \\
4.34105576 & -87.508582 & 46.875 \\
4.34105576 & -87.508582 & 46.875 \\
4.34105576 & -87.508582 & 46.875 \\
4.34105576 & -87.508582 & 46.875 \\
4.34105576 & -87.508582 & 46.875 \\
1.2534072 & 12.1318695 & -380.92559 \\
1.2534072 & 12.1318695 & -380.92559 \\
1.2534072 & 12.1318695 & -380.92559 \\
1.2534072 & 12.1318695 & -380.92559 \\
1.2534072 & 12.1318695 & -380.92559 \\
1.2534072 & 12.1318695 & -380.92559 \\
58.1673812 & -361.97863 & 46.875 \\
58.1673812 & -361.97863 & 46.875 \\
58.1673812 & -361.97863 & 46.875 \\
58.1673812 & -361.97863 & 46.875 \\
58.1673812 & -361.97863 & 46.875 \\
58.1673812 & -361.97863 & 46.875 \\
1.01124535 & -3.2147636 & -296.37377 \\
1.09967782 & 8.21310921 & -356.94592 \\
1.81149234 & 18.0588259 & -425.65932 \\
6.60606732 & 12.0447664 & -519.65929 \\
634.274565 & -7194.0293 & 19905 \\
4.85695888 & -96.747469 & 80.571415 \\
1.6630828 & -30.56747 & -173.84626 \\
1.08540567 & -9.414378 & -266.68912 \\
1.00974932 & 2.78708576 & -326.93937 \\
1.25541446 & 12.169568 & -381.17002 \\
2.18732695 & 19.7457724 & -444.03367 \\
7.01614607 & 10.3495571 & -521.67783 \\
955.686591 & -8832.9666 & 19905 \\
9.74443336 & -175.62632 & 355.706485 \\
2.45307152 & -50.058456 & -94.999232 \\
1.2980968 & -19.00128 & -223.3341 \\
& & \\
\hline
\end{tabular}

\begin{tabular}{cccc} 
& & & \multicolumn{2}{c}{ Source S24 } \\
Line & ai & bi & ci \\
S24C01 & 2.44621225 & -69.237411 & 308.877187 \\
S24C02 & 2.44621225 & -69.237411 & 308.877187 \\
S24C03 & 2.44621225 & -69.237411 & 308.877187 \\
S24C04 & 2.44621225 & -69.237411 & 308.877187 \\
S24C05 & 2.44621225 & -69.237411 & 308.877187 \\
S24C06 & 2.44621225 & -69.237411 & 308.877187
\end{tabular}

$\begin{array}{ccc}\text { ao } & \text { bo } & \text { co } \\ 2.44621225 & -69.237411 & 302.556361 \\ 2.44621225 & -69.237411 & 302.556361 \\ 2.44621225 & -69.237411 & 302.556361 \\ 2.44621225 & -69.237411 & 302.556361 \\ 2.44621225 & -69.237411 & 302.556361 \\ 2.44621225 & -69.237411 & 302.556361\end{array}$




\section{REVISED METHODOLOGY FOR DETERMINING CESIUM-137 CONTENT OF HN-200 GROUT CONTAINERS.}

$\begin{array}{llll}\text { S24C07 } & 1.35224115 & -28.413768 & 53.1958258 \\ \text { S24C08 } & 1.35224115 & -28.413768 & 53.1958258 \\ \text { S24C09 } & 1.35224115 & -28.413768 & 53.1958258 \\ \text { S24C10 } & 1.35224115 & -28.413768 & 53.1958258 \\ \text { S24C11 } & 1.35224115 & -28.413768 & 53.1958258 \\ \text { S24C12 } & 1.35224115 & -28.413768 & 53.1958258 \\ \text { S24C13 } & 1.36273858 & 17.3661093 & -311.95746 \\ \text { S24C14 } & 1.36273858 & 17.3661093 & -311.95746 \\ \text { S24C15 } & 1.36273858 & 17.3661093 & -311.95746 \\ \text { S24C16 } & 1.36273858 & 17.3661093 & -311.95746 \\ \text { S24C17 } & 1.36273858 & 17.3661093 & -311.95746 \\ \text { S24C18 } & 1.36273858 & 17.3661093 & -311.95746 \\ \text { S24C19 } & 30.3723621 & -259.46467 & 53.1958258 \\ \text { S24C20 } & 30.3723621 & -259.46467 & 53.1958258 \\ \text { S24C21 } & 30.3723621 & -259.46467 & 53.1958258 \\ \text { S24C22 } & 30.3723621 & -259.46467 & 53.1958258 \\ \text { S24C23 } & 30.3723621 & -259.46467 & 53.1958258 \\ \text { S24C24 } & 30.3723621 & -259.46467 & 53.1958258 \\ \text { S24R01 } & 1.02026017 & -5.7918767 & -105.86989 \\ \text { S24R02 } & 1.08177917 & 9.68604297 & -233.00085 \\ \text { S24R03 } & 1.77294107 & 21.4736983 & -370.66355 \\ \text { S24R04 } & 6.82937262 & -0.051716 & -519.80797 \\ \text { S24R05 } & 242.490515 & -4442.4875 & 19911.3208 \\ \text { S24R06 } & 4.24310331 & -120.83861 & 605.809069 \\ \text { S24R07 } & 1.56366314 & -37.823583 & 114.71248 \\ \text { S24R08 } & 1.06423632 & -10.761492 & -69.090692 \\ \text { S24R09 } & 1.01620991 & 4.63400679 & -188.62141 \\ \text { S24R10 } & 1.2786491 & 15.8461163 & -294.52527 \\ \text { S24R11 } & 2.21701318 & 23.0100691 & -411.04523 \\ \text { S24R12 } & 6.8277221 & -0.0385685 & -519.80802 \\ \text { S24R13 } & 415.777765 & -5822.157 & 19911.3208 \\ \text { S24R14 } & 11.4751407 & -291.16274 & 1503.4521 \\ \text { S24R15 } & 2.66886815 & -76.21268 & 350.298485 \\ \text { S24R16 } & 1.35474779 & -28.534681 & 53.9996119\end{array}$

\begin{tabular}{ccc}
1.35224115 & -28.413768 & 46.875 \\
1.35224115 & -28.413768 & 46.875 \\
1.35224115 & -28.413768 & 46.875 \\
1.35224115 & -28.413768 & 46.875 \\
1.35224115 & -28.413768 & 46.875 \\
1.35224115 & -28.413768 & 46.875 \\
1.36273858 & 17.3661093 & -318.27829 \\
1.36273858 & 17.3661093 & -318.27829 \\
1.36273858 & 17.3661093 & -318.27829 \\
1.36273858 & 17.3661093 & -318.27829 \\
1.36273858 & 17.3661093 & -318.27829 \\
1.36273858 & 17.3661093 & -318.27829 \\
30.3723621 & -259.46467 & 46.875 \\
30.3723621 & -259.46467 & 46.875 \\
30.3723621 & -259.46467 & 46.875 \\
30.3723621 & -259.46467 & 46.875 \\
30.3723621 & -259.46467 & 46.875 \\
30.3723621 & -259.46467 & 46.875 \\
1.02026017 & -5.7918767 & -112.19072 \\
1.08177917 & 9.68604297 & -239.32167 \\
1.77294107 & 21.4736983 & -376.98438 \\
6.82937262 & -0.051716 & -526.12879 \\
242.490515 & -4442.4875 & 19905 \\
4.24310331 & -120.83861 & 599.488243 \\
1.56366314 & -37.823583 & 108.391654 \\
1.06423632 & -10.761492 & -75.411518 \\
1.01620991 & 4.63400679 & -194.94223 \\
1.2786491 & 15.8461163 & -300.8461 \\
2.21701318 & 23.0100691 & -417.36606 \\
6.8277221 & -0.0385685 & -526.12884 \\
415.777765 & -5822.157 & 19905 \\
11.4751407 & -291.16274 & 1497.13127 \\
2.66886815 & -76.21268 & 343.977659 \\
1.35474779 & -28.534681 & 47.6787862 \\
& & \\
\hline
\end{tabular}

\begin{tabular}{|c|c|c|c|c|c|c|}
\hline \multicolumn{7}{|c|}{ Source S25 } \\
\hline Line & ai & bi & ci & ao & bo & co \\
\hline $\mathrm{S} 25 \mathrm{CO} 1$ & 1.37812855 & -18.102904 & -303.13895 & 1.37812855 & -18.102904 & -309.45977 \\
\hline $\mathrm{S} 25 \mathrm{C} 02$ & 1.37812855 & -18.102904 & -303.13895 & 1.37812855 & -18.102904 & -309.45977 \\
\hline S25C03 & 1.37812855 & -18.102904 & -303.13895 & 1.37812855 & -18.102904 & -309.45977 \\
\hline S25C04 & 1.37812855 & -18.102904 & -303.13895 & 1.37812855 & -18.102904 & -309.45977 \\
\hline S25C05 & 1.37812855 & -18.102904 & -303.13895 & 1.37812855 & -18.102904 & -309.45977 \\
\hline S25C06 & 1.37812855 & -18.102904 & -303.13895 & 1.37812855 & -18.102904 & -309.45977 \\
\hline S25C07 & 3.93304403 & -81.991358 & 53.1958258 & 3.93304403 & -81.991358 & 46.875 \\
\hline
\end{tabular}




\section{REVISED METHODOLOGY FOR DETERMINING CESIUM-137 CONTENT OF HN-200 GROUT CONTAINERS.}

$\begin{array}{llll}\text { S2SC08 } & 3.93304403 & -81.991358 & 53.1958258 \\ \text { S25C09 } & 3.93304403 & -81.991358 & 53.1958258 \\ \text { S25C10 } & 3.93304403 & -81.991358 & 53.1958258 \\ \text { S25C11 } & 3.93304403 & -81.991358 & 53.1958258 \\ \text { S25C12 } & 3.93304403 & -81.991358 & 53.1958258 \\ \text { S25C13 } & 1.08733727 & 4.18127164 & -469.76349 \\ \text { S25C14 } & 1.08733727 & 4.18127164 & -469.76349 \\ \text { S25C15 } & 1.08733727 & 4.18127164 & -469.76349 \\ \text { S25C16 } & 1.08733727 & 4.18127164 & -469.76349 \\ \text { S25C17 } & 1.08733727 & 4.18127164 & -469.76349 \\ \text { S25C18 } & 1.08733727 & 4.18127164 & -469.76349 \\ \text { S25C19 } & 16.9082896 & -190.95038 & 53.1958258 \\ \text { S25C20 } & 16.9082896 & -190.95038 & 53.1958258 \\ \text { S25C21 } & 16.9082896 & -190.95038 & 53.1958258 \\ \text { S25C22 } & 16.9082896 & -190.95038 & 53.1958258 \\ \text { S25C23 } & 16.9082896 & -190.95038 & 53.1958258 \\ \text { S25C24 } & 16.9082896 & -190.95038 & 53.1958258 \\ \text { S25R01 } & 1.00504476 & -1.4421722 & -416.73784 \\ \text { S25R02 } & 1.13327315 & 4.73876442 & -477.68428 \\ \text { S25R03 } & 1.97515576 & 2.4952664 & -518.21183 \\ \text { S25R04 } & 7.99961108 & -66.994103 & -359.50597 \\ \text { S25R05 } & 253.287972 & -4540.7172 & 19911.3208 \\ \text { S25R06 } & 4.76928515 & -100.40972 & 148.893747 \\ \text { S25R07 } & 1.69889055 & -27.715221 & -245.03921 \\ \text { S25R08 } & 1.10330633 & -7.8778079 & -369.62402 \\ \text { S25R09 } & 1.00398718 & 1.13983441 & -438.34554 \\ \text { S25R10 } & 1.2092919 & 5.22831964 & -487.15593 \\ \text { S25R11 } & 2.02127689 & 2.16181868 & -518.66406 \\ \text { S25R12 } & 6.03714878 & -41.704657 & -433.48552 \\ \text { S25R13 } & 330.766788 & -5191.3419 & 19911.3208 \\ \text { S25R14 } & 10.3540502 & -215.55274 & 721.979828 \\ \text { S25R15 } & 2.42497854 & -46.746633 & -136.42552 \\ \text { S25R16 } & 1.27000273 & -14.465514 & -326.05903\end{array}$

$\begin{array}{ccc}3.93304403 & -81.991358 & 46.875 \\ 3.93304403 & -81.991358 & 46.875 \\ 3.93304403 & -81.991358 & 46.875 \\ 3.93304403 & -81.991358 & 46.875 \\ 3.93304403 & -81.991358 & 46.875 \\ 1.08733727 & 4.18127164 & -476.08431 \\ 1.08733727 & 4.18127164 & -476.08431 \\ 1.08733727 & 4.18127164 & -476.08431 \\ 1.08733727 & 4.18127164 & -476.08431 \\ 1.08733727 & 4.18127164 & -476.08431 \\ 1.08733727 & 4.18127164 & -476.08431 \\ 16.9082896 & -190.95038 & 46.875 \\ 16.9082896 & -190.95038 & 46.875 \\ 16.9082896 & -190.95038 & 46.875 \\ 16.9082896 & -190.95038 & 46.875 \\ 16.9082896 & -190.95038 & 46.875 \\ 16.9082896 & -190.95038 & 46.875 \\ 1.00504476 & -1.4421722 & -423.05866 \\ 1.13327315 & 4.73876442 & -484.0051 \\ 1.97515576 & 2.4952664 & -524.53266 \\ 7.99961108 & -66.994103 & -365.82679 \\ 253.287972 & -4540.7172 & 19905 \\ 4.76928515 & -100.40972 & 142.572921 \\ 1.69889055 & -27.715221 & -251.36003 \\ 1.10330633 & -7.8778079 & -375.94484 \\ 1.00398718 & 1.13983441 & -444.66637 \\ 1.2092919 & 5.22831964 & -493.47675 \\ 2.02127689 & 2.16181868 & -524.98488 \\ 6.03714878 & -41.704657 & -439.80634 \\ 330.766788 & -5191.3419 & 19905 \\ 10.3540502 & -215.55274 & 715.659003 \\ 2.42497854 & -46.746633 & -142.74635 \\ 1.27000273 & -14.465514 & -332.37986\end{array}$

$\begin{array}{cccc}\text { Line } & \text { ai } & \text { bi } & \begin{array}{c}\text { Source S26 } \\ \text { ci }\end{array} \\ \text { S26C01 } & 1.66884988 & -32.021188 & -136.55449 \\ \text { S26C02 } & 1.66884988 & -32.021188 & -136.55449 \\ \text { S26C03 } & 1.66884988 & -32.021188 & -136.55449 \\ \text { S26C04 } & 1.66884988 & -32.021188 & -136.55449 \\ \text { S26C05 } & 1.66884988 & -32.021188 & -136.55449 \\ \text { S26C06 } & 1.66884988 & -32.021188 & -136.55449 \\ \text { S26C07 } & 4.53091948 & -89.960673 & 53.1958258 \\ \text { S26C08 } & 4.53091948 & -89.960673 & 53.1958258\end{array}$

$\begin{array}{ccc}\text { ao } & \text { bo } & \text { co } \\ 1.66884988 & -32.021188 & -142.87531 \\ 1.66884988 & -32.021188 & -142.87531 \\ 1.66884988 & -32.021188 & -142.87531 \\ 1.66884988 & -32.021188 & -142.87531 \\ 1.66884988 & -32.021188 & -142.87531 \\ 1.66884988 & -32.021188 & -142.87531 \\ 4.53091948 & -89.960673 & 46.875 \\ 4.53091948 & -89.960673 & 46.875\end{array}$


HNF-4799, Rev 0

August 25, 1999

REVISED METHODOLOGY FOR DETERMINING CESIUM-137 CONTENT OF HN-200 GROUT CONTAINERS.

$\begin{array}{lccc}\text { S26C09 } & 4.53091948 & -89.960673 & 53.1958258 \\ \text { S26C10 } & 4.53091948 & -89.960673 & 53.1958258 \\ \text { S26C11 } & 4.53091948 & -89.960673 & 53.1958258 \\ \text { S26C12 } & 4.53091948 & -89.960673 & 53.1958258 \\ \text { S26C13 } & 1.33431159 & 16.0051675 & -328.24623 \\ \text { S26C14 } & 1.33431159 & 16.0051675 & -328.24623 \\ \text { S26C15 } & 1.33431159 & 16.0051675 & -328.24623 \\ \text { S26C16 } & 1.33431159 & 16.0051675 & -328.24623 \\ \text { S26C17 } & 1.33431159 & 16.0051675 & -328.24623 \\ \text { S26C18 } & 1.33431159 & 16.0051675 & -328.24623 \\ \text { S26C19 } & 96.4957882 & -467.84382 & 53.1958258 \\ \text { S26C20 } & 96.4957882 & -467.84382 & 53.1958258 \\ \text { S26C21 } & 96.4957882 & -467.84382 & 53.1958258 \\ \text { S26C22 } & 96.4957882 & -467.84382 & 53.1958258 \\ \text { S26C23 } & 96.4957882 & -467.84382 & 53.1958258 \\ \text { S26C24 } & 96.4957882 & -467.84382 & 53.1958258 \\ \text { S26R01 } & 1.0136443 & -3.9005648 & -241.03959 \\ \text { S26R02 } & 1.09046487 & 9.01149339 & -295.39219 \\ \text { S26R03 } & 1.76585943 & 22.0892106 & -360.53171 \\ \text { S26R04 } & 6.24298299 & 31.1764002 & -473.46195 \\ \text { S26R05 } & 952.101288 & -8816.365 & 19911.3208 \\ \text { S26R06 } & 4.88004785 & -95.771771 & 71.1789722 \\ \text { S26R07 } & 1.65085871 & -31.514167 & -138.33407 \\ \text { S26R08 } & 1.07987478 & -9.822606 & -217.82418 \\ \text { S26R09 } & 1.01216184 & 3.47676582 & -271.32797 \\ \text { S26R10 } & 1.27123987 & 14.6621755 & -321.66299 \\ \text { S26R11 } & 2.2434704 & 25.9473029 & -384.4485 \\ \text { S26R12 } & 7.35684916 & 29.5316024 & -485.50982 \\ \text { S26R13 } & 1501.24921 & -11072.811 & 19911.3208 \\ \text { S26R14 } & 9.5898044 & -165.64613 & 278.773584 \\ \text { S26R15 } & 2.46318012 & -51.255743 & -70.931145 \\ \text { S26R16 } & 1.30720184 & -20.500712 & -177.78606\end{array}$

\begin{tabular}{ccc}
4.53091948 & -89.960673 & 46.875 \\
4.53091948 & -89.960673 & 46.875 \\
4.53091948 & -89.960673 & 46.875 \\
4.53091948 & -89.960673 & 46.875 \\
1.33431159 & 16.0051675 & -334.56706 \\
1.33431159 & 16.0051675 & -334.56706 \\
1.33431159 & 16.0051675 & -334.56706 \\
1.33431159 & 16.0051675 & -334.56706 \\
1.33431159 & 16.0051675 & -334.56706 \\
1.33431159 & 16.0051675 & -334.56706 \\
96.4957882 & -467.84382 & 46.875 \\
96.4957882 & -467.84382 & 46.875 \\
96.4957882 & -467.84382 & 46.875 \\
96.4957882 & -467.84382 & 46.875 \\
96.4957882 & -467.84382 & 46.875 \\
96.4957882 & -467.84382 & 46.875 \\
1.0136443 & -3.9005648 & -247.36042 \\
1.09046487 & 9.01149339 & -301.71302 \\
1.76585943 & 22.0892106 & -366.85254 \\
6.24298299 & 31.1764002 & -479.78277 \\
952.101288 & -8816.365 & 19905 \\
4.88004785 & -95.771771 & 64.8581464 \\
1.65085871 & -31.514167 & -144.6549 \\
1.07987478 & -9.822606 & -224.14501 \\
1.01216184 & 3.47676582 & -277.6488 \\
1.27123987 & 14.6621755 & -327.98382 \\
2.2434704 & 25.9473029 & -390.76933 \\
7.35684916 & 29.5316024 & -491.83064 \\
1501.24921 & -11072.811 & 19905 \\
9.5898044 & -165.64613 & 272.452758 \\
2.46318012 & -51.255743 & -77.251971 \\
1.30720184 & -20.500712 & -184.10688 \\
& & \\
\hline
\end{tabular}

$\begin{array}{cccc}\text { Line } & \text { ai } & \text { bi } & \begin{array}{c}\text { Source S27 } \\ \text { ci }\end{array} \\ \text { S27C01 } & 1.94842796 & -45.405989 & 23.6448451 \\ \text { S27C02 } & 1.94842796 & -45.405989 & 23.6448451 \\ \text { S27C03 } & 1.94842796 & -45.405989 & 23.6448451 \\ \text { S27C04 } & 1.94842796 & -45.405989 & 23.6448451 \\ \text { S27C05 } & 1.94842796 & -45.405989 & 23.6448451 \\ \text { S27C06 } & 1.94842796 & -45.405989 & 23.6448451 \\ \text { S27C07 } & 394.73539 & -949.97246 & 53.1958258 \\ \text { S27C08 } & 394.73539 & -949.97246 & 53.1958258 \\ \text { S27C09 } & 394.73539 & -949.97246 & 53.1958258\end{array}$

$\begin{array}{ccc}\text { ao } & \text { bo } & \text { co } \\ 1.94842796 & -45.405989 & 17.3240193 \\ 1.94842796 & -45.405989 & 17.3240193 \\ 1.94842796 & -45.405989 & 17.3240193 \\ 1.94842796 & -45.405989 & 17.3240193 \\ 1.94842796 & -45.405989 & 17.3240193 \\ 1.94842796 & -45.405989 & 17.3240193 \\ 394.73539 & -949.97246 & 46.875 \\ 394.73539 & -949.97246 & 46.875 \\ 394.73539 & -949.97246 & 46.875\end{array}$


REVISED METHODOLOGY FOR DETERMINING CESIUM-137 CONTENT OF HN-200 GROUT CONTAINERS.

\begin{tabular}{lccc} 
S27C10 & 394.73539 & -949.97246 & 53.1958258 \\
S27C11 & 394.73539 & -949.97246 & 53.1958258 \\
S27C12 & 394.73539 & -949.97246 & 53.1958258 \\
S27C13 & 1.64831419 & 31.0380417 & -148.32152 \\
S27C14 & 1.64831419 & 31.0380417 & -148.32152 \\
S27C15 & 1.64831419 & 31.0380417 & -148.32152 \\
S27C16 & 1.64831419 & 31.0380417 & -148.32152 \\
S27C17 & 1.64831419 & 31.0380417 & -148.32152 \\
S27C18 & 1.64831419 & 31.0380417 & -148.32152 \\
S27C19 & 2.56103519 & -59.815692 & 53.1958258 \\
S27C20 & 2.56103519 & -59.815692 & 53.1958258 \\
S27C21 & 2.56103519 & -59.815692 & 53.1958258 \\
S27C22 & 2.56103519 & -59.815692 & 53.1958258 \\
S27C23 & 2.56103519 & -59.815692 & 53.1958258 \\
S27C24 & 2.56103519 & -59.815692 & 53.1958258 \\
S27R01 & 1.02250304 & -6.4330575 & -60.045504 \\
S27R02 & 1.34110691 & -26.196975 & -16.826836 \\
S27R03 & 2.5290483 & -59.127456 & 51.7984639 \\
S27R04 & 9.53341632 & -162.0222 & 249.262409 \\
S27R05 & 5224.0003 & -20660.28 & 19911.3208 \\
S27R06 & 8.2276374 & 80.6495468 & -294.82621 \\
S27R07 & 2.39766296 & 43.5489343 & -180.57936 \\
S27R08 & 1.31844096 & 22.3698938 & -126.94707 \\
S27R09 & 1.02111802 & 6.03711325 & -88.343143 \\
S27R10 & 1.0625615 & -10.839985 & -50.249048 \\
S27R11 & 1.59883468 & -35.377138 & 2.68247344 \\
S27R12 & 4.79324339 & -99.415737 & 131.579643 \\
S27R13 & 2882.21766 & -15344.905 & 19911.3208 \\
S27R14 & 5.47854855 & 68.9819846 & -254.17993 \\
S27R15 & 1.6551273 & 31.1851995 & -148.69064 \\
S27R16 & 1.06701449 & 10.620981 & -98.984057 \\
& & & \\
\hline
\end{tabular}

\begin{tabular}{ccc}
394.73539 & -949.97246 & 46.875 \\
394.73539 & -949.97246 & 46.875 \\
394.73539 & -949.97246 & 46.875 \\
1.64831419 & 31.0380417 & -154.64234 \\
1.64831419 & 31.0380417 & -154.64234 \\
1.64831419 & 31.0380417 & -154.64234 \\
1.64831419 & 31.0380417 & -154.64234 \\
1.64831419 & 31.0380417 & -154.64234 \\
1.64831419 & 31.0380417 & -154.64234 \\
2.56103519 & -59.815692 & 46.875 \\
2.56103519 & -59.815692 & 46.875 \\
2.56103519 & -59.815692 & 46.875 \\
2.56103519 & -59.815692 & 46.875 \\
2.56103519 & -59.815692 & 46.875 \\
2.56103519 & -59.815692 & 46.875 \\
1.02250304 & -6.4330575 & -66.36633 \\
1.34110691 & -26.196975 & -23.147662 \\
2.5290483 & -59.127456 & 45.4776382 \\
9.53341632 & -162.0222 & 242.941583 \\
5224.0003 & -20660.28 & 19905 \\
8.2276374 & 80.6495468 & -301.14704 \\
2.39766296 & 43.5489343 & -186.90018 \\
1.31844096 & 22.3698938 & -133.26789 \\
1.02111802 & 6.03711325 & -94.663969 \\
1.0625615 & -10.839985 & -56.569873 \\
1.59883468 & -35.377138 & -3.6383523 \\
4.79324339 & -99.415737 & 125.258818 \\
2882.21766 & -15344.905 & 19905 \\
5.47854855 & 68.9819846 & -260.50076 \\
1.6551273 & 31.1851995 & -155.01146 \\
1.06701449 & 10.620981 & -105.30488 \\
& & \\
\hline
\end{tabular}

$\begin{array}{lccc}\text { Line } & \text { ai } & \text { bi } & \begin{array}{c}\text { Source S28 } \\ \text { ci }\end{array} \\ \text { S28C01 } & 2.05703927 & -50.605755 & 85.8795491 \\ \text { S28C02 } & 2.05703927 & -50.605755 & 85.8795491 \\ \text { S28C03 } & 2.05703927 & -50.605755 & 85.8795491 \\ \text { S28C04 } & 2.05703927 & -50.605755 & 85.8795491 \\ \text { S28C05 } & 2.05703927 & -50.605755 & 85.8795491 \\ \text { S28C06 } & 2.05703927 & -50.605755 & 85.8795491 \\ \text { S28C07 } & 3.58621094 & -76.991142 & 53.1958258 \\ \text { S28C08 } & 3.58621094 & -76.991142 & 53.1958258 \\ \text { S28C09 } & 3.58621094 & -76.991142 & 53.1958258 \\ \text { S28C10 } & 3.58621094 & -76.991142 & 53.1958258\end{array}$

$\begin{array}{ccc}\text { ao } & \text { bo } & \text { co } \\ 2.05703927 & -50.605755 & 79.5587234 \\ 2.05703927 & -50.605755 & 79.5587234 \\ 2.05703927 & -50.605755 & 79.5587234 \\ 2.05703927 & -50.605755 & 79.5587234 \\ 2.05703927 & -50.605755 & 79.5587234 \\ 2.05703927 & -50.605755 & 79.5587234 \\ 3.58621094 & -76.991142 & 46.875 \\ 3.58621094 & -76.991142 & 46.875 \\ 3.58621094 & -76.991142 & 46.875 \\ 3.58621094 & -76.991142 & \dot{46.875}\end{array}$




\section{REVISED METHODOLOGY FOR DETERMINING CESIUM-137 CONTENT} OF HN-200 GROUT CONTAINERS.

$\begin{array}{lccc}\text { S28C11 } & 3.58621094 & -76.991142 & 53.1958258 \\ \text { S28C12 } & 3.58621094 & -76.991142 & 53.1958258 \\ \text { S28C13 } & 1.01796992 & 0.86031003 & -509.51124 \\ \text { S28C14 } & 1.01796992 & 0.86031003 & -509.51124 \\ \text { S28C15 } & 1.01796992 & 0.86031003 & -509.51124 \\ \text { S28C16 } & 1.01796992 & 0.86031003 & -509.51124 \\ \text { S28C17 } & 1.01796992 & 0.86031003 & -509.51124 \\ \text { S28C18 } & 1.01796992 & 0.86031003 & -509.51124 \\ \text { S28C19 } & 1.98320404 & -47.471244 & 53.1958258 \\ \text { S28C20 } & 1.98320404 & -47.471244 & 53.1958258 \\ \text { S28C21 } & 1.98320404 & -47.471244 & 53.1958258 \\ \text { S28C22 } & 1.98320404 & -47.471244 & 53.1958258 \\ \text { S28C23 } & 1.98320404 & -47.471244 & 53.1958258 \\ \text { S28C24 } & 1.98320404 & -47.471244 & 53.1958258 \\ \text { S28R01 } & 1.00207882 & -0.5942837 & -477.33537 \\ \text { S28R02 } & 1.28227462 & -16.429194 & -280.75151 \\ \text { S28R03 } & 2.66792092 & -76.095322 & 348.113477 \\ \text { S28R04 } & 15.4093277 & -573.80797 & 5192.73439 \\ \text { S28R05 } & 66.1277783 & -2307.0622 & 19911.3208 \\ \text { S28R06 } & 4.5487128 & -109.31092 & 321.967345 \\ \text { S28R07 } & 1.79815624 & -19.252145 & -403.71393 \\ \text { S28R08 } & 1.16100293 & -1.3736475 & -516.87815 \\ \text { S28R09 } & 1.00123811 & 0.35394358 & -494.51218 \\ \text { S28R10 } & 1.10613887 & -7.6084914 & -383.45572 \\ \text { S28R11 } & 1.63738722 & -32.540794 & -104.47843 \\ \text { S28R12 } & 3.98771334 & -129.66649 & 887.070374 \\ \text { S28R13 } & 56.5557762 & -2130.7907 & 19911.3208 \\ \text { S28R14 } & 13.1314242 & -407.25877 & 2898.16869 \\ \text { S28R15 } & 2.33195911 & -35.95211 & -277.20412 \\ \text { S28R16 } & 1.18608549 & -1.9553914 & -514.67125 \\ & & & \\ & & & \end{array}$

\begin{tabular}{ccc}
3.58621094 & -76.991142 & 46.875 \\
3.58621094 & -76.991142 & 46.875 \\
1.01796992 & 0.86031003 & -515.83207 \\
1.01796992 & 0.86031003 & -515.83207 \\
1.01796992 & 0.86031003 & -515.83207 \\
1.01796992 & 0.86031003 & -515.83207 \\
1.01796992 & 0.86031003 & -515.83207 \\
1.01796992 & 0.86031003 & -515.83207 \\
1.98320404 & -47.471244 & 46.875 \\
1.98320404 & -47.471244 & 46.875 \\
1.98320404 & -47.471244 & 46.875 \\
1.98320404 & -47.471244 & 46.875 \\
1.98320404 & -47.471244 & 46.875 \\
1.98320404 & -47.471244 & 46.875 \\
1.00207882 & -0.5942837 & -483.65619 \\
1.28227462 & -16.429194 & -287.07233 \\
2.66792092 & -76.095322 & 341.792652 \\
15.4093277 & -573.80797 & 5186.41357 \\
66.1277783 & -2307.0622 & 19905 \\
4.5487128 & -109.31092 & 315.646519 \\
1.79815624 & -19.252145 & -410.03475 \\
1.16100293 & -1.3736475 & -523.19898 \\
1.00123811 & 0.35394358 & -500.833 \\
1.10613887 & -7.6084914 & -389.77655 \\
1.63738722 & -32.540794 & -110.79926 \\
3.98771334 & -129.66649 & 880.749548 \\
56.5557762 & -2130.7907 & 19905 \\
13.1314242 & -407.25877 & 2891.84786 \\
2.33195911 & -35.95211 & -283.52494 \\
1.18608549 & -1.9553914 & -520.99208 \\
& & \\
\hline
\end{tabular}

$\begin{array}{cccc}\text { Line } & \text { ai } & \text { bi } & \begin{array}{c}\text { Source S29 } \\ \text { ci }\end{array} \\ \mathrm{S} 29 \mathrm{C} 01 & 1.02298309 & -1.1003152 & -506.63868 \\ \mathrm{~S} 29 \mathrm{C} 02 & 1.02298309 & -1.1003152 & -506.63868 \\ \mathrm{~S} 29 \mathrm{C} 03 & 1.02298309 & -1.1003152 & -506.63868 \\ \mathrm{~S} 29 \mathrm{C} 04 & 1.02298309 & -1.1003152 & -506.63868 \\ \mathrm{~S} 29 \mathrm{C} 05 & 1.02298309 & -1.1003152 & -506.63868 \\ \mathrm{~S} 29 \mathrm{C} 06 & 1.02298309 & -1.1003152 & -506.63868 \\ \mathrm{~S} 29 \mathrm{C} 07 & 50449.6458 & 10753.0965 & 53.1958258 \\ \mathrm{~S} 29 \mathrm{C} 08 & 50449.6458 & 10753.0965 & 53.1958258 \\ \mathrm{~S} 29 \mathrm{C} 09 & 50449.6458 & 10753.0965 & 53.1958258 \\ \mathrm{~S} 29 \mathrm{C} 10 & 50449.6458 & 10753.0965 & 53.1958258 \\ \mathrm{~S} 29 \mathrm{C} 11 & 50449.6458 & 10753.0965 & 53.1958258\end{array}$

$\begin{array}{ccc}\text { ao } & \text { bo } & \text { co } \\ 1.02298309 & -1.1003152 & -512.95951 \\ 1.02298309 & -1.1003152 & -512.95951 \\ 1.02298309 & -1.1003152 & -512.95951 \\ 1.02298309 & -1.1003152 & -512.95951 \\ 1.02298309 & -1.1003152 & -512.95951 \\ 1.02298309 & -1.1003152 & -512.95951 \\ 50449.6458 & 10753.0965 & 46.875 \\ 50449.6458 & 10753.0965 & 46.875 \\ 50449.6458 & 10753.0965 & 46.875 \\ 50449.6458 & 10753.0965 & 46.875 \\ 50449.6458 & 10753.0965 & 46.875\end{array}$




\section{REVISED METHODOLOGY FOR DETERMINING CESIUM-137 CONTENT OF HN-200 GROUT CONTAINERS.}

\begin{tabular}{llll} 
S29C12 & 50449.6458 & 10753.0965 & 53.1958258 \\
S29C13 & 1.02333274 & 1.11705475 & -506.43833 \\
S29C14 & 1.02333274 & 1.11705475 & -506.43833 \\
S29C15 & 1.02333274 & 1.11705475 & -506.43833 \\
S29C16 & 1.02333274 & 1.11705475 & -506.43833 \\
S29C17 & 1.02333274 & 1.11705475 & -506.43833 \\
S29C18 & 1.02333274 & 1.11705475 & -506.43833 \\
S29C19 & 93170.1558 & 14613.1845 & 53.1958258 \\
S29C20 & 93170.1558 & 14613.1845 & 53.1958258 \\
S29C21 & 93170.1558 & 14613.1845 & 53.1958258 \\
S29C22 & 93170.1558 & 14613.1845 & 53.1958258 \\
S29C23 & 93170.1558 & 14613.1845 & 53.1958258 \\
S29C24 & 93170.1558 & 14613.1845 & 53.1958258 \\
S29R01 & 1.00064863 & -0.1854271 & -506.55583 \\
S29R02 & 1.14927804 & 2.84177406 & -506.28352 \\
S29R03 & 1.9275592 & 7.18411414 & -505.89751 \\
S29R04 & 6.49315596 & 18.067666 & -504.95139 \\
S29R05 & 2376559.33 & 440707.475 & 19911.3208 \\
S29R06 & 6.52957202 & -16.132214 & -508.04187 \\
S29R07 & 1.93088202 & -6.8608251 & -507.1666 \\
S29R08 & 1.14968716 & -2.7916015 & -506.79254 \\
S29R09 & 1.00065027 & 0.1858966 & -506.52228 \\
S29R10 & 1.19545249 & 3.25616569 & -506.24645 \\
S29R11 & 2.07530116 & 7.74896979 & -505.84768 \\
S29R12 & 7.17478801 & 19.2192709 & -504.8529 \\
S29R13 & 2631655.43 & 463757.139 & 19911.3208 \\
S29R14 & 7.13412275 & -16.935174 & -508.11936 \\
S29R15 & 2.07146282 & -7.347525 & -507.21172 \\
S29R16 & 1.19491829 & -3.1812158 & -506.82811 \\
& & & \\
\hline
\end{tabular}

$\begin{array}{lcc}50449.6458 & 10753.0965 & 46.875 \\ 1.02333274 & 1.11705475 & -512.75916 \\ 1.02333274 & 1.11705475 & -512.75916 \\ 1.02333274 & 1.11705475 & -512.75916 \\ 1.02333274 & 1.11705475 & -512.75916 \\ 1.02333274 & 1.11705475 & -512.75916 \\ 1.02333274 & 1.11705475 & -512.75916 \\ 93170.1558 & 14613.1845 & 46.875 \\ 93170.1558 & 14613.1845 & 46.875 \\ 93170.1558 & 14613.1845 & 46.875 \\ 93170.1558 & 14613.1845 & 46.875 \\ 93170.1558 & 14613.1845 & 46.875 \\ 93170.1558 & 14613.1845 & 46.875 \\ 1.00064863 & -0.1854271 & -512.87666 \\ 1.14927804 & 2.84177406 & -512.60435 \\ 1.9275592 & 7.18411414 & -512.21834 \\ 6.49315596 & 18.067666 & -511.27221 \\ 2376559.33 & 440707.475 & 19905 \\ 6.52957202 & -16.132214 & -514.3627 \\ 1.93088202 & -6.8608251 & -513.48742 \\ 1.14968716 & -2.7916015 & -513.11336 \\ 1.00065027 & 0.1858966 & -512.84311 \\ 1.19545249 & 3.25616569 & -512.56728 \\ 2.07530116 & 7.74896979 & -512.16851 \\ 7.17478801 & 19.2192709 & -511.17372 \\ 2631655.43 & 463757.139 & 19905 \\ 7.13412275 & -16.935174 & -514.44019 \\ 2.07146282 & -7.347525 & -513.53255 \\ 1.19491829 & -3.1812158 & -513.14894\end{array}$

$\begin{array}{lccccccc}\text { Line } & \text { ai } & \text { bi } & \begin{array}{c}\text { Source S30 } \\ \text { ci }\end{array} & \text { ao } & \text { bo } & \text { co } \\ \text { S30C01 } & 2.42670825 & -68.303658 & 297.701321 & 2.42670825 & -68.303658 & 291.380495 \\ \text { S30C02 } & 2.42670825 & -68.303658 & 297.701321 & 2.42670825 & -68.303658 & 291.380495 \\ \text { S30C03 } & 2.42670825 & -68.303658 & 297.701321 & 2.42670825 & -68.303658 & 291.380495 \\ \text { S30C04 } & 2.42670825 & -68.303658 & 297.701321 & 2.42670825 & -68.303658 & 291.380495 \\ \text { S30C05 } & 2.42670825 & -68.303658 & 297.701321 & 2.42670825 & -68.303658 & 291.380495 \\ \text { S30C06 } & 2.42670825 & -68.303658 & 297.701321 & 2.42670825 & -68.303658 & 291.380495 \\ \text { S30C07 } & 36.1658004 & -283.90238 & 53.1958258 & 36.1658004 & -283.90238 & 46.875 \\ \text { S30C08 } & 36.1658004 & -283.90238 & 53.1958258 & 36.1658004 & -283.90238 & 46.875 \\ \text { S30C09 } & 36.1658004 & -283.90238 & 53.1958258 & 36.1658004 & -283.90238 & 46.875 \\ \text { S30C10 } & 36.1658004 & -283.90238 & 53.1958258 & 36.1658004 & -283.90238 & 46.875 \\ \text { S30C11 } & 36.1658004 & -283.90238 & 53.1958258 & 36.1658004 & -283.90238 & 46.875 \\ \text { S30C12 } & 36.1658004 & -283.90238 & 53.1958258 & 36.1658004 & -283.90238 & 46.875\end{array}$




\section{REVISED METHODOLOGY FOR DETERMINING CESIUM-137 CONTENT OF HN-200 GROUT CONTAINERS.}

$\begin{array}{lccc}\text { S3OC13 } & 1.39930634 & 19.1167911 & -291.00399 \\ \text { S30C14 } & 1.39930634 & 19.1167911 & -291.00399 \\ \text { S30C15 } & 1.39930634 & 19.1167911 & -291.00399 \\ \text { S30C16 } & 1.39930634 & 19.1167911 & -291.00399 \\ \text { S30C17 } & 1.39930634 & 19.1167911 & -291.00399 \\ \text { S30C18 } & 1.39930634 & 19.1167911 & -291.00399 \\ \text { S30C19 } & 1.31713639 & -26.960741 & 53.1958258 \\ \text { S30C20 } & 1.31713639 & -26.960741 & 53.1958258 \\ \text { S30C21 } & 1.31713639 & -26.960741 & 53.1958258 \\ \text { S30C22 } & 1.31713639 & -26.960741 & 53.1958258 \\ \text { S30C23 } & 1.31713639 & -26.960741 & 53.1958258 \\ \text { S30C24 } & 1.31713639 & -26.960741 & 53.1958258 \\ \text { S30R01 } & 1.02130084 & -6.0893766 & -84.607948 \\ \text { S30R02 } & 1.35687704 & -28.901792 & 65.3469409 \\ \text { S30R03 } & 2.66370674 & -75.573464 & 338.418365 \\ \text { S30R04 } & 11.2937337 & -278.75098 & 1367.31348 \\ \text { S30R05 } & 488.080158 & -6309.2305 & 19911.3208 \\ \text { S30R06 } & 6.98387164 & 8.55872774 & -516.74769 \\ \text { S30R07 } & 2.24201413 & 25.7849589 & -385.98027 \\ \text { S30R08 } & 1.28538207 & 16.9308609 & -268.6938 \\ \text { S30R09 } & 1.01732643 & 4.95319347 & -165.80953 \\ \text { S30R10 } & 1.06270427 & -10.833482 & -51.880107 \\ \text { S30R11 } & 1.56184811 & -37.945937 & 120.887388 \\ \text { S30R12 } & 4.271421 & -119.81269 & 577.198462 \\ \text { S30R13 } & 279.98905 & -4774.9602 & 19911.3208 \\ \text { S30R14 } & 6.62765021 & 10.8862162 & -514.54346 \\ \text { S30R15 } & 1.75260858 & 23.2307311 & -340.54263 \\ \text { S30R16 } & 1.07841318 & 9.92444417 & -205.78372\end{array}$

$$
\begin{array}{ccc}
1.39930634 & 19.1167911 & -297.32481 \\
1.39930634 & 19.1167911 & -297.32481 \\
1.39930634 & 19.1167911 & -297.32481 \\
1.39930634 & 19.1167911 & -297.32481 \\
1.39930634 & 19.1167911 & -297.32481 \\
1.39930634 & 19.1167911 & -297.32481 \\
1.31713639 & -26.960741 & 46.875 \\
1.31713639 & -26.960741 & 46.875 \\
1.31713639 & -26.960741 & 46.875 \\
1.31713639 & -26.960741 & 46.875 \\
1.31713639 & -26.960741 & 46.875 \\
1.31713639 & -26.960741 & 46.875 \\
1.02130084 & -6.0893766 & -90.928774 \\
1.35687704 & -28.901792 & 59.0261151 \\
2.66370674 & -75.573464 & 332.097539 \\
11.2937337 & -278.75098 & 1360.99266 \\
488.080158 & -6309.2305 & 19905 \\
6.98387164 & 8.55872774 & -523.06852 \\
2.24201413 & 25.7849589 & -392.3011 \\
1.28538207 & 16.9308609 & -275.01463 \\
1.01732643 & 4.95319347 & -172.13036 \\
1.06270427 & -10.833482 & -58.200933 \\
1.56184811 & -37.945937 & 114.566563 \\
4.271421 & -119.81269 & 570.877637 \\
279.98905 & -4774.9602 & 19905 \\
6.62765021 & 10.8862162 & -520.86429 \\
1.75260858 & 23.2307311 & -346.86345 \\
1.07841318 & 9.92444417 & -212.10455
\end{array}
$$

Source S31

$\begin{array}{cccc}\text { Line } & \text { ai } & \text { bi } & \text { ci } \\ \text { S31C01 } & 1.93722983 & -44.869878 & 17.2282756 \\ \text { S31C02 } & 1.93722983 & -44.869878 & 17.2282756 \\ \text { S31C03 } & 1.93722983 & -44.869878 & 17.2282756 \\ \text { S31C04 } & 1.93722983 & -44.869878 & 17.2282756 \\ \text { S31C05 } & 1.93722983 & -44.869878 & 17.2282756 \\ \text { S31C06 } & 1.93722983 & -44.869878 & 17.2282756 \\ \text { S31C07 } & 57.3885314 & -359.50437 & 53.1958258 \\ \text { S31C08 } & 57.3885314 & -359.50437 & 53.1958258 \\ \text { S31C09 } & 57.3885314 & -359.50437 & 53.1958258 \\ \text { S31C10 } & 57.3885314 & -359.50437 & 53.1958258 \\ \text { S31C11 } & 57.3885314 & -359.50437 & 53.1958258 \\ \text { S31C12 } & 57.3885314 & -359.50437 & 53.1958258 \\ \text { S31C13 } & 1.3639583 & 17.4245037 & -311.25855\end{array}$

$\begin{array}{ccc}\text { ao } & \text { bo } & \text { co } \\ 1.93722983 & -44.869878 & 10.9074498 \\ 1.93722983 & -44.869878 & 10.9074498 \\ 1.93722983 & -44.869878 & 10.9074498 \\ 1.93722983 & -44.869878 & 10.9074498 \\ 1.93722983 & -44.869878 & 10.9074498 \\ 1.93722983 & -44.869878 & 10.9074498 \\ 57.3885314 & -359.50437 & 46.875 \\ 57.3885314 & -359.50437 & 46.875 \\ 57.3885314 & -359.50437 & 46.875 \\ 57.3885314 & -359.50437 & 46.875 \\ 57.3885314 & -359.50437 & 46.875 \\ 57.3885314 & -359.50437 & 46.875 \\ 1.3639583 & 17.4245037 & -317.57938\end{array}$




\section{REVISED METHODOLOGY FOR DETERMINING CESIUM-137 CONTENT OF HN-200 GROUT CONTAINERS.}

$\begin{array}{lccc}\text { S31C14 } & 1.3639583 & 17.4245037 & -311.25855 \\ \text { S31C15 } & 1.3639583 & 17.4245037 & -311.25855 \\ \text { S31C16 } & 1.3639583 & 17.4245037 & -311.25855 \\ \text { S31C17 } & 1.3639583 & 17.4245037 & -311.25855 \\ \text { S31C18 } & 1.3639583 & 17.4245037 & -311.25855 \\ \text { S31C19 } & 2.22209443 & -52.925037 & 53.1958258 \\ \text { S31C20 } & 2.22209443 & -52.925037 & 53.1958258 \\ \text { S31C21 } & 2.22209443 & -52.925037 & 53.1958258 \\ \text { S31C22 } & 2.22209443 & -52.925037 & 53.1958258 \\ \text { S31C23 } & 2.22209443 & -52.925037 & 53.1958258 \\ \text { S31C24 } & 2.22209443 & -52.925037 & 53.1958258 \\ \text { S31R01 } & 1.0167763 & -4.7959242 & -177.04937 \\ \text { S31R02 } & 1.32841451 & -24.044737 & -79.701619 \\ \text { S31R03 } & 2.5487264 & -61.501976 & 90.7731431 \\ \text { S31R04 } & 10.2983387 & -211.86703 & 687.064413 \\ \text { S31R05 } & 837.499647 & -8268.1635 & 19911.3208 \\ \text { S31R06 } & 7.184459 & 19.7635241 & -504.01863 \\ \text { S31R07 } & 2.24268543 & 25.8597854 & -385.27514 \\ \text { S31R08 } & 1.27750672 & 15.6628975 & -298.7987 \\ \text { S31R09 } & 1.0143589 & 4.10485166 & -226.43946 \\ \text { S31R10 } & 1.07192225 & -10.343466 & -147.92287 \\ \text { S31R11 } & 1.60996921 & -34.573944 & -29.882687 \\ \text { S31R12 } & 4.59579161 & -107.44993 & 282.900201 \\ \text { S31R13 } & 508.08544 & -6437.4927 & 19911.3208 \\ \text { S31R14 } & 6.40627534 & 22.6576276 & -496.06863 \\ \text { S31R15 } & 1.7601887 & 22.5793672 & -352.14319 \\ \text { S31R16 } & 1.08511618 & 9.43660589 & -258.25518\end{array}$

\author{
$\begin{array}{lll}1.3639583 & 17.4245037 & -317.57938\end{array}$ \\ $\begin{array}{llll}1.3639583 & 17.4245037 & -317.57938\end{array}$ \\ $\begin{array}{lll}1.3639583 & 17.4245037 & -317.57938\end{array}$ \\ $\begin{array}{lll}1.3639583 & 17.4245037 & -317.57938\end{array}$ \\ $\begin{array}{lll}1.3639583 & 17.4245037 & -317.57938\end{array}$ \\ $2.22209443 \quad-52.925037 \quad 46.875$ \\ $2.22209443 \quad-52.925037 \quad 46.875$ \\ $2.22209443 \quad-52.925037 \quad 46.875$ \\ $\begin{array}{lll}2.22209443 & -52.925037 & 46.875\end{array}$ \\ $\begin{array}{lll}2.22209443 & -52.925037 & 46.875\end{array}$ \\ $\begin{array}{lllr}2.22209443 & -52.925037 & 46.875\end{array}$ \\ $\begin{array}{llll}1.0167763 & -4.7959242 & -183.3702\end{array}$ \\ $\begin{array}{llll}1.32841451 & -24.044737 & -86.022445\end{array}$ \\ $\begin{array}{llll}2.5487264 & -61.501976 & 84.4523174\end{array}$ \\ $\begin{array}{llll}10.2983387 & -211.86703 & 680.743587\end{array}$ \\ $\begin{array}{lll}837.499647 & -8268.1635 & 19905\end{array}$ \\ $\begin{array}{llll}7.184459 & 19.7635241 & -510.33945\end{array}$ \\ $\begin{array}{llll}2.24268543 & 25.8597854 & -391.59597\end{array}$ \\ $\begin{array}{llll}1.27750672 & 15.6628975 & -305.11953\end{array}$ \\ $\begin{array}{llll}1.0143589 & 4.10485166 & -232.76029\end{array}$ \\ $\begin{array}{llll}1.07192225 & -10.343466 & -154.24369\end{array}$ \\ $\begin{array}{llll}1.60996921 & -34.573944 & -36.203513\end{array}$ \\ $\begin{array}{llll}4.59579161 & -107.44993 & 276.579375\end{array}$ \\ $508.08544 \quad-6437.4927 \quad 19905$ \\ $\begin{array}{llll}6.40627534 & 22.6576276 & -502.38945\end{array}$ \\ $\begin{array}{llll}1.7601887 & 22.5793672 & -358.46402\end{array}$ \\ $\begin{array}{llll}1.08511618 & 9.43660589 & -264.57601\end{array}$
}

$\begin{array}{lccc}\text { Line } & \text { ai } & \text { bi } & \begin{array}{c}\text { Source S32 } \\ \text { ci }\end{array} \\ \mathrm{S} 32 \mathrm{C} 01 & 1.52245843 & -25.012697 & -220.43736 \\ \mathrm{~S} 32 \mathrm{C} 02 & 1.52245843 & -25.012697 & -220.43736 \\ \mathrm{~S} 32 \mathrm{C} 03 & 1.52245843 & -25.012697 & -220.43736 \\ \mathrm{~S} 32 \mathrm{C} 04 & 1.52245843 & -25.012697 & -220.43736 \\ \mathrm{~S} 32 \mathrm{C} 05 & 1.52245843 & -25.012697 & -220.43736 \\ \mathrm{~S} 32 \mathrm{C} 06 & 1.52245843 & -25.012697 & -220.43736 \\ \mathrm{~S} 32 \mathrm{C} 07 & 43.4108726 & 311.779382 & 53.1958258 \\ \mathrm{~S} 32 \mathrm{C} 08 & 43.4108726 & 311.779382 & 53.1958258 \\ \mathrm{~S} 32 \mathrm{C} 09 & 43.4108726 & 311.779382 & 53.1958258 \\ \mathrm{~S} 32 \mathrm{C} 10 & 43.4108726 & 311.779382 & 53.1958258 \\ \mathrm{~S} 32 \mathrm{C} 11 & 43.4108726 & 311.779382 & 53.1958258 \\ \mathrm{~S} 32 \mathrm{C} 12 & 43.4108726 & 311.779382 & 53.1958258 \\ \mathrm{~S} 32 \mathrm{C} 13 & 1.60902824 & 29.1572268 & -170.83252 \\ \mathrm{~S} 32 \mathrm{C} 14 & 1.60902824 & 29.1572268 & -170.83252\end{array}$

$\begin{array}{ccc}\text { ao } & \text { bo } & \text { co } \\ 1.52245843 & -25.012697 & -226.75819 \\ 1.52245843 & -25.012697 & -226.75819 \\ 1.52245843 & -25.012697 & -226.75819 \\ 1.52245843 & -25.012697 & -226.75819 \\ 1.52245843 & -25.012697 & -226.75819 \\ 1.52245843 & -25.012697 & -226.75819 \\ 43.4108726 & 311.779382 & 46.875 \\ 43.4108726 & 311.779382 & 46.875 \\ 43.4108726 & 311.779382 & 46.875 \\ 43.4108726 & 311.779382 & 46.875 \\ 43.4108726 & 311.779382 & 46.875 \\ 43.4108726 & 311.779382 & 46.875 \\ 1.60902824 & 29.1572268 & -177.15335 \\ 1.60902824 & 29.1572268 & -177.15335\end{array}$




\section{REVISED METHODOLOGY FOR DETERMINING CESIUM-137 CONTENT OF HN-200 GROUT CONTAINERS.}

$\begin{array}{lccc}\text { S32C15 } & 1.60902824 & 29.1572268 & -170.83252 \\ \text { S32C16 } & 1.60902824 & 29.1572268 & -170.83252 \\ \text { S32C17 } & 1.60902824 & 29.1572268 & -170.83252 \\ \text { S32C18 } & 1.60902824 & 29.1572268 & -170.83252 \\ \text { S32C19 } & 2088.75016 & 2187.49994 & 53.1958258 \\ \text { S32C20 } & 2088.75016 & 2187.49994 & 53.1958258 \\ \text { S32C21 } & 2088.75016 & 2187.49994 & 53.1958258 \\ \text { S32C22 } & 2088.75016 & 2187.49994 & 53.1958258 \\ \text { S32C23 } & 2088.75016 & 2187.49994 & 53.1958258 \\ \text { S32C24 } & 2088.75016 & 2187.49994 & 53.1958258 \\ \text { S32R01 } & 1.01559613 & -4.4585446 & -201.16147 \\ \text { S32R02 } & 1.07631618 & 10.0658872 & -187.89261 \\ \text { S32R03 } & 1.66399684 & 30.4961154 & -169.6509 \\ \text { S32R04 } & 5.20819389 & 81.4256367 & -125.92566 \\ \text { S32R05 } & 18571.4295 & 38957.1476 & 19911.3208 \\ \text { S32R06 } & 5.50011035 & -67.966999 & -263.17477 \\ \text { S32R07 } & 1.6885391 & -28.551547 & -223.82241 \\ \text { S32R08 } & 1.07846585 & -9.9208203 & -206.22364 \\ \text { S32R09 } & 1.01600157 & 4.57444913 & -192.87792 \\ \text { S32R10 } & 1.30702992 & 20.4722753 & -178.54325 \\ \text { S32R11 } & 2.41260466 & 45.2952989 & -156.70859 \\ \text { S32R12 } & 8.7808206 & 114.495839 & -98.602599 \\ \text { S32R13 } & 30784.7218 & 50157.5991 & 19911.3208 \\ \text { S32R14 } & 8.27608863 & -83.574116 & -279.82225 \\ \text { S32R15 } & 2.36225383 & -39.437834 & -234.37251 \\ \text { S32R16 } & 1.29861847 & -19.086819 & -214.81466\end{array}$

\author{
$\begin{array}{lll}1.60902824 & 29.1572268 & -177.15335\end{array}$ \\ $\begin{array}{lll}1.60902824 & 29.1572268 & -177.15335\end{array}$ \\ $\begin{array}{lll}1.60902824 & 29.1572268 & -177.15335\end{array}$ \\ $\begin{array}{lll}1.60902824 & 29.1572268 & -177.15335\end{array}$ \\ $2088.75016 \quad 2187.49994 \quad 46.875$ \\ $2088.75016 \quad 2187.49994 \quad 46.875$ \\ $2088.75016 \quad 2187.49994 \quad 46.875$ \\ $\begin{array}{lll}2088.75016 & 2187.49994 \quad 46.875\end{array}$ \\ $2088.75016 \quad 2187.49994 \quad 46.875$ \\ $\begin{array}{lll}2088.75016 & 2187.49994 & 46.875\end{array}$ \\ $\begin{array}{lll}1.01559613 & -4.4585446 & -207.4823\end{array}$ \\ $\begin{array}{llll}1.07631618 & 10.0658872 & -194.21343\end{array}$ \\ $\begin{array}{llll}1.66399684 & 30.4961154 & -175.97172\end{array}$ \\ $\begin{array}{llll}5.20819389 & 81.4256367 & -132.24649\end{array}$ \\ $\begin{array}{lll}18571.4295 & 38957.1476 \quad 19905\end{array}$ \\ $\begin{array}{llll}5.50011035 & -67.966999 & -269.49559\end{array}$ \\ $\begin{array}{llll}1.6885391 & -28.551547 & -230.14324\end{array}$ \\ $\begin{array}{llll}1.07846585 & -9.9208203 & -212.54446\end{array}$ \\ $\begin{array}{llll}1.01600157 & 4.57444913 & -199.19874\end{array}$ \\ $\begin{array}{llll}1.30702992 & 20.4722753 & -184.86408\end{array}$ \\ $\begin{array}{llll}2.41260466 & 45.2952989 & -163.02942\end{array}$ \\ $\begin{array}{llll}8.7808206 & 114.495839 & -104.92342\end{array}$ \\ $\begin{array}{llll}30784.7218 & 50157.5991 & 19905\end{array}$ \\ $\begin{array}{llll}8.27608863 & -83.574116 & -286.14307\end{array}$ \\ $\begin{array}{llll}2.36225383 & -39.437834 & -240.69334\end{array}$ \\ $\begin{array}{llll}1.29861847 & -19.086819 & -221.13549\end{array}$
}

$\begin{array}{cccc}\text { Line } & \text { ai } & \text { bi } & \begin{array}{c}\text { Source S33 } \\ \text { ci }\end{array} \\ \text { S33C01 } & 1.68857838 & -32.96569 & -125.24998 \\ \text { S33C02 } & 1.68857838 & -32.96569 & -125.24998 \\ \text { S33C03 } & 1.68857838 & -32.96569 & -125.24998 \\ \text { S33C04 } & 1.68857838 & -32.96569 & -125.24998 \\ \text { S33C05 } & 1.68857838 & -32.96569 & -125.24998 \\ \text { S33C06 } & 1.68857838 & -32.96569 & -125.24998 \\ \text { S33C07 } & 273.484595 & 790.277768 & 53.1958258 \\ \text { S33C08 } & 273.484595 & 790.277768 & 53.1958258 \\ \text { S33C09 } & 273.484595 & 790.277768 & 53.1958258 \\ \text { S33C10 } & 273.484595 & 790.277768 & 53.1958258 \\ \text { S33C11 } & 273.484595 & 790.277768 & 53.1958258 \\ \text { S33C12 } & 273.484595 & 790.277768 & 53.1958258 \\ \text { S33C13 } & 2.10062595 & 52.6924672 & 110.854886 \\ \text { S33C14 } & 2.10062595 & 52.6924672 & 110.854886 \\ \text { S33C15 } & 2.10062595 & 52.6924672 & 110.854886\end{array}$

$\begin{array}{ccc}\text { ao } & \text { bo } & \text { co } \\ 1.68857838 & -32.96569 & -131.5708 \\ 1.68857838 & -32.96569 & -131.5708 \\ 1.68857838 & -32.96569 & -131.5708 \\ 1.68857838 & -32.96569 & -131.5708 \\ 1.68857838 & -32.96569 & -131.5708 \\ 1.68857838 & -32.96569 & -131.5708 \\ 273.484595 & 790.277768 & 46.875 \\ 273.484595 & 790.277768 & 46.875 \\ 273.484595 & 790.277768 & 46.875 \\ 273.484595 & 790.277768 & 46.875 \\ 273.484595 & 790.277768 & 46.875 \\ 273.484595 & 790.277768 & 46.875 \\ 2.10062595 & 52.6924672 & 104.534061 \\ 2.10062595 & 52.6924672 & 104.534061 \\ 2.10062595 & 52.6924672 & 104.534061\end{array}$




\section{REVISED METHODOLOGY FOR DETERMINING CESIUM-137 CONTENT OF HN-200 GROUT CONTAINERS.}

$\begin{array}{llll}\text { S33C16 } & 2.10062595 & 52.6924672 & 110.854886 \\ \text { S33C17 } & 2.10062595 & 52.6924672 & 110.854886 \\ \text { S33C18 } & 2.10062595 & 52.6924672 & 110.854886 \\ \text { S33C19 } & 1.39484816 & 30.0831873 & 53.1958258 \\ \text { S33C20 } & 1.39484816 & 30.0831873 & 53.1958258 \\ \text { S33C21 } & 1.39484816 & 30.0831873 & 53.1958258 \\ \text { S33C22 } & 1.39484816 & 30.0831873 & 53.1958258 \\ \text { S33C23 } & 1.39484816 & 30.0831873 & 53.1958258 \\ \text { S33C24 } & 1.39484816 & 30.0831873 & 53.1958258 \\ \text { S33R01 } & 1.02316812 & -6.6231866 & -46.45721 \\ \text { S33R02 } & 1.32504096 & -23.47657 & -95.900467 \\ \text { S33R03 } & 2.40816997 & -44.776273 & -163.86478 \\ \text { S33R04 } & 8.19692713 & -78.799967 & -304.11065 \\ \text { S33R05 } & 3493.77794 & 16895.135 & 19911.3208 \\ \text { S33R06 } & 9.82987441 & 181.167215 & 409.467871 \\ \text { S33R07 } & 2.57285141 & 64.4269311 & 139.953773 \\ \text { S33R08 } & 1.35375029 & 28.3629013 & 48.7103072 \\ \text { S33R09 } & 1.02505248 & 7.16187711 & -7.9576758 \\ \text { S33R10 } & 1.06328432 & -10.806698 & -58.458715 \\ \text { S33R11 } & 1.64436496 & -32.011253 & -122.23824 \\ \text { S33R12 } & 5.4809012 & -68.871394 & -255.16997 \\ \text { S33R13 } & 1869.05404 & 12355.8007 & 19911.3208 \\ \text { S33R14 } & 4.65222855 & 105.190764 & 237.612807 \\ \text { S33R15 } & 1.57689835 & 36.9195958 & 70.8750709 \\ \text { S33R16 } & 1.05814836 & 11.0227921 & 2.57103328\end{array}$

$\begin{array}{ccc}2.10062595 & 52.6924672 & 104.534061 \\ 2.10062595 & 52.6924672 & 104.534061 \\ 2.10062595 & 52.6924672 & 104.534061 \\ 1.39484816 & 30.0831873 & 46.875 \\ 1.39484816 & 30.0831873 & 46.875 \\ 1.39484816 & 30.0831873 & 46.875 \\ 1.39484816 & 30.0831873 & 46.875 \\ 1.39484816 & 30.0831873 & 46.875 \\ 1.39484816 & 30.0831873 & 46.875 \\ 1.02316812 & -6.6231866 & -52.778036 \\ 1.32504096 & -23.47657 & -102.22129 \\ 2.40816997 & -44.776273 & -170.18561 \\ 8.19692713 & -78.799967 & -310.43148 \\ 3493.77794 & 16895.135 & 19905 \\ 9.82987441 & 181.167215 & 403.147046 \\ 2.57285141 & 64.4269311 & 133.632947 \\ 1.35375029 & 28.3629013 & 42.3894814 \\ 1.02505248 & 7.16187711 & -14.278502 \\ 1.06328432 & -10.806698 & -64.779541 \\ 1.64436496 & -32.011253 & -128.55906 \\ 5.4809012 & -68.871394 & -261.49079 \\ 1869.05404 & 12355.8007 & 19905 \\ 4.65222855 & 105.190764 & 231.291981 \\ 1.57689835 & 36.9195958 & 64.5542451 \\ 1.05814836 & 11.0227921 & -3.7497925\end{array}$

$\begin{array}{lll}2.10062595 & 52.6924672 & 104.534061\end{array}$

$2.10062595 \quad 52.6924672 \quad 104.534061$

$\begin{array}{lll}1.39484816 & 30.0831873 \quad 46.875\end{array}$

$\begin{array}{lll}1.39484816 & 30.0831873 \quad 46.875\end{array}$

$\begin{array}{lll}1.39484816 & 30.0831873 \quad 46.875\end{array}$

$\begin{array}{lll}1.39484816 & 30.0831873 & 46.875\end{array}$

$\begin{array}{lll}1.39484816 & 30.0831873 & 46.875\end{array}$

$\begin{array}{lll}1.39484816 & 30.0831873 \quad 46.875\end{array}$

$\begin{array}{llll}1.02316812 & -6.6231866 & -52.778036\end{array}$

$\begin{array}{llll}1.32504096 & -23.47657 & -102.22129\end{array}$

$\begin{array}{llll}2.40816997 & -44.776273 & -170.18561\end{array}$

$8.19692713-78.799967-310.43148$

$\begin{array}{llll}9.82987441 & 181.167215 & 403.147046\end{array}$

$\begin{array}{llll}2.57285141 & 64.4269311 & 133.632947\end{array}$

$\begin{array}{llll}1.35375029 & 28.3629013 & 42.3894814\end{array}$

$\begin{array}{llll}1.02505248 & 7.16187711 & -14.278502\end{array}$

$\begin{array}{llll}1.06328432 & -10.806698 & -64.779541\end{array}$

$\begin{array}{llll}1.64436496 & -32.011253 & -128.55906\end{array}$

$\begin{array}{llll}5.4809012 & -68.871394 & -261.49079\end{array}$

$\begin{array}{ccc}1869.05404 & 12355.8007 & 19905 \\ 4.65222855 & 105.190764 & 231.29198\end{array}$

$\begin{array}{lll}1.57689835 & 36.9195958 & 64.5542451 \\ 1.05814836 & 11.0227921 & -3.7497925\end{array}$

\section{Source S34}

$\begin{array}{lccc}\text { Line } & \text { ai } & \text { bi } & \text { ci } \\ \text { S34C01 } & 1.19860025 & -9.507987 & -406.00936 \\ \text { S34C02 } & 1.19860025 & -9.507987 & -406.00936 \\ \text { S34C03 } & 1.19860025 & -9.507987 & -406.00936 \\ \text { S34C04 } & 1.19860025 & -9.507987 & -406.00936 \\ \text { S34C05 } & 1.19860025 & -9.507987 & -406.00936 \\ \text { S34C06 } & 1.19860025 & -9.507987 & -406.00936 \\ \text { S34C07 } & 1.2428251 & 23.591501 & 53.1958258 \\ \text { S34C08 } & 1.2428251 & 23.591501 & 53.1958258 \\ \text { S34C09 } & 1.2428251 & 23.591501 & 53.1958258 \\ \text { S34C10 } & 1.2428251 & 23.591501 & 53.1958258 \\ \text { S34C11 } & 1.2428251 & 23.591501 & 53.1958258 \\ \text { S34C12 } & 1.2428251 & 23.591501 & 53.1958258 \\ \text { S34C13 } & 4.00025824 & 143.637363 & 1199.35161 \\ \text { S34C14 } & 4.00025824 & 143.637363 & 1199.35161 \\ \text { S34C15 } & 4.00025824 & 143.637363 & 1199.35161 \\ \text { S34C16 } & 4.00025824 & 143.637363 & 1199.35161\end{array}$

$\begin{array}{ccc}\text { ao } & \text { bo } & \text { co } \\ 1.19860025 & -9.507987 & -412.33019 \\ 1.19860025 & -9.507987 & -412.33019 \\ 1.19860025 & -9.507987 & -412.33019 \\ 1.19860025 & -9.507987 & -412.33019 \\ 1.19860025 & -9.507987 & -412.33019 \\ 1.19860025 & -9.507987 & -412.33019 \\ 1.2428251 & 23.591501 & 46.875 \\ 1.2428251 & 23.591501 & 46.875 \\ 1.2428251 & 23.591501 & 46.875 \\ 1.2428251 & 23.591501 & 46.875 \\ 1.2428251 & 23.591501 & 46.875 \\ 1.2428251 & 23.591501 & 46.875 \\ 4.00025824 & 143.637363 & 1193.03079 \\ 4.00025824 & 143.637363 & 1193.03079 \\ 4.00025824 & 143.637363 & 1193.03079 \\ 4.00025824 & 143.637363 & 1193.03079\end{array}$




\section{REVISED METHODOLOGY FOR DETERMINING CESIUM-137 CONTENT OF HN-200 GROUT CONTAINERS.}

$\begin{array}{lccc}\text { S34C17 } & 4.00025824 & 143.637363 & 1199.35161 \\ \text { S34C18 } & 4.00025824 & 143.637363 & 1199.35161 \\ \text { S34C19 } & 9.36861773 & 138.495497 & 53.1958258 \\ \text { S34C20 } & 9.36861773 & 138.495497 & 53.1958258 \\ \text { S34C21 } & 9.36861773 & 138.495497 & 53.1958258 \\ \text { S34C22 } & 9.36861773 & 138.495497 & 53.1958258 \\ \text { S34C23 } & 9.36861773 & 138.495497 & 53.1958258 \\ \text { S34C24 } & 9.36861773 & 138.495497 & 53.1958258 \\ \text { S34R01 } & 1.01167078 & -3.3363846 & -281.36084 \\ \text { S34R02 } & 1.06660437 & 10.6425082 & -94.674614 \\ \text { S34R03 } & 1.53286732 & 39.8447032 & 225.030418 \\ \text { S34R04 } & 3.79492318 & 135.782934 & 1129.34327 \\ \text { S34R05 } & 80.3565111 & 2546.63944 & 19911.3208 \\ \text { S34R06 } & 9.05155219 & 131.406322 & 16.3500735 \\ \text { S34R07 } & 1.9359795 & -6.3636682 & -508.99153 \\ \text { S34R08 } & 1.10238479 & -7.9640157 & -364.93755 \\ \text { S34R09 } & 1.01735885 & 4.96246193 & -165.14713 \\ \text { S34R10 } & 1.36941686 & 31.0754458 & 133.71064 \\ \text { S34R11 } & 2.84380669 & 98.2295993 & 788.498024 \\ \text { S34R12 } & 14.5004604 & 506.511616 & 4231.03024 \\ \text { S34R13 } & 128.877889 & 3232.7633 & 19911.3208 \\ \text { S34R14 } & 5.68639446 & 59.0664684 & -333.69231 \\ \text { S34R15 } & 2.0497071 & -5.0849279 & -513.65006 \\ \text { S34R16 } & 1.24031671 & -9.8410175 & -419.06017\end{array}$

$\begin{array}{ccc}4.00025824 & 143.637363 & 1193.03079 \\ 4.00025824 & 143.637363 & 1193.03079 \\ 9.36861773 & 138.495497 & 46.875 \\ 9.36861773 & 138.495497 & 46.875 \\ 9.36861773 & 138.495497 & 46.875 \\ 9.36861773 & 138.495497 & 46.875 \\ 9.36861773 & 138.495497 & 46.875 \\ 9.36861773 & 138.495497 & 46.875 \\ 1.01167078 & -3.3363846 & -287.68167 \\ 1.06660437 & 10.6425082 & -100.99544 \\ 1.53286732 & 39.8447032 & 218.709592 \\ 3.79492318 & 135.782934 & 1123.02244 \\ 80.3565111 & 2546.63944 & 19905 \\ 9.05155219 & 131.406322 & 10.0292477 \\ 1.9359795 & -6.3636682 & -515.31236 \\ 1.10238479 & -7.9640157 & -371.25838 \\ 1.01735885 & 4.96246193 & -171.46796 \\ 1.36941686 & 31.0754458 & 127.389814 \\ 2.84380669 & 98.2295993 & 782.177198 \\ 14.5004604 & 506.511616 & 4224.70941 \\ 128.877889 & 3232.7633 & 19905 \\ 5.68639446 & 59.0664684 & -340.01313 \\ 2.0497071 & -5.0849279 & -519.97088 \\ 1.24031671 & -9.8410175 & -425.381\end{array}$

$4.00025824 \quad 143.637363 \quad 1193.03079$

$9.36861773 \quad 138.495497 \quad 46.875$

$9.36861773 \quad 138.495497 \quad 46.875$

$9.36861773 \quad 138.495497 \quad 46.875$

$9.36861773 \quad 138.495497 \quad 46.875$

$9.36861773 \quad 138.495497 \quad 46.875$

$9.36861773 \quad 138.495497 \quad 46.875$

$\begin{array}{llll}1.01167078 & -3.3363846 & -287.68167\end{array}$

$\begin{array}{llll}1.06660437 & 10.6425082 & -100.99544\end{array}$

$\begin{array}{lll}3.79492318 & 135.782934 & 1123.02244\end{array}$

$\begin{array}{lll}80.3565111 & 2546.63944 \quad 19905\end{array}$

$\begin{array}{llll}9.05155219 & 131.406322 & 10.0292477\end{array}$

$\begin{array}{llll}1.10238479 & -7.9640157 & -371.25838\end{array}$

$\begin{array}{llll}1.01735885 & 4.96246193 & -171.46796\end{array}$

$\begin{array}{llll}1.36941686 & 31.0754458 & 127.389814\end{array}$

$\begin{array}{llll}2.84380669 & 98.2295993 & 782.177198\end{array}$

$14.5004604 \quad 506.511616 \quad 4224.70941$

$\begin{array}{llll}128.877889 & 3232.7633 & 19905\end{array}$

$\begin{array}{llll}5.68639446 & 59.0664684 & -340.01313\end{array}$

$\begin{array}{lll}1.24031671 & -9.8410175 & -425.381\end{array}$

$\begin{array}{lccccccc}\text { Line } & \text { ai } & \text { bi } & \text { ci } & \text { ao } & \text { bo } & \text { co } \\ \text { S35C01 } & 5.14456869 & -198.42123 & 1855.04597 & 5.14456869 & -198.42123 & 1848.72514 \\ \text { S35C02 } & 5.14456869 & -198.42123 & 1855.04597 & 5.14456869 & -198.42123 & 1848.72514 \\ \text { S35C03 } & 5.14456869 & -198.42123 & 1855.04597 & 5.14456869 & -198.42123 & 1848.72514 \\ \text { S35C04 } & 5.14456869 & -198.42123 & 1855.04597 & 5.14456869 & -198.42123 & 1848.72514 \\ \text { S35C05 } & 5.14456869 & -198.42123 & 1855.04597 & 5.14456869 & -198.42123 & 1848.72514 \\ \text { S35C06 } & 5.14456869 & -198.42123 & 1855.04597 & 5.14456869 & -198.42123 & 1848.72514 \\ \text { S35C07 } & 1.39647416 & -30.145065 & 53.1958258 & 1.39647416 & -30.145065 & 46.875 \\ \text { S35C08 } & 1.39647416 & -30.145065 & 53.1958258 & 1.39647416 & -30.145065 & 46.875 \\ \text { S35C09 } & 1.39647416 & -30.145065 & 53.1958258 & 1.39647416 & -30.145065 & 46.875 \\ \text { S35C10 } & 1.39647416 & -30.145065 & 53.1958258 & 1.39647416 & -30.145065 & 46.875 \\ \text { S35C11 } & 1.39647416 & -30.145065 & 53.1958258 & 1.39647416 & -30.145065 & 46.875 \\ \text { S35C12 } & 1.39647416 & -30.145065 & 53.1958258 & 1.39647416 & -30.145065 & 46.875 \\ \text { S35C13 } & 1.09532165 & 4.56352392 & -465.1884 & 1.09532165 & 4.56352392 & -471.50923 \\ \text { S35C14 } & 1.09532165 & 4.56352392 & -465.1884 & 1.09532165 & 4.56352392 & -471.50923 \\ \text { S35C15 } & \mathbf{1 . 0 9 5 3 2 1 6 5} & 4.56352392 & -465.1884 & 1.09532165 & 4.56352392 & -471.50923 \\ \text { S35C16 } & 1.09532165 & 4.56352392 & -465.1884 & 1.09532165 & 4.56352392 & -471.50923 \\ \text { S35C17 } & 1.09532165 & 4.56352392 & -465.1884 & 1.09532165 & 4.56352392 & -471.50923\end{array}$




\section{REVISED METHODOLOGY FOR DETERMINING CESIUM-137 CONTENT OF HN-200 GROUT CONTAINERS.}

$\begin{array}{lccc}\text { S35C18 } & 1.09532165 & 4.56352392 & -465.1884 \\ \text { S35C19 } & 5.34889188 & -99.83851 & 53.1958258 \\ \text { S35C20 } & 5.34889188 & -99.83851 & 53.1958258 \\ \text { S35C21 } & 5.34889188 & -99.83851 & 53.1958258 \\ \text { S35C22 } & 5.34889188 & -99.83851 & 53.1958258 \\ \text { S35C23 } & 5.34889188 & -99.83851 & 53.1958258 \\ \text { S35C24 } & 5.34889188 & -99.83851 & 53.1958258 \\ \text { S35R01 } & 1.01049136 & -2.9992188 & -305.45766 \\ \text { S35R02 } & 1.13386685 & 4.67081871 & -479.06508 \\ \text { S35R03 } & 2.11832598 & -12.293734 & -486.02189 \\ \text { S35R04 } & 11.3454241 & -282.28097 & 1405.74249 \\ \text { S35R05 } & 55.4397075 & -2109.2792 & 19911.3208 \\ \text { S35R06 } & 3.71671311 & -138.11737 & 1235.65969 \\ \text { S35R07 } & 1.55254392 & -38.566902 & 153.172788 \\ \text { S35R08 } & 1.07822042 & -9.9376778 & -204.16978 \\ \text { S35R09 } & 1.00638899 & 1.82645306 & -389.27376 \\ \text { S35R10 } & 1.20354658 & 4.40262753 & -496.00133 \\ \text { S35R11 } & 1.92078642 & -7.8411225 & -503.11496 \\ \text { S35R12 } & 5.01249439 & -90.087061 & -14.157617 \\ \text { S35R13 } & 79.040698 & -2525.4382 & 19911.3208 \\ \text { S35R14 } & 16.2798453 & -639.20206 & 6165.12986 \\ \text { S35R15 } & 2.86363354 & -100.76563 & 842.277297 \\ \text { S35R16 } & 1.34835071 & -27.435295 & 20.3770037\end{array}$

$\begin{array}{ccc}1.09532165 & 4.56352392 & -471.50923 \\ 5.34889188 & -99.83851 & 46.875 \\ 5.34889188 & -99.83851 & 46.875 \\ 5.34889188 & -99.83851 & 46.875 \\ 5.34889188 & -99.83851 & 46.875 \\ 5.34889188 & -99.83851 & 46.875 \\ 5.34889188 & -99.83851 & 46.875 \\ 1.01049136 & -2.9992188 & -311.77849 \\ 1.13386685 & 4.67081871 & -485.38591 \\ 2.11832598 & -12.293734 & -492.34272 \\ 11.3454241 & -282.28097 & 1399.42166 \\ 55.4397075 & -2109.2792 & 19905 \\ 3.71671311 & -138.11737 & 1229.33887 \\ 1.55254392 & -38.566902 & 146.851962 \\ 1.07822042 & -9.9376778 & -210.49061 \\ 1.00638899 & 1.82645306 & -395.59459 \\ 1.20354658 & 4.40262753 & -502.32216 \\ 1.92078642 & -7.8411225 & -509.43578 \\ 5.01249439 & -90.087061 & -20.478443 \\ 79.040698 & -2525.4382 & 19905 \\ 16.2798453 & -639.20206 & 6158.80903 \\ 2.86363354 & -100.76563 & 835.956471 \\ 1.34835071 & -27.435295 & 14.056178\end{array}$

TABLE 7

Determine the two possible $X$ intercepts of the line from each source to each measurement point and the inner and outer radius of the cylinder (XZ Plane).

Source S01

$\begin{array}{ccccc}\text { Line } & \mathbf{X 1 i} & \mathbf{X 2 i} & \mathbf{X 1 0} & \mathbf{X 2 0} \\ \text { S01C01 } & 22.7988391 & -22.035169 & 22.9371464 & -22.173477 \\ \text { S01C02 } & 22.7988391 & -22.035169 & 22.9371464 & -22.173477 \\ \text { S01C03 } & 22.7988391 & -22.035169 & 22.9371464 & -22.173477 \\ \text { S01C04 } & 22.7988391 & -22.035169 & 22.9371464 & -22.173477 \\ \text { S01C05 } & 22.7988391 & -22.035169 & 22.9371464 & -22.173477 \\ \text { S01C06 } & 22.7988391 & -22.035169 & 22.9371464 & -22.173477 \\ \text { S01C07 } & 22.5633195 & 0.96064256 & 22.6818899 & 0.84207215 \\ \text { S01C08 } & 22.5633195 & 0.96064256 & 22.6818899 & 0.84207215 \\ \text { S01C09 } & 22.5633195 & 0.96064256 & 22.6818899 & 0.84207215 \\ \text { S01C10 } & 22.5633195 & 0.96064256 & 22.6818899 & 0.84207215 \\ \text { S01C11 } & 22.5633195 & 0.96064256 & 22.6818899 & 0.84207215 \\ \text { S01C12 } & 22.5633195 & 0.96064256 & 22.6818899 & 0.84207215 \\ \text { S01C13 } & 22.7906547 & -22.799295 & 22.9288559 & -22.937496 \\ \text { S01C14 } & 22.7906547 & -22.799295 & 22.9288559 & -22.937496 \\ \text { S01C15 } & 22.7906547 & -22.799295 & 22.9288559 & -22.937496 \\ \text { S01C16 } & 22.7906547 & -22.799295 & 22.9288559 & -22.937496 \\ \text { S01C17 } & 22.7906547 & -22.799295 & 22.9288559 & -22.937496 \\ \text { S01C18 } & 22.7906547 & -22.799295 & 22.9288559 & -22.937496\end{array}$


REVISED METHODOLOGY FOR DETERMINING CESIUM-137 CONTENT OF HN-200 GROUT CONTAINERS.

$\begin{array}{lcc}\text { S01C19 } & 22.37328 & 0.91352136 \\ \text { S01C20 } & 22.37328 & 0.91352136 \\ \text { S01C21 } & 22.37328 & 0.91352136 \\ \text { S01C22 } & 22.37328 & 0.91352136 \\ \text { S01C23 } & 22.37328 & 0.91352136 \\ \text { S01C24 } & 22.37328 & 0.91352136 \\ \text { S01R01 } & 22.792281 & -22.785943 \\ \text { S01R02 } & 22.694871 & -15.889611 \\ \text { S01R03 } & 22.390261 & 0.35981514 \\ \text { S01R04 } & 21.3563984 & 14.4783505 \\ \text { S01R05 } & 20.7362415 & 17.4506411 \\ \text { S01R06 } & 22.3187248 & 7.58244723 \\ \text { S01R07 } & 22.6935706 & -6.2228972 \\ \text { S01R08 } & 22.7902766 & -18.034248 \\ \text { S01R09 } & 22.7915584 & -22.795232 \\ \text { S01R10 } & 22.7274682 & -18.546797 \\ \text { S01R11 } & 22.5623902 & -7.0214122 \\ \text { S01R12 } & 22.1222999 & 6.91019269 \\ \text { S01R13 } & 20.6059164 & 17.2824724 \\ \text { S01R14 } & 21.5248004 & 14.9335835 \\ \text { S01R15 } & 22.5480961 & 1.55294822 \\ \text { S01R16 } & 22.7732846 & -14.799487\end{array}$

22.48585640 .80094493

22.48585640 .80094493

22.48585640 .80094493

22.48585640 .80094493

22.48585640 .80094493

22.48585640 .80094493

$22.9305393-22.924202$

$22.8275318-16.022272$

$22.5038597 \quad 0.24621639$

$\begin{array}{lll}21.4177491 & 14.4169998\end{array}$

$20.7708395 \quad 17.4160431$

$22.4213448 \quad 7.47982728$

$22.8218744 \quad-6.3512009$

$22.9271108-18.171082$

$22.9297923-22.933466$

$22.8621602-18.681489$

$22.6866415 \quad-7.1456635$

$22.2204361 \quad 6.8120565$

$20.6395912 \quad 17.2487976$

$21.5877375 \quad 14.8706465$

$22.6655966 \quad 1.43544767$

$22.9083955-14.934598$

$\begin{array}{lrcccc}\text { Line } & \mathbf{X 1 i} & \mathbf{X} 2 \mathrm{~S} & \mathbf{S} & \mathbf{X 1 0} & \mathbf{X 2 0} \\ \text { S02C01 } & 22.7900376 & -11.164539 & 22.9304085 & -11.30491 \\ \text { S02C02 } & 22.7900376 & -11.164539 & 22.9304085 & -11.30491 \\ \text { S02C03 } & 22.7900376 & -11.164539 & 22.9304085 & -11.30491 \\ \text { S02C04 } & 22.7900376 & -11.164539 & 22.9304085 & -11.30491 \\ \text { S02C05 } & 22.7900376 & -11.164539 & 22.9304085 & -11.30491 \\ \text { S02C06 } & 22.7900376 & -11.164539 & 22.9304085 & -11.30491 \\ \text { S02C07 } & -0.2781886 & -10.748808 & -0.2443647 & -10.782632 \\ \text { S02C08 } & -0.2781886 & -10.748808 & -0.2443647 & -10.782632 \\ \text { S02C09 } & -0.2781886 & -10.748808 & -0.2443647 & -10.782632 \\ \text { S02C10 } & -0.2781886 & -10.748808 & -0.2443647 & -10.782632 \\ \text { S02C11 } & -0.2781886 & -10.748808 & -0.2443647 & -10.782632 \\ \text { S02C12 } & -0.2781886 & -10.748808 & -0.2443647 & -10.782632 \\ \text { S02C13 } & -9.9868456 & -22.730201 & -9.8316905 & -22.885356 \\ \text { S02C14 } & -9.9868456 & -22.730201 & -9.8316905 & -22.885356 \\ \text { S02C15 } & -9.9868456 & -22.730201 & -9.8316905 & -22.885356 \\ \text { S02C16 } & -9.9868456 & -22.730201 & -9.8316905 & -22.885356 \\ \text { S02C17 } & -9.9868456 & -22.730201 & -9.8316905 & -22.885356 \\ \text { S02C18 } & -9.9868456 & -22.730201 & -9.8316905 & -22.885356 \\ \text { S02C19 } & -3.3778989 & -13.468207 & -2.8679392 & -13.978166\end{array}$

74 of 210 


\section{REVISED METHODOLOGY FOR DETERMINING CESIUM-137 CONTENT OF HN-200 GROUT CONTAINERS.}

$\begin{array}{lll}\text { S02C20 } & -3.3778989 & -13.468207 \\ \text { S02C21 } & -3.3778989 & -13.468207 \\ \text { S02C22 } & -3.3778989 & -13.468207 \\ \text { S02C23 } & -3.3778989 & -13.468207 \\ \text { S02C24 } & -3.3778989 & -13.468207 \\ \text { S02R01 } & 16.0758032 & -11.50718 \\ \text { S02R02 } & 22.6898979 & -11.187651 \\ \text { S02R03 } & 19.0691341 & -10.992775 \\ \text { S02R04 } & 7.13976211 & -10.830325 \\ \text { S02R05 } & -7.9427396 & -10.655965 \\ \text { S02R06 } & -10.409684 & -19.662297 \\ \text { S02R07 } & -9.8862829 & -22.491758 \\ \text { S02R08 } & -7.2958373 & -15.904675 \\ \text { S02R09 } & 5.92624594 & -12.045012 \\ \text { S02R10 } & 20.1492365 & -11.341371 \\ \text { S02R11 } & 20.9869906 & -11.039022 \\ \text { S02R12 } & 5.70186202 & -10.814469 \\ \text { S02R13 } & -10.562894 & -13.859811 \\ \text { S02R14 } & -10.116498 & -22.75977 \\ \text { S02R15 } & -8.3752138 & -17.639638 \\ \text { S02R16 } & 2.10584163 & -12.405786\end{array}$

$-2.8679392-13.978166$

$-2.8679392-13.978166$

$-2.8679392-13.978166$

$-2.8679392-13.978166$

$\begin{array}{ll}-2.8679392 & -13.978166\end{array}$

$16.2994838-11.730861$

$22.8360676-11.333821$

$\begin{array}{ll}19.165864 & -11.089505\end{array}$

$7.19465075-10.885213$

$\begin{array}{ll}-7.9328728 & -10.665832\end{array}$

$-10.356746-19.715236$

$-9.7083452-22.669696$

$-6.7910701-16.409442$

$6.26408548-12.382852$

$20.3333801-11.525514$

$21.0955514 \quad-11.147583$

$\begin{array}{ll}5.752654 & -10.865261\end{array}$

$-10.548857-13.873848$

$\begin{array}{ll}-9.9916717 & -22.884597\end{array}$

$\begin{array}{ll}-7.9495708 & -18.065281\end{array}$

$2.50550544 \quad-12.80545$

\begin{tabular}{ccccc} 
& \multicolumn{3}{c}{ Source S03 } & $\mathbf{X}$ \\
Line & $\mathbf{X 1 i}$ & $\mathbf{X 2 i}$ & $\mathbf{X 2 0}$ \\
S03C01 & 22.7962367 & -18.159545 & 22.9351513 & -18.29846 \\
S03C02 & 22.7962367 & -18.159545 & 22.9351513 & -18.29846 \\
S03C03 & 22.7962367 & -18.159545 & 22.9351513 & -18.29846 \\
S03C04 & 22.7962367 & -18.159545 & 22.9351513 & -18.29846 \\
S03C05 & 22.7962367 & -18.159545 & 22.9351513 & -18.29846 \\
S03C06 & 22.7962367 & -18.159545 & 22.9351513 & -18.29846 \\
S03C07 & 2.6436554 & 0.06459974 & 2.65149966 & 0.05675548 \\
S03C08 & 2.6436554 & 0.06459974 & 2.65149966 & 0.05675548 \\
S03C09 & 2.6436554 & 0.06459974 & 2.65149966 & 0.05675548 \\
S03C10 & 2.6436554 & 0.06459974 & 2.65149966 & 0.05675548 \\
S03C11 & 2.6436554 & 0.06459974 & 2.65149966 & 0.05675548 \\
S03C12 & 2.6436554 & 0.06459974 & 2.65149966 & 0.05675548 \\
S03C13 & 19.2618238 & -22.797026 & 19.400554 & -22.935756 \\
S03C14 & 19.2618238 & -22.797026 & 19.400554 & -22.935756 \\
S03C15 & 19.2618238 & -22.797026 & 19.400554 & -22.935756 \\
S03C16 & 19.2618238 & -22.797026 & 19.400554 & -22.935756 \\
S03C17 & 19.2618238 & -22.797026 & 19.400554 & -22.935756 \\
S03C18 & 19.2618238 & -22.797026 & 19.400554 & -22.935756 \\
S03C19 & 4.90452767 & 0.12085581 & 4.91920574 & 0.10617774 \\
S03C20 & 4.90452767 & 0.12085581 & 4.91920574 & 0.10617774
\end{tabular}




\section{REVISED METHODOLOGY FOR DETERMINING CESIUM-137 CONTENT OF HN-200 GROUT CONTAINERS.}

$\begin{array}{lcc}\text { S03C21 } & 4.90452767 & 0.12085581 \\ \text { S03C22 } & 4.90452767 & 0.12085581 \\ \text { S03C23 } & 4.90452767 & 0.12085581 \\ \text { S03C24 } & 4.90452767 & 0.12085581 \\ \text { S03R01 } & 21.9338208 & -21.179913 \\ \text { S03R02 } & 22.642775 & -16.35894 \\ \text { S03R03 } & 19.4012527 & -10.245499 \\ \text { S03R04 } & 11.9136262 & -3.9993537 \\ \text { S03R05 } & 1.95264319 & 1.41665422 \\ \text { S03R06 } & 7.54834941 & -9.5614301 \\ \text { S03R07 } & 12.7941777 & -18.180975 \\ \text { S03R08 } & 17.5433965 & -22.453456 \\ \text { S03R09 } & 21.2594854 & -21.977041 \\ \text { S03R10 } & 22.7984639 & -17.831182 \\ \text { S03R11 } & 20.5509283 & -11.714246 \\ \text { S03R12 } & 13.4012878 & -5.0093336 \\ \text { S03R13 } & 2.16133553 & 1.5683706 \\ \text { S03R14 } & 7.78086186 & -9.9984578 \\ \text { S03R15 } & 13.5193412 & -19.096745 \\ \text { S03R16 } & 18.4853814 & -22.7384\end{array}$

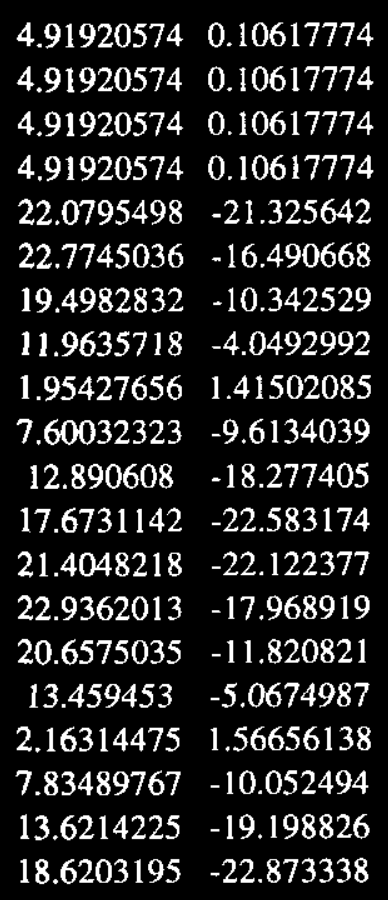

\begin{tabular}{|c|c|c|c|c|}
\hline \multirow[b]{2}{*}{ Line } & \multicolumn{4}{|c|}{ Source su4 } \\
\hline & X1i & $\mathbf{X} 2 \mathbf{i}$ & X10 & $\mathbf{X} 20$ \\
\hline $\mathrm{S} 04 \mathrm{C} 01$ & 22.7665125 & 2.19672244 & 22.9125357 & 2.05069933 \\
\hline $\mathrm{S} 04 \mathrm{C} 02$ & 22.7665125 & 2.19672244 & 22.9125357 & 2.05069933 \\
\hline $\mathrm{S} 04 \mathrm{CO} 3$ & 22.7665125 & 2.19672244 & 22.9125357 & 2.05069933 \\
\hline $\mathrm{S} 04 \mathrm{CO} 4$ & 22.7665125 & 2.19672244 & 22.9125357 & 2.05069933 \\
\hline S04C05 & 22.7665125 & 2.19672244 & 22.9125357 & 2.05069933 \\
\hline S04C06 & 22.7665125 & 2.19672244 & 22.9125357 & 2.05069933 \\
\hline S04C07 & 5.88580696 & 0.1458092 & 5.90351774 & 0.12809843 \\
\hline S04C08 & 5.88580696 & 0.1458092 & 5.90351774 & 0.12809843 \\
\hline S04C09 & 5.88580696 & 0.1458092 & 5.90351774 & 0.12809843 \\
\hline SO4C10 & 5.88580696 & 0.1458092 & 5.90351774 & 0.12809843 \\
\hline SO4C11 & 5.88580696 & 0.1458092 & 5.90351774 & 0.12809843 \\
\hline $\mathrm{S} 04 \mathrm{C} 12$ & 5.88580696 & 0.1458092 & 5.90351774 & 0.12809843 \\
\hline $\mathrm{SO4C13}$ & 8.49804307 & -22.786946 & 8.63914541 & -22.928048 \\
\hline $\mathrm{S} 04 \mathrm{Cl} 14$ & 8.49804307 & -22.786946 & 8.63914541 & -22.928048 \\
\hline $\mathrm{SO} 4 \mathrm{C} 15$ & 8.49804307 & -22.786946 & 8.63914541 & -22.928048 \\
\hline $\mathrm{S} 04 \mathrm{C} 16$ & 8.49804307 & -22.786946 & 8.63914541 & -22.928048 \\
\hline SO4C17 & 8.49804307 & -22.786946 & 8.63914541 & -22.928048 \\
\hline SO4C18 & 8.49804307 & -22.786946 & 8.63914541 & -22.928048 \\
\hline SO4C 19 & 22.2608267 & 1.38179576 & 22.4344367 & 1.20818573 \\
\hline $\mathrm{S} 04 \mathrm{C} 20$ & 22.2608267 & 1.38179576 & 22,4344367 & 1.20818573 \\
\hline $\mathrm{S} 04 \mathrm{C} 21$ & 22.2608267 & 1.38179576 & 22.4344367 & 1.20818573 \\
\hline
\end{tabular}




\section{REVISED METHODOLOGY FOR DETERMINING CESIUM-137 CONTENT OF HN-200 GROUT CONTAINERS.}

$\begin{array}{lcc}\text { SO4C22 } & 22.2608267 & 1.38179576 \\ \text { S04C23 } & 22.2608267 & 1.38179576 \\ \text { S04C24 } & 22.2608267 & 1.38179576 \\ \text { S04R01 } & 13.9399981 & -8.4555723 \\ \text { S04R02 } & 20.1439935 & -0.5912384 \\ \text { S04R03 } & 22.6755037 & 2.82060935 \\ \text { S04R04 } & 16.7877548 & 4.50833497 \\ \text { S04R05 } & 5.62420459 & 4.10695199 \\ \text { S04R06 } & 6.56727797 & -10.235679 \\ \text { S04R07 } & 7.55757825 & -20.445333 \\ \text { S04R08 } & 8.85821684 & -22.407133 \\ \text { S04R09 } & 11.0640354 & -15.775239 \\ \text { S04R10 } & 15.609968 & -5.6265033 \\ \text { S04R11 } & 21.7815794 & 0.88450841 \\ \text { S04R12 } & 20.7847004 & 3.78337997 \\ \text { S04R13 } & 7.34859394 & 5.39511953 \\ \text { S04R14 } & 6.65428146 & -11.442424 \\ \text { S04R15 } & 8.01007256 & -22.322201 \\ \text { S04R16 } & 10.0217644 & -19.208462\end{array}$

\author{
$22.4344367 \quad 1.20818573$ \\ $22.4344367 \quad 1.20818573$ \\ $22.4344367 \quad 1.20818573$ \\ $14.2134795 \quad-8.7290537$ \\ $20.3688282-0.8160731$ \\ 22.79790142 .69821165 \\ $16.8367523 \quad 4.45933749$ \\ 5.629022064 .10213451 \\ $6.61858785-10.286989$ \\ $7.65651699 \quad-20.544272$ \\ $9.01432071-22.563236$ \\ $\begin{array}{ll}11.2936957 & -16.0049\end{array}$ \\ $\begin{array}{lll}15.8854726 & -5.9020079\end{array}$ \\ $\begin{array}{lll}21.970157 & 0.69593081\end{array}$ \\ 20.86673353 .70134678 \\ $7.35501558 \quad 5.38869789$ \\ $6.70985582-11.497998$ \\ $8.12978123 \quad-22.44191$ \\ $10.220886 \quad-19.407584$
}

$\begin{array}{lcccc}\text { Line } & \mathbf{X 1 i} & \mathbf{X 2 i} & \mathbf{X 1 0} & \mathbf{X 2 0} \\ \text { S05C01 } & 22.7991737 & -22.587325 & 22.9374031 & -22.725554 \\ \text { S05C02 } & 22.7991737 & -22.587325 & 22.9374031 & -22.725554 \\ \text { S05C03 } & 22.7991737 & -22.587325 & 22.9374031 & -22.725554 \\ \text { S05C04 } & 22.7991737 & -22.587325 & 22.9374031 & -22.725554 \\ \text { S05C05 } & 22.7991737 & -22.587325 & 22.9374031 & -22.725554 \\ \text { S05C06 } & 22.7991737 & -22.587325 & 22.9374031 & -22.725554 \\ \text { S05C07 } & -0.6681267 & -20.145094 & -0.5863587 & -20.226862 \\ \text { S05C08 } & -0.6681267 & -20.145094 & -0.5863587 & -20.226862 \\ \text { S05C09 } & -0.6681267 & -20.145094 & -0.5863587 & -20.226862 \\ \text { S05C10 } & -0.6681267 & -20.145094 & -0.5863587 & -20.226862 \\ \text { S05C11 } & -0.6681267 & -20.145094 & -0.5863587 & -20.226862 \\ \text { S05C12 } & -0.6681267 & -20.145094 & -0.5863587 & -20.226862 \\ \text { S05C13 } & 20.7237924 & -22.79801 & 20.8622929 & -22.936511 \\ \text { S05C14 } & 20.7237924 & -22.79801 & 20.8622929 & -22.936511 \\ \text { S05C15 } & 20.7237924 & -22.79801 & 20.8622929 & -22.936511 \\ \text { S05C16 } & 20.7237924 & -22.79801 & 20.8622929 & -22.936511 \\ \text { S05C17 } & 20.7237924 & -22.79801 & 20.8622929 & -22.936511 \\ \text { S05C18 } & 20.7237924 & -22.79801 & 20.8622929 & -22.936511 \\ \text { S05C19 } & -0.5433879 & -18.003731 & -0.4770641 & -18.070055 \\ \text { S05C20 } & -0.5433879 & -18.003731 & -0.4770641 & -18.070055 \\ \text { S05C21 } & -0.5433879 & -18.003731 & -0.4770641 & -18.070055 \\ \text { S05C22 } & -0.5433879 & -18.003731 & -0.4770641 & -18.070055\end{array}$




\section{REVISED METHODOLOGY FOR DETERMINING CESIUM-137 CONTENT OF HN-200 GROUT CONTAINERS.}

$\begin{array}{lll}\text { S05C23 } & -0.5433879 & -18.003731 \\ \text { S05C24 } & -0.5433879 & -18.003731 \\ \text { S05R01 } & 22.7217019 & -22.651845 \\ \text { S05R02 } & 18.3154819 & -22.763667 \\ \text { S05R03 } & 8.87004131 & -22.039035 \\ \text { S05R04 } & -2.0005169 & -19.715092 \\ \text { S05R05 } & -10.952192 & -14.314434 \\ \text { S05R06 } & -4.4495565 & -16.757089 \\ \text { S05R07 } & 8.61520171 & -20.060964 \\ \text { S05R08 } & 19.6159409 & -21.849782 \\ \text { S05R09 } & 22.5896746 & -22.688913 \\ \text { S05R10 } & 16.1244399 & -22.670286 \\ \text { S05R11 } & 4.52261862 & -21.377416 \\ \text { S05R12 } & -6.4173562 & -17.762554 \\ \text { S05R13 } & -10.568279 & -13.866142 \\ \text { S05R14 } & 0.09264891 & -18.179309 \\ \text { S05R15 } & 11.6549431 & -20.59145 \\ \text { S05R16 } & 20.2319415 & -21.951757\end{array}$

\author{
$-0.4770641-18.070055$ \\ $-0.4770641-18.070055$ \\ $22.8605493-22.790692$ \\ $\begin{array}{ll}18.451116 & -22.899301\end{array}$ \\ $8.98618526-22.155179$ \\ $-1.9252253-19.790383$ \\ $-10.937454-14.329172$ \\ $-4.4005736-16.806072$ \\ $8.71323451-20.158997$ \\ $19.7435168 \quad-21.977358$ \\ $\begin{array}{ll}22.7287972 & -22.828036\end{array}$ \\ $\begin{array}{ll}16.2566249 & -22.802471\end{array}$ \\ $\begin{array}{ll}4.62535528 & -21.480152\end{array}$ \\ $\begin{array}{ll}-6.367672 & -17.812238\end{array}$ \\ $-10.554233-13.880188$ \\ $0.16153116-18.248192$ \\ $11.7616859-20.698193$ \\ $20.3611484 \quad-22.080964$
}

$\begin{array}{lccccc}\text { Line } & \mathbf{X 1 i} & \mathbf{X} 2 \mathbf{S} & \mathbf{X 1 0} & \mathbf{X} 20 \\ \text { S06C01 } & 22.7922696 & -13.389977 & 22.9321146 & -13.529822 \\ \text { S06C02 } & 22.7922696 & -13.389977 & 22.9321146 & -13.529822 \\ \text { S06C03 } & 22.7922696 & -13.389977 & 22.9321146 & -13.529822 \\ \text { S06C04 } & 22.7922696 & -13.389977 & 22.9321146 & -13.529822 \\ \text { S06C05 } & 22.7922696 & -13.389977 & 22.9321146 & -13.529822 \\ \text { S06C06 } & 22.7922696 & -13.389977 & 22.9321146 & -13.529822 \\ \text { S06C07 } & 13.8696776 & 0.3766013 & 13.9155193 & 0.33075963 \\ \text { S06C08 } & 13.8696776 & 0.3766013 & 13.9155193 & 0.33075963 \\ \text { S06C09 } & 13.8696776 & 0.3766013 & 13.9155193 & 0.33075963 \\ \text { S06C10 } & 13.8696776 & 0.3766013 & 13.9155193 & 0.33075963 \\ \text { S06C11 } & 13.8696776 & 0.3766013 & 13.9155193 & 0.33075963 \\ \text { S06C12 } & 13.8696776 & 0.3766013 & 13.9155193 & 0.33075963 \\ \text { S06C13 } & 20.9569399 & -22.798161 & 21.0954051 & -22.936627 \\ \text { S06C14 } & 20.9569399 & -22.798161 & 21.0954051 & -22.936627 \\ \text { S06C15 } & 20.9569399 & -22.798161 & 21.0954051 & -22.936627 \\ \text { S06C16 } & 20.9569399 & -22.798161 & 21.0954051 & -22.936627 \\ \text { S06C17 } & 20.9569399 & -22.798161 & 21.0954051 & -22.936627 \\ \text { S06C18 } & 20.9569399 & -22.798161 & 21.0954051 & -22.936627 \\ \text { S06C19 } & 20.3188084 & 0.68068286 & 20.4021382 & 0.59735305 \\ \text { S06C20 } & 20.3188084 & 0.68068286 & 20.4021382 & 0.59735305 \\ \text { S06C21 } & 20.3188084 & 0.68068286 & 20.4021382 & 0.59735305 \\ \text { S06C22 } & 20.3188084 & 0.68068286 & 20.4021382 & 0.59735305 \\ \text { S06C23 } & 20.3188084 & 0.68068286 & 20.4021382 & 0.59735305\end{array}$




\section{REVISED METHODOLOGY FOR DETERMINING CESIUM-137 CONTENT OF HN-200 GROUT CONTAINERS.}

$\begin{array}{lcl}\text { SO6C24 } & 20.3188084 & 0.68068286 \\ \text { SO6R01 } & 21.9367934 & -21.185382 \\ \text { SO6R02 } & 22.7951492 & -13.192464 \\ \text { SO6R03 } & 21.7240805 & -3.1382658 \\ \text { SO6R04 } & 17.1532469 & 5.2147229 \\ \text { SO6R05 } & 11.0380164 & 8.24574956 \\ \text { SO6R06 } & 14.9875954 & -3.5513795 \\ \text { S06R07 } & 17.8031974 & -14.777324 \\ \text { SO6R08 } & 19.9408248 & -21.709546 \\ \text { SO6R09 } & 21.590092 & -22.155834 \\ \text { S06R10 } & 22.6497172 & -16.459659 \\ \text { SO6R11 } & 22.5044709 & -7.2961968 \\ \text { SO6R12 } & 19.6577679 & 1.91427586 \\ \text { S06R13 } & 12.0970686 & 9.09776861 \\ \text { SO6R14 } & 14.0973018 & -0.3861021 \\ \text { S06R15 } & 17.5783215 & -13.877692 \\ \text { S06R16 } & 20.1155496 & -22.042179\end{array}$
20.40213820 .59735305
$22.0824953-21.331084$
$22.9346011-13.331916$
$21.8292151-3.2434004$
$17.2024488 \quad 5.16552104$
11.04832598 .23544006
$15.0484302-3.6122143$
$17.9028447-14.876972$
$20.0682713-21.836992$
$\begin{array}{ll}21.7338233 & -22.299566\end{array}$
$22.7943435 \quad-16.604285$
$\begin{array}{ll}22.6274092 & -7.419135\end{array}$
$19.7327664 \quad 1.83927735$
$12.1086721 \quad 9.08616503$
$14.1461596 \quad-0.4349599$
$17.6748823 \quad-13.974253$
$20.2450455-22.171675$

$\begin{array}{lcccc}\text { Line } & \mathbf{X 1 i} & \mathbf{X 2 i} & \mathbf{X 1 0} & \mathbf{X} 20 \\ \text { S07C01 } & 22.7856013 & -7.4648618 & 22.9270227 & -7.6062832 \\ \text { S07C02 } & 22.7856013 & -7.4648618 & 22.9270227 & -7.6062832 \\ \text { S07C03 } & 22.7856013 & -7.4648618 & 22.9270227 & -7.6062832 \\ \text { S07C04 } & 22.7856013 & -7.4648618 & 22.9270227 & -7.6062832 \\ \text { S07C05 } & 22.7856013 & -7.4648618 & 22.9270227 & -7.6062832 \\ \text { S07C06 } & 22.7856013 & -7.4648618 & 22.9270227 & -7.6062832 \\ \text { S07C07 } & -0.0806125 & -3.2927714 & -0.0708234 & -3.3025605 \\ \text { S07C08 } & -0.0806125 & -3.2927714 & -0.0708234 & -3.3025605 \\ \text { S07C09 } & -0.0806125 & -3.2927714 & -0.0708234 & -3.3025605 \\ \text { S07C10 } & -0.0806125 & -3.2927714 & -0.0708234 & -3.3025605 \\ \text { S07C11 } & -0.0806125 & -3.2927714 & -0.0708234 & -3.3025605 \\ \text { S07C12 } & -0.0806125 & -3.2927714 & -0.0708234 & -3.3025605 \\ \text { S07C13 } & 1.8044511 & -22.776235 & 1.94811392 & -22.919897 \\ \text { S07C14 } & 1.8044511 & -22.776235 & 1.94811392 & -22.919897 \\ \text { S07C15 } & 1.8044511 & -22.776235 & 1.94811392 & -22.919897 \\ \text { S07C16 } & 1.8044511 & -22.776235 & 1.94811392 & -22.919897 \\ \text { S07C17 } & 1.8044511 & -22.776235 & 1.94811392 & -22.919897 \\ \text { S07C18 } & 1.8044511 & -22.776235 & 1.94811392 & -22.919897 \\ \text { S07C19 } & -0.6315939 & -19.597561 & -0.5543621 & -19.674792 \\ \text { S07C20 } & -0.6315939 & -19.597561 & -0.5543621 & -19.674792 \\ \text { S07C21 } & -0.6315939 & -19.597561 & -0.5543621 & -19.674792 \\ \text { S07C22 } & -0.6315939 & -19.597561 & -0.5543621 & -19.674792 \\ \text { S07C23 } & -0.6315939 & -19.597561 & -0.5543621 & -19.674792 \\ \text { S07C24 } & -0.6315939 & -19.597561 & -0.5543621 & -19.674792\end{array}$




\section{REVISED METHODOLOGY FOR DETERMINING CESIUM-137 CONTENT OF HN-200 GROUT CONTAINERS.}

$\begin{array}{ccc}\text { SO7R01 } & 16.1913511 & -11.678783 \\ \text { S07R02 } & 22.293676 & -8.1739345 \\ \text { S07R03 } & 20.9386722 & -6.0503898 \\ \text { S07R04 } & 11.6033677 & -4.4877571 \\ \text { S07R05 } & -2.237797 & -3.0804387 \\ \text { S07R06 } & -1.5394075 & -15.189619 \\ \text { S07R07 } & 0.53200845 & -22.248967 \\ \text { S07R08 } & 3.98865062 & -21.311692 \\ \text { S07R09 } & 10.456234 & -15.357824 \\ \text { S07R10 } & 19.2342235 & -10.056453 \\ \text { S07R11 } & 22.5912409 & -6.8712352 \\ \text { S07R12 } & 13.7027622 & -4.7419882 \\ \text { S07R13 } & -2.9042767 & -3.9919128 \\ \text { S07R14 } & -0.8181513 & -18.884538 \\ \text { S07R15 } & 2.29064918 & -22.61114 \\ \text { S07R16 } & 7.8694182 & -17.51982\end{array}$

\author{
$16.4128071-11.900239$ \\ $22.4532156-8.3334741$ \\ $21.0392644-6.150982$ \\ $11.6535334-4.5379228$ \\ $-2.235208-3.0830277$ \\ $-1.4907348-15.238292$ \\ $0.64306754-22.360026$ \\ $4.17630051-21.499342$ \\ $10.6946927-15.596282$ \\ $19.4329172-10.255147$ \\ $22.7162269-6.9962213$ \\ $13.7613894-4.8006153$ \\ $\begin{array}{ll}-2.9009033 & -3.9952862\end{array}$ \\ $\begin{array}{ll}-0.7466513 & -18.956038\end{array}$ \\ $\begin{array}{lll}2.44543745 & -22.765928\end{array}$ \\ $8.09958747-17.74999$
}

$\begin{array}{lcccc}\text { Line } & \mathbf{X} 1 \mathbf{r} & \mathbf{X 2 i} & \mathbf{X} 10 & \mathbf{X 2 0} \\ \text { S08C01 } & 22.795377 & -17.022345 & 22.9344928 & -17.161461 \\ \text { S08C02 } & 22.795377 & -17.022345 & 22.9344928 & -17.161461 \\ \text { S08C03 } & 22.795377 & -17.022345 & 22.9344928 & -17.161461 \\ \text { S08C04 } & 22.795377 & -17.022345 & 22.9344928 & -17.161461 \\ \text { S08C05 } & 22.795377 & -17.022345 & 22.9344928 & -17.161461 \\ \text { S08C06 } & 22.795377 & -17.022345 & 22.9344928 & -17.161461 \\ \text { S08C07 } & -0.0493975 & -2.0243563 & -0.0433994 & -2.0303543 \\ \text { S08C08 } & -0.0493975 & -2.0243563 & -0.0433994 & -2.0303543 \\ \text { S08C09 } & -0.0493975 & -2.0243563 & -0.0433994 & -2.0303543 \\ \text { S08C10 } & -0.0493975 & -2.0243563 & -0.0433994 & -2.0303543 \\ \text { S08C11 } & -0.0493975 & -2.0243563 & -0.0433994 & -2.0303543 \\ \text { S08C12 } & -0.0493975 & -2.0243563 & -0.0433994 & -2.0303543 \\ \text { S08C13 } & 15.6753591 & -22.794293 & 15.8147288 & -22.933663 \\ \text { S08C14 } & 15.6753591 & -22.794293 & 15.8147288 & -22.933663 \\ \text { S08C15 } & 15.6753591 & -22.794293 & 15.8147288 & -22.933663 \\ \text { S08C16 } & 15.6753591 & -22.794293 & 15.8147288 & -22.933663 \\ \text { S08C17 } & 15.6753591 & -22.794293 & 15.8147288 & -22.933663 \\ \text { S08C18 } & 15.6753591 & -22.794293 & 15.8147288 & -22.933663 \\ \text { S08C19 } & -0.1132982 & -4.6043543 & -0.0995385 & -4.6181141 \\ \text { S08C20 } & -0.1132982 & -4.6043543 & -0.0995385 & -4.6181141 \\ \text { S08C21 } & -0.1132982 & -4.6043543 & -0.0995385 & -4.6181141 \\ \text { S08C22 } & -0.1132982 & -4.6043543 & -0.0995385 & -4.6181141 \\ \text { S08C23 } & -0.1132982 & -4.6043543 & -0.0995385 & -4.6181141 \\ \text { S08C24 } & -0.1132982 & -4.6043543 & -0.0995385 & -4.6181141 \\ \text { S08R01 } & 21.3818629 & -20.175355 & 21.5327724 & -20.326265\end{array}$


REVISED METHODOLOGY FOR DETERMINING CESIUM-137 CONTENT OF HN-200 GROUT CONTAINERS.

$\begin{array}{lcl}\text { S08R02 } & 22.6937849 & -15.900601 \\ \text { S08R03 } & 18.9878886 & -11.168984 \\ \text { S08R04 } & 10.347826 & -6.4012695 \\ \text { S08R05 } & -1.1412306 & -1.5734953 \\ \text { S08R06 } & 3.49615755 & -12.229257 \\ \text { S08R07 } & 8.97652489 & -19.922017 \\ \text { S08R08 } & 14.7773112 & -22.77431 \\ \text { S08R09 } & 20.0635651 & -21.319705 \\ \text { S08R10 } & 22.7774489 & -17.267986 \\ \text { S08R11 } & 20.2187117 & -12.178091 \\ \text { S08R12 } & 11.2450605 & -6.8121598 \\ \text { S08R13 } & -1.3019865 & -1.7948344 \\ \text { S08R14 } & 4.48316551 & -13.990103 \\ \text { S08R15 } & 10.6094441 & -21.236345 \\ \text { S08R16 } & 16.6747505 & -22.693853\end{array}$

$\begin{array}{cc}22.826423 & -16.033239 \\ 19.0845624 & -11.265658 \\ 10.3990318 & -6.4524752 \\ -1.1399158 & -1.57481 \\ 3.54589471 & -12.278994 \\ 9.0742946 & -20.019787 \\ 14.9124761 & -22.909475 \\ 20.2150779 & -21.471218 \\ 22.9178495 & -17.408386 \\ 20.32402 & -12.283399 \\ 11.300408 & -6.8675074 \\ -1.3004859 & -1.796335 \\ 4.54225141 & -14.049189 \\ 10.7193576 & -21.346258 \\ 16.8182058 & -22.837309\end{array}$

$\begin{array}{lccccc}\text { Line } & \mathbf{X 1 i} & \mathbf{X} 2 \mathrm{~S} & \mathbf{X} 10 & \mathbf{X} 20 \\ \text { S09C01 } & 22.7992621 & -22.735497 & 22.9374709 & -22.873706 \\ \text { S09C02 } & 22.7992621 & -22.735497 & 22.9374709 & -22.873706 \\ \text { S09C03 } & 22.7992621 & -22.735497 & 22.9374709 & -22.873706 \\ \text { S09C04 } & 22.7992621 & -22.735497 & 22.9374709 & -22.873706 \\ \text { S09C05 } & 22.7992621 & -22.735497 & 22.9374709 & -22.873706 \\ \text { S09C06 } & 22.7992621 & -22.735497 & 22.9374709 & -22.873706 \\ \text { S09C07 } & -0.5135527 & -17.373578 & -0.4509055 & -17.436225 \\ \text { S09C08 } & -0.5135527 & -17.373578 & -0.4509055 & -17.436225 \\ \text { S09C09 } & -0.5135527 & -17.373578 & -0.4509055 & -17.436225 \\ \text { S09C10 } & -0.5135527 & -17.373578 & -0.4509055 & -17.436225 \\ \text { S09C11 } & -0.5135527 & -17.373578 & -0.4509055 & -17.436225 \\ \text { S09C12 } & -0.5135527 & -17.373578 & -0.4509055 & -17.436225 \\ \text { S09C13 } & 22.3051629 & -22.799004 & 22.4434318 & -22.937273 \\ \text { S09C14 } & 22.3051629 & -22.799004 & 22.4434318 & -22.937273 \\ \text { S09C15 } & 22.3051629 & -22.799004 & 22.4434318 & -22.937273 \\ \text { S09C16 } & 22.3051629 & -22.799004 & 22.4434318 & -22.937273 \\ \text { S09C17 } & 22.3051629 & -22.799004 & 22.4434318 & -22.937273 \\ \text { S09C18 } & 22.3051629 & -22.799004 & 22.4434318 & -22.937273 \\ \text { S09C19 } & -0.572652 & -18.576457 & -0.5027158 & -18.646393 \\ \text { S09C20 } & -0.572652 & -18.576457 & -0.5027158 & -18.646393 \\ \text { S09C21 } & -0.572652 & -18.576457 & -0.5027158 & -18.646393 \\ \text { S09C22 } & -0.572652 & -18.576457 & -0.5027158 & -18.646393 \\ \text { S09C23 } & -0.572652 & -18.576457 & -0.5027158 & -18.646393 \\ \text { S09C24 } & -0.572652 & -18.576457 & -0.5027158 & -18.646393 \\ \text { S09R01 } & 22.7772241 & -22.757304 & 22.9156078 & -22.895688 \\ \text { S09R02 } & 20.000901 & -22.12467 & 20.1306967 & -22.254465\end{array}$




\section{REVISED METHODOLOGY FOR DETERMINING CESIUM-137 CONTENT OF HN-200 GROUT CONTAINERS.}

$\begin{array}{lcl}\text { S09R03 } & 11.6255809 & -20.611353 \\ \text { S09R04 } & 0.59846811 & -17.763173 \\ \text { S09R05 } & -9.5991807 & -12.709087 \\ \text { S09R06 } & -4.4704591 & -16.767999 \\ \text { S09R07 } & 6.66411802 & -20.745248 \\ \text { S09R08 } & 17.4754894 & -22.466807 \\ \text { S09R09 } & 22.7415987 & -22.768963 \\ \text { S09R10 } & 19.239318 & -21.993483 \\ \text { S09R11 } & 8.76460214 & -20.003996 \\ \text { S09R12 } & -3.4199689 & -16.213618 \\ \text { S09R13 } & -9.7805191 & -12.928251 \\ \text { S09R14 } & -0.4654769 & -18.618202 \\ \text { S09R15 } & 10.195546 & -21.456788 \\ \text { S09R16 } & 19.0095264 & -22.611898\end{array}$

$11.7324071-20.718179$

$0.66599741-17.830702$

$-9.5867772-12.72149$

$-4.4214711-16.816987$

$6.76398399-20.845114$

$17.6053086-22.596626$

$22.8800513-22.907416$

$\begin{array}{lll}19.3671262 & -22.121291\end{array}$

$\begin{array}{ll}8.86252332 & -20.101917\end{array}$

$-3.3711848-16.262402$

$\begin{array}{ll}-9.7678184 & -12.940951\end{array}$

$\begin{array}{ll}-0.3950196 & -18.688659\end{array}$

$10.3068303-21.568072$

$19.1424603 \quad-22.744832$

$\begin{array}{lccccc}\text { Line } & \text { X1i } & \mathbf{X 2 i} & \text { Source S10 } & \mathbf{X 1 0} & \mathbf{X 2 0} \\ \text { S10C01 } & 22.7962151 & -18.13022 & 22.9351348 & -18.26914 \\ \text { S10C02 } & 22.7962151 & -18.13022 & 22.9351348 & -18.26914 \\ \text { S10C03 } & 22.7962151 & -18.13022 & 22.9351348 & -18.26914 \\ \text { S10C04 } & 22.7962151 & -18.13022 & 22.9351348 & -18.26914 \\ \text { S10C05 } & 22.7962151 & -18.13022 & 22.9351348 & -18.26914 \\ \text { S10C06 } & 22.7962151 & -18.13022 & 22.9351348 & -18.26914 \\ \text { S10C07 } & -0.7001335 & -20.573724 & -0.6143816 & -20.659476 \\ \text { S10C08 } & -0.7001335 & -20.573724 & -0.6143816 & -20.659476 \\ \text { S10C09 } & -0.7001335 & -20.573724 & -0.6143816 & -20.659476 \\ \text { S10C10 } & -0.7001335 & -20.573724 & -0.6143816 & -20.659476 \\ \text { S10C11 } & -0.7001335 & -20.573724 & -0.6143816 & -20.659476 \\ \text { S10C12 } & -0.7001335 & -20.573724 & -0.6143816 & -20.659476 \\ \text { S10C13 } & 7.99977685 & -22.786309 & 8.14103033 & -22.927562 \\ \text { S10C14 } & 7.99977685 & -22.786309 & 8.14103033 & -22.927562 \\ \text { S10C15 } & 7.99977685 & -22.786309 & 8.14103033 & -22.927562 \\ \text { S10C16 } & 7.99977685 & -22.786309 & 8.14103033 & -22.927562 \\ \text { S10C17 } & 7.99977685 & -22.786309 & 8.14103033 & -22.927562 \\ \text { S10C18 } & 7.99977685 & -22.786309 & 8.14103033 & -22.927562 \\ \text { S10C19 } & -0.2696851 & -10.457402 & -0.2368979 & -10.490189 \\ \text { S10C20 } & -0.2696851 & -10.457402 & -0.2368979 & -10.490189 \\ \text { S10C21 } & -0.2696851 & -10.457402 & -0.2368979 & -10.490189 \\ \text { S10C22 } & -0.2696851 & -10.457402 & -0.2368979 & -10.490189 \\ \text { S10C23 } & -0.2696851 & -10.457402 & -0.2368979 & -10.490189 \\ \text { S10C24 } & -0.2696851 & -10.457402 & -0.2368979 & -10.490189 \\ \text { S10R01 } & 21.1551086 & -19.768829 & 21.3082397 & -19.92196 \\ \text { S10R02 } & 14.4221594 & -21.93758 & 14.5796366 & -22.095057 \\ \text { S10R03 } & 5.73854018 & -22.7471 & 5.86948536 & -22.878046\end{array}$




\section{REVISED METHODOLOGY FOR DETERMINING CESIUM-137 CONTENT OF HN-200 GROUT CONTAINERS.}

$\begin{array}{lcc}\text { S10R04 } & -1.8606725 & -19.621879 \\ \text { S10R05 } & -7.4821553 & -10.070163 \\ \text { S10R06 } & 4.44538825 & -11.630439 \\ \text { S10R07 } & 17.5739971 & -14.977122 \\ \text { S10R08 } & 22.794179 & -17.962706 \\ \text { S10R09 } & 18.9550586 & -20.695195 \\ \text { S10R10 } & 10.3690008 & -22.609221 \\ \text { S10R11 } & 1.96819253 & -21.980276 \\ \text { S10R12 } & -4.2940987 & -16.675797 \\ \text { S10R13 } & -6.4359768 & -8.7186335 \\ \text { S10R14 } & 6.165222 & -12.043426 \\ \text { S10R15 } & 17.014179 & -14.805038 \\ \text { S10R16 } & 22.4943134 & -17.329994\end{array}$

Source S11

$\begin{array}{ccccc}\text { Line } & \mathbf{X 1 i} & \mathbf{X 2 i} & \mathbf{X 1 0} & \mathbf{X 2 0} \\ \text { S11C01 } & 22.789198 & -10.396535 & 22.9297671 & -10.537104 \\ \text { S11C02 } & 22.789198 & -10.396535 & 22.9297671 & -10.537104 \\ \text { S11C03 } & 22.789198 & -10.396535 & 22.9297671 & -10.537104 \\ \text { S11C04 } & 22.789198 & -10.396535 & 22.9297671 & -10.537104 \\ \text { S11C05 } & 22.789198 & -10.396535 & 22.9297671 & -10.537104 \\ \text { S11C06 } & 22.789198 & -10.396535 & 22.9297671 & -10.537104 \\ \text { S11C07 } & 22.0818679 & 0.86088994 & 22.1877816 & 0.75497624 \\ \text { S11C08 } & 22.0818679 & 0.86088994 & 22.1877816 & 0.75497624 \\ \text { S11C09 } & 22.0818679 & 0.86088994 & 22.1877816 & 0.75497624 \\ \text { S11C10 } & 22.0818679 & 0.86088994 & 22.1877816 & 0.75497624 \\ \text { S11C11 } & 22.0818679 & 0.86088994 & 22.1877816 & 0.75497624 \\ \text { S11C12 } & 22.0818679 & 0.86088994 & 22.1877816 & 0.75497624 \\ \text { S11C13 } & 21.3935034 & -22.79844 & 21.5319038 & -22.93684 \\ \text { S11C14 } & 21.3935034 & -22.79844 & 21.5319038 & -22.93684 \\ \text { S11C15 } & 21.3935034 & -22.79844 & 21.5319038 & -22.93684 \\ \text { S11C16 } & 21.3935034 & -22.79844 & 21.5319038 & -22.93684 \\ \text { S11C17 } & 21.3935034 & -22.79844 & 21.5319038 & -22.93684 \\ \text { S11C18 } & 21.3935034 & -22.79844 & 21.5319038 & -22.93684 \\ \text { S11C19 } & 16.8778685 & 0.49152195 & 16.9378046 & 0.43158577 \\ \text { S11C20 } & 16.8778685 & 0.49152195 & 16.9378046 & 0.43158577 \\ \text { S11C21 } & 16.8778685 & 0.49152195 & 16.9378046 & 0.43158577 \\ \text { S11C22 } & 16.8778685 & 0.49152195 & 16.9378046 & 0.43158577 \\ \text { S11C23 } & 16.8778685 & 0.49152195 & 16.9378046 & 0.43158577 \\ \text { S11C24 } & 16.8778685 & 0.49152195 & 16.9378046 & 0.43158577 \\ \text { S11R01 } & 21.9986295 & -21.299283 & 22.1437703 & -21.444424 \\ \text { S11R02 } & 20.708879 & -21.501079 & 20.8370818 & -21.629282 \\ \text { S11R03 } & 18.9131401 & -11.328927 & 19.0097678 & -11.425555 \\ \text { S11R04 } & 16.2299997 & 3.45067414 & 16.2787882 & 3.40188563\end{array}$




\section{REVISED METHODOLOGY FOR DETERMINING CESIUM-137 CONTENT OF HN-200 GROUT CONTAINERS.}

$\begin{array}{lcc}\text { S11R05 } & 15.2867133 & 11.8037648 \\ \text { S11R06 } & 21.1349363 & 4.4646368 \\ \text { S11R07 } & 22.7429038 & -5.7838637 \\ \text { S11R08 } & 22.5637585 & -16.110273 \\ \text { S11R09 } & 21.7643918 & -22.249596 \\ \text { S11R10 } & 20.6623555 & -21.329703 \\ \text { S11R11 } & 19.2293939 & -13.375248 \\ \text { S11R12 } & 17.2185327 & -1.2307106 \\ \text { S11R13 } & 14.0604132 & 10.734025 \\ \text { S11R14 } & 18.846497 & 8.64397496 \\ \text { S11R15 } & 22.280316 & -0.344466 \\ \text { S11R16 } & 22.7531751 & -12.073602\end{array}$

\author{
$15.3030824 \quad 11.7873957$ \\ $21.2199425 \quad 4.37963061$ \\ $22.8735956-5.9145555$ \\ $22.7103597-16.256875$ \\ $21.9072936-22.392497$ \\ $20.7898054-21.457153$ \\ $19.3317909-13.477645$ \\ $17.2844636-1.2966414$ \\ $14.0747547 \quad 10.7196836$ \\ $\begin{array}{lll}18.8976192 & 8.59285277\end{array}$ \\ $22.3918848-0.4560347$ \\ $22.8961871-12.216614$
}

$\begin{array}{lccccc}\text { Line } & \mathbf{X} 1 \mathbf{3} & \mathbf{X 2 i} & \text { Source S12 } & \mathbf{X 1 0} & \mathbf{X 2 0} \\ \text { S12C01 } & 22.7486395 & 6.94083223 & 22.899094 & 6.79037766 \\ \text { S12C02 } & 22.7486395 & 6.94083223 & 22.899094 & 6.79037766 \\ \text { S12C03 } & 22.7486395 & 6.94083223 & 22.899094 & 6.79037766 \\ \text { S12C04 } & 22.7486395 & 6.94083223 & 22.899094 & 6.79037766 \\ \text { S12C05 } & 22.7486395 & 6.94083223 & 22.899094 & 6.79037766 \\ \text { S12C06 } & 22.7486395 & 6.94083223 & 22.899094 & 6.79037766 \\ \text { S12C07 } & 22.4477585 & 1.32405081 & 22.6136433 & 1.15816603 \\ \text { S12C08 } & 22.4477585 & 1.32405081 & 22.6136433 & 1.15816603 \\ \text { S12C09 } & 22.4477585 & 1.32405081 & 22.6136433 & 1.15816603 \\ \text { S12C10 } & 22.4477585 & 1.32405081 & 22.6136433 & 1.15816603 \\ \text { S12C11 } & 22.4477585 & 1.32405081 & 22.6136433 & 1.15816603 \\ \text { S12C12 } & 22.4477585 & 1.32405081 & 22.6136433 & 1.15816603 \\ \text { S12C13 } & 20.7147363 & -22.798004 & 20.8532381 & -22.936506 \\ \text { S12C14 } & 20.7147363 & -22.798004 & 20.8532381 & -22.936506 \\ \text { S12C15 } & 20.7147363 & -22.798004 & 20.8532381 & -22.936506 \\ \text { S12C16 } & 20.7147363 & -22.798004 & 20.8532381 & -22.936506 \\ \text { S12C17 } & 20.7147363 & -22.798004 & 20.8532381 & -22.936506 \\ \text { S12C18 } & 20.7147363 & -22.798004 & 20.8532381 & -22.936506 \\ \text { S12C19 } & 19.057042 & 0.59914937 & 19.1302545 & 0.52593682 \\ \text { S12C20 } & 19.057042 & 0.59914937 & 19.1302545 & 0.52593682 \\ \text { S12C21 } & 19.057042 & 0.59914937 & 19.1302545 & 0.52593682 \\ \text { S12C22 } & 19.057042 & 0.59914937 & 19.1302545 & 0.52593682 \\ \text { S12C23 } & 19.057042 & 0.59914937 & 19.1302545 & 0.52593682 \\ \text { S12C24 } & 19.057042 & 0.59914937 & 19.1302545 & 0.52593682 \\ \text { S12R01 } & 21.1687996 & -19.793274 & 21.3217949 & -19.946269 \\ \text { S12R02 } & 20.4442047 & -21.768104 & 20.5729269 & -21.896827 \\ \text { S12R03 } & 19.6486243 & -9.6585845 & 19.745961 & -9.7559213 \\ \text { S12R04 } & 18.4888654 & 7.89583158 & 18.5394139 & 7.84528305 \\ \text { S12R05 } & 19.6711578 & 16.1543692 & 19.6996121 & 16.1259149\end{array}$


REVISED METHODOLOGY FOR DETERMINING CESIUM-137 CONTENT OF HN-200 GROUT CONTAINERS.

$\begin{array}{lcc}\text { S12R06 } & 22.776534 & 10.3590228 \\ \text { S12R07 } & 22.3167178 & -0.8704918 \\ \text { S12R08 } & 21.5758111 & -13.68861 \\ \text { S12R09 } & 20.9637849 & -21.816062 \\ \text { S12R10 } & 20.4088192 & -21.47556 \\ \text { S12R11 } & 19.8122795 & -12.699052 \\ \text { S12R12 } & 19.029568 & 1.01511166 \\ \text { S12R13 } & 17.7193253 & 14.0831125 \\ \text { S12R14 } & 21.4730269 & 14.7920698 \\ \text { S12R15 } & 22.7602163 & 7.25088994 \\ \text { S12R16 } & 21.9818664 & -6.5983893\end{array}$

\author{
$22.9040829 \quad 10.231474$ \\ $22.4847681-1.0385421$ \\ $21.7387613-13.85156$ \\ $21.1105931-21.96287$ \\ $20.5362157-21.602957$ \\ $19.9162577-12.80303$ \\ 19.10166710 .94301253 \\ $17.740982 \quad 14.0614559$ \\ $21.5354495 \quad 14.7296472$ \\ $22.9091723 \quad 7.1019339$ \\ $\begin{array}{ll}22.1503976 & -6.7669205\end{array}$
}

$\begin{array}{ccccc}\text { Line } & \mathbf{X 1 i} & \mathbf{X 2 i} & \mathbf{X 1 0} & \mathbf{X} 20 \\ \text { S13C01 } & 22.7945643 & -16.003796 & 22.9338705 & -16.143102 \\ \text { S13C02 } & 22.7945643 & -16.003796 & 22.9338705 & -16.143102 \\ \text { S13C03 } & 22.7945643 & -16.003796 & 22.9338705 & -16.143102 \\ \text { S13C04 } & 22.7945643 & -16.003796 & 22.9338705 & -16.143102 \\ \text { S13C05 } & 22.7945643 & -16.003796 & 22.9338705 & -16.143102 \\ \text { S13C06 } & 22.7945643 & -16.003796 & 22.9338705 & -16.143102 \\ \text { S13C07 } & 9.98289418 & 0.25603392 & 10.0140178 & 0.2249103 \\ \text { S13C08 } & 9.98289418 & 0.25603392 & 10.0140178 & 0.2249103 \\ \text { S13C09 } & 9.98289418 & 0.25603392 & 10.0140178 & 0.2249103 \\ \text { S13C10 } & 9.98289418 & 0.25603392 & 10.0140178 & 0.2249103 \\ \text { S13C11 } & 9.98289418 & 0.25603392 & 10.0140178 & 0.2249103 \\ \text { S13C12 } & 9.98289418 & 0.25603392 & 10.0140178 & 0.2249103 \\ \text { S13C13 } & 18.7826664 & -22.796688 & 18.9214755 & -22.935497 \\ \text { S13C14 } & 18.7826664 & -22.796688 & 18.9214755 & -22.935497 \\ \text { S13C15 } & 18.7826664 & -22.796688 & 18.9214755 & -22.935497 \\ \text { S13C16 } & 18.7826664 & -22.796688 & 18.9214755 & -22.935497 \\ \text { S13C17 } & 18.7826664 & -22.796688 & 18.9214755 & -22.935497 \\ \text { S13C18 } & 18.7826664 & -22.796688 & 18.9214755 & -22.935497 \\ \text { S13C19 } & 5.03010564 & 0.12402737 & 5.04516911 & 0.1089639 \\ \text { S13C20 } & 5.03010564 & 0.12402737 & 5.04516911 & 0.1089639 \\ \text { S13C21 } & 5.03010564 & 0.12402737 & 5.04516911 & 0.1089639 \\ \text { S13C22 } & 5.03010564 & 0.12402737 & 5.04516911 & 0.1089639 \\ \text { S13C23 } & 5.03010564 & 0.12402737 & 5.04516911 & 0.1089639 \\ \text { S13C24 } & 5.03010564 & 0.12402737 & 5.04516911 & 0.1089639 \\ \text { S13R01 } & 21.62413 & -20.613636 & 21.7727269 & -20.762233 \\ \text { S13R02 } & 18.2748077 & -22.768663 & 18.4106117 & -22.904467 \\ \text { S13R03 } & 13.8362067 & -18.794207 & 13.9376201 & -18.89562 \\ \text { S13R04 } & 8.78048234 & -8.6481557 & 8.83330549 & -8.7009789 \\ \text { S13R05 } & 4.31292457 & 3.13977576 & 4.3165775 & 3.13612283 \\ \text { S13R06 } & 15.3486775 & -3.2000014 & 15.4102132 & -3.261537 \\ & & & & \end{array}$




\section{REVISED METHODOLOGY FOR DETERMINING CESIUM-137 CONTENT OF HN-200 GROUT CONTAINERS.}

$\begin{array}{ccc}\text { S13R07 } & 21.4825659 & -10.144686 \\ \text { S13R08 } & 22.7338864 & -16.909554 \\ \text { S13R09 } & 20.8227309 & -21.738916 \\ \text { S13R10 } & 17.384427 & -22.484196 \\ \text { S13R11 } & 13.287422 & -17.913637 \\ \text { S13R12 } & 8.79246822 & -8.6758901 \\ \text { S13R13 } & 3.84130635 & 2.79393797 \\ \text { S13R14 } & 13.2951356 & -1.7494362 \\ \text { S13R15 } & 20.2549074 & -8.0927895 \\ \text { S13R16 } & 22.7710165 & -14.846972\end{array}$

\author{
$21.5940233-10.256143$ \\ $22.876102-17.051769$ \\ $20.9702535-21.886438$ \\ $17.5143851-22.614154$ \\ $13.3838809-18.010096$ \\ $8.84541164-8.7288335$ \\ $3.8445492 \quad 2.79069512$ \\ $13.3442926-1.7985931$ \\ $20.3533621-8.1912443$ \\ $22.9060124-14.981968$
}

Source S14

$\begin{array}{lcccc}\text { Line } & \mathbf{X 1 i} & \mathbf{X} 2 \mathbf{i} & \mathbf{X 1 0} & \mathbf{X 2 0} \\ \text { S14C01 } & 22.7986988 & -21.807696 & 22.9370388 & -21.946036 \\ \text { S14C02 } & 22.7986988 & -21.807696 & 22.9370388 & -21.946036 \\ \text { S14C03 } & 22.7986988 & -21.807696 & 22.9370388 & -21.946036 \\ \text { S14C04 } & 22.7986988 & -21.807696 & 22.9370388 & -21.946036 \\ \text { S14C05 } & 22.7986988 & -21.807696 & 22.9370388 & -21.946036 \\ \text { S14C06 } & 22.7986988 & -21.807696 & 22.9370388 & -21.946036 \\ \text { S14C07 } & -1.1983961 & -22.732084 & -1.0491108 & -22.881369 \\ \text { S14C08 } & -1.1983961 & -22.732084 & -1.0491108 & -22.881369 \\ \text { S14C09 } & -1.1983961 & -22.732084 & -1.0491108 & -22.881369 \\ \text { S14C10 } & -1.1983961 & -22.732084 & -1.0491108 & -22.881369 \\ \text { S14C11 } & -1.1983961 & -22.732084 & -1.0491108 & -22.881369 \\ \text { S14C12 } & -1.1983961 & -22.732084 & -1.0491108 & -22.881369 \\ \text { S14C13 } & -2.9377498 & -22.764283 & -2.7911804 & -22.910852 \\ \text { S14C14 } & -2.9377498 & -22.764283 & -2.7911804 & -22.910852 \\ \text { S14C1S } & -2.9377498 & -22.764283 & -2.7911804 & -22.910852 \\ \text { S14C16 } & -2.9377498 & -22.764283 & -2.7911804 & -22.910852 \\ \text { S14C17 } & -2.9377498 & -22.764283 & -2.7911804 & -22.910852 \\ \text { S14C18 } & -2.9377498 & -22.764283 & -2.7911804 & -22.910852 \\ \text { S14C19 } & -0.6969486 & -20.533148 & -0.6115935 & -20.618503 \\ \text { S14C20 } & -0.6969486 & -20.533148 & -0.6115935 & -20.618503 \\ \text { S14C21 } & -0.6969486 & -20.533148 & -0.6115935 & -20.618503 \\ \text { S14C22 } & -0.6969486 & -20.533148 & -0.6115935 & -20.618503 \\ \text { S14C23 } & -0.6969486 & -20.533148 & -0.6115935 & -20.618503 \\ \text { S14C24 } & -0.6969486 & -20.533148 & -0.6115935 & -20.618503 \\ \text { S14R01 } & 22.3327774 & -21.919561 & 22.4749501 & -22.061734 \\ \text { S14R02 } & 15.2446872 & -22.282559 & 15.3965806 & -22.434453 \\ \text { S14R03 } & 2.52758086 & -22.647498 & 2.68038867 & -22.800306 \\ \text { S14R04 } & -9.8964447 & -22.740635 & -9.7739772 & -22.863103 \\ \text { S14R05 } & -17.03958 & -20.413361 & -17.007162 & -20.445779 \\ \text { S14R06 } & -10.626162 & -19.759404 & -10.572947 & -19.812619 \\ \text { S14R07 } & 6.24014542 & -20.879942 & 6.34050515 & -20.980302\end{array}$




\section{REVISED METHODOLOGY FOR DETERMINING CESIUM-137 CONTENT OF HN-200 GROUT CONTAINERS.}

$\begin{array}{lll}\text { S14R08 } & 20.3269681 & -21.519565 \\ \text { S14R09 } & 21.3203122 & -22.01003 \\ \text { S14R10 } & 9.26271153 & -22.46784 \\ \text { S14R11 } & -5.9811275 & -22.798087 \\ \text { S14R12 } & -15.43181 & -21.702665 \\ \text { S14R13 } & -15.438319 & -19.028207 \\ \text { S14R14 } & -3.080926 & -20.389626 \\ \text { S14R15 } & 10.8813945 & -21.080965 \\ \text { S14R16 } & 20.6715223 & -21.541561\end{array}$

$20.4543604-21.646958$

$21.4653501-22.155068$

$9.41690336-22.622032$

$\begin{array}{ll}-5.8417801 & -22.937434\end{array}$

$\begin{array}{ll}-15.366888 & -21.767587\end{array}$

$-15.412528-19.053999$

$\begin{array}{rr}-3.0017145 & -20.468838\end{array}$

$10.9904435-21.190014$

$20.7997922-21.669831$

Source S15

$\begin{array}{lcccc}\text { Line } & \mathbf{X 1 i} & \mathbf{X 2 i} & \mathbf{X 1 0} & \mathbf{X 2 o} \\ \text { S15C01 } & 22.6815595 & 14.2999302 & 22.8497782 & 14.1317114 \\ \text { S15C02 } & 22.6815595 & 14.2999302 & 22.8497782 & 14.1317114 \\ \text { S15C03 } & 22.6815595 & 14.2999302 & 22.8497782 & 14.1317114 \\ \text { S15C04 } & 22.6815595 & 14.2999302 & 22.8497782 & 14.1317114 \\ \text { S15C05 } & 22.6815595 & 14.2999302 & 22.8497782 & 14.1317114 \\ \text { S15C06 } & 22.6815595 & 14.2999302 & 22.8497782 & 14.1317114 \\ \text { S15C07 } & 22.4165445 & 1.33438341 & 22.5838069 & 1.16712098 \\ \text { S15C08 } & 22.4165445 & 1.33438341 & 22.5838069 & 1.16712098 \\ \text { S15C09 } & 22.4165445 & 1.33438341 & 22.5838069 & 1.16712098 \\ \text { S15C10 } & 22.4165445 & 1.33438341 & 22.5838069 & 1.16712098 \\ \text { S15C11 } & 22.4165445 & 1.33438341 & 22.5838069 & 1.16712098 \\ \text { S15C12 } & 22.4165445 & 1.33438341 & 22.5838069 & 1.16712098 \\ \text { S15C13 } & 22.1155868 & -22.798888 & 22.2538826 & -22.937184 \\ \text { S15C14 } & 22.1155868 & -22.798888 & 22.2538826 & -22.937184 \\ \text { S15C15 } & 22.1155868 & -22.798888 & 22.2538826 & -22.937184 \\ \text { S15C16 } & 22.1155868 & -22.798888 & 22.2538826 & -22.937184 \\ \text { S15C17 } & 22.1155868 & -22.798888 & 22.2538826 & -22.937184 \\ \text { S15C18 } & 22.1155868 & -22.798888 & 22.2538826 & -22.937184 \\ \text { S15C19 } & 21.8328462 & 0.82531416 & 21.9342745 & 0.72388588 \\ \text { S15C20 } & 21.8328462 & 0.82531416 & 21.9342745 & 0.72388588 \\ \text { S15C21 } & 21.8328462 & 0.82531416 & 21.9342745 & 0.72388588 \\ \text { S15C22 } & 21.8328462 & 0.82531416 & 21.9342745 & 0.72388588 \\ \text { S15C23 } & 21.8328462 & 0.82531416 & 21.9342745 & 0.72388588 \\ \text { S15C24 } & 21.8328462 & 0.82531416 & 21.9342745 & 0.72388588 \\ \text { S15R01 } & 22.1611979 & -21.600046 & 22.3048815 & -21.743729 \\ \text { S15R02 } & 22.0321233 & -19.12911 & 22.1600146 & -19.257001 \\ \text { S15R03 } & 21.8697604 & -2.4918433 & 21.9761799 & -2.5982628 \\ \text { S15R04 } & 21.5467446 & 14.9940117 & 21.6099076 & 14.9308487 \\ \text { S15R05 } & 22.4421966 & 20.2196333 & 22.5052219 & 20.1566081 \\ \text { S15R06 } & 22.6432756 & 13.0675816 & 22.8139905 & 12.8968667 \\ \text { S15R07 } & 22.3855391 & -1.1532025 & 22.5507424 & -1.3184057 \\ \text { S15R08 } & 22.2424044 & -15.139561 & 22.3949805 & -15.292137\end{array}$




\section{REVISED METHODOLOGY FOR DETERMINING CESIUM-137 CONTENT OF HN-200 GROUT CONTAINERS.}

$\begin{array}{lcc}\text { S15R09 } & 22.1395255 & -22.45023 \\ \text { S15R10 } & 22.0447644 & -20.112087 \\ \text { S15R11 } & 21.9343354 & -9.1421341 \\ \text { S15R12 } & 21.7644153 & 5.89598493 \\ \text { S15R13 } & 21.364113 & 18.3144561 \\ \text { S15R14 } & 22.7379345 & 19.3870616 \\ \text { S15R15 } & 22.5523348 & 9.47526346 \\ \text { S15R16 } & 22.3095465 & -8.2649844\end{array}$

\author{
$22.2806797-22.591384$ \\ $22.1742691-20.241592$ \\ $22.0494211-9.2572198$ \\ $21.8565718 \quad 5.80382848$ \\ $21.4043119 \quad 18.2742572$ \\ $22.8416494 \quad 19.2833466$ \\ $22.7246553 \quad 9.30294292$ \\ $22.4686137-8.4240516$
}

\begin{tabular}{|c|c|c|c|c|}
\hline \multirow[b]{2}{*}{ Line } & \multicolumn{4}{|c|}{ Source S16 } \\
\hline & $\mathbf{X 1 i}$ & $\mathbf{X} \mathbf{2 i}$ & X1o & $\mathbf{X} 20$ \\
\hline S16C01 & 22.7738213 & -0.6731098 & 22.9180668 & -0.8173552 \\
\hline $\mathrm{S} 16 \mathrm{C} 02$ & 22.7738213 & -0.6731098 & 22.9180668 & -0.8173552 \\
\hline S16C03 & 22.7738213 & -0.6731098 & 22.9180668 & -0.8173552 \\
\hline S16C04 & 22.7738213 & -0.6731098 & 22.9180668 & -0.8173552 \\
\hline S16C05 & 22.7738213 & -0.6731098 & 22.9180668 & -0.8173552 \\
\hline S16C06 & 22.7738213 & -0.6731098 & 22.9180668 & -0.8173552 \\
\hline S16C07 & 22.796879 & 1.09378443 & 22.9325464 & 0.95811707 \\
\hline S16C08 & 22.796879 & 1.09378443 & 22.9325464 & 0.95811707 \\
\hline S16C09 & 22.796879 & 1.09378443 & 22.9325464 & 0.95811707 \\
\hline S16C10 & 22.796879 & 1.09378443 & 22.9325464 & 0.95811707 \\
\hline S16C11 & 22.796879 & 1.09378443 & 22.9325464 & 0.95811707 \\
\hline $\mathrm{S} 16 \mathrm{C} 12$ & 22.796879 & 1.09378443 & 22.9325464 & 0.95811707 \\
\hline S16C13 & 19.2844325 & -22.797041 & 19.423159 & -22.935768 \\
\hline S16C14 & 19.2844325 & -22.797041 & 19.423159 & -22.935768 \\
\hline S16C15 & 19.2844325 & -22.797041 & 19.423159 & -22.935768 \\
\hline S16C16 & 19.2844325 & -22.797041 & 19.423159 & -22.935768 \\
\hline S16C17 & 19.2844325 & -22.797041 & 19.423159 & -22.935768 \\
\hline $\mathrm{S} 16 \mathrm{C} 18$ & 19.2844325 & -22.797041 & 19.423159 & -22.935768 \\
\hline S16C19 & 15.2480236 & 0.42594763 & 15.2999083 & 0.37406292 \\
\hline S16C20 & 15.2480236 & 0.42594763 & 15.2999083 & 0.37406292 \\
\hline $\mathrm{S} 16 \mathrm{C} 21$ & 15.2480236 & 0.42594763 & 15.2999083 & 0.37406292 \\
\hline S16C22 & 15.2480236 & 0.42594763 & 15.2999083 & 0.37406292 \\
\hline S16C23 & 15.2480236 & 0.42594763 & 15.2999083 & 0.37406292 \\
\hline S16C24 & 15.2480236 & 0.42594763 & 15.2999083 & 0.37406292 \\
\hline S16R01 & 20.6287757 & -18.838712 & 20.787289 & -18.997225 \\
\hline S16R02 & 18.9767235 & -22.622135 & 19.1097763 & -22.755188 \\
\hline S16R03 & 17.2417594 & -14.440426 & 17.3384902 & -14.537157 \\
\hline S16R04 & 15.1035049 & 1.38465015 & 15.1521818 & 1.33597323 \\
\hline S16R05 & 15.3962709 & 11.9015098 & 15.412837 & 11.8849437 \\
\hline S16R06 & 21.9416091 & 6.37214903 & 22.0364657 & 6.27729238 \\
\hline S16R07 & 22.6670846 & -2.666637 & 22.8187577 & -2.8183101 \\
\hline S16R08 & 21.4705363 & -13.490905 & 21.6350465 & -13.655415 \\
\hline S16R09 & 20.0706327 & -21.323639 & 20.2221072 & -21.475114 \\
\hline
\end{tabular}


REVISED METHODOLOGY FOR DETERMINING CESIUM-137 CONTENT OF HN-200 GROUT CONTAINERS.

$\begin{array}{lcc}\text { S16R10 } & 18.7280671 & -22.161109 \\ \text { S16R11 } & 17.3442842 & -15.171553 \\ \text { S16R12 } & 15.7126775 & -2.837329 \\ \text { S16R13 } & 13.4532455 & 10.2190306 \\ \text { S16R14 } & 19.3645814 & 9.7548736 \\ \text { S16R15 } & 22.6949747 & 3.06277858 \\ \text { S16R16 } & 22.199239 & -7.6577263\end{array}$

\author{
$18.8563136-22.289356$ \\ $17.4432912-15.27056$ \\ $15.7749615-2.8996131$ \\ $13.4666837 \quad 10.2055924$ \\ $19.4167609 \quad 9.70269404$ \\ $22.81841 \quad 2.9393433$ \\ $22.3615495 \quad-7.8200368$
}

\begin{tabular}{|c|c|c|c|c|}
\hline \multirow[b]{2}{*}{ Line } & \multicolumn{4}{|c|}{ Source S17 } \\
\hline & $\mathbf{X 1 i}$ & $\mathbf{X} \mathbf{2} \mathbf{i}$ & X1o & $\mathbf{X} 20$ \\
\hline $\mathrm{S} 17 \mathrm{C} 01$ & 22.7991153 & -22.489909 & 22.9373583 & -22.628152 \\
\hline $\mathrm{S} 17 \mathrm{C} 02$ & 22.7991153 & -22.489909 & 22.9373583 & -22.628152 \\
\hline $\mathrm{S} 17 \mathrm{C} 03$ & 22.7991153 & -22.489909 & 22.9373583 & -22.628152 \\
\hline S17C04 & 22.7991153 & -22.489909 & 22.9373583 & -22.628152 \\
\hline S17C05 & 22.7991153 & -22.489909 & 22.9373583 & -22.628152 \\
\hline S17C06 & 22.7991153 & -22.489909 & 22.9373583 & -22.628152 \\
\hline S17C07 & -0.8012248 & -21.638548 & -0.7028258 & -21.736947 \\
\hline S17C08 & -0.8012248 & -21.638548 & -0.7028258 & -21.736947 \\
\hline S17C09 & -0.8012248 & -21.638548 & -0.7028258 & -21.736947 \\
\hline $\mathrm{S} 17 \mathrm{C} 10$ & -0.8012248 & -21.638548 & -0.7028258 & -21.736947 \\
\hline S17C11 & -0.8012248 & -21.638548 & -0.7028258 & -21.736947 \\
\hline S17C12 & -0.8012248 & -21.638548 & -0.7028258 & -21.736947 \\
\hline S17C13 & 18.1470252 & -22.796228 & 18.285942 & -22.935144 \\
\hline S17C14 & 18.1470252 & -22.796228 & 18.285942 & -22.935144 \\
\hline S17C15 & 18.1470252 & -22.796228 & 18.285942 & -22.935144 \\
\hline S17C16 & 18.1470252 & -22.796228 & 18.285942 & -22.935144 \\
\hline S17C17 & 18.1470252 & -22.796228 & 18.285942 & -22.935144 \\
\hline S17C18 & 18.1470252 & -22.796228 & 18.285942 & -22.935144 \\
\hline S17C19 & -0.6144757 & -19.318862 & -0.5393656 & -19.393972 \\
\hline S17C20 & -0.6144757 & -19.318862 & -0.5393656 & -19.393972 \\
\hline S17C21 & -0.6144757 & -19.318862 & -0.5393656 & -19.393972 \\
\hline $\mathrm{S} 17 \mathrm{C} 22$ & -0.6144757 & -19.318862 & -0.5393656 & -19.393972 \\
\hline S17C23 & -0.6144757 & -19.318862 & -0.5393656 & -19.393972 \\
\hline S17C24 & -0.6144757 & -19.318862 & -0.5393656 & -19.393972 \\
\hline S17R01 & 22.6761152 & -22.56543 & 22.8153454 & -22.70466 \\
\hline S17R02 & 17.6606196 & -22.798645 & 17.7991391 & -22.937165 \\
\hline S17R03 & 7.32270886 & -22.498528 & 7.44552253 & -22.621342 \\
\hline S17R04 & -4.1216592 & -20.962672 & -4.0381807 & -21.046151 \\
\hline S17R05 & -12.860563 & -16.447388 & -12.841939 & -16.466011 \\
\hline S17R06 & -6.7323585 & -17.919429 & -6.6825172 & -17.969271 \\
\hline S17R07 & 7.32720534 & -20.524786 & 7.42636872 & -20.623949 \\
\hline S17R08 & 19.4966514 & -21.897348 & 19.6242918 & -22.024988 \\
\hline S17R09 & 22.4475786 & -22.613812 & 22.587335 & -22.753568 \\
\hline S17R 10 & 14.6840471 & -22.778387 & 14.8194406 & -22.91378 \\
\hline
\end{tabular}




\section{REVISED METHODOLOGY FOR DETERMINING CESIUM-137 CONTENT OF HN-200 GROUT CONTAINERS.}

$\begin{array}{lll}\text { S17R11 } & 1.8236222 & -22.009607 \\ \text { S17R12 } & -9.2067353 & -19.110935 \\ \text { S17R13 } & -12.276571 & -15.812573 \\ \text { S17R14 } & -1.250071 & -19.199092 \\ \text { S17R15 } & 11.0625699 & -20.972702 \\ \text { S17R16 } & 20.1950199 & -21.981194\end{array}$

\author{
$1.93149136-22.117476$ \\ $-9.1551107-19.16256$ \\ $-12.259229-15.829915$ \\ $-1.1772408-19.271923$ \\ $11.1710586-21.081191$ \\ $20.3243168-22.110491$
}

\begin{tabular}{lccccc} 
Line & X1i & X2i & Source S18 & X1o & X2o \\
S18C01 & 22.580964 & 17.7951787 & 22.7795945 & 17.5965483 \\
S18C02 & 22.580964 & 17.7951787 & 22.7795945 & 17.5965483 \\
S18C03 & 22.580964 & 17.7951787 & 22.7795945 & 17.5965483 \\
S18C04 & 22.580964 & 17.7951787 & 22.7795945 & 17.5965483 \\
S18C05 & 22.580964 & 17.7951787 & 22.7795945 & 17.5965483 \\
S18C06 & 22.580964 & 17.7951787 & 22.7795945 & 17.5965483 \\
S18C07 & 19.0430897 & 0.59835146 & 19.1162035 & 0.52523765 \\
S18C08 & 19.0430897 & 0.59835146 & 19.1162035 & 0.52523765 \\
S18C09 & 19.0430897 & 0.59835146 & 19.1162035 & 0.52523765 \\
S18C10 & 19.0430897 & 0.59835146 & 19.1162035 & 0.52523765 \\
S18C11 & 19.0430897 & 0.59835146 & 19.1162035 & 0.52523765 \\
S18C12 & 19.0430897 & 0.59835146 & 19.1162035 & 0.52523765 \\
S18C13 & 19.2912783 & -22.797046 & 19.4300038 & -22.935772 \\
S18C14 & 19.2912783 & -22.797046 & 19.4300038 & -22.935772 \\
S18C15 & 19.2912783 & -22.797046 & 19.4300038 & -22.935772 \\
S18C16 & 19.2912783 & -22.797046 & 19.4300038 & -22.935772 \\
S18C17 & 19.2912783 & -22.797046 & 19.4300038 & -22.935772 \\
S18C18 & 19.2912783 & -22.797046 & 19.4300038 & -22.935772 \\
S18C19 & 19.7627569 & 1.91650811 & 20.0114689 & 1.66779608 \\
S18C20 & 19.7627569 & 1.91650811 & 20.0114689 & 1.66779608 \\
S18C21 & 19.7627569 & 1.91650811 & 20.0114689 & 1.66779608 \\
S18C22 & 19.7627569 & 1.91650811 & 20.0114689 & 1.66779608 \\
S18C23 & 19.7627569 & 1.91650811 & 20.0114689 & 1.66779608 \\
S18C24 & 19.7627569 & 1.91650811 & 20.0114689 & 1.66779608 \\
S18R01 & 19.423011 & -16.776446 & 19.5951867 & -16.948621 \\
S18R02 & 19.7214904 & 0.43503943 & 19.9618645 & 0.19466529 \\
S18R03 & 20.9435243 & 15.0103139 & 21.2920172 & 14.661821 \\
S18R04 & 22.5675614 & 18.4007709 & 22.6546036 & 18.3137288 \\
S18R05 & 18.8619943 & 15.2593339 & 18.8871796 & 15.2341486 \\
S18R06 & 19.0394396 & 1.02865081 & 19.1115803 & 0.95651004 \\
S18R07 & 19.1523424 & -13.459349 & 19.2545542 & -13.56156 \\
S18R08 & 19.2493341 & -21.989899 & 19.3771351 & -22.1177 \\
S18R09 & 19.3564666 & -20.923066 & 19.5119307 & -21.07853 \\
S18R10 & 19.5134224 & -10.437082 & 19.7075482 & -10.631208 \\
S18R11 & 19.8683245 & 4.97986013 & 20.1369504 & 4.71123423 \\
& & & & \\
\hline
\end{tabular}




\section{REVISED METHODOLOGY FOR DETERMINING CESIUM-137 CONTENT} OF HN-200 GROUT CONTAINERS.

\author{
S18R12 $21.5436884 \quad 16.4491151$ \\ S18R13 21.5511075 18.5940005 \\ S18R14 $18.9612199 \quad 8.88707606$ \\ S18R15 $19.1318952-10.854932$ \\ $\begin{array}{lll}\text { S18R16 } & 19.2655511 & -22.520314\end{array}$
}

\author{
$21.8618365 \quad 16.130967$ \\ $21.5935171 \quad 18.5515908$ \\ $19.0125512 \quad 8.8357448$ \\ $19.2286729-10.95171$ \\ $19.3975883-22.652352$
}

\begin{tabular}{|c|c|c|c|c|}
\hline \multirow[b]{2}{*}{ Line } & \multicolumn{4}{|c|}{ Source S19 } \\
\hline & $\mathbf{X 1 i}$ & $\mathbf{X} \mathbf{2} \mathbf{i}$ & $\mathbf{X 1 0}$ & $\mathbf{X} 20$ \\
\hline $\mathrm{S} 19 \mathrm{C} 01$ & 22.7992988 & -22.797214 & 22.9374991 & -22.935414 \\
\hline $\mathrm{S} 19 \mathrm{C} 02$ & 22.7992988 & -22.797214 & 22.9374991 & -22.935414 \\
\hline $\mathrm{S} 19 \mathrm{C} 03$ & 22.7992988 & -22.797214 & 22.9374991 & -22.935414 \\
\hline $\mathrm{S} 19 \mathrm{CO} 4$ & 22.7992988 & -22.797214 & 22.9374991 & -22.935414 \\
\hline S19C05 & 22.7992988 & -22.797214 & 22.9374991 & -22.935414 \\
\hline S19C06 & 22.7992988 & -22.797214 & 22.9374991 & -22.935414 \\
\hline $\mathrm{S} 19 \mathrm{C} 07$ & -0.8918512 & -22.263919 & -0.7820221 & -22.373748 \\
\hline S19C08 & -0.8918512 & -22.263919 & -0.7820221 & -22.373748 \\
\hline S19C09 & -0.8918512 & -22.263919 & -0.7820221 & -22.373748 \\
\hline $\mathrm{S} 19 \mathrm{C} 10$ & -0.8918512 & -22.263919 & -0.7820221 & -22.373748 \\
\hline $\mathrm{S} 19 \mathrm{C} 11$ & -0.8918512 & -22.263919 & -0.7820221 & -22.373748 \\
\hline $\mathrm{S} 19 \mathrm{C} 12$ & -0.8918512 & -22.263919 & -0.7820221 & -22.373748 \\
\hline $\mathrm{S} 19 \mathrm{C} 13$ & 22.684386 & -22.799232 & 22.8226019 & -22.937448 \\
\hline$S 19 C 14$ & 22.684386 & -22.799232 & 22.8226019 & -22.937448 \\
\hline S19C15 & 22.684386 & -22.799232 & 22.8226019 & -22.937448 \\
\hline S19C.16 & 22.684386 & -22.799232 & 22.8226019 & -22.937448 \\
\hline S19C17 & 22.684386 & -22.799232 & 22.8226019 & -22.937448 \\
\hline S19C18 & 22.684386 & -22.799232 & 22.8226019 & -22.937448 \\
\hline S19C19 & -0.8707222 & -22.14307 & -0.7635663 & -22.250226 \\
\hline $\mathrm{S}_{19 C_{2} 0}$ & -0.8707222 & -22.14307 & -0.7635663 & -22.250226 \\
\hline S19C21 & -0.8707222 & -22.14307 & -0.7635663 & -22.250226 \\
\hline $\mathrm{S} 19 \mathrm{C} 22$ & -0.8707222 & -22.14307 & -0.7635663 & -22.250226 \\
\hline $\mathrm{S} 19 \mathrm{C} 23$ & -0.8707222 & -22.14307 & -0.7635663 & -22.250226 \\
\hline S19C24 & -0.8707222 & -22.14307 & -0.7635663 & -22.250226 \\
\hline S19R01 & 22.7983552 & -22.797502 & 22.9365631 & -22.93571 \\
\hline S19R02 & 18.3490959 & -22.759235 & 18.4845906 & -22.89473 \\
\hline S19R03 & 7.07260644 & -22.552105 & 7.19661006 & -22.676108 \\
\hline S19R04 & -6.2778707 & -21.907811 & -6.183563 & -22.002118 \\
\hline S19R05 & -16.246871 & -19.751539 & -16.218041 & -19.780368 \\
\hline S19R06 & -13.002373 & -20.777217 & -12.945042 & -20.834548 \\
\hline S19R07 & 0.69401293 & -22.221189 & 0.80464171 & -22.331818 \\
\hline S19R08 & 16.0935594 & -22.673686 & 16.225806 & -22.805933 \\
\hline S19R09 & 22.7962945 & -22.797721 & 22.9345076 & -22.935934 \\
\hline S19R10 & 15.5933851 & -22.721832 & 15.7266724 & -22.855119 \\
\hline S19R11 & 0.14302285 & -22.31302 & 0.25515319 & -22.425151 \\
\hline S19R 12 & -13.219994 & -20.865495 & -13.162159 & -20.92333 \\
\hline
\end{tabular}


REVISED METHODOLOGY FOR DETERMINING CESIUM-137 CONTENT

OF HN-200 GROUT CONTAINERS.
S19R13
S19R 14
S19R15
S19R16

$\begin{array}{ll}-16.145801 & -19.717096\end{array}$

$\begin{array}{ll}-5.8819928 & -21.887368\end{array}$

$7.56506245-22.593073$

$18.724701 \quad-22.853673$

\begin{tabular}{|c|c|c|c|c|}
\hline \multirow[b]{2}{*}{ Line } & \multicolumn{4}{|c|}{ pores } \\
\hline & $\mathbf{X 1 i}$ & $\mathbf{X} \mathbf{2} \mathbf{i}$ & X1o & $\mathbf{X} 20$ \\
\hline $\mathbf{S} 20 \mathrm{CO} 1$ & 22.7938008 & -15.093182 & 22.933286 & -15.232667 \\
\hline $\mathrm{S} 20 \mathrm{CO} 2$ & 22.7938008 & -15.093182 & 22.933286 & -15.232667 \\
\hline $\mathrm{S} 20 \mathrm{C} 03$ & 22.7938008 & -15.093182 & 22.933286 & -15.232667 \\
\hline $\mathrm{S} 20 \mathrm{C} 04$ & 22.7938008 & -15.093182 & 22.933286 & -15.232667 \\
\hline S20C05 & 22.7938008 & -15.093182 & 22.933286 & -15.232667 \\
\hline S20C06 & 22.7938008 & -15.093182 & 22.933286 & -15.232667 \\
\hline S20C07 & 8.75913011 & 0.22182122 & 8.78608703 & 0.19486431 \\
\hline S20C08 & 8.75913011 & 0.22182122 & 8.78608703 & 0.19486431 \\
\hline S20C09 & 8.75913011 & 0.22182122 & 8.78608703 & 0.19486431 \\
\hline $\mathrm{S} 20 \mathrm{C} 10$ & 8.75913011 & 0.22182122 & 8.78608703 & 0.19486431 \\
\hline $\mathrm{S} 20 \mathrm{C} 11$ & 8.75913011 & 0.22182122 & 8.78608703 & 0.19486431 \\
\hline $\mathrm{S} 20 \mathrm{C} 12$ & 8.75913011 & 0.22182122 & 8.78608703 & 0.19486431 \\
\hline$S 20 C 13$ & 17.7239119 & -22.795913 & 17.8629022 & -22.934903 \\
\hline $\mathrm{S} 20 \mathrm{C} 14$ & 17.7239119 & -22.795913 & 17.8629022 & -22.934903 \\
\hline S20C15 & 17.7239119 & -22.795913 & 17.8629022 & -22.934903 \\
\hline $\mathrm{S} 20 \mathrm{C} 16$ & 17.7239119 & -22.795913 & 17.8629022 & -22.934903 \\
\hline S20C17 & 17.7239119 & -22.795913 & 17.8629022 & -22.934903 \\
\hline S20C18 & 17.7239119 & -22.795913 & 17.8629022 & -22.934903 \\
\hline $\mathbf{S 2 0 C 1 9}$ & 4.00604875 & 0.09832532 & 4.01798943 & 0.08638464 \\
\hline $\mathrm{S} 20 \mathrm{C} 20$ & 4.00604875 & 0.09832532 & 4.01798943 & 0.08638464 \\
\hline$S 20 C 21$ & 4.00604875 & 0.09832532 & 4.01798943 & 0.08638464 \\
\hline $\mathrm{S} 20 \mathrm{C} 22$ & 4.00604875 & 0.09832532 & 4.01798943 & 0.08638464 \\
\hline S20C23 & 4.00604875 & 0.09832532 & 4.01798943 & 0.08638464 \\
\hline S20C24 & 4.00604875 & 0.09832532 & 4.01798943 & 0.08638464 \\
\hline S20R01 & 21.3463834 & -20.111513 & 21.4976367 & -20.262766 \\
\hline S20R02 & 17.6510861 & -22.798482 & 17.78965 & -22.937045 \\
\hline S20R03 & 13.0151626 & -19.547782 & 13.1183811 & -19.651001 \\
\hline S20R04 & 7.98323444 & -9.7303162 & 8.0370133 & -9.7840951 \\
\hline S20R05 & 3.54446645 & 2.57672318 & 3.54745316 & 2.57373647 \\
\hline S20R06 & 15.1660867 & -3.3786914 & 15.227263 & -3.4398677 \\
\hline S20R07 & 21.5914025 & -9.9234901 & 21.7036248 & -10.035712 \\
\hline S20R08 & 22.6556094 & -16.486536 & 22.8000874 & -16.631014 \\
\hline S20R09 & 20.3243714 & -21.464489 & 20.4744852 & -21.614602 \\
\hline S20R 10 & 16.5310326 & -22.620628 & 16.6624383 & -22.752034 \\
\hline S20R11 & 12.2750517 & -18.450557 & 12.3715047 & -18.54701 \\
\hline S20R 12 & 7.82966091 & -9.3643069 & 7.881841 & -9.416487 \\
\hline S20R13 & 3.11583682 & 2.26362672 & 3.11845605 & 2.26100749 \\
\hline
\end{tabular}




\section{REVISED METHODOLOGY FOR DETERMINING CESIUM-137 CONTENT OF HN-200 GROUT CONTAINERS.}

\author{
S20R14 $13.0964428 \quad-2.0806223$ \\ S20R15 $20.307355 \quad-7.9476733$ \\ S20R16 $22.790495 \quad-14.329202$
}

$\begin{array}{ll}13.1456928 & -2.1298723 \\ 20.4059358 & -8.0462541 \\ 22.9267853 & -14.465493\end{array}$

Source S21

\begin{tabular}{lcc} 
Line & X1i & X2i \\
S21C01 & 22.7951871 & -16.779497 \\
S21C02 & 22.7951871 & -16.779497 \\
S21C03 & 22.7951871 & -16.779497 \\
S21C04 & 22.7951871 & -16.779497 \\
S21C05 & 22.7951871 & -16.779497 \\
S21C06 & 22.7951871 & -16.779497 \\
S21C07 & 4.450982 & 0.1094489 \\
S21C08 & 4.450982 & 0.1094489 \\
S21C09 & 4.450982 & 0.1094489 \\
S21C10 & 4.450982 & 0.1094489 \\
S21C11 & 4.450982 & 0.1094489 \\
S21C12 & 4.450982 & 0.1094489 \\
S21C13 & 19.0092569 & -22.796849 \\
S21C14 & 19.0092569 & -22.796849 \\
S21C15 & 19.0092569 & -22.796849 \\
S21C16 & 19.0092569 & -22.796849 \\
S21C17 & 19.0092569 & -22.796849 \\
S21C18 & 19.0092569 & -22.796849 \\
S21C19 & 8.60874756 & 0.21770547 \\
S21C20 & 8.60874756 & 0.21770547 \\
S21C21 & 8.60874756 & 0.21770547 \\
S21C22 & 8.60874756 & 0.21770547 \\
S21C23 & 8.60874756 & 0.21770547 \\
S21C24 & 8.60874756 & 0.21770547 \\
S21R01 & 21.7437954 & -20.83164 \\
S21R02 & 22.7403824 & -15.354957 \\
S21R03 & 20.0083981 & -8.7531199 \\
S21R04 & 12.9134514 & -2.3833696 \\
S21R05 & 3.35920445 & 2.44132009 \\
S21R06 & 8.52282884 & -8.8709022 \\
S21R07 & 13.2372513 & -17.941349 \\
S21R08 & 17.5056795 & -22.460912 \\
S21R09 & 20.9964244 & -21.83388 \\
S21R10 & 22.7732976 & -17.223984 \\
S21R11 & 21.2221907 & -10.634844 \\
S21R12 & 14.8096623 & -3.7216366 \\
S21R13 & 3.75156524 & 2.72823386 \\
S21R14 & 8.56000132 & -8.9515422 \\
& & \\
\hline
\end{tabular}

\begin{tabular}{cc}
$\mathbf{X} 10$ & $\mathbf{X} \mathbf{2 0}$ \\
22.9343473 & -16.918658 \\
22.9343473 & -16.918658 \\
22.9343473 & -16.918658 \\
22.9343473 & -16.918658 \\
22.9343473 & -16.918658 \\
22.9343473 & -16.918658 \\
4.46427407 & 0.09615682 \\
4.46427407 & 0.09615682 \\
4.46427407 & 0.09615682 \\
4.46427407 & 0.09615682 \\
4.46427407 & 0.09615682 \\
4.46427407 & 0.09615682 \\
19.1480286 & -22.93562 \\
19.1480286 & -22.93562 \\
19.1480286 & -22.93562 \\
19.1480286 & -22.93562 \\
19.1480286 & -22.93562 \\
19.1480286 & -22.93562 \\
8.63520346 & 0.19124957 \\
8.63520346 & 0.19124957 \\
8.63520346 & 0.19124957 \\
8.63520346 & 0.19124957 \\
8.63520346 & 0.19124957 \\
8.63520346 & 0.19124957 \\
21.8912726 & -20.979118 \\
22.8741953 & -15.48877 \\
20.1063277 & -8.8510495 \\
12.9627934 & -2.4327116 \\
3.36203201 & 2.43849253 \\
8.57554925 & -8.9236226 \\
13.333705 & -18.037802 \\
17.6354534 & -22.590686 \\
21.1430683 & -21.980524 \\
22.913916 & -17.364603 \\
21.3320222 & -10.744675 \\
14.8701658 & -3.7821401 \\
3.7547305 & 2.72506859 \\
8.61307979 & -9.0046206 \\
& \\
\hline
\end{tabular}




\title{
REVISED METHODOLOGY FOR DETERMINING CESIUM-137 CONTENT OF HN-200 GROUT CONTAINERS.
}

\author{
S21R15 $13.8165944-18.813371$ \\ $13.9180482-18.914825$ \\ $\begin{array}{lll}\text { S21R16 } & 18.3919547 & -22.753183\end{array}$ \\ $18.5272728-22.888501$
}

\begin{tabular}{|c|c|c|c|c|}
\hline \multirow[b]{2}{*}{ Line } & \multicolumn{4}{|c|}{ Source S22 } \\
\hline & $\mathbf{X 1 i}$ & $\mathbf{X} 2 \mathbf{i}$ & X1o & $\mathbf{X} 20$ \\
\hline $\mathrm{S} 22 \mathrm{C} 01$ & 22.7966141 & -18.679605 & 22.9354405 & -18.818431 \\
\hline $\mathrm{S} 22 \mathrm{CO} 2$ & 22.7966141 & -18.679605 & 22.9354405 & -18.818431 \\
\hline $\mathrm{S} 22 \mathrm{C} 03$ & 22.7966141 & -18.679605 & 22.9354405 & -18.818431 \\
\hline $\mathrm{S} 22 \mathrm{CO} 4$ & 22.7966141 & -18.679605 & 22.9354405 & -18.818431 \\
\hline $\mathrm{S} 22 \mathrm{COS}$ & 22.7966141 & -18.679605 & 22.9354405 & -18.818431 \\
\hline $\mathrm{S} 22 \mathrm{C} 06$ & 22.7966141 & -18.679605 & 22.9354405 & -18.818431 \\
\hline S22C07 & -1.9875677 & -19.396318 & -1.7282993 & -19.655586 \\
\hline $\mathrm{S} 22 \mathrm{C} 08$ & -1.9875677 & -19.396318 & -1.7282993 & -19.655586 \\
\hline $\mathbf{S} 22 \mathrm{C} 09$ & -1.9875677 & -19.396318 & -1.7282993 & -19.655586 \\
\hline $\mathbf{S} 22 \mathrm{C} 10$ & -1.9875677 & -19.396318 & -1.7282993 & -19.655586 \\
\hline $\mathbf{S} 22 \mathrm{C} 11$ & -1.9875677 & -19.396318 & -1.7282993 & -19.655586 \\
\hline $\mathrm{S} 22 \mathrm{C} 12$ & -1.9875677 & -19.396318 & -1.7282993 & -19.655586 \\
\hline $\mathrm{S} 22 \mathrm{C} 13$ & -16.786563 & -22.621944 & -16.600912 & -22.807595 \\
\hline $\mathrm{S} 22 \mathrm{C} 14$ & -16.786563 & -22.621944 & -16.600912 & -22.807595 \\
\hline $\mathrm{S} 22 \mathrm{C} 15$ & -16.786563 & -22.621944 & -16.600912 & -22.807595 \\
\hline $\mathrm{S} 22 \mathrm{C} 16$ & -16.786563 & -22.621944 & -16.600912 & -22.807595 \\
\hline $\mathrm{S} 22 \mathrm{C} 17$ & -16.786563 & -22.621944 & -16.600912 & -22.807595 \\
\hline $\mathrm{S} 22 \mathrm{C} 18$ & -16.786563 & -22.621944 & -16.600912 & -22.807595 \\
\hline $\mathbf{S} 22 \mathrm{C} 19$ & -0.5594921 & -18.32441 & -0.4911811 & -18.392721 \\
\hline S22C20 & -0.5594921 & -18.32441 & -0.4911811 & -18.392721 \\
\hline S22C21 & -0.5594921 & -18.32441 & -0.4911811 & -18.392721 \\
\hline $\mathrm{S} 22 \mathrm{C} 22$ & -0.5594921 & -18.32441 & -0.4911811 & -18.392721 \\
\hline $\mathrm{S} 22 \mathrm{C} 23$ & -0.5594921 & -18.32441 & -0.4911811 & -18.392721 \\
\hline $\mathrm{S} 22 \mathrm{C} 24$ & -0.5594921 & -18.32441 & -0.4911811 & -18.392721 \\
\hline S22R01 & 20.597598 & -18.784198 & 20.7564404 & -18.943041 \\
\hline S22R02 & 9.7757115 & -19.024268 & 9.97792666 & -19.226484 \\
\hline S22R03 & -5.4538824 & -19.591036 & -5.170916 & -19.874003 \\
\hline S22R04 & -15.789211 & -21.852117 & -15.518742 & -22.122585 \\
\hline S22R05 & -17.783233 & -20.986475 & -17.746665 & -21.023043 \\
\hline S22R06 & -7.3708485 & -18.23389 & -7.320645 & -18.284093 \\
\hline S22R07 & 12.2467849 & -18.464904 & 12.3432406 & -18.561359 \\
\hline S22R08 & 22.7055577 & -18.656112 & 22.8398189 & -18.790374 \\
\hline S22R09 & 15.8975776 & -18.892209 & 16.0764411 & -19.071072 \\
\hline S22R10 & -1.2468459 & -19.362806 & -0.9921093 & -19.617543 \\
\hline S22R11 & -14.558118 & -21.155106 & -14.240691 & -21.472533 \\
\hline S22R12 & -17.547523 & -22.362135 & -17.469571 & -22.440088 \\
\hline S22R 13 & -14.460465 & -18.095089 & -14.437753 & -18.117801 \\
\hline S22R14 & -0.0957776 & -18.329864 & -0.0263735 & -18.399268 \\
\hline S22R 15 & 14.1430252 & -18.48667 & 14.2438268 & -18.587471 \\
\hline
\end{tabular}




\section{REVISED METHODOLOGY FOR DETERMINING CESIUM-137 CONTENT OF HN-200 GROUT CONTAINERS.}

Source S23

\begin{tabular}{|c|c|c|c|c|}
\hline Line & $\mathbf{X 1 i}$ & $\mathbf{X} \mathbf{2} \mathbf{i}$ & X10 & $\mathbf{X} 20$ \\
\hline S23C01 & 22.7816541 & -4.7903478 & 22.9240162 & -4.9327099 \\
\hline $\mathrm{S} 23 \mathrm{C} 02$ & 22.7816541 & -4.7903478 & 22.9240162 & -4.9327099 \\
\hline $\mathrm{S} 23 \mathrm{CO} 3$ & 22.7816541 & -4.7903478 & 22.9240162 & $-4,9327099$ \\
\hline S23C04 & 22.7816541 & -4.7903478 & 22.9240162 & -4.9327099 \\
\hline $\mathrm{S} 23 \mathrm{C} 05$ & 22.7816541 & -4.7903478 & 22.9240162 & -4.9327099 \\
\hline S23C06 & 22.7816541 & -4.7903478 & 22.9240162 & -4.9327099 \\
\hline $\mathrm{S} 23 \mathrm{C} 07$ & 19.5309432 & 0.62742089 & 19.6076576 & 0.5507065 \\
\hline S23C08 & 19.5309432 & 0.62742089 & 19.6076576 & 0.5507065 \\
\hline S23C09 & 19.5309432 & 0.62742089 & 19.6076576 & 0.5507065 \\
\hline $\mathrm{S} 23 \mathrm{C} 10$ & 19.5309432 & 0.62742089 & 19.6076576 & 0.5507065 \\
\hline $\mathrm{S} 23 \mathrm{C} 11$ & 19.5309432 & 0.62742089 & 19.6076576 & 0.5507065 \\
\hline $\mathrm{S} 23 \mathrm{C} 12$ & 19.5309432 & 0.62742089 & 19.6076576 & 0.5507065 \\
\hline $\mathrm{S} 23 \mathrm{C} 13$ & 13.1128941 & -22.792007 & 13.2528009 & -22.931914 \\
\hline $\mathrm{S} 23 \mathrm{C} 14$ & 13.1128941 & -22.792007 & 13.2528009 & -22.931914 \\
\hline $\mathrm{S} 23 \mathrm{C} 15$ & 13.1128941 & -22.792007 & 13.2528009 & -22.931914 \\
\hline S23C16 & 13.1128941 & -22.792007 & 13.2528009 & -22.931914 \\
\hline $\mathrm{S} 23 \mathrm{C} 17$ & 13.1128941 & -22.792007 & 13.2528009 & -22.931914 \\
\hline S23C18 & 13.1128941 & -22.792007 & 13.2528009 & -22.931914 \\
\hline S23C19 & 6.07244873 & 0.1506032 & 6.09074226 & 0.13230966 \\
\hline $\mathrm{S} 23 \mathrm{C} 20$ & 6.07244873 & 0.1506032 & 6.09074226 & 0.13230966 \\
\hline $\mathrm{S} 23 \mathrm{C} 21$ & 6.07244873 & 0.1506032 & 6.09074226 & 0.13230966 \\
\hline $\mathrm{S} 23 \mathrm{C} 22$ & 6.07244873 & 0.1506032 & 6.09074226 & 0.13230966 \\
\hline $\mathrm{S} 23 \mathrm{C} 23$ & 6.07244873 & 0.1506032 & 6.09074226 & 0.13230966 \\
\hline S23C24 & 6.07244873 & 0.1506032 & 6.09074226 & 0.13230966 \\
\hline S23R01 & 18.5999159 & -15.420901 & 18.782661 & -15.603647 \\
\hline S23R02 & 14.5081707 & -21.976821 & 14.6650375 & -22.133688 \\
\hline S23R03 & 11.025895 & -20.994927 & 11.1344962 & -21.103529 \\
\hline S23R04 & 7.95053467 & -9.7738231 & 8.00435466 & -9.8276431 \\
\hline S23R05 & 6.54785053 & 4.79428687 & 6.5535152 & 4.7886222 \\
\hline S23R06 & 18.9765974 & 0.94275366 & 19.048475 & 0.87087606 \\
\hline S23R07 & 22.7983829 & -4.4183777 & 22.9373181 & -4.5573129 \\
\hline S23R08 & 20.4206112 & -11.747008 & 20.6006388 & -11.927036 \\
\hline S23R09 & 16.4924484 & -19.252624 & 16.6667221 & -19.426898 \\
\hline S23R10 & 13.0996932 & -22.793359 & 13.2394229 & -22.933089 \\
\hline S23R11 & 10.3351083 & -19.362461 & 10.4320974 & -19.45945 \\
\hline S23R12 & 7.86459734 & -9.339703 & 7.91680356 & -9.3919093 \\
\hline S23R13 & 5.34344527 & 3.89908957 & 5.34800999 & 3.89452485 \\
\hline S23R14 & 15.6491773 & 2.37406843 & 15.6978617 & 2.32538408 \\
\hline S23R15 & 22.0461773 & -1.6397377 & 22.1544683 & -1.7480287 \\
\hline S23R16 & 22.1763667 & -7.5385676 & 22.33934 & -7.7015409 \\
\hline
\end{tabular}




\section{REVISED METHODOLOGY FOR DETERMINING CESIUM-137 CONTENT OF HN-200 GROUT CONTAINERS.}

\begin{tabular}{|c|c|c|c|c|}
\hline \multirow[b]{2}{*}{ Line } & \multicolumn{4}{|c|}{ Source S24 } \\
\hline & X1i & $\mathbf{X} \mathbf{2 i}$ & X1o & $\mathbf{X} 20$ \\
\hline S24C01 & 22.7549004 & 5.54902598 & 22.9037888 & 5.40013753 \\
\hline $\mathrm{S} 24 \mathrm{C} 02$ & 22.7549004 & 5.54902598 & 22.9037888 & 5.40013753 \\
\hline $\mathrm{S} 24 \mathrm{C} 03$ & 22.7549004 & 5.54902598 & 22.9037888 & 5.40013753 \\
\hline S24C04 & 22.7549004 & 5.54902598 & 22.9037888 & 5.40013753 \\
\hline $\mathrm{S} 24 \mathrm{C} 05$ & 22.7549004 & 5.54902598 & 22.9037888 & 5.40013753 \\
\hline S24C06 & 22.7549004 & 5.54902598 & 22.9037888 & 5.40013753 \\
\hline S24C07 & 18.9347429 & 2.07760984 & 19.2076169 & 1.80473586 \\
\hline S24C08 & 18.9347429 & 2.07760984 & 19.2076169 & 1.80473586 \\
\hline S24C09 & 18.9347429 & 2.07760984 & 19.2076169 & 1.80473586 \\
\hline $\mathrm{S} 24 \mathrm{C} 10$ & 18.9347429 & 2.07760984 & 19.2076169 & 1.80473586 \\
\hline S24C11 & 18.9347429 & 2.07760984 & 19.2076169 & 1.80473586 \\
\hline $\mathrm{S} 24 \mathrm{C} 12$ & 18.9347429 & 2.07760984 & 19.2076169 & 1.80473586 \\
\hline $\mathrm{S} 24 \mathrm{C} 13$ & 10.0452639 & -22.788801 & 10.185927 & -22.929464 \\
\hline S24C14 & 10.0452639 & -22.788801 & 10.185927 & -22.929464 \\
\hline S24C15 & 10.0452639 & -22.788801 & 10.185927 & -22.929464 \\
\hline S24C16 & 10.0452639 & -22.788801 & 10.185927 & -22.929464 \\
\hline S24C17 & 10.0452639 & -22.788801 & 10.185927 & -22.929464 \\
\hline S24C18 & 10.0452639 & -22.788801 & 10.185927 & -22.929464 \\
\hline S24C19 & 8.33259529 & 0.21019322 & 8.35813684 & 0.18465167 \\
\hline $\mathrm{S} 24 \mathrm{C} 20$ & 8.33259529 & 0.21019322 & 8.35813684 & 0.18465167 \\
\hline $\mathrm{S} 24 \mathrm{C} 21$ & 8.33259529 & 0.21019322 & 8.35813684 & 0.18465167 \\
\hline $\mathrm{S} 24 \mathrm{C} 22$ & 8.33259529 & 0.21019322 & 8.35813684 & 0.18465167 \\
\hline S24C23 & 8.33259529 & 0.21019322 & 8.35813684 & 0.18465167 \\
\hline S24C24 & 8.33259529 & 0.21019322 & 8.35813684 & 0.18465167 \\
\hline S24R01 & 13.4131291 & -7.7362666 & 13.7021113 & -8.0252488 \\
\hline S24R02 & 10.8668039 & -19.820612 & 11.0560404 & -20.009848 \\
\hline S24R03 & 9.62019446 & -21.732102 & 9.733498 & -21.845406 \\
\hline S24R04 & 8.72809655 & -8.720524 & 8.78097974 & -8.7734071 \\
\hline S24R05 & 10.5003368 & 7.81991598 & 10.5100264 & 7.81022631 \\
\hline S24R06 & 21.9844731 & 6.49435616 & 22.0800524 & 6.39877683 \\
\hline S24R07 & 20.6336648 & 3.55542155 & 20.8671663 & 3.32191999 \\
\hline S24R08 & 14.5682381 & -4.4563002 & 14.8754685 & -4.7635306 \\
\hline S24R09 & 11.5334003 & -16.093488 & 11.7567378 & -16.316826 \\
\hline S24R10 & 10.1967618 & -22.58962 & 10.3468496 & -22.739707 \\
\hline S24R11 & 9.38229404 & -19.761154 & 9.47979616 & -19.858656 \\
\hline S24R12 & 8.72818916 & -8.7225403 & 8.78107874 & -8.7754299 \\
\hline S24R 13 & 8.06548924 & 5.93756113 & 8.07260964 & 5.93044073 \\
\hline S24R 14 & 18.1578268 & 7.21552119 & 18.2079366 & 7.16541139 \\
\hline S24R 15 & 22.7992567 & 5.7569234 & 22.9371105 & 5.61906958 \\
\hline S24R 16 & 18.9604835 & 2.10224235 & 19.2328432 & 1.82988269 \\
\hline
\end{tabular}




\section{REVISED METHODOLOGY FOR DETERMINING CESIUM-137 CONTENT OF HN-200 GROUT CONTAINERS.}

\begin{tabular}{|c|c|c|c|c|}
\hline \multirow[b]{2}{*}{ Line } & \multicolumn{4}{|c|}{ Source S25 } \\
\hline & $\mathbf{X 1 i}$ & X2i & X1o & $\mathbf{X} 20$ \\
\hline $\mathrm{S} 25 \mathrm{C} 01$ & 22.7883466 & -9.6524862 & 22.9291171 & -9.7932567 \\
\hline $\mathrm{S} 25 \mathrm{C} 02$ & 22.7883466 & -9.6524862 & 22.9291171 & -9.7932567 \\
\hline $\mathrm{S} 25 \mathrm{C} 03$ & 22.7883466 & -9.6524862 & 22.9291171 & -9.7932567 \\
\hline S25C04 & 22.7883466 & -9.6524862 & 22.9291171 & -9.7932567 \\
\hline $\mathrm{S} 25 \mathrm{C} 05$ & 22.7883466 & -9.6524862 & 22.9291171 & -9.7932567 \\
\hline $\mathrm{S} 25 \mathrm{C} 06$ & 22.7883466 & -9.6524862 & 22.9291171 & -9.7932567 \\
\hline $\mathrm{S} 25 \mathrm{C} 07$ & 20.1764398 & 0.67035401 & 20.2584847 & 0.58830903 \\
\hline $\mathrm{S} 25 \mathrm{C} 08$ & 20.1764398 & 0.67035401 & 20.2584847 & 0.58830903 \\
\hline $\mathrm{S} 25 \mathrm{C} 09$ & 20.1764398 & 0.67035401 & 20.2584847 & 0.58830903 \\
\hline $\mathrm{S} 25 \mathrm{C} 10$ & 20.1764398 & 0.67035401 & 20.2584847 & 0.58830903 \\
\hline $\mathrm{S} 25 \mathrm{C} 11$ & 20.1764398 & 0.67035401 & 20.2584847 & 0.58830903 \\
\hline $\mathrm{S} 25 \mathrm{C} 12$ & 20.1764398 & 0.67035401 & 20.2584847 & 0.58830903 \\
\hline $\mathrm{S} 25 \mathrm{C} 13$ & 18.9513847 & -22.796808 & 19.0901659 & -22.935589 \\
\hline $\mathrm{S} 25 \mathrm{C} 14$ & 18.9513847 & -22.796808 & 19.0901659 & -22.935589 \\
\hline $\mathrm{S} 25 \mathrm{C} 15$ & 18.9513847 & -22.796808 & 19.0901659 & -22.935589 \\
\hline $\mathrm{S} 25 \mathrm{C} 16$ & 18.9513847 & -22.796808 & 19.0901659 & -22.935589 \\
\hline $\mathrm{S} 25 \mathrm{C} 17$ & 18.9513847 & -22.796808 & 19.0901659 & -22.935589 \\
\hline $\mathrm{S} 25 \mathrm{C} 18$ & 18.9513847 & -22.796808 & 19.0901659 & -22.935589 \\
\hline $\mathrm{S} 25 \mathrm{C} 19$ & 11.0074815 & 0.28581822 & 11.0422356 & 0.25106409 \\
\hline $\mathrm{S} 25 \mathrm{C} 20$ & 11.0074815 & 0.28581822 & 11.0422356 & 0.25106409 \\
\hline$S 25 C 21$ & 11.0074815 & 0.28581822 & 11.0422356 & 0.25106409 \\
\hline $\mathrm{S} 25 \mathrm{C} 22$ & 11.0074815 & 0.28581822 & 11.0422356 & 0.25106409 \\
\hline $\mathrm{S} 25 \mathrm{C} 23$ & 11.0074815 & 0.28581822 & 11.0422356 & 0.25106409 \\
\hline $\mathrm{S} 25 \mathrm{C} 24$ & 11.0074815 & 0.28581822 & 11.0422356 & 0.25106409 \\
\hline S25R01 & 21.0929618 & -19.658029 & 21.2467117 & -19.811778 \\
\hline S25R02 & 18.5461109 & -22.727596 & 18.6808057 & -22.86229 \\
\hline S25R03 & 15.5783351 & -16.841662 & 15.676746 & -16.940072 \\
\hline S25R04 & 12.0913976 & -3.7167276 & 12.1412238 & -3.7665538 \\
\hline S25R05 & 10.2802758 & 7.64681772 & 10.2897181 & 7.63737541 \\
\hline S25R06 & 19.4481526 & 1.6052579 & 19.5221231 & 1.53128741 \\
\hline S25R07 & 22.6747509 & -6.3610331 & 22.8023274 & -6.4886096 \\
\hline S25R08 & 22.2184263 & -15.078244 & 22.3714046 & -15.231223 \\
\hline S25R09 & 20.3351438 & -21.470452 & 20.4852004 & -21.620508 \\
\hline S25R10 & 18.025323 & -22.348778 & 18.1543718 & -22.477827 \\
\hline S25R11 & 15.4929652 & -16.562496 & 15.5902243 & -16.659756 \\
\hline S25R12 & 12.604585 & -5.6965795 & 12.6616161 & -5.7536106 \\
\hline S25R 13 & 9.02420187 & 6.67066871 & 9.03229359 & 6.66257698 \\
\hline S25R 14 & 16.6236181 & 4.19458736 & 16.6725419 & 4.14566356 \\
\hline S25R 15 & 21.8516906 & -2.574558 & 21.9579394 & -2.6808068 \\
\hline S25R 16 & 22.7001502 & -11.310006 & 22.845865 & -11.455721 \\
\hline
\end{tabular}




\section{REVISED METHODOLOGY FOR DETERMINING CESIUM-137 CONTENT OF HN-200 GROUT CONTAINERS.}

\begin{tabular}{|c|c|c|c|c|}
\hline \multirow[b]{2}{*}{ Line } & \multicolumn{4}{|c|}{ Source S26 } \\
\hline & $\mathbf{X 1 i}$ & $\mathbf{X} 2 \mathbf{i}$ & X1o & X20 \\
\hline $\mathrm{S} 26 \mathrm{CO} 1$ & 22.7796263 & -3.5920478 & 22.9224738 & -3.7348954 \\
\hline $\mathrm{S} 26 \mathrm{C} 02$ & 22.7796263 & -3.5920478 & 22.9224738 & -3.7348954 \\
\hline $\mathrm{S} 26 \mathrm{C} 03$ & 22.7796263 & -3.5920478 & 22.9224738 & -3.7348954 \\
\hline S26C04 & 22.7796263 & -3.5920478 & 22.9224738 & -3.7348954 \\
\hline S26C05 & 22.7796263 & -3.5920478 & 22.9224738 & -3.7348954 \\
\hline S26C06 & 22.7796263 & -3.5920478 & 22.9224738 & -3.7348954 \\
\hline s26C07 & 19.2447697 & 0.61006835 & 19.319334 & 0.53550408 \\
\hline S26C08 & 19.2447697 & 0.61006835 & 19.319334 & 0.53550408 \\
\hline S26C09 & 19.2447697 & 0.61006835 & 19.319334 & 0.53550408 \\
\hline $\mathrm{S} 26 \mathrm{C} 10$ & 19.2447697 & 0.61006835 & 19.319334 & 0.53550408 \\
\hline S26C11 & 19.2447697 & 0.61006835 & 19.319334 & 0.53550408 \\
\hline $\mathrm{S} 26 \mathrm{C} 12$ & 19.2447697 & 0.61006835 & 19.319334 & 0.53550408 \\
\hline $\mathrm{S} 26 \mathrm{C} 13$ & 10.794563 & -22.789638 & 10.9350282 & -22.930103 \\
\hline S26C14 & 10.794563 & -22.789638 & 10.9350282 & -22.930103 \\
\hline $\mathrm{S} 26 \mathrm{C} 15$ & 10.794563 & -22.789638 & 10.9350282 & -22.930103 \\
\hline S26C16 & 10.794563 & -22.789638 & 10.9350282 & -22.930103 \\
\hline $\mathrm{S} 26 \mathrm{C} 17$ & 10.794563 & -22.789638 & 10.9350282 & -22.930103 \\
\hline $\mathrm{S} 26 \mathrm{C} 18$ & 10.794563 & -22.789638 & 10.9350282 & -22.930103 \\
\hline S26C19 & 4.7318303 & 0.11650379 & 4.74597955 & 0.10235453 \\
\hline $\mathrm{S} 26 \mathrm{C} 20$ & 4.7318303 & 0.11650379 & 4.74597955 & 0.10235453 \\
\hline$S 26 C 21$ & 4.7318303 & 0.11650379 & 4.74597955 & 0.10235453 \\
\hline S26C22 & 4.7318303 & 0.11650379 & 4.74597955 & 0.10235453 \\
\hline $\mathrm{S} 26 \mathrm{C} 23$ & 4.7318303 & 0.11650379 & 4.74597955 & 0.10235453 \\
\hline S26C24 & 4.7318303 & 0.11650379 & 4.74597955 & 0.10235453 \\
\hline S26R01 & 17.464202 & -13.616141 & 17.6635564 & -13.815496 \\
\hline S26R02 & 12.8374191 & -21.10132 & 13.0073597 & -21.27126 \\
\hline S26R03 & 9.34313503 & -21.852178 & 9.45745962 & -21.966502 \\
\hline S26R04 & 6.56253519 & -11.556366 & 6.61824307 & -11.612074 \\
\hline S26R05 & 5.35342909 & 3.90647332 & 5.35800276 & 3.90189965 \\
\hline S26R06 & 18.8514523 & 0.77371821 & 18.9228189 & 0.70235167 \\
\hline S26R07 & 22.7696829 & -3.6801225 & 22.9136568 & -3.8240964 \\
\hline S26R08 & 19.4610117 & -10.364952 & 19.6559855 & -10.559925 \\
\hline S26R09 & 14.7451161 & -18.180106 & 14.9337044 & -18.368694 \\
\hline S26R 10 & 11.1531621 & -22.686922 & 11.2994611 & -22.833221 \\
\hline S26R11 & 8.52814816 & -20.093847 & 8.62624784 & -20.191947 \\
\hline S26R 12 & 6.36087088 & -10.375035 & 6.41205163 & -10.426216 \\
\hline S26R 13 & 4.26854197 & 3.10718937 & 4.27215613 & 3.10357521 \\
\hline S26R 14 & 15.3834738 & 1.88967646 & 15.4321444 & 1.84100591 \\
\hline S26R15 & 22.1111247 & -1.3023568 & 22.2202166 & -1.4114487 \\
\hline S26R 16 & 21.8946818 & -6.2117849 & 22.0656796 & -6.3827828 \\
\hline
\end{tabular}




\section{REVISED METHODOLOGY FOR DETERMINING CESIUM-137 CONTENT OF HN-200 GROUT CONTAINERS.}

\begin{tabular}{|c|c|c|c|c|}
\hline \multirow[b]{2}{*}{ Line } & \multicolumn{4}{|c|}{ Source S27 } \\
\hline & X1i & $\mathbf{X 2 i}$ & X10 & $\mathbf{X} 20$ \\
\hline$S 27 C 01$ & 22.7709791 & 0.5329303 & 22.9159135 & 0.3879959 \\
\hline S27C02 & 22.7709791 & 0.5329303 & 22.9159135 & 0.3879959 \\
\hline $\mathrm{S} 27 \mathrm{C} 03$ & 22.7709791 & 0.5329303 & 22.9159135 & 0.3879959 \\
\hline S27C04 & 22.7709791 & 0.5329303 & 22.9159135 & 0.3879959 \\
\hline $\mathrm{S} 27 \mathrm{C} 05$ & 22.7709791 & 0.5329303 & 22.9159135 & 0.3879959 \\
\hline S27C06 & 22.7709791 & 0.5329303 & 22.9159135 & 0.3879959 \\
\hline S27C07 & 2.34924115 & 0.05736459 & 2.35620675 & 0.05039899 \\
\hline S27C08 & 2.34924115 & 0.05736459 & 2.35620675 & 0.0503989 \\
\hline S27C09 & 2.34924115 & 0.05736459 & 2.35620675 & 0.0503989 \\
\hline $\mathrm{S} 27 \mathrm{C} 10$ & 2.34924115 & 0.05736459 & 2.35620675 & 0.0503989 \\
\hline S27C11 & 2.34924115 & 0.05736459 & 2.35620675 & 0.05039899 \\
\hline S27C12 & 2.34924115 & 0.05736459 & 2.35620675 & 0.05039899 \\
\hline S27C13 & 3.95007789 & -22.780251 & 4.09277568 & -22.922949 \\
\hline S27C14 & 3.95007789 & -22.780251 & 4.09277568 & -22.922949 \\
\hline S27C15 & 3.95007789 & -22.780251 & 4.09277568 & -22.922949 \\
\hline $\mathrm{S} 27 \mathrm{C} 16$ & 3.95007789 & -22.780251 & 4.09277568 & -22.922949 \\
\hline S27C17 & 3.95007789 & -22.780251 & 4.09277568 & -22.922949 \\
\hline $\mathrm{S} 27 \mathrm{C} 18$ & 3.95007789 & -22.780251 & 4.09277568 & -22.922949 \\
\hline S27C19 & 22.4300145 & 0.92604577 & 22.5441814 & 0.81187892 \\
\hline $\mathrm{S} 27 \mathrm{C} 20$ & 22.4300145 & 0.92604577 & 22.5441814 & 0.81187892 \\
\hline $\mathrm{S} 27 \mathrm{C} 21$ & 22.4300145 & 0.92604577 & 22.5441814 & 0.81187892 \\
\hline$S 27 C 22$ & 22.4300145 & 0.92604577 & 22.5441814 & 0.81187892 \\
\hline $\mathrm{S} 27 \mathrm{C} 23$ & 22.4300145 & 0.92604577 & 22.5441814 & 0.81187892 \\
\hline S27C24 & 22.4300145 & 0.92604577 & 22.5441814 & 0.81187892 \\
\hline S27R01 & 11.4294418 & -5.1379617 & 11.7945222 & -5.5030422 \\
\hline S27R02 & 20.1563287 & -0.6224832 & 20.3807296 & -0.8468841 \\
\hline S27R03 & 22.4677387 & 0.91159176 & 22.583065 & 0.79626549 \\
\hline S27R04 & 15.2845615 & 1.7106267 & 15.3332319 & 1.66195628 \\
\hline S27R05 & 2.29169199 & 1.66318527 & 2.29361127 & 1.661266 \\
\hline S27R06 & 2.83545071 & -12.637724 & 2.8849424 & -12.687215 \\
\hline S27R07 & 3.47987398 & -21.64295 & 3.58437355 & -21.747449 \\
\hline S27R08 & 4.4878467 & -21.454775 & 4.67134759 & -21.638276 \\
\hline S27R09 & 6.8037272 & -12.715985 & 7.1158567 & -13.028115 \\
\hline S27R 10 & 13.6629649 & -3.4612168 & 14.0035741 & -3.801826 \\
\hline S27R11 & 22.0507398 & 0.07608669 & 22.2291976 & -0.1023711 \\
\hline S27R 12 & 19.3199401 & 1.42086707 & 19.3933132 & 1.34749392 \\
\hline S27R13 & 3.08376704 & 2.24022548 & 3.08635888 & 2.23763364 \\
\hline S27R14 & 2.97962529 & -15.570914 & 3.04161263 & -15.632901 \\
\hline S27R15 & 3.94288065 & -22.784452 & 4.0850099 & -22.926582 \\
\hline S27R16 & 5.86450724 & -15.818431 & 6.13435195 & -16.088276 \\
\hline
\end{tabular}




\section{REVISED METHODOLOGY FOR DETERMINING CESIUM-137 CONTENT OF HN-200 GROUT CONTAINERS.}

\begin{tabular}{|c|c|c|c|c|}
\hline \multirow[b]{2}{*}{ Line } & \multicolumn{4}{|c|}{ Source S28 } \\
\hline & $\mathbf{X 1 i}$ & $\mathbf{X} \mathbf{2} \mathbf{i}$ & X10 & $\mathbf{X} 20$ \\
\hline $\mathrm{S} 28 \mathrm{C} 01$ & 22.7675474 & 1.83371114 & 22.9133176 & 1.68794094 \\
\hline $\mathrm{S} 28 \mathrm{C} 02$ & 22.7675474 & 1.83371114 & 22.9133176 & 1.68794094 \\
\hline $\mathrm{S} 28 \mathrm{CO} 3$ & 22.7675474 & 1.83371114 & 22.9133176 & 1.68794094 \\
\hline $\mathrm{S} 28 \mathrm{C} 04$ & 22.7675474 & 1.83371114 & 22.9133176 & 1.68794094 \\
\hline S28C05 & 22.7675474 & 1.83371114 & 22.9133176 & 1.68794094 \\
\hline S28C06 & 22.7675474 & 1.83371114 & 22.9133176 & 1.68794094 \\
\hline $\mathrm{S} 28 \mathrm{C} 07$ & 20.7539306 & 0.71472893 & 20.8415023 & 0.62715722 \\
\hline S28C08 & 20.7539306 & 0.71472893 & 20.8415023 & 0.62715722 \\
\hline $\mathrm{S} 28 \mathrm{CO}$ & 20.7539306 & 0.71472893 & 20.8415023 & 0.62715722 \\
\hline $\mathrm{S} 28 \mathrm{C} 10$ & 20.7539306 & 0.71472893 & 20.8415023 & 0.62715722 \\
\hline $\mathrm{S} 28 \mathrm{C} 11$ & 20.7539306 & 0.71472893 & 20.8415023 & 0.62715722 \\
\hline $\mathrm{S} 28 \mathrm{C} 12$ & 20.7539306 & 0.71472893 & 20.8415023 & 0.62715722 \\
\hline $\mathrm{S} 28 \mathrm{C} 13$ & 21.9536658 & -22.798789 & 22.0919847 & -22.937108 \\
\hline S28C14 & 21.9536658 & -22.798789 & 22.0919847 & -22.937108 \\
\hline $\mathrm{S} 28 \mathrm{C} 15$ & 21.9536658 & -22.798789 & 22.0919847 & -22.937108 \\
\hline $\mathrm{S} 28 \mathrm{C} 16$ & 21.9536658 & -22.798789 & 22.0919847 & -22.937108 \\
\hline $\mathrm{S} 28 \mathrm{C} 17$ & 21.9536658 & -22.798789 & 22.0919847 & -22.937108 \\
\hline $\mathrm{S} 28 \mathrm{C} 18$ & 21.9536658 & -22.798789 & 22.0919847 & -22.937108 \\
\hline $\mathbf{S 2 8 C 1 9}$ & 22.7580162 & 1.17862528 & 22.9047145 & 1.03192706 \\
\hline $\mathrm{S} 28 \mathrm{C} 20$ & 22.7580162 & 1.17862528 & 22.9047145 & 1.03192706 \\
\hline $\mathbf{S} 28 \mathrm{C} 21$ & 22.7580162 & 1.17862528 & 22.9047145 & 1.03192706 \\
\hline $\mathrm{S} 28 \mathrm{C} 22$ & 22.7580162 & 1.17862528 & 22.9047145 & 1.03192706 \\
\hline $\mathrm{S} 28 \mathrm{C} 23$ & 22.7580162 & 1.17862528 & 22.9047145 & 1.03192706 \\
\hline $\mathrm{S} 28 \mathrm{C} 24$ & 22.7580162 & 1.17862528 & 22.9047145 & 1.03192706 \\
\hline S28R01 & 22.123872 & -21.530821 & 22.2678879 & -21.674837 \\
\hline S28R02 & 22.5304231 & -9.7178837 & 22.6825625 & -9.8700231 \\
\hline S28R03 & 22.7992946 & 5.72303597 & 22.9369274 & 5.58540321 \\
\hline S28R04 & 21.7293497 & 15.5083534 & 21.7946024 & 15.4431007 \\
\hline S28R05 & 19.2295891 & 15.6583549 & 19.2561568 & 15.6317873 \\
\hline S28R06 & 20.5941823 & 3.43699386 & 20.674795 & 3.35638117 \\
\hline S28R07 & 21.2647228 & -10.55812 & 21.3748026 & -10.6682 \\
\hline S28R08 & 21.6996253 & -20.516469 & 21.8281959 & -20.64504 \\
\hline S28R09 & 22.0478261 & -22.401332 & 22.1894028 & -22.542909 \\
\hline S28R10 & 22.3730344 & -15.494611 & 22.5233401 & -15.644917 \\
\hline S28R11 & 22.6862399 & -2.8126308 & 22.836743 & -2.9631339 \\
\hline S28R12 & 22.729714 & 9.78678783 & 22.8510431 & 9.66545866 \\
\hline S28R13 & 20.5123114 & 17.1636047 & 20.5453601 & 17.1305559 \\
\hline S28R14 & 19.9526052 & 11.061454 & 20.0064177 & 11.0076415 \\
\hline S28R15 & 21.061231 & -5.6441038 & 21.1623456 & -5.7452184 \\
\hline S28R16 & 21.6714561 & -20.022847 & 21.7988814 & -20.150272 \\
\hline
\end{tabular}




\section{REVISED METHODOLOGY FOR DETERMINING CESIUM-137 CONTENT OF HN-200 GROUT CONTAINERS.}

\begin{tabular}{|c|c|c|c|c|}
\hline \multirow[b]{2}{*}{ Line } & \multicolumn{4}{|c|}{ Source S29 } \\
\hline & $\mathbf{X 1 i}$ & $\mathbf{X} 2 \mathbf{i}$ & $\mathbf{X 1 0}$ & $\mathbf{X} 20$ \\
\hline$\$ 29 \mathrm{C} 01$ & 22.7986463 & -21.723052 & 22.9369985 & -21.861404 \\
\hline $\mathrm{S} 29 \mathrm{C} 02$ & 22.7986463 & -21.723052 & 22.9369985 & -21.861404 \\
\hline $\mathrm{S} 29 \mathrm{C} 03$ & 22.7986463 & -21.723052 & 22.9369985 & -21.861404 \\
\hline $\mathrm{S} 29 \mathrm{CO} 4$ & 22.7986463 & -21.723052 & 22.9369985 & -21.861404 \\
\hline $\mathrm{S} 29 \mathrm{COS}$ & 22.7986463 & -21.723052 & 22.9369985 & -21.861404 \\
\hline $\mathbf{S} 29 \mathrm{C} 06$ & 22.7986463 & -21.723052 & 22.9369985 & -21.861404 \\
\hline S29C07 & -0.0050675 & -0.2080776 & -0.0044522 & -0.2086929 \\
\hline $\mathrm{S} 29 \mathrm{COB}$ & -0.0050675 & -0.2080776 & -0.0044522 & -0.2086929 \\
\hline S29C09 & -0.0050675 & -0.2080776 & -0.0044522 & -0.2086929 \\
\hline $\mathbf{S} 29 \mathrm{C} 10$ & -0.0050675 & -0.2080776 & -0.0044522 & -0.2086929 \\
\hline$S 29 C 11$ & -0.0050675 & -0.2080776 & -0.0044522 & -0.2086929 \\
\hline $\mathrm{S} 29 \mathrm{C} 12$ & -0.0050675 & -0.2080776 & -0.0044522 & -0.2086929 \\
\hline$S 29 C 13$ & 21.7070512 & -22.798636 & 21.8454058 & -22.936991 \\
\hline $\mathrm{S} 29 \mathrm{C} 14$ & 21.7070512 & -22.798636 & 21.8454058 & -22.936991 \\
\hline$S 29 C 15$ & 21.7070512 & -22.798636 & 21.8454058 & -22.936991 \\
\hline $\mathrm{S} 29 \mathrm{C} 16$ & 21.7070512 & -22.798636 & 21.8454058 & -22.936991 \\
\hline $\mathrm{S} 29 \mathrm{C} 17$ & 21.7070512 & -22.798636 & 21.8454058 & -22.936991 \\
\hline S29C18 & 21.7070512 & -22.798636 & 21.8454058 & -22.936991 \\
\hline$S 29 C 19$ & -0.0037289 & -0.1531151 & -0.0032762 & -0.1535679 \\
\hline$S 29 C 20$ & -0.0037289 & -0.1531151 & -0.0032762 & -0.1535679 \\
\hline $\mathrm{S} 29 \mathrm{C} 21$ & -0.0037289 & -0.1531151 & -0.0032762 & -0.1535679 \\
\hline $\mathrm{S} 29 \mathrm{C} 22$ & -0.0037289 & -0.1531151 & -0.0032762 & -0.1535679 \\
\hline$S 29 C 23$ & -0.0037289 & -0.1531151 & -0.0032762 & -0.1535679 \\
\hline S29C24 & -0.0037289 & -0.1531151 & -0.0032762 & -0.1535679 \\
\hline S29R01 & 22.5923438 & -22.407037 & 22.7322823 & -22.546975 \\
\hline S29R02 & 19.7886933 & -22.261353 & 19.9190813 & -22.391741 \\
\hline S29R03 & 14.4437639 & -18.170816 & 14.5439995 & -18.271052 \\
\hline S29R04 & 7.53633392 & -10.318905 & 7.59068802 & -10.373259 \\
\hline S29R05 & -0.0779305 & -0.1075088 & -0.0778408 & -0.1075985 \\
\hline S29R06 & 10.1421886 & -7.6715498 & 10.1963657 & -7.7257269 \\
\hline S29R07 & 18.0804962 & -14.527288 & 18.1805805 & -14.627373 \\
\hline S29R08 & 22.2445965 & -19.816456 & 22.3749044 & -19.946764 \\
\hline S29R09 & 22.4060398 & -22.591816 & 22.5459828 & -22.731759 \\
\hline S29R 10 & 19.2616726 & -21.985466 & 19.3894648 & -22.113258 \\
\hline S29R11 & 13.8566667 & -17.590568 & 13.9532226 & -17.687124 \\
\hline S29R12 & 7.15526856 & -9.8339917 & 7.20696621 & -9.8856894 \\
\hline S29R 13 & -0.0740572 & -0.1021654 & -0.073972 & -0.1022506 \\
\hline S29R14 & 9.70938781 & -7.3355608 & 9.76121041 & -7.3873834 \\
\hline S29R15 & 17.5215944 & -13.974572 & 17.6181793 & -14.071157 \\
\hline S29R16 & 21.9690915 & -19.306804 & 22.0968521 & -19.434565 \\
\hline
\end{tabular}

Source S30 


\section{REVISED METHODOLOGY FOR DETERMINING CESIUM-137 CONTENT} OF HN-200 GROUT CONTAINERS.

\begin{tabular}{|c|c|c|c|c|}
\hline Line & $\mathbf{X 1 i}$ & $\mathbf{X} \mathbf{2} \mathbf{i}$ & X10 & $\mathbf{X} 20$ \\
\hline S30C01 & 22.7555477 & 5.39108155 & 22.9042751 & 5.24235418 \\
\hline $\mathrm{S} 30 \mathrm{C} 02$ & 22.7555477 & 5.39108155 & 22.9042751 & 5.24235418 \\
\hline S30C03 & 22.7555477 & 5.39108155 & 22.9042751 & 5.24235418 \\
\hline S30C04 & 22.7555477 & 5.39108155 & 22.9042751 & 5.24235418 \\
\hline $\mathrm{S} 30 \mathrm{C} 05$ & 22.7555477 & 5.39108155 & 22.9042751 & 5.24235418 \\
\hline$\$ 30 \mathrm{C06}$ & 22.7555477 & 5.39108155 & 22.9042751 & 5.24235418 \\
\hline S30C07 & 7.65795027 & 0.19207327 & 7.68128698 & 0.16873657 \\
\hline S30C08 & 7.65795027 & 0.19207327 & 7.68128698 & 0.16873657 \\
\hline S30C09 & 7.65795027 & 0.19207327 & 7.68128698 & 0.16873657 \\
\hline S30C10 & 7.65795027 & 0.19207327 & 7.68128698 & 0.16873657 \\
\hline S30C11 & 7.65795027 & 0.19207327 & 7.68128698 & 0.16873657 \\
\hline $\mathrm{S} 30 \mathrm{C} 12$ & 7.65795027 & 0.19207327 & 7.68128698 & 0.16873657 \\
\hline S30C13 & 9.12610067 & -22.78772 & 9.26701941 & -22.928639 \\
\hline$S 30 C 14$ & 9.12610067 & -22.78772 & 9.26701941 & -22.928639 \\
\hline S30C15 & 9.12610067 & -22.78772 & 9.26701941 & -22.928639 \\
\hline S30C16 & 9.12610067 & -22.78772 & 9.26701941 & -22.928639 \\
\hline $\mathrm{S} 30 \mathrm{C} 17$ & 9.12610067 & -22.78772 & 9.26701941 & -22.928639 \\
\hline S30C18 & 9.12610067 & -22.78772 & 9.26701941 & -22.928639 \\
\hline S30C19 & 18.2570517 & 2.21215809 & 18.550768 & 1.91844184 \\
\hline$S 30 C 20$ & 18.2570517 & 2.21215809 & 18.550768 & 1.91844184 \\
\hline$S 30 C 21$ & 18.2570517 & 2.21215809 & 18.550768 & 1.91844184 \\
\hline S30C22 & 18.2570517 & 2.21215809 & 18.550768 & 1.91844184 \\
\hline $530 C 23$ & 18.2570517 & 2.21215809 & 18.550768 & 1.91844184 \\
\hline S30C24 & 18.2570517 & 2.21215809 & 18.550768 & 1.91844184 \\
\hline S30R01 & 12.5588057 & -6.5964327 & 12.8766291 & -6.9142561 \\
\hline S30R02 & 18.7287982 & 2.57143089 & 19.0121414 & 2.28808772 \\
\hline S30R03 & 22.799026 & 5.57251464 & 22.9356913 & 5.43584935 \\
\hline S30R04 & 17.9294193 & 6.75249592 & 17.9792711 & 6.70264408 \\
\hline S30R05 & 7.45287974 & 5.47374805 & 7.45940172 & 5.46722608 \\
\hline S30R06 & 8.01088324 & -9.2363822 & 8.06320013 & -9.2886991 \\
\hline S30R07 & 8.57527323 & -20.076074 & 8.67333653 & -20.174138 \\
\hline S30R08 & 9.30156948 & -22.47342 & 9.45558207 & -22.627433 \\
\hline S30R09 & 10.5621965 & -15.43103 & 10.7990684 & -15.667902 \\
\hline S30R 10 & 13.7458098 & -3.5515514 & 14.083093 & -3.8888346 \\
\hline S30R 11 & 20.5244052 & 3.77113101 & 20.7625847 & 3.53295145 \\
\hline S30R 12 & 21.8714572 & 6.17838643 & 21.9651934 & 6.08465027 \\
\hline S30R13 & 9.79034086 & 7.2637554 & 9.79924457 & 7.25485169 \\
\hline S30R 14 & 8.02804197 & -9.6705873 & 8.08176473 & -9.7243101 \\
\hline S30R15 & 8.80721376 & -22.062159 & 8.9236067 & -22.178552 \\
\hline S30R16 & 9.95859721 & -19.161419 & 10.1585032 & -19.361325 \\
\hline
\end{tabular}

Source S31

Line

$\mathbf{X 1 i} \quad \mathbf{X 2 i}$

X10

$\mathbf{X} 20$ 


\section{REVISED METHODOLOGY FOR DETERMINING CESIUM-137 CONTENT OF HN-200 GROUT CONTAINERS.}

\begin{tabular}{|c|c|c|c|c|}
\hline S31C01 & 22.7713305 & 0.39054605 & 22.9161796 & 0.245697 \\
\hline S31C02 & 22.7713305 & 0.39054605 & 22.9161796 & 0.245697 \\
\hline S31C03 & 22.7713305 & 0.39054605 & 22.9161796 & 0.245697 \\
\hline S31C04 & 22.7713305 & 0.39054605 & 22.9161796 & 0.245697 \\
\hline S31C05 & 22.7713305 & 0.39054605 & 22.9161796 & 0.245697 \\
\hline S31C06 & 22.7713305 & 0.39054605 & 22.9161796 & 0.245697 \\
\hline S31C07 & 6.11275342 & 0.15164063 & 6.13117307 & 0.13322097 \\
\hline S31C08 & 6.11275342 & 0.15164063 & 6.13117307 & 0.13322097 \\
\hline S31C09 & 6.11275342 & 0.15164063 & 6.13117307 & 0.13322097 \\
\hline S31C10 & 6.11275342 & 0.15164063 & 6.13117307 & 0.13322097 \\
\hline S31C11 & 6.11275342 & 0.15164063 & 6.13117307 & 0.13322097 \\
\hline $\mathrm{S} 31 \mathrm{C} 12$ & 6.11275342 & 0.15164063 & 6.13117307 & 0.13322097 \\
\hline S31C13 & 10.0138115 & -22.788765 & 10.154483 & -22.929436 \\
\hline S31C14 & 10.0138115 & -22.788765 & 10.154483 & -22.929436 \\
\hline S31C15 & 10.0138115 & -22.788765 & 10.154483 & -22.929436 \\
\hline S31C16 & 10.0138115 & -22.788765 & 10.154483 & -22.929436 \\
\hline S31C17 & 10.0138115 & -22.788765 & 10.154483 & -22.929436 \\
\hline S31C18 & 10.0138115 & -22.788765 & 10.154483 & -22.929436 \\
\hline S31C19 & 22.7660945 & 1.05154172 & 22.8963104 & 0.92132587 \\
\hline S31C20 & 22.7660945 & 1.05154172 & 22.8963104 & 0.92132587 \\
\hline S31C21 & 22.7660945 & 1.05154172 & 22.8963104 & 0.92132587 \\
\hline S31C22 & 22.7660945 & 1.05154172 & 22.8963104 & 0.92132587 \\
\hline S31C23 & 22.7660945 & 1.05154172 & 22.8963104 & 0.92132587 \\
\hline S31C24 & 22.7660945 & 1.05154172 & 22.8963104 & 0.92132587 \\
\hline S31R01 & 15.7632537 & -11.04646 & 15.9931584 & -11.276365 \\
\hline S31R02 & 20.9624688 & -2.8621415 & 21.1605388 & -3.0602115 \\
\hline S31R03 & 22.5511725 & 1.57930143 & 22.6687664 & 1.46170747 \\
\hline S31R04 & 16.5390932 & 4.03383928 & 16.5879831 & 3.98494933 \\
\hline S31R05 & 5.70533407 & 4.16710418 & 5.71022497 & 4.16221328 \\
\hline S31R06 & 7.11254608 & -9.8634177 & 7.16421454 & -9.9150861 \\
\hline S31R07 & 8.55355142 & -20.084275 & 8.65163144 & -20.182355 \\
\hline S31R08 & 10.3461522 & -22.606673 & 10.4956216 & -22.756142 \\
\hline S31R09 & 13.0540313 & -17.100776 & 13.2592796 & -17.306024 \\
\hline S31R10 & 17.5241668 & -7.8747114 & 17.7542472 & -8.1047918 \\
\hline S31R11 & 22.3069829 & -0.8320726 & 22.4754288 & -1.0005186 \\
\hline S31R12 & 20.3560922 & 3.02397724 & 20.4350849 & 2.94498452 \\
\hline S31R13 & 7.30660894 & 5.36348977 & 7.31299031 & 5.3571084 \\
\hline S31R14 & 7.20724109 & -10.744028 & 7.26203716 & -10.798824 \\
\hline S31R15 & 9.11664815 & -21.944459 & 9.23183161 & -22.059642 \\
\hline S31R16 & 11.6800423 & -20.376444 & 11.860735 & -20.557136 \\
\hline
\end{tabular}

Source S32

$\begin{array}{ccccc}\text { Line } & \text { X1i } & \text { X2i } & \text { X1o } & \text { X2o } \\ \text { S32C01 } & 22.784051 & -6.3549014 & 22.9258412 & -6.4966916\end{array}$




\section{REVISED METHODOLOGY FOR DETERMINING CESIUM-137 CONTENT OF HN-200 GROUT CONTAINERS.}

$\begin{array}{lll}\text { S32C02 } & 22.784051 & -6.3549014 \\ \text { S32C03 } & 22.784051 & -6.3549014 \\ \text { S32C04 } & 22.784051 & -6.3549014 \\ \text { S32C05 } & 22.784051 & -6.3549014 \\ \text { S32C06 } & 22.784051 & -6.3549014 \\ \text { S32C07 } & -0.1748783 & -7.0071792 \\ \text { S32C08 } & -0.1748783 & -7.0071792 \\ \text { S32C09 } & -0.1748783 & -7.0071792 \\ \text { S32C10 } & -0.1748783 & -7.0071792 \\ \text { S32C11 } & -0.1748783 & -7.0071792 \\ \text { S32C12 } & -0.1748783 & -7.0071792 \\ \text { S32C13 } & 4.66042651 & -22.781443 \\ \text { S32C14 } & 4.66042651 & -22.781443 \\ \text { S32C15 } & 4.66042651 & -22.781443 \\ \text { S32C16 } & 4.66042651 & -22.781443 \\ \text { S32C17 } & 4.66042651 & -22.781443 \\ \text { S32C18 } & 4.66042651 & -22.781443 \\ \text { S32C19 } & -0.0249106 & -1.0223664 \\ \text { S32C20 } & -0.0249106 & -1.0223664 \\ \text { S32C21 } & -0.0249106 & -1.0223664 \\ \text { S32C22 } & -0.0249106 & -1.0223664 \\ \text { S32C23 } & -0.0249106 & -1.0223664 \\ \text { S32C24 } & -0.0249106 & -1.0223664 \\ \text { S32R01 } & 16.4390019 & -12.048926 \\ \text { S32R02 } & 9.33947414 & -18.69164 \\ \text { S32R03 } & 4.47187561 & -22.798903 \\ \text { S32R04 } & 1.4179155 & -17.052056 \\ \text { S32R05 } & -0.8817222 & -1.2159702 \\ \text { S32R06 } & 15.4536743 & -3.0962861 \\ \text { S32R07 } & 22.7385113 & -5.8294882 \\ \text { S32R08 } & 19.1725942 & -9.9735822 \\ \text { S32R09 } & 11.7097469 & -16.21215 \\ \text { S32R10 } & 6.23738024 & -21.900583 \\ \text { S32R11 } & 2.98508838 & -21.759527 \\ \text { S32R12 } & 0.81077585 & -13.850086 \\ \text { S32R13 } & -0.6847905 & -0.9445112 \\ \text { S32R14 } & 12.7500829 & -2.6518203 \\ \text { S32R15 } & 21.3435167 & -4.6485139 \\ \text { S32R16 } & 22.1618643 & -7.4640759\end{array}$

\begin{tabular}{ll}
22.9258412 & -6.4966916 \\
22.9258412 & -6.4966916 \\
22.9258412 & -6.4966916 \\
22.9258412 & -6.4966916 \\
22.9258412 & -6.4966916 \\
-0.1536331 & -7.0284244 \\
-0.1536331 & -7.0284244 \\
-0.1536331 & -7.0284244 \\
-0.1536331 & -7.0284244 \\
-0.1536331 & -7.0284244 \\
-0.1536331 & -7.0284244 \\
4.80283913 & -22.923855 \\
4.80283913 & -22.923855 \\
4.80283913 & -22.923855 \\
4.80283913 & -22.923855 \\
4.80283913 & -22.923855 \\
4.80283913 & -22.923855 \\
-0.0218859 & -1.025391 \\
-0.0218859 & -1.025391 \\
-0.0218859 & -1.025391 \\
-0.0218859 & -1.025391 \\
-0.0218859 & -1.025391 \\
-0.0218859 & -1.025391 \\
16.6558217 & -12.265745 \\
9.5474359 & -18.899602 \\
4.61046256 & -22.93749 \\
1.48339172 & -17.117533 \\
-0.8807071 & -1.2169854 \\
15.5154214 & -3.1580332 \\
22.8689494 & -5.9599263 \\
19.3723135 & -10.173301 \\
11.9308066 & -16.43321 \\
6.40821133 & -22.071415 \\
3.09051747 & -21.864956 \\
0.85971225 & -13.899022 \\
-0.6840024 & -0.9452993 \\
12.799512 & -2.7012494 \\
21.4460576 & -4.7510548 \\
22.3252565 & -7.6274682 \\
& \\
\hline
\end{tabular}

Source \$33

$\begin{array}{ccccc}\text { Line } & \mathbf{X 1 i} & \mathbf{X 2 i} & \mathbf{X 1 0} & \mathbf{X 2 0} \\ \text { S33C01 } & 22.7790246 & -3.2562767 & 22.9220165 & -3.3992686 \\ \text { S33C02 } & 22.7790246 & -3.2562767 & 22.9220165 & -3.3992686\end{array}$




\section{REVISED METHODOLOGY FOR DETERMINING CESIUM-137 CONTENT OF HN-200 GROUT CONTAINERS.}

\begin{tabular}{|c|c|c|c|c|}
\hline $\mathrm{S} 33 \mathrm{C} 03$ & 22.7790246 & -3.2562767 & 22.9220165 & -3.3992686 \\
\hline S33C04 & 22.7790246 & -3.2562767 & 22.9220165 & -3.3992686 \\
\hline S33C05 & 22.7790246 & -3.2562767 & 22.9220165 & -3.3992686 \\
\hline S33C06 & 22.7790246 & -3.2562767 & 22.9220165 & -3.3992686 \\
\hline S33C07 & -0.0689584 & -2.8207026 & -0.0605848 & -2.8290762 \\
\hline $\mathrm{S} 33 \mathrm{CO}$ & -0.0689584 & -2.8207026 & -0.0605848 & -2.8290762 \\
\hline S33C09 & -0.0689584 & -2.8207026 & -0.0605848 & -2.8290762 \\
\hline S33C10 & -0.0689584 & -2.8207026 & -0.0605848 & -2.8290762 \\
\hline S33C11 & -0.0689584 & -2.8207026 & -0.0605848 & -2.8290762 \\
\hline $\mathrm{S} 33 \mathrm{C} 12$ & -0.0689584 & -2.8207026 & -0.0605848 & -2.8290762 \\
\hline $\mathrm{S} 33 \mathrm{C} 13$ & -2.3180156 & -22.766159 & -2.1719059 & -22.912268 \\
\hline $\mathrm{S} 33 \mathrm{C} 14$ & -2.3180156 & -22.766159 & -2.1719059 & -22.912268 \\
\hline S33C15 & -2.3180156 & -22.766159 & -2.1719059 & -22.912268 \\
\hline $\mathrm{S} 33 \mathrm{C} 16$ & -2.3180156 & -22.766159 & -2.1719059 & -22.912268 \\
\hline $\mathrm{S} 33 \mathrm{C} 17$ & -2.3180156 & -22.766159 & -2.1719059 & -22.912268 \\
\hline $\mathrm{S} 33 \mathrm{C} 18$ & -2.3180156 & -22.766159 & -2.1719059 & -22.912268 \\
\hline$S 33 C 19$ & -1.9434092 & -19.623947 & -1.690719 & -19.876637 \\
\hline $\mathrm{S} 33 \mathrm{C} 20$ & -1.9434092 & -19.623947 & -1.690719 & -19.876637 \\
\hline S33C21 & -1.9434092 & -19.623947 & -1.690719 & -19.876637 \\
\hline S33C22 & -1.9434092 & -19.623947 & -1.690719 & -19.876637 \\
\hline $\mathrm{S} 33 \mathrm{C} 23$ & -1.9434092 & -19.623947 & -1.690719 & -19.876637 \\
\hline S33C24 & -1.9434092 & -19.623947 & -1.690719 & -19.876637 \\
\hline S33R01 & 10.7119588 & -4.2387444 & 11.1143342 & -4.6411197 \\
\hline S33R02 & 21.1410719 & -3.4234534 & 21.3337546 & -3.6161361 \\
\hline S33R03 & 21.7255311 & -3.1320457 & 21.8306776 & -3.2371922 \\
\hline S33R04 & 12.565847 & -2.9524924 & 12.6153798 & -3.0020253 \\
\hline S33R05 & -2.0343434 & -2.8014348 & -2.0319921 & -2.803786 \\
\hline S33R06 & -2.6376542 & -15.792613 & -2.5889539 & -15.841314 \\
\hline S33R07 & -2.402857 & -22.638205 & -2.2821685 & -22.758893 \\
\hline S33R08 & -1.8874259 & -19.063928 & -1.6197649 & -19.331589 \\
\hline S33R09 & 0.97504422 & -7.9618837 & 1.61867511 & -8.6055146 \\
\hline S33R 10 & 14.0708364 & -3.9073286 & 14.3956268 & -4.232119 \\
\hline S33R 11 & 22.7367385 & -3.2694951 & 22.8837159 & -3.4164725 \\
\hline S33R 12 & 15.5581132 & -2.992407 & 15.6200741 & -3.0543678 \\
\hline S33R 13 & -2.7835725 & -3.8271517 & -2.7803419 & -3.8303823 \\
\hline S33R 14 & -2.5454283 & -20.065405 & -2.4682191 & -20.142614 \\
\hline S33R15 & -2.1098425 & -21.302951 & -1.9032215 & -21.509572 \\
\hline S33R 16 & -0.2387175 & -10.17834 & 0.32974735 & -10.746805 \\
\hline
\end{tabular}

\begin{tabular}{|c|c|c|c|c|}
\hline & \multicolumn{4}{|c|}{ Source S34 } \\
\hline Line & $\mathbf{X 1 i}$ & $\mathbf{X} 2 \mathbf{i}$ & X10 & $\mathbf{X 2 0}$ \\
\hline $\mathrm{S} 34 \mathrm{C} 01$ & 22.7936002 & -14.861025 & 22.9331325 & -15.000557 \\
\hline $\mathrm{S} 34 \mathrm{C} 02$ & 22.7936002 & -14.861025 & 22.9331325 & -15.000557 \\
\hline $\mathrm{S} 34 \mathrm{C} 03$ & 22.7936002 & -14.861025 & 22.9331325 & -15.000557 \\
\hline
\end{tabular}


REVISED METHODOLOGY FOR DETERMINING CESIUM-137 CONTENT OF HN-200 GROUT CONTAINERS.

\begin{tabular}{lll} 
S34C04 & 22.7936002 & -14.861025 \\
S34C05 & 22.7936002 & -14.861025 \\
S34C06 & 22.7936002 & -14.861025 \\
S34C07 & -2.615162 & -16.366995 \\
S34C08 & -2.615162 & -16.366995 \\
S34C09 & -2.615162 & -16.366995 \\
S34C10 & -2.615162 & -16.366995 \\
S34C11 & -2.615162 & -16.366995 \\
S34C12 & -2.615162 & -16.366995 \\
S34C13 & -13.209038 & -22.697984 \\
S34C14 & -13.209038 & -22.697984 \\
S34C15 & -13.209038 & -22.697984 \\
S34C16 & -13.209038 & -22.697984 \\
S34C17 & -13.209038 & -22.697984 \\
S34C18 & -13.209038 & -22.697984 \\
S34C19 & -0.3946327 & -14.388284 \\
S34C20 & -0.3946327 & -14.388284 \\
S34C21 & -0.3946327 & -14.388284 \\
S34C22 & -0.3946327 & -14.388284 \\
S34C23 & -0.3946327 & -14.388284 \\
S34C24 & -0.3946327 & -14.388284 \\
S34R01 & 18.4070519 & -15.109156 \\
S34R02 & 5.67182216 & -15.649756 \\
S34R03 & -8.294351 & -17.699225 \\
S34R04 & -13.150703 & -22.629451 \\
S34R05 & -14.028005 & -17.663757 \\
S34R06 & -0.1255089 & -14.392037 \\
S34R07 & 17.9411589 & -14.654105 \\
S34R08 & 22.1618802 & -14.937528 \\
S34R09 & 10.5332867 & -15.411076 \\
S34R10 & -5.7698151 & -16.922651 \\
S34R11 & -12.686928 & -21.85466 \\
S34R12 & -13.824898 & -21.105829 \\
S34R13 & -10.867671 & -14.216254 \\
S34R14 & 4.06142705 & -14.448759 \\
S34R15 & 17.1191758 & -14.638369 \\
S34R16 & 22.7714908 & -14.837213 \\
& & \\
\hline
\end{tabular}
$22.9331325-15.000557$
$22.9331325-15.000557$
$22.9331325-15.000557$
$-2.2547755-16.727381$
$-2.2547755-16.727381$
$-2.2547755-16.727381$
$-2.2547755-16.727381$
$-2.2547755-16.727381$
$-2.2547755-16.727381$
$\begin{array}{ll}-13.045342 & -22.861681\end{array}$
$\begin{array}{ll}-13.045342 & -22.861681\end{array}$
$\begin{array}{ll}-13.045342 & -22.861681\end{array}$
$\begin{array}{ll}-13.045342 & -22.861681\end{array}$
$\begin{array}{ll}-13.045342 & -22.861681\end{array}$
$\begin{array}{ll}-13.045342 & -22.861681\end{array}$
$\begin{array}{ll}-0.3465843 & -14.436333\end{array}$
$\begin{array}{ll}-0.3465843 & -14.436333\end{array}$
$-0.3465843-14.436333$
$-0.3465843-14.436333$
$-0.3465843-14.436333$
$-0.3465843 \quad-14.436333$
$\begin{array}{ll}18.592441 & -15.294545\end{array}$
$5.94623057-15.924165$
$\begin{array}{ll}-7.8746357 & -18.11894\end{array}$
$\begin{array}{ll}-12.978125 & -22.802028\end{array}$
$\begin{array}{ll}-14.006497 & -17.685265\end{array}$
$-0.0767279-14.440818$
$18.0410185-14.753965$
$22.3157933-15.091441$
$10.7705892-15.648378$
$\begin{array}{ll}-5.3702692 & -17.322197\end{array}$
$\begin{array}{ll}-12.450577 & -22.091011\end{array}$
$-13.765513 \quad-21.165214$
$-10.853088-14.230837$
$4.12128529-14.508618$
$17.2159843-14.735177$
$22.9065103-14.972232$

\begin{tabular}{|c|c|c|c|c|}
\hline \multirow[b]{2}{*}{ Line } & \multicolumn{4}{|c|}{ Source S35 } \\
\hline & $\mathbf{X 1 i}$ & $\mathbf{X}_{2 \mathbf{i}}$ & X1o & X2o \\
\hline $\mathrm{S} 35 \mathrm{C} 01$ & 22.6475534 & 15.9215159 & 22.8255141 & 15.743555 \\
\hline $\mathrm{S} 35 \mathrm{C} 02$ & 22.6475534 & 15.9215159 & 22.8255141 & 15.743555 \\
\hline S35C03 & 22.6475534 & 15.9215159 & 22.8255141 & 15.743555 \\
\hline S35C04 & 22.6475534 & 15.9215159 & 22.8255141 & 15.743555 \\
\hline
\end{tabular}


REVISED METHODOLOGY FOR DETERMINING CESIUM-137 CONTENT OF HN-200 GROUT CONTAINERS.

\begin{tabular}{|c|c|c|c|c|}
\hline S35C05 & 22.6475534 & 15.9215159 & 22.8255141 & 15.7435551 \\
\hline S35C06 & 22.6475534 & 15.9215159 & 22.8255141 & 15.7435551 \\
\hline S35C07 & 19.6477607 & 1.93879367 & 19.8997668 & 1.68678757 \\
\hline S35C08 & 19.6477607 & 1.93879367 & 19.8997668 & 1.68678757 \\
\hline S35C09 & 19.6477607 & 1.93879367 & 19.8997668 & 1.68678757 \\
\hline $\mathrm{S} 35 \mathrm{C} 10$ & 19.6477607 & 1.93879367 & 19.8997668 & 1.68678757 \\
\hline S35C11 & 19.6477607 & 1.93879367 & 19.8997668 & 1.68678757 \\
\hline S35C12 & 19.6477607 & 1.93879367 & 19.8997668 & 1.68678757 \\
\hline S35C13 & 18.6302008 & -22.796579 & 18.7690355 & -22.935413 \\
\hline S35C14 & 18.6302008 & -22.796579 & 18.7690355 & -22.935413 \\
\hline S35C15 & 18.6302008 & -22.796579 & 18.7690355 & -22.935413 \\
\hline S35C16 & 18.6302008 & -22.796579 & 18.7690355 & -22.935413 \\
\hline $\mathrm{S} 35 \mathrm{C} 17$ & 18.6302008 & -22.796579 & 18.7690355 & -22.935413 \\
\hline S35C18 & 18.6302008 & -22.796579 & 18.7690355 & -22.935413 \\
\hline $\mathrm{S} 35 \mathrm{C} 19$ & 18.1163055 & 0.54896429 & 18.1833172 & 0.48195262 \\
\hline S35C20 & 18.1163055 & 0.54896429 & 18.1833172 & 0.48195262 \\
\hline S35C21 & 18.1163055 & 0.54896429 & 18.1833172 & 0.48195262 \\
\hline S35C22 & 18.1163055 & 0.54896429 & 18.1833172 & 0.48195262 \\
\hline S35C23 & 18.1163055 & 0.54896429 & 18.1833172 & 0.48195262 \\
\hline S35C24 & 18.1163055 & 0.54896429 & 18.1833172 & 0.48195262 \\
\hline S35R01 & 18.9336427 & -15.965563 & 19.1119677 & -16.143888 \\
\hline S35R02 & 18.5981916 & -22.717563 & 18.7326799 & -22.852051 \\
\hline S35R03 & 18.3243692 & -12.520856 & 18.4208048 & -12.617291 \\
\hline S35R04 & 17.9952233 & 6.88537815 & 18.045146 & 6.83545546 \\
\hline S35R05 & 20.675064 & 17.3712982 & 20.7092208 & 17.3371415 \\
\hline S35R06 & 22.1551628 & 15.0059983 & 22.3856151 & 14.7755461 \\
\hline S35R07 & 19.87782 & 4.9632822 & 20.1459721 & 4.6951301 \\
\hline S35R08 & 19.1202684 & -9.9035279 & 19.3208637 & -10.104123 \\
\hline S35R09 & 18.7807886 & -20.595647 & 18.9396517 & -20.75451 \\
\hline S35R10 & 18.5538567 & -22.211902 & 18.6822816 & -22.340327 \\
\hline S35R I1 & 18.3536316 & -14.271386 & 18.4541875 & -14.371942 \\
\hline S35R 12 & 18.1283053 & -0.1558042 & 18.1970149 & -0.2245137 \\
\hline S35R 13 & 17.7938347 & 14.1572777 & 17.8156937 & 14.1354187 \\
\hline S35R14 & 22.2213984 & 17.0420003 & 22.2953063 & 16.9680924 \\
\hline S35R 15 & 21.5209144 & 13.6671177 & 21.7925641 & 13.395468 \\
\hline S35R16 & 19.5752774 & 0.77202174 & 19.8213657 & 0.52593347 \\
\hline
\end{tabular}

TABLE 8

Determine the coordinates $(\mathbf{X}, \mathbf{Z}, \mathbf{Y})$ where the line from each source to each measurement point intercepts the inner and outer radius of the cylinder.

Source S01

$\begin{array}{cccc}\text { Line } & \text { XINTi } & \text { ZINTi } & \text { YINTi } \\ \text { S01C01 } & 22.7988391 & 0.14497214 & 43.6204096 \\ \text { S01C02 } & 22.7988391 & 0.14497214 & 36.8629491 \\ \text { S01C03 } & 22.7988391 & 0.14497214 & 30.1054886 \\ \text { S01C04 } & 22.7988391 & 0.14497214 & 23.3480281 \\ \text { S01C05 } & 22.7988391 & 0.14497214 & 16.5905676\end{array}$

$\begin{array}{ccc}\text { XINTo } & \text { ZINTo } & \text { YINTo } \\ 22.9371464 & 0.12736312 & 44.3345734 \\ 22.9371464 & 0.12736312 & 37.3047231 \\ 22.9371464 & 0.12736312 & 30.2748728 \\ 22.9371464 & 0.12736312 & 23.2450225 \\ 22.9371464 & 0.12736312 & 16.2151722\end{array}$




\section{REVISED METHODOLOGY FOR DETERMINING CESIUM-137 CONTENT OF HN-200 GROUT CONTAINERS.}

$\begin{array}{llll}\text { S01C06 } & 22.7988391 & 0.14497214 & 9.83310714 \\ \text { S01C07 } & 0.96064256 & 22.7790528 & 48.3296031 \\ \text { S01C08 } & 0.96064256 & 22.7790528 & 39.7760052 \\ \text { S01C09 } & 0.96064256 & 22.7790528 & 31.2224072 \\ \text { S01C10 } & 0.96064256 & 22.7790528 & 22.6688093 \\ \text { S01C11 } & 0.96064256 & 22.7790528 & 14.1152114 \\ \text { S01C12 } & 0.96064256 & 22.7790528 & 5.56161342 \\ \text { S01C13 } & -22.799295 & 0.01529211 & 48.8798026 \\ \text { S01C14 } & -22.799295 & 0.01529211 & 40.1163526 \\ \text { S01C15 } & -22.799295 & 0.01529211 & 31.3529026 \\ \text { S01C16 } & -22.799295 & 0.01529211 & 22.5894526 \\ \text { S01C17 } & -22.799295 & 0.01529211 & 13.8260026 \\ \text { S01C18 } & -22.799295 & 0.01529211 & 5.06255264 \\ \text { S01C19 } & 0.91352136 & -22.780991 & 48.3870131 \\ \text { S01C20 } & 0.91352136 & -22.780991 & 39.8115184 \\ \text { S01C21 } & 0.91352136 & -22.780991 & 31.2360236 \\ \text { S01C22 } & 0.91352136 & -22.780991 & 22.6605289 \\ \text { S01C23 } & 0.91352136 & -22.780991 & 14.0850342 \\ \text { S01C24 } & 0.91352136 & -22.780991 & 5.50953942 \\ \text { S01R01 } & 22.792281 & 0.56569342 & 25.9268599 \\ \text { S01R02 } & 22.694871 & 2.17965866 & 25.9283934 \\ \text { S01R03 } & 22.390261 & 4.29933635 & 25.9347164 \\ \text { S01R04 } & 21.3563984 & 7.98200035 & 25.9510491 \\ \text { S01R05 } & 17.4506411 & 14.6725324 & 25.9871962 \\ \text { S01R06 } & 7.58244723 & 21.5015017 & 26.0381046 \\ \text { S01R07 } & -6.2228972 & 21.9336187 & 26.0832804 \\ \text { S01R08 } & -18.034248 & 13.9489774 & 26.1125085 \\ \text { S01R09 } & -22.795232 & 0.43067568 & 26.1233241 \\ \text { S01R10 } & -18.546797 & -13.25988 & 26.1153741 \\ \text { S01R11 } & -7.0214122 & -21.691193 & 26.0888933 \\ \text { S01R12 } & 6.91019269 & -21.72688 & 26.0457886 \\ \text { S01R13 } & 17.2824724 & -14.870246 & 25.9945472 \\ \text { S01R14 } & 21.5248004 & -7.5160526 & 25.9550842 \\ \text { S01R15 } & 22.5480961 & -3.3751214 & 25.9363519 \\ \text { S01R16 } & 22.7732846 & -1.0888468 & 25.9289825 \\ & & & \\ \text { S0 } & & & \end{array}$

\begin{abstract}
$\begin{array}{lll}22.9371464 & 0.12736312 & 9.18532191\end{array}$
$\begin{array}{lll}0.84207215 & 22.9220379 & 48.4740631\end{array}$

$\begin{array}{llll}0.84207215 & 22.9220379 & 39.8653666\end{array}$

$\begin{array}{lll}0.84207215 & 22.9220379 & 31.25667\end{array}$

$\begin{array}{llll}0.84207215 & 22.9220379 & 22.6479735\end{array}$

$\begin{array}{llll}0.84207215 & 22.9220379 & 14.0392769\end{array}$

$0.84207215 \quad 22.92203795 .43058036$

$\begin{array}{llll}-22.937496 & 0.01343534 & 48.9551072\end{array}$

$\begin{array}{lll}-22.937496 & 0.01343534 & 40.1629352\end{array}$

$\begin{array}{llll}-22.937496 & 0.01343534 & 31.3707632\end{array}$

$\begin{array}{llll}-22.937496 & 0.01343534 & 22.5785913\end{array}$

$\begin{array}{llll}-22.937496 & 0.01343534 & 13.7864193\end{array}$

$\begin{array}{llll}-22.937496 & 0.01343534 & 4.99424733\end{array}$

$\begin{array}{llll}0.80094493 & -22.923512 & 48.5241704\end{array}$

$\begin{array}{llll}0.80094493 & -22.923512 & 39.8963624\end{array}$

$\begin{array}{llll}0.80094493 & -22.923512 & 31.2685544\end{array}$

$\begin{array}{llll}0.80094493 & -22.923512 & 22.6407464\end{array}$

$\begin{array}{llll}0.80094493 & -22.923512 & 14.0129384\end{array}$

$\begin{array}{llll}0.80094493 & -22.923512 & 5.38513035\end{array}$

$\begin{array}{llll}22.9305393 & 0.56504244 & 25.9278071\end{array}$

$\begin{array}{llll}22.8275318 & 2.24336777 & 25.9293901\end{array}$

$\begin{array}{llll}22.5038597 & 4.4390543 & 25.9358935\end{array}$

$\begin{array}{llll}21.4177491 & 8.21029416 & 25.9525191\end{array}$

$\begin{array}{llll}17.4160431 & 14.9268332 & 25.9887085\end{array}$

$\begin{array}{lll}7.47982728 & 21.6836595 & 26.0392776\end{array}$

$\begin{array}{lll}-6.3512009 & 22.0406704 & 26.0841822\end{array}$

$\begin{array}{lll}-18.171082 & 13.9978808 & 26.1132735\end{array}$

$\begin{array}{llll}-22.933466 & 0.43018015 & 26.1240452\end{array}$

$\begin{array}{llll}-18.681489 & -13.309053 & 26.1161272\end{array}$

$\begin{array}{llll}-7.1456635 & -21.796064 & 26.0897667\end{array}$

$\begin{array}{llll}6.8120565 & -21.902621 & 26.0469103\end{array}$

$\begin{array}{llll}17.2487976 & -15.119785 & 25.9960192\end{array}$

$\begin{array}{llll}21.5877375 & -7.752322 & 25.9565923\end{array}$

$\begin{array}{llll}22.6655966 & -3.5213117 & 25.9375694\end{array}$

$\begin{array}{llll}22.9083955 & -1.1551277 & 25.9299976\end{array}$
\end{abstract}

$\begin{array}{cccc}\text { Line } & \text { XINTi } & \text { ZINTi } & \begin{array}{c}\text { Source S02 } \\ \text { YINTi }\end{array} \\ \text { S02C01 } & 22.7900376 & -0.6498203 & 48.4827436 \\ \text { S02C02 } & 22.7900376 & -0.6498203 & 39.7816042 \\ \text { S02C03 } & 22.7900376 & -0.6498203 & 31.0804647 \\ \text { S02C04 } & 22.7900376 & -0.6498203 & 22.3793253 \\ \text { S02C05 } & 22.7900376 & -0.6498203 & 13.6781858 \\ \text { S02C06 } & 22.7900376 & -0.6498203 & 4.97704638\end{array}$

$\begin{array}{ccc}\text { XINTo } & \text { ZINTo } & \text { YINTo } \\ 22.9304085 & -0.5703267 & 48.6071862 \\ 22.9304085 & -0.5703267 & 39.8694867 \\ 22.9304085 & -0.5703267 & 31.1317871 \\ 22.9304085 & -0.5703267 & 22.3940876 \\ 22.9304085 & -0.5703267 & 13.6563881 \\ 22.9304085 & -0.5703267 & 4.91868854\end{array}$




\section{REVISED METHODOLOGY FOR DETERMINING CESIUM-137 CONTENT OF HN-200 GROUT CONTAINERS.}

\begin{tabular}{llll} 
S02C07 & -0.2781886 & 22.7976028 & 48.6973678 \\
S02C08 & -0.2781886 & 22.7976028 & 39.9331738 \\
S02C09 & -0.2781886 & 22.7976028 & 31.1689797 \\
S02C10 & -0.2781886 & 22.7976028 & 22.4047856 \\
S02C11 & -0.2781886 & 22.7976028 & 13.6405916 \\
S02C12 & -0.2781886 & 22.7976028 & 4.8763975 \\
S02C13 & -22.730201 & -1.7737055 & 46.7233656 \\
S02C14 & -22.730201 & -1.7737055 & 38.5391152 \\
S02C15 & -22.730201 & -1.7737055 & 30.3548648 \\
S02C16 & -22.730201 & -1.7737055 & 22.1706144 \\
S02C17 & -22.730201 & -1.7737055 & 13.986364 \\
S02C18 & -22.730201 & -1.7737055 & 5.8021136 \\
S02C19 & -3.3778989 & -22.54768 & 39.7540562 \\
S02C20 & -3.3778989 & -22.54768 & 33.6173247 \\
S02C21 & -3.3778989 & -22.54768 & 27.4805932 \\
S02C22 & -3.3778989 & -22.54768 & 21.3438617 \\
S02C23 & -3.3778989 & -22.54768 & 15.2071301 \\
S02C24 & -3.3778989 & -22.54768 & 9.07039862 \\
S02R01 & 16.0758032 & -16.167147 & 20.2364869 \\
S02R02 & 22.6898979 & -2.230832 & 20.7064926 \\
S02R03 & 19.0691341 & 12.4970479 & 20.961508 \\
S02R04 & 7.13976211 & 21.6525259 & 21.0093343 \\
S02R05 & -7.9427396 & 21.3710311 & 20.8521692 \\
S02R06 & -19.662297 & 11.5413232 & 20.4835854 \\
S02R07 & -22.491758 & -3.7321443 & 19.9009097 \\
S02R08 & -15.904675 & -16.335526 & 19.2091999 \\
S02R09 & -12.045012 & -19.357835 & 18.9510043 \\
S02R10 & -11.341371 & -19.778306 & 18.9129427 \\
S02R11 & -11.039022 & -19.948636 & 18.9026852 \\
S02R12 & -10.814469 & -20.071256 & 18.9011622 \\
S02R13 & -10.562894 & -20.204786 & 18.9065905 \\
S02R14 & -10.116498 & -20.431949 & 18.9264424 \\
S02R15 & -8.3752138 & -21.205279 & 19.0242441 \\
S02R16 & 2.10584163 & -22.701839 & 19.56904 \\
& & & \\
\hline
\end{tabular}

$\begin{array}{ccc}-0.2443647 & 22.9361983 & 48.7949567 \\ -0.2443647 & 22.9361983 & 40.0020919 \\ -0.2443647 & 22.9361983 & 31.2092271 \\ -0.2443647 & 22.9361983 & 22.4163624 \\ -0.2443647 & 22.9361983 & 13.6234976 \\ -0.2443647 & 22.9361983 & 4.83063284 \\ -22.885356 & -1.5457591 & 47.0802027 \\ -22.885356 & -1.5457591 & 38.7911168 \\ -22.885356 & -1.5457591 & 30.502031 \\ -22.885356 & -1.5457591 & 22.2129451 \\ -22.885356 & -1.5457591 & 13.9238593 \\ -22.885356 & -1.5457591 & 5.63477345 \\ -2.8679392 & -22.757501 & 41.2253964 \\ -2.8679392 & -22.757501 & 34.6563987 \\ -2.8679392 & -22.757501 & 28.087401 \\ -2.8679392 & -22.757501 & 21.5184033 \\ -2.8679392 & -22.757501 & 14.9494056 \\ -2.8679392 & -22.757501 & 8.38040783 \\ 16.2994838 & -16.138641 & 20.2479715 \\ 22.8360676 & -2.154744 & 20.7145698 \\ 19.165864 & 12.6015302 & 20.9683361 \\ 7.19465075 & 21.7799428 & 21.0159596 \\ -7.9328728 & 21.5220453 & 20.8594957 \\ -19.715236 & 11.7234116 & 20.4930534 \\ -22.669696 & -3.4948251 & 19.9164188 \\ -16.409442 & -16.026825 & 19.2419702 \\ -12.382852 & -19.307871 & 18.9711338 \\ -11.525514 & -19.831576 & 18.9248976 \\ -11.147583 & -20.046454 & 18.9121474 \\ -10.865261 & -20.200866 & 18.9102463 \\ -10.548857 & -20.36788 & 18.9170133 \\ -9.9916717 & -20.646922 & 18.9415094 \\ -7.9495708 & -21.515883 & 19.0542897 \\ 2.50550544 & -22.800249 & 19.591125\end{array}$

$\begin{array}{ccccccc}\text { Line } & \text { XINTi } & \text { ZINTi } & \text { YINTi } & \text { Xource S03 } & & \\ \text { S03C01 } & 22.7962367 & -0.373728 & 49.1080659 & 22.9351513 & -0.3282378 & 49.1557722 \\ \text { S03C02 } & 22.7962367 & -0.373728 & 40.571428 & 22.9351513 & -0.3282378 & 40.5627338 \\ \text { S03C03 } & 22.7962367 & -0.373728 & 32.03479 & 22.9351513 & -0.3282378 & 31.9696953 \\ \text { S03C04 } & 22.7962367 & -0.373728 & 23.498152 & 22.9351513 & -0.3282378 & 23.3766569 \\ \text { S03C05 } & 22.7962367 & -0.373728 & 14.961514 & 22.9351513 & -0.3282378 & 14.7836185 \\ \text { S03C06 } & 22.7962367 & -0.373728 & 6.42487605 & 22.9351513 & -0.3282378 & 6.19058006 \\ \text { S03C07 } & 0.06459974 & 22.7992085 & 49.2222318 & 0.05675548 & 22.9374298 & 49.2559609\end{array}$




\section{REVISED METHODOLOGY FOR DETERMINING CESIUM-137 CONTENT OF HN-200 GROUT CONTAINERS.}

\begin{tabular}{|c|c|c|c|c|c|c|}
\hline S03C08 & 0.06459974 & 22.7992085 & 40.5506218 & 0.05675548 & 22.9374298 & 40.5444749 \\
\hline S03C09 & 0.06459974 & 22.7992085 & 31.8790118 & 0.05675548 & 22.9374298 & 31.8329889 \\
\hline $\mathrm{S} 03 \mathrm{C} 10$ & 0.06459974 & 22.7992085 & 23.2074018 & 0.05675548 & 22.9374298 & 23.1215029 \\
\hline S03C11 & 0.06459974 & 22.7992085 & 14.5357918 & 0.05675548 & 22.9374298 & 14.4100169 \\
\hline $\mathrm{S} 03 \mathrm{C} 12$ & 0.06459974 & 22.7992085 & 5.86418185 & 0.05675548 & 22.9374298 & 5.69853097 \\
\hline $\mathrm{S} 03 \mathrm{C} 13$ & -22.797026 & -0.3220296 & 49.1622828 & -22.935756 & -0.2828571 & 49.2033636 \\
\hline S03C14 & -22.797026 & -0.3220296 & 40.5615472 & -22.935756 & -0.2828571 & 40.5540605 \\
\hline S03C15 & -22.797026 & -0.3220296 & 31.9608117 & -22.935756 & -0.2828571 & 31.9047573 \\
\hline S03C16 & -22.797026 & -0.3220296 & 23.3600761 & -22.935756 & -0.2828571 & 23.2554542 \\
\hline $\mathrm{S} 03 \mathrm{C} 17$ & -22.797026 & -0.3220296 & 14.7593405 & -22.935756 & -0.2828571 & 14.6061511 \\
\hline S03C18 & -22.797026 & -0.3220296 & 6.15860492 & -22.935756 & -0.2828571 & 5.95684792 \\
\hline S03C19 & 0.12085581 & -22.79898 & 48.98034 & 0.10617774 & -22.937254 & 49.0434533 \\
\hline $\mathrm{S} 03 \mathrm{C} 20$ & 0.12085581 & -22.79898 & 40.5947054 & 0.10617774 & -22.937254 & 40.5832033 \\
\hline S03C2I & 0.12085581 & -22.79898 & 32.2090707 & 0.10617774 & -22.937254 & 32.1229532 \\
\hline $\mathrm{S} 03 \mathrm{C} 22$ & 0.12085581 & -22.79898 & 23.823436 & 0.10617774 & -22.937254 & 23.6627032 \\
\hline $\mathrm{S} 03 \mathrm{C} 23$ & 0.12085581 & -22.79898 & 15.4378013 & 0.10617774 & -22.937254 & 15.2024532 \\
\hline $\mathrm{S} 03 \mathrm{C} 24$ & 0.12085581 & -22.79898 & 7.05216664 & 0.10617774 & -22.937254 & 6.74220313 \\
\hline S03R01 & 21.9338208 & -6.2221849 & 39.7252425 & 22.0795498 & -6.2146913 & 39.709616 \\
\hline S03R02 & 22.642775 & 2.66698743 & 39.4623093 & 22.7745036 & 2.72963205 & 39.4470044 \\
\hline S03R03 & 19.4012527 & 11.9749519 & 39.1997561 & 19.4982832 & 12.0808053 & 39.1849649 \\
\hline S03R04 & 11.9136262 & 19.4389709 & 38.9864976 & 11.9635718 & 19.5704332 & 38.9722136 \\
\hline S03R05 & 1.41665422 & 22.7552449 & 38.8624151 & 1.41502085 & 22.8938119 & 38.8484498 \\
\hline S03R06 & -9.5614301 & 20.6975152 & 38.8497507 & -9.6134039 & 20.8257382 & 38.8358186 \\
\hline S03R07 & -18.180975 & 13.7571888 & 38.9507199 & -18.277405 & 13.8587654 & 38.9365263 \\
\hline S03R08 & -22.453456 & 3.95605698 & 39.1473724 & -22.583174 & 4.01611309 & 39.1326999 \\
\hline S03R09 & -21.977041 & -6.0677645 & 39.4032268 & -22.122377 & -6.060474 & 39.3880237 \\
\hline S03R 10 & -17.831182 & -14.20764 & 39.6702028 & -17.968919 & -14.256467 & 39.6546231 \\
\hline S03R11 & -11.714246 & -19.559768 & 39.9025557 & -11.820821 & -19.656986 & 39.886869 \\
\hline S03R12 & -5.0093336 & -22.242182 & 40.0699816 & -5.0674987 & -22.370726 & 40.0543899 \\
\hline S03R 13 & 1.5683706 & -22.745292 & 40.1595925 & 1.56656138 & -22.883942 & 40.1441236 \\
\hline S03R14 & 7.78086186 & -21.430499 & 40.1684346 & 7.83489767 & -21.557905 & 40.1529808 \\
\hline S03R 15 & 13.5193412 & -18.358526 & 40.096384 & 13.6214225 & -18.454966 & 40.0808229 \\
\hline S03R 16 & 18.4853814 & -13.345365 & 39.9453368 & 18.6203195 & -13.394499 & 39.9296589 \\
\hline
\end{tabular}

$\begin{array}{cccc}\text { Line } & \text { XINTi } & \text { ZINTi } & \begin{array}{c}\text { Source S04 } \\ \text { YINTi }\end{array} \\ \text { S04C01 } & 22.7665125 & -1.2222876 & 48.1755365 \\ \text { S04C02 } & 22.7665125 & -1.2222876 & 39.7479495 \\ \text { S04C03 } & 22.7665125 & -1.2222876 & 31.3203625 \\ \text { S04C04 } & 22.7665125 & -1.2222876 & 22.8927754 \\ \text { S04C05 } & 22.7665125 & -1.2222876 & 14.4651884 \\ \text { S04C06 } & 22.7665125 & -1.2222876 & 6.0376014 \\ \text { S04C07 } & 0.1458092 & 22.7988337 & 48.9505425 \\ \text { S04C08 } & 0.1458092 & 22.7988337 & 40.1880097\end{array}$

$\begin{array}{ccc}\text { XINTo } & \text { ZINTo } & \text { YINTo } \\ 22.9125357 & -1.0698673 & 48.3406982 \\ 22.9125357 & -1.0698673 & 39.8417308 \\ 22.9125357 & -1.0698673 & 31.3427634 \\ 22.9125357 & -1.0698673 & 22.843796 \\ 22.9125357 & -1.0698673 & 14.3448286 \\ 22.9125357 & -1.0698673 & 5.84586123 \\ 0.12809843 & 22.9371423 & 49.0172826 \\ 0.12809843 & 22.9371423 & 40.2259057\end{array}$




\section{REVISED METHODOLOGY FOR DETERMINING CESIUM-137 CONTENT} OF HN-200 GROUT CONTAINERS.

\begin{tabular}{llll} 
SO4C09 & 0.1458092 & 22.7988337 & 31.4254768 \\
S04C10 & 0.1458092 & 22.7988337 & 22.662944 \\
SO4C11 & 0.1458092 & 22.7988337 & 13.9004112 \\
S04C12 & 0.1458092 & 22.7988337 & 5.13787837 \\
S04C13 & -22.786946 & -0.7504587 & 48.6868074 \\
S04C14 & -22.786946 & -0.7504587 & 40.0382569 \\
S04C15 & -22.786946 & -0.7504587 & 31.3897063 \\
S04C16 & -22.786946 & -0.7504587 & 22.7411558 \\
S04C17 & -22.786946 & -0.7504587 & 14.0926053 \\
S04C18 & -22.786946 & -0.7504587 & 5.44405472 \\
S04C19 & 1.38179576 & -22.757388 & 44.2929346 \\
S04C20 & 1.38179576 & -22.757388 & 37.5433491 \\
S04C21 & 1.38179576 & -22.757388 & 30.7937635 \\
S04C22 & 1.38179576 & -22.757388 & 24.044178 \\
S04C23 & 1.38179576 & -22.757388 & 17.2945924 \\
S04C24 & 1.38179576 & -22.757388 & 10.5450069 \\
S04R01 & 13.9399981 & -18.04119 & 28.5576695 \\
S04R02 & 20.1439935 & -10.678371 & 28.453117 \\
S04R03 & 22.6755037 & 2.3726803 & 28.3299571 \\
S04R04 & 16.7877548 & 15.4265799 & 28.2345854 \\
S04R05 & 4.10695199 & 22.4263467 & 28.1810541 \\
SO4R06 & -10.235679 & 20.372505 & 28.1716289 \\
S04R07 & -20.445333 & 10.0894222 & 28.2064293 \\
SO4R08 & -22.407133 & -4.2105213 & 28.284628 \\
S04R09 & -15.775239 & -16.460556 & 28.399295 \\
SO4R10 & -5.6265033 & -22.094129 & 28.5194848 \\
S04R11 & 0.88450841 & -22.782136 & 28.5917282 \\
S04R12 & 3.78337997 & -22.483196 & 28.6198532 \\
S04R13 & 5.39511953 & -22.151767 & 28.6299119 \\
S04R14 & 6.65428146 & -21.806619 & 28.6313988 \\
S04R15 & 8.01007256 & -21.345885 & 28.6255152 \\
S04R16 & 10.0217644 & -20.478582 & 28.6071593 \\
& & & \\
\hline
\end{tabular}

$\begin{array}{lll}0.12809843 & 22.9371423 & 31.4345288\end{array}$

$\begin{array}{lll}0.12809843 & 22.9371423 & 22.643152\end{array}$

$\begin{array}{lll}0.12809843 & 22.9371423 & 13.8517751\end{array}$

$\begin{array}{llll}0.12809843 & 22.9371423 & 5.06039822\end{array}$

$\begin{array}{llll}-22.928048 & -0.6584235 & 48.7865362\end{array}$

$\begin{array}{lll}-22.928048 & -0.6584235 & 40.0948844\end{array}$

$\begin{array}{lll}-22.928048 & -0.6584235 & 31.4032326\end{array}$

$\begin{array}{lll}-22.928048 & -0.6584235 & 22.7115808\end{array}$

$\begin{array}{lll}-22.928048 & -0.6584235 & 14.019929\end{array}$

$\begin{array}{lll}-22.928048 & -0.6584235 & 5.3282772\end{array}$

$\begin{array}{llll}1.20818573 & -22.905659 & 44.9471549\end{array}$

$\begin{array}{llll}1.20818573 & -22.905659 & 37.9148253\end{array}$

$\begin{array}{llll}1.20818573 & -22.905659 & 30.8824957\end{array}$

$\begin{array}{llll}1.20818573 & -22.905659 & 23.8501661\end{array}$

$\begin{array}{lll}1.20818573 & -22.905659 & 16.8178365\end{array}$

$\begin{array}{llll}1.20818573 & -22.905659 & 9.78550689\end{array}$

$\begin{array}{llll}14.2134795 & -18.002942 & 28.5538372\end{array}$

$\begin{array}{llll}20.3688282 & -10.547025 & 28.4496954\end{array}$

$\begin{array}{llll}22.7979014 & 2.52677605 & 28.3274903\end{array}$

$\begin{array}{llll}16.8367523 & 15.5773129 & 28.2326667\end{array}$

$\begin{array}{llll}4.10213451 & 22.567707 & 28.1793755\end{array}$

$\begin{array}{lll}-10.286989 & 20.5013846 & 28.1699884\end{array}$

$\begin{array}{lll}-20.544272 & 10.2010689 & 28.2046421\end{array}$

$\begin{array}{lll}-22.563236 & -4.1266532 & 28.2824432\end{array}$

$\begin{array}{llll}-16.0049 & -16.430827 & 28.3963163\end{array}$

$\begin{array}{lll}-5.9020079 & -22.16518 & 28.5156289\end{array}$

$\begin{array}{llll}0.69593081 & -22.92694 & 28.5883217\end{array}$

$\begin{array}{llll}3.70134678 & -22.636893 & 28.6172303\end{array}$

$\begin{array}{llll}5.38869789 & -22.295534 & 28.6276743\end{array}$

$\begin{array}{llll}6.70985582 & -21.934146 & 28.6292226\end{array}$

$\begin{array}{llll}8.12978123 & -21.44844 & 28.6231027\end{array}$

$\begin{array}{lll}10.220886 & -20.53442 & 28.604129\end{array}$

\section{Source S05}

$\begin{array}{cccc}\text { Line } & \text { XINTi } & \text { ZINTi } & \text { YINTi } \\ \text { S05C01 } & 22.7991737 & 0.07589067 & 49.0896331 \\ \text { S05C02 } & 22.7991737 & 0.07589067 & 40.3706661 \\ \text { S05C03 } & 22.7991737 & 0.07589067 & 31.6516992 \\ \text { S05C04 } & 22.7991737 & 0.07589067 & 22.9327322 \\ \text { S05C05 } & 22.7991737 & 0.07589067 & 14.2137652 \\ \text { S05C06 } & 22.7991737 & 0.07589067 & 5.4947983 \\ \text { S05C07 } & -0.6681267 & 22.7895083 & 48.7985223 \\ \text { S05C08 } & -0.6681267 & 22.7895083 & 40.2789178 \\ \text { S05C09 } & -0.6681267 & 22.7895083 & 31.7593133\end{array}$

\section{XINTo}

22.9374031

22.9374031

22.9374031

22.9374031

22.9374031

22.9374031

$-0.5863587$

$-0.5863587$

$-0.5863587$

\begin{tabular}{cc} 
ZINTo & YINTo \\
0.0666751 & 49.1394648 \\
0.0666751 & 40.3863714 \\
0.0666751 & 31.633278 \\
0.0666751 & 22.8801846 \\
0.0666751 & 14.1270912 \\
0.0666751 & 5.37399781 \\
22.9300041 & 48.8843719 \\
22.9300041 & 40.3059747 \\
\hline 2.9300041 & 31.7275774
\end{tabular}

$22.9300041 \quad 31.7275774$ 


\section{REVISED METHODOLOGY FOR DETERMINING CESIUM-137 CONTENT OF HN-200 GROUT CONTAINERS.}

$\begin{array}{llll}\text { S0SC10 } & -0.6681267 & 22.7895083 & 23.2397088 \\ \text { S05C11 } & -0.6681267 & 22.7895083 & 14.7201043 \\ \text { S05C12 } & -0.6681267 & 22.7895083 & 6.20049976 \\ \text { S05C13 } & -22.79801 & 0.24249407 & 48.1887512 \\ \text { S05C14 } & -22.79801 & 0.24249407 & 40.0867384 \\ \text { S0SC15 } & -22.79801 & 0.24249407 & 31.9847256 \\ \text { S05C16 } & -22.79801 & 0.24249407 & 23.8827128 \\ \text { S05C17 } & -22.79801 & 0.24249407 & 15.7807 \\ \text { S05C18 } & -22.79801 & 0.24249407 & 7.67868718 \\ \text { S05C19 } & -0.5433879 & -22.792824 & 48.9294877 \\ \text { S05C20 } & -0.5433879 & -22.792824 & 40.3201937 \\ \text { S05C21 } & -0.5433879 & -22.792824 & 31.7108996 \\ \text { S05C22 } & -0.5433879 & -22.792824 & 23.1016056 \\ \text { S05C23 } & -0.5433879 & -22.792824 & 14.4923116 \\ \text { S05C24 } & -0.5433879 & -22.792824 & 5.88301762 \\ \text { S05R01 } & 22.7217019 & 1.87945336 & 34.1801318 \\ \text { S05R02 } & 18.3154819 & 13.5775993 & 34.3090476 \\ \text { S05R03 } & 8.87004131 & 21.0031057 & 34.5490583 \\ \text { S05R04 } & -2.0005169 & 22.7113631 & 34.8548633 \\ \text { S05R05 } & -10.952192 & 19.9964391 & 35.1569228 \\ \text { S05R06 } & -16.757089 & 15.4598848 & 35.3923538 \\ \text { S05R07 } & -20.060964 & 10.8335504 & 35.5396159 \\ \text { S05R08 } & -21.849782 & 6.5111533 & 35.6079956 \\ \text { S05R09 } & -22.688913 & 2.24082433 & 35.6087567 \\ \text { S05R10 } & -22.670286 & -2.4220228 & 35.5420469 \\ \text { S05R11 } & -21.377416 & -7.9255395 & 35.3967824 \\ \text { S05R12 } & -17.762554 & -14.293346 & 35.1633353 \\ \text { S05R13 } & -10.568279 & -20.201969 & 34.862231 \\ \text { S05R14 } & 0.09264891 & -22.799112 & 34.5556617 \\ \text { S05R15 } & 11.6549431 & -19.595162 & 34.3134929 \\ \text { S05R16 } & 20.2319415 & -10.510786 & 34.1816833\end{array}$

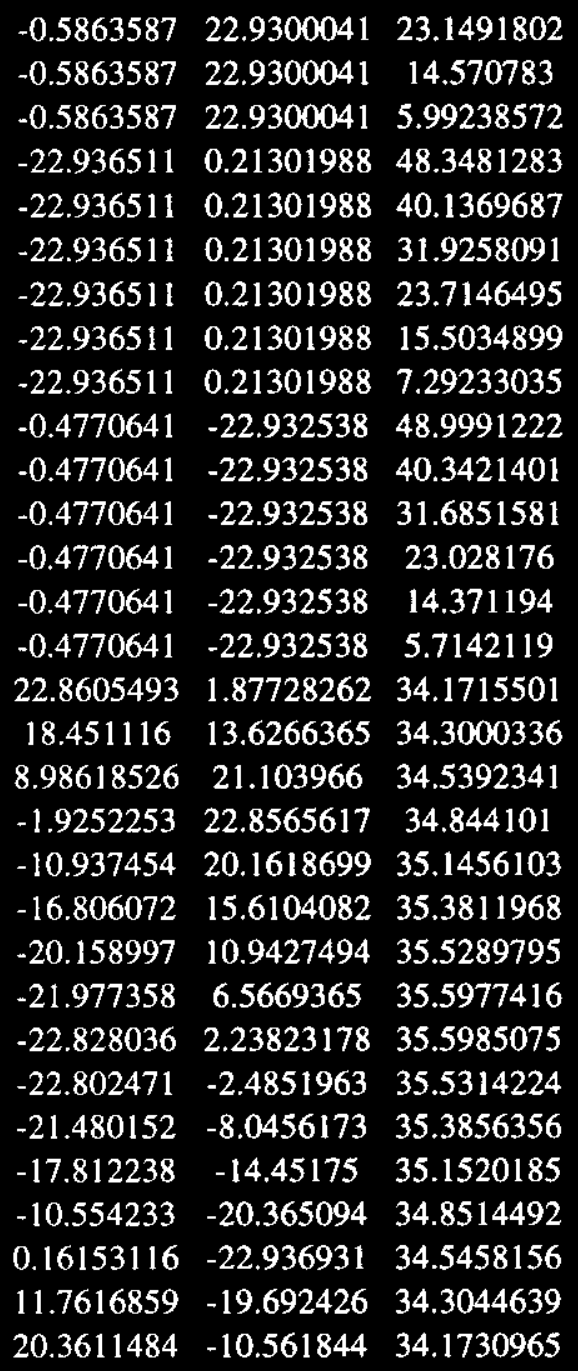

\section{Source S06}

$\begin{array}{cccc}\text { Line } & \text { XINTi } & \text { ZINTi } & \text { YINTi } \\ \text { S06C01 } & 22.7922696 & -0.5661525 & 47.8508277 \\ \text { S06C02 } & 22.7922696 & -0.5661525 & 39.5982915 \\ \text { S06C03 } & 22.7922696 & -0.5661525 & 31.3457554 \\ \text { SO6C04 } & 22.7922696 & -0.5661525 & 23.0932192 \\ \text { S06C05 } & 22.7922696 & -0.5661525 & 14.8406831 \\ \text { S06C06 } & 22.7922696 & -0.5661525 & 6.58814691 \\ \text { S06C07 } & 0.3766013 & 22.7961894 & 48.7630887 \\ \text { S06C08 } & 0.3766013 & 22.7961894 & 40.0970833 \\ \text { S06C09 } & 0.3766013 & 22.7961894 & 31.4310778 \\ \text { S06C10 } & 0.3766013 & 22.7961894 & 22.7650723\end{array}$

$\begin{array}{ccc}\text { XINTo } & \text { ZINTo } & \text { YINTo } \\ 22.9321146 & -0.4970191 & 48.0522094 \\ 22.9321146 & -0.4970191 & 39.7083999 \\ 22.9321146 & -0.4970191 & 31.3645903 \\ 22.9321146 & -0.4970191 & 23.0207808 \\ 22.9321146 & -0.4970191 & 14.6769712 \\ 22.9321146 & -0.4970191 & 6.33316163 \\ 0.33075963 & 22.9351151 & 48.852789 \\ 0.33075963 & 22.9351151 & 40.1461282 \\ 0.33075963 & 22.9351151 & 31.4394673 \\ 0.33075963 & 22.9351151 & 22.7328065\end{array}$


REVISED METHODOLOGY FOR DETERMINING CESIUM-137 CONTENT OF HN-200 GROUT CONTAINERS.

\begin{tabular}{|c|c|c|c|}
\hline & & & \\
\hline $36 \mathrm{C} 12$ & .3766013 & & \\
\hline $6 \mathrm{C} 13$ & 22.798161 & -0.2278597 & \\
\hline & & & \\
\hline & & & \\
\hline & & & \\
\hline & & & \\
\hline 60 & 22.7 & -0.2 & \\
\hline & & -22 & \\
\hline 0 & & -22 & \\
\hline & & -22 & \\
\hline & & -22 & \\
\hline & & -22 & \\
\hline & & & \\
\hline & & & \\
\hline & & & \\
\hline & & 6.9 & \\
\hline 61 & & 15. & 29. \\
\hline & & 21. & \\
\hline & & 076 & \\
\hline & & & \\
\hline & & 6.9 & \\
\hline & & & 29. \\
\hline & -16 & -15 & 29. \\
\hline 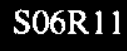 & -7.2 & -21 & 29. \\
\hline & 86 & -22 & 29. \\
\hline & & & \\
\hline & & & \\
\hline & & -14.519321 & \\
\hline 6R16 & 20.1155496 & -10.731856 & 29.40626 \\
\hline
\end{tabular}

$\begin{array}{llll}0.33075963 & 22.9351151 & 14.0261456\end{array}$

$\begin{array}{llll}0.33075963 & 22.9351151 & 5.31948479\end{array}$

$\begin{array}{llll}-22.936627 & -0.2001676 & 48.9169222\end{array}$

$\begin{array}{llll}-22.936627 & -0.2001676 & 40.1811939\end{array}$

$\begin{array}{llll}-22.936627 & -0.2001676 & 31.4454656\end{array}$

$\begin{array}{llll}-22.936627 & -0.2001676 & 22.7097373\end{array}$

$\begin{array}{llll}-22.936627 & -0.2001676 & 13.974009\end{array}$

$\begin{array}{llll}-22.936627 & -0.2001676 & 5.23828069\end{array}$

$\begin{array}{llll}0.59735305 & -22.92972 & 48.3311347\end{array}$

$\begin{array}{llll}0.59735305 & -22.92972 & 39.8609062\end{array}$

$\begin{array}{llll}0.59735305 & -22.92972 & 31.3906778\end{array}$

$\begin{array}{llll}0.59735305 & -22.92972 & 22.9204493\end{array}$

$\begin{array}{llll}0.59735305 & -22.92972 & 14.4502209\end{array}$

$\begin{array}{llll}0.59735305 & -22.92972 & 5.97999242\end{array}$

$\begin{array}{llll}22.0824953 & -6.2042168 & 29.3827965\end{array}$

$\begin{array}{llll}22.9346011 & -0.3646629 & 29.3393727\end{array}$

$\begin{array}{llll}21.8292151 & 7.04373997 & 29.271145\end{array}$

$\begin{array}{llll}17.2024488 & 15.1724969 & 29.184739\end{array}$

$\begin{array}{lll}8.23544006 & 21.4080927 & 29.0975664\end{array}$

$\begin{array}{lll}-3.6122143 & 22.6512873 & 29.0289536\end{array}$

$\begin{array}{lll}-14.876972 & 17.4586546 & 28.991894\end{array}$

$\begin{array}{llll}-21.836992 & 7.01959166 & 28.9921326\end{array}$

$\begin{array}{llll}-22.299566 & -5.3719907 & 29.0296364\end{array}$

$\begin{array}{llll}-16.604285 & -15.824874 & 29.0985781\end{array}$

$\begin{array}{llll}-7.419135 & -21.7045 & 29.1858688\end{array}$

$\begin{array}{llll}1.83927735 & -22.863638 & 29.2721378\end{array}$

$\begin{array}{llll}9.08616503 & -21.061114 & 29.3400712\end{array}$

$\begin{array}{llll}14.1461596 & -18.055888 & 29.3831868\end{array}$

$\begin{array}{lll}17.6748823 & -14.61942 & 29.4033168\end{array}$

$\begin{array}{lll}20.2450455 & -10.782719 & 29.4031933\end{array}$

\begin{tabular}{|c|c|c|c|c|c|c|}
\hline \multicolumn{7}{|c|}{ Source S07 } \\
\hline Line & XINTi & ZINTi & YINTi & XINTo & ZINTo & YINTo \\
\hline S07C01 & 22.7856013 & -0.790224 & 47.4485048 & 22.9270227 & -0.6932063 & 47.7003719 \\
\hline $\mathrm{S} 07 \mathrm{C} 02$ & 22.7856013 & -0.790224 & 38.8333049 & 22.9270227 & -0.6932063 & 39.0379292 \\
\hline S07C03 & 22.7856013 & -0.790224 & 30.218105 & 22.9270227 & -0.6932063 & 30.3754864 \\
\hline $\mathrm{S} 07 \mathrm{C} 04$ & 22.7856013 & -0.790224 & 21.6029051 & 22.9270227 & -0.6932063 & 21.7130436 \\
\hline S07C05 & 22.7856013 & -0.790224 & 12.9877052 & 22.9270227 & -0.6932063 & 13.0506009 \\
\hline S07C06 & 22.7856013 & -0.790224 & 4.37250524 & 22.9270227 & -0.6932063 & 4.38815809 \\
\hline S07C07 & -0.0806125 & 22.7991575 & 48.2123984 & -0.0708234 & 22.9373907 & 48.3687567 \\
\hline S07C08 & -0.0806125 & 22.7991575 & 39.4539146 & -0.0708234 & 22.9373907 & 39.5809446 \\
\hline $\mathrm{S} 07 \mathrm{C} 09$ & -0.0806125 & 22.7991575 & 30.6954307 & -0.0708234 & 22.9373907 & 30.7931325 \\
\hline $\mathrm{S} 07 \mathrm{C} 10$ & -0.0806125 & 22.7991575 & 21.9369468 & -0.0708234 & 22.9373907 & 22.0053205 \\
\hline S07C11 & -0.0806125 & 22.7991575 & 13.178463 & -0.0708234 & 22.9373907 & 13.2175084 \\
\hline
\end{tabular}

113 of 210

ATTACHMENT 1 


\section{REVISED METHODOLOGY FOR DETERMINING CESIUM-137 CONTENT} OF HN-200 GROUT CONTAINERS.

$\begin{array}{lccc}\text { S07C12 } & -0.0806125 & 22.7991575 & 4.41997912 \\ \text { S07C13 } & -22.776235 & -1.0252909 & 46.8382494 \\ \text { S07C14 } & -22.776235 & -1.0252909 & 38.3375155 \\ \text { S07C15 } & -22.776235 & -1.0252909 & 29.8367815 \\ \text { S07C16 } & -22.776235 & -1.0252909 & 21.3360475 \\ \text { S07C17 } & -22.776235 & -1.0252909 & 12.8353135 \\ \text { S07C18 } & -22.776235 & -1.0252909 & 4.33457956 \\ \text { S07C19 } & -0.6315939 & -22.79055 & 39.4117231 \\ \text { S07C20 } & -0.6315939 & -22.79055 & 32.3039868 \\ \text { S07C21 } & -0.6315939 & -22.79055 & 25.1962504 \\ \text { S07C22 } & -0.6315939 & -22.79055 & 18.0885141 \\ \text { S07C23 } & -0.6315939 & -22.79055 & 10.9807778 \\ \text { S07C24 } & -0.6315939 & -22.79055 & 3.87304146 \\ \text { S07R01 } & 16.1913511 & -16.051425 & 4.83675342 \\ \text { S07R02 } & 22.293676 & -4.7749439 & 6.24413707 \\ \text { S07R03 } & 20.9386722 & 9.02109125 & 7.32265381 \\ \text { S07R04 } & 11.6033677 & 19.6257468 & 7.90536628 \\ \text { S07R05 } & -2.237797 & 22.6892121 & 7.95368773 \\ \text { S07R06 } & -15.189619 & 17.0024569 & 7.46567592 \\ \text { S07R07 } & -22.248967 & 4.97911076 & 6.46959467 \\ \text { S07R08 } & -21.311692 & -8.1006085 & 5.09750544 \\ \text { S07R09 } & -15.357824 & -16.850677 & 3.74562126 \\ \text { S07R10 } & -10.056453 & -20.46157 & 2.89692462 \\ \text { S07R11 } & -6.8712352 & -21.739232 & 2.51305774 \\ \text { S07R12 } & -4.7419882 & -22.300709 & 2.36634444 \\ \text { S07R13 } & -2.9042767 & -22.613564 & 2.35561052 \\ \text { S07R14 } & -0.8181513 & -22.784616 & 2.47384997 \\ \text { S07R15 } & 2.29064918 & -22.683937 & 2.80167413 \\ \text { S07R16 } & 7.8694182 & -21.398139 & 3.54941315\end{array}$

$\begin{array}{cccc}\text { Line } & \text { XINTi } & \text { ZINTi } & \begin{array}{c}\text { Source S08 } \\ \text { YINTi }\end{array} \\ \text { S08C01 } & 22.795377 & -0.4229253 & 48.2114852 \\ \text { S08C02 } & 22.795377 & -0.4229253 & 39.6164254 \\ \text { S08C03 } & 22.795377 & -0.4229253 & 31.0213657 \\ \text { S08C04 } & 22.795377 & -0.4229253 & 22.4263059 \\ \text { S08C05 } & 22.795377 & -0.4229253 & 13.8312462 \\ \text { S08C06 } & 22.795377 & -0.4229253 & 5.23618644 \\ \text { S08C07 } & -0.0493975 & 22.7992465 & 48.5221989 \\ \text { S08C08 } & -0.0493975 & 22.7992465 & 39.8294915 \\ \text { S08C09 } & -0.0493975 & 22.7992465 & 31.136784 \\ \text { S08C10 } & -0.0493975 & 22.7992465 & 22.4440766 \\ \text { S08C11 } & -0.0493975 & 22.7992465 & 13.7513692 \\ \text { S08C12 } & -0.0493975 & 22.7992465 & 5.05866172\end{array}$

$\begin{array}{lll}-0.0708234 & 22.9373907 & 4.42969635 \\ -22.919897 & -0.8984498 & 47.1675407 \\ -22.919897 & -0.8984498 & 38.6050414 \\ -22.919897 & -0.8984498 & 30.0425421 \\ -22.919897 & -0.8984498 & 21.4800427 \\ -22.919897 & -0.8984498 & 12.9175434 \\ -22.919897 & -0.8984498 & 4.3550441 \\ -0.5543621 & -22.9308 & 40.645326 \\ -0.5543621 & -22.9308 & 33.3062021 \\ -0.5543621 & -22.9308 & 25.9670782 \\ -0.5543621 & -22.9308 & 18.6279543 \\ -0.5543621 & -22.9308 & 11.2888304 \\ -0.5543621 & -22.9308 & 3.94970647 \\ 16.4128071 & -16.023379 & 4.87504113 \\ 22.4532156 & -4.6884981 & 6.27394207 \\ 21.0392644 & 9.13664374 & 7.34704113 \\ 11.6535334 & 19.7566208 & 7.92730209 \\ -2.235208 & 22.8283322 & 7.97543395 \\ -15.238292 & 17.1441931 & 7.48943236 \\ -22.360026 & 5.11450249 & 6.49816906 \\ -21.499342 & -7.9941977 & 5.13419402 \\ -15.596282 & -16.819182 & 3.78861866 \\ -10.255147 & -20.517331 & 2.93577243 \\ -6.9962213 & -21.844491 & 2.54521541 \\ -4.8006153 & -22.429512 & 2.39495946 \\ -2.9009033 & -22.753322 & 2.38394506 \\ -0.7466513 & -22.925344 & 2.50511454 \\ 2.44543745 & -22.80677 & 2.8392006 \\ 8.09958747 & -21.45986 & 3.59241312\end{array}$

$\begin{array}{llll}-0.0708234 & 22.9373907 & 4.42969635\end{array}$

$\begin{array}{lll}-22.919897 & -0.8984498 & 47.1675407\end{array}$

$\begin{array}{llll}-22.919897 & -0.8984498 & 38.6050414\end{array}$

$\begin{array}{lll}-22.919897 & -0.8984498 & 30.0425421\end{array}$

$\begin{array}{lll}-22.919897 & -0.8984498 & 21.4800427\end{array}$

$\begin{array}{lll}-22.919897 & -0.8984498 & 12.9175434\end{array}$

$\begin{array}{lll}-22.919897 & -0.8984498 & 4.3550441\end{array}$

$-0.5543621 \quad-22.9308 \quad 40.645326$

$\begin{array}{lll}-0.5543621 & -22.9308 & 33.3062021\end{array}$

$\begin{array}{lll}-0.5543621 & -22.9308 & 25.9670782\end{array}$

$\begin{array}{lll}-0.5543621 & -22.9308 & 18.6279543\end{array}$

$\begin{array}{lll}-0.5543621 & -22.9308 & 11.2888304\end{array}$

$\begin{array}{lll}-0.5543621 & -22.9308 & 3.94970647\end{array}$

$\begin{array}{llll}16.4128071 & -16.023379 & 4.87504113\end{array}$

$\begin{array}{llll}22.4532156 & -4.6884981 & 6.27394207\end{array}$

$\begin{array}{llll}21.0392644 & 9.13664374 & 7.34704113\end{array}$

$\begin{array}{llll}11.6535334 & 19.7566208 & 7.92730209\end{array}$

$\begin{array}{llll}-2.235208 & 22.8283322 & 7.97543395\end{array}$

$\begin{array}{llll}-15.238292 & 17.1441931 & 7.48943236\end{array}$

$\begin{array}{llll}-22.360026 & 5.11450249 & 6.49816906\end{array}$

$\begin{array}{llll}-21.499342 & -7.9941977 & 5.13419402\end{array}$

$\begin{array}{lll}-15.596282 & -16.819182 & 3.78861866\end{array}$

$\begin{array}{lll}-10.255147 & -20.517331 & 2.93577243\end{array}$

$\begin{array}{llll}-6.9962213 & -21.844491 & 2.54521541\end{array}$

$\begin{array}{lll}-4.8006153 & -22.429512 & 2.39495946\end{array}$

$\begin{array}{llll}-2.9009033 & -22.753322 & 2.38394506\end{array}$

$\begin{array}{lll}-0.7466513 & -22.925344 & 2.50511454\end{array}$

$\begin{array}{lll}8.09958747 & -21.45986 & 3.59241312\end{array}$
XINTo ZINTo YINTo

$\begin{array}{llll}22.9344928 & -0.3714111 & 48.3684321\end{array}$

$\begin{array}{llll}22.9344928 & -0.3714111 & 39.7240488\end{array}$

$\begin{array}{llll}22.9344928 & -0.3714111 & 31.0796655\end{array}$

$\begin{array}{llll}22.9344928 & -0.3714111 & 22.4352822\end{array}$

$\begin{array}{llll}22.9344928 & -0.3714111 & 13.7908989\end{array}$

$\begin{array}{llll}22.9344928 & -0.3714111 & 5.14651559\end{array}$

$\begin{array}{llll}-0.0433994 & 22.9374589 & 48.6409284\end{array}$

$\begin{array}{lll}-0.0433994 & 22.9374589 & 39.910908\end{array}$

$\begin{array}{lll}-0.0433994 & 22.9374589 & 31.1808875\end{array}$

$\begin{array}{lll}-0.0433994 & 22.9374589 & 22.4508671\end{array}$

$\begin{array}{lll}-0.0433994 & 22.9374589 & 13.7208467\end{array}$

$\begin{array}{lll}-0.0433994 & 22.9374589 & 4.99082621\end{array}$ 
REVISED METHODOLOGY FOR DETERMINING CESIUM-137 CONTENT OF HN-200 GROUT CONTAINERS.

$\begin{array}{lccc}\text { S08C13 } & -22.794293 & -0.477789 & 48.0443332 \\ \text { S08C14 } & -22.794293 & -0.477789 & 39.5018041 \\ \text { S08C15 } & -22.794293 & -0.477789 & 30.9592751 \\ \text { S08C16 } & -22.794293 & -0.477789 & 22.416746 \\ \text { S08C17 } & -22.794293 & -0.477789 & 13.8742169 \\ \text { S08C18 } & -22.794293 & -0.477789 & 5.33168788 \\ \text { S08C19 } & -0.1132982 & -22.799018 & 47.257311 \\ \text { S08C20 } & -0.1132982 & -22.799018 & 38.9621186 \\ \text { S08C21 } & -0.1132982 & -22.799018 & 30.6669262 \\ \text { S08C22 } & -0.1132982 & -22.799018 & 22.3717338 \\ \text { S08C23 } & -0.1132982 & -22.799018 & 14.0765414 \\ \text { S08C24 } & -0.1132982 & -22.799018 & 5.78134904 \\ \text { S08R01 } & 21.3818629 & -7.9135338 & 21.7930474 \\ \text { S08R02 } & 22.6937849 & 2.19093794 & 21.9267776 \\ \text { S08R03 } & 18.9878886 & 12.6201492 & 22.0357199 \\ \text { S08R04 } & 10.347826 & 20.3157716 & 22.0989679 \\ \text { S08R05 } & -1.1412306 & 22.7707196 & 22.1055117 \\ \text { S08R06 } & -12.229257 & 19.2419685 & 22.0542728 \\ \text { S08R07 } & -19.922017 & 11.0869881 & 21.9540061 \\ \text { S08R08 } & -22.77431 & 1.0671876 & 21.8235551 \\ \text { S08R09 } & -21.319705 & -8.0794964 & 21.6890903 \\ \text { S08R10 } & -17.267986 & -14.887067 & 21.575331 \\ \text { S08R11 } & -12.178091 & -19.274392 & 21.4963377 \\ \text { S08R12 } & -6.8121598 & -21.757816 & 21.455335 \\ \text { S08R13 } & -1.3019865 & -22.762094 & 21.4512801 \\ \text { S08R14 } & 4.48316551 & -22.354179 & 21.4839621 \\ \text { S08R15 } & 10.6094441 & -20.180381 & 21.5545271 \\ \text { S08R16 } & 16.6747505 & -15.548658 & 21.6613215 \\ & & & \end{array}$
$-22.933663 \quad-0.4195412 \quad 48.2217953$
$\begin{array}{lll}-22.933663 & -0.4195412 & 39.6234954\end{array}$
$\begin{array}{lll}-22.933663 & -0.4195412 & 31.0251955\end{array}$
$\begin{array}{lll}-22.933663 & -0.4195412 & 22.4268956\end{array}$
$\begin{array}{lll}-22.933663 & -0.4195412 & 13.8285957\end{array}$
$-22.933663 \quad-0.4195412 \quad 5.2302958$
$\begin{array}{llll}-0.0995385 & -22.937284 & 47.5296796\end{array}$
$\begin{array}{lll}-0.0995385 & -22.937284 & 39.1488902\end{array}$
$\begin{array}{lll}-0.0995385 & -22.937284 & 30.7681008\end{array}$
$\begin{array}{lll}-0.0995385 & -22.937284 & 22.3873114\end{array}$
$\begin{array}{lll}-0.0995385 & -22.937284 & 14.006522\end{array}$
$\begin{array}{llll}-0.0995385 & -22.937284 & 5.62573263\end{array}$
$\begin{array}{llll}21.5327724 & -7.9037093 & 21.7992014\end{array}$
$\begin{array}{llll}22.826423 & 2.25462185 & 21.9326274\end{array}$
$\begin{array}{llll}19.0845624 & 12.7243225 & 22.0412721\end{array}$
$\begin{array}{lll}10.3990318 & 20.4447804 & 22.1043377\end{array}$
$\begin{array}{lll}-1.1399158 & 22.9091575 & 22.1108623\end{array}$
$\begin{array}{lll}-12.278994 & 19.3740859 & 22.0597719\end{array}$
$\begin{array}{lll}-20.019787 & 11.1954023 & 21.9597843\end{array}$
$\begin{array}{lll}-22.909475 & 1.13352239 & 21.8296483\end{array}$
$\begin{array}{llll}-21.471218 & -8.0694308 & 21.6953952\end{array}$
$\begin{array}{lll}-17.408386 & -14.935762 & 21.5816602\end{array}$
$\begin{array}{llll}-12.283399 & -19.371294 & 21.5025615\end{array}$
$\begin{array}{lll}-6.8675074 & -21.885298 & 21.4614545\end{array}$
$\begin{array}{llll}-1.3004859 & -22.900604 & 21.4573872\end{array}$
$\begin{array}{llll}4.54225141 & -22.483257 & 21.4901582\end{array}$
$\begin{array}{llll}10.7193576 & -20.278666 & 21.5608397\end{array}$
$\begin{array}{llll}16.8182058 & -15.597335 & 21.6676482\end{array}$

$\begin{array}{lccc}\text { Line } & \text { XINTi } & \text { ZINTi } & \begin{array}{c}\text { Source S09 } \\ \text { YINTi }\end{array} \\ \text { S09C01 } & 22.7992621 & -0.0415679 & 49.3702662 \\ \text { S09C02 } & 22.7992621 & -0.0415679 & 40.6607853 \\ \text { S09C03 } & 22.7992621 & -0.0415679 & 31.9513044 \\ \text { S09C04 } & 22.7992621 & -0.0415679 & 23.2418235 \\ \text { S09C05 } & 22.7992621 & -0.0415679 & 14.5323427 \\ \text { S09C06 } & 22.7992621 & -0.0415679 & 5.82286177 \\ \text { S09C07 } & -0.5135527 & 22.7935154 & 49.3177339 \\ \text { S09C08 } & -0.5135527 & 22.7935154 & 40.7258911 \\ \text { S09C09 } & -0.5135527 & 22.7935154 & 32.1340483 \\ \text { S09C10 } & -0.5135527 & 22.7935154 & 23.5422056 \\ \text { S09C11 } & -0.5135527 & 22.7935154 & 14.9503628 \\ \text { S09C12 } & -0.5135527 & 22.7935154 & 6.35852001 \\ \text { S09C13 } & -22.799004 & -0.1162311 & 49.1372419\end{array}$

$\begin{array}{ccc}\text { XINTo } & \text { ZINTo } & \text { YINTo } \\ 22.9374709 & -0.0365206 & 49.3860189 \\ 22.9374709 & -0.0365206 & 40.6412622 \\ 22.9374709 & -0.0365206 & 31.8965055 \\ 22.9374709 & -0.0365206 & 23.1517487 \\ 22.9374709 & -0.0365206 & 14.406992 \\ 22.9374709 & -0.0365206 & 5.66223527 \\ -0.4509055 & 22.9330676 & 49.3399681 \\ -0.4509055 & 22.9330676 & 40.6983351 \\ -0.4509055 & 22.9330676 & 32.0567021 \\ -0.4509055 & 22.9330676 & 23.4150691 \\ -0.4509055 & 22.9330676 & 14.7734361 \\ -0.4509055 & 22.9330676 & 6.13180315 \\ -22.937273 & -0.102115 & 49.1812984\end{array}$




\section{REVISED METHODOLOGY FOR DETERMINING CESIUM-137 CONTENT OF HN-200 GROUT CONTAINERS.}

\begin{tabular}{lccc} 
S09C14 & -22.799004 & -0.1162311 & 40.9495835 \\
S09C15 & -22.799004 & -0.1162311 & 32.761925 \\
S09C16 & -22.799004 & -0.1162311 & 24.5742665 \\
S09C17 & -22.799004 & -0.1162311 & 16.3866081 \\
S09C18 & -22.799004 & -0.1162311 & 8.19894964 \\
S09C19 & -0.572652 & -22.792107 & 49.2967588 \\
S09C20 & -0.572652 & -22.792107 & 40.7518865 \\
S09C21 & -0.572652 & -22.792107 & 32.2070142 \\
S09C22 & -0.572652 & -22.792107 & 23.6621418 \\
S09C23 & -0.572652 & -22.792107 & 15.1172695 \\
S09C24 & -0.572652 & -22.792107 & 6.57239719 \\
S09R01 & 22.7772241 & -1.0030659 & 41.340282 \\
S09R02 & 20.000901 & 10.9440412 & 41.3887574 \\
S09R03 & 11.6255809 & 19.6125967 & 41.6563913 \\
S09R04 & 0.59846811 & 22.7914439 & 42.0985638 \\
S09R05 & -9.5991807 & 20.6800341 & 42.6279515 \\
S09R06 & -16.767999 & 15.4480514 & 43.1300923 \\
S09R07 & -20.745248 & 9.45741825 & 43.514702 \\
S09R08 & -22.466807 & 3.87951985 & 43.7522364 \\
S09R09 & -22.768963 & -1.175754 & 43.8521489 \\
S09R10 & -21.993483 & -6.0078927 & 43.8256696 \\
S09R11 & -20.003996 & -10.938384 & 43.6694059 \\
S09R12 & -16.213618 & -16.028932 & 43.3694905 \\
S09R13 & -9.7805191 & -20.594891 & 42.9279023 \\
S09R14 & -0.4654769 & -22.794548 & 42.4004053 \\
S09R15 & 10.195546 & -20.392619 & 41.8947087 \\
S09R16 & 19.0095264 & -12.587533 & 41.5182684 \\
& & & \\
\hline
\end{tabular}

$\begin{array}{lcc}-22.937273 & -0.102115 & 40.8949821 \\ -22.937273 & -0.102115 & 32.6086658 \\ -22.937273 & -0.102115 & 24.3223494 \\ -22.937273 & -0.102115 & 16.0360331 \\ -22.937273 & -0.102115 & 7.74971675 \\ -0.5027158 & -22.93199 & 49.32158 \\ -0.5027158 & -22.93199 & 40.7211244 \\ -0.5027158 & -22.93199 & 32.1206688 \\ -0.5027158 & -22.93199 & 23.5202131 \\ -0.5027158 & -22.93199 & 14.9197575 \\ -0.5027158 & -22.93199 & 6.31930188 \\ 22.9156078 & -1.0019107 & 41.323479 \\ 20.1306967 & 10.994724 & 41.3718012 \\ 11.7324071 & 19.709884 & 41.6385886 \\ 0.66599741 & 22.9278292 & 42.0794057 \\ -9.5867772 & 20.8380088 & 42.6074348 \\ -16.816987 & 15.5986491 & 43.1089377 \\ -20.845114 & 9.57131776 & 43.4938593 \\ -22.596626 & 3.93972093 & 43.7320957 \\ -22.907416 & -1.1743994 & 43.8324446 \\ -22.121291 & -6.0644348 & 43.8058409 \\ -20.101917 & -11.047255 & 43.6489691 \\ -16.262402 & -16.176006 & 43.3484241 \\ -9.7678184 & -20.753762 & 42.9068941 \\ -0.3950196 & -22.934098 & 42.3804166 \\ 10.3068303 & -20.491417 & 41.876163 \\ 19.1424603 & -12.637053 & 41.5009022\end{array}$

Source S10

$\begin{array}{cccc}\text { Line } & \text { XINTi } & \text { ZINTi } & \text { YINTi } \\ \text { S10C01 } & 22.7962151 & 0.37504127 & 49.3911218 \\ \text { S10C02 } & 22.7962151 & 0.37504127 & 40.7111387 \\ \text { S10C03 } & 22.7962151 & 0.37504127 & 32.0311557 \\ \text { S10C04 } & 22.7962151 & 0.37504127 & 23.3511727 \\ \text { S10C05 } & 22.7962151 & 0.37504127 & 14.6711896 \\ \text { S10C06 } & 22.7962151 & 0.37504127 & 5.9912066 \\ \text { S10C07 } & -0.7001335 & 22.7885474 & 49.2372569 \\ \text { S10C08 } & -0.7001335 & 22.7885474 & 41.0095164 \\ \text { S10C09 } & -0.7001335 & 22.7885474 & 32.781776 \\ \text { S10C10 } & -0.7001335 & 22.7885474 & 24.5540355 \\ \text { S10C11 } & -0.7001335 & 22.7885474 & 16.326295 \\ \text { S10C12 } & -0.7001335 & 22.7885474 & 8.09855453 \\ \text { S10C13 } & -22.786309 & 0.76955981 & 49.2765891 \\ \text { S10C14 } & -22.786309 & 0.76955981 & 40.9332427\end{array}$

$\begin{array}{ccc}\text { XINTo } & \text { ZINTo } & \text { YINTo } \\ 22.9351348 & 0.32939043 & 49.4043747 \\ 22.9351348 & 0.32939043 & 40.6854385 \\ 22.9351348 & 0.32939043 & 31.9665023 \\ 22.9351348 & 0.32939043 & 23.2475661 \\ 22.9351348 & 0.32939043 & 14.5286299 \\ 22.9351348 & 0.32939043 & 5.80969371 \\ -0.6143816 & 22.9292704 & 49.2694375 \\ -0.6143816 & 22.9292704 & 40.9471112 \\ -0.6143816 & 22.9292704 & 32.6247849 \\ -0.6143816 & 22.9292704 & 24.3024585 \\ -0.6143816 & 22.9292704 & 15.9801322 \\ -0.6143816 & 22.9292704 & 7.65780584 \\ -22.927562 & 0.67513329 & 49.3040021 \\ -22.927562 & 0.67513329 & 40.880083\end{array}$




\section{REVISED METHODOLOGY FOR DETERMINING CESIUM-137 CONTENT OF HN-200 GROUT CONTAINERS.}

$\begin{array}{lccc}\text { S10C15 } & -22.786309 & 0.76955981 & 32.5898963 \\ \text { S10C16 } & -22.786309 & 0.76955981 & 24.2465499 \\ \text { S10C17 } & -22.786309 & 0.76955981 & 15.9032035 \\ \text { S10C18 } & -22.786309 & 0.76955981 & 7.55985706 \\ \text { S10C19 } & -0.2696851 & -22.797705 & 49.3987937 \\ \text { S10C20 } & -0.2696851 & -22.797705 & 40.6962611 \\ \text { S10C21 } & -0.2696851 & -22.797705 & 31.9937285 \\ \text { S10C22 } & -0.2696851 & -22.797705 & 23.2911959 \\ \text { S10C23 } & -0.2696851 & -22.797705 & 14.5886633 \\ \text { S10C24 } & -0.2696851 & -22.797705 & 5.88613064 \\ \text { S10R01 } & 21.1551086 & 8.50114465 & 42.6182712 \\ \text { S10R02 } & 14.4221594 & 17.6581256 & 43.2672529 \\ \text { S10R03 } & 5.73854018 & 22.0652949 & 43.9329823 \\ \text { S10R04 } & -1.8606725 & 22.7232475 & 44.4651448 \\ \text { S10R05 } & -7.4821553 & 21.5366068 & 44.8037487 \\ \text { S10R06 } & -11.630439 & 19.6097165 & 44.9696244 \\ \text { S10R07 } & -14.977122 & 17.1899355 & 44.9933114 \\ \text { S10R08 } & -17.962706 & 14.040985 & 44.880172 \\ \text { S10R09 } & -20.695195 & 9.56645159 & 44.6067774 \\ \text { S10R10 } & -22.609221 & 2.9378904 & 44.1418168 \\ \text { S10R11 } & -21.980276 & -6.056034 & 43.5093606 \\ \text { S10R12 } & -16.675797 & -15.547536 & 42.8352871 \\ \text { S10R13 } & -6.4359768 & -21.872043 & 42.2794167 \\ \text { S10R14 } & 6.165222 & -21.9499 & 41.9513772 \\ \text { S10R15 } & 17.014179 & -15.176488 & 41.9006539 \\ \text { S10R16 } & 22.4943134 & -3.7167117 & 42.1338318\end{array}$

$\begin{array}{lll}-22.927562 & 0.67513329 & 32.4561638\end{array}$

$\begin{array}{llll}-22.927562 & 0.67513329 & 24.0322447\end{array}$

$\begin{array}{llll}-22.927562 & 0.67513329 & 15.6083256\end{array}$

$\begin{array}{lll}-22.927562 & 0.67513329 & 7.18440652\end{array}$

$\begin{array}{lll}-0.2368979 & -22.936277 & 49.411098\end{array}$

$\begin{array}{llll}-0.2368979 & -22.936277 & 40.6724005\end{array}$

$\begin{array}{lll}-0.2368979 & -22.936277 & 31.933703\end{array}$

$\begin{array}{llll}-0.2368979 & -22.936277 & 23.1950055\end{array}$

$\begin{array}{lll}-0.2368979 & -22.936277 & 14.456308\end{array}$

$\begin{array}{lll}-0.2368979 & -22.936277 & 5.71761046\end{array}$

$\begin{array}{llll}21.3082397 & 8.49045518 & 42.5983181\end{array}$

$\begin{array}{llll}14.5796366 & 17.7077131 & 43.2451413\end{array}$

$\begin{array}{llll}5.86948536 & 22.1738145 & 43.9093807\end{array}$

$\begin{array}{lll}-1.7858547 & 22.8678733 & 44.4417113\end{array}$

$\begin{array}{lll}-7.472946 & 21.686032 & 44.7815274\end{array}$

$-11.680584 \quad 19.7406396 \quad 44.9484112$

$\begin{array}{llll}-15.076438 & 17.2866981 & 44.9722668\end{array}$

$\begin{array}{llll}-18.099855 & 14.0898596 & 44.8583783\end{array}$

$\begin{array}{llll}-20.853011 & 9.55410121 & 44.5837242\end{array}$

$\begin{array}{lll}-22.758597 & 2.8592257 & 44.1180802\end{array}$

$\begin{array}{lll}-22.087821 & -6.1852314 & 43.4865722\end{array}$

$\begin{array}{llll}-16.724743 & -15.697511 & 42.8145815\end{array}$

$\begin{array}{llll}-6.4281997 & -22.018337 & 42.2606512\end{array}$

$\begin{array}{llll}6.22152272 & -22.077626 & 41.9337434\end{array}$

$\begin{array}{llll}17.1110603 & -15.275488 & 41.883192\end{array}$

$\begin{array}{llll}22.6243559 & -3.7772247 & 42.1155723\end{array}$

\section{Source S11}

Line

S11C01

$\mathrm{S} 11 \mathrm{C} 02$

S11C03

S11C04

S11C05

S11C06

S11C07

S11C08

S11C09

S11C10

S11C11

S11C12

$\mathrm{S} 11 \mathrm{C} 13$

S11C14

S11C15

\section{XINTi}

22.789198

22.789198

22.789198

22.789198

22.789198

22.789198

0.86088994

0.86088994

0.86088994

0.86088994

0.86088994

0.86088994

$-22.79844$

$-22.79844$

$-22.79844$

\section{ZINTi}

0.6786289

0.6786289

0.6786289

0.6786289

0.6786289

0.6786289

$227830408 \quad 48.1607056$

$22.7830408-48.1607056$

$22.7830408 \quad 39.7542399$

$22.7830408 \quad 31.3477741$

$22.7830408 \quad 22.9413084$

$22.7830408 \quad 14.5348426$

$\begin{array}{lll}22.7830408 & 6.12837688\end{array}$

$0.19805618 \quad 48.8746585$

$0.19805618 \quad 40.1517907$

$0.19805618 \quad 31.4289229$
XINTo ZINTo YINTo $\begin{array}{llll}22.9297671 & 0.59555467 & 47.6195989\end{array}$ $\begin{array}{llll}22.9297671 & 0.59555467 & 39.4529351\end{array}$ $\begin{array}{llll}22.9297671 & 0.59555467 & 31.2862713\end{array}$ $\begin{array}{llll}22.9297671 & 0.59555467 & 23.1196075\end{array}$ $\begin{array}{llll}22.9297671 & 0.59555467 & 14.9529437\end{array}$ $\begin{array}{llll}22.9297671 & 0.59555467 & 6.78627986\end{array}$ $\begin{array}{llll}0.75497624 & 22.9250718 & 48.3254765\end{array}$ $\begin{array}{llll}0.75497624 & 22.9250718 & 39.8459894\end{array}$ $\begin{array}{lll}0.75497624 & 22.9250718 & 31.3665022\end{array}$ $\begin{array}{llll}0.75497624 & 22.9250718 & 22.887015\end{array}$ $\begin{array}{llll}0.75497624 & 22.9250718 & 14.4075279\end{array}$ $\begin{array}{llll}0.75497624 & 22.9250718 & 5.92804068\end{array}$ $\begin{array}{lll}-22.93684 & 0.17399156 & 48.95064\end{array}$ $\begin{array}{llll}-22.93684 & 0.17399156 & 40.1940995\end{array}$ $\begin{array}{llll}-22.93684 & 0.17399156 & 31.4375591\end{array}$ 


\section{REVISED METHODOLOGY FOR DETERMINING CESIUM-137 CONTENT OF HN-200 GROUT CONTAINERS.}

$\begin{array}{lcccccc}\text { S11C16 } & -22.79844 & 0.19805618 & 22.7060551 & -22.93684 & 0.17399156 & 22.6810186 \\ \text { S11C17 } & -22.79844 & 0.19805618 & 13.9831873 & -22.93684 & 0.17399156 & 13.9244781 \\ \text { S11C18 } & -22.79844 & 0.19805618 & 5.26031953 & -22.93684 & 0.17399156 & 5.16793769 \\ \text { S11C19 } & 0.49152195 & -22.794001 & 48.7353348 & 0.43158577 & -22.933439 & 48.8285781 \\ \text { S11C20 } & 0.49152195 & -22.794001 & 40.074211 & 0.43158577 & -22.933439 & 40.1261317 \\ \text { S11C21 } & 0.49152195 & -22.794001 & 31.4130872 & 0.43158577 & -22.933439 & 31.4236853 \\ \text { S11C22 } & 0.49152195 & -22.794001 & 22.7519634 & 0.43158577 & -22.933439 & 22.721239 \\ \text { S11C23 } & 0.49152195 & -22.794001 & 14.0908396 & 0.43158577 & -22.933439 & 14.0187926 \\ \text { S11C24 } & 0.49152195 & -22.794001 & 5.4297158 & 0.43158577 & -22.933439 & 5.31634627 \\ \text { S11R01 } & 21.9986295 & 5.98902181 & 29.0235839 & 22.1437703 & 5.98183428 & 29.0208553 \\ \text { S11R02 } & 20.708879 & 9.5367925 & 29.034673 & 20.8370818 & 9.5887918 & 29.0320425 \\ \text { S11R03 } & 18.9131401 & 12.7318975 & 29.0291979 & 19.0097678 & 12.835795 & 29.0265173 \\ \text { S11R04 } & 16.2299997 & 16.0123449 & 29.0055265 & 16.2787882 & 16.1595161 & 29.002666 \\ \text { S11R05 } & 11.8037648 & 19.5058765 & 28.9578785 & 11.7873957 & 19.6770478 & 28.9548167 \\ \text { S11R06 } & 4.4646368 & 22.3578867 & 28.8821901 & 4.37963061 & 22.5155 & 28.8791266 \\ \text { S11R07 } & -5.7838637 & 22.0534578 & 28.7886995 & -5.9145555 & 22.1618352 & 28.7859033 \\ \text { S11R08 } & -16.110273 & 16.132798 & 28.7009991 & -16.256875 & 16.1815616 & 28.6985323 \\ \text { S11R09 } & -22.249596 & 4.97630102 & 28.6391295 & -22.392497 & 4.97040878 & 28.6368927 \\ \text { S11R10 } & -21.329703 & -8.0530646 & 28.6131734 & -21.457153 & -8.1067562 & 28.6110289 \\ \text { S11R11 } & -13.375248 & -18.46377 & 28.6262812 & -13.477645 & -18.560226 & 28.6240904 \\ \text { S11R12 } & -1.2307106 & -22.766059 & 28.676939 & -1.2966414 & -22.900822 & 28.674563 \\ \text { S11R13 } & 10.734025 & -20.114393 & 28.7577851 & 10.7196836 & -20.278493 & 28.7551026 \\ \text { S11R14 } & 18.846497 & -12.83034 & 28.8521184 & 18.8976192 & -13.000342 & 28.8491211 \\ \text { S11R15 } & 22.280316 & -4.8368996 & 28.935788 & 22.3918848 & -4.9731682 & 28.9326929 \\ \text { S11R16 } & 22.7531751 & 1.4495186 & 28.9927275 & 22.8961871 & 1.3760534 & 28.9897932\end{array}$

\begin{tabular}{|c|c|c|c|c|c|c|}
\hline \multicolumn{7}{|c|}{ Source S12 } \\
\hline Line & XINTi & ZINTi & YINTi & XINTo & ZINTo & YINTo \\
\hline $\mathrm{S} 12 \mathrm{C} 01$ & 22.7486395 & 1.51903989 & 44.6026552 & 22.899094 & 1.32679994 & 45.2224318 \\
\hline $\mathrm{S} 12 \mathrm{C} 02$ & 22.7486395 & 1.51903989 & 37.1649737 & 22.899094 & 1.32679994 & 37.5870332 \\
\hline $\mathrm{S} 12 \mathrm{C} 03$ & 22.7486395 & 1.51903989 & 29.7272921 & 22.899094 & 1.32679994 & 29.9516347 \\
\hline S12C04 & 22.7486395 & 1.51903989 & 22.2896106 & 22.899094 & 1.32679994 & 22.3162361 \\
\hline S12C05 & 22.7486395 & 1.51903989 & 14.851929 & 22.899094 & 1.32679994 & 14.6808375 \\
\hline S12C06 & 22.7486395 & 1.51903989 & 7.41424743 & 22.899094 & 1.32679994 & 7.04543896 \\
\hline $\mathrm{S} 12 \mathrm{C} 07$ & 1.32405081 & 22.7608209 & 47.3141266 & 1.15816603 & 22.9082421 & 47.5879856 \\
\hline S12C08 & 1.32405081 & 22.7608209 & 39.0114495 & 1.15816603 & 22.9082421 & 39.1979437 \\
\hline$S 12 C 09$ & 1.32405081 & 22.7608209 & 30.7087723 & 1.15816603 & 22.9082421 & 30.8079019 \\
\hline $\mathrm{S} 12 \mathrm{C} 10$ & 1.32405081 & 22.7608209 & 22.4060951 & 1.15816603 & 22.9082421 & 22.41786 \\
\hline $\mathrm{S} 12 \mathrm{C} 11$ & 1.32405081 & 22.7608209 & 14.1034179 & 1.15816603 & 22.9082421 & 14.0278182 \\
\hline $\mathrm{S} 12 \mathrm{C} 12$ & 1.32405081 & 22.7608209 & 5.80074073 & 1.15816603 & 22.9082421 & 5.63777637 \\
\hline $\mathrm{S} 12 \mathrm{C} 13$ & -22.798004 & 0.24304779 & 48.7164203 & -22.936506 & 0.21350616 & 48.8116617 \\
\hline $\mathrm{S} 12 \mathrm{C} 14$ & -22.798004 & 0.24304779 & 39.9663927 & -22.936506 & 0.21350616 & 40.0312509 \\
\hline $\mathrm{S} 12 \mathrm{C} 15$ & -22.798004 & 0.24304779 & 31.2163651 & -22.936506 & 0.21350616 & 31.25084 \\
\hline $\mathrm{S} 12 \mathrm{C} 16$ & -22.798004 & 0.24304779 & 22.4663375 & -22.936506 & 0.21350616 & 22.4704291 \\
\hline
\end{tabular}




\section{REVISED METHODOLOGY FOR DETERMINING CESIUM-137 CONTENT OF HN-200 GROUT CONTAINERS.}

$\begin{array}{lccc}\text { S12C17 } & -22.798004 & 0.24304779 & 13.7163099 \\ \text { S12C18 } & -22.798004 & 0.24304779 & 4.9662823 \\ \text { S12C19 } & 0.59914937 & -22.791426 & 48.5108653 \\ \text { S12C20 } & 0.59914937 & -22.791426 & 39.8264125 \\ \text { S12C21 } & 0.59914937 & -22.791426 & 31.1419597 \\ \text { S12C22 } & 0.59914937 & -22.791426 & 22.4575069 \\ \text { S12C23 } & 0.59914937 & -22.791426 & 13.7730541 \\ \text { S12C24 } & 0.59914937 & -22.791426 & 5.08860127 \\ \text { S12R01 } & 21.1687996 & 8.46699492 & 21.46509 \\ \text { S12R02 } & 20.4442047 & 10.0917083 & 21.4474236 \\ \text { S12R03 } & 19.6486243 & 11.5645858 & 21.4544938 \\ \text { S12R04 } & 18.4888654 & 13.3405373 & 21.4913283 \\ \text { S12R05 } & 16.1543692 & 16.0886431 & 21.5867025 \\ \text { S12R06 } & 10.3590228 & 20.3100647 & 21.8000509 \\ \text { S12R07 } & -0.8704918 & 22.782676 & 22.1181863 \\ \text { S12R08 } & -13.68861 & 18.2326643 & 22.4151413 \\ \text { S12R09 } & -21.816062 & 6.6232569 & 22.6159103 \\ \text { S12R10 } & -21.47556 & -7.6556124 & 22.7003131 \\ \text { S12R11 } & -12.699052 & -18.93521 & 22.6649608 \\ \text { S12R12 } & 1.01511166 & -22.77669 & 22.510975 \\ \text { S12R13 } & 14.0831125 & -17.929697 & 22.2487225 \\ \text { S12R14 } & 21.4730269 & -7.6627147 & 21.9246981 \\ \text { S12R15 } & 22.7602163 & 1.33440485 & 21.6568612 \\ \text { S12R16 } & 21.9818664 & 6.05025866 & 21.5204759 \\ & & & \end{array}$

$\begin{array}{ccc}-22.936506 & 0.21350616 & 13.6900182 \\ -22.936506 & 0.21350616 & 4.90960726 \\ 0.52593682 & -22.93147 & 48.6317318 \\ 0.52593682 & -22.93147 & 39.9087209 \\ 0.52593682 & -22.93147 & 31.1857101 \\ 0.52593682 & -22.93147 & 22.4626993 \\ 0.52593682 & -22.93147 & 13.7396885 \\ 0.52593682 & -22.93147 & 5.01667763 \\ 21.3217949 & 8.45635663 & 21.4717302 \\ 20.5729269 & 10.1431545 & 21.453539 \\ 19.745961 & 11.6715865 & 21.4608243 \\ 18.5394139 & 13.5062592 & 21.4986691 \\ 16.1259149 & 16.3120745 & 21.5957972 \\ 10.231474 & 20.5291463 & 21.8097553 \\ -1.0385421 & 22.9139769 & 22.1259544 \\ -13.85156 & 18.2828662 & 22.4211088 \\ -21.96287 & 6.61522902 & 22.6209211 \\ -21.602957 & -7.7098102 & 22.7049786 \\ -12.80303 & -19.031851 & 22.6697672 \\ 0.94301253 & -22.918107 & 22.5164606 \\ 14.0614559 & -18.12193 & 22.2556444 \\ 21.5354495 & -7.896412 & 21.9337633 \\ 22.9091723 & 1.13961874 & 21.6665488 \\ 22.1503976 & 5.95724689 & 21.5284826\end{array}$

$\begin{array}{lll}-22.936506 & 0.21350616 & 13.6900182\end{array}$

$\begin{array}{llll}-22.936506 & 0.21350616 & 4.90960726\end{array}$

$\begin{array}{llll}0.52593682 & -22.93147 & 48.6317318\end{array}$

$\begin{array}{llll}0.52593682 & -22.93147 & 39.9087209\end{array}$

$\begin{array}{lll}0.52593682 & -22.93147 & 31.1857101\end{array}$

$\begin{array}{lll}0.52593682 & -22.93147 & 22.4626993\end{array}$

$\begin{array}{llll}0.52593682 & -22: 93147 & 13.7396885\end{array}$

$\begin{array}{llll}0.52593682 & -22.93147 & 5.01667763\end{array}$

$\begin{array}{llll}21.3217949 & 8.45635663 & 21.4717302\end{array}$

$\begin{array}{llll}20.5729269 & 10.1431545 & 21.453539\end{array}$

$\begin{array}{llll}19.745961 & 11.6715865 & 21.4608243\end{array}$

$\begin{array}{lll}18.5394139 & 13.5062592 & 21.4986691\end{array}$

$\begin{array}{llll}16.1259149 & 16.3120745 & 21.5957972\end{array}$

$\begin{array}{lll}10.231474 & 20.5291463 & 21.8097553\end{array}$

$\begin{array}{lll}-1.0385421 & 22.9139769 & 22.1259544\end{array}$

$\begin{array}{lll}-13.85156 & 18.2828662 & 22.4211088\end{array}$

$\begin{array}{lll}-21.96287 & 6.61522902 \quad 22.6209211\end{array}$

$\begin{array}{llll}-12.80303 & -19.031851 & 22.6697672\end{array}$

$\begin{array}{llll}0.94301253 & -22.918107 & 22.5164606\end{array}$

$\begin{array}{llll}14.0614559 & -18.12193 & 22.2556444\end{array}$

$\begin{array}{lll}21.5354495 & -7.896412 & 21.9337633\end{array}$

$\begin{array}{llll}22.1503976 & 5.95724689 & 21.5284826\end{array}$

$\begin{array}{cccc}\text { Line } & \text { XINTi } & \text { ZINTi } & \begin{array}{c}\text { Source S13 } \\ \text { YINTi }\end{array} \\ \text { S13C01 } & 22.7945643 & 0.46466894 & 49.0493973 \\ \text { S13C02 } & 22.7945643 & 0.46466894 & 40.5530104 \\ \text { S13C03 } & 22.7945643 & 0.46466894 & 32.0566235 \\ \text { S13C04 } & 22.7945643 & 0.46466894 & 23.5602366 \\ \text { S13C05 } & 22.7945643 & 0.46466894 & 15.0638497 \\ \text { S13C06 } & 22.7945643 & 0.46466894 & 6.56746275 \\ \text { S13C07 } & 0.25603392 & 22.7978623 & 48.9129828 \\ \text { S13C08 } & 0.25603392 & 22.7978623 & 40.5690587 \\ \text { S13C09 } & 0.25603392 & 22.7978623 & 32.2251345 \\ \text { S13C10 } & 0.25603392 & 22.7978623 & 23.8812103 \\ \text { S13C11 } & 0.25603392 & 22.7978623 & 15.5372862 \\ \text { S13C12 } & 0.25603392 & 22.7978623 & 7.19336202 \\ \text { S13C13 } & -22.796688 & 0.3451158 & 49.1653314 \\ \text { S13C14 } & -22.796688 & 0.3451158 & 40.5393715 \\ \text { S13C15 } & -22.796688 & 0.3451158 & 31.9134117 \\ \text { S13C16 } & -22.796688 & 0.3451158 & 23.2874518 \\ \text { S13C17 } & -22.796688 & 0.3451158 & 14.6614919\end{array}$

$\begin{array}{ccc}\text { XINTo } & \text { ZINTo } & \text { YINTo } \\ 22.9338705 & 0.408033 & 49.1043188 \\ 22.9338705 & 0.408033 & 40.5465493 \\ 22.9338705 & 0.408033 & 31.9887797 \\ 22.9338705 & 0.408033 & 23.4310102 \\ 22.9338705 & 0.408033 & 14.8732406 \\ 22.9338705 & 0.408033 & 6.31547107 \\ 0.2249103 & 22.9363973 & 48.9843409 \\ 0.2249103 & 22.9363973 & 40.5606638 \\ 0.2249103 & 22.9363973 & 32.1369868 \\ 0.2249103 & 22.9363973 & 23.7133097 \\ 0.2249103 & 22.9363973 & 15.2896326 \\ 0.2249103 & 22.9363973 & 6.86595547 \\ -22.935497 & 0.30312357 & 49.2060525 \\ -22.935497 & 0.30312357 & 40.534581 \\ -22.935497 & 0.30312357 & 31.8631095 \\ -22.935497 & 0.30312357 & 23.191638 \\ -22.935497 & 0.30312357 & 14.5201665\end{array}$


REVISED METHODOLOGY FOR DETERMINING CESIUM-137 CONTENT OF HN-200 GROUT CONTAINERS.

$\begin{array}{lccc}\text { S13C18 } & -22.796688 & 0.3451158 & 6.03553203 \\ \text { S13C19 } & 0.12402737 & -22.798963 & 49.2156385 \\ \text { S13C20 } & 0.12402737 & -22.798963 & 40.5334532 \\ \text { S13C21 } & 0.12402737 & -22.798963 & 31.851268 \\ \text { S13C22 } & 0.12402737 & -22.798963 & 23.1690828 \\ \text { S13C23 } & 0.12402737 & -22.798963 & 14.4868976 \\ \text { S13C24 } & 0.12402737 & -22.798963 & 5.80471235 \\ \text { S13R01 } & 21.62413 & 7.22530858 & 39.5381003 \\ \text { S13R02 } & 18.2748077 & 13.6322956 & 39.7594445 \\ \text { S13R03 } & 13.8362067 & 18.1209124 & 39.8920433 \\ \text { S13R04 } & 8.78048234 & 21.0407037 & 39.9346895 \\ \text { S13R05 } & 3.13977576 & 22.5820701 & 39.8886714 \\ \text { S13R06 } & -3.2000014 & 22.5736145 & 39.7526355 \\ \text { S13R07 } & -10.144686 & 20.4179683 & 39.5281183 \\ \text { S13R08 } & -16.909554 & 15.2929748 & 39.2333713 \\ \text { S13R09 } & -21.738916 & 6.87223575 & 38.9131954 \\ \text { S13R10 } & -22.484196 & -3.7774366 & 38.6290129 \\ \text { S13R11 } & -17.913637 & -14.103535 & 38.4363401 \\ \text { S13R12 } & -8.6758901 & -21.084046 & 38.3700867 \\ \text { S13R13 } & 2.79393797 & -22.627461 & 38.4414823 \\ \text { S13R14 } & 13.2951356 & -18.52154 & 38.6384093 \\ \text { S13R15 } & 20.2549074 & -10.466461 & 38.9250693 \\ \text { S13R16 } & 22.7710165 & -1.1352922 & 39.2453643\end{array}$

$\begin{array}{lll}-22.935497 & 0.30312357 & 5.84869503\end{array}$

$\begin{array}{llll}0.1089639 & -22.937241 & 49.250175\end{array}$

$\begin{array}{llll}0.1089639 & -22.937241 & 40.5293902\end{array}$

$\begin{array}{llll}0.1089639 & -22.937241 & 31.8086055\end{array}$

$\begin{array}{llll}0.1089639 & -22.937241 & 23.0878208\end{array}$

$\begin{array}{llll}0.1089639 & -22.937241 & 14.3670361\end{array}$

$\begin{array}{lll}0.1089639 & -22.937241 & 5.6462514\end{array}$

$\begin{array}{llll}21.7727269 & 7.21645829 & 39.5224362\end{array}$

$\begin{array}{llll}18.4106117 & 13.6813115 & 39.7439172\end{array}$

$\begin{array}{llll}13.9376201 & 18.2173448 & 39.8767654\end{array}$

$\begin{array}{llll}8.83330549 & 21.1684109 & 39.9195225\end{array}$

$\begin{array}{llll}3.13612283 & 22.7220959 & 39.8733854\end{array}$

$\begin{array}{llll}-3.261537 & 22.70443331 & 39.7370991\end{array}$

$\begin{array}{lll}-10.256143 & 20.5168329 & 39.5124551\end{array}$

$\begin{array}{llll}-17.051769 & 15.3416451 & 39.2179534\end{array}$

$\begin{array}{llll}-21.886438 & 6.86387089 & 38.8983904\end{array}$

$\begin{array}{lll}-22.614154 & -3.8378327 & 38.6149239\end{array}$

$\begin{array}{lll}-18.010096 & -14.204413 & 38.4227846\end{array}$

$\begin{array}{lll}-8.7288335 & -21.211704 & 38.3567196\end{array}$

$\begin{array}{llll}2.79069512 & -22.767102 & 38.4279123\end{array}$

$\begin{array}{llll}13.3442926 & -18.656333 & 38.624295\end{array}$

$\begin{array}{llll}20.3533621 & -10.576841 & 38.9102371\end{array}$

$\begin{array}{llll}22.9060124 & -1.2014579 & 39.2299293\end{array}$

\section{Source S14}

Line

S14C01

$\mathrm{S} 14 \mathrm{C} 02$

$\mathrm{S} 14 \mathrm{C} 03$

S14C04

S14C05

S14C06

S14C07

S14C08

S14C09

S14C10

S14C11

S14C12

S14C13

S14C14

S14C15

S14C16

S14C17

S14C18

\section{XINTi}

22.7986988

22.7986988

22.7986988

22.7986988

22.7986988

22.7986988

$-1.1983961$

$-1.1983961$

$-1.1983961$

$-1.1983961$

$-1.1983961$

$-1.1983961$

$-22.764283$

$-22.764283$

$-22.764283$

$-22.764283$

$-22.764283$

$-22.764283$
ZINT

0.1655667

0.1655667

0.1655667

0.1655667

0.1655667

0.1655667

22.7677827

22.7677827

22.7677827

22.7677827

22.7677827

22.7677827

1.2631365

1.2631365

1.2631365

1.2631365

1.2631365

1.2631365
YINTi

49.2413831

40.4843749

31.7273668

22.9703586

14.2133505

5.4563423

48.8707228

40.4619804

32.053238

23.6444955

15.2357531

6.82701071

47.5269674

40.3807935

33.2346196

26.0884457

18.9422718

11.7960979
XINTo ZINTo YINTo $\begin{array}{llll}22.9370388 & 0.14545389 & 49.2727995\end{array}$ $\begin{array}{llll}22.9370388 & 0.14545389 & 40.486273\end{array}$ $\begin{array}{lll}22.9370388 & 0.14545389 & 31.6997466\end{array}$ $\begin{array}{llll}22.9370388 & 0.14545389 & 22.9132201\end{array}$ $\begin{array}{llll}22.9370388 & 0.14545389 & 14.1266936\end{array}$ $\begin{array}{llll}22.9370388 & 0.14545389 & 5.34016717\end{array}$ $\begin{array}{llll}-1.0491108 & 22.9134954 & 48.9491124\end{array}$ $\begin{array}{lll}-1.0491108 & 22.9134954 & 40.4667165\end{array}$ $\begin{array}{lll}-1.0491108 & 22.9134954 & 31.9843206\end{array}$ $\begin{array}{lll}-1.0491108 & 22.9134954 & 23.5019247\end{array}$ $\begin{array}{llll}-1.0491108 & 22.9134954 & 15.0195288\end{array}$ $\begin{array}{llll}-1.0491108 & 22.9134954 & 6.53713284\end{array}$ $\begin{array}{llll}-22.910852 & 1.10533354 & 47.7734573\end{array}$ $\begin{array}{llll}-22.910852 & 1.10533354 & 40.3956859\end{array}$ $\begin{array}{llll}-22.910852 & 1.10533354 & 33.0179145\end{array}$ $\begin{array}{lll}-22.910852 & 1.10533354 & 25.6401431\end{array}$ $\begin{array}{llll}-22.910852 & 1.10533354 & 18.2623717\end{array}$ $\begin{array}{llll}-22.910852 & 1.10533354 & 10.8846003\end{array}$ 
REVISED METHODOLOGY FOR DETERMINING CESIUM-137 CONTENT OF HN-200 GROUT CONTAINERS.

$\begin{array}{lccc}\text { S14C19 } & -0.6969486 & -22.788645 & 49.1340326 \\ \text { S14C20 } & -0.6969486 & -22.788645 & 40.477889 \\ \text { S14C21 } & -0.6969486 & -22.788645 & 31.8217454 \\ \text { S14C22 } & -0.6969486 & -22.788645 & 23.1656018 \\ \text { S14C23 } & -0.6969486 & -22.788645 & 14.5094582 \\ \text { S14C24 } & -0.6969486 & -22.788645 & 5.85331454 \\ \text { S14R01 } & 22.3327774 & 4.58858755 & 36.6055981 \\ \text { S14R02 } & 15.2446872 & 16.9530998 & 36.9867255 \\ \text { S14R03 } & 2.52758086 & 22.6587602 & 37.6285104 \\ \text { S14R04 } & -9.8964447 & 20.5394367 & 38.4143389 \\ \text { S14R05 } & -17.03958 & 15.1479638 & 39.0532676 \\ \text { S14R06 } & -19.759404 & 11.3742704 & 39.3729826 \\ \text { S14R07 } & -20.879942 & 9.15620563 & 39.501759 \\ \text { S14R08 } & -21.519565 & 7.53102814 & 39.5419465 \\ \text { S14R09 } & -22.01003 & 5.94698809 & 39.5232388 \\ \text { S14R10 } & -22.46784 & 3.87353335 & 39.4322075 \\ \text { S14R11 } & -22.798087 & 0.23518226 & 39.1967451 \\ \text { S14R12 } & -21.702665 & -6.9858721 & 38.6709371 \\ \text { S14R13 } & -15.438319 & -16.77696 & 37.8970929 \\ \text { S14R14 } & -3.080926 & -22.590174 & 37.1836401 \\ \text { S14R15 } & 10.8813945 & -20.035053 & 36.7063175 \\ \text { S14R16 } & 20.6715223 & -9.617497 & 36.5111505\end{array}$

$\begin{array}{ccc}-0.6115935 & -22.929345 & 49.1788525 \\ -0.6115935 & -22.929345 & 40.4805969 \\ -0.6115935 & -22.929345 & 31.7823413 \\ -0.6115935 & -22.929345 & 23.0840857 \\ -0.6115935 & -22.929345 & 14.3858301 \\ -0.6115935 & -22.929345 & 5.68757454 \\ 22.4749501 & 4.58317838 & 36.59398 \\ 15.3965806 & 17.0021826 & 36.9734145 \\ 2.68038867 & 22.7803517 & 37.6116416 \\ -9.7739772 & 20.750862 & 38.3922246 \\ -17.007162 & 15.3910798 & 39.029861 \\ -19.812619 & 11.5580723 & 39.3537576 \\ -20.980302 & 9.2712375 & 39.4858003 \\ -21.646958 & 7.58538924 & 39.5272041 \\ -22.155068 & 5.9398554 & 39.5079188 \\ -22.622032 & 3.79112073 & 39.4143636 \\ -22.937434 & 0.05482973 & 39.1745867 \\ -21.767587 & -7.2319485 & 38.6474827 \\ -15.412528 & -16.987728 & 37.8784701 \\ -3.0017145 & -22.740242 & 37.1693367 \\ 10.9904435 & -20.133034 & 36.6942794 \\ 20.7997922 & -9.6694131 & 36.4999097\end{array}$

$\begin{array}{lll}-0.6115935 & -22.929345 & 49.1788525\end{array}$

$\begin{array}{llll}-0.6115935 & -22.929345 & 40.4805969\end{array}$

$\begin{array}{llll}-0.6115935 & -22.929345 & 31.7823413\end{array}$

$\begin{array}{lll}-0.6115935 & -22.929345 & 23.0840857\end{array}$

$\begin{array}{llll}-0.6115935 & -22.929345 & 14.3858301\end{array}$

$\begin{array}{llll}-0.6115935 & -22.929345 & 5.68757454\end{array}$

$\begin{array}{llll}22.4749501 & 4.58317838 & 36.59398\end{array}$

$\begin{array}{lll}15.3965806 & 17.0021826 & 36.9734145\end{array}$

$\begin{array}{llll}2.68038867 & 22.7803517 & 37.6116416\end{array}$

$\begin{array}{llll}-9.7739772 & 20.750862 & 38.3922246\end{array}$

$\begin{array}{lll}-17.007162 & 15.3910798 & 39.029861\end{array}$

$\begin{array}{llll}-19.812619 & 11.5580723 & 39.3537576\end{array}$

$\begin{array}{llll}-20.980302 & 9.2712375 & 39.4858003\end{array}$

$\begin{array}{llll}-21.646958 & 7.58538924 & 39.5272041\end{array}$

$\begin{array}{llll}-22.155068 & 5.9398554 & 39.5079188\end{array}$

$\begin{array}{llll}-22.622032 & 3.79112073 & 39.4143636\end{array}$

$\begin{array}{llll}-22.937434 & 0.05482973 & 39.1745867\end{array}$

$\begin{array}{llll}-21.767587 & -7.2319485 & 38.6474827\end{array}$

$\begin{array}{lll}-15.412528 & -16.987728 & 37.8784701\end{array}$

$\begin{array}{llll}-3.0017145 & -22.740242 & 37.1693367\end{array}$

$\begin{array}{llll}20.7997922 & -9.6694131 & 36.4999097\end{array}$

$\begin{array}{lccc}\text { Line } & \text { XINTi } & \text { ZINTi } & \begin{array}{c}\text { Source S15 } \\ \text { YINTi }\end{array} \\ \text { S15C01 } & 22.6815595 & 2.3140746 & 32.898817 \\ \text { S15C02 } & 22.6815595 & 2.3140746 & 27.7501653 \\ \text { S15C03 } & 22.6815595 & 2.3140746 & 22.6015136 \\ \text { S15C04 } & 22.6815595 & 2.3140746 & 17.4528619 \\ \text { S15C05 } & 22.6815595 & 2.3140746 & 12.3042102 \\ \text { S15C06 } & 22.6815595 & 2.3140746 & 7.15555843 \\ \text { S15C07 } & 1.33438341 & 22.7602175 & 47.0352261 \\ \text { S15C08 } & 1.33438341 & 22.7602175 & 38.6070349 \\ \text { S15C09 } & 1.33438341 & 22.7602175 & 30.1788437 \\ \text { S15C10 } & 1.33438341 & 22.7602175 & 21.7506525 \\ \text { S15C11 } & 1.33438341 & 22.7602175 & 13.3224613 \\ \text { S15C12 } & 1.33438341 & 22.7602175 & 4.89427017 \\ \text { S15C13 } & -22.798888 & 0.13700898 & 48.5170969 \\ \text { S15C14 } & -22.798888 & 0.13700898 & 39.7451229 \\ \text { S15C15 } & -22.798888 & 0.13700898 & 30.973149 \\ \text { S15C16 } & -22.798888 & 0.13700898 & 22.201175 \\ \text { S15C17 } & -22.798888 & 0.13700898 & 13.4292011 \\ \text { S15C18 } & -22.798888 & 0.13700898 & 4.65722715 \\ \text { S15C19 } & 0.82531416 & -22.784357 & 47.975541\end{array}$

$\begin{array}{ccc}\text { XINTo } & \text { ZINTo } & \text { YINTo } \\ 22.8497782 & 2.00413094 & 35.1223544 \\ 22.8497782 & 2.00413094 & 29.4578589 \\ 22.8497782 & 2.00413094 & 23.7933634 \\ 22.8497782 & 2.00413094 & 18.1288679 \\ 22.8497782 & 2.00413094 & 12.4643724 \\ 22.8497782 & 2.00413094 & 6.79987694 \\ 1.16712098 & 22.9077876 & 47.3441808 \\ 1.16712098 & 22.9077876 & 38.8443145 \\ 1.16712098 & 22.9077876 & 30.3444481 \\ 1.16712098 & 22.9077876 & 21.8445818 \\ 1.16712098 & 22.9077876 & 13.3447155 \\ 1.16712098 & 22.9077876 & 4.84484915 \\ -22.937184 & 0.12036786 & 48.6364804 \\ -22.937184 & 0.12036786 & 39.8368104 \\ -22.937184 & 0.12036786 & 31.0371404 \\ -22.937184 & 0.12036786 & 22.2374703 \\ -22.937184 & 0.12036786 & 13.4378003 \\ -22.937184 & 0.12036786 & 4.63813033 \\ 0.72388588 & -22.926075 & 48.1628918\end{array}$


REVISED METHODOLOGY FOR DETERMINING CESIUM-137 CONTENT OF HN-200 GROUT CONTAINERS.

$\begin{array}{llll}\text { S15C20 } & 0.82531416 & -22.784357 & 39.3292039 \\ \text { S15C21 } & 0.82531416 & -22.784357 & 30.6828668 \\ \text { S15C22 } & 0.82531416 & -22.784357 & 22.0365297 \\ \text { S15C23 } & 0.82531416 & -22.784357 & 13.3901926 \\ \text { S15C24 } & 0.82531416 & -22.784357 & 4.74385551 \\ \text { S15R01 } & 22.1611979 & 5.35624756 & 10.8580806 \\ \text { S15R02 } & 22.0321233 & 5.86460774 & 10.8543694 \\ \text { S15R03 } & 21.8697604 & 6.44373048 & 10.8793952 \\ \text { S15R04 } & 21.5467446 & 7.45291072 & 10.9647287 \\ \text { S15R05 } & 20.2196333 & 10.534444 & 11.3037249 \\ \text { S15R06 } & 13.0675816 & 18.6827833 & 12.3873673 \\ \text { S15R07 } & -1.1532025 & 22.7701165 & 13.6175763 \\ \text { S15R08 } & -15.139561 & 17.0470457 & 14.4942016 \\ \text { S15R09 } & -22.45023 & 3.97432279 & 14.958234 \\ \text { S15R10 } & -20.112087 & -10.738344 & 15.0154435 \\ \text { S15R11 } & -9.1421341 & -20.886107 & 14.6675683 \\ \text { S15R12 } & 5.89598493 & -22.023747 & 13.9073169 \\ \text { S15R13 } & 18.3144561 & -13.578983 & 12.7591366 \\ \text { S15R14 } & 22.7379345 & -1.67165 & 11.5318938 \\ \text { S15R15 } & 22.5523348 & 3.34668155 & 11.0161698 \\ \text { S15R16 } & 22.3095465 & 4.70023561 & 10.8944496\end{array}$

$\begin{array}{cccc}\text { Line } & \text { XINTi } & \text { ZINTi } & \begin{array}{c}\text { Source S16 } \\ \text { YINTi }\end{array} \\ \text { S16C01 } & 22.7738213 & 1.07756348 & 48.4370401 \\ \text { S16C02 } & 22.7738213 & 1.07756348 & 40.3756945 \\ \text { S16C03 } & 22.7738213 & 1.07756348 & 32.314349 \\ \text { S16C04 } & 22.7738213 & 1.07756348 & 24.2530034 \\ \text { S16C05 } & 22.7738213 & 1.07756348 & 16.1916579 \\ \text { S16C06 } & 22.7738213 & 1.07756348 & 8.13031231 \\ \text { S16C07 } & 1.09378443 & 22.773048 & 48.6277176 \\ \text { S16C08 } & 1.09378443 & 22.773048 & 40.3979929 \\ \text { S16C09 } & 1.09378443 & 22.773048 & 32.1682682 \\ \text { S16C10 } & 1.09378443 & 22.773048 & 23.9385435 \\ \text { S16C11 } & 1.09378443 & 22.773048 & 15.7088188 \\ \text { S16C12 } & 1.09378443 & 22.773048 & 7.47909406 \\ \text { S16C13 } & -22.797041 & 0.32091274 & 49.1834364 \\ \text { S16C14 } & -22.797041 & 0.32091274 & 40.4629802 \\ \text { S16C15 } & -22.797041 & 0.32091274 & 31.742524 \\ \text { S16C16 } & -22.797041 & 0.32091274 & 23.0220677 \\ \text { S16C17 } & -22.797041 & 0.32091274 & 14.3016115 \\ \text { S16C18 } & -22.797041 & 0.32091274 & 5.5811553 \\ \text { S16C19 } & 0.42594763 & -22.795321 & 49.1603109 \\ \text { S16C20 } & 0.42594763 & -22.795321 & 40.4602758\end{array}$

$\begin{array}{lll}0.72388588 & -22.926075 & 39.4730907 \\ 0.72388588 & -22.926075 & 30.7832897 \\ 0.72388588 & -22.926075 & 22.0934886 \\ 0.72388588 & -22.926075 & 13.4036876 \\ 0.72388588 & -22.926075 & 4.71388651 \\ 22.3048815 & 5.3498754 & 10.8769867 \\ 22.1600146 & 5.92137306 & 10.8728463 \\ 21.9761799 & 6.57087703 & 10.9007196 \\ 21.6099076 & 7.69030564 & 10.9948027 \\ 20.1566081 & 10.9471483 & 11.3518714 \\ 12.8968667 & 18.9683878 & 12.4235486 \\ -1.3184057 & 22.8995789 & 13.6392891 \\ -15.292137 & 17.0961821 & 14.5101953 \\ -22.591384 & 3.96966676 & 14.9720484 \\ -20.241592 & -10.789202 & 15.0290188 \\ -9.2572198 & -20.986491 & 14.6826941 \\ 5.80382848 & -22.19109 & 13.9268486 \\ 18.2742572 & -13.862916 & 12.7898456 \\ 22.8416494 & -2.094745 & 11.5812759 \\ 22.7246553 & 3.11752268 & 11.0506994 \\ 22.4686137 & 4.6141416 & 10.9174306\end{array}$

$\begin{array}{lll}0.72388588 & -22.926075 & 39.4730907\end{array}$

$\begin{array}{llll}0.72388588 & -22.926075 & 30.7832897\end{array}$

$\begin{array}{llll}0.72388588 & -22.926075 & 22.0934886\end{array}$

$\begin{array}{llll}0.72388588 & -22.926075 & 13.4036876\end{array}$

$\begin{array}{llll}0.72388588 & -22.926075 & 4.71388651\end{array}$

$\begin{array}{llll}22.3048815 & 5.3498754 & 10.8769867\end{array}$

$22.1600146 \quad 5.92137306 \quad 10.8728463$

$21.9761799 \quad 6.57087703 \quad 10.9007196$

$21.6099076 \quad 7.69030564 \quad 10.9948027$

$\begin{array}{llll}20.1566081 & 10.9471483 & 11.3518714\end{array}$

$\begin{array}{llll}12.8968667 & 18.9683878 & 12.4235486\end{array}$

$\begin{array}{llll}-1.3184057 & 22.8995789 & 13.6392891\end{array}$

$\begin{array}{llll}-15.292137 & 17.0961821 & 14.5101953\end{array}$

$\begin{array}{llll}-22.591384 & 3.96966676 & 14.9720484\end{array}$

$\begin{array}{llll}-20.241592 & -10.789202 & 15.0290188\end{array}$

$\begin{array}{llll}-9.2572198 & -20.986491 & 14.6826941\end{array}$

$\begin{array}{llll}5.80382848 & -22.19109 & 13.9268486\end{array}$

$\begin{array}{llll}18.2742572 & -13.862916 & 12.7898456\end{array}$

$\begin{array}{llll}22.8416494 & -2.094745 & 11.5812759\end{array}$

$\begin{array}{llll}22.4686137 & 4.6141416 & 10.9174306\end{array}$ 
REVISED METHODOLOGY FOR DETERMINING CESIUM-137 CONTENT OF HN-200 GROUT CONTAINERS.

$\begin{array}{lccc}\text { S16C21 } & 0.42594763 & -22.795321 & 31.7602407 \\ \text { S16C22 } & 0.42594763 & -22.795321 & 23.0602056 \\ \text { S16C23 } & 0.42594763 & -22.795321 & 14.3601705 \\ \text { S16C24 } & 0.42594763 & -22.795321 & 5.66013541 \\ \text { S16R01 } & 20.6287757 & 9.70884612 & 38.5508458 \\ \text { S16R02 } & 18.9767235 & 12.6369319 & 38.6557026 \\ \text { S16R03 } & 17.2417594 & 14.9174332 & 38.6735112 \\ \text { S16R04 } & 15.1035049 & 17.0789994 & 38.6120491 \\ \text { S16R05 } & 11.9015098 & 19.4463916 & 38.4449652 \\ \text { S16R06 } & 6.37214903 & 21.890724 & 38.1156331 \\ \text { S16R07 } & -2.666637 & 22.6428162 & 37.6043446 \\ \text { S16R08 } & -13.490905 & 18.3794331 & 37.0303139 \\ \text { S16R09 } & -21.323639 & 8.06910744 & 36.5577868 \\ \text { S16R10 } & -22.161109 & -5.356615 & 36.2785609 \\ \text { S16R11 } & -15.171553 & -17.01858 & 36.2250242 \\ \text { S16R12 } & -2.837329 & -22.622061 & 36.4017276 \\ \text { S16R13 } & 10.2190306 & -20.380861 & 36.7916846 \\ \text { S16R14 } & 19.3645814 & -12.034163 & 37.3355383 \\ \text { S16R15 } & 22.6949747 & -2.1785782 & 37.8978794 \\ \text { S16R16 } & 22.199239 & 5.19633208 & 38.3164188\end{array}$

$\begin{array}{lccl}\text { Line } & \text { XINTi } & \text { ZINTi } & \begin{array}{c}\text { Source S17 } \\ \text { YINTi }\end{array} \\ \text { S17C01 } & 22.7991153 & 0.09178379 & 48.2911655 \\ \text { S17C02 } & 22.7991153 & 0.09178379 & 39.5577816 \\ \text { S17C03 } & 22.7991153 & 0.09178379 & 30.8243977 \\ \text { S17C04 } & 22.7991153 & 0.09178379 & 22.0910138 \\ \text { S17C05 } & 22.7991153 & 0.09178379 & 13.3576299 \\ \text { S17C06 } & 22.7991153 & 0.09178379 & 4.62424599 \\ \text { S17C07 } & -0.8012248 & 22.7852171 & 47.2436792 \\ \text { S17C08 } & -0.8012248 & 22.7852171 & 38.741325 \\ \text { S17C09 } & -0.8012248 & 22.7852171 & 30.2389708 \\ \text { S17C10 } & -0.8012248 & 22.7852171 & 21.7366167 \\ \text { S17C11 } & -0.8012248 & 22.7852171 & 13.2342625 \\ \text { S17C12 } & -0.8012248 & 22.7852171 & 4.73190834 \\ \text { S17C13 } & -22.796228 & 0.374289 & 44.5704425 \\ \text { S17C14 } & -22.796228 & 0.374289 & 36.6576876 \\ \text { S17C15 } & -22.796228 & 0.374289 & 28.7449327 \\ \text { S17C16 } & -22.796228 & 0.374289 & 20.8321778 \\ \text { S17C17 } & -22.796228 & 0.374289 & 12.9194229 \\ \text { S17C18 } & -22.796228 & 0.374289 & 5.00666797 \\ \text { S17C19 } & -0.6144757 & -22.791018 & 47.7695814 \\ \text { S17C20 } & -0.6144757 & -22.791018 & 39.1512362 \\ \text { S17C21 } & -0.6144757 & -22.791018 & 30.5328909\end{array}$

$\begin{array}{ccc}0.37406292 & -22.93445 & 31.7285408 \\ 0.37406292 & -22.93445 & 22.9919669 \\ 0.37406292 & -22.93445 & 14.2553931 \\ 0.37406292 & -22.93445 & 5.51881923 \\ 20.787289 & 9.69626337 & 38.5355518 \\ 19.1097763 & 12.686424 & 38.6416941 \\ 17.3384902 & 15.016846 & 38.6597528 \\ 15.1521818 & 17.2203453 & 38.5974667 \\ 11.8849437 & 19.6182828 & 38.4286866 \\ 6.27729238 & 22.0618337 & 38.0979801 \\ -2.8183101 & 22.7636999 & 37.5876147 \\ -13.655415 & 18.4298275 & 37.01605 \\ -21.475114 & 8.05905707 & 36.5455709 \\ -22.289356 & -5.4141976 & 36.2674412 \\ -15.27056 & -17.115458 & 36.2141035 \\ -2.8996131 & -22.753487 & 36.3901364 \\ 10.2055924 & -20.542025 & 36.7784796 \\ 19.4167609 & -12.2114 & 37.3199066 \\ 22.81841 & -2.3343245 & 37.8803228 \\ 22.3615495 & 5.1078379 & 38.2993298\end{array}$




\section{REVISED METHODOLOGY FOR DETERMINING CESIUM-137 CONTENT OF HN-200 GROUT CONTAINERS.}

$\begin{array}{llll}\text { S17C22 } & -0.6144757 & -22.791018 & 21.9145457 \\ \text { S17C23 } & -0.6144757 & -22.791018 & 13.2962005 \\ \text { S17C24 } & -0.6144757 & -22.791018 & 4.67785525 \\ \text { S17R01 } & 22.6761152 & 2.36682946 & 12.956831 \\ \text { S17R02 } & 17.6606196 & 14.4191052 & 12.6553677 \\ \text { S17R03 } & 7.32270886 & 21.5913412 & 12.10224 \\ \text { S17R04 } & -4.1216592 & 22.4236484 & 11.3998997 \\ \text { S17R05 } & -12.860563 & 18.8258861 & 10.7248292 \\ \text { S17R06 } & -17.919429 & 14.0961744 & 10.2339446 \\ \text { S17R07 } & -20.524786 & 9.92679411 & 9.95254381 \\ \text { S17R08 } & -21.897348 & 6.34934886 & 9.83167634 \\ \text { S17R09 } & -22.613812 & 2.90234151 & 9.83602814 \\ \text { S17R10 } & -22.778387 & -0.9763081 & 9.96698631 \\ \text { S17R11 } & -22.009607 & -5.9485516 & 10.2621907 \\ \text { S17R12 } & -19.110935 & -12.43303 & 10.7689847 \\ \text { S17R13 } & -12.276571 & -19.211816 & 11.4525246 \\ \text { S17R14 } & -1.250071 & -22.765004 & 12.1492719 \\ \text { S17R15 } & 11.0625699 & -19.935587 & 12.6865701 \\ \text { S17R16 } & 20.1950199 & -10.581552 & 12.9676279\end{array}$

$\begin{array}{lll}-0.5393656 & -22.931158 & 21.9861084 \\ -0.5393656 & -22.931158 & 13.3211118 \\ -0.5393656 & -22.931158 & 4.6561152 \\ 22.8153454 & 2.36408931 & 12.9727998 \\ 17.7991391 & 14.46788 & 12.6724345 \\ 7.44552253 & 21.6954627 & 12.1214289 \\ -4.0381807 & 22.5792383 & 11.4216843 \\ -12.841939 & 19.0056175 & 10.7480359 \\ -17.969271 & 14.2560239 & 10.2563256 \\ -20.623949 & 10.0390049 & 9.97322349 \\ -22.024988 & 6.40537187 & 9.85127937 \\ -22.753568 & 2.89897043 & 9.85567378 \\ -22.91378 & -1.0428702 & 9.98778005 \\ -22.117476 & -6.0783337 & 10.2846858 \\ -19.16256 & -12.606555 & 10.7921665 \\ -12.259229 & -19.386599 & 11.4741348 \\ -1.1772408 & -22.90727 & 12.1682777 \\ 11.1710586 & -20.033381 & 12.7035208 \\ 20.3243168 & -10.632547 & 12.9835584\end{array}$

$\begin{array}{cccc}\text { Line } & \text { XINTi } & \text { ZINTi } & \begin{array}{c}\text { Source S18 } \\ \text { YINTi }\end{array} \\ \text { S18C01 } & 22.580964 & -3.1477208 & 39.2781683 \\ \text { S18C02 } & 22.580964 & -3.1477208 & 32.6383147 \\ \text { S18C03 } & 22.580964 & -3.1477208 & 25.9984612 \\ \text { S18C04 } & 22.580964 & -3.1477208 & 19.3586076 \\ \text { S18C05 } & 22.580964 & -3.1477208 & 12.7187541 \\ \text { S18C06 } & 22.580964 & -3.1477208 & 6.07890051 \\ \text { S18C07 } & 0.59835146 & 22.791447 & 48.2570618 \\ \text { S18C08 } & 0.59835146 & 22.791447 & 39.5440471 \\ \text { S18C09 } & 0.59835146 & 22.791447 & 30.8310325 \\ \text { S18C10 } & 0.59835146 & 22.791447 & 22.1180179 \\ \text { S18C11 } & 0.59835146 & 22.791447 & 13.4050033 \\ \text { S18C12 } & 0.59835146 & 22.791447 & 4.69198867 \\ \text { S18C13 } & -22.797046 & -0.320574 & 48.4589757 \\ \text { S18C14 } & -22.797046 & -0.320574 & 39.6993406 \\ \text { S18C15 } & -22.797046 & -0.320574 & 30.9397055 \\ \text { S18C16 } & -22.797046 & -0.320574 & 22.1800705 \\ \text { S18C17 } & -22.797046 & -0.320574 & 13.4204354 \\ \text { S18C18 } & -22.797046 & -0.320574 & 4.66080032 \\ \text { S18C19 } & 1.91650811 & -22.718606 & 45.5188929 \\ \text { S18C20 } & 1.91650811 & -22.718606 & 37.4381015 \\ \text { S18C21 } & 1.91650811 & -22.718606 & 29.3573101 \\ \text { S18C22 } & 1.91650811 & -22.718606 & 21.2765188\end{array}$

$\begin{array}{ccc}\text { XINTo } & \text { ZINTo } & \text { YINTo } \\ 22.7795945 & -2.6868165 & 40.7748976 \\ 22.7795945 & -2.6868165 & 33.7894602 \\ 22.7795945 & -2.6868165 & 26.8040227 \\ 22.7795945 & -2.6868165 & 19.8185853 \\ 22.7795945 & -2.6868165 & 12.8331478 \\ 22.7795945 & -2.6868165 & 5.84771037 \\ 0.52523765 & 22.9314856 & 48.408939 \\ 0.52523765 & 22.9314856 & 39.660857 \\ 0.52523765 & 22.9314856 & 30.912775 \\ 0.52523765 & 22.9314856 & 22.1646931 \\ 0.52523765 & 22.9314856 & 13.4166111 \\ 0.52523765 & 22.9314856 & 4.66852917 \\ -22.935772 & -0.2815792 & 48.5856065 \\ -22.935772 & -0.2815792 & 39.7967333 \\ -22.935772 & -0.2815792 & 31.0078601 \\ -22.935772 & -0.2815792 & 22.2189869 \\ -22.935772 & -0.2815792 & 13.4301137 \\ -22.935772 & -0.2815792 & 4.64124048 \\ 1.66779608 & -22.876787 & 46.0355353 \\ 1.66779608 & -22.876787 & 37.8354549 \\ 1.66779608 & -22.876787 & 29.6353746 \\ 1.66779608 & -22.876787 & 21.4352942\end{array}$




\section{REVISED METHODOLOGY FOR DETERMINING CESIUM-137 CONTENT OF HN-200 GROUT CONTAINERS.}

$\begin{array}{lccc}\text { S18C23 } & 1.91650811 & -22.718606 & 13.1957274 \\ \text { S18C24 } & 1.91650811 & -22.718606 & 5.11493597 \\ \text { S18R01 } & 19.423011 & -11.939628 & 10.6069114 \\ \text { S18R02 } & 19.7214904 & -11.439882 & 10.6579331 \\ \text { S18R03 } & 20.9435243 & -9.0098207 & 10.9504927 \\ \text { S18R04 } & 22.5675614 & 3.24241451 & 12.2383638 \\ \text { S18R05 } & 15.2593339 & 16.9399176 & 13.5524878 \\ \text { S18R06 } & 1.02865081 & 22.776083 & 14.4389336 \\ \text { S18R07 } & -13.459349 & 18.4025546 & 14.8848538 \\ \text { S18R08 } & -21.989899 & 6.02099948 & 14.9062401 \\ \text { S18R09 } & -20.923066 & -9.0572287 & 14.5040873 \\ \text { S18R10 } & -10.437082 & -20.270062 & 13.6631003 \\ \text { S18R11 } & 4.97986013 & -22.248799 & 12.3876841 \\ \text { S18R12 } & 16.4491151 & -15.787169 & 11.0323727 \\ \text { S18R13 } & 18.5940005 & -13.193606 & 10.6683972 \\ \text { S18R14 } & 18.9612199 & -12.660182 & 10.6096594 \\ \text { S18R15 } & 19.1318952 & -12.400753 & 10.5932812 \\ \text { S18R16 } & 19.2655511 & -12.192072 & 10.5926198\end{array}$

$\begin{array}{ccc}1.66779608 & -22.876787 & 13.2352139 \\ 1.66779608 & -22.876787 & 5.03513355 \\ 19.5951867 & -11.922985 & 10.6294144 \\ 19.9618645 & -11.298357 & 10.6923667 \\ 21.2920172 & -8.5310557 & 11.0192074 \\ 22.6546036 & 3.5913568 & 12.2776739 \\ 15.2341486 & 17.1478752 & 13.5742702 \\ 0.95651004 & 22.9175477 & 14.4548703 \\ -13.56156 & 18.4989995 & 14.898696 \\ -22.1177 & 6.07752136 & 14.9199921 \\ -21.07853 & -9.0456884 & 14.5196904 \\ -10.631208 & -20.325017 & 13.6839892 \\ 4.71123423 & -22.448456 & 12.4240633 \\ 16.130967 & -16.307078 & 11.1026554 \\ 18.5515908 & -13.489529 & 10.7050765 \\ 19.0125512 & -12.831672 & 10.6328418 \\ 19.2286729 & -12.505481 & 10.6123635 \\ 19.3975883 & -12.241833 & 10.6115341\end{array}$

$\begin{array}{lccc}\text { Line } & \text { XINTi } & \text { ZINTi } & \begin{array}{c}\text { Source S19 } \\ \text { YINTi }\end{array} \\ \text { S19C01 } & 22.7992988 & 0.00751195 & 48.7339354 \\ \text { S19C02 } & 22.7992988 & 0.00751195 & 39.9767033 \\ \text { S19C03 } & 22.7992988 & 0.00751195 & 31.2194713 \\ \text { S19C04 } & 22.7992988 & 0.00751195 & 22.4622392 \\ \text { S19C05 } & 22.7992988 & 0.00751195 & 13.7050071 \\ \text { S19C06 } & 22.7992988 & 0.00751195 & 4.94777506 \\ \text { S19C07 } & -0.8918512 & 22.7818498 & 48.1127768 \\ \text { S19C08 } & -0.8918512 & 22.7818498 & 39.5523916 \\ \text { S19C09 } & -0.8918512 & 22.7818498 & 30.9920063 \\ \text { S19C10 } & -0.8918512 & 22.7818498 & 22.4316211 \\ \text { S19C11 } & -0.8918512 & 22.7818498 & 13.8712358 \\ \text { S19C12 } & -0.8918512 & 22.7818498 & 5.31085059 \\ \text { S19C13 } & -22.799232 & 0.05581734 & 43.8077814 \\ \text { S19C14 } & -22.799232 & 0.05581734 & 36.6116608 \\ \text { S19C15 } & -22.799232 & 0.05581734 & 29.4155401 \\ \text { S19C16 } & -22.799232 & 0.05581734 & 22.2194195 \\ \text { S19C17 } & -22.799232 & 0.05581734 & 15.0232989 \\ \text { S19C18 } & -22.799232 & 0.05581734 & 7.82717821 \\ \text { S19C19 } & -0.8707222 & -22.782667 & 48.1456418 \\ \text { S19C20 } & -0.8707222 & -22.782667 & 39.5748415 \\ \text { S19C21 } & -0.8707222 & -22.782667 & 31.0040413 \\ \text { S19C22 } & -0.8707222 & -22.782667 & 22.4332411 \\ \text { S19C23 } & -0.8707222 & -22.782667 & 13.8624408\end{array}$

$\begin{array}{ccc}\text { XINTo } & \text { ZINTo } & \text { YINTo } \\ 22.9374991 & 0.00659985 & 48.8269509 \\ 22.9374991 & 0.00659985 & 40.0402419 \\ 22.9374991 & 0.00659985 & 31.253533 \\ 22.9374991 & 0.00659985 & 22.4668241 \\ 22.9374991 & 0.00659985 & 13.6801152 \\ 22.9374991 & 0.00659985 & 4.89340626 \\ -0.7820221 & 22.9241651 & 48.2836097 \\ -0.7820221 & 22.9241651 & 39.6690871 \\ -0.7820221 & 22.9241651 & 31.0545644 \\ -0.7820221 & 22.9241651 & 22.4400418 \\ -0.7820221 & 22.9241651 & 13.8255191 \\ -0.7820221 & 22.9241651 & 5.21099645 \\ -22.937448 & 0.04903963 & 44.4989674 \\ -22.937448 & 0.04903963 & 37.0838081 \\ -22.937448 & 0.04903963 & 29.6686487 \\ -22.937448 & 0.04903963 & 22.2534894 \\ -22.937448 & 0.04903963 & 14.8383301 \\ -22.937448 & 0.04903963 & 7.42317071 \\ -0.7635663 & -22.924787 & 48.3123166 \\ -0.7635663 & -22.924787 & 39.6886967 \\ -0.7635663 & -22.924787 & 31.0650767 \\ -0.7635663 & -22.924787 & 22.4414568 \\ -0.7635663 & -22.924787 & 13.8178368\end{array}$




\section{REVISED METHODOLOGY FOR DETERMINING CESIUM-137 CONTENT OF HN-200 GROUT CONTAINERS.}

$\begin{array}{llll}\text { S19C24 } & -0.8707222 & -22.782667 & 5.29164057 \\ \text { S19R01 } & 22.7983552 & 0.2075557 & 22.5391459 \\ \text { S19R02 } & 18.3490959 & 13.532138 & 22.4760757 \\ \text { S19R03 } & 7.07260644 & 21.6745546 & 22.2994498 \\ \text { S19R04 } & -6.2778707 & 21.9179474 & 22.027887 \\ \text { S19R05 } & -16.246871 & 15.9952266 & 21.7225623 \\ \text { S19R06 } & -20.777217 & 9.38697758 & 21.4906329 \\ \text { S19R07 } & -22.221189 & 5.10165019 & 21.3704722 \\ \text { S19R08 } & -22.673686 & 2.38998679 & 21.3193252 \\ \text { S19R09 } & -22.797721 & 0.26834734 & 21.3058158 \\ \text { S19R10 } & -22.721832 & -1.8778839 & 21.3217156 \\ \text { S19R11 } & -22.31302 & -4.6837162 & 21.3767373 \\ \text { S19R12 } & -20.865495 & -9.1890809 & 21.5043196 \\ \text { S19R13 } & -16.174335 & -16.06857 & 21.7450076 \\ \text { S19R14 } & -5.9745828 & -22.002555 & 22.0513736 \\ \text { S19R15 } & 7.44280854 & -21.550236 & 22.3169776 \\ \text { S19R16 } & 18.5901811 & -13.198987 & 22.4851373\end{array}$

\author{
$-0.7635663 \quad-22.924787 \quad 5.19421689$ \\ $\begin{array}{llll}22.9365631 & 0.20731693 & 22.5439901\end{array}$ \\ $\begin{array}{lll}18.4845906 & 13.5811935 & 22.4811685\end{array}$ \\ $\begin{array}{lll}7.19661006 & 21.7792954 & 22.3053209\end{array}$ \\ $\begin{array}{lll}-6.183563 & 22.0882877 & 22.0351902\end{array}$ \\ $\begin{array}{lll}-16.218041 & 16.2204825 & 21.7314833\end{array}$ \\ $\begin{array}{lll}-20.834548 & 9.59429667 & 21.4995215\end{array}$ \\ $\begin{array}{llll}-22.331818 & 5.23629789 & 21.3780197\end{array}$ \\ $\begin{array}{lll}-22.805933 & 2.45323038 & 21.325891\end{array}$ \\ $\begin{array}{llll}-22.935934 & 0.26803862 & 21.312079\end{array}$ \\ $\begin{array}{llll}-22.855119 & -1.9422776 & 21.328333\end{array}$ \\ $\begin{array}{llll}-22.425151 & -4.8209456 & 21.3843873\end{array}$ \\ $\begin{array}{llll}-20.92333 & -9.3991056 & 21.5132863\end{array}$ \\ $\begin{array}{llll}-16.145801 & -16.292392 & 21.7538374\end{array}$ \\ $\begin{array}{lll}-5.8819928 & -22.1705 & 22.0585438\end{array}$ \\ $\begin{array}{llll}7.56506245 & -21.65407 & 22.3227659\end{array}$ \\ $\begin{array}{lll}18.724701 & -13.248188 & 22.4901935\end{array}$
}




\section{REVISED METHODOLOGY FOR DETERMINING CESIUM-137 CONTENT OF HN-200 GROUT CONTAINERS.}

$\begin{array}{lccc}\text { S2OR01 } & 21.3463834 & 8.00874499 & 42.1143235 \\ \text { S20R02 } & 17.6510861 & 14.430774 & 42.4239033 \\ \text { S20R03 } & 13.0151626 & 18.7193382 & 42.6202701 \\ \text { S20R04 } & 7.98323444 & 21.3559371 & 42.7018322 \\ \text { S20R05 } & 2.57672318 & 22.6532245 & 42.672338 \\ \text { S20R06 } & -3.3786914 & 22.5475614 & 42.5301212 \\ \text { S20R07 } & -9.9234901 & 20.5263836 & 42.2720329 \\ \text { S20R08 } & -16.486536 & 15.7480855 & 41.9111664 \\ \text { S20R09 } & -21.464489 & 7.68659899 & 41.4963047 \\ \text { S20R10 } & -22.620628 & -2.8487286 & 41.1064627 \\ \text { S20R11 } & -18.450557 & -13.39347 & 40.8199273 \\ \text { S20R12 } & -9.3643069 & -20.787444 & 40.689675 \\ \text { S20R13 } & 2.26362672 & -22.68665 & 40.737622 \\ \text { S20R14 } & 13.0964428 & -18.662563 & 40.9558767 \\ \text { S20R15 } & 20.307355 & -10.364334 & 41.3060175 \\ \text { S20R16 } & 22.790495 & -0.6335773 & 41.7197911\end{array}$

Source S21

$\begin{array}{lccc}\text { Line } & \text { XINTi } & \text { ZINTi } & \text { YINTi } \\ \text { S21C01 } & 22.7951871 & -0.4330437 & 47.8794392 \\ \text { S21C02 } & 22.7951871 & -0.4330437 & 39.3719239 \\ \text { S21C03 } & 22.7951871 & -0.4330437 & 30.8644085 \\ \text { S21C04 } & 22.7951871 & -0.4330437 & 22.3568932 \\ \text { S21C05 } & 22.7951871 & -0.4330437 & 13.8493779 \\ \text { S21C06 } & 22.7951871 & -0.4330437 & 5.34186257 \\ \text { S21C07 } & 0.1094489 & 22.7990373 & 48.4414591 \\ \text { S21C08 } & 0.1094489 & 22.7990373 & 39.7631472 \\ \text { S21C09 } & 0.1094489 & 22.7990373 & 31.0848354 \\ \text { S21C10 } & 0.1094489 & 22.7990373 & 22.4065235 \\ \text { S21C11 } & 0.1094489 & 22.7990373 & 13.7282116 \\ \text { S21C12 } & 0.1094489 & 22.7990373 & 5.04989973 \\ \text { S21C13 } & -22.796849 & -0.3343315 & 48.2488459 \\ \text { S21C14 } & -22.796849 & -0.3343315 & 39.6290687 \\ \text { S21C15 } & -22.796849 & -0.3343315 & 31.0092916 \\ \text { S21C16 } & -22.796849 & -0.3343315 & 22.3895144 \\ \text { S21C17 } & -22.796849 & -0.3343315 & 13.7697372 \\ \text { S21C18 } & -22.796849 & -0.3343315 & 5.14996007 \\ \text { S21C19 } & 0.21770547 & -22.798261 & 47.3944498 \\ \text { S21C20 } & 0.21770547 & -22.798261 & 39.0343217 \\ \text { S21C21 } & 0.21770547 & -22.798261 & 30.6741935 \\ \text { S21C22 } & 0.21770547 & -22.798261 & 22.3140653 \\ \text { S21C23 } & 0.21770547 & -22.798261 & 13.9539371 \\ \text { S21C24 } & 0.21770547 & -22.798261 & 5.59380891 \\ \text { S21R01 } & 21.7437954 & -6.8567808 & 20.8016865\end{array}$

$\begin{array}{ccc}21.4976367 & 7.9987825 & 42.0952111 \\ 17.78965 & 14.4795462 & 42.4049197 \\ 13.1183811 & 18.8158705 & 42.6016673 \\ 8.0370133 & 21.4833732 & 42.6834689 \\ 2.57373647 & 22.7926476 & 42.6538822 \\ -3.4398677 & 22.6780999 & 42.5113108 \\ -10.035712 & 20.6255517 & 42.2529221 \\ -16.631014 & 15.7967802 & 41.892213 \\ -21.614602 & 7.67710005 & 41.4780815 \\ -22.752034 & -2.9109888 & 41.0892241 \\ -18.54701 & -13.495827 & 40.8035019 \\ -9.416487 & -20.915513 & 40.6736307 \\ 2.26100749 & -22.825791 & 40.721437 \\ 13.1456928 & -18.796799 & 40.9390598 \\ 20.4059358 & -10.475051 & 41.2882505 \\ 22.9267853 & -0.7010152 & 41.701119\end{array}$

$\begin{array}{ccc}\text { XINTo } & \text { ZINTo } & \text { YINTo } \\ 22.9343473 & -0.380289 & 48.0768611 \\ 22.9343473 & -0.380289 & 39.5093497 \\ 22.9343473 & -0.380289 & 30.9418383 \\ 22.9343473 & -0.380289 & 22.3743269 \\ 22.9343473 & -0.380289 & 13.8068155 \\ 22.9343473 & -0.380289 & 5.23930416 \\ 0.09615682 & 22.9372984 & 48.5700141 \\ 0.09615682 & 22.9372984 & 39.8526347 \\ 0.09615682 & 22.9372984 & 31.1352552 \\ 0.09615682 & 22.9372984 & 22.4178758 \\ 0.09615682 & 22.9372984 & 13.7004963 \\ 0.09615682 & 22.9372984 & 4.98311689 \\ -22.93562 & -0.2936567 & 48.4010613 \\ -22.93562 & -0.2936567 & 39.7350262 \\ -22.93562 & -0.2936567 & 31.0689911 \\ -22.93562 & -0.2936567 & 22.4029561 \\ -22.93562 & -0.2936567 & 13.736921 \\ -22.93562 & -0.2936567 & 5.07088594 \\ 0.19124957 & -22.936703 & 47.6503195 \\ 0.19124957 & -22.936703 & 39.2124331 \\ 0.19124957 & -22.936703 & 30.7745467 \\ 0.19124957 & -22.936703 & 22.3366603 \\ 0.19124957 & -22.936703 & 13.898774 \\ 0.19124957 & -22.936703 & 5.46088758 \\ 21.8912726 & -6.848437 & 20.8089248\end{array}$


REVISED METHODOLOGY FOR DETERMINING CESIUM-137 CONTENT OF HN-200 GROUT CONTAINERS.

$\begin{array}{llll}\text { S21R02 } & 22.7403824 & 1.6380139 & 20.9320662 \\ \text { S21R03 } & 20.0083981 & 10.9303287 & 21.0717936 \\ \text { S21R04 } & 12.9134514 & 18.7896475 & 21.1945065 \\ \text { S21R05 } & 2.44132009 & 22.6682164 & 21.276552 \\ \text { S21R06 } & -8.8709022 & 21.0027421 & 21.3030535 \\ \text { S21R07 } & -17.941349 & 14.0682652 & 21.2694584 \\ \text { S21R08 } & -22.460912 & 3.91350279 & 21.181561 \\ \text { S21R09 } & -21.83388 & -6.564279 & 21.0554342 \\ \text { S21R10 } & -17.223984 & -14.937953 & 20.9154793 \\ \text { S21R11 } & -10.634844 & -20.167007 & 20.7877642 \\ \text { S21R12 } & -3.7216366 & -22.493499 & 20.6910428 \\ \text { S21R13 } & 2.72823386 & -22.635477 & 20.6332904 \\ \text { S21R14 } & 8.56000132 & -21.131362 & 20.6157154 \\ \text { S21R15 } & 13.8165944 & -18.13587 & 20.6380812 \\ \text { S21R16 } & 18.3919547 & -13.47383 & 20.7006387\end{array}$

$\begin{array}{lll}22.8741953 & 1.7029661 & 20.9391878\end{array}$

$\begin{array}{llll}20.1063277 & 11.0392251 & 21.0786532\end{array}$

$\begin{array}{llll}12.9627934 & 18.9233954 & 21.2010665\end{array}$

$\begin{array}{lll}2.43849253 & 22.8075132 & 21.2828914\end{array}$

$\begin{array}{lll}-8.9236226 & 21.1304961 & 21.3093195\end{array}$

$\begin{array}{lll}-18.037802 & 14.1692127 & 21.2758173\end{array}$

$\begin{array}{lll}-22.590686 & 3.97363937 & 21.1881547\end{array}$

$\begin{array}{lll}-21.980524 & -6.5563303 & 21.0623298\end{array}$

$\begin{array}{lll}-17.364603 & -14.986643 & 20.922624\end{array}$

$\begin{array}{lll}-10.744675 & -20.265262 & 20.795005\end{array}$

$\begin{array}{lll}-3.7821401 & -22.623535 & 20.6982339\end{array}$

$\begin{array}{llll}2.72506859 & -22.77505 & 20.6403869\end{array}$

$\begin{array}{llll}8.61307979 & -21.258969 & 20.6227722\end{array}$

$\begin{array}{llll}13.9180482 & -18.232302 & 20.6451876\end{array}$

$\begin{array}{lll}18.5272728 & -13.522909 & 20.7078405\end{array}$

\begin{tabular}{|c|c|c|c|c|c|c|}
\hline \multicolumn{7}{|c|}{ Source 522} \\
\hline Line & XINTi & ZINTi & YINTi & XINTo & ZINTo & YINTo \\
\hline $\mathrm{S} 22 \mathrm{C} 01$ & 22.7966141 & 0.34994873 & 49.5147832 & 22.9354405 & 0.30736593 & 49.5129843 \\
\hline $\mathrm{S} 22 \mathrm{CO} 2$ & 22.7966141 & 0.34994873 & 40.7597505 & 22.9354405 & 0.30736593 & 40.7281433 \\
\hline $\mathrm{S} 22 \mathrm{C} 03$ & 22.7966141 & 0.34994873 & 32.0047177 & 22.9354405 & 0.30736593 & 31.9433022 \\
\hline $\mathrm{S} 22 \mathrm{C} 04$ & 22.7966141 & 0.34994873 & 23.249685 & 22.9354405 & 0.30736593 & 23.1584611 \\
\hline $\mathrm{S} 22 \mathrm{C05}$ & 22.7966141 & 0.34994873 & 14.4946522 & 22.9354405 & 0.30736593 & 14.37362 \\
\hline S22C06 & 22.7966141 & 0.34994873 & 5.7396195 & 22.9354405 & 0.30736593 & 5.58877891 \\
\hline S22C07 & -1.9875677 & 22.7125 & 49.5600453 & -1.7282993 & 22.8722952 & 49.5522127 \\
\hline $\mathrm{S} 22 \mathrm{C08}$ & -1.9875677 & 22.7125 & 41.5550345 & -1.7282993 & 22.8722952 & 41.4174104 \\
\hline$\$ 22 C 09$ & -1.9875677 & 22.7125 & 33.5500237 & -1.7282993 & 22.8722952 & 33.2826082 \\
\hline $\mathrm{S} 22 \mathrm{C} 10$ & -1.9875677 & 22.7125 & 25.5450129 & -1.7282993 & 22.8722952 & 25.147806 \\
\hline $\mathrm{S} 22 \mathrm{C} 11$ & -1.9875677 & 22.7125 & 17.5400022 & -1.7282993 & 22.8722952 & 17.0130037 \\
\hline $\mathrm{S} 22 \mathrm{C} 12$ & -1.9875677 & 22.7125 & 9.53499137 & -1.7282993 & 22.8722952 & 8.87820151 \\
\hline $\mathrm{S} 22 \mathrm{C} 13$ & -22.621944 & 2.83826274 & 49.6198993 & -22.807595 & 2.43772737 & 49.6029792 \\
\hline $\mathrm{S} 22 \mathrm{C} 14$ & -22.621944 & 2.83826274 & 42.6067088 & -22.807595 & 2.43772737 & 42.3094102 \\
\hline $\mathrm{S} 22 \mathrm{C} 15$ & -22.621944 & 2.83826274 & 35.5935182 & -22.807595 & 2.43772737 & 35.0158413 \\
\hline $\mathrm{S} 22 \mathrm{C} 16$ & -22.621944 & 2.83826274 & 28.5803277 & -22.807595 & 2.43772737 & 27.7222724 \\
\hline $\mathrm{S} 22 \mathrm{C} 17$ & -22.621944 & 2.83826274 & 21.5671371 & -22.807595 & 2.43772737 & 20.4287035 \\
\hline $\mathrm{S} 22 \mathrm{C} 18$ & -22.621944 & 2.83826274 & 14.5539466 & -22.807595 & 2.43772737 & 13.1351346 \\
\hline S22C19 & -0.5594921 & -22.792434 & 49.5169025 & -0.4911811 & -22.93224 & 49.5148388 \\
\hline $\mathrm{S} 22 \mathrm{C} 20$ & -0.5594921 & -22.792434 & 40.7969878 & -0.4911811 & -22.93224 & 40.7607272 \\
\hline $\mathrm{S} 22 \mathrm{C} 21$ & -0.5594921 & -22.792434 & 32.0770732 & -0.4911811 & -22.93224 & 32.0066157 \\
\hline $\mathrm{S} 22 \mathrm{C} 22$ & -0.5594921 & -22.792434 & 23.3571585 & -0.4911811 & -22.93224 & 23.2525041 \\
\hline $\mathbf{S} 22 \mathbf{C} 23$ & -0.5594921 & -22.792434 & 14.6372439 & -0.4911811 & -22.93224 & 14.4983926 \\
\hline $\mathbf{S} 22 \mathrm{C} 24$ & -0.5594921 & -22.792434 & 5.91732919 & -0.4911811 & -22.93224 & 5.74428102 \\
\hline S22R0I & 20.597598 & 9.77481653 & 44.4591436 & 20.7564404 & 9.76212521 & 44.4361506 \\
\hline S22R02 & 9.7757115 & 20.5971732 & 45.7343047 & 9.97792666 & 20.6535684 & 45.7029106 \\
\hline
\end{tabular}




\section{REVISED METHODOLOGY FOR DETERMINING CESIUM-137 CONTENT OF HN-200 GROUT CONTAINERS.}

$\begin{array}{llcc}\text { S22R03 } & -5.4538824 & 22.1373722 & 47.5926487 \\ \text { S22R04 } & -15.789211 & 16.447155 & 49.341565 \\ \text { S22R05 } & -17.783233 & 14.26761 & 49.7905314 \\ \text { S22R06 } & -18.23389 & 13.6869772 & 49.8809372 \\ \text { S22R07 } & -18.464904 & 13.3736837 & 49.9066933 \\ \text { S22R08 } & -18.656112 & 13.1056304 & 49.9047087 \\ \text { S22R09 } & -18.892209 & 12.7629353 & 49.8727515 \\ \text { S22R10 } & -19.362806 & 12.037019 & 49.7604128 \\ \text { S22R11 } & -21.155106 & 8.50115137 & 49.1525679 \\ \text { S22R12 } & -22.362135 & -4.4433091 & 47.2623221 \\ \text { S22R13 } & -14.460465 & -17.62677 & 45.4854459 \\ \text { S22R14 } & -0.0957776 & -22.799099 & 44.311856 \\ \text { S22R15 } & 14.1430252 & -17.882475 & 43.7583592 \\ \text { S22R16 } & 22.1912737 & -5.230244 & 43.806775\end{array}$

$\begin{array}{lccc}\text { Line } & \text { XINTi } & \text { ZINTi } & \begin{array}{c}\text { Source S23 } \\ \text { YINTi }\end{array} \\ \text { S23C01 } & 22.7816541 & 0.89683833 & 49.6536215 \\ \text { S23C02 } & 22.7816541 & 0.89683833 & 41.2058264 \\ \text { S23C03 } & 22.7816541 & 0.89683833 & 32.7580313 \\ \text { S23C04 } & 22.7816541 & 0.89683833 & 24.3102362 \\ \text { S23C05 } & 22.7816541 & 0.89683833 & 15.8624411 \\ \text { S23C06 } & 22.7816541 & 0.89683833 & 7.41464606 \\ \text { S23C07 } & 0.62742089 & 22.7906653 & 49.8080726 \\ \text { S23C08 } & 0.62742089 & 22.7906653 & 41.9154642 \\ \text { S23C09 } & 0.62742089 & 22.7906653 & 34.0228559 \\ \text { S23C10 } & 0.62742089 & 22.7906653 & 26.1302476 \\ \text { S23C11 } & 0.62742089 & 22.7906653 & 18.2376393 \\ \text { S23C12 } & 0.62742089 & 22.7906653 & 10.3450309 \\ \text { S23C13 } & -22.792007 & 0.57663634 & 49.5987734 \\ \text { S23C14 } & -22.792007 & 0.57663634 & 40.9538222 \\ \text { S23C15 } & -22.792007 & 0.57663634 & 32.3088711 \\ \text { S23C16 } & -22.792007 & 0.57663634 & 23.6639199 \\ \text { S23C17 } & -22.792007 & 0.57663634 & 15.0189688 \\ \text { S23C18 } & -22.792007 & 0.57663634 & 6.37401763 \\ \text { S23C19 } & 0.1506032 & -22.798803 & 49.5739483 \\ \text { S23C20 } & 0.1506032 & -22.798803 & 40.8397615 \\ \text { S23C21 } & 0.1506032 & -22.798803 & 32.1055746 \\ \text { S23C22 } & 0.1506032 & -22.798803 & 23.3713878 \\ \text { S23C23 } & 0.1506032 & -22.798803 & 14.6372009 \\ \text { S23C24 } & 0.1506032 & -22.798803 & 5.90301407 \\ \text { S23R01 } & 18.5999159 & 13.1852649 & 49.5302589 \\ \text { S23R02 } & 14.5081707 & 17.5875258 & 50.1321829 \\ \text { S23R03 } & 11.025895 & 19.9558943 & 50.4442467 \\ & & & \end{array}$

$\begin{array}{llll}-5.170916 & 22.3470475 & 47.5372841\end{array}$

$\begin{array}{llll}-15.518742 & 16.8907531 & 49.2548805\end{array}$

$\begin{array}{lll}-17.746665 & 14.5321983 & 49.7431524\end{array}$

$\begin{array}{lll}-18.284093 & 13.8499401 & 49.8490922\end{array}$

$\begin{array}{llll}-18.561359 & 13.4760842 & 49.8796546\end{array}$

$\begin{array}{llll}-18.790374 & 13.154876 & 49.8772946\end{array}$

$\begin{array}{lll}-19.071072 & 12.7445322 & 49.8394104\end{array}$

$\begin{array}{lll}-19.617543 & 11.8861655 & 49.7083995\end{array}$

$-21.472533 \quad 8.06593127 \quad 49.0635859$

$\begin{array}{llll}-22.440088 & -4.750933 & 47.2128753\end{array}$

$\begin{array}{llll}-14.437753 & -17.823585 & 45.4560198\end{array}$

$\begin{array}{llll}-0.0263735 & -22.937485 & 44.2896121\end{array}$

$\begin{array}{llll}14.2438268 & -17.978941 & 43.7386366\end{array}$

$\begin{array}{llll}22.3196232 & -5.2880363 & 43.7868486\end{array}$

$\begin{array}{ccc}\text { XINTo } & \text { ZINTo } & \text { YINTo } \\ 22.9240162 & 0.78637743 & 49.6347004 \\ 22.9240162 & 0.78637743 & 41.1188919 \\ 22.9240162 & 0.78637743 & 32.6030834 \\ 22.9240162 & 0.78637743 & 24.0872748 \\ 22.9240162 & 0.78637743 & 15.5714663 \\ 22.9240162 & 0.78637743 & 7.05565778 \\ 0.5507065 & 22.9308881 & 49.7704047 \\ 0.5507065 & 22.9308881 & 41.7423963 \\ 0.5507065 & 22.9308881 & 33.7143878 \\ 0.5507065 & 22.9308881 & 25.6863793 \\ 0.5507065 & 22.9308881 & 17.6583709 \\ 0.5507065 & 22.9308881 & 9.63036239 \\ -22.931914 & 0.50620785 & 49.5867095 \\ -22.931914 & 0.50620785 & 40.8983939 \\ -22.931914 & 0.50620785 & 32.2100782 \\ -22.931914 & 0.50620785 & 23.5217625 \\ -22.931914 & 0.50620785 & 14.8334469 \\ -22.931914 & 0.50620785 & 6.14513119 \\ 0.13230966 & -22.937118 & 49.5649659 \\ 0.13230966 & -22.937118 & 40.7984912 \\ 0.13230966 & -22.937118 & 32.0320164 \\ 0.13230966 & -22.937118 & 23.2655416 \\ 0.13230966 & -22.937118 & 14.4990669 \\ 0.13230966 & -22.937118 & 5.73259212 \\ 18.782661 & 13.1658858 & 49.4967777 \\ 14.6650375 & 17.6370514 & 50.1009798 \\ 11.1344962 & 20.0537254 & 50.41567\end{array}$




\section{REVISED METHODOLOGY FOR DETERMINING CESIUM-137 CONTENT OF HN-200 GROUT CONTAINERS.}

$\begin{array}{llll}\text { S23R04 } & 7.95053467 & 21.3681323 & 50.5520235 \\ \text { S23R05 } & 4.79428687 & 22.2895243 & 50.4937873 \\ \text { S23R06 } & 0.94275366 & 22.7798002 & 50.248465 \\ \text { S23R07 } & -4.4183777 & 22.3670744 & 49.739935 \\ \text { S23R08 } & -11.747008 & 19.5401096 & 48.9019788 \\ \text { S23R09 } & -19.252624 & 12.2124746 & 47.8495635 \\ \text { S23R10 } & -22.793359 & 0.52045533 & 46.8680785 \\ \text { S23R11 } & -19.362461 & -12.037574 & 46.1853537 \\ \text { S23R12 } & -9.339703 & -20.79851 & 45.90607 \\ \text { S23R13 } & 3.89908957 & -22.463419 & 46.0603075 \\ \text { S23R14 } & 15.6491773 & -16.58045 & 46.6323197 \\ \text { S23R15 } & 22.0461773 & -5.8115529 & 47.5444323 \\ \text { S23R16 } & 22.1763667 & 5.29309379 & 48.6068665\end{array}$

8.0043546621 .495562650 .5246215

$\begin{array}{llll}4.7886222 & 22.4320753 & 50.465733\end{array}$

$0.87087606 \quad 22.9209616 \quad 50.2181103$

$\begin{array}{llll}-4.5573129 & 22.4802092 & 49.706888\end{array}$

$\begin{array}{lll}-11.927036 & 19.5927213 & 48.8688314\end{array}$

$\begin{array}{llll}-19.426898 & 12.1952671 & 47.8198339\end{array}$

$\begin{array}{llll}-22.933089 & 0.44983794 & 46.8423509\end{array}$

$\begin{array}{lll}-19.45945 & -12.143257 & 46.162284\end{array}$

$\begin{array}{lll}-9.3919093 & -20.926561 & 45.8840227\end{array}$

$\begin{array}{llll}3.89452485 & -22.604459 & 46.0377006\end{array}$

$\begin{array}{llll}15.6978617 & -16.724415 & 46.6075325\end{array}$

$\begin{array}{llll}22.1544683 & -5.9420906 & 47.5159372\end{array}$

$\begin{array}{llll}22.33934 & 5.20411321 & 48.5744487\end{array}$

\section{Source S24}

$\begin{array}{lccc}\text { Line } & \text { XINTi } & \text { ZINTi } & \text { YINTi } \\ \text { S24C01 } & 22.7549004 & 1.42217731 & 48.2810524 \\ \text { S24C02 } & 22.7549004 & 1.42217731 & 39.9472743 \\ \text { S24C03 } & 22.7549004 & 1.42217731 & 31.6134963 \\ \text { S24C04 } & 22.7549004 & 1.42217731 & 23.2797182 \\ \text { S24C05 } & 22.7549004 & 1.42217731 & 14.9459402 \\ \text { S24C06 } & 22.7549004 & 1.42217731 & 6.6121621 \\ \text { S24C07 } & 2.07760984 & 22.7044405 & 45.2030117 \\ \text { S24C08 } & 2.07760984 & 22.7044405 & 38.5515522 \\ \text { S24C09 } & 2.07760984 & 22.7044405 & 31.9000928 \\ \text { S24C10 } & 2.07760984 & 22.7044405 & 25.2486333 \\ \text { S24C11 } & 2.07760984 & 22.7044405 & 18.5971738 \\ \text { S24C12 } & 2.07760984 & 22.7044405 & 11.9457144 \\ \text { S24C13 } & -22.788801 & 0.69183603 & 48.9070276 \\ \text { S24C14 } & -22.788801 & 0.69183603 & 40.2311196 \\ \text { S24C15 } & -22.788801 & 0.69183603 & 31.5552117 \\ \text { S24C16 } & -22.788801 & 0.69183603 & 22.8793037 \\ \text { S24C17 } & -22.788801 & 0.69183603 & 14.2033958 \\ \text { S24C18 } & -22.788801 & 0.69183603 & 5.52748781 \\ \text { S24C19 } & 0.21019322 & -22.798331 & 49.0652707 \\ \text { S24C20 } & 0.21019322 & -22.798331 & 40.3028742 \\ \text { S24C21 } & 0.21019322 & -22.798331 & 31.5404777 \\ \text { S24C22 } & 0.21019322 & -22.798331 & 22.7780811 \\ \text { S24C23 } & 0.21019322 & -22.798331 & 14.0156846 \\ \text { S24C24 } & 0.21019322 & -22.798331 & 5.25328804 \\ \text { S24R01 } & 13.4131291 & 18.4362699 & 32.7794565 \\ \text { S24R02 } & 10.8668039 & 20.0429702 & 32.8861328 \\ \text { S24R03 } & 9.62019446 & 20.670267 & 32.921308 \\ \text { S24R04 } & 8.72809655 & 21.0624882 & 32.9301984 \\ & & & \end{array}$

$\begin{array}{ccc}\text { XINTo } & \text { ZINTo } & \text { YINTo } \\ 22.9037888 & 1.2431262 & 48.434517 \\ 22.9037888 & 1.2431262 & 40.0168621 \\ 22.9037888 & 1.2431262 & 31.5992072 \\ 22.9037888 & 1.2431262 & 23.1815522 \\ 22.9037888 & 1.2431262 & 14.7638973 \\ 22.9037888 & 1.2431262 & 6.3462424 \\ 1.80473586 & 22.8663909 & 45.7673796 \\ 1.80473586 & 22.8663909 & 38.807462 \\ 1.80473586 & 22.8663909 & 31.8475444 \\ 1.80473586 & 22.8663909 & 24.8876269 \\ 1.80473586 & 22.8663909 & 17.9277093 \\ 1.80473586 & 22.8663909 & 10.9677917 \\ -22.929464 & 0.60711779 & 48.9796396 \\ -22.929464 & 0.60711779 & 40.2640452 \\ -22.929464 & 0.60711779 & 31.5484508 \\ -22.929464 & 0.60711779 & 22.8328564 \\ -22.929464 & 0.60711779 & 14.117262 \\ -22.929464 & 0.60711779 & 5.40166759 \\ 0.18465167 & -22.936757 & 49.1180967 \\ 0.18465167 & -22.936757 & 40.3268279 \\ 0.18465167 & -22.936757 & 31.535559 \\ 0.18465167 & -22.936757 & 22.7442902 \\ 0.18465167 & -22.936757 & 13.9530214 \\ 0.18465167 & -22.936757 & 5.16175253 \\ 13.7021113 & 18.3951367 & 32.7660041 \\ 11.0560404 & 20.0970863 & 32.8765513 \\ 9.733498 & 20.7698802 & 32.9136621 \\ 8.78097974 & 21.1901699 & 32.9230891\end{array}$


REVISED METHODOLOGY FOR DETERMINING CESIUM-137 CONTENT OF HN-200 GROUT CONTAINERS.

$\begin{array}{lccc}\text { S24R05 } & 7.81991598 & 21.4162787 & 32.9212917 \\ \text { S24R06 } & 6.49435616 & 21.8547802 & 32.8860834 \\ \text { S24R07 } & 3.55542155 & 22.5203698 & 32.7793018 \\ \text { S24R08 } & -4.4563002 & 22.3595498 & 32.475973 \\ \text { S24R09 } & -16.093488 & 16.1495421 & 32.0315977 \\ \text { S24R10 } & -22.58962 & 3.08499093 & 31.6622557 \\ \text { S24R11 } & -19.761154 & -11.37123 & 31.4356528 \\ \text { S24R12 } & -8.7225403 & -21.06479 & 31.3602491 \\ \text { S24R13 } & 5.93756113 & -22.012575 & 31.435782 \\ \text { S24R14 } & 18.1578268 & -13.787727 & 31.6625142 \\ \text { S24R15 } & 22.7992567 & 0.044422 & 32.0319656 \\ \text { S24R16 } & 18.9604835 & 12.6612853 & 32.4763316\end{array}$

$\begin{array}{lll}7.81022631 & 21.5668559 & 32.9136448\end{array}$

$\begin{array}{llll}6.39877683 & 22.0269054 & 32.8764995\end{array}$

$\begin{array}{lll}3.32191999 & 22.695677 & 32.765846\end{array}$

$\begin{array}{lll}-4.7635306 & 22.437417 & 32.4621863\end{array}$

$\begin{array}{lll}-16.316826 & 16.1211072 & 32.0222983\end{array}$

$\begin{array}{llll}-22.739707 & 3.00576375 & 31.6555206\end{array}$

$\begin{array}{lll}-19.858656 & -11.478793 & 31.4300341\end{array}$

$\begin{array}{lll}-8.7754299 & -21.192469 & 31.3549457\end{array}$

$\begin{array}{llll}5.93044073 & -22.15759 & 31.4301627\end{array}$

$\begin{array}{llll}18.2079366 & -13.949909 & 31.6557777\end{array}$

$\begin{array}{llll}22.9371105 & -0.133664 & 32.022663\end{array}$

$\begin{array}{lll}19.2328432 & 12.4990659 & 32.4625414\end{array}$

\section{Source S25}

$\begin{array}{lccc}\text { Line } & \text { XINTi } & \text { ZINTi } & \text { YINTi } \\ \text { S25C01 } & 22.7883466 & 0.70663921 & 48.7867317 \\ \text { S25C02 } & 22.7883466 & 0.70663921 & 40.4522625 \\ \text { S25C03 } & 22.7883466 & 0.70663921 & 32.1177934 \\ \text { S25C04 } & 22.7883466 & 0.70663921 & 23.7833243 \\ \text { S25C05 } & 22.7883466 & 0.70663921 & 15.4488551 \\ \text { S25C06 } & 22.7883466 & 0.70663921 & 7.11438598 \\ \text { S25C07 } & 0.67035401 & 22.7894429 & 48.7300122 \\ \text { S25C08 } & 0.67035401 & 22.7894429 & 40.4484664 \\ \text { S25C09 } & 0.67035401 & 22.7894429 & 32.1669206 \\ \text { S25C10 } & 0.67035401 & 22.7894429 & 23.8853749 \\ \text { S25C11 } & 0.67035401 & 22.7894429 & 15.6038291 \\ \text { S25C12 } & 0.67035401 & 22.7894429 & 7.32228331 \\ \text { S25C13 } & -22.796808 & 0.33710743 & 49.1597301 \\ \text { S25C14 } & -22.796808 & 0.33710743 & 40.4772265 \\ \text { S25C15 } & -22.796808 & 0.33710743 & 31.7947229 \\ \text { S25C16 } & -22.796808 & 0.33710743 & 23.1122193 \\ \text { S25C17 } & -22.796808 & 0.33710743 & 14.4297157 \\ \text { S25C18 } & -22.796808 & 0.33710743 & 5.74721205 \\ \text { S25C19 } & 0.28581822 & -22.797508 & 49.171701 \\ \text { S25C20 } & 0.28581822 & -22.797508 & 40.4780277 \\ \text { S25C21 } & 0.28581822 & -22.797508 & 31.7843543 \\ \text { S25C22 } & 0.28581822 & -22.797508 & 23.090681 \\ \text { S25C23 } & 0.28581822 & -22.797508 & 14.3970077 \\ \text { S25C24 } & 0.28581822 & -22.797508 & 5.70333434 \\ \text { S25R01 } & 21.0929618 & 8.65419217 & 38.6178788 \\ \text { S25R02 } & 18.5461109 & 13.260839 & 38.7789712 \\ \text { S25R03 } & 15.5783351 & 16.6470284 & 38.8390483 \\ \text { S25R04 } & 12.0913976 & 19.3288951 & 38.8089879 \\ \text { S25R05 } & 7.64681772 & 21.4786931 & 38.6830573\end{array}$

XINTo ZINTo YINTo $\begin{array}{llll}22.9291171 & 0.6200764 & 48.8741065\end{array}$ $\begin{array}{llll}22.9291171 & 0.6200764 & 40.4581103\end{array}$ $\begin{array}{llll}22.9291171 & 0.6200764 & 32.0421141\end{array}$ $\begin{array}{llll}22.9291171 & 0.6200764 & 23.6261179\end{array}$ $\begin{array}{llll}22.9291171 & 0.6200764 & 15.2101217\end{array}$ $\begin{array}{llll}22.9291171 & 0.6200764 & 6.79412551\end{array}$ $\begin{array}{llll}0.58830903 & 22.9299542 & 48.8242514\end{array}$ $\begin{array}{llll}0.58830903 & 22.9299542 & 40.4547736\end{array}$ $\begin{array}{lll}0.58830903 & 22.9299542 & 32.0852959\end{array}$ $\begin{array}{lll}0.58830903 & 22.9299542 & 23.7158181\end{array}$ $\begin{array}{lll}0.58830903 & 22.9299542 & 15.3463403\end{array}$ $\begin{array}{llll}0.58830903 & 22.9299542 & 6.97686257\end{array}$ $\begin{array}{llll}-22.935589 & 0.2960936 & 49.2011287\end{array}$ $\begin{array}{llll}-22.935589 & 0.2960936 & 40.4799972\end{array}$ $\begin{array}{llll}-22.935589 & 0.2960936 & 31.7588657\end{array}$ $\begin{array}{llll}-22.935589 & 0.2960936 & 23.0377342\end{array}$ $\begin{array}{lll}-22.935589 & 0.2960936 & 14.3166028\end{array}$ $\begin{array}{llll}-22.935589 & 0.2960936 & 5.59547127\end{array}$ $\begin{array}{llll}0.25106409 & -22.936126 & 49.2116206\end{array}$ $\begin{array}{llll}0.25106409 & -22.936126 & 40.4806994\end{array}$ $\begin{array}{llll}0.25106409 & -22.936126 & 31.7497782\end{array}$ $\begin{array}{llll}0.25106409 & -22.936126 & 23.018857\end{array}$ $\begin{array}{llll}0.25106409 & -22.936126 & 14.2879358\end{array}$ $\begin{array}{llll}0.25106409 & -22.936126 & 5.55701461\end{array}$ $\begin{array}{llll}21.2467117 & 8.64327186 & 38.6029032\end{array}$ $\begin{array}{llll}18.6808057 & 13.3100115 & 38.7646973\end{array}$ $\begin{array}{lll}15.676746 & 16.7442092 & 38.8251327\end{array}$ $\begin{array}{llll}12.1412238 & 19.4607192 & 38.7948861\end{array}$ $\begin{array}{llll}7.63737541 & 21.6286709 & 38.6683223\end{array}$ 
REVISED METHODOLOGY FOR DETERMINING CESIUM-137 CONTENT OF HN-200 GROUT CONTAINERS.

$\begin{array}{lccc}\text { S25R06 } & 1.6052579 & 22.7427181 & 38.4437972 \\ \text { S25R07 } & -6.3610331 & 21.8939567 & 38.0877268 \\ \text { S25R08 } & -15.078244 & 17.1013048 & 37.6641543 \\ \text { S25R09 } & -21.470452 & 7.66992773 & 37.2681131 \\ \text { S25R10 } & -22.348778 & -4.5100092 & 36.9858112 \\ \text { S25R11 } & -16.562496 & -15.668178 & 36.8665242 \\ \text { S25R12 } & -5.6965795 & -22.076165 & 36.9273046 \\ \text { S25R13 } & 6.67066871 & -21.801611 & 37.1597515 \\ \text { S25R14 } & 16.6236181 & -15.603314 & 37.5262833 \\ \text { S25R15 } & 21.8516906 & -6.5047444 & 37.951979 \\ \text { S25R16 } & 22.7001502 & 2.12397314 & 38.3387595\end{array}$

\begin{tabular}{|c|c|c|c|}
\hline Line & XINTi & ZINTi & YINTi \\
\hline $\mathrm{S} 26 \mathrm{C} 01$ & 22.7796263 & 0.94694669 & 48.6061867 \\
\hline $\mathrm{S} 26 \mathrm{C} 02$ & 22.7796263 & 0.94694669 & 40.1317363 \\
\hline $\mathrm{S} 26 \mathrm{CO} 3$ & 22.7796263 & 0.94694669 & 31.6572 \\
\hline S26C04 & 22.7796263 & 0.94694669 & 23.1828356 \\
\hline S26C05 & 22.7796263 & 0.94694669 & 14.7083852 \\
\hline S26C06 & 22.7796263 & 0.94694669 & 6.23393483 \\
\hline S26C07 & 0.61006835 & 22.7911364 & 47.2274235 \\
\hline S26C08 & 0.61006835 & 22.7911364 & 39.5636664 \\
\hline $\mathrm{S} 26 \mathrm{C} 09$ & 0.61006835 & 22.7911364 & 31.8999094 \\
\hline $\mathrm{S} 26 \mathrm{C} 10$ & 0.61006835 & 22.7911364 & 24.2361524 \\
\hline $\mathrm{S} 26 \mathrm{C} 11$ & 0.610 & 22.79 & 16.5723953 \\
\hline $\mathrm{S} 26 \mathrm{C} 12$ & 0.6100 & 22.7911364 & 8.90863832 \\
\hline S26C13 & -22.789638 & 0.66369025 & 48.8735496 \\
\hline $\mathrm{S} 26 \mathrm{C} 14$ & -22.789638 & 0.66369025 & 40.2418936 \\
\hline S26C15 & -22.789638 & 0.66369025 & 31.6102376 \\
\hline S26C16 & -22.789638 & 0.66369025 & 22.9785816 \\
\hline S26C17 & -22.789638 & 0.66369025 & 14.3469257 \\
\hline $\mathrm{S} 26 \mathrm{C} 18$ & -22.789638 & 0.66369025 & 5.71526971 \\
\hline S26C19 & 0.11650379 & -22.799002 & 49.0660097 \\
\hline S26C20 & 0.11650379 & -22.799002 & 40.3211899 \\
\hline S26C21 & 0.11650379 & -22.799002 & 31.5763701 \\
\hline S26C22 & 0.11650379 & -22.799002 & 22.8315503 \\
\hline $\mathrm{S} 26 \mathrm{C} 23$ & 0.11650379 & -22.799002 & 14.0867305 \\
\hline S26C24 & 0.11650379 & -22.799002 & 5.34191067 \\
\hline S26R01 & 17.464202 & 14.6563886 & 33.47745 \\
\hline S26R02 & 12.8374191 & 18.8416759 & 33.6857253 \\
\hline S26R03 & 9.34313503 & 20.7969687 & 33.7917044 \\
\hline S26R04 & 6.56253519 & 21.8344043 & 33.832517 \\
\hline S26R05 & 3.90647332 & 22.4621358 & 33.8266401 \\
\hline S26R06 & 0.77371821 & 22.7861677 & 33.7713601 \\
\hline
\end{tabular}

$\begin{array}{lll}1.53128741 & 22.8863292 & 38.4284346\end{array}$ $\begin{array}{lll}-6.4886096 & 22.0006102 & 38.0724548\end{array}$ $\begin{array}{llll}-15.231223 & 17.1504741 & 37.6498814\end{array}$ $\begin{array}{llll}-21.620508 & 7.66045254 & 37.2551194\end{array}$ $\begin{array}{llll}-22.477827 & -4.569047 & 36.9737709\end{array}$ $\begin{array}{lll}-16.659756 & -15.766466 & 36.8548814\end{array}$ $\begin{array}{lll}-5.7536106 & -22.204163 & 36.91546\end{array}$ $\begin{array}{lll}6.66257698 & -21.948553 & 37.1471241\end{array}$ $\begin{array}{llll}16.6725419 & -15.752944 & 37.512437\end{array}$ $\begin{array}{llll}21.9579394 & -6.6315763 & 37.9369551\end{array}$ $\begin{array}{llll}22.845865 & 2.04825711 & 38.3233178\end{array}$ 


\section{REVISED METHODOLOGY FOR DETERMINING CESIUM-137 CONTENT OF HN-200 GROUT CONTAINERS.}

$\begin{array}{llll}\text { S26R07 } & -3.6801225 & 22.5003284 & 33.6422863 \\ \text { S26R08 } & -10.364952 & 20.3070397 & 33.4022927 \\ \text { S26R09 } & -18.180106 & 13.7583364 & 33.0652338 \\ \text { S26R10 } & -22.686922 & 2.26089543 & 32.7287125 \\ \text { S26R11 } & -20.093847 & -10.772437 & 32.4807113 \\ \text { S26R12 } & -10.375035 & -20.30189 & 32.3602469 \\ \text { S26R13 } & 3.10718937 & -22.586577 & 32.3787242 \\ \text { S26R14 } & 15.3834738 & -16.827264 & 32.5346775 \\ \text { S26R15 } & 22.1111247 & -5.5593387 & 32.8115515 \\ \text { S26R16 } & 21.8946818 & 6.35853681 & 33.1588094\end{array}$

\begin{tabular}{|c|c|c|c|}
\hline & & & Source S27 \\
\hline Line & XINTi & ZINTi & YINTi \\
\hline $\mathrm{S} 27 \mathrm{C} 01$ & 22.7709791 & -1.1360429 & 47.5872449 \\
\hline $\mathrm{S} 27 \mathrm{C} 02$ & 22.7709791 & -1.1360429 & 39.0717766 \\
\hline $\mathrm{S} 27 \mathrm{CO3}$ & 22.7709791 & -1.1360429 & 30.5563083 \\
\hline S27C04 & 22.7709791 & -1.1360429 & 22.04084 \\
\hline $\mathrm{S} 27 \mathrm{C} 05$ & 22.7709791 & -1.1360429 & 13.5253718 \\
\hline S27C06 & 22.7709791 & -1.1360429 & 5.00990346 \\
\hline $\mathrm{S} 27 \mathrm{C} 07$ & 0.05736459 & 22.7992278 & 48.6020829 \\
\hline $\mathrm{S} 27 \mathrm{C08}$ & 0.05736459 & 22.7992278 & 39.8295398 \\
\hline $\mathbf{S} 27 \mathrm{C} 09$ & 0.05736459 & 22.7992278 & 31.0569966 \\
\hline $\mathbf{S} 27 \mathrm{C} 10$ & 0.05736459 & 22.7992278 & 22.2844535 \\
\hline $\mathrm{S} 27 \mathrm{C} 11$ & 0.05736459 & 22.7992278 & 13.5119104 \\
\hline $\mathrm{S} 27 \mathrm{C} 12$ & 0.05736459 & 22.7992278 & 4.73936732 \\
\hline S27C13 & -22.780251 & -0.9317932 & 47.93114 \\
\hline S27C14 & $-22.78025 !$ & -0.9317932 & 39.3285576 \\
\hline S27C15 & -22.780251 & -0.9317932 & 30.7259751 \\
\hline $\mathrm{S} 27 \mathrm{C} 16$ & -22.780251 & -0.9317932 & 22.1233926 \\
\hline S27C17 & -22.780251 & -0.9317932 & 13.5208102 \\
\hline $\mathrm{S} 27 \mathrm{C} 18$ & -22.780251 & -0.9317932 & 4.91822768 \\
\hline S27C19 & 0.92604577 & -22.780486 & 35.0047797 \\
\hline S27C20 & 0.92604577 & -22.780486 & 29.6766528 \\
\hline S27C21 & 0.92604577 & -22.780486 & 24.3485258 \\
\hline S27C22 & 0.92604577 & -22.780486 & 19.0203989 \\
\hline S27C23 & 0.92604577 & -22.780486 & 13.6922719 \\
\hline S27C24 & 0.92604577 & -22.780486 & 8.36414499 \\
\hline S27R01 & 11.4294418 & -19.727543 & 14.803364 \\
\hline S27R02 & 20.1563287 & -10.655069 & 15.7323624 \\
\hline S27R03 & 22.4677387 & 3.87411878 & 16.5835989 \\
\hline S27R04 & 15.2845615 & 16.9171588 & 17.1433504 \\
\hline S27R05 & 1.66318527 & 22.7385553 & 17.3864403 \\
\hline S27R06 & -12.637724 & 18.9761962 & 17.3151455 \\
\hline S27R07 & -21.64295 & 7.16873771 & 16.9281988 \\
\hline
\end{tabular}

\author{
$-3.824096422 .6164806 \quad 33.6320975$ \\ $-10.559925 \quad 20.3621434 \quad 33.3916345$ \\ $\begin{array}{lll}-18.368694 & 13.7375388 & 33.0556874\end{array}$ \\ $\begin{array}{llll}-22.833221 & 2.18470189 & 32.7207151\end{array}$ \\ $-20.191947-10.881829 \quad 32.4737689$ \\ $\begin{array}{lll}-10.426216 & -20.430931 & 32.3537689\end{array}$ \\ $\begin{array}{llll}3.10357521 & -22.726564 & 32.3721771\end{array}$ \\ $15.4321444-16.969909 \quad 32.5275165$ \\ $22.2202166 \quad-5.6912986 \quad 32.8031769$ \\ $\begin{array}{llll}22.0656796 & 6.26375989 & 33.1488615\end{array}$
}

$\begin{array}{ccc}\text { XINTo } & \text { ZINTo } & \text { YINTo } \\ 22.9159135 & -0.9948952 & 47.8248952 \\ 22.9159135 & -0.9948952 & 39.2492262 \\ 22.9159135 & -0.9948952 & 30.6735573 \\ 22.9159135 & -0.9948952 & 22.0978884 \\ 22.9159135 & -0.9948952 & 13.5222194 \\ 22.9159135 & -0.9948952 & 4.9465505 \\ 0.05039899 & 22.9374446 & 48.711141 \\ 0.05039899 & 22.9374446 & 39.9109516 \\ 0.05039899 & 22.9374446 & 31.1107891 \\ 0.05039899 & 22.9374446 & 22.3106267 \\ 0.05039899 & 22.9374446 & 13.5104642 \\ 0.05039899 & 22.9374446 & 4.7103017 \\ -22.922949 & -0.8168958 & 48.1245927 \\ -22.922949 & -0.8168958 & 39.4730055 \\ -22.922949 & -0.8168958 & 30.8214184 \\ -22.922949 & -0.8168958 & 22.1698312 \\ -22.922949 & -0.8168958 & 13.5182441 \\ -22.922949 & -0.8168958 & 4.86665695 \\ 0.81187892 & -22.923127 & 36.791812 \\ 0.81187892 & -22.923127 & 31.011001 \\ 0.81187892 & -22.923127 & 25.2301899 \\ 0.81187892 & -22.923127 & 19.4493789 \\ 0.81187892 & -22.923127 & 13.6685678 \\ 0.81187892 & -22.923127 & 7.88775679 \\ 11.7945222 & -19.672777 & 14.8365291 \\ 20.3807296 & -10.524009 & 15.7544567 \\ 22.583065 & 4.01672513 & 16.5985148 \\ 15.3332319 & 17.0593349 & 17.1552129 \\ 1.661266 & 22.8772617 & 17.3972456 \\ -12.687215 & 19.109251 & 17.3262468 \\ -21.747449 & 7.29228002 & 16.9411207\end{array}$


REVISED METHODOLOGY FOR DETERMINING CESIUM-137 CONTENT OF HN-200 GROUT CONTAINERS.

$\begin{array}{llll}\text { S27R08 } & -21.454775 & -7.7136691 & 16.2282287 \\ \text { S27R09 } & -12.715985 & -18.923842 & 15.2900702 \\ \text { S27R10 } & -3.4612168 & -22.535041 & 14.5164719 \\ \text { S27R11 } & 0.07608669 & -22.799173 & 14.2425342 \\ \text { S27R12 } & 1.42086707 & -22.754982 & 14.161693 \\ \text { S27R13 } & 2.24022548 & -22.688972 & 14.1377641 \\ \text { S27R14 } & 2.97962529 & -22.603759 & 14.1442766 \\ \text { S27R15 } & 3.94288065 & -22.455774 & 14.1876614 \\ \text { S27R16 } & 5.86450724 & -22.03215 & 14.3252028\end{array}$

Source S28

\begin{tabular}{lccc} 
Line & XINTi & ZNNTi & YINTi \\
S28C01 & 22.7675474 & -1.2028565 & 40.3866391 \\
S28C02 & 22.7675474 & -1.2028565 & 33.2934201 \\
S28C03 & 22.7675474 & -1.2028565 & 26.200201 \\
S28C04 & 22.7675474 & -1.2028565 & 19.106982 \\
S28C05 & 22.7675474 & -1.2028565 & 12.0137629 \\
S28C06 & 22.7675474 & -1.2028565 & 4.92054391 \\
S28C07 & 0.71472893 & 22.7880943 & 47.8305163 \\
S28C08 & 0.71472893 & 22.7880943 & 39.179821 \\
S28C09 & 0.71472893 & 22.7880943 & 30.5291257 \\
S28C10 & 0.71472893 & 22.7880943 & 21.8784304 \\
S28C11 & 0.71472893 & 22.7880943 & 13.2277351 \\
S28C12 & 0.71472893 & 22.7880943 & 4.57703977 \\
S28C13 & -22.798789 & -0.1526464 & 48.3434847 \\
S28C14 & -22.798789 & -0.1526464 & 39.5854614 \\
S28C15 & -22.798789 & -0.1526464 & 30.8274382 \\
S28C16 & -22.798789 & -0.1526464 & 22.0694149 \\
S28C17 & -22.798789 & -0.1526464 & 13.3113917 \\
S28C18 & -22.798789 & -0.1526464 & 4.5533684 \\
S28C19 & 1.17862528 & -22.768815 & 46.7469343 \\
S28C20 & 1.17862528 & -22.768815 & 38.322956 \\
S28C21 & 1.17862528 & -22.768815 & 29.8989776 \\
S28C22 & 1.17862528 & -22.768815 & 21.4749993 \\
S28C23 & 1.17862528 & -22.768815 & 13.0510209 \\
S28C24 & 1.17862528 & -22.768815 & 4.62704259 \\
S28R01 & 22.123872 & -5.5083909 & 7.08850319 \\
S28R02 & 22.5304231 & -3.4911481 & 7.21878604 \\
S28R03 & 22.7992946 & -0.0156726 & 7.55978694 \\
S28R04 & 21.7293497 & 6.9024229 & 8.33586145 \\
S28R05 & 15.6583549 & 16.5717833 & 9.51885963 \\
S28R06 & 3.43699386 & 22.5387478 & 10.6364521 \\
S28R07 & -10.55812 & 20.2072806 & 11.3988544 \\
S28R08 & -20.516469 & 9.94397142 & 11.7281505 \\
& & & \\
\hline
\end{tabular}

\author{
$\begin{array}{lll}-21.638276 & -7.6101185 & 16.2456855\end{array}$ \\ $\begin{array}{lll}-13.028115 & -18.878483 & 15.3175387\end{array}$ \\ $\begin{array}{lll}-3.801826 & -22.620235 & 14.5488746\end{array}$ \\ $\begin{array}{lll}-0.1023711 & -22.937272 & 14.2646014\end{array}$ \\ $\begin{array}{lll}1.34749392 & -22.897886 & 14.1781512\end{array}$ \\ $\begin{array}{llll}2.23763364 & -22.828095 & 14.1523558\end{array}$ \\ $\begin{array}{llll}3.04161263 & -22.73494 & 14.1593848\end{array}$ \\ $\begin{array}{lll}4.0850099 & -22.570813 & 14.2060438\end{array}$ \\ $\begin{array}{llll}6.13435195 & -22.102005 & 14.3517714\end{array}$
}

$\begin{array}{ccc}\text { XINTo } & \text { ZINTo } & \text { YINTo } \\ 22.9133176 & -1.0529866 & 41.522118 \\ 22.9133176 & -1.0529866 & 34.1913236 \\ 22.9133176 & -1.0529866 & 26.8605293 \\ 22.9133176 & -1.0529866 & 19.5297349 \\ 22.9133176 & -1.0529866 & 12.1989406 \\ 22.9133176 & -1.0529866 & 4.86814626 \\ 0.62715722 & 22.9289245 & 48.0350687 \\ 0.62715722 & 22.9289245 & 39.3415751 \\ 0.62715722 & 22.9289245 & 30.6480814 \\ 0.62715722 & 22.9289245 & 21.9545878 \\ 0.62715722 & 22.9289245 & 13.2610942 \\ 0.62715722 & 22.9289245 & 4.56760052 \\ -22.937108 & -0.1341045 & 48.4839664 \\ -22.937108 & -0.1341045 & 39.6965502 \\ -22.937108 & -0.1341045 & 30.9091341 \\ -22.937108 & -0.1341045 & 22.121718 \\ -22.937108 & -0.1341045 & 13.3343019 \\ -22.937108 & -0.1341045 & 4.54688575 \\ 1.03192706 & -22.914276 & 47.0895961 \\ 1.03192706 & -22.914276 & 38.5939229 \\ 1.03192706 & -22.914276 & 30.0982497 \\ 1.03192706 & -22.914276 & 21.6025765 \\ 1.03192706 & -22.914276 & 13.1069034 \\ 1.03192706 & -22.914276 & 4.61123017 \\ 22.2678879 & -5.5018246 & 7.11194069 \\ 22.6825625 & -3.4103172 & 7.24591615 \\ 22.9369274 & 0.1620774 & 7.59353049 \\ 21.7946024 & 7.15011971 & 8.37230537 \\ 15.6317873 & 16.7861887 & 9.5480958 \\ 3.35638117 & 22.6906062 & 10.6587952 \\ -10.6682 & 20.3056255 & 11.4175239 \\ -20.64504 & 9.9955606 & 11.7454659\end{array}$




\section{REVISED METHODOLOGY FOR DETERMINING CESIUM-137 CONTENT OF HN-200 GROUT CONTAINERS.}

$\begin{array}{lccc}\text { S28R09 } & -22.401332 & -4.2412738 & 11.6113155 \\ \text { S28R10 } & -15.494611 & -16.724985 & 11.051826 \\ \text { S28R11 } & -2.8126308 & -22.625145 & 10.0852217 \\ \text { S28R12 } & 9.78678783 & -20.591913 & 8.87650456 \\ \text { S28R13 } & 17.1636047 & -15.00729 & 7.86241684 \\ \text { S28R14 } & 19.9526052 & -11.031846 & 7.34354199 \\ \text { S28R15 } & 21.061231 & -8.7311298 & 7.13366436 \\ \text { S28R16 } & 21.6714561 & -7.0820952 & 7.06561163\end{array}$

\begin{tabular}{|c|c|c|c|}
\hline & & & Source S29 \\
\hline Line & XINTi & ZnNTi & YINTi \\
\hline $\mathrm{S} 29 \mathrm{CO} 1$ & 22.7986463 & 0.1726522 & 48.9713048 \\
\hline $\mathrm{S} 29 \mathrm{C} 02$ & 22.7986463 & 0.1726522 & 40.3978798 \\
\hline $\mathrm{S} 29 \mathrm{C} 03$ & 22.7986463 & 0.1726522 & 31.8244549 \\
\hline $\mathrm{S} 29 \mathrm{C} 04$ & 22.7986463 & 0.1726522 & 23.2510299 \\
\hline $\mathrm{S} 29 \mathrm{C} 05$ & 22.7986463 & 0.1726522 & 14.6776049 \\
\hline S29C06 & 22.7986463 & 0.1726522 & 6.10417993 \\
\hline $\mathrm{S} 29 \mathrm{C} 07$ & -0.0050675 & 22.7992994 & 48.8744159 \\
\hline $\mathrm{S} 29 \mathrm{C} 08$ & -0.0050675 & 22.7992994 & 40.3791652 \\
\hline $\mathrm{S} 29 \mathrm{C} 09$ & -0.0050675 & 22.7992994 & 31.8839146 \\
\hline $\mathrm{S} 29 \mathrm{C} 10$ & -0.0050675 & 22.7992994 & 23.388664 \\
\hline$S 29 C 11$ & -0.0050675 & 22.7992994 & 14.8934133 \\
\hline $\mathrm{S} 29 \mathrm{C} 12$ & -0.0050675 & 22.7992994 & 6.39816268 \\
\hline $\mathrm{S} 29 \mathrm{C} 13$ & -22.798636 & 0.17396208 & 48.9672937 \\
\hline $\mathrm{S} 29 \mathrm{C} 14$ & -22.798636 & 0.17396208 & 40.3971051 \\
\hline $\mathrm{s} 29 \mathrm{C} 15$ & -22.798636 & 0.17396208 & 31.8269164 \\
\hline $\mathrm{S} 29 \mathrm{C} 16$ & -22.798636 & 0.17396208 & 23.2567278 \\
\hline $\mathrm{S} 29 \mathrm{C} 17$ & -22.798636 & 0.17396208 & 14.6865392 \\
\hline $\mathrm{S} 29 \mathrm{C} 18$ & -22.798636 & 0.17396208 & 6.11635053 \\
\hline $\mathbf{S} 29 \mathrm{C} 19$ & -0.0037289 & -22.7993 & 49.0396647 \\
\hline$\$ 29 C 20$ & -0.0037289 & -22.7993 & 40.4110839 \\
\hline $\mathbf{S} 29 \mathrm{C} 21$ & -0.0037289 & -22.7993 & 31.7825031 \\
\hline $\mathbf{S} 29 \mathrm{C} 22$ & -0.0037289 & -22.7993 & 23.1539223 \\
\hline $\mathbf{S} 29 \mathrm{C} 23$ & -0.0037289 & -22.7993 & 14.5253415 \\
\hline S29C24 & -0.0037289 & -22.7993 & 5.8967607 \\
\hline S29R01 & 22.5923438 & 3.06497672 & 36.5065263 \\
\hline S29R02 & 19.7886933 & 11.3232371 & 36.6011272 \\
\hline S29R03 & 14.4437639 & 17.6404581 & 36.6795105 \\
\hline S29R04 & 7.53633392 & 21.5177079 & 36.7313757 \\
\hline S29R05 & -0.0779305 & 22.7991668 & 36.7507788 \\
\hline S29R06 & -7.6715498 & 21.469872 & 36.7356952 \\
\hline S29R07 & -14.527288 & 17.5717379 & 36.6876891 \\
\hline S29R08 & -19.816456 & 11.2745792 & 36.6121777 \\
\hline S29R09 & -22.591816 & 3.06886764 & 36.5189119 \\
\hline
\end{tabular}

\author{
$\begin{array}{lll}-22.542909 & -4.2362922 & 11.6290967\end{array}$ \\ $\begin{array}{lll}-15.644917 & -16.773953 & 11.0720686\end{array}$ \\ $\begin{array}{lll}-2.9631339 & -22.745302 & 10.110747\end{array}$ \\ $\begin{array}{llll}9.66545866 & -20.80163 & 8.91013289\end{array}$ \\ $\begin{array}{llll}17.1305559 & -15.253621 & 7.8987851\end{array}$ \\ $\begin{array}{llll}20.0064177 & -11.219276 & 7.37359652\end{array}$ \\ $\begin{array}{llll}21.1623456 & -8.8478266 & 7.1584547\end{array}$ \\ $\begin{array}{llll}21.7988814 & -7.1370635 & 7.08833462\end{array}$
}

$\begin{array}{ccc}\text { XINTo } & \text { ZINTo } & \text { YINTo } \\ 22.9369985 & 0.15167776 & 49.0355327 \\ 22.9369985 & 0.15167776 & 40.4102858 \\ 22.9369985 & 0.15167776 & 31.7850389 \\ 22.9369985 & 0.15167776 & 23.159792 \\ 22.9369985 & 0.15167776 & 14.534545 \\ 22.9369985 & 0.15167776 & 5.90929813 \\ -0.0044522 & 22.9374996 & 48.9503742 \\ -0.0044522 & 22.9374996 & 40.393837 \\ -0.0044522 & 22.9374996 & 31.8372998 \\ -0.0044522 & 22.9374996 & 23.2807625 \\ -0.0044522 & 22.9374996 & 14.7242253 \\ -0.0044522 & 22.9374996 & 6.16768807 \\ -22.936991 & 0.15282834 & 49.0320094 \\ -22.936991 & 0.15282834 & 40.4096052 \\ -22.936991 & 0.15282834 & 31.7872011 \\ -22.936991 & 0.15282834 & 23.1647969 \\ -22.936991 & 0.15282834 & 14.5423928 \\ -22.936991 & 0.15282834 & 5.91998862 \\ -0.0032762 & -22.9375 & 49.0955585 \\ -0.0032762 & -22.9375 & 40.4218801 \\ -0.0032762 & -22.9375 & 31.7482016 \\ -0.0032762 & -22.9375 & 23.0745232 \\ -0.0032762 & -22.9375 & 14.4008447 \\ -0.0032762 & -22.9375 & 5.72716627 \\ 22.7322823 & 3.06141274 & 36.4951813 \\ 19.9190813 & 11.3736145 & 36.5896861 \\ 14.5439995 & 17.7369948 & 36.6680213 \\ 7.59068802 & 21.6451002 & 36.7198726 \\ -0.0778408 & 22.9373679 & 36.7392744 \\ -7.7257269 & 21.5972695 & 36.7241916 \\ -14.627373 & 17.6683014 & 36.6761967 \\ -19.946764 & 11.3249946 & 36.600728 \\ -22.731759 & 3.06529904 & 36.5075522\end{array}$




\section{REVISED METHODOLOGY FOR DETERMINING CESIUM-137 CONTENT OF HN-200 GROUT CONTAINERS.}

$\begin{array}{llll}\text { S29R10 } & -21.985466 & -6.0371651 & 36.4215946 \\ \text { S29R11 } & -17.590568 & -14.504482 & 36.3359416 \\ \text { S29R12 } & -9.8339917 & -20.569411 & 36.2765353 \\ \text { S29R13 } & -0.0740572 & -22.79918 & 36.2537189 \\ \text { S29R14 } & 9.70938781 & -20.628521 & 36.2714844 \\ \text { S29R15 } & 17.5215944 & -14.587728 & 36.3267235 \\ \text { S29R16 } & 21.9690915 & -6.0964827 & 36.4098053\end{array}$

\begin{tabular}{|c|c|c|c|}
\hline & & & Source S30 \\
\hline Line & XINTi & ZINTi & YINTi \\
\hline S30C01 & 22.7555477 & -1.4117816 & 46.8527578 \\
\hline $\mathrm{S} 30 \mathrm{C} 02$ & 22.7555477 & -1.4117816 & 38.4949514 \\
\hline $\mathrm{S} 30 \mathrm{CO} 3$ & 22.7555477 & -1.4117816 & 30.1371449 \\
\hline $\mathrm{S} 30 \mathrm{CO} 4$ & 22.7555477 & -1.4117816 & 21.7793385 \\
\hline S30C05 & 22.7555477 & -1.4117816 & 13.4215321 \\
\hline S30C06 & 22.7555477 & -1.4117816 & 5.06372563 \\
\hline$\$ 30 C 07$ & 0.19207327 & 22.7984909 & 48.5335291 \\
\hline S30C08 & 0.19207327 & 22.7984909 & 39.7679849 \\
\hline$\$ 30 C 09$ & 0.19207327 & 22.7984909 & 31.0024408 \\
\hline $\mathrm{S} 30 \mathrm{C} 10$ & 0.19207327 & 22.7984909 & 22.2368966 \\
\hline S30C11 & 0.19207327 & 22.7984909 & 13.4713525 \\
\hline $\mathrm{S} 30 \mathrm{C} 12$ & 0.19207327 & 22.7984909 & 4.70580831 \\
\hline$S 30 C 13$ & -22.78772 & -0.7265537 & 48.1376338 \\
\hline $\mathrm{S} 30 \mathrm{C} 14$ & -22.78772 & -0.7265537 & 39.4681297 \\
\hline$S 30 \mathrm{C} 15$ & -22.78772 & -0.7265537 & 30.7986257 \\
\hline S30C16 & -22.78772 & -0.7265537 & 22.1291216 \\
\hline S30C17 & -22.78772 & -0.7265537 & 13.4596176 \\
\hline$\$ 30 C 18$ & -22.78772 & -0.7265537 & 4.79011352 \\
\hline S30C19 & 2.21215809 & -22.691726 & 38.3689014 \\
\hline$S 30 C 20$ & 2.21215809 & -22.691726 & 32.0691907 \\
\hline $\mathrm{S} 30 \mathrm{C} 21$ & 2.21215809 & -22.691726 & 25.7694801 \\
\hline S30C22 & 2.21215809 & -22.691726 & 19.4697694 \\
\hline S30C23 & 2.21215809 & -22.691726 & 13.1700588 \\
\hline S30C24 & 2.21215809 & -22.691726 & 6.87034812 \\
\hline S30R01 & 12.5588057 & -19.028518 & 12.9492312 \\
\hline S30R02 & 18.7287982 & -13.001546 & 13.7072274 \\
\hline S30R03 & 22.799026 & 0.11176611 & 14.7627462 \\
\hline S30R04 & 17.9294193 & 14.0834657 & 15.6010483 \\
\hline S30R05 & 5.47374805 & 22.1324685 & 16.0968303 \\
\hline S30R06 & -9.2363822 & 20.8445994 & 16.2400014 \\
\hline S30R07 & -20.076074 & 10.8055224 & 16.0324406 \\
\hline S30R08 & -22.47342 & -3.8410231 & 15.4720168 \\
\hline S30R09 & -15.43103 & -16.783664 & 14.5773508 \\
\hline S30R 10 & -3.5515514 & -22.520981 & 13.52464 \\
\hline
\end{tabular}

$\begin{array}{ccc}-22.113258 & -6.093662 & 36.4103659 \\ -17.687124 & -14.604607 & 36.3248544 \\ -9.8856894 & -20.697876 & 36.2655581 \\ -0.073972 & -22.937381 & 36.2427863 \\ 9.76121041 & -20.756871 & 36.260517 \\ 17.6181793 & -14.687705 & 36.3156527 \\ 22.0968521 & -6.1528884 & 36.3985948\end{array}$

\begin{tabular}{ccc} 
XINTo & ZINTo & YINTo \\
22.9042751 & -1.2341344 & 47.1858655 \\
22.9042751 & -1.2341344 & 38.7472506 \\
22.9042751 & -1.2341344 & 30.3086357 \\
22.9042751 & -1.2341344 & 21.8700207 \\
22.9042751 & -1.2341344 & 13.4314058 \\
22.9042751 & -1.2341344 & 4.99279093 \\
0.16873657 & 22.9368793 & 48.6509543 \\
0.16873657 & 22.9368793 & 39.856924 \\
0.16873657 & 22.9368793 & 31.0628937 \\
0.16873657 & 22.9368793 & 22.2688634 \\
0.16873657 & 22.9368793 & 13.4748331 \\
0.16873657 & 22.9368793 & 4.68080281 \\
-22.928639 & -0.6375062 & 48.3046075 \\
-22.928639 & -0.6375062 & 39.5945973 \\
-22.928639 & -0.6375062 & 30.8845872 \\
-22.928639 & -0.6375062 & 22.174577 \\
-22.928639 & -0.6375062 & 13.4645669 \\
-22.928639 & -0.6375062 & 4.75455676 \\
1.91844184 & -22.857132 & 39.8468176 \\
1.91844184 & -22.857132 & 33.1885798 \\
1.91844184 & -22.857132 & 26.530342 \\
1.91844184 & -22.857132 & 19.8721041 \\
1.91844184 & -22.857132 & 13.2138663 \\
1.91844184 & -22.857132 & 6.55562841 \\
12.8766291 & -18.982132 & 12.9828732 \\
19.0121414 & -12.832279 & 13.739837 \\
22.9356913 & 0.28804351 & 14.783676 \\
17.9792711 & 14.2434096 & 15.6161636 \\
5.46722608 & 22.2764078 & 16.1095236 \\
-9.2886991 & 20.9725767 & 16.2520957 \\
-20.174138 & 10.9148097 & 16.0454165 \\
-22.627433 & -3.7587478 & 15.4878673 \\
-15.667902 & -16.752485 & 14.5999643 \\
-3.8888346 & -22.605439 & 13.5593521 \\
& & \\
\hline
\end{tabular}




\section{REVISED METHODOLOGY FOR DETERMINING CESIUM-137 CONTENT OF HN-200 GROUT CONTAINERS.}

$\begin{array}{lccc}\text { S30R11 } & 3.77113101 & -22.485254 & 12.8769297 \\ \text { S30R12 } & 6.17838643 & -21.946198 & 12.6764946 \\ \text { S30R13 } & 7.2637554 & -21.611246 & 12.6130653 \\ \text { S30R14 } & 8.02804197 & -21.339134 & 12.5989043 \\ \text { S30R15 } & 8.80721376 & -21.029528 & 12.6199479 \\ \text { S30R16 } & 9.95859721 & -20.509374 & 12.6978782\end{array}$

$\begin{array}{cccc}\text { Line } & \text { XINTi } & \text { ZINTi } & \text { YINTi } \\ \text { S31C01 } & 22.7713305 & -1.128976 & 47.7092241 \\ \text { S31C02 } & 22.7713305 & -1.128976 & 39.2802492 \\ \text { S31C03 } & 22.7713305 & -1.128976 & 30.8512743 \\ \text { S31C04 } & 22.7713305 & -1.128976 & 22.4222994 \\ \text { S31C05 } & 22.7713305 & -1.128976 & 13.9933245 \\ \text { S31C06 } & 22.7713305 & -1.128976 & 5.56434955 \\ \text { S31C07 } & 0.15164063 & 22.7987957 & 48.7298485 \\ \text { S31C08 } & 0.15164063 & 22.7987957 & 39.9754269 \\ \text { S31C09 } & 0.15164063 & 22.7987957 & 31.2210052 \\ \text { S31C10 } & 0.15164063 & 22.7987957 & 22.4665836 \\ \text { S31C11 } & 0.15164063 & 22.7987957 & 13.712162 \\ \text { S31C12 } & 0.15164063 & 22.7987957 & 4.95774038 \\ \text { S31C13 } & -22.788765 & -0.6930199 & 48.4007354 \\ \text { S31C14 } & -22.788765 & -0.6930199 & 39.7512581 \\ \text { S31C15 } & -22.788765 & -0.6930199 & 31.1017809 \\ \text { S31C16 } & -22.788765 & -0.6930199 & 22.4523036 \\ \text { S31C17 } & -22.788765 & -0.6930199 & 13.8028263 \\ \text { S31C18 } & -22.788765 & -0.6930199 & 5.15334907 \\ \text { S31C19 } & 1.05154172 & -22.775038 & 44.159436 \\ \text { S31C20 } & 1.05154172 & -22.775038 & 36.8623828 \\ \text { S31C21 } & 1.05154172 & -22.775038 & 29.5653296 \\ \text { S31C22 } & 1.05154172 & -22.775038 & 22.2682763 \\ \text { S31C23 } & 1.05154172 & -22.775038 & 14.9712231 \\ \text { S31C24 } & 1.05154172 & -22.775038 & 7.67416987 \\ \text { S31R01 } & 15.7632537 & -16.472034 & 21.6820594 \\ \text { S31R02 } & 20.9624688 & -8.9656557 & 21.9420528 \\ \text { S31R03 } & 22.5511725 & 3.35450447 & 22.2493907 \\ \text { S31R04 } & 16.5390932 & 15.6928798 & 22.4987568 \\ \text { S31R05 } & 4.16710418 & 22.4152476 & 22.6449095 \\ \text { S31R06 } & -9.8634177 & 20.5553174 & 22.6764009 \\ \text { S31R07 } & -20.084275 & 10.7902728 & 22.5918622 \\ \text { S31R08 } & -22.606673 & -2.9574361 & 22.3957871 \\ \text { S31R09 } & -17.100776 & -15.078844 & 22.1107014 \\ \text { S31R10 } & -7.8747114 & -21.396191 & 21.8097122 \\ \text { S31R11 } & -0.8320726 & -22.784111 & 21.6034011 \\ & & & \\ \text { S3 } & & \end{array}$

\author{
$\begin{array}{llll}3.53295145 & -22.663785 & 12.9084461\end{array}$ \\ $\begin{array}{llll}6.08465027 & -22.11574 & 12.698164\end{array}$ \\ $\begin{array}{lll}7.25485169 & -21.759964 & 12.6303939\end{array}$ \\ $\begin{array}{llll}8.08176473 & -21.466578 & 12.6151933\end{array}$ \\ $\begin{array}{lll}8.9236067 & -21.130503 & 12.6377731\end{array}$ \\ $\begin{array}{lll}10.1585032 & -20.565352 & 12.720885\end{array}$
}

$\begin{array}{ccc}\text { XINTo } & \text { ZINTo } & \text { YINTo } \\ 22.9161796 & -0.9887468 & 47.9316551 \\ 22.9161796 & -0.9887468 & 39.4317535 \\ 22.9161796 & -0.9887468 & 30.931852 \\ 22.9161796 & -0.9887468 & 22.4319505 \\ 22.9161796 & -0.9887468 & 13.932049 \\ 22.9161796 & -0.9887468 & 5.43214748 \\ 0.13322097 & 22.9371131 & 48.8233981 \\ 0.13322097 & 22.9371131 & 40.0391463 \\ 0.13322097 & 22.9371131 & 31.2548945 \\ 0.13322097 & 22.9371131 & 22.4706427 \\ 0.13322097 & 22.9371131 & 13.6863909 \\ 0.13322097 & 22.9371131 & 4.90213905 \\ -22.929436 & -0.6081542 & 48.5353489 \\ -22.929436 & -0.6081542 & 39.8429474 \\ -22.929436 & -0.6081542 & 31.1505459 \\ -22.929436 & -0.6081542 & 22.4581444 \\ -22.929436 & -0.6081542 & 13.7657429 \\ -22.929436 & -0.6081542 & 5.07334139 \\ 0.92132587 & -22.918989 & 44.8207755 \\ 0.92132587 & -22.918989 & 37.3128408 \\ 0.92132587 & -22.918989 & 29.8049061 \\ 0.92132587 & -22.918989 & 22.2969714 \\ 0.92132587 & -22.918989 & 14.7890367 \\ 0.92132587 & -22.918989 & 7.28110202 \\ 15.9931584 & -16.442256 & 21.6912212 \\ 21.1605388 & -8.8521468 & 21.9506249 \\ 22.6687664 & 3.50084762 & 22.2561309 \\ 16.5879831 & 15.8419608 & 22.5042033 \\ 4.16221328 & 22.5567038 & 22.6497276 \\ -9.9150861 & 20.6838094 & 22.6810953 \\ -20.182355 & 10.8996082 & 22.5968979 \\ -22.756142 & -2.8786973 & 22.4017334 \\ -17.306024 & -15.054249 & 22.1182684 \\ -8.1047918 & -21.457895 & 21.8188654 \\ -1.0005186 & -22.915669 & 21.6120496\end{array}$


REVISED METHODOLOGY FOR DETERMINING CESIUM-137 CONTENT OF HN-200 GROUT CONTAINERS.

\author{
$\begin{array}{llll}\text { S31R12 } & 3.02397724 & -22.597868 & 21.5055197\end{array}$ \\ $\begin{array}{lllll}\text { S31R13 } & 5.36348977 & -22.159446 & 21.4663282\end{array}$ \\ $\begin{array}{llll}\text { S31R14 } & 7.20724109 & -21.630158 & 21.4591541\end{array}$ \\ $\begin{array}{lllll}\text { S31R15 } & 9.11664815 & -20.897244 & 21.4793597\end{array}$ \\ $\begin{array}{llll}\text { S31R16 } & 11.6800423 & -19.580212 & 21.5403281\end{array}$
}

\author{
$\begin{array}{lll}2.94498452 & -22.747659 & 21.5126965\end{array}$ \\ $\begin{array}{llll}5.3571084 & -22.303145 & 21.4726146\end{array}$ \\ $\begin{array}{llll}7.26203716 & -21.757567 & 21.4652586\end{array}$ \\ $\begin{array}{llll}9.23183161 & -20.997671 & 21.4859617\end{array}$ \\ $\begin{array}{llll}11.860735 & -19.632928 & 21.5481481\end{array}$
}

Source S32

\begin{tabular}{lccc} 
Line & XINTi & ZINTi & YINTi \\
S32C01 & 22.784051 & 0.83372779 & 48.4207048 \\
S32C02 & 22.784051 & 0.83372779 & 39.8383762 \\
S32C03 & 22.784051 & 0.83372779 & 31.2560475 \\
S32C04 & 22.784051 & 0.83372779 & 22.6737188 \\
S32C05 & 22.784051 & 0.83372779 & 14.0913901 \\
S32C06 & 22.784051 & 0.83372779 & 5.50906143 \\
S32C07 & -0.1748783 & 22.7986293 & 45.0651277 \\
S32C08 & -0.1748783 & 22.7986293 & 37.7813579 \\
S32C09 & -0.1748783 & 22.7986293 & 30.4975881 \\
S32C10 & -0.1748783 & 22.7986293 & 23.2138183 \\
S32C11 & -0.1748783 & 22.7986293 & 15.9300485 \\
S32C12 & -0.1748783 & 22.7986293 & 8.64627877 \\
S32C13 & -22.781443 & 0.9021901 & 48.3320775 \\
S32C14 & -22.781443 & 0.9021901 & 39.7840463 \\
S32C15 & -22.781443 & 0.9021901 & 31.2360151 \\
S32C16 & -22.781443 & 0.9021901 & 22.6879839 \\
S32C17 & -22.781443 & 0.9021901 & 14.1399526 \\
S32C18 & -22.781443 & 0.9021901 & 5.59192142 \\
S32C19 & -0.0249106 & -22.799286 & 48.8682724 \\
S32C20 & -0.0249106 & -22.799286 & 40.1127417 \\
S32C21 & -0.0249106 & -22.799286 & 31.3572109 \\
S32C22 & -0.0249106 & -22.799286 & 22.6016802 \\
S32C23 & -0.0249106 & -22.799286 & 13.8461495 \\
S32C24 & -0.0249106 & -22.799286 & 5.0906187 \\
S32R01 & 16.4390019 & 15.7976991 & 26.3044336 \\
S32R02 & 9.33947414 & 20.798613 & 26.2823829 \\
S32R03 & 4.47187561 & 22.35644 & 26.2700734 \\
S32R04 & 1.4179155 & 22.7551664 & 26.2645732 \\
S32R05 & -0.8817222 & 22.7822441 & 26.2628309 \\
S32R06 & -3.0962861 & 22.5880741 & 26.2638311 \\
S32R07 & -5.8294882 & 22.0414416 & 26.2681529 \\
S32R08 & -9.9735822 & 20.5020911 & 26.2782928 \\
S32R09 & -16.21215 & 16.0304166 & 26.2978699 \\
S32R10 & -21.900583 & 6.33817978 & 26.3243765 \\
S32R11 & -21.759527 & -6.8066915 & 26.3487454 \\
S32R12 & -13.850086 & -18.110307 & 26.3651452 \\
& & & \\
\hline
\end{tabular}

XINTo ZINTo YINTo $\begin{array}{lll}22.9258412 & 0.73123999 & 48.5533795\end{array}$ $\begin{array}{llll}22.9258412 & 0.73123999 & 39.9197077\end{array}$ $\begin{array}{llll}22.9258412 & 0.73123999 & 31.2860359\end{array}$ $\begin{array}{llll}22.9258412 & 0.73123999 & 22.652364\end{array}$ $\begin{array}{llll}22.9258412 & 0.73123999 & 14.0186922\end{array}$ $\begin{array}{llll}22.9258412 & 0.73123999 & 5.38502037\end{array}$ $\begin{array}{lll}-0.1536331 & 22.9369855 & 45.6039\end{array}$ $\begin{array}{lll}-0.1536331 & 22.9369855 & 38.1116332\end{array}$ $\begin{array}{lll}-0.1536331 & 22.9369855 & 30.6193665\end{array}$ $\begin{array}{lll}-0.1536331 & 22.9369855 & 23.1270998\end{array}$ $\begin{array}{lll}-0.1536331 & 22.9369855 & 15.6348331\end{array}$ $\begin{array}{llll}-0.1536331 & 22.9369855 & 8.14256637\end{array}$ $\begin{array}{llll}-22.923855 & 0.79105092 & 48.4759518\end{array}$ $\begin{array}{lll}-22.923855 & 0.79105092 & 39.8722434\end{array}$ $\begin{array}{lll}-22.923855 & 0.79105092 & 31.2685349\end{array}$ $\begin{array}{lll}-22.923855 & 0.79105092 & 22.6648265\end{array}$ $\begin{array}{llll}-22.923855 & 0.79105092 & 14.061118\end{array}$ $\begin{array}{llll}-22.923855 & 0.79105092 & 5.45740958\end{array}$ $\begin{array}{lll}-0.0218859 & -22.93749 & 48.9449775\end{array}$ $\begin{array}{lll}-0.0218859 & -22.93749 & 40.159763\end{array}$ $\begin{array}{lll}-0.0218859 & -22.93749 & 31.3745486\end{array}$ $\begin{array}{lll}-0.0218859 & -22.93749 & 22.5893341\end{array}$ $\begin{array}{lll}-0.0218859 & -22.93749 & 13.8041196\end{array}$ $\begin{array}{lll}-0.0218859 & -22.93749 & 5.01890512\end{array}$ $\begin{array}{llll}16.6558217 & 15.7706217 & 26.3051973\end{array}$ $\begin{array}{llll}9.5474359 & 20.8560632 & 26.2831754\end{array}$ $\begin{array}{llll}4.61046256 & 22.469369 & 26.2707619\end{array}$ $\begin{array}{llll}1.48339172 & 22.8894835 & 26.2651697\end{array}$ $\begin{array}{lll}-0.8807071 & 22.920586 & 26.2633918\end{array}$ $\begin{array}{lll}-3.1580332 & 22.719061 & 26.2644128\end{array}$ $\begin{array}{lll}-5.9599263 & 22.1496769 & 26.2688128\end{array}$ $\begin{array}{lll}-10.173301 & 20.558036 & 26.2790645\end{array}$ $\begin{array}{lll}-16.43321 & 16.0024532 & 26.2986586\end{array}$ $\begin{array}{llll}-22.071415 & 6.24352179 & 26.3250366\end{array}$ $\begin{array}{llll}-21.864956 & -6.9319972 & 26.3492788\end{array}$ $\begin{array}{llll}-13.899022 & -18.246811 & 26.3656062\end{array}$ 
REVISED METHODOLOGY FOR DETERMINING CESIUM-137 CONTENT OF HN-200 GROUT CONTAINERS.

\author{
S32R 13 \\ S32R14 \\ S32R 15 \\ S32R 16 \\ $\begin{array}{lll}-0.6847905 & -22.789014 & 26.3716396\end{array}$ \\ $\begin{array}{llll}12.7500829 & -18.900885 & 26.3678154\end{array}$ \\ $\begin{array}{llll}21.3435167 & -8.0163816 & 26.3538946\end{array}$ \\ $\begin{array}{llll}22.1618643 & 5.35348973 & 26.3312428\end{array}$
}

\author{
$\begin{array}{lll}-0.6840024 & -22.927299 & 26.372075\end{array}$ \\ $\begin{array}{llll}12.799512 & -19.034217 & 26.3682657\end{array}$ \\ $\begin{array}{llll}21.4460576 & -8.1360628 & 26.3544041\end{array}$ \\ $\begin{array}{llll}22.3252565 & 5.26420241 & 26.3318654\end{array}$
}

\begin{tabular}{|c|c|c|c|c|c|c|}
\hline \multicolumn{7}{|c|}{ Source S33 } \\
\hline Line & XINTi & ZINTi & YINTi & XINTo & ZINTo & YINTo \\
\hline S33COI & 22.7790246 & -0.9613101 & 48.6473191 & 22.9220165 & -0.8426546 & 48.7525664 \\
\hline $\mathrm{S} 33 \mathrm{C} 02$ & 22.7790246 & -0.9613101 & 40.0373576 & 22.9220165 & -0.8426546 & 40.094462 \\
\hline $\mathrm{S} 33 \mathrm{C} 03$ & 22.7790246 & -0.9613101 & 31.427396 & 22.9220165 & -0.8426546 & 31.4363576 \\
\hline S33CO4 & 22.7790246 & -0.9613101 & 22.8174345 & 22.9220165 & -0.8426546 & 22.7782532 \\
\hline $\mathrm{S} 33 \mathrm{C} 05$ & 22.7790246 & -0.9613101 & 14.2074729 & 22.9220165 & -0.8426546 & 14.1201488 \\
\hline S33C06 & 22.7790246 & -0.9613101 & 5.5975114 & 22.9220165 & -0.8426546 & 5.46204441 \\
\hline $\mathrm{S} 33 \mathrm{C} 07$ & -0.0689584 & 22.7991957 & 49.0143802 & -0.0605848 & 22.93742 & 49.073349 \\
\hline$\$ 33 C 08$ & -0.0689584 & 22.7991957 & 40.2365153 & -0.0605848 & 22.93742 & 40.2685103 \\
\hline S33C09 & -0.0689584 & 22.7991957 & 31.4586505 & -0.0605848 & 22.93742 & 31.4636716 \\
\hline $\mathrm{S} 33 \mathrm{C} 10$ & -0.0689584 & 22.7991957 & 22.6807857 & -0.0605848 & 22.93742 & 22.6588329 \\
\hline S33C11 & -0.0689584 & 22.7991957 & 13.9029208 & -0.0605848 & 22.93742 & 13.8539942 \\
\hline $\mathrm{S} 33 \mathrm{C} 12$ & -0.0689584 & 22.7991957 & 5.125056 & -0.0605848 & 22.93742 & 5.04915546 \\
\hline S33C13 & -22.766159 & -1.2288627 & 48.4100003 & -22.912268 & -1.075578 & 48.5459636 \\
\hline $\mathbf{S 3 3 C 1 4}$ & -22.766159 & -1.2288627 & 39.9085946 & -22.912268 & -1.075578 & 39.9823647 \\
\hline S33C15 & -22.766159 & -1.2288627 & 31.4071888 & -22.912268 & -1.075578 & 31.4187658 \\
\hline S33C16 & -22.766159 & -1.2288627 & 22.9057831 & -22.912268 & -1.075578 & 22.8551669 \\
\hline $\mathrm{S} 33 \mathrm{C} 17$ & -22.766159 & -1.2288627 & 14.4043774 & -22.912268 & -1.075578 & 14.291568 \\
\hline $\mathrm{S} 33 \mathrm{C} 18$ & -22.766159 & -1.2288627 & 5.90297172 & -22.912268 & -1.075578 & 5.72796913 \\
\hline S33C19 & -1.9434092 & -22.716321 & 35.8141031 & -1.690719 & -22.875104 & 37.5936007 \\
\hline$\$ 33 C 20$ & -1.9434092 & -22.716321 & 33.0743891 & -1.690719 & -22.875104 & 34.0398981 \\
\hline $\mathrm{S} 33 \mathrm{C} 21$ & -1.9434092 & -22.716321 & 30.3346751 & -1.690719 & -22.875104 & 30.4861955 \\
\hline $\mathrm{S} 33 \mathrm{C} 22$ & -1.9434092 & -22.716321 & 27.5949611 & -1.690719 & -22.875104 & 26.9324929 \\
\hline $\mathrm{S} 33 \mathrm{C} 23$ & -1.9434092 & -22.716321 & 24.855247 & -1.690719 & -22.875104 & 23.3787902 \\
\hline S33C24 & -1.9434092 & -22.716321 & 22.115533 & -1.690719 & -22.875104 & 19.8250876 \\
\hline S33R01 & 10.7119588 & -20.126153 & 29.539738 & 11.1143342 & -20.064907 & 29.5312485 \\
\hline S33R02 & 21.1410719 & -8.5359921 & 29.2789559 & 21.3337546 & -8.4261391 & 29.2745626 \\
\hline S33R03 & 21.7255311 & 6.91443259 & 29.0988535 & 21.8306776 & 7.03920606 & 29.0957409 \\
\hline S33R04 & 12.565847 & 19.0238685 & 29.0032693 & 12.6153798 & 19.1567507 & 29.0006203 \\
\hline S33R05 & -2.0343434 & 22.7083581 & 28.9887876 & -2.0319921 & 22.8473174 & 28.9862001 \\
\hline S33R06 & -15.792613 & 16.4438878 & 29.0547001 & -15.841314 & 16.5886012 & 29.0518153 \\
\hline S33R07 & -22.638205 & 2.7055064 & 29.2038492 & -22.758893 & 2.85686592 & 29.2000734 \\
\hline S33R08 & -19.063928 & -12.504988 & 29.4376859 & -19.331589 & -12.345792 & 29.4313193 \\
\hline S33R09 & -7.9618837 & -21.363906 & 29.7113062 & -8.6055146 & -21.262032 & 29.6971852 \\
\hline S33R 10 & -3.9073286 & -22.461987 & 29.7982052 & -4.232119 & -22.543693 & 29.7904796 \\
\hline S33R11 & -3.2694951 & -22.563654 & 29.8098103 & -3.4164725 & -22.681636 & 29.8052121 \\
\hline S33R 12 & -2.992407 & -22.60207 & 29.8129312 & -3.0543678 & -22.73323 & 29.8092609 \\
\hline S33R 13 & -2.7835725 & -22.628738 & 29.8133116 & -2.7803419 & -22.768369 & 29.8097563 \\
\hline
\end{tabular}




\section{REVISED METHODOLOGY FOR DETERMINING CESIUM-137 CONTENT OF HN-200 GROUT CONTAINERS.}

$\begin{array}{llll}\text { S33R 14 } & -2.5454283 & -22.656762 & 29.8114007 \\ \text { S33R 15 } & -2.1098425 & -22.701468 & 29.8044391 \\ \text { S33R16 } & -0.2387175 & -22.79805 & 29.7664292\end{array}$

\begin{tabular}{lccc} 
Line & XINTi & ZINTi & YINTi \\
S34C01 & 22.7936002 & 0.50977423 & 48.1766687 \\
S34C02 & 22.7936002 & 0.50977423 & 39.4470363 \\
S34C03 & 22.7936002 & 0.50977423 & 30.7174039 \\
S34C04 & 22.7936002 & 0.50977423 & 21.9877715 \\
S34C05 & 22.7936002 & 0.50977423 & 13.2581391 \\
S34C06 & 22.7936002 & 0.50977423 & 4.52850672 \\
S34C07 & -2.615162 & 22.6488191 & 41.3532266 \\
S34C08 & -2.615162 & 22.6488191 & 34.0176802 \\
S34C09 & -2.615162 & 22.6488191 & 26.6821339 \\
S34C10 & -2.615162 & 22.6488191 & 19.3465875 \\
S34C11 & -2.615162 & 22.6488191 & 12.0110412 \\
S34C12 & -2.615162 & 22.6488191 & 4.67549482 \\
S34C13 & -22.697984 & 2.1469968 & 43.9265758 \\
S34C14 & -22.697984 & 2.1469968 & 36.0652728 \\
S34C15 & -22.697984 & 2.1469968 & 28.2039698 \\
S34C16 & -22.697984 & 2.1469968 & 20.3426667 \\
S34C17 & -22.697984 & 2.1469968 & 12.4813637 \\
S34C18 & -22.697984 & 2.1469968 & 4.62006067 \\
S34C19 & -0.3946327 & -22.795884 & 48.2706372 \\
S34C20 & -0.3946327 & -22.795884 & 39.5218062 \\
S34C21 & -0.3946327 & -22.795884 & 30.7729753 \\
S34C22 & -0.3946327 & -22.795884 & 22.0241444 \\
S34C23 & -0.3946327 & -22.795884 & 13.2753134 \\
S34C24 & -0.3946327 & -22.795884 & 4.52648248 \\
S34R01 & 18.4070519 & 13.4531974 & 9.8626638 \\
S34R02 & 5.67182216 & 22.0825387 & 8.33561884 \\
S34R03 & -8.294351 & 21.2370389 & 6.52982303 \\
S34R04 & -13.150703 & 18.6243684 & 5.75524827 \\
S34R05 & -14.028005 & 17.972845 & 5.61861832 \\
S34R06 & -14.392037 & 17.6826853 & 5.58095624 \\
S34R07 & -14.654105 & 17.466118 & 5.5747453 \\
S34R08 & -14.937528 & 17.2243528 & 5.59288441 \\
S34R09 & -15.411076 & 16.8019887 & 5.65904126 \\
S34R10 & -16.922651 & 15.2784803 & 5.9514881 \\
S34R11 & -21.85466 & 6.49476171 & 7.33911825 \\
S34R12 & -21.105829 & -8.6227643 & 9.10694106 \\
S34R13 & -10.867671 & -20.0425 & 10.3792541 \\
S34R14 & 4.06142705 & -22.434636 & 11.0811394 \\
& & & \\
\hline
\end{tabular}

$\begin{array}{lll}-2.4682191 & -22.804315 & 29.8072717 \\ -1.9032215 & -22.858404 & 29.7983226 \\ 0.32974735 & -22.93513 & 29.7534678\end{array}$

\begin{tabular}{ccc} 
XINTo & ZINTo & YINTo \\
22.9331325 & 0.44759223 & 48.338088 \\
22.9331325 & 0.44759223 & 39.5754763 \\
22.9331325 & 0.44759223 & 30.8128646 \\
22.9331325 & 0.44759223 & 22.0502529 \\
22.9331325 & 0.44759223 & 13.2876412 \\
22.9331325 & 0.44759223 & 4.52502948 \\
-2.2547755 & 22.8264078 & 42.4759054 \\
-2.2547755 & 22.8264078 & 34.9109864 \\
-2.2547755 & 22.8264078 & 27.3460674 \\
-2.2547755 & 22.8264078 & 19.7811484 \\
-2.2547755 & 22.8264078 & 12.2162295 \\
-2.2547755 & 22.8264078 & 4.65131048 \\
-22.861681 & 1.86345382 & 44.6626297 \\
-22.861681 & 1.86345382 & 36.6509447 \\
-22.861681 & 1.86345382 & 28.6392598 \\
-22.861681 & 1.86345382 & 20.6275748 \\
-22.861681 & 1.86345382 & 12.6158898 \\
-22.861681 & 1.86345382 & 4.60420486 \\
-0.3465843 & -22.934881 & 48.4203178 \\
-0.3465843 & -22.934881 & 39.6409059 \\
-0.3465843 & -22.934881 & 30.8614939 \\
-0.3465843 & -22.934881 & 22.082082 \\
-0.3465843 & -22.934881 & 13.3026701 \\
-0.3465843 & -22.934881 & 4.52325812 \\
18.592441 & 13.4331696 & 9.88780405 \\
5.94623057 & 22.1533575 & 8.37560027 \\
-7.8746357 & 21.5434217 & 6.60742195 \\
-12.978125 & 18.9128836 & 5.8086483 \\
-14.006497 & 18.1644417 & 5.65101712 \\
-14.440818 & 17.8211024 & 5.60657527 \\
-14.753965 & 17.5627282 & 5.5992142 \\
-15.091441 & 17.2736014 & 5.62068813 \\
-15.648378 & 16.7707234 & 5.69828755 \\
-17.322197 & 15.0356376 & 6.02366429 \\
-22.091011 & 6.17382823 & 7.39703201 \\
-21.165214 & -8.8409627 & 9.13812922 \\
-10.853088 & -20.207409 & 10.4012215 \\
4.12128529 & -22.564218 & 11.0996611 \\
& & \\
\hline
\end{tabular}




\section{REVISED METHODOLOGY FOR DETERMINING CESIUM-137 CONTENT OF HN-200 GROUT CONTAINERS.}

$\begin{array}{llll}\text { S34R15 } & 17.1191758 & -15.057951 & 11.2283938\end{array}$

$\begin{array}{lllll}\text { S34R } 16 & 22.7714908 & -1.1257391 & 10.8270488\end{array}$ $\begin{array}{lll}17.2159843 & -15.157137 & 11.2462922\end{array}$

$\begin{array}{llll}22.9065103 & -1.1919285 & 10.8467212\end{array}$

$\begin{array}{lccc}\text { Line } & \text { XINTi } & \text { ZINTi } & \text { YINTi } \\ \text { S35C01 } & 22.6475534 & 2.62610088 & 45.5337179 \\ \text { S35C02 } & 22.6475534 & 2.62610088 & 38.3752176 \\ \text { S35C03 } & 22.6475534 & 2.62610088 & 31.2167174 \\ \text { S35C04 } & 22.6475534 & 2.62610088 & 24.0582172 \\ \text { S35C05 } & 22.6475534 & 2.62610088 & 16.899717 \\ \text { S35C06 } & 22.6475534 & 2.62610088 & 9.74121674 \\ \text { S35C07 } & 1.93879367 & 22.7167154 & 47.3686394 \\ \text { S35C08 } & 1.93879367 & 22.7167154 & 39.3582059 \\ \text { S35C09 } & 1.93879367 & 22.7167154 & 31.3477725 \\ \text { S35C10 } & 1.93879367 & 22.7167154 & 23.337339 \\ \text { S35C11 } & 1.93879367 & 22.7167154 & 15.3269056 \\ \text { S35C12 } & 1.93879367 & 22.7167154 & 7.31647212 \\ \text { S35C13 } & -22.796579 & 0.35225037 & 48.9679853 \\ \text { S35C14 } & -22.796579 & 0.35225037 & 40.2149937 \\ \text { S35C15 } & -22.796579 & 0.35225037 & 31.4620021 \\ \text { S35C16 } & -22.796579 & 0.35225037 & 22.7090105 \\ \text { S35C17 } & -22.796579 & 0.35225037 & 13.9560189 \\ \text { S35C18 } & -22.796579 & 0.35225037 & 5.20302728 \\ \text { S35C19 } & 0.54896429 & -22.79269 & 48.8965109 \\ \text { S35C20 } & 0.54896429 & -22.79269 & 40.176704 \\ \text { S35C21 } & 0.54896429 & -22.79269 & 31.4568972 \\ \text { S35C22 } & 0.54896429 & -22.79269 & 22.7370903 \\ \text { S35C23 } & 0.54896429 & -22.79269 & 14.0172835 \\ \text { S35C24 } & 0.54896429 & -22.79269 & 5.29747662 \\ \text { S35R01 } & 18.9336427 & 12.7013879 & 30.0805768 \\ \text { S35R02 } & 18.5981916 & 13.1876969 & 30.0871218 \\ \text { S35R03 } & 18.3243692 & 13.5656026 & 30.0876255 \\ \text { S35R04 } & 17.9952233 & 13.9992863 & 30.0826293 \\ \text { S35R05 } & 17.3712982 & 14.7663834 & 30.0655356 \\ \text { S35R06 } & 15.0059983 & 17.164734 & 29.9932725 \\ \text { S35R07 } & 4.9632822 & 22.2525035 & 29.7562944 \\ \text { S35R08 } & -9.9035279 & 20.5360224 & 29.4963963 \\ \text { S35R09 } & -20.595647 & 9.77892744 & 29.3142449 \\ \text { S35R10 } & -22.211902 & -5.1419358 & 29.2196651 \\ \text { S35R11 } & -14.271386 & -17.780203 & 29.2109569 \\ \text { S35R12 } & -0.1558042 & -22.798768 & 29.2878239 \\ \text { S35R13 } & 14.1572777 & -17.871194 & 29.4520985 \\ \text { S35R14 } & 22.2213984 & -5.1007383 & 29.6989095 \\ \text { S35R15 } & 21.5209144 & 7.5271723 & 29.958693 \\ & & & \end{array}$

$\begin{array}{ccc}\text { XINTo } & \text { ZINTo } & \text { YINTo } \\ 22.8255141 & 2.26380463 & 46.0809052 \\ 22.8255141 & 2.26380463 & 38.6683521 \\ 22.8255141 & 2.26380463 & 31.255799 \\ 22.8255141 & 2.26380463 & 23.8432459 \\ 22.8255141 & 2.26380463 & 16.4306928 \\ 22.8255141 & 2.26380463 & 9.01813972 \\ 1.68678757 & 22.8753941 & 47.6456755 \\ 1.68678757 & 22.8753941 & 39.5066173 \\ 1.68678757 & 22.8753941 & 31.3675591 \\ 1.68678757 & 22.8753941 & 23.228501 \\ 1.68678757 & 22.8753941 & 15.0894428 \\ 1.68678757 & 22.8753941 & 6.95038461 \\ -22.935413 & 0.30938627 & 49.0327243 \\ -22.935413 & 0.30938627 & 40.2496751 \\ -22.935413 & 0.30938627 & 31.4666259 \\ -22.935413 & 0.30938627 & 22.6835767 \\ -22.935413 & 0.30938627 & 13.9005276 \\ -22.935413 & 0.30938627 & 5.11747838 \\ 0.48195262 & -22.932436 & 48.9701784 \\ 0.48195262 & -22.932436 & 40.2161685 \\ 0.48195262 & -22.932436 & 31.4621587 \\ 0.48195262 & -22.932436 & 22.7081489 \\ 0.48195262 & -22.932436 & 13.9541391 \\ 0.48195262 & -22.932436 & 5.20012923 \\ 19.1119677 & 12.6831225 & 30.0757872 \\ 18.7326799 & 13.2369033 & 30.0831661 \\ 18.4208048 & 13.6675842 & 30.0837358 \\ 18.045146 & 14.1598592 & 30.0780966 \\ 17.3371415 & 15.0184031 & 30.0590163 \\ 14.7755461 & 17.5445759 & 29.98255 \\ 4.6951301 & 22.4518298 & 29.7486918 \\ -10.104123 & 20.5921247 & 29.4918863 \\ -20.75451 & 9.76622933 & 29.3109151 \\ -22.340327 & -5.1998761 & 29.2167777 \\ -14.371942 & -17.876694 & 29.208106 \\ -0.2245137 & -22.936401 & 29.284627 \\ 14.1354187 & -18.064298 & 29.4479264 \\ 22.2953063 & -5.3896402 & 29.6921989 \\ 21.7925641 & 7.15632988 & 29.9477361\end{array}$




\section{REVISED METHODOLOGY FOR DETERMINING CESIUM-137 CONTENT OF HN-200 GROUT CONTAINERS.}

TABLE 9. Calculate Microshield input data sheets for the simulations from each source to each

\begin{tabular}{lcccccr} 
& & \multicolumn{3}{c}{ measuring point in inches. } & \\
& Cs-137 & Ba-137m & Source 01 & X Posit & Z Posit & Y Posit \\
Line & 29.4975 & 27.9047 & & 19.3677 & 0.5818 & 25.9034 \\
SO1C01 & 18.0515 & 0.7276 & 24.0421 & 5.2629 & \\
S01C02 & 11.4924 & 0.4633 & 15.3063 & 3.3506 & \\
S01C03 & 5.4425 & 0.2194 & 7.2487 & 1.5868 & \\
S01C04 & 4.3004 & 0.1733 & 5.7275 & 1.2538 & \\
S01C05 & 9.9344 & 0.4005 & 13.2312 & 2.8964 & \\
S01C06 & 16.4383 & 0.6626 & 21.8935 & 4.7926 & \\
S01C07 & 36.5304 & 0.2353 & 38.4369 & 1.6712 & \\
S01C08 & 31.9998 & 0.2061 & 33.6698 & 1.4639 & \\
S01C09 & 29.3228 & 0.1889 & 30.8532 & 1.3414 & \\
S01C10 & 29.0172 & 0.1869 & 30.5316 & 1.3275 & \\
S01C11 & 31.1528 & 0.2007 & 32.7787 & 1.4252 & \\
S01C12 & 35.2892 & 0.2273 & 37.1309 & 1.6144 & \\
S01C13 & 48.0239 & 0.1574 & 49.3202 & 1.1389 & \\
S01C14 & 44.5015 & 0.1459 & 45.7027 & 1.0554 & \\
S01C15 & 42.5215 & 0.1394 & 43.6692 & 1.0084 & \\
S01C16 & 42.3008 & 0.1386 & 43.4426 & 1.0032 & \\
S01C17 & 43.8662 & 0.1438 & 45.0502 & 1.0403 & \\
S01C18 & 47.0395 & 0.1542 & 48.3093 & 1.1156 & \\
S01C19 & 37.3081 & 0.2276 & 39.1549 & 1.6192 & \\
S01C20 & 32.8605 & 0.2005 & 34.4872 & 1.4262 & \\
S01C21 & 30.2459 & 0.1845 & 31.7431 & 1.3127 & \\
S01C22 & 29.9482 & 0.1827 & 31.4307 & 1.2998 & \\
S01C23 & 32.0320 & 0.1954 & 33.6177 & 1.3902 & \\
S01C24 & 36.0872 & 0.2201 & 37.8736 & 1.5663 & \\
S01R01 & 3.4247 & 0.1383 & 123.5741 & 120.0111 & \\
S01R02 & 3.6910 & 0.1472 & 125.0134 & 121.1752 & \\
S01R03 & 4.7913 & 0.1801 & 129.5169 & 124.5455 & \\
S01R04 & 7.6629 & 0.2364 & 136.1426 & 128.2433 & \\
S01R05 & 14.2208 & 0.2566 & 143.6696 & 129.1922 & \\
S01R06 & 24.0113 & 0.2091 & 150.9054 & 126.6850 & \\
S01R07 & 33.3288 & 0.1671 & 156.8590 & 123.3631 & \\
S01R08 & 39.7194 & 0.1453 & 160.8071 & 120.9424 & \\
S01R09 & 42.1638 & 0.1382 & 162.3085 & 120.0064 & \\
S01R10 & 40.3627 & 0.1434 & 161.2024 & 120.6963 & \\
S01R11 & 34.5327 & 0.1626 & 157.6070 & 122.9118 & \\
S01R12 & 25.5517 & 0.2013 & 151.9203 & 126.1673 & \\
S01R13 & 15.5924 & 0.2518 & 144.8227 & 128.9785 & \\
S01R14 & 8.3804 & 0.2445 & 137.2666 & 128.6417 & \\
S01R15 & 5.0767 & 0.1876 & 130.4218 & 125.1575 & \\
S01R16 & 3.7934 & 0.1505 & 125.5215 & 121.5776 & \\
& & & & & & \\
& &
\end{tabular}


REVISED METHODOLOGY FOR DETERMINING CESIUM-137 CONTENT OF HN-200 GROUT CONTAINERS.

\begin{tabular}{|c|c|c|c|c|c|c|}
\hline & Cs-137 & Ba-137m & Source 02 & X Posit & Z Posit & Y Posit \\
\hline & 13.8502 & 13.1023 & & -10.6176 & -19.5689 & 18.8660 \\
\hline Line & Shield 1 & Shield 2 & Total Dist & Air Gap & & \\
\hline $\mathrm{S} 02 \mathrm{CO}$ & 48.4887 & 0.2037 & 50.1542 & 1.4617 & & \\
\hline $\mathrm{S} 02 \mathrm{C} 02$ & 43.7203 & 0.1837 & 45.2220 & 1.3180 & & \\
\hline $\mathrm{SO2C03}$ & 40.2889 & 0.1693 & 41.6728 & 1.2145 & & \\
\hline $\mathrm{S} 02 \mathrm{C} 04$ & 38.5532 & 0.1620 & 39.8774 & 1.1622 & & \\
\hline S02C05 & 38.7417 & 0.1628 & 40.0723 & 1.1679 & & \\
\hline S02C06 & 40.8278 & 0.1715 & 42.2301 & 1.2308 & & \\
\hline S02C07 & 52.8369 & 0.1728 & 54.2585 & 1.2488 & & \\
\hline S02C08 & 48.4320 & 0.1584 & 49.7351 & 1.1447 & & \\
\hline S02C09 & 45.3122 & 0.1482 & 46.5313 & 1.0709 & & \\
\hline SO2C10 & 43.7533 & 0.1431 & 44.9305 & 1.0341 & & \\
\hline SO2C11 & 43.9219 & 0.1437 & 45.1036 & 1.0381 & & \\
\hline $\mathrm{S} 02 \mathrm{C} 12$ & 45.7989 & 0.1498 & 47.0311 & 1.0824 & & \\
\hline SO2C13 & 35.2054 & 0.4510 & 38.7144 & 3.0581 & & \\
\hline S02C14 & 29.1619 & 0.3735 & 32.0686 & 2.5331 & & \\
\hline $\mathrm{S} 02 \mathrm{C} 15$ & 24.4004 & 0.3126 & 26.8325 & 2.1195 & & \\
\hline S02C16 & 21.7786 & 0.2790 & 23.9493 & 1.8918 & & \\
\hline S02C17 & 22.0725 & 0.2827 & 24.2725 & 1.9173 & & \\
\hline $\mathrm{S} 02 \mathrm{C} 18$ & 25.1803 & 0.3225 & 27.6901 & 2.1873 & & \\
\hline S02C19 & 22.3069 & 1.5713 & 32.7149 & 8.8367 & & \\
\hline SO2C20 & 16.7000 & 1.1763 & 24.4918 & 6.6155 & & \\
\hline S02C21 & 11.6404 & 0.8199 & 17.0715 & 4.6112 & & \\
\hline $\mathrm{S} 02 \mathrm{C} 22$ & 8.2114 & 0.5784 & 12.0426 & 3.2528 & & \\
\hline S02C23 & 8.6414 & 0.6087 & 12.6733 & 3.4232 & & \\
\hline S02C24 & 12.5395 & 0.8833 & 18.3902 & 4.9674 & & \\
\hline SO2R01 & 26.9442 & 0.2258 & 154.9977 & 127.8277 & & \\
\hline S02R02 & 37.5951 & 0.1650 & 161.0405 & 123.2805 & & \\
\hline SO2R03 & 43.7484 & 0.1425 & 164.5935 & 120.7025 & & \\
\hline S02R04 & 44.9347 & 0.1389 & 165.2844 & 120.2108 & & \\
\hline S02R05 & 41.0753 & 0.1515 & 163.0437 & 121.8168 & & \\
\hline S02R06 & 32.4387 & 0.1899 & 158.1007 & 125.4721 & & \\
\hline S02R07 & 19.8210 & 0.2970 & 150.9915 & 130.8735 & & \\
\hline S02R08 & 6.2069 & 0.5926 & 142.5676 & 135.7681 & & \\
\hline S02R09 & 1.4454 & 0.3421 & 133.9912 & 132.2037 & & \\
\hline SO2R10 & 0.7549 & 0.1921 & 126.6644 & 125.7174 & & \\
\hline SO2R 11 & 0.5684 & 0.1464 & 122.0120 & 121.2972 & & \\
\hline SO2R 12 & 0.5407 & 0.1395 & 121.0742 & 120.3940 & & \\
\hline SO2R 13 & 0.6395 & 0.1640 & 124.0754 & 123.2718 & & \\
\hline SO2R 14 & 0.9998 & 0.2490 & 130.3153 & 129.0665 & & \\
\hline SO2R 15 & 2.7805 & 0.5278 & 138.4901 & 135.1819 & & \\
\hline S02R16 & 13.1223 & 0.4122 & 147.1475 & 133.6130 & & \\
\hline
\end{tabular}




\section{REVISED METHODOLOGY FOR DETERMINING CESIUM-137 CONTENT OF HN-200 GROUT CONTAINERS.}

\begin{tabular}{|c|c|c|c|c|c|c|}
\hline & Cs-137 & $\mathrm{Ba}-137 \mathrm{~m}$ & Source $\mathbf{0 3}$ & X Posit & Z Posit & Y Posit \\
\hline & 23.5916 & 22.3177 & & 1.7704 & -7.2590 & \\
\hline Line & Shield 1 & Shield 2 & Total Dist & Air Gap & & \\
\hline $\mathrm{S} 03 \mathrm{C} 01$ & 23.2729 & 0.1538 & 24.5362 & 1.1095 & & \\
\hline $\mathrm{S} 03 \mathrm{CO} 2$ & 22.1635 & 0.1464 & 23.3666 & 1.0566 & & \\
\hline $\mathrm{S} 03 \mathrm{C} 03$ & 24.2191 & 0.1600 & 25.5337 & 1.1546 & & \\
\hline S03C04 & 28.7690 & 0.1901 & 30.3305 & 1.3715 & & \\
\hline $\mathrm{S} 03 \mathrm{COS}$ & 34.8496 & 0.2302 & 36.7412 & 1.6614 & & \\
\hline $\mathrm{S} 03 \mathrm{C} 06$ & 41.7981 & 0.2762 & 44.0668 & 1.9926 & & \\
\hline S03C07 & 30.9872 & 0.1425 & 32.1607 & 1.0310 & & \\
\hline S03C08 & 30.1363 & 0.1386 & 31.2775 & 1.0027 & & \\
\hline S03C09 & 31.7265 & 0.1459 & 32.9280 & 1.0556 & & \\
\hline $\mathrm{S} 03 \mathrm{C} 10$ & 35.4309 & 0.1629 & 36.7726 & 1.1788 & & \\
\hline $\mathrm{S} 03 \mathrm{C} 11$ & 40.6757 & 0.1870 & 42.2161 & 1.3533 & & \\
\hline $\mathrm{S} 03 \mathrm{C} 12$ & 46.9476 & 0.2159 & 48.7255 & 1.5620 & & \\
\hline $\mathrm{S} 03 \mathrm{C} 13$ & 26.5444 & 0.1499 & 27.7767 & 1.0824 & & \\
\hline S03C14 & 25.5625 & 0.1443 & 26.7491 & 1.0423 & & \\
\hline $\mathrm{S} 03 \mathrm{C} 15$ & 27.3901 & 0.1547 & 28.6616 & 1.1168 & & \\
\hline $\mathrm{S} 03 \mathrm{C} 16$ & 31.5427 & 0.1781 & 33.0070 & 1.2862 & & \\
\hline $\mathrm{S} 03 \mathrm{C} 17$ & 37.2507 & 0.2104 & 38.9799 & 1.5189 & & \\
\hline S03C18 & 43.9116 & 0.2480 & 45.9500 & 1.7905 & & \\
\hline $\mathrm{S} 03 \mathrm{C} 19$ & 17.1616 & 0.1527 & 18.4190 & 1.1046 & & \\
\hline $\mathrm{S} 03 \mathrm{C} 20$ & 15.6806 & 0.1395 & 16.8295 & 1.0093 & & \\
\hline $\mathrm{S} 03 \mathrm{C} 21$ & 18.3815 & 0.1636 & 19.7282 & 1.1831 & & \\
\hline $\mathrm{S} 03 \mathrm{C} 22$ & 23.8855 & 0.2125 & 25.6354 & 1.5374 & & \\
\hline $\mathrm{S} 03 \mathrm{C} 23$ & 30.7212 & 0.2734 & 32.9719 & 1.9774 & & \\
\hline S03C24 & 38.1799 & 0.3397 & 40.9771 & 2.4575 & & \\
\hline S03R01 & 20.3055 & 0.1468 & 142.1618 & 121.7096 & & \\
\hline S03R02 & 23.2392 & 0.1467 & 145.0607 & 121.6749 & & \\
\hline S03R03 & 26.2300 & 0.1444 & 147.7349 & 121.3605 & & \\
\hline S03R04 & 28.7068 & 0.1414 & 149.7986 & 120.9504 & & \\
\hline S03R05 & 30.1684 & 0.1393 & 150.9678 & 120.6601 & & \\
\hline S03R06 & 30.3184 & 0.1391 & 151.0860 & 120.6285 & & \\
\hline S03R07 & 29.1267 & 0.1408 & 150.1378 & 120.8703 & & \\
\hline S03R08 & 26.8344 & 0.1437 & 148.2492 & 121.2711 & & \\
\hline S03R09 & 23.9068 & 0.1463 & 145.6785 & 121.6254 & & \\
\hline S03R10 & 20.9147 & 0.1470 & 142.7920 & 121.7304 & & \\
\hline S03R11 & 18.3599 & 0.1451 & 140.0237 & 121.5187 & & \\
\hline S03R12 & 16.5458 & 0.1419 & 137.8135 & 121.1258 & & \\
\hline S03R 13 & 15.5837 & 0.1395 & 136.5318 & 120.8086 & & \\
\hline S03R14 & 15.4891 & 0.1393 & 136.4009 & 120.7726 & & \\
\hline S03R15 & 16.2617 & 0.1413 & 137.4440 & 121.0410 & & \\
\hline S03R16 & 17.8942 & 0.1445 & 139.4789 & 121.4402 & & \\
\hline
\end{tabular}


HNF-4799, Rev 0

August 25, 1999

\section{REVISED METHODOLOGY FOR DETERMINING CESIUM-137 CONTENT OF HN-200 GROUT CONTAINERS.}

\begin{tabular}{|c|c|c|c|c|c|c|}
\hline & $\begin{array}{c}\text { Cs-137 } \\
20.6982\end{array}$ & $\begin{array}{c}\text { Ba-137m } \\
19.5805\end{array}$ & Source 04 & $\begin{array}{l}\text { X Posit } \\
5.5262\end{array}$ & $\begin{array}{c}\text { Z Posit } \\
-19.2179\end{array}$ & $\begin{array}{l}\text { Y Posit } \\
28.6756\end{array}$ \\
\hline Line & Shield 1 & Shield 2 & Total Dist & Air Gap & & \\
\hline S04C01 & 31.6437 & 0.2680 & 33.7929 & 1.8813 & & \\
\hline $\mathrm{S} 04 \mathrm{C} 02$ & 27.2703 & 0.2310 & 29.1225 & 1.6213 & & \\
\hline $\mathrm{S} 04 \mathrm{C} 03$ & 25.0613 & 0.2123 & 26.7635 & 1.4899 & & \\
\hline S04C04 & 25.5835 & 0.2167 & 27.3211 & 1.5210 & & \\
\hline SO4C05 & 28.6881 & 0.2430 & 30.6366 & 1.7056 & & \\
\hline S04C06 & 33.6682 & 0.2852 & 35.9550 & 2.0016 & & \\
\hline $\mathrm{S} 04 \mathrm{C} 07$ & 46.9620 & 0.1546 & 48.2347 & 1.1181 & & \\
\hline S04C08 & 43.8964 & 0.1445 & 45.0860 & 1.0451 & & \\
\hline S04C09 & 42.4490 & 0.1397 & 43.5994 & 1.0106 & & \\
\hline $\mathrm{S} 04 \mathrm{Cl} 10$ & 42.7844 & 0.1408 & 43.9439 & 1.0186 & & \\
\hline S04C11 & 44.8627 & 0.1477 & 46.0785 & 1.0681 & & \\
\hline S04C12 & 48.4601 & 0.1595 & 49.7734 & 1.1538 & & \\
\hline $\mathrm{S} 04 \mathrm{C} 13$ & 39.2827 & 0.1958 & 40.8790 & 1.4006 & & \\
\hline SO4C14 & 35.6622 & 0.1777 & 37.1114 & 1.2715 & & \\
\hline S04C15 & 33.9123 & 0.1690 & 35.2904 & 1.2091 & & \\
\hline S04C16 & 34.3205 & 0.1710 & 35.7152 & 1.2236 & & \\
\hline S04C17 & 36.8150 & 0.1835 & 38.3110 & 1.3126 & & \\
\hline $\mathrm{S} 04 \mathrm{C} 18$ & 41.0169 & 0.2044 & 42.6837 & 1.4624 & & \\
\hline S04C19 & 16.5410 & 0.6929 & 22.0561 & 4.8221 & & \\
\hline S04C20 & 10.4087 & 0.4360 & 13.8791 & 3.0344 & & \\
\hline S04C21 & 5.8473 & 0.2449 & 7.7968 & 1.7046 & & \\
\hline S04C22 & 7.1522 & 0.2996 & 9.5368 & 2.0850 & & \\
\hline S04C23 & 12.6187 & 0.5286 & 16.8259 & 3.6786 & & \\
\hline S04C24 & 18.9320 & 0.7931 & 25.2442 & 5.5191 & & \\
\hline S04R01 & 8.4965 & 0.2762 & 138.7621 & 129.9894 & & \\
\hline S04R02 & 16.9309 & 0.2604 & 146.5523 & 129.3611 & & \\
\hline S04R03 & 27.5749 & 0.1968 & 153.6308 & 125.8591 & & \\
\hline S04R04 & 36.4316 & 0.1585 & 159.0781 & 122.4881 & & \\
\hline SO4R05 & 41.6714 & 0.1415 & 162.2610 & 120.4482 & & \\
\hline S04R06 & 42.6156 & 0.1387 & 162.8342 & 120.0798 & & \\
\hline S04R07 & 39.1620 & 0.1492 & 160.7376 & 121.4265 & & \\
\hline S04R08 & 31.7119 & 0.1772 & 156.1943 & 124.3052 & & \\
\hline S04R09 & 21.4809 & 0.2316 & 149.7147 & 128.0022 & & \\
\hline SO4R 10 & 11.5186 & 0.2845 & 142.0978 & 130.2947 & & \\
\hline S04R11 & 5.8528 & 0.2378 & 134.4136 & 128.3229 & & \\
\hline S04R 12 & 3.7017 & 0.1742 & 127.9200 & 124.0441 & & \\
\hline S04R 13 & 2.9371 & 0.1439 & 123.8579 & 120.7769 & & \\
\hline S04R14 & 2.8242 & 0.1391 & 123.1033 & 120.1400 & & \\
\hline S04R 15 & 3.2712 & 0.1576 & 125.8286 & 122.3998 & & \\
\hline S04R 16 & 4.6695 & 0.2068 & 131.4257 & 126.5494 & & \\
\hline
\end{tabular}




\section{REVISED METHODOLOGY FOR DETERMINING CESIUM-137 CONTENT OF HN-200 GROUT CONTAINERS.}

\begin{tabular}{|c|c|c|c|c|c|c|}
\hline & $\begin{array}{c}\text { Cs-137 } \\
10.9510\end{array}$ & $\begin{array}{c}\text { Ba-137m } \\
10.3596\end{array}$ & Source 05 & $\begin{array}{c}\text { X Posit } \\
-12.5171\end{array}$ & $\begin{array}{l}\text { Z Posit } \\
2.4304\end{array}$ & $\begin{array}{l}\text { Y Posit } \\
36.3581\end{array}$ \\
\hline Line & Shield 1 & Shield 2 & Total Dist & Air Gap & & \\
\hline $\mathrm{S} 05 \mathrm{C} 0 \mathrm{I}$ & 37.6148 & 0.1472 & 38.8272 & 1.0652 & & \\
\hline $\mathrm{S} 05 \mathrm{C} 02$ & 35.6214 & 0.1394 & 36.7695 & 1.0087 & & \\
\hline S05C03 & 35.7062 & 0.1398 & 36.8571 & 1.0111 & & \\
\hline S05C04 & 37.8553 & 0.1482 & 39.0754 & 1.0720 & & \\
\hline S05C05 & 41.7511 & 0.1634 & 43.0968 & 1.1823 & & \\
\hline S05C06 & 46.9609 & 0.1838 & 48.4745 & 1.3299 & & \\
\hline S05C07 & 26.6394 & 0.1838 & 28.1415 & 1.3183 & & \\
\hline S05C08 & 23.8802 & 0.1648 & 25.2267 & 1.1817 & & \\
\hline S05C09 & 24.0008 & 0.1656 & 25.3542 & 1.1877 & & \\
\hline $\mathrm{S} 05 \mathrm{C} 10$ & 26.9626 & 0.1861 & 28.4830 & 1.3343 & & \\
\hline S0sC11 & 31.9859 & 0.2207 & 33.7894 & 1.5829 & & \\
\hline $\mathrm{S} 05 \mathrm{C} 12$ & 38.2671 & 0.2641 & 40.4249 & 1.8937 & & \\
\hline $\mathrm{S} 05 \mathrm{C} 13$ & 15.8256 & 0.2132 & 17.5796 & 1.5408 & & \\
\hline S05C14 & 11.1529 & 0.1502 & 12.3890 & 1.0859 & & \\
\hline S05C15 & 11.3847 & 0.1534 & 12.6465 & 1.1085 & & \\
\hline S05C16 & 16.3132 & 0.2198 & 18.1213 & 1.5883 & & \\
\hline S05C17 & 23.1066 & 0.3113 & 25.6676 & 2.2497 & & \\
\hline S05C18 & 30.5450 & 0.4115 & 33.9304 & 2.9740 & & \\
\hline S05C19 & 30.6205 & 0.1696 & 32.0102 & 1.2200 & & \\
\hline $\mathrm{S} 05 \mathrm{C} 20$ & 28.2007 & 0.1562 & 29.4804 & 1.1236 & & \\
\hline S05C21 & 28.3050 & 0.1568 & 29.5896 & 1.1278 & & \\
\hline $\mathrm{S} 05 \mathrm{C} 22$ & 30.9082 & 0.1712 & 32.3108 & 1.2315 & & \\
\hline $\mathrm{S} 05 \mathrm{C} 23$ & 35.4640 & 0.1964 & 37.0734 & 1.4130 & & \\
\hline S05C24 & 41.3317 & 0.2289 & 43.2074 & 1.6468 & & \\
\hline S05R01 & 35.3103 & 0.1391 & 155.7702 & 120.3207 & & \\
\hline S05R02 & 32.8497 & 0.1445 & 154.0327 & 121.0384 & & \\
\hline S05R03 & 28.3836 & 0.1541 & 150.7481 & 122.2104 & & \\
\hline S05R04 & 22.8949 & 0.1639 & 146.3335 & 123.2747 & & \\
\hline SOSR05 & 17.6765 & 0.1665 & 141.3904 & 123.5474 & & \\
\hline SO5R06 & 13.7360 & 0.1587 & 136.6556 & 122.7609 & & \\
\hline S05R07 & 11.3223 & 0.1471 & 132.9079 & 121.4385 & & \\
\hline S05R08 & 10.2135 & 0.1396 & 130.8213 & 120.4682 & & \\
\hline S05R09 & 10.2012 & 0.1395 & 130.7965 & 120.4558 & & \\
\hline SOSR 10 & 11.2827 & 0.1469 & 132.8381 & 121.4085 & & \\
\hline S05R 11 & 13.6629 & 0.1584 & 136.5543 & 122.7330 & & \\
\hline S05R 12 & 17.5677 & 0.1664 & 141.2747 & 123.5406 & & \\
\hline SO5R13 & 22.7653 & 0.1641 & 146.2218 & 123.2924 & & \\
\hline S05R14 & 28.2627 & 0.1544 & 150.6562 & 122.2391 & & \\
\hline S05R 15 & 32.7657 & 0.1447 & 153.9726 & 121.0623 & & \\
\hline S05R 16 & 35.2804 & 0.1392 & 155.7493 & 120.3297 & & \\
\hline
\end{tabular}




\section{REVISED METHODOLOGY FOR DETERMINING CESIUM-137 CONTENT} OF HN-200 GROUT CONTAINERS.

\begin{tabular}{|c|c|c|c|c|c|c|}
\hline & Cs-137 & Ba-137m & Source 06 & X Posit & $\begin{array}{l}2 \text { Posit } \\
68160\end{array}$ & Y Posit \\
\hline & 16.3182 & 15.4370 & & 10.1481 & -6.8169 & \\
\hline Line & Shield 1 & Shield 2 & Total Dist & Air Gap & & \\
\hline S06C01 & 23.0321 & 0.2547 & 25.1182 & 1.8314 & & \\
\hline $\mathrm{SO6CO} 2$ & 17.2644 & 0.1909 & 18.8281 & 1.3728 & & \\
\hline $\mathrm{S} 06 \mathrm{CO} 3$ & 14.2073 & 0.1571 & 15.4941 & 1.1297 & & \\
\hline S06C04 & 15.5513 & 0.1720 & 16.9599 & 1.2365 & & \\
\hline S06C05 & 20.4462 & 0.2261 & 22.2981 & 1.6258 & & \\
\hline S06C06 & 27.0271 & 0.2989 & 29.4750 & 2.1490 & & \\
\hline $\mathrm{S} 06 \mathrm{CO} 7$ & 36.5787 & 0.1716 & 37.9885 & 1.2382 & & \\
\hline \$06C08 & 32.8893 & 0.1543 & 34.1569 & 1.1133 & & \\
\hline S06C09 & 31.2348 & 0.1465 & 32.4386 & 1.0573 & & \\
\hline $\mathrm{SO6C10}$ & 31.9330 & 0.1498 & 33.1638 & 1.0809 & & \\
\hline SO6C 11 & 34.8428 & 0.1635 & 36.1857 & 1.1794 & & \\
\hline $\mathrm{S} 06 \mathrm{C} 12$ & 39.4782 & 0.1852 & 40.9997 & 1.3363 & & \\
\hline SO6C13 & 38.6945 & 0.1626 & 40.0326 & 1.1755 & & \\
\hline SO6C 14 & 35.1995 & 0.1479 & 36.4167 & 1.0693 & & \\
\hline S06C15 & 33.6466 & 0.1414 & 34.8102 & 1.0221 & & \\
\hline S06C16 & 34.3007 & 0.1442 & 35.4869 & 1.0420 & & \\
\hline $\mathrm{S} 06 \mathrm{C} 17$ & 37.0450 & 0.1557 & 38.3261 & 1.1254 & & \\
\hline SO6C18 & 41.4666 & 0.1743 & 42.9006 & 1.2597 & & \\
\hline SO6C19 & 26.2285 & 0.2309 & 28.1142 & 1.6549 & & \\
\hline SO6C20 & 21.1504 & 0.1862 & 22.6711 & 1.3345 & & \\
\hline $\mathrm{S} 06 \mathrm{C} 21$ & 18.6480 & 0.1641 & 19.9887 & 1.1766 & & \\
\hline $\mathrm{SO6C} 22$ & 19.7269 & 0.1736 & 21.1452 & 1.2447 & & \\
\hline $\mathrm{SO6C23}$ & 23.9071 & 0.2104 & 25.6259 & 1.5084 & & \\
\hline S06C24 & 29.9156 & 0.2633 & 32.0665 & 1.8875 & & \\
\hline S06R01 & 11.8070 & 0.1459 & 132.9957 & 121.0428 & & \\
\hline S06R02 & 14.1692 & 0.1562 & 136.5813 & 122.2559 & & \\
\hline S06R03 & 17.9670 & 0.1632 & 141.1225 & 122.9924 & & \\
\hline S06R04 & 22.9367 & 0.1611 & 145.8738 & 122.7761 & & \\
\hline S06R05 & 28.1424 & 0.1525 & 150.1257 & 121.8307 & & \\
\hline S06R06 & 32.3846 & 0.1438 & 153.2957 & 120.7673 & & \\
\hline S06R07 & 34.7320 & 0.1389 & 154.9785 & 120.1076 & & \\
\hline S06R08 & 34.7168 & 0.1389 & 154.9676 & 120.1119 & & \\
\hline S06R09 & 32.3417 & 0.1439 & 153.2646 & 120.7790 & & \\
\hline SO6R 10 & 28.0809 & 0.1526 & 150.0781 & 121.8446 & & \\
\hline S06R 11 & 22.8705 & 0.1612 & 145.8161 & 122.7845 & & \\
\hline S06R 12 & 17.9109 & 0.1631 & 141.0628 & 122.9887 & & \\
\hline SO6R 13 & 14.1309 & 0.1561 & 136.5290 & 122.2421 & & \\
\hline S06R 14 & 11.7860 & 0.1458 & 132.9598 & 121.0280 & & \\
\hline S06R 15 & 10.7047 & 0.1391 & 130.9946 & 120.1507 & & \\
\hline S06R 16 & 10.7113 & 0.1392 & 131.0073 & 120.1568 & & \\
\hline
\end{tabular}

Cs-137 Ba-137m Source $07 \quad X$ Posit $\quad Z$ Posit $\quad$ Y Posit 


\section{REVISED METHODOLOGY FOR DETERMINING CESIUM-137 CONTENT} OF HN-200 GROUT CONTAINERS.

$\begin{array}{lccccc} & 25.9358 & 24.5353 & & -3.0040 & -18.4824 \\ \text { Line } & \text { Shield 1 } & \text { Shield 2 } & \text { Total Dist } & \text { Air Gap } & \\ \text { S07C01 } & 55.5673 & 0.3047 & 58.0492 & 2.1772 \\ \text { S07C02 } & 48.6882 & 0.2670 & 50.8629 & 1.9077 \\ \text { S07C03 } & 42.4477 & 0.2328 & 44.3436 & 1.6632 \\ \text { S07C04 } & 37.1688 & 0.2038 & 38.8289 & 1.4563 \\ \text { S07C05 } & 33.3117 & 0.1827 & 34.7996 & 1.3052 \\ \text { S07C06 } & 31.4048 & 0.1722 & 32.8075 & 1.2305 \\ \text { S07C07 } & 62.3945 & 0.2089 & 64.1150 & 1.5116 \\ \text { S07C08 } & 56.1412 & 0.1880 & 57.6894 & 1.3601 \\ \text { S07C09 } & 50.6362 & 0.1696 & 52.0325 & 1.2267 \\ \text { S07C10 } & 46.1480 & 0.1545 & 47.4206 & 1.1180 \\ \text { S07C11 } & 42.9962 & 0.1440 & 44.1819 & 1.0417 \\ \text { S07C12 } & 41.4865 & 0.1389 & 42.6305 & 1.0051 \\ \text { S07C13 } & 52.4367 & 0.3810 & 55.5165 & 2.6987 \\ \text { S07C14 } & 45.2920 & 0.3291 & 47.9521 & 2.3310 \\ \text { S07C15 } & 38.6994 & 0.2812 & 40.9723 & 1.9917 \\ \text { S07C16 } & 32.9916 & 0.2397 & 34.9292 & 1.6980 \\ \text { S07C17 } & 28.7014 & 0.2085 & 30.3871 & 1.4772 \\ \text { S07C18 } & 26.5259 & 0.1927 & 28.0838 & 1.3652 \\ \text { S07C19 } & 38.2115 & 1.2439 & 48.3844 & 8.9289 \\ \text { S07C20 } & 31.1763 & 1.0149 & 39.4763 & 7.2850 \\ \text { S07C21 } & 24.1836 & 0.7873 & 30.6219 & 5.6510 \\ \text { S07C22 } & 17.2849 & 0.5627 & 21.8866 & 4.0390 \\ \text { S07C23 } & 10.6645 & 0.3472 & 13.5037 & 2.4920 \\ \text { S07C24 } & 5.4530 & 0.1775 & 6.9047 & 1.2742 \\ \text { S07R01 } & 19.6312 & 0.2265 & 149.2554 & 129.3977 \\ \text { S07R02 } & 29.1582 & 0.1839 & 155.6719 & 126.3298 \\ \text { S07R03 } & 36.9240 & 0.1551 & 160.5047 & 123.4255 \\ \text { S07R04 } & 41.3086 & 0.1419 & 163.1824 & 121.7319 \\ \text { S07R05 } & 41.6786 & 0.1408 & 163.4076 & 121.5882 \\ \text { S07R06 } & 37.9873 & 0.1517 & 161.1559 & 123.0168 \\ \text { S07R07 } & 30.7462 & 0.1774 & 156.6757 & 125.7521 \\ \text { S07R08 } & 21.3487 & 0.2188 & 150.4891 & 128.9216 \\ \text { S07R09 } & 12.6587 & 0.2443 & 143.3863 & 130.4833 \\ \text { S07R10 } & 7.4536 & 0.2100 & 136.3929 & 128.7294 \\ \text { S07R11 } & 5.1529 & 0.1665 & 130.6716 & 125.3521 \\ \text { S07R12 } & 4.2802 & 0.1444 & 127.3118 & 122.8873 \\ \text { S07R13 } & 4.2164 & 0.1426 & 127.0227 & 122.6636 \\ \text { S07R14 } & 4.9194 & 0.1609 & 129.8675 & 124.7872 \\ \text { S07R15 } & 6.8800 & 0.2011 & 135.2389 & 128.1577 \\ \text { S07R16 } & 11.4394 & 0.2421 & 142.0909 & 130.4094 \\ & & & & \\ & & & & \\ \text { S0 } & \end{array}$

$\begin{array}{cccccc}\text { Cs-137 } & \text { Ba-137m } & \text { Source 08 } & \text { X Posit } & \text { Z Posit } & \text { Y Posit } \\ 25.1678 & 23.8087 & & -1.4468 & -9.3997 & 20.8621\end{array}$




\section{REVISED METHODOLOGY FOR DETERMINING CESIUM-137 CONTENT} OF HN-200 GROUT CONTAINERS.

\begin{tabular}{|c|c|c|c|c|}
\hline Line & Shield 1 & Shield 2 & Total Dist & Air Gap \\
\hline S08C01 & 37.6331 & 0.2160 & 39.4061 & 1.5571 \\
\hline $\mathrm{S} 08 \mathrm{CO} 2$ & 31.9373 & 0.1833 & 33.4419 & 1.3214 \\
\hline $\mathrm{S} 08 \mathrm{C} 03$ & 27.7754 & 0.1594 & 29.0840 & 1.1492 \\
\hline S08C04 & 25.8981 & 0.1486 & 27.1182 & 1.0715 \\
\hline S08C05 & 26.7899 & 0.1537 & 28.0520 & 1.1084 \\
\hline S08C06 & 30.2065 & 0.1733 & 31.6296 & 1.2498 \\
\hline $\mathrm{S} 08 \mathrm{C} 07$ & 42.4712 & 0.1823 & 43.9726 & 1.3191 \\
\hline S08C08 & 37.3964 & 0.1605 & 38.7183 & 1.1615 \\
\hline S08C09 & 33.8274 & 0.1452 & 35.0233 & 1.0506 \\
\hline S08C10 & 32.2681 & 0.1385 & 33.4088 & 1.0022 \\
\hline s08C11 & 33.0044 & 0.1417 & 34.1711 & 1.0251 \\
\hline $\mathrm{S} 08 \mathrm{C} 12$ & 35.8953 & 0.1541 & 37.1643 & 1.1148 \\
\hline $\mathrm{S} 08 \mathrm{C} 13$ & 35.6958 & 0.2330 & 37.6074 & 1.6785 \\
\hline $\mathrm{S} 08 \mathrm{C} 14$ & 29.7112 & 0.1940 & 31.3023 & 1.3971 \\
\hline S08C15 & 25.2442 & 0.1648 & 26.5961 & 1.1871 \\
\hline S08C16 & 23.1891 & 0.1514 & 24.4309 & 1.0904 \\
\hline S08C17 & 24.1692 & 0.1578 & 25.4635 & 1.1365 \\
\hline $\mathrm{S} 08 \mathrm{C} 18$ & 27.8660 & 0.1819 & 29.3583 & 1.3104 \\
\hline S08C19 & 29.6315 & 0.3058 & 32.1492 & 2.2119 \\
\hline S08C20 & 22.5595 & 0.2328 & 24.4762 & 1.6840 \\
\hline $\mathrm{S} 08 \mathrm{C} 21$ & 16.6569 & 0.1719 & 18.0722 & 1.2434 \\
\hline S08C22 & 13.5498 & 0.1398 & 14.7011 & 1.0115 \\
\hline $\mathrm{S} 08 \mathrm{C} 23$ & 15.0786 & 0.1556 & 16.3597 & 1.1256 \\
\hline S08C24 & 20.2175 & 0.2086 & 21.9353 & 1.5092 \\
\hline S08R01 & 22.8959 & 0.1514 & 144.8097 & 121.7624 \\
\hline S08R02 & 26.8000 & 0.1473 & 148.2116 & 121.2643 \\
\hline S08R03 & 30.0637 & 0.1422 & 150.8271 & 120.6211 \\
\hline S08R04 & 31.9946 & 0.1389 & 152.3060 & 120.1725 \\
\hline S08R05 & 32.1959 & 0.1385 & 152.4578 & 120.1234 \\
\hline S08R06 & 30.6273 & 0.1413 & 151.2632 & 120.4947 \\
\hline S08R07 & 27.6086 & 0.1461 & 148.8755 & 121.1208 \\
\hline S08R08 & 23.7770 & 0.1507 & 145.6106 & 121.6829 \\
\hline S08R09 & 19.9339 & 0.1520 & 141.9248 & 121.8389 \\
\hline S08R 10 & 16.7610 & 0.1487 & 138.3683 & 121.4586 \\
\hline S08R 11 & 14.5970 & 0.1432 & 135.5127 & 120.7725 \\
\hline S08R 12 & 13.4856 & 0.1391 & 133.8483 & 120.2235 \\
\hline S08R13 & 13.3761 & 0.1387 & 133.6753 & 120.1606 \\
\hline S08R 14 & 14.2607 & 0.1421 & 135.0256 & 120.6228 \\
\hline S08R 15 & 16.1881 & 0.1476 & 137.6539 & 121.3183 \\
\hline S08R 16 & 19.1530 & 0.1516 & 141.1029 & 121.7982 \\
\hline
\end{tabular}

$\begin{array}{ccccccc} & \text { Cs-137 } & \text { Ba-137m } & \text { Source 09 } & \text { X Posit } & \text { Z Posit } & \text { Y Posit } \\ \text { Line } & 24.9347 & 23.5882 & & -11.3240 & -1.2877 & 45.4810 \\ & \text { Shield 1 } & \text { Shield 2 } & \text { Total Dist } & \text { Air Gap } & & \end{array}$




\section{REVISED METHODOLOGY FOR DETERMINING CESIUM-137 CONTENT OF HN-200 GROUT CONTAINERS.}

\begin{tabular}{lllll} 
SO9C01 & 34.3668 & 0.1392 & 35.5132 & 1.0072 \\
S09C02 & 34.4846 & 0.1397 & 35.6348 & 1.0106 \\
S09C03 & 36.7288 & 0.1488 & 37.9539 & 1.0764 \\
S09C04 & 40.7496 & 0.1650 & 42.1089 & 1.1942 \\
S09C05 & 46.0844 & 0.1867 & 47.6216 & 1.3506 \\
S09C06 & 52.3327 & 0.2120 & 54.0784 & 1.5337 \\
S09C07 & 26.6738 & 0.1546 & 27.9410 & 1.1126 \\
S09C08 & 26.8213 & 0.1554 & 28.0955 & 1.1187 \\
S09C09 & 29.5789 & 0.1714 & 30.9841 & 1.2337 \\
S09C10 & 34.3232 & 0.1989 & 35.9537 & 1.4316 \\
S09C11 & 40.3595 & 0.2339 & 42.2768 & 1.6834 \\
S09C12 & 47.1947 & 0.2735 & 49.4367 & 1.9685 \\
S09C13 & 12.1003 & 0.1458 & 13.3008 & 1.0547 \\
S09C14 & 12.3928 & 0.1493 & 13.6224 & 1.0802 \\
S09C15 & 17.1704 & 0.2069 & 18.8740 & 1.4967 \\
S09C16 & 23.8776 & 0.2877 & 26.2466 & 2.0813 \\
S09C17 & 31.2974 & 0.3771 & 34.4026 & 2.7281 \\
S09C18 & 39.0256 & 0.4702 & 42.8975 & 3.4017 \\
S09C19 & 24.3432 & 0.1583 & 25.6398 & 1.1382 \\
S09C20 & 24.5029 & 0.1594 & 25.8080 & 1.1457 \\
S09C21 & 27.4632 & 0.1786 & 28.9260 & 1.2841 \\
S09C22 & 32.4668 & 0.2112 & 34.1961 & 1.5181 \\
S09C23 & 38.7296 & 0.2519 & 40.7925 & 1.8109 \\
S09C24 & 45.7374 & 0.2975 & 48.1735 & 2.1386 \\
S09R01 & 34.3529 & 0.1394 & 155.3999 & 120.9076 \\
S09R02 & 33.8764 & 0.1404 & 155.0599 & 121.0431 \\
S09R03 & 31.2751 & 0.1456 & 153.1707 & 121.7500 \\
S09R04 & 27.0812 & 0.1534 & 149.9694 & 122.7347 \\
S09R05 & 22.2193 & 0.1598 & 145.8767 & 123.4976 \\
S09R06 & 17.7553 & 0.1598 & 141.4678 & 123.5527 \\
S09R07 & 14.4251 & 0.1529 & 137.4158 & 122.8378 \\
S09R08 & 12.4037 & 0.1445 & 134.3946 & 121.8464 \\
S09R09 & 11.5608 & 0.1399 & 132.9459 & 121.2452 \\
S09R10 & 11.7838 & 0.1412 & 133.3422 & 121.4173 \\
S09R11 & 13.1057 & 0.1478 & 135.5083 & 122.2547 \\
S09R12 & 15.6739 & 0.1564 & 139.0428 & 123.2126 \\
S09R13 & 19.5363 & 0.1608 & 143.3309 & 123.6338 \\
S09R14 & 24.2887 & 0.1576 & 147.6841 & 123.2378 \\
S09R15 & 28.9991 & 0.1500 & 151.4617 & 122.3126 \\
S09R16 & 32.6115 & 0.1429 & 154.1487 & 121.3942 \\
& & & & \\
\hline
\end{tabular}

$\begin{array}{ccccccc} & \text { Cs-137 } & \text { Ba-137m } & \text { Source 10 } & \text { X Posit } & \text { Z Posit } & \text { Y Posit } \\ & 26.8139 & 25.3659 & & -8.1594 & 10.5475 & 46.4380 \\ \text { Line } & \text { Shield 1 } & \text { Shield 2 } & \text { Total Dist } & \text { Air Gap } & & \\ \text { S10C01 } & 32.7178 & 0.1468 & 33.9240 & 1.0594 & & \end{array}$




\section{REVISED METHODOLOGY FOR DETERMINING CESIUM-137 CONTENT OF HN-200 GROUT CONTAINERS.}

\begin{tabular}{lllll} 
S10C02 & 33.0836 & 0.1485 & 34.3034 & 1.0713 \\
S10C03 & 35.6271 & 0.1599 & 36.9406 & 1.1536 \\
S10C04 & 39.9341 & 0.1792 & 41.4064 & 1.2931 \\
S10C05 & 45.5067 & 0.2042 & 47.1844 & 1.4735 \\
S10C06 & 51.9391 & 0.2331 & 53.8540 & 1.6818 \\
S10C07 & 14.6055 & 0.1679 & 15.9764 & 1.2030 \\
S10C08 & 15.3282 & 0.1762 & 16.7669 & 1.2625 \\
S10C09 & 19.7984 & 0.2276 & 21.6567 & 1.6307 \\
S10C10 & 26.1609 & 0.3007 & 28.6163 & 2.1547 \\
S10C11 & 33.3496 & 0.3834 & 36.4798 & 2.7468 \\
S10C12 & 40.9316 & 0.4705 & 44.7734 & 3.3713 \\
S10C13 & 17.8216 & 0.1721 & 19.2243 & 1.2305 \\
S10C14 & 18.4352 & 0.1780 & 19.8861 & 1.2729 \\
S10C15 & 22.3902 & 0.2162 & 24.1524 & 1.5460 \\
S10C16 & 28.3198 & 0.2735 & 30.5487 & 1.9554 \\
S10C17 & 35.2410 & 0.3403 & 38.0145 & 2.4333 \\
S10C18 & 42.6739 & 0.4121 & 46.0325 & 2.9465 \\
S10C19 & 34.3935 & 0.1429 & 35.5692 & 1.0327 \\
S10C20 & 34.7436 & 0.1444 & 35.9312 & 1.0432 \\
S10C21 & 37.1858 & 0.1545 & 38.4569 & 1.1165 \\
S10C22 & 41.3512 & 0.1718 & 42.7647 & 1.2416 \\
S10C23 & 46.7817 & 0.1944 & 48.3808 & 1.4047 \\
S10C24 & 53.0905 & 0.2206 & 54.9052 & 1.5941 \\
S10R01 & 29.6331 & 0.1548 & 152.7388 & 122.9509 \\
S10R02 & 23.8860 & 0.1666 & 148.3162 & 124.2636 \\
S10R03 & 18.2233 & 0.1717 & 143.2266 & 124.8316 \\
S10R04 & 13.8498 & 0.1645 & 138.2153 & 124.2010 \\
S10R05 & 11.1306 & 0.1513 & 134.0944 & 122.8125 \\
S10R06 & 9.8147 & 0.1418 & 131.5986 & 121.6421 \\
S10R07 & 9.6276 & 0.1402 & 131.2063 & 121.4385 \\
S10R08 & 10.5231 & 0.1472 & 132.9949 & 122.3246 \\
S10R09 & 12.7067 & 0.1600 & 136.6162 & 123.7495 \\
S10R10 & 16.4916 & 0.1705 & 141.4052 & 124.7431 \\
S10R11 & 21.8007 & 0.1696 & 146.5582 & 124.5879 \\
S10R12 & 27.6850 & 0.1591 & 151.2933 & 123.4492 \\
S10R13 & 32.7306 & 0.1477 & 154.9575 & 122.0793 \\
S10R14 & 35.7967 & 0.1407 & 157.0826 & 121.1452 \\
S10R15 & 36.2770 & 0.1396 & 157.4105 & 120.9939 \\
S10R16 & 34.0829 & 0.1446 & 155.9021 & 121.6746 \\
& & & & \\
\hline
\end{tabular}

$\begin{array}{ccccccc} & \text { Cs-137 } & \text { Ba-137m } & \text { Source 11 } & \text { X Posit } & \text { Z Posit } & \text { Y Posit } \\ & 12.5933 & 11.9133 & & 13.0540 & 6.4320 & 29.1917 \\ \text { Line } & \text { Shield 1 } & \text { Shield 2 } & \text { Total Dist } & \text { Air Gap } & & \\ \text { S11C01 } & 21.3977 & 0.3090 & 23.9217 & 2.2150 & & \\ \text { S11C02 } & 15.1720 & 0.2191 & 16.9616 & 1.5705 & & \end{array}$




\section{REVISED METHODOLOGY FOR DETERMINING CESIUM-137 CONTENT OF HN-200 GROUT CONTAINERS.}

\begin{tabular}{|c|c|c|c|c|}
\hline $\mathrm{S} 11 \mathrm{C03}$ & 11.4951 & 0.1660 & 12.8510 & 1.1899 \\
\hline S11C04 & 12.7947 & 0.1847 & 14.3038 & 1.3244 \\
\hline S11C05 & 18.0246 & 0.2603 & 20.1507 & 1.8658 \\
\hline S11C06 & 24.8131 & 0.3583 & 27.7399 & 2.5685 \\
\hline S11C07 & 27.8541 & 0.2420 & 29.8207 & 1.7247 \\
\hline S11C08 & 22.9695 & 0.1995 & 24.5912 & 1.4222 \\
\hline S11C09 & 20.5104 & 0.1782 & 21.9586 & 1.2700 \\
\hline S11C10 & 21.3330 & 0.1853 & 22.8392 & 1.3209 \\
\hline S11C11 & 25.1168 & 0.2182 & 26.8902 & 1.5552 \\
\hline S11C12 & 30.7888 & 0.2674 & 32.9626 & 1.9064 \\
\hline S11C13 & 41.3724 & 0.1597 & 42.6869 & 1.1547 \\
\hline S11C14. & 38.0050 & 0.1467 & 39.2125 & 1.0607 \\
\hline S11C15 & 36.4591 & 0.1407 & 37.6174 & 1.0176 \\
\hline S11C16 & 36.9638 & 0.1427 & 38.1382 & 1.0317 \\
\hline S11C17 & 39.4406 & 0.1523 & 40.6937 & 1.1008 \\
\hline S11C18 & 43.5543 & 0.1681 & 44.9380 & 1.2156 \\
\hline S11C19 & 37.3353 & 0.1781 & 38.7961 & 1.2827 \\
\hline S11C20 & 33.6215 & 0.1604 & 34.9369 & 1.1551 \\
\hline S11C21 & 31.8890 & 0.1521 & 33.1367 & 1.0955 \\
\hline S11C22 & 32.4568 & 0.1549 & 33.7267 & 1.1151 \\
\hline S11C23 & 35.2138 & 0.1680 & 36.5916 & 1.2098 \\
\hline S11C24 & 39.7065 & 0.1894 & 41.2601 & 1.3641 \\
\hline S11R01 & 8.9571 & 0.1453 & 130.0656 & 120.9631 \\
\hline S11R02 & 8.2620 & 0.1384 & 128.4424 & 120.0420 \\
\hline S11R03 & 8.6049 & 0.1419 & 129.2668 & 120.5199 \\
\hline S11R04 & 10.0948 & 0.1551 & 132.3706 & 122.1207 \\
\hline S11R05 & 13.1356 & 0.1720 & 137.1500 & 123.8424 \\
\hline S11R06 & 18.0972 & 0.1791 & 142.7522 & 124.4759 \\
\hline S11R07 & 24.4757 & 0.1698 & 148.2818 & 123.6363 \\
\hline S11R08 & 30.7393 & 0.1545 & 152.9476 & 122.0538 \\
\hline S11R09 & 35.3379 & 0.1430 & 156.1432 & 120.6622 \\
\hline S11R10 & 37.3148 & 0.1383 & 157.4810 & 120.0279 \\
\hline S11R11 & 36.3129 & 0.1407 & 156.8052 & 120.3516 \\
\hline S11R12 & 32.5091 & 0.1500 & 154.1938 & 121.5347 \\
\hline S11R13 & 26.6511 & 0.1647 & 149.9587 & 123.1429 \\
\hline S11R14 & 20.1173 & 0.1775 & 144.6358 & 124.3410 \\
\hline SI1R15 & 14.5663 & 0.1761 & 138.9614 & 124.2189 \\
\hline S11R16 & 10.9059 & 0.1608 & 133.8087 & 122.7420 \\
\hline
\end{tabular}

$\begin{array}{ccccccc} & \text { Cs-137 } & \text { Ba-137m } & \text { Source 12 } & \text { X Posit } & \text { Z Posit } & \text { Y Posit } \\ & 17.5307 & 16.5841 & & 17.0889 & 8.7507 & 21.2880 \\ \text { Line } & \text { Shield 1 } & \text { Shield 2 } & \text { Total Dist } & \text { Air Gap } & & \\ \text { S12C01 } & 25.0580 & 0.6661 & 30.3215 & 4.5974 & & \\ \text { S12C02 } & 18.3414 & 0.4876 & 22.1941 & 3.3651 & & \\ \text { S12C03 } & 12.4720 & 0.3315 & 15.0918 & 2.2883 & & \end{array}$




\section{REVISED METHODOLOGY FOR DETERMINING CESIUM-137 CONTENT OF HN-200 GROUT CONTAINERS.}

\begin{tabular}{|c|c|c|c|c|}
\hline S12C04 & 9.2376 & 0.2456 & 11.1780 & 1.6948 \\
\hline $\mathrm{S} 12 \mathrm{C} 05$ & 11.2140 & 0.2981 & 13.5695 & 2.0574 \\
\hline $\mathrm{S} 12 \mathrm{C} 06$ & 16.6376 & 0.4423 & 20.1325 & 3.0525 \\
\hline $\mathrm{S} 12 \mathrm{C} 07$ & 33.4988 & 0.3525 & 36.3123 & 2.4610 \\
\hline S12C08 & 27.5487 & 0.2899 & 29.8625 & 2.0239 \\
\hline $\mathrm{S} 12 \mathrm{C} 09$ & 23.0990 & 0.2431 & 25.0390 & 1.6970 \\
\hline $\mathrm{S} 12 \mathrm{C} 10$ & 21.1202 & 0.2222 & 22.8941 & 1.5516 \\
\hline S12C11 & 22.2807 & 0.2344 & 24.1521 & 1.6369 \\
\hline $\mathrm{S} 12 \mathrm{C} 12$ & 26.1662 & 0.2753 & 28.3638 & 1.9223 \\
\hline $\mathrm{S} 12 \mathrm{C} 13$ & 49.1494 & 0.1707 & 50.5535 & 1.2334 \\
\hline S12C14 & 44.8578 & 0.1558 & 46.1393 & 1.1257 \\
\hline S12C15 & 41.9752 & 0.1458 & 43.1743 & 1.0534 \\
\hline S12C16 & 40.8011 & 0.1417 & 41.9667 & 1.0239 \\
\hline S12C17 & 41.4810 & 0.1440 & 42.6660 & 1.0410 \\
\hline $\mathrm{S} 12 \mathrm{C} 18$ & 43.9288 & 0.1525 & 45.1838 & 1.1024 \\
\hline S12C19 & 44.8096 & 0.1989 & 46.4377 & 1.4292 \\
\hline $\mathrm{S} 12 \mathrm{C} 20$ & 40.1309 & 0.1782 & 41.5890 & 1.2800 \\
\hline S12C21 & 36.9312 & 0.1640 & 38.2731 & 1.1779 \\
\hline $\mathrm{S} 12 \mathrm{C} 22$ & 35.6116 & 0.1581 & 36.9055 & 1.1358 \\
\hline$S 12 C 23$ & 36.3771 & 0.1615 & 37.6988 & 1.1602 \\
\hline $\mathrm{S} 12 \mathrm{C} 24$ & 39.1055 & 0.1736 & 40.5263 & 1.2473 \\
\hline S12R01 & 4.0936 & 0.1535 & 126.2707 & 122.0236 \\
\hline S12R02 & 3.6169 & 0.1388 & 123.9307 & 120.1751 \\
\hline S12R03 & 3.8076 & 0.1448 & 124.9250 & 120.9726 \\
\hline S12R04 & 4.8029 & 0.1734 & 129.0305 & 124.0541 \\
\hline S12R05 & 7.4032 & 0.2254 & 135.3808 & 127.7521 \\
\hline S12R06 & 13.3855 & 0.2537 & 142.7862 & 129.1470 \\
\hline S12R07 & 22.8062 & 0.2134 & 150.0503 & 127.0307 \\
\hline S12R08 & 32.2247 & 0.1706 & 156.1590 & 123.7637 \\
\hline S12R09 & 38.9857 & 0.1471 & 160.3585 & 121.2257 \\
\hline S12R10 & 41.9330 & 0.1385 & 162.1736 & 120.1021 \\
\hline S12R11 & 40.6906 & 0.1420 & 161.4091 & 120.5764 \\
\hline S12R12 & 35.4096 & 0.1588 & 158.1463 & 122.5779 \\
\hline S12R13 & 26.8663 & 0.1936 & 152.7454 & 125.6855 \\
\hline S12R14 & 17.0008 & 0.2421 & 145.8466 & 128.6037 \\
\hline S12R15 & 9.3435 & 0.2454 & 138.3621 & 128.7731 \\
\hline S12R16 & 5.5935 & 0.1927 & 131.4286 & 125.6424 \\
\hline
\end{tabular}

$\begin{array}{ccccccc} & \text { Cs-137 } & \text { Ba-137m } & \text { Source 13 } & \text { X Posit } & \text { Z Posit } & \text { Y Posit } \\ & 17.9331 & 16.9647 & & 3.5123 & 8.3040 & 41.4473 \\ \text { Line } & \text { Shield 1 } & \text { Shield 2 } & \text { Total Dist } & \text { Air Gap } & & \\ \text { S13C01 } & 22.1597 & 0.1601 & 23.4732 & 1.1534 & & \\ \text { S13C02 } & 20.8342 & 0.1505 & 22.0691 & 1.0844 & & \\ \text { S13C03 } & 22.8353 & 0.1650 & 24.1888 & 1.1886 & & \\ \text { S13C04 } & 27.4447 & 0.1983 & 29.0715 & 1.4285 & & \end{array}$




\section{REVISED METHODOLOGY FOR DETERMINING CESIUM-137 CONTENT OF HN-200 GROUT CONTAINERS.}

$\begin{array}{lllll}\text { S13C05 } & 33.6058 & 0.2428 & 35.5978 & 1.7492 \\ \text { S13C06 } & 40.6186 & 0.2935 & 43.0262 & 2.1142 \\ \text { S13C07 } & 16.6256 & 0.1589 & 17.9328 & 1.1483 \\ \text { S13C08 } & 14.8810 & 0.1422 & 16.0511 & 1.0278 \\ \text { S13C09 } & 17.4849 & 0.1671 & 18.8598 & 1.2077 \\ \text { S13C10 } & 23.0053 & 0.2199 & 24.8142 & 1.5890 \\ \text { S13C11 } & 29.8665 & 0.2855 & 32.2148 & 2.0629 \\ \text { S13C12 } & 37.3364 & 0.3569 & 40.2722 & 2.5789 \\ \text { S13C13 } & 28.5495 & 0.1506 & 29.7874 & 1.0873 \\ \text { S13C14 } & 27.5014 & 0.1451 & 28.6940 & 1.0474 \\ \text { S13C15 } & 29.0930 & 0.1535 & 30.3545 & 1.1080 \\ \text { S13C16 } & 32.9437 & 0.1738 & 34.3722 & 1.2547 \\ \text { S13C17 } & 38.3795 & 0.2025 & 40.0437 & 1.4617 \\ \text { S13C18 } & 44.8275 & 0.2365 & 46.7713 & 1.7073 \\ \text { S13C19 } & 32.2370 & 0.1433 & 33.4170 & 1.0367 \\ \text { S13C20 } & 31.3003 & 0.1392 & 32.4461 & 1.0066 \\ \text { S13C21 } & 32.7255 & 0.1455 & 33.9235 & 1.0524 \\ \text { S13C22 } & 36.2349 & 0.1611 & 37.5613 & 1.1653 \\ \text { S13C23 } & 41.3006 & 0.1836 & 42.8125 & 1.3282 \\ \text { S13C24 } & 47.4265 & 0.2109 & 49.1626 & 1.5252 \\ \text { S13R01 } & 18.2441 & 0.1497 & 140.4435 & 122.0496 \\ \text { S13R02 } & 15.7852 & 0.1452 & 137.4494 & 121.5190 \\ \text { S13R03 } & 14.3309 & 0.1408 & 135.4247 & 120.9530 \\ \text { S13R04 } & 13.8660 & 0.1390 & 134.7255 & 120.7205 \\ \text { S13R05 } & 14.3677 & 0.1409 & 135.4788 & 120.9703 \\ \text { S13R06 } & 15.8602 & 0.1454 & 137.5480 & 121.5424 \\ \text { S13R07 } & 18.3560 & 0.1498 & 140.5696 & 122.0638 \\ \text { S13R08 } & 21.6979 & 0.1511 & 144.0403 & 122.1913 \\ \text { S13R09 } & 25.4184 & 0.1485 & 147.4195 & 121.8526 \\ \text { S13R10 } & 28.8049 & 0.1440 & 150.2149 & 121.2660 \\ \text { S13R11 } & 31.1486 & 0.1402 & 152.0429 & 120.7541 \\ \text { S13R12 } & 31.9637 & 0.1388 & 152.6626 & 120.5600 \\ \text { S13R13 } & 31.0855 & 0.1403 & 151.9946 & 120.7687 \\ \text { S13R14 } & 28.6916 & 0.1442 & 150.1246 & 121.2889 \\ \text { S13R15 } & 25.2786 & 0.1487 & 147.2993 & 121.8720 \\ \text { S13R16 } & 21.5604 & 0.1511 & 143.9071 & 122.1956\end{array}$

$\begin{array}{ccccccc} & \text { Cs-137 } & \text { Ba-137m } & \text { Source 14 } & \text { X Posit } & \text { Z Posit } & \text { Y Posit } \\ & 26.2138 & 24.7983 & & -18.2417 & 6.1323 & 39.9213 \\ \text { Line } & \text { Shield 1 } & \text { Shield 2 } & \text { Total Dist } & \text { Air Gap } & & \\ \text { S14C01 } & 42.5063 & 0.1433 & 43.6858 & 1.0362 & & \\ \text { S14C02 } & 41.4757 & 0.1398 & 42.6266 & 1.0111 & & \\ \text { S14C03 } & 42.2736 & 0.1425 & 43.4466 & 1.0305 & & \\ \text { S14C04 } & 44.8024 & 0.1510 & 46.0456 & 1.0922 & & \\ \text { S14C05 } & 48.7936 & 0.1645 & 50.1476 & 1.1895 & & \end{array}$




\section{REVISED METHODOLOGY FOR DETERMINING CESIUM-137 CONTENT OF HN-200 GROUT CONTAINERS.}

\begin{tabular}{|c|c|c|c|c|}
\hline S14C06 & 53.9236 & 0.1818 & 55.4198 & 1.3145 \\
\hline S14C07 & 25.4422 & 0.2229 & 27.2312 & 1.5661 \\
\hline S14C08 & 23.8224 & 0.2087 & 25.4975 & 1.4664 \\
\hline S14C09 & 25.0823 & 0.2197 & 26.8459 & 1.5440 \\
\hline S14C10 & 28.8470 & 0.2527 & 30.8753 & 1.7757 \\
\hline S14C11 & 34.3014 & 0.3005 & 36.7133 & 2.1114 \\
\hline $\mathrm{S} 14 \mathrm{C} 12$ & 40.7731 & 0.3571 & 43.6400 & 2.5098 \\
\hline S14C13 & 10.0999 & 0.3273 & 12.7200 & 2.2928 \\
\hline S14C14 & 6.6613 & 0.2159 & 8.3894 & 1.5122 \\
\hline S14C15 & 9.4273 & 0.3055 & 11.8729 & 2.1401 \\
\hline S14C16 & 15.3463 & 0.4974 & 19.3274 & 3.4837 \\
\hline S14C17 & 22.0064 & 0.7132 & 27.7152 & 4.9956 \\
\hline S14C18 & 28.8996 & 0.9366 & 36.3966 & 6.5604 \\
\hline S14C19 & 35.0587 & 0.1706 & 36.4514 & 1.2221 \\
\hline $\mathrm{S} 14 \mathrm{C} 20$ & 33.8312 & 0.1646 & 35.1751 & 1.1793 \\
\hline S14C21 & 34.7828 & 0.1692 & 36.1645 & 1.2125 \\
\hline S14C22 & 37.7491 & 0.1836 & 39.2486 & 1.3159 \\
\hline $\mathrm{S} 14 \mathrm{C} 23$ & 42.3084 & 0.2058 & 43.9891 & 1.4748 \\
\hline S14C24 & 48.0090 & 0.2336 & 49.9161 & 1.6735 \\
\hline S14R01 & 40.7390 & 0.1427 & 161.8327 & 120.9510 \\
\hline S14R02 & 35.3135 & 0.1602 & 158.4992 & 123.0255 \\
\hline S14R03 & 26.6410 & 0.1960 & 153.0452 & 126.2082 \\
\hline S14R04 & 16.7177 & 0.2453 & 146.1197 & 129.1567 \\
\hline S14R05 & 9.1368 & 0.2464 & 138.6431 & 129.2599 \\
\hline S14R06 & 5.4847 & 0.1923 & 131.7562 & 126.0792 \\
\hline S14R07 & 4.0349 & 0.1535 & 126.6804 & 122.4920 \\
\hline S14R08 & 3.5839 & 0.1393 & 124.4436 & 120.7203 \\
\hline S14R09 & 3.7938 & 0.1460 & 125.5393 & 121.5995 \\
\hline S14R 10 & 4.8168 & 0.1757 & 129.7226 & 124.7301 \\
\hline S14RII & 7.4874 & 0.2290 & 136.1144 & 128.3980 \\
\hline S14R12 & 13.6245 & 0.2556 & 143.5234 & 129.6433 \\
\hline S14R13 & 23.1687 & 0.2132 & 150.7583 & 127.3763 \\
\hline S14R14 & 32.5933 & 0.1703 & 156.8127 & 124.0491 \\
\hline S14R15 & 39.2839 & 0.1471 & 160.9412 & 121.5102 \\
\hline S14R16 & 42.1180 & 0.1388 & 162.6768 & 120.4199 \\
\hline
\end{tabular}

$\begin{array}{ccccccc} & \text { Cs-137 } & \text { Ba-137m } & \text { Source 15 } & \text { X Posit } & \text { Z Posit } & \text { Y Posit } \\ & 22.7469 & 21.5186 & & 21.0026 & 5.4076 & 10.7056 \\ \text { Line } & \text { Shield 1 } & \text { Shicld 2 } & \text { Total Dist } & \text { Air Gap } & & \\ \text { S15C01 } & 22.4706 & 2.2513 & 39.2793 & 14.5573 & & \\ \text { S15C02 } & 17.4042 & 1.7437 & 30.4230 & 11.2751 & & \\ \text { S15C03 } & 12.4057 & 1.2429 & 21.6855 & 8.0369 & & \\ \text { S15C04 } & 7.6101 & 0.7625 & 13.3028 & 4.9302 & & \\ \text { S15C05 } & 3.8658 & 0.3873 & 6.7576 & 2.5044 & & \\ \text { S15C06 } & 4.9992 & 0.5009 & 8.7388 & 3.2387 & & \end{array}$




\section{REVISED METHODOLOGY FOR DETERMINING CESIUM-137 CONTENT OF HN-200 GROUT CONTAINERS.}

\begin{tabular}{lcccc} 
S15C07 & 44.8084 & 0.3811 & 47.8484 & 2.6590 \\
S15C08 & 38.2941 & 0.3257 & 40.8922 & 2.2724 \\
S15C09 & 32.6673 & 0.2778 & 34.8837 & 1.9385 \\
S15C10 & 28.4595 & 0.2420 & 30.3903 & 1.6888 \\
S15C11 & 26.3590 & 0.2242 & 28.1473 & 1.5642 \\
S15C12 & 26.8649 & 0.2285 & 28.6875 & 1.5942 \\
S15C13 & 58.1038 & 0.1835 & 59.6142 & 1.3269 \\
S15C14 & 52.8170 & 0.1668 & 54.1900 & 1.2062 \\
S15C15 & 48.5502 & 0.1533 & 49.8122 & 1.1088 \\
S15C16 & 45.5905 & 0.1439 & 46.7756 & 1.0412 \\
S15C17 & 44.2014 & 0.1396 & 45.3504 & 1.0094 \\
S15C18 & 44.5301 & 0.1406 & 45.6877 & 1.0170 \\
S15C19 & 50.9014 & 0.2559 & 52.9835 & 1.8262 \\
S15C20 & 44.9580 & 0.2260 & 46.7969 & 1.6129 \\
S15C21 & 40.0125 & 0.2011 & 41.6491 & 1.4355 \\
S15C22 & 36.4733 & 0.1833 & 37.9651 & 1.3085 \\
S15C23 & 34.7724 & 0.1748 & 36.1947 & 1.2475 \\
S15C24 & 35.1774 & 0.1768 & 36.6163 & 1.2620 \\
S15R01 & 1.1698 & 0.1451 & 123.1048 & 121.7900 \\
S15R02 & 1.1362 & 0.1411 & 122.5570 & 121.2797 \\
S15R03 & 1.3623 & 0.1672 & 125.7785 & 124.2491 \\
S15R04 & 2.1322 & 0.2475 & 132.0340 & 129.6543 \\
S15R05 & 5.2206 & 0.4203 & 140.0464 & 134.4055 \\
S15R06 & 15.5571 & 0.3347 & 148.4194 & 132.5277 \\
S15R07 & 28.2986 & 0.2110 & 155.9209 & 127.4113 \\
S15R08 & 38.1586 & 0.1611 & 161.5994 & 123.2797 \\
S15R09 & 43.6839 & 0.1419 & 164.8120 & 120.9862 \\
S15R10 & 44.3811 & 0.1398 & 165.2197 & 120.6988 \\
S15R11 & 40.1965 & 0.1535 & 162.7808 & 122.4308 \\
S15R12 & 31.4792 & 0.1920 & 157.7492 & 126.0779 \\
S15R13 & 19.2856 & 0.2884 & 150.6811 & 131.1071 \\
S15R14 & 7.3356 & 0.4384 & 142.4410 & 134.6671 \\
S15R15 & 2.5973 & 0.2888 & 134.1882 & 131.3022 \\
S15R16 & 1.4981 & 0.1823 & 127.2926 & 125.6122 \\
& & & & \\
\hline
\end{tabular}

$\begin{array}{ccccccr} & \text { Cs-137 } & \text { Ba-137m } & \text { Source 16 } & \text { X Posit } & \text { Z Posit } & \text { Y Posit } \\ & 27.6556 & 26.1622 & & 12.7799 & 10.3319 & 39.3081 \\ \text { Line } & \text { Shield 1 } & \text { Shield 2 } & \text { Total Dist } & \text { Air Gap } & & \\ \text { S16C01 } & 16.3969 & 0.2367 & 18.3061 & 1.6726 & & \\ \text { S16C02 } & 13.6624 & 0.1972 & 15.2532 & 1.3936 & & \\ \text { S16C03 } & 15.3112 & 0.2210 & 17.0940 & 1.5618 & & \\ \text { S16C04 } & 20.3022 & 0.2930 & 22.6661 & 2.0709 & & \\ \text { S16C05 } & 26.8308 & 0.3873 & 29.9550 & 2.7369 & & \\ \text { S16C06 } & 34.0232 & 0.4911 & 37.9848 & 3.4706 & & \\ \text { S16C07 } & 19.4474 & 0.2258 & 21.2677 & 1.5944 & & \\ & & & & & \end{array}$




\section{REVISED METHODOLOGY FOR DETERMINING CESIUM-137 CONTENT OF HN-200 GROUT CONTAINERS.}

\begin{tabular}{lcccc} 
S16C08 & 17.1037 & 0.1986 & 18.7045 & 1.4023 \\
S16C09 & 18.5021 & 0.2148 & 20.2338 & 1.5169 \\
S16C10 & 22.9690 & 0.2667 & 25.1188 & 1.8832 \\
S16C11 & 29.1252 & 0.3381 & 31.8512 & 2.3879 \\
S16C12 & 36.1170 & 0.4193 & 39.4974 & 2.9611 \\
S16C13 & 38.2552 & 0.1492 & 39.4815 & 1.0771 \\
S16C14 & 36.9767 & 0.1442 & 38.1620 & 1.0411 \\
S16C15 & 37.7250 & 0.1471 & 38.9344 & 1.0622 \\
S16C16 & 40.3878 & 0.1575 & 41.6825 & 1.1372 \\
S16C17 & 44.6236 & 0.1740 & 46.0541 & 1.2565 \\
S16C18 & 50.0345 & 0.1951 & 51.6384 & 1.4088 \\
S16C19 & 36.7028 & 0.1541 & 37.9683 & 1.1113 \\
S16C20 & 35.3746 & 0.1486 & 36.5942 & 1.0711 \\
S16C21 & 36.1525 & 0.1518 & 37.3990 & 1.0947 \\
S16C22 & 38.9105 & 0.1634 & 40.2521 & 1.1782 \\
S16C23 & 43.2716 & 0.1817 & 44.7636 & 1.3102 \\
S16C24 & 48.8080 & 0.2050 & 50.4908 & 1.4778 \\
S16R01 & 7.9099 & 0.1597 & 131.1695 & 123.0999 \\
S16R02 & 6.6437 & 0.1426 & 127.8798 & 121.0934 \\
S16R03 & 6.4295 & 0.1394 & 127.2281 & 120.6593 \\
S16R04 & 7.1699 & 0.1502 & 129.3522 & 122.0321 \\
S16R05 & 9.1973 & 0.1735 & 133.8106 & 124.4398 \\
S16R06 & 13.2698 & 0.1964 & 139.7434 & 126.2772 \\
S16R07 & 19.8257 & 0.1947 & 146.1293 & 126.1089 \\
S16R08 & 27.5701 & 0.1726 & 151.9998 & 124.2571 \\
S16R09 & 34.2890 & 0.1523 & 156.5643 & 122.1229 \\
S16R10 & 38.4211 & 0.1410 & 159.2626 & 120.7004 \\
S16R11 & 39.2280 & 0.1389 & 159.7837 & 120.4167 \\
S16R12 & 36.5829 & 0.1459 & 158.0690 & 121.3402 \\
S16R13 & 30.9219 & 0.1623 & 154.3131 & 123.2290 \\
S16R14 & 23.3985 & 0.1854 & 148.9617 & 125.3778 \\
S16R15 & 16.0253 & 0.1995 & 142.7027 & 126.4780 \\
S16R16 & 10.7741 & 0.1857 & 136.4326 & 125.4728 \\
& & & & \\
\hline
\end{tabular}

$\begin{array}{ccccccc} & \text { Cs-137 } & \text { Ba-137m } & \text { Source 17 } & \text { X Posit } & \text { Z Posit } & \text { Y Posit } \\ & 28.5227 & 26.9825 & & -14.4903 & 3.0983 & 8.6941 \\ \text { Line } & \text { Shield 1 } & \text { Shield 2 } & \text { Total Dist } & \text { Air Gap } & & \\ \text { S17C01 } & 54.4744 & 0.2020 & 56.1375 & 1.4611 & & \\ \text { S17C02 } & 48.4985 & 0.1798 & 49.9791 & 1.3008 & & \\ \text { S17C03 } & 43.4659 & 0.1611 & 44.7929 & 1.1658 & & \\ \text { S17C04 } & 39.7368 & 0.1473 & 40.9499 & 1.0658 & \\ \text { S17C05 } & 37.6999 & 0.1398 & 38.8509 & 1.0112 & \\ \text { S17C06 } & 37.6311 & 0.1395 & 38.7799 & 1.0093 & \\ \text { S17C07 } & 45.3986 & 0.3263 & 48.0558 & 2.3309 & & \\ \text { S17C08 } & 38.4422 & 0.2763 & 40.6922 & 1.9737 & & \\ & & & & & & \end{array}$


REVISED METHODOLOGY FOR DETERMINING CESIUM-137 CONTENT OF HN-200 GROUT CONTAINERS.

$\begin{array}{lcccc}\text { S17C09 } & 32.2358 & 0.2317 & 34.1226 & 1.6551 \\ \text { S17C10 } & 27.2960 & 0.1962 & 28.8937 & 1.4014 \\ \text { S17C11 } & 24.4045 & 0.1754 & 25.8329 & 1.2530 \\ \text { S17C12 } & 24.3036 & 0.1747 & 25.7261 & 1.2478 \\ \text { S17C13 } & 36.9259 & 0.6176 & 41.9997 & 4.4562 \\ \text { S17C14 } & 29.2980 & 0.4900 & 33.3236 & 3.5357 \\ \text { S17C15 } & 21.8734 & 0.3658 & 24.8789 & 2.6397 \\ \text { S17C16 } & 14.9580 & 0.2502 & 17.0133 & 1.8051 \\ \text { S17C17 } & 9.7089 & 0.1624 & 11.0429 & 1.1717 \\ \text { S17C18 } & 9.4872 & 0.1587 & 10.7907 & 1.1449 \\ \text { S17C19 } & 48.8844 & 0.2646 & 51.0492 & 1.9002 \\ \text { S17C20 } & 42.3135 & 0.2290 & 44.1873 & 1.6448 \\ \text { S17C21 } & 36.6023 & 0.1981 & 38.2232 & 1.4228 \\ \text { S17C22 } & 32.2114 & 0.1744 & 33.6378 & 1.2521 \\ \text { S17C23 } & 29.7317 & 0.1609 & 31.0483 & 1.1557 \\ \text { S17C24 } & 29.6467 & 0.1605 & 30.9595 & 1.1524 \\ \text { S17R01 } & 37.4172 & 0.1402 & 158.4901 & 120.9328 \\ \text { S17R02 } & 34.3152 & 0.1478 & 156.4124 & 121.9494 \\ \text { S17R03 } & 28.7996 & 0.1622 & 152.5764 & 123.6146 \\ \text { S17R04 } & 22.0975 & 0.1779 & 147.4572 & 125.1818 \\ \text { S17R05 } & 15.9417 & 0.1822 & 141.7427 & 125.6188 \\ \text { S17R06 } & 11.6226 & 0.1689 & 136.2834 & 124.4919 \\ \text { S17R07 } & 9.1993 & 0.1512 & 131.9898 & 122.6393 \\ \text { S17R08 } & 8.1687 & 0.1408 & 129.6560 & 121.3465 \\ \text { S17R09 } & 8.2057 & 0.1412 & 129.7471 & 121.4002 \\ \text { S17R10 } & 9.3228 & 0.1523 & 132.2444 & 122.7692 \\ \text { S17R11 } & 11.8678 & 0.1703 & 136.6525 & 124.6144 \\ \text { S17R12 } & 16.3364 & 0.1825 & 142.1611 & 125.6422 \\ \text { S17R13 } & 22.5887 & 0.1770 & 147.8595 & 125.0938 \\ \text { S17R14 } & 29.2601 & 0.1610 & 152.9060 & 123.4850 \\ \text { S17R15 } & 34.6330 & 0.1470 & 156.6274 & 121.8473 \\ \text { S17R16 } & 37.5296 & 0.1399 & 158.5646 & 120.8951\end{array}$

$\begin{array}{ccccccc} & \text { Cs-137 } & \text { Ba-137m } & \text { Source 18 } & \text { X Posit } & \text { Z Posit } & \text { Y Posit } \\ & 23.2528 & 21.9972 & & 18.7646 & -12.0033 & 10.5209 \\ \text { Line } & \text { Shield 1 } & \text { Shield 2 } & \text { Total Dist } & \text { Air Gap } & & \\ \text { S18C01 } & 30.3310 & 1.5786 & 41.1122 & 9.2026 & & \\ \text { S18C02 } & 24.1281 & 1.2558 & 32.7045 & 7.3206 & & \\ \text { S18C03 } & 18.2357 & 0.9491 & 24.7176 & 5.5328 & & \\ \text { S18C04 } & 13.0802 & 0.6808 & 17.7296 & 3.9686 & & \\ \text { S18C05 } & 9.8902 & 0.5148 & 13.4057 & 3.0007 & & \\ \text { S18C06 } & 10.6168 & 0.5526 & 14.3906 & 3.2212 & & \\ \text { S18C07 } & 54.4491 & 0.2191 & 56.2425 & 1.5743 & & \\ \text { S18C08 } & 48.8163 & 0.1965 & 50.4242 & 1.4114 & & \\ \text { S18C09 } & 44.1949 & 0.1779 & 45.6506 & 1.2778 & & \end{array}$




\section{REVISED METHODOLOGY FOR DETERMINING CESIUM-137 CONTENT OF HN-200 GROUT CONTAINERS.}

$\begin{array}{lcccc}\text { S18C10 } & 40.9290 & 0.1647 & 42.2771 & 1.1834 \\ \text { S18C11 } & 39.3574 & 0.1584 & 40.6537 & 1.1379 \\ \text { S18C12 } & 39.6820 & 0.1597 & 40.9890 & 1.1473 \\ \text { S18C13 } & 57.4731 & 0.1918 & 59.0502 & 1.3852 \\ \text { S18C14 } & 52.1080 & 0.1739 & 53.5378 & 1.2559 \\ \text { S18C15 } & 47.7576 & 0.1594 & 49.0680 & 1.1511 \\ \text { S18C16 } & 44.7190 & 0.1493 & 45.9461 & 1.0778 \\ \text { S18C17 } & 43.2697 & 0.1444 & 44.4570 & 1.0429 \\ \text { S18C18 } & 43.5683 & 0.1454 & 44.7638 & 1.0501 \\ \text { S18C19 } & 40.2932 & 0.5948 & 44.8766 & 3.9886 \\ \text { S18C20 } & 33.5144 & 0.4947 & 37.3267 & 3.3176 \\ \text { S18C21 } & 27.4497 & 0.4052 & 30.5722 & 2.7173 \\ \text { S18C22 } & 22.6795 & 0.3348 & 25.2594 & 2.2451 \\ \text { S18C23 } & 20.1453 & 0.2974 & 22.4368 & 1.9942 \\ \text { S18C24 } & 20.6857 & 0.3054 & 23.0388 & 2.0477 \\ \text { S18R01 } & 0.6671 & 0.1744 & 125.8029 & 124.9614 \\ \text { S18R02 } & 1.1189 & 0.2811 & 132.4683 & 131.0684 \\ \text { S18R03 } & 3.7273 & 0.5961 & 140.7976 & 136.4741 \\ \text { S18R04 } & 15.8064 & 0.3618 & 149.3589 & 133.1907 \\ \text { S18R05 } & 29.3119 & 0.2106 & 156.9144 & 127.3919 \\ \text { S18R06 } & 39.2367 & 0.1596 & 162.5231 & 123.1268 \\ \text { S18R07 } & 44.5190 & 0.1412 & 165.5604 & 120.9002 \\ \text { S18R08 } & 44.7776 & 0.1404 & 165.7101 & 120.7921 \\ \text { S18R09 } & 39.9957 & 0.1567 & 162.9571 & 122.8048 \\ \text { S18R10 } & 30.5115 & 0.2028 & 157.5866 & 126.8723 \\ \text { S18R11 } & 17.2764 & 0.3367 & 150.1915 & 132.5784 \\ \text { S18R12 } & 4.4655 & 0.6136 & 141.6803 & 136.6012 \\ \text { S18R13 } & 1.2115 & 0.3012 & 133.2639 & 131.7512 \\ \text { S18R14 } & 0.6914 & 0.1805 & 126.3631 & 125.4911 \\ \text { S18R15 } & 0.5460 & 0.1439 & 122.3567 & 121.6668 \\ \text { S18R16 } & 0.5401 & 0.1424 & 122.1538 & 121.4713\end{array}$

$\begin{array}{ccccccc} & \text { Cs-137 } & \text { Ba-137m } & \text { Source 19 } & \text { X Posit } & \text { Z Posit } & \text { Y Posit } \\ \text { Line } & 15.3416 & 14.5131 & & -18.2584 & 0.2785 & 21.1001 \\ \text { S19C01 } & 49.4918 & 0.1666 & 50.8638 & 1.2054 & & \\ \text { S19C02 } & 45.1900 & 0.1521 & 46.4427 & 1.1006 & & \\ \text { S19C03 } & 42.2872 & 0.1423 & 43.4595 & 1.0299 & & \\ \text { S19C04 } & 41.0812 & 0.1383 & 42.2200 & 1.0006 & & \\ \text { S19C05 } & 41.7192 & 0.1404 & 42.8758 & 1.0161 & & \\ \text { S19C06 } & 44.1215 & 0.1485 & 45.3446 & 1.0746 & & \\ \text { S19C07 } & 39.2133 & 0.2480 & 41.2271 & 1.7658 & & \\ \text { S19C08 } & 33.8893 & 0.2143 & 35.6297 & 1.5260 & & \\ \text { S19C09 } & 30.0973 & 0.1903 & 31.6429 & 1.3553 & & \\ \text { S19C10 } & 28.4565 & 0.1800 & 29.9178 & 1.2814 & & \\ & & & & & & \end{array}$


HNF-4799, Rev 0

August 25, 1999

\section{REVISED METHODOLOGY FOR DETERMINING CESIUM-137 CONTENT OF HN-200 GROUT CONTAINERS.}

$\begin{array}{lcccc}\text { S19C11 } & 29.3301 & 0.1855 & 30.8363 & 1.3207 \\ \text { S19C12 } & 32.5161 & 0.2056 & 34.1860 & 1.4642 \\ \text { S19C13 } & 23.1583 & 0.7049 & 28.9635 & 5.1003 \\ \text { S19C14 } & 16.1641 & 0.4920 & 20.2160 & 3.5599 \\ \text { S19C15 } & 9.4771 & 0.2885 & 11.8527 & 2.0872 \\ \text { S19C16 } & 4.6821 & 0.1425 & 5.8557 & 1.0312 \\ \text { S19C17 } & 7.5892 & 0.2310 & 9.4917 & 1.6714 \\ \text { S19C18 } & 14.0300 & 0.4270 & 17.5469 & 3.0899 \\ \text { S19C19 } & 39.5678 & 0.2438 & 41.5492 & 1.7376 \\ \text { S19C20 } & 34.2850 & 0.2113 & 36.0019 & 1.5056 \\ \text { S19C21 } & 30.5325 & 0.1882 & 32.0615 & 1.3408 \\ \text { S19C22 } & 28.9124 & 0.1782 & 30.3602 & 1.2697 \\ \text { S19C23 } & 29.7747 & 0.1835 & 31.2657 & 1.3075 \\ \text { S19C24 } & 32.9250 & 0.2029 & 34.5738 & 1.4459 \\ \text { S19R01 } & 41.0820 & 0.1383 & 161.2951 & 120.0748 \\ \text { S19R02 } & 38.9572 & 0.1442 & 159.9635 & 120.8621 \\ \text { S19R03 } & 33.1797 & 0.1624 & 156.3043 & 122.9622 \\ \text { S19R04 } & 24.7520 & 0.1948 & 150.7329 & 125.7861 \\ \text { S19R05 } & 15.8572 & 0.2273 & 143.9336 & 127.8492 \\ \text { S19R06 } & 9.4584 & 0.2153 & 136.8411 & 127.1674 \\ \text { S19R07 } & 6.2482 & 0.1744 & 130.5733 & 124.1507 \\ \text { S19R08 } & 4.8991 & 0.1467 & 126.2685 & 121.2226 \\ \text { S19R09 } & 4.5440 & 0.1384 & 124.8074 & 120.1250 \\ \text { S19R10 } & 4.9620 & 0.1482 & 126.5095 & 121.3993 \\ \text { S19R11 } & 6.4140 & 0.1774 & 131.0037 & 124.4123 \\ \text { S19R12 } & 9.8283 & 0.2180 & 137.3775 & 127.3312 \\ \text { S19R13 } & 16.4920 & 0.2258 & 144.4857 & 127.7679 \\ \text { S19R14 } & 25.4606 & 0.1919 & 151.2200 & 125.5675 \\ \text { S19R15 } & 33.7420 & 0.1605 & 156.6641 & 122.7615 \\ \text { S19R16 } & 39.2604 & 0.1433 & 160.1538 & 120.7501\end{array}$

$\begin{array}{lcccccc} & \text { Cs-137 } & \text { Ba-137m } & \text { Source 20 } & \text { X Posit } & \text { Z Posit } & \text { Y Posit } \\ \text { Line } & 16.4988 & 15.6078 & & 2.8644 & 9.2261 & 44.4497 \\ \text { S20C01 } & 22.2739 & 0.1559 & 23.5521 & 1.1223 & & \\ \text { S20C02 } & 22.0741 & 0.1545 & 23.3409 & 1.1123 & & \\ \text { S20C03 } & 24.9660 & 0.1747 & 26.3987 & 1.2580 & \\ \text { S20C04 } & 30.0704 & 0.2105 & 31.7960 & 1.5152 & \\ \text { S20C05 } & 36.4698 & 0.2553 & 38.5627 & 1.8377 & \\ \text { S20C06 } & 43.5977 & 0.3051 & 46.0997 & 2.1968 & \\ \text { S20C07 } & 14.5909 & 0.1488 & 15.8157 & 1.0760 & \\ \text { S20C08 } & 14.2991 & 0.1459 & 15.4994 & 1.0544 & \\ \text { S20C09 } & 18.2733 & 0.1864 & 19.8072 & 1.3475 & & \\ \text { S20C10 } & 24.5203 & 0.2501 & 26.5786 & 1.8082 & & \\ \text { S20C11 } & 31.7247 & 0.3236 & 34.3877 & 2.3394 & & \\ & & & & & & \end{array}$




\section{REVISED METHODOLOGY FOR DETERMINING CESIUM-137 CONTENT OF HN-200 GROUT CONTAINERS.}

\begin{tabular}{|c|c|c|c|c|}
\hline $\mathrm{S} 20 \mathrm{C} 12$ & 39.3643 & 0.4016 & 42.6686 & 2.9028 \\
\hline S20C13 & 27.5654 & 0.1493 & 28.7918 & .1 .0770 \\
\hline S20C14 & 27.4002 & 0.1484 & 28.6192 & 1.0706 \\
\hline S20C 15 & 29.8360 & 0.1616 & 31.1634 & 1.1658 \\
\hline S20C16 & 34.3234 & 0.1859 & 35.8504 & 1.3411 \\
\hline S20C17 & 40.1808 & 0.2176 & 41.9684 & 1.5699 \\
\hline S20C18 & 46.8977 & 0.2540 & 48.9841 & 1.8324 \\
\hline S20C 19 & 32.5123 & 0.1404 & 33.6680 & 1.0154 \\
\hline S20C20 & 32.3699 & 0.1397 & 33.5206 & 1.0109 \\
\hline S20C21 & 34.4912 & 0.1489 & 35.7173 & 1.0772 \\
\hline S20C22 & 38.5038 & 0.1662 & 39.8725 & 1.2025 \\
\hline S20C23 & 43.8921 & 0.1895 & 45.4523 & 1.3708 \\
\hline S20C24 & 50.2151 & 0.2168 & 52.0001 & 1.5682 \\
\hline S20R01 & 18.6687 & 0.1528 & 141.4881 & 122.6666 \\
\hline S20R02 & 15.8063 & 0.1481 & 138.1010 & 122.1465 \\
\hline S20R03 & 14.0181 & 0.1425 & 135.6235 & 121.4628 \\
\hline S20R04 & 13.2812 & 0.1395 & 134.4902 & 121.0695 \\
\hline S20R05 & 13.5473 & 0.1407 & 134.9082 & 121.2202 \\
\hline S20R06 & 14.8365 & 0.1454 & 136.8004 & 121.8185 \\
\hline S20R07 & 17.2037 & 0.1510 & 139.8276 & 122.4729 \\
\hline S20R08 & 20.5776 & 0.1536 & 143.4744 & 122.7432 \\
\hline S20R09 & 24.5558 & 0.1515 & 147.1618 & 122.4545 \\
\hline S20R 10 & 28.3983 & 0.1464 & 150.3448 & 121.8001 \\
\hline S20R11 & 31.2913 & 0.1416 & 152.5836 & 121.1507 \\
\hline S20R12 & 32.6265 & 0.1392 & 153.5833 & 120.8175 \\
\hline S20R 13 & 32.1335 & 0.1401 & 153.2163 & 120.9427 \\
\hline S20R 14 & 29.9112 & 0.1440 & 151.5292 & 121.4741 \\
\hline S20R15 & 26.4183 & 0.1493 & 148.7405 & 122.1729 \\
\hline S20R 16 & 22.3990 & 0.1532 & 145.2258 & 122.6736 \\
\hline
\end{tabular}

$\begin{array}{ccccccc} & \text { Cs-137 } & \text { Ba-137m } & \text { Source 21 } & \text { X Posit } & \text { Z Posit } & \text { Y Posit } \\ \text { Line } & \text { Sh.6466 } & 27.0997 & & 3.0621 & -7.9137 & 19.8848 \\ \text { S21C01 } & 35.0579 & 0.2472 & 37.0874 & 1.7822 & & \\ \text { S21C02 } & 28.7246 & 0.2026 & 30.3875 & 1.4602 & & \\ \text { S21C03 } & 23.7888 & 0.1678 & 25.1659 & 1.2093 & & \\ \text { S21C04 } & 21.2478 & 0.1498 & 22.4777 & 1.0802 & & \\ \text { S21C05 } & 21.9495 & 0.1548 & 23.2201 & 1.1158 & & \\ \text { S21C06 } & 25.6291 & 0.1807 & 27.1127 & 1.3029 & & \\ \text { S21C07 } & 42.0414 & 0.1893 & 43.5998 & 1.3691 & & \\ \text { S21C08 } & 36.7034 & 0.1652 & 38.0640 & 1.1953 & & \\ \text { S21C09 } & 32.8243 & 0.1478 & 34.0410 & 1.0690 & & \\ \text { S21C10 } & 30.9573 & 0.1394 & 32.1048 & 1.0082 & & \\ \text { S21C11 } & 31.4626 & 0.1416 & 32.6289 & 1.0246 & & \\ \text { S21C12 } & 34.2354 & 0.1541 & 35.5045 & 1.1149 & & \end{array}$




\section{REVISED METHODOLOGY FOR DETERMINING CESIUM-137 CONTENT OF HN-200 GROUT CONTAINERS.}

$\begin{array}{lllll}\text { S21C13 } & 39.1236 & 0.2100 & 40.8493 & 1.5158 \\ \text { S21C14 } & 33.4061 & 0.1793 & 34.8797 & 1.2943 \\ \text { S21C15 } & 29.1528 & 0.1564 & 30.4388 & 1.1295 \\ \text { S21C16 } & 27.0630 & 0.1452 & 28.2568 & 1.0485 \\ \text { S21C17 } & 27.6320 & 0.1483 & 28.8508 & 1.0706 \\ \text { S21C18 } & 30.7123 & 0.1648 & 32.0671 & 1.1899 \\ \text { S21C19 } & 31.4074 & 0.2921 & 33.8112 & 2.1118 \\ \text { S21C20 } & 24.4202 & 0.2271 & 26.2893 & 1.6420 \\ \text { S21C21 } & 18.6025 & 0.1730 & 20.0263 & 1.2508 \\ \text { S21C22 } & 15.3473 & 0.1427 & 16.5220 & 1.0319 \\ \text { S21C23 } & 16.2731 & 0.1514 & 17.5186 & 1.0942 \\ \text { S21C24 } & 20.8296 & 0.1937 & 22.4238 & 1.4005 \\ \text { S21R01 } & 18.7340 & 0.1479 & 140.2672 & 121.3853 \\ \text { S21R02 } & 21.8990 & 0.1489 & 143.5523 & 121.5044 \\ \text { S21R03 } & 25.3709 & 0.1466 & 146.7345 & 121.2169 \\ \text { S21R04 } & 28.4927 & 0.1427 & 149.3499 & 120.7145 \\ \text { S21R05 } & 30.6199 & 0.1395 & 151.0384 & 120.2790 \\ \text { S21R06 } & 31.3141 & 0.1383 & 151.5763 & 120.1239 \\ \text { S21R07 } & 30.4347 & 0.1398 & 150.8939 & 120.3195 \\ \text { S21R08 } & 28.1601 & 0.1432 & 149.0797 & 120.7765 \\ \text { S21R09 } & 24.9600 & 0.1470 & 146.3750 & 121.2680 \\ \text { S21R10 } & 21.4925 & 0.1490 & 143.1546 & 121.5131 \\ \text { S21R11 } & 18.4001 & 0.1475 & 139.8912 & 121.3435 \\ \text { S21R12 } & 16.1009 & 0.1436 & 137.0953 & 120.8508 \\ \text { S21R13 } & 14.7445 & 0.1398 & 135.2329 & 120.3485 \\ \text { S21R14 } & 14.3341 & 0.1384 & 134.6296 & 120.1571 \\ \text { S21R15 } & 14.8566 & 0.1402 & 135.3941 & 120.3974 \\ \text { S21R16 } & 16.3274 & 0.1441 & 137.3891 & 120.9175\end{array}$

$\begin{array}{lcccccc} & \text { Cs-137 } & \text { Ba-137m } & \text { Source 22 } & \begin{array}{c}\text { X Posit } \\ \text { Z Posit }\end{array} & \text { Y Posit } \\ & 20.3724 & 19.2723 & & -17.9782 & 12.8570 & 50.0431 \\ \text { Line } & \text { Shield 1 } & \text { Shield 2 } & \text { Total Dist } & \text { Air Gap } & & \\ \text { S22C01 } & 42.6531 & 0.1452 & 43.8466 & 1.0482 & & \\ \text { S22C02 } & 43.6485 & 0.1486 & 44.8698 & 1.0727 & & \\ \text { S22C03 } & 46.3076 & 0.1577 & 47.6033 & 1.1380 & \\ \text { S22C04 } & 50.3676 & 0.1715 & 51.7769 & 1.2378 & \\ \text { S22C05 } & 55.5221 & 0.1890 & 57.0756 & 1.3645 & \\ \text { S22C06 } & 61.4964 & 0.2094 & 63.2171 & 1.5113 & \\ \text { S22C07 } & 18.7900 & 0.3047 & 21.1255 & 2.0309 & \\ \text { S22C08 } & 20.6126 & 0.3342 & 23.1747 & 2.2279 & \\ \text { S22C09 } & 24.9971 & 0.4053 & 28.1041 & 2.7017 & \\ \text { S22C10 } & 30.8705 & 0.5005 & 34.7076 & 3.3365 & \\ \text { S22C11 } & 37.5404 & 0.6087 & 42.2066 & 4.0574 & & \\ \text { S22C12 } & 44.6513 & 0.7240 & 50.2013 & 4.8260 & & \\ \text { S22C13 } & 11.0507 & 0.4418 & 14.1813 & 2.6888 & & \\ & & & & & \end{array}$




\section{REVISED METHODOLOGY FOR DETERMINING CESIUM-137 CONTENT OF HN-200 GROUT CONTAINERS.}

$\begin{array}{lllll}\text { S22C14 } & 13.3131 & 0.5322 & 17.0847 & 3.2393 \\ \text { S22C15 } & 18.1860 & 0.7271 & 23.3380 & 4.4250 \\ \text { S22C16 } & 24.1369 & 0.9650 & 30.9748 & 5.8729 \\ \text { S22C17 } & 30.5421 & 1.2210 & 39.1946 & 7.4314 \\ \text { S22C18 } & 37.1675 & 1.4859 & 47.6969 & 9.0435 \\ \text { S22C19 } & 39.6808 & 0.1556 & 40.9554 & 1.1189 \\ \text { S22C20 } & 40.7404 & 0.1598 & 42.0490 & 1.1488 \\ \text { S22C21 } & 43.5554 & 0.1708 & 44.9544 & 1.2282 \\ \text { S22C22 } & 47.8167 & 0.1875 & 49.3525 & 1.3484 \\ \text { S22C23 } & 53.1777 & 0.2085 & 54.8858 & 1.4995 \\ \text { S22C24 } & 59.3412 & 0.2327 & 61.2473 & 1.6733 \\ \text { S22R01 } & 39.0995 & 0.1610 & 163.1004 & 123.8399 \\ \text { S22R02 } & 29.1334 & 0.2123 & 157.4927 & 128.1470 \\ \text { S22R03 } & 15.7794 & 0.3565 & 149.9915 & 133.8556 \\ \text { S22R04 } & 4.2630 & 0.5267 & 141.5391 & 136.7493 \\ \text { S22R05 } & 1.4463 & 0.2713 & 133.3669 & 131.6493 \\ \text { S22R06 } & 0.8835 & 0.1735 & 126.8846 & 125.8276 \\ \text { S22R07 } & 0.7228 & 0.1433 & 123.4061 & 122.5401 \\ \text { S22R08 } & 0.7352 & 0.1456 & 123.7229 & 122.8421 \\ \text { S22R09 } & 0.9345 & 0.1829 & 127.7603 & 126.6429 \\ \text { S22R10 } & 1.6338 & 0.3006 & 134.6123 & 132.6778 \\ \text { S22R11 } & 5.4643 & 0.5460 & 142.9231 & 136.9127 \\ \text { S22R12 } & 18.0624 & 0.3212 & 151.2981 & 132.9145 \\ \text { S22R13 } & 31.0227 & 0.2003 & 158.5487 & 127.3258 \\ \text { S22R14 } & 40.2987 & 0.1564 & 163.7825 & 123.3275 \\ \text { S22R15 } & 44.9019 & 0.1409 & 166.4193 & 121.3764 \\ \text { S22R16 } & 44.4930 & 0.1422 & 166.1838 & 121.5486\end{array}$

$\begin{array}{ccccccc} & \text { Cs-137 } & \text { Ba-137m } & \text { Source 23 } & \text { X Posit } & \text { Z Posit } & \text { Y Posit } \\ & 13.0146 & 12.3118 & & 5.0992 & 14.6169 & 52.0038 \\ \text { Line } & \text { Shield 1 } & \text { Shield 2 } & \text { Total Dist } & \text { Air Gap } & & \\ \text { S23C01 } & 22.5041 & 0.1812 & 23.9751 & 1.2898 & & \\ \text { S23C02 } & 24.8497 & 0.2001 & 26.4740 & 1.4243 & & \\ \text { S23C03 } & 29.5180 & 0.2376 & 31.4474 & 1.6918 & & \\ \text { S23C04 } & 35.6068 & 0.2867 & 37.9343 & 2.0408 & \\ \text { S23C05 } & 42.5101 & 0.3422 & 45.2888 & 2.4365 & \\ \text { S23C06 } & 49.8909 & 0.4017 & 53.1521 & 2.8595 & \\ \text { S23C07 } & 9.5722 & 0.1642 & 10.9153 & 1.1788 & \\ \text { S23C08 } & 13.7325 & 0.2356 & 15.6592 & 1.6912 & \\ \text { S23C09 } & 20.2514 & 0.3474 & 23.0928 & 2.4940 & \\ \text { S23C10 } & 27.4999 & 0.4718 & 31.3584 & 3.3867 & & \\ \text { S23C11 } & 35.0280 & 0.6009 & 39.9426 & 4.3138 & & \\ \text { S23C12 } & 42.6879 & 0.7323 & 48.6773 & 5.2571 & \\ \text { S23C13 } & 31.3182 & 0.1571 & 32.6045 & 1.1291 & & \\ \text { S23C14 } & 33.1232 & 0.1662 & 34.4836 & 1.1942 & & \\ & & & & & \end{array}$




\section{REVISED METHODOLOGY FOR DETERMINING CESIUM-137 CONTENT OF HN-200 GROUT CONTAINERS.}

$\begin{array}{lcccc}\text { S23C15 } & 36.9180 & 0.1852 & 38.4342 & 1.3310 \\ \text { S23C16 } & 42.1687 & 0.2115 & 43.9005 & 1.5203 \\ \text { S23C17 } & 48.4038 & 0.2428 & 50.3917 & 1.7451 \\ \text { S23C18 } & 55.2913 & 0.2774 & 57.5621 & 1.9935 \\ \text { S23C19 } & 37.8197 & 0.1398 & 38.9707 & 1.0112 \\ \text { S23C20 } & 39.3581 & 0.1455 & 40.5559 & 1.0523 \\ \text { S23C21 } & 42.6657 & 0.1577 & 43.9642 & 1.1408 \\ \text { S23C22 } & 47.3734 & 0.1751 & 48.8151 & 1.2666 \\ \text { S23C23 } & 53.1101 & 0.1963 & 54.7265 & 1.4200 \\ \text { S23C24 } & 59.5794 & 0.2202 & 61.3926 & 1.5930 \\ \text { S23R01 } & 13.7999 & 0.1868 & 140.8929 & 126.9062 \\ \text { S23R02 } & 10.0427 & 0.1674 & 135.5090 & 125.2988 \\ \text { S23R03 } & 8.1279 & 0.1489 & 131.6171 & 123.3402 \\ \text { S23R04 } & 7.4710 & 0.1410 & 129.9621 & 122.3500 \\ \text { S23R05 } & 7.8257 & 0.1454 & 130.8813 & 122.9102 \\ \text { S23R06 } & 9.3268 & 0.1613 & 134.1858 & 124.6977 \\ \text { S23R07 } & 12.4809 & 0.1822 & 139.2285 & 126.5654 \\ \text { S23R08 } & 17.8228 & 0.1905 & 145.1076 & 127.0943 \\ \text { S23R09 } & 24.8203 & 0.1776 & 150.8849 & 125.8870 \\ \text { S23R10 } & 31.6714 & 0.1587 & 155.7382 & 123.9081 \\ \text { S23R11 } & 36.6427 & 0.1453 & 159.0410 & 122.2530 \\ \text { S23R12 } & 38.7288 & 0.1400 & 160.3961 & 121.5273 \\ \text { S23R13 } & 37.5728 & 0.1429 & 159.6470 & 121.9312 \\ \text { S23R14 } & 33.3681 & 0.1540 & 156.8796 & 123.3575 \\ \text { S23R15 } & 26.9149 & 0.1720 & 152.4221 & 125.3353 \\ \text { S23R16 } & 19.7510 & 0.1885 & 146.8361 & 126.8966\end{array}$

$\begin{array}{ccccccc} & \text { Cs-137 } & \text { Ba-137m } & \text { Source 24 } & \text { X Posit } & \text { Z Posit } & \text { Y Posit } \\ & 12.5419 & 11.8646 & & 7.9617 & 19.2122 & 33.0332 \\ \text { Line } & \text { Shield 1 } & \text { Shield 2 } & \text { Total Dist } & \text { Air Gap } & & \\ \text { S24C01 } & 27.7095 & 0.2789 & 29.9247 & 1.9363 & & \\ \text { S24C02 } & 24.1480 & 0.2430 & 26.0785 & 1.6874 & & \\ \text { S24C03 } & 23.1806 & 0.2333 & 25.0337 & 1.6198 & & \\ \text { S24C04 } & 25.1088 & 0.2527 & 27.1161 & 1.7545 & \\ \text { S24C05 } & 29.3679 & 0.2956 & 31.7156 & 2.0522 & \\ \text { S24C06 } & 35.1197 & 0.3535 & 37.9273 & 2.4541 & \\ \text { S24C07 } & 13.9615 & 0.6475 & 18.8911 & 4.2821 & \\ \text { S24C08 } & 8.7904 & 0.4076 & 11.8941 & 2.6961 & \\ \text { S24C09 } & 6.9356 & 0.3216 & 9.3845 & 2.1272 & \\ \text { S24C10 } & 10.3643 & 0.4806 & 14.0238 & 3.1789 & \\ \text { S24C11 } & 15.9756 & 0.7409 & 21.6163 & 4.8999 & & \\ \text { S24C12 } & 22.1698 & 1.0281 & 29.9977 & 6.7998 & & \\ \text { S24C13 } & 39.2502 & 0.1795 & 40.7164 & 1.2867 & \\ \text { S24C14 } & 36.6116 & 0.1675 & 37.9793 & 1.2002 & \\ \text { S24C15 } & 35.9275 & 0.1643 & 37.2696 & 1.1777 & & \\ & & & & & \end{array}$




\section{REVISED METHODOLOGY FOR DETERMINING CESIUM-137 CONTENT OF HN-200 GROUT CONTAINERS.}

$\begin{array}{lcccc}\text { S24C16 } & 37.3055 & 0.1706 & 38.6991 & 1.2229 \\ \text { S24C17 } & 40.5359 & 0.1854 & 42.0502 & 1.3288 \\ \text { S24C18 } & 45.2235 & 0.2069 & 46.9129 & 1.4825 \\ \text { S24C19 } & 45.6289 & 0.1503 & 46.8662 & 1.0869 \\ \text { S24C20 } & 43.3338 & 0.1428 & 44.5089 & 1.0323 \\ \text { S24C21 } & 42.7458 & 0.1408 & 43.9049 & 1.0183 \\ \text { S24C22 } & 43.9334 & 0.1448 & 45.1247 & 1.0465 \\ \text { S24C23 } & 46.7615 & 0.1541 & 48.0295 & 1.1139 \\ \text { S24C24 } & 50.9578 & 0.1679 & 52.3396 & 1.2139 \\ \text { S24R01 } & 5.5122 & 0.2922 & 136.4809 & 130.6765 \\ \text { S24R02 } & 3.0251 & 0.1971 & 129.2226 & 126.0005 \\ \text { S24R03 } & 2.2111 & 0.1511 & 124.1373 & 121.7751 \\ \text { S24R04 } & 2.0054 & 0.1384 & 122.3024 & 120.1586 \\ \text { S24R05 } & 2.2115 & 0.1511 & 124.1403 & 121.7778 \\ \text { S24R06 } & 3.0262 & 0.1971 & 129.2281 & 126.0047 \\ \text { S24R07 } & 5.5158 & 0.2923 & 136.4877 & 130.6796 \\ \text { S24R08 } & 12.8228 & 0.3172 & 144.5825 & 131.4425 \\ \text { S24R09 } & 24.2701 & 0.2253 & 152.2471 & 127.7516 \\ \text { S24R10 } & 34.5739 & 0.1698 & 158.4541 & 123.7104 \\ \text { S24R11 } & 41.3093 & 0.1453 & 162.4690 & 121.0144 \\ \text { S24R12 } & 43.6280 & 0.1383 & 163.8546 & 120.0883 \\ \text { S24R13 } & 41.3054 & 0.1453 & 162.4666 & 121.0160 \\ \text { S24R14 } & 34.5664 & 0.1699 & 158.4497 & 123.7134 \\ \text { S24R15 } & 24.2602 & 0.2254 & 152.2410 & 127.7554 \\ \text { S24R16 } & 12.8139 & 0.3173 & 144.5756 & 131.4443\end{array}$

$\begin{array}{lcccccc} & \text { Cs-137 } & \text { Ba-137m } & \text { Source 25 } & \text { X Posit } & \text { Z Posit } & \text { Y Posit } \\ \text { Line } & \text { Shield 1 } & \text { Shield 2 } & \text { Total Dist } & \text { Air Gap } & & \\ \text { S25C01 } & 19.1100 & 0.1869 & 20.6360 & 1.3391 & & \\ \text { S25C02 } & 16.9046 & 0.1654 & 18.2545 & 1.1845 & & \\ \text { S25C03 } & 18.5813 & 0.1818 & 20.0650 & 1.3020 & \\ \text { S25C04 } & 23.3171 & 0.2281 & 25.1791 & 1.6339 & \\ \text { S25C05 } & 29.6823 & 0.2903 & 32.0525 & 2.0799 & \\ \text { S25C06 } & 36.8418 & 0.3604 & 39.7837 & 2.5815 & \\ \text { S25C07 } & 17.7090 & 0.1880 & 19.2454 & 1.3483 & \\ \text { S25C08 } & 15.3358 & 0.1628 & 16.6663 & 1.1676 & \\ \text { S25C09 } & 17.1445 & 0.1820 & 18.6318 & 1.3053 & \\ \text { S25C10 } & 22.1325 & 0.2350 & 24.0525 & 1.6851 & \\ \text { S25C11 } & 28.6867 & 0.3046 & 31.1754 & 2.1841 & \\ \text { S25C12 } & 35.9608 & 0.3818 & 39.0805 & 2.7379 & \\ \text { S25C13 } & 33.8328 & 0.1505 & 35.0699 & 1.0867 & \\ \text { S25C14 } & 32.5339 & 0.1447 & 33.7236 & 1.0449 & \\ \text { S25C15 } & 33.5116 & 0.1491 & 34.7370 & 1.0763 & \\ \text { S25C16 } & 36.5837 & 0.1628 & 37.9215 & 1.1750 & \end{array}$




\section{REVISED METHODOLOGY FOR DETERMINING CESIUM-137 CONTENT OF HN-200 GROUT CONTAINERS.}

$\begin{array}{lllll}\text { S25C17 } & 41.2854 & 0.1837 & 42.7951 & 1.3260 \\ \text { S25C18 } & 47.1314 & 0.2097 & 48.8549 & 1.5138 \\ \text { S25C19 } & 34.6317 & 0.1484 & 35.8520 & 1.0719 \\ \text { S25C20 } & 33.3606 & 0.1429 & 34.5361 & 1.0325 \\ \text { S25C21 } & 34.3172 & 0.1470 & 35.5264 & 1.0622 \\ \text { S25C22 } & 37.3305 & 0.1599 & 38.6459 & 1.1554 \\ \text { S25C23 } & 41.9598 & 0.1798 & 43.4383 & 1.2987 \\ \text { S25C24 } & 47.7372 & 0.2045 & 49.4193 & 1.4775 \\ \text { S25R01 } & 12.7874 & 0.1549 & 135.5141 & 122.5718 \\ \text { S25R02 } & 10.8572 & 0.1441 & 132.2928 & 121.2915 \\ \text { S25R03 } & 10.1430 & 0.1390 & 130.9027 & 120.6208 \\ \text { S25R04 } & 10.5000 & 0.1416 & 131.6136 & 120.9720 \\ \text { S25R05 } & 12.0037 & 0.1510 & 134.2866 & 122.1319 \\ \text { S25R06 } & 14.9002 & 0.1623 & 138.4179 & 123.3554 \\ \text { S25R07 } & 19.3174 & 0.1670 & 143.2848 & 123.8004 \\ \text { S25R08 } & 24.7556 & 0.1613 & 148.1120 & 123.1951 \\ \text { S25R09 } & 30.0389 & 0.1509 & 152.2015 & 122.0117 \\ \text { S25R10 } & 33.9324 & 0.1424 & 155.0096 & 120.9347 \\ \text { S25R11 } & 35.6115 & 0.1388 & 156.1854 & 120.4351 \\ \text { S25R12 } & 34.7534 & 0.1406 & 155.5867 & 120.6926 \\ \text { S25R13 } & 31.5204 & 0.1477 & 153.2856 & 121.6175 \\ \text { S25R14 } & 26.5721 & 0.1580 & 149.5655 & 122.8354 \\ \text { S25R15 } & 21.0376 & 0.1661 & 144.9099 & 123.7061 \\ \text { S25R16 } & 16.1894 & 0.1649 & 139.9722 & 123.6178\end{array}$

$\begin{array}{lcccccc} & \text { Cs-137 } & \text { Ba-137m } & \text { Source 26 } & \text { X Posit } & \text { Z Posit } & \text { Y Posit } \\ \text { Line } & 24.8512 & 23.5093 & & 4.1090 & 16.2164 & 34.1935 \\ \text { Shield 1 } & \text { Shield 2 } & \text { Total Dist } & \text { Air Gap } & & \\ \text { S26C02 } & 28.0976 & 0.2150 & 29.8401 & 1.5275 & & \\ \text { S26C03 } & 24.8397 & 0.1900 & 26.3802 & 1.3504 & & \\ \text { S26C04 } & 24.2524 & 0.1856 & 25.7565 & 1.3185 & & \\ \text { S26C05 } & 31.0068 & 0.2029 & 28.1581 & 1.4414 & & \\ \text { S26C06 } & 36.9254 & 0.2372 & 32.9297 & 1.6857 & \\ \text { S26C07 } & 15.0117 & 0.3199 & 39.2154 & 2.0074 & \\ \text { S26C08 } & 9.1820 & 0.1957 & 17.6292 & 2.2975 & \\ \text { S26C09 } & 7.7930 & 0.1661 & 9.1517 & 1.4053 & \\ \text { S26C10 } & 12.4346 & 0.2650 & 14.6027 & 1.9031 & \\ \text { S26C11 } & 19.1304 & 0.4077 & 22.4660 & 2.9279 & \\ \text { S26C12 } & 26.3590 & 0.5617 & 30.9549 & 4.0342 & & \\ \text { S26C13 } & 34.3646 & 0.1795 & 35.8311 & 1.2870 & \\ \text { S26C14 } & 31.6545 & 0.1653 & 33.0053 & 1.1855 & & \\ \text { S26C15 } & 31.1785 & 0.1628 & 32.5090 & 1.1677 & & \\ \text { S26C16 } & 33.0333 & 0.1725 & 34.4429 & 1.2371 & & \\ \text { S26C17 } & 36.8688 & 0.1925 & 38.4421 & 1.3808 & & \end{array}$




\section{REVISED METHODOLOGY FOR DETERMINING CESIUM-137 CONTENT OF HN-200 GROUT CONTAINERS.}

$\begin{array}{lcccc}\text { S26C18 } & 42.1478 & 0.2201 & 43.9464 & 1.5785 \\ \text { S26C19 } & 41.9444 & 0.1486 & 43.1684 & 1.0753 \\ \text { S26C20 } & 39.6950 & 0.1407 & 40.8533 & 1.0177 \\ \text { S26C21 } & 39.3064 & 0.1393 & 40.4534 & 1.0077 \\ \text { S26C22 } & 40.8318 & 0.1447 & 42.0233 & 1.0468 \\ \text { S26C23 } & 44.0729 & 0.1562 & 45.3590 & 1.1299 \\ \text { S26C24 } & 48.6884 & 0.1725 & 50.1091 & 1.2482 \\ \text { S26R01 } & 13.4651 & 0.2010 & 139.9705 & 126.3044 \\ \text { S26R02 } & 9.1288 & 0.1777 & 133.8173 & 124.5107 \\ \text { S26R03 } & 6.9670 & 0.1522 & 129.0649 & 121.9457 \\ \text { S26R04 } & 6.1410 & 0.1394 & 126.6250 & 120.3445 \\ \text { S26R05 } & 6.2598 & 0.1414 & 127.0060 & 120.6049 \\ \text { S26R06 } & 7.3800 & 0.1579 & 130.1266 & 122.5887 \\ \text { S26R07 } & 10.0231 & 0.1853 & 135.3475 & 125.1392 \\ \text { S26R08 } & 15.0617 & 0.2029 & 141.6953 & 126.4307 \\ \text { S26R09 } & 22.4526 & 0.1900 & 148.1251 & 125.4825 \\ \text { S26R10 } & 30.2477 & 0.1651 & 153.7065 & 123.2937 \\ \text { S26R11 } & 36.2920 & 0.1471 & 157.7183 & 121.2792 \\ \text { S26R12 } & 39.3285 & 0.1390 & 159.6837 & 120.2162 \\ \text { S26R13 } & 38.8583 & 0.1402 & 159.3808 & 120.3823 \\ \text { S26R14 } & 34.9535 & 0.1509 & 156.8433 & 121.7389 \\ \text { S26R15 } & 28.2873 & 0.1714 & 152.3609 & 123.9022 \\ \text { S26R16 } & 20.3612 & 0.1958 & 146.4760 & 125.9190\end{array}$

$\begin{array}{lcccccc} & \text { Cs-137 } & \text { Ba-137m } & \text { Source 27 } & \text { X Posit } & \text { Z Posit } & \text { Y Posit } \\ & 10.2138 & 9.6622 & & 2.2698 & -21.1016 & 13.9713 \\ \text { Line } & \text { Shield 1 } & \text { Shield 2 } & \text { Total Dist } & \text { Air Gap } & & \\ \text { S27C01 } & 44.1470 & 0.3121 & 46.6589 & 2.1999 & & \\ \text { S27C02 } & 38.0652 & 0.2691 & 40.2311 & 1.8968 & \\ \text { S27C03 } & 33.0754 & 0.2338 & 34.9574 & 1.6482 & \\ \text { S27C04 } & 29.7328 & 0.2102 & 31.4246 & 1.4816 & \\ \text { S27C05 } & 28.6203 & 0.2023 & 30.2488 & 1.4262 & \\ \text { S27C06 } & 29.9871 & 0.2120 & 31.6934 & 1.4943 & \\ \text { S27C07 } & 55.9595 & 0.1762 & 57.4105 & 1.2748 & \\ \text { S27C08 } & 50.9983 & 0.1606 & 52.3206 & 1.1617 & \\ \text { S27C09 } & 47.1603 & 0.1485 & 48.3831 & 1.0743 & \\ \text { S27C10 } & 44.7357 & 0.1408 & 45.8956 & 1.0191 & \\ \text { S27C11 } & 43.9589 & 0.1384 & 45.0987 & 1.0014 & \\ \text { S27C12 } & 44.9155 & 0.1414 & 46.0801 & 1.0232 & \\ \text { S27C13 } & 46.7718 & 0.2664 & 48.9325 & 1.8943 & \\ \text { S27C14 } & 40.9551 & 0.2333 & 42.8471 & 1.6587 & \\ \text { S27C15 } & 36.2636 & 0.2066 & 37.9388 & 1.4687 & \\ \text { S27C16 } & 33.1780 & 0.1890 & 34.7108 & 1.3437 & \\ \text { S27C17 } & 32.1641 & 0.1832 & 33.6500 & 1.3027 & \\ \text { S27C18 } & 33.4108 & 0.1903 & 34.9543 & 1.3532 & \\ & & & & & \end{array}$




\section{REVISED METHODOLOGY FOR DETERMINING CESIUM-137 CONTENT OF HN-200 GROUT CONTAINERS.}

$\begin{array}{lcccc}\text { S27C19 } & 21.1432 & 1.7963 & 35.7139 & 12.7744 \\ \text { S27C20 } & 15.8519 & 1.3468 & 26.7763 & 9.5775 \\ \text { S27C21 } & 10.5977 & 0.9004 & 17.9011 & 6.4030 \\ \text { S27C22 } & 5.4880 & 0.4663 & 9.2700 & 3.3158 \\ \text { S27C23 } & 2.1685 & 0.1842 & 3.6629 & 1.3102 \\ \text { S27C24 } & 6.0054 & 0.5102 & 10.1439 & 3.6284 \\ \text { S27R01 } & 9.2994 & 0.3707 & 142.8145 & 133.1444 \\ \text { S27R02 } & 20.7884 & 0.2608 & 150.8438 & 129.7946 \\ \text { S27R03 } & 32.2268 & 0.1840 & 157.6439 & 125.2331 \\ \text { S27R04 } & 40.3097 & 0.1507 & 162.3877 & 121.9272 \\ \text { S27R05 } & 43.9771 & 0.1391 & 164.5517 & 120.4354 \\ \text { S27R06 } & 42.8911 & 0.1424 & 163.9097 & 120.8762 \\ \text { S27R07 } & 37.1453 & 0.1623 & 160.5280 & 123.2204 \\ \text { S27R08 } & 27.3347 & 0.2114 & 154.7670 & 127.2209 \\ \text { S27R09 } & 15.2005 & 0.3166 & 147.2879 & 131.7708 \\ \text { S27R10 } & 5.9327 & 0.3526 & 139.0533 & 132.7681 \\ \text { S27R11 } & 2.7871 & 0.2267 & 131.2941 & 128.2803 \\ \text { S27R12 } & 1.8683 & 0.1615 & 125.3792 & 123.3494 \\ \text { S27R13 } & 1.5964 & 0.1399 & 122.5253 & 120.7890 \\ \text { S27R14 } & 1.6704 & 0.1459 & 123.3828 & 121.5665 \\ \text { S27R15 } & 2.1633 & 0.1838 & 127.7516 & 125.4045 \\ \text { S27R16 } & 3.7300 & 0.2800 & 134.6731 & 130.6631\end{array}$

$\begin{array}{ccccccc} & \text { Cs-137 } & \text { Ba-137m } & \text { Source 28 } & \text { X Posit } & \text { Z Posit } & \text { Y Posit } \\ \text { Line } & 15.5133 & 14.6756 & & 18.4153 & -5.6775 & 6.4850 \\ \text { Shield 1 } & \text { Shield 2 } & \text { Total Dist } & \text { Air Gap } & & \\ \text { S28C02 } & 34.4715 & 1.1546 & 43.7381 & 8.1120 & & \\ \text { S28C03 } & 27.5256 & 0.9219 & 34.9249 & 6.4774 & \\ \text { S28C04 } & 14.6798 & 0.6926 & 26.2389 & 4.8665 & \\ \text { S28C05 } & 8.3386 & 0.4716 & 17.8664 & 3.3136 & \\ \text { S28C06 } & 6.4352 & 0.2793 & 10.5801 & 1.9623 & \\ \text { S28C07 } & 53.2264 & 0.2633 & 55.3757 & 1.8859 & \\ \text { S28C08 } & 46.8247 & 0.2317 & 48.7154 & 1.6591 & \\ \text { S28C09 } & 41.2519 & 0.2041 & 42.9176 & 1.4616 & \\ \text { S28C10 } & 36.8858 & 0.1825 & 38.3752 & 1.3069 & \\ \text { S28C11 } & 34.1916 & 0.1692 & 35.5722 & 1.2115 & \\ \text { S28C12 } & 33.5744 & 0.1661 & 34.9301 & 1.1896 & \\ \text { S28C13 } & 59.0022 & 0.1980 & 60.6324 & 1.4322 & \\ \text { S28C14 } & 53.1486 & 0.1784 & 54.6170 & 1.2901 & \\ \text { S28C15 } & 48.1838 & 0.1617 & 49.5151 & 1.1696 & \\ \text { S28C16 } & 44.4072 & 0.1490 & 45.6342 & 1.0779 & \\ \text { S28C17 } & 42.1394 & 0.1414 & 43.3037 & 1.0229 & \\ \text { S28C18 } & 41.6276 & 0.1397 & 42.7777 & 1.0104 & \\ \text { S28C19 } & 47.0132 & 0.4001 & 50.2279 & 2.8146 & \end{array}$




\section{REVISED METHODOLOGY FOR DETERMINING CESIUM-137 CONTENT} OF HN-200 GROUT CONTAINERS.

$\begin{array}{lcccc}\text { S28C20 } & 40.0359 & 0.3407 & 42.7735 & 2.3969 \\ \text { S28C21 } & 33.7259 & 0.2870 & 36.0320 & 2.0191 \\ \text { S28C22 } & 28.5293 & 0.2428 & 30.4801 & 1.7080 \\ \text { S28C23 } & 25.1462 & 0.2140 & 26.8657 & 1.5055 \\ \text { S28C24 } & 24.3448 & 0.2072 & 26.0095 & 1.4575 \\ \text { S28R01 } & 3.7611 & 0.1461 & 126.2881 & 122.3809 \\ \text { S28R02 } & 4.7173 & 0.1744 & 130.2709 & 125.3793 \\ \text { S28R03 } & 7.2409 & 0.2273 & 136.5221 & 129.0539 \\ \text { S28R04 } & 13.1401 & 0.2587 & 143.8681 & 130.4692 \\ \text { S28R05 } & 22.6238 & 0.2180 & 151.1165 & 128.2748 \\ \text { S28R06 } & 32.2140 & 0.1734 & 157.2491 & 124.8617 \\ \text { S28R07 } & 39.1616 & 0.1488 & 161.5035 & 122.1932 \\ \text { S28R08 } & 42.2754 & 0.1396 & 163.3953 & 120.9803 \\ \text { S28R09 } & 41.1624 & 0.1428 & 162.7195 & 121.4144 \\ \text { S28R10 } & 35.9554 & 0.1594 & 159.5487 & 123.4340 \\ \text { S28R11 } & 27.4009 & 0.1943 & 154.2338 & 126.6385 \\ \text { S28R12 } & 17.3957 & 0.2446 & 147.4051 & 129.7648 \\ \text { S28R13 } & 9.5137 & 0.2512 & 139.9647 & 130.1998 \\ \text { S28R14 } & 5.6365 & 0.1973 & 133.0377 & 127.2039 \\ \text { S28R15 } & 4.0922 & 0.1564 & 127.8395 & 123.5909 \\ \text { S28R16 } & 3.5934 & 0.1406 & 125.4125 & 121.6785\end{array}$

$\begin{array}{lcccccc} & \text { Cs-137 } & \text { Ba-137m } & \text { Source 29 } & \text { X Posit } & \text { Z Posit } & \text { Y Posit } \\ \text { Line } & 18.0653 & 17.0898 & & -0.0904 & 3.6427 & 38.3454 \\ \text { Shield 1 } & \text { Shield 2 } & \text { Total Dist } & \text { Air Gap } & & \\ \text { S29C02 } & 25.4727 & 0.1540 & 26.7401 & 1.1134 & & \\ \text { S29C03 } & 23.2413 & 0.1405 & 24.3977 & 1.0159 & \\ \text { S29C04 } & 24.0514 & 0.1454 & 25.2481 & 1.0513 & \\ \text { S29C05 } & 33.1076 & 0.1670 & 29.0118 & 1.2080 & \\ \text { S29C06 } & 39.6919 & 0.2001 & 34.7549 & 1.4472 & \\ \text { S29C07 } & 21.8596 & 0.1577 & 23.1584 & 1.1411 & \\ \text { S29C08 } & 19.2645 & 0.1390 & 20.4091 & 1.0056 & \\ \text { S29C09 } & 20.2172 & 0.1459 & 21.4184 & 1.0554 & \\ \text { S29C10 } & 24.3041 & 0.1753 & 25.7481 & 1.2687 & \\ \text { S29C11 } & 30.2817 & 0.2185 & 32.0809 & 1.5807 & \\ \text { S29C12 } & 37.2507 & 0.2687 & 39.4639 & 1.9445 & \\ \text { S29C13 } & 25.3085 & 0.1542 & 26.5778 & 1.1151 & \\ \text { S29C14 } & 23.0631 & 0.1405 & 24.2198 & 1.0161 & \\ \text { S29C15 } & 23.8786 & 0.1455 & 25.0762 & 1.0521 & \\ \text { S29C16 } & 27.4839 & 0.1675 & 28.8623 & 1.2109 & \\ \text { S29C17 } & 32.9764 & 0.2009 & 34.6302 & 1.4529 & \\ \text { S29C18 } & 39.5779 & 0.2411 & 41.5628 & 1.7438 & \\ \text { S29C19 } & 28.5228 & 0.1491 & 29.7506 & 1.0787 & \\ \text { S29C20 } & 26.5227 & 0.1386 & 27.6643 & 1.0031 & \end{array}$




\section{REVISED METHODOLOGY FOR DETERMINING CESIUM-137 CONTENT OF HN-200 GROUT CONTAINERS.}

$\begin{array}{llllc}\text { S29C21 } & 27.2444 & 0.1424 & 28.4171 & 1.0303 \\ \text { S29C22 } & 30.4954 & 0.1594 & 31.8080 & 1.1533 \\ \text { S29C23 } & 35.5891 & 0.1860 & 37.1210 & 1.3459 \\ \text { S29C24 } & 41.8581 & 0.2188 & 43.6599 & 1.5830 \\ \text { S29R01 } & 22.7645 & 0.1404 & 143.5433 & 120.6384 \\ \text { S29R02 } & 21.3825 & 0.1402 & 142.1415 & 120.6188 \\ \text { S29R03 } & 20.2473 & 0.1396 & 140.9283 & 120.5414 \\ \text { S29R04 } & 19.5010 & 0.1390 & 140.0952 & 120.4552 \\ \text { S29R05 } & 19.2228 & 0.1387 & 139.7767 & 120.4152 \\ \text { S29R06 } & 19.4390 & 0.1389 & 140.0246 & 120.4467 \\ \text { S29R07 } & 20.1294 & 0.1395 & 140.7987 & 120.5297 \\ \text { S29R08 } & 21.2219 & 0.1402 & 141.9735 & 120.6114 \\ \text { S29R09 } & 22.5828 & 0.1404 & 143.3633 & 120.6401 \\ \text { S29R10 } & 24.0166 & 0.1402 & 144.7532 & 120.5965 \\ \text { S29R11 } & 25.2906 & 0.1395 & 145.9347 & 120.5046 \\ \text { S29R12 } & 26.1810 & 0.1389 & 146.7346 & 120.4147 \\ \text { S29R13 } & 26.5245 & 0.1386 & 147.0381 & 120.3750 \\ \text { S29R14 } & 26.2569 & 0.1389 & 146.8020 & 120.4062 \\ \text { S29R15 } & 25.4284 & 0.1395 & 146.0598 & 120.4920 \\ \text { S29R16 } & 24.1913 & 0.1401 & 144.9180 & 120.5866\end{array}$

$\begin{array}{lcccccc} & \text { Cs-137 } & \text { Ba-137m } & \text { Source 30 } & \text { X Posit } & \text { Z Posit } & \text { Y Posit } \\ \text { Line } & \text { 15.0401 } & 14.2280 & & 7.3731 & -19.7854 & 12.4003 \\ \text { Shield 1 } & \text { Shield 2 } & \text { Total Dist } & \text { Air Gap } & & \\ \text { S30C02 } & 41.9664 & 0.4058 & 45.1910 & 2.8188 & & \\ \text { S30C03 } & 29.4279 & 0.3425 & 38.1501 & 2.3797 & \\ \text { S30C04 } & 25.7328 & 0.2882 & 32.1036 & 2.0025 & \\ \text { S30C05 } & 23.9844 & 0.2488 & 27.7100 & 1.7284 & \\ \text { S30C06 } & 25.0606 & 0.2423 & 26.9862 & 1.6833 & \\ \text { S30C07 } & 56.3077 & 0.1830 & 57.8138 & 1.3231 & \\ \text { S30C08 } & 51.1267 & 0.1662 & 52.4942 & 1.2014 & \\ \text { S30C09 } & 47.0212 & 0.1528 & 48.2789 & 1.1049 & \\ \text { S30C10 } & 44.2912 & 0.1439 & 45.4759 & 1.0407 & \\ \text { S30C11 } & 43.1984 & 0.1404 & 44.3538 & 1.0151 & \\ \text { S30C12 } & 43.8652 & 0.1426 & 45.0385 & 1.0307 & \\ \text { S30C13 } & 50.4982 & 0.2359 & 52.4233 & 1.6891 & \\ \text { S30C14 } & 44.7837 & 0.2092 & 46.4909 & 1.4980 & \\ \text { S30C15 } & 40.1424 & 0.1876 & 41.6727 & 1.3427 & \\ \text { S30C16 } & 36.9806 & 0.1728 & 38.3903 & 1.2370 & \\ \text { S30C17 } & 35.6936 & 0.1668 & 37.0543 & 1.1939 & \\ \text { S30C18 } & 36.4805 & 0.1704 & 37.8712 & 1.2203 & \\ \text { S30C19 } & 26.6355 & 1.5159 & 38.0525 & 9.9011 & \\ \text { S30C20 } & 20.5413 & 1.1690 & 29.3461 & 7.6357 & \\ \text { S30C21 } & 14.6225 & 0.8322 & 20.8902 & 5.4355 & \\ & & & & \end{array}$


REVISED METHODOLOGY FOR DETERMINING CESIUM-137 CONTENT OF HN-200 GROUT CONTAINERS.

$\begin{array}{lcccc}\text { S30C22 } & 9.2228 & 0.5249 & 13.1760 & 3.4283 \\ \text { S30C23 } & 5.9728 & 0.3399 & 8.5330 & 2.2202 \\ \text { S30C24 } & 8.1032 & 0.4612 & 11.5766 & 3.0122 \\ \text { S30R01 } & 5.2693 & 0.3229 & 137.7501 & 132.1578 \\ \text { S30R02 } & 13.2921 & 0.3317 & 145.9453 & 132.3215 \\ \text { S30R03 } & 25.2871 & 0.2240 & 153.5967 & 128.0856 \\ \text { S30R04 } & 35.6199 & 0.1682 & 159.6929 & 123.9048 \\ \text { S30R05 } & 42.1233 & 0.1446 & 163.5207 & 121.2527 \\ \text { S30R06 } & 44.0614 & 0.1388 & 164.6665 & 120.4663 \\ \text { S30R07 } & 41.2608 & 0.1474 & 163.0116 & 121.6035 \\ \text { S30R08 } & 33.9775 & 0.1753 & 158.7284 & 124.5755 \\ \text { S30R09 } & 23.1036 & 0.2400 & 152.2847 & 128.9411 \\ \text { S30R10 } & 11.3179 & 0.3494 & 144.4496 & 132.7822 \\ \text { S30R11 } & 4.5266 & 0.2993 & 136.2857 & 131.4598 \\ \text { S30R12 } & 2.4845 & 0.1949 & 129.0886 & 126.4092 \\ \text { S30R13 } & 1.8415 & 0.1500 & 124.2044 & 122.2129 \\ \text { S30R14 } & 1.6978 & 0.1393 & 122.6811 & 120.8440 \\ \text { S30R15 } & 1.9113 & 0.1551 & 124.8718 & 122.8054 \\ \text { S30R16 } & 2.7014 & 0.2089 & 130.2728 & 127.3626\end{array}$

$\begin{array}{lcccccc} & \text { Cs-137 } & \text { Ba-137m } & \text { Source 31 } & \text { X Posit } & \text { Z Posit } & \text { Y Posit } \\ \text { Line } & \text { Sh.3550 } & 21.1478 & & 5.5574 & -17.7939 & 21.2754 \\ \text { S31C01 } & 35.6762 & 0.3002 & 38.0931 & 2.1167 & & \\ \text { S31C02 } & 29.9703 & 0.2522 & 32.0006 & 1.7782 & \\ \text { S31C03 } & 25.8019 & 0.2171 & 27.5499 & 1.5309 & \\ \text { S31C04 } & 23.9866 & 0.2018 & 25.6116 & 1.4231 & \\ \text { S31C05 } & 25.0414 & 0.2107 & 26.7378 & 1.4857 & \\ \text { S31C06 } & 28.6510 & 0.2411 & 30.5919 & 1.6999 & \\ \text { S31C07 } & 49.3025 & 0.1680 & 50.6856 & 1.2150 & \\ \text { S31C08 } & 45.0187 & 0.1534 & 46.2816 & 1.1095 & \\ \text { S31C09 } & 42.1415 & 0.1436 & 43.3237 & 1.0386 & \\ \text { S31C10 } & 40.9684 & 0.1396 & 42.1177 & 1.0096 & \\ \text { S31C11 } & 41.6436 & 0.1419 & 42.8118 & 1.0263 & \\ \text { S31C12 } & 44.0824 & 0.1502 & 45.3190 & 1.0864 & \\ \text { S31C13 } & 42.7987 & 0.2124 & 44.5331 & 1.5220 & \\ \text { S31C14 } & 37.9118 & 0.1881 & 39.4482 & 1.3482 & \\ \text { S31C15 } & 34.5326 & 0.1714 & 35.9321 & 1.2281 & \\ \text { S31C16 } & 33.1260 & 0.1644 & 34.4684 & 1.1780 & \\ \text { S31C17 } & 33.9379 & 0.1684 & 35.3133 & 1.2069 & \\ \text { S31C18 } & 36.8220 & 0.1827 & 38.3143 & 1.3095 & \\ \text { S31C19 } & 23.8494 & 0.6892 & 29.4153 & 4.8766 & \\ \text { S31C20 } & 16.9726 & 0.4905 & 20.9336 & 3.4705 & \\ \text { S31C21 } & 10.6695 & 0.3083 & 13.1594 & 2.1816 & \\ \text { S31C22 } & 6.7897 & 0.1962 & 8.3742 & 1.3883 & \\ & & & & & \end{array}$




\section{REVISED METHODOLOGY FOR DETERMINING CESIUM-137 CONTENT OF HN-200 GROUT CONTAINERS.}

$\begin{array}{lcccc}\text { S31C23 } & 9.2117 & 0.2662 & 11.3615 & 1.8836 \\ \text { S31C24 } & 15.1692 & 0.4384 & 18.7094 & 3.1017 \\ \text { S31R01 } & 10.2992 & 0.2320 & 138.6359 & 128.1047 \\ \text { S31R02 } & 17.7680 & 0.2285 & 145.9023 & 127.9059 \\ \text { S31R03 } & 27.1477 & 0.1879 & 152.5850 & 125.2495 \\ \text { S31R04 } & 35.2628 & 0.1570 & 157.7981 & 122.3784 \\ \text { S31R05 } & 40.2565 & 0.1416 & 160.9206 & 120.5225 \\ \text { S31R06 } & 41.3573 & 0.1386 & 161.6051 & 120.1092 \\ \text { S31R07 } & 38.4224 & 0.1470 & 159.7778 & 121.2084 \\ \text { S31R08 } & 31.8526 & 0.1690 & 155.6375 & 123.6158 \\ \text { S31R09 } & 22.8355 & 0.2069 & 149.6573 & 126.6150 \\ \text { S31R10 } & 13.9170 & 0.2384 & 142.5823 & 128.4270 \\ \text { S31R11 } & 8.1138 & 0.2139 & 135.4070 & 127.0793 \\ \text { S31R12 } & 5.4359 & 0.1695 & 129.2940 & 123.6887 \\ \text { S31R13 } & 4.3740 & 0.1440 & 125.3865 & 120.8685 \\ \text { S31R14 } & 4.1800 & 0.1388 & 124.5030 & 120.1842 \\ \text { S31R15 } & 4.7266 & 0.1530 & 126.8396 & 121.9601 \\ \text { S31R16 } & 6.3834 & 0.1884 & 131.8870 & 125.3152\end{array}$

$\begin{array}{ccccccc} & \text { Cs-137 } & \text { Ba-137m } & \text { Source 32 } & \text { X Posit } & \text { Z Posit } & \text { Y Posit } \\ & 21.6866 & 20.5156 & & -0.9171 & 17.9652 & 26.2433 \\ \text { Line } & \text { Shield 1 } & \text { Shield 2 } & \text { Total Dist } & \text { Air Gap } & & \\ \text { S32C01 } & 36.7024 & 0.2196 & 38.4886 & 1.5666 & & \\ \text { S32C02 } & 32.2499 & 0.1929 & 33.8194 & 1.3766 & & \\ \text { S32C03 } & 29.6708 & 0.1775 & 31.1148 & 1.2665 & \\ \text { S32C04 } & 29.4614 & 0.1763 & 30.8952 & 1.2575 & \\ \text { S32C05 } & 31.6686 & 0.1895 & 33.2098 & 1.3517 & \\ \text { S32C06 } & 35.8488 & 0.2145 & 37.5935 & 1.5302 & \\ \text { S32C07 } & 19.4467 & 0.5567 & 24.0288 & 4.0254 & \\ \text { S32C08 } & 12.5315 & 0.3587 & 15.4843 & 2.5940 & \\ \text { S32C09 } & 6.4817 & 0.1855 & 8.0089 & 1.3417 & \\ \text { S32C10 } & 5.7524 & 0.1647 & 7.1079 & 1.1908 & \\ \text { S32C11 } & 11.4139 & 0.3267 & 14.1032 & 2.3627 & \\ \text { S32C12 } & 18.2638 & 0.5228 & 22.5672 & 3.7806 & \\ \text { S32C13 } & 35.4558 & 0.2309 & 37.3305 & 1.6437 & \\ \text { S32C14 } & 30.8634 & 0.2010 & 32.4953 & 1.4308 & \\ \text { S32C15 } & 28.1802 & 0.1836 & 29.6702 & 1.3065 & \\ \text { S32C16 } & 27.9614 & 0.1821 & 29.4398 & 1.2963 & \\ \text { S32C17 } & 30.2603 & 0.1971 & 31.8603 & 1.4029 & \\ \text { S32C18 } & 34.5786 & 0.2252 & 36.4069 & 1.6031 & \\ \text { S32C19 } & 46.6308 & 0.1581 & 47.9328 & 1.1439 & \\ \text { S32C20 } & 43.0686 & 0.1460 & 44.2711 & 1.0565 & \\ \text { S32C21 } & 41.0937 & 0.1393 & 42.2411 & 1.0081 & \\ \text { S32C22 } & 40.9365 & 0.1388 & 42.0796 & 1.0042 & \\ \text { S32C23 } & 42.6172 & 0.1445 & 43.8072 & 1.0455 & \end{array}$


REVISED METHODOLOGY FOR DETERMINING CESIUM-137 CONTENT OF HN-200 GROUT CONTAINERS.

$\begin{array}{lcccc}\text { S32C24 } & 45.9345 & 0.1557 & 47.2170 & 1.1268 \\ \text { S32R01 } & 17.4910 & 0.2185 & 144.9729 & 127.2634 \\ \text { S32R02 } & 10.6408 & 0.2158 & 137.9558 & 127.0992 \\ \text { S32R03 } & 6.9516 & 0.1788 & 131.5629 & 124.4326 \\ \text { S32R04 } & 5.3288 & 0.1494 & 126.9269 & 121.4486 \\ \text { S32R05 } & 4.8172 & 0.1383 & 124.9767 & 120.0211 \\ \text { S32R06 } & 5.1108 & 0.1448 & 126.1340 & 120.8783 \\ \text { S32R07 } & 6.3834 & 0.1695 & 130.1462 & 123.5933 \\ \text { S32R08 } & 9.4052 & 0.2074 & 136.1887 & 126.5761 \\ \text { S32R09 } & 15.4171 & 0.2228 & 143.1531 & 127.5132 \\ \text { S32R10 } & 23.9896 & 0.1953 & 149.9271 & 125.7422 \\ \text { S32R11 } & 32.3738 & 0.1638 & 155.5672 & 123.0296 \\ \text { S32R12 } & 38.3239 & 0.1450 & 159.3722 & 120.9033 \\ \text { S32R13 } & 40.7551 & 0.1383 & 160.9061 & 120.0127 \\ \text { S32R14 } & 39.3181 & 0.1422 & 160.0005 & 120.5402 \\ \text { S32R15 } & 34.2139 & 0.1576 & 156.7544 & 122.3829 \\ \text { S32R16 } & 26.3002 & 0.1862 & 151.5340 & 125.0476\end{array}$

$\begin{array}{ccccccc} & \text { Cs-137 } & \text { Ba-137m } & \text { Source 33 } & \text { X Posit } & \text { Z Posit } & \text { Y Posit } \\ & 17.8281 & 16.8653 & & -2.7939 & -22.1819 & 29.8247 \\ \text { Line } & \text { Shield 1 } & \text { Shield 2 } & \text { Total Dist } & \text { Air Gap } & & \\ \text { S33C01 } & 38.1914 & 0.2135 & 39.9215 & 1.5166 & \\ \text { S33C02 } & 34.7647 & 0.1944 & 36.3396 & 1.3805 & \\ \text { S33C03 } & 33.2695 & 0.1860 & 34.7766 & 1.3211 & \\ \text { S33C04 } & 33.9616 & 0.1899 & 35.5001 & 1.3486 & \\ \text { S33C05 } & 36.7176 & 0.2053 & 38.3810 & 1.4580 & \\ \text { S33C06 } & 41.1247 & 0.2300 & 42.9877 & 1.6330 & \\ \text { S33C07 } & 48.9793 & 0.1505 & 50.2187 & 1.0890 & \\ \text { S33C08 } & 46.2507 & 0.1421 & 47.4212 & 1.0283 & \\ \text { S33C09 } & 45.0932 & 0.1386 & 46.2343 & 1.0026 & \\ \text { S33C10 } & 45.6263 & 0.1402 & 46.7809 & 1.0144 & \\ \text { S33C11 } & 47.7936 & 0.1469 & 49.0031 & 1.0626 & \\ \text { S33C12 } & 51.3887 & 0.1579 & 52.6891 & 1.1425 & \\ \text { S33C13 } & 34.3996 & 0.2517 & 36.4171 & 1.7658 & \\ \text { S33C14 } & 30.6530 & 0.2242 & 32.4507 & 1.5735 & \\ \text { S33C15 } & 28.9901 & 0.2121 & 30.6903 & 1.4881 & \\ \text { S33C16 } & 29.7622 & 0.2177 & 31.5078 & 1.5278 & \\ \text { S33C17 } & 32.7980 & 0.2399 & 34.7215 & 1.6836 & \\ \text { S33C18 } & 37.5522 & 0.2747 & 39.7546 & 1.9277 & \\ \text { S33C19 } & 6.0731 & 1.8043 & 19.9501 & 12.0727 & \\ \text { S33C20 } & 3.4014 & 1.0106 & 11.1736 & 6.7617 & \\ \text { S33C21 } & 1.1265 & 0.3347 & 3.7006 & 2.2394 & \\ \text { S33C22 } & 2.4455 & 0.7266 & 8.0336 & 4.8615 & \\ \text { S33C23 } & 5.0699 & 1.5063 & 16.6548 & 10.0786 & \\ \text { S33C24 } & 7.7743 & 2.3098 & 25.5388 & 15.4546 & \\ & & & & & \\ \end{array}$




\section{REVISED METHODOLOGY FOR DETERMINING CESIUM-137 CONTENT OF HN-200 GROUT CONTAINERS.}

$\begin{array}{lllll}\text { S33R01 } & 13.6644 & 0.4071 & 147.4420 & 133.3705 \\ \text { S33R02 } & 27.5571 & 0.2218 & 155.2579 & 127.4790 \\ \text { S33R03 } & 38.0569 & 0.1632 & 161.2115 & 122.9914 \\ \text { S33R04 } & 43.9831 & 0.1418 & 164.6348 & 120.5099 \\ \text { S33R05 } & 44.9045 & 0.1390 & 165.1716 & 120.1282 \\ \text { S33R06 } & 40.7616 & 0.1527 & 162.7677 & 121.8533 \\ \text { S33R07 } & 31.8365 & 0.1936 & 157.6693 & 125.6391 \\ \text { S33R08 } & 18.9342 & 0.3115 & 150.4300 & 131.1843 \\ \text { S33R09 } & 5.2335 & 0.6518 & 141.9215 & 136.0362 \\ \text { S33R10 } & 1.1484 & 0.3350 & 133.3260 & 131.8426 \\ \text { S33R11 } & 0.6100 & 0.1885 & 126.0624 & 125.2638 \\ \text { S33R12 } & 0.4649 & 0.1451 & 121.5575 & 120.9475 \\ \text { S33R13 } & 0.4471 & 0.1397 & 120.8271 & 120.2402 \\ \text { S33R14 } & 0.5361 & 0.1666 & 124.0464 & 123.3437 \\ \text { S33R15 } & 0.8593 & 0.2595 & 130.4656 & 129.3468 \\ \text { S33R16 } & 2.6291 & 0.5849 & 138.7503 & 135.5363\end{array}$

$\begin{array}{lcccccc} & \text { Cs-137 } & \text { Ba-137m } & \text { Source 34 } & \text { X Posit } & \text { Z Posit } & \text { Y Posit } \\ \text { Line } & 14.6776 & 13.8850 & & -14.1407 & 16.9694 & 5.4489 \\ \text { Shield 1 } & \text { Shield 2 } & \text { Total Dist } & \text { Air Gap } & & \\ \text { S34C02 } & 58.8279 & 0.2222 & 60.6498 & 1.5997 & \\ \text { S34C03 } & 52.8292 & 0.1996 & 54.4654 & 1.4366 & \\ \text { S34C04 } & 43.6818 & 0.1801 & 49.1586 & 1.2966 & \\ \text { S34C05 } & 41.1830 & 0.1650 & 45.0405 & 1.1880 & \\ \text { S34C06 } & 40.4463 & 0.1556 & 42.4585 & 1.1199 & \\ \text { S34C07 } & 38.1341 & 1.1924 & 41.6990 & 1.0999 & \\ \text { S34C08 } & 31.3252 & 0.9795 & 38.4329 & 6.1283 & \\ \text { S34C09 } & 24.8182 & 0.7760 & 30.4495 & 4.8553 & \\ \text { S34C10 } & 18.9272 & 0.5918 & 23.2218 & 3.7028 & \\ \text { S34C11 } & 14.4276 & 0.4511 & 17.7012 & 2.8225 & \\ \text { S34C12 } & 12.8721 & 0.4025 & 15.7928 & 2.5182 & \\ \text { S34C13 } & 42.1125 & 0.8056 & 48.2124 & 5.2943 & \\ \text { S34C14 } & 35.0755 & 0.6710 & 40.1561 & 4.4097 & \\ \text { S34C15 } & 28.4732 & 0.5447 & 32.5975 & 3.5796 & \\ \text { S34C16 } & 22.6882 & 0.4340 & 25.9745 & 2.8523 & \\ \text { S34C17 } & 18.5037 & 0.3540 & 21.1839 & 2.3263 & \\ \text { S34C18 } & 17.1353 & 0.3278 & 19.6173 & 2.1542 & \\ \text { S34C19 } & 60.0327 & 0.2098 & 61.7562 & 1.5136 & \\ \text { S34C20 } & 54.1405 & 0.1892 & 55.6948 & 1.3651 & \\ \text { S34C21 } & 49.1074 & 0.1717 & 50.5172 & 1.2382 & \\ \text { S34C22 } & 45.2213 & 0.1581 & 46.5196 & 1.1402 & \\ \text { S34C23 } & 42.7958 & 0.1496 & 44.0244 & 1.0790 & \\ \text { S34C24 } & 42.0842 & 0.1471 & 43.2924 & 1.0611 & \\ \text { S34R01 } & 33.0333 & 0.1882 & 159.4216 & 126.2001 & \\ & & & & & \end{array}$


REVISED METHODOLOGY FOR DETERMINING CESIUM-137 CONTENT OF HN-200 GROUT CONTAINERS.

$\begin{array}{lcccc}\text { S34R02 } & 20.6643 & 0.2862 & 152.4830 & 131.5326 \\ \text { S34R03 } & 7.3185 & 0.5254 & 144.2255 & 136.3815 \\ \text { S34R04 } & 1.9527 & 0.3404 & 135.7862 & 133.4932 \\ \text { S34R05 } & 1.0239 & 0.1955 & 128.5366 & 127.3172 \\ \text { S34R06 } & 0.7678 & 0.1490 & 123.8707 & 122.9539 \\ \text { S34R07 } & 0.7254 & 0.1411 & 122.8173 & 121.9508 \\ \text { S34R08 } & 0.8490 & 0.1640 & 125.6248 & 124.6118 \\ \text { S34R09 } & 1.2985 & 0.2425 & 131.6447 & 130.1036 \\ \text { S34R10 } & 3.2941 & 0.4731 & 139.6226 & 135.8554 \\ \text { S34R11 } & 13.1452 & 0.4028 & 148.1371 & 134.5891 \\ \text { S34R12 } & 26.7741 & 0.2283 & 155.9091 & 128.9067 \\ \text { S34R13 } & 37.4820 & 0.1670 & 161.9380 & 124.2890 \\ \text { S34R14 } & 43.7689 & 0.1439 & 165.5343 & 121.6215 \\ \text { S34R15 } & 45.1257 & 0.1397 & 166.3175 & 121.0520 \\ \text { S34R16 } & 41.4592 & 0.1517 & 164.2071 & 122.5962\end{array}$

\begin{tabular}{|ccccccc} 
& Cs-137 & Ba-137m & Source 35 & X Posit & Z Posit & Y Posit \\
Line & 28.0146 & 26.5018 & & 17.6331 & 12.8346 & 30.1155 \\
S35C01 & 19.1593 & 0.6800 & 24.0880 & 4.2487 & & \\
S35C02 & 14.0563 & 0.4989 & 17.6723 & 3.1171 & \\
S35C03 & 11.4268 & 0.4055 & 14.3662 & 2.5340 & \\
S35C04 & 12.8860 & 0.4573 & 16.2009 & 2.8576 & \\
S35C05 & 17.4360 & 0.6188 & 21.9214 & 3.8666 & \\
S35C06 & 23.3339 & 0.8281 & 29.3364 & 5.1744 & \\
S35C07 & 25.3306 & 0.4067 & 28.4598 & 2.7225 & \\
S35C08 & 20.7219 & 0.3327 & 23.2817 & 2.2271 & \\
S35C09 & 18.5873 & 0.2985 & 20.8834 & 1.9977 & \\
S35C10 & 19.7462 & 0.3171 & 22.1855 & 2.1223 & \\
S35C11 & 23.7207 & 0.3809 & 26.6510 & 2.5494 & \\
S35C12 & 29.3899 & 0.4719 & 33.0205 & 3.1588 & \\
S35C13 & 46.3226 & 0.1591 & 47.6298 & 1.1481 & \\
S35C14 & 43.5014 & 0.1494 & 44.7290 & 1.0782 & \\
S35C15 & 42.3342 & 0.1454 & 43.5288 & 1.0493 & \\
S35C16 & 42.9561 & 0.1475 & 44.1683 & 1.0647 & \\
S35C17 & 45.2935 & 0.1555 & 46.5716 & 1.1226 & \\
S35C18 & 49.1019 & 0.1686 & 50.4876 & 1.2170 & \\
S35C19 & 43.7481 & 0.1716 & 45.1539 & 1.2342 & \\
S35C20 & 40.7725 & 0.1599 & 42.0827 & 1.1502 & \\
S35C21 & 39.5344 & 0.1551 & 40.8048 & 1.1153 & \\
S35C22 & 40.1947 & 0.1577 & 41.4863 & 1.1339 & \\
S35C23 & 42.6653 & 0.1674 & 44.0362 & 1.2036 & \\
S35C24 & 46.6595 & 0.1830 & 48.1588 & 1.3163 & \\
S35R01 & 1.3078 & 0.1793 & 126.0049 & 124.5178 & \\
S35R02 & 1.0280 & 0.1433 & 121.8887 & 120.7174 & \\
& & & & & \\
& & & & \\
& &
\end{tabular}




\section{REVISED METHODOLOGY FOR DETERMINING CESIUM-137 CONTENT OF HN-200 GROUT CONTAINERS.}

$\begin{array}{lcccc}\text { S35R03 } & 1.0065 & 0.1404 & 121.4876 & 120.3408 \\ \text { S35R04 } & 1.2201 & 0.1682 & 124.8969 & 123.5086 \\ \text { S35R05 } & 1.9501 & 0.2544 & 131.3355 & 129.1310 \\ \text { S35R06 } & 5.0662 & 0.4444 & 139.4896 & 133.9790 \\ \text { S3SR07 } & 15.7908 & 0.3342 & 147.9463 & 131.8213 \\ \text { S35R08 } & 28.6000 & 0.2083 & 155.4707 & 126.6624 \\ \text { S35R09 } & 38.3591 & 0.1594 & 161.1179 & 122.5994 \\ \text { S35R10 } & 43.7217 & 0.1409 & 164.2539 & 120.3913 \\ \text { S35R11 } & 44.2265 & 0.1394 & 164.5509 & 120.1850 \\ \text { S35R12 } & 39.8355 & 0.1539 & 161.9782 & 121.9889 \\ \text { S35R13 } & 30.9090 & 0.1944 & 156.8031 & 125.6997 \\ \text { S35R14 } & 18.5176 & 0.2983 & 149.5956 & 130.7797 \\ \text { S35R15 } & 6.5809 & 0.4598 & 141.2378 & 134.1971 \\ \text { S35R16 } & 2.2559 & 0.2858 & 132.9099 & 130.3681\end{array}$

TABLE 10-Calculate Microshield input data sheets for the simulations from each source to each measuring point in centimeters.

$\begin{array}{ccccccc} & \text { Cs-137 } & \text { Ba-137m } & \text { Source 01 } & \text { X Posit } & \text { Z Posit } & \text { Y Posit } \\ \text { Line } & 29.4975 & 27.9047 & & 49.1940 & 1.4778 & 65.7946 \\ \text { Shield 1 } & \text { Shield 2 } & \text { Total Dist } & \text { Air Gap } & & \\ \text { S01C02 } & 45.8508 & 1.8482 & 61.0669 & 13.3679 & \\ \text { S01C03 } & 13.1907 & 1.1767 & 38.8780 & 8.5106 & \\ \text { S01C04 } & 10.9230 & 0.5572 & 18.4117 & 4.0304 & \\ \text { S01C05 } & 25.2334 & 1.0171 & 33.6074 & 7.3568 & \\ \text { S01C06 } & 41.7533 & 1.6831 & 55.6096 & 12.1732 & \\ \text { S01C07 } & 92.7873 & 0.5977 & 97.6297 & 4.2448 & \\ \text { S01C08 } & 81.2795 & 0.5236 & 85.5213 & 3.7183 & \\ \text { S01C09 } & 74.4800 & 0.4798 & 78.3670 & 3.4073 & \\ \text { S01C10 } & 73.7038 & 0.4748 & 77.5503 & 3.3717 & \\ \text { S01C11 } & 79.1282 & 0.5097 & 83.2578 & 3.6199 & \\ \text { S01C12 } & 89.6345 & 0.5774 & 94.3124 & 4.1005 & \\ \text { S01C13 } & 121.9806 & 0.3998 & 125.2732 & 2.8928 & \\ \text { S01C14 } & 113.0338 & 0.3705 & 116.0849 & 2.6806 & \\ \text { S01C15 } & 108.0045 & 0.3540 & 110.9198 & 2.5614 & \\ \text { S01C16 } & 107.4441 & 0.3521 & 110.3443 & 2.5481 & \\ \text { S01C17 } & 111.4201 & 0.3652 & 114.4276 & 2.6424 & \\ \text { S01C18 } & 119.4804 & 0.3916 & 122.7055 & 2.8335 & \\ \text { S01C19 } & 94.7624 & 0.5781 & 99.4534 & 4.1129 & \\ \text { S01C20 } & 83.4657 & 0.5092 & 87.5974 & 3.6226 & \\ \text { S01C21 } & 76.8246 & 0.4687 & 80.6276 & 3.3343 & \\ \text { S01C22 } & 76.0684 & 0.4640 & 79.8339 & 3.3015 & \\ \text { S01C23 } & 81.3614 & 0.4963 & 85.3889 & 3.5312 & \\ \text { S01C24 } & 91.6615 & 0.5592 & 96.1990 & 3.9783 & \\ \text { S01R01 } & 8.6987 & 0.3512 & 313.8781 & 304.8282 & \\ \text { S01R02 } & 9.3752 & 0.3738 & 317.5340 & 307.7850 & \\ \text { S01R03 } & 12.1700 & 0.4574 & 328.9729 & 316.3455 & \\ & & & & & \\ & & & & \end{array}$




\section{REVISED METHODOLOGY FOR DETERMINING CESIUM-137 CONTENT OF HN-200 GROUT CONTAINERS.}

$\begin{array}{lcccc}\text { S01R04 } & 19.4637 & 0.6005 & 345.8021 & 325.7379 \\ \text { S01R05 } & 36.1208 & 0.6519 & 364.9209 & 328.1482 \\ \text { S01R06 } & 60.9888 & 0.5311 & 383.2997 & 321.7799 \\ \text { S01R07 } & 84.6552 & 0.4244 & 398.4220 & 313.3423 \\ \text { S01R08 } & 100.8873 & 0.3691 & 408.4500 & 307.1936 \\ \text { S01R09 } & 107.0960 & 0.3511 & 412.2635 & 304.8164 \\ \text { S01R10 } & 102.5212 & 0.3642 & 409.4541 & 306.5687 \\ \text { S01R11 } & 87.7130 & 0.4130 & 400.3219 & 312.1959 \\ \text { S01R12 } & 64.9012 & 0.5113 & 385.8775 & 320.4650 \\ \text { S01R13 } & 39.6047 & 0.6396 & 367.8497 & 327.6054 \\ \text { S01R14 } & 21.2862 & 0.6211 & 348.6573 & 326.7500 \\ \text { S01R15 } & 12.8949 & 0.4764 & 331.2714 & 317.9000 \\ \text { S01R16 } & 9.6352 & 0.3823 & 318.8247 & 308.8072\end{array}$

$\begin{array}{lcccccc} & \text { Cs-137 } & \text { Ba-137m } & \text { Source 02 } & \text { X Posit } & \text { Z Posit } & \text { Y Posit } \\ & 13.8502 & 13.1023 & & -26.9687 & -49.7051 & 47.9195 \\ \text { Line } & \text { Shield 1 } & \text { Shield 2 } & \text { Total Dist } & \text { Air Gap } & & \\ \text { S02C01 } & 123.1614 & 0.5175 & 127.3917 & 3.7128 & \\ \text { S02C02 } & 111.0497 & 0.4666 & 114.8639 & 3.3477 & \\ \text { S02C03 } & 102.3339 & 0.4300 & 105.8488 & 3.0849 & \\ \text { S02C04 } & 97.9251 & 0.4115 & 101.2886 & 2.9520 & \\ \text { S02C05 } & 98.4038 & 0.4135 & 101.7838 & 2.9664 & \\ \text { S02C06 } & 103.7025 & 0.4357 & 107.2644 & 3.1262 & \\ \text { S02C07 } & 134.2058 & 0.4390 & 137.8167 & 3.1719 & \\ \text { S02C08 } & 123.0172 & 0.4024 & 126.3270 & 2.9074 & \\ \text { S02C09 } & 115.0929 & 0.3765 & 118.1895 & 2.7201 & \\ \text { S02C10 } & 111.1334 & 0.3636 & 114.1235 & 2.6266 & \\ \text { S02C11 } & 111.5616 & 0.3650 & 114.5632 & 2.6367 & \\ \text { S02C12 } & 116.3291 & 0.3806 & 119.4590 & 2.7494 & \\ \text { S02C13 } & 89.4217 & 1.1454 & 98.3347 & 7.7675 & \\ \text { S02C14 } & 74.0713 & 0.9488 & 81.4542 & 6.4341 & \\ \text { S02C15 } & 61.9770 & 0.7939 & 68.1545 & 5.3836 & \\ \text { S02C16 } & 55.3175 & 0.7086 & 60.8312 & 4.8051 & \\ \text { S02C17 } & 56.0641 & 0.7181 & 61.6522 & 4.8699 & \\ \text { S02C18 } & 63.9580 & 0.8193 & 70.3329 & 5.5556 & \\ \text { S02C19 } & 56.6596 & 3.9911 & 83.0958 & 22.4451 & \\ \text { S02C20 } & 42.4180 & 2.9879 & 62.2093 & 16.8034 & \\ \text { S02C21 } & 29.5666 & 2.0826 & 43.3617 & 11.7125 & \\ \text { S02C22 } & 20.8569 & 1.4691 & 30.5882 & 8.2622 & \\ \text { S02C23 } & 21.9491 & 1.5461 & 32.1901 & 8.6949 & \\ \text { S02C24 } & 31.8504 & 2.2435 & 46.7111 & 12.6172 & \\ \text { S02R01 } & 68.4382 & 0.5735 & 393.6941 & 324.6823 & \\ \text { S02R02 } & 95.4914 & 0.4191 & 409.0429 & 313.1324 & \\ \text { S02R03 } & 111.1209 & 0.3621 & 418.0674 & 306.5844 & \\ \text { S02R04 } & 114.1341 & 0.3528 & 419.8225 & 305.3356 & \\ & & & & & \end{array}$




\section{REVISED METHODOLOGY FOR DETERMINING CESIUM-137 CONTENT OF HN-200 GROUT CONTAINERS.}

$\begin{array}{lcccc}\text { S02R05 } & 104.3313 & 0.3848 & 414.1309 & 309.4148 \\ \text { S02R06 } & 82.3944 & 0.4823 & 401.5757 & 318.6991 \\ \text { S02R07 } & 50.3452 & 0.7544 & 383.5184 & 332.4187 \\ \text { S02R08 } & 15.7655 & 1.5052 & 362.1218 & 344.8510 \\ \text { S02R09 } & 3.6714 & 0.8690 & 340.3378 & 335.7974 \\ \text { S02R10 } & 1.9174 & 0.4878 & 321.7275 & 319.3222 \\ \text { S02R11 } & 1.4438 & 0.3719 & 309.9106 & 308.0948 \\ \text { S02R12 } & 1.3733 & 0.3543 & 307.5285 & 305.8008 \\ \text { S02R13 } & 1.6243 & 0.4166 & 315.1514 & 313.1105 \\ \text { S02R14 } & 2.5395 & 0.6326 & 331.0010 & 327.8289 \\ \text { S02R15 } & 7.0624 & 1.3406 & 351.7649 & 343.3620 \\ \text { S02R16 } & 33.3307 & 1.0470 & 373.7546 & 339.3769\end{array}$

\begin{tabular}{|ccccccc} 
& Cs-137 & Ba-137m & Source 03 & X Posit & Z Posit & Y Posit \\
& 23.5916 & 22.3177 & & 4.4969 & -18.4379 & 106.3939 \\
Line & Shield 1 & Shield 2 & Total Dist & Air Gap & & \\
S03C01 & 59.1133 & 0.3906 & 62.3219 & 2.8181 & \\
S03C02 & 56.2954 & 0.3719 & 59.3511 & 2.6837 & \\
S03C03 & 61.5165 & 0.4064 & 64.8555 & 2.9326 & \\
S03C04 & 73.0732 & 0.4828 & 77.0396 & 3.4836 & \\
S03C05 & 88.5179 & 0.5848 & 93.3226 & 4.2199 & \\
S03C06 & 106.1671 & 0.7014 & 111.9298 & 5.0612 & \\
S03C07 & 78.7075 & 0.3619 & 81.6881 & 2.6187 & \\
S03C08 & 76.5461 & 0.3520 & 79.4448 & 2.5468 & \\
S03C09 & 80.5854 & 0.3706 & 83.6372 & 2.6812 & \\
S03C10 & 89.9945 & 0.4138 & 93.4025 & 2.9942 & \\
S03C11 & 103.3164 & 0.4751 & 107.2289 & 3.4374 & \\
S03C12 & 119.2469 & 0.5484 & 123.7627 & 3.9675 & \\
S03C13 & 67.4229 & 0.3807 & 70.5528 & 2.7492 & \\
S03C14 & 64.9287 & 0.3666 & 67.9428 & 2.6475 & \\
S03C15 & 69.5709 & 0.3929 & 72.8005 & 2.8368 & \\
S03C16 & 80.1185 & 0.4524 & 83.8378 & 3.2668 & \\
S03C17 & 94.6167 & 0.5343 & 99.0090 & 3.8580 & \\
S03C18 & 111.5354 & 0.6298 & 116.7131 & 4.5479 & \\
S03C19 & 43.5906 & 0.3879 & 46.7842 & 2.8058 & \\
S03C20 & 39.8288 & 0.3544 & 42.7469 & 2.5636 & \\
S03C21 & 46.6891 & 0.4154 & 50.1097 & 3.0052 & \\
S03C22 & 60.6692 & 0.5398 & 65.1140 & 3.9050 & \\
S03C23 & 78.0318 & 0.6943 & 83.7487 & 5.0226 & \\
S03C24 & 96.9769 & 0.8629 & 104.0818 & 6.2420 & \\
S03R01 & 51.5758 & 0.3728 & 361.0909 & 309.1423 & \\
S03R02 & 59.0276 & 0.3725 & 368.4543 & 309.0542 & \\
S03R03 & 66.6243 & 0.3667 & 375.2465 & 308.2556 & \\
S03R04 & 72.9153 & 0.3590 & 380.4883 & 307.2140 & \\
S03R05 & 76.6277 & 0.3538 & 383.4581 & 306.4767 & \\
& & & & & \\
& & &
\end{tabular}




\section{REVISED METHODOLOGY FOR DETERMINING CESIUM-137 CONTENT OF HN-200 GROUT CONTAINERS.}

$\begin{array}{lllll}\text { S03R06 } & 77.0088 & 0.3532 & 383.7585 & 306.3965 \\ \text { S03R07 } & 73.9818 & 0.3576 & 381.3500 & 307.0107 \\ \text { S03R08 } & 68.1593 & 0.3650 & 376.5529 & 308.0287 \\ \text { S03R09 } & 60.7232 & 0.3716 & 370.0234 & 308.9286 \\ \text { S03R10 } & 53.1232 & 0.3733 & 362.6917 & 309.1951 \\ \text { S03R11 } & 46.6341 & 0.3686 & 355.6601 & 308.6574 \\ \text { S03R12 } & 42.0263 & 0.3606 & 350.0463 & 307.6594 \\ \text { S03R13 } & 39.5825 & 0.3544 & 346.7907 & 306.8538 \\ \text { S03R14 } & 39.3422 & 0.3537 & 346.4582 & 306.7623 \\ \text { S03R15 } & 41.3047 & 0.3589 & 349.1078 & 307.4441 \\ \text { S03R16 } & 45.4514 & 0.3669 & 354.2763 & 308.4580\end{array}$

$\begin{array}{lcccccc} & \text { Cs-137 } & \text { Ba-137m } & \text { Source 04 } & \text { X Posit } & \text { Z Posit } & \text { Y Posit } \\ \text { Line } & \text { Sh.6982 } & 19.5805 & & 14.0365 & -48.8135 & 72.8360 \\ \text { S04C01 } & 80.3749 & 0.6808 & 85.8341 & 4.7784 & \\ \text { S04C02 } & 69.2666 & 0.5867 & 73.9713 & 4.1180 & \\ \text { S04C03 } & 63.6556 & 0.5392 & 67.9792 & 3.7844 & \\ \text { S04C04 } & 64.9820 & 0.5504 & 69.3956 & 3.8633 & \\ \text { S04C05 } & 72.8678 & 0.6172 & 77.8171 & 4.3321 & \\ \text { S04C06 } & 85.5173 & 0.7243 & 91.3258 & 5.0841 & \\ \text { S04C07 } & 119.2835 & 0.3927 & 122.5161 & 2.8400 & \\ \text { S04C08 } & 111.4968 & 0.3670 & 114.5184 & 2.6546 & \\ \text { S04C09 } & 107.8205 & 0.3549 & 110.7424 & 2.5670 & \\ \text { S04C10 } & 108.6725 & 0.3577 & 111.6175 & 2.5873 & \\ \text { S04C11 } & 113.9512 & 0.3751 & 117.0394 & 2.7130 & \\ \text { S04C12 } & 123.0886 & 0.4052 & 126.4243 & 2.9306 & \\ \text { S04C13 } & 99.7780 & 0.4973 & 103.8327 & 3.5574 & \\ \text { S04C14 } & 90.5819 & 0.4514 & 94.2629 & 3.2295 & \\ \text { S04C15 } & 86.1373 & 0.4293 & 89.6377 & 3.0711 & \\ \text { S04C16 } & 87.1741 & 0.4344 & 90.7165 & 3.1080 & \\ \text { S04C17 } & 93.5100 & 0.4660 & 97.3100 & 3.3339 & \\ \text { S04C18 } & 104.1828 & 0.5192 & 108.4165 & 3.7144 & \\ \text { S04C19 } & 42.0142 & 1.7600 & 56.0224 & 12.2482 & \\ \text { S04C20 } & 26.4381 & 1.1075 & 35.2530 & 7.7074 & \\ \text { S04C21 } & 14.8520 & 0.6222 & 19.8039 & 4.3297 & \\ \text { S04C22 } & 18.1665 & 0.7610 & 24.2235 & 5.2960 & \\ \text { S04C23 } & 32.0514 & 1.3427 & 42.7378 & 9.3438 & \\ \text { S04C24 } & 48.0873 & 2.0144 & 64.1204 & 14.0186 & \\ \text { S04R01 } & 21.5812 & 0.7015 & 352.4557 & 330.1730 & \\ \text { S04R02 } & 43.0044 & 0.6614 & 372.2430 & 328.5771 & \\ \text { S04R03 } & 70.0402 & 0.4999 & 390.2222 & 319.6822 & \\ \text { S04R04 } & 92.5362 & 0.4026 & 404.0585 & 311.1197 & \\ \text { S04R05 } & 105.8453 & 0.3593 & 412.1429 & 305.9383 & \\ \text { S04R06 } & 108.2437 & 0.3524 & 413.5989 & 305.0028 & \\ & & & & & \\ & & & & \\ & & \end{array}$




\section{REVISED METHODOLOGY FOR DETERMINING CESIUM-137 CONTENT} OF HN-200 GROUT CONTAINERS.

$\begin{array}{lllll}\text { S04R07 } & 99.4714 & 0.3789 & 408.2736 & 308.4233 \\ \text { S04R08 } & 80.5482 & 0.4501 & 396.7336 & 315.7352 \\ \text { S04R09 } & 54.5615 & 0.5883 & 380.2754 & 325.1256 \\ \text { S04R10 } & 29.2573 & 0.7227 & 360.9285 & 330.9485 \\ \text { S04R11 } & 14.8662 & 0.6040 & 341.4104 & 325.9403 \\ \text { S04R12 } & 9.4023 & 0.4426 & 324.9169 & 315.0720 \\ \text { S04R13 } & 7.4603 & 0.3656 & 314.5991 & 306.7732 \\ \text { S04R14 } & 7.1734 & 0.3534 & 312.6823 & 305.1555 \\ \text { S04R15 } & 8.3088 & 0.4004 & 319.6047 & 310.8955 \\ \text { S04R16 } & 11.8606 & 0.5253 & 333.8214 & 321.4355\end{array}$

$\begin{array}{ccccccc} & \text { Cs-137 } & \text { Ba-137m } & \text { Source 05 } & \text { X Posit } & \text { Z Posit } & \text { Y Posit } \\ \text { Line } & 10.9510 & 10.3596 & & -31.7933 & 6.1732 & 92.3496 \\ \text { Shield 1 } & \text { Shield 2 } & \text { Total Dist } & \text { Air Gap } & & \\ \text { S05C02 } & 95.5415 & 0.3740 & 98.6211 & 2.7056 & \\ \text { S05C03 } & 90.4782 & 0.3541 & 93.3946 & 2.5622 & \\ \text { S05C04 } & 96.6937 & 0.3550 & 93.6169 & 2.5683 & \\ \text { S05C05 } & 106.0477 & 0.3763 & 99.2516 & 2.7229 & \\ \text { S05C06 } & 119.2807 & 0.4151 & 109.4659 & 3.0031 & \\ \text { S05C07 } & 67.6640 & 0.4669 & 71.4793 & 3.3778 & \\ \text { S05C08 } & 60.6557 & 0.4186 & 64.0759 & 3.0016 & \\ \text { S05C09 } & 60.9621 & 0.4207 & 64.3996 & 3.0168 & \\ \text { S05C10 } & 68.4851 & 0.4726 & 72.3468 & 3.3891 & \\ \text { S05C11 } & 81.2441 & 0.5607 & 85.8252 & 4.0205 & \\ \text { S05C12 } & 97.1985 & 0.6708 & 102.6793 & 4.8100 & \\ \text { S05C13 } & 40.1969 & 0.5415 & 44.6522 & 3.9137 & \\ \text { S05C14 } & 28.3284 & 0.3816 & 31.4682 & 2.7582 & \\ \text { S05C15 } & 28.9171 & 0.3896 & 32.1222 & 2.8155 & \\ \text { S05C16 } & 41.4355 & 0.5582 & 46.0280 & 4.0343 & \\ \text { S05C17 } & 58.6908 & 0.7907 & 65.1958 & 5.7143 & \\ \text { S05C18 } & 77.5842 & 1.0452 & 86.1833 & 7.5539 & \\ \text { S05C19 } & 77.7762 & 0.4308 & 81.3058 & 3.0988 & \\ \text { S05C20 } & 71.6297 & 0.3968 & 74.8803 & 2.8539 & \\ \text { S05C21 } & 71.8948 & 0.3982 & 75.1575 & 2.8645 & \\ \text { S05C22 } & 78.5067 & 0.4349 & 82.0695 & 3.1279 & \\ \text { S05C23 } & 90.0785 & 0.4990 & 94.1664 & 3.5890 & \\ \text { S05C24 } & 104.9826 & 0.5815 & 109.7469 & 4.1828 & \\ \text { S05R01 } & 89.6882 & 0.3534 & 395.6562 & 305.6147 & \\ \text { S05R02 } & 83.4383 & 0.3670 & 391.2430 & 307.4376 & \\ \text { S05R03 } & 72.0943 & 0.3915 & 382.9003 & 310.4145 & \\ \text { S05R04 } & 58.1530 & 0.4163 & 371.6870 & 313.1177 & \\ \text { S05R05 } & 44.8983 & 0.4228 & 359.1316 & 313.8105 & \\ \text { S05R06 } & 34.8895 & 0.4031 & 347.1053 & 311.8127 & \\ \text { S05R07 } & 28.7586 & 0.3737 & 337.5860 & 308.4537 & \\ & & & & & \\ & & \end{array}$




\section{REVISED METHODOLOGY FOR DETERMINING CESIUM-137 CONTENT OF HN-200 GROUT CONTAINERS.}

$\begin{array}{lllll}\text { SO5R08 } & 25.9422 & 0.3546 & 332.2860 & 305.9892 \\ \text { S05R09 } & 25.9110 & 0.3544 & 332.2230 & 305.9576 \\ \text { S05R10 } & 28.6581 & 0.3731 & 337.4089 & 308.3777 \\ \text { S05R11 } & 34.7038 & 0.4024 & 346.8479 & 311.7417 \\ \text { S05R12 } & 44.6221 & 0.4227 & 358.8379 & 313.7931 \\ \text { S05R13 } & 57.8238 & 0.4168 & 371.4033 & 313.1627 \\ \text { S05R14 } & 71.7873 & 0.3921 & 382.6666 & 310.4872 \\ \text { S05R15 } & 83.2249 & 0.3675 & 391.0905 & 307.4981 \\ \text { S05R16 } & 89.6123 & 0.3536 & 395.6031 & 305.6373\end{array}$

\begin{tabular}{|c|c|c|c|c|c|c|}
\hline & Cs-137 & Ba-137m & Source 06 & X Posit & Z Posit & Y Posit \\
\hline & 16.3182 & 15.4370 & & 25.7762 & -17.3149 & 75.2927 \\
\hline Line & Shield 1 & Shield 2 & Total Dist & Air Gap & & \\
\hline S06C01 & 58.5016 & 0.6470 & 63.8003 & 4.6517 & & \\
\hline S06C02 & 43.8515 & 0.4850 & 47.8233 & 3.4868 & & \\
\hline $\mathrm{S} 06 \mathrm{C} 03$ & 36.0865 & 0.3991 & 39.3550 & 2.8694 & & \\
\hline S06C04 & 39.5003 & 0.4369 & 43.0780 & 3.1408 & & \\
\hline S06C05 & 51.9334 & 0.5744 & 56.6373 & 4.1294 & & \\
\hline S06C06 & 68.6488 & 0.7593 & 74.8666 & 5.4585 & & \\
\hline S06C07 & 92.9099 & 0.4359 & 96.4908 & 3.1449 & & \\
\hline S06C08 & 83.5389 & 0.3919 & 86.7586 & 2.8277 & & \\
\hline S06C09 & 79.3365 & 0.3722 & 82.3941 & 2.6855 & & \\
\hline SO6C10 & 81.1099 & 0.3805 & 84.2360 & 2.7455 & & \\
\hline S06C11 & 88.5008 & 0.4152 & 91.9117 & 2.9957 & & \\
\hline $\mathrm{S} 06 \mathrm{C} 12$ & 100.2746 & 0.4704 & 104.1392 & 3.3942 & & \\
\hline S06C13 & 98.2839 & 0.4131 & 101.6828 & 2.9858 & & \\
\hline S06C14 & 89.4066 & 0.3758 & 92.4985 & 2.7161 & & \\
\hline S06C15 & 85.4624 & 0.3592 & 88.4178 & 2.5963 & & \\
\hline S06C16 & 87.1238 & 0.3662 & 90.1366 & 2.6467 & & \\
\hline S06C17 & 94.0943 & 0.3955 & 97.3482 & 2.8585 & & \\
\hline S06C18 & 105.3251 & 0.4427 & 108.9674 & 3.1997 & & \\
\hline S06C19 & 66.6203 & 0.5864 & 71.4101 & 4.2034 & & \\
\hline $\mathrm{S} 06 \mathrm{C} 20$ & 53.7221 & 0.4728 & 57.5846 & 3.3896 & & \\
\hline S06C21 & 47.3659 & 0.4169 & 50.7713 & 2.9886 & & \\
\hline SO6C22 & 50.1063 & 0.4410 & 53.7088 & 3.1615 & & \\
\hline S06C23 & 60.7240 & 0.5345 & 65.0899 & 3.8314 & & \\
\hline S06C24 & 75.9857 & 0.6688 & 81.4489 & 4.7944 & & \\
\hline S06R01 & 29.9898 & 0.3707 & 337.8091 & 307.4487 & & \\
\hline S06R02 & 35.9897 & 0.3968 & 346.9165 & 310.5299 & & \\
\hline SO6R03 & 45.6362 & 0.4145 & 358.4513 & 312.4006 & & \\
\hline S06R04 & 58.2591 & 0.4092 & 370.5195 & 311.8512 & & \\
\hline S06R05 & 71.4818 & 0.3874 & 381.3193 & 309.4501 & & \\
\hline S06R06 & 82.2568 & 0.3653 & 389.3711 & 306.7490 & & \\
\hline S06R07 & 88.2194 & 0.3527 & 393.6454 & 305.0733 & & \\
\hline S06R08 & 88.1806 & 0.3528 & 393.6177 & 305.0843 & & \\
\hline
\end{tabular}




\section{REVISED METHODOLOGY FOR DETERMINING CESIUM-137 CONTENT} OF HN-200 GROUT CONTAINERS.

$\begin{array}{lllll}\text { SO6R09 } & 82.1480 & 0.3655 & 389.2921 & 306.7787 \\ \text { S06R10 } & 71.3254 & 0.3877 & 381.1984 & 309.4853 \\ \text { S06R11 } & 58.0911 & 0.4094 & 370.3730 & 311.8726 \\ \text { S06R12 } & 45.4938 & 0.4144 & 358.2994 & 312.3913 \\ \text { S06R13 } & 35.8924 & 0.3965 & 346.7837 & 310.4948 \\ \text { S06R14 } & 29.9363 & 0.3704 & 337.7179 & 307.4112 \\ \text { S06R15 } & 27.1901 & 0.3534 & 332.7263 & 305.1829 \\ \text { S06R16 } & 27.2068 & 0.3535 & 332.7586 & 305.1983\end{array}$

\begin{tabular}{|c|c|c|c|c|c|c|}
\hline & $\begin{array}{c}\text { Cs-137 } \\
25.9358\end{array}$ & $\begin{array}{c}\text { Ba-137m } \\
24.5353\end{array}$ & Source 07 & $\begin{array}{l}\text { X Posit } \\
-7.6301\end{array}$ & $\begin{array}{c}\text { Z Posit } \\
-46.9452\end{array}$ & $\begin{array}{l}\text { Y Posit } \\
3.8559\end{array}$ \\
\hline Line & Shield 1 & Shield 2 & Total Dist & Air Gap & & \\
\hline $\mathrm{S} 07 \mathrm{C} 01$ & 141.1409 & 0.7740 & 147.4450 & 5.5301 & & \\
\hline $\mathrm{S} 07 \mathrm{C} 02$ & 123.6681 & 0.6782 & 129.1917 & 4.8455 & & \\
\hline $\mathrm{S} 07 \mathrm{C03}$ & 107.8172 & 0.5912 & 112.6329 & 4.2244 & & \\
\hline S07C04 & 94.4086 & 0.5177 & 98.6254 & 3.6991 & & \\
\hline S07C05 & 84.6117 & 0.4640 & 88.3909 & 3.3152 & & \\
\hline S07C06 & 79.7683 & 0.4374 & 83.3311 & 3.1254 & & \\
\hline S07C07 & 158.4820 & 0.5307 & 162.8522 & 3.8395 & & \\
\hline S07C08 & 142.5988 & 0.4775 & 146.5310 & 3.4547 & & \\
\hline S07C09 & 128.6161 & 0.4307 & 132.1627 & 3.1159 & & \\
\hline S07C10 & 117.2160 & 0.3925 & 120.4483 & 2.8397 & & \\
\hline S07C11 & 109.2104 & 0.3657 & 112.2219 & 2.6458 & & \\
\hline $\mathrm{S} 07 \mathrm{C} 12$ & 105.3758 & 0.3529 & 108.2815 & 2.5529 & & \\
\hline $\mathrm{S} 07 \mathrm{C} 13$ & 133.1893 & 0.9677 & 141.0118 & 6.8548 & & \\
\hline S07C14 & 115.0416 & 0.8359 & 121.7983 & 5.9208 & & \\
\hline S07C15 & 98.2964 & 0.7142 & 104.0696 & 5.0589 & & \\
\hline S07C16 & 83.7986 & 0.6089 & 88.7203 & 4.3128 & & \\
\hline $\mathrm{S} 07 \mathrm{C} 17$ & 72.9016 & 0.5297 & 77.1833 & 3.7520 & & \\
\hline S07C18 & 67.3758 & 0.4895 & 71.3329 & 3.4676 & & \\
\hline S07C19 & 97.0572 & 3.1596 & 122.8964 & 22.6795 & & \\
\hline $\mathrm{S} 07 \mathrm{C} 20$ & 79.1878 & 2.5779 & 100.2697 & 18.5039 & & \\
\hline S07C21 & 61.4263 & 1.9997 & 77.7796 & 14.3536 & & \\
\hline $\mathrm{S} 07 \mathrm{C} 22$ & 43.9037 & 1.4293 & 55.5920 & 10.2591 & & \\
\hline S07C23 & 27.0879 & 0.8818 & 34.2994 & 6.3297 & & \\
\hline S07C24 & 13.8505 & 0.4509 & 17.5379 & 3.2365 & & \\
\hline S07R01 & 49.8633 & 0.5753 & 379.1087 & 328.6701 & & \\
\hline S07R02 & 74.0619 & 0.4671 & 395.4067 & 320.8778 & & \\
\hline S07R03 & 93.7871 & 0.3940 & 407.6819 & 313.5008 & & \\
\hline S07R04 & 104.9239 & 0.3603 & 414.4832 & 309.1990 & & \\
\hline S07R05 & 105.8636 & 0.3577 & 415.0553 & 308.8341 & & \\
\hline S07R06 & 96.4878 & 0.3854 & 409.3359 & 312.4627 & & \\
\hline S07R07 & 78.0953 & 0.4507 & 397.9563 & 319.4103 & & \\
\hline S07R08 & 54.2256 & 0.5558 & 382.2422 & 327.4608 & & \\
\hline S07R09 & 32.1530 & 0.6206 & 364.2012 & 331.4276 & & \\
\hline
\end{tabular}




\section{REVISED METHODOLOGY FOR DETERMINING CESIUM-137 CONTENT OF HN-200 GROUT CONTAINERS.}

$\begin{array}{lllll}\text { S07R10 } & 18.9321 & 0.5334 & 346.4381 & 326.9726 \\ \text { S07R11 } & 13.0885 & 0.4230 & 331.9059 & 318.3944 \\ \text { S07R12 } & 10.8717 & 0.3667 & 323.3721 & 312.1337 \\ \text { S07R13 } & 10.7097 & 0.3623 & 322.6375 & 311.5655 \\ \text { S07R14 } & 12.4954 & 0.4087 & 329.8635 & 316.9595 \\ \text { S07R15 } & 17.4751 & 0.5109 & 343.5067 & 325.5207 \\ \text { S07R16 } & 29.0560 & 0.6151 & 360.9109 & 331.2398\end{array}$

\begin{tabular}{|c|c|c|c|c|c|c|}
\hline & $\begin{array}{r}\text { Cs-137 } \\
25.1678\end{array}$ & $\begin{array}{c}\text { Ba-137m } \\
23.8087\end{array}$ & Source 08 & $\begin{array}{l}\text { X Posit } \\
-3.6748\end{array}$ & $\begin{array}{c}\text { Z Posit } \\
-23.8753\end{array}$ & $\begin{array}{r}\text { Y Posit } \\
52.9898\end{array}$ \\
\hline Line & Shield 1 & Shield 2 & Total Dist & Air Gap & & \\
\hline $\mathrm{S} 08 \mathrm{C} 01$ & 95.5881 & 0.5485 & 100.0916 & 3.9549 & & \\
\hline $\mathrm{S} 08 \mathrm{C} 02$ & 81.1206 & 0.4655 & 84.9425 & 3.3563 & & \\
\hline $\mathrm{S} 08 \mathrm{CO} 3$ & 70.5496 & 0.4049 & 73.8734 & 2.9190 & & \\
\hline S08C04 & 65.7811 & 0.3775 & 68.8803 & 2.7217 & & \\
\hline S08C05 & 68.0463 & 0.3905 & 71.2521 & 2.8154 & & \\
\hline S08C06 & 76.7245 & 0.4403 & 80.3393 & 3.1744 & & \\
\hline S08C07 & 107.8770 & 0.4631 & 111.6905 & 3.3505 & & \\
\hline S08C08 & 94.9868 & 0.4077 & 98.3446 & 2.9501 & & \\
\hline S08C09 & 85.9217 & 0.3688 & 88.9591 & 2.6686 & & \\
\hline S08C10 & 81.9609 & 0.3518 & 84.8583 & 2.5456 & & \\
\hline S08C11 & 83.8311 & 0.3598 & 86.7946 & 2.6036 & & \\
\hline S08C12 & 91.1742 & 0.3914 & 94.3972 & 2.8317 & & \\
\hline $\mathrm{S} 08 \mathrm{C} 13$ & 90.6674 & 0.5919 & 95.5228 & 4.2635 & & \\
\hline SosCt4 & 75.4665 & 0.4927 & 79.5079 & 3.5487 & & \\
\hline S08C15 & 64.1203 & 0.4186 & 67.5541 & 3.0152 & & \\
\hline S08C16 & 58.9004 & 0.3845 & 62.0546 & 2.7697 & & \\
\hline S08C17 & 61.3897 & 0.4008 & 64.6773 & 2.8868 & & \\
\hline S08C 18 & 70.7796 & 0.4621 & 74.5700 & 3.3283 & & \\
\hline S08C19 & 75.2640 & 0.7766 & 81.6589 & 5.6182 & & \\
\hline S08C20 & 57.3010 & 0.5913 & 62.1697 & 4.2773 & & \\
\hline S08C21 & 42.3086 & 0.4366 & 45.9034 & 3.1582 & & \\
\hline $\mathrm{S} 08 \mathrm{C} 22$ & 34.4166 & 0.3551 & 37.3408 & 2.5691 & & \\
\hline $\mathrm{S} 08 \mathrm{C} 23$ & 38.2996 & 0.3952 & 41.5537 & 2.8589 & & \\
\hline S08C24 & 51.3525 & 0.5299 & 55.7157 & 3.8333 & & \\
\hline S08R01 & 58.1555 & 0.3844 & 367.8165 & 309.2765 & & \\
\hline SO8R02 & 68.0721 & 0.3740 & 376.4574 & 308.0112 & & \\
\hline SO8R03 & 76.3618 & 0.3613 & 383.1008 & 306.3777 & & \\
\hline S08R04 & 81.2662 & 0.3528 & 386.8572 & 305.2381 & & \\
\hline SO8R05 & 81.7776 & 0.3519 & 387.2429 & 305.1134 & & \\
\hline S08R06 & 77.7933 & 0.3588 & 384.2086 & 306.0564 & & \\
\hline S08R07 & 70.1258 & 0.3711 & 378.1437 & 307.6468 & & \\
\hline S08R08 & 60.3936 & 0.3827 & 369.8510 & 309.0747 & & \\
\hline S08R09 & 50.6321 & 0.3860 & 360.4890 & 309.4709 & & \\
\hline SO8R 10 & 42.5729 & 0.3778 & 351.4556 & 308.5049 & & \\
\hline
\end{tabular}




\section{REVISED METHODOLOGY FOR DETERMINING CESIUM-137 CONTENT OF HN-200 GROUT CONTAINERS.}

$\begin{array}{lllll}\text { SO8R11 } & 37.0764 & 0.3638 & 344.2023 & 306.7621 \\ \text { S08R12 } & 34.2535 & 0.3533 & 339.9746 & 305.3678 \\ \text { S08R13 } & 33.9754 & 0.3522 & 339.5354 & 305.2078 \\ \text { S08R14 } & 36.2223 & 0.3609 & 342.9650 & 306.3818 \\ \text { S08R15 } & 41.1177 & 0.3749 & 349.6410 & 308.1484 \\ \text { S08R16 } & 48.6486 & 0.3851 & 358.4013 & 309.3675\end{array}$

\begin{tabular}{|ccccccc} 
& Cs-137 & Ba-137m & Source 09 & X Posit & Z Posit & Y Posit \\
& 24.9347 & 23.5882 & & -28.7630 & -3.2708 & 115.5217 \\
Line & Shield 1 & Shield 2 & Total Dist & Air Gap & & \\
S09C01 & 87.2917 & 0.3536 & 90.2034 & 2.5582 & \\
S09C02 & 87.5908 & 0.3548 & 90.5125 & 2.5670 & \\
S09C03 & 93.2911 & 0.3779 & 96.4029 & 2.7340 & \\
S09C04 & 103.5040 & 0.4192 & 106.9565 & 3.0333 & \\
S09C05 & 117.0543 & 0.4741 & 120.9588 & 3.4304 & \\
S09C06 & 132.9252 & 0.5384 & 137.3591 & 3.8956 & \\
S09C07 & 67.7515 & 0.3926 & 70.9701 & 2.8259 & \\
S09C08 & 68.1262 & 0.3948 & 71.3625 & 2.8416 & \\
S09C09 & 75.1305 & 0.4354 & 78.6996 & 3.1337 & \\
S09C10 & 87.1809 & 0.5052 & 91.3225 & 3.6363 & \\
S09C11 & 102.5132 & 0.5941 & 107.3831 & 4.2758 & \\
S09C12 & 119.8745 & 0.6947 & 125.5692 & 5.0000 & \\
S09C13 & 30.7347 & 0.3703 & 33.7840 & 2.6790 & \\
S09C14 & 31.4777 & 0.3793 & 34.6008 & 2.7438 & \\
S09C15 & 43.6128 & 0.5255 & 47.9398 & 3.8015 & \\
S09C16 & 60.6490 & 0.7308 & 66.6663 & 5.2865 & \\
S09C17 & 79.4955 & 0.9579 & 87.3827 & 6.9293 & \\
S09C18 & 99.1250 & 1.1944 & 108.9597 & 8.6403 & \\
S09C19 & 61.8316 & 0.4022 & 65.1250 & 2.8911 & \\
S09C20 & 62.2375 & 0.4048 & 65.5524 & 2.9101 & \\
S09C21 & 69.7565 & 0.4538 & 73.4720 & 3.2617 & \\
S09C22 & 82.4656 & 0.5364 & 86.8580 & 3.8560 & \\
S09C23 & 98.3733 & 0.6399 & 103.6129 & 4.5998 & \\
S09C24 & 116.1729 & 0.7557 & 122.3607 & 5.4321 & \\
S09R01 & 87.2563 & 0.3541 & 394.7157 & 307.1053 & \\
S09R02 & 86.0461 & 0.3565 & 393.8521 & 307.4494 & \\
S09R03 & 79.4389 & 0.3698 & 389.0536 & 309.2450 & \\
S09R04 & 68.7863 & 0.3896 & 380.9222 & 311.7463 & \\
S09R05 & 56.4370 & 0.4059 & 370.5267 & 313.6838 & \\
S09R06 & 45.0984 & 0.4058 & 359.3281 & 313.8239 & \\
S09R07 & 36.6398 & 0.3884 & 349.0362 & 312.0080 & \\
S09R08 & 31.5053 & 0.3671 & 341.3623 & 309.4899 & \\
S09R09 & 29.3645 & 0.3552 & 337.6827 & 307.9629 & \\
S09R10 & 29.9308 & 0.3585 & 338.6893 & 308.3999 & \\
S09R11 & 33.2884 & 0.3755 & 344.1910 & 310.5270 & \\
& & & & & \\
& & & &
\end{tabular}




\section{REVISED METHODOLOGY FOR DETERMINING CESIUM-137 CONTENT OF HN-200 GROUT CONTAINERS.}

$\begin{array}{lllll}\text { SO9R 12 } & 39.8116 & 0.3972 & 353.1688 & 312.9600 \\ \text { S09R13 } & 49.6222 & 0.4083 & 364.0604 & 314.0299 \\ \text { SO9R14 } & 61.6933 & 0.4003 & 375.1176 & 313.0240 \\ \text { S09R15 } & 73.6578 & 0.3809 & 384.7128 & 310.6741 \\ \text { S09R16 } & 82.8333 & 0.3630 & 391.5376 & 308.3413\end{array}$

$\begin{array}{lcccccr} & \text { Cs-137 } & \text { Ba-137m } & \text { Source 10 } & \text { X Posit } & \text { Z Posit } & \text { Y Posit } \\ \text { Line } & 26.8139 & 25.3659 & & -20.7250 & 26.7906 & 117.9524 \\ \text { S10C01 } & 8 \text { Shield 1 } & \text { Shield 2 } & \text { Total Dist } & \text { Air Gap } & & \\ \text { S10C02 } & 84.0324 & 0.3729 & 86.1670 & 2.6909 & \\ \text { S10C03 } & 90.4927 & 0.3771 & 87.1306 & 2.7210 & \\ \text { S10C04 } & 101.4326 & 0.4561 & 93.8290 & 2.9302 & \\ \text { S10C05 } & 115.5870 & 0.5187 & 105.1722 & 3.2845 & \\ \text { S10C06 } & 131.9253 & 0.5920 & 136.789 & 3.7428 & 4.2718 & \\ \text { S10C07 } & 37.0980 & 0.4265 & 40.5800 & 3.0556 & \\ \text { S10C08 } & 38.9336 & 0.4476 & 42.5879 & 3.2067 & \\ \text { S10C09 } & 50.2879 & 0.5781 & 55.0080 & 4.1419 & \\ \text { S10C10 } & 66.4486 & 0.7639 & 72.6855 & 5.4730 & \\ \text { S10C11 } & 84.7080 & 0.9738 & 92.6588 & 6.9769 & \\ \text { S10C12 } & 103.9662 & 1.1952 & 113.7246 & 8.5631 & \\ \text { S10C13 } & 45.2670 & 0.4371 & 48.8297 & 3.1255 & \\ \text { S10C14 } & 46.8253 & 0.4522 & 50.5106 & 3.2331 & \\ \text { S10C15 } & 56.8712 & 0.5492 & 61.3471 & 3.9268 & \\ \text { S10C16 } & 71.9323 & 0.6947 & 77.5937 & 4.9667 & \\ \text { S10C17 } & 89.5120 & 0.8644 & 96.5570 & 6.1805 & \\ \text { S10C18 } & 108.3917 & 1.0468 & 116.9225 & 7.4841 & \\ \text { S10C19 } & 87.3596 & 0.3630 & 90.3457 & 2.6231 & \\ \text { S10C20 } & 88.2487 & 0.3667 & 91.2652 & 2.6498 & \\ \text { S10C21 } & 94.4520 & 0.3925 & 97.6805 & 2.8360 & \\ \text { S10C22 } & 105.0321 & 0.4365 & 108.6222 & 3.1537 & \\ \text { S10C23 } & 118.8255 & 0.4938 & 122.8872 & 3.5679 & \\ \text { S10C24 } & 134.8498 & 0.5604 & 139.4592 & 4.0490 & \\ \text { S10R01 } & 75.2680 & 0.3932 & 387.9566 & 312.2954 & \\ \text { S10R02 } & 60.6706 & 0.4231 & 376.7231 & 315.6295 & \\ \text { S10R03 } & 46.2872 & 0.4361 & 363.7955 & 317.0722 & \\ \text { S10R04 } & 35.1784 & 0.4179 & 351.0668 & 315.4706 & \\ \text { S10R05 } & 28.2717 & 0.3844 & 340.5998 & 311.9436 & \\ \text { S10R06 } & 24.9293 & 0.3602 & 334.2604 & 308.9709 & \\ \text { S10R07 } & 24.4540 & 0.3562 & 333.2639 & 308.4537 & \\ \text { S10R08 } & 26.7287 & 0.3739 & 337.8071 & 310.7045 & \\ \text { S10R09 } & 32.2751 & 0.4063 & 347.0052 & 314.3238 & \\ \text { S10R10 } & 41.8888 & 0.4330 & 359.1692 & 316.8474 & \\ \text { S10R11 } & 55.3737 & 0.4309 & 372.2579 & 316.4533 & \\ \text { S10R12 } & 70.3198 & 0.4041 & 384.2849 & 313.5610 & \\ & & & & & \\ & & & & \end{array}$




\section{REVISED METHODOLOGY FOR DETERMINING CESIUM-137 CONTENT OF HN-200 GROUT CONTAINERS.}

$\begin{array}{lllll}\text { S10R13 } & 83.1356 & 0.3752 & 393.5921 & 310.0813 \\ \text { S10R14 } & 90.9237 & 0.3574 & 398.9897 & 307.7087 \\ \text { S10R15 } & 92.1436 & 0.3546 & 399.8227 & 307.3246 \\ \text { S10R16 } & 86.5706 & 0.3673 & 395.9914 & 309.0535\end{array}$

\begin{tabular}{|c|c|c|c|c|c|c|}
\hline & Cs-137 & Ba-137m & Source 11 & X Posit & Z Posit & Y Posit \\
\hline & 12.5933 & 11.9133 & & 33.1572 & 16.3372 & 74.1470 \\
\hline Line & Shield 1 & Shield 2 & Total Dist & Air Gap & & \\
\hline S11C01 & 54.3502 & 0.7848 & 60.7610 & 5.6260 & & \\
\hline S11C02 & 38.5370 & 0.5564 & 43.0826 & 3.9891 & & \\
\hline S11C03 & 29.1976 & 0.4216 & 32.6416 & 3.0224 & & \\
\hline S11C04 & 32.4984 & 0.4693 & 36.3317 & 3.3641 & & \\
\hline S11C05 & 45.7826 & 0.6611 & 51.1828 & 4.7392 & & \\
\hline S1ICO6 & 63.0253 & 0.9100 & 70.4594 & 6.5240 & & \\
\hline S11C07 & 70.7495 & 0.6146 & 75.7447 & 4.3807 & & \\
\hline S11C08 & 58.3425 & 0.5068 & 62.4617 & 3.6125 & & \\
\hline $511 C 09$ & 52.0965 & 0.4525 & 55.7748 & 3.2257 & & \\
\hline $\mathrm{S} 1 \mathrm{C} 10$ & 54.1859 & 0.4707 & 58.0116 & 3.3551 & & \\
\hline S11C11 & 63.7967 & 0.5542 & 68.3011 & 3.9502 & & \\
\hline S11C12 & 78.2035 & 0.6793 & 83.7250 & 4.8422 & & \\
\hline S11C13 & 105.0860 & 0.4057 & 108.4246 & 2.9330 & & \\
\hline S11C14 & 96.5328 & 0.3726 & 99.5997 & 2.6943 & & \\
\hline S11C15 & 92.6061 & 0.3575 & 95.5483 & 2.5847 & & \\
\hline S11C16 & 93.8881 & 0.3624 & 96.8710 & 2.6205 & & \\
\hline S11C17 & 100.1791 & 0.3867 & 103.3619 & 2.7960 & & \\
\hline S11C18 & 110.6278 & 0.4271 & 114.1425 & 3.0877 & & \\
\hline S11C19 & 94.8317 & 0.4524 & 98.5421 & 3.2580 & & \\
\hline S11C20 & 85.3985 & 0.4074 & 88.7398 & 2.9339 & & \\
\hline S11C21 & 80.9981 & 0.3864 & 84.1672 & 2.7827 & & \\
\hline $\mathrm{S} 11 \mathrm{C} 22$ & 82.4403 & 0.3933 & 85.6659 & 2.8322 & & \\
\hline$S 11 C 23$ & 89.4430 & 0.4267 & 92.9426 & 3.0728 & & \\
\hline S11C24 & 100.8545 & 0.4812 & 104.8006 & 3.4649 & & \\
\hline S11R01 & 22.7512 & 0.3692 & 330.3665 & 307.2462 & & \\
\hline S11R02 & 20.9856 & 0.3515 & 326.2437 & 304.9067 & & \\
\hline S11R03 & 21.8565 & 0.3605 & 328.3376 & 306.1206 & & \\
\hline S11R04 & 25.6408 & 0.3939 & 336.2214 & 310.1867 & & \\
\hline S1IR05 & 33.3645 & 0.4368 & 348.3611 & 314.5597 & & \\
\hline S11R06 & 45.9669 & 0.4549 & 362.5906 & 316.1688 & & \\
\hline S11R07 & 62.1682 & 0.4313 & 376.6357 & 314.0362 & & \\
\hline S11R08 & 78.0778 & 0.3925 & 388.4868 & 310.0166 & & \\
\hline S11R09 & 89.7584 & 0.3633 & 396.6036 & 306.4820 & & \\
\hline S11R10 & 94.7795 & 0.3513 & 400.0017 & 304.8709 & & \\
\hline S11R11 & 92.2347 & 0.3574 & 398.2851 & 305.6931 & & \\
\hline S11R12 & 82.5732 & 0.3811 & 391.6523 & 308.6980 & & \\
\hline S11R13 & 67.6937 & 0.4185 & 380.8951 & 312.7829 & & \\
\hline
\end{tabular}




\section{REVISED METHODOLOGY FOR DETERMINING CESIUM-137 CONTENT OF HN-200 GROUT CONTAINERS.}

$\begin{array}{lllll}\text { S11R14 } & 51.0979 & 0.4510 & 367.3750 & 315.8262 \\ \text { S11R15 } & 36.9984 & 0.4474 & 352.9619 & 315.5161 \\ \text { S11R16 } & 27.7009 & 0.4084 & 339.8741 & 311.7647\end{array}$

$\begin{array}{lcccccc} & \text { Cs-137 } & \text { Ba-137m } & \text { Source 12 } & \text { X Posit } & \text { Z Posit } & \text { Y Posit } \\ \text { Line } & 17.5307 & 16.5841 & & 43.4057 & 22.2267 & 54.0716 \\ \text { S12C01 } & 63 \text { ield 1 } & \text { Shield 2 } & \text { Total Dist } & \text { Air Gap } & & \\ \text { S12C02 } & 46.5872 & 1.6919 & 77.0167 & 11.6774 & \\ \text { S12C03 } & 31.6789 & 0.8421 & 38.3332 & 5.8122 & \\ \text { S12C04 } & 23.4635 & 0.6237 & 28.3921 & 4.3049 & \\ \text { S12C05 } & 28.4835 & 0.7572 & 34.4665 & 5.2259 & \\ \text { S12C06 } & 42.2596 & 1.1234 & 51.1364 & 7.7534 & \\ \text { S12C07 } & 85.0870 & 0.8953 & 92.2333 & 6.2509 & \\ \text { S12C08 } & 69.9738 & 0.7363 & 75.8508 & 5.1406 & \\ \text { S12C09 } & 58.6715 & 0.6174 & 63.5992 & 4.3103 & \\ \text { S12C10 } & 53.6453 & 0.5645 & 58.1509 & 3.9411 & \\ \text { S12C11 } & 56.5931 & 0.5955 & 61.3462 & 4.1576 & \\ \text { S12C12 } & 66.4621 & 0.6993 & 72.0441 & 4.8827 & \\ \text { S12C13 } & 124.8394 & 0.4335 & 128.4058 & 3.1329 & \\ \text { S12C14 } & 113.9389 & 0.3956 & 117.1939 & 2.8594 & \\ \text { S12C15 } & 106.6169 & 0.3702 & 109.6628 & 2.6756 & \\ \text { S12C16 } & 103.6348 & 0.3599 & 106.5955 & 2.6008 & \\ \text { S12C17 } & 105.3617 & 0.3659 & 108.3717 & 2.6441 & \\ \text { S12C18 } & 11.5792 & 0.3874 & 114.7668 & 2.8002 & \\ \text { S12C19 } & 113.8164 & 0.5053 & 117.9518 & 3.6302 & \\ \text { S12C20 } & 101.9324 & 0.4526 & 105.6361 & 3.2511 & \\ \text { S12C21 } & 93.8053 & 0.4165 & 97.2137 & 2.9919 & \\ \text { S12C22 } & 90.4534 & 0.4016 & 93.7400 & 2.8850 & \\ \text { S12C23 } & 92.3977 & 0.4102 & 95.7550 & 2.9470 & \\ \text { S12C24 } & 99.3279 & 0.4410 & 102.9369 & 3.1680 & \\ \text { S12R01 } & 10.3978 & 0.3899 & 320.7275 & 309.9398 & \\ \text { S12R02 } & 9.1870 & 0.3524 & 314.7841 & 305.2447 & \\ \text { S12R03 } & 9.6714 & 0.3678 & 317.3095 & 307.2704 & \\ \text { S12R04 } & 12.1994 & 0.4405 & 327.7374 & 315.0975 & \\ \text { S12R05 } & 18.8043 & 0.5726 & 343.8673 & 324.4904 & \\ \text { S12R06 } & 33.9992 & 0.6444 & 362.6769 & 328.0333 & \\ \text { S12R07 } & 57.9278 & 0.5420 & 381.1277 & 322.6579 & \\ \text { S12R08 } & 81.8507 & 0.4334 & 396.6439 & 314.3598 & \\ \text { S12R09 } & 99.0236 & 0.3737 & 407.3106 & 307.9133 & \\ \text { S12R10 } & 106.5098 & 0.3519 & 411.9210 & 305.0594 & \\ \text { S12R11 } & 103.3541 & 0.3608 & 409.9791 & 306.2642 & \\ \text { S12R12 } & 89.9403 & 0.4034 & 401.6916 & 311.3479 & \\ \text { S12R13 } & 68.2405 & 0.4917 & 387.9734 & 319.2412 & \\ \text { S12R14 } & 43.1819 & 0.6148 & 370.4503 & 326.6535 & \\ & & & & & \\ & & \end{array}$




\section{REVISED METHODOLOGY FOR DETERMINING CESIUM-137 CONTENT OF HN-200 GROUT CONTAINERS.}

$\begin{array}{lllll}\text { S12R15 } & 23.7326 & 0.6233 & 351.4396 & 327.0838 \\ \text { S12R16 } & 14.2076 & 0.4894 & 333.8286 & 319.1316\end{array}$

$\begin{array}{lcccccr} & \text { Cs-137 } & \text { Ba-137m } & \text { Source 13 } & \text { X Posit } & \text { Z Posit } & \text { Y Posit } \\ & 17.9331 & 16.9647 & & 8.9211 & 21.0922 & 105.2762 \\ \text { Line } & \text { Shield 1 } & \text { Shield 2 } & \text { Total Dist } & \text { Air Gap } & & \\ \text { S13C01 } & 56.2858 & 0.4066 & 59.6220 & 2.9296 & \\ \text { S13C02 } & 52.9188 & 0.3823 & 56.0555 & 2.7544 & \\ \text { S13C03 } & 58.0015 & 0.4190 & 61.4395 & 3.0189 & \\ \text { S13C04 } & 69.7095 & 0.5036 & 73.8415 & 3.6283 & \\ \text { S13C05 } & 85.3588 & 0.6167 & 90.4183 & 4.4429 & \\ \text { S13C06 } & 103.1712 & 0.7454 & 109.2865 & 5.3700 & \\ \text { S13C07 } & 42.2290 & 0.4036 & 45.5494 & 2.9168 & \\ \text { S13C08 } & 37.7978 & 0.3613 & 40.7699 & 2.6107 & \\ \text { S13C09 } & 44.4117 & 0.4245 & 47.9038 & 3.0676 & \\ \text { S13C10 } & 58.4334 & 0.5585 & 63.0280 & 4.0361 & \\ \text { S13C11 } & 75.8608 & 0.7251 & 81.8257 & 5.2398 & \\ \text { S13C12 } & 94.8345 & 0.9064 & 102.2913 & 6.5503 & \\ \text { S13C13 } & 72.5157 & 0.3826 & 75.6601 & 2.7618 & \\ \text { S13C14 } & 69.8537 & 0.3686 & 72.8827 & 2.6604 & \\ \text { S13C15 } & 73.8961 & 0.3899 & 77.1004 & 2.8144 & \\ \text { S13C16 } & 83.6770 & 0.4415 & 87.3054 & 3.1869 & \\ \text { S13C17 } & 97.4839 & 0.5143 & 101.7111 & 3.7128 & \\ \text { S13C18 } & 113.8617 & 0.6007 & 118.7990 & 4.3365 & \\ \text { S13C19 } & 81.8819 & 0.3640 & 84.8793 & 2.6333 & \\ \text { S13C20 } & 79.5029 & 0.3535 & 82.4131 & 2.5568 & \\ \text { S13C21 } & 83.1229 & 0.3695 & 86.1656 & 2.6732 & \\ \text { S13C22 } & 92.0368 & 0.4092 & 95.4058 & 2.9599 & \\ \text { S13C23 } & 104.9036 & 0.4664 & 108.7436 & 3.3737 & \\ \text { S13C24 } & 120.4633 & 0.5356 & 124.8729 & 3.8740 & \\ \text { S13R01 } & 46.3401 & 0.3802 & 356.7264 & 310.0061 & \\ \text { S13R02 } & 40.0944 & 0.3688 & 349.1214 & 308.6582 & \\ \text { S13R03 } & 36.4004 & 0.3576 & 343.9787 & 307.2207 & \\ \text { S13R04 } & 35.2196 & 0.3531 & 342.2029 & 306.6302 & \\ \text { S13R05 } & 36.4939 & 0.3579 & 344.1163 & 307.2644 & \\ \text { S13R06 } & 40.2850 & 0.3693 & 349.3719 & 308.7176 & \\ \text { S13R07 } & 46.6242 & 0.3805 & 357.0468 & 310.0420 & \\ \text { S13R08 } & 55.1126 & 0.3838 & 365.8623 & 310.3659 & \\ \text { S13R09 } & 64.5627 & 0.3772 & 374.4454 & 309.5056 & \\ \text { S13R10 } & 73.1644 & 0.3658 & 381.5459 & 308.0157 & \\ \text { S13R11 } & 79.1174 & 0.3562 & 386.1889 & 306.7153 & \\ \text { S13R12 } & 81.1879 & 0.3527 & 387.7631 & 306.2225 & \\ \text { S13R13 } & 78.9572 & 0.3565 & 386.0662 & 306.7526 & \\ \text { S13R14 } & 72.8766 & 0.3662 & 381.3165 & 308.0737 & \\ \text { S13R15 } & 64.2078 & 0.3776 & 374.1401 & 309.5548 & \\ & & & & & \\ & & & & \\ & & \end{array}$




\section{REVISED METHODOLOGY FOR DETERMINING CESIUM-137 CONTENT OF HN-200 GROUT CONTAINERS.}

$\begin{array}{lllll}\text { S13R } 16 & 54.7633 & 0.3839 & 365.5240 & 310.3768\end{array}$

\begin{tabular}{|c|c|c|c|c|c|c|}
\hline & $\begin{array}{c}\text { Cs-137 } \\
26.2138\end{array}$ & $\begin{array}{c}\text { Ba-137m } \\
24.7983\end{array}$ & Source 14 & $\begin{array}{c}\text { X Posit } \\
-46.3340\end{array}$ & $\begin{array}{l}\text { Z Posit } \\
15.5761\end{array}$ & $\begin{array}{c}\text { Y Posit } \\
101.4000\end{array}$ \\
\hline Line & Shield 1 & Shield 2 & Total Dist & Air Gap & & \\
\hline$S 14 C 01$ & 107.9659 & 0.3639 & 110.9618 & 2.6319 & & \\
\hline S14C02 & 105.3484 & 0.3551 & 108.2716 & 2.5681 & & \\
\hline $\mathrm{S} 14 \mathrm{C} 03$ & 107.3750 & 0.3619 & 110.3545 & 2.6175 & & \\
\hline S14C04 & 113.7980 & 0.3836 & 116.9557 & 2.7741 & & \\
\hline S14C05 & 123.9358 & 0.4178 & 127.3748 & 3.0212 & & \\
\hline S14C06 & 136.9659 & 0.4617 & 140.7664 & 3.3389 & & \\
\hline S14C07 & 64.6233 & 0.5660 & 69.1672 & 3.9779 & & \\
\hline S14C08 & 60.5089 & 0.5300 & 64.7636 & 3.7247 & & \\
\hline S14C09 & 63.7090 & 0.5580 & 68.1887 & 3.9216 & & \\
\hline S14C10 & 73.2713 & 0.6418 & 78.4234 & 4.5103 & & \\
\hline S14C11 & 87.1257 & 0.7631 & 93.2519 & 5.3631 & & \\
\hline $\mathrm{S} 14 \mathrm{C} 12$ & 103.5636 & 0.9071 & 110.8457 & 6.3749 & & \\
\hline $\mathrm{S} 14 \mathrm{C} 13$ & 25.6539 & 0.8314 & 32.3089 & 5.8236 & & \\
\hline S14C14 & 16.9198 & 0.5483 & 21.3090 & 3.8409 & & \\
\hline S14C15 & 23.9453 & 0.7760 & 30.1571 & 5.4357 & & \\
\hline S14C16 & 38.9796 & 1.2633 & 49.0915 & 8.8486 & & \\
\hline $\mathrm{S} 14 \mathrm{C} 17$ & 55.8962 & 1.8115 & 70.3965 & 12.6888 & & \\
\hline S14C18 & 73.4050 & 2.3790 & 92.4474 & 16.6634 & & \\
\hline S14C19 & 89.0492 & 0.4332 & 92.5866 & 3.1042 & & \\
\hline $\mathrm{S} 14 \mathrm{C} 20$ & 85.9313 & 0.4181 & 89.3448 & 2.9955 & & \\
\hline$S 14 C 21$ & 88.3483 & 0.4298 & 91.8579 & 3.0797 & & \\
\hline $\mathrm{S} 14 \mathrm{C} 22$ & 95.8827 & 0.4665 & 99.6915 & 3.3424 & & \\
\hline $\mathrm{S} 14 \mathrm{C} 23$ & 107.4634 & 0.5228 & 111.7322 & 3.7461 & & \\
\hline $\mathrm{S} 14 \mathrm{C} 24$ & 121.9429 & 0.5933 & 126.7870 & 4.2508 & & \\
\hline S14R01 & 103.4771 & 0.3626 & 411.0551 & 307.2154 & & \\
\hline S14R02 & 89.6962 & 0.4069 & 402.5879 & 312.4849 & & \\
\hline S14R03 & 67.6682 & 0.4979 & 388.7349 & 320.5687 & & \\
\hline S14R04 & 42.4629 & 0.6231 & 371.1441 & 328.0581 & & \\
\hline S14R05 & 23.2074 & 0.6258 & 352.1534 & 328.3202 & & \\
\hline S14R06 & 13.9312 & 0.4885 & 334.6608 & 320.2411 & & \\
\hline S14R07 & 10.2485 & 0.3899 & 321.7681 & 311.1297 & & \\
\hline S14R08 & 9.1032 & 0.3538 & 316.0866 & 306.6297 & & \\
\hline S14R09 & 9.6362 & 0.3709 & 318.8699 & 308.8628 & & \\
\hline S14R10 & 12.2346 & 0.4464 & 329.4953 & 316.8143 & & \\
\hline S14R11 & 19.0180 & 0.5816 & 345.7306 & 326.1310 & & \\
\hline S14R12 & 34.6063 & 0.6492 & 364.5494 & 329.2939 & & \\
\hline S14R13 & 58.8486 & 0.5414 & 382.9260 & 323.5359 & & \\
\hline S14R14 & 82.7871 & 0.4325 & 398.3044 & 315.0847 & & \\
\hline S14R15 & 99.7811 & 0.3736 & 408.7906 & 308.6359 & & \\
\hline S14R 16 & 106.9797 & 0.3526 & 413.1990 & 305.8667 & & \\
\hline
\end{tabular}




\section{REVISED METHODOLOGY FOR DETERMINING CESIUM-137 CONTENT OF HN-200 GROUT CONTAINERS.}

\begin{tabular}{|c|c|c|c|c|c|c|}
\hline & $\begin{array}{c}\text { Cs-137 } \\
22.7469\end{array}$ & $\begin{array}{c}\text { Ba- } 137 \mathrm{~m} \\
21.5186\end{array}$ & Source 15 & $\begin{array}{l}\text { X Posit } \\
53.3465\end{array}$ & Z Posit & Y Posit \\
\hline Line & Shield 1 & Shicld 2 & Total Dist & Air Gap & & \\
\hline S15C01 & 57.0753 & 5.7184 & 99.7693 & 36.9757 & & \\
\hline $\mathrm{S} 15 \mathrm{C} 02$ & 44.2066 & 4.4291 & 77.2745 & 28.6388 & & \\
\hline $\mathrm{S} 15 \mathrm{CO} 3$ & 31.5105 & 3.1570 & 55.0813 & 20.4138 & & \\
\hline S15C04 & 19.3298 & 1.9367 & 33.7890 & 12.5226 & & \\
\hline $\mathrm{S} 15 \mathrm{CO5}$ & 9.8192 & 0.9838 & 17.1642 & 6.3613 & & \\
\hline S15C06 & 12.6980 & 1.2722 & 22.1965 & 8.2263 & & \\
\hline S15C07 & 113.8132 & 0.9679 & 121.5349 & 6.7537 & & \\
\hline S15C08 & 97.2670 & 0.8272 & 103.8661 & 5.7719 & & \\
\hline S15C09 & 82.9751 & 0.7056 & 88.6045 & 4.9238 & & \\
\hline S15C10 & 72.2871 & 0.6147 & 77.1914 & 4.2896 & & \\
\hline S15C11 & 66.9519 & 0.5694 & 71.4942 & 3.9730 & & \\
\hline $\mathrm{S} 15 \mathrm{C} 12$ & 68.2368 & 0.5803 & 72.8663 & 4.0492 & & \\
\hline $\mathrm{S} 15 \mathrm{C} 13$ & 147.5837 & 0.4660 & 151.4201 & 3.3704 & & \\
\hline S15C14 & 134.1553 & 0.4236 & 137.6426 & 3.0638 & & \\
\hline S15C15 & 123.3174 & 0.3894 & 126.5231 & 2.8163 & & \\
\hline S15C16 & 115.7999 & 0.3656 & 118.8101 & 2.6446 & & \\
\hline $\mathrm{S} 15 \mathrm{C} 17$ & 112.2716 & 0.3545 & 115.1901 & 2.5640 & & \\
\hline S15C18 & 113.1065 & 0.3571 & 116.0466 & 2.5831 & & \\
\hline S15C19 & 129.2896 & 0.6499 & 134.5780 & 4.6384 & & \\
\hline S15C20 & 114.1932 & 0.5740 & 118.8641 & 4.0968 & & \\
\hline S15C21 & 101.6317 & 0.5109 & 105.7888 & 3.6462 & & \\
\hline $\mathrm{S} 15 \mathrm{C} 22$ & 92.6421 & 0.4657 & 96.4314 & 3.3237 & & \\
\hline $\mathrm{S} 15 \mathrm{C} 23$ & 88.3218 & 0.4440 & 91.9344 & 3.1687 & & \\
\hline S15C24 & 89.3507 & 0.4492 & 93.0054 & 3.2056 & & \\
\hline S15R01 & 2.9712 & 0.3685 & 312.6862 & 309.3466 & & \\
\hline S15R02 & 2.8859 & 0.3585 & 311.2949 & 308.0504 & & \\
\hline S15R03 & 3.4601 & 0.4246 & 319.4775 & 315.5928 & & \\
\hline S15R04 & 5.4159 & 0.6286 & 335.3665 & 329.3220 & & \\
\hline S15R05 & 13,2604 & 1.0675 & 355.7179 & 341.3900 & & \\
\hline S15R06 & 39.5149 & 0.8501 & 376.9854 & 336.6204 & & \\
\hline S15R07 & 71.8786 & 0.5360 & 396.0392 & 323.6246 & & \\
\hline S15R08 & 96.9230 & 0.4092 & 410.4625 & 313.1304 & & \\
\hline S15R09 & 110.9571 & 0.3604 & 418.6226 & 307.3050 & & \\
\hline S15R 10 & 112.7280 & 0.3551 & 419.6581 & 306.5750 & & \\
\hline S15R11 & 102.0992 & 0.3898 & 413.4632 & 310.9742 & & \\
\hline S15R12 & 79.9572 & 0.4878 & 400.6828 & 320.2379 & & \\
\hline S15R 13 & 48.9854 & 0.7325 & 382.7301 & 333.0121 & & \\
\hline S15R14 & 18.6323 & 1.1136 & 361.8002 & 342.0544 & & \\
\hline S15R15 & 6.5970 & 0.7335 & 340.8381 & 333.5076 & & \\
\hline S15R16 & 3.8051 & 0.4631 & 323.3233 & 319.0550 & & \\
\hline
\end{tabular}




\section{REVISED METHODOLOGY FOR DETERMINING CESIUM-137 CONTENT OF HN-200 GROUT CONTAINERS.}

\begin{tabular}{|c|c|c|c|c|c|c|}
\hline & $\begin{array}{c}\text { Cs-137 } \\
27.6556\end{array}$ & $\begin{array}{c}\text { Ba-137m } \\
26.1622\end{array}$ & Source 16 & $\begin{array}{l}\text { X Posit } \\
32.4610\end{array}$ & $\begin{array}{l}\text { Z Posit } \\
26.2430\end{array}$ & $\begin{array}{r}\text { Y Posit } \\
99.8427\end{array}$ \\
\hline Line & Shield 1 & Shield 2 & Total Dist & Air Gap & & \\
\hline S16C01 & 41.6481 & 0.6011 & 46.4975 & 4.2483 & & \\
\hline S16C02 & 34.7024 & 0.5009 & 38.7431 & 3.5398 & & \\
\hline $\mathrm{S} 16 \mathrm{C} 03$ & 38.8905 & 0.5613 & 43.4189 & 3.9670 & & \\
\hline S16C04 & 51.5675 & 0.7443 & 57.5719 & 5.2602 & & \\
\hline S16C05 & 68.1502 & 0.9836 & 76.0856 & 6.9517 & & \\
\hline S16C06 & 86.4189 & 1.2473 & 96.4814 & 8.8152 & & \\
\hline S16C07 & 49.3965 & 0.5735 & 54.0199 & 4.0499 & & \\
\hline S16C08 & 43.4434 & 0.5043 & 47.5095 & 3.5618 & & \\
\hline S16C09 & 46.9952 & 0.5456 & 51.3938 & 3.8530 & & \\
\hline S16C10 & 58.3411 & 0.6773 & 63.8017 & 4.7832 & & \\
\hline S16C11 & 73.9780 & 0.8588 & 80.9021 & 6.0653 & & \\
\hline $\mathrm{S} 16 \mathrm{C} 12$ & 91.7371 & 1.0650 & 100.3234 & 7.5213 & & \\
\hline $\mathrm{S} 16 \mathrm{C} 13$ & 97.1683 & 0.3789 & 100.2831 & 2.7359 & & \\
\hline S16C14 & 93.9207 & 0.3662 & 96.9315 & 2.6445 & & \\
\hline S16C15 & 95.8216 & 0.3736 & 98.8933 & 2.6980 & & \\
\hline S16C16 & 102.5851 & 0.4000 & 105.8735 & 2.8885 & & \\
\hline S16C17 & 113.3440 & 0.4420 & 116.9773 & 3.1914 & & \\
\hline S16C18 & 127.0876 & 0.4956 & 131.1615 & 3.5784 & & \\
\hline S16C19 & 93.2252 & 0.3915 & 96.4395 & 2.8227 & & \\
\hline S16C20 & 89.8514 & 0.3774 & 92.9494 & 2.7206 & & \\
\hline S16C21 & 91.8274 & 0.3857 & 94.9934 & 2.7804 & & \\
\hline S16C22 & 98.8327 & 0.4151 & 102.2403 & 2.9925 & & \\
\hline S16C23 & 109.9099 & 0.4616 & 113.6995 & 3.3279 & & \\
\hline S16C24 & 123.9723 & 0.5207 & 128.2467 & 3.7537 & & \\
\hline S16R01 & 20.0911 & 0.4058 & 333.1706 & 312.6738 & & \\
\hline S16R02 & 16.8751 & 0.3623 & 324.8147 & 307.5772 & & \\
\hline S16R03 & 16.3308 & 0.3540 & 323.1595 & 306.4746 & & \\
\hline S16R04 & 18.2115 & 0.3815 & 328.5545 & 309.9615 & & \\
\hline S16R05 & 23.3612 & 0.4406 & 339.8790 & 316.0772 & & \\
\hline S16R06 & 33.7054 & 0.4990 & 354.9483 & 320.7440 & & \\
\hline S16R07 & 50.3573 & 0.4945 & 371.1685 & 320.3167 & & \\
\hline S16R08 & 70.0279 & 0.4385 & 386.0796 & 315.6131 & & \\
\hline S16R09 & 87.0941 & 0.3868 & 397.6732 & 310.1923 & & \\
\hline S16R10 & 97.5897 & 0.3582 & 404.5269 & 306.5790 & & \\
\hline S16R11 & 99.6392 & 0.3529 & 405.8507 & 305.8585 & & \\
\hline S16R12 & 92.9206 & 0.3706 & 401.4953 & 308.2042 & & \\
\hline S16R13 & 78.5416 & 0.4121 & 391.9553 & 313.0016 & & \\
\hline S16R14 & 59.4321 & 0.4710 & 378.3626 & 318.4595 & & \\
\hline S16R15 & 40.7041 & 0.5067 & 362.4649 & 321.2540 & & \\
\hline S16R16 & 27.3662 & 0.4716 & 346.5387 & 318.7010 & & \\
\hline
\end{tabular}




\section{REVISED METHODOLOGY FOR DETERMINING CESIUM-137 CONTENT OF HN-200 GROUT CONTAINERS.}

\begin{tabular}{|c|c|c|c|c|c|c|}
\hline & $\begin{array}{c}\text { Cs-137 } \\
28.5227\end{array}$ & $\begin{array}{c}\text { Ba-137m } \\
26.9825\end{array}$ & Source 17 & $\begin{array}{c}\text { X Posit } \\
-36.8053\end{array}$ & $\begin{array}{l}\text { Z Posit } \\
7.8697\end{array}$ & $\begin{array}{l}\text { Y Posit } \\
22.0830\end{array}$ \\
\hline Line & Shield 1 & Shield 2 & Total Dist & Air Gap & & \\
\hline S17C01 & 138.3651 & 0.5130 & 142.5892 & 3.7111 & & \\
\hline $\mathrm{S} 17 \mathrm{C} 02$ & 123.1862 & 0.4567 & 126.9469 & 3.3040 & & \\
\hline $\mathrm{S} 17 \mathrm{C}^{\circ} 3^{\circ}$ & 110.4035 & 0.4093 & 113.7739 & 2.9611 & & \\
\hline S17C04 & 100.9315 & 0.3742 & 104.0128 & 2.7071 & & \\
\hline $\mathrm{S} 17 \mathrm{C05}$ & 95.7579 & 0.3550 & 98.6812 & 2.5683 & & \\
\hline S17C06 & 95.5830 & 0.3544 & 98.5010 & 2.5636 & & \\
\hline S17C07 & 115.3125 & 0.8289 & 122.0618 & 5.9204 & & \\
\hline S17C08 & 97.6431 & 0.7019 & 103.3582 & 5.0132 & & \\
\hline S17C09 & 81.8789 & 0.5886 & 86.6713 & 4.2038 & & \\
\hline S17C10 & 69.3319 & 0.4984 & 73.3899 & 3.5597 & & \\
\hline S17C11 & 61.9874 & 0.4456 & 65.6155 & 3.1826 & & \\
\hline $\mathrm{S} 17 \mathrm{C} 12$ & 61.7311 & 0.4437 & 65.3442 & 3.1694 & & \\
\hline S17C13 & 93.7918 & 1.5687 & 106.6791 & 11.3187 & & \\
\hline S17C14 & 74.4169 & 1.2446 & 84.6420 & 8.9806 & & \\
\hline S17C15 & 55.5584 & 0.9292 & 63.1924 & 6.7047 & & \\
\hline S17C16 & 37.9933 & 0.6354 & 43.2138 & 4.5850 & & \\
\hline S17C17 & 24.6606 & 0.4124 & 28.0490 & 2.9760 & & \\
\hline S17C18 & 24.0974 & 0.4030 & 27.4085 & 2.9081 & & \\
\hline S17C19 & 124.1665 & 0.6721 & 129.6650 & 4.8265 & & \\
\hline S17C20 & 107.4763 & 0.5818 & 112.2357 & 4.1777 & & \\
\hline S17C21 & 92.9698 & 0.5032 & 97.0869 & 3.6138 & & \\
\hline S17C22 & 81.8170 & 0.4429 & 85.4401 & 3.1803 & & \\
\hline $\mathrm{S} 17 \mathrm{C} 23$ & 75.5185 & 0.4088 & 78.8628 & 2.9355 & & \\
\hline S17C24 & 75.3025 & 0.4076 & 78.6372 & 2.9271 & & \\
\hline S17R01 & 95.0397 & 0.3560 & 402.5649 & 307.1692 & & \\
\hline S17R02 & 87.1606 & 0.3755 & 397.2875 & 309.7513 & & \\
\hline S17R03 & 73.1509 & 0.4119 & 387.5439 & 313.9812 & & \\
\hline S17R04 & 56.1276 & 0.4519 & 374.5413 & 317.9618 & & \\
\hline S17R05 & 40.4919 & 0.4627 & 360.0264 & 319.0718 & & \\
\hline S17R06 & 29.5213 & 0.4291 & 346.1599 & 316.2095 & & \\
\hline S17R07 & 23.3663 & 0.3840 & 335.2540 & 311.5037 & & \\
\hline S17R08 & 20.7486 & 0.3575 & 329.3261 & 308.2200 & & \\
\hline S17R09 & 20.8426 & 0.3586 & 329.5576 & 308.3564 & & \\
\hline S17R10 & 23.6800 & 0.3868 & 335.9007 & 311.8338 & & \\
\hline S17R11 & 30.1442 & 0.4324 & 347.0973 & 316.5206 & & \\
\hline S17R 12 & 41.4944 & 0.4636 & 361.0892 & 319.1311 & & \\
\hline S17R13 & 57.3753 & 0.4495 & 375.5632 & 317.7383 & & \\
\hline S17R14 & 74.3206 & 0.4088 & 388.3812 & 313.6518 & & \\
\hline S17R15 & 87.9679 & 0.3735 & 397.8336 & 309.4922 & & \\
\hline S17R16 & 95.3252 & 0.3553 & 402.7541 & 307.0736 & & \\
\hline
\end{tabular}




\section{REVISED METHODOLOGY FOR DETERMINING CESIUM-137 CONTENT OF HN-200 GROUT CONTAINERS.}

\begin{tabular}{|c|c|c|c|c|c|c|}
\hline & $\begin{array}{c}\text { Cs-137 } \\
23.2528\end{array}$ & $\begin{array}{c}\text { Ba-137m } \\
21.9972\end{array}$ & Source 18 & $\begin{array}{l}\text { X Posit } \\
47.6621\end{array}$ & $\begin{array}{c}\text { Z Posit } \\
-30.4883\end{array}$ & $\begin{array}{l}\text { Y Posit } \\
26.7230\end{array}$ \\
\hline Line & Shield 1 & Shield 2 & Total Dist & Air Gap & & \\
\hline S18C01 & 77.0407 & 4.0097 & 104.4249 & 23.3745 & & \\
\hline $\mathrm{S} 18 \mathrm{C} 02$ & 61.2855 & 3.1897 & 83.0695 & 18.5943 & & \\
\hline S18C03 & 46.3187 & 2.4107 & 62.7828 & 14.0533 & & \\
\hline S18C04 & 33.2237 & 1.7292 & 45.0331 & 10.0802 & & \\
\hline S $18 \mathrm{C} 05$ & 25.1211 & 1.3075 & 34.0505 & 7.6219 & & \\
\hline S18C06 & 26.9667 & 1.4035 & 36.5520 & 8.1818 & & \\
\hline S18C07 & 138.3008 & 0.5566 & 142.8560 & 3.9987 & & \\
\hline S18C08 & 123.9934 & 0.4990 & 128.0774 & 3.5850 & & \\
\hline S18C09 & 112.2550 & 0.4518 & 115.9524 & 3.2456 & & \\
\hline S18C10 & 103.9595 & 0.4184 & 107.3837 & 3.0058 & & \\
\hline S18C11 & 99.9677 & 0.4023 & 103.2604 & 2.8904 & & \\
\hline $\mathrm{S} 18 \mathrm{C} 12$ & 100.7923 & 0.4057 & 104.1121 & 2.9142 & & \\
\hline S18C13 & 145.9816 & 0.4873 & 149.9874 & 3.5185 & & \\
\hline S18C14 & 132.3542 & 0.4418 & 135.9860 & 3.1900 & & \\
\hline S18C15 & 121.3042 & 0.4049 & 124.6328 & 2.9237 & & \\
\hline S18C16 & 113.5864 & 0.3791 & 116.7032 & 2.7377 & & \\
\hline S18C17 & 109.9049 & 0.3668 & 112.9207 & 2.6490 & & \\
\hline S18C18 & 110.6634 & 0.3694 & 113.7001 & 2.6672 & & \\
\hline S18C19 & 102.3446 & 1.5108 & 113.9866 & 10.1311 & & \\
\hline S18C20 & 85.1265 & 1.2566 & 94.8099 & 8.4267 & & \\
\hline S18C21 & 69.7223 & 1.0292 & 77.6534 & 6.9018 & & \\
\hline S18C22 & 57.6060 & 0.8504 & 64.1588 & 5.7024 & & \\
\hline S18C23 & 51.1689 & 0.7554 & 56.9895 & 5.0652 & & \\
\hline S18C24 & 52.5418 & 0.7756 & 58.5186 & 5.2011 & & \\
\hline S18R01 & 1.6943 & 0.4431 & 319.5394 & 317.4020 & & \\
\hline S18R02 & 2.8419 & 0.7139 & 336.4695 & 332.9137 & & \\
\hline S18R03 & 9.4675 & 1.5142 & 357.6258 & 346.6441 & & \\
\hline S18R04 & 40.1484 & 0.9189 & 379.3717 & 338.3044 & & \\
\hline S18R05 & 74.4522 & 0.5349 & 398.5627 & 323.5756 & & \\
\hline S18R06 & 99.6612 & 0.4054 & 412.8087 & 312.7421 & & \\
\hline S18R07 & 113.0783 & 0.3587 & 420.5235 & 307.0866 & & \\
\hline S18R08 & 113.7351 & 0.3567 & 420.9037 & 306.8119 & & \\
\hline S18R09 & 101.5891 & 0.3979 & 413.9111 & 311.9241 & & \\
\hline S18R10 & 77.4992 & 0.5152 & 400.2699 & 322.2555 & & \\
\hline S18R11 & 43.8821 & 0.8551 & 381.4863 & 336.7491 & & \\
\hline S18R12 & 11.3424 & 1.5585 & 359.8679 & 346.9670 & & \\
\hline S18R13 & 3.0772 & 0.7650 & 338.4903 & 334.6480 & & \\
\hline S18R14 & 1.7562 & 0.4585 & 320.9622 & 318.7474 & & \\
\hline S18R15 & 1.3869 & 0.3654 & 310.7861 & 309.0337 & & \\
\hline S18R16 & 1.3720 & 0.3616 & 310.2706 & 308.5371 & & \\
\hline
\end{tabular}


HNF-4799, Rev 0

August 25, 1999

\section{REVISED METHODOLOGY FOR DETERMINING CESIUM-137 CONTENT OF HN-200 GROUT CONTAINERS.}

$\begin{array}{ccccccc} & \text { Cs-137 } & \text { Ba-137m } & \text { Source 19 } & \text { X Posit } & \text { Z Posit } & \text { Y Posit } \\ \text { Line } & 15.3416 & 14.5131 & & -46.3763 & 0.7074 & \text { 53.5943 } \\ \text { S19C01 } & 125.7091 & \text { Shield 2 } & \text { Total Dist } & \text { Air Gap } & & \\ \text { S19C02 } & 114.7825 & 0.3864 & 129.1940 & 3.0618 & \\ \text { S19C03 } & 107.4095 & 0.3615 & 110.3875 & 2.7956 & \\ \text { S19C04 } & 104.3462 & 0.3512 & 107.2389 & 2.6161 & \\ \text { S19C05 } & 105.9669 & 0.3567 & 108.9045 & 2.5809 & \\ \text { S19C06 } & 112.0686 & 0.3772 & 115.1754 & 2.7295 & \\ \text { S19C07 } & 99.6018 & 0.6299 & 104.7168 & 4.4851 & \\ \text { S19C08 } & 86.0788 & 0.5444 & 90.4994 & 3.8762 & \\ \text { S19C09 } & 76.4472 & 0.4835 & 80.3731 & 3.4424 & \\ \text { S19C10 } & 72.2795 & 0.4571 & 75.9913 & 3.2548 & \\ \text { S19C11 } & 74.4985 & 0.4711 & 78.3243 & 3.3547 & \\ \text { S19C12 } & 82.5910 & 0.5223 & 86.8324 & 3.7191 & \\ \text { S19C13 } & 58.8221 & 1.7905 & 73.5672 & 12.9547 & \\ \text { S19C14 } & 41.0567 & 1.2497 & 51.3486 & 9.0421 & \\ \text { S19C15 } & 24.0718 & 0.7327 & 30.1060 & 5.3015 & \\ \text { S19C16 } & 11.8924 & 0.3620 & 14.8735 & 2.6191 & \\ \text { S19C17 } & 19.2766 & 0.5868 & 24.1088 & 4.2454 & \\ \text { S19C18 } & 35.6361 & 1.0847 & 44.5691 & 7.8483 & \\ \text { S19C19 } & 100.5021 & 0.6194 & 105.5350 & 4.4135 & \\ \text { S19C20 } & 87.0840 & 0.5367 & 91.4449 & 3.8242 & \\ \text { S19C21 } & 77.5526 & 0.4779 & 81.4362 & 3.4057 & \\ \text { S19C22 } & 73.4374 & 0.4526 & 77.1150 & 3.2249 & \\ \text { S19C23 } & 75.6277 & 0.4661 & 79.4149 & 3.3211 & \\ \text { S19C24 } & 83.6295 & 0.5154 & 87.8174 & 3.6725 & \\ \text { S19R01 } & 104.3483 & 0.3513 & 409.6896 & 304.9900 & \\ \text { S19R02 } & 98.9512 & 0.3662 & 406.3073 & 306.9898 & \\ \text { S19R03 } & 84.2763 & 0.4126 & 397.0130 & 312.3241 & \\ \text { S19R04 } & 62.8700 & 0.4949 & 382.8615 & 319.4966 & \\ \text { S19R05 } & 40.2772 & 0.5773 & 365.5914 & 324.7369 & \\ \text { S19R06 } & 24.0244 & 0.5468 & 347.5763 & 323.0051 & \\ \text { S19R07 } & 15.8704 & 0.4431 & 331.6561 & 315.3427 & \\ \text { S19R08 } & 12.4437 & 0.3727 & 320.7219 & 307.9054 & \\ \text { S19R09 } & 11.5417 & 0.3514 & 317.0107 & 305.1175 & \\ \text { S19R10 } & 12.6034 & 0.3764 & 321.3341 & 308.3543 & \\ \text { S19R11 } & 16.2917 & 0.4505 & 332.7494 & 316.0072 & \\ \text { S19R12 } & 24.9638 & 0.5538 & 348.9389 & 323.4212 & \\ \text { S19R13 } & 41.8896 & 0.5735 & 366.9936 & 324.5304 & \\ \text { S19R14 } & 64.6699 & 0.4875 & 384.0989 & 318.9415 & \\ \text { S19R15 } & 85.7048 & 0.4077 & 397.9267 & 311.8143 & \\ \text { S19R16 } & 99.7214 & 0.3640 & 406.7908 & 306.7053 & \\ & & & & & \\ & & & \end{array}$

$\begin{array}{llllll}\text { Cs-137 } & \text { Ba-137m } & \text { Source } 20 & \text { X Posit } & \text { Z Posit } & \text { Y Posit }\end{array}$ 
REVISED METHODOLOGY FOR DETERMINING CESIUM-137 CONTENT OF HN-200 GROUT CONTAINERS.

\begin{tabular}{lcccc} 
Line & 16.4988 & 15.6078 & & 7.2755 \\
S20C01 & 56.5757 & Shield 2 & Total Dist & Air Gap \\
S20C02 & 56.0683 & 0.3960 & 59.8224 & 2.8508 \\
S20C03 & 63.4136 & 0.4438 & 59.2859 & 2.8252 \\
S20C04 & 76.3788 & 0.5346 & 80.7619 & 3.8486 \\
S20C05 & 92.6333 & 0.6483 & 97.9493 & 4.6677 \\
S20C06 & 110.7382 & 0.7751 & 117.0931 & 5.5799 \\
S20C07 & 37.0608 & 0.3781 & 40.1718 & 2.7329 \\
S20C08 & 36.3196 & 0.3705 & 39.3684 & 2.6783 \\
S20C09 & 46.4142 & 0.4735 & 50.3103 & 3.4226 \\
S20C10 & 62.2815 & 0.6353 & 67.5096 & 4.5927 \\
S20C11 & 80.5807 & 0.8220 & 87.3448 & 5.9421 \\
S20C12 & 99.9853 & 1.0200 & 108.3783 & 7.3730 \\
S20C13 & 70.0162 & 0.3792 & 73.1311 & 2.7357 \\
S20C14 & 69.5966 & 0.3770 & 72.6929 & 2.7193 \\
S20C15 & 75.7835 & 0.4105 & 79.1550 & 2.9610 \\
S20C16 & 87.1814 & 0.4722 & 91.0600 & 3.4063 \\
S20C17 & 102.0592 & 0.5528 & 106.5997 & 3.9877 \\
S20C18 & 119.1201 & 0.6452 & 124.4196 & 4.6543 \\
S20C19 & 82.5811 & 0.3565 & 85.5167 & 2.5791 \\
S20C20 & 82.2195 & 0.3549 & 85.1422 & 2.5678 \\
S20C21 & 87.6076 & 0.3782 & 90.7219 & 2.7360 \\
S20C22 & 97.7997 & 0.4222 & 101.2762 & 3.0543 \\
S20C23 & 111.4859 & 0.4813 & 115.4489 & 3.4818 \\
S20C24 & 127.5463 & 0.5506 & 132.0802 & 3.9833 \\
S20R01 & 47.4186 & 0.3881 & 359.3798 & 311.5732 \\
S20R02 & 40.1481 & 0.3762 & 350.7765 & 310.2522 \\
S20R03 & 35.6060 & 0.3621 & 344.4837 & 308.5156 \\
S20R04 & 33.7343 & 0.3544 & 341.6052 & 307.5165 \\
S20R05 & 34.4102 & 0.3573 & 342.6669 & 307.8994 \\
S20R06 & 37.6848 & 0.3693 & 347.4730 & 309.4190 \\
S20R07 & 43.6975 & 0.3835 & 355.1621 & 311.0812 \\
S20R08 & 52.2671 & 0.3902 & 364.4251 & 311.7677 \\
S20R09 & 62.3716 & 0.3848 & 373.7909 & 311.0344 \\
S20R10 & 72.1317 & 0.3719 & 381.8759 & 309.3723 \\
S20R11 & 79.4799 & 0.3597 & 387.5624 & 307.7228 \\
S20R12 & 82.8714 & 0.3536 & 390.1015 & 306.8765 \\
S20R13 & 81.6191 & 0.3559 & 389.1695 & 307.1944 \\
S20R14 & 75.9744 & 0.3657 & 384.8842 & 308.5441 \\
S20R15 & 67.1024 & 0.3792 & 377.8008 & 310.3192 \\
S20R16 & 56.8935 & 0.3891 & 368.8736 & 311.5909 \\
& & & & \\
\hline
\end{tabular}

$\begin{array}{cccccc}\text { Cs-137 } & \text { Ba-137m } & \text { Source 21 } & \text { X Posit } & \text { Z Posit } & \text { Y Posit } \\ 28.6466 & 27.0997 & & 7.7777 & -20.1009 & 50.5073\end{array}$ 


\section{REVISED METHODOLOGY FOR DETERMINING CESIUM-137 CONTENT} OF HN-200 GROUT CONTAINERS.

$\begin{array}{lcccc}\text { Line } & \text { Shield 1 } & \text { Shield 2 } & \text { Total Dist } & \text { Air Gap } \\ \text { S21C01 } & 89.0471 & 0.6280 & 94.2019 & 4.5268 \\ \text { S21C02 } & 72.9606 & 0.5145 & 77.1841 & 3.7090 \\ \text { S21C03 } & 60.4236 & 0.4261 & 63.9214 & 3.0717 \\ \text { S21C04 } & 53.9693 & 0.3806 & 57.0935 & 2.7436 \\ \text { S21C05 } & 55.7518 & 0.3932 & 58.9792 & 2.8342 \\ \text { S21C06 } & 65.0980 & 0.4591 & 68.8664 & 3.3093 \\ \text { S21C07 } & 106.7851 & 0.4807 & 110.7434 & 3.4776 \\ \text { S21C08 } & 93.2267 & 0.4197 & 96.6825 & 3.0361 \\ \text { S21C09 } & 83.3737 & 0.3753 & 86.4642 & 2.7152 \\ \text { S21C10 } & 78.6314 & 0.3540 & 81.5461 & 2.5607 \\ \text { S21C11 } & 79.9150 & 0.3598 & 82.8773 & 2.6025 \\ \text { S21C12 } & 86.9580 & 0.3915 & 90.1814 & 2.8319 \\ \text { S21C13 } & 99.3739 & 0.5333 & 103.7573 & 3.8501 \\ \text { S21C14 } & 84.8516 & 0.4554 & 88.5944 & 3.2875 \\ \text { S21C15 } & 74.0482 & 0.3974 & 77.3145 & 2.8689 \\ \text { S21C16 } & 68.7400 & 0.3689 & 71.7722 & 2.6633 \\ \text { S21C17 } & 70.1852 & 0.3766 & 73.2811 & 2.7193 \\ \text { S21C18 } & 78.0093 & 0.4186 & 81.4503 & 3.0224 \\ \text { S21C19 } & 79.7747 & 0.7420 & 85.8805 & 5.3638 \\ \text { S21C20 } & 62.0272 & 0.5769 & 66.7747 & 4.1706 \\ \text { S21C21 } & 47.2502 & 0.4395 & 50.8667 & 3.1770 \\ \text { S21C22 } & 38.9823 & 0.3626 & 41.9659 & 2.6211 \\ \text { S21C23 } & 41.3337 & 0.3844 & 44.4973 & 2.7792 \\ \text { S21C24 } & 52.9071 & 0.4921 & 56.9566 & 3.5573 \\ \text { S21R01 } & 47.5844 & 0.3756 & 356.2787 & 308.3186 \\ \text { S21R02 } & 55.6235 & 0.3782 & 364.6229 & 308.6211 \\ \text { S21R03 } & 64.4422 & 0.3724 & 372.7056 & 307.8909 \\ \text { S21R04 } & 72.3715 & 0.3625 & 379.3487 & 306.6147 \\ \text { S21R05 } & 77.7745 & 0.3543 & 383.6376 & 305.5088 \\ \text { S21R06 } & 79.5377 & 0.3514 & 385.0038 & 305.1147 \\ \text { S21R07 } & 77.3041 & 0.3550 & 383.2706 & 305.6114 \\ \text { S21R08 } & 71.5266 & 0.3637 & 378.6624 & 306.7722 \\ \text { S21R09 } & 63.3984 & 0.3734 & 371.7925 & 308.0207 \\ \text { S21R10 } & 54.5909 & 0.3784 & 363.6127 & 308.6433 \\ \text { S21R11 } & 46.7363 & 0.3748 & 355.3236 & 308.2125 \\ \text { S21R12 } & 40.8963 & 0.3648 & 348.2220 & 306.9610 \\ \text { S21R13 } & 37.4511 & 0.3551 & 343.4914 & 305.6853 \\ \text { S21R14 } & 36.4087 & 0.3515 & 341.9592 & 305.1991 \\ \text { S21R15 } & 37.7358 & 0.3560 & 343.9011 & 305.8094 \\ \text { S21R16 } & 41.4717 & 0.3661 & 348.9682 & 307.1305\end{array}$

$\begin{array}{lcccccc} & \text { Cs-137 } & \text { Ba-137m } & \text { Source 22 } & \text { X Posit } & \text { Z Posit } & \text { Y Posit } \\ \text { Line } & 20.3724 & 19.2723 & & -45.6646 & 32.6567 & 127.1095 \\ & \text { Shield 1 } & \text { Shield 2 } & \text { Total Dist } & \text { Air Gap } & & \end{array}$




\section{REVISED METHODOLOGY FOR DETERMINING CESIUM-137 CONTENT OF HN-200 GROUT CONTAINERS.}

$\begin{array}{lcccc}\text { S22C01 } & 108.3390 & 0.3689 & 111.3703 & 2.6625 \\ \text { S22C02 } & 110.8672 & 0.3775 & 113.9693 & 2.7246 \\ \text { S22C03 } & 117.6213 & 0.4005 & 120.9124 & 2.8906 \\ \text { S22C04 } & 127.9338 & 0.4356 & 131.5134 & 3.1440 \\ \text { S22C05 } & 141.0262 & 0.4802 & 144.9721 & 3.4658 \\ \text { S22C06 } & 156.2009 & 0.5318 & 160.5715 & 3.8387 \\ \text { S22C07 } & 47.7266 & 0.7738 & 53.6589 & 5.1584 \\ \text { S22C08 } & 52.3560 & 0.8489 & 58.8636 & 5.6587 \\ \text { S22C09 } & 63.4926 & 1.0295 & 71.3844 & 6.8624 \\ \text { S22C10 } & 78.4111 & 1.2713 & 88.1573 & 8.4748 \\ \text { S22C11 } & 95.3527 & 1.5460 & 107.2047 & 10.3059 \\ \text { S22C12 } & 113.4144 & 1.8389 & 127.5113 & 12.2581 \\ \text { S22C13 } & 28.0688 & 1.1222 & 36.0206 & 6.8296 \\ \text { S22C14 } & 33.8153 & 1.3519 & 43.3951 & 8.2279 \\ \text { S22C15 } & 46.1924 & 1.8467 & 59.2786 & 11.2394 \\ \text { S22C16 } & 61.3078 & 2.4510 & 78.6760 & 14.9172 \\ \text { S22C17 } & 77.5770 & 3.1014 & 99.5542 & 18.8758 \\ \text { S22C18 } & 94.4054 & 3.7742 & 121.1501 & 22.9705 \\ \text { S22C19 } & 100.7893 & 0.3953 & 104.0267 & 2.8421 \\ \text { S22C20 } & 103.4807 & 0.4058 & 106.8045 & 2.9180 \\ \text { S22C21 } & 110.6307 & 0.4339 & 114.1841 & 3.1196 \\ \text { S22C22 } & 121.4543 & 0.4763 & 125.3554 & 3.4248 \\ \text { S22C23 } & 135.0714 & 0.5297 & 139.4099 & 3.8088 \\ \text { S22C24 } & 150.7266 & 0.5911 & 155.5680 & 4.2503 \\ \text { S22R01 } & 99.3128 & 0.4089 & 414.2750 & 314.5532 \\ \text { S22R02 } & 73.9989 & 0.5392 & 400.0315 & 325.4934 \\ \text { S22R03 } & 40.0796 & 0.9055 & 380.9784 & 339.9932 \\ \text { S22R04 } & 10.8280 & 1.3379 & 359.5092 & 347.3433 \\ \text { S22R05 } & 3.6735 & 0.6890 & 338.7519 & 334.3893 \\ \text { S22R06 } & 2.2441 & 0.4406 & 322.2868 & 319.6021 \\ \text { S22R07 } & 1.8360 & 0.3639 & 313.4516 & 311.2517 \\ \text { S22R08 } & 1.8675 & 0.3699 & 314.2563 & 312.0189 \\ \text { S22R09 } & 2.3736 & 0.4645 & 324.5112 & 321.6731 \\ \text { S22R10 } & 4.1499 & 0.7635 & 341.9152 & 337.0017 \\ \text { S22R11 } & 13.8794 & 1.3868 & 363.0246 & 347.7583 \\ \text { S22R12 } & 45.8786 & 0.8158 & 384.2971 & 337.6027 \\ \text { S22R13 } & 78.7976 & 0.5087 & 402.7138 & 323.4075 \\ \text { S22R14 } & 102.3587 & 0.3973 & 416.0077 & 313.2517 \\ \text { S22R15 } & 114.0510 & 0.3579 & 422.7050 & 308.2961 \\ \text { S22R16 } & 113.0122 & 0.3611 & 422.1068 & 308.7335\end{array}$

$\begin{array}{ccccccc} & \text { Cs-137 } & \text { Ba-137m } & \text { Source 23 } & \text { X Posit } & \text { Z Posit } & \text { Y Posit } \\ & 13.0146 & 12.3118 & & 12.9519 & 37.1270 & 132.0896 \\ \text { Line } & \text { Shield 1 } & \text { Shield 2 } & \text { Total Dist } & \text { Air Gap } & & \\ \text { S23C01 } & 57.1604 & 0.4602 & 60.8968 & 3.2762 & & .\end{array}$


REVISED METHODOLOGY FOR DETERMINING CESIUM-137 CONTENT OF HN-200 GROUT CONTAINERS.

\begin{tabular}{lcccc} 
S23C02 & 63.1182 & 0.5082 & 67.2440 & 3.6177 \\
S23C03 & 74.9756 & 0.6036 & 79.8765 & 4.2973 \\
S23C04 & 90.4412 & 0.7281 & 96.3531 & 5.1837 \\
S23C05 & 107.9756 & 0.8693 & 115.0336 & 6.1887 \\
S23C06 & 126.7229 & 1.0202 & 135.0063 & 7.2632 \\
S23C07 & 24.3135 & 0.4171 & 27.7248 & 2.9943 \\
S23C08 & 34.8804 & 0.5984 & 39.7744 & 4.2956 \\
S23C09 & 51.4386 & 0.8824 & 58.6558 & 6.3348 \\
S23C10 & 69.8498 & 1.1983 & 79.6502 & 8.6022 \\
S23C11 & 88.9710 & 1.5263 & 101.4543 & 10.9570 \\
S23C12 & 108.4273 & 1.8601 & 123.6404 & 13.3530 \\
S23C13 & 79.5483 & 0.3990 & 82.8154 & 2.8680 \\
S23C14 & 84.1331 & 0.4220 & 87.5884 & 3.0333 \\
S23C15 & 93.7717 & 0.4704 & 97.6229 & 3.3808 \\
S23C16 & 107.1084 & 0.5373 & 111.5073 & 3.8617 \\
S23C17 & 122.9455 & 0.6167 & 127.9949 & 4.4327 \\
S23C18 & 140.4398 & 0.7045 & 146.2077 & 5.0634 \\
S23C19 & 96.0621 & 0.3551 & 98.9856 & 2.5684 \\
S23C20 & 99.9696 & 0.3696 & 103.0120 & 2.6729 \\
S23C21 & 108.3709 & 0.4006 & 111.6691 & 2.8975 \\
S23C22 & 120.3284 & 0.4448 & 123.9905 & 3.2172 \\
S23C23 & 134.8997 & 0.4987 & 139.0052 & 3.6068 \\
S23C24 & 151.3317 & 0.5594 & 155.9373 & 4.0461 \\
S23R01 & 35.0518 & 0.4745 & 357.8680 & 322.3417 \\
S23R02 & 25.5085 & 0.4253 & 344.1927 & 318.2589 \\
S23R03 & 20.6448 & 0.3783 & 334.3073 & 313.2842 \\
S23R04 & 18.9764 & 0.3582 & 330.1037 & 310.7691 \\
S23R05 & 19.8773 & 0.3693 & 332.4386 & 312.1920 \\
S23R06 & 23.6901 & 0.4097 & 340.8320 & 316.7323 \\
S23R07 & 31.7015 & 0.4628 & 353.6405 & 321.4762 \\
S23R08 & 45.2699 & 0.4838 & 368.5732 & 322.8195 \\
S23R09 & 63.0437 & 0.4512 & 383.2478 & 319.7529 \\
S23R10 & 80.4454 & 0.4030 & 395.5750 & 314.7265 \\
S23R11 & 93.0725 & 0.3690 & 403.9642 & 310.5227 \\
S23R12 & 98.3711 & 0.3557 & 407.4061 & 308.6794 \\
S23R13 & 95.4350 & 0.3630 & 405.5033 & 309.7053 \\
S23R14 & 84.7550 & 0.3911 & 398.4743 & 313.3281 \\
S23R15 & 68.3637 & 0.4368 & 387.1522 & 318.3516 \\
S23R16 & 50.1676 & 0.4788 & 372.9638 & 322.3174 \\
& & & & \\
\hline
\end{tabular}

$\begin{array}{ccccccr} & \text { Cs-137 } & \text { Ba-137m } & \text { Source 24 } & \text { X Posit } & \text { Z Posit } & \text { Y Posit } \\ & 12.5419 & 11.8646 & & 20.2228 & 48.7990 & 83.9044 \\ \text { Line } & \text { Shield 1 } & \text { Shield 2 } & \text { Total Dist } & \text { Air Gap } & & \\ \text { S24C01 } & 70.3822 & 0.7084 & 76.0088 & 4.9181 & & \\ \text { S24C02 } & 61.3360 & 0.6173 & 66.2393 & 4.2860 & & \end{array}$




\section{REVISED METHODOLOGY FOR DETERMINING CESIUM-137 CONTENT OF HN-200 GROUT CONTAINERS.}

$\begin{array}{lcccc}\text { S24C03 } & 58.8786 & 0.5926 & 63.5855 & 4.1143 \\ \text { S24C04 } & 63.7764 & 0.6419 & 68.8749 & 4.4565 \\ \text { S24C05 } & 74.5944 & 0.7508 & 80.5577 & 5.2125 \\ \text { S24C06 } & 89.2041 & 0.8978 & 96.3353 & 6.2334 \\ \text { S24C07 } & 35.4621 & 1.6445 & 47.9833 & 10.8767 \\ \text { S24C08 } & 22.3276 & 1.0354 & 30.2111 & 6.8481 \\ \text { S24C09 } & 17.6165 & 0.8170 & 23.8366 & 5.4032 \\ \text { S24C10 } & 26.3253 & 1.2208 & 35.6204 & 8.0743 \\ \text { S24C11 } & 40.5779 & 1.8818 & 54.9054 & 12.4457 \\ \text { S24C12 } & 56.3114 & 2.6114 & 76.1942 & 17.2714 \\ \text { S24C13 } & 99.6955 & 0.4560 & 103.4197 & 3.2681 \\ \text { S24C14 } & 92.9935 & 0.4254 & 96.4673 & 3.0484 \\ \text { S24C15 } & 91.2558 & 0.4174 & 94.6647 & 2.9915 \\ \text { S24C16 } & 94.7560 & 0.4334 & 98.2957 & 3.1062 \\ \text { S24C17 } & 102.9613 & 0.4710 & 106.8075 & 3.3752 \\ \text { S24C18 } & 114.8677 & 0.5254 & 119.1586 & 3.7655 \\ \text { S24C19 } & 115.8975 & 0.3819 & 119.0402 & 2.7608 \\ \text { S24C20 } & 110.0679 & 0.3627 & 113.0525 & 2.6220 \\ \text { S24C21 } & 108.5742 & 0.3578 & 111.5184 & 2.5864 \\ \text { S24C22 } & 111.5907 & 0.3677 & 114.6167 & 2.6582 \\ \text { S24C23 } & 118.7742 & 0.3914 & 121.9950 & 2.8293 \\ \text { S24C24 } & 129.4328 & 0.4265 & 132.9425 & 3.0833 \\ \text { S24R01 } & 14.0009 & 0.7422 & 346.6615 & 331.9184 \\ \text { S24R02 } & 7.6837 & 0.5005 & 328.2254 & 320.0412 \\ \text { S24R03 } & 5.6162 & 0.3837 & 315.3086 & 309.3088 \\ \text { S24R04 } & 5.0936 & 0.3515 & 310.6480 & 305.2029 \\ \text { S24R05 } & 5.6171 & 0.3837 & 315.3164 & 309.3155 \\ \text { S24R06 } & 7.6866 & 0.5007 & 328.2393 & 320.0520 \\ \text { S24R07 } & 14.0101 & 0.7424 & 346.6788 & 331.9263 \\ \text { S24R08 } & 32.5699 & 0.8058 & 367.2395 & 333.8638 \\ \text { S24R09 } & 61.6460 & 0.5723 & 386.7076 & 324.4892 \\ \text { S24R10 } & 87.8176 & 0.4314 & 402.4735 & 314.2245 \\ \text { S24R11 } & 104.9256 & 0.3690 & 412.6712 & 307.3766 \\ \text { S24R12 } & 110.8151 & 0.3513 & 416.1906 & 305.0243 \\ \text { S24R13 } & 104.9156 & 0.3691 & 412.6652 & 307.3805 \\ \text { S24R14 } & 87.7986 & 0.4315 & 402.4622 & 314.2321 \\ \text { S24R15 } & 61.6210 & 0.5725 & 386.6922 & 324.4987 \\ \text { S24R16 } & 32.5474 & 0.8060 & 367.2219 & 333.8686 \\ & & & & \\ & & & \end{array}$

$\begin{array}{ccccccc} & \text { Cs-137 } & \text { Ba-137m } & \text { Source 25 } & \text { X Posit } & \text { Z Posit } & \text { Y Posit } \\ & 19.7625 & 18.6953 & & 21.3295 & 24.2720 & 101.2303 \\ \text { Line } & \text { Shield 1 } & \text { Shield 2 } & \text { Total Dist } & \text { Air Gap } & & \\ \text { S25C01 } & 48.5395 & 0.4748 & 52.4155 & 3.4012 & & \\ \text { S25C02 } & 42.9376 & 0.4200 & 46.3663 & 3.0087 & & \\ \text { S25C03 } & 47.1964 & 0.4617 & 50.9652 & 3.3071 & & \end{array}$




\section{REVISED METHODOLOGY FOR DETERMINING CESIUM-137 CONTENT} OF HN-200 GROUT CONTAINERS.

\begin{tabular}{|c|c|c|c|c|}
\hline $\mathrm{S} 25 \mathrm{C} 04$ & 59.2255 & 0.5793 & 63.9548 & 4.1500 \\
\hline S25C05 & 75.3931 & 0.7375 & 81.4134 & 5.2829 \\
\hline $\mathrm{S} 25 \mathrm{C} 06$ & 93.5782 & 0.9154 & 101.0507 & 6.5571 \\
\hline S25C07 & 44.9810 & 0.4776 & 48.8832 & 3.4247 \\
\hline $\mathrm{S} 25 \mathrm{CO} 08$ & 38.9530 & 0.4136 & 42.3323 & 2.9657 \\
\hline S25C09 & 43.5469 & 0.4624 & 47.3248 & 3.3155 \\
\hline S25C10 & 56.2164 & 0.5969 & 61.0934 & 4.2801 \\
\hline $\mathrm{S} 25 \mathrm{C} 11$ & 72.8642 & 0.7737 & 79.1855 & 5.5476 \\
\hline $\mathrm{S} 25 \mathrm{C} 12$ & 91.3403 & 0.9698 & 99.2644 & 6.9543 \\
\hline $\mathbf{S} 25 \mathrm{C} 13$ & 85.9352 & 0.3823 & 89.0777 & 2.7601 \\
\hline S25C14 & 82.6362 & 0.3676 & 85.6580 & 2.6541 \\
\hline S25C15 & 85.1195 & 0.3787 & 88.2321 & 2.7339 \\
\hline $\mathrm{S} 25 \mathrm{C} 16$ & 92.9227 & 0.4134 & 96.3207 & 2.9845 \\
\hline $\mathrm{S} 25 \mathrm{C} 17$ & 104.8649 & 0.4665 & 108.6996 & 3.3681 \\
\hline $\mathrm{S} 25 \mathrm{C} 18$ & 119.7138 & 0.5326 & 124.0914 & 3.8450 \\
\hline S25C19 & 87.9645 & 0.3769 & 91.0640 & 2.7226 \\
\hline $\mathrm{S} 25 \mathrm{C} 20$ & 84.7360 & 0.3630 & 87.7217 & 2.6227 \\
\hline$\$ 25 C 21$ & 87.1656 & 0.3735 & 90.2370 & 2.6979 \\
\hline $\mathrm{S} 25 \mathrm{C} 22$ & 94.8195 & 0.4063 & 98.1605 & 2.9348 \\
\hline $\mathrm{S} 25 \mathrm{C} 23$ & 106.5778 & 0.4566 & 110.3332 & 3.2987 \\
\hline $\mathrm{S} 25 \mathrm{C} 24$ & 121.2525 & 0.5195 & 125.5249 & 3.7529 \\
\hline S25R01 & 32.4800 & 0.3934 & 344.2058 & 311.3324 \\
\hline S25R02 & 27.5772 & 0.3660 & 336.0237 & 308.0804 \\
\hline S25R03 & 25.7632 & 0.3531 & 332.4930 & 306.3767 \\
\hline S25R04 & 26.6699 & 0.3597 & 334.2987 & 307.2690 \\
\hline S25R05 & 30.4894 & 0.3835 & 341.0880 & 310.2151 \\
\hline S25R06 & 37.8465 & 0.4122 & 351.5814 & 313.3227 \\
\hline S25R07 & 49.0662 & 0.4241 & 363.9433 & 314.4530 \\
\hline S25R08 & 62.8792 & 0.4097 & 376.2044 & 312.9154 \\
\hline S25R09 & 76.2989 & 0.3833 & 386.5919 & 309.9097 \\
\hline S25R 10 & 86.1884 & 0.3618 & 393.7244 & 307.1743 \\
\hline S25R11 & 90.4533 & 0.3525 & 396.7108 & 305.9051 \\
\hline S2SR 12 & 88.2736 & 0.3572 & 395.1901 & 306.5593 \\
\hline S25R13 & 80.0619 & 0.3752 & 389.3455 & 308.9084 \\
\hline S25R 14 & 67.4931 & 0.4014 & 379.8965 & 312.0019 \\
\hline S25R15 & 53.4355 & 0.4220 & 368.0711 & 314.2136 \\
\hline S25R16 & 41.1212 & 0.4189 & 355.5293 & 313.9892 \\
\hline
\end{tabular}

$\begin{array}{ccccccr} & \text { Cs-137 } & \text { Ba-137m } & \text { Source 26 } & \text { X Posit } & \text { Z Posit } & \text { Y Posit } \\ & 24.8512 & 23.5093 & & 10.4368 & 41.1896 & 86.8515 \\ \text { Line } & \text { Shield 1 } & \text { Shield 2 } & \text { Total Dist } & \text { Air Gap } & & \\ \text { S26C01 } & 71.3678 & 0.5460 & 75.7938 & 3.8799 & & \\ \text { S26C02 } & 63.0929 & 0.4827 & 67.0056 & 3.4300 & & \\ \text { S26C03 } & 61.6012 & 0.4713 & 65.4215 & 3.3489 & & \\ \text { S26C04 } & 67.3451 & 0.5153 & 71.5216 & 3.6612 & & \end{array}$




\section{REVISED METHODOLOGY FOR DETERMINING CESIUM-137 CONTENT OF HN-200 GROUT CONTAINERS.}

\begin{tabular}{|c|c|c|c|c|}
\hline $\mathrm{S} 26 \mathrm{C05}$ & 78.7571 & 0.6026 & 83.6413 & 4.2816 \\
\hline $\mathbf{S} 26 \mathrm{C} 06$ & 93.7906 & 0.7176 & 99.6071 & 5.0989 \\
\hline $\mathrm{S} 26 \mathrm{C07}$ & 38.1298 & 0.8126 & 44.7781 & 5.8357 \\
\hline $\mathbf{S} 26 \mathrm{C0} 8$ & 23.3222 & 0.4970 & 27.3886 & 3.5694 \\
\hline $\mathrm{S} 26 \mathrm{C} 09$ & 19.7941 & 0.4218 & 23.2454 & 3.0295 \\
\hline $\mathrm{S} 26 \mathrm{C} 10$ & 31.5838 & 0.6731 & 37.0907 & 4.8338 \\
\hline $\mathrm{S} 26 \mathrm{C} 11$ & 48.5913 & 1.0355 & 57.0636 & 7.4368 \\
\hline $\mathrm{S} 26 \mathrm{C} 12$ & 66.9517 & 1.4268 & 78.6254 & 10.2468 \\
\hline $\mathrm{S} 26 \mathrm{C} 13$ & 87.2861 & 0.4558 & 91.0109 & 3.2690 \\
\hline $\mathrm{S} 26 \mathrm{Cl} 14$ & 80.4024 & 0.4199 & 83.8334 & 3.0112 \\
\hline S26C15 & 79.1933 & 0.4135 & 82.5728 & 2.9659 \\
\hline $\mathrm{S} 26 \mathrm{C} 16$ & 83.9045 & 0.4382 & 87.4850 & 3.1424 \\
\hline $\mathrm{S} 26 \mathrm{C} 17$ & 93.6468 & 0.4890 & 97.6431 & 3.5072 \\
\hline $\mathrm{S} 26 \mathrm{C} 18$ & 107.0553 & 0.5590 & 111.6237 & 4.0094 \\
\hline S26C19 & 106.5388 & 0.3776 & 109.6476 & 2.7313 \\
\hline $\mathrm{S} 26 \mathrm{C} 20$ & 100.8252 & 0.3573 & 103.7673 & 2.5848 \\
\hline S26C21 & 99.8382 & 0.3538 & 102.7515 & 2.5595 \\
\hline S26C22 & 103.7128 & 0.3676 & 106.7392 & 2.6589 \\
\hline $\mathbf{S} 26 \mathrm{C} 23$ & 111.9453 & 0.3967 & 115.2119 & 2.8699 \\
\hline S26C24 & 123.6684 & 0.4383 & 127.2772 & 3.1705 \\
\hline S26R01 & 34.2013 & 0.5105 & 355.5250 & 320.8132 \\
\hline S26R02 & 23.1872 & 0.4515 & 339.8959 & 316.2573 \\
\hline S26R03 & 17.6962 & 0.3865 & 327.8248 & 309.7420 \\
\hline S26R04 & 15.5982 & 0.3542 & 321.6274 & 305.6750 \\
\hline S26R05 & 15.8999 & 0.3591 & 322.5953 & 306.3364 \\
\hline S26R06 & 18.7452 & 0.4011 & 330.5215 & 311.3752 \\
\hline S26R07 & 25.4586 & 0.4706 & 343.7828 & 317.8536 \\
\hline S26R08 & 38.2567 & 0.5153 & 359.9060 & 321.1340 \\
\hline S26R09 & 57.0296 & 0.4825 & 376.2378 & 318.7257 \\
\hline S26R10 & 76.8291 & 0.4195 & 390.4145 & 313.1659 \\
\hline S26R11 & 92.1816 & 0.3736 & 400.6045 & 308.0492 \\
\hline S26R 12 & 99.8945 & 0.3530 & 405.5966 & 305.3491 \\
\hline S26R 13 & 98.7001 & 0.3561 & 404.8273 & 305.7712 \\
\hline S26R14 & 88.7820 & 0.3833 & 398.3821 & 309.2169 \\
\hline S26R 15 & 71.8496 & 0.4354 & 386.9967 & 314.7116 \\
\hline S26R 16 & 51.7175 & 0.4972 & 372.0489 & 319.8342 \\
\hline
\end{tabular}

$\begin{array}{ccccccc} & \text { Cs-137 } & \text { Ba-137m } & \text { Source 27 } & \text { X Posit } & \text { Z Posit } & \text { Y Posit } \\ & 10.2138 & 9.6622 & & 5.7653 & -53.5980 & 35.4870 \\ \text { Line } & \text { Shield 1 } & \text { Shield 2 } & \text { Total Dist } & \text { Air Gap } & & \\ \text { S27C01 } & 112.1333 & 0.7927 & 118.5137 & 5.5877 & & \\ \text { S27C02 } & 96.6855 & 0.6835 & 102.1869 & 4.8179 & & \\ \text { S27C03 } & 84.0116 & 0.5939 & 88.7919 & 4.1864 & & \\ \text { S27C04 } & 75.5213 & 0.5339 & 79.8185 & 3.7633 & & \\ \text { S27C05 } & 72.6955 & 0.5139 & 76.8319 & 3.6225 & & \end{array}$




\section{REVISED METHODOLOGY FOR DETERMINING CESIUM-137 CONTENT OF HN-200 GROUT CONTAINERS.}

\begin{tabular}{|c|c|c|c|c|}
\hline S27C06 & 76.1673 & 0.5385 & 80.5012 & 3.7955 \\
\hline $\mathrm{S} 27 \mathrm{C} 07$ & 142.1372 & 0.4475 & 145.8226 & 3.2379 \\
\hline S27C08 & 129.5357 & 0.4078 & 132.8943 & 2.9508 \\
\hline $\mathrm{S} 27 \mathrm{C} 09$ & 119.7873 & 0.3771 & 122.8931 & 2.7287 \\
\hline $\mathrm{S} 27 \mathrm{C} 10$ & 113.6287 & 0.3577 & 116.5749 & 2.5884 \\
\hline $\mathrm{S} 27 \mathrm{C} 11$ & 111.6557 & 0.3515 & 114.5507 & 2.5435 \\
\hline $\mathrm{S} 27 \mathrm{C} 12$ & 114.0854 & 0.3592 & 117.0435 & 2.5989 \\
\hline $\mathrm{S} 27 \mathrm{C} 13$ & 118.8003 & 0.6767 & 124.2886 & 4.8115 \\
\hline $\mathrm{S} 27 \mathrm{C} 14$ & 104.0259 & 0.5926 & 108.8316 & 4.2131 \\
\hline S27C15 & 92.1094 & 0.5247 & 96.3647 & 3.7305 \\
\hline S27C16 & 84.2722 & 0.4801 & 88.1654 & 3.4131 \\
\hline S27C17 & 81.6968 & 0.4654 & 85.4710 & 3.3088 \\
\hline S27C18 & 84.8635 & 0.4834 & 88.7840 & 3.4371 \\
\hline $\mathrm{S} 27 \mathrm{C} 19$ & 53.7036 & 4.5627 & 90.7134 & 32.4471 \\
\hline $\mathrm{S} 27 \mathrm{C} 20$ & 40.2639 & 3.4209 & 68.0117 & 24.3269 \\
\hline S27C21 & 26.9182 & 2.2870 & 45.4689 & 16.2637 \\
\hline S27C22 & 13.9395 & 1.1843 & 23.5459 & 8.4221 \\
\hline S27C23 & 5.5079 & 0.4680 & 9.3037 & 3.3278 \\
\hline S27C24 & 15.2536 & 1.2960 & 25.7656 & 9.2160 \\
\hline S27R01 & 23.6206 & 0.9415 & 362.7488 & 338.1868 \\
\hline S27R02 & 52.8026 & 0.6625 & 383.1433 & 329.6783 \\
\hline S27R03 & 81.8561 & 0.4674 & 400.4155 & 318.0921 \\
\hline S27R04 & 102.3866 & 0.3829 & 412.4647 & 309.6952 \\
\hline S27R05 & 111.7019 & 0.3534 & 417.9614 & 305.9060 \\
\hline S27R06 & 108.9433 & 0.3617 & 416.3306 & 307.0256 \\
\hline S27R07 & 94.3491 & 0.4123 & 407.7412 & 312.9798 \\
\hline S27R08 & 69.4301 & 0.5370 & 393.1083 & 323.1411 \\
\hline S27R09 & 38.6093 & 0.8042 & 374.1113 & 334.6979 \\
\hline S27R 10 & 15.0690 & 0.8956 & 353.1954 & 337.2309 \\
\hline S27R 11 & 7.0792 & 0.5759 & 333.4869 & 325.8318 \\
\hline S27R12 & 4.7456 & 0.4102 & 318.4631 & 313.3074 \\
\hline S27R13 & 4.0548 & 0.3554 & 311.2142 & 306.8040 \\
\hline S27R 14 & 4.2429 & 0.3705 & 313.3922 & 308.7788 \\
\hline S27R15 & 5.4948 & 0.4668 & 324.4891 & 318.5275 \\
\hline$S 27 R 16$ & 9.4743 & 0.7112 & 342.0698 & 331.8843 \\
\hline
\end{tabular}

$\begin{array}{ccccccc} & \text { Cs-137 } & \text { Ba-137m } & \text { Source 28 } & \text { X Posit } & \text { Z Posit } & \text { Y Posit } \\ & 15.5133 & 14.6756 & & 46.7749 & -14.4208 & 16.4718 \\ \text { Line } & \text { Shield 1 } & \text { Shield 2 } & \text { Total Dist } & \text { Air Gap } & & \\ \text { S28C01 } & 87.5577 & 2.9326 & 111.0948 & 20.6044 & & \\ \text { S28C02 } & 69.9150 & 2.3417 & 88.7093 & 16.4527 & & \\ \text { S28C03 } & 52.5267 & 1.7593 & 66.6468 & 12.3608 & & \\ \text { S28C04 } & 35.7662 & 1.1979 & 45.3807 & 8.4166 & & \\ \text { S28C05 } & 21.1800 & 0.7094 & 26.8735 & 4.9842 & & \\ \text { S28C06 } & 16.3453 & 0.5475 & 20.7393 & 3.8465 & & \end{array}$




\section{REVISED METHODOLOGY FOR DETERMINING CESIUM-137 CONTENT OF HN-200 GROUT CONTAINERS.}

$\begin{array}{lcccc}\text { S28C07 } & 135.1952 & 0.6689 & 140.6542 & 4.7902 \\ \text { S28C08 } & 118.9348 & 0.5884 & 123.7372 & 4.2140 \\ \text { S28C09 } & 104.7799 & 0.5184 & 109.0108 & 3.7125 \\ \text { S28C10 } & 93.6898 & 0.4635 & 97.4729 & 3.3196 \\ \text { S28C11 } & 86.8466 & 0.4297 & 90.3534 & 3.0771 \\ \text { S28C12 } & 85.2790 & 0.4219 & 88.7224 & 3.0216 \\ \text { S28C13 } & 149.8657 & 0.5030 & 154.0063 & 3.6377 \\ \text { S28C14 } & 134.9974 & 0.4531 & 138.7272 & 3.2768 \\ \text { S28C15 } & 122.3870 & 0.4107 & 125.7684 & 2.9707 \\ \text { S28C16 } & 112.7944 & 0.3785 & 115.9108 & 2.7379 \\ \text { S28C17 } & 107.0340 & 0.3592 & 109.9913 & 2.5980 \\ \text { S28C18 } & 105.7341 & 0.3549 & 108.6555 & 2.5665 \\ \text { S28C19 } & 119.4136 & 1.0163 & 127.5790 & 7.1491 \\ \text { S28C20 } & 101.6912 & 0.8655 & 108.6448 & 6.0881 \\ \text { S28C21 } & 85.6637 & 0.7291 & 91.5213 & 5.1285 \\ \text { S28C22 } & 72.4643 & 0.6167 & 77.4193 & 4.3383 \\ \text { S28C23 } & 63.8713 & 0.5436 & 68.2388 & 3.8238 \\ \text { S28C24 } & 61.8358 & 0.5263 & 66.0641 & 3.7020 \\ \text { S28R01 } & 9.5533 & 0.3710 & 320.7717 & 310.8474 \\ \text { S28R02 } & 11.9818 & 0.4430 & 330.8882 & 318.4634 \\ \text { S28R03 } & 18.3919 & 0.5774 & 346.7662 & 327.7969 \\ \text { S28R04 } & 33.3759 & 0.6572 & 365.4249 & 331.3918 \\ \text { S28R05 } & 57.4644 & 0.5538 & 383.8360 & 325.8179 \\ \text { S28R06 } & 81.8235 & 0.4404 & 399.4127 & 317.1488 \\ \text { S28R07 } & 99.4704 & 0.3779 & 410.2190 & 310.3707 \\ \text { S28R08 } & 107.3794 & 0.3546 & 415.0239 & 307.2899 \\ \text { S28R09 } & 104.5525 & 0.3627 & 413.3076 & 308.3925 \\ \text { S28R10 } & 91.3266 & 0.4048 & 405.2536 & 313.5222 \\ \text { S28R11 } & 69.5984 & 0.4934 & 391.7537 & 321.6619 \\ \text { S28R12 } & 44.1852 & 0.6213 & 374.4091 & 329.6026 \\ \text { S28R13 } & 24.1647 & 0.6380 & 355.5103 & 330.7076 \\ \text { S28R14 } & 14.3166 & 0.5012 & 337.9156 & 323.0979 \\ \text { S28R15 } & 10.3943 & 0.3972 & 324.7124 & 313.9209 \\ \text { S28R16 } & 9.1272 & 0.3572 & 318.5477 & 309.0633\end{array}$

$\begin{array}{ccccccr} & \text { Cs-137 } & \text { Ba-137m } & \text { Source 29 } & \text { X Posit } & \text { Z Posit } & \text { Y Posit } \\ & 18.0653 & 17.0898 & & -0.2295 & 9.2524 & 97.3974 \\ \text { Line } & \text { Shield 1 } & \text { Shield 2 } & \text { Total Dist } & \text { Air Gap } & & \\ \text { S29C01 } & 64.7006 & 0.3911 & 67.9198 & 2.8281 & & \\ \text { S29C02 } & 59.0330 & 0.3568 & 61.9702 & 2.5804 & & \\ \text { S29C03 } & 61.0906 & 0.3693 & 64.1302 & 2.6703 & & \\ \text { S29C04 } & 70.1973 & 0.4243 & 73.6900 & 3.0684 & & \\ \text { S29C05 } & 84.0933 & 0.5083 & 88.2774 & 3.6758 & & \\ \text { S29C06 } & 100.8174 & 0.6094 & 105.8336 & 4.4068 & & \\ \text { S29C07 } & 55.5235 & 0.4006 & 58.8224 & 2.8984 & & \end{array}$




\section{REVISED METHODOLOGY FOR DETERMINING CESIUM-137 CONTENT OF HN-200 GROUT CONTAINERS.}

$\begin{array}{lcccc}\text { S29C08 } & 48.9318 & 0.3530 & 51.8391 & 2.5543 \\ \text { S29C09 } & 51.3517 & 0.3705 & 54.4028 & 2.6806 \\ \text { S29C10 } & 61.7324 & 0.4454 & 65.4003 & 3.2225 \\ \text { S29C11 } & 76.9155 & 0.5549 & 81.4855 & 4.0151 \\ \text { S29C12 } & 94.6167 & 0.6826 & 100.2384 & 4.9391 \\ \text { S29C13 } & 64.2837 & 0.3917 & 67.5076 & 2.8323 \\ \text { S29C14 } & 58.5803 & 0.3569 & 61.5182 & 2.5810 \\ \text { S29C15 } & 60.6517 & 0.3695 & 63.6935 & 2.6723 \\ \text { S29C16 } & 69.8092 & 0.4253 & 73.3103 & 3.0757 \\ \text { S29C17 } & 83.7600 & 0.5103 & 87.9607 & 3.6904 \\ \text { S29C18 } & 100.5279 & 0.6125 & 105.5696 & 4.4292 \\ \text { S29C19 } & 72.4480 & 0.3787 & 75.5665 & 2.7399 \\ \text { S29C20 } & 67.3676 & 0.3521 & 70.2674 & 2.5478 \\ \text { S29C21 } & 69.2008 & 0.3617 & 72.1795 & 2.6171 \\ \text { S29C22 } & 77.4582 & 0.4048 & 80.7924 & 2.9294 \\ \text { S29C23 } & 90.3962 & 0.4725 & 94.2874 & 3.4187 \\ \text { S29C24 } & 106.3196 & 0.5557 & 110.8962 & 4.0209 \\ \text { S29R01 } & 57.8217 & 0.3567 & 364.6001 & 306.4217 \\ \text { S29R02 } & 54.3115 & 0.3562 & 361.0394 & 306.3717 \\ \text { S29R03 } & 51.4282 & 0.3547 & 357.9579 & 306.1751 \\ \text { S29R04 } & 49.5325 & 0.3530 & 355.8417 & 305.9562 \\ \text { S29R05 } & 48.8258 & 0.3522 & 355.0327 & 305.8546 \\ \text { S29R06 } & 49.3751 & 0.3528 & 355.6625 & 305.9345 \\ \text { S29R07 } & 51.1286 & 0.3545 & 357.6286 & 306.1455 \\ \text { S29R08 } & 53.9036 & 0.3561 & 360.6127 & 306.3530 \\ \text { S29R09 } & 57.3602 & 0.3567 & 364.1427 & 306.4258 \\ \text { S29R10 } & 61.0021 & 0.3560 & 367.6732 & 306.3150 \\ \text { S29R11 } & 64.2381 & 0.3544 & 370.6742 & 306.0817 \\ \text { S29R12 } & 66.4997 & 0.3528 & 372.7059 & 305.8534 \\ \text { S29R13 } & 67.3721 & 0.3521 & 373.4768 & 305.7526 \\ \text { S29R14 } & 66.6926 & 0.3527 & 372.8770 & 305.8317 \\ \text { S29R15 } & 64.5881 & 0.3542 & 370.9920 & 306.0497 \\ \text { S29R16 } & 61.4458 & 0.3559 & 368.0917 & 306.2900\end{array}$

$\begin{array}{lcccccc} & \text { Cs-137 } & \text { Ba-137m } & \text { Source 30 } & \text { X Posit } & \text { Z Posit } & \text { Y Posit } \\ & 15.0401 & 14.2280 & & 18.7276 & -50.2548 & 31.4968 \\ \text { Line } & \text { Shield 1 } & \text { Shield 2 } & \text { Total Dist } & \text { Air Gap } & & \\ \text { S30C01 } & 106.5947 & 1.0306 & 114.7852 & 7.1599 & & \\ \text { S30C02 } & 89.9870 & 0.8701 & 96.9014 & 6.0443 & & \\ \text { S30C03 } & 75.7246 & 0.7322 & 81.5431 & 5.0863 & & \\ \text { S30C04 } & 65.3612 & 0.6320 & 70.3834 & 4.3902 & \\ \text { S30C05 } & 60.9204 & 0.5890 & 65.6014 & 4.0920 & \\ \text { S30C06 } & 63.6540 & 0.6154 & 68.5450 & 4.2756 & & \\ \text { S30C07 } & 143.0216 & 0.4648 & 146.8471 & 3.3607 & & \\ \text { S30C08 } & 129.8619 & 0.4220 & 133.3353 & 3.0514 & & \end{array}$




\section{REVISED METHODOLOGY FOR DETERMINING CESIUM-137 CONTENT OF HN-200 GROUT CONTAINERS.}

\begin{tabular}{lcccc} 
S30C09 & 119.4338 & 0.3881 & 122.6283 & 2.8064 \\
S30C10 & 112.4996 & 0.3656 & 115.5087 & 2.6435 \\
S30C11 & 109.7238 & 0.3566 & 112.6587 & 2.5783 \\
S30C12 & 111.4176 & 0.3621 & 114.3978 & 2.6181 \\
S30C13 & 128.2654 & 0.5993 & 133.1551 & 4.2904 \\
S30C14 & 113.7506 & 0.5315 & 118.0869 & 3.8049 \\
S30C15 & 101.9616 & 0.4764 & 105.8486 & 3.4106 \\
S30C16 & 93.9306 & 0.4389 & 97.5114 & 3.1419 \\
S30C17 & 90.6618 & 0.4236 & 94.1179 & 3.0326 \\
S30C18 & 92.6605 & 0.4329 & 96.1928 & 3.0994 \\
S30C19 & 67.6542 & 3.8503 & 96.6532 & 25.1488 \\
S30C20 & 52.1750 & 2.9694 & 74.5391 & 19.3948 \\
S30C21 & 37.1411 & 2.1138 & 53.0611 & 13.8063 \\
S30C22 & 23.4258 & 1.3332 & 33.4670 & 8.7080 \\
S30C23 & 15.1709 & 0.8634 & 21.6738 & 5.6394 \\
S30C24 & 20.5822 & 1.1714 & 29.4045 & 7.6509 \\
S30R01 & 13.3841 & 0.8203 & 349.8852 & 335.6808 \\
S30R02 & 33.7620 & 0.8424 & 370.7011 & 336.0967 \\
S30R03 & 64.2292 & 0.5690 & 390.1356 & 325.3374 \\
S30R04 & 90.4746 & 0.4273 & 405.6201 & 314.7182 \\
S30R05 & 106.9933 & 0.3674 & 415.3425 & 307.9818 \\
S30R06 & 111.9161 & 0.3525 & 418.2529 & 305.9843 \\
S30R07 & 104.8023 & 0.3744 & 414.0496 & 308.8728 \\
S30R08 & 86.3028 & 0.4453 & 403.1701 & 316.4219 \\
S30R09 & 58.6832 & 0.6096 & 386.8032 & 327.5105 \\
S30R10 & 28.7475 & 0.8875 & 366.9019 & 337.2669 \\
S30R11 & 11.4977 & 0.7603 & 346.1658 & 333.9078 \\
S30R12 & 6.3106 & 0.4951 & 327.8851 & 321.0794 \\
S30R13 & 4.6774 & 0.3810 & 315.4791 & 310.4207 \\
S30R14 & 4.3125 & 0.3537 & 311.6100 & 306.9438 \\
S30R15 & 4.8546 & 0.3940 & 317.1743 & 311.9256 \\
S30R16 & 6.8616 & 0.5305 & 330.8930 & 323.5009 \\
& & & & \\
\hline
\end{tabular}

$\begin{array}{ccccccc} & \text { Cs-137 } & \text { Ba-137m } & \text { Source 31 } & \text { X Posit } & \text { Z Posit } & \text { Y Posit } \\ & 22.3550 & 21.1478 & & 14.1157 & -45.1966 & 54.0394 \\ \text { Line } & \text { Shield 1 } & \text { Shield 2 } & \text { Total Dist } & \text { Air Gap } & & \\ \text { S31C01 } & 90.6176 & 0.7625 & 96.7565 & 5.3764 & & \\ \text { S31C02 } & 76.1245 & 0.6406 & 81.2816 & 4.5165 & & \\ \text { S31C03 } & 65.5369 & 0.5515 & 69.9768 & 3.8884 & & \\ \text { S31C04 } & 60.9260 & 0.5127 & 65.0535 & 3.6148 & \\ \text { S31C05 } & 63.6051 & 0.5352 & 67.9140 & 3.7737 & \\ \text { S31C06 } & 72.7734 & 0.6124 & 77.7035 & 4.3177 & \\ \text { S31C07 } & 125.2285 & 0.4267 & 128.7414 & 3.0862 & \\ \text { S31C08 } & 114.3475 & 0.3896 & 117.5552 & 2.8180 & & \\ \text { S31C09 } & 107.0395 & 0.3647 & 110.0421 & 2.6379 & & \\ & & & & & & \end{array}$




\section{REVISED METHODOLOGY FOR DETERMINING CESIUM-137 CONTENT OF HN-200 GROUT CONTAINERS.}

$\begin{array}{lcccc}\text { S31C10 } & 104.0598 & 0.3546 & 106.9788 & 2.5645 \\ \text { S31C11 } & 105.7749 & 0.3604 & 108.7421 & 2.6068 \\ \text { S31C12 } & 111.9692 & 0.3815 & 115.1102 & 2.7594 \\ \text { S31C13 } & 108.7087 & 0.5395 & 113.1142 & 3.8660 \\ \text { S31C14 } & 96.2959 & 0.4779 & 100.1983 & 3.4245 \\ \text { S31C15 } & 87.7129 & 0.4353 & 91.2675 & 3.1193 \\ \text { S31C16 } & 84.1399 & 0.4176 & 87.5497 & 2.9922 \\ \text { S31C17 } & 86.2023 & 0.4278 & 89.6957 & 3.0656 \\ \text { S31C18 } & 93.5280 & 0.4641 & 97.3182 & 3.3261 \\ \text { S31C19 } & 60.5775 & 1.7507 & 74.7148 & 12.3866 \\ \text { S31C20 } & 43.1104 & 1.2459 & 53.1713 & 8.8150 \\ \text { S31C21 } & 27.1004 & 0.7832 & 33.4250 & 5.5414 \\ \text { S31C22 } & 17.2458 & 0.4984 & 21.2705 & 3.5263 \\ \text { S31C23 } & 23.3978 & 0.6762 & 28.8582 & 4.7843 \\ \text { S31C24 } & 38.5299 & 1.1135 & 47.5218 & 7.8784 \\ \text { S31R01 } & 26.1599 & 0.5893 & 352.1351 & 325.3858 \\ \text { S31R02 } & 45.1306 & 0.5803 & 370.5918 & 324.8809 \\ \text { S31R03 } & 68.9550 & 0.4772 & 387.5658 & 318.1336 \\ \text { S31R04 } & 89.5674 & 0.3987 & 400.8073 & 310.8411 \\ \text { S31R05 } & 102.2515 & 0.3597 & 408.7384 & 306.1271 \\ \text { S31R06 } & 105.0476 & 0.3520 & 410.4769 & 305.0773 \\ \text { S31R07 } & 97.5930 & 0.3733 & 405.8356 & 307.8693 \\ \text { S31R08 } & 80.9057 & 0.4294 & 395.3192 & 313.9842 \\ \text { S31R09 } & 58.0022 & 0.5254 & 380.1296 & 321.6020 \\ \text { S31R10 } & 35.3491 & 0.6055 & 362.1592 & 326.2045 \\ \text { S31R11 } & 20.6091 & 0.5433 & 343.9339 & 322.7814 \\ \text { S31R12 } & 13.8071 & 0.4305 & 328.4068 & 314.1692 \\ \text { S31R13 } & 11.1099 & 0.3657 & 318.4816 & 307.0060 \\ \text { S31R14 } & 10.6172 & 0.3526 & 316.2376 & 305.2678 \\ \text { S31R15 } & 12.0056 & 0.3885 & 322.1727 & 309.7786 \\ \text { S31R16 } & 16.2139 & 0.4785 & 334.9929 & 318.3005 \\ & & & & \\ & & & & \\ \text { S31 } & & \end{array}$

$\begin{array}{lcccccc} & \text { Cs-137 } & \text { Ba-137m } & \text { Source 32 } & \text { X Posit } & \text { Z Posit } & \text { Y Posit } \\ & 21.6866 & 20.5156 & & -2.3294 & 45.6316 & 66.6580 \\ \text { Line } & \text { Shield 1 } & \text { Shield 2 } & \text { Total Dist } & \text { Air Gap } & & \\ \text { S32C01 } & 93.2242 & 0.5577 & 97.7610 & 3.9792 & & \\ \text { S32C02 } & 81.9148 & 0.4900 & 85.9013 & 3.4965 & & \\ \text { S32C03 } & 75.3639 & 0.4509 & 79.0316 & 3.2168 & & \\ \text { S32C04 } & 74.8319 & 0.4477 & 78.4737 & 3.1941 & & \\ \text { S32C05 } & 80.4382 & 0.4812 & 84.3529 & 3.4334 & & \\ \text { S32C06 } & 91.0561 & 0.5447 & 95.4874 & 3.8866 & & \\ \text { S32C07 } & 49.3946 & 1.4139 & 61.0332 & 10.2246 & \\ \text { S32C08 } & 31.8301 & 0.9111 & 39.3301 & 6.5888 & & \\ \text { S32C09 } & 16.4634 & 0.4713 & 20.3426 & 3.4079 & & \\ \text { S32C10 } & 14.6112 & 0.4182 & 18.0540 & 3.0245 & & \\ & & & & & & \end{array}$


REVISED METHODOLOGY FOR DETERMINING CESIUM-137 CONTENT OF HN-200 GROUT CONTAINERS.

$\begin{array}{lcccc}\text { S32C11 } & 28.9912 & 0.8299 & 35.8222 & 6.0011 \\ \text { S32C12 } & 46.3902 & 1.3279 & 57.3208 & 9.6027 \\ \text { S32C13 } & 90.0576 & 0.5866 & 94.8194 & 4.1751 \\ \text { S32C14 } & 78.3930 & 0.5106 & 82.5380 & 3.6343 \\ \text { S32C15 } & 71.5777 & 0.4662 & 75.3623 & 3.3184 \\ \text { S32C16 } & 71.0218 & 0.4626 & 74.7771 & 3.2926 \\ \text { S32C17 } & 76.8613 & 0.5006 & 80.9252 & 3.5633 \\ \text { S32C18 } & 87.8295 & 0.5721 & 92.4734 & 4.0718 \\ \text { S32C19 } & 118.4422 & 0.4016 & 121.7493 & 2.9056 \\ \text { S32C20 } & 109.3941 & 0.3709 & 112.4486 & 2.6836 \\ \text { S32C21 } & 104.3780 & 0.3539 & 107.2924 & 2.5605 \\ \text { S32C22 } & 103.9788 & 0.3525 & 106.8821 & 2.5507 \\ \text { S32C23 } & 108.2478 & 0.3670 & 111.2702 & 2.6555 \\ \text { S32C24 } & 116.6736 & 0.3956 & 119.9313 & 2.8622 \\ \text { S32R01 } & 44.4271 & 0.5550 & 368.2312 & 323.2490 \\ \text { S32R02 } & 27.0276 & 0.5480 & 350.4076 & 322.8320 \\ \text { S32R03 } & 17.6570 & 0.4541 & 334.1698 & 316.0587 \\ \text { S32R04 } & 13.5352 & 0.3795 & 322.3943 & 308.4795 \\ \text { S32R05 } & 12.2357 & 0.3514 & 317.4408 & 304.8537 \\ \text { S32R06 } & 12.9814 & 0.3678 & 320.3803 & 307.0310 \\ \text { S32R07 } & 16.2139 & 0.4305 & 330.5714 & 313.9270 \\ \text { S32R08 } & 23.8892 & 0.5268 & 345.9194 & 321.5034 \\ \text { S32R09 } & 39.1593 & 0.5660 & 363.6089 & 323.8835 \\ \text { S32R10 } & 60.9336 & 0.4961 & 380.8149 & 319.3852 \\ \text { S32R11 } & 82.2296 & 0.4159 & 395.1408 & 312.4953 \\ \text { S32R12 } & 97.3427 & 0.3683 & 404.8054 & 307.0944 \\ \text { S32R13 } & 103.5179 & 0.3513 & 408.7015 & 304.8324 \\ \text { S32R14 } & 99.8680 & 0.3612 & 406.4012 & 306.1720 \\ \text { S32R15 } & 86.9032 & 0.4003 & 398.1561 & 310.8525 \\ \text { S32R16 } & 66.8025 & 0.4729 & 384.8964 & 317.6210\end{array}$

$\begin{array}{ccccccr} & \text { Cs-137 } & \text { Ba-137m } & \text { Source 33 } & \text { X Posit } & \text { Z Posit } & \text { Y Posit } \\ & 17.8281 & 16.8653 & & -7.0965 & -56.3420 & 75.7547 \\ \text { Line } & \text { Shield 1 } & \text { Shield 2 } & \text { Total Dist } & \text { Air Gap } & & \\ \text { S33C01 } & 97.0060 & 0.5424 & 101.4005 & 3.8520 & & \\ \text { S33C02 } & 88.3024 & 0.4937 & 92.3026 & 3.5064 & & \\ \text { S33C03 } & 84.5044 & 0.4725 & 88.3325 & 3.3556 & \\ \text { S33C04 } & 86.2624 & 0.4823 & 90.1702 & 3.4254 & \\ \text { S33C05 } & 93.2628 & 0.5215 & 97.4877 & 3.7034 & \\ \text { S33C06 } & 104.4568 & 0.5841 & 109.1888 & 4.1479 & \\ \text { S33C07 } & 124.4073 & 0.3823 & 127.5556 & 2.7660 & \\ \text { S33C08 } & 117.4768 & 0.3610 & 120.4497 & 2.6119 & \\ \text { S33C09 } & 114.5366 & 0.3520 & 117.4351 & 2.5465 & \\ \text { S33C10 } & 115.8908 & 0.3561 & 118.8236 & 2.5766 & & \\ \text { S33C11 } & 121.3957 & 0.3730 & 124.4678 & 2.6990 & & \\ & & & & & \end{array}$




\section{REVISED METHODOLOGY FOR DETERMINING CESIUM-137 CONTENT OF HN-200 GROUT CONTAINERS.}

$\begin{array}{lcccc}\text { S33C12 } & 130.5272 & 0.4011 & 133.8304 & 2.9021 \\ \text { S33C13 } & 87.3750 & 0.6392 & 92.4994 & 4.4852 \\ \text { S33C14 } & 77.8586 & 0.5696 & 82.4249 & 3.9967 \\ \text { S33C15 } & 73.6348 & 0.5387 & 77.9534 & 3.7799 \\ \text { S33C16 } & 75.5961 & 0.5530 & 80.0297 & 3.8806 \\ \text { S33C17 } & 83.3068 & 0.6094 & 88.1926 & 4.2764 \\ \text { S33C18 } & 95.3826 & 0.6978 & 100.9767 & 4.8963 \\ \text { S33C19 } & 15.4256 & 4.5830 & 50.6732 & 30.6646 \\ \text { S33C20 } & 8.6396 & 2.5669 & 28.3811 & 17.1746 \\ \text { S33C21 } & 2.8614 & 0.8501 & 9.3996 & 5.6881 \\ \text { S33C22 } & 6.2117 & 1.8455 & 20.4054 & 12.3482 \\ \text { S33C23 } & 12.8777 & 3.8260 & 42.3033 & 25.5996 \\ \text { S33C24 } & 19.7468 & 5.8669 & 64.8684 & 39.2548 \\ \text { S33R01 } & 34.7076 & 1.0340 & 374.5026 & 338.7610 \\ \text { S33R02 } & 69.9949 & 0.5635 & 394.3551 & 323.7967 \\ \text { S33R03 } & 96.6645 & 0.4145 & 409.4772 & 312.3982 \\ \text { S33R04 } & 111.7170 & 0.3603 & 418.1724 & 306.0951 \\ \text { S33R05 } & 114.0573 & 0.3531 & 419.5360 & 305.1256 \\ \text { S33R06 } & 103.5345 & 0.3879 & 413.4299 & 309.5074 \\ \text { S33R07 } & 80.8648 & 0.4918 & 400.4800 & 319.1234 \\ \text { S33R08 } & 48.0930 & 0.7912 & 382.0923 & 333.2081 \\ \text { S33R09 } & 13.2932 & 1.6556 & 360.4806 & 345.5319 \\ \text { S33R10 } & 2.9170 & 0.8509 & 338.6480 & 334.8801 \\ \text { S33R11 } & 1.5495 & 0.4789 & 320.1984 & 318.1701 \\ \text { S33R12 } & 1.1807 & 0.3686 & 308.7559 & 307.2066 \\ \text { S33R13 } & 1.1357 & 0.3549 & 306.9007 & 305.4102 \\ \text { S33R14 } & 1.3617 & 0.4231 & 315.0780 & 313.2931 \\ \text { S33R15 } & 2.1825 & 0.6592 & 331.3826 & 328.5408 \\ \text { S33R16 } & 6.6779 & 1.4857 & 352.4257 & 344.2622 \\ & & & & \end{array}$

$\begin{array}{ccccccc} & \text { Cs-137 } & \text { Ba-137m } & \text { Source 34 } & \text { X Posit } & \text { Z Posit } & \text { Y Posit } \\ & 14.6776 & 13.8850 & & -35.9173 & 43.1022 & 13.8403 \\ \text { Line } & \text { Shield 1 } & \text { Shield 2 } & \text { Total Dist } & \text { Air Gap } & & \\ \text { S34C01 } & 149.4228 & 0.5645 & 154.0506 & 4.0633 & & \\ \text { S34C02 } & 134.1863 & 0.5069 & 138.3422 & 3.6490 & & \\ \text { S34C03 } & 121.1117 & 0.4575 & 124.8627 & 3.2934 & \\ \text { S34C04 } & 110.9660 & 0.4192 & 114.4028 & 3.0175 & \\ \text { S34C05 } & 104.6049 & 0.3952 & 107.8446 & 2.8446 & \\ \text { S34C06 } & 102.7336 & 0.3881 & 105.9154 & 2.7937 & \\ \text { S34C07 } & 96.8607 & 3.0287 & 118.8386 & 18.9492 & \\ \text { S34C08 } & 79.5659 & 2.4879 & 97.6196 & 15.5658 & \\ \text { S34C09 } & 63.0382 & 1.9711 & 77.3417 & 12.3324 & \\ \text { S34C10 } & 48.0750 & 1.5032 & 58.9834 & 9.4051 & & \\ \text { S34C11 } & 36.6460 & 1.1459 & 44.9611 & 7.1692 & & \\ \text { S34C12 } & 32.6952 & 1.0223 & 40.1138 & 6.3963 & & \end{array}$




\section{REVISED METHODOLOGY FOR DETERMINING CESIUM-137 CONTENT OF HN-200 GROUT CONTAINERS.}

$\begin{array}{lcccc}\text { S34C13 } & 106.9657 & 2.0462 & 122.4595 & 13.4476 \\ \text { S34C14 } & 89.0918 & 1.7043 & 101.9966 & 11.2005 \\ \text { S34C15 } & 72.3219 & 1.3835 & 82.7976 & 9.0922 \\ \text { S34C16 } & 57.6280 & 1.1024 & 65.9753 & 7.2449 \\ \text { S34C17 } & 46.9993 & 0.8991 & 53.8071 & 5.9087 \\ \text { S34C18 } & 43.5236 & 0.8326 & 49.8279 & 5.4717 \\ \text { S34C19 } & 152.4831 & 0.5330 & 156.8607 & 3.8446 \\ \text { S34C20 } & 137.5168 & 0.4807 & 141.4647 & 3.4673 \\ \text { S34C21 } & 124.7328 & 0.4360 & 128.3137 & 3.1449 \\ \text { S34C22 } & 114.8621 & 0.4015 & 118.1597 & 2.8961 \\ \text { S34C23 } & 108.7013 & 0.3800 & 111.8220 & 2.7407 \\ \text { S34C24 } & 106.8939 & 0.3736 & 109.9627 & 2.6952 \\ \text { S34R01 } & 83.9045 & 0.4779 & 404.9308 & 320.5484 \\ \text { S34R02 } & 52.4872 & 0.7270 & 387.3069 & 334.0927 \\ \text { S34R03 } & 18.5890 & 1.3345 & 366.3327 & 346.4091 \\ \text { S34R04 } & 4.9597 & 0.8646 & 344.8971 & 339.0727 \\ \text { S34R05 } & 2.6008 & 0.4966 & 326.4830 & 323.3856 \\ \text { S34R06 } & 1.9501 & 0.3784 & 314.6315 & 312.3030 \\ \text { S34R07 } & 1.8425 & 0.3583 & 311.9559 & 309.7550 \\ \text { S34R08 } & 2.1564 & 0.4165 & 319.0869 & 316.5141 \\ \text { S34R09 } & 3.2982 & 0.6161 & 334.3775 & 330.4632 \\ \text { S34R10 } & 8.3671 & 1.2017 & 354.6414 & 345.0727 \\ \text { S34R11 } & 33.3888 & 1.0230 & 376.2682 & 341.8564 \\ \text { S34R12 } & 68.0062 & 0.5798 & 396.0090 & 327.4230 \\ \text { S34R13 } & 95.2042 & 0.4242 & 411.3224 & 315.6940 \\ \text { S34R14 } & 111.1729 & 0.3656 & 420.4571 & 308.9186 \\ \text { S34R15 } & 114.6193 & 0.3550 & 422.4464 & 307.4722 \\ \text { S34R16 } & 105.3063 & 0.3852 & 417.0859 & 311.3944\end{array}$

$\begin{array}{ccccccc} & \text { Cs-137 } & \text { Ba-137m } & \text { Source 35 } & \text { X Posit } & \text { Z Posit } & \text { Y Posit } \\ \text { Line } & \text { 28.0146 } & 26.5018 & & 44.7881 & 32.5999 & 76.4934 \\ \text { Shield 1 } & \text { Shield 2 } & \text { Total Dist } & \text { Air Gap } & & \\ \text { S35C01 } & 48.6646 & 1.7271 & 61.1835 & 10.7917 & & \\ \text { S35C03 } & 35.7031 & 1.2671 & 44.8876 & 7.9174 & & \\ \text { S35C04 } & 29.0239 & 1.0301 & 36.4903 & 6.4363 & & \\ \text { S35C05 } & 44.7304 & 1.1616 & 41.1502 & 7.2582 & \\ \text { S35C06 } & 59.2680 & 2.5717 & 55.6803 & 9.8211 & \\ \text { S35C07 } & 64.3397 & 1.0331 & 74.5145 & 13.1431 & \\ \text { S35C08 } & 52.6335 & 0.8451 & 59.1356 & 5.6569 & \\ \text { S35C09 } & 47.2117 & 0.7581 & 53.0439 & 5.0742 & \\ \text { S35C10 } & 50.1553 & 0.8054 & 56.3512 & 5.3906 & & \\ \text { S35C11 } & 60.2505 & 0.9675 & 67.6936 & 6.4756 & & \\ \text { S35C12 } & 74.6503 & 1.1987 & 83.8721 & 8.0232 & & \\ \text { S35C13 } & 117.6595 & 0.4040 & 120.9798 & 2.9163 & & \\ & & & & & \end{array}$




\section{REVISED METHODOLOGY FOR DETERMINING CESIUM-137 CONTENT OF HN-200 GROUT CONTAINERS.}

$\begin{array}{lcccc}\text { S35C14 } & 110.4935 & 0.3794 & 113.6116 & 2.7387 \\ \text { S35C15 } & 107.5288 & 0.3693 & 110.5633 & 2.6652 \\ \text { S35C16 } & 109.1085 & 0.3747 & 112.1875 & 2.7044 \\ \text { S35C17 } & 115.0454 & 0.3951 & 118.2920 & 2.8515 \\ \text { S35C18 } & 124.7189 & 0.4283 & 128.2385 & 3.0913 \\ \text { S35C19 } & 11.1202 & 0.4359 & 114.6908 & 3.1348 \\ \text { S35C20 } & 103.5622 & 0.4062 & 106.8900 & 2.9215 \\ \text { S35C21 } & 100.4175 & 0.3939 & 103.6442 & 2.8328 \\ \text { S35C22 } & 102.0945 & 0.4005 & 105.3751 & 2.8801 \\ \text { S35C23 } & 108.3698 & 0.4251 & 111.8520 & 3.0572 \\ \text { S35C24 } & 118.5151 & 0.4649 & 122.3233 & 3.3434 \\ \text { S35R01 } & 3.3218 & 0.4555 & 320.0525 & 316.2752 \\ \text { S35R02 } & 2.6112 & 0.3639 & 309.5972 & 306.6221 \\ \text { S35R03 } & 2.5564 & 0.3566 & 308.5786 & 305.6655 \\ \text { S35R04 } & 3.0991 & 0.4273 & 317.2382 & 313.7119 \\ \text { S35R05 } & 4.9532 & 0.6462 & 333.5922 & 327.9928 \\ \text { S35R06 } & 12.8682 & 1.1288 & 354.3037 & 340.3066 \\ \text { S35R07 } & 40.1087 & 0.8489 & 375.7836 & 334.8260 \\ \text { S35R08 } & 72.6441 & 0.5292 & 394.8957 & 321.7224 \\ \text { S35R09 } & 97.4320 & 0.4049 & 409.2395 & 311.4026 \\ \text { S35R10 } & 111.0530 & 0.3579 & 417.2050 & 305.7940 \\ \text { S35R11 } & 112.3353 & 0.3541 & 417.9592 & 305.2699 \\ \text { S35R12 } & 101.1822 & 0.3908 & 411.4247 & 309.8517 \\ \text { S35R13 } & 78.5089 & 0.4937 & 398.2798 & 319.2771 \\ \text { S35R14 } & 47.0347 & 0.7576 & 379.9728 & 332.1804 \\ \text { S35R15 } & 16.7155 & 1.1680 & 358.7441 & 340.8607 \\ \text { S35R16 } & 5.7301 & 0.7260 & 337.5912 & 331.1351\end{array}$




\section{REVISED METHODOLOGY FOR DETERMINING CESIUM-137 CONTENT OF HN-200 GROUT CONTAINERS.}

\section{TABLE 1 \\ DATA FOR HN-200 GROUT CONTAINER LINER DRAWING NUMBER H-3-49415}

$\begin{array}{rcccc}\text { Outer Height } & 54.0000 & \text { in } & 137.1600 & \mathrm{~cm} \\ \text { Inner Height (note 1) } & 53.5000 & \text { in } & 135.8900 & \mathrm{~cm} \\ \text { Outer Radius } & 22.9375 & \text { in } & 58.2613 & \mathrm{~cm} \\ \text { Inner Radius } & 22.7993 & \text { in } & 57.9102 & \mathrm{~cm} \\ \text { Wall Thickness } & 0.1382 & \text { in } & 0.3510 & \mathrm{~cm} \\ & & & & \\ \text { Volume (inner) } & 87366.8507 & \text { in }^{3} & 1431686.17 & \mathrm{~cm}^{3} \\ & 50.5595201 & \mathrm{ft}^{3} & 1.43168617 & \mathrm{~m}^{3}\end{array}$

Note 1 - The top and bottom closure plates for the container are constructed of $0.125^{\prime \prime}$ steel. A bottom stiffening plate, constructed of $0.25^{\text {" }}$ steel, is inserted into the container. This yields an internal height of $53.5^{\prime \prime}$ for the container.

Note 2 - For purposes of the coordinates and calculations presented in this attachment, the cylinder of interest is placed in an $\mathrm{X}, \mathrm{Z}, \mathrm{Y}$ coordinate frame to match the coordinate frame use in Microshield version 5.03. The circular portion of the cylinder is centered at $X=0, Z=0$. The bottom of the inner height of the cylinder is placed at $Y=0$. This placement matches the orientation used in Microshield for a cylindrical source. 


\section{REVISED METHODOLOGY FOR DETERMINING CESIUM-137 CONTENT OF HN-200 GROUT CONTAINERS.}

TABLE 2

Coordinates of current contact measuring points.

$\begin{array}{cccc}\text { Point } & \mathbf{X} & \mathbf{Z} & \mathbf{Y} \\ \text { C01 } & 23.9375 & 0.0000 & 49.5000 \\ \text { C02 } & 23.9375 & 0.0000 & 40.5000 \\ \text { C03 } & 23.9375 & 0.0000 & 31.5000 \\ \text { C04 } & 23.9375 & 0.0000 & 22.5000 \\ \text { C05 } & 23.9375 & 0.0000 & 13.5000 \\ \text { C06 } & 23.9375 & 0.0000 & 4.5000 \\ \text { C07 } & 0.0000 & 23.9375 & 49.5000 \\ \text { C08 } & 0.0000 & 23.9375 & 40.5000 \\ \text { C09 } & 0.0000 & 23.9375 & 31.5000 \\ \text { C10 } & 0.0000 & 23.9375 & 22.5000 \\ \text { C11 } & 0.0000 & 23.9375 & 13.5000 \\ \text { C12 } & 0.0000 & 23.9375 & 4.5000 \\ \text { C13 } & -23.9375 & 0.0000 & 49.5000 \\ \text { C14 } & -23.9375 & 0.0000 & 40.5000 \\ \text { C15 } & -23.9375 & 0.0000 & 31.5000 \\ \text { C16 } & -23.9375 & 0.0000 & 22.5000 \\ \text { C17 } & -23.9375 & 0.0000 & 13.5000 \\ \text { C18 } & -23.9375 & 0.0000 & 4.5000 \\ \text { C19 } & 0.0000 & -23.9375 & 49.5000 \\ \text { C20 } & 0.0000 & -23.9375 & 40.5000 \\ \text { C21 } & 0.0000 & -23.9375 & 31.5000 \\ \text { C22 } & 0.0000 & -23.9375 & 22.5000 \\ \text { C23 } & 0.0000 & -23.9375 & 13.5000 \\ \text { C24 } & 0.0000 & -23.9375 & 4.5000\end{array}$

Note 1 - The radiation readings taken for this container are normally taken with an RO- 7 radiation survey instrument. The coordinates for the contact readings are set at the center of the active portion of the RO-7 probe when the probe face is on contact with the surface of the container.

Note 2 - The center of the active portion of the RO-7 radiation survey instrument probe is one inch from the face of the probe. 
HNF-4799, Rev 0

August 25, 1999

\section{REVISED METHODOLOGY FOR DETERMINING CESIUM-137 CONTENT OF HN-200 GROUT CONTAINERS.}

TABLE 3

Coordinates of Remote Measuring Points.

$\begin{array}{lccc}\text { Point } & \mathbf{X} & \mathbf{Z} & \mathbf{Y} \\ \text { R01 } & 142.9375 & 0.0000 & 26.7500 \\ \text { R02 } & 132.0570 & 54.6998 & 26.7500 \\ \text { R03 } & 101.0721 & 101.0721 & 26.7500 \\ \text { R04 } & 54.6998 & 132.0570 & 26.7500 \\ \text { R05 } & 0.0000 & 142.9375 & 26.7500 \\ \text { R06 } & -54.6998 & 132.0570 & 26.7500 \\ \text { R07 } & -101.0721 & 101.0721 & 26.7500 \\ \text { R08 } & -132.0570 & 54.6998 & 26.7500 \\ \text { R09 } & -142.9375 & 0.0000 & 26.7500 \\ \text { R10 } & -132.0570 & -54.6998 & 26.7500 \\ \text { R11 } & -101.0721 & -101.0721 & 26.7500 \\ \text { R12 } & -54.6998 & -132.0570 & 26.7500 \\ \text { R13 } & 0.0000 & -142.9375 & 26.7500 \\ \text { R14 } & 54.6998 & -132.0570 & 26.7500 \\ \text { R15 } & 101.0721 & -101.0721 & 26.7500 \\ \text { R16 } & 132.0570 & -54.6998 & 26.7500\end{array}$

Note $\mathrm{t}$ - Remote Measuring Points (R) are 120 inches (10 feet) from the surface of the container. 


\section{REVISED METHODOLOGY FOR DETERMINING CESIUM-137 CONTENT OF HN-200 GROUT CONTAINERS.}

TABLE 4

\begin{tabular}{lccccc}
\multicolumn{5}{c}{ Coordinates and source strengths for random sources. } \\
Source & $\mathbf{X}$ & $\mathbf{Z}$ & $\mathbf{Y}$ & Validity & Strength \\
S01 & 19.3677 & 0.5818 & 25.9034 & Valid & 29.4975 \\
S02 & -10.6176 & -19.5689 & 18.8660 & Valid & 13.8502 \\
S03 & 1.7704 & -7.2590 & 41.8874 & Valid & 23.5916 \\
S04 & 5.5262 & -19.2179 & 28.6756 & Valid & 20.6982 \\
S05 & -12.5171 & 2.4304 & 36.3581 & Valid & 10.9510 \\
S06 & 10.1481 & -6.8169 & 29.6428 & Valid & 16.3182 \\
S07 & -3.0040 & -18.4824 & 1.5181 & Valid & 25.9358 \\
S08 & -1.4468 & -9.3997 & 20.8621 & Valid & 25.1678 \\
S09 & -11.3240 & -1.2877 & 45.4810 & Valid & 24.9347 \\
S10 & -8.1594 & 10.5475 & 46.4380 & Valid & 26.8139 \\
S11 & 13.0540 & 6.4320 & 29.1917 & Valid & 12.5933 \\
S12 & 17.0889 & 8.7507 & 21.2880 & Valid & 17.5307 \\
S13 & 3.5123 & 8.3040 & 41.4473 & Valid & 17.9331 \\
S14 & -18.2417 & 6.1323 & 39.9213 & Valid & 26.2138 \\
S15 & 21.0026 & 5.4076 & 10.7056 & Valid & 22.7469 \\
S16 & 12.7799 & 10.3319 & 39.3081 & Valid & 27.6556 \\
S17 & -14.4903 & 3.0983 & 8.6941 & Valid & 28.5227 \\
S18 & 18.7646 & -12.0033 & 10.5209 & Valid & 23.2528 \\
S19 & -18.2584 & 0.2785 & 21.1001 & Valid & 15.3416 \\
S20 & 2.8644 & 9.2261 & 44.4497 & Valid & 16.4988 \\
S21 & 3.0621 & -7.9137 & 19.8848 & Valid & 28.6466 \\
S22 & -17.9782 & 12.8570 & 50.0431 & Valid & 20.3724 \\
S23 & 5.0992 & 14.6169 & 52.0038 & Valid & 13.0146 \\
S24 & 7.9617 & 19.2122 & 33.0332 & Valid & 12.5419 \\
S25 & 8.3975 & 9.5559 & 39.8544 & Valid & 19.7625 \\
S26 & 4.1090 & 16.2164 & 34.1935 & Valid & 24.8512 \\
S27 & 2.2698 & -21.1016 & 13.9713 & Valid & 10.2138 \\
S28 & 18.4153 & -5.6775 & 6.4850 & Valid & 15.5133 \\
S29 & -0.0904 & 3.6427 & 38.3454 & Valid & 18.0653 \\
S30 & 7.3731 & -19.7854 & 12.4003 & Valid & 15.0401 \\
S31 & 5.5574 & -17.7939 & 21.2754 & Valid & 22.3550 \\
S32 & -0.9171 & 17.9652 & 26.2433 & Valid & 21.6866 \\
S33 & -2.7939 & -22.1819 & 29.8247 & Valid & 17.8281 \\
S34 & -14.1407 & 16.9694 & 5.4489 & Valid & 14.6776 \\
S35 & 17.6331 & 12.8346 & 30.1155 & Valid & 28.0146 \\
& & & & & \\
\hline
\end{tabular}

TABLE 5 - CALCULATION OF CONVERSION FACTORS FOR A GROUTED HN-200 GROUT 


\section{REVISED METHODOLOGY FOR DETERMINING CESIUM-137 CONTENT OF HN-200 GROUT CONTAINERS.} CONTAINER ASSUMING A HOMOGENEOUS MIXTURE OF ACTIVITY AND GROUT.

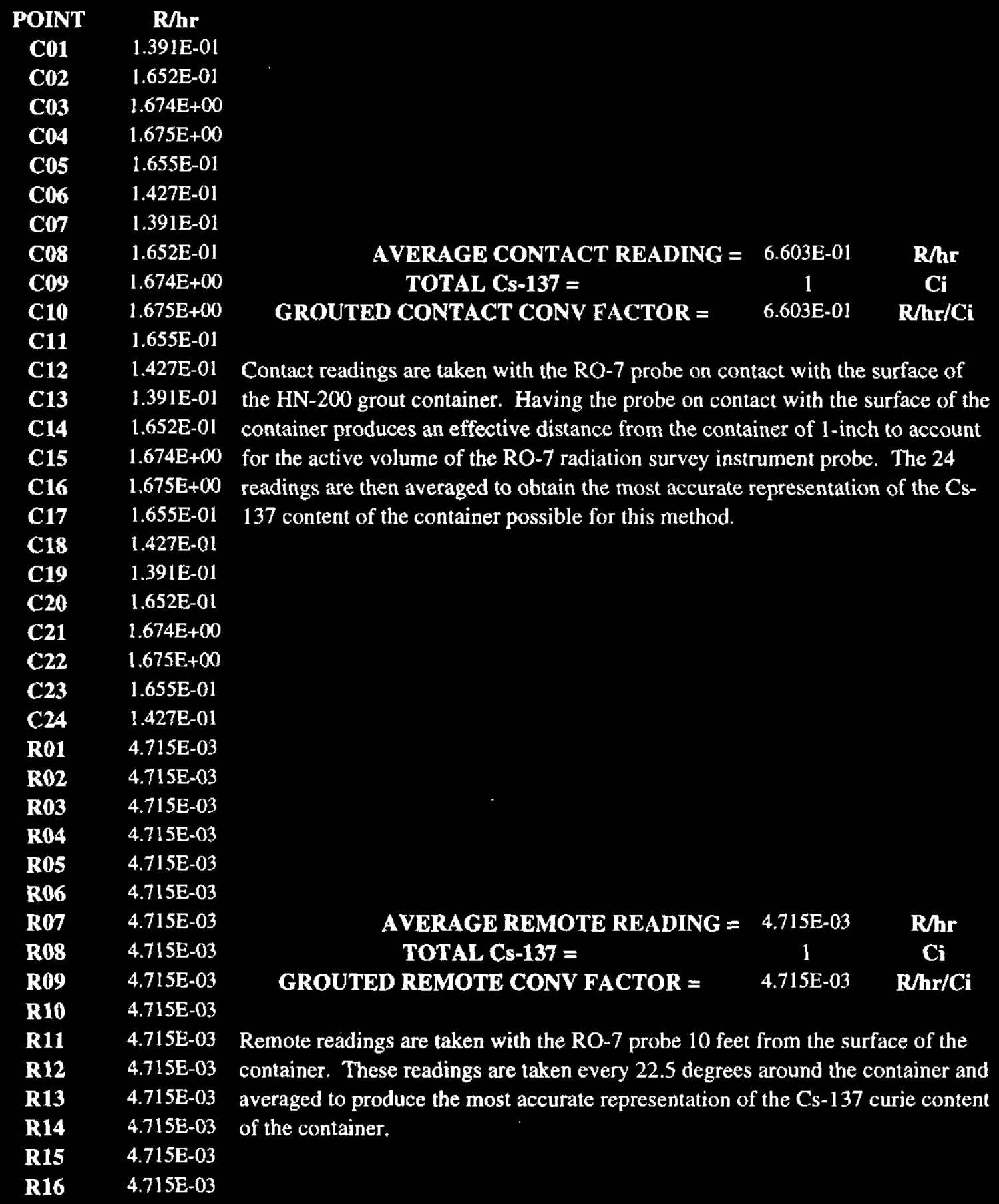

\section{TABLE 6 - RESULTS OF MICROSHIELD CALCULATION FOR EACH SOURCE TO EACH MEASUREMENT POINT FROM A GROUTED HN-200 GROUT CONTAINER.}




\section{REVISED METHODOLOGY FOR DETERMINING CESIUM-137 CONTENT OF HN-200 GROUT CONTAINERS.}

\begin{tabular}{|c|c|c|c|c|c|c|c|}
\hline \multicolumn{8}{|c|}{ SOURCE } \\
\hline OINT & S01 & $\mathrm{S} 02$ & S03 & S04 & S05 & S06 & S07 \\
\hline C01 & $4.851 \mathrm{E}-01$ & 3.042E-05 & $1.977 \mathrm{E}-01$ & $9.152 \mathrm{E}-03$ & $8.594 \mathrm{E}-04$ & $1.233 \mathrm{E}-01$ & $5.093 \mathrm{E}-06$ \\
\hline $\mathrm{CO2}$ & $7.441 E+00$ & $1.442 \mathrm{E}-04$ & $2.896 \mathrm{E}-01$ & $4.065 \mathrm{E}-02$ & $1.657 \mathrm{E}-03$ & $9.642 \mathrm{E}-01$ & $4.827 \mathrm{E}-05$ \\
\hline $\mathrm{CO3}$ & $1.373 \mathrm{E}+02$ & $4.435 \mathrm{E}-04$ & $1.430 \mathrm{E}-01$ & 8.680E-02 & $1.611 \mathrm{E}-03$ & $3.008 \mathrm{E}+00$ & $3.745 \mathrm{E}-04$ \\
\hline $\mathrm{CO4}$ & $2.734 \mathrm{E}+02$ & $7.842 \mathrm{E}-04$ & $3.038 \mathrm{E}-02$ & $7.253 \mathrm{E}-02$ & $7.941 \mathrm{E}-04$ & $1.817 \mathrm{E}+00$ & $2.139 \mathrm{E}-03$ \\
\hline $\mathrm{C} 05$ & $1.483 \mathrm{E}+01$ & $7.371 \mathrm{E}-04$ & $3.929 \mathrm{E}-03$ & $2.503 \mathrm{E}-02$ & $2.214 \mathrm{E}-04$ & $3.064 \mathrm{E}-01$ & $7.707 \mathrm{E}-03$ \\
\hline $\mathrm{C} 06$ & $9.309 \mathrm{E}-01$ & $3.717 \mathrm{E}-04$ & $3.902 \mathrm{E}-04$ & $4.615 \mathrm{E}-03$ & $4.043 \mathrm{E}-05$ & $3.071 \mathrm{E}-02$ & $1.458 \mathrm{E}-02$ \\
\hline $\mathrm{CO} 7$ & $2.836 \mathrm{E}-03$ & $7.996 \mathrm{E}-06$ & $1.598 \mathrm{E}-02$ & $8.010 \mathrm{E}-05$ & $2.868 \mathrm{E}-02$ & $1.717 \mathrm{E}-03$ & 6.778E-07 \\
\hline $\mathrm{C08}$ & $1.290 \mathrm{E}-02$ & $3.332 \mathrm{E}-05$ & $2.125 \mathrm{E}-02$ & $2.171 \mathrm{E}-04$ & $7.364 \mathrm{E}-02$ & $5.833 E-03$ & $5.108 \mathrm{E}-06$ \\
\hline $\mathrm{CO9}$ & $3.180 \mathrm{E}-02$ & $9.178 \mathrm{E}-05$ & $1.249 \mathrm{E}-02$ & $3.479 \mathrm{E}-04$ & $7.066 \mathrm{E}-02$ & $1.013 \mathrm{E}-02$ & $3.034 \mathrm{E}-05$ \\
\hline C10 & $3.526 E-02$ & $1.524 \mathrm{E}-04$ & $3.648 \mathrm{E}-03$ & $3.118 \mathrm{E}-04$ & $2.569 \mathrm{E}-02$ & $8.022 \mathrm{E}-03$ & $1.302 \mathrm{E}-04$ \\
\hline C11 & $1.715 \mathrm{E}-02$ & $1.443 \mathrm{E}-04$ & $6.476 \mathrm{E}-04$ & $1.585 \mathrm{E}-04$ & $4.687 \mathrm{E}-03$ & $3.049 \mathrm{E}-03$ & $3.632 \mathrm{E}-04$ \\
\hline $\mathrm{C} 12$ & $4.290 \mathrm{E}-03$ & $7.834 \mathrm{E}-05$ & 8.302E-05 & $4.926 \mathrm{E}-05$ & $5.736 \mathrm{E}-04$ & $6.601 \mathrm{E}-04$ & $5.943 \mathrm{E}-04$ \\
\hline C13 & $8.096 \mathrm{E}-05$ & $1.434 \mathrm{E}-03$ & $6.767 \mathrm{E}-02$ & 8.765E-04 & $1.018 \mathrm{E}+00$ & $8.816 \mathrm{E}-04$ & $1.221 \mathrm{E}-05$ \\
\hline C14 & $2.543 E-04$ & $1.152 \mathrm{E}-02$ & $9.444 \mathrm{E}-02$ & $2.899 \mathrm{E}-03$ & $6.201 \mathrm{E}+00$ & $2.789 \mathrm{E}-03$ & $1.290 \mathrm{E}-04$ \\
\hline C15 & $4.846 \mathrm{E}-04$ & $6.075 \mathrm{E}-02$ & $5.081 \mathrm{E}-02$ & $5.181 \mathrm{E}-03$ & $5.647 \mathrm{E}+00$ & $4.663 \mathrm{E}-03$ & $1.151 \mathrm{E}-03$ \\
\hline C16 & $5.208 \mathrm{E}-04$ & $1.530 \mathrm{E}-01$ & $1.254 \mathrm{E}-02$ & $4.524 \mathrm{E}-03$ & $8.482 \mathrm{E}-01$ & $3.755 \mathrm{E}-03$ & $7.765 \mathrm{E}-03$ \\
\hline $\mathrm{Cl7}$ & 3.127E-04 & $1.378 \mathrm{E}-01$ & $1.873 \mathrm{E}-03$ & $1.979 \mathrm{E}-03$ & $7.286 \mathrm{E}-02$ & $1.517 \mathrm{E}-03$ & $3.312 \mathrm{E}-02$ \\
\hline C18 & $1.114 \mathrm{E}-04$ & $4.621 \mathrm{E}-02$ & $2.078 \mathrm{E}-04$ & $4.952 \mathrm{E}-04$ & $5.390 \mathrm{E}-03$ & $3.550 \mathrm{E}-04$ & $6.949 \mathrm{E}-02$ \\
\hline C19 & $2.236 \mathrm{E}-03$ & $1.562 \mathrm{E}-02$ & $1.557 \mathrm{E}+00$ & $6.070 \mathrm{E}-01$ & $7.981 \mathrm{E}-03$ & 4.501E-02 & $2.784 \mathrm{E}-04$ \\
\hline $\mathrm{C} 20$ & $9.847 \mathrm{E}-03$ & $1.838 \mathrm{E}-01$ & $2.680 \mathrm{E}+\infty 0$ & $8.256 \mathrm{E}+00$ & $1.803 \mathrm{E}-02$ & $2.610 \mathrm{E}-01$ & $3.784 \mathrm{E}-03$ \\
\hline C21 & $2.369 \mathrm{E}-02$ & $1.878 \mathrm{E}+00$ & $1.002 \mathrm{E}+00$ & $7.616 \mathrm{E}+01$ & $1.741 \mathrm{E}-02$ & $6.325 \mathrm{E}-01$ & $5.260 \mathrm{E}-02$ \\
\hline $\mathrm{C} 22$ & $2.620 \mathrm{E}-02$ & $1.014 \mathrm{E}+01$ & $1.452 \mathrm{E}-01$ & $3.839 E+01$ & $7.246 \mathrm{E}-03$ & $4.308 \mathrm{E}-01$ & $7.465 \mathrm{E}-01$ \\
\hline $\mathrm{C} 23$ & $1.300 \mathrm{E}-02$ & $8.133 \mathrm{E}+00$ & $1.390 \mathrm{E}-02$ & $3.142 \mathrm{E}+00$ & $1.584 \mathrm{E}-03$ & $1.002 \mathrm{E}-01$ & $1.130 \mathrm{E}+01$ \\
\hline $\mathrm{C} 24$ & $3.356 \mathrm{E}-03$ & $1.229 \mathrm{E}+00$ & $1.120 \mathrm{E}-03$ & $2.309 \mathrm{E}-01$ & $2.274 \mathrm{E}-04$ & $1.273 \mathrm{E}-02$ & $1.372 \mathrm{E}+02$ \\
\hline R01 & $6.719 \mathrm{E}-01$ & $1.026 \mathrm{E}-03$ & $1.206 \mathrm{E}-02$ & $1.401 \mathrm{E}-01$ & $7.999 \mathrm{E}-05$ & $6.868 \mathrm{E}-02$ & $1.294 \mathrm{E}-02$ \\
\hline R02 & $6.295 \mathrm{E}-01$ & $6.058 \mathrm{E}-05$ & $5.622 \mathrm{E}-03$ & $1.978 \mathrm{E}-02$ & $1.922 \mathrm{E}-04$ & $3.828 \mathrm{E}-02$ & $1.125 \mathrm{E}-03$ \\
\hline $\mathbf{R 0 3}$ & $4.858 \mathrm{E}-01$ & $1.110 \mathrm{E}-05$ & $2.550 \mathrm{E}-03$ & $1.372 \mathrm{E}-03$ & $6.436 \mathrm{E}-04$ & $1.463 \mathrm{E}-02$ & $1.386 \mathrm{E}-04$ \\
\hline R04 & $2.529 \mathrm{E}-01$ & $7.969 \mathrm{E}-06$ & $1.311 \mathrm{E}-03$ & $1.281 \mathrm{E}-04$ & $2.739 \mathrm{E}-03$ & $4.077 \mathrm{E}-03$ & $4.138 \mathrm{E}-05$ \\
\hline R05 & $5.556 \mathrm{E}-02$ & $2.331 \mathrm{E}-05$ & $8.822 \mathrm{E}-04$ & $3.026 \mathrm{E}-05$ & $1.045 \mathrm{E}-02$ & $1.032 \mathrm{E}-03$ & $3.733 \mathrm{E}-05$ \\
\hline R06 & $4.974 \mathrm{E}-03$ & $2.432 \mathrm{E}-04$ & $8.469 \mathrm{E}-04$ & $2.327 \mathrm{E}-05$ & $2.822 \mathrm{E}-02$ & $3.275 \mathrm{E}-04$ & $1.036 \mathrm{E}-04$ \\
\hline R07 & $4.271 \mathrm{E}-04$ & $5.955 \mathrm{E}-03$ & $1.170 \mathrm{E}-03$ & $6.060 \mathrm{E}-05$ & $5.113 \mathrm{E}-02$ & $1.723 \mathrm{E}-04$ & 7.373E-04 \\
\hline R08 & $7.450 \mathrm{E}-05$ & $1.043 \mathrm{E}-01$ & $2.170 \mathrm{E}-03$ & $4.588 \mathrm{E}-04$ & $6.705 \mathrm{E}-02$ & $1.730 \mathrm{E}-04$ & $8.436 \mathrm{E}-03$ \\
\hline R09 & $3.781 \mathrm{E}-05$ & $3.039 \mathrm{E}-01$ & $4.718 \mathrm{E}-03$ & $6.490 \mathrm{E}-03$ & $6.725 \mathrm{E}-02$ & $3.313 \mathrm{E}-04$ & $7.054 \mathrm{E}-02$ \\
\hline R10 & $6.235 \mathrm{E}-05$ & $3.890 \mathrm{E}-01$ & $1.030 \mathrm{E}-02$ & $7.063 \mathrm{E}-02$ & $5.163 \mathrm{E}-02$ & $1.049 \mathrm{E}-03$ & $2.359 E-01$ \\
\hline R11 & $3.083 E-04$ & $4.307 \mathrm{E}-01$ & $1.995 \mathrm{E}-02$ & $2.532 \mathrm{E}-01$ & $2.873 E-02$ & $4.148 \mathrm{E}-03$ & $3.998 \mathrm{E}-01$ \\
\hline R12 & $3.350 \mathrm{E}-03$ & $4.389 \mathrm{E}-01$ & $3.186 \mathrm{E}-02$ & $4.138 \mathrm{E}-01$ & $1.074 \mathrm{E}-02$ & $1.484 \mathrm{E}-02$ & $4.910 \mathrm{E}-01$ \\
\hline R13 & $4.001 E-02$ & $4.130 \mathrm{E}-01$ & $4.078 \mathrm{E}-02$ & $4.977 \mathrm{E}-01$ & $2.832 \mathrm{E}-03$ & $3.865 \mathrm{E}-02$ & $4.985 \mathrm{E}-01$ \\
\hline R14 & $2.149 \mathrm{E}-01$ & 3.508E-01 & $4.178 \mathrm{E}-02$ & $5.122 \mathrm{E}-01$ & $6.648 \mathrm{E}-04$ & $6.904 \mathrm{E}-02$ & $4.221 \mathrm{E}-01$ \\
\hline R15 & $4.549 \mathrm{E}-01$ & $2.123 \mathrm{E}-01$ & $3.428 \mathrm{E}-02$ & $4.584 \mathrm{E}-01$ & $1.966 \mathrm{E}-04$ & $9.004 \mathrm{E}-02$ & $2.689 \mathrm{E}-01$ \\
\hline R16 & $6.142 \mathrm{E}-01$ & $2.709 \mathrm{E}-02$ & $2.251 \mathrm{E}-02$ & $3.309 \mathrm{E}-01$ & $9.879 \mathrm{E}-05$ & $8.989 \mathrm{E}-02$ & $9.392 \mathrm{E}-02$ \\
\hline
\end{tabular}

$\begin{array}{cccccccc}\text { POINT } & \text { S08 } & \text { S09 } & \text { S10 } & \text { S11 } & \text { S12 } & \text { S13 } & \text { S14 } \\ \text { C01 } & 1.751 E-03 & 5.663 E-03 & 1.027 E-02 & 1.480 \mathrm{E}-01 & 3.344 \mathrm{E}-02 & 2.150 \mathrm{E}-01 & 4.300 \mathrm{E}-04\end{array}$


HNF-4799, Rev 0

August 25, 1999

\section{REVISED METHODOLOGY FOR DETERMINING CESIUM-137 CONTENT OF HN-200 GROUT CONTAINERS.}

\begin{tabular}{|c|c|c|c|c|c|c|c|}
\hline C02 & $1.166 \mathrm{E}-02$ & $5.447 \mathrm{E}-03$ & $9.090 \mathrm{E}-03$ & $1.455 \mathrm{E}+00$ & $4.133 \mathrm{E}-01$ & $3.406 \mathrm{E}-01$ & $6.019 \mathrm{E}-04$ \\
\hline $\mathrm{CO3}$ & $4.737 \mathrm{E}-02$ & $2.597 \mathrm{E}-03$ & $3.912 \mathrm{E}-03$ & $6.067 \mathrm{E}+00$ & 4. $192 \mathrm{E}+00$ & $1.703 \mathrm{E}-01$ & $4.639 \mathrm{E}-04$ \\
\hline $\mathrm{CO4}$ & 8.953E-02 & $6.932 \mathrm{E}-04$ & $9.462 \mathrm{E}-04$ & $3.627 \mathrm{E}+00$ & $1.654 \mathrm{E}+01$ & $3.513 \mathrm{E}-02$ & $2.035 \mathrm{E}-04$ \\
\hline C05 & $6.615 \mathrm{E}-02$ & $1.212 \mathrm{E}-04$ & $1.524 \mathrm{E}-04$ & $5.014 \mathrm{E}-01$ & $7.058 E+00$ & $4.371 \mathrm{E}-03$ & $5.564 \mathrm{E}-05$ \\
\hline $\mathrm{COS}$ & $2.086 \mathrm{E}-02$ & $1.587 \mathrm{E}-05$ & $1.870 \mathrm{E}-05$ & $4.408 \mathrm{E}-02$ & $7.970 \mathrm{E}-01$ & $4.207 \mathrm{E}-04$ & $1.056 \mathrm{E}-05$ \\
\hline $\mathrm{C} 07$ & $3.915 \mathrm{E}-04$ & $6.799 \mathrm{E}-02$ & $4.200 \mathrm{E}+00$ & $2.000 \mathrm{E}-02$ & $3.689 \mathrm{E}-03$ & $1.408 \mathrm{E}+00$ & $9.517 \mathrm{E}-02$ \\
\hline $\mathrm{COS}$ & $2.072 \mathrm{E}-03$ & $6.467 \mathrm{E}-02$ & $3.202 \mathrm{E}+00$ & $1.075 \mathrm{E}-01$ & $2.838 \mathrm{E}-02$ & $2.685 \mathrm{E}+00$ & $1.664 \mathrm{E}-01$ \\
\hline $\mathrm{COS}$ & $6.739 \mathrm{E}-03$ & $2.545 \mathrm{E}-02$ & $6.241 \mathrm{E}-01$ & $2.534 \mathrm{E}-01$ & $1.329 \mathrm{E}-01$ & $1.029 \mathrm{E}+00$ & $1.077 \mathrm{E}-01$ \\
\hline $\mathrm{C} 10$ & $1.131 \mathrm{E}-02$ & $5.192 \mathrm{E}-03$ & $6.683 \mathrm{E}-02$ & $1.899 \mathrm{E}-01$ & $2.659 \mathrm{E}-01$ & $1.456 \mathrm{E}-01$ & $2.959 \mathrm{E}-02$ \\
\hline C11 & $8.855 \mathrm{E}-03$ & $7.012 \mathrm{E}-04$ & $5.600 \mathrm{E}-03$ & $5.122 \mathrm{E}-02$ & $1.769 \mathrm{E}-01$ & $1.367 \mathrm{E}-02$ & $4.642 \mathrm{E}-03$ \\
\hline $\mathrm{Cl2}$ & $3.400 \mathrm{E}-03$ & $7.389 \mathrm{E}-05$ & $4.257 \mathrm{E}-04$ & $7.345 \mathrm{E}-03$ & $4.578 \mathrm{E}-02$ & $1.085 \mathrm{E}-03$ & $5.285 \mathrm{E}-04$ \\
\hline C13 & $3.175 \mathrm{E}-03$ & $1.005 \mathrm{E}+01$ & $1.358 \mathrm{E}+00$ & $2.892 \mathrm{E}-04$ & $3.289 \mathrm{E}-05$ & $2.659 \mathrm{E}-02$ & $1.479 \mathrm{E}+01$ \\
\hline C14 & $2.366 \mathrm{E}-02$ & $8.959 E+00$ & $1.088 \mathrm{E}+00$ & 8.723E-04 & $1.327 \mathrm{E}-04$ & $3.788 \mathrm{E}-02$ & $7.386 \mathrm{E}+01$ \\
\hline C15 & $1.079 \mathrm{E}-01$ & $1.473 E+00$ & $2.689 \mathrm{E}-01$ & $1.450 \mathrm{E}-03$ & 3.396E-04 & $2.214 \mathrm{E}-02$ & $1.993 E+01$ \\
\hline C16 & $2.179 \mathrm{E}-01$ & $1.343 \mathrm{E}-01$ & $3.460 \mathrm{E}-02$ & $1.228 \mathrm{E}-03$ & $4.983 \mathrm{E}-04$ & $6.091 \mathrm{E}-03$ & $1.656 \mathrm{E}+00$ \\
\hline $\mathrm{Cl7}$ & $1.557 \mathrm{E}-01$ & $1.020 \mathrm{E}-02$ & $3.274 \mathrm{E}-03$ & $5.446 \mathrm{E}-04$ & $3.990 \mathrm{E}-04$ & $1.003 \mathrm{E}-03$ & $1.236 \mathrm{E}+00$ \\
\hline C18 & $4.420 \mathrm{E}-02$ & $7.273 \mathrm{E}-04$ & 2.686E-04 & $1.418 \mathrm{E}-04$ & $1.796 \mathrm{E}-04$ & $1.201 \mathrm{E}-04$ & $9.105 \mathrm{E}-03$ \\
\hline C19 & $1.999 \mathrm{E}-02$ & $1.456 \mathrm{E}-01$ & $6.000 \mathrm{E}-03$ & $1.027 \mathrm{E}-03$ & $1.256 \mathrm{E}-04$ & $8.074 \mathrm{E}-03$ & $4.516 \mathrm{E}-03$ \\
\hline $\mathrm{C} 20$ & $2.312 \mathrm{E}-01$ & $1.378 \mathrm{E}-01$ & $5.344 \mathrm{E}-03$ & $3.514 \mathrm{E}-03$ & $5.818 \mathrm{E}-04$ & $1.103 \mathrm{E}-02$ & $6.787 \mathrm{E}-03$ \\
\hline $\mathrm{C21}$ & $1.904 \mathrm{E}+00$ & $5.027 \mathrm{E}-02$ & $2.387 \mathrm{E}-03$ & $6.256 \mathrm{E}-03$ & $1.668 \mathrm{E}-03$ & $6.863 \mathrm{E}-03$ & $4.948 \mathrm{E}-03$ \\
\hline C22 & $6.080 \mathrm{E}+00$ & $9.278 \mathrm{E}-03$ & $6.077 \mathrm{E}-04$ & $5.177 \mathrm{E}-03$ & $2.579 \mathrm{E}-03$ & $2.145 \mathrm{E}-03$ & $1.855 \mathrm{E}-03$ \\
\hline $\mathrm{C} 23$ & $3.418 \mathrm{E}+00$ & $1.148 \mathrm{E}-03$ & $1.030 \mathrm{E}-04$ & $2.072 \mathrm{E}-03$ & $2.003 \mathrm{E}-03$ & $4.051 \mathrm{E}-04$ & $4.136 \mathrm{E}-04$ \\
\hline C24 & $5.267 \mathrm{E}-01$ & $1.131 \mathrm{E}-04$ & $1.323 \mathrm{E}-05$ & $4.701 \mathrm{E}-04$ & $8.151 \mathrm{E}-04$ & $5.461 \mathrm{E}-05$ & $6.393 \mathrm{E}-05$ \\
\hline R01 & $6.521 \mathrm{E}-03$ & $2.896 \mathrm{E}-04$ & $1.106 \mathrm{E}-03$ & $9.996 \mathrm{E}-02$ & $3.447 \mathrm{E}-01$ & $1.542 \mathrm{E}-02$ & $4.964 \mathrm{E}-05$ \\
\hline $\mathbf{R} 02$ & $2.326 \mathrm{E}-03$ & $3.301 \mathrm{E}-04$ & $5.077 \mathrm{E}-03$ & $1.181 \mathrm{E}-01$ & $3.867 \mathrm{E}-01$ & $2.900 \mathrm{E}-02$ & $2.204 \mathrm{E}-04$ \\
\hline $\mathbf{R} 03$ & $9.657 \mathrm{E}-04$ & $6.710 \mathrm{E}-04$ & $2.174 \mathrm{E}-02$ & $1.088 \mathrm{E}-01$ & 3.692E-01 & 4.194E-02 & $2.232 \mathrm{E}-03$ \\
\hline R04 & $5.710 \mathrm{E}-04$ & $2.078 \mathrm{E}-03$ & $6.542 \mathrm{E}-02$ & $7.602 E-02$ & $2.918 \mathrm{E}-01$ & $4.714 \mathrm{E}-02$ & $2.696 \mathrm{E}-02$ \\
\hline R05 & 5.405E-04 & $7.455 \mathrm{E}-03$ & $1.275 \mathrm{E}-01$ & $3.632 \mathrm{E}-02$ & $1.612 \mathrm{E}-01$ & $4.155 \mathrm{E}-02$ & $1.607 \mathrm{E}-01$ \\
\hline R06 & $8.287 \mathrm{E}-04$ & 2.349E-02 & $1.756 \mathrm{E}-01$ & $1.051 \mathrm{E}-02$ & $4.052 \mathrm{E}-02$ & $2.845 \mathrm{E}-02$ & $3.687 \mathrm{E}-01$ \\
\hline R07 & $1.874 \mathrm{E}-03$ & $5.472 \mathrm{E}-02$ & $1.838 \mathrm{E}-01$ & $2.049 \mathrm{E}-03$ & $4.023 \mathrm{E}-03$ & $1.499 \mathrm{E}-02$ & $5,165 \mathrm{E}-01$ \\
\hline R08 & $5.179 \mathrm{E}-03$ & $9.045 E-02$ & $1.479 \mathrm{E}-01$ & 3.873E-04 & $3.421 \mathrm{E}-04$ & $6.319 \mathrm{E}-03$ & $5.759 \mathrm{E}-01$ \\
\hline R09 & $1.405 \mathrm{E}-02$ & $1.114 \mathrm{E}-01$ & 8.672E-02 & $1.108 \mathrm{E}-04$ & $5.424 \mathrm{E}-05$ & $2.384 \mathrm{E}-03$ & $5.472 \mathrm{E}-01$ \\
\hline R10 & $3.181 \mathrm{E}-02$ & $1.054 \mathrm{E}-01$ & $3.375 \mathrm{E}-02$ & $6.429 \mathrm{E}-05$ & $2.397 \mathrm{E}-05$ & $9.628 \mathrm{E}-04$ & $4.300 \mathrm{E}-01$ \\
\hline R11 & $5.519 E-02$ & $7.602 \mathrm{E}-02$ & $8.708 \mathrm{E}-03$ & $8.473 \mathrm{E}-05$ & 3.385E-05 & $5.099 \mathrm{E}-04$ & $2.340 \mathrm{E}-01$ \\
\hline R12 & $7.297 \mathrm{E}-02$ & 3.995E-02 & $1.865 \mathrm{E}-03$ & $2.398 \mathrm{E}-04$ & $1.445 \mathrm{E}-04$ & $4.083 \mathrm{E}-04$ & $5.671 \mathrm{E}-02$ \\
\hline R13 & $7.500 \mathrm{E}-02$ & $1.488 \mathrm{E}-02$ & $4.779 \mathrm{E}-04$ & $1.157 \mathrm{E}-03$ & $1.418 \mathrm{E}-03$ & $5.187 \mathrm{E}-04$ & $5.445 \mathrm{E}-03$ \\
\hline R14 & $6.007 \mathrm{E}-02$ & $4.349 \mathrm{E}-03$ & $2.066 \mathrm{E}-04$ & $6.288 \mathrm{E}-03$ & $1.698 \mathrm{E}-02$ & $9.927 \mathrm{E}-04$ & 4.601E-04 \\
\hline R15 & $3.684 \mathrm{E}-02$ & $1.242 \mathrm{E}-03$ & $1.810 \mathrm{E}-04$ & 2.553E-02 & $1.036 \mathrm{E}-01$ & $2.473 \mathrm{E}-03$ & $7.425 \mathrm{E}-05$ \\
\hline R16 & $1.719 \mathrm{E}-02$ & $4.664 \mathrm{E}-04$ & 3.305E-04 & $6.249 \mathrm{E}-02$ & $2.432 \mathrm{E}-01$ & $6.548 \mathrm{E}-03$ & $3.384 \mathrm{E}-05$ \\
\hline
\end{tabular}

\begin{tabular}{cccccccc} 
& \multicolumn{9}{c}{ SOURCE } \\
POINT & S15 & S16 & S17 & S18 & S19 & S20 & S21 \\
C01 & $7.437 \mathrm{E}-03$ & $2.020 \mathrm{E}+00$ & $9.333 \mathrm{E}-06$ & $1.946 \mathrm{E}-03$ & $2.597 \mathrm{E}-05$ & $1.919 \mathrm{E}-01$ & $4.335 \mathrm{E}-03$ \\
C02 & $8.533 \mathrm{E}-02$ & $5.704 \mathrm{E}+00$ & $6.488 \mathrm{E}-05$ & $2.387 \mathrm{E}-02$ & $1.050 \mathrm{E}-04$ & $2.056 \mathrm{E}-01$ & $3.662 \mathrm{E}-02$
\end{tabular}




\section{REVISED METHODOLOGY FOR DETERMINING CESIUM-137 CONTENT OF HN-200 GROUT CONTAINERS.}

\begin{tabular}{|c|c|c|c|c|c|c|c|}
\hline $\mathrm{COB}$ & $1.011 \mathrm{E}+00$ & $3.040 \mathrm{E}+00$ & $3.341 \mathrm{E}-04$ & $2.689 \mathrm{E}-01$ & $2.704 \mathrm{E}-04$ & $7.615 \mathrm{E}-02$ & $1.974 \mathrm{E}-01$ \\
\hline C04 & $1.296 \mathrm{E}+01$ & $4.826 \mathrm{E}-01$ & $1.131 \mathrm{E}-03$ & $2.434 \mathrm{E}+00$ & $4.008 \mathrm{E}-04$ & $1.340 \mathrm{E}-02$ & $4.743 \mathrm{E}-01$ \\
\hline C05 & $1.389 \mathrm{E}+02$ & $4.770 \mathrm{E}-02$ & $2.205 \mathrm{E}-03$ & $1.028 \mathrm{E}+01$ & $3.254 \mathrm{E}-04$ & $1.563 \mathrm{E}-03$ & $3.718 \mathrm{E}-01$ \\
\hline $\mathrm{CO6}$ & $6.281 E+01$ & $3.890 \mathrm{E}-03$ & $2.256 \mathrm{E}-03$ & $7.343 \mathrm{E}+00$ & $1.487 \mathrm{E}-04$ & $1.464 \mathrm{E}-04$ & $1.051 \mathrm{E}-01$ \\
\hline $\mathrm{C} 07$ & $1.216 \mathrm{E}-04$ & $7.284 E-01$ & $1.377 \mathrm{E}-04$ & $7.465 \mathrm{E}-06$ & $6.094 \mathrm{E}-04$ & $2.700 \mathrm{E}+00$ & $5.056 \mathrm{E}-04$ \\
\hline C08 & $1.070 \mathrm{E}-03$ & $1.706 \mathrm{E}+00$ & $1.384 \mathrm{E}-03$ & 4.654E-05 & $3.584 \mathrm{E}-03$ & $3.013 E+\infty$ & $2.926 \mathrm{E}-03$ \\
\hline C09 & $7.103 \mathrm{E}-03$ & $1.024 \mathrm{E}+00$ & $1.105 \mathrm{E}-02$ & $2.099 \mathrm{E}-04$ & $1.280 \mathrm{E}-02$ & $6.952 \mathrm{E}-01$ & $1.058 \mathrm{E}-02$ \\
\hline C10 & $2.971 \mathrm{E}-02$ & $2.094 \mathrm{E}-01$ & $5.893 \mathrm{E}-02$ & $6.108 \mathrm{E}-04$ & $2.230 \mathrm{E}-02$ & $7.699 \mathrm{E}-01$ & $1.970 \mathrm{E}-02$ \\
\hline C11 & $6.101 \mathrm{E}-02$ & $2.458 \mathrm{E}-02$ & $1.583 \mathrm{E}-01$ & $1.023 \mathrm{E}-03$ & $1.659 \mathrm{E}-02$ & $6.440 \mathrm{E}-03$ & 1.664E-02 \\
\hline C12 & $5.129 \mathrm{E}-02$ & $2.237 \mathrm{E}-03$ & $1.639 \mathrm{E}-01$ & $9.193 \mathrm{E}-04$ & $5.676 \mathrm{E}-03$ & $4.837 \mathrm{E}-04$ & $6.619 \mathrm{E}-03$ \\
\hline C13 & $2.418 \mathrm{E}-06$ & $1.760 \mathrm{E}-03$ & $1.277 \mathrm{E}-03$ & $2.981 \mathrm{E}-06$ & $4.990 \mathrm{E}-02$ & $3.387 \mathrm{E}-02$ & $1.246 \mathrm{E}-03$ \\
\hline C14 & $1.335 \mathrm{E}-05$ & $2.680 \mathrm{E}-03$ & $1.839 \mathrm{E}-02$ & $1.690 \mathrm{E}-05$ & $7.332 \mathrm{E}-01$ & $3.582 \mathrm{E}-02$ & 8.297E-03 \\
\hline C15 & $5.314 \mathrm{E}-05$ & $2.095 \mathrm{E}-03$ & $2.580 \mathrm{E}-01$ & $6.927 \mathrm{E}-05$ & $1.177 \mathrm{E}+01$ & $1.575 \mathrm{E}-02$ & $3.450 \mathrm{E}-02$ \\
\hline C16 & $1.389 \mathrm{E}-04$ & $8.745 E-04$ & $3.329 E+00$ & $1.860 \mathrm{E}-04$ & $1.311 \mathrm{E}+02$ & $3.511 \mathrm{E}-03$ & $6.987 \mathrm{E}-02$ \\
\hline C17 & $2.181 \mathrm{E}-04$ & $2.190 \mathrm{E}-04$ & $2.709 \mathrm{E}+01$ & $2.981 \mathrm{E}-04$ & $2.808 \mathrm{E}+01$ & $5.047 \mathrm{E}-04$ & $5.764 \mathrm{E}-02$ \\
\hline C18 & $1.960 \mathrm{E}-04$ & $3.761 \mathrm{E}-05$ & $2.977 \mathrm{E}+01$ & 2.705E-04 & $1.727 \mathrm{E}+00$ & $5.546 \mathrm{E}-05$ & 2.042E-02 \\
\hline C19 & $2.130 \mathrm{E}-05$ & $2.879 \mathrm{E}-03$ & $5.004 \mathrm{E}-05$ & $3.740 \mathrm{E}-04$ & $5.476 \mathrm{E}-04$ & $6.827 \mathrm{E}-03$ & $1.308 \mathrm{E}-02$ \\
\hline $\mathrm{C} 20$ & $1.488 \mathrm{E}-04$ & $4.463 \mathrm{E}-03$ & $4.324 \mathrm{E}-04$ & $3.866 \mathrm{E}-03$ & $3.169 \mathrm{E}-03$ & $7.158 \mathrm{E}-03$ & $1.440 \mathrm{E}-01$ \\
\hline C21 & $7.544 \mathrm{E}-04$ & $3.452 \mathrm{E}-03$ & $2.850 \mathrm{E}-03$ & $3.207 \mathrm{E}-02$ & $1.115 \mathrm{E}-02$ & $3.541 \mathrm{E}-03$ & $1.107 \mathrm{E}+00$ \\
\hline $\mathrm{C} 22$ & $2.430 \mathrm{E}-03$ & $1.392 \mathrm{E}-03$ & $1.228 \mathrm{E}-02$ & $1.730 \mathrm{E}-01$ & $1.927 \mathrm{E}-02$ & $9.421 \mathrm{E}-04$ & $3.635 E+00$ \\
\hline $\mathrm{C} 23$ & $4.269 \mathrm{E}-03$ & $3.333 \mathrm{E}-04$ & $2.821 \mathrm{E}-02$ & $4.282 \mathrm{E}-01$ & $1.440 \mathrm{E}-02$ & $1.609 \mathrm{E}-04$ & $2.582 \mathrm{E}+00$ \\
\hline C24 & $3.732 \mathrm{E}-03$ & $5.473 \mathrm{E}-05$ & $2.903 \mathrm{E}-02$ & $3.525 \mathrm{E}-01$ & $4.994 \mathrm{E}-03$ & 2.045E-05 & $5.024 \mathrm{E}-01$ \\
\hline R01 & $6.645 \mathrm{E}-01$ & $2.609 \mathrm{E}-01$ & $1.395 \mathrm{E}-04$ & $6.716 \mathrm{E}-01$ & $2.678 \mathrm{E}-05$ & $1.258 \mathrm{E}-02$ & $2.200 \mathrm{E}-02$ \\
\hline R02 & $6.737 \mathrm{E}-01$ & $3.517 \mathrm{E}-01$ & $3.269 \mathrm{E}-04$ & $5.556 \mathrm{E}-01$ & $4.826 \mathrm{E}-05$ & $2.622 \mathrm{E}-02$ & $9.696 \mathrm{E}-03$ \\
\hline $\mathbf{R} 03$ & $6.195 \mathrm{E}-01$ & $3.700 \mathrm{E}-01$ & $1.453 \mathrm{E}-03$ & $2.808 \mathrm{E}-01$ & $2.341 \mathrm{E}-04$ & $4.121 \mathrm{E}-02$ & $3.899 \mathrm{E}-03$ \\
\hline R04 & $5.000 \mathrm{E}-01$ & $3.106 \mathrm{E}-01$ & $8.422 \mathrm{E}-03$ & $2.499 \mathrm{E}-02$ & $2.186 \mathrm{E}-03$ & $4.955 \mathrm{E}-02$ & $1.691 \mathrm{E}-03$ \\
\hline R05 & $2.468 \mathrm{E}-01$ & $1.926 \mathrm{E}-01$ & $4.015 \mathrm{E}-02$ & $9.231 \mathrm{E}-04$ & $2.033 \mathrm{E}-02$ & $4.636 \mathrm{E}-02$ & $9.500 \mathrm{E}-04$ \\
\hline R06 & $2.705 \mathrm{E}-02$ & $7.269 \mathrm{E}-02$ & $1.162 \mathrm{E}-01$ & $6.438 \mathrm{E}-05$ & $9.317 \mathrm{E}-02$ & $3.353 \mathrm{E}-02$ & $7.864 \mathrm{E}-04$ \\
\hline R07 & $1.192 \mathrm{E}-03$ & $1.424 \mathrm{E}-02$ & $2.084 \mathrm{E}-01$ & $1.491 \mathrm{E}-05$ & $1.955 \mathrm{E}-01$ & $1.833 \mathrm{E}-02$ & $9,991 \mathrm{E}-04$ \\
\hline R08 & $8.522 \mathrm{E}-05$ & $1.931 \mathrm{E}-03$ & $2.667 \mathrm{E}-01$ & $1.387 \mathrm{E}-05$ & $2.679 \mathrm{E}-01$ & $7.690 \mathrm{E}-03$ & $1.849 \mathrm{E}-03$ \\
\hline 09 & $1.852 \mathrm{E}-05$ & $3.168 \mathrm{E}-04$ & $2.643 \mathrm{E}-01$ & $5.226 \mathrm{E}-05$ & $2.917 \mathrm{E}-01$ & $2.729 \mathrm{E}-03$ & $4.347 \mathrm{E}-03$ \\
\hline R10 & $1.525 \mathrm{E}-05$ & $1.019 \mathrm{E}-04$ & $2.023 \mathrm{E}-01$ & $6.738 \mathrm{E}-04$ & $2.639 \mathrm{E}-01$ & $9.803 \mathrm{E}-04$ & $1.078 \mathrm{E}-02$ \\
\hline R11 & $4.870 \mathrm{E}-05$ & $8.154 \mathrm{E}-05$ & $1.095 \mathrm{E}-01$ & $1.789 \mathrm{E}-02$ & $1.881 \mathrm{E}-01$ & $4.477 \mathrm{E}-04$ & $2.398 \mathrm{E}-02$ \\
\hline R12 & $5.161 \mathrm{E}-04$ & $1.691 \mathrm{E}-04$ & $3.636 \mathrm{E}-02$ & $2.405 \mathrm{E}-01$ & $8.548 \mathrm{E}-02$ & $3.111 \mathrm{E}-04$ & $4.334 \mathrm{E}-02$ \\
\hline $\mathbf{R} 13$ & $1.130 \mathrm{E}-02$ & $7.886 \mathrm{E}-04$ & $7.424 \mathrm{E}-03$ & $5.390 \mathrm{E}-01$ & $1.740 \mathrm{E}-02$ & $3.559 \mathrm{E}-04$ & $6.122 \mathrm{E}-02$ \\
\hline R14 & 1.583E-01 & $5.739 \mathrm{E}-03$ & $1.285 \mathrm{E}-03$ & 6.631E-01 & $1.820 \mathrm{E}-03$ & $6.514 \mathrm{E}-04$ & $6.792 \mathrm{E}-02$ \\
\hline R15 & $4.466 \mathrm{E}-01$ & $3,696 \mathrm{E}-02$ & $2.997 \mathrm{E}-04$ & $7.204 \mathrm{E}-01$ & $2.011 \mathrm{E}-04$ & $1,666 \mathrm{E}-03$ & $5.951 \mathrm{E}-02$ \\
\hline R16 & $5.932 \mathrm{E}-01$ & $1.324 \mathrm{E}-01$ & $1.352 \mathrm{E}-04$ & $7.233 \mathrm{E}-01$ & $4.438 \mathrm{E}-05$ & $4.797 \mathrm{E}-03$ & $4.089 \mathrm{E}-02$ \\
\hline
\end{tabular}

\begin{tabular}{cccccccc} 
& \multicolumn{9}{c}{ SOURCE } \\
POINT & S22 & S23 & S24 & S25 & S26 & S27 & S28 \\
C01 & $3.178 E-04$ & $1.340 \mathrm{E}-01$ & $1.965 \mathrm{E}-02$ & $6.266 \mathrm{E}-01$ & $3.822 \mathrm{E}-02$ & $7.533 \mathrm{E}-05$ & $6.354 \mathrm{E}-04$ \\
C02 & $2.298 \mathrm{E}-04$ & $5.971 \mathrm{E}-02$ & $6.735 \mathrm{E}-02$ & $1.392 \mathrm{E}+00$ & $1.165 \mathrm{E}-01$ & $5.670 \mathrm{E}-04$ & $8.576 \mathrm{E}-03$ \\
C03 & $9.670 \mathrm{E}-05$ & $1.209 \mathrm{E}-02$ & $9.427 \mathrm{E}-02$ & $7.574 \mathrm{E}-01$ & $1.425 \mathrm{E}-01$ & $3.007 \mathrm{E}-03$ & $1.166 \mathrm{E}-01$
\end{tabular}




\section{REVISED METHODOLOGY FOR DETERMINING CESIUM-137 CONTENT OF HN-200 GROUT CONTAINERS.}

\begin{tabular}{|c|c|c|c|c|c|c|c|}
\hline $\mathrm{CO4}$ & $2.588 \mathrm{E}-05$ & $1.546 \mathrm{E}-03$ & $4.827 \mathrm{E}-02$ & $1.427 \mathrm{E}-01$ & $6.564 \mathrm{E}-02$ & $9.287 \mathrm{E}-03$ & $1.587 \mathrm{E}+00$ \\
\hline $\cos$ & $4.877 \mathrm{E}-06$ & $1.540 \mathrm{E}-04$ & $1.111 \mathrm{E}-02$ & $1.587 \mathrm{E}-02$ & $1.423 \mathrm{E}-02$ & $1.354 \mathrm{E}-02$ & $1.894 \mathrm{E}+01$ \\
\hline C06 & $7.076 \mathrm{E}-07$ & $1.331 \mathrm{E}-05$ & $1.564 \mathrm{E}-03$ & $1.394 \mathrm{E}-03$ & $1.945 \mathrm{E}-03$ & $8.520 \mathrm{E}-03$ & $4.807 \mathrm{E}+01$ \\
\hline $\mathrm{CO7}$ & 5.838E-01 & $1.298 \mathrm{E}+01$ & $9.759 \mathrm{E}-01$ & $1.008 \mathrm{E}+00$ & $2.476 E+00$ & $2.172 \mathrm{E}-06$ & $6.848 \mathrm{E}-06$ \\
\hline $\mathrm{C} 08$ & $3.008 \mathrm{E}-01$ & $2.409 \mathrm{E}+00$ & $9.825 \mathrm{E}+00$ & $2.419 \mathrm{E}+00$ & $2.668 \mathrm{E}+01$ & $1.080 \mathrm{E}-05$ & 5.538E-05 \\
\hline $\mathrm{COP}$ & $6.270 \mathrm{E}-02$ & $2.083 \mathrm{E}-01$ & $2.454 \mathrm{E}+0 \mathrm{l}$ & $1.239 \mathrm{E}+00$ & $4.984 \mathrm{E}+01$ & $3.747 \mathrm{E}-05$ & $3.444 \mathrm{E}-04$ \\
\hline C10 & $7.880 \mathrm{E}-03$ & $1.554 \mathrm{E}-02$ & $4.733 \mathrm{E}+00$ & $2.085 \mathrm{E}-01$ & $6.850 \mathrm{E}+00$ & $8.237 \mathrm{E}-05$ & $1.452 \mathrm{E}-03$ \\
\hline C11 & $7.730 \mathrm{E}-04$ & $1.101 \mathrm{E}-03$ & $4.161 \mathrm{E}-01$ & $2.142 \mathrm{E}-02$ & $5.163 \mathrm{E}-01$ & $1.060 \mathrm{E}-04$ & $3.546 \mathrm{E}-03$ \\
\hline C12 & $6.652 \mathrm{E}-05$ & $7.711 \mathrm{E}-05$ & $3.341 \mathrm{E}-02$ & $1.785 \mathrm{E}-03$ & $3.678 \mathrm{E}-02$ & $7.769 \mathrm{E}-05$ & $4.354 \mathrm{E}-03$ \\
\hline C13 & $6.738 \mathrm{E}+00$ & $7.721 \mathrm{E}-03$ & $5.513 \mathrm{E}-04$ & $5.235 \mathrm{E}-03$ & $5.278 \mathrm{E}-03$ & $3.507 \mathrm{E}-05$ & $1.211 \mathrm{E}-06$ \\
\hline C14 & $2.550 \mathrm{E}+00$ & $4.227 \mathrm{E}-03$ & $1.315 \mathrm{E}-03$ & $8.060 \mathrm{E}-03$ & $1.302 \mathrm{E}-02$ & $2.378 \mathrm{E}-04$ & 8.039E-06 \\
\hline C15 & $3.443 \mathrm{E}-01$ & $1.200 \mathrm{E}-03$ & $1.649 \mathrm{E}-03$ & $5.824 \mathrm{E}-03$ & $1.528 \mathrm{E}-02$ & $1.123 \mathrm{E}-03$ & $4.018 \mathrm{E}-05$ \\
\hline C16 & $3.304 \mathrm{E}-02$ & $2.127 \mathrm{E}-04$ & $1.046 \mathrm{E}-03$ & $2.107 \mathrm{E}-03$ & $8.221 \mathrm{E}-03$ & $3.137 \mathrm{E}-03$ & $1.371 \mathrm{E}-04$ \\
\hline $\mathrm{C} 17$ & $2.768 \mathrm{E}-03$ & $2.757 \mathrm{E}-05$ & $3.612 \mathrm{E}-04$ & $4.486 \mathrm{E}-04$ & $2.300 \mathrm{E}-03$ & $4.402 \mathrm{E}-03$ & $2.871 \mathrm{E}-04$ \\
\hline C18 & $2.205 \mathrm{E}-04$ & $2.913 \mathrm{E}-06$ & $7.773 \mathrm{E}-05$ & $6.626 \mathrm{E}-05$ & $4.032 \mathrm{E}-04$ & $2.902 \mathrm{E}-03$ & 3.393E-04 \\
\hline C19 & $8.111 \mathrm{E}-04$ & $9.681 \mathrm{E}-04$ & $7.484 E-05$ & $4.056 \mathrm{E}-03$ & $4.838 \mathrm{E}-04$ & $9.975 \mathrm{E}-03$ & $3.979 \mathrm{E}-05$ \\
\hline C20 & $5.732 \mathrm{E}-04$ & $5.844 \mathrm{E}-04$ & $1.580 \mathrm{E}-04$ & $6.181 \mathrm{E}-03$ & $1.009 \mathrm{E}-03$ & $1.150 \mathrm{E}-01$ & 4.068E-04 \\
\hline C21 & $2.283 \mathrm{E} \times 04$ & $1.981 \mathrm{E}-04$ & $1.913 \mathrm{E}-04$ & $4.502 \mathrm{E}-03$ & $1.146 \mathrm{E}-03$ & $1.466 \mathrm{E}+00$ & 4E-03 \\
\hline C22 & $5.691 \mathrm{E}-05$ & $4.272 \mathrm{E}-05$ & $1.299 \mathrm{E}-04$ & $1.664 \mathrm{E}-03$ & $6.959 \mathrm{E}-04$ & $2.363 \mathrm{E}+01$ & $1.976 \mathrm{E}-02$ \\
\hline $\mathrm{C} 23$ & $9.968 \mathrm{E}-06$ & $6.633 \mathrm{E}-06$ & $5.180 \mathrm{E}-05$ & $3.637 \mathrm{E}-04$ & $2.416 \mathrm{E}-04$ & $3.033 E+02$ & $6.305 \mathrm{E}-02$ \\
\hline $\mathrm{C} 24$ & $1.352 \mathrm{E}-06$ & $8.166 \mathrm{E}-07$ & $1.328 \mathrm{E}-05$ & $5.507 \mathrm{E}-05$ & $5.384 \mathrm{E}-05$ & $1.729 \mathrm{E}+01$ & $8.310 \mathrm{E}-02$ \\
\hline R01 & $5.802 \mathrm{E}-05$ & $3.018 \mathrm{E}-02$ & $1.511 \mathrm{E}-01$ & $6.416 \mathrm{E}-02$ & $6.204 \mathrm{E}-02$ & $5.073 \mathrm{E}-02$ & 3.215E-01 \\
\hline $\mathbf{R} \mathbf{0 2}$ & 8.395E-04 & $7.485 \mathrm{E}-02$ & $2.655 \mathrm{E}-01$ & $1.030 \mathrm{E}-01$ & $1.747 \mathrm{E}-01$ & $3.618 \mathrm{E}-03$ & $2.566 \mathrm{E}-01$ \\
\hline $\mathbf{R} 03$ & $2.197 \mathrm{E}-02$ & $1.181 \mathrm{E}-01$ & $3.250 \mathrm{E}-01$ & $1.227 \mathrm{E}-01$ & $2.904 \mathrm{E}-01$ & $1.922 \mathrm{E}-04$ & $4 \mathrm{E}-01$ \\
\hline R04 & $2.346 \mathrm{E}-01$ & $1.381 \mathrm{E}-01$ & $3,441 \mathrm{E}-01$ & $1.124 \mathrm{E}-0 \mathrm{l}$ & $3.530 \mathrm{E}-01$ & 2.138E-05 & $3.711 \mathrm{E}-02$ \\
\hline R05 & $4.670 \mathrm{E}-01$ & $1.269 \mathrm{E}-01$ & $3.249 \mathrm{E}-01$ & $7.780 \mathrm{E}-02$ & $3.432 \mathrm{E}-01$ & $7.722 \mathrm{E}-06$ & 3.653E-03 \\
\hline R06 & $5.680 \mathrm{E}-01$ & $8.881 \mathrm{E}-02$ & $2.654 \mathrm{E}-01$ & $3.797 \mathrm{E}-02$ & $2.636 \mathrm{E}-01$ & $1.045 \mathrm{E}-05$ & $2.983 \mathrm{E}-04$ \\
\hline R07 & $6.145 \mathrm{E}-01$ & $4.161 \mathrm{E}-02$ & $1.510 \mathrm{E}-01$ & $1.236 \mathrm{E}-02$ & $1.414 \mathrm{E}-01$ & $5.095 \mathrm{E}-05$ & $4.501 \mathrm{E}-05$ \\
\hline R08 & $6.106 \mathrm{E}-01$ & $1.108 \mathrm{E}-02$ & $3.001 \mathrm{E}-02$ & 3.032E-03 & $4.202 \mathrm{E}-02$ & $6.976 \mathrm{E}-04$ & 1.899E-05 \\
\hline $\mathbf{R} 09$ & $5.554 \mathrm{E}-01$ & $1.856 \mathrm{E}-03$ & $1.909 \mathrm{E}-03$ & $7.415 \mathrm{E}-04$ & $6.567 \mathrm{E}-03$ & $1.368 \mathrm{E}-02$ & $2.588 \mathrm{E}-05$ \\
\hline R10 & $4.431 \mathrm{E}-01$ & $3.001 \mathrm{E}-04$ & $1.271 \mathrm{E}-04$ & $2.572 \mathrm{E}-04$ & $8.502 \mathrm{E}-04$ & $1.047 \mathrm{E}-01$ & $1.084 \mathrm{E}-04$ \\
\hline R11 & $1.828 \mathrm{E}-01$ & $7.741 \mathrm{E}-05$ & $2.010 \mathrm{E}-05$ & $1.623 \mathrm{E}-04$ & $1.649 \mathrm{E}-04$ & $2.121 \mathrm{E}-01$ & $1.071 \mathrm{E}-03$ \\
\hline R12 & $1.301 \mathrm{E}-02$ & 4.355E-05 & $1.054 \mathrm{E}-05$ & $2.054 \mathrm{E}-04$ & $7.138 \mathrm{E}-05$ & $2.673 \mathrm{E}-01$ & $1.334 \mathrm{E}-02$ \\
\hline R13 & $5.111 \mathrm{E}-04$ & $5.992 \mathrm{E}-05$ & $2.012 \mathrm{E}-05$ & $4.964 E-04$ & $8.130 \mathrm{E}-05$ & $2.903 \mathrm{E}-01$ & 8.604E-02 \\
\hline R14 & $4.174 \mathrm{E}-05$ & $1.895 \mathrm{E}-04$ & $1.274 \mathrm{E}-04$ & $1.877 \mathrm{E}-03$ & $2.738 \mathrm{E}-04$ & 2.835E-01 & 2.077E-01 \\
\hline R15 & $1.164 \mathrm{E}-05$ & $1.071 \mathrm{E}-03$ & $1.914 \mathrm{E}-03$ & 7.953E-03 & $1.435 \mathrm{E}-03$ & $2.471 \mathrm{E}-01$ & $2.970 \mathrm{E}-01$ \\
\hline R16 & $1.304 \mathrm{E}-05$ & $6.806 \mathrm{E}-03$ & $3.007 \mathrm{E}-02$ & $2.743 \mathrm{E}-02$ & $1.117 \mathrm{E}-02$ & $1.707 \mathrm{E}-01$ & $3.348 \mathrm{E}-01$ \\
\hline
\end{tabular}

\begin{tabular}{cccccccc}
\multicolumn{8}{c}{ SOURCE } \\
POINT & S29 & S30 & S31 & S32 & S33 & S34 & S35 \\
C01 & $7.320 \mathrm{E}-02$ & $1.915 \mathrm{E}-04$ & $2.541 \mathrm{E}-03$ & $2.025 \mathrm{E}-03$ & $1.042 \mathrm{E}-03$ & $1.166 \mathrm{E}-06$ & $3.693 \mathrm{E}-01$ \\
C02 & $1.567 \mathrm{E}-01$ & $1.729 \mathrm{E}-03$ & $1.749 \mathrm{E}-02$ & $8.939 \mathrm{E}-03$ & $3.246 \mathrm{E}-03$ & $8.140 \mathrm{E}-06$ & $2.861 \mathrm{E}+00$ \\
C03 & $1.188 \mathrm{E}-01$ & $1.167 \mathrm{E}-02$ & $7.281 \mathrm{E}-02$ & $2.127 \mathrm{E}-02$ & $5.343 \mathrm{E}-03$ & $4.332 \mathrm{E}-05$ & $8.629 \mathrm{E}+00$ \\
C04 & $3.510 \mathrm{E}-02$ & $4.747 \mathrm{E}-02$ & $1.360 \mathrm{E}-01$ & $2.283 \mathrm{E}-02$ & $4.241 \mathrm{E}-03$ & $1.592 \mathrm{E}-04$ & $4.653 \mathrm{E}+00$
\end{tabular}




\section{REVISED METHODOLOGY FOR DETERMINING CESIUM-137 CONTENT OF HN-200 GROUT CONTAINERS.}

\begin{tabular}{|c|c|c|c|c|c|c|c|}
\hline $\mathrm{C05}$ & $5.562 \mathrm{E}-03$ & 8.693E-02 & $9.458 \mathrm{E}-02$ & $1.086 \mathrm{E}-02$ & $1.697 \mathrm{E}-03$ & $3.608 \mathrm{E}-04$ & $7.278 \mathrm{E}-01$ \\
\hline $\mathrm{C} 06$ & $6.229 \mathrm{E}-04$ & $5.989 \mathrm{E}-02$ & $2.744 \mathrm{E}-02$ & $2.689 \mathrm{E}-03$ & $3.953 \mathrm{E}-04$ & $4.591 \mathrm{E}-04$ & $7.340 \mathrm{E}-02$ \\
\hline $\mathrm{C} 07$ & $2.402 \mathrm{E}-01$ & $2.832 \mathrm{E}-06$ & $4.011 \mathrm{E}-05$ & $3.080 \mathrm{E}-01$ & $3.648 \mathrm{E}-05$ & $1.839 \mathrm{E}-04$ & $7.705 \mathrm{E}-02$ \\
\hline C08 & $5.953 \mathrm{E}-01$ & $1.513 \mathrm{E}-05$ & $1.613 \mathrm{E}-04$ & $4.728 \mathrm{E}+00$ & $8.838 \mathrm{E}-05$ & $2.269 \mathrm{E}-03$ & $3.996 \mathrm{E}-01$ \\
\hline C09 & $4.257 \mathrm{E}-01$ & $5.729 \mathrm{E}-05$ & $4.120 \mathrm{E}-04$ & $7.100 \mathrm{E}+01$ & $1.287 \mathrm{E}-04$ & $2.590 \mathrm{E}-02$ & $8.688 \mathrm{E}-01$ \\
\hline C10 & $1.035 \mathrm{E}-01$ & $1.391 \mathrm{E}-04$ & $6.043 \mathrm{E}-04$ & $1.040 \mathrm{E}+02$ & $1.082 \mathrm{E}-04$ & $2.436 \mathrm{E}-01$ & $5.689 \mathrm{E}-01$ \\
\hline C11 & $1.350 \mathrm{E}-02$ & $1.986 \mathrm{E}-04$ & $4.847 \mathrm{E}-04$ & $7.524 \mathrm{E}+00$ & $5.358 \mathrm{E}-05$ & $1.443 E+00$ & $1.366 \mathrm{E}-01$ \\
\hline C12 & $1.301 \mathrm{E}-03$ & $1.598 \mathrm{E}-04$ & $2.188 \mathrm{E}-04$ & $4.843 \mathrm{E}-01$ & $1.672 \mathrm{E}-05$ & $2.725 \mathrm{E}+00$ & $1.835 \mathrm{E}-02$ \\
\hline $\mathrm{C13}$ & $7.724 \mathrm{E}-02$ & $1.653 \mathrm{E}-05$ & $2.982 \mathrm{E}-04$ & $2.970 \mathrm{E}-03$ & $3.320 \mathrm{E}-03$ & $9.465 \mathrm{E}-05$ & $1.320 \mathrm{E}-04$ \\
\hline C14 & $1.663 \mathrm{E}-01$ & $1.069 \mathrm{E}-04$ & $1.490 \mathrm{E}-03$ & $1.384 \mathrm{E}-02$ & $1.172 \mathrm{E}-02$ & $1.111 \mathrm{E}-03$ & $3.308 \mathrm{E}-04$ \\
\hline C15 & $1.258 \mathrm{E}-01$ & $4.898 \mathrm{E}-04$ & $4.559 \mathrm{E}-03$ & $3.430 \mathrm{E}-02$ & $2.060 \mathrm{E}-02$ & $1.150 \mathrm{E}-02$ & 4.840E-04 \\
\hline C16 & $3.688 \mathrm{E}-02$ & $1.388 \mathrm{E}-03$ & $7.277 \mathrm{E}-03$ & $3.694 \mathrm{E}-02$ & $1.585 \mathrm{E}-02$ & $9.185 \mathrm{E}-02$ & $3.951 \mathrm{E}-04$ \\
\hline $\mathrm{C17}$ & $5.796 \mathrm{E}-03$ & $2.124 \mathrm{E}-03$ & $5.555 \mathrm{E}-03$ & $1.696 \mathrm{E}-02$ & $5.686 \mathrm{E}-03$ & $4.242 \mathrm{E}-01$ & $1.845 \mathrm{E}-04$ \\
\hline C18 & $6.448 \mathrm{E}-04$ & $1.637 \mathrm{E}-03$ & $2.135 \mathrm{E}-03$ & $3.980 \mathrm{E}-03$ & $1.157 \mathrm{E}-03$ & $7.082 \mathrm{E}-01$ & 5.354E-05 \\
\hline C19 & $2.709 \mathrm{E}-02$ & $4.279 \mathrm{E}-03$ & $6.018 \mathrm{E}-02$ & $9.277 \mathrm{E}-05$ & $2.224 \mathrm{E}+00$ & $8.108 E-07$ & $2.948 \mathrm{E}-04$ \\
\hline $\mathrm{C} 20$ & $5.324 E-02$ & $5.371 E-02$ & $8.220 \mathrm{E}-01$ & $2.958 \mathrm{E}-04$ & $2.624 \mathrm{E}+01$ & $5.452 \mathrm{E}-06$ & $7.800 \mathrm{E}-04$ \\
\hline C21 & $4.171 \mathrm{E}-02$ & $6.680 \mathrm{E}-01$ & $1.064 \mathrm{E}+01$ & 5.634E-04 & $5.374 E+02$ & $2.786 \mathrm{E}-05$ & $1.170 \mathrm{E}-03$ \\
\hline $\mathrm{C} 22$ & $1.395 \mathrm{E}-02$ & $7.846 \mathrm{E}+00$ & $6.279 \mathrm{E}+01$ & $5.930 \mathrm{E}-04$ & $7.523 \mathrm{E}+01$ & 9.854E-05 & $9.425 \mathrm{E}-04$ \\
\hline $\mathrm{C} 23$ & $2.553 \mathrm{E}-03$ & $4.182 \mathrm{E}+01$ & $2.012 \mathrm{E}+01$ & $3.426 \mathrm{E}-04$ & $5.362 E+00$ & $2.172 \mathrm{E}-04$ & 4.199E-04 \\
\hline $\mathrm{C} 24$ & $3.221 \mathrm{E}-03$ & $1.360 \mathrm{E}+01$ & $1.676 \mathrm{E}+00$ & $1.163 \mathrm{E}-04$ & $5.311 \mathrm{E}-01$ & $2.740 \mathrm{E}-04$ & $1.141 \mathrm{E}-04$ \\
\hline R01 & $4.985 \mathrm{E}-03$ & $1.810 \mathrm{E}-01$ & $1.094 \mathrm{E}-01$ & $1.941 \mathrm{E}-02$ & $3.086 \mathrm{E}-02$ & $2.168 \mathrm{E}-04$ & $7.601 \mathrm{E}-01$ \\
\hline R02 & $7.154 \mathrm{E}-03$ & $3.131 \mathrm{E}-02$ & $1.828 \mathrm{E}-02$ & $1.014 \mathrm{E}-01$ & $1.128 \mathrm{E}-03$ & $5.094 \mathrm{E}-03$ & 8.463E-01 \\
\hline $\mathbf{R 0 3}$ & $9.614 \mathrm{E}-03$ & $1.740 \mathrm{E}-03$ & $1.697 \mathrm{E}-03$ & $2.391 \mathrm{E}-01$ & $6.882 \mathrm{E}-05$ & $9.235 \mathrm{E}-02$ & $8.546 \mathrm{E}-01$ \\
\hline R04 & $1.167 \mathrm{E}-02$ & $1.135 \mathrm{E}-04$ & $1.930 \mathrm{E}-04$ & $3.489 \mathrm{E}-01$ & $1.340 \mathrm{E}-05$ & $2.961 \mathrm{E}-01$ & $7.837 \mathrm{E}-01$ \\
\hline R05 & $1.255 \mathrm{E}-02$ & 1.904E-05 & $4.893 \mathrm{E}-05$ & $3.941 \mathrm{E}-01$ & $1.036 \mathrm{E}-05$ & $3.901 \mathrm{E}-01$ & $6.330 \mathrm{E}-01$ \\
\hline R06 & $1.186 \mathrm{E}-02$ & $1.110 \mathrm{E}-05$ & $3.604 \mathrm{E}-05$ & $3.674 \mathrm{E}-01$ & $3.275 \mathrm{E}-05$ & $4.377 \mathrm{E}-01$ & $3.087 \mathrm{E}-01$ \\
\hline R07 & $9.913 \mathrm{E}-03$ & $2.419 \mathrm{E}-05$ & $8.122 \mathrm{E}-05$ & $2.727 \mathrm{E}-01$ & $3.676 \mathrm{E}-04$ & $4.472 \mathrm{E}-0 \mathrm{I}$ & $3.175 \mathrm{E}-02$ \\
\hline R08 & $7.460 \mathrm{E}-03$ & $1.770 \mathrm{E}-04$ & $4.857 \mathrm{E}-04$ & $1.354 \mathrm{E}-01$ & $9.427 \mathrm{E}-03$ & $4.204 \mathrm{E}-01$ & $1.369 \mathrm{E}-03$ \\
\hline R09 & $5.228 \mathrm{E}-03$ & $3.016 \mathrm{E}-03$ & $5.159 \mathrm{E}-03$ & $3.237 \mathrm{E}-02$ & $1.547 \mathrm{E}-01$ & $3.551 \mathrm{E}-01$ & $1.002 \mathrm{E}-04$ \\
\hline R10 & $3.586 \mathrm{E}-03$ & $4.859 \mathrm{E}-02$ & $4.671 \mathrm{E}-02$ & $3.786 \mathrm{E}-03$ & $4.090 \mathrm{E}-01$ & $2.125 \mathrm{E}-01$ & $2.276 \mathrm{E}-05$ \\
\hline R11 & $2.559 E-03$ & $2.134 \mathrm{E}-01$ & $1.813 \mathrm{E}-01$ & $4.134 \mathrm{E}-04$ & $5.126 \mathrm{E}-01$ & $2.846 \mathrm{E}-02$ & $1.977 \mathrm{E}-05$ \\
\hline R12 & $2.018 \mathrm{E}-03$ & $3.416 \mathrm{E}-01$ & 3.352E-01 & $8.154 \mathrm{E}-05$ & $5.620 \mathrm{E}-01$ & $1.119 \mathrm{E}-03$ & $6.682 \mathrm{E}-05$ \\
\hline R13 & $1.840 \mathrm{E}-03$ & $4.044 \mathrm{E}-01$ & $4.304 \mathrm{E}-01$ & $4.161 \mathrm{E}-05$ & $5.701 \mathrm{E}-01$ & $6.529 \mathrm{E}-05$ & $7.461 \mathrm{E}-04$ \\
\hline R14 & $1.977 \mathrm{E}-03$ & $4.224 \mathrm{E}-01$ & $4.510 \mathrm{E}-01$ & $6.197 \mathrm{E}-05$ & 5.345E-01 & $1.154 \mathrm{E}-05$ & $1.682 \mathrm{E}-02$ \\
\hline R15 & $2.467 \mathrm{E}-03$ & $3.964 \mathrm{E}-01$ & $3.957 \mathrm{E}-01$ & $2.512 \mathrm{E}-04$ & $4.543 \mathrm{E}-01$ & $7.901 \mathrm{E}-06$ & $2.251 \mathrm{E}-01$ \\
\hline R16 & $3.424 \mathrm{E}-03$ & $3.242 \mathrm{E}-01$ & 2.695E-01 & 2.081E-03 & $2.675 \mathrm{E}-01$ & $2.191 \mathrm{E}-05$ & $5.864 \mathrm{E}-01$ \\
\hline
\end{tabular}

TABLE 7 - TOTAL RADIATION LEVEL AT EACH MEASUREMENT POINT FOR EACH SET OF SOURCES TO BE ANALYZED.

$\begin{array}{cccccccc}\text { POINT } & \text { S01-S35 } & \text { S01-S30 } & \text { S06-S35 } & \text { S01-S25 } & \text { S11-S35 } & \text { S01-S20 } & \text { S16-S35 } \\ \text { C01 } & 4.724 E+00 & 4.349 E+00 & 4.031 E+00 & 4.237 E+00 & 3.890 E+00 & 3.452 E+00 & 3.486 E+00 \\ \text { C02 } & 2.172 E+01 & 1.883 E+01 & 1.395 E+01 & 1.855 E+01 & 1.296 E+01 & 1.699 E+01 & 1.066 E+01\end{array}$


REVISED METHODOLOGY FOR DETERMINING CESIUM-137 CONTENT OF HN-200 GROUT CONTAINERS.

\begin{tabular}{|c|c|c|c|c|c|c|c|}
\hline 03 & $1.656 \mathrm{E}+02$ & $1.569 \mathrm{E}+02$ & $2.807 E+01$ & $1.565 \mathrm{E}+02$ & $2.501 E+01$ & $1.554 \mathrm{E}+02$ & $1.357 \mathrm{E}+01$ \\
\hline 204 & $3.187 \mathrm{E}+02$ & $3.139 \mathrm{E}+02$ & $4.523 \mathrm{E}+01$ & $3.122 E+02$ & $4.332 \mathrm{E}+01$ & $3.115 \mathrm{E}+02$ & $1.016 \mathrm{E}+01$ \\
\hline 05 & $923 \mathrm{E}+02$ & $.915 \mathrm{E}+02$ & $1.775 E+02$ & $1.724 E+02$ & $1.771 E+02$ & $1.720 E+02$ & $.063 E+01$ \\
\hline 06 & $1.204 \mathrm{E}+02$ & $1.203 \mathrm{E}+02$ & $1.194 \mathrm{E}+02$ & $7.211 \mathrm{E}+01$ & $194 E+02$ & $7.200 \mathrm{E}+01$ & $.570 \mathrm{E}+0$ \\
\hline 07 & $2.792 \mathrm{E}+01$ & $2.754 \mathrm{E}+01$ & $2.788 \mathrm{E}+01$ & $2.482 \mathrm{E}+01$ & $2.361 \mathrm{E}+01$ & $9.274 \mathrm{E}+00$ & $.208 \mathrm{E}+0$ \\
\hline 208 & $5.846 \mathrm{E}+01$ & $5.333 \mathrm{E}+01$ & $5.835 E+01$ & $2.605 E+01$ & $5.507 \mathrm{E}+01$ & $1.109 \mathrm{E}+01$ & $5.209 \mathrm{E}+0$ \\
\hline 09 & $1.523 \mathrm{E}+02$ & $8.038 \mathrm{E}+01$ & $1.522 \mathrm{E}+02$ & $3.012 \mathrm{E}+01$ & $1.515 \mathrm{E}+02$ & $4.055 \mathrm{E}+00$ & $1.500 \mathrm{E}+0$ \\
\hline 10 & $1.186 \mathrm{E}+02$ & $1.382 E+01$ & $1.186 \mathrm{E}+02$ & $6.863 E+00$ & $1.185 \mathrm{E}+02$ & $1.878 \mathrm{E}+00$ & $1.178 \mathrm{E}+0$ \\
\hline 11 & $1.065 \mathrm{E}+01$ & $1.545 \mathrm{E}+00$ & $1.063 \mathrm{E}+01$ & $1.012 \mathrm{E}+00$ & $1.061 \mathrm{E}+01$ & $5.557 \mathrm{E}-01$ & $1.030 \mathrm{E}+0$ \\
\hline 12 & $3.602 \mathrm{E}+00$ & $3.741 \mathrm{E}-01$ & $3.597 \mathrm{E}+00$ & $3.314 \mathrm{E}-01$ & $3.592 \mathrm{E}+00$ & $2.895 \mathrm{E}-01$ & $36 \mathrm{E}+0$ \\
\hline 13 & $425 \mathrm{E}+01$ & $3.424 \mathrm{E}+01$ & $3.316 \mathrm{E}+01$ & $3.416 \mathrm{E}+01$ & $2.175 \mathrm{E}+01$ & $2.740 \mathrm{E}+01$ & $.929 E+C$ \\
\hline 14 & $9.385 E+01$ & $9.382 \mathrm{E}+01$ & $8.754 \mathrm{E}+01$ & $9.364 \mathrm{E}+01$ & $7.747 \mathrm{E}+01$ & $9.107 \mathrm{E}+01$ & $3.570 \mathrm{E}+0$ \\
\hline 15 & $4.022 \mathrm{E}+01$ & $4.015 E+01$ & $3.446 \mathrm{E}+01$ & $4.001 E+01$ & $3.260 \mathrm{E}+01$ & $3.962 E+01$ & $1.265 \mathrm{E}+01$ \\
\hline 16 & $1.378 \mathrm{E}+02$ & $1.377 \mathrm{E}+02$ & $1.368 E+02$ & $1.376 \mathrm{E}+02$ & $1.364 \mathrm{E}+02$ & $1.375 \mathrm{E}+02$ & $1.347 \mathrm{E}+02$ \\
\hline 17 & $5.736 \mathrm{E}+01$ & $5.690 \mathrm{E}+01$ & $5.714 E+01$ & $5.689 \mathrm{E}+01$ & $5.694 \mathrm{E}+01$ & $5.683 \mathrm{E}+01$ & $5.570 \mathrm{E}+01$ \\
\hline 18 & $3.242 \mathrm{E}+01$ & $3.170 \mathrm{E}+01$ & $3.236 \mathrm{E}+01$ & $3.170 \mathrm{E}+01$ & $3.225 \mathrm{E}+01$ & $3.167 \mathrm{E}+01$ & $3.224 \mathrm{E}+01$ \\
\hline 19 & $4.777 \mathrm{E}+00$ & $2.492 \mathrm{E}+00$ & $2.587 \mathrm{E}+00$ & $2.450 \mathrm{E}+00$ & $2.370 \mathrm{E}+00$ & $2.431 \mathrm{E}+00$ & $2.356 \mathrm{E}+0$ \\
\hline 20 & $3.927 \mathrm{E}+01$ & $1.220 \mathrm{E}+01$ & $2.812 \mathrm{E}+01$ & $1.198 \mathrm{E}+01$ & $2.748 \mathrm{E}+01$ & $1.183 \mathrm{E}+01$ & $2.746 \mathrm{E}+0$ \\
\hline 21 & $6.331 \mathrm{E}+02$ & $8.509 E+01$ & $5.540 \mathrm{E}+02$ & $8.291 E+01$ & $5.514 \mathrm{E}+02$ & $8.180 \mathrm{E}+01$ & $5.514 \mathrm{E}+0$ \\
\hline 22 & $2.294 \mathrm{E}+02$ & $9.134 \mathrm{E}+01$ & $1.807 \mathrm{E}+02$ & $5.983 \mathrm{E}+01$ & $1.734 \mathrm{E}+02$ & $5.620 \mathrm{E}+01$ & $1.734 \mathrm{E}+02$ \\
\hline 23 & $3.999 \mathrm{E}+02$ & $3.744 \mathrm{E}+02$ & $3.886 \mathrm{E}+02$ & $2.919 \mathrm{E}+01$ & $3.737 \mathrm{E}+02$ & $2.660 \mathrm{E}+01$ & $3.737 \mathrm{E}+02$ \\
\hline 24 & $1.733 E+02$ & $1.711 \mathrm{E}+02$ & $1.718 E+02$ & $\mathrm{E}+02$ & $3.408 \mathrm{E}+01$ & $6 \mathrm{E}+02$ & $3,407 \mathrm{E}+01$ \\
\hline 01 & $4.792 \mathrm{E}+00$ & $\mathrm{E}+00$ & $3.967 \mathrm{E}+00$ & $2 \mathrm{E}+00$ & $3.878 \mathrm{E}+00$ & $5 \mathrm{E}+00$ & $2.753 \mathrm{E}+00$ \\
\hline 02 & $4.743 E+\infty 0$ & $3.771 \mathrm{E}+\infty 0$ & $4.088 E+00$ & $3.298 \mathrm{E}+00$ & $4.041 E+00$ & $2.844 E+00$ & $2.833 \mathrm{E}+0$ \\
\hline 03 & $4.590 \mathrm{E}+00$ & $3.402 \mathrm{E}+00$ & $4.099 \mathrm{E}+00$ & $2.956 \mathrm{E}+00$ & $4.061 \mathrm{E}+00$ & $2,364 \mathrm{E}+00$ & $2.920 \mathrm{E}+0$ \\
\hline 04 & $4.329 \mathrm{E}+00$ & $2.900 \mathrm{E}+00$ & $4.072 \mathrm{E}+00$ & $2.498 \mathrm{E}+00$ & $3.999 \mathrm{E}+00$ & $1.667 \mathrm{E}+00$ & $3.057 \mathrm{E}+0$ \\
\hline 05 & $3.925 \mathrm{E}+00$ & $2.507 \mathrm{E}+00$ & $3.858 \mathrm{E}+00$ & $2.148 E+00$ & $3.721 \mathrm{E}+00$ & $1.150 \mathrm{E}+00$ & $3.075 \mathrm{E}+00$ \\
\hline 06 & $3.376 \mathrm{E}+00$ & $2.262 \mathrm{E}+00$ & $3.342 \mathrm{E}+00$ & $1.987 \mathrm{E}+00$ & $3.141 \mathrm{E}+00$ & $1.026 \mathrm{E}+00$ & $2.666 \mathrm{E}+00$ \\
\hline 07 & $2.999 \mathrm{E}+00$ & $2.247 \mathrm{E}+00$ & $2.941 \mathrm{E}+00$ & $2.096 \mathrm{E}+00$ & $2.699 \mathrm{E}+00$ & $1.275 \mathrm{E}+00$ & $2.160 E+00$ \\
\hline R08 & $2.827 \mathrm{E}+00$ & $2.260 \mathrm{E}+00$ & $2.653 \mathrm{E}+00$ & $2.210 \mathrm{E}+00$ & $2.401 \mathrm{E}+00$ & $1.553 \mathrm{E}+00$ & $1.818 \mathrm{E}+00$ \\
\hline 209 & $2.915 \mathrm{E}+00$ & $2.367 \mathrm{E}+00$ & $2.532 \mathrm{E}+00$ & $2.339 \mathrm{E}+00$ & $2.249 \mathrm{E}+00$ & $1.774 \mathrm{E}+00$ & $1.699 \mathrm{E}+00$ \\
\hline 10 & $3.113 E+00$ & $2.441 E+00$ & $2.591 \mathrm{E}+00$ & $2.283 \mathrm{E}+00$ & $2.183 E+00$ & $1.829 \mathrm{E}+00$ & $1.752 \mathrm{E}+0$ \\
\hline 1 & $187 \mathrm{E}+00$ & $2.464 \mathrm{E}+00$ & $2.454 \mathrm{E}+00$ & $2.034 \mathrm{E}+\infty 0$ & $1.910 \mathrm{E}+00$ & $1.827 \mathrm{E}+00$ & $1.675 \mathrm{E}+0$ \\
\hline 12 & $3.520 \mathrm{E}+00$ & $2.621 E+00$ & $2.621 \mathrm{E}+00$ & $1.997 \mathrm{E}+00$ & $2.000 \mathrm{E}+00$ & $1.940 \mathrm{E}+00$ & $1.942 \mathrm{E}+0$ \\
\hline & $4.053 E+00$ & $3.052 \mathrm{E}+00$ & $3.059 \mathrm{E}+00$ & $2.269 \mathrm{E}+00$ & $2.431 E+00$ & $2.207 \mathrm{E}+00$ & $2.411 \mathrm{E}+0$ \\
\hline & $520 \mathrm{E}+00$ & $3.518 \mathrm{E}+00$ & $3.400 \mathrm{E}+00$ & $2.602 \mathrm{E}+00$ & $2.844 \mathrm{E}+00$ & $2.532 \mathrm{E}+00$ & $2.661 \mathrm{E}+0$ \\
\hline & $985 \mathrm{E}+00$ & $3.910 \mathrm{E}+00$ & $3.825 \mathrm{E}+00$ & $2.966 \mathrm{E}+00$ & $3.428 \mathrm{E}+00$ & $2.895 \mathrm{E}+00$ & $2.850 \mathrm{E}+0$ \\
\hline & $5.038 \mathrm{E}+00$ & $3.912 \mathrm{E}+00$ & $4.043 \mathrm{E}+00$ & $3.068 \mathrm{E}+00$ & $3.841 \mathrm{E}+00$ & $2.963 \mathrm{E}+00$ & $2.936 \mathrm{E}+\mathrm{C}$ \\
\hline
\end{tabular}

\section{SOURCE}

$\begin{array}{ccc}\text { POINT } & \text { S01-S18 } & \text { S19-S35 } \\ \text { C01 } & 3.260 \mathrm{E}+00 & 1.464 \mathrm{E}+00 \\ \text { C02 } & 1.679 \mathrm{E}+01 & 4.936 \mathrm{E}+00 \\ \text { C03 } & 1.553 \mathrm{E}+02 & 1.026 \mathrm{E}+01 \\ \text { C04 } & 3.115 \mathrm{E}+02 & 7.241 \mathrm{E}+00 \\ \text { C05 } & 1.720 \mathrm{E}+02 & 2.030 \mathrm{E}+01 \\ \text { C06 } & 7.200 \mathrm{E}+01 & 4.835 \mathrm{E}+01\end{array}$

$\begin{array}{cc}\text { S01-S12 } & \text { S13-S24 } \\ 1.015 \mathrm{E}+00 & 2.595 \mathrm{E}+00 \\ 1.063 \mathrm{E}+01 & 6.524 \mathrm{E}+00 \\ 1.509 \mathrm{E}+02 & 4.871 \mathrm{E}+00 \\ 2.956 \mathrm{E}+02 & 1.645 \mathrm{E}+01 \\ 2.280 \mathrm{E}+01 & 1.496 \mathrm{E}+02 \\ 1.844 \mathrm{E}+00 & 7.027 \mathrm{E}+01\end{array}$

\begin{abstract}
S25-S35
$1.114 \mathrm{E}+00$

$4.567 \mathrm{E}+00$

$9.878 \mathrm{E}+00$

$6.703 \mathrm{E}+00$

$1.991 \mathrm{E}+01$

$4.825 \mathrm{E}+01$
\end{abstract}

S01-S10
$8.338 \mathrm{E}-01$
$8.763 \mathrm{E}+00$
$1.406 \mathrm{E}+02$
$2.754 \mathrm{E}+02$
$1.524 \mathrm{E}+01$
$1.003 \mathrm{E}+00$

S13-S22

$2.441 E+00$

$6.397 \mathrm{E}+00$

$4.765 \mathrm{E}+00$

$1.640 \mathrm{E}+01$

$1.496 \mathrm{E}+02$

$7.026 \mathrm{E}+01$ 


\section{REVISED METHODOLOGY FOR DETERMINING CESIUM-137 CONTENT OF HN-200 GROUT CONTAINERS.}

\begin{tabular}{|c|c|c|c|c|c|c|c|}
\hline $\mathrm{CO7}$ & $6.573 E+00$ & $2.135 \mathrm{E}+01$ & $4.341 E+\infty 0$ & $1.947 \mathrm{E}+01$ & $4.110 \mathrm{E}+\infty 0$ & $4.318 \mathrm{E}+00$ & $5.517 \mathrm{E}+00$ \\
\hline C08 & $8.078 \mathrm{E}+00$ & $5.038 \mathrm{E}+01$ & $3.519 \mathrm{E}+\infty 0$ & $2.011 \mathrm{E}+01$ & $3.482 \mathrm{E}+01$ & $3.383 E+00$ & $7.880 \mathrm{E}+00$ \\
\hline C09 & $3.347 \mathrm{E}+00$ & $1.489 \mathrm{E}+02$ & $1.168 \mathrm{E}+00$ & $2.771 \mathrm{E}+01$ & $1.234 \mathrm{E}+02$ & $7.818 \mathrm{E}-01$ & $2.960 \mathrm{E}+00$ \\
\hline $\mathrm{C10}$ & $1.086 \mathrm{E}+00$ & $1.175 \mathrm{E}+02$ & $6.123 \mathrm{E}-01$ & $6.042 \mathrm{E}+00$ & $1.120 \mathrm{E}+02$ & $1.565 \mathrm{E}-01$ & $1.294 \mathrm{E}+00$ \\
\hline C11 & $5.327 \mathrm{E}-01$ & $1.012 \mathrm{E}+01$ & $2.695 \mathrm{E}-01$ & $7.209 \mathrm{E}-01$ & $9.659 \mathrm{E}+00$ & $4.136 \mathrm{E}-02$ & $3.037 \mathrm{E}-01$ \\
\hline $\mathrm{C} 12$ & $2.833 \mathrm{E}-01$ & $3.319 \mathrm{E}+00$ & $6.335 \mathrm{E}-02$ & 2.663E-01 & $3.272 \mathrm{E}+00$ & $1.023 \mathrm{E}-02$ & $2.328 \mathrm{E}-01$ \\
\hline $\mathrm{C13}$ & $2.732 \mathrm{E}+01$ & $6.926 \mathrm{E}+00$ & $1.250 \mathrm{E}+01$ & $2.165 \mathrm{E}+01$ & $9.462 \mathrm{E}-02$ & $1.250 \mathrm{E}+01$ & $2.164 E+01$ \\
\hline C14 & $9.030 \mathrm{E}+01$ & $3.549 \mathrm{E}+00$ & $1.638 \mathrm{E}+01$ & $7.725 \mathrm{E}+01$ & $2.162 \mathrm{E}-01$ & $1.638 \mathrm{E}+01$ & $7.725 \mathrm{E}+01$ \\
\hline C15 & $2.783 \mathrm{E}+01$ & $1.239 \mathrm{E}+01$ & $7.622 E+00$ & $3.238 \mathrm{E}+01$ & $2.200 \mathrm{E}-01$ & $7.620 \mathrm{E}+00$ & $3.238 \mathrm{E}+01$ \\
\hline C16 & $6.411 \mathrm{E}+00$ & $1.314 \mathrm{E}+02$ & $1.419 \mathrm{E}+00$ & $1.362 E+02$ & $2.042 \mathrm{E}-01$ & $1.417 \mathrm{E}+00$ & $1.362 \mathrm{E}+02$ \\
\hline C17 & $2.875 \mathrm{E}+01$ & $2.861 \mathrm{E}+01$ & $4.196 \mathrm{E}-01$ & $5.647 \mathrm{E}+01$ & $4.679 \mathrm{E}-01$ & $4.186 \mathrm{E}-01$ & $5.647 \mathrm{E}+01$ \\
\hline C18 & $2.995 \mathrm{E}+01$ & $2.469 \mathrm{E}+00$ & $1.678 \mathrm{E}-01$ & $3.153 \mathrm{E}+01$ & $7.215 \mathrm{E}-01$ & $1.675 \mathrm{E}-01$ & $3.153 E+01$ \\
\hline C19 & $2.424 \mathrm{E}+00$ & $2.353 \mathrm{E}+00$ & $2.408 \mathrm{E}+00$ & $3.822 \mathrm{E}-02$ & $2.330 E+00$ & $2.407 \mathrm{E}+00$ & $3.718 \mathrm{E}-02$ \\
\hline C20 & $1.182 E+01$ & $2.745 \mathrm{E}+01$ & $1.179 E+01$ & $1.824 \mathrm{E}-01$ & $2.729 \mathrm{E}+01$ & $1.179 \mathrm{E}+01$ & $1.816 \mathrm{E}-01$ \\
\hline C21 & $8.178 E+01$ & $5.513 \mathrm{E}+02$ & $8.173 \mathrm{E}+01$ & $1.173 \mathrm{E}+\infty 0$ & $5.502 \mathrm{E}+02$ & $8.172 \mathrm{E}+01$ & $1.173 \mathrm{E}+00$ \\
\hline $\mathrm{C22}$ & $5.618 \mathrm{E}+01$ & $1.732 \mathrm{E}+02$ & $5.598 \mathrm{E}+01$ & $3.849 \mathrm{E}+00$ & $1.695 \mathrm{E}+02$ & $5.598 \mathrm{E}+01$ & $3.848 \mathrm{E}+00$ \\
\hline $\mathrm{C23}$ & $2.659 \mathrm{E}+01$ & $3.733 \mathrm{E}+02$ & $2.613 \mathrm{E}+01$ & $3.058 \mathrm{E}+00$ & $3.707 \mathrm{E}+02$ & $2.612 \mathrm{E}+01$ & $3.058 \mathrm{E}+00$ \\
\hline $\mathrm{C} 24$ & $1.396 \mathrm{E}+02$ & $3.369 \mathrm{E}+01$ & $1.392 \mathrm{E}+02$ & $8.929 \mathrm{E}-01$ & $3.318 \mathrm{E}+01$ & $1.392 \mathrm{E}+02$ & $8.929 \mathrm{E}-01$ \\
\hline R01 & $2.972 \mathrm{E}+00$ & $1.820 \mathrm{E}+00$ & $1.359 \mathrm{E}+00$ & $1.829 \mathrm{E}+00$ & $1.604 \mathrm{E}+00$ & $9.147 \mathrm{E}-01$ & $1.647 \mathrm{E}+00$ \\
\hline R02 & $2.818 \mathrm{E}+00$ & $1.926 \mathrm{E}+00$ & $1.207 \mathrm{E}+00$ & $1.988 E+00$ & $1.549 \mathrm{E}+00$ & $7.023 \mathrm{E}-01$ & $1.647 \mathrm{E}+00$ \\
\hline R03 & $2.322 \mathrm{E}+00$ & $2.267 \mathrm{E}+00$ & $1.007 \mathrm{E}+00$ & $1.826 \mathrm{E}+00$ & $1.757 \mathrm{E}+00$ & 5.285E-01 & $1.383 E+00$ \\
\hline 204 & $1.615 E+00$ & $2.713 E+00$ & $6.971 \mathrm{E}-01$ & $1.688 \mathrm{E}+00$ & $1.943 \mathrm{E}+00$ & $3.293 \mathrm{E}-01$ & $1.206 \mathrm{E}+00$ \\
\hline 205 & $1.084 E+00$ & $2.841 \mathrm{E}+00$ & $4.010 \mathrm{E}-01$ & $1.669 \mathrm{E}+00$ & $1.854 \mathrm{E}+00$ & $2.035 \mathrm{E}-01$ & $1.217 \mathrm{E}+00$ \\
\hline 206 & $8.988 \mathrm{E}-01$ & $2.477 \mathrm{E}+00$ & 2.857E-01 & $1.663 \mathrm{E}+00$ & $1.428 \mathrm{E}+00$ & 2.347E-01 & $1.309 \mathrm{E}+00$ \\
\hline 107 & $1.061 \mathrm{E}+00$ & $1.938 \mathrm{E}+00$ & $3.061 \mathrm{E}-01$ & $1.777 \mathrm{E}+00$ & $9.159 \mathrm{E}-01$ & $3.000 \mathrm{E}-01$ & $1.585 \mathrm{E}+00$ \\
\hline 208 & $1.278 \mathrm{E}+00$ & $1.550 \mathrm{E}+00$ & $4.269 \mathrm{E}-01$ & $1.780 E+00$ & $6.205 E-01$ & $4.262 \mathrm{E}-01$ & $1.739 \mathrm{E}+00$ \\
\hline R09 & $1.480 \mathrm{E}+00$ & $1.435 E+00$ & $6.656 \mathrm{E}-01$ & $1.672 E+00$ & $5.767 \mathrm{E}-01$ & $6.654 \mathrm{E}-01$ & $1.668 \mathrm{E}+00$ \\
\hline R10 & $1.564 \mathrm{E}+00$ & $1.549 \mathrm{E}+00$ & $9.296 \mathrm{E}-01$ & $1.353 E+00$ & $8.301 \mathrm{E}-01$ & $9.295 \mathrm{E}-01$ & $1.353 \mathrm{E}+00$ \\
\hline R11 & $1.639 \mathrm{E}+00$ & $1.548 \mathrm{E}+00$ & $1.277 \mathrm{E}+00$ & $7.575 \mathrm{E}-01$ & $1.152 \mathrm{E}+00$ & $1.277 \mathrm{E}+00$ & $7.574 \mathrm{E}-01$ \\
\hline 12 & $1.854 \mathrm{E}+00$ & $1.665 \mathrm{E}+00$ & $1.520 \mathrm{E}+00$ & $4.769 \mathrm{E}-01$ & $1.523 \mathrm{E}+00$ & $1.519 \mathrm{E}+00$ & $4.768 \mathrm{E}-01$ \\
\hline 13 & $2.189 \mathrm{E}+00$ & $1.864 \mathrm{E}+00$ & $1.624 \mathrm{E}+00$ & $6.440 \mathrm{E}-01$ & $1.785 \mathrm{E}+00$ & $1.622 \mathrm{E}+00$ & $6.440 \mathrm{E}-01$ \\
\hline 14 & $2.529 \mathrm{E}+00$ & $1.991 \mathrm{E}+00$ & $1.699 \mathrm{E}+00$ & $9.006 \mathrm{E}-01$ & $1.920 \mathrm{E}+00$ & $1.676 \mathrm{E}+00$ & $9.003 \mathrm{E}-01$ \\
\hline & $2.893 \mathrm{E}+00$ & $2.092 \mathrm{E}+00$ & $1.686 \mathrm{E}+00$ & $1.271 \mathrm{E}+00$ & $2.028 \mathrm{E}+00$ & $1.557 \mathrm{E}+00$ & $1.268 \mathrm{E}+00$ \\
\hline 1 & $2.958 \mathrm{E}+00$ & $2.080 \mathrm{E}+00$ & $1.502 \mathrm{E}+00$ & $1.538 \mathrm{E}+00$ & $1.997 \mathrm{E}+00$ & $1.197 \mathrm{E}+00$ & $1.501 \mathrm{E}+00$ \\
\hline
\end{tabular}

\begin{tabular}{ccccccc} 
& & \multicolumn{5}{c}{ SOURCE } \\
POINT & S23-S32 & S01-S07 & S08-S14 & S15-S21 & S22-S28 & S29-S35 \\
C01 & $8.971 E-01$ & $8.161 E-01$ & $4.146 E-01$ & $2.226 E+00$ & $8.195 E-01$ & $4.483 E-01$ \\
C02 & $1.830 E+00$ & $8.737 E+00$ & $2.236 E+00$ & $6.056 E+00$ & $1.645 E+00$ & $3.049 E+00$ \\
C03 & $1.350 E+00$ & $1.405 E+02$ & $1.048 E+01$ & $4.594 E+00$ & $1.126 E+00$ & $8.859 E+00$ \\
C04 & $2.096 E+00$ & $2.753 E+02$ & $2.029 E+01$ & $1.637 E+01$ & $1.854 E+00$ & $4.899 E+00$ \\
C05 & $1.919 E+01$ & $1.517 E+01$ & $7.630 E+00$ & $1.496 E+02$ & $1.899 E+01$ & $9.278 E-01$ \\
C06 & $4.817 E+01$ & $9.816 E-01$ & $8.624 E-01$ & $7.026 E+01$ & $4.808 E+01$ & $1.649 E-01$ \\
C07 & $1.799 E+01$ & $4.930 E-02$ & $5.795 E+00$ & $3.430 E+00$ & $1.802 E+01$ & $6.255 E-01$
\end{tabular}




\section{REVISED METHODOLOGY FOR DETERMINING CESIUM-137 CONTENT OF HN-200 GROUT CONTAINERS.}

\begin{tabular}{|c|c|c|c|c|c|c|}
\hline $\mathrm{COB}$ & $4.666 \mathrm{E}+01$ & $1.139 \mathrm{E}-01$ & $6.256 \mathrm{E}+00$ & $4.728 \mathrm{E}+00$ & $4.163 \mathrm{E}+01$ & $5.725 E+00$ \\
\hline CO9 & $1.473 \mathrm{E}+02$ & $1.256 \mathrm{E}-01$ & $2.179 \mathrm{E}+00$ & $1.761 E+00$ & $7.589 E+01$ & $7.232 \mathrm{E}+01$ \\
\hline C10 & $1.159 \mathrm{E}+02$ & $7.321 \mathrm{E}-02$ & $7.143 \mathrm{E}-01$ & $1.111 \mathrm{E}+00$ & $1.182 \mathrm{E}+01$ & $1.049 \mathrm{E}+02$ \\
\hline C11 & $8.497 \mathrm{E}+00$ & $2.620 \mathrm{E}-02$ & $2.616 \mathrm{E}-01$ & $2.846 \mathrm{E}-01$ & $9.593 \mathrm{E}-01$ & $9.118 \mathrm{E}+00$ \\
\hline C12 & $5.625 \mathrm{E}-01$ & $6.329 \mathrm{E}-03$ & $5.864 \mathrm{E}-02$ & $2.311 \mathrm{E}-01$ & $7.655 \mathrm{E}-02$ & $3.229 \mathrm{E}+00$ \\
\hline C13 & $9.935 \mathrm{E}-02$ & $1.089 \mathrm{E}+00$ & $2.623 \mathrm{E}+01$ & 8.806E-02 & $6.757 \mathrm{E}+00$ & $8.407 \mathrm{E}-02$ \\
\hline C14 & $2.086 \mathrm{E}-01$ & $6.313 \mathrm{E}+00$ & $8.397 \mathrm{E}+01$ & $7.984 \mathrm{E}-01$ & $2.577 \mathrm{E}+00$ & $1.949 \mathrm{E}-01$ \\
\hline $\mathrm{C15}$ & $1.903 \mathrm{E}-01$ & $5.770 \mathrm{E}+00$ & $2.180 \mathrm{E}+01$ & $1.208 \mathrm{E}+01$ & $3.694 \mathrm{E}-01$ & $1.977 \mathrm{E}-01$ \\
\hline C16 & $9.735 \mathrm{E}-02$ & $1.030 \mathrm{E}+00$ & $2.051 E+00$ & $1.345 \mathrm{E}+02$ & $4.790 \mathrm{E}-02$ & $1.906 \mathrm{E}-01$ \\
\hline C17 & $3.826 \mathrm{E}-02$ & $2.495 \mathrm{E}-01$ & $1.407 \mathrm{E}+00$ & $5.523 E+01$ & $1.059 \mathrm{E}-02$ & 4.605E-01 \\
\hline C18 & $1.219 \mathrm{E}-02$ & $1.223 \mathrm{E}-01$ & $5.474 \mathrm{E}-02$ & $3.152 \mathrm{E}+01$ & $4.012 \mathrm{E}-03$ & $7.178 \mathrm{E}-01$ \\
\hline C19 & $1.072 \mathrm{E}-01$ & $2.235 \mathrm{E}+00$ & $1.853 \mathrm{E}-01$ & $2.378 \mathrm{E}-02$ & $1.641 \mathrm{E}-02$ & $2.316 \mathrm{E}+00$ \\
\hline C20 & $1.053 \mathrm{E}+00$ & $1.141 \mathrm{E}+01$ & $3.963 \mathrm{E}-01$ & $1.632 \mathrm{E}-01$ & $1.239 \mathrm{E}-01$ & $2.717 \mathrm{E}+01$ \\
\hline C21 & $1.283 \mathrm{E}+01$ & $7.977 \mathrm{E}+01$ & $1.976 \mathrm{E}+00$ & $1.161 \mathrm{E}+00$ & $1.476 \mathrm{E}+00$ & $5.488 \mathrm{E}+02$ \\
\hline $\mathrm{C} 22$ & $9.430 \mathrm{E}+01$ & $4.989 \mathrm{E}+01$ & $6.102 E+00$ & $3.844 \mathrm{E}+00$ & $2.365 \mathrm{E}+01$ & $1.459 \mathrm{E}+02$ \\
\hline $\mathrm{C} 23$ & $3.653 E+02$ & $2.270 \mathrm{E}+01$ & $3.424 \mathrm{E}+\infty 0$ & $3.058 \mathrm{E}+00$ & $3.034 \mathrm{E}+02$ & $6.731 \mathrm{E}+01$ \\
\hline C24 & $3.265 \mathrm{E}+01$ & $1.387 \mathrm{E}+02$ & $5.282 \mathrm{E}-01$ & $8.927 \mathrm{E}-01$ & $1.737 \mathrm{E}+01$ & $1.581 \mathrm{E}+01$ \\
\hline R01 & $9.945 \mathrm{E}-01$ & $9.068 \mathrm{E}-01$ & $4.680 \mathrm{E}-01$ & $1.632 \mathrm{E}+00$ & $6.798 \mathrm{E}-01$ & $1.106 \mathrm{E}+00$ \\
\hline R02 & $1.036 \mathrm{E}+00$ & $6.946 \mathrm{E}-01$ & $5.418 \mathrm{E}-01$ & $1.617 \mathrm{E}+00$ & $8.791 \mathrm{E}-01$ & $1.011 \mathrm{E}+00$ \\
\hline R03 & $1.253 \mathrm{E}+00$ & $5.051 \mathrm{E}-01$ & $5.455 \mathrm{E}-01$ & $1.317 \mathrm{E}+00$ & $1.023 \mathrm{E}+00$ & $1.199 \mathrm{E}+00$ \\
\hline R04 & $1.346 \mathrm{E}+00$ & $2.612 E-01$ & $5.100 \mathrm{E}-01$ & $8.974 \mathrm{E}-01$ & $1.219 \mathrm{E}+00$ & $1.441 \mathrm{E}+00$ \\
\hline R05 & $1.283 \mathrm{E}+00$ & $6.802 \mathrm{E}-02$ & 5.353E-01 & $5.481 \mathrm{E}-01$ & $1.343 \mathrm{E}+00$ & $1.430 \mathrm{E}+00$ \\
\hline R06 & $1.035 E+00$ & $3.474 \mathrm{E}-02$ & $6.481 \mathrm{E}-01$ & $3.435 \mathrm{E}-01$ & $1.224 \mathrm{E}+00$ & $1.126 \mathrm{E}+00$ \\
\hline R07 & $6.292 \mathrm{E}-01$ & $5.965 \mathrm{E}-02$ & $7.780 \mathrm{E}-01$ & 4.387E-01 & $9.610 \mathrm{E}-01$ & $7.620 \mathrm{E}-01$ \\
\hline R08 & $2.304 \mathrm{E}-01$ & $1.827 \mathrm{E}-01$ & $8.265 E-01$ & $5.462 \mathrm{E}-01$ & $6.975 \mathrm{E}-01$ & $5.747 \mathrm{E}-01$ \\
\hline R09 & 7.055E-02 & $4.533 \mathrm{E}-01$ & $7.619 \mathrm{E}-01$ & $5.635 \mathrm{E}-01$ & $5.802 \mathrm{E}-01$ & 5.557E-01 \\
\hline R10 & $2.090 \mathrm{E}-01$ & $7.586 \mathrm{E}-01$ & $6.020 \mathrm{E}-01$ & $4.788 \mathrm{E}-01$ & $5.494 \mathrm{E}-01$ & $7.242 \mathrm{E}-01$ \\
\hline R11 & $6.113 \mathrm{E}-01$ & $1.137 \mathrm{E}+00$ & $3.745 \mathrm{E}-01$ & 3.400E-01 & $3.964 \mathrm{E}-01$ & $9.388 \mathrm{E}-01$ \\
\hline R12 & $9.599 \mathrm{E}-01$ & $1.404 \mathrm{E}+00$ & $1.723 \mathrm{E}-01$ & $4.067 \mathrm{E}-01$ & $2.940 \mathrm{E}-01$ & $1.242 \mathrm{E}+00$ \\
\hline R13 & $1.214 \mathrm{E}+00$ & $1.531 \mathrm{E}+00$ & $9,890 \mathrm{E}-02$ & 6.375E-01 & $3.775 \mathrm{E}-01$ & $1.408 \mathrm{E}+00$ \\
\hline 14 & $1.369 \mathrm{E}+00$ & $1.611 \mathrm{E}+00$ & $8.935 \mathrm{E}-02$ & $8.988 \mathrm{E}-01$ & $4.937 \mathrm{E}-01$ & $1.427 \mathrm{E}+00$ \\
\hline 15 & $1.351 \mathrm{E}+00$ & $1.519 E+00$ & $1.699 \mathrm{E}-01$ & $1.266 \mathrm{E}+00$ & $5.565 \mathrm{E}-01$ & $1.474 \mathrm{E}+00$ \\
\hline R16 & $1.180 \mathrm{E}+00$ & $1.179 \mathrm{E}+00$ & 3.303E-01 & $1.495 \mathrm{E}+00$ & $5.810 \mathrm{E}-01$ & $1.453 \mathrm{E}+00$ \\
\hline
\end{tabular}

$\begin{array}{cccccccc}\text { POINT } & \text { S01-S05 } & \text { S06-S10 } & \text { S11-S15 } & \text { S16-S20 } & \text { S21-S25 } & \text { S26-S30 } & \text { S31-S35 } \\ \text { C01 } & 6.928 E-01 & 1.410 E-01 & 4.043 E-01 & 2.214 E+00 & 7.849 \mathrm{E}-01 & 1.123 \mathrm{E}-01 & 3.749 \mathrm{E}-01 \\ \text { C02 } & 7.773 \mathrm{E}+00 & 9.904 \mathrm{E}-01 & 2.295 \mathrm{E}+00 & 5.934 \mathrm{E}+00 & 1.556 \mathrm{E}+00 & 2.841 \mathrm{E}-01 & 2.891 \mathrm{E}+00 \\ \text { C03 } & 1.375 \mathrm{E}+02 & 3.062 \mathrm{E}+00 & 1.144 \mathrm{E}+01 & 3.386 \mathrm{E}+00 & 1.061 \mathrm{E}+00 & 3.926 \mathrm{E}-01 & 8.728 \mathrm{E}+00 \\ \text { C04 } & 2.735 \mathrm{E}+02 & 1.910 \mathrm{E}+00 & 3.316 \mathrm{E}+01 & 2.932 \mathrm{E}+00 & 6.668 \mathrm{E}-01 & 1.744 \mathrm{E}+00 & 4.816 \mathrm{E}+00 \\ \text { C05 } & 1.486 \mathrm{E}+01 & 3.805 \mathrm{E}-01 & 1.465 \mathrm{E}+02 & 1.033 \mathrm{E}+01 & 3.989 \mathrm{E}-01 & 1.906 \mathrm{E}+01 & 8.353 \mathrm{E}-01 \\ \text { C06 } & 9.363 \mathrm{E}-01 & 6.618 \mathrm{E}-02 & 6.365 \mathrm{E}+01 & 7.349 \mathrm{E}+00 & 1.081 \mathrm{E}-01 & 4.814 \mathrm{E}+01 & 1.044 \mathrm{E}-01 \\ \text { C07 } & 4.758 \mathrm{E}-02 & 4.270 \mathrm{E}+00 & 1.527 \mathrm{E}+00 & 3.429 \mathrm{E}+00 & 1.555 \mathrm{E}+01 & 2.716 \mathrm{E}+00 & 3.853 \mathrm{E}-01 \\ \text { C08 } & 1.080 \mathrm{E}-01 & 3.275 \mathrm{E}+00 & 2.988 \mathrm{E}+00 & 4.724 \mathrm{E}+00 & 1.496 \mathrm{E}+01 & 2.728 \mathrm{E}+01 & 5.130 \mathrm{E}+00\end{array}$




\section{REVISED METHODOLOGY FOR DETERMINING CESIUM-137 CONTENT OF HN-200 GROUT CONTAINERS.}

\begin{tabular}{|c|c|c|c|c|c|c|c|}
\hline $\mathrm{CO9}$ & $1.154 \mathrm{E}-01$ & $6.664 \mathrm{E}-01$ & $1.530 \mathrm{E}+00$ & $1.743 \mathrm{E}+00$ & $2.606 \mathrm{E}+01$ & $5.027 \mathrm{E}+01$ & $7.190 \mathrm{E}+01$ \\
\hline C10 & $6.506 \mathrm{E}-02$ & $9.148 \mathrm{E}-02$ & $6.607 \mathrm{E}-01$ & $1.061 E+00$ & $4.985 \mathrm{E}+00$ & $6.955 E+00$ & $1.048 \mathrm{E}+02$ \\
\hline C11 & $2.279 \mathrm{E}-02$ & $1.857 \mathrm{E}-02$ & $3.074 \mathrm{E}-0 \mathrm{I}$ & $2.069 \mathrm{E}-01$ & $4.560 \mathrm{E}-01$ & $5.337 \mathrm{E}-01$ & $.104 \mathrm{E}+00$ \\
\hline $\mathrm{C} 12$ & $5.074 \mathrm{E}-03$ & $5.154 \mathrm{E}-03$ & $1.060 \mathrm{E}-01$ & $1.732 \mathrm{E}-01$ & 4.196E-02 & $4.267 \mathrm{E}-02$ & $3.228 \mathrm{E}+00$ \\
\hline C13 & $1.088 \mathrm{E}+00$ & $1.141 \mathrm{E}+01$ & $1.482 \mathrm{E}+01$ & $8.681 E-02$ & $6.753 \mathrm{E}+00$ & $8.257 \mathrm{E}-02$ & $6.815 \mathrm{E}-03$ \\
\hline C14 & $6.310 \mathrm{E}+00$ & $1.007 \mathrm{E}+01$ & $7.390 \mathrm{E}+01$ & $7.901 \mathrm{E}-01$ & $2.572 \mathrm{E}+00$ & $1.797 \mathrm{E}-01$ & $2.849 \mathrm{E}-02$ \\
\hline C15 & $5.764 E+00$ & $1.856 \mathrm{E}+00$ & $1.995 \mathrm{E}+01$ & $1.205 \mathrm{E}+01$ & $3.875 \mathrm{E}-01$ & $1.427 \mathrm{E}-01$ & $7.144 \mathrm{E}-02$ \\
\hline C16 & $1.019 \mathrm{E}+\infty 0$ & $3.983 \mathrm{E}-01$ & $1.664 \mathrm{E}+00$ & $1.344 \mathrm{E}+02$ & $1.063 \mathrm{E}-01$ & $4.976 \mathrm{E}-02$ & $1.523 \mathrm{E}-01$ \\
\hline C17 & $2.148 \mathrm{E}-01$ & $2.038 \mathrm{E}-01$ & $1.238 \mathrm{E}+00$ & $5.517 \mathrm{E}+01$ & $6.125 \mathrm{E}-02$ & $1.491 \mathrm{E}-02$ & $4.526 \mathrm{E}-01$ \\
\hline C18 & $5.241 \mathrm{E}-02$ & $1.150 \mathrm{E}-01$ & $9.743 \mathrm{E}-03$ & $3.150 \mathrm{E}+01$ & $2.079 \mathrm{E}-02$ & $5.926 \mathrm{E}-03$ & 55E-01 \\
\hline C19 & $2.190 \mathrm{E}+00$ & $2.169 \mathrm{E}-01$ & $1.376 \mathrm{E}-02$ & $1.068 \mathrm{E}-02$ & $1.899 \mathrm{E}-02$ & $4.187 \mathrm{E}-02$ & $2.285 \mathrm{E}+00$ \\
\hline C20 & $1.115 \mathrm{E}+01$ & $6.391 \mathrm{E}-01$ & $2.206 \mathrm{E}-02$ & $1.909 \mathrm{E}-02$ & $1.515 \mathrm{E}-01$ & $2.234 \mathrm{E}-01$ & $2.706 \mathrm{E}+01$ \\
\hline C21 & $7.908 \mathrm{E}+01$ & $2.642 \mathrm{E}+00$ & $2.049 \mathrm{E}-02$ & $5.306 \mathrm{E}-02$ & $1.112 E+\infty 0$ & $2.180 \mathrm{E}+00$ & $5.480 \mathrm{E}+02$ \\
\hline $\mathrm{C} 22$ & $4.871 \mathrm{E}+01$ & $7.267 \mathrm{E}+00$ & $1.419 \mathrm{E}-02$ & $2.069 \mathrm{E}-01$ & $3.637 \mathrm{E}+00$ & $3.151 E+01$ & $1.380 \mathrm{E}+02$ \\
\hline $\mathrm{C} 23$ & $1.130 \mathrm{E}+01$ & $1.482 \mathrm{E}+01$ & $9.163 \mathrm{E}-03$ & $4.713 \mathrm{E}-01$ & $2.582 \mathrm{E}+00$ & $3.452 \mathrm{E}+02$ & $2.548 \mathrm{E}+01$ \\
\hline C2A & $1.465 \mathrm{E}+00$ & $1.377 \mathrm{E}+02$ & $5.136 \mathrm{E}-03$ & $3.866 \mathrm{E}-01$ & $5.025 \mathrm{E}-01$ & $3.098 \mathrm{E}+01$ & $2.208 \mathrm{E}+00$ \\
\hline R01 & $8.252 \mathrm{E}-01$ & $8.954 \mathrm{E}-02$ & $1.125 \mathrm{E}+00$ & $9.452 \mathrm{E}-01$ & $2.675 \mathrm{E}-01$ & $6.203 \mathrm{E}-01$ & $9.200 \mathrm{E}-01$ \\
\hline R02 & $6.552 \mathrm{E}-01$ & $4.714 \mathrm{E}-02$ & $1.208 \mathrm{E}+00$ & $9.339 \mathrm{E}-01$ & $9 \mathrm{E}-01$ & $4.734 \mathrm{E}-01$ & $9.722 \mathrm{E}-01$ \\
\hline $\mathbf{R 0 3}$ & 4.904E-01 & $3.815 \mathrm{E}-02$ & $1.142 \mathrm{E}+00$ & $6.937 \mathrm{E}-01$ & $5.917 \mathrm{E}-01$ & 4.463E-01 & $1.188 \mathrm{E}+00$ \\
\hline R04 & $2.571 \mathrm{E}-01$ & $7.219 \mathrm{E}-02$ & $9.419 \mathrm{E}-01$ & $3.957 \mathrm{E}-01$ & $8.309 \mathrm{E}-01$ & $4.019 \mathrm{E}-01$ & $1.429 \mathrm{E}+00$ \\
\hline R05 & $6.695 \mathrm{E}-02$ & $1.366 \mathrm{E}-01$ & $6.466 \mathrm{E}-0 \mathrm{I}$ & $3.004 \mathrm{E}-01$ & $9.976 \mathrm{E}-01$ & $3.594 \mathrm{E}-01$ & $1.417 \mathrm{E}+00$ \\
\hline R06 & $3.431 \mathrm{E}-02$ & $2.003 \mathrm{E}-01$ & $4.752 \mathrm{E}-01$ & $3.157 \mathrm{E}-01$ & $9.610 \mathrm{E}-01$ & $2.758 \mathrm{E}-01$ & $1.114 \mathrm{E}+00$ \\
\hline R07 & $5.874 \mathrm{E}-02$ & $2.413 \mathrm{E}-01$ & $5.388 \mathrm{E}-01$ & $4.365 \mathrm{E}-01$ & $8.205 \mathrm{E}-01$ & $1.514 \mathrm{E}-01$ & $7.521 \mathrm{E}-01$ \\
\hline R08 & $1.741 \mathrm{E}-01$ & $2.521 \mathrm{E}-01$ & $5.830 \mathrm{E}-01$ & $5.442 \mathrm{E}-01$ & $6.566 \mathrm{E}-01$ & $5.037 \mathrm{E}-02$ & $5.671 \mathrm{E}-01$ \\
\hline R09 & $3.824 \mathrm{E}-01$ & $2.830 \mathrm{E}-01$ & $5.498 \mathrm{E}-01$ & $5.591 \mathrm{E}-01$ & $5.643 \mathrm{E}-01$ & $2.852 \mathrm{E}-02$ & $5.474 \mathrm{E}-01$ \\
\hline R10 & $5.216 \mathrm{E}-01$ & 4.079E-01 & 4.311E-01 & $4.680 \mathrm{E}-01$ & 4.546E-01 & $1.578 \mathrm{E}-01$ & $6.720 \mathrm{E}-01$ \\
\hline R11 & $7.329 \mathrm{E}-01$ & $5.439 \mathrm{E}-01$ & $2.347 \mathrm{E}-01$ & $3.160 \mathrm{E}-01$ & $2.070 \mathrm{E}-01$ & 4.293E-01 & $7.228 \mathrm{E}-01$ \\
\hline R12 & $8.987 \mathrm{E}-01$ & $6.206 \mathrm{E}-01$ & $5.802 \mathrm{E}-02$ & $3.628 \mathrm{E}-01$ & $5.661 \mathrm{E}-02$ & $6.243 \mathrm{E}-01$ & $8.985 \mathrm{E}-01$ \\
\hline R13 & $9.943 \mathrm{E}-01$ & $6.275 \mathrm{E}-01$ & $1.984 \mathrm{E}-02$ & $5.650 \mathrm{E}-01$ & $6.231 \mathrm{E}-02$ & $7.827 \mathrm{E}-01$ & $1.001 \mathrm{E}+00$ \\
\hline R14 & $1.120 \mathrm{E}+00$ & $5.558 \mathrm{E}-01$ & $1.830 \mathrm{E}-01$ & $6.726 \mathrm{E}-01$ & $7.016 \mathrm{E}-02$ & $9.159 \mathrm{E}-01$ & $1.002 \mathrm{E}+00$ \\
\hline R15 & $1.160 \mathrm{E}+00$ & $3.972 \mathrm{E}-01$ & $5.783 \mathrm{E}-01$ & $7.595 \mathrm{E}-01$ & $7.046 \mathrm{E}-02$ & $9.444 \mathrm{E}-01$ & $1.075 \mathrm{E}+00$ \\
\hline R16 & $9.948 \mathrm{E}-01$ & $2.018 \mathrm{E}-01$ & $9.055 \mathrm{E}-01$ & $8.607 \mathrm{E}-01$ & $1.052 \mathrm{E}-01$ & 8.443E-01 & $1.126 \mathrm{E}+00$ \\
\hline
\end{tabular}

\begin{tabular}{cccccccc} 
& \multicolumn{7}{c}{ SOURCE } \\
POINT & S01-S03 & S04-S06 & S07-S09 & S10-S12 & S13-S15 & S16-S18 & S19-S21 \\
C01 & $6.828 E-01$ & $1.333 E-01$ & $7.419 E-03$ & $1.917 E-01$ & $2.229 E-01$ & $2.022 \mathrm{E}+00$ & $1.963 \mathrm{E}-01$ \\
C02 & $7.731 \mathrm{E}+00$ & $1.007 \mathrm{E}+00$ & $1.716 \mathrm{E}-02$ & $1.877 \mathrm{E}+00$ & $4.265 \mathrm{E}-01$ & $5.728 \mathrm{E}+00$ & $2.423 \mathrm{E}-01$ \\
C03 & $1.374 \mathrm{E}+02$ & $3.096 \mathrm{E}+00$ & $5.034 \mathrm{E}-02$ & $1.026 \mathrm{E}+01$ & $1.182 \mathrm{E}+00$ & $3.309 \mathrm{E}+00$ & $2.738 \mathrm{E}-01$ \\
C04 & $2.734 \mathrm{E}+02$ & $1.890 \mathrm{E}+00$ & $9.236 \mathrm{E}-02$ & $2.017 \mathrm{E}+01$ & $1.300 \mathrm{E}+01$ & $2.918 \mathrm{E}+00$ & $4.881 \mathrm{E}-01$ \\
C05 & $1.483 \mathrm{E}+01$ & $3.317 \mathrm{E}-01$ & $7.398 \mathrm{E}-02$ & $7.560 \mathrm{E}+00$ & $1.389 \mathrm{E}+02$ & $1.033 \mathrm{E}+01$ & $3.737 \mathrm{E}-01$ \\
C06 & $9.317 \mathrm{E}-01$ & $3.537 \mathrm{E}-02$ & $3.546 \mathrm{E}-02$ & $8.411 \mathrm{E}-01$ & $6.281 \mathrm{E}+01$ & $7.349 \mathrm{E}+00$ & $1.054 \mathrm{E}-01$ \\
C07 & $1.882 \mathrm{E}-02$ & $3.048 \mathrm{E}-02$ & $6.838 \mathrm{E}-02$ & $4.224 \mathrm{E}+00$ & $1.503 \mathrm{E}+00$ & $7.285 \mathrm{E}-01$ & $2.701 \mathrm{E}+00$ \\
C08 & $3.418 \mathrm{E}-02$ & $7.969 \mathrm{E}-02$ & $6.675 \mathrm{E}-02$ & $3.338 \mathrm{E}+00$ & $2.852 \mathrm{E}+00$ & $1.707 \mathrm{E}+00$ & $3.020 \mathrm{E}+00$ \\
C09 & $4.438 \mathrm{E}-02$ & $8.114 \mathrm{E}-02$ & $3.222 \mathrm{E}-02$ & $1.010 \mathrm{E}+00$ & $1.144 \mathrm{E}+00$ & $1.035 \mathrm{E}+00$ & $7.186 \mathrm{E}-01$
\end{tabular}




\section{REVISED METHODOLOGY FOR DETERMINING CESIUM-137 CONTENT OF HN-200 GROUT CONTAINERS.}

\begin{tabular}{|c|c|c|c|c|c|c|c|}
\hline C10 & $3.906 \mathrm{E}-02$ & $3.402 \mathrm{E}-02$ & $1.663 \mathrm{E}-02$ & $5.226 \mathrm{E}-01$ & $2.049 \mathrm{E}-01$ & 2.689E-01 & $8.119 \mathrm{E}-01$ \\
\hline C11 & $1.794 \mathrm{E}-02$ & $7.895 \mathrm{E}-03$ & $9.919 \mathrm{E}-03$ & 2.337E-01 & $7.932 \mathrm{E}-02$ & $1.839 \mathrm{E}-01$ & $3.967 \mathrm{E}-02$ \\
\hline $\mathrm{C} 12$ & $4.451 \mathrm{E}-03$ & $1.283 \mathrm{E}-03$ & $4.068 \mathrm{E}-03$ & $5.355 \mathrm{E}-02$ & $5.290 \mathrm{E}-02$ & $1.671 \mathrm{E}-01$ & $1.278 \mathrm{E}-02$ \\
\hline $\mathrm{C13}$ & $6.918 \mathrm{E}-02$ & $1.020 \mathrm{E}+00$ & $1.005 E+01$ & $1.358 \mathrm{E}+\infty 0$ & $1.482 \mathrm{E}+01$ & $3.040 \mathrm{E}-03$ & 8.502E-02 \\
\hline C14 & $1.062 \mathrm{E}-01$ & $6.207 \mathrm{E}+00$ & $8.983 E+00$ & $1.089 \mathrm{E}+00$ & $7.390 \mathrm{E}+01$ & $2.109 \mathrm{E}-02$ & $7.773 \mathrm{E}-01$ \\
\hline C15 & $1.120 \mathrm{E}-01$ & $5.657 \mathrm{E}+00$ & $1.582 E+00$ & $2.707 \mathrm{E}-01$ & $1.995 \mathrm{E}+01$ & $2.602 \mathrm{E}-01$ & $1.182 \mathrm{E}+01$ \\
\hline C16 & $1.661 \mathrm{E}-01$ & $8.565 \mathrm{E}-01$ & $3.600 \mathrm{E}-01$ & 3.633E-02 & $1.662 \mathrm{E}+00$ & $3.330 \mathrm{E}+00$ & $1.312 E+02$ \\
\hline C17 & $1.400 \mathrm{E}-01$ & $7.636 \mathrm{E}-02$ & $1.990 \mathrm{E}-01$ & $4.218 \mathrm{E}-03$ & $1.237 \mathrm{E}+00$ & $2.709 E+01$ & $2.814 \mathrm{E}+01$ \\
\hline C18 & $4.653 \mathrm{E}-02$ & $6.240 \mathrm{E}-03$ & $1.144 \mathrm{E}-01$ & $5.900 \mathrm{E}-04$ & $9.421 \mathrm{E}-03$ & $2.977 \mathrm{E}+01$ & $1.747 \mathrm{E}+00$ \\
\hline C19 & $1.575 E+00$ & $6.600 \mathrm{E}-01$ & $1.659 \mathrm{E}-01$ & $7.153 \mathrm{E}-03$ & $1.261 \mathrm{E}-02$ & $3.303 \mathrm{E}-03$ & $2.045 \mathrm{E}-02$ \\
\hline $\mathrm{C20}$ & $2.874 \mathrm{E}+00$ & $8.535 \mathrm{E}+00$ & $3.728 \mathrm{E}-01$ & $9.440 \mathrm{E}-03$ & $1.797 \mathrm{E}-02$ & $8.761 \mathrm{E}-03$ & $1.543 E-01$ \\
\hline C21 & $2.904 E+00$ & $7.681 \mathrm{E}+01$ & $2.007 \mathrm{E}+\infty 0$ & $1.031 \mathrm{E}-02$ & $1.257 \mathrm{E}-02$ & $3.837 \mathrm{E}-02$ & $1.122 \mathrm{E}+00$ \\
\hline $\mathrm{C} 22$ & $1.031 \mathrm{E}+01$ & $3.883 \mathrm{E}+01$ & $6.836 \mathrm{E}+00$ & $8.364 \mathrm{E}-03$ & $6.430 \mathrm{E}-03$ & $1.867 \mathrm{E}-01$ & $3.655 \mathrm{E}+00$ \\
\hline $\mathrm{C} 23$ & $8.160 \mathrm{E}+00$ & $3.244 \mathrm{E}+00$ & $1.472 \mathrm{E}+01$ & $4.178 \mathrm{E}-03$ & $5.088 \mathrm{E}-03$ & $4.567 \mathrm{E}-01$ & $2.597 \mathrm{E}+00$ \\
\hline C24 & $1.233 \mathrm{E}+00$ & $2.439 \mathrm{E}-01$ & $1.377 \mathrm{E}+02$ & $1.298 \mathrm{E}-03$ & $3.851 \mathrm{E}-03$ & $3.816 \mathrm{E}-01$ & $5.074 \mathrm{E}-01$ \\
\hline R01 & $6.850 \mathrm{E}-01$ & $2.089 \mathrm{E}-01$ & $1.975 \mathrm{E}-02$ & $4.458 \mathrm{E}-01$ & $6.800 \mathrm{E}-01$ & $9.326 \mathrm{E}-01$ & $3.461 \mathrm{E}-02$ \\
\hline R02 & $6.352 \mathrm{E}-01$ & $5.825 \mathrm{E}-02$ & $3.781 \mathrm{E}-03$ & $5.099 \mathrm{E}-01$ & $7.029 \mathrm{E}-01$ & $9.076 \mathrm{E}-01$ & $3.596 \mathrm{E}-02$ \\
\hline $\mathbf{R} 03$ & $4.884 \mathrm{E}-01$ & $1.665 \mathrm{E}-02$ & $1.775 \mathrm{E}-03$ & $4.997 \mathrm{E}-01$ & $6.637 \mathrm{E}-01$ & $6.523 \mathrm{E}-01$ & 4.534E-02 \\
\hline R04 & $2.542 \mathrm{E}-01$ & $6.944 \mathrm{E}-03$ & $2.690 \mathrm{E}-03$ & $4.332 \mathrm{E}-01$ & $5.741 \mathrm{E}-01$ & $3.440 \mathrm{E}-01$ & $5.343 \mathrm{E}-02$ \\
\hline R05 & $5.647 \mathrm{E}-02$ & $1.151 \mathrm{E}-02$ & $8.033 \mathrm{E}-03$ & $3.250 \mathrm{E}-01$ & 4.491E-01 & $2.337 \mathrm{E}-01$ & $6.764 \mathrm{E}-02$ \\
\hline R06 & $6.064 \mathrm{E}-03$ & $2.857 \mathrm{E}-02$ & $2.442 \mathrm{E}-02$ & $2.266 \mathrm{E}-01$ & $4.242 \mathrm{E}-01$ & $1.890 \mathrm{E}-01$ & $1.275 \mathrm{E}-01$ \\
\hline R07 & $7.552 \mathrm{E}-03$ & $5.136 \mathrm{E}-02$ & 5.733E-02 & $1.899 \mathrm{E}-01$ & $5.327 \mathrm{E}-01$ & $2.227 \mathrm{E}-01$ & $2.148 \mathrm{E}-01$ \\
\hline R08 & $1.065 \mathrm{E}-01$ & $6.768 \mathrm{E}-02$ & $1.041 \mathrm{E}-01$ & $1.486 \mathrm{E}-01$ & $5.823 \mathrm{E}-01$ & $2.686 \mathrm{E}-01$ & $2.774 \mathrm{E}-01$ \\
\hline R09 & $3.087 \mathrm{E}-01$ & $7.407 \mathrm{E}-02$ & $1.960 \mathrm{E}-01$ & 8.689E-02 & $5.496 \mathrm{E}-01$ & $2.647 \mathrm{E}-01$ & $2.988 \mathrm{E}-01$ \\
\hline R10 & $3.994 \mathrm{E}-01$ & $1.233 \mathrm{E}-01$ & $3.731 \mathrm{E}-01$ & 3.384E-02 & $4.310 \mathrm{E}-01$ & $2.031 \mathrm{E}-01$ & $2.757 \mathrm{E}-01$ \\
\hline R11 & $4.510 \mathrm{E}-01$ & $2.861 \mathrm{E}-01$ & $5.310 \mathrm{E}-01$ & $8.827 \mathrm{E}-03$ & $2.346 \mathrm{E}-01$ & $1.275 \mathrm{E}-01$ & $2.125 \mathrm{E}-01$ \\
\hline R12 & 4.741E-01 & 4.394E-01 & $6.039 \mathrm{E}-01$ & $2.249 \mathrm{E}-03$ & $5.763 \mathrm{E}-02$ & $2.770 \mathrm{E}-01$ & $1.291 \mathrm{E}-01$ \\
\hline R13 & $4.938 \mathrm{E}-01$ & $5.392 \mathrm{E}-01$ & $5.884 \mathrm{E}-01$ & 3.053E-03 & $1.726 \mathrm{E}-02$ & $5.472 \mathrm{E}-01$ & $7.898 \mathrm{E}-02$ \\
\hline R14 & $6.075 \mathrm{E}-01$ & $5.819 \mathrm{E}-01$ & 4.865E- 01 & $2.347 \mathrm{E}-02$ & $1.598 \mathrm{E}-01$ & $6.701 \mathrm{E}-01$ & $7.039 \mathrm{E}-02$ \\
\hline R15 & $7.015 \mathrm{E}-01$ & $5.486 \mathrm{E}-01$ & $3.070 \mathrm{E}-01$ & $1.293 \mathrm{E}-01$ & $4.491 \mathrm{E}-01$ & $7.577 \mathrm{E}-01$ & $6.138 \mathrm{E}-02$ \\
\hline R16 & $6.638 \mathrm{E}-01$ & $4.209 \mathrm{E}-01$ & $1.116 \mathrm{E}-01$ & $3.060 \mathrm{E}-01$ & $5.998 \mathrm{E}-01$ & $8.558 \mathrm{E}-01$ & $4.573 \mathrm{E}-02$ \\
\hline
\end{tabular}

$\begin{array}{cccccc}\text { POINT } & \text { S22-S24 } & \text { S25-S27 } & \text { S28-S30 } & \text { S31-S33 } & \text { S34-S35 } \\ \text { C01 } & 1.540 \mathrm{E}-01 & 6.649 \mathrm{E}-01 & 7.403 \mathrm{E}-02 & 5.608 \mathrm{E}-03 & 3.693 \mathrm{E}-01 \\ \text { C02 } & 1.273 \mathrm{E}-01 & 1.509 \mathrm{E}+00 & 1.670 \mathrm{E}-01 & 2.968 \mathrm{E}-02 & 2.861 \mathrm{E}+00 \\ \text { C03 } & 1.065 \mathrm{E}-01 & 9.029 \mathrm{E}-01 & 2.471 \mathrm{E}-01 & 9.942 \mathrm{E}-02 & 8.629 \mathrm{E}+00 \\ \text { C04 } & 4.984 \mathrm{E}-02 & 2.176 \mathrm{E}-01 & 1.670 \mathrm{E}+00 & 1.631 \mathrm{E}-01 & 4.653 \mathrm{E}+00 \\ \text { C05 } & 1.127 \mathrm{E}-02 & 4.364 \mathrm{E}-02 & 1.903 \mathrm{E}+01 & 1.071 \mathrm{E}-01 & 7.282 \mathrm{E}-01 \\ \text { C06 } & 1.578 \mathrm{E}-03 & 1.186 \mathrm{E}-02 & 4.813 \mathrm{E}+01 & 3.052 \mathrm{E}-02 & 7.386 \mathrm{E}-02 \\ \text { C07 } & 1.454 \mathrm{E}+01 & 3.484 \mathrm{E}+00 & 2.402 \mathrm{E}-01 & 3.081 \mathrm{E}-01 & 7.723 \mathrm{E}-02 \\ \text { C08 } & 1.253 \mathrm{E}+01 & 2.910 \mathrm{E}+01 & 5.954 \mathrm{E}-01 & 4.728 \mathrm{E}+00 & 4.019 \mathrm{E}-01 \\ \text { C09 } & 2.481 \mathrm{E}+01 & 5.108 \mathrm{E}+01 & 4.261 \mathrm{E}-01 & 7.100 \mathrm{E}+01 & 8.947 \mathrm{E}-01 \\ \text { C10 } & 4.756 \mathrm{E}+00 & 7.059 \mathrm{E}+00 & 1.051 \mathrm{E}-01 & 1.040 \mathrm{E}+02 & 8.125 \mathrm{E}-01\end{array}$




\section{REVISED METHODOLOGY FOR DETERMINING CESIUM-137 CONTENT OF HN-200 GROUT CONTAINERS.}

\begin{tabular}{|c|c|c|c|c|c|}
\hline C11 & 4.180E-01 & $5.378 \mathrm{E}-01$ & $1.724 \mathrm{E}-02$ & $7.525 \mathrm{E}+00$ & $1.580 \mathrm{E}+00$ \\
\hline C12 & $3.355 \mathrm{E}-02$ & $3.864 \mathrm{E}-02$ & $5.815 \mathrm{E}-03$ & $4.845 \mathrm{E}-01$ & $2.743 \mathrm{E}+00$ \\
\hline C13 & $6.746 \mathrm{E}+00$ & $1.055 \mathrm{E}-02$ & $7.726 \mathrm{E}-02$ & $6.588 \mathrm{E}-03$ & $2.267 \mathrm{E}-04$ \\
\hline C14 & $2.556 \mathrm{E}+00$ & $2.132 \mathrm{E}-02$ & $1.664 \mathrm{E}-01$ & 2.705E-02 & $1.442 \mathrm{E}-03$ \\
\hline C15 & $3.471 \mathrm{E}-01$ & $2.223 \mathrm{E}-02$ & $1.263 \mathrm{E}-01$ & $5.946 \mathrm{E}-02$ & $1.198 \mathrm{E}-02$ \\
\hline C16 & $3.430 \mathrm{E}-02$ & $1.347 \mathrm{E}-02$ & $3.841 \mathrm{E}-02$ & $6.007 \mathrm{E}-02$ & $9.225 \mathrm{E}-02$ \\
\hline C17 & $3.157 \mathrm{E}-03$ & $7.151 \mathrm{E}-03$ & $8.207 \mathrm{E}-03$ & $2.820 \mathrm{E}-02$ & $4.244 \mathrm{E}-01$ \\
\hline C18 & $3.011 \mathrm{E}-04$ & $3.371 \mathrm{E}-03$ & $2.621 \mathrm{E}-03$ & $7.272 \mathrm{E}-03$ & $7.083 \mathrm{E}-01$ \\
\hline C19 & $1.854 \mathrm{E}-03$ & $1.451 \mathrm{E}-02$ & $3.141 \mathrm{E}-02$ & $2.284 \mathrm{E}+00$ & $2.956 \mathrm{E}-04$ \\
\hline $\mathrm{C20}$ & $1.316 \mathrm{E}-03$ & $1.222 \mathrm{E}-01$ & $1.074 \mathrm{E}-01$ & $2.706 \mathrm{E}+01$ & $7.855 \mathrm{E}-04$ \\
\hline C21 & $6.177 \mathrm{E}-04$ & $1.472 \mathrm{E}+00$ & $7.131 \mathrm{E}-01$ & $5.480 \mathrm{E}+02$ & $1.198 \mathrm{E}-03$ \\
\hline $\mathrm{C22}$ & $2.295 \mathrm{E}-04$ & $2.363 \mathrm{E}+01$ & $7.880 \mathrm{E}+00$ & $1.380 \mathrm{E}+02$ & $1.041 \mathrm{E}-03$ \\
\hline $\mathrm{C} 23$ & $6.840 \mathrm{E}-05$ & $3.033 \mathrm{E}+02$ & 4.189E+01 & $2.548 \mathrm{E}+01$ & $6.371 \mathrm{E}-04$ \\
\hline C24 & $1.545 \mathrm{E}-05$ & $1.729 \mathrm{E}+01$ & $1.369 \mathrm{E}+01$ & $2.207 \mathrm{E}+00$ & $3.881 \mathrm{E}-04$ \\
\hline R01 & $1.813 \mathrm{E}-01$ & $1.769 \mathrm{E}-01$ & $5.075 \mathrm{E}-01$ & $1.597 \mathrm{E}-01$ & $7.603 \mathrm{E}-01$ \\
\hline R02 & $3.412 \mathrm{E}-01$ & $2.813 \mathrm{E}-01$ & $2.951 \mathrm{E}-01$ & $1.208 \mathrm{E}-01$ & $8.514 \mathrm{E}-01$ \\
\hline $\mathbf{R 0 3}$ & $4.651 \mathrm{E}-01$ & $4.133 \mathrm{E}-01$ & $1.558 \mathrm{E}-01$ & $2.409 \mathrm{E}-01$ & $9.470 \mathrm{E}-01$ \\
\hline R04 & $7.168 \mathrm{E}-01$ & $4.654 \mathrm{E}-01$ & $4.889 \mathrm{E}-02$ & $3.491 \mathrm{E}-01$ & $1.080 \mathrm{E}+00$ \\
\hline R05 & $9.188 \mathrm{E}-01$ & $4.210 \mathrm{E}-01$ & $1.622 \mathrm{E}-02$ & $3.942 \mathrm{E}-01$ & $1.023 \mathrm{E}+00$ \\
\hline R06 & $9.222 \mathrm{E}-01$ & $3.016 \mathrm{E}-01$ & $1.217 \mathrm{E}-02$ & $3.675 \mathrm{E}-01$ & $7.464 \mathrm{E}-01$ \\
\hline R07 & $8.071 \mathrm{E}-01$ & $1.538 \mathrm{E}-01$ & $9.982 \mathrm{E}-03$ & $2.731 \mathrm{E}-01$ & $4.790 \mathrm{E}-01$ \\
\hline R08 & $6.517 \mathrm{E}-01$ & $4.575 \mathrm{E}-02$ & $7.656 \mathrm{E}-03$ & $1.453 \mathrm{E}-01$ & $4.218 \mathrm{E}-01$ \\
\hline R09 & $5.592 \mathrm{E}-01$ & $2.099 \mathrm{E}-02$ & $8.270 \mathrm{E}-03$ & $1.922 \mathrm{E}-01$ & $3.552 \mathrm{E}-01$ \\
\hline R10 & $4.435 \mathrm{E}-01$ & $1.058 \mathrm{E}-01$ & $5.228 \mathrm{E}-02$ & 4.595E-01 & $2.125 \mathrm{E}-01$ \\
\hline R11 & $1.829 \mathrm{E}-01$ & $2.124 \mathrm{E}-01$ & $2.170 \mathrm{E}-01$ & $6.943 \mathrm{E}-01$ & $2.848 \mathrm{E}-02$ \\
\hline R12 & $1.306 \mathrm{E}-02$ & $2.676 \mathrm{E}-01$ & $3.570 \mathrm{E}-01$ & 8.973E-01 & $1.186 \mathrm{E}-03$ \\
\hline $\mathbf{R} 13$ & $5.911 \mathrm{E}-04$ & $2.909 \mathrm{E}-01$ & $4.923 \mathrm{E}-01$ & $1.001 \mathrm{E}+00$ & $8.114 \mathrm{E}-04$ \\
\hline R14 & $3.586 \mathrm{E}-04$ & $2.857 \mathrm{E}-01$ & $6.321 \mathrm{E}-01$ & $9.856 \mathrm{E}-01$ & $1.683 \mathrm{E}-02$ \\
\hline R15 & $2.997 \mathrm{E}-03$ & $2.565 \mathrm{E}-01$ & $6.959 \mathrm{E}-01$ & 8.503E-01 & $2.251 \mathrm{E}-01$ \\
\hline R16 & $3.689 \mathrm{E}-02$ & $2.093 \mathrm{E}-01$ & $6.624 \mathrm{E}-01$ & 5.391E-01 & $5.864 \mathrm{E}-01$ \\
\hline
\end{tabular}

TABLE 8 - ESTIMATES OF CURIE CONTENT FOR VARIOUS SOURCE SETS COMPARED TO ACTUAL CURIE CONTENT FOR A GROUTED CONTAINER. MULTIPLE SOURCE SET ESTIMATE AND ERROR - CONTACT MEASUREMENT

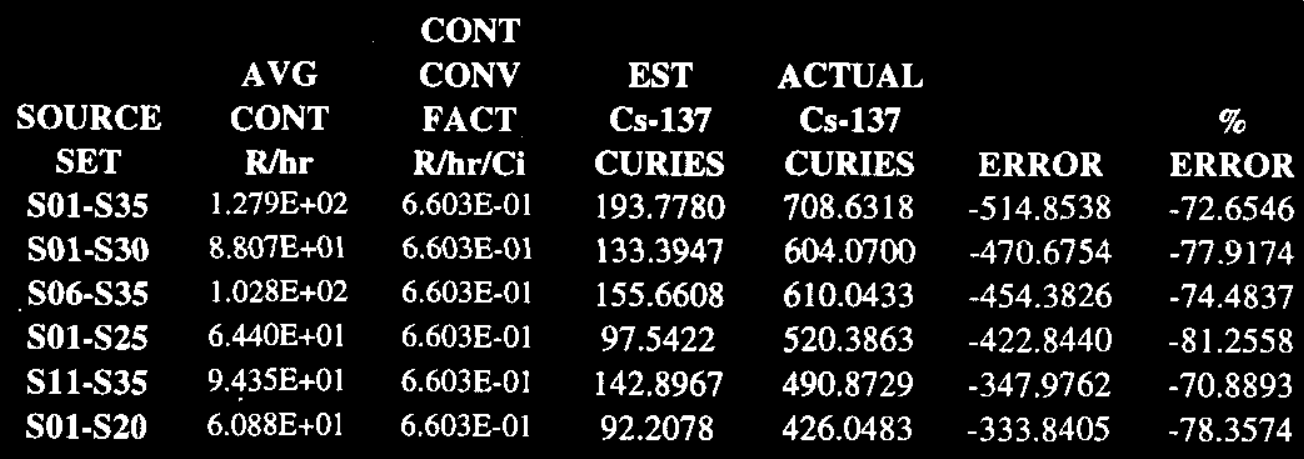




\section{REVISED METHODOLOGY FOR DETERMINING CESIUM-137 CONTENT OF HN-200 GROUT CONTAINERS.}

\begin{tabular}{|c|c|c|c|c|c|c|}
\hline S16-S25 & $7.867 \mathrm{E}+01$ & $6.603 \mathrm{E}-01$ & 119.1554 & 393.8550 & -274.6996 & -69.7464 \\
\hline S01-S18 & $5.332 \mathrm{E}+01$ & $6.603 \mathrm{E}-01$ & 80.7628 & 394.2079 & -313.4452 & -79.5126 \\
\hline S19.S35 & $7.462 \mathrm{E}+01$ & $6.603 \mathrm{E}-01$ & 113.0152 & 314.4239 & -201.4086 & -64.0564 \\
\hline SO1-S12 & $3.535 E+01$ & $6.603 \mathrm{E}-01$ & 53.5439 & 247.8830 & -194.3390 & -78.3995 \\
\hline S13-S24 & $2.872 E+01$ & $6.603 \mathrm{E}-01$ & 43.5015 & 252.7408 & -209.2393 & -82.7881 \\
\hline S25-S35 & $6.387 \mathrm{E}+01$ & $6.603 \mathrm{E}-01$ & 96.7326 & 208.0080 & -111.2755 & -53.4957 \\
\hline S01-S10 & $3.359 \mathrm{E}+01$ & $6.603 \mathrm{E}-01$ & 50.8813 & 217.7589 & -166.8776 & -76.6341 \\
\hline S13-S22 & $2.636 \mathrm{E}+01$ & $6.603 \mathrm{E}-01$ & 39.9291 & 227.1843 & -187.2552 & -82.4243 \\
\hline S23-\$32 & $3.822 E+01$ & $6.603 \mathrm{E}-01$ & 57.8887 & 173.0444 & -115.1557 & -66.5469 \\
\hline S01-S07 & $3.172 \mathrm{E}+01$ & $6.603 \mathrm{E}-01$ & 48.0388 & 140.8425 & -92.8038 & -65.8919 \\
\hline S08-\$14 & $8.555 E+00$ & $6.603 \mathrm{E}-01$ & 12.9566 & 151.1873 & -138.2307 & -91.4301 \\
\hline S15-S21 & $2.100 \mathrm{E}+01$ & $6.603 \mathrm{E}-01$ & 31.8070 & 162.6650 & -130.8580 & -80.4463 \\
\hline S22-S28 & $2.403 \mathrm{E}+01$ & $6.603 \mathrm{E}-01$ & 36.3937 & 116.2697 & -79.8760 & -68.6989 \\
\hline S29-S35 & $4.264 \mathrm{E}+01$ & $6.603 \mathrm{E}-01$ & 64.5819 & 137.6673 & -73.0 & -53.0884 \\
\hline S01-S05 & $2.517 E+01$ & $6.603 \mathrm{E}-01$ & 38.1 & 885 & & -61.3370 \\
\hline S06-S10 & $8.427 E+00$ & $6.603 \mathrm{E}-01$ & 12.7641 & 119.1704 & 063 & -89.2892 \\
\hline S11-S15 & $1.568 \mathrm{E}+01$ & $6.603 \mathrm{E}-01$ & 23.7 & 97.0179 & 767 & -75.5290 \\
\hline S16-S20 & $1.161 \mathrm{E}+01$ & $6.603 \mathrm{E}-01$ & 17.5853 & 111.2715 & -93.6862 & -84.1961 \\
\hline S21-S25 & $3.522 \mathrm{E}+00$ & $6.603 \mathrm{E}-01$ & 5.3344 & 94.3380 & -89.0036 & -94.3454 \\
\hline S26-S30 & $2.367 \mathrm{E}+01$ & $6.603 \mathrm{E}-01$ & 35.8524 & 83.6838 & -47.8313 & -57.1573 \\
\hline S31-S35 & $3.987 \mathrm{E}+01$ & $6.603 E-01$ & 60.3834 & 104.5618 & 1784 & -42.2510 \\
\hline S01-S03 & $1.929 \mathrm{E}+01$ & $6.603 \mathrm{E}-01$ & 29.2 & 66. & 263 & -56.3589 \\
\hline S04-S06 & $6.203 E+00$ & $6.603 \mathrm{E}-01$ & 9.3949 & 47.9673 & -38.5725 & -80.4140 \\
\hline S07-S09 & $7.650 \mathrm{E}+00$ & $6.603 \mathrm{E}-01$ & 11.5861 & 76.0383 & -64.4522 & -84.7628 \\
\hline S10-S12 & $2.212 \mathrm{E}+00$ & $6.603 \mathrm{E}-01$ & 3.3499 & 56.9379 & -53.5881 & -94.1166 \\
\hline S13-S15 & $1.392 \mathrm{E}+01$ & $6.603 \mathrm{E}-01$ & 21.0786 & 66.8938 & -45.8152 & -68.4894 \\
\hline S16-S18 & $4.054 \mathrm{E}+00$ & $6.603 \mathrm{E}-01$ & 6.1402 & 79.4311 & -73.2909 & -92.2698 \\
\hline S19-S21 & $7.949 \mathrm{E}+00$ & $6.603 \mathrm{E}-01$ & 12.0397 & 60.4869 & -48.4473 & -80.0954 \\
\hline S22-S24 & $2.801 \mathrm{E}+00$ & $6.603 \mathrm{E}-01$ & 4.2430 & 45.9289 & -41.6859 & -90.7618 \\
\hline S25-S27 & $1.836 \mathrm{E}+01$ & $6.603 \mathrm{E}-01$ & 27.8024 & 54.8275 & -27.0251 & -49.2911 \\
\hline S28-S30 & $5.643 E+00$ & $6.603 E-01$ & 8.5468 & 48.6188 & -40.0719 & -82.4207 \\
\hline S31-S33 & $3.882 \mathrm{E}+01$ & $6.603 \mathrm{E}-01$ & 58.8015 & 61.8697 & -3.0682 & -4.9592 \\
\hline S34-S35 & $1.044 E+00$ & $6.603 \mathrm{E}-01$ & 1.5819 & 42.6921 & -41.1102 & -96.2947 \\
\hline
\end{tabular}

MULTIPLE SOURCE SET ESTIMATE AND ERROR - REMOTE MEASUREMENT REMOTE

$\begin{array}{ccccccc}\text { SOURCE } & \text { AVG } & \text { ROMOTE } & \text { FACT } & \begin{array}{c}\text { EST } \\ \text { Cs-137 }\end{array} & \begin{array}{c}\text { ACTUAL } \\ \text { Cs-137 }\end{array} & \\ \text { SET } & \text { R/hr } & \text { R/hr/Ci } & \text { CURIES } & \text { CURIES } & \text { ERROR } & \text { ERROR } \\ \text { S01-S35 } & 3.932 E+00 & 4.715 E-03 & 833.9262 & 708.6318 & 125.2944 & 17.6812 \\ \text { S01-S30 } & 2.969 E+00 & 4.715 E-03 & 629.7304 & 604.0700 & 25.6603 & 4.2479 \\ \text { S06-S35 } & 3.347 E+00 & 4.715 E-03 & 709.7623 & 610.0433 & 99.7189 & 16.3462 \\ \text { S01-S25 } & 2.500 E+00 & 4.715 E-03 & 530.2328 & 520.3863 & 9.8465 & 1.8922 \\ \text { S11-S35 } & 3.052 E+00 & 4.715 E-03 & 647.2612 & 490.8729 & 156.3883 & 31.8592 \\ \text { S01-S20 } & 2.052 E+00 & 4.715 E-03 & 435.1891 & 426.0483 & 9.1408 & 2.1455 \\ \text { S16-S25 } & 2.451 E+00 & 4.715 E-03 & 519.7471 & 393.8550 & 125.8920 & 31.9641\end{array}$




\section{REVISED METHODOLOGY FOR DETERMINING CESIUM-137 CONTENT OF HN-200 GROUT CONTAINERS.}

\begin{tabular}{|c|c|c|c|c|c|c|}
\hline S01-S18 & $1.947 \mathrm{E}+00$ & $4.715 \mathrm{E}-03$ & 412.9800 & 394.2079 & 18.7721 & 4.7620 \\
\hline S19-S35 & $1.985 \mathrm{E}+00$ & $4.715 \mathrm{E}-03$ & 420.9462 & 314.4239 & 106.5223 & 33.8786 \\
\hline S01-S12 & $1.037 \mathrm{E}+00$ & $4.715 \mathrm{E}-03$ & 219.9637 & 247.8830 & -27.9193 & -11.2631 \\
\hline S13-S24 & $1.427 \mathrm{E}+00$ & $4.715 \mathrm{E}-03$ & 302.6797 & 252.7408 & 49.9389 & 19.7589 \\
\hline S25-S35 & $1.468 \mathrm{E}+00$ & $4.715 \mathrm{E}-03$ & 311.2829 & 208.0080 & 103.2748 & 49.6494 \\
\hline S01-S10 & $8.801 \mathrm{E}-01$ & $4.715 \mathrm{E}-03$ & 186.6650 & 217.7589 & -31.0939 & -14.2790 \\
\hline S13-S22 & $1.269 \mathrm{E}+00$ & $4.715 \mathrm{E}-03$ & 269.1267 & 227.1843 & 41.9423 & 18.4618 \\
\hline S23-S32 & $9.233 \mathrm{E}-01$ & $4.715 \mathrm{E}-03$ & 195.8188 & 173.0444 & 22.7745 & 13.1611 \\
\hline S01-S07 & $7.692 \mathrm{E}-01$ & $4.715 E-03$ & 163.1298 & 140.8425 & 22.2872 & 15.8242 \\
\hline S08-S14 & $4.658 \mathrm{E}-01$ & $4.715 \mathrm{E}-03$ & 98.7850 & 151.1873 & -52.4023 & -34.6605 \\
\hline S15-S21 & 8.391E-01 & $4.715 \mathrm{E}-03$ & 177.9648 & 162.6650 & 15.2998 & 9.4057 \\
\hline S22-S28 & $7.410 \mathrm{E}-01$ & $4.715 \mathrm{E}-03$ & 157.1532 & 116.2697 & 40.8835 & 35.1626 \\
\hline S29-S35 & $1.117 \mathrm{E}+00$ & $4.715 \mathrm{E}-03$ & 236.8935 & 137.6673 & 99.2262 & 72.0768 \\
\hline S01-S05 & $5.854 \mathrm{E}-01$ & $4.715 \mathrm{E}-03$ & 124.1640 & 98.5885 & 25.5755 & 25.9416 \\
\hline S06-S10 & $2.947 \mathrm{E}-01$ & $4.715 \mathrm{E}-03$ & 62.5010 & 119.1704 & -56.6694 & -47.5532 \\
\hline S11-S15 & $6.012 \mathrm{E}-01$ & $4.715 E-03$ & 127.5142 & 97.0179 & 30.4963 & 31.4337 \\
\hline S16-S20 & $5.706 \mathrm{E}-01$ & $4.715 \mathrm{E}-03$ & 121.0099 & 111.2715 & 9.7384 & 8.7519 \\
\hline S21-S25 & $4.481 \mathrm{E}-01$ & $4.715 E-03$ & 95.0437 & 94.3380 & 0.7058 & 0.7481 \\
\hline S26-S30 & $4.691 \mathrm{E}-01$ & $4.715 \mathrm{E}-03$ & 99.4976 & 83.6838 & 15.8138 & 18.8971 \\
\hline S31-S35 & $9.628 \mathrm{E}-01$ & $4.715 \mathrm{E}-03$ & 204.1959 & 104.5618 & 99.6341 & 95.2872 \\
\hline S01-S03 & $3.962 \mathrm{E}-01$ & $4.715 \mathrm{E}-03$ & 84.0272 & 66.9394 & 17.0878 & 25.5273 \\
\hline S04-S06 & $2.165 \mathrm{E}-01$ & $4.715 \mathrm{E}-03$ & 45.9077 & 47.9673 & -2.0596 & -4.2938 \\
\hline S07-S09 & $2.137 \mathrm{E}-01$ & $4.715 \mathrm{E}-03$ & 45.3252 & 76.0383 & -30.7131 & -40.3916 \\
\hline S10-S12 & $2.108 \mathrm{E}-01$ & 4.715E-03 & 44.7035 & 56.9379 & -12.2344 & -21.4873 \\
\hline S13-S15 & $4.442 \mathrm{E}-01$ & $4.715 \mathrm{E}-03$ & 94.2155 & 66.8938 & 27.3217 & 40.8433 \\
\hline S16-S18 & $4.658 \mathrm{E}-01$ & $4.715 \mathrm{E}-03$ & 98.8008 & 79.4311 & 19.3697 & 24.3855 \\
\hline S19-S21 & $1.268 \mathrm{E}-01$ & $4.715 \mathrm{E}-03$ & 26.8996 & 60.4869 & -33.5873 & -55.5282 \\
\hline S22-S2A & $3.902 \mathrm{E}-01$ & $4.715 \mathrm{E}-03$ & 82.7637 & 45.9289 & 36.8348 & 80.1996 \\
\hline S25-S27 & $2.443 \mathrm{E}-01$ & 4.715E-03 & 51.8058 & 54.8275 & -3.0217 & -5.5113 \\
\hline S28-S30 & $2.607 \mathrm{E}-01$ & $4.715 \mathrm{E}-03$ & 55.2812 & 48.6188 & 6.6625 & 13.7035 \\
\hline S31-S33 & $4.793 \mathrm{E}-01$ & $4.715 \mathrm{E}-03$ & 101.6609 & 61.8697 & 39.7912 & 64.3145 \\
\hline S34-S35 & 4.835E-01 & $4.715 \mathrm{E}-03$ & 102.5350 & 42.6921 & 59.8429 & 140.1731 \\
\hline \multicolumn{7}{|c|}{ Average \% Error = } \\
\hline
\end{tabular}

\begin{tabular}{|c|c|c|c|c|c|c|}
\hline SOURCE & $\begin{array}{c}\text { AVG } \\
\text { CONT } \\
\text { R/hr }\end{array}$ & $\begin{array}{c}\text { CONT } \\
\text { CONV } \\
\text { FACT } \\
\text { R/hr/Ci }\end{array}$ & $\begin{array}{c}\text { EST } \\
\text { Cs-137 } \\
\text { CURIES }\end{array}$ & $\begin{array}{c}\text { ACTUAL } \\
\text { Cs-137 } \\
\text { CURIES }\end{array}$ & ERROR & $\begin{array}{c}\% \\
\text { ERROR }\end{array}$ \\
\hline S01 & $1.811 \mathrm{E}+01$ & 6.603E-01 & 27.4247 & 29.4975 & -2.0728 & -7.0272 \\
\hline S02 & $9.164 \mathrm{E}-01$ & $6.603 \mathrm{E}-01$ & 1.3879 & 13.8502 & -12.4623 & -89.9790 \\
\hline S03 & $2.644 \mathrm{E}-01$ & $6.603 \mathrm{E}-01$ & 0.4005 & 23.5916 & -23.1912 & -98.3025 \\
\hline S04 & $5.293 \mathrm{E}+00$ & $6.603 \mathrm{E}-01$ & 8.0173 & 20.6982 & -12.6809 & -61.2658 \\
\hline S05 & $5.856 \mathrm{E}-01$ & 6.603E-01 & 0.8869 & 10.9510 & -10.0641 & -91.9011 \\
\hline S06 & $3.240 \mathrm{E}-01$ & $6.603 \mathrm{E}-01$ & 0.4907 & 16.3182 & -15.8275 & -96.9931 \\
\hline S07 & $6.227 \mathrm{E}+00$ & 6.603E-01 & 9.4308 & 25.9358 & -16.5050 & -63.6379 \\
\hline S08 & $5.418 \mathrm{E}-01$ & $6.603 \mathrm{E}-01$ & 0.8206 & 25.1678 & -24.3473 & -96.7397 \\
\hline S09 & $8.813 \mathrm{E}-01$ & $6.603 \mathrm{E}-01$ & 1.3347 & 24.9347 & -23.5999 & -94.6471 \\
\hline S10 & $4.538 \mathrm{E}-01$ & $6.603 \mathrm{E}-01$ & 0.6873 & 26.8139 & -26.1266 & -97.4368 \\
\hline
\end{tabular}




\section{REVISED METHODOLOGY FOR DETERMINING CESIUM-137 CONTENT} OF HN-200 GROUT CONTAINERS.

$\begin{array}{lllllll}\text { S11 } & 5.206 E-01 & 6.603 E-01 & 0.7885 & 12.5933 & -11.8048 & -93.7386 \\ \text { S12 } & 1.237 E+00 & 6.603 E-01 & 1.8741 & 17.5307 & -15.6566 & -89.3097 \\ \text { S13 } & 2.571 E-01 & 6.603 E-01 & 0.3894 & 17.9331 & -17.5437 & -97.8285 \\ \text { S14 } & 4.663 E+00 & 6.603 E-01 & 7.0621 & 26.2138 & -19.1517 & -73.0598 \\ \text { S15 } & 8.997 E+00 & 6.603 E-01 & 13.6272 & 22.7469 & -9.1198 & -40.0923 \\ \text { S16 } & 6.255 E-01 & 6.603 E-01 & 0.9474 & 27.6556 & -26.7082 & -96.5742 \\ \text { S17 } & 2.539 E+00 & 6.603 E-01 & 3.8457 & 28.5227 & -24.6770 & -86.5170 \\ \text { S18 } & 8.894 E-01 & 6.603 E-01 & 1.3471 & 23.2528 & -21.9058 & -94.2069 \\ \text { S19 } & 7.232 E+00 & 6.603 E-01 & 10.9540 & 15.3416 & -4.3876 & -28.5995 \\ \text { S20 } & 3.242 E-01 & 6.603 E-01 & 0.4911 & 16.4988 & -16.0077 & -97.0234 \\ \text { S21 } & 3.926 E-01 & 6.603 E-01 & 0.5946 & 28.6466 & -28.0520 & -97.9244 \\ \text { S22 } & 4.428 E-01 & 6.603 E-01 & 0.6706 & 20.3724 & -19.7018 & -96.7082 \\ \text { S23 } & 6.599 E-01 & 6.603 E-01 & 0.9994 & 13.0146 & -12.0152 & -92.3208 \\ \text { S24 } & 1.699 E+00 & 6.603 E-01 & 2.5730 & 12.5419 & -9.9689 & -79.4850 \\ \text { S25 } & 3.280 E-01 & 6.603 E-01 & 0.4968 & 19.7625 & -19.2657 & -97.4862 \\ \text { S26 } & 3.618 E+00 & 6.603 E-01 & 5.4794 & 24.8512 & -19.3719 & -77.9513 \\ \text { S27 } & 1.441 E+01 & 6.603 E-01 & 21.8262 & 10.2138 & 11.6125 & 113.6943 \\ \text { S28 } & 2.871 E+00 & 6.603 E-01 & 4.3483 & 15.5133 & -11.1650 & -71.9705 \\ \text { S29 } & 9.683 E-02 & 6.603 E-01 & 0.1467 & 18.0653 & -17.9187 & -99.1882 \\ \text { S30 } & 2.675 E+00 & 6.603 E-01 & 4.0519 & 15.0401 & -10.9882 & -73.0595 \\ \text { S31 } & 4.020 E+00 & 6.603 E-01 & 6.0887 & 22.3550 & -16.2662 & -72.7633 \\ \text { S32 } & 7.843 E+00 & 6.603 E-01 & 11.8783 & 21.6866 & -9.8083 & -45.2275 \\ \text { S33 } & 2.696 E+01 & 6.603 E-01 & 40.8344 & 17.8281 & 23.0063 & 129.0456 \\ \text { S34 } & 2.366 E-01 & 6.603 E-01 & 0.3584 & 14.6776 & -14.3192 & -97.5585 \\ \text { S35 } & 8.078 E-01 & 6.603 E-01 & 1.2235 & 28.0146 & -26.7910 & -95.6325 \\ & & & & \text { Average } \% \text { Error }= & -69.9833\end{array}$

INDIVIDUAL SOURCE ESTIMATE AND ERROR - REMOTE MEASUREMENT REMOTE

$\begin{array}{ccccccc} & \text { AVG } & \text { CONV } & \text { EST } & \text { ACTUAL } & & \\ \text { SOURCE } & \text { REMOTE } & \text { FACT } & \text { Cs-137 } & \text { Cs-137 } & & \text { \% } \\ \text { S01 } & 2.143 E-01 & \text { R/hr/Ci } & \text { CURIES } & \text { CURIES } & \text { ERROR } & \text { ERROR } \\ \text { S02 } & 1.673 E-01 & 4.715 E-03 & 45.4521 & 29.4975 & 15.9546 & 54.0878 \\ \text { S03 } & 1.455 E-02 & 4.715 E-03 & 3.0854 & 13.8502 & 21.6391 & 156.2369 \\ \text { S04 } & 1.691 E-01 & 4.715 E-03 & 35.8599 & 23.5916 & -20.5059 & -86.9201 \\ \text { S05 } & 2.017 E-02 & 4.715 E-03 & 4.2769 & 10.9510 & -6.6741 & -60.9453 \\ \text { S06 } & 2.721 E-02 & 4.715 E-03 & 5.7709 & 16.3182 & -10.5472 & -64.6349 \\ \text { S07 } & 1.565 E-01 & 4.715 E-03 & 33.1948 & 25.9358 & 7.2590 & 27.9883 \\ \text { S08 } & 2.387 E-02 & 4.715 E-03 & 5.0626 & 25.1678 & -20.1052 & -79.8844 \\ \text { S09 } & 3.332 E-02 & 4.715 E-03 & 7.0678 & 24.9347 & -17.8669 & -71.6549 \\ \text { S10 } & 5.377 E-02 & 4.715 E-03 & 11.4049 & 26.8139 & -15.4090 & -57.4666 \\ \text { S11 } & 3.426 E-02 & 4.715 E-03 & 7.2655 & 12.5933 & -5.3278 & -42.3066\end{array}$




\section{REVISED METHODOLOGY FOR DETERMINING CESIUM-137 CONTENT OF HN-200 GROUT CONTAINERS.}

$\begin{array}{lcccccc}\text { S12 } & 1.227 E-01 & 4.715 E-03 & 26.0331 & 17.5307 & 8.5024 & 48.5002 \\ \text { S13 } & 1.498 E-02 & 4.715 E-03 & 3.1761 & 17.9331 & -14.7570 & -82.2891 \\ \text { S14 } & 1.828 E-01 & 4.715 E-03 & 38.7750 & 26.2138 & 12.5612 & 47.9182 \\ \text { S15 } & 2.464 E-01 & 4.715 E-03 & 52.2644 & 22.7469 & 29.5175 & 129.7646 \\ \text { S16 } & 1.095 E-01 & 4.715 E-03 & 23.2134 & 27.6556 & -4.4422 & -16.0626 \\ \text { S17 } & 7.896 E-02 & 4.715 E-03 & 16.7470 & 28.5227 & -11.7757 & -41.2853 \\ \text { S18 } & 2.774 E-01 & 4.715 E-03 & 58.8404 & 23.2528 & 35.5876 & 153.0464 \\ \text { S19 } & 8.925 E-02 & 4.715 E-03 & 18.9295 & 15.3416 & 3.5879 & 23.3869 \\ \text { S20 } & 1.546 E-02 & 4.715 E-03 & 3.2795 & 16.4988 & -13.2192 & -80.1225 \\ \text { S21 } & 2.212 E-02 & 4.715 E-03 & 4.6906 & 28.6466 & -23.9560 & -83.6260 \\ \text { S22 } & 2.320 E-01 & 4.715 E-03 & 49.2107 & 20.3724 & 28.8383 & 141.5556 \\ \text { S23 } & 4.000 E-02 & 4.715 E-03 & 8.4840 & 13.0146 & -4.5306 & -34.8117 \\ \text { S24 } & 1.182 E-01 & 4.715 E-03 & 25.0690 & 12.5419 & 12.5272 & 99.8826 \\ \text { S25 } & 3.578 E-02 & 4.715 E-03 & 7.5894 & 19.7625 & -12.1731 & -61.5969 \\ \text { S26 } & 1.057 E-01 & 4.715 E-03 & 22.4148 & 24.8512 & -2.4364 & -9.8040 \\ \text { S27 } & 1.028 E-01 & 4.715 E-03 & 21.8015 & 10.2138 & 11.5878 & 113.4528 \\ \text { S28 } & 1.065 E-01 & 4.715 E-03 & 22.5837 & 15.5133 & 7.0703 & 45.5760 \\ \text { S29 } & 6.144 E-03 & 4.715 E-03 & 1.3031 & 18.0653 & -16.7623 & -92.7868 \\ \text { S30 } & 1.480 E-01 & 4.715 E-03 & 31.3945 & 15.0401 & 16.3544 & 108.7382 \\ \text { S31 } & 1.403 E-01 & 4.715 E-03 & 29.7613 & 22.3550 & 7.4063 & 33.1304 \\ \text { S32 } & 1.198 E-01 & 4.715 E-03 & 25.4175 & 21.6866 & 3.7309 & 17.2035 \\ \text { S33 } & 2.192 E-01 & 4.715 E-03 & 46.4821 & 17.8281 & 28.6540 & 160.7242 \\ \text { S34 } & 1.679 E-01 & 4.715 E-03 & 35.6104 & 14.6776 & 20.9328 & 142.6175 \\ \text { S35 } & 3.155 E-01 & 4.715 E-03 & 66.9246 & 28.0146 & 38.9101 & 138.8924 \\ & & & & \text { Average \% Error = } & 21.4216\end{array}$

TABLE 9 - CALCULATION OF CONVERSION FACTORS FOR AN UNGROUTED HN-200 GROUT CONTAINER ASSUMING A HOMOGENEOUS MIXTURE OF ACTIVITY AND A MATERIAL WITH LITTLE OR NO SHIELDING VALUE (e.g., PAPER).

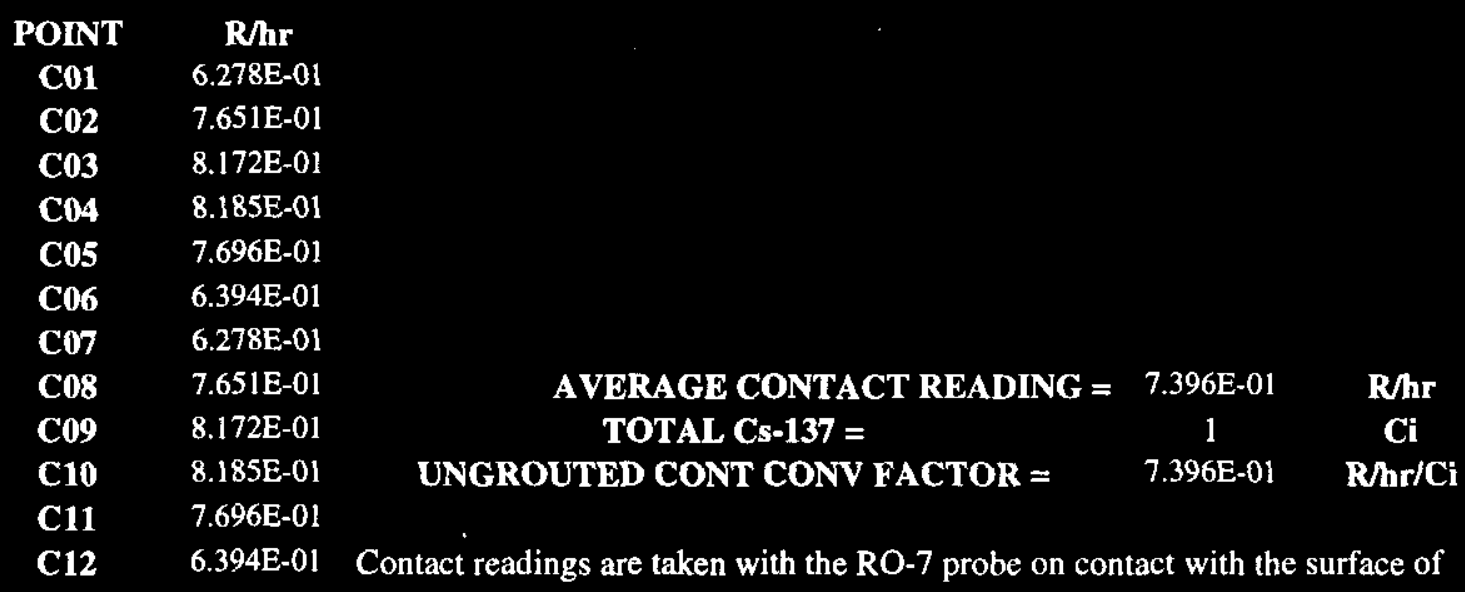




\section{REVISED METHODOLOGY FOR DETERMINING CESIUM-137 CONTENT OF HN-200 GROUT CONTAINERS.}

\begin{tabular}{|c|c|c|c|c|}
\hline C13 & $6.278 \mathrm{E}-01$ & \multirow{2}{*}{\multicolumn{3}{|c|}{$\begin{array}{l}\text { the HN- } 200 \text { grout container. Having the probe on contact with the surface of the } \\
\text { container produces an effective distance from the container of } 1 \text {-inch to account }\end{array}$}} \\
\hline C14 & $7.651 \mathrm{E}-01$ & & & \\
\hline C15 & $8.172 \mathrm{E}-01$ & \multirow{3}{*}{\multicolumn{3}{|c|}{$\begin{array}{l}\text { for the active volume of the RO- } 7 \text { radiation survey instrument probe. The } 24 \\
\text { readings are then averaged to obtain the most accurate representation of the Cs- } \\
137 \text { content of the container possible for this method. }\end{array}$}} \\
\hline C16 & 8.185E-01 & & & \\
\hline C17 & $7.696 \mathrm{E}-01$ & & & \\
\hline C18 & 6.394E-01 & & & \\
\hline C19 & $6.278 \mathrm{E}-01$ & & & \\
\hline $\mathbf{C 2 0}$ & 7.651E-01 & & & \\
\hline C21 & 8.172E-01 & & & \\
\hline $\mathrm{C22}$ & 8.185E-01 & & & \\
\hline $\mathbf{C 2 3}$ & $7.696 \mathrm{E}-01$ & & & \\
\hline $\mathrm{C24}$ & $6.394 \mathrm{E}-01$ & & & \\
\hline $\mathbf{R 0 1}$ & $2.264 \mathrm{E}-02$ & & & \\
\hline $\mathbf{R} 02$ & $2.264 \mathrm{E}-02$ & & & \\
\hline $\mathbf{R} \mathbf{0 3}$ & $2.264 \mathrm{E}-02$ & & & \\
\hline R04 & $2.264 \mathrm{E}-02$ & & & \\
\hline R05 & $2.264 \mathrm{E}-02$ & & & \\
\hline R06 & 2.264E-02 & & & \\
\hline $\mathbf{R} 07$ & $2.264 \mathrm{E}-02$ & AVERAGE REMOTE READING = & $2.264 \mathrm{E}-02$ & $\mathrm{R} / \mathrm{hr}$ \\
\hline $\mathbf{R} 08$ & $2.264 \mathrm{E}-02$ & TOTAL Cs-137 = & 1 & $\mathbf{C i}$ \\
\hline R09 & $2.264 \mathrm{E}-02$ & UNGROUTED REMOTE CONV FACT = & $2.264 \mathrm{E}-02$ & $\mathbf{R} / \mathbf{h r} / \mathrm{Ci}$ \\
\hline $\mathbf{R} 10$ & $2.264 \mathrm{E}-02$ & & & \\
\hline R11 & $2.264 \mathrm{E}-02$ & \multirow{5}{*}{\multicolumn{3}{|c|}{$\begin{array}{l}\text { Remote readings are taken with the RO- } 7 \text { probe } 10 \text { feet from the surface of the } \\
\text { container. These readings are taken every } 22.5 \text { degrees around the container and } \\
\text { averaged to produce the most accurate representation of the Cs- } 137 \text { curie content } \\
\text { of the container. }\end{array}$}} \\
\hline R12 & $2.264 \mathrm{E}-02$ & & & \\
\hline $\mathbf{R} 13$ & $2.264 \mathrm{E}-02$ & & & \\
\hline R14 & $2.264 \mathrm{E}-02$ & & & \\
\hline R15 & $2.264 \mathrm{E}-02$ & & & \\
\hline R16 & $2.264 \mathrm{E}-02$ & & & \\
\hline
\end{tabular}

\section{TABLE 10 - RESULTS OF MICROSHIELD CALCULATION FOR EACH SOURCE TO EACH MEASUREMENT POINT FROM AN UNGROUTED HN-200 GROUT CONTAINER.}

$\begin{array}{cccccccc}\text { POINT } & \text { S01 } & \text { S02 } & \text { S03 } & \text { S04 } & \text { S05 } & \text { S06 } & \text { S07 } \\ \text { C01 } & 1.764 \mathrm{E}+01 & 2.534 \mathrm{E}+00 & 1.841 \mathrm{E}+01 & 8.152 \mathrm{E}+00 & 3.417 \mathrm{E}+00 & 1.170 \mathrm{E}+01 & 3.410 \mathrm{E}+00 \\ \mathbf{C 0 2} & 5.142 \mathrm{E}+01 & 3.142 \mathrm{E}+00 & 2.035 \mathrm{E}+01 & 1.113 \mathrm{E}+01 & 3.822 \mathrm{E}+00 & 2.133 \mathrm{E}+01 & 4.506 \mathrm{E}+00 \\ \text { C03 } & 2.576 \mathrm{E}+02 & 3.720 \mathrm{E}+00 & 1.696 \mathrm{E}+01 & 1.328 \mathrm{E}+01 & 3.804 \mathrm{E}+00 & 3.191 \mathrm{E}+01 & 6.007 \mathrm{E}+00 \\ \mathbf{C 0 4} & 4.198 \mathrm{E}+02 & 4.075 \mathrm{E}+00 & 1.188 \mathrm{E}+01 & 1.272 \mathrm{E}+01 & 3.373 \mathrm{E}+00 & 2.648 \mathrm{E}+01 & 7.923 \mathrm{E}+00 \\ \mathbf{C 0 5} & 7.138 \mathrm{E}+01 & 4.034 \mathrm{E}+00 & 7.971 \mathrm{E}+00 & 1.001 \mathrm{E}+01 & 2.756 \mathrm{E}+00 & 1.501 \mathrm{E}+01 & 9.945 \mathrm{E}+00 \\ \text { C06 } & 2.223 \mathrm{E}+01 & 3.620 \mathrm{E}+00 & 5.444 \mathrm{E}+00 & 7.154 \mathrm{E}+00 & 2.161 \mathrm{E}+00 & 8.353 \mathrm{E}+00 & 1.123 \mathrm{E}+01 \\ \mathbf{C 0 7} & 9.088 \mathrm{E}+00 & 2.190 \mathrm{E}+00 & 1.075 \mathrm{E}+01 & 4.171 \mathrm{E}+00 & 6.421 \mathrm{E}+00 & 5.272 \mathrm{E}+00 & 2.896 \mathrm{E}+00 \\ \text { C08 } & 1.198 \mathrm{E}+01 & 2.621 \mathrm{E}+00 & 1.139 \mathrm{E}+01 & 4.793 \mathrm{E}+00 & 8.049 \mathrm{E}+00 & 6.565 \mathrm{E}+00 & 3.606 \mathrm{E}+00 \\ \text { C09 } & 1.436 \mathrm{E}+01 & 3.007 \mathrm{E}+00 & 1.025 \mathrm{E}+01 & 5.135 \mathrm{E}+00 & 7.966 \mathrm{E}+00 & 7.300 \mathrm{E}+00 & 4.466 \mathrm{E}+00 \\ \mathbf{C 1 0} & 1.467 \mathrm{E}+01 & 3.231 \mathrm{E}+00 & 8.161 \mathrm{E}+00 & 5.053 \mathrm{E}+00 & 6.263 \mathrm{E}+00 & 6.976 \mathrm{E}+00 & 5.408 \mathrm{E}+00 \\ \mathbf{C 1 1} & 1.266 \mathrm{E}+01 & 3.206 \mathrm{E}+00 & 6.134 \mathrm{E}+00 & 4.583 \mathrm{E}+00 & 4.391 \mathrm{E}+00 & 5.828 \mathrm{E}+00 & 6.256 \mathrm{E}+00 \\ \text { C12 } & 9.769 \mathrm{E}+00 & 2.941 \mathrm{E}+00 & 4.553 \mathrm{E}+00 & 3.910 \mathrm{E}+00 & 3.017 \mathrm{E}+00 & 4.502 \mathrm{E}+00 & 6.733 \mathrm{E}+00 \\ \mathbf{C 1 3} & 5.680 \mathrm{E}+00 & 3.793 \mathrm{E}+00 & 1.438 \mathrm{E}+01 & 5.721 \mathrm{E}+00 & 1.629 \mathrm{E}+01 & 4.762 \mathrm{E}+00 & 3.591 \mathrm{E}+00\end{array}$




\section{REVISED METHODOLOGY FOR DETERMINING CESIUM-137 CONTENT OF HN-200 GROUT CONTAINERS.}

\begin{tabular}{|c|c|c|c|c|c|c|c|}
\hline C14 & $6.644 \mathrm{E}+00$ & $5.784 \mathrm{E}+00$ & $1.554 \mathrm{E}+01$ & $6.991 \mathrm{E}+00$ & $3.359 \mathrm{E}+01$ & $5.788 \mathrm{E}+00$ & $4.954 \mathrm{E}+00$ \\
\hline C15 & $7.296 \mathrm{E}+00$ & $8.513 \mathrm{E}+00$ & $1.348 \mathrm{E}+01$ & $7.757 \mathrm{E}+00$ & $3.219 \mathrm{E}+01$ & $6.351 E+00$ & $6.912 \mathrm{E}+00$ \\
\hline C16 & $7.375 \mathrm{E}+00$ & $1.082 \mathrm{E}+01$ & $1.007 \mathrm{E}+01$ & $7.567 \mathrm{E}+00$ & $1.529 \mathrm{E}+01$ & $6.104 E+00$ & $663 \mathrm{E}+00$ \\
\hline $\mathrm{C17}$ & $6.844 E+00$ & $1.052 \mathrm{E}+01$ & $7.134 \mathrm{E}+00$ & $6.545 \mathrm{E}+00$ & $7.360 \mathrm{E}+00$ & $5.210 \mathrm{E}+00$ & $1.292 \mathrm{E}+01$ \\
\hline C18 & $5.927 \mathrm{E}+00$ & $7.963 \mathrm{E}+00$ & $5.060 \mathrm{E}+00$ & $5.230 \mathrm{E}+00$ & $3.996 \mathrm{E}+00$ & $4.128 \mathrm{E}+00$ & $1.522 \mathrm{E}+0$ \\
\hline C19 & $8.783 \mathrm{E}+00$ & $2.215 \mathrm{E}+00$ & $3.269 \mathrm{E}+01$ & $1.506 \mathrm{E}+01$ & $4.988 \mathrm{E}+00$ & $9.419 \mathrm{E}+\infty 0$ & $2.535 \mathrm{E}+00$ \\
\hline C20 & $1.144 \mathrm{E}+01$ & $5.619 \mathrm{E}+00$ & $3.935 \mathrm{E}+01$ & $4.459 E+01$ & $5.912 \mathrm{E}+00$ & $1.473 \mathrm{E}+01$ & $4.624 \mathrm{E}+0$ \\
\hline C21 & $1.359 E+01$ & $1.540 \mathrm{E}+01$ & $2.838 \mathrm{E}+01$ & $1.547 \mathrm{E}+02$ & $5.867 \mathrm{E}+00$ & $1.911 \mathrm{E}+01$ & $9.161 \mathrm{E}+0$ \\
\hline $\mathrm{C} 22$ & $1.387 \mathrm{E}+01$ & $3.642 \mathrm{E}+01$ & $1.650 \mathrm{E}+01$ & $1.013 \mathrm{E}+02$ & $4.893 \mathrm{E}+00$ & $1.702 \mathrm{E}+01$ & $2.083 \mathrm{E}+\mathrm{C}$ \\
\hline $\mathrm{C23}$ & $1.206 \mathrm{E}+01$ & $3.227 \mathrm{E}+01$ & $9.742 \mathrm{E}+00$ & $2.872 E+01$ & $3.680 \mathrm{E}+00$ & $1.143 \mathrm{E}+01$ & $6.212 \mathrm{E}+0$ \\
\hline $\mathrm{C} 24$ & $9.414 \mathrm{E}+00$ & $1.265 \mathrm{E}+01$ & $6.147 \mathrm{E}+00$ & $1.072 \mathrm{E}+01$ & $2.676 \mathrm{E}+00$ & $7.151 \mathrm{E}+00$ & $35 \mathrm{E}+\mathrm{C}$ \\
\hline R01 & $9.071 \mathrm{E}-01$ & $2.615 \mathrm{E}-01$ & $5.458 \mathrm{E}-01$ & $4.789 \mathrm{E}-0 \mathrm{l}$ & $2.114 \mathrm{E}-01$ & $4.317 \mathrm{E}-01$ & $5.281 \mathrm{E}-01$ \\
\hline R02 & 8.833E-01 & $2.477 \mathrm{E}-01$ & $5.241 \mathrm{E}-01$ & $4.317 \mathrm{E}-01$ & $2.158 \mathrm{E}-01$ & $4.077 \mathrm{E}-01$ & $4.931 \mathrm{E}-01$ \\
\hline R03 & $8.126 \mathrm{E}-01$ & $2.391 \mathrm{E}-01$ & $5.057 \mathrm{E}-01$ & $4.021 \mathrm{E}-01$ & $2.246 \mathrm{E}-01$ & $3.808 \mathrm{E}-01$ & $4.687 \mathrm{E}-01$ \\
\hline R04 & $7.198 \mathrm{E}-01$ & $2.374 \mathrm{E}-01$ & $4.923 \mathrm{E}-01$ & $3.803 \mathrm{E}-01$ & $2.375 \mathrm{E}-01$ & $3.565 \mathrm{E}-01$ & $4.556 \mathrm{E}-01$ \\
\hline 205 & $6.412 \mathrm{E}-01$ & $2.428 \mathrm{E}-01$ & $4.851 \mathrm{E}-01$ & $3.678 \mathrm{E}-01$ & $2.543 \mathrm{E}-01$ & $3.376 \mathrm{E}-01$ & 4.545E-01 \\
\hline 206 & $5.913 \mathrm{E}-01$ & $2.547 \mathrm{E}-01$ & $4.843 \mathrm{E}-01$ & $3.656 \mathrm{E}-01$ & $2.731 \mathrm{E}-01$ & $3.248 \mathrm{E}-01$ & 4.655E-01 \\
\hline 207 & $5.558 \mathrm{E}-01$ & $2.684 \mathrm{E}-01$ & $4.902 \mathrm{E}-01$ & $3.738 \mathrm{E}-01$ & $2.900 \mathrm{E}-01$ & $3.183 \mathrm{E}-01$ & $4.879 \mathrm{E}-01$ \\
\hline 08 & $5.330 \mathrm{E}-01$ & $2.541 \mathrm{E}-01$ & $5.023 \mathrm{E}-01$ & $3.918 \mathrm{E}-01$ & $3.002 \mathrm{E}-01$ & $3.184 \mathrm{E}-01$ & $5.209 \mathrm{E}-01$ \\
\hline R09 & $5.245 \mathrm{E}-01$ & $3.341 \mathrm{E}-01$ & $5.197 \mathrm{E}-01$ & $4.180 \mathrm{E}-01$ & $3.003 \mathrm{E}-01$ & $3.249 \mathrm{E}-01$ & $5.686 \mathrm{E}-01$ \\
\hline R10 & $5.308 \mathrm{E}-01$ & $3.972 \mathrm{E}-01$ & $5.409 \mathrm{E}-01$ & $4.552 \mathrm{E}-01$ & $2.903 \mathrm{E}-01$ & $3.378 \mathrm{E}-01$ & $6.368 \mathrm{E}-01$ \\
\hline R11 & $5.514 \mathrm{E}-01$ & $4.356 \mathrm{E}-01$ & $5.630 \mathrm{E}-01$ & $5.179 E-01$ & $2.735 \mathrm{E}-01$ & $3.568 \mathrm{E}-01$ & $7.054 \mathrm{E}-01$ \\
\hline R12 & $5.851 \mathrm{E}-01$ & 4.435E-01 & $5.820 \mathrm{E}-01$ & $5.858 \mathrm{E}-01$ & $2.547 \mathrm{E}-01$ & $3.811 \mathrm{E}-01$ & $7.495 \mathrm{E}-01$ \\
\hline R13 & $6.321 \mathrm{E}-01$ & $4.184 \mathrm{E}-01$ & $5.935 \mathrm{E}-01$ & $6.322 \mathrm{E}-01$ & $2.379 \mathrm{E}-01$ & $4.080 \mathrm{E}-01$ & $7.534 \mathrm{E}-01$ \\
\hline 14 & $7.059 \mathrm{E}-01$ & $3.673 \mathrm{E}-01$ & $5.947 \mathrm{E}-01$ & $6.411 \mathrm{E}-01$ & $2.248 \mathrm{E}-01$ & $4.320 \mathrm{E}-01$ & $7.157 \mathrm{E}-01$ \\
\hline & $7.991 \mathrm{E}-01$ & $2.804 \mathrm{E}-01$ & $5.853 \mathrm{E}-01$ & $6.093 \mathrm{E}-01$ & $2.160 \mathrm{E}-01$ & $4.462 \mathrm{E}-01$ & $6.499 \mathrm{E}-01$ \\
\hline 16 & $8.750 \mathrm{E}-01$ & $2.657 \mathrm{E}-01$ & $5.676 \mathrm{E}-01$ & $5.482 \mathrm{E}-01$ & $2.115 \mathrm{E}-01$ & $4.461 \mathrm{E}-01$ & $5.795 \mathrm{E}-0$ \\
\hline
\end{tabular}




\section{REVISED METHODOLOGY FOR DETERMINING CESIUM-137 CONTENT OF HN-200 GROUT CONTAINERS.}

\begin{tabular}{|c|c|c|c|c|c|c|c|}
\hline C18 & $1.357 \mathrm{E}+01$ & $5.497 \mathrm{E}+00$ & $5.308 \mathrm{E}+00$ & $2.910 \mathrm{E}+00$ & $4.030 \mathrm{E}+00$ & $3.728 \mathrm{E}+00$ & $5.855 E+00$ \\
\hline C19 & $1.080 \mathrm{E}+01$ & $1.778 \mathrm{E}+01$ & $9.989 \mathrm{E}+\infty 0$ & $3.891 \mathrm{E}+00$ & $3.749 E+00$ & $7.569 \mathrm{E}+00$ & $9.202 \mathrm{E}+00$ \\
\hline C20 & $1.916 \mathrm{E}+01$ & $1.755 \mathrm{E}+01$ & $9.783 \mathrm{E}+00$ & $4.831 \mathrm{E}+00$ & $4.712 \mathrm{E}+00$ & $8.041 E+00$ & $9.905 \mathrm{E}+00$ \\
\hline C21 & $3.597 \mathrm{E}+01$ & $1.386 \mathrm{E}+01$ & $8.506 E+00$ & $5.387 \mathrm{E}+00$ & $5.595 \mathrm{E}+00$ & $7.338 \mathrm{E}+00$ & $9.353 \mathrm{E}+00$ \\
\hline $\mathrm{C} 22$ & $5.502 \mathrm{E}+01$ & $9.797 \mathrm{E}+00$ & $6.833 \mathrm{E}+00$ & $5.195 \mathrm{E}+00$ & $6.031 \mathrm{E}+00$ & $5.949 \mathrm{E}+00$ & $7.897 \mathrm{E}+00$ \\
\hline $\mathrm{C} 23$ & $4.416 \mathrm{E}+01$ & $6.778 \mathrm{E}+00$ & $5.292 \mathrm{E}+00$ & $4.391 E+00$ & $5.773 E+00$ & $4.540 \mathrm{E}+00$ & $6.233 \mathrm{E}+00$ \\
\hline C24 & $2.407 E+01$ & $4.775 \mathrm{E}+00$ & $4.067 \mathrm{E}+00$ & $3.425 \mathrm{E}+00$ & $4.972 \mathrm{E}+00$ & $3.406 \mathrm{E}+00$ & $4.789 \mathrm{E}+00$ \\
\hline R01 & $5.601 \mathrm{E}-01$ & $4.837 \mathrm{E}-01$ & 5.354E-01 & $3.485 \mathrm{E}-01$ & $5.133 \mathrm{E}-01$ & $4.247 \mathrm{E}-01$ & $4.681 \mathrm{E}-01$ \\
\hline R02 & $5.354 \mathrm{E}-01$ & 4.857E-01 & $5.655 \mathrm{E}-01$ & $3.583 \mathrm{E}-01$ & $5.359 \mathrm{E}-01$ & $4.442 \mathrm{E}-01$ & $4.849 \mathrm{E}-01$ \\
\hline R03 & 5.179E-01 & $4.968 \mathrm{E}-01$ & $6.054 \mathrm{E}-01$ & 3.533E-01 & $5.261 \mathrm{E}-01$ & $4.584 \mathrm{E}-01$ & $5.133 \mathrm{E}-01$ \\
\hline R04 & $5.085 \mathrm{E}-01$ & $5.168 \mathrm{E}-01$ & $6.521 \mathrm{E}-01$ & $3.352 \mathrm{E}-01$ & $4.878 \mathrm{E}-01$ & $4.635 \mathrm{E}-01$ & $5.531 \mathrm{E}-01$ \\
\hline R05 & $5.075 \mathrm{E}-01$ & $5.450 \mathrm{E}-01$ & $6.964 \mathrm{E}-01$ & $3.102 \mathrm{E}-01$ & 4.344E-01 & $4.580 \mathrm{E}-01$ & $6.144 \mathrm{E}-01$ \\
\hline R06 & $5.151 \mathrm{E}-01$ & $5.797 \mathrm{E}-01$ & $7.257 \mathrm{E}-01$ & $2.854 \mathrm{E}-01$ & $3.862 \mathrm{E}-01$ & 4.435E-01 & $6.945 \mathrm{E}-01$ \\
\hline R07 & $5.308 \mathrm{E}-01$ & $6.161 \mathrm{E}-01$ & $7.305 \mathrm{E}-01$ & $2.654 \mathrm{E}-01$ & $3.549 \mathrm{E}-01$ & $4.239 \mathrm{E}-01$ & $7.625 \mathrm{E}-01$ \\
\hline R0B & $5.541 \mathrm{E}-01$ & $6.463 \mathrm{E}-01$ & $7.091 \mathrm{E}-01$ & $2.508 \mathrm{E}-01$ & $3.382 \mathrm{E}-01$ & $4.034 \mathrm{E}-01$ & $7.945 \mathrm{E}-01$ \\
\hline R09 & $5.831 \mathrm{E}-01$ & $6.617 \mathrm{E}-01$ & $6.686 \mathrm{E}-01$ & $2.416 \mathrm{E}-01$ & $3.183 \mathrm{E}-01$ & $3.854 \mathrm{E}-01$ & $7.787 \mathrm{E}-01$ \\
\hline R10 & $6.143 \mathrm{E}-01$ & $6.574 \mathrm{E}-01$ & $6.215 \mathrm{E}-01$ & $2.379 \mathrm{E}-01$ & $3.122 \mathrm{E}-01$ & $3.718 \mathrm{E}-01$ & $7.210 \mathrm{E}-01$ \\
\hline R11 & $6.419 \mathrm{E}-01$ & $6.349 \mathrm{E}-01$ & $5.785 \mathrm{E}-01$ & $2.398 \mathrm{E}-01$ & $3.148 \mathrm{E}-01$ & $3.634 \mathrm{E}-01$ & $6.417 \mathrm{E}-01$ \\
\hline R12 & 6.591E-01 & $6.010 \mathrm{E}-01$ & $5.449 \mathrm{E}-01$ & $2.472 \mathrm{E}-01$ & $3.259 \mathrm{E}-01$ & $3.606 \mathrm{E}-01$ & $5.712 \mathrm{E}-01$ \\
\hline R13 & $6.609 \mathrm{E}-01$ & $5.645 \mathrm{E}-01$ & $5.215 \mathrm{E}-01$ & $2.599 \mathrm{E}-01$ & $3.450 \mathrm{E}-01$ & $3.636 \mathrm{E}-01$ & $5.257 \mathrm{E}-01$ \\
\hline R14 & $6.468 \mathrm{E}-01$ & $5.322 \mathrm{E}-01$ & $5.088 \mathrm{E}-01$ & $2.782 \mathrm{E}-01$ & $3.717 \mathrm{E}-01$ & $3.772 \mathrm{E}-01$ & $4.936 \mathrm{E}-01$ \\
\hline $\mathbf{R} 15$ & $6.210 \mathrm{E}-01$ & $5.073 \mathrm{E}-01$ & $5.068 \mathrm{E}-01$ & $3.016 \mathrm{E}-01$ & $4.217 \mathrm{E}-01$ & $3.860 \mathrm{E}-01$ & $4.726 \mathrm{E}-01$ \\
\hline R16 & $5.900 \mathrm{E}-01$ & $4.910 \mathrm{E}-01$ & $5.158 \mathrm{E}-01$ & $3.273 \mathrm{E}-01$ & $4.667 \mathrm{E}-01$ & $4.042 \mathrm{E}-01$ & $4.639 \mathrm{E}-01$ \\
\hline
\end{tabular}

$\begin{array}{lc}\text { POINT } & \text { S15 } \\ \text { C01 } & 1.313 \mathrm{E}+00 \\ \text { C02 } & 3.587 \mathrm{E}+00 \\ \text { C03 } & 1.112 \mathrm{E}+01 \\ \text { C04 } & 4.342 \mathrm{E}+01 \\ \text { C05 } & 2.128 \mathrm{E}+02 \\ \text { C06 } & 1.191 \mathrm{E}+02 \\ \text { C07 } & 4.242 \mathrm{E}+00 \\ \text { C08 } & 5.985 \mathrm{E}+00 \\ \text { C09 } & 8.377 \mathrm{E}+00 \\ \text { C10 } & 1.119 \mathrm{E}+01 \\ \text { C11 } & 1.313 \mathrm{E}+01 \\ \text { C12 } & 1.262 \mathrm{E}+01 \\ \text { C13 } & 2.967 \mathrm{E}+00 \\ \text { C14 } & 3.614 \mathrm{E}+00 \\ \text { C15 } & 4.300 \mathrm{E}+00 \\ \text { C16 } & 4.895 \mathrm{E}+00 \\ \text { C17 } & 5.216 \mathrm{E}+00 \\ \text { C18 } & 5.137 \mathrm{E}+00\end{array}$

S16
$3.759 \mathrm{E}+01$
$5.496 \mathrm{E}+01$
$4.337 \mathrm{E}+01$
$2.400 \mathrm{E}+01$
$1.314 \mathrm{E}+01$
$7.685 \mathrm{E}+00$
$2.796 \mathrm{E}+01$
$3.653 \mathrm{E}+01$
$3.102 \mathrm{E}+01$
$1.974 \mathrm{E}+01$
$1.195 \mathrm{E}+01$
$7.410 \mathrm{E}+00$
$8.340 \mathrm{E}+00$
$8.994 \mathrm{E}+00$
$8.583 \mathrm{E}+00$
$7.458 \mathrm{E}+00$
$6.070 \mathrm{E}+00$
$4.789 \mathrm{E}+00$

SOURCE

$\begin{array}{ccc}\mathbf{S} 17 & \mathbf{S 1 8} & \mathbf{S 1 9} \\ 4.167 \mathrm{E}+00 & 2.336 \mathrm{E}+00 & 2.768 \mathrm{E}+00 \\ 5.303 \mathrm{E}+00 & 4.934 \mathrm{E}+00 & 3.338 \mathrm{E}+00 \\ 6.651 \mathrm{E}+00 & 1.117 \mathrm{E}+01 & 3.827 \mathrm{E}+00 \\ 8.001 \mathrm{E}+00 & 2.641 \mathrm{E}+01 & 4.062 \mathrm{E}+00 \\ 8.915 \mathrm{E}+00 & 5.127 \mathrm{E}+01 & 3.935 \mathrm{E}+00 \\ 8.948 \mathrm{E}+00 & 4.349 \mathrm{E}+01 & 3.507 \mathrm{E}+00 \\ 5.430 \mathrm{E}+00 & 3.363 \mathrm{E}+00 & 4.089 \mathrm{E}+00 \\ 7.720 \mathrm{E}+00 & 4.221 \mathrm{E}+00 & 5.545 \mathrm{E}+00 \\ 1.117 \mathrm{E}+01 & 5.187 \mathrm{E}+00 & 7.096 \mathrm{E}+00 \\ 1.579 \mathrm{E}+01 & 6.079 \mathrm{E}+00 & 7.970 \mathrm{E}+00 \\ 1.991 \mathrm{E}+01 & 6.591 \mathrm{E}+00 & 7.486 \mathrm{E}+00 \\ 2.008 \mathrm{E}+01 & 6.480 \mathrm{E}+00 & 6.044 \mathrm{E}+00 \\ 5.998 \mathrm{E}+00 & 3.081 \mathrm{E}+00 & 6.417 \mathrm{E}+00 \\ 1.031 \mathrm{E}+01 & 3.775 \mathrm{E}+00 & 1.507 \mathrm{E}+01 \\ 1.989 \mathrm{E}+01 & 4.520 \mathrm{E}+00 & 4.881 \mathrm{E}+01 \\ 4.467 \mathrm{E}+01 & 5.176 \mathrm{E}+00 & 2.113 \mathrm{E}+02 \\ 1.096 \mathrm{E}+02 & 5.539 \mathrm{E}+00 & 7.778 \mathrm{E}+01 \\ 1.149 \mathrm{E}+02 & 5.461 \mathrm{E}+00 & 2.078 \mathrm{E}+01\end{array}$

S20

$1.396 \mathrm{E}+01$

$1.422 \mathrm{E}+01$

$1.103 \mathrm{E}+01$

$7.501 \mathrm{E}+00$

$5.013 \mathrm{E}+00$

$3.441 \mathrm{E}+00$

$3.106 \mathrm{E}+01$

$3.237 \mathrm{E}+01$

$1.952 \mathrm{E}+01$

$1.058 \mathrm{E}+01$

$6.146 \mathrm{E}+00$

$3.826 \mathrm{E}+00$

$9.362 \mathrm{E}+00$

$9.478 \mathrm{E}+00$

$7.953 \mathrm{E}+00$

$5.954 \mathrm{E}+00$

4.291E+00

$3.106 \mathrm{E}+00$
S21

$9.439 \mathrm{E}+00$

$1.430 \mathrm{E}+01$

2.113E+01

$2.667 \mathrm{E}+01$

$2.495 \mathrm{E}+01$

$1.812 \mathrm{E}+01$

$6.977 \mathrm{E}+00$

$9.240 \mathrm{E}+00$

$1.163 \mathrm{E}+01$

$1.312 \mathrm{E}+01$

$1.269 \mathrm{E}+01$

$1.067 \mathrm{E}+01$

$7.888 \mathrm{E}+00$

$1.095 \mathrm{E}+01$

$1.450 \mathrm{E}+01$

$1.690 \mathrm{E}+01$

$1.619 \mathrm{E}+01$

$1.303 \mathrm{E}+01$ 


\section{REVISED METHODOLOGY FOR DETERMINING CESIUM-137 CONTENT OF HN-200 GROUT CONTAINERS.}

\begin{tabular}{|c|c|c|c|c|c|c|c|}
\hline C19 & $3.657 \mathrm{E}+00$ & $9,002 \mathrm{E}+00$ & $4.924 E+00$ & $4.344 \mathrm{E}+00$ & $4.032 \mathrm{E}+00$ & $6.867 \mathrm{E}+00$ & $1.117 \mathrm{E}+01$ \\
\hline C20 & $4.742 \mathrm{E}+00$ & $9.712 \mathrm{E}+00$ & $6.663 \mathrm{E}+00$ & $6.677 \mathrm{E}+00$ & $5.437 \mathrm{E}+00$ & $6.930 \mathrm{E}+00$ & $1.894 \mathrm{E}+01$ \\
\hline C21 & $6.045 E+00$ & $9.287 \mathrm{E}+00$ & $9.011 \mathrm{E}+00$ & $1.049 \mathrm{E}+01$ & $6.917 \mathrm{E}+00$ & $6.082 \mathrm{E}+00$ & $3.332 \mathrm{E}+01$ \\
\hline C22 & $7.325 \mathrm{E}+00$ & $7.981 \mathrm{E}+00$ & $1.174 \mathrm{E}+01$ & $1.600 E+01$ & $7.744 \mathrm{E}+00$ & $4.847 \mathrm{E}+00$ & $4.952 E+01$ \\
\hline $\mathrm{C} 23$ & $8.086 E+00$ & $6.407 E+00$ & $1.385 \mathrm{E}+01$ & $2.056 \mathrm{E}+01$ & $7.287 \mathrm{E}+00$ & $3.697 \mathrm{E}+00$ & $4.390 \mathrm{E}+01$ \\
\hline $\mathrm{C} 24$ & $7.895 \mathrm{E}+00$ & $4.991 \mathrm{E}+00$ & $1.394 \mathrm{E}+01$ & $1.944 E+01$ & $5.915 E+00$ & $2.794 \mathrm{E}+00$ & $2.637 \mathrm{E}+01$ \\
\hline R01 & $7.031 \mathrm{E}-01$ & $7.484 \mathrm{E}-01$ & $5.317 \mathrm{E}-01$ & $6.805 \mathrm{E}-01$ & $2.763 \mathrm{E}-01$ & $3.845 \mathrm{E}-01$ & $6.806 \mathrm{E}-01$ \\
\hline R02 & $7.104 \mathrm{E}-01$ & $7.926 \mathrm{E}-01$ & $5.444 \mathrm{E}-01$ & $5.895 \mathrm{E}-01$ & $2.803 \mathrm{E}-01$ & 4.044E-01 & $6.494 \mathrm{E}-0 \mathrm{l}$ \\
\hline $\mathbf{R} 03$ & $6.678 \mathrm{E}-01$ & $8.108 \mathrm{E}-01$ & $5.692 \mathrm{E}-01$ & $4.366 \mathrm{E}-01$ & $2.916 \mathrm{E}-01$ & $4.202 \mathrm{E}-01$ & $6.219 \mathrm{E}-01$ \\
\hline R04 & $5.879 \mathrm{E}-01$ & $7.724 \mathrm{E}-01$ & $6.060 \mathrm{E}-01$ & $4.457 \mathrm{E}-01$ & $3.099 \mathrm{E}-01$ & $4.278 \mathrm{E}-01$ & $6.011 \mathrm{E}-01$ \\
\hline R05 & $4.799 \mathrm{E}-01$ & $7.153 \mathrm{E}-01$ & $6.550 \mathrm{E}-01$ & $4.307 \mathrm{E}-01$ & 3.359E-01 & $4.250 \mathrm{E}-01$ & 5.884E-01 \\
\hline R06 & 4.485E-01 & $6.500 \mathrm{E}-01$ & $7.123 \mathrm{E}-01$ & $4.091 \mathrm{E}-01$ & 3.735E-01 & $4,125 \mathrm{E}-01$ & $5.845 \mathrm{E}-01$ \\
\hline $\mathbf{R} 07$ & $4.267 \mathrm{E}-01$ & $5.946 \mathrm{E}-01$ & $7.647 \mathrm{E}-01$ & $3.969 \mathrm{E}-01$ & $4.167 \mathrm{E}-01$ & $3.940 \mathrm{E}-01$ & 5.895E-01 \\
\hline R08 & $4.046 \mathrm{E}-01$ & $5.539 \mathrm{E}-01$ & $7.957 \mathrm{E}-01$ & $3.963 \mathrm{E}-01$ & $4.503 \mathrm{E}-01$ & $3.738 \mathrm{E}-01$ & $6.032 \mathrm{E}-01$ \\
\hline $\mathbf{R} 09$ & 3.917E-01 & $5.259 \mathrm{E}-01$ & $7.944 \mathrm{E}-01$ & $4.074 E-01$ & $4.624 \mathrm{E}-01$ & $3.555 \mathrm{E}-01$ & $6.249 \mathrm{E}-01$ \\
\hline R10 & $3.901 \mathrm{E}-01$ & $5.103 \mathrm{E}-01$ & $7.614 \mathrm{E}-01$ & $4.283 \mathrm{E}-01$ & $4.484 \mathrm{E}-01$ & $3.411 \mathrm{E}-01$ & $6.530 \mathrm{E}-01$ \\
\hline R11 & $3.999 \mathrm{E}-01$ & $5.074 \mathrm{E}-01$ & $7.081 \mathrm{E}-01$ & $4.472 \mathrm{E}-01$ & $4.135 \mathrm{E}-01$ & $3.318 \mathrm{E}-01$ & $6.843 \mathrm{E}-01$ \\
\hline $\mathbf{R} 12$ & $4.198 \mathrm{E}-01$ & $5.172 \mathrm{E}-01$ & $6.511 \mathrm{E}-01$ & $4.264 E-01$ & $3.702 \mathrm{E}-01$ & $3.277 \mathrm{E}-01$ & $7.137 \mathrm{E}-01$ \\
\hline $\mathbf{R} 13$ & $4.440 \mathrm{E}-01$ & $5.395 \mathrm{E}-01$ & $6.029 \mathrm{E}-01$ & $5.781 \mathrm{E}-01$ & 3.335E-01 & $3.292 \mathrm{E}-01$ & $7.346 E-01$ \\
\hline R14 & $4.590 \mathrm{E}-01$ & $5.741 \mathrm{E}-01$ & $5.670 \mathrm{E}-01$ & $6.730 \mathrm{E}-01$ & $3.082 E-01$ & $3.361 \mathrm{E}-01$ & $7.416 \mathrm{E}-01$ \\
\hline R15 & $5.603 \mathrm{E}-01$ & $6.225 \mathrm{E}-01$ & $5.431 \mathrm{E}-01$ & $7.279 \mathrm{E}-01$ & $2.905 \mathrm{E}-01$ & 3.482E-01 & $7.328 \mathrm{E}-01$ \\
\hline R16 & 6.483E-01 & $6.849 \mathrm{E}-01$ & $5.312 \mathrm{E}-01$ & $7.307 \mathrm{E}-01$ & 2.797E-01 & $3.648 \mathrm{E}-01$ & $7.105 E-01$ \\
\hline
\end{tabular}

\begin{tabular}{|c|c|c|c|c|c|c|c|}
\hline \multicolumn{8}{|c|}{ SOURCE } \\
\hline POINT & $\mathbf{S 2 2}$ & $\mathbf{S 2 3}$ & $\$ 24$ & $\mathbf{S 2 5}$ & S26 & S27 & $\mathbf{S 2 8}$ \\
\hline C01 & $4.987 \mathrm{E}+00$ & $1.053 \mathrm{E}+01$ & $6.276 E+00$ & $2.153 \mathrm{E}+01$ & $1.281 \mathrm{E}+01$ & $2.074 \mathrm{E}+00$ & $2.005 \mathrm{E}+00$ \\
\hline $\mathrm{CO2}$ & $4.756 \mathrm{E}+00$ & $8.571 \mathrm{E}+00$ & $8.377 \mathrm{E}+00$ & $2.775 \mathrm{E}+01$ & $1.655 \mathrm{E}+01$ & $2.863 E+00$ & $3.807 \mathrm{E}+00$ \\
\hline $\mathrm{CO3}$ & $4.211 \mathrm{E}+00$ & $5.988 \mathrm{E}+\infty 0$ & $9.125 E+00$ & $2.282 \mathrm{E}+01$ & $1.739 \mathrm{E}+01$ & $3.807 \mathrm{E}+00$ & $7.974 \mathrm{E}+00$ \\
\hline $\mathrm{C} 04$ & $3.540 \mathrm{E}+00$ & $4.039 \mathrm{E}+00$ & $7.720 \mathrm{E}+00$ & $1.424 \mathrm{E}+01$ & $1.445 \mathrm{E}+01$ & $4.755 \mathrm{E}+00$ & $1.975 \mathrm{E}+01$ \\
\hline $\mathrm{CO5}$ & $2.893 \mathrm{E}+00$ & $2.771 \mathrm{E}+00$ & $5.552 \mathrm{E}+00$ & $8.581 E+00$ & $1.043 \mathrm{E}+01$ & $5.147 \mathrm{E}+00$ & $6.216 \mathrm{E}+01$ \\
\hline C06 & $2.340 \mathrm{E}+00$ & $1.943 \mathrm{E}+00$ & $3.786 E+\infty 0$ & $5.399 E+00$ & $7.227 \mathrm{E}+00$ & $4.671 E+00$ & $1.069 \mathrm{E}+02$ \\
\hline $\mathrm{CO} 7$ & $2.027 \mathrm{E}+01$ & $5.115 \mathrm{E}+01$ & $1.282 \mathrm{E}+01$ & $2.475 \mathrm{E}+01$ & $3.531 \mathrm{E}+01$ & $1.440 \mathrm{E}+00$ & $2.277 \mathrm{E}+00$ \\
\hline $\mathrm{COS}$ & $1.665 \mathrm{E}+01$ & $2.419 \mathrm{E}+01$ & $3.741 \mathrm{E}+01$ & $3.332 \mathrm{E}+01$ & $9.891 \mathrm{E}+01$ & $1.745 E+00$ & $2.978 \mathrm{E}+00$ \\
\hline $\mathrm{CO9}$ & $1.088 \mathrm{E}+01$ & $1.065 \mathrm{E}+01$ & $6.288 \mathrm{E}+01$ & $2.647 \mathrm{E}+01$ & $1.389 \mathrm{E}+02$ & $2.050 \mathrm{E}+00$ & $3.878 \mathrm{E}+00$ \\
\hline $\mathrm{C} 10$ & $6.745 E+00$ & $5.370 \mathrm{E}+00$ & $2.579 E+01$ & $1.557 \mathrm{E}+01$ & $5.254 \mathrm{E}+01$ & $2.286 \mathrm{E}+00$ & $4.891 \mathrm{E}+00$ \\
\hline C11 & $4.266 \mathrm{E}+00$ & $3.059 E+00$ & $9.169 \mathrm{E}+00$ & $9.023 E+00$ & $2.075 \mathrm{E}+01$ & $2.369 \mathrm{E}+00$ & $5.722 \mathrm{E}+00$ \\
\hline C12 & $2.793 E+00$ & $1.887 \mathrm{E}+\infty 0$ & $3.837 \mathrm{E}+00$ & $5.527 \mathrm{E}+00$ & $9.973 E+00$ & $2.267 \mathrm{E}+00$ & $5.941 \mathrm{E}+00$ \\
\hline C13 & $4.190 \mathrm{E}+01$ & $5.741 \mathrm{E}+\infty 0$ & $3.516 E+00$ & $7.552 \mathrm{E}+00$ & $8.999 E+00$ & $1.918 \mathrm{E}+00$ & $1.945 \mathrm{E}+\infty 0$ \\
\hline C14 & $2.736 \mathrm{E}+01$ & $5.114 \mathrm{E}+00$ & $4.060 \mathrm{E}+\infty 0$ & $8.158 E+\infty 0$ & $1.066 \mathrm{E}+01$ & $2.534 \mathrm{E}+00$ & $2.416 \mathrm{E}+00$ \\
\hline C15 & $1.294 \mathrm{E}+01$ & $4.087 \mathrm{E}+00$ & $4.221 \mathrm{E}+00$ & $7.701 \mathrm{E}+00$ & $1.100 \mathrm{E}+01$ & $3.265 \mathrm{E}+00$ & $2.959 \mathrm{E}+00$ \\
\hline C16 & $6.148 \mathrm{E}+00$ & $3.100 \mathrm{E}+00$ & $3.906 \mathrm{E}+00$ & $6.428 \mathrm{E}+00$ & $9.765 \mathrm{E}+00$ & $3.927 \mathrm{E}+00$ & $3.501 \mathrm{E}+00$ \\
\hline C17 & $3.099 \mathrm{E}+00$ & $2.325 \mathrm{E}+00$ & $3.289 E+00$ & $5.006 E+00$ & $7.778 \mathrm{E}+00$ & $4.188 \mathrm{E}+00$ & $3.899 E+00$ \\
\hline C18 & $1.653 \mathrm{E}+00$ & $1.758 \mathrm{E}+00$ & $2.621 \mathrm{E}+00$ & $3.803 \mathrm{E}+00$ & $5.889 \mathrm{E}+00$ & $3.871 E+00$ & $3.999 \mathrm{E}+00$ \\
\hline C19 & $5.695 \mathrm{E}+00$ & $4.043 \mathrm{E}+00$ & $2.682 \mathrm{E}+\infty 0$ & $7.231 \mathrm{E}+00$ & $6.269 E+00$ & $1.112 \mathrm{E}+00$ & $2.596 \mathrm{E}+00$ \\
\hline
\end{tabular}




\section{REVISED METHODOLOGY FOR DETERMINING CESIUM-137 CONTENT OF HN-200 GROUT CONTAINERS.}

$\begin{array}{llllllll}\text { C20 } & 5.394 \mathrm{E}+00 & 3.725 \mathrm{E}+00 & 2.982 \mathrm{E}+00 & 7.809 \mathrm{E}+00 & 7.021 \mathrm{E}+00 & 2.987 \mathrm{E}+00 & 3.707 \mathrm{E}+00 \\ \text { C21 } & 4.699 \mathrm{E}+00 & 3.154 \mathrm{E}+00 & 3.067 \mathrm{E}+00 & 7.368 \mathrm{E}+00 & 7.165 \mathrm{E}+00 & 9.721 \mathrm{E}+00 & 5.336 \mathrm{E}+00 \\ \text { C22 } & 3.873 \mathrm{E}+00 & 2.541 \mathrm{E}+00 & 2.899 \mathrm{E}+00 & 6.195 \mathrm{E}+00 & 6.625 \mathrm{E}+00 & 4.849 \mathrm{E}+01 & 7.584 \mathrm{E}+00 \\ \text { C23 } & 3.106 \mathrm{E}+00 & 2.005 \mathrm{E}+00 & 2.550 \mathrm{E}+00 & 4.866 \mathrm{E}+00 & 5.661 \mathrm{E}+00 & 3.540 \mathrm{E}+02 & 9.870 \mathrm{E}+00 \\ \text { C24 } & 2.471 \mathrm{E}+00 & 1.579 \mathrm{E}+00 & 2.136 \mathrm{E}+00 & 3.724 \mathrm{E}+00 & 4.609 \mathrm{E}+00 & 3.945 \mathrm{E}+01 & 1.056 \mathrm{E}+01 \\ \text { R01 } & 3.557 \mathrm{E}-01 & 3.020 \mathrm{E}-01 & 2.982 \mathrm{E}-01 & 5.018 \mathrm{E}-01 & 5.812 \mathrm{E}-01 & 2.132 \mathrm{E}-01 & 4.554 \mathrm{E}-01 \\ \text { R02 } & 3.744 \mathrm{E}-01 & 3.290 \mathrm{E}-01 & 3.449 \mathrm{E}-01 & 5.288 \mathrm{E}-01 & 6.417 \mathrm{E}-01 & 2.010 \mathrm{E}-01 & 4.233 \mathrm{E}-01 \\ \text { R03 } & 3.884 \mathrm{E}-01 & 3.512 \mathrm{E}-01 & 3.803 \mathrm{E}-01 & 5.412 \mathrm{E}-01 & 6.967 \mathrm{E}-01 & 1.893 \mathrm{E}-01 & 3.777 \mathrm{E}-01 \\ \text { R04 } & 3.951 \mathrm{E}-01 & 3.613 \mathrm{E}-01 & 3.938 \mathrm{E}-01 & 5.348 \mathrm{E}-01 & 7.273 \mathrm{E}-01 & 1.806 \mathrm{E}-01 & 3.360 \mathrm{E}-01 \\ \text { R05 } & 5.114 \mathrm{E}-01 & 3.557 \mathrm{E}-01 & 3.803 \mathrm{E}-01 & 5.118 \mathrm{E}-01 & 7.224 \mathrm{E}-01 & 1.766 \mathrm{E}-01 & 3.091 \mathrm{E}-01 \\ \text { R06 } & 5.863 \mathrm{E}-01 & 3.363 \mathrm{E}-01 & 3.449 \mathrm{E}-01 & 4.796 \mathrm{E}-01 & 6.838 \mathrm{E}-01 & 1.778 \mathrm{E}-01 & 2.902 \mathrm{E}-01 \\ \text { R07 } & 6.270 \mathrm{E}-01 & 3.098 \mathrm{E}-01 & 2.982 \mathrm{E}-01 & 4.466 \mathrm{E}-01 & 6.254 \mathrm{E}-01 & 1.840 \mathrm{E}-01 & 2.775 \mathrm{E}-01 \\ \text { R08 } & 6.232 \mathrm{E}-01 & 2.842 \mathrm{E}-01 & 2.631 \mathrm{E}-01 & 4.188 \mathrm{E}-01 & 5.667 \mathrm{E}-01 & 1.944 \mathrm{E}-01 & 2.721 \mathrm{E}-01 \\ \text { R09 } & 5.762 \mathrm{E}-01 & 2.641 \mathrm{E}-01 & 2.455 \mathrm{E}-01 & 3.980 \mathrm{E}-01 & 5.209 \mathrm{E}-01 & 2.065 \mathrm{E}-01 & 2.740 \mathrm{E}-01 \\ \text { R10 } & 4.965 \mathrm{E}-01 & 2.496 \mathrm{E}-01 & 2.313 \mathrm{E}-01 & 3.849 \mathrm{E}-01 & 4.881 \mathrm{E}-01 & 2.273 \mathrm{E}-01 & 2.833 \mathrm{E}-01 \\ \text { R11 } & 3.829 \mathrm{E}-01 & 2.405 \mathrm{E}-01 & 2.220 \mathrm{E}-01 & 3.796 \mathrm{E}-01 & 4.666 \mathrm{E}-01 & 2.690 \mathrm{E}-01 & 2.993 \mathrm{E}-01 \\ \text { R12 } & 3.895 \mathrm{E}-01 & 2.369 \mathrm{E}-01 & 2.188 \mathrm{E}-01 & 3.823 \mathrm{E}-01 & 4.565 \mathrm{E}-01 & 3.024 \mathrm{E}-01 & 3.217 \mathrm{E}-01 \\ \text { R13 } & 3.710 \mathrm{E}-01 & 2.388 \mathrm{E}-01 & 2.220 \mathrm{E}-01 & 3.928 \mathrm{E}-01 & 4.580 \mathrm{E}-01 & 3.193 \mathrm{E}-01 & 3.561 \mathrm{E}-01 \\ \text { R14 } & 3.533 \mathrm{E}-01 & 2.464 \mathrm{E}-01 & 2.313 \mathrm{E}-01 & 4.111 \mathrm{E}-01 & 4.712 \mathrm{E}-01 & 3.142 \mathrm{E}-01 & 4.024 \mathrm{E}-01 \\ \text { R15 } & 3.442 \mathrm{E}-01 & 2.593 \mathrm{E}-01 & 2.455 \mathrm{E}-01 & 4.368 \mathrm{E}-01 & 4.956 \mathrm{E}-01 & 2.888 \mathrm{E}-01 & 4.426 \mathrm{E}-01 \\ \text { R16 } & 3.450 \mathrm{E}-01 & 2.778 \mathrm{E}-01 & 2.632 \mathrm{E}-01 & 4.685 \mathrm{E}-01 & 5.316 \mathrm{E}-01 & 2.506 \mathrm{E}-01 & 4.627 \mathrm{E}-01\end{array}$

POINT
C01
C02
C03
C04
C05
C06
C07
C08
C09
C10
C11
C12
C13
C14
C15
C16
C17
C18
C19
C20

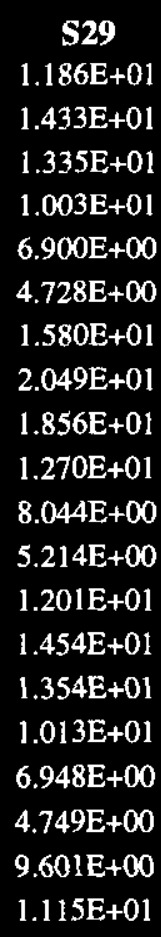

\begin{abstract}
S30
3.101E+00

$4.515 \mathrm{E}+00$

$6.515 \mathrm{E}+00$

$8.877 \mathrm{E}+00$

$1.028 \mathrm{E}+01$

9.383E +00

$2.086 \mathrm{E}+00$

$2.547 \mathrm{E}+00$

$3.028 \mathrm{E}+00$

$3.424 \mathrm{E}+00$

$3.605 \mathrm{E}+00$

$3.493 \mathrm{E}+00$

$2.488 \mathrm{E}+00$

$3.197 \mathrm{E}+00$

$4.013 \mathrm{E}+00$

$4.755 \mathrm{E}+00$

$5.116 \mathrm{E}+00$

$4.891 \mathrm{E}+00$

$1.868 \mathrm{E}+00$

$4.274 \mathrm{E}+00$
\end{abstract}

\begin{abstract}
$2.982 \mathrm{E}+00$
$3.067 \mathrm{E}+00$

$2.899 \mathrm{E}+00$

$2.550 \mathrm{E}+00$

$2.136 \mathrm{E}+00$

3.803E-01

3.449E-01

2.982E-01

2.313E-01

2.220E-01

2.188E-01

2.220E-0]

2.313E-01

2.632E-01
\end{abstract}

5.316E-0
SOURCE

$\begin{array}{cc}\text { S31 } & \text { S32 } \\ 6.845 E+00 & 6.703 E+00 \\ 9.878 E+00 & 8.772 E+00 \\ 1.351 E+01 & 1.042 E+01 \\ 1.572 \mathrm{E}+01 & 1.058 \mathrm{E}+01 \\ 1.438 \mathrm{E}+01 & 9.109 \mathrm{E}+00 \\ 1.086 \mathrm{E}+01 & 7.040 \mathrm{E}+00 \\ 4.059 \mathrm{E}+00 & 1.450 \mathrm{E}+01 \\ 4.896 \mathrm{E}+00 & 3.924 \mathrm{E}+01 \\ 5.609 \mathrm{E}+00 & 1.571 \mathrm{E}+02 \\ 5.944 \mathrm{E}+00 & 2.010 \mathrm{E}+02 \\ 5.748 \mathrm{E}+00 & 4.803 \mathrm{E}+01 \\ 5.113 \mathrm{E}+00 & 1.678 \mathrm{E}+01 \\ 5.173 \mathrm{E}+00 & 7.096 \mathrm{E}+00 \\ 6.655 \mathrm{E}+00 & 9.473 \mathrm{E}+00 \\ 8.074 \mathrm{E}+00 & 1.144 \mathrm{E}+01 \\ 8.798 \mathrm{E}+00 & 1.163 \mathrm{E}+01 \\ 8.369 \mathrm{E}+00 & 9.869 \mathrm{E}+00 \\ 7.070 \mathrm{E}+00 & 7.477 \mathrm{E}+00 \\ 9.161 \mathrm{E}+00 & 4.420 \mathrm{E}+00 \\ 2.049 \mathrm{E}+01 & 5.206 \mathrm{E}+00\end{array}$

S33

$5.133 \mathrm{E}+00$

$6.241 \mathrm{E}+00$

$6.837 \mathrm{E}+00$

$6.551 \mathrm{E}+00$

$5.571 \mathrm{E}+00$

$4.399 \mathrm{E}+00$

$3.319 \mathrm{E}+00$

$3.735 \mathrm{E}+00$

$3.934 \mathrm{E}+00$

$3.841 \mathrm{E}+00$

$3.491 \mathrm{E}+00$

$3.006 \mathrm{E}+00$

$6.083 \mathrm{E}+00$

$7.741 \mathrm{E}+00$

$8.695 \mathrm{E}+00$

$8.232 \mathrm{E}+00$

$6.721 \mathrm{E}+00$

$5.059 \mathrm{E}+00$

$6.186 \mathrm{E}+00$

$3.997 \mathrm{E}+01$

$\begin{array}{cc}\text { S34 } & \text { S35 } \\ 1.823 \mathrm{E}+00 & 1.724 \mathrm{E}+01 \\ 2.280 \mathrm{E}+00 & 3.587 \mathrm{E}+01 \\ 2.821 \mathrm{E}+00 & 5.733 \mathrm{E}+01 \\ 3.380 \mathrm{E}+00 & 4.374 \mathrm{E}+01 \\ 3.818 \mathrm{E}+00 & 2.166 \mathrm{E}+01 \\ 3.962 \mathrm{E}+00 & 1.047 \mathrm{E}+01 \\ 1.604 \mathrm{E}+00 & 1.458 \mathrm{E}+01 \\ 2.841 \mathrm{E}+00 & 2.271 \mathrm{E}+01 \\ 5.286 \mathrm{E}+00 & 2.859 \mathrm{E}+01 \\ 1.028 \mathrm{E}+01 & 2.515 \mathrm{E}+01 \\ 1.926 \mathrm{E}+01 & 1.688 \mathrm{E}+01 \\ 2.490 \mathrm{E}+01 & 1.042 \mathrm{E}+01 \\ 2.060 \mathrm{E}+00 & 5.781 \mathrm{E}+00 \\ 3.263 \mathrm{E}+00 & 6.580 \mathrm{E}+00 \\ 5.366 \mathrm{E}+00 & 6.959 \mathrm{E}+00 \\ 9.028 \mathrm{E}+00 & 6.753 \mathrm{E}+00 \\ 1.422 \mathrm{E}+01 & 6.055 \mathrm{E}+00 \\ 1.679 \mathrm{E}+01 & 5.126 \mathrm{E}+00 \\ 1.766 \mathrm{E}+00 & 6.403 \mathrm{E}+00 \\ 2.189 \mathrm{E}+00 & 7.405 \mathrm{E}+00\end{array}$




\section{REVISED METHODOLOGY FOR DETERMINING CESIUM-137 CONTENT OF HN-200 GROUT CONTAINERS.}

$\begin{array}{llllllll}\text { C21 } & 1.055 \mathrm{E}+01 & 1.106 \mathrm{E}+01 & 5.727 \mathrm{E}+01 & 5.734 \mathrm{E}+00 & 5.722 \mathrm{E}+02 & 2.679 \mathrm{E}+00 & 7.891 \mathrm{E}+00 \\ \text { C22 } & 8.366 \mathrm{E}+00 & 3.412 \mathrm{E}+01 & 1.475 \mathrm{E}+02 & 5.779 \mathrm{E}+00 & 9.572 \mathrm{E}+01 & 3.176 \mathrm{E}+00 & 7.626 \mathrm{E}+00 \\ \text { C23 } & 6.079 \mathrm{E}+00 & 9.058 \mathrm{E}+01 & 7.806 \mathrm{E}+01 & 5.320 \mathrm{E}+00 & 1.170 \mathrm{E}+01 & 3.558 \mathrm{E}+00 & 6.743 \mathrm{E}+00 \\ \text { C24 } & 4.339 \mathrm{E}+00 & 4.591 \mathrm{E}+01 & 2.646 \mathrm{E}+01 & 4.559 \mathrm{E}+00 & 2.299 \mathrm{E}+00 & 3.683 \mathrm{E}+00 & 5.604 \mathrm{E}+00 \\ \text { R01 } & 4.109 \mathrm{E}-01 & 3.470 \mathrm{E}-01 & 5.269 \mathrm{E}-01 & 4.696 \mathrm{E}-01 & 3.417 \mathrm{E}-01 & 2.656 \mathrm{E}-01 & 8.158 \mathrm{E}-01 \\ \text { R02 } & 4.191 \mathrm{E}-01 & 3.073 \mathrm{E}-01 & 4.761 \mathrm{E}-01 & 5.193 \mathrm{E}-01 & 3.359 \mathrm{E}-01 & 2.800 \mathrm{E}-01 & 8.839 \mathrm{E}-01 \\ \text { R03 } & 4.265 \mathrm{E}-01 & 2.894 \mathrm{E}-01 & 4.418 \mathrm{E}-01 & 5.792 \mathrm{E}-01 & 3.184 \mathrm{E}-01 & 2.743 \mathrm{E}-01 & 8.907 \mathrm{E}-01 \\ \text { R04 } & 4.317 \mathrm{E}-01 & 2.732 \mathrm{E}-01 & 4.178 \mathrm{E}-01 & 6.294 \mathrm{E}-01 & 3.077 \mathrm{E}-01 & 3.451 \mathrm{E}-01 & 8.338 \mathrm{E}-01 \\ \text { R05 } & 4.337 \mathrm{E}-01 & 2.628 \mathrm{E}-01 & 4.039 \mathrm{E}-01 & 6.519 \mathrm{E}-01 & 3.060 \mathrm{E}-01 & 4.082 \mathrm{E}-01 & 7.299 \mathrm{E}-01 \\ \text { R06 } & 4.321 \mathrm{E}-01 & 2.598 \mathrm{E}-01 & 4.010 \mathrm{E}-01 & 6.384 \mathrm{E}-01 & 3.135 \mathrm{E}-01 & 4.474 \mathrm{E}-01 & 5.875 \mathrm{E}-01 \\ \text { R07 } & 4.273 \mathrm{E}-01 & 2.642 \mathrm{E}-01 & 4.089 \mathrm{E}-01 & 5.940 \mathrm{E}-01 & 3.292 \mathrm{E}-01 & 4.565 \mathrm{E}-01 & 5.561 \mathrm{E}-01 \\ \text { R08 } & 4.201 \mathrm{E}-01 & 2.759 \mathrm{E}-01 & 4.276 \mathrm{E}-01 & 5.346 \mathrm{E}-01 & 3.461 \mathrm{E}-01 & 4.325 \mathrm{E}-01 & 5.291 \mathrm{E}-01 \\ \text { R09 } & 4.119 \mathrm{E}-01 & 2.926 \mathrm{E}-01 & 4.561 \mathrm{E}-01 & 4.809 \mathrm{E}-01 & 3.178 \mathrm{E}-01 & 3.823 \mathrm{E}-01 & 5.016 \mathrm{E}-01 \\ \text { R10 } & 4.040 \mathrm{E}-01 & 3.106 \mathrm{E}-01 & 4.968 \mathrm{E}-01 & 4.428 \mathrm{E}-01 & 4.362 \mathrm{E}-01 & 3.021 \mathrm{E}-01 & 4.859 \mathrm{E}-01 \\ \text { R11 } & 3.976 \mathrm{E}-01 & 3.577 \mathrm{E}-01 & 5.561 \mathrm{E}-01 & 4.160 \mathrm{E}-01 & 5.169 \mathrm{E}-01 & 2.794 \mathrm{E}-01 & 4.844 \mathrm{E}-01 \\ \text { R12 } & 3.933 \mathrm{E}-01 & 4.148 \mathrm{E}-01 & 6.204 \mathrm{E}-01 & 3.991 \mathrm{E}-01 & 5.652 \mathrm{E}-01 & 2.736 \mathrm{E}-01 & 4.973 \mathrm{E}-01 \\ \text { R13 } & 3.918 \mathrm{E}-01 & 4.558 \mathrm{E}-01 & 6.662 \mathrm{E}-01 & 3.924 \mathrm{E}-01 & 5.732 \mathrm{E}-01 & 2.594 \mathrm{E}-01 & 5.228 \mathrm{E}-01 \\ \text { R14 } & 3.930 \mathrm{E}-01 & 4.691 \mathrm{E}-01 & 6.770 \mathrm{E}-01 & 3.963 \mathrm{E}-01 & 5.383 \mathrm{E}-01 & 2.503 \mathrm{E}-01 & 5.528 \mathrm{E}-01 \\ \text { R15 } & 3.969 \mathrm{E}-01 & 4.500 \mathrm{E}-01 & 6.488 \mathrm{E}-01 & 4.106 \mathrm{E}-01 & 4.698 \mathrm{E}-01 & 2.484 \mathrm{E}-01 & 5.678 \mathrm{E}-01 \\ \text { R16 } & 4.031 \mathrm{E}-01 & 4.051 \mathrm{E}-01 & 5.920 \mathrm{E}-01 & 4.349 \mathrm{E}-01 & 3.472 \mathrm{E}-01 & 2.537 \mathrm{E}-01 & 7.043 \mathrm{E}-01\end{array}$

TABLE 11 - TOTAL RADIATION LEVEL AT EACH MEASUREMENT POINT FOR EACH SET OF SOURCES TO BE ANALYZED.

\begin{tabular}{|c|c|c|c|c|c|c|c|}
\hline \multicolumn{8}{|c|}{ SOURCES } \\
\hline POINT & S01-S35 & S01-S30 & S06-S35 & S01.S25 & S11-S35 & S01-S20 & S16-S35 \\
\hline $\mathrm{C} 01$ & $3.158 \mathrm{E}+02$ & $2.781 \mathrm{E}+02$ & $2.657 \mathrm{E}+02$ & $2.462 \mathrm{E}+02$ & $2.228 \mathrm{E}+02$ & $1.935 \mathrm{E}+02$ & $1.832 \mathrm{E}+02$ \\
\hline $\mathrm{CO2}$ & $4.599 \mathrm{E}+02$ & $3.968 E+02$ & $3.700 \mathrm{E}+02$ & $3.547 \mathrm{E}+02$ & $3.137 \mathrm{E}+02$ & $2.910 \mathrm{E}+02$ & $2.516 \mathrm{E}+02$ \\
\hline $\mathrm{C} 03$ & $7.454 \mathrm{E}+02$ & $6.545 \mathrm{E}+02$ & $4.500 \mathrm{E}+02$ & $6.054 \mathrm{E}+02$ & $3.808 \mathrm{E}+02$ & $5.421 \mathrm{E}+02$ & $2.793 \mathrm{E}+02$ \\
\hline $\mathrm{CO4}$ & $9.316 \mathrm{E}+02$ & $8.516 \mathrm{E}+02$ & $4.797 \mathrm{E}+02$ & $7.937 \mathrm{E}+02$ & $4.154 \mathrm{E}+02$ & $7.375 E+02$ & $2.640 \mathrm{E}+02$ \\
\hline C05 & $7.037 \mathrm{E}+02$ & $6.492 \mathrm{E}+02$ & $6.076 E+02$ & $5.543 \mathrm{E}+02$ & $5.570 \mathrm{E}+02$ & $5.095 \mathrm{E}+02$ & $2.765 \mathrm{E}+02$ \\
\hline C06 & $5.007 \mathrm{E}+02$ & $4.639 E+02$ & $4.601 \mathrm{E}+02$ & $3.310 \mathrm{E}+02$ & $4.206 \mathrm{E}+02$ & $2.994 \mathrm{E}+02$ & $2.683 \mathrm{E}+02$ \\
\hline $\mathrm{C} 07$ & $4.526 \mathrm{E}+02$ & $4.145 \mathrm{E}+02$ & $4.199 \mathrm{E}+02$ & $3.576 \mathrm{E}+02$ & $3.416 \mathrm{E}+02$ & $2.416 \mathrm{E}+02$ & $2.828 \mathrm{E}+02$ \\
\hline C08 & $5.992 \mathrm{E}+02$ & $5.258 \mathrm{E}+02$ & $5.604 \mathrm{E}+02$ & $1 \mathrm{E}+02$ & $4.831 \mathrm{E}+02$ & $2.783 \mathrm{E}+02$ & $4.073 \mathrm{E}+02$ \\
\hline Co9 & $7.373 \mathrm{E}+02$ & $5.368 \mathrm{E}+02$ & $6.966 \mathrm{E}+02$ & $3.704 \mathrm{E}+02$ & $6.369 \mathrm{E}+02$ & $2.479 \mathrm{E}+02$ & $5.634 \mathrm{E}+02$ \\
\hline C10 & $5.962 E+02$ & $3.500 \mathrm{E}+02$ & $5.588 \mathrm{E}+02$ & $2.741 E+02$ & $5.123 \mathrm{E}+02$ & $2.075 \mathrm{E}+02$ & $4.488 \mathrm{E}+02$ \\
\hline C11 & $4.209 E+02$ & $3.275 E+02$ & $3.900 \mathrm{E}+02$ & $\mathrm{E}+02$ & $2.754 \mathrm{E}+02$ & $2.488 \mathrm{E}+02$ & $2.242 \mathrm{E}+02$ \\
\hline $\mathrm{C12}$ & $2.480 \mathrm{E}+02$ & $1.878 \mathrm{E}+02$ & $2.238 \mathrm{E}+02$ & $1.609 \mathrm{E}+02$ & $1.940 \mathrm{E}+02$ & $1.362 \mathrm{E}+02$ & $1.557 \mathrm{E}+02$ \\
\hline C13 & $4.062 E+02$ & $3.801 \mathrm{E}+02$ & $3.604 \mathrm{E}+02$ & $3.527 \mathrm{E}+02$ & $2.436 \mathrm{E}+02$ & $2.861 \mathrm{E}+02$ & $1.533 \mathrm{E}+02$ \\
\hline C14 & $5.490 \mathrm{E}+02$ & $5.153 \mathrm{E}+02$ & $4.805 \mathrm{E}+02$ & $4.820 \mathrm{E}+02$ & $3.630 \mathrm{E}+02$ & $4.263 \mathrm{E}+02$ & $1.703 \mathrm{E}+02$ \\
\hline C15 & $4.656 \mathrm{E}+02$ & $4.251 \mathrm{E}+02$ & $3.964 \mathrm{E}+02$ & $3.903 \mathrm{E}+02$ & $3.132 \mathrm{E}+02$ & $3.469 E+02$ & $2.085 \mathrm{E}+02$ \\
\hline C16 & $5.422 \mathrm{E}+02$ & $4.977 \mathrm{E}+02$ & $4.910 \mathrm{E}+02$ & $4.656 \mathrm{E}+02$ & $4.264 \mathrm{E}+02$ & $4.292 \mathrm{E}+02$ & $3.876 \mathrm{E}+02$ \\
\hline C17 & $4.286 \mathrm{E}+02$ & $3.834 E+02$ & $3.902 E+02$ & $3.555 E+02$ & $3.367 \mathrm{E}+02$ & $3.255 \mathrm{E}+02$ & $3.064 \mathrm{E}+02$ \\
\hline C18 & $3.304 \mathrm{E}+02$ & $2.889 \mathrm{E}+02$ & $3.022 \mathrm{E}+02$ & $2.655 \mathrm{E}+02$ & $2.585 \mathrm{E}+02$ & $2.426 \dot{\mathrm{E}}+02$ & $2.368 \mathrm{E}+02$ \\
\hline
\end{tabular}




\section{REVISED METHODOLOGY FOR DETERMINING CESIUM-137 CONTENT OF HN-200 GROUT CONTAINERS.}

\begin{tabular}{|c|c|c|c|c|c|c|c|}
\hline C19 & $2.517 \mathrm{E}+02$ & $2.238 \mathrm{E}+02$ & $1.880 E+02$ & $2.023 \mathrm{E}+02$ & $1.374 \mathrm{E}+02$ & $1.715 E+02$ & $1.094 \mathrm{E}+02$ \\
\hline C20 & $3.837 \mathrm{E}+02$ & $3.084 \mathrm{E}+02$ & $2.767 \mathrm{E}+02$ & $2.793 \mathrm{E}+02$ & $2.109 \mathrm{E}+02$ & $2.404 \mathrm{E}+02$ & $1.787 \mathrm{E}+02$ \\
\hline $\mathrm{C} 21$ & $1.121 \mathrm{E}+03$ & $4.755 \mathrm{E}+02$ & $9.033 \mathrm{E}+02$ & $4.317 \mathrm{E}+02$ & $8.167 \mathrm{E}+02$ & $3.800 \mathrm{E}+02$ & $7.830 \mathrm{E}+02$ \\
\hline $\mathrm{C} 22$ & $7.932 \mathrm{E}+02$ & $5.334 \mathrm{E}+02$ & $6.202 E+02$ & $4.282 \mathrm{E}+02$ & $5.107 \mathrm{E}+02$ & $3.632 \mathrm{E}+02$ & $4.783 E+02$ \\
\hline $\mathrm{C23}$ & $9.251 \mathrm{E}+02$ & $8.197 E+02$ & $8.386 \mathrm{E}+02$ & $3.535 \mathrm{E}+02$ & $7.088 \mathrm{E}+02$ & $2.971 E+02$ & $6.798 \mathrm{E}+02$ \\
\hline $\mathrm{C} 24$ & $5.905 \mathrm{E}+02$ & $5.479 \mathrm{E}+02$ & $5.489 \mathrm{E}+02$ & $4.430 \mathrm{E}+02$ & $2.553 \mathrm{E}+02$ & $4.067 \mathrm{E}+02$ & $2.308 \mathrm{E}+02$ \\
\hline R01 & $1.659 \mathrm{E}+01$ & $1.417 \mathrm{E}+01$ & $1.418 \mathrm{E}+01$ & $1.216 \mathrm{E}+01$ & $1.164 \mathrm{E}+01$ & $1.002 \mathrm{E}+01$ & $9.187 E+00$ \\
\hline R02 & $1.665 \mathrm{E}+01$ & $1.415 \mathrm{E}+01$ & $1.435 \mathrm{E}+01$ & $1.216 \mathrm{E}+01$ & $1.186 \mathrm{E}+01$ & $9.935 \mathrm{E}+00$ & $9.325 \mathrm{E}+00$ \\
\hline $\mathbf{R} 03$ & $1.647 \mathrm{E}+01$ & $1.396 \mathrm{E}+01$ & $1.428 \mathrm{E}+01$ & $1.198 \mathrm{E}+01$ & $1.181 \mathrm{E}+01$ & $9.701 E+00$ & $9.295 \mathrm{E}+00$ \\
\hline R04 & $1.631 \mathrm{E}+01$ & $1.378 \mathrm{E}+01$ & $1.425 \mathrm{E}+01$ & $1.183 E+01$ & $1.176 \mathrm{E}+01$ & $9.546 \mathrm{E}+00$ & $9.331 E+\infty 0$ \\
\hline R05 & $1.614 E+01$ & $1.364 \mathrm{E}+01$ & $1.415 \mathrm{E}+01$ & $1.174 \mathrm{E}+01$ & $1.161 \mathrm{E}+01$ & $9.391 \mathrm{E}+00$ & $9.314 \mathrm{E}+00$ \\
\hline R06 & $1.596 \mathrm{E}+01$ & $1.357 \mathrm{E}+01$ & $1.399 \mathrm{E}+01$ & $1.173 \mathrm{E}+01$ & $1.138 \mathrm{E}+01$ & $9.395 \mathrm{E}+00$ & $9.121 \mathrm{E}+\infty$ \\
\hline R07 & $1.586 \mathrm{E}+01$ & $1.351 \mathrm{E}+01$ & $1.388 \mathrm{E}+01$ & $1.173 \mathrm{E}+01$ & $1.119 \mathrm{E}+01$ & $9.462 \mathrm{E}+00$ & $8.961 \mathrm{E}+00$ \\
\hline R08 & $1.568 \mathrm{E}+01$ & $1.341 \mathrm{E}+01$ & $1.370 \mathrm{E}+01$ & $1.168 \mathrm{E}+01$ & $1.095 \mathrm{E}+01$ & $9.492 E+00$ & $8.762 \mathrm{E}+00$ \\
\hline R09 & $1.552 \mathrm{E}+01$ & $1.338 \mathrm{E}+01$ & $1.342 \mathrm{E}+01$ & $1.167 E+01$ & $1.061 \mathrm{E}+01$ & $9.565 \mathrm{E}+00$ & $8.499 \mathrm{E}+00$ \\
\hline R10 & $1.550 \mathrm{E}+01$ & $1.333 \mathrm{E}+01$ & $1.328 \mathrm{E}+01$ & $1.162 E+01$ & $1.041 \mathrm{E}+01$ & $9.605 E+\infty 0$ & $8.382 E+00$ \\
\hline R11 & $1.558 \mathrm{E}+01$ & $1.333 \mathrm{E}+01$ & $1.324 \mathrm{E}+01$ & $1.154 \mathrm{E}+01$ & $1.032 \mathrm{E}+01$ & $9.627 \mathrm{E}+00$ & $8.360 E+00$ \\
\hline R12 & $1.579 \mathrm{E}+01$ & $1.343 \mathrm{E}+01$ & $1.334 \mathrm{E}+01$ & $1.155 \mathrm{E}+01$ & $1.040 \mathrm{E}+01$ & $9.604 \mathrm{E}+00$ & $8.478 E+00$ \\
\hline R13 & $1.610 \mathrm{E}+01$ & $1.368 \mathrm{E}+01$ & $1.358 \mathrm{E}+01$ & $1.170 \mathrm{E}+01$ & $1.068 \mathrm{E}+01$ & $9.744 \mathrm{E}+00$ & $8.737 \mathrm{E}+00$ \\
\hline R14 & $1.626 \mathrm{E}+01$ & $1.384 \mathrm{E}+01$ & $1.372 \mathrm{E}+01$ & $1.179 \mathrm{E}+01$ & $1.089 \mathrm{E}+01$ & $9.807 E+\infty 0$ & $8.907 E+00$ \\
\hline R15 & $1.633 \mathrm{E}+01$ & $1.399 \mathrm{E}+01$ & $1.384 \mathrm{E}+01$ & $1.191 \mathrm{E}+01$ & $1.111 \mathrm{E}+01$ & $9.896 E+00$ & $8.970 \mathrm{E}+00$ \\
\hline R16 & $1.644 \mathrm{E}+01$ & $1.411 \mathrm{E}+01$ & $1.397 \mathrm{E}+01$ & $1.206 \mathrm{E}+01$ & $1.135 \mathrm{E}+01$ & $9.992 \mathrm{E}+00$ & $9.042 \mathrm{E}+00$ \\
\hline
\end{tabular}

\begin{tabular}{|c|c|c|c|c|c|c|c|}
\hline POINT & S01-S18 & S19-S35 & S01-S12 & S13-S24 & S25-S35 & S01-S10 & S13-S22 \\
\hline $\mathrm{C} 01$ & $1.767 \mathrm{E}+02$ & $1.391 \mathrm{E}+02$ & $1.096 \mathrm{E}+02$ & $1.151 \mathrm{E}+02$ & $9.112 \mathrm{E}+01$ & $9.300 \mathrm{E}+01$ & $9.828 \mathrm{E}+01$ \\
\hline $\mathrm{CO2}$ & $2.734 \mathrm{E}+02$ & $1.864 E+02$ & $1.805 \mathrm{E}+02$ & $1.465 \mathrm{E}+02$ & $1.329 \mathrm{E}+02$ & $1.461 \mathrm{E}+02$ & $1.295 \mathrm{E}+02$ \\
\hline $\mathrm{C} 03$ & $5.273 \mathrm{E}+02$ & $2.181 \mathrm{E}+02$ & $4.341 E+02$ & $1.485 \mathrm{E}+02$ & $1.628 \mathrm{E}+02$ & $3.646 \mathrm{E}+02$ & $1.334 E+02$ \\
\hline $\mathrm{C} 04$ & $7.260 \mathrm{E}+02$ & $2.056 \mathrm{E}+02$ & $6.085 \mathrm{E}+02$ & $1.710 \mathrm{E}+02$ & $1.521 \mathrm{E}+02$ & $5.162 \mathrm{E}+02$ & $1.592 \mathrm{E}+02$ \\
\hline C05 & $5.006 \mathrm{E}+02$ & $2.032 \mathrm{E}+02$ & $2.032 \mathrm{E}+02$ & $3.425 \mathrm{E}+02$ & $1.580 \mathrm{E}+02$ & $1.468 \mathrm{E}+02$ & $3.342 \mathrm{E}+02$ \\
\hline C06 & $2.925 \mathrm{E}+02$ & $2.082 \mathrm{E}+02$ & $1.050 \mathrm{E}+02$ & $2.206 \mathrm{E}+02$ & $1.750 \mathrm{E}+02$ & $8.004 \mathrm{E}+01$ & $2.149 \mathrm{E}+02$ \\
\hline $\mathrm{C} 07$ & $2.065 \mathrm{E}+02$ & $2.461 \mathrm{E}+02$ & $1.231 \mathrm{E}+02$ & $2.097 \mathrm{E}+02$ & $1.197 \mathrm{E}+02$ & $1.109 \mathrm{E}+02$ & $1.457 \mathrm{E}+02$ \\
\hline $\mathrm{Cos}$ & $2.404 \mathrm{E}+02$ & $3.588 \mathrm{E}+02$ & $1.345 \mathrm{E}+02$ & $2.313 E+02$ & $2.334 \mathrm{E}+02$ & $1.161 \mathrm{E}+02$ & $1.697 \mathrm{E}+02$ \\
\hline C09 & $2.212 \mathrm{E}+02$ & $5.161 E+02$ & $1.252 \mathrm{E}+02$ & $2.187 \mathrm{E}+02$ & $3.934 \mathrm{E}+02$ & $1.004 \mathrm{E}+02$ & $1.451 \mathrm{E}+02$ \\
\hline C10 & $1.890 \mathrm{E}+02$ & $4.072 \mathrm{E}+02$ & $1.104 \mathrm{E}+02$ & $1.482 \mathrm{E}+02$ & $3.376 \mathrm{E}+02$ & $8.386 \mathrm{E}+01$ & $1.170 \mathrm{E}+02$ \\
\hline C11 & $2.352 \mathrm{E}+02$ & $1.857 \mathrm{E}+02$ & $1.673 \mathrm{E}+02$ & $1.108 \mathrm{E}+02$ & $1.429 \mathrm{E}+02$ & $1.456 \mathrm{E}+02$ & $9.853 \mathrm{E}+01$ \\
\hline C12 & $1.263 \mathrm{E}+02$ & $1.217 \mathrm{E}+02$ & $6.897 \mathrm{E}+01$ & $8.640 \mathrm{E}+01$ & $9.263 \mathrm{E}+01$ & $5.398 \mathrm{E}+01$ & $8.068 \mathrm{E}+01$ \\
\hline C13 & $2.703 \mathrm{E}+02$ & $1.359 \mathrm{E}+02$ & $1.691 \mathrm{E}+02$ & $1.761 E+02$ & $6.111 \mathrm{E}+01$ & $1.626 \mathrm{E}+02$ & $1.668 \mathrm{E}+02$ \\
\hline C14 & $4.018 \mathrm{E}+02$ & $1.472 \mathrm{E}+02$ & $1.937 \mathrm{E}+02$ & $2.801 E+02$ & $7.522 \mathrm{E}+01$ & $1.860 \mathrm{E}+02$ & $2.709 \mathrm{E}+02$ \\
\hline C15 & $2.901 E+02$ & $1.755 \mathrm{E}+02$ & $1.611 \mathrm{E}+02$ & $2.215 \mathrm{E}+02$ & $8.301 E+01$ & $1.525 \mathrm{E}+02$ & $2.132 \mathrm{E}+02$ \\
\hline C16 & $2.119 \mathrm{E}+02$ & $3.303 \mathrm{E}+02$ & $1.246 \mathrm{E}+02$ & $3.347 \mathrm{E}+02$ & $8.295 \mathrm{E}+01$ & $1.158 \mathrm{E}+02$ & $3.277 \mathrm{E}+02$ \\
\hline C17 & $2.435 \mathrm{E}+02$ & $1.851 \mathrm{E}+02$ & $9.998 \mathrm{E}+01$ & $2.505 E+02$ & $7.817 \mathrm{E}+01$ & $9.188 \mathrm{E}+01$ & $2.448 \mathrm{E}+02$ \\
\hline C18 & $2.187 \mathrm{E}+02$ & $1.117 \mathrm{E}+02$ & $7.884 \mathrm{E}+01$ & $1.828 \mathrm{E}+02$ & $6.872 \mathrm{E}+01$ & $7.190 \mathrm{E}+01$ & $1.784 \mathrm{E}+02$ \\
\hline C19 & $1.606 \mathrm{E}+02$ & $9.110 \mathrm{E}+01$ & $1.219 \mathrm{E}+02$ & $7.319 \mathrm{E}+01$ & $5.661 \mathrm{E}+01$ & $1.143 E+02$ & $6.646 \mathrm{E}+01$ \\
\hline C20 & $2.280 \mathrm{E}+02$ & $1.556 \mathrm{E}+02$ & $1.823 \mathrm{E}+02$ & $8.915 \mathrm{E}+01$ & $1.122 \mathrm{E}+02$ & $1.728 \mathrm{E}+02$ & $8.244 \mathrm{E}+01$ \\
\hline C21 & $3.671 \mathrm{E}+02$ & $7.542 \mathrm{E}+02$ & $3.155 \mathrm{E}+02$ & $1.088 \mathrm{E}+02$ & $6.970 \mathrm{E}+02$ & $3.045 \mathrm{E}+02$ & $1.025 E+02$ \\
\hline $\mathrm{C} 22$ & $3.506 \mathrm{E}+02$ & $4.426 \mathrm{E}+02$ & $2.937 \mathrm{E}+02$ & $1.283 E+02$ & $3.712 \mathrm{E}+02$ & $2.825 E+02$ & $1.229 \mathrm{E}+02$ \\
\hline
\end{tabular}


HNF-4799, Rev 0

August 25, 1999

REVISED METHODOLOGY FOR DETERMINING CESIUM-137 CONTENT OF HN-200 GROUT CONTAINERS.

$\begin{array}{llllllll}\mathbf{C 2 3} & 2.861 \mathrm{E}+02 & 6.390 \mathrm{E}+02 & 2.264 \mathrm{E}+02 & 1.222 \mathrm{E}+02 & 5.764 \mathrm{E}+02 & 2.163 \mathrm{E}+02 & 1.177 \mathrm{E}+02 \\ \mathbf{C} 24 & 3.980 \mathrm{E}+02 & 1.925 \mathrm{E}+02 & 3.436 \mathrm{E}+02 & 9.573 \mathrm{E}+01 & 1.512 \mathrm{E}+02 & 3.352 \mathrm{E}+02 & 9.201 \mathrm{E}+01 \\ \text { R01 } & 9.362 \mathrm{E}+00 & 7.226 \mathrm{E}+00 & 5.806 \mathrm{E}+00 & 5.854 \mathrm{E}+00 & 4.929 \mathrm{E}+00 & 4.944 \mathrm{E}+00 & 5.254 \mathrm{E}+00 \\ \text { R02 } & 9.250 \mathrm{E}+00 & 7.399 \mathrm{E}+00 & 5.684 \mathrm{E}+00 & 5.948 \mathrm{E}+00 & 5.016 \mathrm{E}+00 & 4.790 \mathrm{E}+00 & 5.275 \mathrm{E}+00 \\ \text { R03 } & 8.989 \mathrm{E}+00 & 7.479 \mathrm{E}+00 & 5.533 \mathrm{E}+00 & 5.910 \mathrm{E}+00 & 5.025 \mathrm{E}+00 & 4.654 \mathrm{E}+00 & 5.178 \mathrm{E}+00 \\ \text { R04 } & 8.808 \mathrm{E}+00 & 7.506 \mathrm{E}+00 & 5.380 \mathrm{E}+00 & 5.918 \mathrm{E}+00 & 5.017 \mathrm{E}+00 & 4.557 \mathrm{E}+00 & 5.163 \mathrm{E}+00 \\ \text { R05 } & 8.630 \mathrm{E}+00 & 7.513 \mathrm{E}+00 & 5.277 \mathrm{E}+00 & 5.950 \mathrm{E}+00 & 4.916 \mathrm{E}+00 & 4.532 \mathrm{E}+00 & 5.214 \mathrm{E}+00 \\ \text { R06 } & 8.609 \mathrm{E}+00 & 7.349 \mathrm{E}+00 & 5.251 \mathrm{E}+00 & 5.996 \mathrm{E}+00 & 4.711 \mathrm{E}+00 & 4.580 \mathrm{E}+00 & 5.315 \mathrm{E}+00 \\ \text { R07 } & 8.651 \mathrm{E}+00 & 7.205 \mathrm{E}+00 & 5.282 \mathrm{E}+00 & 6.005 \mathrm{E}+00 & 4.570 \mathrm{E}+00 & 4.662 \mathrm{E}+00 & 5.397 \mathrm{E}+00 \\ \text { R08 } & 8.668 \mathrm{E}+00 & 7.016 \mathrm{E}+00 & 5.319 \mathrm{E}+00 & 5.946 \mathrm{E}+00 & 4.418 \mathrm{E}+00 & 4.730 \mathrm{E}+00 & 5.399 \mathrm{E}+00 \\ \text { R09 } & 8.747 \mathrm{E}+00 & 6.771 \mathrm{E}+00 & 5.463 \mathrm{E}+00 & 5.812 \mathrm{E}+00 & 4.243 \mathrm{E}+00 & 4.904 \mathrm{E}+00 & 5.303 \mathrm{E}+00 \\ \text { R10 } & 8.815 \mathrm{E}+00 & 6.682 \mathrm{E}+00 & 5.632 \mathrm{E}+00 & 5.603 \mathrm{E}+00 & 4.262 \mathrm{E}+00 & 5.082 \mathrm{E}+00 & 5.122 \mathrm{E}+00 \\ \text { R11 } & 8.881 \mathrm{E}+00 & 6.698 \mathrm{E}+00 & 5.814 \mathrm{E}+00 & 5.343 \mathrm{E}+00 & 4.423 \mathrm{E}+00 & 5.259 \mathrm{E}+00 & 4.880 \mathrm{E}+00 \\ \text { R12 } & 8.906 \mathrm{E}+00 & 6.883 \mathrm{E}+00 & 5.960 \mathrm{E}+00 & 5.203 \mathrm{E}+00 & 4.627 \mathrm{E}+00 & 5.387 \mathrm{E}+00 & 4.747 \mathrm{E}+00 \\ \text { R13 } & 9.081 \mathrm{E}+00 & 7.017 \mathrm{E}+00 & 6.027 \mathrm{E}+00 & 5.283 \mathrm{E}+00 & 4.788 \mathrm{E}+00 & 5.422 \mathrm{E}+00 & 4.822 \mathrm{E}+00 \\ \text { R14 } & 9.163 \mathrm{E}+00 & 7.093 \mathrm{E}+00 & 6.019 \mathrm{E}+00 & 5.361 \mathrm{E}+00 & 4.876 \mathrm{E}+00 & 5.369 \mathrm{E}+00 & 4.883 \mathrm{E}+00 \\ \text { R15 } & 9.257 \mathrm{E}+00 & 7.077 \mathrm{E}+00 & 5.945 \mathrm{E}+00 & 5.533 \mathrm{E}+00 & 4.856 \mathrm{E}+00 & 5.221 \mathrm{E}+00 & 5.028 \mathrm{E}+00 \\ \text { R16 } & 9.348 \mathrm{E}+00 & 7.095 \mathrm{E}+00 & 5.884 \mathrm{E}+00 & 5.704 \mathrm{E}+00 & 4.854 \mathrm{E}+00 & 5.090 \mathrm{E}+00 & 5.163 \mathrm{E}+00\end{array}$

\begin{tabular}{|c|c|c|c|c|c|c|}
\hline & & & & & S22-S28 & \\
\hline $\mathrm{C} 01$ & $8.373 \mathrm{E}+01$ & & $7 \mathrm{E}+01$ & & & \\
\hline $\mathrm{CO2}$ & $1.054 \mathrm{E}+02$ & $1.157 \mathrm{E}+02$ & $895 \mathrm{E}+01$ & $1.006 \mathrm{E}+02$ & $7.267 \mathrm{E}+01$ & $\mathrm{E}+0$ \\
\hline $\mathrm{C} 03$ & $1.109 \mathrm{E}+02$ & $3.333 \mathrm{E}+02$ & $1.217 \mathrm{E}+02$ & $1.083 E+02$ & $7.132 \mathrm{E}+01$ & $88 \mathrm{E}+0$ \\
\hline C04 & $1.102 \mathrm{E}+02$ & $4.863 \mathrm{E}+02$ & $1.379 \mathrm{E}+02$ & $1.401 \mathrm{E}+02$ & $6.849 \mathrm{E}+01$ & $888 \mathrm{E}+01$ \\
\hline C05 & $1.353 \mathrm{E}+02$ & $1.211 \mathrm{E}+02$ & $9.335 \mathrm{E}+01$ & $3.200 \mathrm{E}+02$ & $9.753 \mathrm{E}+01$ & 7.172E+01 \\
\hline $\mathrm{CO6}$ & $1.619 \mathrm{E}+02$ & $6.019 \mathrm{E}+01$ & $5.309 \mathrm{E}+01$ & $2.043 \mathrm{E}+02$ & $1.323 \mathrm{E}+02$ & $084 E+01$ \\
\hline $\mathrm{C} 07$ & $1.642 \mathrm{E}+02$ & $4.079 \mathrm{E}+01$ & $1.247 \mathrm{E}+02$ & $8.312 E+01$ & $1.480 \mathrm{E}+02$ & $5.595 \mathrm{E}+01$ \\
\hline $\mathrm{C} 08$ & $2.657 \mathrm{E}+02$ & 4.90 & $1.369 \mathrm{E}+02$ & $1.016 \mathrm{E}+02$ & $2.152 \mathrm{E}+02$ & $9.646 \mathrm{E}+01$ \\
\hline $\mathrm{COS}$ & $4.291 E+02$ & $5.248 \mathrm{E}+01$ & $1.130 \mathrm{E}+02$ & $9.400 \mathrm{E}+01$ & $2.557 \mathrm{E}+02$ & $2.221 E+02$ \\
\hline C10 & $3.295 E+02$ & $4.976 \mathrm{E}+01$ & $8.641 E+01$ & $8.447 \mathrm{E}+01$ & $1.132 \mathrm{E}+02$ & $2.623 \mathrm{E}+0$ \\
\hline C11 & $1.155 \mathrm{E}+02$ & $4.306 \mathrm{E}+01$ & $1.406 \mathrm{E}+02$ & $7.790 \mathrm{E}+01$ & $5.436 \mathrm{E}+01$ & $1.051 \mathrm{E}+0$ \\
\hline C12 & $6.003 E+01$ & $\mathrm{E}+01$ & $4.430 \mathrm{E}+01$ & $\mathrm{E}+01$ & $\mathrm{E}+01$ & $6.893 \mathrm{E}+0$ \\
\hline C13 & $5.644 \mathrm{E}+01$ & $5.422 E+01$ & $E+02$ & $E+01$ & $E+01$ & $4.069 \mathrm{E}+0$ \\
\hline C14 & $6.681 E+01$ & $7.929 \mathrm{E}+01$ & $2.958 \mathrm{E}+02$ & $\mathrm{E}+01$ & $6.030 \mathrm{E}+01$ & $5.145 E+0$ \\
\hline C15 & $7.030 \mathrm{E}+01$ & $8.250 \mathrm{E}+01$ & $1.703 \mathrm{E}+02$ & $1.086 \mathrm{E}+02$ & $4.617 \mathrm{E}+01$ & $5.809 E+0$ \\
\hline C16 & $6.594 E+01$ & $6.689 \mathrm{E}+01$ & $8.281 \mathrm{E}+01$ & $2.964 \mathrm{E}+02$ & $3.678 \mathrm{E}+01$ & $5.933 E+0$ \\
\hline $\mathrm{C} 17$ & $5.679 \mathrm{E}+01$ & $5.653 \mathrm{E}+01$ & $6.051 \mathrm{E}+01$ & $2.247 \mathrm{E}+02$ & $2.958 \mathrm{E}+01$ & $5.730 \mathrm{E}+0$ \\
\hline C18 & $4.613 \mathrm{E}+01$ & $4.752 E+01$ & $4.090 \mathrm{E}+01$ & $1.672 \mathrm{E}+02$ & $2.359 \mathrm{E}+01$ & $5.116 \mathrm{E}+01$ \\
\hline C19 & $4.898 \mathrm{E}+01$ & $7.569 \mathrm{E}+01$ & $6.298 \mathrm{E}+01$ & $4.400 \mathrm{E}+01$ & $2.963 \mathrm{E}+01$ & $3.941 \mathrm{E}+01$ \\
\hline $\mathrm{C} 20$ & $6.935 E+01$ & $1.263 \mathrm{E}+02$ & $7.398 \mathrm{E}+01$ & $5.910 \mathrm{E}+01$ & $3.363 \mathrm{E}+01$ & $9.068 \mathrm{E}+01$ \\
\hline $\mathrm{C} 21$ & $1.204 \mathrm{E}+02$ & $2.462 \mathrm{E}+02$ & $8.601 E+01$ & $8.115 E+01$ & $4.051 \mathrm{E}+01$ & $6.674 \mathrm{E}+02$ \\
\hline 22 & $701 \mathrm{E}+02$ & $2.108 \mathrm{E}+02$ & $9.672 \mathrm{E}+01$ & $1.052 \mathrm{E}+02$ & $7.821 \mathrm{E}+01$ & $3.023 E+0$ \\
\hline & $5.590 \mathrm{E}+02$ & $1.600 \mathrm{E}+02$ & $7.717 \mathrm{E}+01$ & $1.038 \mathrm{E}+02$ & $3.821 E+02$ & $2.020 \mathrm{E}+$ \\
\hline
\end{tabular}




\section{REVISED METHODOLOGY FOR DETERMINING CESIUM-137 CONTENT OF HN-200 GROUT CONTAINERS.}

$\begin{array}{lllllll}\mathbf{C} 24 & 1.433 \mathrm{E}+02 & 3.023 \mathrm{E}+02 & 4.950 \mathrm{E}+01 & 8.135 \mathrm{E}+01 & 6.453 \mathrm{E}+01 & 9.285 \mathrm{E}+01 \\ \mathbf{R} 01 & 4.106 \mathrm{E}+00 & 3.365 \mathrm{E}+00 & 3.334 \mathrm{E}+00 & 4.005 \mathrm{E}+00 & 2.708 \mathrm{E}+00 & 3.178 \mathrm{E}+00 \\ \mathbf{R} 02 & 4.191 \mathrm{E}+00 & 3.203 \mathrm{E}+00 & 3.410 \mathrm{E}+00 & 3.971 \mathrm{E}+00 & 2.843 \mathrm{E}+00 & 3.222 \mathrm{E}+00 \\ \mathbf{R 0 3} & 4.273 \mathrm{E}+00 & 3.034 \mathrm{E}+00 & 3.471 \mathrm{E}+00 & 3.818 \mathrm{E}+00 & 2.925 \mathrm{E}+00 & 3.220 \mathrm{E}+00 \\ \mathbf{R} 04 & 4.286 \mathrm{E}+00 & 2.879 \mathrm{E}+00 & 3.517 \mathrm{E}+00 & 3.751 \mathrm{E}+00 & 2.929 \mathrm{E}+00 & 3.239 \mathrm{E}+00 \\ \mathbf{R} 05 & 4.208 \mathrm{E}+00 & 2.783 \mathrm{E}+00 & 3.566 \mathrm{E}+00 & 3.630 \mathrm{E}+00 & 2.967 \mathrm{E}+00 & 3.196 \mathrm{E}+00 \\ \mathbf{R} 06 & 4.044 \mathrm{E}+00 & 2.759 \mathrm{E}+00 & 3.630 \mathrm{E}+00 & 3.590 \mathrm{E}+00 & 2.899 \mathrm{E}+00 & 3.080 \mathrm{E}+00 \\ \mathbf{R} 07 & 3.836 \mathrm{E}+00 & 2.784 \mathrm{E}+00 & 3.684 \mathrm{E}+00 & 3.583 \mathrm{E}+00 & 2.769 \mathrm{E}+00 & 3.036 \mathrm{E}+00 \\ \mathbf{R 0 8} & 3.658 \mathrm{E}+00 & 2.821 \mathrm{E}+00 & 3.696 \mathrm{E}+00 & 3.578 \mathrm{E}+00 & 2.623 \mathrm{E}+00 & 2.966 \mathrm{E}+00 \\ \mathbf{R} 09 & 3.551 \mathrm{E}+00 & 2.990 \mathrm{E}+00 & 3.637 \mathrm{E}+00 & 3.562 \mathrm{E}+00 & 2.485 \mathrm{E}+00 & 2.843 \mathrm{E}+00 \\ \mathbf{R} 10 & 3.519 \mathrm{E}+00 & 3.189 \mathrm{E}+00 & 3.536 \mathrm{E}+00 & 3.533 \mathrm{E}+00 & 2.361 \mathrm{E}+00 & 2.878 \mathrm{E}+00 \\ \text { R11 } & 3.604 \mathrm{E}+00 & 3.404 \mathrm{E}+00 & 3.415 \mathrm{E}+00 & 3.492 \mathrm{E}+00 & 2.260 \mathrm{E}+00 & 3.008 \mathrm{E}+00 \\ \text { R12 } & 3.746 \mathrm{E}+00 & 3.582 \mathrm{E}+00 & 3.310 \mathrm{E}+00 & 3.426 \mathrm{E}+00 & 2.308 \mathrm{E}+00 & 3.164 \mathrm{E}+00 \\ \text { R13 } & 3.893 \mathrm{E}+00 & 3.676 \mathrm{E}+00 & 3.241 \mathrm{E}+00 & 3.562 \mathrm{E}+00 & 2.358 \mathrm{E}+00 & 3.262 \mathrm{E}+00 \\ \text { R14 } & 4.012 \mathrm{E}+00 & 3.682 \mathrm{E}+00 & 3.209 \mathrm{E}+00 & 3.659 \mathrm{E}+00 & 2.430 \mathrm{E}+00 & 3.277 \mathrm{E}+00 \\ \text { R15 } & 4.075 \mathrm{E}+00 & 3.586 \mathrm{E}+00 & 3.217 \mathrm{E}+00 & 3.825 \mathrm{E}+00 & 2.513 \mathrm{E}+00 & 3.192 \mathrm{E}+00 \\ \text { R16 } & 4.090 \mathrm{E}+00 & 3.494 \mathrm{E}+00 & 3.259 \mathrm{E}+00 & 3.950 \mathrm{E}+00 & 2.599 \mathrm{E}+00 & 3.140 \mathrm{E}+00\end{array}$

\begin{tabular}{|c|c|c|c|c|c|c|c|}
\hline \multicolumn{8}{|c|}{ SOURCE } \\
\hline POINT & S01-S05 & S06-S10 & S11-S15 & S16-S20 & S21-S25 & S26-S30 & S31-S35 \\
\hline C01 & $5.015 \mathrm{E}+01$ & $4.284 \mathrm{E}+01$ & $3.965 \mathrm{E}+01$ & $6.082 \mathrm{E}+01$ & $5.276 \mathrm{E}+01$ & $3.185 \mathrm{E}+01$ & $3.774 \mathrm{E}+01$ \\
\hline $\mathrm{CO2}$ & $8.986 E+01$ & $5.627 \mathrm{E}+01$ & $6.210 \mathrm{E}+01$ & $8.276 E+01$ & $6.375 \mathrm{E}+01$ & $4.207 \mathrm{E}+01$ & $6.304 \mathrm{E}+01$ \\
\hline $\mathrm{CO3}$ & $2.954 \mathrm{E}+02$ & $6.920 \mathrm{E}+01$ & $1.015 E+02$ & $7.605 \mathrm{E}+01$ & $6.327 \mathrm{E}+01$ & $4.904 \mathrm{E}+01$ & $9.092 E+01$ \\
\hline C04 & $4.518 \mathrm{E}+02$ & $6.434 \mathrm{E}+01$ & $1.514 \mathrm{E}+02$ & $6.997 \mathrm{E}+01$ & $5.621 \mathrm{E}+01$ & $5.786 \mathrm{E}+01$ & $7.997 \mathrm{E}+01$ \\
\hline C05 & $9.615 \mathrm{E}+01$ & $5.061 \mathrm{E}+01$ & $2.805 \mathrm{E}+02$ & $8.227 E+0 t$ & $4.475 \mathrm{E}+01$ & $9.492 \mathrm{E}+01$ & $5.454 E+01$ \\
\hline COS & $4.061 \mathrm{E}+01$ & $3.943 E+01$ & $1.523 \mathrm{E}+02$ & $6.707 \mathrm{E}+01$ & $3.159 \mathrm{E}+01$ & $1.329 \mathrm{E}+02$ & $3.673 \mathrm{E}+01$ \\
\hline $\mathrm{CO}$ & $3.262 \mathrm{E}+01$ & $7.831 \mathrm{E}+01$ & $5.878 \mathrm{E}+01$ & $7.190 \mathrm{E}+01$ & $1.160 \mathrm{E}+02$ & $5.691 \mathrm{E}+01$ & $3.806 \mathrm{E}+01$ \\
\hline $\mathrm{COB}$ & $3.883 \mathrm{E}+01$ & $7.730 \mathrm{E}+01$ & $7.577 \mathrm{E}+01$ & $8.639 E+01$ & $1.208 \mathrm{E}+02$ & $1.267 \mathrm{E}+02$ & $7.342 E+01$ \\
\hline C09 & $4.072 \mathrm{E}+01$ & $5.967 \mathrm{E}+01$ & $7.348 \mathrm{E}+01$ & $7.399 \mathrm{E}+01$ & $1.225 \mathrm{E}+02$ & $1.664 \mathrm{E}+02$ & $2.005 \mathrm{E}+02$ \\
\hline C10 & $3.738 \mathrm{E}+01$ & $4.649 \mathrm{E}+01$ & $6.350 \mathrm{E}+01$ & $6.016 \mathrm{E}+01$ & $6.660 \mathrm{E}+01$ & $7.584 \mathrm{E}+01$ & $2.462 \mathrm{E}+02$ \\
\hline C11 & $3.097 \mathrm{E}+01$ & $1.146 \mathrm{E}+02$ & $5.118 \mathrm{E}+01$ & $5.208 \mathrm{E}+01$ & $3.821 \mathrm{E}+01$ & $4.049 \mathrm{E}+01$ & $9.341 \mathrm{E}+01$ \\
\hline C12 & $2.419 \mathrm{E}+01$ & $2.979 \mathrm{E}+01$ & $3.837 \mathrm{E}+01$ & $4.384 \mathrm{E}+01$ & $2.471 \mathrm{E}+01$ & $2.689 \mathrm{E}+01$ & $6.022 \mathrm{E}+01$ \\
\hline $\mathrm{C13}$ & $4.586 \mathrm{E}+01$ & $1.168 \mathrm{E}+02$ & $9.026 \mathrm{E}+01$ & $3.320 \mathrm{E}+01$ & $6.660 \mathrm{E}+01$ & $2.736 \mathrm{E}+01$ & $2.619 \mathrm{E}+01$ \\
\hline C14 & $6.855 \mathrm{E}+01$ & $1.175 \mathrm{E}+02$ & $1.927 \mathrm{E}+02$ & $4.763 \mathrm{E}+01$ & $5.564 \mathrm{E}+01$ & $3.335 \mathrm{E}+01$ & $3.371 \mathrm{E}+01$ \\
\hline C15 & $6.924 \mathrm{E}+01$ & $8.323 \mathrm{E}+01$ & $1.047 \mathrm{E}+02$ & $8.976 \mathrm{E}+01$ & $4.345 \mathrm{E}+01$ & $3.478 \mathrm{E}+01$ & $4.053 \mathrm{E}+01$ \\
\hline C16 & $5.112 \mathrm{E}+01$ & $6.466 \mathrm{E}+01$ & $3.882 E+01$ & $2.746 \mathrm{E}+02$ & $3.648 \mathrm{E}+01$ & $3.208 \mathrm{E}+01$ & $4.444 \mathrm{E}+01$ \\
\hline C17 & $3.840 \mathrm{E}+01$ & $5.348 \mathrm{E}+01$ & $3.038 \mathrm{E}+01$ & $2.033 E+02$ & $2.991 \mathrm{E}+01$ & $2.793 \mathrm{E}+01$ & $4.523 \mathrm{E}+01$ \\
\hline C18 & $2.818 \mathrm{E}+01$ & $4.372 \mathrm{E}+01$ & $2.166 \mathrm{E}+01$ & $1.490 \mathrm{E}+02$ & $2.287 \mathrm{E}+01$ & $2.340 \mathrm{E}+01$ & $4.152 \mathrm{E}+01$ \\
\hline C19 & $6.374 \mathrm{E}+01$ & $5.052 \mathrm{E}+01$ & $2.807 \mathrm{E}+0 \mathrm{l}$ & $2.917 \mathrm{E}+01$ & $3.082 \mathrm{E}+01$ & $2.145 \mathrm{E}+01$ & $2.794 \mathrm{E}+01$ \\
\hline $\mathrm{C20}$ & $1.069 E+02$ & $6.585 \mathrm{E}+01$ & $3.223 E+01$ & $3.542 \mathrm{E}+01$ & $3.885 \mathrm{E}+01$ & $2.914 \mathrm{E}+01$ & $7.526 \mathrm{E}+01$ \\
\hline C21 & $2.179 \mathrm{E}+02$ & $8.661 E+01$ & $3.372 E+01$ & $4.179 \mathrm{E}+01$ & $5.161 \mathrm{E}+01$ & $4.383 \mathrm{E}+01$ & $6.458 \mathrm{E}+02$ \\
\hline C22 & $1.730 \mathrm{E}+02$ & $1.095 \mathrm{E}+02$ & $3.240 \mathrm{E}+01$ & $4.831 \mathrm{E}+01$ & $6.503 \mathrm{E}+01$ & $1.052 \mathrm{E}+02$ & $2.598 \mathrm{E}+02$ \\
\hline $\mathrm{C23}$ & $8.647 \mathrm{E}+01$ & $1.298 \mathrm{E}+02$ & $2.902 E+01$ & $5.180 \mathrm{E}+01$ & $5.643 \mathrm{E}+01$ & $4.662 \mathrm{E}+02$ & $1.054 \mathrm{E}+02$ \\
\hline C24 & $4.161 \mathrm{E}+01$ & $2.936 \mathrm{E}+02$ & $2.449 \mathrm{E}+01$ & $4.708 \mathrm{E}+01$ & $3.628 \mathrm{E}+01$ & $1.049 \mathrm{E}+02$ & $4.261 \mathrm{E}+01$ \\
\hline
\end{tabular}




\section{REVISED METHODOLOGY FOR DETERMINING CESIUM-137 CONTENT OF HN-200 GROUT CONTAINERS.}

$\begin{array}{llllllll}\text { R01 } & 2.405 \mathrm{E}+00 & 2.539 \mathrm{E}+00 & 2.458 \mathrm{E}+00 & 2.621 \mathrm{E}+00 & 2.138 \mathrm{E}+00 & 2.008 \mathrm{E}+00 & 2.420 \mathrm{E}+00 \\ \text { R02 } & 2.303 \mathrm{E}+00 & 2.487 \mathrm{E}+00 & 2.534 \mathrm{E}+00 & 2.611 \mathrm{E}+00 & 2.227 \mathrm{E}+00 & 1.992 \mathrm{E}+00 & 2.495 \mathrm{E}+00 \\ \text { R03 } & 2.184 \mathrm{E}+00 & 2.470 \mathrm{E}+00 & 2.519 \mathrm{E}+00 & 2.528 \mathrm{E}+00 & 2.283 \mathrm{E}+00 & 1.980 \mathrm{E}+00 & 2.504 \mathrm{E}+00 \\ \text { R04 } & 2.067 \mathrm{E}+00 & 2.490 \mathrm{E}+00 & 2.428 \mathrm{E}+00 & 2.562 \mathrm{E}+00 & 2.286 \mathrm{E}+00 & 1.949 \mathrm{E}+00 & 2.534 \mathrm{E}+00 \\ \text { R05 } & 1.991 \mathrm{E}+00 & 2.541 \mathrm{E}+00 & 2.297 \mathrm{E}+00 & 2.562 \mathrm{E}+00 & 2.348 \mathrm{E}+00 & 1.905 \mathrm{E}+00 & 2.500 \mathrm{E}+00 \\ \mathbf{R 0 6} & 1.969 \mathrm{E}+00 & 2.611 \mathrm{E}+00 & 2.258 \mathrm{E}+00 & 2.557 \mathrm{E}+00 & 2.332 \mathrm{E}+00 & 1.844 \mathrm{E}+00 & 2.388 \mathrm{E}+00 \\ \text { R07 } & 1.978 \mathrm{E}+00 & 2.684 \mathrm{E}+00 & 2.233 \mathrm{E}+00 & 2.567 \mathrm{E}+00 & 2.271 \mathrm{E}+00 & 1.778 \mathrm{E}+00 & 2.345 \mathrm{E}+00 \\ \text { R08 } & 1.981 \mathrm{E}+00 & 2.749 \mathrm{E}+00 & 2.192 \mathrm{E}+00 & 2.570 \mathrm{E}+00 & 2.193 \mathrm{E}+00 & 1.729 \mathrm{E}+00 & 2.270 \mathrm{E}+00 \\ \text { R09 } & 2.097 \mathrm{E}+00 & 2.807 \mathrm{E}+00 & 2.116 \mathrm{E}+00 & 2.546 \mathrm{E}+00 & 2.109 \mathrm{E}+00 & 1.706 \mathrm{E}+00 & 2.139 \mathrm{E}+00 \\ \text { R10 } & 2.214 \mathrm{E}+00 & 2.868 \mathrm{E}+00 & 2.033 \mathrm{E}+00 & 2.490 \mathrm{E}+00 & 2.015 \mathrm{E}+00 & 1.713 \mathrm{E}+00 & 2.164 \mathrm{E}+00 \\ \text { R11 } & 2.341 \mathrm{E}+00 & 2.918 \mathrm{E}+00 & 1.960 \mathrm{E}+00 & 2.408 \mathrm{E}+00 & 1.909 \mathrm{E}+00 & 1.790 \mathrm{E}+00 & 2.253 \mathrm{E}+00 \\ \text { R12 } & 2.451 \mathrm{E}+00 & 2.936 \mathrm{E}+00 & 1.925 \mathrm{E}+00 & 2.293 \mathrm{E}+00 & 1.941 \mathrm{E}+00 & 1.889 \mathrm{E}+00 & 2.356 \mathrm{E}+00 \\ \text { R13 } & 2.514 \mathrm{E}+00 & 2.908 \mathrm{E}+00 & 1.938 \mathrm{E}+00 & 2.383 \mathrm{E}+00 & 1.959 \mathrm{E}+00 & 1.981 \mathrm{E}+00 & 2.414 \mathrm{E}+00 \\ \text { R14 } & 2.534 \mathrm{E}+00 & 2.836 \mathrm{E}+00 & 1.980 \mathrm{E}+00 & 2.458 \mathrm{E}+00 & 1.984 \mathrm{E}+00 & 2.050 \mathrm{E}+00 & 2.415 \mathrm{E}+00 \\ \text { R15 } & 2.490 \mathrm{E}+00 & 2.731 \mathrm{E}+00 & 2.142 \mathrm{E}+00 & 2.532 \mathrm{E}+00 & 2.019 \mathrm{E}+00 & 2.074 \mathrm{E}+00 & 2.345 \mathrm{E}+00 \\ \text { R16 } & 2.468 \mathrm{E}+00 & 2.622 \mathrm{E}+00 & 2.310 \mathrm{E}+00 & 2.591 \mathrm{E}+00 & 2.065 \mathrm{E}+00 & 2.053 \mathrm{E}+00 & 2.332 \mathrm{E}+00\end{array}$

\begin{tabular}{|c|c|c|c|c|c|c|c|}
\hline \multicolumn{8}{|c|}{ SOURCE } \\
\hline OINT & S01-S03 & S04-S06 & S07-S09 & S10-S12 & S13-S15 & S16-S18 & S19-S21 \\
\hline C01 & $3.858 E+01$ & $2.327 E+01$ & $2.017 \mathrm{E}+01$ & $2.759 \mathrm{E}+01$ & $2.303 \mathrm{E}+01$ & $4.409 \mathrm{E}+01$ & $2.617 \mathrm{E}+01$ \\
\hline $\mathrm{CO2}$ & $7.491 \mathrm{E}+01$ & $3.628 \mathrm{E}+01$ & $2.422 E+01$ & $4.511 \mathrm{E}+01$ & $2.771 \mathrm{E}+01$ & $6.520 \mathrm{E}+01$ & $3.186 \mathrm{E}+01$ \\
\hline $\mathrm{CO3}$ & $2.783 \mathrm{E}+02$ & $4.899 \mathrm{E}+01$ & $2.809 \mathrm{E}+01$ & $7.873 \mathrm{E}+01$ & $3.200 \mathrm{E}+01$ & $6.119 \mathrm{E}+01$ & $3.599 \mathrm{E}+01$ \\
\hline $\mathrm{CO4}$ & $4.358 \mathrm{E}+02$ & $4.257 \mathrm{E}+01$ & $3.059 \mathrm{E}+01$ & $9.960 \mathrm{E}+01$ & $5.903 \mathrm{E}+01$ & $5.841 E+01$ & $3.823 E+01$ \\
\hline $\mathrm{COS}$ & $8.339 E+01$ & $2.778 \mathrm{E}+01$ & $3.006 \mathrm{E}+01$ & $6.194 \mathrm{E}+01$ & $2.241 \mathrm{E}+02$ & $7.333 \mathrm{E}+01$ & $3.390 \mathrm{E}+01$ \\
\hline CO6 & $3.129 \mathrm{E}+01$ & $1.767 \mathrm{E}+01$ & $2.687 \mathrm{E}+01$ & $2.917 \mathrm{E}+01$ & $1.274 \mathrm{E}+02$ & $6.012 \mathrm{E}+01$ & $2.507 \mathrm{E}+01$ \\
\hline $\mathrm{C} 07$ & $2,203 \mathrm{E}+01$ & $1.586 \mathrm{E}+01$ & $2.393 \mathrm{E}+01$ & $6.132 \mathrm{E}+01$ & $4.657 \mathrm{E}+01$ & $3.675 \mathrm{E}+01$ & $4.213 E+01$ \\
\hline $\mathrm{C} 08$ & $2.599 \mathrm{E}+01$ & $1.941 \mathrm{E}+01$ & $2.629 \mathrm{E}+01$ & $6.284 E+01$ & $5.739 \mathrm{E}+01$ & $4.847 \mathrm{E}+01$ & $4.716 \mathrm{E}+01$ \\
\hline C09 & $2.762 E+01$ & $2.040 \mathrm{E}+01$ & $2.624 \mathrm{E}+01$ & $5.099 \mathrm{E}+01$ & $4.862 \mathrm{E}+01$ & $4.738 \mathrm{E}+01$ & $3.825 \mathrm{E}+01$ \\
\hline C10 & $2.606 \mathrm{E}+01$ & $1.829 E+01$ & $2.496 \mathrm{E}+01$ & $4.107 \mathrm{E}+01$ & $3.698 \mathrm{E}+01$ & $4.161 E+01$ & $3.167 \mathrm{E}+01$ \\
\hline C11 & $2.200 \mathrm{E}+01$ & $1.480 \mathrm{E}+01$ & $2.277 E+01$ & $1.077 \mathrm{E}+02$ & $2.949 \mathrm{E}+01$ & $3.845 \mathrm{E}+01$ & $2.632 \mathrm{E}+01$ \\
\hline $\mathrm{C} 12$ & $1.726 \mathrm{E}+01$ & $1.143 \mathrm{E}+01$ & $1.986 \mathrm{E}+01$ & $2.042 \mathrm{E}+01$ & $2.337 \mathrm{E}+01$ & $3.397 \mathrm{E}+01$ & $2.054 \mathrm{E}+01$ \\
\hline C13 & $2.385 E+01$ & $2.677 \mathrm{E}+01$ & $7.815 \mathrm{E}+01$ & $4.029 \mathrm{E}+01$ & $8.383 \mathrm{E}+01$ & $1.742 \mathrm{E}+01$ & $2.367 \mathrm{E}+01$ \\
\hline C14 & $2.797 \mathrm{E}+01$ & $4.637 E+01$ & $8.009 \mathrm{E}+01$ & $3.928 \mathrm{E}+01$ & $1.850 \mathrm{E}+02$ & $2.308 \mathrm{E}+01$ & $3.550 \mathrm{E}+01$ \\
\hline C15 & $2.929 E+01$ & $4.630 \mathrm{E}+01$ & $5.579 \mathrm{E}+01$ & $2.971 \mathrm{E}+01$ & $9.603 E+01$ & $3.299 \mathrm{E}+01$ & $7.126 \mathrm{E}+01$ \\
\hline C16 & $2.827 \mathrm{E}+01$ & $2.896 \mathrm{E}+01$ & $4.565 \mathrm{E}+01$ & $2.167 \mathrm{E}+01$ & $3.005 E+01$ & $5.730 \mathrm{E}+01$ & $2.342 \mathrm{E}+02$ \\
\hline C17 & $2.450 \mathrm{E}+01$ & $1.912 \mathrm{E}+01$ & $4.015 \mathrm{E}+01$ & $1.622 \mathrm{E}+01$ & $2.228 \mathrm{E}+01$ & $1.212 \mathrm{E}+02$ & $9.826 \mathrm{E}+01$ \\
\hline C18 & $1.895 \mathrm{E}+01$ & $1.335 \mathrm{E}+01$ & $3.429 \mathrm{E}+01$ & $1.225 \mathrm{E}+01$ & $1.472 \mathrm{E}+01$ & $1.252 \mathrm{E}+02$ & $3.692 \mathrm{E}+01$ \\
\hline C19 & $4.369 E+01$ & $2.947 \mathrm{E}+01$ & $3.112 \mathrm{E}+01$ & $1.763 \mathrm{E}+01$ & $2.043 \mathrm{E}+01$ & $1.827 \mathrm{E}+01$ & $2.207 \mathrm{E}+01$ \\
\hline C20 & $5.641 E+01$ & $6.523 \mathrm{E}+01$ & $4.133 \mathrm{E}+01$ & $1.933 \mathrm{E}+01$ & $2.269 \mathrm{E}+01$ & $2.305 \mathrm{E}+01$ & $3.131 \mathrm{E}+01$ \\
\hline C21 & $5.737 \mathrm{E}+01$ & $1.797 \mathrm{E}+02$ & $5.899 \mathrm{E}+01$ & $1.949 \mathrm{E}+01$ & $2.274 \mathrm{E}+01$ & $2.879 \mathrm{E}+01$ & $4.632 \mathrm{E}+01$ \\
\hline $\mathrm{C} 22$ & $6.679 E+01$ & $1.232 \mathrm{E}+02$ & $8.565 E+01$ & $1.806 \mathrm{E}+01$ & $2.117 \mathrm{E}+01$ & $3.572 \mathrm{E}+01$ & $6.211 \mathrm{E}+01$ \\
\hline $\mathrm{C} 23$ & $5.407 \mathrm{E}+01$ & $4.383 \mathrm{E}+01$ & $1.131 \mathrm{E}+02$ & $1.546 \mathrm{E}+01$ & $1.886 \mathrm{E}+01$ & $4.082 \mathrm{E}+01$ & $5.488 \mathrm{E}+01$ \\
\hline C2A & $2.821 \mathrm{E}+01$ & $2.055 \mathrm{E}+01$ & $2.823 \mathrm{E}+02$ & $1.246 \mathrm{E}+01$ & $1.609 \mathrm{E}+01$ & $3.837 \mathrm{E}+01$ & $3.508 E+01$ \\
\hline R01 & $1.714 \mathrm{E}+00$ & $1.122 \mathrm{E}+00$ & $1.572 \mathrm{E}+00$ & $1.397 \mathrm{E}+00$ & $1.596 \mathrm{E}+00$ & $1.961 E+00$ & $1.341 \mathrm{E}+00$ \\
\hline
\end{tabular}




\section{REVISED METHODOLOGY FOR DETERMINING CESIUM-137 CONTENT OF HN-200 GROUT CONTAINERS.}

$\begin{array}{llllllll}\text { R02 } & 1.655 \mathrm{E}+00 & 1.055 \mathrm{E}+00 & 1.514 \mathrm{E}+00 & 1.460 \mathrm{E}+00 & 1.640 \mathrm{E}+00 & 1.927 \mathrm{E}+00 & 1.334 \mathrm{E}+00 \\ \text { R03 } & 1.557 \mathrm{E}+00 & 1.008 \mathrm{E}+00 & 1.483 \mathrm{E}+00 & 1.485 \mathrm{E}+00 & 1.640 \mathrm{E}+00 & 1.817 \mathrm{E}+00 & 1.334 \mathrm{E}+00 \\ \text { R04 } & 1.450 \mathrm{E}+00 & 9.743 \mathrm{E}-01 & 1.481 \mathrm{E}+00 & 1.475 \mathrm{E}+00 & 1.605 \mathrm{E}+00 & 1.824 \mathrm{E}+00 & 1.339 \mathrm{E}+00 \\ \text { R05 } & 1.369 \mathrm{E}+00 & 9.597 \mathrm{E}-01 & 1.507 \mathrm{E}+00 & 1.441 \mathrm{E}+00 & 1.552 \mathrm{E}+00 & 1.801 \mathrm{E}+00 & 1.349 \mathrm{E}+00 \\ \text { R06 } & 1.330 \mathrm{E}+00 & 9.635 \mathrm{E}-01 & 1.560 \mathrm{E}+00 & 1.397 \mathrm{E}+00 & 1.587 \mathrm{E}+00 & 1.771 \mathrm{E}+00 & 1.371 \mathrm{E}+00 \\ \text { R07 } & 1.314 \mathrm{E}+00 & 9.821 \mathrm{E}-01 & 1.635 \mathrm{E}+00 & 1.351 \mathrm{E}+00 & 1.613 \mathrm{E}+00 & 1.756 \mathrm{E}+00 & 1.400 \mathrm{E}+00 \\ \text { R08 } & 1.289 \mathrm{E}+00 & 1.010 \mathrm{E}+00 & 1.721 \mathrm{E}+00 & 1.298 \mathrm{E}+00 & 1.603 \mathrm{E}+00 & 1.746 \mathrm{E}+00 & 1.427 \mathrm{E}+00 \\ \text { R09 } & 1.378 \mathrm{E}+00 & 1.043 \mathrm{E}+00 & 1.813 \mathrm{E}+00 & 1.229 \mathrm{E}+00 & 1.556 \mathrm{E}+00 & 1.728 \mathrm{E}+00 & 1.443 \mathrm{E}+00 \\ \text { R10 } & 1.469 \mathrm{E}+00 & 1.083 \mathrm{E}+00 & 1.909 \mathrm{E}+00 & 1.172 \mathrm{E}+00 & 1.483 \mathrm{E}+00 & 1.700 \mathrm{E}+00 & 1.443 \mathrm{E}+00 \\ \text { R11 } & 1.550 \mathrm{E}+00 & 1.148 \mathrm{E}+00 & 1.982 \mathrm{E}+00 & 1.133 \mathrm{E}+00 & 1.405 \mathrm{E}+00 & 1.663 \mathrm{E}+00 & 1.430 \mathrm{E}+00 \\ \text { R12 } & 1.611 \mathrm{E}+00 & 1.222 \mathrm{E}+00 & 2.010 \mathrm{E}+00 & 1.118 \mathrm{E}+00 & 1.352 \mathrm{E}+00 & 1.595 \mathrm{E}+00 & 1.412 \mathrm{E}+00 \\ \text { R13 } & 1.644 \mathrm{E}+00 & 1.278 \mathrm{E}+00 & 1.979 \mathrm{E}+00 & 1.126 \mathrm{E}+00 & 1.333 \mathrm{E}+00 & 1.721 \mathrm{E}+00 & 1.397 \mathrm{E}+00 \\ \text { R14 } & 1.668 \mathrm{E}+00 & 1.298 \mathrm{E}+00 & 1.895 \mathrm{E}+00 & 1.159 \mathrm{E}+00 & 1.330 \mathrm{E}+00 & 1.814 \mathrm{E}+00 & 1.386 \mathrm{E}+00 \\ \text { R15 } & 1.665 \mathrm{E}+00 & 1.272 \mathrm{E}+00 & 1.778 \mathrm{E}+00 & 1.230 \mathrm{E}+00 & 1.419 \mathrm{E}+00 & 1.894 \mathrm{E}+00 & 1.372 \mathrm{E}+00 \\ \text { R16 } & 1.708 \mathrm{E}+00 & 1.206 \mathrm{E}+00 & 1.661 \mathrm{E}+00 & 1.310 \mathrm{E}+00 & 1.516 \mathrm{E}+00 & 1.947 \mathrm{E}+00 & 1.355 \mathrm{E}+00\end{array}$

\begin{tabular}{|c|c|c|c|c|c|}
\hline OINT & S22-S24 & S25-S27 & S28-S30 & S31-S33 & S34-S35 \\
\hline $\mathrm{CO1}$ & $2.179 \mathrm{E}+01$ & $3.641 \mathrm{E}+01$ & $1.697 \mathrm{E}+01$ & $1.868 \mathrm{E}+01$ & $1.906 \mathrm{E}+01$ \\
\hline $\mathrm{CO2}$ & $2.170 \mathrm{E}+01$ & $4.716 \mathrm{E}+01$ & $2.265 \mathrm{E}+01$ & $2.489 \mathrm{E}+01$ & $3.815 E+01$ \\
\hline $\mathrm{CO3}$ & $1.932 E+01$ & $4.402 \mathrm{E}+01$ & $2.784 \mathrm{E}+01$ & $3.077 \mathrm{E}+01$ & $6.015 \mathrm{E}+01$ \\
\hline CO4 & $1.530 \mathrm{E}+01$ & $3.345 \mathrm{E}+01$ & $3.866 \mathrm{E}+01$ & $3.285 \mathrm{E}+01$ & $4.712 \mathrm{E}+01$ \\
\hline $\cos$ & $1.122 \mathrm{E}+01$ & $2.416 \mathrm{E}+01$ & $7.934 E+01$ & $2.906 \mathrm{E}+01$ & $2.548 \mathrm{E}+01$ \\
\hline $\mathrm{CO6}$ & $8.069 \mathrm{E}+00$ & $1.730 \mathrm{E}+01$ & $1.210 \mathrm{E}+02$ & $2.230 \mathrm{E}+01$ & $1.443 \mathrm{E}+01$ \\
\hline $\mathrm{CO}$ & $8.424 E+01$ & $6.150 \mathrm{E}+01$ & $2.016 \mathrm{E}+01$ & $2.188 \mathrm{E}+01$ & $1.618 \mathrm{E}+01$ \\
\hline $\mathrm{COB}$ & $7.825 \mathrm{E}+01$ & $1.340 \mathrm{E}+02$ & $2.602 \mathrm{E}+01$ & $4.787 \mathrm{E}+01$ & $2.555 \mathrm{E}+01$ \\
\hline C09 & $8.441 \mathrm{E}+01$ & $1.674 \mathrm{E}+02$ & $2.547 \mathrm{E}+01$ & $1.666 \mathrm{E}+02$ & $3.388 E+01$ \\
\hline C10 & $3.791 \mathrm{E}+01$ & $7.040 \mathrm{E}+01$ & $2.102 \mathrm{E}+01$ & $2.108 \mathrm{E}+02$ & $3.543 \mathrm{E}+01$ \\
\hline C11 & $1.649 \mathrm{E}+01$ & $3.214 \mathrm{E}+01$ & $1.737 \mathrm{E}+01$ & $5.727 \mathrm{E}+01$ & $3.614 \mathrm{E}+01$ \\
\hline C12 & $8.517 E+00$ & $1.777 \mathrm{E}+01$ & $1.465 \mathrm{E}+01$ & $2.490 \mathrm{E}+01$ & $3.532 \mathrm{E}+01$ \\
\hline $\mathrm{C} 13$ & $5.116 \mathrm{E}+01$ & $1.847 \mathrm{E}+01$ & $1.644 \mathrm{E}+01$ & $1.835 \mathrm{E}+01$ & $7.841 \mathrm{E}+00$ \\
\hline C14 & $3.653 \mathrm{E}+01$ & $2.135 \mathrm{E}+01$ & $2.015 E+01$ & $2.387 \mathrm{E}+01$ & $9.843 \mathrm{E}+00$ \\
\hline C15 & $2.125 E+01$ & $2.197 \mathrm{E}+01$ & $2.051 \mathrm{E}+01$ & $2.821 \mathrm{E}+01$ & $1.233 \mathrm{E}+01$ \\
\hline C16 & $1.315 \mathrm{E}+01$ & $2.012 \mathrm{E}+01$ & $1.839 \mathrm{E}+01$ & $2.866 \mathrm{E}+01$ & $1.578 \mathrm{E}+01$ \\
\hline C17 & $8.713 \mathrm{E}+00$ & $1.697 \mathrm{E}+01$ & $1.596 \mathrm{E}+01$ & $2.496 \mathrm{E}+01$ & $2.028 \mathrm{E}+01$ \\
\hline C18 & $6.032 \mathrm{E}+00$ & $1.356 \mathrm{E}+01$ & $1.364 \mathrm{E}+01$ & $1.961 \mathrm{E}+01$ & $2.192 \mathrm{E}+01$ \\
\hline C19 & $1.242 \mathrm{E}+01$ & $1.461 \mathrm{E}+01$ & $1.407 \mathrm{E}+01$ & $1.977 \mathrm{E}+01$ & $8.169 \mathrm{E}+00$ \\
\hline C20 & $1.210 \mathrm{E}+01$ & $1.782 \mathrm{E}+01$ & $1.913 \mathrm{E}+01$ & $6.567 \mathrm{E}+01$ & $9.594 \mathrm{E}+00$ \\
\hline C21 & $1.092 \mathrm{E}+01$ & $2.425 \mathrm{E}+01$ & $2.695 \mathrm{E}+01$ & $6.352 E+02$ & $1.057 \mathrm{E}+01$ \\
\hline $\mathrm{C} 22$ & $9.313 E+00$ & $6.131 \mathrm{E}+01$ & $5.007 \mathrm{E}+01$ & $2.490 \mathrm{E}+02$ & $1.080 \mathrm{E}+01$ \\
\hline C23 & $7.661 \mathrm{E}+00$ & $3.645 \mathrm{E}+02$ & $1.065 \mathrm{E}+02$ & $9.508 \mathrm{E}+01$ & $1.030 \mathrm{E}+01$ \\
\hline C24 & $6.186 \mathrm{E}+00$ & $4.778 \mathrm{E}+01$ & $6.081 E+01$ & $3.332 E+01$ & $9.287 \mathrm{E}+00$ \\
\hline 01 & $9.559 \mathrm{E}-01$ & $1.296 \mathrm{E}+00$ & $1.213 \mathrm{E}+00$ & $1.338 \mathrm{E}+00$ & $1.081 \mathrm{E}+00$ \\
\hline R02 & $1.048 \mathrm{E}+00$ & $1.372 \mathrm{E}+00$ & $1.150 E+\infty 0$ & $1.331 \mathrm{E}+00$ & $1.164 \mathrm{E}+00$ \\
\hline
\end{tabular}




\section{REVISED METHODOLOGY FOR DETERMINING CESIUM-137 CONTENT} OF HN-200 GROUT CONTAINERS.

$\begin{array}{llllll}\mathbf{R} 03 & 1.120 \mathrm{E}+00 & 1.427 \mathrm{E}+00 & 1.094 \mathrm{E}+00 & 1.339 \mathrm{E}+00 & 1.165 \mathrm{E}+00 \\ \text { R04 } & 1.150 \mathrm{E}+00 & 1.443 \mathrm{E}+00 & 1.041 \mathrm{E}+00 & 1.355 \mathrm{E}+00 & 1.179 \mathrm{E}+00 \\ \text { R05 } & 1.247 \mathrm{E}+00 & 1.411 \mathrm{E}+00 & 1.006 \mathrm{E}+00 & 1.362 \mathrm{E}+00 & 1.138 \mathrm{E}+00 \\ \mathbf{R} 06 & 1.268 \mathrm{E}+00 & 1.341 \mathrm{E}+00 & 9.821 \mathrm{E}-01 & 1.353 \mathrm{E}+00 & 1.035 \mathrm{E}+00 \\ \mathbf{R} 07 & 1.235 \mathrm{E}+00 & 1.256 \mathrm{E}+00 & 9.690 \mathrm{E}-01 & 1.332 \mathrm{E}+00 & 1.013 \mathrm{E}+00 \\ \mathbf{R} 08 & 1.171 \mathrm{E}+00 & 1.180 \mathrm{E}+00 & 9.681 \mathrm{E}-01 & 1.308 \mathrm{E}+00 & 9.616 \mathrm{E}-01 \\ \text { R09 } & 1.086 \mathrm{E}+00 & 1.125 \mathrm{E}+00 & 9.785 \mathrm{E}-01 & 1.255 \mathrm{E}+00 & 8.839 \mathrm{E}-01 \\ \text { R10 } & 9.774 \mathrm{E}-01 & 1.100 \mathrm{E}+00 & 9.979 \mathrm{E}-01 & 1.376 \mathrm{E}+00 & 7.880 \mathrm{E}-01 \\ \text { R11 } & 8.454 \mathrm{E}-01 & 1.115 \mathrm{E}+00 & 1.055 \mathrm{E}+00 & 1.489 \mathrm{E}+00 & 7.638 \mathrm{E}-01 \\ \text { R12 } & 8.452 \mathrm{E}-01 & 1.141 \mathrm{E}+00 & 1.130 \mathrm{E}+00 & 1.585 \mathrm{E}+00 & 7.709 \mathrm{E}-01 \\ \text { R13 } & 8.318 \mathrm{E}-01 & 1.170 \mathrm{E}+00 & 1.204 \mathrm{E}+00 & 1.632 \mathrm{E}+00 & 7.822 \mathrm{E}-01 \\ \text { R14 } & 8.310 \mathrm{E}-01 & 1.197 \mathrm{E}+00 & 1.265 \mathrm{E}+00 & 1.612 \mathrm{E}+00 & 8.031 \mathrm{E}-01 \\ \text { R15 } & 8.490 \mathrm{E}-01 & 1.221 \mathrm{E}+00 & 1.290 \mathrm{E}+00 & 1.529 \mathrm{E}+00 & 8.162 \mathrm{E}-01 \\ \text { R16 } & 8.860 \mathrm{E}-01 & 1.251 \mathrm{E}+00 & 1.271 \mathrm{E}+00 & 1.374 \mathrm{E}+00 & 9.580 \mathrm{E}-01\end{array}$

TABLE 12 - ESTIMATES OF CURIE CONTENT FOR VARIOUS SOURCE SETS COMPARED TO ACTUAL CURIE CONTENT FOR AN UNGROUTED CONTAINER. MULTIPLE SOURCE SET ESTIMATE AND ERROR - CONTACT MEASUREMENT

\begin{tabular}{|c|c|c|c|c|c|c|}
\hline $\begin{array}{l}\text { SOURCE } \\
\text { SET }\end{array}$ & $\begin{array}{l}\text { AVG } \\
\text { CONT } \\
\text { R/hr }\end{array}$ & $\begin{array}{l}\text { CONT } \\
\text { CONV } \\
\text { FACT } \\
\text { R/hr/Ci }\end{array}$ & $\begin{array}{c}\text { EST } \\
\text { Cs-137 } \\
\text { CURIES }\end{array}$ & $\begin{array}{c}\text { ACTUAL } \\
\text { Cs-137 } \\
\text { CURIES }\end{array}$ & ERROR & $\begin{array}{c}\% \\
\text { ERROR }\end{array}$ \\
\hline S01-S35 & $5.624 \mathrm{E}+02$ & $7.396 \mathrm{E}-01$ & 760.4690 & 708.6318 & 51.8372 & 7.3151 \\
\hline S01-S30 & $4.598 \mathrm{E}+02$ & $7.396 \mathrm{E}-01$ & 621.7013 & 604.0700 & 17.6313 & 2.9187 \\
\hline S06-S35 & $4.700 \mathrm{E}+02$ & $7.396 \mathrm{E}-0 \mathrm{t}$ & 635.4184 & 610.0433 & 25.3751 & 4.1596 \\
\hline S01.525 & $3.827 \mathrm{E}+02$ & $7.396 \mathrm{E}-01$ & 517.3990 & 520.3863 & -2.9872 & -0.5740 \\
\hline S11-S35 & $3.890 \mathrm{E}+02$ & $7.396 \mathrm{E}-01$ & 525.9006 & 490.8729 & 35.0277 & 7.1358 \\
\hline S01-S20 & 3.270E+02 & $7.396 \mathrm{E}-01$ & 442.1841 & 426.0483 & 16.1358 & 3.7873 \\
\hline S16-S25 & $3.137 \mathrm{E}+02$ & $7.396 \mathrm{E}-01$ & 424.1038 & 393.8550 & 30.2488 & 7.6802 \\
\hline S01-S18 & $2.976 \mathrm{E}+02$ & $7.396 \mathrm{E}-01$ & 402.3437 & 394.2079 & 8.1357 & 2.0638 \\
\hline S19-S35 & $2.649 \mathrm{E}+02$ & 7.396E-01 & 358.1253 & 314.4239 & 43.7015 & 13.8989 \\
\hline S01-S12 & $1.950 \mathrm{E}+02$ & $7.396 \mathrm{E}-01$ & 263.7183 & 247.8830 & 15.8354 & 6.3882 \\
\hline S13-S24 & $1.755 \mathrm{E}+02$ & $7.396 \mathrm{E}-01$ & 237.2968 & 252.7408 & -15.4440 & -6.1106 \\
\hline S25-S35 & $1.919 \mathrm{E}+02$ & 7.396E-01 & 259.4539 & 208.0080 & 51.4458 & 24.7326 \\
\hline S01-S10 & $1.735 \mathrm{E}+02$ & $7.396 \mathrm{E}-01$ & 234.5684 & 217.7589 & 16.8095 & 7.7193 \\
\hline S13-S22 & $1.588 \mathrm{E}+02$ & $7.396 \mathrm{E}-01$ & 214.7626 & 227.1843 & -12.4217 & -5.4677 \\
\hline S23-S32 & $1.519 \mathrm{E}+02$ & $7.396 \mathrm{E}-01$ & 205.3553 & 173.0444 & 32.3109 & 18.6720 \\
\hline S01-S07 & $1.234 \mathrm{E}+02$ & 7.396E-01 & 166.7874 & 140.8425 & 25.9449 & 18.4212 \\
\hline S08-S14 & $1.041 E+02$ & $7.396 E-01$ & 140.8031 & 151.1873 & -10.3843 & -6.8685 \\
\hline S15-S21 & $1.179 \mathrm{E}+02$ & $7.396 \mathrm{E}-01$ & 159.4727 & 162.6650 & -3.1923 & -1.9625 \\
\hline S22-S28 & $9.241 \mathrm{E}+01$ & $7.396 \mathrm{E}-01$ & 124.9411 & 116.2697 & 8.6714 & 7.4580 \\
\hline S29-S35 & $1.246 \mathrm{E}+02$ & $7.396 \mathrm{E}-01$ & 168.4647 & 137.6673 & 30.7974 & 22.3709 \\
\hline S01-S05 & $9.249 \mathrm{E}+01$ & $7.396 \mathrm{E}-01$ & 125.0506 & 98.5885 & 26.4621 & 26.8409 \\
\hline S06-S10 & $8.100 \mathrm{E}+01$ & 7.396E-01 & 109.5178 & 119.1704 & -9.6526 & -8.0998 \\
\hline
\end{tabular}




\section{REVISED METHODOLOGY FOR DETERMINING CESIUM-137 CONTENT OF HN-200 GROUT CONTAINERS.}

$\begin{array}{lllllll}\text { S11-S15 } & 7.529 E+01 & 7.396 E-01 & 101.7968 & 97.0179 & 4.7789 & 4.9258 \\ \text { S16-S20 } & 7.826 E+01 & 7.396 E-01 & 105.8189 & 111.2715 & -5.4526 & -4.9002 \\ \text { S21-S25 } & 5.563 E+01 & 7.396 E-01 & 75.2149 & 94.3380 & -19.1230 & -20.2708 \\ \text { S26-S30 } & 7.714 E+01 & 7.396 E-01 & 104.3023 & 83.6838 & 20.6185 & 24.6386 \\ \text { S31-S35 } & 1.026 E+02 & 7.396 E-01 & 138.7677 & 104.5618 & 34.2059 & 32.7136 \\ \text { S01-S03 } & 6.427 E+01 & 7.396 E-01 & 86.9014 & 66.9394 & 19.9620 & 29.8210 \\ \text { S04-S06 } & 3.915 E+01 & 7.396 E-01 & 52.9336 & 47.9673 & 4.9663 & 10.3535 \\ \text { S07-S09 } & 5.211 E+01 & 7.396 E-01 & 70.4577 & 76.0383 & -5.5806 & -7.3392 \\ \text { S10-S12 } & 3.951 E+01 & 7.396 E-01 & 53.4256 & 56.9379 & -3.5123 & -6.1687 \\ \text { S13-S15 } & 5.373 E+01 & 7.396 E-01 & 72.6469 & 66.8938 & 5.7530 & 8.6002 \\ \text { S16-S18 } & 4.880 E+01 & 7.396 E-01 & 65.9785 & 79.4311 & -13.4527 & -16.9363 \\ \text { S19-S21 } & 4.787 E+01 & 7.396 E-01 & 64.7196 & 60.4869 & 4.2326 & 6.9976 \\ \text { S22-S24 } & 2.511 E+01 & 7.396 E-01 & 33.9519 & 45.9289 & -11.9770 & -26.0773 \\ \text { S25-S27 } & 5.535 E+01 & 7.396 E-01 & 74.8399 & \mathbf{5 4 . 8 2 7 5} & 20.0125 & 36.5009 \\ \text { S28-S30 } & 3.391 E+01 & 7.396 E-01 & 45.8462 & 48.6188 & -2.7725 & -\mathbf{5 . 7 0 2 6} \\ \text { S31-S33 } & \mathbf{8 . 0 4 0 E + 0 1} & 7.396 E-01 & 108.7065 & \mathbf{6 1 . 8 6 9 7} & 46.8368 & 75.7023 \\ \text { S34-S35 } & 2.223 E+01 & 7.396 E-01 & 30.0612 & 42.6921 & -12.6309 & -29.5860 \\ & & & & \text { Average } \% \text { Error }= & 6.8142\end{array}$

MULTIPLE SOURCE SET ESTIMATE AND ERROR - REMOTE MEASUREMENT REMOTE

\begin{tabular}{|c|c|c|c|c|c|c|}
\hline $\begin{array}{c}\text { SOURCE } \\
\text { SET }\end{array}$ & $\begin{array}{c}\text { AVG } \\
\text { REMOTE } \\
\text { R/hr }\end{array}$ & $\begin{array}{l}\text { CONV } \\
\text { FACT } \\
\text { R/hr/Ci }\end{array}$ & $\begin{array}{c}\text { EST } \\
\text { Cs-137 } \\
\text { CURIES }\end{array}$ & $\begin{array}{c}\text { ACTUAL } \\
\text { Cs-137 } \\
\text { CURIES }\end{array}$ & ERROR & $\begin{array}{c}\% \\
\text { ERROR }\end{array}$ \\
\hline S01-S35 & $1.607 \mathrm{E}+01$ & 2.264E-02 & 709.9558 & 708.6318 & 1.3240 & 0.1868 \\
\hline S01-S30 & $1.371 \mathrm{E}+01$ & $2.264 \mathrm{E}-02$ & 605.4053 & 604.0700 & 1.3352 & 0.2210 \\
\hline S06-S35 & $1.382 E+01$ & $2.264 \mathrm{E}-02$ & 610.6073 & 610.0433 & 0.5640 & 0.0925 \\
\hline S01-S25 & $1.180 \mathrm{E}+01$ & $2.264 \mathrm{E}-02$ & 521.3715 & 520.3863 & 0.9852 & 0.1893 \\
\hline S11-S35 & $1.112 \mathrm{E}+01$ & $2.264 \mathrm{E}-02$ & 491.3635 & 490.8729 & 0.4905 & 0.0999 \\
\hline S01-S20 & $9.674 \mathrm{E}+00$ & $2.264 \mathrm{E}-02$ & 427.2965 & 426.0483 & 1.2483 & 0.2930 \\
\hline S16-S25 & $8.917 \mathrm{E}+00$ & $2.264 \mathrm{E}-02$ & 393.8557 & 393.8550 & 0.0007 & 0.0002 \\
\hline S01-S18 & $8.948 \mathrm{E}+00$ & $2.264 \mathrm{E}-02$ & 395.2253 & 394.2079 & 1.0173 & 0.2581 \\
\hline S19-S35 & $7.126 \mathrm{E}+00$ & $2.264 \mathrm{E}-02$ & 314.7306 & 314.4239 & 0.3067 & 0.0975 \\
\hline S01-S12 & $5.642 \mathrm{E}+00$ & $2.264 \mathrm{E}-02$ & 249.2176 & 247.8830 & 1.3347 & 0.5384 \\
\hline S13-S24 & $5.710 \mathrm{E}+00$ & $2.264 \mathrm{E}-02$ & 252.2295 & 252.7408 & -0.5114 & -0.2023 \\
\hline S25-S35 & $4.721 \mathrm{E}+00$ & $2.264 \mathrm{E}-02$ & 208.5087 & 208.0080 & 0.5007 & 0.2407 \\
\hline S01-S10 & $4.949 E+00$ & $2.264 \mathrm{E}-02$ & 218.5924 & 217.7589 & 0.8335 & 0.3827 \\
\hline S13-S22 & $5.134 \mathrm{E}+00$ & $2.264 \mathrm{E}-02$ & 226.7596 & 227.1843 & -0.4247 & -0.1869 \\
\hline S23-S32 & $3.943 E+00$ & $2.264 \mathrm{E}-02$ & 174.1685 & 173.0444 & 1.1242 & 0.6496 \\
\hline S01-S07 & $3.202 \mathrm{E}+00$ & $2.264 \mathrm{E}-02$ & 141.4250 & 140.8425 & 0.5825 & 0.4136 \\
\hline S08-S14 & $3.446 \mathrm{E}+00$ & $2.264 \mathrm{E}-02$ & 152.1983 & 151.1873 & 1.0109 & 0.6687 \\
\hline S15-S21 & $3.683 \mathrm{E}+00$ & $2.264 \mathrm{E}-02$ & 162.6982 & 162.6650 & 0.0332 & 0.0204 \\
\hline S22-S28 & $2.623 \mathrm{E}+00$ & $2.264 \mathrm{E}-02$ & 115.8784 & 116.2697 & -0.3913 & -0.3365 \\
\hline S29-S35 & $3.119 \mathrm{E}+00$ & $2.264 \mathrm{E}-02$ & 137.7559 & 137.6673 & 0.0886 & 0.0644 \\
\hline S01-S05 & $2.249 \mathrm{E}+00$ & $2.264 \mathrm{E}-02$ & 99.3485 & 98.5885 & 0.7600 & 0.7709 \\
\hline S06-S10 & $2.700 \mathrm{E}+00$ & $2.264 \mathrm{E}-02$ & 119.2439 & 119.1704 & 0.0735 & 0.0617 \\
\hline S11-S15 & $2.208 \mathrm{E}+00$ & $2.264 \mathrm{E}-02$ & 97.5077 & 97.0179 & 0.4898 & 0.5049 \\
\hline
\end{tabular}




\section{REVISED METHODOLOGY FOR DETERMINING CESIUM-137 CONTENT OF HN-200 GROUT CONTAINERS.}

$\begin{array}{lllllll}\text { S16-S20 } & 2.517 E+00 & 2.264 E-02 & 111.1964 & 111.2715 & -0.0750 & -0.0674 \\ \text { S21-S25 } & 2.130 \mathrm{E}+00 & 2.264 \mathrm{E}-02 & 94.0749 & 94.3380 & -0.2630 & -0.2788 \\ \text { S26-S30 } & 1.903 \mathrm{E}+00 & 2.264 \mathrm{E}-02 & 84.0338 & 83.6838 & 0.3500 & 0.4183 \\ \text { S31-S35 } & 2.367 \mathrm{E}+00 & 2.264 \mathrm{E}-02 & 104.5506 & 104.5618 & -0.0112 & -0.0107 \\ \text { S01-S03 } & 1.523 \mathrm{E}+00 & 2.264 \mathrm{E}-02 & 67.2825 & 66.9394 & 0.3431 & 0.5126 \\ \text { S04-S06 } & 1.102 \mathrm{E}+00 & 2.264 \mathrm{E}-02 & 48.6537 & 47.9673 & 0.6863 & 1.4308 \\ \text { S07-S09 } & 1.719 \mathrm{E}+00 & 2.264 \mathrm{E}-02 & 75.9157 & 76.0383 & -0.1226 & -0.1613 \\ \text { S10-S12 } & 1.299 \mathrm{E}+00 & 2.264 \mathrm{E}-02 & 57.3658 & 56.9379 & 0.4279 & 0.7515 \\ \text { S13-S15 } & 1.514 \mathrm{E}+00 & 2.264 \mathrm{E}-02 & 66.8825 & 66.8938 & -0.0114 & -0.0170 \\ \text { S16-S18 } & 1.791 \mathrm{E}+00 & 2.264 \mathrm{E}-02 & 79.1252 & 79.4311 & -0.3060 & -0.3852 \\ \text { S19-S21 } & 1.383 \mathrm{E}+00 & 2.264 \mathrm{E}-02 & 61.0962 & 60.4869 & 0.6093 & 1.0073 \\ \text { S22-S24 } & 1.022 \mathrm{E}+00 & 2.264 \mathrm{E}-02 & 45.1256 & 45.9289 & -0.8033 & -1.7490 \\ \text { S25-S27 } & 1.253 \mathrm{E}+00 & 2.264 \mathrm{E}-02 & 55.3393 & 54.8275 & 0.5118 & 0.9335 \\ \text { S28-S30 } & 1.101 \mathrm{E}+00 & 2.264 \mathrm{E}-02 & 48.6189 & 48.6188 & 0.0001 & 0.0002 \\ \text { S31-S33 } & 1.411 \mathrm{E}+00 & 2.264 \mathrm{E}-02 & \mathbf{6 2 . 3 0 6 5} & 61.8697 & 0.4368 & 0.7060 \\ \text { S34-S35 } & 9.564 \mathrm{E}-01 & 2.264 \mathrm{E}-02 & 42.2441 & 42.6921 & -0.4480 & -1.0495 \\ & & & & & \end{array}$

Average \% Error $=0.1813$

INDIVIDUAL SOURCE ESTIMATE AND ERROR - CONTACT MEASUREMENT

\begin{tabular}{|c|c|c|c|c|c|c|}
\hline DURCE & $\begin{array}{c}\text { AVG } \\
\text { CONT } \\
\text { R/hr }\end{array}$ & $\begin{array}{l}\text { CONT } \\
\text { CONV } \\
\mathrm{FACT} \\
\mathrm{R} / \mathrm{hr} / \mathrm{Ci}\end{array}$ & $\begin{array}{c}\text { EST } \\
\text { Cs-137 } \\
\text { CURIES }\end{array}$ & $\begin{array}{c}\text { ACTUAL } \\
\text { Cs-137 } \\
\text { CURIES }\end{array}$ & ERROR & $\begin{array}{c}\% \\
\text { ERROR }\end{array}$ \\
\hline S01 & $4.256 \mathrm{E}+01$ & $7.396 \mathrm{E}-01$ & 57.5491 & 29.4975 & 28.0516 & 95.0982 \\
\hline S02 & $7.929 \mathrm{E}+00$ & $7.396 \mathrm{E}-01$ & 10.7202 & 13.8502 & -3.1300 & -22.5990 \\
\hline S03 & $1.378 E+01$ & $7.396 \mathrm{E}-01$ & 18.6320 & 23.5916 & -4.9596 & -21.0227 \\
\hline S04 & $2.021 \mathrm{E}+01$ & $7.396 \mathrm{E}-01$ & 27.3229 & 20.6982 & 6.6247 & 32.0062 \\
\hline S05 & $8.007 \mathrm{E}+00$ & $7.396 \mathrm{E}-01$ & 10.8263 & 10.9510 & -0.1246 & -1.1381 \\
\hline S06 & $1.093 \mathrm{E}+01$ & $7.396 \mathrm{E}-01$ & 14.7844 & 16.3182 & -1.5338 & -9.3993 \\
\hline S07 & $1.993 \mathrm{E}+01$ & $7.396 \mathrm{E}-01$ & 26.9524 & 25.9358 & 1.0166 & 3.9196 \\
\hline S08 & $1.687 \mathrm{E}+01$ & $7.396 \mathrm{E}-01$ & 22.8160 & 25.1678 & -2.3518 & -9.3445 \\
\hline s09 & $1.530 \mathrm{E}+01$ & $7.396 \mathrm{E}-01$ & 20.6893 & 24.9347 & -4.2453 & -17.0258 \\
\hline S10 & $1.795 \mathrm{E}+01$ & $7.396 \mathrm{E}-01$ & 24.2757 & 26.8139 & -2.5382 & -9.4661 \\
\hline S11 & $9.033 \mathrm{E}+00$ & $7.396 \mathrm{E}-01$ & 12.2127 & 12.5933 & -0.3807 & -3.0227 \\
\hline S12 & $1.253 \mathrm{E}+01$ & $7.396 \mathrm{E}-01$ & 16.9373 & 17.5307 & -0.5935 & -3.3853 \\
\hline S13 & $1.073 \mathrm{E}+01$ & $7.396 \mathrm{E}-01$ & 14.5112 & 17.9331 & -3.4219 & -19.0813 \\
\hline S14 & $2.172 \mathrm{E}+0 \mathrm{I}$ & $7.396 \mathrm{E}-01$ & 29.3609 & 26.2138 & 3.1471 & 12.0055 \\
\hline S15 & $2.128 \mathrm{E}+01$ & $7.396 \mathrm{E}-01$ & 28.7747 & 22.7469 & 6.0278 & 26.4994 \\
\hline S16 & $1.696 \mathrm{E}+01$ & $7.396 \mathrm{E}-01$ & 22.9273 & 27.6556 & -4.7283 & -17.0970 \\
\hline S17 & $2.032 E+01$ & $7.396 \mathrm{E}-01$ & 27.4687 & 28.5227 & -1.0540 & -3.6953 \\
\hline S18 & $1.152 \mathrm{E}+01$ & $7.396 \mathrm{E}-01$ & 15.5824 & 23.2528 & -7.6704 & -32.9870 \\
\hline S19 & $1.988 \mathrm{E}+01$ & $7.396 \mathrm{E}-01$ & 26.8814 & 15.3416 & $1 \mathrm{I} .5398$ & 75.2195 \\
\hline $\mathbf{s 2 0}$ & $9.585 E+00$ & $7.396 \mathrm{E}-01$ & 12.9590 & 16.4988 & -3.5397 & -21.4546 \\
\hline S21 & $1.840 \mathrm{E}+01$ & $7.396 \mathrm{E}-01$ & 24.8791 & 28.6466 & -3.7675 & -13.1516 \\
\hline $\mathbf{S 2 2}$ & $8.445 E+00$ & $7.396 \mathrm{E}-01$ & 11.4177 & 20.3724 & -8.9547 & -43.9550 \\
\hline S23 & $7.055 \mathrm{E}+00$ & $7.396 \mathrm{E}-01$ & 9.5389 & 13.0146 & -3.4757 & -26.7060 \\
\hline S24 & $9.611 E+00$ & $7.396 \mathrm{E}-01$ & 12.9953 & 12.5419 & 0.4534 & 3.6149 \\
\hline S25 & $1.212 \mathrm{E}+01$ & $7.396 \mathrm{E}-01$ & 16.3839 & 19.7625 & -3.3785 & -17.0958 \\
\hline S26 & $2.195 \mathrm{E}+01$ & $7.396 \mathrm{E}-01$ & 29.6715 & 24.8512 & 4.8203 & 19.3965 \\
\hline
\end{tabular}




\section{REVISED METHODOLOGY FOR DETERMINING CESIUM-137 CONTENT OF HN-200 GROUT CONTAINERS.}

$\begin{array}{lcccccc}\text { S27 } & 2.129 \mathrm{E}+01 & 7.396 \mathrm{E}-01 & 28.7845 & 10.2138 & 18.5708 & 181.8213 \\ \mathbf{S 2 8} & 1.194 \mathrm{E}+01 & 7.396 \mathrm{E}-01 & 16.1492 & 15.5133 & 0.6359 & 4.0991 \\ \mathbf{S 2 9} & 1.058 \mathrm{E}+01 & 7.396 \mathrm{E}-01 & 14.3100 & 18.0653 & -3.7554 & -20.7876 \\ \mathbf{S 3 0} & 1.138 \mathrm{E}+01 & 7.396 \mathrm{E}-01 & 15.3870 & 15.0401 & 0.3469 & 2.3065 \\ \mathbf{S 3 1} & 2.024 \mathrm{E}+01 & 7.396 \mathrm{E}-01 & 27.3595 & 22.3550 & 5.0045 & 22.3866 \\ \mathbf{S 3 2} & 2.572 \mathrm{E}+01 & 7.396 \mathrm{E}-01 & 34.7754 & 21.6866 & 13.0887 & 60.3540 \\ \mathbf{S 3 3} & 3.444 \mathrm{E}+01 & 7.396 \mathrm{E}-01 & 46.5716 & 17.8281 & 28.7435 & 161.2262 \\ \mathbf{S 3 4} & 6.251 \mathrm{E}+00 & 7.396 \mathrm{E}-01 & 8.4524 & 14.6776 & -6.2252 & -42.4130 \\ \mathbf{S 3 5} & 1.598 \mathrm{E}+01 & 7.396 \mathrm{E}-01 & 21.6089 & 28.0146 & -6.4057 & -22.8656\end{array}$

Average \% Error $=9.2074$

INDIVIDUAL SOURCE ESTIMATE AND ERROR - REMOTE MEASUREMENT REMOTE

$\begin{array}{ccccccc} & \text { AVG } & \text { CONV } & \text { EST } & \text { ACTUAL } & & \\ \text { SOURCE } & \text { REMOTE } & \text { FACT } & \text { Cs-137 } & \text { Cs-137 } & & \text { \% } \\ \text { S01 } & \text { R.780E-01 } & 2.264 \text { E-02 } & 29.9470 & 29.4975 & 0.4495 & 1.5238 \\ \text { S02 } & 3.092 E-01 & 2.264 E-02 & 13.6592 & 13.8502 & -0.1910 & -1.3793 \\ \text { S03 } & 5.360 E-01 & 2.264 E-02 & 23.6763 & 23.5916 & 0.0847 & 0.3589 \\ \text { S04 } & 4.750 E-01 & 2.264 E-02 & 20.9797 & 20.6982 & 0.2816 & 1.3603 \\ \text { S05 } & 2.510 E-01 & 2.264 E-02 & 11.0863 & 10.9510 & 0.1353 & 1.2356 \\ \text { S06 } & 3.755 E-01 & 2.264 E-02 & 16.5876 & 16.3182 & 0.2694 & 1.6511 \\ \text { S07 } & 5.771 E-01 & 2.264 E-02 & 25.4889 & 25.9358 & -0.4469 & -1.7232 \\ \text { S08 } & 5.779 E-01 & 2.264 E-02 & 25.5259 & 25.1678 & 0.3581 & 1.4228 \\ \text { S09 } & 5.638 E-01 & 2.264 E-02 & 24.9009 & 24.9347 & -0.0338 & -0.1354 \\ \text { S10 } & 6.054 E-01 & 2.264 E-02 & 26.7406 & 26.8139 & -0.0733 & -0.2735 \\ \text { S11 } & 2.900 E-01 & 2.264 E-02 & 12.8108 & 12.5933 & 0.2175 & 1.7271 \\ \text { S12 } & 4.033 E-01 & 2.264 E-02 & 17.8144 & 17.5307 & 0.2837 & 1.6184 \\ \text { S13 } & 4.082 E-01 & 2.264 E-02 & 18.0317 & 17.9331 & 0.0986 & 0.5498 \\ \text { S14 } & 5.971 E-01 & 2.264 E-02 & 26.3740 & 26.2138 & 0.1601 & 0.6109 \\ \text { S15 } & 5.089 E-01 & 2.264 E-02 & 22.4768 & 22.7469 & -0.2701 & -1.1875 \\ \text { S16 } & 6.325 E-01 & 2.264 E-02 & 27.9367 & 27.6556 & 0.2811 & 1.0165 \\ \text { S17 } & 6.461 E-01 & 2.264 E-02 & 28.5396 & 28.5227 & 0.0169 & 0.0593 \\ \text { S18 } & 5.128 E-01 & 2.264 E-02 & 22.6488 & 23.2528 & -0.6040 & -2.5977 \\ \text { S19 } & 3.526 E-01 & 2.264 E-02 & 15.5723 & 15.3416 & 0.2307 & 1.5037 \\ \text { S20 } & 3.735 E-01 & 2.264 E-02 & 16.4990 & 16.4988 & 0.0002 & 0.0014 \\ \text { S21 } & 6.571 E-01 & 2.264 E-02 & 29.0250 & 28.6466 & 0.3784 & 1.3208 \\ \text { S22 } & 4.450 E-01 & 2.264 E-02 & 19.6558 & 20.3724 & -0.7167 & -3.5178 \\ \text { S23 } & 2.902 E-01 & 2.264 E-02 & 12.8172 & 13.0146 & -0.1974 & -1.5170 \\ \text { S24 } & 2.865 E-01 & 2.264 E-02 & 12.6527 & 12.5419 & 0.1108 & 0.8833 \\ \text { S25 } & 4.511 E-01 & 2.264 E-02 & 19.9244 & 19.7625 & 0.1619 & 0.8192 \\ \text { S26 } & 5.709 E-01 & 2.264 E-02 & 25.2145 & 24.8512 & 0.3633 & 1.4617 \\ \text { S27 } & 2.309 E-01 & 2.264 E-02 & 10.2004 & 10.2138 & -0.0133 & -0.1305 \\ & & & & & & \end{array}$




\begin{tabular}{|c|c|c|c|c|c|c|}
\hline S28 & $3.490 \mathrm{E}-01$ & $2.264 \mathrm{E}-02$ & 15.4135 & 15.5133 & -0.0998 & -0.6431 \\
\hline $\mathbf{S} 29$ & $4.121 \mathrm{E}-01$ & $2.264 \mathrm{E}-02$ & 18.2006 & 18.0653 & 0.1353 & 0.7489 \\
\hline S30 & $3.397 \mathrm{E}-01$ & $2.264 \mathrm{E}-02$ & 15.0047 & 15.0401 & -0.0354 & -0.2356 \\
\hline S31 & $5.136 \mathrm{E}-01$ & $2.264 \mathrm{E}-02$ & 22.6850 & 22.3550 & 0.3300 & 1.4761 \\
\hline S32 & $4.993 \mathrm{E}-0 \mathrm{I}$ & $2.264 \mathrm{E}-02$ & 22.0555 & 21.6866 & 0.3689 & 1.7011 \\
\hline S33 & $3.977 \mathrm{E}-01$ & $2.264 \mathrm{E}-02$ & 17.5660 & 17.8281 & -0.2621 & -1.4701 \\
\hline S34 & $3.224 \mathrm{E}-01$ & $2.264 \mathrm{E}-02$ & 14.2414 & 14.6776 & -0.4362 & -2.9718 \\
\hline S35 & $6.340 \mathrm{E}-01$ & $2.264 \mathrm{E}-02$ & 28.0027 & 28.0146 & -0.0118 & -0.0423 \\
\hline
\end{tabular}




\section{REVISED METHODOLOGY FOR DETERMINING CESIUM-137 CONTENT OF HN-200 GROUT CONTAINERS.}

\section{THIS ATTACHMENT CALCULATES MICROSHIELD INPUT DATA SHEETS FOR READINGS \\ TAKEN ON HN-200 GROUT CONTAINERS WITH THE MEASUREMENT PROBE 5 FEET FROM THE SURFACE OF THE CONTAINER.}

TABLE 1

Coordinates of Remote Measuring Points.

$\begin{array}{cccc}\text { Point } & \mathbf{X} & \mathbf{Z} & \mathbf{Y} \\ \text { R01 } & 82.9375 & 0.0000 & 26.7500 \\ \text { R02 } & 76.6243 & 31.7388 & 26.7500 \\ \text { R03 } & 58.6457 & 58.6457 & 26.7500 \\ \text { R04 } & 31.7388 & 76.6243 & 26.7500 \\ \text { R05 } & 0.0000 & 82.9375 & 26.7500 \\ \text { R06 } & -31.7388 & 76.6243 & 26.7500 \\ \text { R07 } & -58.6457 & 58.6457 & 26.7500 \\ \text { R08 } & -76.6243 & 31.7388 & 26.7500 \\ \text { R09 } & -82.9375 & 0.0000 & 26.7500 \\ \text { R10 } & -76.6243 & -31.7388 & 26.7500 \\ \text { R11 } & -58.6457 & -58.6457 & 26.7500 \\ \text { R12 } & -31.7388 & -76.6243 & 26.7500 \\ \text { R13 } & 0.0000 & -82.9375 & 26.7500 \\ \text { R14 } & 31.7388 & -76.6243 & 26.7500 \\ \text { R15 } & \mathbf{5 8 . 6 4 5 7} & -\mathbf{5 8 . 6 4 5 7} & 26.7500 \\ \text { R16 } & 76.6243 & -31.7388 & 26.7500\end{array}$

Note 1 - Remote Measuring Points (R) are 60 inches (5 feet) from the surface of the container. 


\section{REVISED METHODOLOGY FOR DETERMINING CESIUM-137 CONTENT OF HN-200 GROUT CONTAINERS.}

TABLE 2

\begin{tabular}{|c|c|c|c|c|c|}
\hline \multicolumn{6}{|c|}{ Coordinates and source strengths for random sources. } \\
\hline Source & $\mathbf{X}$ & $\mathbf{Z}$ & $\mathbf{Y}$ & Validity & Strength \\
\hline S01 & 19.3677 & 0.5818 & 25.9034 & Valid & 29.4975 \\
\hline $\mathrm{SO2}$ & -10.6176 & -19.5689 & 18.8660 & Valid & 13.8502 \\
\hline $\mathrm{SO3}$ & 1.7704 & -7.2590 & 41.8874 & Valid & 23.5916 \\
\hline SO4 & 5.5262 & -19.2179 & 28.6756 & Valid & 20.6982 \\
\hline S05 & -12.5171 & 2.4304 & 36.3581 & Valid & 10.9510 \\
\hline SO6 & 10.1481 & -6.8169 & 29.6428 & Valid & 16.3182 \\
\hline S07 & -3.0040 & -18.4824 & 1.5181 & Valid & 25.9358 \\
\hline S08 & -1.4468 & -9.3997 & 20.8621 & Valid & 25.1678 \\
\hline S09 & -11.3240 & -1.2877 & 45.4810 & Valid & 24.9347 \\
\hline$\$ 10$ & -8.1594 & 10.5475 & 46.4380 & Valid & 26.8139 \\
\hline S11 & 13.0540 & 6.4320 & 29.1917 & Valid & 12.5933 \\
\hline S12 & 17.0889 & 8.7507 & 21.2880 & Valid & 17.5307 \\
\hline$S 13$ & 3.5123 & 8.3040 & 41.4473 & Valid & 17.9331 \\
\hline S14 & -18.2417 & 6.1323 & 39.9213 & Valid & 26.2138 \\
\hline S15 & 21.0026 & 5.4076 & 10.7056 & Valid & 22.7469 \\
\hline S16 & 12.7799 & 10.3319 & 39.3081 & Valid & 27.6556 \\
\hline $\mathbf{S 1 7}$ & -14.4903 & 3.0983 & 8.6941 & Valid & 28.5227 \\
\hline S18 & 18.7646 & -12.0033 & 10.5209 & Valid & 23.2528 \\
\hline S19 & -18.2584 & 0.2785 & 21.1001 & Valid & 15.3416 \\
\hline S20 & 2.8644 & 9.2261 & 44.4497 & Valid & 16.4988 \\
\hline S21 & 3.0621 & -7.9137 & 19.8848 & Valid & 28.6466 \\
\hline $\mathbf{S} 22$ & -17.9782 & 12.8570 & 50.0431 & Valid & 20.3724 \\
\hline S23 & 5.0992 & 14.6169 & 52.0038 & Valid & 13.0146 \\
\hline S24 & 7.9617 & 19.2122 & 33.0332 & Valid & 12.5419 \\
\hline $\mathbf{S} 25$ & 8.3975 & 9.5559 & 39.8544 & Valid & 19.7625 \\
\hline S26 & 4.1090 & 16.2164 & 34.1935 & Valid & 24.8512 \\
\hline S27 & 2.2698 & -21.1016 & 13.9713 & Valid & 10.2138 \\
\hline S28 & 18.4153 & -5.6775 & 6.4850 & Valid & 15.5133 \\
\hline S29 & -0.0904 & 3.6427 & 38.3454 & Valid & 18.0653 \\
\hline S30 & 7.3731 & -19.7854 & 12.4003 & Valid & 15.0401 \\
\hline S31 & 5.5574 & -17.7939 & 21.2754 & Valid & 22.3550 \\
\hline S32 & -0.9171 & 17.9652 & 26.2433 & Valid & 21.6866 \\
\hline S33 & -2.7939 & -22.1819 & 29.8247 & Valid & 17.8281 \\
\hline S34 & -14.1407 & 16.9694 & 5.4489 & Valid & 14.6776 \\
\hline S35 & 17.6331 & 12.8346 & 30.1155 & Valid & 28.0146 \\
\hline
\end{tabular}

TABLE 3 - Calculate Microshield input data sheets for the simulations from each source to each measuring point in inches. 
REVISED METHODOLOGY FOR DETERMINING CESIUM-137 CONTENT OF HN-200 GROUT CONTAINERS.

$\begin{array}{lcccccr} & \text { Cs-137 } & \text { Ba-137m } & \text { Source 01 } & \text { X Posit } & \text { Z Posit } & \text { Y Posit } \\ \text { Line } & \text { Shield 1 } & \text { Shield 2 } & \text { Total Dist } & \text { Air Gap } & & \\ \text { S01R01 } & 3.4254 & 0.1383 & 63.5781 & 60.0144 & & \\ \text { S01R02 } & 3.7651 & 0.1496 & 65.1904 & 61.2757 & & \\ \text { S01R03 } & 5.1838 & 0.1903 & 70.1063 & 64.7323 & & \\ \text { S01R04 } & 8.8835 & 0.2490 & 77.0469 & 67.9143 & \\ \text { S01R05 } & 16.4904 & 0.2480 & 84.6066 & 67.8683 & \\ \text { S01R06 } & 26.1607 & 0.1983 & 91.6245 & 65.2655 & \\ \text { S01R07 } & 34.5576 & 0.1625 & 97.2534 & 62.5333 & \\ \text { S01R08 } & 40.0868 & 0.1442 & 100.9254 & 60.6944 & \\ \text { S01R09 } & 42.1666 & 0.1382 & 102.3104 & 60.0056 & \\ \text { S01R10 } & 40.6358 & 0.1426 & 101.2907 & 60.5123 & & \\ \text { S01R11 } & 35.6106 & 0.1587 & 97.9526 & 62.1832 & & \\ \text { S01R12 } & 27.5858 & 0.1915 & 92.5925 & 64.8152 & & \\ \text { S01R13 } & 17.9345 & 0.2410 & 85.7397 & 67.5642 & \\ \text { S01R14 } & 9.7811 & 0.2549 & 78.1956 & 68.1595 & & \\ \text { S01R15 } & 5.5544 & 0.1991 & 71.0730 & 65.3196 & & \\ \text { S01R16 } & 3.8961 & 0.1538 & 65.7545 & 61.7046 & & \\ & & & & & \end{array}$

$\begin{array}{ccccccc} & \text { Cs-137 } & \text { Ba-137m } & \text { Source 02 } & \text { X Posit } & \text { Z Posit } & \text { Y Posit } \\ \text { Line } & 13.8502 & 13.1023 & & -10.6176 & -19.5689 & 18.8660 \\ \text { S02R01 } & 29.7139 & 0.2070 & 95.9044 & 65.9835 & & \\ \text { S02R02 } & 38.8848 & 0.1603 & 101.5175 & 62.4723 & & \\ \text { S02R03 } & 44.0530 & 0.1421 & 104.7716 & 60.5766 & & \\ \text { S02R04 } & 45.0402 & 0.1390 & 105.4010 & 60.2217 & \\ \text { S02R05 } & 41.8180 & 0.1494 & 103.3560 & 61.3886 & \\ \text { S02R06 } & 34.4851 & 0.1799 & 98.7998 & 64.1349 & \\ \text { S02R07 } & 23.3624 & 0.2582 & 92.1216 & 68.5010 & \\ \text { S02R08 } & 9.7760 & 0.4995 & 83.9734 & 73.6978 & \\ \text { S02R09 } & 1.9814 & 0.4339 & 75.3344 & 72.9191 & \\ \text { S02R10 } & 0.8431 & 0.2131 & 67.5807 & 66.5244 & \\ \text { S02R11 } & 0.5827 & 0.1500 & 62.4167 & 61.6841 & & \\ \text { S02R12 } & 0.5454 & 0.1407 & 61.3480 & 60.6619 & & \\ \text { S02R13 } & 0.6798 & 0.1739 & 64.7338 & 63.8801 & \\ \text { S02R14 } & 1.2155 & 0.2961 & 71.4950 & 69.9835 & & \\ \text { S02R15 } & 4.5803 & 0.6074 & 79.9159 & 74.7282 & & \\ \text { S02R16 } & 17.1086 & 0.3377 & 88.4388 & 70.9925 & & \\ & & & & & \end{array}$

Cs-137 Ba-137m Source 03 X Posit Z Posit Y Posit 


\section{REVISED METHODOLOGY FOR DETERMINING CESIUM-137 CONTENT} OF HN-200 GROUT CONTAINERS.

$\begin{array}{ccccccc}\text { Line } & 23.5916 & 22.3177 & & 1.7704 & -7.2590 & 41.8874 \\ \text { SO3R01 } & \text { Shield 1 } & \text { Shield 2 } & \text { Total Dist } & \text { Air Gap } & & \\ \text { S03R02 } & 23.8022 & 0.1485 & 82.8850 & 61.9343 & \\ \text { S03R03 } & 26.7065 & 0.1481 & 85.7500 & 61.8309 & \\ \text { S03R04 } & 29.0771 & 0.1455 & 88.3593 & 61.5073 & \\ \text { S03R05 } & 30.4533 & 0.1405 & 90.3529 & 61.1333 & \\ \text { S03R06 } & 30.5937 & 0.1403 & 91.5751 & 60.8812 & \\ \text { S03R07 } & 29.4741 & 0.1419 & 90.6790 & 60.8543 & \\ \text { S03R08 } & 27.2896 & 0.1448 & 88.8578 & 61.0630 & \\ \text { S03R09 } & 24.4338 & 0.1476 & 86.3555 & 61.7741 & \\ \text { S03R10 } & 21.4266 & 0.1487 & 83.5113 & 61.9360 & \\ \text { S03R11 } & 18.7783 & 0.1471 & 80.7455 & 61.8201 & \\ \text { S03R12 } & 16.8486 & 0.1440 & 78.5083 & 61.5157 & \\ \text { S03R13 } & 15.8079 & 0.1414 & 77.1978 & 61.2485 & \\ \text { S03R14 } & 15.7049 & 0.1412 & 77.0635 & 61.2175 & \\ \text { S03R15 } & 16.5426 & 0.1433 & 78.1315 & 61.4456 & \\ \text { S03R16 } & 18.2870 & 0.1465 & 80.1966 & 61.7631 & \end{array}$

$\begin{array}{ccccccc} & \text { Cs-137 } & \text { Ba-137m } & \text { Source 04 } & \text { X Posit } & \text { Z Posit } & \text { Y Posit } \\ \text { Line } & \text { Shieg82 } & 19.5805 & & 5.5262 & -19.2179 & 28.6756 \\ \text { S04R01 } & 10.2463 & \text { Shield 2 } & \text { Total Dist } & \text { Air Gap } & & \\ \text { S04R02 } & 19.5488 & 0.2843 & 79.7844 & 69.2538 & & \\ \text { S04R03 } & 29.5836 & 0.1869 & 94.4942 & 67.7015 & & \\ \text { S04R04 } & 37.3753 & 0.1552 & 99.3808 & 61.8503 & & \\ \text { S04R05 } & 41.8506 & 0.1410 & 102.3229 & 60.3314 & \\ \text { S04R06 } & 42.6488 & 0.1387 & 102.8500 & 60.0626 & \\ \text { S04R07 } & 39.7175 & 0.1474 & 100.9182 & 61.0532 & \\ \text { S04R08 } & 33.2652 & 0.1707 & 96.6902 & 63.2543 & \\ \text { S04R09 } & 23.9702 & 0.2166 & 90.5475 & 66.3607 & \\ \text { S04R10 } & 13.8198 & 0.2776 & 83.1215 & 69.0240 & \\ \text { S04R11 } & 6.8398 & 0.2567 & 75.3411 & 68.2447 & \\ \text { S04R12 } & 3.9740 & 0.1841 & 68.4681 & 64.3100 & \\ \text { S04R13 } & 2.9766 & 0.1456 & 63.9877 & 60.8655 & \\ \text { S04R14 } & 2.8312 & 0.1394 & 63.1372 & 60.1666 & \\ \text { S04R15 } & 3.4097 & 0.1631 & 66.1811 & 62.6083 & \\ \text { S04R16 } & 5.2603 & 0.2235 & 72.2179 & 66.7341 & & \\ & & & & & \end{array}$

$\begin{array}{cccccc}\text { Cs-137 } & \text { Ba-137m } & \text { Source 05 } & \text { X Posit } & \text { Z Posit } & \text { Y Posit } \\ 10.9510 & 10.3596 & & -12.5171 & 2.4304 & 36.3581\end{array}$ 
REVISED METHODOLOGY FOR DETERMINING CESIUM-137 CONTENT OF HN-200 GROUT CONTAINERS.

$\begin{array}{ccccc}\text { Line } & \text { Shield 1 } & \text { Shield 2 } & \text { Total Dist } & \text { Air Gap } \\ \text { S05R01 } & 35.4544 & 0.1395 & 95.9677 & 60.3738 \\ \text { S05R02 } & 33.2396 & 0.1444 & 94.3265 & 60.9425 \\ \text { S05R03 } & 29.1265 & 0.1533 & 91.1955 & 61.9156 \\ \text { S05R04 } & 23.8601 & 0.1633 & 86.9232 & 62.8998 \\ \text { S05R05 } & 18.5556 & 0.1676 & 82.0390 & 63.3157 \\ \text { S05R06 } & 14.3013 & 0.1612 & 77.2433 & 62.7809 \\ \text { S05R07 } & 11.5796 & 0.1492 & 73.3507 & 61.6220 \\ \text { S05R08 } & 10.3019 & 0.1407 & 71.1410 & 60.6984 \\ \text { S05R09 } & 10.2877 & 0.1406 & 71.1144 & 60.6862 \\ \text { S05R10 } & 11.5343 & 0.1489 & 73.2775 & 61.5943 \\ \text { S05R11 } & 14.2201 & 0.1609 & 77.1392 & 62.7582 \\ \text { S05R12 } & 18.4413 & 0.1676 & 81.9233 & 63.3145 \\ \text { S05R13 } & 23.7322 & 0.1635 & 86.8140 & 62.9182 \\ \text { S05R14 } & 29.0133 & 0.1536 & 91.1073 & 61.9404 \\ \text { S05R15 } & 33.1633 & 0.1445 & 94.2695 & 60.9617 \\ \text { S05R16 } & 35.4277 & 0.1396 & 95.9481 & 60.3809\end{array}$

$\begin{array}{ccccccr} & \text { Cs-137 } & \text { Ba-137m } & \text { Source 06 } & \text { X Posit } & \text { Z Posit } & \text { Y Posit } \\ \text { Line } & \text { 16.3182 } & 15.4370 & & 10.1481 & -6.8169 & 29.6428 \\ \text { Shield 1 } & \text { Shield 2 } & \text { Total Dist } & \text { Air Gap } & & \\ \text { S06R01 } & 11.9888 & 0.1470 & 73.1651 & 61.0294 & & \\ \text { S06R03 } & 14.6287 & 0.1577 & 76.9025 & 62.1161 & & \\ \text { S06R04 } & 18.7011 & 0.1636 & 81.5214 & 62.6567 & \\ \text { S06R05 } & 23.7384 & 0.1601 & 86.2378 & 62.3393 & \\ \text { S06R06 } & 32.7368 & 0.1514 & 90.3726 & 61.4843 & \\ \text { S06R07 } & 34.7732 & 0.1433 & 93.4094 & 60.6127 & \\ \text { S06R08 } & 34.7595 & 0.1388 & 95.0069 & 60.0948 & \\ \text { S06R09 } & 32.6144 & 0.1434 & 94.9966 & 60.0982 & \\ \text { S06R10 } & 28.6791 & 0.1515 & 90.3267 & 60.6219 & \\ \text { S06R11 } & 23.6732 & 0.1602 & 86.1811 & 62.3477 & \\ \text { S06R12 } & 18.6425 & 0.1635 & 81.4614 & 62.6553 & \\ \text { S06R13 } & 14.5865 & 0.1576 & 76.8486 & 62.1045 & \\ \text { S06R14 } & 11.9649 & 0.1468 & 73.1273 & 61.0156 & \\ \text { S06R15 } & 10.7302 & 0.1393 & 71.0395 & 60.1700 & \\ \text { S06R16 } & 10.7378 & 0.1394 & 71.0532 & 60.1760 & & \\ & & & & & \end{array}$

$\begin{array}{lcccccc} & \text { Cs-137 } & \text { Ba-137m } & \text { Source 07 } & \text { X Posit } & \text { Z Posit } & \text { Y Posit } \\ & 25.9358 & 24.5353 & & -3.0040 & -18.4824 & 1.5181 \\ \text { Line } & \text { Shield 1 } & \text { Shield 2 } & \text { Total Dist } & \text { Air Gap } & & \end{array}$




\section{REVISED METHODOLOGY FOR DETERMINING CESIUM-137 CONTENT OF HN-200 GROUT CONTAINERS.}

$\begin{array}{lcccc}\text { S07R01 } & 22.3793 & 0.2222 & 91.4559 & 68.8544 \\ \text { S07R02 } & 31.4434 & 0.1812 & 97.4653 & 65.8407 \\ \text { S07R03 } & 38.3710 & 0.1558 & 101.9121 & 63.3853 \\ \text { S07R04 } & 42.1682 & 0.1441 & 104.3503 & 62.0379 \\ \text { S07R05 } & 42.4856 & 0.1432 & 104.5546 & 61.9258 \\ \text { S07R06 } & 39.2983 & 0.1528 & 102.5067 & 63.0556 \\ \text { S07R07 } & 32.8853 & 0.1754 & 98.3941 & 65.3333 \\ \text { S07R08 } & 24.0805 & 0.2141 & 92.6216 & 68.3270 \\ \text { S07R09 } & 14.9639 & 0.2508 & 85.8348 & 70.6202 \\ \text { S07R10 } & 8.6446 & 0.2295 & 78.9451 & 70.0710 \\ \text { S07R11 } & 5.6645 & 0.1805 & 73.1146 & 67.2695 \\ \text { S07R12 } & 4.5354 & 0.1528 & 69.5904 & 64.9022 \\ \text { S07R13 } & 4.4533 & 0.1506 & 69.2830 & 64.6792 \\ \text { S07R14 } & 5.3616 & 0.1736 & 72.2786 & 66.7434 \\ \text { S07R15 } & 7.9063 & 0.2205 & 77.7845 & 69.6578 \\ \text { S07R16 } & 13.5526 & 0.2522 & 84.5757 & 70.7709\end{array}$

$\begin{array}{ccccccr} & \text { Cs-137 } & \text { Ba-137m } & \text { Source 08 } & \text { X Posit } & \text { Z Posit } & \text { Y Posit } \\ \text { Line } & \text { 25.1678 } & 23.8087 & & -1.4468 & -9.3997 & 20.8621 \\ \text { S08R01 } & 23.4033 & 0.1513 & 85.1101 & 61.5556 & & \\ \text { S08R02 } & 27.2028 & 0.1470 & 88.4428 & 61.0931 & & \\ \text { S08R03 } & 30.2796 & 0.1421 & 90.9723 & 60.5505 & & \\ \text { S08R04 } & 32.0641 & 0.1390 & 92.3909 & 60.1878 & \\ \text { S08R05 } & 32.2488 & 0.1387 & 92.5361 & 60.1485 & \\ \text { S08R06 } & 30.8030 & 0.1412 & 91.3915 & 60.4473 & \\ \text { S08R07 } & 27.9727 & 0.1458 & 89.0874 & 60.9688 & \\ \text { S08R08 } & 24.2735 & 0.1505 & 85.8994 & 61.4754 & \\ \text { S08R09 } & 20.4164 & 0.1523 & 82.2421 & 61.6734 & \\ \text { S08R10 } & 17.0996 & 0.1496 & 78.6471 & 61.3980 & \\ \text { S08R11 } & 14.7613 & 0.1440 & 75.7070 & 60.8017 & \\ \text { S08R12 } & 13.5358 & 0.1395 & 73.9690 & 60.2937 & \\ \text { S08R13 } & 13.4142 & 0.1390 & 73.7873 & 60.2341 & \\ \text { S08R14 } & 14.3923 & 0.1428 & 75.2003 & 60.6653 & & \\ \text { S08R15 } & 16.4867 & 0.1484 & 77.9162 & 61.2811 & \\ \text { S08R16 } & 19.6119 & 0.1521 & 81.4174 & 61.6534 & & \\ & & & & & \end{array}$

$\begin{array}{ccccccc} & \text { Cs-137 } & \text { Ba-137m } & \text { Source 09 } & \text { X Posit } & \text { Z Posit } & \text { Y Posit } \\ & 24.9347 & 23.5882 & & -11.3240 & -1.2877 & 45.4810 \\ \text { Line } & \text { Shield 1 } & \text { Shield 2 } & \text { Total Dist } & \text { Air Gap } & & \\ \text { S09R01 } & 34.7785 & 0.1411 & 96.1132 & 61.1936 & & \end{array}$




\section{REVISED METHODOLOGY FOR DETERMINING CESIUM-137 CONTENT OF HN-200 GROUT CONTAINERS.}

$\begin{array}{lllll}\text { SO9R02 } & 34.3466 & 0.1420 & 95.7941 & 61.3055 \\ \text { SO9R03 } & 31.9648 & 0.1469 & 94.0140 & 61.9023 \\ \text { SO9R04 } & 28.0271 & 0.1545 & 90.9700 & 62.7883 \\ \text { S09R05 } & 23.2724 & 0.1616 & 87.0228 & 63.5888 \\ \text { S09R06 } & 18.6738 & 0.1631 & 82.6916 & 63.8546 \\ \text { S09R07 } & 15.0668 & 0.1571 & 78.6270 & 63.4032 \\ \text { S09R08 } & 12.7989 & 0.1483 & 75.5363 & 62.5890 \\ \text { S09R09 } & 11.8366 & 0.1432 & 74.0338 & 62.0541 \\ \text { S09R10 } & 12.0921 & 0.1446 & 74.4463 & 62.2096 \\ \text { S09R11 } & 13.5931 & 0.1519 & 76.6820 & 62.9370 \\ \text { S09R12 } & 16.4381 & 0.1604 & 80.2696 & 63.6711 \\ \text { S09R13 } & 20.5390 & 0.1635 & 84.5326 & 63.8302 \\ \text { S09R14 } & 25.3247 & 0.1590 & 88.7742 & 63.2905 \\ \text { S09R15 } & 29.8442 & 0.1511 & 92.3934 & 62.3981 \\ \text { S09R16 } & 33.1936 & 0.1444 & 94.9369 & 61.5990\end{array}$

$\begin{array}{ccccccc} & \text { Cs-137 } & \text { Ba-137m } & \text { Source 10 } & \text { X Posit } & \text { Z Posit } & \text { Y Posit } \\ \text { Line } & \text { Sh.8139 } & 25.3659 & & -8.1594 & 10.5475 & 46.4380 \\ \text { S10R01 } & 30.7104 & 0.1555 & 93.7951 & 62.9291 & & \\ \text { S10R02 } & 25.2285 & 0.1676 & 89.5822 & 64.1861 & & \\ \text { S10R03 } & 19.4920 & 0.1749 & 84.6403 & 64.9734 & & \\ \text { S10R04 } & 14.7546 & 0.1700 & 79.6595 & 64.7349 & \\ \text { S10R05 } & 11.6575 & 0.1567 & 75.4620 & 63.6478 & \\ \text { S10R06 } & 10.1205 & 0.1459 & 72.8680 & 62.6016 & \\ \text { S10R07 } & 9.9001 & 0.1442 & 72.4563 & 62.4120 & \\ \text { S10R08 } & 10.9507 & 0.1521 & 74.3245 & 63.2216 & \\ \text { S10R09 } & 13.4666 & 0.1656 & 78.0425 & 64.4102 & \\ \text { S10R10 } & 17.6518 & 0.1746 & 82.8443 & 65.0179 & & \\ \text { S10R11 } & 23.1612 & 0.1712 & 87.8873 & 64.5549 & & \\ \text { S10R12 } & 28.8833 & 0.1598 & 92.4258 & 63.3827 & & \\ \text { S10R13 } & 33.5609 & 0.1488 & 95.8834 & 62.1738 & & \\ \text { S10R14 } & 36.3261 & 0.1423 & 97.8693 & 61.4008 & \\ \text { S10R15 } & 36.7548 & 0.1414 & 98.1745 & 61.2784 & & \\ \text { S10R16 } & 34.7868 & 0.1459 & 96.7679 & 61.8351 & & \\ & & & & & & \end{array}$

$\begin{array}{ccccccc} & \text { Cs-137 } & \text { Ba-137m } & \text { Source 11 } & \text { X Posit } & \text { Z Posit } & \text { Y Posit } \\ & 12.5933 & 11.9133 & & 13.0540 & 6.4320 & 29.1917 \\ \text { Line } & \text { Shield 1 } & \text { Shield 2 } & \text { Total Dist } & \text { Air Gap } & & \\ \text { S11R01 } & 9.0908 & 0.1466 & 70.2213 & 60.9839 & & \\ \text { S11R02 } & 8.2684 & 0.1385 & 68.4659 & 60.0590 & & \end{array}$




\section{REVISED METHODOLOGY FOR DETERMINING CESIUM-137 CONTENT OF HN-200 GROUT CONTAINERS.}

$\begin{array}{lllll}\text { S11R03 } & 8.6747 & 0.1426 & 69.3602 & 60.5429 \\ \text { S11R04 } & 10.4244 & 0.1576 & 72.6777 & 62.0957 \\ \text { S11R05 } & 13.8957 & 0.1745 & 77.6496 & 63.5794 \\ \text { S11R06 } & 19.2322 & 0.1785 & 83.3026 & 63.8919 \\ \text { S11R07 } & 25.5857 & 0.1673 & 88.7304 & 62.9774 \\ \text { S11R08 } & 31.4435 & 0.1528 & 93.2127 & 61.6164 \\ \text { S11R09 } & 35.5810 & 0.1425 & 96.2378 & 60.5142 \\ \text { S11R10 } & 37.3270 & 0.1383 & 97.4945 & 60.0291 \\ \text { S11R11 } & 36.4443 & 0.1404 & 96.8603 & 60.2755 \\ \text { S11R12 } & 33.0496 & 0.1487 & 94.3965 & 61.1982 \\ \text { S11R13 } & 27.6549 & 0.1623 & 90.3508 & 62.5336 \\ \text { S11R14 } & 21.2978 & 0.1760 & 85.1671 & 63.6933 \\ \text { S11R15 } & 15.4760 & 0.1778 & 79.4963 & 63.8425 \\ \text { S11R16 } & 11.3645 & 0.1637 & 74.1900 & 62.6618\end{array}$

$\begin{array}{ccccc} & \text { Cs-137 } & \text { Ba-137m } & \text { Source 12 } & \text { X Posit } \\ \text { Line } & 17.5307 & 16.5841 & & 17.0889 \\ \text { Shield 1 } & \text { Shield 2 } & \text { Total Dist } & \text { Air Gap } \\ \text { S12R01 } & 4.2380 & 0.1578 & 66.6517 & 62.2558 \\ \text { S12R03 } & 3.6302 & 0.1392 & 64.0527 & 60.2833 \\ \text { S12R04 } & 3.8728 & 0.1469 & 65.1638 & 61.1441 \\ \text { S12R05 } & 5.1497 & 0.1824 & 69.6511 & 64.3190 \\ \text { S12R06 } & 15.5374 & 0.2386 & 76.3253 & 67.5953 \\ \text { S12R07 } & 25.0133 & 0.2484 & 83.7902 & 68.0044 \\ \text { S12R08 } & 33.5869 & 0.1657 & 90.8574 & 65.6413 \\ \text { S12R09 } & 39.4679 & 0.1459 & 100.5569 & 60.9430 \\ \text { S12R10 } & 41.9827 & 0.1386 & 102.2320 & 60.1108 \\ \text { S12R11 } & 40.9258 & 0.1416 & 101.5275 & 60.4601 \\ \text { S12R12 } & 36.3789 & 0.1558 & 98.5031 & 61.9685 \\ \text { S12R13 } & 28.7794 & 0.1853 & 93.4269 & 64.4622 \\ \text { S12R14 } & 19.3408 & 0.2314 & 86.7948 & 67.2226 \\ \text { S12R15 } & 10.8955 & 0.2531 & 79.3667 & 68.2182 \\ \text { S12R16 } & 6.1711 & 0.2049 & 72.2060 & 65.8300\end{array}$

$\begin{array}{ccccccc} & \text { Cs-137 } & \text { Ba-137m } & \text { Source 13 } & \text { X Posit } & \text { Z Posit } & \text { Y Posit } \\ & 17.9331 & 16.9647 & & 3.5123 & 8.3040 & 41.4473 \\ \text { Line } & \text { Shield 1 } & \text { Shield 2 } & \text { Total Dist } & \text { Air Gap } & & \\ \text { S13R01 } & 18.7858 & 0.1517 & 81.1994 & 62.2619 & & \\ \text { S13R02 } & 16.1593 & 0.1475 & 78.1701 & 61.8634 & & \\ \text { S13R03 } & 14.5678 & 0.1428 & 76.0920 & 61.3814 & & \end{array}$




\section{REVISED METHODOLOGY FOR DETERMINING CESIUM-137 CONTENT OF HN-200 GROUT CONTAINERS.}

$\begin{array}{lllll}\text { S13R04 } & 14.0530 & 0.1409 & 75.3685 & 61.1746 \\ \text { S13R05 } & 14.6084 & 0.1429 & 76.1479 & 61.3965 \\ \text { S13R06 } & 16.2407 & 0.1476 & 78.2707 & 61.8824 \\ \text { S13R07 } & 18.9034 & 0.1518 & 81.3259 & 62.2707 \\ \text { S13R08 } & 22.3452 & 0.1525 & 84.7766 & 62.2788 \\ \text { S13R09 } & 26.0308 & 0.1494 & 88.0825 & 61.9023 \\ \text { S13R10 } & 29.2736 & 0.1449 & 90.7816 & 61.3631 \\ \text { S13R11 } & 31.4656 & 0.1413 & 92.5305 & 60.9236 \\ \text { S13R12 } & 32.2191 & 0.1400 & 93.1207 & 60.7617 \\ \text { S13R13 } & 31.4071 & 0.1414 & 92.4844 & 60.9359 \\ \text { S13R14 } & 29.1666 & 0.1451 & 90.6949 & 61.3832 \\ \text { S13R15 } & 25.8949 & 0.1496 & 87.9658 & 61.9213 \\ \text { S13R16 } & 22.2062 & 0.1526 & 84.6452 & 62.2864\end{array}$

$\begin{array}{ccccccc} & \text { Cs-137 } & \text { Ba-137m } & \text { Source 14 } & \text { X Posit } & \text { Z Posit } & \text { Y Posit } \\ \text { Line } & \text { S6.2138 } & 24.7983 & & -18.2417 & 6.1323 & 39.9213 \\ \text { S14R01 } & 41.1628 & 0.1428 & 102.2171 & 60.9114 & & \\ \text { S14R02 } & 36.4830 & 0.1577 & 99.1400 & 62.4993 & & \\ \text { S14R03 } & 28.7441 & 0.1883 & 94.0363 & 65.1038 & \\ \text { S14R04 } & 19.1924 & 0.2361 & 87.4109 & 67.9824 & \\ \text { S14R05 } & 10.7449 & 0.2568 & 80.0330 & 69.0313 & \\ \text { S14R06 } & 6.1003 & 0.2066 & 72.9711 & 66.6642 & \\ \text { S14R07 } & 4.2199 & 0.1595 & 67.5546 & 63.1752 & \\ \text { S14R08 } & 3.6416 & 0.1415 & 65.0976 & 61.3145 & \\ \text { S14R09 } & 3.9102 & 0.1501 & 66.3071 & 62.2468 & \\ \text { S14R10 } & 5.2314 & 0.1870 & 70.8253 & 65.4069 & \\ \text { S14R11 } & 8.6853 & 0.2443 & 77.4736 & 68.5440 & \\ \text { S14R12 } & 15.9245 & 0.2511 & 84.8782 & 68.7026 & \\ \text { S14R13 } & 25.5166 & 0.2036 & 91.8677 & 66.1475 & \\ \text { S14R14 } & 34.0953 & 0.1662 & 97.5715 & 63.3100 & \\ \text { S14R15 } & 39.9174 & 0.1465 & 101.3970 & 61.3331 & \\ \text { S14R16 } & 42.3372 & 0.1395 & 102.9916 & 60.5149 & & \\ & & & & & \end{array}$

$\begin{array}{ccccccc} & \text { Cs-137 } & \text { Ba-137m } & \text { Source 15 } & \text { X Posit } & \text { Z Posit } & \text { Y Posit } \\ & 22.7469 & 21.5186 & & 21.0026 & 5.4076 & 10.7056 \\ \text { Line } & \text { Shield 1 } & \text { Shield 2 } & \text { Total Dist } & \text { Air Gap } & & \\ \text { S15R01 } & 1.2138 & 0.1504 & 64.2075 & 62.8433 & & \\ \text { S15R02 } & 1.1689 & 0.1452 & 63.5966 & 62.2825 & & \\ \text { S15R03 } & 1.4757 & 0.1802 & 67.1470 & 65.4911 & & \\ \text { S15R04 } & 2.5994 & 0.2902 & 73.7869 & 70.8973 & & \end{array}$




\section{REVISED METHODOLOGY FOR DETERMINING CESIUM-137 CONTENT OF HN-200 GROUT CONTAINERS.}

$\begin{array}{lcccc}\text { S15R05 } & 7.4544 & 0.4442 & 81.9110 & 74.0124 \\ \text { S15R06 } & 19.2291 & 0.2943 & 90.0605 & 70.5370 \\ \text { S15R07 } & 30.9572 & 0.1981 & 97.1368 & 65.9815 \\ \text { S15R08 } & 39.4954 & 0.1583 & 102.3805 & 62.7267 \\ \text { S15R09 } & 44.1590 & 0.1424 & 105.3100 & 61.0086 \\ \text { S15R10 } & 44.7424 & 0.1406 & 105.6801 & 60.7971 \\ \text { S15R11 } & 41.2242 & 0.1520 & 103.4606 & 62.0844 \\ \text { S15R12 } & 33.7467 & 0.1832 & 98.8348 & 64.9048 \\ \text { S15R13 } & 22.7887 & 0.2578 & 92.2138 & 69.1673 \\ \text { S15R14 } & 10.3360 & 0.4173 & 84.2729 & 73.5197 \\ \text { S15R15 } & 3.3266 & 0.3440 & 76.0083 & 72.3376 \\ \text { S15R16 } & 1.6652 & 0.2009 & 68.7827 & 66.9166\end{array}$

$\begin{array}{ccccccc} & \text { Cs-137 } & \text { Ba-137m } & \text { Source 16 } & \text { X Posit } & \text { Z Posit } & \text { Y Posit } \\ \text { Line } & \text { 27.6556 } & \text { 26.1622 } & & 12.7799 & 10.3319 & 39.3081 \\ \text { S16R01 } & 8.3230 & 0.1653 & 72.0176 & 63.5293 & & \\ \text { S16R02 } & 6.7801 & 0.1452 & 68.4987 & 61.5733 & & \\ \text { S16R03 } & 6.5180 & 0.1413 & 67.7909 & 61.1316 & \\ \text { S16R04 } & 7.4227 & 0.1542 & 70.0844 & 62.5075 & \\ \text { S16R05 } & 9.8747 & 0.1803 & 74.7837 & 64.7287 & \\ \text { S16R06 } & 14.5944 & 0.2003 & 80.8351 & 66.0404 & \\ \text { S16R07 } & 21.5366 & 0.1923 & 87.1410 & 65.4121 & \\ \text { S16R08 } & 29.0030 & 0.1697 & 92.7851 & 63.6124 & \\ \text { S16R09 } & 35.0999 & 0.1513 & 97.0890 & 61.8378 & \\ \text { S16R10 } & 38.7339 & 0.1414 & 99.6030 & 60.7278 & \\ \text { S16R11 } & 39.4353 & 0.1396 & 100.0861 & 60.5113 & \\ \text { S16R12 } & 37.1263 & 0.1457 & 98.4937 & 61.2217 & \\ \text { S16R13 } & 32.0781 & 0.1602 & 94.9748 & 62.7365 & \\ \text { S16R14 } & 25.0561 & 0.1821 & 89.8806 & 64.6424 & \\ \text { S16R15 } & 17.6027 & 0.2001 & 83.7812 & 65.9784 & & \\ \text { S16R16 } & 11.7398 & 0.1922 & 77.4839 & 65.5519 & & \\ & & & & & \end{array}$

$\begin{array}{ccccccc} & \text { Cs-137 } & \text { Ba-137m } & \text { Source 17 } & \text { X Posit } & \text { Z Posit } & \text { Y Posit } \\ \text { Line } & 28.5227 & 26.9825 & & -14.4903 & 3.0983 & 8.6941 \\ \text { Shield 1 } & \text { Shield 2 } & \text { Total Dist } & \text { Air Gap } & & \\ \text { S17R01 } & 37.8611 & 0.1415 & 99.1352 & 61.1326 & & \\ \text { S17R03 } & 35.1040 & 0.1484 & 97.2016 & 61.9492 & & \\ \text { S17R04 } & 30.0711 & 0.1617 & 93.5970 & 63.3642 & & \\ \text { S17R05 } & 17.6444 & 0.1779 & 88.7086 & 64.8863 & & \\ & 17.2854 & 0.1858 & 83.1281 & 65.6569 & & \end{array}$




\section{REVISED METHODOLOGY FOR DETERMINING CESIUM-137 CONTENT OF HN-200 GROUT CONTAINERS.}

$\begin{array}{lcccc}\text { S17R06 } & 12.4618 & 0.1754 & 77.6505 & 65.0134 \\ \text { S17R07 } & 9.6194 & 0.1565 & 73.2204 & 63.4446 \\ \text { S17R08 } & 8.3873 & 0.1444 & 70.7597 & 62.2280 \\ \text { S17R09 } & 8.4317 & 0.1449 & 70.8565 & 62.2798 \\ \text { S17R10 } & 9.7663 & 0.1578 & 73.4866 & 63.5625 \\ \text { S17R11 } & 12.7444 & 0.1766 & 78.0259 & 65.1049 \\ \text { S17R12 } & 17.7101 & 0.1858 & 83.5417 & 65.6458 \\ \text { S17R13 } & 24.1304 & 0.1768 & 89.0962 & 64.7890 \\ \text { S17R14 } & 30.4988 & 0.1605 & 93.9086 & 63.2493 \\ \text { S17R15 } & 35.3887 & 0.1477 & 97.4023 & 61.8659 \\ \text { S17R16 } & 37.9602 & 0.1413 & 99.2044 & 61.1029\end{array}$

$\begin{array}{ccccccc} & \text { Cs-137 } & \text { Ba-137m } & \text { Source 18 } & \text { X Posit } & \text { Z Posit } & \text { Y Posit } \\ & 23.2528 & 21.9972 & & 18.7646 & -12.0033 & 10.5209 \\ \text { Line } & \text { Shield 1 } & \text { Shield 2 } & \text { Total Dist } & \text { Air Gap } & & \\ \text { S18R01 } & 0.7334 & 0.1911 & 67.2728 & 66.3483 & & \\ \text { S18R02 } & 1.4355 & 0.3482 & 74.3270 & 72.5432 & & \\ \text { S18R03 } & 6.3650 & 0.6069 & 82.7355 & 75.7636 & & \\ \text { S18R04 } & 19.8449 & 0.3054 & 91.0306 & 70.8803 & \\ \text { S18R05 } & 32.0166 & 0.1971 & 98.1287 & 65.9150 & \\ \text { S18R06 } & 40.5319 & 0.1570 & 103.2900 & 62.6011 & \\ \text { S18R07 } & 44.9644 & 0.1418 & 106.0519 & 60.9457 & \\ \text { S18R08 } & 45.1799 & 0.1411 & 106.1875 & 60.8665 & \\ \text { S18R09 } & 41.1726 & 0.1546 & 103.6860 & 62.3588 & \\ \text { S18R10 } & 33.0608 & 0.1911 & 98.7518 & 65.4998 & \\ \text { S18R11 } & 21.2204 & 0.2880 & 91.8219 & 70.3135 & \\ \text { S18R12 } & 7.5339 & 0.5777 & 83.6053 & 75.4938 & \\ \text { S18R13 } & 1.5949 & 0.3787 & 75.1476 & 73.1740 & \\ \text { S18R14 } & 0.7681 & 0.1996 & 67.8792 & 66.9115 & \\ \text { S18R15 } & 0.5658 & 0.1490 & 63.4776 & 62.7628 & \\ \text { S18R16 } & 0.5578 & 0.1470 & 63.2505 & 62.5457 & \end{array}$

$\begin{array}{ccccccc} & \text { Cs-137 } & \text { Ba-137m } & \text { Source 19 } & \text { X Posit } & \text { Z Posit } & \text { Y Posit } \\ \text { Line } & \text { Sh.3416 } & 14.5131 & & -18.2584 & 0.2785 & 21.1001 \\ \text { S19R01 } & 41.1212 & \text { Shield 2 } & \text { Total Dist } & \text { Air Gap } & & \\ \text { S19R02 } & 39.2983 & 0.1384 & 101.3539 & 60.0943 & & \\ \text { S19R03 } & 34.2588 & 0.1591 & 100.1219 & 60.6802 & & \\ \text { S19R04 } & 26.6081 & 0.1876 & 91.4348 & 64.6391 & & \\ \text { S19R05 } & 17.8410 & 0.2223 & 84.8399 & 66.7766 & & \\ \text { S19R06 } & 10.6596 & 0.2233 & 77.7324 & 66.8496 & & \\ & & & & & \end{array}$




\section{REVISED METHODOLOGY FOR DETERMINING CESIUM-137 CONTENT} OF HN-200 GROUT CONTAINERS.

$\begin{array}{lllll}\text { S19R07 } & 6.6976 & 0.1824 & 71.2024 & 64.3225 \\ \text { S19R08 } & 5.0020 & 0.1492 & 66.5451 & 61.3940 \\ \text { S19R09 } & 4.5568 & 0.1387 & 64.9260 & 60.2305 \\ \text { S19R10 } & 5.0809 & 0.1510 & 66.8102 & 61.5784 \\ \text { S19R11 } & 6.9059 & 0.1857 & 71.6597 & 64.5681 \\ \text { S19R12 } & 11.1023 & 0.2253 & 78.2795 & 66.9519 \\ \text { S19R13 } & 18.5041 & 0.2201 & 85.3826 & 66.6584 \\ \text { S19R14 } & 27.2697 & 0.1849 & 91.9004 & 64.4458 \\ \text { S19R15 } & 34.7553 & 0.1574 & 97.0475 & 62.1348 \\ \text { S19R16 } & 39.5593 & 0.1427 & 100.2983 & 60.5963\end{array}$

\begin{tabular}{|c|c|c|c|c|c|c|}
\hline & $\begin{array}{c}\text { Cs-137 } \\
16.4988\end{array}$ & $\begin{array}{c}\text { Ba-137m } \\
15.6078\end{array}$ & Source 20 & $\begin{array}{c}\text { X Posit } \\
2.8644\end{array}$ & $\begin{array}{l}\text { Z Posit } \\
9.2261\end{array}$ & $\begin{array}{l}\text { Y Posit } \\
44.4497\end{array}$ \\
\hline Line & Shield 1 & Shield 2 & Total Dist & Air Gap & & \\
\hline S20R01 & 19.3906 & 0.1555 & 82.5234 & 62.9772 & & \\
\hline S20R02 & 16.3352 & 0.1513 & 79.1242 & 62.6376 & & \\
\hline S20R03 & 14.3697 & 0.1456 & 76.5972 & 62.0820 & & \\
\hline S20R04 & 13.5470 & 0.1423 & 75.4290 & 61.7397 & & \\
\hline S20R05 & 13.8449 & 0.1436 & 75.8608 & 61.8723 & & \\
\hline S20R06 & 15.2747 & 0.1486 & 77.8022 & 62.3789 & & \\
\hline S20R07 & 17.8406 & 0.1540 & 80.8645 & 62.8699 & & \\
\hline S20R08 & 21.3689 & 0.1559 & 84.4899 & 62.9651 & & \\
\hline S20R09 & 25.3558 & 0.1531 & 88.0929 & 62.5841 & & \\
\hline S20R10 & 29.0593 & 0.1477 & 91.1584 & 61.9514 & & \\
\hline S20R11 & 31.7694 & 0.1430 & 93.2917 & 61.3793 & & \\
\hline S20R 12 & 33.0008 & 0.1408 & 94.2388 & 61.0972 & & \\
\hline S2OR 13 & 32.5474 & 0.1416 & 93.8915 & 61.2024 & & \\
\hline S20R 14 & 30.4841 & 0.1453 & 92.2892 & 61.6598 & & \\
\hline S20R 15 & 27.1671 & 0.1507 & 89.6182 & 62.3004 & & \\
\hline S20R16 & 23.2157 & 0.1551 & 86.2086 & 62.8378 & & \\
\hline
\end{tabular}

$\begin{array}{ccccccr} & \text { Cs-137 } & \text { Ba-137m } & \text { Source 21 } & \text { X Posit } & \text { Z Posit } & \text { Y Posit } \\ \text { Line } & 28.6466 & 27.0997 & & 3.0621 & -7.9137 & 19.8848 \\ \text { S21R01 } & 19.0850 & 0.1485 & 80.5595 & 61.3261 & & \\ \text { S21R02 } & 22.3224 & 0.1491 & 83.8502 & 61.3786 & & \\ \text { S21R03 } & 25.7489 & 0.1466 & 86.9875 & 61.0920 & & \\ \text { S21R04 } & 28.7355 & 0.1427 & 89.5330 & 60.6548 & & \\ \text { S21R05 } & 30.7271 & 0.1396 & 91.1617 & 60.2949 & & \\ \text { S21R06 } & 31.3702 & 0.1386 & 91.6783 & 60.1695 & & \\ \text { S21R07 } & 30.5550 & 0.1399 & 91.0228 & 60.3278 & & \\ \end{array}$




\section{REVISED METHODOLOGY FOR DETERMINING CESIUM-137 CONTENT OF HN-200 GROUT CONTAINERS.}

$\begin{array}{lllll}\text { S21R08 } & 28.4211 & 0.1432 & 89.2714 & 60.7072 \\ \text { S21R09 } & 25.3494 & 0.1470 & 86.6354 & 61.1389 \\ \text { S21R10 } & 21.9130 & 0.1493 & 83.4547 & 61.3924 \\ \text { S21R11 } & 18.7364 & 0.1482 & 80.1793 & 61.2947 \\ \text { S21R12 } & 16.2975 & 0.1443 & 77.3264 & 60.8845 \\ \text { S21R13 } & 14.8263 & 0.1403 & 75.3994 & 60.4328 \\ \text { S21R14 } & 14.3764 & 0.1388 & 74.7705 & 60.2553 \\ \text { S21R15 } & 14.9488 & 0.1407 & 75.5672 & 60.4777 \\ \text { S21R16 } & 16.5409 & 0.1449 & 77.6284 & 60.9426\end{array}$

$\begin{array}{ccccccc} & \text { Cs-137 } & \text { Ba-137m } & \text { Source 22 } & \text { X Posit } & \text { Z Posit } & \text { Y Posit } \\ \text { Line } & \text { Shicicid 1 } & \text { Shield 2 } & \text { Total Dist } & \text { Air Gap } & & \\ \text { S22R01 } & 40.7334 & 0.1595 & 104.3640 & 63.4711 & & \\ \text { S22R02 } & 32.1273 & 0.2005 & 99.2407 & 66.9129 & & \\ \text { S22R03 } & 19.9427 & 0.3079 & 92.2518 & 72.0013 & \\ \text { S22R04 } & 6.8506 & 0.5542 & 84.1464 & 76.7416 & \\ \text { S22R05 } & 1.8533 & 0.3350 & 76.0070 & 73.8186 & \\ \text { S22R06 } & 0.9874 & 0.1931 & 69.2690 & 68.0885 & \\ \text { S22R07 } & 0.7640 & 0.1514 & 65.5212 & 64.6058 & \\ \text { S22R08 } & 0.7809 & 0.1546 & 65.8670 & 64.9315 & \\ \text { S22R09 } & 1.0604 & 0.2063 & 70.1967 & 68.9300 & \\ \text { S22R10 } & 2.1697 & 0.3776 & 77.2704 & 74.7231 & \\ \text { S22R11 } & 8.6588 & 0.5218 & 85.4930 & 76.3124 & \\ \text { S22R12 } & 22.1087 & 0.2820 & 93.4817 & 71.0910 & \\ \text { S22R13 } & 33.7826 & 0.1912 & 100.2116 & 66.2378 & \\ \text { S22R14 } & 41.7501 & 0.1557 & 104.9821 & 63.0763 & \\ \text { S22R15 } & 45.6210 & 0.1427 & 107.3612 & 61.5975 & \\ \text { S22R16 } & 45.2791 & 0.1438 & 107.1494 & 61.7265 & & \\ & & & & & \end{array}$

$\begin{array}{cccccc}\text { Cs-137 } & \text { Ba-137m } & \text { Source 23 } & \begin{array}{c}\text { X Posit } \\ \text { Z Posit }\end{array} & \text { Y Posit } \\ 13.0146 & 12.3118 & & 5.0992 & 14.6169 & 52.0038 \\ \text { Shield 1 } & \text { Shield 2 } & \text { Total Dist } & \text { Air Gap } & & \\ 15.3065 & 0.1952 & 83.1277 & 67.6260 & & \\ 10.9414 & 0.1778 & 77.7609 & 66.6417 & & \\ 8.6293 & 0.1571 & 73.7802 & 64.9938 & & \\ 7.8271 & 0.1477 & 72.0579 & 64.0830 & & \\ 8.2607 & 0.1530 & 73.0168 & 64.6032 & & \\ 10.0824 & 0.1712 & 76.4179 & 66.1643 & & \\ 13.8035 & 0.1918 & 81.4843 & 67.4889 & & \\ 19.6928 & 0.1948 & 87.2332 & 67.3456 & & \end{array}$




\section{REVISED METHODOLOGY FOR DETERMINING CESIUM-137 CONTENT OF HN-200 GROUT CONTAINERS.}

$\begin{array}{lllll}\text { S23R09 } & 26.7455 & 0.1785 & 92.7462 & 65.8222 \\ \text { S23R10 } & 33.1755 & 0.1599 & 97.2899 & 63.9546 \\ \text { S23R11 } & 37.6491 & 0.1475 & 100.3423 & 62.5457 \\ \text { S23R12 } & 39.4898 & 0.1428 & 101.5862 & 61.9536 \\ \text { S23R13 } & 38.4722 & 0.1454 & 100.8991 & 62.2815 \\ \text { S23R14 } & 34.7175 & 0.1555 & 98.3483 & 63.4753 \\ \text { S23R15 } & 28.7510 & 0.1728 & 94.1934 & 65.2696 \\ \text { S23R16 } & 21.7002 & 0.1913 & 88.8957 & 67.0042\end{array}$

$\begin{array}{ccccccc} & \text { Cs-137 } & \text { Ba-137m } & \text { Source 24 } & \text { X Posit } & \text { Z Posit } & \text { Y Posit } \\ \text { Line } & 12.5419 & 11.8646 & & 7.9617 & 19.2122 & 33.0332 \\ \text { S24R01 } & \text { Shield 1 } & \text { Shield 2 } & \text { Total Dist } & \text { Air Gap } & & \\ \text { S24R02 } & 3.3756 & 0.3185 & 77.6528 & 70.4587 & & \\ \text { S24R03 } & 2.2825 & 0.2151 & 70.0781 & 66.4821 & & \\ \text { S24R04 } & 2.0129 & 0.1354 & 64.5240 & 62.0860 & \\ \text { S24R05 } & 2.2830 & 0.1555 & 62.4578 & 60.3060 & \\ \text { S24R06 } & 3.3825 & 0.2151 & 70.0840 & 62.0889 & \\ \text { S24R07 } & 6.8806 & 0.3186 & 77.6597 & 70.4605 & \\ \text { S24R08 } & 15.7304 & 0.2941 & 85.7391 & 69.7146 & \\ \text { S24R09 } & 26.8459 & 0.2094 & 93.1196 & 66.0643 & \\ \text { S24R10 } & 35.9710 & 0.1645 & 98.9459 & 62.8103 & \\ \text { S24R11 } & 41.7238 & 0.1443 & 102.6542 & 60.7861 & & \\ \text { S24R12 } & 43.6758 & 0.1385 & 103.9242 & 60.1100 & \\ \text { S24R13 } & 41.7205 & 0.1443 & 102.6520 & 60.7872 & \\ \text { S24R14 } & 35.9646 & 0.1646 & 98.9417 & 62.8126 & \\ \text { S24R15 } & 26.8369 & 0.2095 & 93.1139 & 66.0675 & \\ \text { S24R16 } & 15.7209 & 0.2942 & 85.7323 & 69.7172 & & \\ & & & & & \end{array}$

$\begin{array}{ccccccc} & \text { Cs-137 } & \text { Ba-137m } & \text { Source 25 } & \text { X Posit } & \text { Z Posit } & \text { Y Posit } \\ \text { Line } & \mathbf{1 9 . 7 6 2 5} & 18.6953 & & \mathbf{8 . 3 9 7 5} & 9.5559 & 39.8544 \\ \text { Shield 1 } & \text { Shield 2 } & \text { Total Dist } & \text { Air Gap } & & \\ \text { S25R01 } & 13.2970 & 0.1581 & 76.2841 & \mathbf{6 2 . 8 2 9 0} & & \\ \text { S25R03 } & 11.0957 & 0.1465 & 72.9295 & 61.6873 & & \\ \text { S25R04 } & 10.2684 & 0.1407 & 71.4592 & 61.0501 & & \\ \text { S25R05 } & 10.6827 & 0.1437 & 72.2130 & 61.3865 & & \\ \text { S25R06 } & 12.4096 & 0.1541 & 75.0140 & 62.4504 & \\ \text { S25R07 } & 15.6432 & 0.1652 & 79.2516 & 63.4431 & \\ \text { S25R08 } & 25.7322 & 0.1681 & 84.1209 & 63.6206 & \\ \text { S25R09 } & 30.7575 & 0.1610 & 88.8398 & \mathbf{6 2 . 9 2 0 0} & & \\ & & 0.1507 & 92.7638 & 61.8556 & & \end{array}$




\section{REVISED METHODOLOGY FOR DETERMINING CESIUM-137 CONTENT OF HN-200 GROUT CONTAINERS.}

$\begin{array}{lllll}\text { S25R10 } & 34.3142 & 0.1429 & 95.4237 & 60.9666 \\ \text { S25R11 } & 35.8213 & 0.1396 & 96.5296 & 60.5687 \\ \text { S25R12 } & 35.0529 & 0.1412 & 95.9670 & 60.7729 \\ \text { S25R13 } & 32.1218 & 0.1477 & 93.7938 & 61.5242 \\ \text { S25R14 } & 27.5033 & 0.1577 & 90.2418 & 62.5808 \\ \text { S25R15 } & 22.0856 & 0.1666 & 85.7209 & 63.4687 \\ \text { S25R16 } & 17.0414 & 0.1674 & 80.8201 & 63.6113\end{array}$

$\begin{array}{ccccccc} & \text { Cs-137 } & \text { Ba-137m } & \text { Source 26 } & \text { X Posit } & \text { Z Posit } & \text { Y Posit } \\ \text { Line } & \text { Sh.8512 } & 23.5093 & & 4.1090 & 16.2164 & 34.1935 \\ \text { S26R01 } & 14.8147 & 0.2033 & 80.8227 & 65.8047 & & \\ \text { S26R02 } & 9.8092 & 0.1840 & 74.5307 & 64.5374 & & \\ \text { S26R03 } & 7.1928 & 0.1556 & 69.4975 & 62.1491 & \\ \text { S26R04 } & 6.1823 & 0.1403 & 66.8426 & 60.5200 & \\ \text { S26R05 } & 6.3277 & 0.1426 & 67.2607 & 60.7903 & \\ \text { S26R06 } & 7.6966 & 0.1623 & 70.6370 & 62.7782 & \\ \text { S26R07 } & 10.8718 & 0.1914 & 76.1170 & 65.0539 & \\ \text { S26R08 } & 16.5617 & 0.2031 & 82.5483 & 65.7834 & \\ \text { S26R09 } & 24.0966 & 0.1855 & 88.8565 & 64.5743 & \\ \text { S26R10 } & 31.3941 & 0.1621 & 94.1965 & 62.6402 & \\ \text { S26R11 } & 36.7831 & 0.1462 & 97.9688 & 61.0395 & \\ \text { S26R12 } & 39.4284 & 0.1391 & 99.7991 & 60.2316 & \\ \text { S26R13 } & 39.0211 & 0.1402 & 99.5178 & 60.3565 & \\ \text { S26R14 } & 35.6051 & 0.1495 & 97.1504 & 61.3958 & \\ \text { S26R15 } & 29.6025 & 0.1677 & 92.9193 & 63.1490 & \\ \text { S26R16 } & 22.0418 & 0.1918 & 87.2558 & 65.0222 & & \\ & & & & & \end{array}$

$\begin{array}{ccccccc} & \text { Cs-137 } & \text { Ba-137m } & \text { Source 27 } & \text { X Posit } & \text { Z Posit } & \text { Y Posit } \\ \text { Line } & \text { 10.2138 } & \text { 9.6622 } & & 2.2698 & -21.1016 & 13.9713 \\ \text { Shield 1 } & \text { Shield 2 } & \text { Total Dist } & \text { Air Gap } & & \\ \text { S27R01 } & 12.1881 & 0.3522 & 84.3555 & 71.8152 & & \\ \text { S27R03 } & 23.9084 & 0.2378 & 92.1086 & 67.9625 & & \\ \text { S27R04 } & 41.1772 & 0.1765 & 98.4945 & 64.1407 & \\ \text { S27R05 } & 44.2030 & 0.145 & 0.1494 & 102.8692 & 61.6053 & \\ \text { S27R06 } & 43.2918 & 0.1424 & 104.8455 & 60.5028 & \\ \text { S27R07 } & 38.4219 & 0.1589 & 101.1614 & 62.5806 & \\ \text { S27R08 } & 29.8677 & 0.1984 & 95.8107 & 65.7445 & \\ \text { S27R09 } & 18.5288 & 0.2851 & 88.7066 & 69.8927 & & \\ \text { S27R10 } & 7.8783 & 0.3751 & 80.6271 & 72.3737 & & \\ & & & & & \end{array}$




\section{REVISED METHODOLOGY FOR DETERMINING CESIUM-137 CONTENT OF HN-200 GROUT CONTAINERS.}

$\begin{array}{lllll}\text { S27R11 } & 3.2773 & 0.2570 & 72.6881 & 69.1538 \\ \text { S27R12 } & 1.9878 & 0.1709 & 66.3525 & 64.1938 \\ \text { S27R13 } & 1.6249 & 0.1424 & 63.1833 & 61.4160 \\ \text { S27R14 } & 1.7228 & 0.1502 & 64.1443 & 62.2712 \\ \text { S27R15 } & 2.3916 & 0.2006 & 68.9282 & 66.3360 \\ \text { S27R16 } & 4.6689 & 0.3207 & 76.1908 & 71.2012\end{array}$

$\begin{array}{ccccccc} & \text { Cs-137 } & \text { Ba-137m } & \text { Source 28 } & \text { X Posit } & \text { Z Posit } & \text { Y Posit } \\ \text { Line } & \text { Sh.5133 } & 14.6756 & & 18.4153 & -5.6775 & 6.4850 \\ \text { S28R01 } & 3.9423 & 0.1527 & 67.8676 & 63.7726 & & \\ \text { S28R02 } & 5.1872 & 0.1883 & 72.1036 & 66.7281 & & \\ \text { S28R03 } & 8.4877 & 0.2465 & 78.5279 & 69.7937 & \\ \text { S28R04 } & 15.5736 & 0.2582 & 85.8008 & 69.9689 & \\ \text { S28R05 } & 25.2468 & 0.2098 & 92.7492 & 67.2926 & \\ \text { S28R06 } & 34.0465 & 0.1702 & 98.4870 & 64.2703 & \\ \text { S28R07 } & 40.1196 & 0.1491 & 102.4038 & 62.1351 & \\ \text { S28R08 } & 42.7854 & 0.1412 & 104.1306 & 61.2040 & \\ \text { S28R09 } & 41.8360 & 0.1439 & 103.5147 & 61.5348 & \\ \text { S28R10 } & 37.3406 & 0.1582 & 100.6101 & 63.1113 & \\ \text { S28R11 } & 29.7072 & 0.1884 & 95.6802 & 65.7847 & \\ \text { S28R12 } & 20.0983 & 0.2370 & 89.2164 & 68.8811 & \\ \text { S28R13 } & 11.3475 & 0.2635 & 81.9689 & 70.3579 & \\ \text { S28R14 } & 6.3942 & 0.2154 & 74.9776 & 68.3680 & \\ \text { S28R15 } & 4.3714 & 0.1659 & 69.5326 & 64.9954 & \\ \text { S28R16 } & 3.7259 & 0.1458 & 66.9190 & 63.0473 & & \\ & & & & & \end{array}$

$\begin{array}{ccccccc} & \text { Cs-137 } & \text { Ba-137m } & \text { Source 29 } & \text { X Posit } & \text { Z Posit } & \text { Y Posit } \\ \text { Line } & \text { Sh.0653 } & 17.0898 & & -0.0904 & 3.6427 & 38.3454 \\ \text { S29R01 } & 22.9780 & 0.1413 & 83.9127 & 60.7934 & & \\ \text { S29R02 } & 21.5828 & 0.1412 & 82.5166 & 60.7926 & & \\ \text { S29R03 } & 20.4212 & 0.1406 & 81.3001 & 60.7383 & & \\ \text { S29R04 } & 19.6496 & 0.1400 & 80.4604 & 60.6708 & \\ \text { S29R05 } & 19.3603 & 0.1397 & 80.1382 & 60.6383 & \\ \text { S29R06 } & 19.5852 & 0.1399 & 80.3890 & 60.6639 & \\ \text { S29R07 } & 20.2997 & 0.1405 & 81.1697 & 60.7294 & \\ \text { S29R08 } & 21.4194 & 0.1411 & 82.3486 & 60.7881 & \\ \text { S29R09 } & 22.7957 & 0.1413 & 83.7339 & 60.7969 & & \\ \text { S29R10 } & 24.2251 & 0.1410 & 85.1102 & 60.7441 & & \\ \text { S29R11 } & 25.4784 & 0.1403 & 86.2730 & 60.6542 & & \end{array}$


REVISED METHODOLOGY FOR DETERMINING CESIUM-137 CONTENT OF HN-200 GROUT CONTAINERS.

$\begin{array}{lllll}\text { S29R12 } & 26.3454 & 0.1397 & 87.0567 & 60.5716 \\ \text { S29R13 } & 26.6780 & 0.1394 & 87.3532 & 60.5358 \\ \text { S29R14 } & 26.4190 & 0.1397 & 87.1225 & 60.5639 \\ \text { S29R15 } & 25.6130 & 0.1403 & 86.3957 & 60.6424 \\ \text { S29R16 } & 24.3978 & 0.1409 & 85.2727 & 60.7340\end{array}$

$\begin{array}{ccccccc} & \text { Cs-137 } & \text { Ba-137m } & \text { Source 30 } & \text { X Posit } & \text { Z Posit } & \text { Y Posit } \\ \text { Line } & \text { 15.0401 } & 14.2280 & & 7.3731 & -19.7854 & 12.4003 \\ \text { Shield 1 } & \text { Shield 2 } & \text { Total Dist } & \text { Air Gap } & & \\ \text { S30R01 } & 6.8508 & 0.3557 & 79.4189 & 72.2124 & & \\ \text { S30R03 } & 16.5466 & 0.3029 & 87.5008 & 70.6512 & & \\ \text { S30R04 } & 28.0445 & 0.2090 & 94.7957 & 66.5422 & & \\ \text { S30R05 } & 47.1445 & 0.1640 & 100.4710 & 63.1625 & \\ \text { S30R06 } & 44.6834 & 0.1445 & 103.9820 & 61.1542 & \\ \text { S30R07 } & 41.9553 & 0.1396 & 105.0261 & 60.5739 & \\ \text { S30R08 } & 35.7258 & 0.1698 & 103.5173 & 61.4152 & \\ \text { S30R09 } & 26.0548 & 0.2218 & 99.5802 & 63.6845 & \\ \text { S30R10 } & 14.4271 & 0.3246 & 86.0486 & 67.2829 & \\ \text { S30R11 } & 5.7728 & 0.3377 & 77.9392 & 71.2968 & \\ \text { S30R12 } & 2.8008 & 0.2157 & 70.4721 & 67.4557 & & \\ \text { S30R13 } & 1.9217 & 0.1562 & 65.1803 & 63.1024 & \\ \text { S30R14 } & 1.7314 & 0.1420 & 63.4844 & 61.6110 & \\ \text { S30R15 } & 2.0149 & 0.1629 & 65.9160 & 63.7381 & \\ \text { S30R16 } & 3.1067 & 0.2339 & 71.7254 & 68.3847 & & \\ & & & & & \end{array}$

$\begin{array}{ccccccc} & \text { Cs-137 } & \text { Ba-137m } & \text { Source 31 } & \text { X Posit } & \text { Z Posit } & \text { Y Posit } \\ \text { Line } & 22.3550 & 21.1478 & & 5.5574 & -17.7939 & 21.2754 \\ \text { S31R01 } & \text { Shield 1 } & \text { Shield 2 } & \text { Total Dist } & \text { Air Gap } & & \\ \text { S31R02 } & 11.8035 & 0.2378 & 79.5882 & 67.5470 & & \\ \text { S31R03 } & 28.9098 & 0.2201 & 86.7985 & 66.6463 & & \\ \text { S31R04 } & 36.1534 & 0.1809 & 93.2275 & 64.1369 & & \\ \text { S31R05 } & 40.4694 & 0.1413 & 98.1338 & 61.8261 & \\ \text { S31R06 } & 41.4095 & 0.1386 & 101.0331 & 60.4224 & \\ \text { S31R07 } & 38.8943 & 0.1458 & 99.9751 & 60.9350 & \\ \text { S31R08 } & 33.1500 & 0.1646 & 96.1108 & 62.7962 & \\ \text { S31R09 } & 24.8922 & 0.1980 & 90.4319 & 65.3417 & \\ \text { S31R10 } & 15.8991 & 0.2350 & 83.5360 & 67.4019 & & \\ \text { S31R11 } & 9.1583 & 0.2246 & 76.2946 & 66.9117 & & \\ \text { S31R12 } & 5.7855 & 0.1770 & 69.8712 & 63.9087 & & \end{array}$


REVISED METHODOLOGY FOR DETERMINING CESIUM-137 CONTENT OF HN-200 GROUT CONTAINERS.

$\begin{array}{lllll}\text { S31R13 } & 4.4403 & 0.1458 & 65.6090 & 61.0229 \\ \text { S31R14 } & 4.1958 & 0.1393 & 64.6255 & 60.2904 \\ \text { S31R15 } & 4.8860 & 0.1569 & 67.2102 & 62.1673 \\ \text { S31R16 } & 6.9879 & 0.1988 & 72.6288 & 65.4421\end{array}$

$\begin{array}{ccccccr} & \text { Cs-137 } & \text { Ba-137m } & \text { Source 32 } & \text { X Posit } & \text { Z Posit } & \text { Y Posit } \\ \text { Line } & \text { Shield 1 } & \text { Shield 2 } & \text { Total Dist } & \text { Air Gap } & & \\ \text { S32R01 } & 19.4232 & 0.2126 & 85.7589 & 66.1231 & & \\ \text { S32R02 } & 11.9468 & 0.2211 & 78.7568 & 66.5889 & & \\ \text { S32R03 } & 7.4807 & 0.1865 & 72.1310 & 64.4638 & & \\ \text { S32R04 } & 5.4570 & 0.1521 & 67.1383 & 61.5292 & \\ \text { S32R05 } & 4.8189 & 0.1384 & 64.9807 & 60.0235 & \\ \text { S32R06 } & 5.1850 & 0.1464 & 66.2656 & 60.9342 & \\ \text { S32R07 } & 6.7734 & 0.1760 & 70.6240 & 63.6746 & \\ \text { S32R08 } & 10.4828 & 0.2149 & 76.9516 & 66.2539 & \\ \text { S32R09 } & 17.2630 & 0.2191 & 83.9664 & 66.4843 & \\ \text { S32R10 } & 25.7938 & 0.1882 & 90.5667 & 64.5847 & \\ \text { S32R11 } & 33.4572 & 0.1601 & 95.9275 & 62.3102 & & \\ \text { S32R12 } & 38.6691 & 0.1440 & 99.4857 & 60.6726 & \\ \text { S32R13 } & 40.7601 & 0.1383 & 100.9081 & 60.0097 & \\ \text { S32R14 } & 39.5267 & 0.1416 & 100.0692 & 60.4009 & \\ \text { S32R15 } & 35.0850 & 0.1548 & 97.0423 & 61.8026 & & \\ \text { S32R16 } & 27.9539 & 0.1798 & 92.1054 & 63.9717 & & \\ & & & & & \end{array}$

$\begin{array}{cccccr}\text { Cs-137 } & \text { Ba-137m } & \text { Source 33 } & \text { X Posit } & \text { Z Posit } & \text { Y Posit } \\ 17.8281 & 16.8653 & & -2.7939 & -22.1819 & 29.8247 \\ \text { Shield 1 } & \text { Shield 2 } & \text { Total Dist } & \text { Air Gap } & & \\ 17.6639 & 0.3309 & 88.6079 & 70.6131 & & \\ 30.2193 & 0.2036 & 96.0424 & 65.6196 & & \\ 39.2272 & 0.1586 & 101.5745 & 62.1887 & & \\ 44.1943 & 0.1413 & 104.7121 & 60.3766 & & \\ 44.9601 & 0.1389 & 105.2015 & 60.1025 & \\ 41.5034 & 0.1501 & 103.0045 & 61.3509 & \\ 33.9267 & 0.1823 & 98.2953 & 64.1863 & \\ 22.5513 & 0.2670 & 91.4758 & 68.6576 & \\ 8.7404 & 0.5468 & 83.2135 & 73.9262 & \\ 1.5615 & 0.4279 & 74.5098 & 72.5204 & & \\ 0.6751 & 0.2075 & 66.7719 & 65.8892 & & \\ 0.4730 & 0.1476 & 61.7352 & 61.1147 & \\ 0.4492 & 0.1403 & 60.8975 & 60.3080 & & \end{array}$


REVISED METHODOLOGY FOR DETERMINING CESIUM-137 CONTENT OF HN-200 GROUT CONTAINERS.

$\begin{array}{lllll}\text { S33R14 } & 0.5705 & 0.1768 & 64.5441 & 63.7968 \\ \text { S33R15 } & 1.0577 & 0.3122 & 71.5115 & 70.1415 \\ \text { S33R16 } & 4.5971 & 0.6590 & 80.0502 & 74.7941\end{array}$

$\begin{array}{ccccccc} & \text { Cs-137 } & \text { Ba-137m } & \text { Source 34 } & \text { X Posit } & \text { Z Posit } & \text { Y Posit } \\ \text { Line } & \text { 14.6776 } & 13.8850 & & -14.1407 & 16.9694 & 5.4489 \\ \text { Shield 1 } & \text { Shield 2 } & \text { Total Dist } & \text { Air Gap } & & \\ \text { S34R01 } & 35.4234 & 0.1810 & 100.8259 & 65.2215 & & \\ \text { S34R03 } & 24.4333 & 0.2555 & 94.3936 & 69.7048 & & \\ \text { S34R04 } & 11.0068 & 0.4623 & 86.5361 & 75.0670 & & \\ \text { S34R05 } & 2.7029 & 0.4300 & 78.2136 & 75.0807 & \\ \text { S34R06 } & 1.1783 & 0.2230 & 70.7495 & 69.3481 & \\ \text { S34R07 } & 0.7581 & 0.1581 & 65.7430 & 64.7692 & \\ \text { S34R08 } & 0.9279 & 0.1474 & 64.5860 & 63.6804 & \\ \text { S34R09 } & 1.5964 & 0.1787 & 67.6467 & 66.5401 & \\ \text { S34R10 } & 5.1498 & 0.5493 & 73.9912 & 72.1036 & \\ \text { S34R11 } & 17.2894 & 0.3418 & 90.2888 & 76.3401 & \\ \text { S34R12 } & 29.9416 & 0.2122 & 97.5869 & 67.4331 & & \\ \text { S34R13 } & 39.2440 & 0.1640 & 103.1265 & 63.7185 & \\ \text { S34R14 } & 44.5553 & 0.1449 & 106.3881 & 61.6880 & & \\ \text { S34R15 } & 45.6896 & 0.1414 & 107.0945 & 61.2636 & & \\ \text { S34R16 } & 42.6150 & 0.1514 & 105.1879 & 62.4215 & & \\ & & & & & \end{array}$

$\begin{array}{ccccccr} & \text { Cs-137 } & \text { Ba-137m } & \text { Source 35 } & \text { X Posit } & \text { Z Posit } & \text { Y Posit } \\ \text { Line } & \text { 28.0146 } & 26.5018 & & 17.6331 & 12.8346 & 30.1155 \\ \text { S35R01 } & 1.4227 & 0.1936 & 66.6387 & 65.0224 & & \\ \text { S35R02 } & 1.0415 & 0.1450 & 62.0375 & 60.8510 & & \\ \text { S35R03 } & 1.0129 & 0.1413 & 61.5793 & 60.4252 & & \\ \text { S35R04 } & 1.3012 & 0.1785 & 65.4173 & 63.9376 & \\ \text { S35R05 } & 2.3691 & 0.2968 & 72.3648 & 69.6989 & \\ \text { S35R06 } & 7.3253 & 0.4581 & 80.7344 & 72.9510 & \\ \text { S35R07 } & 19.3180 & 0.2891 & 89.0418 & 69.4347 & \\ \text { S35R08 } & 30.9753 & 0.1941 & 96.1933 & 65.0239 & \\ \text { S35R09 } & 39.3757 & 0.1557 & 101.4421 & 61.9108 & \\ \text { S35R10 } & 43.8843 & 0.1405 & 104.3196 & 60.2948 & & \\ \text { S35R11 } & 44.3055 & 0.1392 & 104.5907 & 60.1460 & & \\ \text { S35R12 } & 40.6236 & 0.1512 & 102.2341 & 61.4593 & \\ \text { S35R13 } & 32.9895 & 0.1834 & 97.4400 & 64.2671 & \\ \text { S35R14 } & 21.8961 & 0.2618 & 90.6267 & 68.4687 & & \end{array}$


HNF-4799, Rev 0

August 25, 1999

\section{REVISED METHODOLOGY FOR DETERMINING CESIUM-137 CONTENT OF HN-200 GROUT CONTAINERS.}

$\begin{array}{lllll}\text { S35R15 } & 9.4154 & 0.4352 & 82.4790 & 72.6285 \\ \text { S35R 16 } & 2.8499 & 0.3385 & 74.0140 & 70.8257\end{array}$

TABLE 4- Calculate Microshield input data sheets for the simulations from each source to each measuring point in centimeters.

$\begin{array}{ccccccc} & \text { Cs-137 } & \text { Ba-137m } & \text { Source 01 } & \text { X Posit } & \text { Z Posit } & \text { Y Posit } \\ \text { Line } & 29.4975 & 27.9047 & & 49.1940 & 1.4778 & 65.7946 \\ \text { Shield 1 } & \text { Shield 2 } & \text { Total Dist } & \text { Air Gap } & & \\ \text { S01R01 } & 8.7005 & 0.3513 & 161.4884 & 152.4366 & & \\ \text { S01R03 } & 9.5634 & 0.3799 & 165.5837 & 155.6403 & & \\ \text { S01R04 } & 13.1668 & 0.4833 & 178.0700 & 164.4200 & \\ \text { S01R05 } & 41.5641 & 0.6325 & 195.6990 & 172.5024 & \\ \text { S01R06 } & 66.4481 & 0.5037 & 232.7263 & 165.7745 & \\ \text { S01R07 } & 87.7762 & 0.4128 & 247.0236 & 158.8347 & \\ \text { S01R08 } & 101.8204 & 0.3663 & 256.3506 & 154.1638 & \\ \text { S01R09 } & 107.1031 & 0.3511 & 259.8683 & 152.4141 & \\ \text { S01R10 } & 103.2149 & 0.3622 & 257.2784 & 153.7012 & \\ \text { S01R11 } & 90.4509 & 0.4032 & 248.7995 & 157.9454 & & \\ \text { S01R12 } & 70.0679 & 0.4864 & 235.1850 & 164.6307 & & \\ \text { S01R13 } & 45.5537 & 0.6122 & 217.7789 & 171.6130 & & \\ & & & & & \end{array}$




\section{REVISED METHODOLOGY FOR DETERMINING CESIUM-137 CONTENT OF HN-200 GROUT CONTAINERS.}

$\begin{array}{lcccc}\text { SO1R14 } & 24.8440 & 0.6475 & 198.6167 & 173.1252 \\ \text { S01R15 } & 14.1081 & 0.5058 & 180.5255 & 165.9117 \\ \text { SO1R16 } & 9.8961 & 0.3906 & 167.0165 & 156.7298\end{array}$

$\begin{array}{ccccccc} & \text { Cs-137 } & \text { Ba-137m } & \text { Source 02 } & \text { X Posit } & \text { Z Posit } & \text { Y Posit } \\ & 13.8502 & 13.1023 & & -26.9687 & -49.7051 & 47.9195 \\ \text { Line } & \text { Shield 1 } & \text { Shield 2 } & \text { Total Dist } & \text { Air Gap } & & \\ \text { S02R01 } & 75.4733 & 0.5259 & 243.5973 & 167.5981 & & \\ \text { S02R02 } & 98.7674 & 0.4072 & 257.8543 & 158.6798 & \\ \text { S02R03 } & 111.8946 & 0.3608 & 266.1199 & 153.8645 & \\ \text { S02R04 } & 114.4021 & 0.3531 & 267.7184 & 152.9632 & \\ \text { S02R05 } & 106.2177 & 0.3795 & 262.5242 & 155.9269 & \\ \text { S02R06 } & 87.5920 & 0.4568 & 250.9515 & 162.9026 & \\ \text { S02R07 } & 59.3405 & 0.6558 & 233.9888 & 173.9924 & \\ \text { S02R08 } & 24.8311 & 1.2688 & 213.2923 & 187.1924 & \\ \text { S02R09 } & 5.0328 & 1.1021 & 191.3493 & 185.2144 & \\ \text { S02R10 } & 2.1416 & 0.5413 & 171.6549 & 168.9720 & \\ \text { S02R11 } & 1.4799 & 0.3810 & 158.5385 & 156.6776 & \\ \text { S02R12 } & 1.3853 & 0.3574 & 155.8239 & 154.0813 & \\ \text { S02R13 } & 1.7266 & 0.4418 & 164.4239 & 162.2555 & \\ \text { S02R14 } & 3.0873 & 0.7521 & 181.5974 & 177.7580 & \\ \text { S02R15 } & 11.6339 & 1.5429 & 202.9865 & 189.8097 & \\ \text { S02R16 } & 43.4559 & 0.8577 & 224.6345 & 180.3209 & & \\ & & & & & \end{array}$

$\begin{array}{ccccccc} & \text { Cs-137 } & \text { Ba-137m } & \text { Source 03 } & \text { X Posit } & \text { Z Posit } & \text { Y Posit } \\ & 23.5916 & 22.3177 & & 4.4969 & -18.4379 & 106.3939 \\ \text { Line } & \text { Shield 1 } & \text { Shield 2 } & \text { Total Dist } & \text { Air Gap } & & \\ \text { S03R01 } & 52.8376 & 0.3773 & 210.5279 & 157.3131 & & \\ \text { S03R02 } & 60.3785 & 0.3761 & 217.8051 & 157.0505 & & \\ \text { S03R03 } & 67.8345 & 0.3696 & 224.4326 & 156.2285 & & \\ \text { S03R04 } & 73.8558 & 0.3620 & 229.4964 & 155.2787 & \\ \text { S03R05 } & 77.3515 & 0.3568 & 232.3466 & 154.6383 & \\ \text { S03R06 } & 77.7081 & 0.3563 & 232.6343 & 154.5699 & \\ \text { S03R07 } & 74.8642 & 0.3605 & 230.3247 & 155.1000 & \\ \text { S03R08 } & 69.3157 & 0.3679 & 225.6988 & 156.0152 & \\ \text { S03R09 } & 62.0618 & 0.3750 & 219.3429 & 156.9061 & \\ \text { S03R10 } & 54.4236 & 0.3776 & 212.1187 & 157.3175 & \\ \text { S03R11 } & 47.6970 & 0.3736 & 205.0937 & 157.0231 & & \\ \text { S03R12 } & 42.7955 & 0.3657 & 199.4111 & 156.2498 & & \\ \text { S03R13 } & 40.1521 & 0.3593 & 196.0825 & 155.5712 & \\ \text { S03R14 } & 39.8904 & 0.3585 & 195.7413 & 155.4923 & & \\ \text { S03R15 } & 42.0181 & 0.3640 & 198.4539 & 156.0718 & & \\ \text { S03R16 } & 46.4491 & 0.3721 & 203.6994 & 156.8783 & & \\ & & & & & \end{array}$


HNF-4799, Rev 0

August 25, 1999

\section{REVISED METHODOLOGY FOR DETERMINING CESIUM-137 CONTENT OF HN-200 GROUT CONTAINERS.}

$\begin{array}{ccccccc} & \text { Cs-137 } & \text { Ba-137m } & \text { Source 04 } & \text { X Posit } & \text { Z Posit } & \text { Y Posit } \\ \text { Line } & 20.6982 & 19.5805 & & 14.0365 & -48.8135 & 72.8360 \\ \text { Shield 1 } & \text { Shield 2 } & \text { Total Dist } & \text { Air Gap } & & \\ \text { S04R01 } & 26.0256 & 0.7222 & 202.6524 & 175.9045 & & \\ \text { S04R03 } & 49.6540 & 0.6194 & 222.2353 & 171.9619 & & \\ \text { S04R04 } & 75.1423 & 0.4748 & 239.4634 & 163.8462 & \\ \text { S04R05 } & 106.3005 & 0.3580 & 259.9002 & 153.2416 & \\ \text { S04R06 } & 108.3279 & 0.3522 & 261.2389 & 152.5589 & \\ \text { S04R07 } & 100.8825 & 0.3745 & 256.3322 & 155.0752 & \\ \text { S04R08 } & 84.4937 & 0.4336 & 245.5931 & 160.6659 & \\ \text { S04R09 } & 60.8844 & 0.5502 & 229.9907 & 168.5561 & \\ \text { S04R10 } & 35.1024 & 0.7051 & 211.1285 & 175.3211 & \\ \text { S04R11 } & 17.3730 & 0.6519 & 191.3665 & 173.3416 & \\ \text { S04R12 } & 10.0939 & 0.4676 & 173.9089 & 163.3474 & \\ \text { S04R13 } & 7.5606 & 0.3698 & 162.5289 & 154.5984 & \\ \text { S04R14 } & 7.1912 & 0.3542 & 160.3684 & 152.8230 & \\ \text { S04R15 } & 8.6607 & 0.4144 & 168.1001 & 159.0250 & \\ \text { S04R16 } & 13.3611 & 0.5677 & 183.4335 & 169.5047 & & \\ & & & & & \end{array}$

$\begin{array}{cccccc}\text { Cs-137 } & \text { Ba-137m } & \text { Source 05 } & \begin{array}{c}\text { X Posit } \\ \text { Z Posit }\end{array} & \text { Y Posit } \\ \text { 10.9510 } & 10.3596 & & -31.7933 & 6.1732 & 92.3496 \\ \text { Shield 1 } & \text { Shield 2 } & \text { Total Dist } & \text { Air Gap } & & \\ 90.0541 & 0.3543 & 243.7579 & 153.3495 & & \\ 84.4285 & 0.3667 & 239.5892 & 154.7941 & & \\ 73.9814 & 0.3895 & 231.6365 & 157.2657 & & \\ 60.6046 & 0.4148 & 220.7848 & 159.7654 & & \\ 47.1313 & 0.4257 & 208.3789 & 160.8220 & \\ 36.3252 & 0.4094 & 196.1980 & 159.4634 & \\ 29.4121 & 0.3789 & 186.3109 & 156.5199 & \\ 26.1669 & 0.3574 & 180.6981 & 154.1738 & \\ 26.1307 & 0.3571 & 180.6306 & 154.1429 & \\ 29.2971 & 0.3782 & 186.1248 & 156.4495 & & \\ 36.1190 & 0.4087 & 195.9335 & 159.4058 & & \\ 46.8409 & 0.4256 & 208.0853 & 160.8187 & \\ 60.2799 & 0.4153 & 220.5075 & 159.8123 & \\ 73.6938 & 0.3901 & 231.4125 & 157.3286 & \\ 84.2348 & 0.3671 & 239.4446 & 154.8426 & \\ 89.9862 & 0.3545 & 243.7081 & 153.3674 & & \end{array}$




\section{REVISED METHODOLOGY FOR DETERMINING CESIUM-137 CONTENT OF HN-200 GROUT CONTAINERS.}

$\begin{array}{ccccccc} & \text { Cs-137 } & \text { Ba-137m } & \text { Source 06 } & \text { X Posit } & \text { Z Posit } & \text { Y Posit } \\ & 16.3182 & 15.4370 & & 25.7762 & -17.3149 & 75.2927 \\ \text { Line } & \text { Shield 1 } & \text { Shield 2 } & \text { Total Dist } & \text { Air Gap } & & \\ \text { S06R01 } & 30.4515 & 0.3733 & 185.8394 & 155.0146 & & \\ \text { S06R02 } & 37.1568 & 0.4005 & 195.3323 & 157.7750 & & \\ \text { S06R03 } & 47.5008 & 0.4154 & 207.0643 & 159.1480 & \\ \text { S06R04 } & 60.2956 & 0.4067 & 219.0440 & 158.3417 & \\ \text { S06R05 } & 72.9916 & 0.3846 & 229.5463 & 156.1702 & \\ \text { S06R06 } & 82.9397 & 0.3640 & 237.2598 & 153.9562 & \\ \text { S06R07 } & 88.3240 & 0.3526 & 241.3175 & 152.6409 & \\ \text { S06R08 } & 88.2892 & 0.3527 & 241.2914 & 152.6495 & \\ \text { S06R09 } & 82.8406 & 0.3642 & 237.1844 & 153.9796 & \\ \text { S06R10 } & 72.8448 & 0.3849 & 229.4299 & 156.2003 & \\ \text { S06R11 } & 60.1300 & 0.4070 & 218.9000 & 158.3630 & \\ \text { S06R12 } & 47.3520 & 0.4154 & 206.9119 & 159.1445 & \\ \text { S06R13 } & 37.0496 & 0.4002 & 195.1953 & 157.7455 & \\ \text { S06R14 } & 30.3908 & 0.3730 & 185.7433 & 154.9795 & \\ \text { S06R15 } & 27.2547 & 0.3539 & 180.4403 & 152.8318 & \\ \text { S06R16 } & 27.2739 & 0.3540 & 180.4751 & 152.8471 & & \\ & & & & & \end{array}$

$\begin{array}{cccccc}\text { Cs-137 } & \text { Ba-137m } & \text { Source 07 } & \text { X Posit } & \text { Z Posit } & \text { Y Posit } \\ 25.9358 & 24.5353 & & -7.6301 & -46.9452 & 3.8559 \\ \text { Shield 1 } & \text { Shield 2 } & \text { Total Dist } & \text { Air Gap } & & \\ 56.8435 & 0.5645 & 232.2981 & 174.8901 & & \\ 79.8661 & 0.4602 & 247.5618 & 167.2355 & & \\ 97.4623 & 0.3957 & 258.8567 & 160.9987 & & \\ 107.1074 & 0.3661 & 265.0498 & 157.5764 & & \\ 107.9134 & 0.3638 & 265.5687 & 157.2914 & \\ 99.8177 & 0.3881 & 260.3670 & 160.1611 & \\ 83.5286 & 0.4456 & 249.9209 & 165.9467 & \\ 61.1644 & 0.5439 & 235.2589 & 173.5507 & \\ 38.0082 & 0.6369 & 218.0204 & 179.3752 & \\ 21.9572 & 0.5830 & 200.5206 & 177.9804 & \\ 14.3879 & 0.4585 & 185.7111 & 170.8646 & & \\ 11.5199 & 0.3880 & 176.7596 & 164.8517 & \\ 11.3114 & 0.3824 & 175.9789 & 164.2851 & \\ 13.6184 & 0.4409 & 183.5877 & 169.5283 & \\ 20.0819 & 0.5600 & 197.5726 & 176.9307 & \\ 34.4236 & 0.6407 & 214.8223 & 179.7580 & & \\ & & & & \end{array}$


HNF-4799, Rev 0

August 25, 1999

\section{REVISED METHODOLOGY FOR DETERMINING CESIUM-137 CONTENT OF HN-200 GROUT CONTAINERS.}

$\begin{array}{ccccccc} & \text { Cs-137 } & \text { Ba-137m } & \text { Source 08 } & \text { X Posit } & \text { Z Posit } & \text { Y Posit } \\ & 25.1678 & 23.8087 & & -3.6748 & -23.8753 & 52.9898 \\ \text { Line } & \text { Shield 1 } & \text { Shield 2 } & \text { Total Dist } & \text { Air Gap } & & \\ \text { S08R01 } & 59.4443 & 0.3842 & 216.1796 & 156.3511 & & \\ \text { S08R02 } & 69.0952 & 0.3733 & 224.6448 & 155.1764 & & \\ \text { S08R03 } & 76.9102 & 0.3610 & 231.0695 & 153.7983 & \\ \text { S08R04 } & 81.4429 & 0.3531 & 234.6729 & 152.8769 & \\ \text { S08R05 } & 81.9120 & 0.3523 & 235.0416 & 152.7773 & \\ \text { S08R06 } & 78.2397 & 0.3587 & 232.1344 & 153.5360 & \\ \text { S08R07 } & 71.0508 & 0.3704 & 226.2820 & 154.8608 & \\ \text { S08R08 } & 61.6546 & 0.3823 & 218.1845 & 156.1476 & \\ \text { S08R09 } & 51.8576 & 0.3869 & 208.8949 & 156.6504 & \\ \text { S08R10 } & 43.4329 & 0.3799 & 199.7636 & 155.9508 & \\ \text { S08R11 } & 37.4937 & 0.3657 & 192.2958 & 154.4364 & \\ \text { S08R12 } & 34.3809 & 0.3543 & 187.8814 & 153.1461 & \\ \text { S08R13 } & 34.0720 & 0.3531 & 187.4197 & 152.9946 & \\ \text { S08R14 } & 36.5563 & 0.3626 & 191.0089 & 154.0899 & \\ \text { S08R15 } & 41.8761 & 0.3770 & 197.9072 & 155.6540 & \\ \text { S08R16 } & 49.8142 & 0.3864 & 206.8002 & 156.5996 & \\ & & & & & \end{array}$

$\begin{array}{ccccccc} & \text { Cs-137 } & \text { Ba-137m } & \text { Source 09 } & \begin{array}{c}\text { X Posit } \\ \text { Z Posit }\end{array} & \text { Y Posit } \\ \text { Line } & 24.9347 & 23.5882 & & -28.7630 & -3.2708 & 115.5217 \\ \text { Shield 1 } & \text { Shield 2 } & \text { Total Dist } & \text { Air Gap } & & \\ \text { S09R02 } & 88.3374 & 0.3583 & 244.1274 & 155.4317 & & \\ \text { S09R03 } & 87.2404 & 0.3606 & 243.3170 & 155.7160 & & \\ \text { S09R04 } & 71.1905 & 0.3731 & 238.7955 & 157.2319 & \\ \text { S09R05 } & 59.1120 & 0.3925 & 231.0638 & 159.4824 & \\ \text { S09R06 } & 47.4315 & 0.4143 & 210.0386 & 161.5154 & \\ \text { S09R07 } & 38.2696 & 0.3990 & 199.7126 & 161.0441 & \\ \text { S09R08 } & 32.5093 & 0.3767 & 191.8622 & 158.9762 & \\ \text { S09R09 } & 30.0649 & 0.3636 & 188.0458 & 157.6173 & \\ \text { S09R10 } & 30.7138 & 0.3673 & 189.0935 & 158.0123 & \\ \text { S09R11 } & 34.5264 & 0.3858 & 194.7723 & 159.8600 & \\ \text { S09R12 } & 41.7529 & 0.4073 & 203.8848 & 161.7246 & \\ \text { S09R13 } & 52.1690 & 0.4152 & 214.7129 & 162.1287 & \\ \text { S09R14 } & 64.3247 & 0.4039 & 225.4864 & 160.7578 & \\ \text { S09R15 } & 75.8042 & 0.3838 & 234.6793 & 158.4912 & \\ \text { S09R16 } & 84.3117 & 0.3667 & 241.1398 & 156.4614 & & \\ & & & & & \end{array}$


REVISED METHODOLOGY FOR DETERMINING CESIUM-137 CONTENT OF HN-200 GROUT CONTAINERS.

$\begin{array}{ccccccc} & \text { Cs-137 } & \text { Ba-137m } & \text { Source 10 } & \text { X Posit } & \text { Z Posit } & \text { Y Posit } \\ \text { Line } & 26.8139 & 25.3659 & & -20.7250 & 26.7906 & 117.9524 \\ \text { S10R01 } & 78.0045 & 0.3950 & 238.2395 & 159.8400 & & \\ \text { S10R02 } & 64.0805 & 0.4256 & 227.5388 & 163.0327 & & \\ \text { S10R03 } & 49.5098 & 0.4441 & 214.9864 & 165.0325 & & \\ \text { S10R04 } & 37.4768 & 0.4317 & 202.3351 & 164.4266 & \\ \text { S10R05 } & 29.6101 & 0.3979 & 191.6734 & 161.6653 & \\ \text { S10R06 } & 25.7060 & 0.3707 & 185.0848 & 159.0081 & \\ \text { S10R07 } & 25.1463 & 0.3662 & 184.0390 & 158.5265 & \\ \text { S10R08 } & 27.8148 & 0.3864 & 188.7841 & 160.5829 & \\ \text { S10R09 } & 34.2052 & 0.4207 & 198.2278 & 163.6019 & \\ \text { S10R10 } & 44.8357 & 0.4436 & 210.4246 & 165.1454 & \\ \text { S10R11 } & 58.8296 & 0.4348 & 223.2337 & 163.9694 & & \\ \text { S10R12 } & 73.3636 & 0.4058 & 234.7614 & 160.9921 & & \\ \text { S10R13 } & 85.2446 & 0.3780 & 243.5439 & 157.9214 & \\ \text { S10R14 } & 92.2684 & 0.3615 & 248.5881 & 155.9582 & & \\ \text { S10R15 } & 93.3572 & 0.3590 & 249.3633 & 155.6471 & & \\ \text { S10R16 } & 88.3586 & 0.3706 & 245.7905 & 157.0612 & & \\ & & & & & \end{array}$

$\begin{array}{cccccc}\text { Cs-137 } & \text { Ba-137m } & \text { Source 11 } & \begin{array}{c}\text { X Posit } \\ \text { Z Posit }\end{array} & \text { Y Posit } \\ \text { 12.5933 } & 11.9133 & & 33.1572 & 16.3372 & 74.1470 \\ \text { Shield 1 } & \text { Shield 2 } & \text { Total Dist } & \text { Air Gap } & & \\ 23.0905 & 0.3724 & 178.3621 & 154.8992 & & \\ 21.0017 & 0.3517 & 173.9034 & 152.5499 & & \\ 22.0337 & 0.3623 & 176.1749 & 153.7789 & & \\ 26.4781 & 0.4002 & 184.6013 & 157.7231 & & \\ 35.2952 & 0.4432 & 197.2301 & 161.4917 & & \\ 48.8497 & 0.4534 & 211.5886 & 162.2855 & & \\ 64.9876 & 0.4250 & 225.3752 & 159.9626 & & \\ 79.8665 & 0.3881 & 236.7601 & 156.5056 & \\ 90.3759 & 0.3619 & 244.4439 & 153.7061 & & \\ 94.8107 & 0.3514 & 247.6359 & 152.4739 & & \\ 92.5686 & 0.3567 & 246.0251 & 153.0999 & & \\ 83.9460 & 0.3778 & 239.7672 & 155.4433 & & \\ 70.2435 & 0.4123 & 229.4911 & 158.8353 & \\ 54.0964 & 0.4470 & 216.3243 & 161.7809 & & \\ 39.3090 & 0.4517 & 201.9207 & 162.1600 & & \\ 28.8659 & 0.4157 & 188.4425 & 159.1609 & & \\ & & & & \end{array}$




\section{REVISED METHODOLOGY FOR DETERMINING CESIUM-137 CONTENT} OF HN-200 GROUT CONTAINERS.

$\begin{array}{ccccccc} & \text { Cs-137 } & \text { Ba-137m } & \text { Source 12 } & \text { X Posit } & \text { Z Posit } & \text { Y Posit } \\ \text { Line } & 17.5307 & 16.5841 & & 43.4057 & 22.2267 & 54.0716 \\ \text { S12R01 } & 10.7646 & 0.4009 & 169.2953 & 158.1298 & & \\ \text { S12R02 } & 9.2206 & 0.3537 & 162.6940 & 153.1197 & & \\ \text { S12R03 } & 9.8370 & 0.3731 & 165.5160 & 155.3059 & & \\ \text { S12R04 } & 13.0803 & 0.4632 & 176.9139 & 163.3704 & \\ \text { S12R05 } & 21.5682 & 0.6060 & 193.8662 & 171.6920 & \\ \text { S12R06 } & 39.4650 & 0.6308 & 212.8272 & 172.7313 & \\ \text { S12R07 } & 63.5338 & 0.5151 & 230.7778 & 166.7289 & \\ \text { S12R08 } & 85.3107 & 0.4209 & 245.4808 & 159.7492 & \\ \text { S12R09 } & 100.2486 & 0.3705 & 255.4144 & 154.7953 & \\ \text { S12R10 } & 106.6360 & 0.3521 & 259.6694 & 152.6813 & \\ \text { S12R11 } & 103.9515 & 0.3596 & 257.8798 & 153.5687 & & \\ \text { S12R12 } & 92.4023 & 0.3956 & 250.1979 & 157.3999 & \\ \text { S12R13 } & 73.0998 & 0.4705 & 237.3043 & 163.7340 & \\ \text { S12R14 } & 49.1256 & 0.5878 & 220.4589 & 170.7455 & \\ \text { S12R15 } & 27.6745 & 0.6429 & 201.5915 & 173.2741 & & \\ \text { S12R16 } & 15.6745 & 0.5206 & 183.4031 & 167.2081 & & \\ & & & & & \end{array}$

\begin{tabular}{|c|c|c|c|c|c|}
\hline Cs-137 & Ba-137m & Source 13 & X Posit & Z Posit & Y Posit \\
\hline 17.9331 & 16.9647 & & 8.9211 & 21.0922 & 105.2762 \\
\hline Shield 1 & Shield 2 & Total Dist & Air Gap & & \\
\hline 47.7159 & 0.3854 & 206.2464 & 158.1451 & & \\
\hline 41.0446 & 0.3745 & 198.5522 & 157.1330 & & \\
\hline 37.0022 & 0.3627 & 193.2737 & 155.9088 & & \\
\hline 35.6947 & 0.3579 & 191.4359 & 155.3834 & & \\
\hline 37.1054 & 0.3631 & 193.4157 & 155.9472 & & \\
\hline 41.2513 & 0.3750 & 198.8077 & 157.1814 & & \\
\hline 48.0146 & 0.3857 & 206.5679 & 158.1676 & & \\
\hline 56.7569 & 0.3874 & 215.3325 & 158.1882 & & \\
\hline 66.1182 & 0.3796 & 223.7296 & 157.2318 & & \\
\hline 74.3550 & 0.3680 & 230.5854 & 155.8624 & & \\
\hline 79.9226 & 0.3588 & 235.0274 & 154.7460 & & \\
\hline 81.8365 & 0.3555 & 236.5267 & 154.3346 & & \\
\hline 79.7741 & 0.3591 & 234.9104 & 154.7773 & & \\
\hline 74.0833 & 0.3684 & 230.3651 & 155.9134 & & \\
\hline 65.7729 & 0.3800 & 223.4330 & 157.2801 & & \\
\hline 56.4038 & 0.3875 & 214.9988 & 158.2075 & & \\
\hline
\end{tabular}

Cs-137 Ba-137m Source $14 \quad$ X Posit $\quad$ Z Posit $\quad$ Y Posit 


\section{REVISED METHODOLOGY FOR DETERMINING CESIUM-137 CONTENT OF HN-200 GROUT CONTAINERS.}

$\begin{array}{ccccccc}\text { Line } & 26.2138 & 24.7983 & & -46.3340 & 15.5761 & 101.4000 \\ \text { Shield 1 } & \text { Shield 2 } & \text { Total Dist } & \text { Air Gap } & & \\ \text { S14R02 } & 104.5535 & 0.3628 & 259.6313 & 154.7149 & \\ \text { S14R03 } & 73.6669 & 0.4005 & 251.8156 & 158.7482 & \\ \text { S14R04 } & 48.7487 & 0.5996 & 222.0236 & 172.6752 & \\ \text { S14R05 } & 27.2920 & 0.6523 & 203.2838 & 175.3395 & \\ \text { S14R06 } & 15.4947 & 0.5247 & 185.3465 & 169.3271 & \\ \text { S14R07 } & 10.7185 & 0.4052 & 171.5886 & 160.4650 & \\ \text { S14R08 } & 9.2498 & 0.3594 & 165.3479 & 155.7387 & \\ \text { S14R09 } & 9.9319 & 0.3812 & 168.4200 & 158.1069 & \\ \text { S14R10 } & 13.2877 & 0.4750 & 179.8963 & 166.1336 & \\ \text { S14R11 } & 22.0607 & 0.6204 & 196.7828 & 174.1018 & \\ \text { S14R12 } & 40.4481 & 0.6378 & 215.5906 & 174.5047 & \\ \text { S14R13 } & 64.8122 & 0.5171 & 233.3439 & 168.0147 & \\ \text { S14R14 } & 86.6020 & 0.4223 & 247.8315 & 160.8073 & \\ \text { S14R15 } & 101.3901 & 0.3722 & 257.5484 & 155.7860 & \\ \text { S14R16 } & 107.5364 & 0.3543 & 261.5986 & 153.7079 & \end{array}$

$\begin{array}{cccccr}\text { Cs-137 } & \text { Ba-137m } & \text { Source 15 } & \text { X Posit } & \text { Z Posit } & \text { Y Posit } \\ 22.7469 & 21.5186 & & 53.3465 & 13.7354 & 27.1923 \\ \text { Shield 1 } & \text { Shield 2 } & \text { Total Dist } & \text { Air Gap } & & \\ 3.0831 & 0.3820 & 163.0870 & 159.6219 & & \\ 2.9691 & 0.3687 & 161.5354 & 158.1976 & & \\ 3.7483 & 0.4577 & 170.5534 & 166.3474 & & \\ 6.6024 & 0.7371 & 187.4187 & 180.0791 & \\ 18.9341 & 1.1282 & 208.0539 & 187.9916 & \\ 48.8419 & 0.7476 & 228.7536 & 179.1641 & \\ 78.6312 & 0.5032 & 246.7275 & 167.5931 & \\ 100.3184 & 0.4021 & 260.0463 & 159.3259 & \\ 112.1640 & 0.3617 & 267.4875 & 154.9618 & \\ 113.6457 & 0.3571 & 268.4275 & 154.4246 & \\ 104.7096 & 0.3861 & 262.7899 & 157.6943 & & \\ 85.7167 & 0.4653 & 251.0403 & 164.8583 & \\ 57.8834 & 0.6548 & 234.2231 & 175.6849 & \\ 26.2533 & 1.0598 & 214.0532 & 186.7401 & \\ 8.4496 & 0.8738 & 193.0610 & 183.7376 & \\ 4.2295 & 0.5102 & 174.7080 & 169.9683 & & \\ & & & & \end{array}$

$\begin{array}{cccccc}\text { Cs-137 } & \text { Ba-137m } & \text { Source 16 } & \text { X Posit } & \text { Z Posit } & \text { Y Posit } \\ 27.6556 & 26.1622 & & 32.4610 & 26.2430 & 99.8427\end{array}$ 


\section{REVISED METHODOLOGY FOR DETERMINING CESIUM-137 CONTENT OF HN-200 GROUT CONTAINERS.}

$\begin{array}{ccccc}\text { Line } & \text { Shield 1 } & \text { Shield 2 } & \text { Total Dist } & \text { Air Gap } \\ \text { S16R01 } & 21.1405 & 0.4199 & 182.9248 & 161.3644 \\ \text { S16R02 } & 17.2216 & 0.3689 & 173.9866 & 156.3962 \\ \text { S16R03 } & 16.5558 & 0.3588 & 172.1888 & 155.2742 \\ \text { S16R04 } & 18.8537 & 0.3918 & 178.0145 & 158.7690 \\ \text { S16R05 } & 25.0818 & 0.4579 & 189.9507 & 164.4110 \\ \text { S16R06 } & 37.0697 & 0.5089 & 205.3211 & 167.7425 \\ \text { S16R07 } & 54.7031 & 0.4884 & 221.3381 & 166.1466 \\ \text { S16R08 } & 73.6676 & 0.4310 & 235.6742 & 161.5756 \\ \text { S16R09 } & 89.1538 & 0.3843 & 246.6061 & 157.0681 \\ \text { S16R10 } & 98.3840 & 0.3591 & 252.9917 & 154.2486 \\ \text { S16R11 } & 100.1656 & 0.3545 & 254.2188 & 153.6986 \\ \text { S16R12 } & 94.3009 & 0.3700 & 250.1739 & 155.5030 \\ \text { S16R13 } & 81.4784 & 0.4069 & 241.2360 & 159.3507 \\ \text { S16R14 } & 63.6425 & 0.4625 & 228.2968 & 164.1917 \\ \text { S16R15 } & 44.7108 & 0.5082 & 212.8042 & 167.5851 \\ \text { S16R16 } & 29.8191 & 0.4881 & 196.8091 & 166.5018\end{array}$

$\begin{array}{ccccccc} & \text { Cs-137 } & \text { Ba-137m } & \text { Source 17 } & \text { X Posit } & \text { Z Posit } & \text { Y Posit } \\ \text { Line } & \text { Sh.5227 } & 26.9825 & & -36.8053 & 7.8697 & 22.0830 \\ \text { S17R01 } & 96.1672 & 0.3594 & 251.8034 & 155.2768 & & \\ \text { S17R02 } & 89.1643 & 0.3770 & 246.8922 & 157.3509 & & \\ \text { S17R03 } & 76.3806 & 0.4107 & 237.7364 & 160.9451 & & \\ \text { S17R04 } & 60.0569 & 0.4518 & 225.3199 & 164.8112 & & \\ \text { S17R05 } & 43.9049 & 0.4720 & 211.1454 & 166.7686 & \\ \text { S17R06 } & 31.6530 & 0.4454 & 197.2323 & 165.1339 & \\ \text { S17R07 } & 24.4333 & 0.3975 & 185.9799 & 161.1492 & \\ \text { S17R08 } & 21.3037 & 0.3668 & 179.7296 & 158.0591 & \\ \text { S17R09 } & 21.4166 & 0.3680 & 179.9754 & 158.1908 & \\ \text { S17R10 } & 24.8063 & 0.4007 & 186.6559 & 161.4489 & & \\ \text { S17R11 } & 32.3707 & 0.4486 & 198.1857 & 165.3664 & & \\ \text { S17R12 } & 44.9837 & 0.4720 & 212.1960 & 166.7402 & & \\ \text { S17R13 } & 61.2913 & 0.4491 & 226.3045 & 164.5641 & & \\ \text { S17R14 } & 77.4669 & 0.4078 & 238.5280 & 160.6532 & & \\ \text { S17R15 } & 89.8873 & 0.3751 & 247.4018 & 157.1394 & & \\ \text { S17R16 } & 96.4188 & 0.3588 & 251.9791 & 155.2015 & & \\ & & & & & & \end{array}$

$\begin{array}{lcccccc} & \text { Cs-137 } & \text { Ba-137m } & \text { Source 18 } & \text { X Posit } & \text { Z Posit } & \text { Y Posit } \\ \text { Line } & 23.2528 & 21.9972 & & 47.6621 & -30.4883 & 26.7230 \\ & \text { Shield 1 } & \text { Shield 2 } & \text { Total Dist } & \text { Air Gap } & & \end{array}$




\section{REVISED METHODOLOGY FOR DETERMINING CESIUM-137 CONTENT} OF HN-200 GROUT CONTAINERS.

$\begin{array}{lcccc}\text { S18R01 } & 1.8627 & 0.4854 & 170.8728 & 168.5247 \\ \text { S18R02 } & 3.6463 & 0.8845 & 188.7905 & 184.2598 \\ \text { S18R03 } & 16.1672 & 1.5416 & 210.1482 & 192.4395 \\ \text { S18R04 } & 50.4061 & 0.7756 & 231.2176 & 180.0359 \\ \text { S18R05 } & 81.3222 & 0.5006 & 249.2469 & 167.4242 \\ \text { S18R06 } & 102.9510 & 0.3987 & 262.3566 & 159.0069 \\ \text { S18R07 } & 114.2096 & 0.3602 & 269.3719 & 154.8022 \\ \text { S18R08 } & 114.7569 & 0.3585 & 269.7164 & 154.6010 \\ \text { S18R09 } & 104.5785 & 0.3927 & 263.3623 & 158.3912 \\ \text { S18R10 } & 83.9745 & 0.4855 & 250.8296 & 166.3696 \\ \text { S18R11 } & 53.8997 & 0.7314 & 233.2275 & 178.5963 \\ \text { S18R12 } & 19.1360 & 1.4674 & 212.3576 & 191.7542 \\ \text { S18R13 } & 4.0509 & 0.9619 & 190.8749 & 185.8621 \\ \text { S18R14 } & 1.9509 & 0.5070 & 172.4133 & 169.9553 \\ \text { S18R15 } & 1.4371 & 0.3785 & 161.2331 & 159.4175 \\ \text { S18R16 } & 1.4169 & 0.3734 & 160.6562 & 158.8660\end{array}$

\begin{tabular}{|c|c|c|c|c|c|c|}
\hline & Cs-137 & Ba-137m & Source 19 & X Posit & Z Posit & Y Posit \\
\hline & 15.3416 & 14.5131 & & -46.3763 & 0.7074 & 53.5943 \\
\hline Line & Shield 1 & Shield 2 & Total Dist & Air Gap & & \\
\hline S19R01 & 104.4478 & 0.3516 & 257.4388 & 152.6394 & & \\
\hline S19R02 & 99.8176 & 0.3644 & 254.3097 & 154.1276 & & \\
\hline S19R03 & 87.0172 & 0.4040 & 245.6443 & 158.2231 & & \\
\hline S19R04 & 67.5846 & 0.4764 & 232.2444 & 164.1834 & & \\
\hline S19R05 & 45.3160 & 0.5647 & 215.4933 & 169.6125 & & \\
\hline S19R06 & 27.0753 & 0.5671 & 197.4403 & 169.7979 & & \\
\hline S19R07 & 17.0120 & 0.4632 & 180.8542 & 163.3790 & & \\
\hline S19R08 & 12.7050 & 0.3789 & 169.0246 & 155.9407 & & \\
\hline S19R09 & 11.5742 & 0.3524 & 164.9120 & 152.9855 & & \\
\hline S19R10 & 12.9054 & 0.3834 & 169.6980 & 156.4091 & & \\
\hline S19R11 & 17.5410 & 0.4717 & 182.0157 & 164.0029 & & \\
\hline S19R12 & 28.1999 & 0.5723 & 198.8299 & 170.0578 & & \\
\hline S19R13 & 47.0003 & 0.5591 & 216.8718 & 169.3124 & & \\
\hline S19R14 & 69.2649 & 0.4697 & 233.4270 & 163.6924 & & \\
\hline S19R15 & 88.2786 & 0.3998 & 246.5007 & 157.8223 & & \\
\hline S19R16 & 100.4807 & 0.3625 & 254.7577 & 153.9145 & & \\
\hline
\end{tabular}

$\begin{array}{ccccccc} & \text { Cs-137 } & \text { Ba-137m } & \text { Source 20 } & \text { X Posit } & \text { Z Posit } & \text { Y Posit } \\ & 16.4988 & 15.6078 & & 7.2755 & 23.4343 & 112.9023 \\ \text { Line } & \text { Shield 1 } & \text { Shield 2 } & \text { Total Dist } & \text { Air Gap } & & \\ \text { S20R01 } & 49.2522 & 0.3950 & 209.6094 & 159.9622 & & \end{array}$




\section{REVISED METHODOLOGY FOR DETERMINING CESIUM-137 CONTENT OF HN-200 GROUT CONTAINERS.}

$\begin{array}{lllll}\text { S20R02 } & 41.4914 & 0.3844 & 200.9753 & 159.0996 \\ \text { S20R03 } & 36.4989 & 0.3698 & 194.5570 & 157.6883 \\ \text { S20R04 } & 34.4094 & 0.3615 & 191.5896 & 156.8187 \\ \text { S20R05 } & 35.1661 & 0.3646 & 192.6864 & 157.1557 \\ \text { S20R06 } & 38.7977 & 0.3774 & 197.6175 & 158.4425 \\ \text { S20R07 } & 45.3151 & 0.3912 & 205.3958 & 159.6894 \\ \text { S20R08 } & 54.2769 & 0.3960 & 214.6044 & 159.9315 \\ \text { S20R09 } & 64.4037 & 0.3887 & 223.7560 & 158.9635 \\ \text { S20R10 } & 73.8105 & 0.3753 & 231.5423 & 157.3565 \\ \text { S20R11 } & 80.6942 & 0.3633 & 236.9610 & 155.9035 \\ \text { S20R12 } & 83.8219 & 0.3576 & 239.3665 & 155.1870 \\ \text { S20R13 } & 82.6705 & 0.3597 & 238.4844 & 155.4542 \\ \text { S20R14 } & 77.4295 & 0.3691 & 234.4146 & 156.6160 \\ \text { S20R15 } & 69.0044 & 0.3827 & 227.6302 & 158.2430 \\ \text { S20R16 } & 58.9679 & 0.3939 & 218.9700 & 159.6081\end{array}$

$\begin{array}{ccccccc} & \text { Cs-137 } & \text { Ba-137m } & \text { Source 21 } & \text { X Posit } & \text { Z Posit } & \text { Y Posit } \\ \text { Line } & \text { 28.6466 } & 27.0997 & & 7.7777 & -20.1009 & 50.5073 \\ \text { S21R01 } & 48.4758 & 0.3773 & 204.6212 & 155.7682 & & \\ \text { S21R02 } & 56.6989 & 0.3788 & 212.9795 & 155.9018 & & \\ \text { S21R03 } & 65.4022 & 0.3723 & 220.9483 & 155.1738 & \\ \text { S21R04 } & 72.9883 & 0.3625 & 227.4139 & 154.0632 & \\ \text { S21R05 } & 78.0469 & 0.3547 & 231.5507 & 153.1491 & \\ \text { S21R06 } & 79.6804 & 0.3520 & 232.8629 & 152.8305 & \\ \text { S21R07 } & 77.6097 & 0.3554 & 231.1978 & 153.2327 & \\ \text { S21R08 } & 72.1895 & 0.3636 & 226.7493 & 154.1962 & \\ \text { S21R09 } & 64.3876 & 0.3734 & 220.0539 & 155.2929 & \\ \text { S21R10 } & 55.6590 & 0.3791 & 211.9749 & 155.9368 & \\ \text { S21R11 } & 47.5904 & 0.3765 & 203.6554 & 155.6885 & \\ \text { S21R12 } & 41.3957 & 0.3666 & 196.4091 & 154.6467 & \\ \text { S21R13 } & 37.6589 & 0.3564 & 191.5145 & 153.4992 & \\ \text { S21R14 } & 36.5159 & 0.3525 & 189.9170 & 153.0486 & \\ \text { S21R15 } & 37.9700 & 0.3574 & 191.9407 & 153.6133 & \\ \text { S21R16 } & 42.0139 & 0.3680 & 197.1760 & 154.7942 & & \\ & & & & & \end{array}$

$\begin{array}{ccccccc} & \text { Cs-137 } & \text { Ba-137m } & \text { Source 22 } & \text { X Posit } & \text { Z Posit } & \text { Y Posit } \\ & 20.3724 & 19.2723 & & -45.6646 & 32.6567 & 127.1095 \\ \text { Line } & \text { Shield 1 } & \text { Shield 2 } & \text { Total Dist } & \text { Air Gap } & & \\ \text { S22R01 } & 103.4629 & 0.4052 & 265.0846 & 161.2165 & \ldots & \\ \text { S22R02 } & 81.6034 & 0.5093 & 252.0715 & 169.9587 & & \end{array}$




\section{REVISED METHODOLOGY FOR DETERMINING CESIUM-137 CONTENT OF HN-200 GROUT CONTAINERS.}

$\begin{array}{lcccc}\text { S22R03 } & 50.6544 & 0.7821 & 234.3196 & 182.8832 \\ \text { S22R04 } & 17.4005 & 1.4077 & 213.7319 & 194.9237 \\ \text { S22R05 } & 4.7075 & 0.8510 & 193.0578 & 187.4993 \\ \text { S22R06 } & 2.5080 & 0.4904 & 175.9433 & 172.9449 \\ \text { S22R07 } & 1.9406 & 0.3845 & 166.4238 & 164.0986 \\ \text { S22R08 } & 1.9835 & 0.3927 & 167.3021 & 164.9259 \\ \text { S22R09 } & 2.6935 & 0.5241 & 178.2997 & 175.0822 \\ \text { S22R10 } & 5.5110 & 0.9592 & 196.2669 & 189.7967 \\ \text { S22R11 } & 21.9933 & 1.3254 & 217.1522 & 193.8335 \\ \text { S22R12 } & 56.1562 & 0.7162 & 237.4434 & 180.5710 \\ \text { S22R13 } & 85.8079 & 0.4856 & 254.5375 & 168.2440 \\ \text { S22R14 } & 106.0453 & 0.3955 & 266.6546 & 160.2138 \\ \text { S22R15 } & 115.8774 & 0.3625 & 272.6974 & 156.4576 \\ \text { S22R16 } & 115.0088 & 0.3652 & 272.1594 & 156.7854\end{array}$

$\begin{array}{ccccccc} & \text { Cs-137 } & \text { Ba-137m } & \text { Source 23 } & \text { X Posit } & \text { Z Posit } & \text { Y Posit } \\ & 13.0146 & 12.3118 & & 12.9519 & 37.1270 & 132.0896 \\ \text { Line } & \text { Shield 1 } & \text { Shield 2 } & \text { Total Dist } & \text { Air Gap } & & \\ \text { S23R01 } & 38.8784 & 0.4959 & 211.1443 & 171.7700 & & \\ \text { S23R02 } & 27.7911 & 0.4516 & 197.5126 & 169.2700 & & \\ \text { S23R03 } & 21.9183 & 0.3992 & 187.4017 & 165.0842 & \\ \text { S23R04 } & 19.8809 & 0.3752 & 183.0270 & 162.7709 & \\ \text { S23R05 } & 20.9821 & 0.3885 & 185.4627 & 164.0921 & \\ \text { S23R06 } & 25.6093 & 0.4349 & 194.1016 & 168.0574 & \\ \text { S23R07 } & 35.0610 & 0.4873 & 206.9702 & 171.4219 & \\ \text { S23R08 } & 50.0197 & 0.4948 & 221.5724 & 171.0579 & \\ \text { S23R09 } & 67.9335 & 0.4535 & 235.5754 & 167.1885 & \\ \text { S23R10 } & 84.2658 & 0.4061 & 247.1165 & 162.4446 & \\ \text { S23R11 } & 95.6286 & 0.3747 & 254.8694 & 158.8661 & \\ \text { S23R12 } & 100.3041 & 0.3626 & 258.0289 & 157.3622 & \\ \text { S23R13 } & 97.7194 & 0.3692 & 256.2836 & 158.1950 & \\ \text { S23R14 } & 88.1824 & 0.3950 & 249.8047 & 161.2273 & \\ \text { S23R15 } & 73.0275 & 0.4388 & 239.2512 & 165.7849 & \\ \text { S23R16 } & 55.1185 & 0.4860 & 225.7951 & 170.1907 & & \\ & & & & & \end{array}$

$\begin{array}{cccccc}\text { Cs-137 } & \text { Ba-137m } & \text { Source 24 } & \text { X Posit } & \text { Z Posit } & \text { Y Posit } \\ 12.5419 & 11.8646 & & 20.2228 & 48.7990 & 83.9044 \\ \text { Shield 1 } & \text { Shield 2 } & \text { Total Dist } & \text { Air Gap } & & \\ 17.4640 & 0.8090 & 197.2380 & 178.9650 & & \\ 8.5876 & 0.5462 & 177.9984 & 168.8645 & & \\ 5.7976 & 0.3948 & 163.8909 & 157.6985 & & \end{array}$




\section{REVISED METHODOLOGY FOR DETERMINING CESIUM-137 CONTENT OF HN-200 GROUT CONTAINERS.}

$\begin{array}{lcccc}\text { S24R04 } & 5.1128 & 0.3528 & 158.6428 & 153.1772 \\ \text { S24R05 } & 5.7988 & 0.3949 & 163.8995 & 157.7058 \\ \text { S24R06 } & 8.5916 & 0.5464 & 178.0132 & 168.8752 \\ \text { S24R07 } & 17.4768 & 0.8092 & 197.2557 & 178.9697 \\ \text { S24R08 } & 39.9551 & 0.7471 & 217.7773 & 177.0751 \\ \text { S24R09 } & 68.1886 & 0.5319 & 236.5238 & 167.8033 \\ \text { S24R10 } & 91.3664 & 0.4179 & 251.3225 & 159.5382 \\ \text { S24R11 } & 105.9785 & 0.3665 & 260.7417 & 154.3967 \\ \text { S24R12 } & 110.9365 & 0.3517 & 263.9676 & 152.6794 \\ \text { S24R13 } & 105.9700 & 0.3665 & 260.7361 & 154.3995 \\ \text { S24R14 } & 91.3500 & 0.4180 & 251.3120 & 159.5440 \\ \text { S24R15 } & 68.1658 & 0.5320 & 236.5093 & 167.8115 \\ \text { S24R16 } & 39.9311 & 0.7473 & 217.7601 & 177.0817\end{array}$

$\begin{array}{ccccccc} & \text { Cs-137 } & \text { Ba-137m } & \text { Source 25 } & \text { X Posit } & \text { Z Posit } & \text { Y Posit } \\ \text { Line } & 19.7625 & 18.6953 & & 21.3295 & 24.2720 & 101.2303 \\ \text { S25R01 } & 33.7744 & 0.4016 & 193.7616 & 159.5855 & & \\ \text { S25R02 } & 28.1830 & 0.3721 & 185.2409 & 156.6857 & & \\ \text { S25R03 } & 26.0817 & 0.3574 & 181.5064 & 155.0673 & & \\ \text { S25R04 } & 27.1341 & 0.3651 & 183.4210 & 155.9218 & \\ \text { S25R05 } & 31.5204 & 0.3913 & 190.5356 & 158.6239 & \\ \text { S25R06 } & 39.7338 & 0.4196 & 201.2990 & 161.1456 & \\ \text { S25R07 } & 51.6437 & 0.4269 & 213.6670 & 161.5964 & \\ \text { S25R08 } & 65.4271 & 0.4090 & 225.6530 & 159.8169 & \\ \text { S25R09 } & 78.1239 & 0.3829 & 235.6200 & 157.1131 & \\ \text { S25R10 } & 87.1581 & 0.3629 & 242.3761 & 154.8551 & \\ \text { S25R11 } & 90.9862 & 0.3545 & 245.1853 & 153.8446 & \\ \text { S25R12 } & 89.0344 & 0.3588 & 243.7563 & 154.3631 & \\ \text { S25R13 } & 81.5895 & 0.3753 & 238.2362 & 156.2715 & \\ \text { S25R14 } & 69.8584 & 0.4004 & 229.2140 & 158.9553 & \\ \text { S25R15 } & 56.0974 & 0.4232 & 217.7311 & 161.2105 & \\ \text { S25R16 } & 43.2852 & 0.4252 & 205.2830 & 161.5726 & \end{array}$

$\begin{array}{cccccr}\text { Cs-137 } & \text { Ba-137m } & \text { Source 26 } & \begin{array}{c}\text { X Posit } \\ \text { Z Posit }\end{array} & \text { Y Posit } \\ 24.8512 & 23.5093 & & 10.4368 & 41.1896 & 86.8515 \\ \text { Shield 1 } & \text { Shield 2 } & \text { Total Dist } & \text { Air Gap } & & \\ 37.6294 & 0.5164 & 205.2897 & 167.1439 & & \\ 24.9155 & 0.4673 & 189.3079 & 163.9251 & & \\ 18.2698 & 0.3952 & 176.5237 & 157.8587 & & \\ 15.7029 & 0.3563 & 169.7801 & 153.7208 & & \end{array}$




\section{REVISED METHODOLOGY FOR DETERMINING CESIUM-137 CONTENT OF HN-200 GROUT CONTAINERS.}

$\begin{array}{lclll}\text { S26R05 } & 16.0724 & 0.3623 & 170.8421 & 154.4073 \\ \text { S26R06 } & 19.5493 & 0.4122 & 179.4180 & 159.4565 \\ \text { S26R07 } & 27.6143 & 0.4861 & 193.3373 & 165.2369 \\ \text { S26R08 } & 42.0667 & 0.5160 & 209.6726 & 167.0899 \\ \text { S26R09 } & 61.2055 & 0.4712 & 225.6954 & 164.0187 \\ \text { S26R10 } & 79.7411 & 0.4116 & 239.2590 & 159.1062 \\ \text { S26R11 } & 93.4292 & 0.3713 & 248.8408 & 155.0403 \\ \text { S26R12 } & 100.1483 & 0.3534 & 253.4898 & 152.9881 \\ \text { S26R13 } & 99.1137 & 0.3561 & 252.7751 & 153.3054 \\ \text { S26R14 } & 90.4370 & 0.3797 & 246.7621 & 155.9454 \\ \text { S26R15 } & 75.1904 & 0.4260 & 236.0150 & 160.3986 \\ \text { S26R16 } & 55.9862 & 0.4871 & 221.6298 & 165.1564\end{array}$

$\begin{array}{ccccccr} & \text { Cs-137 } & \text { Ba-137m } & \text { Source 27 } & \text { X Posit } & \text { Z Posit } & \text { Y Posit } \\ \text { Line } & 10.2138 & 9.6622 & & 5.7653 & -53.5980 & 35.4870 \\ \text { S27R01 } & 30.9578 & 0.8947 & 214.2630 & 182.4105 & & \\ \text { S27R02 } & 60.7273 & 0.6039 & 233.9558 & 172.6246 & & \\ \text { S27R03 } & 86.8102 & 0.4484 & 250.1760 & 162.9175 & \\ \text { S27R04 } & 104.4309 & 0.3794 & 261.2877 & 156.4774 & \\ \text { S27R05 } & 112.2757 & 0.3548 & 266.3076 & 153.6771 & \\ \text { S27R06 } & 109.9612 & 0.3617 & 264.8214 & 154.4985 & \\ \text { S27R07 } & 97.5916 & 0.4037 & 256.9499 & 158.9546 & \\ \text { S27R08 } & 75.8639 & 0.5040 & 243.3591 & 166.9911 & \\ \text { S27R09 } & 47.0631 & 0.7242 & 225.3147 & 177.5274 & \\ \text { S27R10 } & 20.0110 & 0.9527 & 204.7928 & 183.8291 & \\ \text { S27R11 } & 8.3242 & 0.6528 & 184.6277 & 175.6507 & \\ \text { S27R12 } & 5.0491 & 0.4341 & 168.5353 & 163.0521 & \\ \text { S27R13 } & 4.1272 & 0.3617 & 160.4856 & 155.9968 & \\ \text { S27R14 } & 4.3760 & 0.3816 & 162.9265 & 158.1689 & \\ \text { S27R15 } & 6.0746 & 0.5095 & 175.0777 & 168.4936 & \\ \text { S27R16 } & 11.8590 & 0.8146 & 193.5246 & 180.8510 & \\ & & & & & \end{array}$

$\begin{array}{ccccccc} & \text { Cs-137 } & \text { Ba-137m } & \text { Source 28 } & \text { X Posit } & \text { Z Posit } & \text { Y Posit } \\ & 15.5133 & 14.6756 & & 46.7749 & -14.4208 & 16.4718 \\ \text { Line } & \text { Shield 1 } & \text { Shicld 2 } & \text { Total Dist } & \text { Air Gap } & & \\ \text { S28R01 } & 10.0134 & 0.3879 & 172.3838 & 161.9825 & & \\ \text { S28R02 } & 13.1756 & 0.4783 & 183.1432 & 169.4894 & & \\ \text { S28R03 } & 21.5587 & 0.6261 & 199.4608 & 177.2760 & & \\ \text { S28R04 } & 39.5570 & 0.6558 & 217.9339 & 177.7211 & & \\ \text { S28R05 } & 64.1268 & 0.5330 & 235.5829 & 170.9231 & & \end{array}$




\section{REVISED METHODOLOGY FOR DETERMINING CESIUM-137 CONTENT OF HN-200 GROUT CONTAINERS.}

$\begin{array}{lcccc}\text { S28R06 } & 86.4780 & 0.4324 & 250.1570 & 163.2466 \\ \text { S28R07 } & 101.9038 & 0.3787 & 260.1057 & 157.8232 \\ \text { S28R08 } & 108.6748 & 0.3586 & 264.4917 & 155.4583 \\ \text { S28R09 } & 106.2635 & 0.3656 & 262.9274 & 156.2984 \\ \text { S28R10 } & 94.8452 & 0.4018 & 255.5497 & 160.3026 \\ \text { S28R11 } & 75.4562 & 0.4785 & 243.0278 & 167.0931 \\ \text { S28R12 } & 51.0496 & 0.6019 & 226.6096 & 174.9580 \\ \text { S28R13 } & 28.8227 & 0.6694 & 208.2011 & 178.7089 \\ \text { S28R14 } & 16.2412 & 0.5471 & 190.4431 & 173.6547 \\ \text { S28R15 } & 11.1033 & 0.4213 & 176.6129 & 165.0883 \\ \text { S28R16 } & 9.4637 & 0.3703 & 169.9742 & 160.1402\end{array}$

$\begin{array}{ccccccc} & \text { Cs-137 } & \text { Ba-137m } & \text { Source 29 } & \text { X Posit } & \text { Z Posit } & \text { Y Posit } \\ \text { Line } & \text { 18.0653 } & 17.0898 & & -0.2295 & 9.2524 & 97.3974 \\ \text { Shield 1 } & \text { Shield 2 } & \text { Total Dist } & \text { Air Gap } & & \\ \text { S29R02 } & 58.3641 & 0.3590 & 213.1384 & 154.4153 & & \\ \text { S29R03 } & 54.8203 & 0.3587 & 209.5921 & 154.4131 & & \\ \text { S29R04 } & 49.8700 & 0.3572 & 206.5024 & 154.2752 & \\ \text { S29R05 } & 49.1751 & 0.3555 & 204.3693 & 154.1038 & \\ \text { S29R06 } & 49.7465 & 0.3554 & 203.5511 & 154.0212 & \\ \text { S29R07 } & 51.5613 & 0.3570 & 206.1882 & 154.0863 & \\ \text { S29R08 } & 54.4052 & 0.3585 & 209.1654 & 154.2527 & \\ \text { S29R09 } & 57.9011 & 0.3590 & 212.6842 & 154.4241 & \\ \text { S29R10 } & 61.5318 & 0.3582 & 216.1799 & 154.2900 & \\ \text { S29R11 } & 64.7151 & 0.3565 & 219.1333 & 154.0618 & \\ \text { S29R12 } & 66.9173 & 0.3549 & 221.1239 & 153.8518 & \\ \text { S29R13 } & 67.7621 & 0.3542 & 221.8772 & 153.7610 & \\ \text { S29R14 } & 67.1043 & 0.3547 & 221.2912 & 153.8322 & \\ \text { S29R15 } & 65.0571 & 0.3563 & 219.4451 & 154.0318 & \\ \text { S29R16 } & 61.9705 & 0.3580 & 216.5928 & 154.2642 & & \\ & & & & & \end{array}$

$\begin{array}{ccccccc} & \text { Cs-137 } & \text { Ba-137m } & \text { Source 30 } & \text { X Posit } & \text { Z Posit } & \text { Y Posit } \\ & 15.0401 & 14.2280 & & 18.7276 & -50.2548 & 31.4968 \\ \text { Line } & \text { Shield 1 } & \text { Shield 2 } & \text { Total Dist } & \text { Air Gap } & & \\ \text { S30R01 } & 17.4011 & 0.9034 & 201.7239 & 183.4194 & & \\ \text { S30R02 } & 42.0284 & 0.7694 & 222.2520 & 179.4542 & & \\ \text { S30R03 } & 71.2331 & 0.5308 & 240.7811 & 169.0171 & & \\ \text { S30R04 } & 94.3471 & 0.4166 & 255.1964 & 160.4327 & & \\ \text { S30R05 } & 108.4158 & 0.3670 & 264.1144 & 155.3315 & & \\ \text { S30R06 } & 112.5541 & 0.3545 & 266.7662 & 153.8576 & & \end{array}$




\section{REVISED METHODOLOGY FOR DETERMINING CESIUM-137 CONTENT OF HN-200 GROUT CONTAINERS.}

$\begin{array}{lllll}\text { S30R07 } & 106.5665 & 0.3729 & 262.9338 & 155.9945 \\ \text { S30R08 } & 90.7436 & 0.4313 & 252.9336 & 161.7587 \\ \text { S30R09 } & 66.1791 & 0.5634 & 237.6410 & 170.8986 \\ \text { S30R10 } & 36.6448 & 0.8246 & 218.5634 & 181.0940 \\ \text { S30R11 } & 14.6629 & 0.8577 & 197.9655 & 182.4450 \\ \text { S30R12 } & 7.1139 & 0.5478 & 178.9991 & 171.3374 \\ \text { S30R13 } & 4.8811 & 0.3966 & 165.5579 & 160.2801 \\ \text { S30R14 } & 4.3978 & 0.3607 & 161.2503 & 156.4918 \\ \text { S30R15 } & 5.1179 & 0.4139 & 167.4267 & 161.8949 \\ \text { S30R16 } & 7.8911 & 0.5942 & 182.1825 & 173.6972\end{array}$

$\begin{array}{ccccccc} & \text { Cs-137 } & \text { Ba-137m } & \text { Source 31 } & \text { X Posit } & \text { Z Posit } & \text { Y Posit } \\ \text { Line } & \text { Shield 1 } & \text { Shicld 2 } & \text { Total Dist } & \text { Air Gap } & & \\ \text { S31R01 } & 29.9808 & 0.6040 & 202.1541 & 171.5693 & & \\ \text { S31R02 } & 50.6275 & 0.5591 & 220.4681 & 169.2815 & & \\ \text { S31R03 } & 73.4308 & 0.4594 & 236.7978 & 162.9076 & \\ \text { S31R04 } & 91.8297 & 0.3920 & 249.2599 & 157.0382 & \\ \text { S31R05 } & 102.7922 & 0.3588 & 256.6240 & 153.4730 & \\ \text { S31R06 } & 105.1803 & 0.3522 & 258.2292 & 152.6968 & \\ \text { S31R07 } & 98.7916 & 0.3703 & 253.9368 & 154.7748 & \\ \text { S31R08 } & 84.2009 & 0.4181 & 244.1215 & 159.5025 & \\ \text { S31R09 } & 63.2261 & 0.5030 & 229.6971 & 165.9680 & \\ \text { S31R10 } & 40.3837 & 0.5968 & 212.1813 & 171.2008 & \\ \text { S31R11 } & 23.2622 & 0.5704 & 193.7884 & 169.9558 & & \\ \text { S31R12 } & 14.6951 & 0.4496 & 177.4729 & 162.3282 & \\ \text { S31R13 } & 11.2784 & 0.3703 & 166.6468 & 154.9981 & \\ \text { S31R14 } & 10.6572 & 0.3539 & 164.1487 & 153.1376 & \\ \text { S31R15 } & 12.4104 & 0.3986 & 170.7139 & 157.9048 & \\ \text { S31R16 } & 17.7493 & 0.5048 & 184.4771 & 166.2230 & & \\ & & & & & \end{array}$

$\begin{array}{cccccc}\text { Cs-137 } & \text { Ba-137m } & \text { Source 32 } & \text { X Posit } & \text { Z Posit } & \text { Y Posit } \\ 21.6866 & 20.5156 & & -2.3294 & 45.6316 & 66.6580 \\ \text { Shield 1 } & \text { Shield 2 } & \text { Total Dist } & \text { Air Gap } & & \\ 49.3350 & 0.5399 & 217.8277 & 167.9528 & & \\ 30.3450 & 0.5615 & 200.0423 & 169.1358 & & \\ 19.0011 & 0.4736 & 183.2128 & 163.7381 & & \\ 13.8608 & 0.3863 & 170.5313 & 156.2842 & & \\ 12.2400 & 0.3515 & 165.0511 & 152.4596 & & \\ 13.1698 & 0.3719 & 168.3145 & 154.7729 & & \\ 17.2046 & 0.4470 & 179.3850 & 161.7335 & & \end{array}$


REVISED METHODOLOGY FOR DETERMINING CESIUM-137 CONTENT OF HN-200 GROUT CONTAINERS.

$\begin{array}{lclll}\text { S32R08 } & 26.6264 & 0.5458 & 195.4571 & 168.2849 \\ \text { S32R09 } & 43.8480 & 0.5565 & 213.2747 & 168.8701 \\ \text { S32R10 } & 65.5162 & 0.4780 & 230.0394 & 164.0452 \\ \text { S32R11 } & 84.9812 & 0.4066 & 243.6558 & 158.2680 \\ \text { S32R12 } & 98.2196 & 0.3658 & 252.6937 & 154.1083 \\ \text { S32R13 } & 103.5308 & 0.3512 & 256.3067 & 152.4247 \\ \text { S32R14 } & 100.3977 & 0.3597 & 254.1757 & 153.4182 \\ \text { S32R15 } & 89.1158 & 0.3932 & 246.4875 & 156.9785 \\ \text { S32R16 } & 71.0029 & 0.4567 & 233.9478 & 162.4881\end{array}$

$\begin{array}{ccccccc} & \text { Cs-137 } & \text { Ba-137m } & \text { Source 33 } & \text { X Posit } & \text { Z Posit } & \text { Y Posit } \\ \text { Line } & 17.8281 & 16.8653 & & -7.0965 & -56.3420 & 75.7547 \\ \text { S33R01 } & 44.8664 & 0.8404 & 225.0641 & 179.3573 & & \\ \text { S33R02 } & 76.7569 & 0.5170 & 243.9478 & 166.6738 & & \\ \text { S33R03 } & 99.6370 & 0.4028 & 257.9992 & 157.9594 & \\ \text { S33R04 } & 112.2535 & 0.3588 & 265.9688 & 153.3565 & \\ \text { S33R05 } & 114.1986 & 0.3528 & 267.2117 & 152.6603 & \\ \text { S33R06 } & 105.4187 & 0.3814 & 261.6314 & 155.8313 & \\ \text { S33R07 } & 86.1738 & 0.4632 & 249.6702 & 163.0332 & \\ \text { S33R08 } & 57.2802 & 0.6781 & 232.3485 & 174.3903 & \\ \text { S33R09 } & 22.2007 & 1.3890 & 211.3623 & 187.7726 & \\ \text { S33R10 } & 3.9663 & 1.0868 & 189.2550 & 184.2018 & \\ \text { S33R11 } & 1.7148 & 0.5272 & 169.6006 & 167.3587 & \\ \text { S33R12 } & 1.2013 & 0.3748 & 156.8074 & 155.2313 & \\ \text { S33R13 } & 1.1409 & 0.3565 & 154.6796 & 153.1823 & \\ \text { S33R14 } & 1.4489 & 0.4491 & 163.9420 & 162.0439 & \\ \text { S33R15 } & 2.6866 & 0.7931 & 181.6391 & 178.1594 & \\ \text { S33R16 } & 11.6768 & 1.6739 & 203.3276 & 189.9770 & \end{array}$

$\begin{array}{ccccccc} & \text { Cs-137 } & \text { Ba-137m } & \text { Source 34 } & \text { X Posit } & \text { Z Posit } & \text { Y Posit } \\ \text { Line } & 14.6776 & 13.8850 & & -35.9173 & 43.1022 & 13.8403 \\ \text { Shield 1 } & \text { Shield 2 } & \text { Total Dist } & \text { Air Gap } & & \\ \text { S34R01 } & 89.9755 & 0.4598 & 256.0978 & 165.6625 & & \\ \text { S34R03 } & 62.0605 & 0.6490 & 239.7597 & 177.0501 & & \\ \text { S34R04 } & 27.9573 & 1.1743 & 219.8018 & 190.6702 & & \\ \text { S34R05 } & 2.8654 & 1.0922 & 198.6626 & 190.7049 & & \\ \text { S34R06 } & 2.0719 & 0.5665 & 179.7037 & 176.1441 & \\ \text { S34R07 } & 1.9257 & 0.4017 & 166.9872 & 164.5136 & \\ \text { S34R08 } & 2.3569 & 0.3745 & 164.0484 & 161.7483 & & \\ & & & 171.8227 & 169.0118 & & \end{array}$




\section{REVISED METHODOLOGY FOR DETERMINING CESIUM-137 CONTENT OF HN-200 GROUT CONTAINERS.}

$\begin{array}{lcccc}\text { S34R09 } & 4.0549 & 0.7396 & 187.9377 & 183.1432 \\ \text { S34R10 } & 13.0806 & 1.3952 & 208.3795 & 193.9038 \\ \text { S34R11 } & 43.9152 & 0.8681 & 229.3336 & 184.5503 \\ \text { S34R12 } & 76.0516 & 0.5391 & 247.8708 & 171.2801 \\ \text { S34R13 } & 99.6798 & 0.4166 & 261.9413 & 161.8449 \\ \text { S34R14 } & 113.1704 & 0.3680 & 270.2259 & 156.6874 \\ \text { S34R15 } & 116.0515 & 0.3590 & 272.0200 & 155.6094 \\ \text { S34R16 } & 108.2421 & 0.3844 & 267.1773 & 158.5507\end{array}$

$\begin{array}{ccccccc} & \text { Cs-137 } & \text { Ba-137m } & \text { Source 35 } & \text { X Posit } & \text { Z Posit } & \text { Y Posit } \\ \text { Line } & 28.0146 & 26.5018 & & 44.7881 & 32.5999 & 76.4934 \\ \text { S35R01 } & 3.6137 & 0.4918 & 169.2623 & 165.1568 & & \\ \text { S35R02 } & 2.6454 & 0.3684 & 157.5753 & 154.5615 & & \\ \text { S35R03 } & 2.5727 & 0.3588 & 156.4115 & 153.4801 & \\ \text { S35R04 } & 3.3051 & 0.4534 & 166.1599 & 162.4014 & \\ \text { S35R05 } & 6.0175 & 0.7538 & 183.8067 & 177.0353 & \\ \text { S35R06 } & 18.6062 & 1.1636 & 205.0653 & 185.2955 & \\ \text { S35R07 } & 49.0677 & 0.7343 & 226.1662 & 176.3642 & \\ \text { S35R08 } & 78.6772 & 0.4931 & 244.3311 & 165.1607 & \\ \text { S35R09 } & 100.0142 & 0.3954 & 257.6630 & 157.2534 & \\ \text { S35R10 } & 111.4662 & 0.3569 & 264.9717 & 153.1487 & \\ \text { S35R11 } & 112.5359 & 0.3537 & 265.6604 & 152.7709 & \\ \text { S35R12 } & 103.1840 & 0.3839 & 259.6746 & 156.1066 & \\ \text { S35R13 } & 83.7934 & 0.4657 & 247.4975 & 163.2384 & \\ \text { S35R14 } & 55.6161 & 0.6650 & 230.1917 & 173.9106 & \\ \text { S35R15 } & 23.9150 & 1.1054 & 209.4968 & 184.4763 & \\ \text { S35R16 } & 7.2387 & 0.8597 & 187.9956 & 179.8972 & & \\ & & & & & \end{array}$


HNF-4799, Rev 0

August 25, 1999

REVISED METHODOLOGY FOR DETERMINING CESIUM-137 CONTENT OF HN-200 GROUT CONTAINERS. 


\section{REVISED METHODOLOGY FOR DETERMINING CESIUM-137 CONTENT OF HN-200 GROUT CONTAINERS.}

\section{THIS ATTACHMENT CALCULATES THE GEOMETRIC ERRORS FOR MEASURING HN-200 GROUT CONTAINERS (BOTH GROUTED AND UNGROUTED) AT 5 FEET FROM THE SURFACE OF THE CONTAINER USING THE OUTPUT OF THE MICROSHIELD DATA SHEETS CALCULATED IN ATTACHMENT 3.}

\section{TABLE 1 \\ DATA FOR HN-200 GROUT CONTAINER LINER DRAWING NUMBER H-3-49415}

$\begin{array}{rcccc}\text { Outer Height } & 54.0000 & \text { in } & 137.1600 & \mathrm{~cm} \\ \text { Inner Height (note 1) } & 53.5000 & \text { in } & 135.8900 & \mathrm{~cm} \\ \text { Outer Radius } & 22.9375 & \text { in } & 58.2613 & \mathrm{~cm} \\ \text { Inner Radius } & 22.7993 & \text { in } & 57.9102 & \mathrm{~cm} \\ \text { Wall Thickness } & 0.1382 & \text { in } & 0.3510 & \mathrm{~cm} \\ & & & & \\ \text { Volume (inner) } & 87366.8507 & \text { in }^{3} & 1431686.17 & \mathrm{~cm}^{3} \\ & 50.5595201 & \mathrm{ft}^{3} & 1.43168617 & \mathrm{~m}^{3}\end{array}$

Note 1 - The top and bottom closure plates for the container are constructed of $0.125^{\prime \prime}$ steel. A bottom stiffening plate, constructed of $0.25^{\prime \prime}$ steel, is inserted into the container. This yields an internal height of $53.5^{\prime \prime}$ for the container.

Note 2 - For purposes of the coordinates and calculations presented in this attachment, the cylinder of interest is placed in an $\mathrm{X}, \mathrm{Z}, \mathrm{Y}$ coordinate frame to match the coordinate frame use in Microshield version 5.03. The circular portion of the cylinder is centered at $X=0, Z=0$. The bottom of the inner height of the cylinder is placed at $Y=0$. This placement matches the orientation used in Microshield for a cylindrical source. 
REVISED METHODOLOGY FOR DETERMINING CESIUM-137 CONTENT OF HN-200 GROUT CONTAINERS.

TABLE 2

\begin{tabular}{lccccc}
\multicolumn{5}{c}{ Coordinates and source strengths for random sources. } \\
Source & $\mathbf{X}$ & $\mathbf{Z}$ & $\mathbf{Y}$ & Validity & Strength \\
S01 & 19.3677 & 0.5818 & 25.9034 & Valid & 29.4975 \\
S02 & -10.6176 & -19.5689 & 18.8660 & Valid & 13.8502 \\
S03 & 1.7704 & -7.2590 & 41.8874 & Valid & 23.5916 \\
S04 & 5.5262 & -19.2179 & 28.6756 & Valid & 20.6982 \\
S05 & -12.5171 & 2.4304 & 36.3581 & Valid & 10.9510 \\
S06 & 10.1481 & -6.8169 & 29.6428 & Valid & 16.3182 \\
S07 & -3.0040 & -18.4824 & 1.5181 & Valid & 25.9358 \\
S08 & -1.4468 & -9.3997 & 20.8621 & Valid & 25.1678 \\
S09 & -11.3240 & -1.2877 & 45.4810 & Valid & 24.9347 \\
S10 & -8.1594 & 10.5475 & 46.4380 & Valid & 26.8139 \\
S11 & 13.0540 & 6.4320 & 29.1917 & Valid & 12.5933 \\
S12 & 17.0889 & 8.7507 & 21.2880 & Valid & 17.5307 \\
S13 & 3.5123 & 8.3040 & 41.4473 & Valid & 17.9331 \\
S14 & -18.2417 & 6.1323 & 39.9213 & Valid & 26.2138 \\
S15 & 21.0026 & 5.4076 & 10.7056 & Valid & 22.7469 \\
S16 & 12.7799 & 10.3319 & 39.3081 & Valid & 27.6556 \\
S17 & -14.4903 & 3.0983 & 8.6941 & Valid & 28.5227 \\
S18 & 18.7646 & -12.0033 & 10.5209 & Valid & 23.2528 \\
S19 & -18.2584 & 0.2785 & 21.1001 & Valid & 15.3416 \\
S20 & 2.8644 & 9.2261 & 44.4497 & Valid & 16.4988 \\
S21 & 3.0621 & -7.9137 & 19.8848 & Valid & 28.6466 \\
S22 & -17.9782 & 12.8570 & 50.0431 & Valid & 20.3724 \\
S23 & 5.0992 & 14.6169 & 52.0038 & Valid & 13.0146 \\
S24 & 7.9617 & 19.2122 & 33.0332 & Valid & 12.5419 \\
S25 & 8.3975 & 9.5559 & 39.8544 & Valid & 19.7625 \\
S26 & 4.1090 & 16.2164 & 34.1935 & Valid & 24.8512 \\
S27 & 2.2698 & -21.1016 & 13.9713 & Valid & 10.2138 \\
S28 & 18.4153 & -5.6775 & 6.4850 & Valid & 15.5133 \\
S29 & -0.0904 & 3.6427 & 38.3454 & Valid & 18.0653 \\
S30 & 7.3731 & -19.7854 & 12.4003 & Valid & 15.0401 \\
S31 & 5.5574 & -17.7939 & 21.2754 & Valid & 22.3550 \\
S32 & -0.9171 & 17.9652 & 26.2433 & Valid & 21.6866 \\
S33 & -2.7939 & -22.1819 & 29.8247 & Valid & 17.8281 \\
S34 & -14.1407 & 16.9694 & 5.4489 & Valid & 14.6776 \\
S35 & 17.6331 & 12.8346 & 30.1155 & Valid & 28.0146 \\
& & & & & \\
& & & & &
\end{tabular}




\section{REVISED METHODOLOGY FOR DETERMINING CESIUM-137 CONTENT OF HN-200 GROUT CONTAINERS.}

\section{TABLE 3 - CALCULATION OF CONVERSION FACTORS FOR A GROUTED HN-200 GROUT CONTAINER ASSUMING A HOMOGENEOUS MIXTURE OF ACTIVITY AND GROUT.}

\begin{tabular}{|c|c|c|c|c|}
\hline POINT & $\mathbf{R} / \mathbf{h r}$ & & & \\
\hline R01 & $1.479 \mathrm{E}-02$ & & & \\
\hline R02 & $1.479 \mathrm{E}-02$ & & & \\
\hline $\mathbf{R} 03$ & $1.479 \mathrm{E}-02$ & & & \\
\hline R04 & $1.479 \mathrm{E}-02$ & & & \\
\hline R05 & $1.479 \mathrm{E}-02$ & & & \\
\hline R06 & $1.479 \mathrm{E}-02$ & & & \\
\hline R07 & $1.479 \mathrm{E}-02$ & AVERAGE 5 FOOT READING = & $1.479 \mathrm{E}-02$ & $\mathbf{R} / \mathbf{h r}$ \\
\hline $\mathbf{R} 08$ & $1.479 \mathrm{E}-02$ & TOTAL Cs-137 = & I & \\
\hline $\mathbf{R} 09$ & $1.479 \mathrm{E}-02$ & GROUTED 5 FOOT CONV FACTOR = & $1.479 \mathrm{E}-02$ & $\mathbf{R} / \mathbf{h r} / \mathbf{C i}$ \\
\hline $\mathbf{R} 10$ & $1.479 \mathrm{E}-02$ & & & \\
\hline R11 & $1.479 \mathrm{E}-02$ & \multirow{6}{*}{\multicolumn{3}{|c|}{$\begin{array}{l}5 \text { FOOT readings are taken with the RO- } 7 \text { probe } 5 \text { feet from the surface of the } \\
\text { container. These readings are taken every } 22.5 \text { degrees around the container and } \\
\text { averaged to produce the most accurate representation of the Cs- } 137 \text { curie content } \\
\text { of the container. }\end{array}$}} \\
\hline $\mathbf{R} 12$ & $1.479 \mathrm{E}-02$ & & & \\
\hline R13 & $1.479 \mathrm{E}-02$ & & & \\
\hline R14 & $1.479 \mathrm{E}-02$ & & & \\
\hline R15 & $1.479 \mathrm{E}-02$ & & & \\
\hline $\mathbf{R} 16$ & $1.479 \mathrm{E}-02$ & & & \\
\hline
\end{tabular}




\section{REVISED METHODOLOGY FOR DETERMINING CESIUM-137 CONTENT OF HN-200 GROUT CONTAINERS. POINT FROM A GROUTED HN-200 GROUT CONTAINER.}

$\begin{array}{cccccccc}\text { POIT } & \text { S01 } & \text { S02 } & \text { S03 } & \text { S04 } & \text { S05 } & \text { S06 } & \text { S07 } \\ \text { R01 } & 2.553 E+00 & 1.347 E-03 & 3.172 E-02 & 2.968 E-01 & 2.513 E-04 & 2.202 E-01 & 1.783 E-02 \\ \text { R02 } & 2.302 E+00 & 1.094 E-04 & 1.422 E-02 & 3.042 E-02 & 4.677 E-04 & 1.098 E-01 & 1.596 E-03 \\ \text { R03 } & 1.555 E+00 & 2.552 E-05 & 6.372 E-03 & 2.209 E-03 & 1.467 E-03 & 3.714 E-02 & 2.351 E-04 \\ \text { R04 } & 6.205 E-01 & 1.927 E-05 & 3.306 E-03 & 2.587 E-04 & 6.172 E-03 & 9.669 E-03 & 8.070 E-05 \\ \text { R05 } & 9.630 E-02 & 4.804 E-05 & 2.253 E-03 & 7.340 E-05 & 2.538 E-02 & 2.471 E-03 & 7.376 E-05 \\ \text { R06 } & 8.002 E-03 & 3.697 E-04 & 2.166 E-03 & 5.852 E-05 & 7.836 E-02 & 8.317 E-04 & 1.813 E-04 \\ \text { R07 } & 8.145 E-04 & 7.046 E-03 & 2.960 E-03 & 1.341 E-04 & 1.601 E-01 & 4.519 E-04 & 1.076 E-03 \\ \text { R08 } & 1.735 E-04 & 1.604 E-01 & 5.426 E-03 & 8.084 E-04 & 2.245 E-01 & 4.609 E-04 & 1.147 E-02 \\ \text { R09 } & 9.628 E-05 & 8.615 E-01 & 1.188 E-02 & 9.821 E-03 & 2.253 E-01 & 8.409 E-04 & 1.173 E-01 \\ \text { R10 } & 1.486 E-04 & 1.349 E-01 & 2.681 E-02 & 1.261 E-01 & 1.621 E-01 & 2.511 E-03 & 5.546 E-01 \\ \text { R11 } & 6.081 E-04 & 1.647 E-01 & 5.473 E-02 & 6.701 E-01 & 8.005 E-02 & 9.840 E-03 & 1.169 E+00 \\ \text { R12 } & 5.468 E-03 & 1.713 E-01 & 9.219 E-02 & 1.389 E+00 & 2.616 E-02 & 3.773 E-02 & 1.582 E+00 \\ \text { R13 } & 6.691 E-02 & 1.514 E-01 & 1.221 E-02 & 1.865 E+00 & 6.388 E-03 & 1.110 E-01 & 1.619 E+00 \\ \text { R14 } & 4.992 E-02 & 1.123 E-01 & 1.255 E-02 & 1.956 E+00 & 1.514 E-03 & 2.216 E-01 & 1.265 E+00 \\ \text { R15 } & 1.413 E+00 & 4.470 E-01 & 1.001 E-02 & 1.631 E+00 & 4.778 E-04 & 3.074 E-01 & 6.651 E-01 \\ \text { R16 } & 2.215 E+00 & 3.232 E-02 & 6.249 E-02 & 9.896 E-01 & 2.532 E-04 & 3.068 E-01 & 1.664 E-01\end{array}$

$\begin{array}{lccccccc}\text { POINT } & \text { S08 } & \text { S09 } & \text { S10 } & \text { S11 } & \text { S12 } & \text { S13 } & \text { S14 } \\ \text { R01 } & 1.683 E-02 & 6.825 E-04 & 2.233 E-03 & 3.366 E-01 & 1.216 E+00 & 4.085 E-02 & 1.122 E-04 \\ \text { R02 } & 5.960 E-03 & 7.704 E-04 & 1.002 E-02 & 4.188 E-01 & 1.454 E+00 & 8.284 E-02 & 4.176 E-04 \\ \text { R03 } & 2.539 E-03 & 1.499 E-03 & 4.616 E-02 & 3.758 E-01 & 1.351 E+00 & 1.269 E-01 & 3.497 E-03 \\ \text { R04 } & 1.542 E-03 & 4.466 E-03 & 1.612 E-01 & 2.372 E-01 & 9.474 E-01 & 1.456 E-01 & 4.246 E-02 \\ \text { R05 } & 1.464 E-03 & 1.627 E-02 & 3.615 E-01 & 9.635 E-02 & 4.101 E-01 & 1.255 E-01 & 3.455 E-01 \\ \text { R06 } & 2.194 E-03 & 5.554 E-02 & 5.410 E-01 & 2.374 E-02 & 7.301 E-02 & 8.104 E-02 & 1.077 E+00 \\ \text { R07 } & 4.819 E-03 & 1.452 E-01 & 5.733 E-01 & 4.380 E-03 & 6.449 E-03 & 3.958 E-02 & 1.772 E+00 \\ \text { R08 } & 1.329 E-02 & 2.642 E-01 & 4.349 E-01 & 8.781 E-04 & 6.329 E-04 & 1.570 E-02 & 2.098 E+00 \\ \text { R09 } & 3.761 E-02 & 3.407 E-01 & 2.256 E-01 & 2.767 E-04 & 1.227 E-04 & 5.770 E-03 & 1.937 E+00 \\ \text { R10 } & 9.177 E-02 & 3.185 E-01 & 7.508 E-02 & 1.693 E-04 & 6.024 E-05 & 2.357 E-03 & 1.347 E+00 \\ \text { R11 } & 1.720 E-01 & 2.143 E-01 & 1.744 E-02 & 2.171 E-04 & 8.129 E-05 & 1.280 E-03 & 5.698 E-01 \\ \text { R12 } & 2.386 E-01 & 1.008 E-01 & 3.698 E-03 & 5.618 E-04 & 2.917 E-04 & 1.036 E-03 & 9.685 E-02 \\ \text { R13 } & 2.465 E-01 & 3.379 E-02 & 1.011 E-03 & 2.495 E-03 & 2.357 E-03 & 1.301 E-03 & 8.288 E-03 \\ \text { R14 } & 1.898 E-01 & 9.350 E-03 & 4.662 E-04 & 1.376 E-02 & 2.793 E-02 & 2.429 E-03 & 8.099 E-04 \\ \text { R15 } & 1.082 E-01 & 2.703 E-03 & 4.132 E-04 & 6.382 E-02 & 2.284 E-01 & 5.989 E-03 & 1.595 E-04 \\ \text { R16 } & 4.668 E-02 & 1.064 E-03 & 7.180 E-04 & 1.857 E-01 & 7.278 E-01 & 1.630 E-02 & 8.043 E-05\end{array}$

$\begin{array}{lllll}\mathbf{S 1 7} & \mathbf{S 1 8} & \mathbf{S 1 9} & \mathbf{S 2 0} & \text { 'S21 }\end{array}$




\section{REVISED METHODOLOGY FOR DETERMINING CESIUM-137 CONTENT OF HN-200 GROUT CONTAINERS.}

$\begin{array}{llllllll}\text { R01 } & 2.438 \mathrm{E}+00 & 8.027 \mathrm{E}-01 & 3.195 \mathrm{E}-04 & 2.330 \mathrm{E}+00 & 6.794 \mathrm{E}-05 & 3.130 \mathrm{E}-02 & 6.191 \mathrm{E}-02 \\ \text { R02 } & 2.500 \mathrm{E}+00 & 1.203 \mathrm{E}+00 & 6.930 \mathrm{E}-04 & 1.663 \mathrm{E}+00 & 1.138 \mathrm{E}-04 & 7.106 \mathrm{E}-02 & 2.588 \mathrm{E}-02 \\ \text { R03 } & 2.148 \mathrm{E}+00 & 1.291 \mathrm{E}+00 & 2.802 \mathrm{E}-03 & 5.023 \mathrm{E}-01 & 4.661 \mathrm{E}-04 & 1.202 \mathrm{E}-01 & 1.019 \mathrm{E}-02 \\ \text { R04 } & 1.484 \mathrm{E}+00 & 1.015 \mathrm{E}+00 & 1.601 \mathrm{E}-02 & 2.739 \mathrm{E}-02 & 3.773 \mathrm{E}-03 & 1.496 \mathrm{E}-01 & 4.469 \mathrm{E}-03 \\ \text { R05 } & 4.696 \mathrm{E}-01 & 5.391 \mathrm{E}-01 & 8.551 \mathrm{E}-02 & 1.189 \mathrm{E}-03 & 3.712 \mathrm{E}-02 & 1.382 \mathrm{E}-01 & 2.565 \mathrm{E}-03 \\ \text { R06 } & 3.221 \mathrm{E}-02 & 1.621 \mathrm{E}-01 & 2.995 \mathrm{E}-01 & 1.137 \mathrm{E}-04 & 2.256 \mathrm{E}-01 & 9.440 \mathrm{E}-02 & 2.143 \mathrm{E}-03 \\ \text { R07 } & 1.570 \mathrm{E}-03 & 2.672 \mathrm{E}-02 & 6.248 \mathrm{E}-01 & 3.252 \mathrm{E}-05 & 6.078 \mathrm{E}-01 & 4.744 \mathrm{E}-02 & 2.692 \mathrm{E}-03 \\ \text { R08 } & 1.500 \mathrm{E}-04 & 3.624 \mathrm{E}-03 & 8.631 \mathrm{E}-01 & 3.058 \mathrm{E}-05 & 9.541 \mathrm{E}-01 & 1.842 \mathrm{E}-02 & 4.877 \mathrm{E}-03 \\ \text { R09 } & 4.027 \mathrm{E}-05 & 6.718 \mathrm{E}-04 & 8.531 \mathrm{E}-01 & 9.501 \mathrm{E}-05 & 1.083 \mathrm{E}+00 & 6.278 \mathrm{E}-03 & 1.137 \mathrm{E}-02 \\ \text { R10 } & 3.412 \mathrm{E}-05 & 2.423 \mathrm{E}-04 & 6.014 \mathrm{E}-01 & 8.962 \mathrm{E}-04 & 9.333 \mathrm{E}-01 & 2.267 \mathrm{E}-03 & 2.890 \mathrm{E}-02 \\ \text { R11 } & 9.235 \mathrm{E}-05 & 1.987 \mathrm{E}-04 & 2.785 \mathrm{E}-01 & 1.957 \mathrm{E}-02 & 5.763 \mathrm{E}-01 & 1.066 \mathrm{E}-03 & 6.801 \mathrm{E}-02 \\ \text { R12 } & 7.361 \mathrm{E}-04 & 3.809 \mathrm{E}-04 & 7.649 \mathrm{E}-02 & 4.010 \mathrm{E}-01 & 2.021 \mathrm{E}-01 & 7.556 \mathrm{E}-04 & 1.314 \mathrm{E}-01 \\ \text { R13 } & 1.334 \mathrm{E}-02 & 1.555 \mathrm{E}-03 & 1.407 \mathrm{E}-02 & 1.573 \mathrm{E}+00 & 3.132 \mathrm{E}-02 & 8.578 \mathrm{E}-04 & 1.952 \mathrm{E}-01 \\ \text { R14 } & 2.517 \mathrm{E}-01 & 1.054 \mathrm{E}-02 & 2.490 \mathrm{E}-03 & 2.273 \mathrm{E}+00 & 3.158 \mathrm{E}-03 & 1.526 \mathrm{E}-03 & 2.203 \mathrm{E}-01 \\ \text { R15 } & 1.221 \mathrm{E}+00 & 7.448 \mathrm{E}-02 & 6.399 \mathrm{E}-04 & 2.677 \mathrm{E}+00 & 4.060 \mathrm{E}-04 & 3.823 \mathrm{E}-03 & 1.889 \mathrm{E}-01 \\ \text { R16 } & 1.991 \mathrm{E}+00 & 3.353 \mathrm{E}-01 & 3.107 \mathrm{E}-04 & 2.699 \mathrm{E}+00 & 1.057 \mathrm{E}-04 & 1.121 \mathrm{E}-02 & 1.230 \mathrm{E}-01\end{array}$

$\begin{array}{ccccc}\text { POINT } & \text { S22 } & \text { S23 } & \text { S24 } & \text { S25 } \\ \text { R01 } & 9.206 \mathrm{E}-05 & 6.154 \mathrm{E}-02 & 3.600 \mathrm{E}-01 & 1.822 \mathrm{E}-01 \\ \text { R02 } & 9.849 \mathrm{E}-04 & 1.884 \mathrm{E}-01 & 8.563 \mathrm{E}-01 & 3.284 \mathrm{E}-01 \\ \text { R03 } & 2.275 \mathrm{E}-02 & 3.416 \mathrm{E}-01 & 1.197 \mathrm{E}+00 & 4.043 \mathrm{E}-01 \\ \text { R04 } & 4.058 \mathrm{E}-01 & 4.212 \mathrm{E}-01 & 1.324 \mathrm{E}+00 & 3.622 \mathrm{E}-01 \\ \text { R05 } & 1.338 \mathrm{E}+00 & 3.760 \mathrm{E}-01 & 1.197 \mathrm{E}+00 & 2.299 \mathrm{E}-01 \\ \text { R06 } & 1.880 \mathrm{E}+00 & 2.348 \mathrm{E}-01 & 8.559 \mathrm{E}-01 & 9.826 \mathrm{E}-02 \\ \text { R07 } & 2.176 \mathrm{E}+00 & 9.061 \mathrm{E}-02 & 3.596 \mathrm{E}-01 & 2.830 \mathrm{E}-02 \\ \text { R08 } & 2.149 \mathrm{E}+00 & 1.964 \mathrm{E}-02 & 4.535 \mathrm{E}-02 & 6.609 \mathrm{E}-03 \\ \text { R09 } & 1.808 \mathrm{E}+00 & 3.034 \mathrm{E}-03 & 2.724 \mathrm{E}-03 & 1.672 \mathrm{E}-03 \\ \text { R10 } & 1.216 \mathrm{E}+00 & 5.211 \mathrm{E}-04 & 2.283 \mathrm{E}-04 & 6.200 \mathrm{E}-04 \\ \text { R11 } & 2.815 \mathrm{E}-01 & 1.496 \mathrm{E}-04 & 4.556 \mathrm{E}-05 & 4.061 \mathrm{E}-04 \\ \text { R12 } & 1.338 \mathrm{E}-02 & 8.905 \mathrm{E}-05 & 2.619 \mathrm{E}-05 & 5.040 \mathrm{E}-04 \\ \text { R13 } & 6.287 \mathrm{E}-04 & 1.186 \mathrm{E}-04 & 4.560 \mathrm{E}-05 & 1.144 \mathrm{E}-03 \\ \text { R14 } & 6.920 \mathrm{E}-05 & 3.395 \mathrm{E}-04 & 2.287 \mathrm{E}-04 & 4.105 \mathrm{E}-03 \\ \text { R15 } & 2.315 \mathrm{E}-05 & 1.760 \mathrm{E}-03 & 2.731 \mathrm{E}-03 & 1.775 \mathrm{E}-02 \\ \text { R16 } & 2.551 \mathrm{E}-05 & 1.161 \mathrm{E}-02 & 4.564 \mathrm{E}-02 & 6.782 \mathrm{E}-02\end{array}$

$\begin{array}{ccc}\text { S26 } & \text { S27 } & \text { S28 } \\ 1.381 \mathrm{E}-01 & 8.056 \mathrm{E}-02 & 1.087 \mathrm{E}+00 \\ 4.926 \mathrm{E}-01 & 4.640 \mathrm{E}-03 & 7.738 \mathrm{E}-01 \\ 9.664 \mathrm{E}-01 & 2.991 \mathrm{E}-04 & 3.406 \mathrm{E}-01 \\ 1.267 \mathrm{E}+00 & 4.337 \mathrm{E}-05 & 6.043 \mathrm{E}-02 \\ 1.218 \mathrm{E}+00 & 1.809 \mathrm{E}-05 & 5.116 \mathrm{E}-03 \\ 8.471 \mathrm{E}-01 & 2.343 \mathrm{E}-05 & 4.742 \mathrm{E}-04 \\ 3.760 \mathrm{E}-01 & 9.231 \mathrm{E}-05 & 8.727 \mathrm{E}-05 \\ 8.805 \mathrm{E}-02 & 9.656 \mathrm{E}-04 & 4.108 \mathrm{E}-05 \\ 1.230 \mathrm{E}-02 & 1.785 \mathrm{E}-02 & 5.376 \mathrm{E}-05 \\ 1.699 \mathrm{E}-03 & 2.132 \mathrm{E}-01 & 1.902 \mathrm{E}-04 \\ 3.793 \mathrm{E}-04 & 6.399 \mathrm{E}-01 & 1.553 \mathrm{E}-03 \\ 1.800 \mathrm{E}-04 & 9.433 \mathrm{E}-01 & 1.933 \mathrm{E}-02 \\ 2.020 \mathrm{E}-04 & 1.092 \mathrm{E}+00 & 1.702 \mathrm{E}-01 \\ 5.277 \mathrm{E}-04 & 1.046 \mathrm{E}+00 & 5.692 \mathrm{E}-01 \\ 2.781 \mathrm{E}-03 & 8.243 \mathrm{E}-01 & 9.630 \mathrm{E}-01 \\ 2.115 \mathrm{E}-02 & 4.490 \mathrm{E}-01 & 1.158 \mathrm{E}+00\end{array}$

SOURCE

$\begin{array}{cccccccc}\text { POINT } & \text { S29 } & \text { S30 } & \text { S31 } & \text { S32 } & \text { S33 } & \text { S34 } & \text { S35 } \\ \text { R01 } & 1.397 \mathrm{E}-02 & 4.014 \mathrm{E}-01 & 2.420 \mathrm{E}-01 & 3.544 \mathrm{E}-02 & 3.655 \mathrm{E}-02 & 2.919 \mathrm{E}-04 & 2.683 \mathrm{E}+00\end{array}$


REVISED METHODOLOGY FOR DETERMINING CESIUM-137 CONTENT OF HN-200 GROUT CONTAINERS.

$\begin{array}{llllllll}\text { R02 } & 2.041 \mathrm{E}-02 & 4.262 \mathrm{E}-02 & 3.124 \mathrm{E}-02 & 2.364 \mathrm{E}-01 & 1.520 \mathrm{E}-03 & 5.444 \mathrm{E}-03 & 3.274 \mathrm{E}+00 \\ \text { R03 } & 2.797 \mathrm{E}-02 & 2.311 \mathrm{E}-03 & 2.933 \mathrm{E}-03 & 7.228 \mathrm{E}-01 & 1.285 \mathrm{E}-04 & 1.274 \mathrm{E}-01 & 3.337 \mathrm{E}+00 \\ \text { R04 } & 3.447 \mathrm{E}-02 & 1.935 \mathrm{E}-04 & 3.989 \mathrm{E}-04 & 1.227 \mathrm{E}+00 & 3.166 \mathrm{E}-05 & 7.686 \mathrm{E}-01 & 2.835 \mathrm{E}+00 \\ \text { R05 } & 3.728 \mathrm{E}-02 & 4.090 \mathrm{E}-05 & 1.186 \mathrm{E}-04 & 1.468 \mathrm{E}+00 & 2.546 \mathrm{E}-05 & 1.260 \mathrm{E}+00 & 1.949 \mathrm{E}+00 \\ \text { R06 } & 3.508 \mathrm{E}-02 & 2.565 \mathrm{E}-05 & 9.090 \mathrm{E}-05 & 1.323 \mathrm{E}+00 & 6.781 \mathrm{E}-05 & 1.550 \mathrm{E}+00 & 6.030 \mathrm{E}-01 \\ \text { R07 } & 2.890 \mathrm{E}-02 & 5.025 \mathrm{E}-05 & 1.850 \mathrm{E}-04 & 8.662 \mathrm{E}-01 & 5.565 \mathrm{E}-04 & 1.616 \mathrm{E}+00 & 3.995 \mathrm{E}-02 \\ \text { R08 } & 2.134 \mathrm{E}-02 & 2.868 \mathrm{E}-04 & 9.182 \mathrm{E}-04 & 3.405 \mathrm{E}-01 & 1.115 \mathrm{E}-02 & 1.437 \mathrm{E}+00 & 1.972 \mathrm{E}-03 \\ \text { R09 } & 1.469 \mathrm{E}-02 & 3.909 \mathrm{E}-03 & 8.610 \mathrm{E}-03 & 6.175 \mathrm{E}-02 & 2.497 \mathrm{E}-01 & 1.071 \mathrm{E}+00 & 1.951 \mathrm{E}-04 \\ \text { R10 } & 9.947 \mathrm{E}-03 & 7.079 \mathrm{E}-02 & 8.732 \mathrm{E}-02 & 6.696 \mathrm{E}-03 & 1.199 \mathrm{E}+00 & 4.258 \mathrm{E}-01 & 5.466 \mathrm{E}-05 \\ \text { R11 } & 7.054 \mathrm{E}-03 & 5.155 \mathrm{E}-01 & 4.643 \mathrm{E}-01 & 8.286 \mathrm{E}-04 & 1.810 \mathrm{E}+00 & 3.132 \mathrm{E}-02 & 4.849 \mathrm{E}-05 \\ \text { R12 } & 5.555 \mathrm{E}-03 & 1.096 \mathrm{E}+00 & 1.084 \mathrm{E}+00 & 1.932 \mathrm{E}-04 & 2.182 \mathrm{E}+00 & 1.290 \mathrm{E}-03 & 1.374 \mathrm{E}-04 \\ \text { R13 } & 5.067 \mathrm{E}-03 & 1.459 \mathrm{E}+00 & 1.565 \mathrm{E}+00 & 1.070 \mathrm{E}-04 & 2.249 \mathrm{E}+00 & 1.014 \mathrm{E}-04 & 1.141 \mathrm{E}-03 \\ \text { R14 } & 5.443 \mathrm{E}-03 & 1.577 \mathrm{E}+00 & 1.681 \mathrm{E}+00 & 1.517 \mathrm{E}-04 & 1.970 \mathrm{E}+00 & 2.274 \mathrm{E}-05 & 2.114 \mathrm{E}-02 \\ \text { R15 } & 6.797 \mathrm{E}-03 & 1.409 \mathrm{E}+00 & 1.380 \mathrm{E}+00 & 5.272 \mathrm{E}-04 & 1.456 \mathrm{E}+00 & 1.648 \mathrm{E}-05 & 3.864 \mathrm{E}-01 \\ \text { R16 } & 9.488 \mathrm{E}-03 & 1.005 \mathrm{E}+00 & 7.965 \mathrm{E}-01 & 3.745 \mathrm{E}-03 & 5.478 \mathrm{E}-01 & 3.939 \mathrm{E}-05 & 1.705 \mathrm{E}+00\end{array}$

TABLE 5 - TOTAL RADIATION LEVEL AT EACH MEASURMENT POINT FROM A GROUTED HN-200 GROUT CONTAINER FOR EACH SET OF SOURCES TO BE ANALYZED. 


\section{REVISED METHODOLOGY FOR DETERMINING CESIUM-137 CONTENT OF HN-200 GROUT CONTAINERS.}

\begin{tabular}{cccccccc} 
& \multicolumn{7}{c}{ SOURCE SET } \\
POINT & S01-S35 & S01-S30 & S06-S35 & S01-S25 & S11-S35 & S01-S20 & S16-S35 \\
R01 & $1.572 \mathrm{E}+01$ & $1.272 \mathrm{E}+01$ & $1.284 \mathrm{E}+01$ & $1.100 \mathrm{E}+01$ & $1.258 \mathrm{E}+01$ & $1.034 \mathrm{E}+01$ & $8.548 \mathrm{E}+00$ \\
R02 & $1.615 \mathrm{E}+01$ & $1.260 \mathrm{E}+01$ & $1.380 \mathrm{E}+01$ & $1.127 \mathrm{E}+01$ & $1.368 \mathrm{E}+01$ & $9.869 \mathrm{E}+00$ & $9.221 \mathrm{E}+00$ \\
R03 & $1.508 \mathrm{E}+01$ & $1.089 \mathrm{E}+01$ & $1.351 \mathrm{E}+01$ & $9.550 \mathrm{E}+00$ & $1.343 \mathrm{E}+01$ & $7.575 \mathrm{E}+00$ & $9.420 \mathrm{E}+00$ \\
R04 & $1.359 \mathrm{E}+01$ & $8.755 \mathrm{E}+00$ & $1.296 \mathrm{E}+01$ & $7.393 \mathrm{E}+00$ & $1.278 \mathrm{E}+01$ & $4.876 \mathrm{E}+00$ & $9.923 \mathrm{E}+00$ \\
R05 & $1.184 \mathrm{E}+01$ & $7.158 \mathrm{E}+00$ & $1.171 \mathrm{E}+01$ & $5.897 \mathrm{E}+00$ & $1.133 \mathrm{E}+01$ & $2.754 \mathrm{E}+00$ & $9.882 \mathrm{E}+00$ \\
R06 & $1.019 \mathrm{E}+01$ & $6.711 \mathrm{E}+00$ & $1.010 \mathrm{E}+01$ & $5.829 \mathrm{E}+00$ & $9.499 \mathrm{E}+00$ & $2.757 \mathrm{E}+00$ & $8.212 \mathrm{E}+00$ \\
R07 & $9.612 \mathrm{E}+00$ & $7.089 \mathrm{E}+00$ & $9.441 \mathrm{E}+00$ & $6.684 \mathrm{E}+00$ & $8.716 \mathrm{E}+00$ & $4.027 \mathrm{E}+00$ & $6.892 \mathrm{E}+00$ \\
R08 & $9.198 \mathrm{E}+00$ & $7.406 \mathrm{E}+00$ & $8.807 \mathrm{E}+00$ & $7.296 \mathrm{E}+00$ & $8.082 \mathrm{E}+00$ & $5.070 \mathrm{E}+00$ & $5.967 \mathrm{E}+00$ \\
R09 & $8.984 \mathrm{E}+00$ & $7.593 \mathrm{E}+00$ & $7.875 \mathrm{E}+00$ & $7.544 \mathrm{E}+00$ & $7.153 \mathrm{E}+00$ & $5.717 \mathrm{E}+00$ & $5.210 \mathrm{E}+00$ \\
R10 & $7.641 \mathrm{E}+00$ & $5.922 \mathrm{E}+00$ & $7.191 \mathrm{E}+00$ & $5.627 \mathrm{E}+00$ & $6.149 \mathrm{E}+00$ & $4.380 \mathrm{E}+00$ & $4.799 \mathrm{E}+00$ \\
R11 & $7.821 \mathrm{E}+00$ & $5.514 \mathrm{E}+00$ & $6.851 \mathrm{E}+00$ & $4.350 \mathrm{E}+00$ & $5.268 \mathrm{E}+00$ & $4.000 \mathrm{E}+00$ & $4.697 \mathrm{E}+00$ \\
R12 & $9.905 \mathrm{E}+00$ & $6.637 \mathrm{E}+00$ & $8.220 \mathrm{E}+00$ & $4.573 \mathrm{E}+00$ & $6.258 \mathrm{E}+00$ & $4.427 \mathrm{E}+00$ & $6.158 \mathrm{E}+00$ \\
R13 & $1.250 \mathrm{E}+01$ & $8.685 \mathrm{E}+00$ & $1.040 \mathrm{E}+01$ & $5.959 \mathrm{E}+00$ & $8.388 \mathrm{E}+00$ & $5.762 \mathrm{E}+00$ & $8.360 \mathrm{E}+00$ \\
R14 & $1.350 \mathrm{E}+01$ & $9.829 \mathrm{E}+00$ & $1.137 \mathrm{E}+01$ & $6.631 \mathrm{E}+00$ & $9.683 \mathrm{E}+00$ & $6.406 \mathrm{E}+00$ & $9.386 \mathrm{E}+00$ \\
R15 & $1.550 \mathrm{E}+01$ & $1.228 \mathrm{E}+01$ & $1.200 \mathrm{E}+01$ & $9.072 \mathrm{E}+00$ & $1.092 \mathrm{E}+01$ & $8.861 \mathrm{E}+00$ & $9.396 \mathrm{E}+00$ \\
R16 & $1.573 \mathrm{E}+01$ & $1.268 \mathrm{E}+01$ & $1.243 \mathrm{E}+01$ & $1.004 \mathrm{E}+01$ & $1.191 \mathrm{E}+01$ & $9.788 \mathrm{E}+00$ & $8.990 \mathrm{E}+00$
\end{tabular}

$\begin{array}{cc}\text { POINT } & \text { S01-S18 } \\ \text { R01 } & 1.031 \mathrm{E}+01 \\ \text { R02 } & 9.798 \mathrm{E}+00 \\ \text { R03 } & 7.454 \mathrm{E}+00 \\ \text { R04 } & 4.722 \mathrm{E}+00 \\ \text { R05 } & 2.579 \mathrm{E}+00 \\ \text { R06 } & 2.437 \mathrm{E}+00 \\ \text { R07 } & 3.371 \mathrm{E}+00 \\ \text { R08 } & 4.098 \mathrm{E}+00 \\ \text { R09 } & 4.628 \mathrm{E}+00 \\ \text { R10 } & 3.445 \mathrm{E}+00 \\ \text { R11 } & 3.423 \mathrm{E}+00 \\ \text { R12 } & 4.224 \mathrm{E}+00 \\ \text { R13 } & 5.730 \mathrm{E}+00 \\ \text { R14 } & 6.401 \mathrm{E}+00 \\ \text { R15 } & 8.857 \mathrm{E}+00 \\ \text { R16 } & 9.777 \mathrm{E}+00\end{array}$

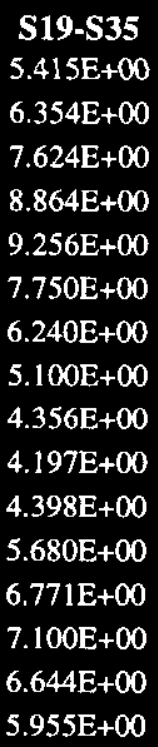

$5.955 \mathrm{E}+00$

\begin{tabular}{cc}
\multicolumn{2}{c}{ SOURCE SET } \\
S01-S12 & S13-S24 \\
$4.693 \mathrm{E}+00$ & $6.127 \mathrm{E}+00$ \\
$4.348 \mathrm{E}+00$ & $6.593 \mathrm{E}+00$ \\
$3.379 \mathrm{E}+00$ & $5.767 \mathrm{E}+00$ \\
$1.992 \mathrm{E}+00$ & $5.039 \mathrm{E}+00$ \\
$1.012 \mathrm{E}+00$ & $4.655 \mathrm{E}+00$ \\
$7.855 \mathrm{E}-01$ & $4.945 \mathrm{E}+00$ \\
$9.067 \mathrm{E}-01$ & $5.749 \mathrm{E}+00$ \\
$1.117 \mathrm{E}+00$ & $6.172 \mathrm{E}+00$ \\
$1.831 \mathrm{E}+00$ & $5.711 \mathrm{E}+00$ \\
$1.493 \mathrm{E}+00$ & $4.133 \mathrm{E}+00$ \\
$2.553 \mathrm{E}+00$ & $1.797 \mathrm{E}+00$ \\
$3.648 \mathrm{E}+00$ & $9.242 \mathrm{E}-01$ \\
$4.118 \mathrm{E}+00$ & $1.840 \mathrm{E}+00$ \\
$3.860 \mathrm{E}+00$ & $2.767 \mathrm{E}+00$ \\
$4.878 \mathrm{E}+00$ & $4.177 \mathrm{E}+00$ \\
$4.735 \mathrm{E}+00$ & $5.234 \mathrm{E}+00$
\end{tabular}

S25-S35

$4.901 \mathrm{E}+00$

$5.211 \mathrm{E}+00$

$5.932 \mathrm{E}+00$

$6.555 \mathrm{E}+00$

$6.167 \mathrm{E}+00$

$4.457 \mathrm{E}+00$

$2.956 \mathrm{E}+00$

$1.909 \mathrm{E}+00$

$1.442 \mathrm{E}+00$

$2.015 \mathrm{E}+00$

$3.471 \mathrm{E}+00$

$5.332 \mathrm{E}+00$

$6.543 \mathrm{E}+00$

$6.875 \mathrm{E}+00$

$6.447 \mathrm{E}+00$

$5.764 \mathrm{E}+00$
S01-S10

$3.141 \mathrm{E}+00$

$2.475 \mathrm{E}+00$

$1.653 \mathrm{E}+00$

8.072E-01

$5.058 \mathrm{E}-01$

6.887E-01

$8.959 \mathrm{E}-01$

$1.116 \mathrm{E}+00$

$1.831 \mathrm{E}+00$

$1.493 \mathrm{E}+00$

$2.553 \mathrm{E}+00$

$3.647 \mathrm{E}+00$

$4.113 \mathrm{E}+00$

$3.819 \mathrm{E}+00$

$4.585 \mathrm{E}+00$

$3.821 \mathrm{E}+00$
S13-S22

$5.705 \mathrm{E}+00$

$5.548 \mathrm{E}+00$

$4.228 \mathrm{E}+00$

$3.294 \mathrm{E}+00$

$3.082 \mathrm{E}+00$

$3.854 \mathrm{E}+00$

$5.299 \mathrm{E}+00$

$6.107 \mathrm{E}+00$

$5.705 \mathrm{E}+00$

4.132E +00

$1.796 \mathrm{E}+00$

9.241E-01

$1.840 \mathrm{E}+00$

$2.766 \mathrm{E}+00$

$4.172 \mathrm{E}+00$

$5.176 \mathrm{E}+00$

\section{SOURCE SET}

$\begin{array}{ccc}\text { POINT } & \text { S23-S32 } & \text { S01-S07 } \\ \text { R01 } & 2.602 \mathrm{E}+00 & 3.121 \mathrm{E}+00 \\ \text { R02 } & 2.975 \mathrm{E}+00 & 2.459 \mathrm{E}+00\end{array}$

$\begin{array}{cc}\text { S08-S14 } & \text { S15-S21 } \\ 1.613 E+00 & 5.664 E+00 \\ 1.973 E+00 & 5.464 E+00\end{array}$

S22-S28

$1.909 \mathrm{E}+00$

S29-S35

$2.645 \mathrm{E}+00 \quad 3.612 \mathrm{E}+00$ 


\section{REVISED METHODOLOGY FOR DETERMINING CESIUM-137 CONTENT OF HN-200 GROUT CONTAINERS.}

$\begin{array}{lllllll}\text { R03 } & 4.006 \mathrm{E}+00 & 1.602 \mathrm{E}+00 & 1.907 \mathrm{E}+00 & 4.075 \mathrm{E}+00 & 3.273 \mathrm{E}+00 & 4.221 \mathrm{E}+00 \\ \text { R04 } & 4.697 \mathrm{E}+00 & 6.400 \mathrm{E}-01 & 1.540 \mathrm{E}+00 & 2.700 \mathrm{E}+00 & 3.841 \mathrm{E}+00 & 4.866 \mathrm{E}+00 \\ \text { R05 } & 4.531 \mathrm{E}+00 & 1.266 \mathrm{E}-01 & 1.357 \mathrm{E}+00 & 1.273 \mathrm{E}+00 & 4.364 \mathrm{E}+00 & 4.714 \mathrm{E}+00 \\ \text { R06 } & 3.395 \mathrm{E}+00 & 8.997 \mathrm{E}-02 & 1.854 \mathrm{E}+00 & 8.161 \mathrm{E}-01 & 3.917 \mathrm{E}+00 & 3.511 \mathrm{E}+00 \\ \mathbf{R} 07 & 1.750 \mathrm{E}+00 & 1.726 \mathrm{E}-01 & 2.546 \mathrm{E}+00 & 1.311 \mathrm{E}+00 & 3.031 \mathrm{E}+00 & 2.552 \mathrm{E}+00 \\ \text { R08 } & 5.237 \mathrm{E}-01 & 4.032 \mathrm{E}-01 & 2.828 \mathrm{E}+00 & 1.844 \mathrm{E}+00 & 2.310 \mathrm{E}+00 & 1.813 \mathrm{E}+00 \\ \text { R09 } & 1.266 \mathrm{E}-01 & 1.227 \mathrm{E}+00 & 2.547 \mathrm{E}+00 & 1.955 \mathrm{E}+00 & 1.846 \mathrm{E}+00 & 1.410 \mathrm{E}+00 \\ \text { R10 } & 3.912 \mathrm{E}-01 & 1.007 \mathrm{E}+00 & 1.835 \mathrm{E}+00 & 1.567 \mathrm{E}+00 & 1.432 \mathrm{E}+00 & 1.800 \mathrm{E}+00 \\ \text { R11 } & 1.630 \mathrm{E}+00 & 2.149 \mathrm{E}+00 & 9.751 \mathrm{E}-01 & 9.437 \mathrm{E}-01 & 9.239 \mathrm{E}-01 & 2.829 \mathrm{E}+00 \\ \text { R12 } & 3.149 \mathrm{E}+00 & 3.304 \mathrm{E}+00 & 4.418 \mathrm{E}-01 & 8.129 \mathrm{E}-01 & 9.768 \mathrm{E}-01 & 4.369 \mathrm{E}+00 \\ \text { R13 } & 4.293 \mathrm{E}+00 & 3.832 \mathrm{E}+00 & 2.957 \mathrm{E}-01 & 1.829 \mathrm{E}+00 & 1.264 \mathrm{E}+00 & 5.279 \mathrm{E}+00 \\ \text { R14 } & 4.884 \mathrm{E}+00 & 3.619 \mathrm{E}+00 & 2.445 \mathrm{E}-01 & 2.763 \mathrm{E}+00 & 1.620 \mathrm{E}+00 & 5.255 \mathrm{E}+00 \\ \text { R15 } & 4.609 \mathrm{E}+00 & 4.474 \mathrm{E}+00 & 4.097 \mathrm{E}-01 & 4.166 \mathrm{E}+00 & 1.812 \mathrm{E}+00 & 4.639 \mathrm{E}+00 \\ \text { R16 } & 3.568 \mathrm{E}+00 & 3.773 \mathrm{E}+00 & 9.783 \mathrm{E}-01 & 5.160 \mathrm{E}+00 & 1.753 \mathrm{E}+00 & 4.068 \mathrm{E}+00\end{array}$

\begin{tabular}{|c|c|}
\hline POINT & S01-S05 \\
\hline R01 & $2.883 E+00$ \\
\hline $\mathrm{R} 02$ & $2.347 \mathrm{E}+00$ \\
\hline $\mathbf{R 0 3}$ & $1.565 \mathrm{E}+00$ \\
\hline R04 & 6.303E-01 \\
\hline R05 & $1.241 \mathrm{E}-01$ \\
\hline R06 & $8.896 \mathrm{E}-02$ \\
\hline R07 & $1.711 \mathrm{E}-01$ \\
\hline R08 & $3.913 \mathrm{E}-01$ \\
\hline R09 & $1.109 \mathrm{E}+00$ \\
\hline R10 & $4.501 \mathrm{E}-01$ \\
\hline R11 & $9.702 \mathrm{E}-01$ \\
\hline R12 & $1.684 \mathrm{E}+00$ \\
\hline $\mathbf{R 1 3}$ & $2.102 \mathrm{E}+00$ \\
\hline R14 & $2.132 E+00$ \\
\hline R15 & $3.501 E+00$ \\
\hline R16 & $3.300 \mathrm{E}+00$ \\
\hline
\end{tabular}

S06-S10
$2.578 \mathrm{E}-01$
$1.281 \mathrm{E}-01$
$8.757 \mathrm{E}-02$
$1.770 \mathrm{E}-01$
$3.818 \mathrm{E}-01$
$5.997 \mathrm{E}-01$
$7.248 \mathrm{E}-01$
$7.243 \mathrm{E}-01$
$7.221 \mathrm{E}-01$
$1.042 \mathrm{E}+00$
$1.583 \mathrm{E}+00$
$1.963 \mathrm{E}+00$
$2.011 \mathrm{E}+00$
$1.686 \mathrm{E}+00$
$1.084 \mathrm{E}+00$
$5.217 \mathrm{E}-01$

\section{SOURCE SET}

$\begin{array}{ccc}\text { S11-S15 } & \text { S16-S20 } & \text { S21-S25 } \\ 4.032 \mathrm{E}+00 & 3.164 \mathrm{E}+00 & 6.657 \mathrm{E}-01 \\ 4.456 \mathrm{E}+00 & 2.938 \mathrm{E}+00 & 1.400 \mathrm{E}+00 \\ 4.005 \mathrm{E}+00 & 1.917 \mathrm{E}+00 & 1.976 \mathrm{E}+00 \\ 2.857 \mathrm{E}+00 & 1.212 \mathrm{E}+00 & 2.518 \mathrm{E}+00 \\ 1.447 \mathrm{E}+00 & 8.011 \mathrm{E}-01 & 3.143 \mathrm{E}+00 \\ 1.287 \mathrm{E}+00 & 7.817 \mathrm{E}-01 & 3.071 \mathrm{E}+00 \\ 1.824 \mathrm{E}+00 & 1.307 \mathrm{E}+00 & 2.657 \mathrm{E}+00 \\ 2.115 \mathrm{E}+00 & 1.839 \mathrm{E}+00 & 2.225 \mathrm{E}+00 \\ 1.943 \mathrm{E}+00 & 1.943 \mathrm{E}+00 & 1.827 \mathrm{E}+00 \\ 1.350 \mathrm{E}+00 & 1.538 \mathrm{E}+00 & 1.246 \mathrm{E}+00 \\ 5.715 \mathrm{E}-01 & 8.756 \mathrm{E}-01 & 3.501 \mathrm{E}-01 \\ 9.948 \mathrm{E}-02 & 6.807 \mathrm{E}-01 & 1.454 \mathrm{E}-01 \\ 2.778 \mathrm{E}-02 & 1.621 \mathrm{E}+00 & 1.971 \mathrm{E}-01 \\ 2.966 \mathrm{E}-01 & 2.291 \mathrm{E}+00 & 2.250 \mathrm{E}-01 \\ 1.519 \mathrm{E}+00 & 2.756 \mathrm{E}+00 & 2.112 \mathrm{E}-01 \\ 2.921 \mathrm{E}+00 & 3.046 \mathrm{E}+00 & 2.481 \mathrm{E}-01\end{array}$

$\begin{array}{cc}\text { S26-S30 } & \text { S31-S35 } \\ 1.721 \mathrm{E}+00 & 2.997 \mathrm{E}+00 \\ 1.334 \mathrm{E}+00 & 3.549 \mathrm{E}+00 \\ 1.338 \mathrm{E}+00 & 4.190 \mathrm{E}+00 \\ 1.362 \mathrm{E}+00 & 4.831 \mathrm{E}+00 \\ 1.260 \mathrm{E}+00 & 4.677 \mathrm{E}+00 \\ 8.827 \mathrm{E}-01 & 3.476 \mathrm{E}+00 \\ 4.051 \mathrm{E}-01 & 2.523 \mathrm{E}+00 \\ 1.107 \mathrm{E}-01 & 1.792 \mathrm{E}+00 \\ 4.880 \mathrm{E}-02 & 1.391 \mathrm{E}+00 \\ 2.958 \mathrm{E}-01 & 1.719 \mathrm{E}+00 \\ 1.164 \mathrm{E}+00 & 2.306 \mathrm{E}+00 \\ 2.064 \mathrm{E}+00 & 3.268 \mathrm{E}+00 \\ 2.726 \mathrm{E}+00 & 3.815 \mathrm{E}+00 \\ 3.198 \mathrm{E}+00 & 3.672 \mathrm{E}+00 \\ 3.206 \mathrm{E}+00 & 3.223 \mathrm{E}+00 \\ 2.643 \mathrm{E}+00 & 3.053 \mathrm{E}+00\end{array}$

\begin{tabular}{cccccccc} 
& \multicolumn{7}{c}{ SOURCE SET } \\
POINT & S01-S03 & S04-S06 & S07-S09 & S10-S12 & S13-S15 & S16-S18 & S19-S21 \\
R01 & $2.586 \mathrm{E}+00$ & $5.173 \mathrm{E}-01$ & $3.534 \mathrm{E}-02$ & $1.555 \mathrm{E}+00$ & $2.479 \mathrm{E}+00$ & $3.133 \mathrm{E}+00$ & $9.328 \mathrm{E}-02$ \\
R02 & $2.316 \mathrm{E}+00$ & $1.407 \mathrm{E}-01$ & $8.326 \mathrm{E}-03$ & $1.883 \mathrm{E}+00$ & $2.583 \mathrm{E}+00$ & $2.867 \mathrm{E}+00$ & $9.705 \mathrm{E}-02$ \\
R03 & $1.561 \mathrm{E}+00$ & $4.082 \mathrm{E}-02$ & $4.273 \mathrm{E}-03$ & $1.773 \mathrm{E}+00$ & $2.278 \mathrm{E}+00$ & $1.796 \mathrm{E}+00$ & $1.309 \mathrm{E}-01$
\end{tabular}


REVISED METHODOLOGY FOR DETERMINING CESIUM-137 CONTENT OF HN-200 GROUT CONTAINERS.

$\begin{array}{llllllll}\text { R04 } & 6.238 \mathrm{E}-01 & 1.610 \mathrm{E}-02 & 6.089 \mathrm{E}-03 & 1.346 \mathrm{E}+00 & 1.672 \mathrm{E}+00 & 1.058 \mathrm{E}+00 & 1.578 \mathrm{E}-01 \\ \text { R05 } & 9.860 \mathrm{E}-02 & 2.792 \mathrm{E}-02 & 1.781 \mathrm{E}-02 & 8.680 \mathrm{E}-01 & 9.406 \mathrm{E}-01 & 6.258 \mathrm{E}-01 & 1.779 \mathrm{E}-01 \\ \text { R06 } & 1.054 \mathrm{E}-02 & 7.925 \mathrm{E}-02 & 5.792 \mathrm{E}-02 & 6.378 \mathrm{E}-01 & 1.190 \mathrm{E}+00 & 4.617 \mathrm{E}-01 & 3.221 \mathrm{E}-01 \\ \text { R07 } & 1.082 \mathrm{E}-02 & 1.607 \mathrm{E}-01 & 1.511 \mathrm{E}-01 & 5.841 \mathrm{E}-01 & 1.813 \mathrm{E}+00 & 6.516 \mathrm{E}-01 & 6.579 \mathrm{E}-01 \\ \text { R08 } & 1.660 \mathrm{E}-01 & 2.258 \mathrm{E}-01 & 2.890 \mathrm{E}-01 & 4.364 \mathrm{E}-01 & 2.114 \mathrm{E}+00 & 8.668 \mathrm{E}-01 & 9.774 \mathrm{E}-01 \\ \text { R09 } & 8.735 \mathrm{E}-01 & 2.360 \mathrm{E}-01 & 4.956 \mathrm{E}-01 & 2.260 \mathrm{E}-01 & 1.943 \mathrm{E}+00 & 8.539 \mathrm{E}-01 & 1.101 \mathrm{E}+00 \\ \text { R10 } & 1.619 \mathrm{E}-01 & 2.907 \mathrm{E}-01 & 9.649 \mathrm{E}-01 & 7.531 \mathrm{E}-02 & 1.349 \mathrm{E}+00 & 6.025 \mathrm{E}-01 & 9.645 \mathrm{E}-01 \\ \text { R11 } & 2.200 \mathrm{E}-01 & 7.600 \mathrm{E}-01 & 1.555 \mathrm{E}+00 & 1.774 \mathrm{E}-02 & 5.712 \mathrm{E}-01 & 2.983 \mathrm{E}-01 & 6.454 \mathrm{E}-01 \\ \text { R12 } & 2.690 \mathrm{E}-01 & 1.453 \mathrm{E}+00 & 1.921 \mathrm{E}+00 & 4.552 \mathrm{E}-03 & 9.862 \mathrm{E}-02 & 4.779 \mathrm{E}-01 & 3.343 \mathrm{E}-01 \\ \text { R13 } & 2.305 \mathrm{E}-01 & 1.982 \mathrm{E}+00 & 1.899 \mathrm{E}+00 & 5.863 \mathrm{E}-03 & 2.293 \mathrm{E}-02 & 1.589 \mathrm{E}+00 & 2.274 \mathrm{E}-01 \\ \text { R14 } & 1.748 \mathrm{E}-01 & 2.179 \mathrm{E}+00 & 1.464 \mathrm{E}+00 & 4.216 \mathrm{E}-02 & 2.549 \mathrm{E}-01 & 2.286 \mathrm{E}+00 & 2.250 \mathrm{E}-01 \\ \text { R15 } & 1.870 \mathrm{E}+00 & 1.939 \mathrm{E}+00 & 7.760 \mathrm{E}-01 & 2.926 \mathrm{E}-01 & 1.227 \mathrm{E}+00 & 2.752 \mathrm{E}+00 & 1.931 \mathrm{E}-01 \\ \text { R16 } & 2.310 \mathrm{E}+00 & 1.297 \mathrm{E}+00 & 2.141 \mathrm{E}-01 & 9.142 \mathrm{E}-01 & 2.007 \mathrm{E}+00 & 3.035 \mathrm{E}+00 & 1.343 \mathrm{E}-01\end{array}$

$\begin{array}{cccccc}\text { POINT } & \text { S22-S24 } & \text { S25-S27 } & \text { S28-S30 } & \text { S31-S33 } & \text { S34-S35 } \\ \text { R01 } & 4.216 \mathrm{E}-01 & 4.009 \mathrm{E}-01 & 1.502 \mathrm{E}+00 & 3.140 \mathrm{E}-01 & 2.683 \mathrm{E}+00 \\ \text { R02 } & 1.046 \mathrm{E}+00 & 8.256 \mathrm{E}-01 & 8.368 \mathrm{E}-01 & 2.692 \mathrm{E}-01 & 3.279 \mathrm{E}+00 \\ \text { R03 } & 1.561 \mathrm{E}+00 & 1.371 \mathrm{E}+00 & 3.709 \mathrm{E}-01 & 7.259 \mathrm{E}-01 & 3.464 \mathrm{E}+00 \\ \text { R04 } & 2.151 \mathrm{E}+00 & 1.629 \mathrm{E}+00 & 9.509 \mathrm{E}-02 & 1.227 \mathrm{E}+00 & 3.604 \mathrm{E}+00 \\ \text { R05 } & 2.911 \mathrm{E}+00 & 1.448 \mathrm{E}+00 & 4.244 \mathrm{E}-02 & 1.468 \mathrm{E}+00 & 3.209 \mathrm{E}+00 \\ \text { R06 } & 2.971 \mathrm{E}+00 & 9.454 \mathrm{E}-01 & 3.558 \mathrm{E}-02 & 1.323 \mathrm{E}+00 & 2.153 \mathrm{E}+00 \\ \text { R07 } & 2.626 \mathrm{E}+00 & 4.044 \mathrm{E}-01 & 2.904 \mathrm{E}-02 & 8.669 \mathrm{E}-01 & 1.656 \mathrm{E}+00 \\ \text { R08 } & 2.214 \mathrm{E}+00 & 9.562 \mathrm{E}-02 & 2.167 \mathrm{E}-02 & 3.526 \mathrm{E}-01 & 1.439 \mathrm{E}+00 \\ \text { R09 } & 1.814 \mathrm{E}+00 & 3.182 \mathrm{E}-02 & 1.865 \mathrm{E}-02 & 3.201 \mathrm{E}-01 & 1.071 \mathrm{E}+00 \\ \text { R10 } & 1.217 \mathrm{E}+00 & 2.155 \mathrm{E}-01 & 8.093 \mathrm{E}-02 & 1.293 \mathrm{E}+00 & 4.259 \mathrm{E}-01 \\ \text { R11 } & 2.817 \mathrm{E}-01 & 6.407 \mathrm{E}-01 & 5.241 \mathrm{E}-01 & 2.275 \mathrm{E}+00 & 3.137 \mathrm{E}-02 \\ \text { R12 } & 1.350 \mathrm{E}-02 & 9.440 \mathrm{E}-01 & 1.121 \mathrm{E}+00 & 3.266 \mathrm{E}+00 & 1.427 \mathrm{E}-03 \\ \text { R13 } & 7.929 \mathrm{E}-04 & 1.093 \mathrm{E}+00 & 1.634 \mathrm{E}+00 & 3.814 \mathrm{E}+00 & 1.242 \mathrm{E}-03 \\ \text { R14 } & 6.374 \mathrm{E}-04 & 1.051 \mathrm{E}+00 & 2.152 \mathrm{E}+00 & 3.651 \mathrm{E}+00 & 2.116 \mathrm{E}-02 \\ \text { R15 } & 4.514 \mathrm{E}-03 & 8.448 \mathrm{E}-01 & 2.379 \mathrm{E}+00 & 2.837 \mathrm{E}+00 & 3.864 \mathrm{E}-01 \\ \text { R16 } & 5.728 \mathrm{E}-02 & 5.380 \mathrm{E}-01 & 2.172 \mathrm{E}+00 & 1.348 \mathrm{E}+00 & 1.705 \mathrm{E}+00\end{array}$

TABLE 6 - ESTIMATES OF CURIE CONTENT FOR VARIOUS SOURCE SETS COMPARED TO ACTUAL CURIE CONTENT FOR A GROUTED CONTAINER. MULTIPLE SOURCE SET ESTIMATE AND ERROR - 5 FOOT MEASUREMENT 5 FOOT

\begin{tabular}{|c|c|c|c|c|c|c|}
\hline Sou & $\begin{array}{c}\text { AVG } \\
5 \text { FOOT }\end{array}$ & $\begin{array}{l}\text { CONV } \\
\text { FACT }\end{array}$ & $\begin{array}{c}\text { EST } \\
\text { Cs-137 }\end{array}$ & $\begin{array}{l}\text { ACTUAL } \\
\text { - }\end{array}$ & & $\%$ \\
\hline SET & $\mathbf{R} / \mathbf{h r}$ & $\mathrm{R} / \mathrm{hr} / \mathrm{Ci}$ & CURIDS & CURIES & ERROR & ERROR \\
\hline & $1.206 \mathrm{E}+01$ & $1.479 \mathrm{E}-02$ & 815.3966 & 708.6318 & 106.7648 & 15.0663 \\
\hline
\end{tabular}




\section{REVISED METHODOLOGY FOR DETERMINING CESIUM-137 CONTENT OF HN-200 GROUT CONTAINERS.}

\begin{tabular}{|c|c|c|c|c|c|c|}
\hline S01-S30 & $8.905 E+00$ & $1.479 \mathrm{E}-02$ & 602.0648 & 604.0700 & -2.0052 & -0.3320 \\
\hline S06-S35 & $1.059 \mathrm{E}+01$ & $1.479 \mathrm{E}-02$ & 716.3037 & 610.0433 & 106.2604 & 17.4185 \\
\hline S01-S25 & $7.420 \mathrm{E}+00$ & $1.479 \mathrm{E}-02$ & 501.6577 & 520.3863 & -18.7285 & -3.5990 \\
\hline S11-S35 & $9.738 \mathrm{E}+00$ & $1.479 \mathrm{E}-02$ & 658.4350 & 490.8729 & 167.5621 & 34.1355 \\
\hline S01-S20 & $6.038 \mathrm{E}+00$ & $1.479 \mathrm{E}-02$ & 408.2395 & 426.0483 & -17.8087 & -4.1800 \\
\hline S16-S35 & $7.816 \mathrm{E}+00$ & $1.479 \mathrm{E}-02$ & 528.4853 & 393.8550 & 134.6302 & 34.1827 \\
\hline S01-S18 & $5.703 \mathrm{E}+00$ & $1.479 \mathrm{E}-02$ & 385.6012 & 394.2079 & -8.6067 & -2.1833 \\
\hline S19-S35 & $6.357 \mathrm{E}+\infty 0$ & $1.479 \mathrm{E}-02$ & 429.7954 & 314.4239 & 115.3715 & 36.6930 \\
\hline S01-S12 & $2.834 \mathrm{E}+00$ & $1.479 \mathrm{E}-02$ & 191.6404 & 247.8830 & -56.2426 & -22.6892 \\
\hline S13-S24 & $4.477 \mathrm{E}+00$ & $1.479 \mathrm{E}-02$ & 302.6889 & 252.7408 & 49.9481 & 19.7626 \\
\hline S25-S35 & $4.749 \mathrm{E}+00$ & $1.479 \mathrm{E}-02$ & 321.0673 & 208.0080 & 113.0592 & 54.3533 \\
\hline S01-S10 & $2.321 \mathrm{E}+00$ & $1.479 \mathrm{E}-02$ & 156.9616 & 217.7589 & -60.7973 & -27.9195 \\
\hline S13-S22 & $3.977 \mathrm{E}+00$ & $1.479 \mathrm{E}-02$ & 268.8898 & 227.1843 & 41.7055 & 18.3575 \\
\hline S23-S32 & $2.946 \mathrm{E}+00$ & $1.479 \mathrm{E}-02$ & 199.1662 & 173.0444 & 26.1219 & 15.0955 \\
\hline S01-S07 & $2.000 \mathrm{E}+00$ & $1.479 \mathrm{E}-02$ & 135.2224 & 140.8425 & -5.6201 & -3.9904 \\
\hline S08-S14 & $1.459 \mathrm{E}+00$ & $1.479 \mathrm{E}-02$ & 98.6486 & 151.1873 & -52.5387 & -34.7508 \\
\hline S15-S21 & $2.647 \mathrm{E}+00$ & $1.479 \mathrm{E}-02$ & 178.9401 & 162.6650 & 16.2751 & 10.0053 \\
\hline S22-S28 & $2.307 E+00$ & $1.479 \mathrm{E}-02$ & 156.0109 & 116.2697 & 39.7412 & 34.1802 \\
\hline S29-S35 & $3.647 \mathrm{E}+00$ & $1.479 \mathrm{E}-02$ & 246.5747 & 137.6673 & 108.9074 & 79.1091 \\
\hline S01-S05 & $1.466 \mathrm{E}+00$ & $1.479 \mathrm{E}-02$ & 99.0929 & 98.5885 & 0.5044 & 0.5116 \\
\hline S06-S10 & 8.559E-01 & $1.479 \mathrm{E}-02$ & 57.8688 & 119.1704 & -61.3016 & -51.4403 \\
\hline S11-S15 & $1.922 \mathrm{E}+00$ & $1.479 \mathrm{E}-02$ & 129.9497 & 97.0179 & 32.9318 & 33.9441 \\
\hline S16-S20 & $1.794 \mathrm{E}+00$ & $1.479 \mathrm{E}-02$ & 121.3282 & 111.2715 & 10.0567 & 9.0380 \\
\hline S21-S25 & $1.382 E+00$ & 1.479E-02 & 93.4182 & 94.3380 & -0.9198 & -0.9750 \\
\hline S26-S30 & $1.485 \mathrm{E}+00$ & $1.479 \mathrm{E}-02$ & 100.4071 & 83.6838 & 16.7233 & 19.9839 \\
\hline S31-S35 & $3.155 \mathrm{E}+00$ & $1.479 \mathrm{E}-02$ & 213.3318 & 104.5618 & 108.7700 & 104.0246 \\
\hline S01-S03 & $8.427 \mathrm{E}-01$ & $1.479 \mathrm{E}-02$ & 56.9769 & 66.9394 & -9.9624 & -14.8828 \\
\hline S04-S06 & $7.091 \mathrm{E}-01$ & $1.479 \mathrm{E}-02$ & 47.9423 & 47.9673 & -0.0250 & -0.0522 \\
\hline S07-S09 & $6.163 \mathrm{E}-01$ & $1.479 \mathrm{E}-02$ & 41.6691 & 76.0383 & -34.3692 & -45.1999 \\
\hline S10-S12 & $6.663 \mathrm{E}-01$ & $1.479 \mathrm{E}-02$ & 45.0521 & 56.9379 & -11.8859 & -20.8751 \\
\hline S13-S15 & $1.409 \mathrm{E}+00$ & $1.479 \mathrm{E}-02$ & 95.2710 & 66.8938 & 28.3771 & 42.4211 \\
\hline S16-S18 & $1.460 \mathrm{E}+00$ & $1.479 \mathrm{E}-02$ & 98.6898 & 79.4311 & 19.2587 & 24.2458 \\
\hline S19-S21 & $4.024 \mathrm{E}-01$ & $1.479 \mathrm{E}-02$ & 27.2099 & 60.4869 & -33.2771 & -55.0153 \\
\hline S22-S24 & $1.206 \mathrm{E}+00$ & $1.479 \mathrm{E}-02$ & 81.5183 & 45.9289 & 35.5894 & 77.4879 \\
\hline S25-S27 & $7.799 \mathrm{E}-01$ & $1.479 \mathrm{E}-02$ & 52.7335 & 54.8275 & -2.0940 & -3.8192 \\
\hline S28-S30 & $8.135 E-01$ & $1.479 \mathrm{E}-02$ & 55.0020 & 48.6188 & 6.3832 & 13.1290 \\
\hline S31-S33 & $1.584 \mathrm{E}+00$ & $1.479 \mathrm{E}-02$ & 107.1310 & 61.8697 & 45.2613 & 73.1559 \\
\hline S34-S35 & $1.571 \mathrm{E}+00$ & $1.479 \mathrm{E}-02$ & 106.2008 & 42.6921 & 63.5087 & 148.7598 \\
\hline
\end{tabular}

Average \% Error $=15.9784$

INDIVIDUAL SOURCE ESTIMATE AND ERROR - 5 FOOT MEASUREMENT 5 FOOT

\begin{tabular}{|c|c|c|c|c|c|c|}
\hline & $\begin{array}{c}\text { AVG } \\
5 \text { FOOT }\end{array}$ & $\begin{array}{l}\text { CONV } \\
\text { FACT }\end{array}$ & $\begin{array}{c}\text { EST } \\
\text { Cs-137 }\end{array}$ & $\begin{array}{c}\text { ACTUAL } \\
\text { Cs-137 }\end{array}$ & & $\%$ \\
\hline SOURCE & R/hr & $\mathrm{R} / \mathrm{hr} / \mathrm{Ci}$ & CURIES & CURIES & ERROR & ERROR \\
\hline S01 & $6.804 \mathrm{E}-01$ & $1.479 \mathrm{E}-02$ & 46.0063 & 29.4975 & 16.5088 & 55.9668 \\
\hline S02 & $1.403 \mathrm{E}-01$ & $1.479 \mathrm{E}-02$ & 9.4861 & 13.8502 & -4.3641 & -31.5095 \\
\hline S03 & $2.196 \mathrm{E}-02$ & $1.479 \mathrm{E}-02$ & 1.4845 & 23.5916 & -22.1071 & -93.7075 \\
\hline S04 & $5.605 \mathrm{E}-01$ & $1.479 \mathrm{E}-02$ & 37.8946 & 20.6982 & 17.1965 & 83.0820 \\
\hline
\end{tabular}




\section{REVISED METHODOLOGY FOR DETERMINING CESIUM-137 CONTENT OF HN-200 GROUT CONTAINERS.}

\begin{tabular}{|c|c|c|c|c|c|c|}
\hline S05 & $6.243 \mathrm{E}-02$ & $1.479 \mathrm{E}-02$ & 4.2214 & 10.9510 & -6.7296 & -61.4523 \\
\hline S06 & $8.617 \mathrm{E}-02$ & $1.479 \mathrm{E}-02$ & 5.8263 & 16.3182 & -10.4918 & -64.2954 \\
\hline $\mathbf{S} 07$ & $4.482 \mathrm{E}-01$ & $1.479 \mathrm{E}-02$ & 30.3032 & 25.9358 & 4.3673 & 16.8390 \\
\hline S08 & $7.374 \mathrm{E}-02$ & $1.479 \mathrm{E}-02$ & 4.9856 & 25.1678 & -20.1822 & -80.1905 \\
\hline S09 & $9.436 E-02$ & $1.479 \mathrm{E}-02$ & 6.3803 & 24.9347 & -18.5544 & -74.4119 \\
\hline S10 & $1.534 \mathrm{E}-01$ & $1.479 \mathrm{E}-02$ & 10.3733 & 26.8139 & -16.4406 & -61.3137 \\
\hline S11 & $1.100 \mathrm{E}-01$ & $1.479 \mathrm{E}-02$ & 7.4406 & 12.5933 & -5.1527 & -40.9162 \\
\hline S12 & $4.029 \mathrm{E}-01$ & $1.479 \mathrm{E}-02$ & 27.2381 & 17.5307 & 9.7074 & 55.3739 \\
\hline S13 & $4.340 \mathrm{E}-02$ & $1.479 \mathrm{E}-02$ & 2.9347 & 17.9331 & -14.9984 & -83.6352 \\
\hline S14 & $5.812 \mathrm{E}-01$ & $1,479 \mathrm{E}-02$ & 39.2959 & 26.2138 & 13.0821 & 49.9052 \\
\hline S15 & $7.845 \mathrm{E}-01$ & $1.479 \mathrm{E}-02$ & 53.0404 & 22.7469 & 30.2934 & 133.1759 \\
\hline S16 & $3.417 \mathrm{E}-01$ & $1.479 \mathrm{E}-02$ & 23.1010 & 27.6556 & -4.5546 & -16.4691 \\
\hline S17 & $2.325 E-01$ & $1.479 \mathrm{E}-02$ & 15.7190 & 28.5227 & -12.8038 & -44.8897 \\
\hline S18 & 8.855E-01 & $1.479 \mathrm{E}-02$ & 59.8699 & 23.2528 & 36.6171 & 157.4737 \\
\hline S19 & $2.912 \mathrm{E}-01$ & $1.479 \mathrm{E}-02$ & 19.6870 & 15.3416 & 4.3454 & 28.3245 \\
\hline $\mathbf{S 2 0}$ & $4.365 \mathrm{E}-02$ & $1.479 \mathrm{E}-02$ & 2.9513 & 16.4988 & -13.5474 & -82.1118 \\
\hline S21 & $6.761 \mathrm{E}-02$ & $1.479 \mathrm{E}-02$ & 4.5715 & 28.6466 & -24.0751 & -84.0416 \\
\hline $\mathbf{S 2 2}$ & $7.058 \mathrm{E}-01$ & $1.479 \mathrm{E}-02$ & 47.7191 & 20.3724 & 27.3467 & 134.2341 \\
\hline $\mathbf{S 2 3}$ & $1.095 \mathrm{E}-01$ & $1.479 \mathrm{E}-02$ & 7.4012 & 13.0146 & -5.6135 & -43.1319 \\
\hline S24 & $3.904 \mathrm{E}-01$ & $1.479 \mathrm{E}-02$ & 26.3980 & 12.5419 & 13.8561 & 110.4786 \\
\hline $\mathbf{S 2 5}$ & $1.084 \mathrm{E}-01$ & $1.479 \mathrm{E}-02$ & 7.3284 & 19.7625 & -12.4341 & -62.9176 \\
\hline S26 & 3.395E-01 & $1.479 \mathrm{E}-02$ & 22.9567 & 24.8512 & -1.8946 & -7.6236 \\
\hline $\mathbf{S 2 7}$ & $3.320 \mathrm{E}-01$ & $1.479 \mathrm{E}-02$ & 22.4484 & 10.2138 & 12.2347 & 119.7861 \\
\hline $\mathbf{S 2 8}$ & $3.218 \mathrm{E}-01$ & $1.479 \mathrm{E}-02$ & 21.7591 & 15.5133 & 6.2458 & 40.2609 \\
\hline $\mathbf{S 2 9}$ & $1.772 \mathrm{E}-02$ & $1.479 \mathrm{E}-02$ & 1.1979 & 18.0653 & -16.8675 & -93.3693 \\
\hline S30 & 4.739E-01 & $1.479 \mathrm{E}-02$ & 32.0450 & 15.0401 & 17.0049 & 113.0632 \\
\hline S31 & $4.590 \mathrm{E}-01$ & $1.479 \mathrm{E}-02$ & $31.037 !$ & 22.3550 & 8.6821 & 38.8375 \\
\hline S32 & $3.933 \mathrm{E}-01$ & $1.479 \mathrm{E}-02$ & 26.5946 & 21.6866 & 4.9079 & 22.6311 \\
\hline S33 & $7.321 \mathrm{E}-01$ & $1.479 \mathrm{E}-02$ & 49.4994 & 17.8281 & 31.6713 & 177.6486 \\
\hline S34 & $5.184 \mathrm{E}-01$ & $1.479 \mathrm{E}-02$ & 35.0504 & 14.6776 & 20.3728 & 138.8024 \\
\hline S35 & $1.052 \mathrm{E}+00$ & $1.479 \mathrm{E}-02$ & 71.1504 & 28.0146 & 43.1359 & 153.9767 \\
\hline & & & & \multicolumn{2}{|c|}{ Average \% Error = } & 17.2535 \\
\hline
\end{tabular}

TABLE 7 - CALCULATION OF CONVERSION FACTORS FOR AN UNGROUTED HN-200 GROUT CONTAINER ASSUMING A HOMOGENEOUS MIXTURE OF ACTIVITY AND A MATERIAL WITH LITTLE OR NO SHIELDING VALUE (e.g., PAPER).

$\begin{array}{cc}\text { POINT } & \text { R/hr } \\ \text { R01 } & 6.751 \mathrm{E}-02 \\ \text { R02 } & 6.751 \mathrm{E}-02\end{array}$




\section{REVISED METHODOLOGY FOR DETERMINING CESIUM-137 CONTENT OF HN-200 GROUT CONTAINERS.}

\begin{tabular}{|c|c|c|c|c|}
\hline $\mathbf{R} 0 \mathbf{3}$ & $6.751 \mathrm{E}-02$ & & & \\
\hline $\mathbf{R} 04$ & $6.751 \mathrm{E}-02$ & & & \\
\hline R05 & 6.751E-02 & & & \\
\hline R06 & 6.751E-02 & & & \\
\hline $\mathbf{R} 07$ & $6.751 \mathrm{E}-02$ & AVERAGE 5 FOOT READING = & $6.751 \mathrm{E}-02$ & $\mathbf{R} / \mathbf{h r}$ \\
\hline R08 & $6.751 \mathrm{E}-02$ & TOTAL Cs-137 = & 1 & \\
\hline R09 & $6.751 \mathrm{E}-02$ & GROUTED 5 FOOT CONV FACTOR = & $6.751 \mathrm{E}-02$ & $\mathrm{R} / \mathrm{hr} / \mathrm{Ci}$ \\
\hline R10 & $6.751 \mathrm{E}-02$ & & & \\
\hline R11 & $6.751 \mathrm{E}-02$ & \multirow{6}{*}{\multicolumn{3}{|c|}{$\begin{array}{l}5 \text { FOOT readings are taken with the RO- } 7 \text { probe } 5 \text { feet from the surface of the } \\
\text { container. These readings are taken every } 22.5 \text { degrees around the container and } \\
\text { averaged to produce the most accurate representation of the Cs- } 137 \text { curie content } \\
\text { of the container. }\end{array}$}} \\
\hline $\mathbf{R} 12$ & 6.751E-02 & & & \\
\hline $\mathbf{R} 13$ & $6.751 \mathrm{E}-02$ & & & \\
\hline $\mathbf{R} 14$ & 6.751E-02 & & & \\
\hline R15 & $6.751 \mathrm{E}-02$ & & & \\
\hline R16 & $6.751 \mathrm{E}-02$ & & & \\
\hline
\end{tabular}

TABLE 8 - MICROSHIELD CALCULATION FOR FACH SOURCE TO EACH MEASURMENT POINT FROM AN UNGROUTED HN-200 GROUT CONTAINER.

\begin{tabular}{cccccccc} 
& \multicolumn{7}{c}{ SOURCE } \\
POINT & S01 & S02 & S03 & S04 & S05 & S06 & S07 \\
R01 & $3.439 \mathrm{E}+00$ & $6.903 \mathrm{E}-01$ & $1.610 \mathrm{E}+00$ & $1.449 \mathrm{E}+00$ & $5.591 \mathrm{E}-01$ & $1.431 \mathrm{E}+00$ & $1.414 \mathrm{E}+00$ \\
R02 & $3.257 \mathrm{E}+00$ & $6.267 \mathrm{E}-01$ & $1.505 \mathrm{E}+00$ & $1.223 \mathrm{E}+00$ & $5.777 \mathrm{E}-01$ & $1.290 \mathrm{E}+00$ & $1.264 \mathrm{E}+00$ \\
R03 & $2.773 \mathrm{E}+00$ & $5.923 \mathrm{E}-01$ & $1.418 \mathrm{E}+00$ & $1.076 \mathrm{E}+00$ & $6.161 \mathrm{E}-01$ & $1.145 \mathrm{E}+00$ & $1.166 \mathrm{E}+00$ \\
R04 & $2.245 \mathrm{E}+00$ & $5.859 \mathrm{E}-01$ & $1.358 \mathrm{E}+00$ & $9.793 \mathrm{E}-01$ & $6.758 \mathrm{E}-01$ & $1.024 \mathrm{E}+00$ & $1.117 \mathrm{E}+00$ \\
R05 & $1.862 \mathrm{E}+00$ & $6.070 \mathrm{E}-01$ & $1.325 \mathrm{E}+00$ & $9.286 \mathrm{E}-01$ & $7.577 \mathrm{E}-01$ & $9.355 \mathrm{E}-01$ & $1.113 \mathrm{E}+00$
\end{tabular}




\section{REVISED METHODOLOGY FOR DETERMINING CESIUM-137 CONTENT OF HN-200 GROUT CONTAINERS.}

$\begin{array}{llllllll}\text { R06 } & 1.616 \mathrm{E}+00 & 6.570 \mathrm{E}-01 & 1.322 \mathrm{E}+00 & 9.198 \mathrm{E}-01 & 8.570 \mathrm{E}-01 & 8.782 \mathrm{E}-01 & 1.154 \mathrm{E}+00 \\ \text { R07 } & 1.454 \mathrm{E}+00 & 7.341 \mathrm{E}-01 & 1.348 \mathrm{E}+00 & 9.524 \mathrm{E}-01 & 9.548 \mathrm{E}-01 & 8.502 \mathrm{E}-01 & 1.242 \mathrm{E}+00 \\ \text { R08 } & 1.359 \mathrm{E}+00 & 7.800 \mathrm{E}-01 & 1.403 \mathrm{E}+00 & 1.029 \mathrm{E}+00 & 1.018 \mathrm{E}+00 & 8.504 \mathrm{E}-01 & 1.383 \mathrm{E}+00 \\ \text { R09 } & 1.325 \mathrm{E}+00 & 1.008 \mathrm{E}+00 & 1.484 \mathrm{E}+00 & 1.154 \mathrm{E}+00 & 1.019 \mathrm{E}+00 & 8.787 \mathrm{E}-01 & 1.589 \mathrm{E}+00 \\ \text { R10 } & 1.350 \mathrm{E}+00 & 1.389 \mathrm{E}+00 & 1.586 \mathrm{E}+00 & 1.339 \mathrm{E}+00 & 9.569 \mathrm{E}-01 & 9.364 \mathrm{E}-01 & 1.894 \mathrm{E}+00 \\ \text { R11 } & 1.435 \mathrm{E}+00 & 1.668 \mathrm{E}+00 & 1.698 \mathrm{E}+00 & 1.643 \mathrm{E}+00 & 8.594 \mathrm{E}-01 & 1.026 \mathrm{E}+00 & 2.249 \mathrm{E}+00 \\ \text { R12 } & 1.587 \mathrm{E}+00 & 1.733 \mathrm{E}+00 & 1.799 \mathrm{E}+00 & 2.045 \mathrm{E}+00 & 7.598 \mathrm{E}-01 & 1.147 \mathrm{E}+00 & 2.510 \mathrm{E}+00 \\ \text { R13 } & 1.817 \mathrm{E}+00 & 1.537 \mathrm{E}+00 & 1.862 \mathrm{E}+00 & 2.376 \mathrm{E}+00 & 6.774 \mathrm{E}-01 & 1.292 \mathrm{E}+00 & 2.534 \mathrm{E}+00 \\ \text { R14 } & 2.175 \mathrm{E}+00 & 1.203 \mathrm{E}+00 & 1.869 \mathrm{E}+00 & 2.446 \mathrm{E}+00 & 6.172 \mathrm{E}-01 & 1.433 \mathrm{E}+00 & 2.308 \mathrm{E}+00 \\ \text { R15 } & 2.689 \mathrm{E}+00 & 8.065 \mathrm{E}-01 & 1.816 \mathrm{E}+00 & 2.206 \mathrm{E}+00 & 5.784 \mathrm{E}-01 & 1.523 \mathrm{E}+00 & 1.957 \mathrm{E}+00 \\ \text { R16 } & 3.196 \mathrm{E}+00 & 7.722 \mathrm{E}-01 & 1.722 \mathrm{E}+00 & 1.811 \mathrm{E}+00 & 5.593 \mathrm{E}-01 & 1.522 \mathrm{E}+00 & 1.635 \mathrm{E}+00\end{array}$

$\begin{array}{cccccccc}\text { POINT } & \text { S08 } & \text { S09 } & \text { S10 } & \text { S11 } & \text { S12 } & \text { S13 } & \text { S14 } \\ \text { R01 } & 1.627 \mathrm{E}+00 & 1.268 \mathrm{E}+00 & 1.425 \mathrm{E}+00 & 1.199 \mathrm{E}+00 & 1.846 \mathrm{E}+00 & 1.274 \mathrm{E}+00 & 1.178 \mathrm{E}+00 \\ \text { R02 } & 1.509 \mathrm{E}+00 & 1.276 \mathrm{E}+00 & 1.555 \mathrm{E}+00 & 1.266 \mathrm{E}+00 & 2.013 \mathrm{E}+00 & 1.377 \mathrm{E}+00 & 1.245 \mathrm{E}+00 \\ \mathbf{R 0 3} & 1.429 \mathrm{E}+00 & 1.323 \mathrm{E}+00 & 1.738 \mathrm{E}+00 & 1.231 \mathrm{E}+00 & 1.939 \mathrm{E}+00 & 1.456 \mathrm{E}+00 & 1.369 \mathrm{E}+00 \\ \text { R04 } & 1.387 \mathrm{E}+00 & 1.409 \mathrm{E}+00 & 1.966 \mathrm{E}+00 & 1.115 \mathrm{E}+00 & 1.675 \mathrm{E}+00 & 1.485 \mathrm{E}+00 & 1.557 \mathrm{E}+00 \\ \text { R05 } & 1.383 \mathrm{E}+00 & 1.536 \mathrm{E}+00 & 2.202 \mathrm{E}+00 & 9.703 \mathrm{E}-01 & 1.365 \mathrm{E}+00 & 1.454 \mathrm{E}+00 & 1.843 \mathrm{E}+00 \\ \text { R06 } & 1.416 \mathrm{E}+00 & 1.701 \mathrm{E}+00 & 2.372 \mathrm{E}+00 & 8.415 \mathrm{E}-01 & 1.128 \mathrm{E}+00 & 1.374 \mathrm{E}+00 & 2.260 \mathrm{E}+00 \\ \text { R07 } & 1.488 \mathrm{E}+00 & 1.886 \mathrm{E}+00 & 2.401 \mathrm{E}+00 & 7.446 \mathrm{E}-01 & 9.754 \mathrm{E}-01 & 1.270 \mathrm{E}+00 & 2.685 \mathrm{E}+00 \\ \text { R08 } & 1.598 \mathrm{E}+00 & 2.050 \mathrm{E}+00 & 2.274 \mathrm{E}+00 & 6.782 \mathrm{E}-01 & 8.738 \mathrm{E}-01 & 1.168 \mathrm{E}+00 & 2.912 \mathrm{E}+00 \\ \text { R09 } & 1.743 \mathrm{E}+00 & 2.139 \mathrm{E}+00 & 2.052 \mathrm{E}+00 & 6.385 \mathrm{E}-01 & 8.129 \mathrm{E}-01 & 1.083 \mathrm{E}+00 & 2.797 \mathrm{E}+00 \\ \text { R10 } & 1.908 \mathrm{E}+00 & 2.114 \mathrm{E}+00 & 1.814 \mathrm{E}+00 & 6.231 \mathrm{E}-01 & 7.886 \mathrm{E}-01 & 1.021 \mathrm{E}+00 & 2.417 \mathrm{E}+00 \\ \text { R11 } & 2.064 \mathrm{E}+00 & 1.987 \mathrm{E}+00 & 1.614 \mathrm{E}+00 & 6.308 \mathrm{E}-01 & 7.987 \mathrm{E}-01 & 9.843 \mathrm{E}-01 & 1.977 \mathrm{E}+00 \\ \text { R12 } & 2.166 \mathrm{E}+00 & 1.807 \mathrm{E}+00 & 1.465 \mathrm{E}+00 & 6.622 \mathrm{E}-01 & 8.442 \mathrm{E}-01 & 9.723 \mathrm{E}-01 & 1.642 \mathrm{E}+00 \\ \text { R13 } & 2.177 \mathrm{E}+00 & 1.627 \mathrm{E}+00 & 1.366 \mathrm{E}+00 & 7.194 \mathrm{E}-01 & 9.284 \mathrm{E}-01 & 9.853 \mathrm{E}-01 & 1.426 \mathrm{E}+00 \\ \text { R14 } & 2.093 \mathrm{E}+00 & 1.477 \mathrm{E}+00 & 1.315 \mathrm{E}+00 & 8.057 \mathrm{E}-01 & 1.058 \mathrm{E}+00 & 1.023 \mathrm{E}+00 & 1.282 \mathrm{E}+00 \\ \text { R15 } & 1.945 \mathrm{E}+00 & 1.368 \mathrm{E}+00 & 1.307 \mathrm{E}+00 & 9.245 \mathrm{E}-01 & 1.255 \mathrm{E}+00 & 1.086 \mathrm{E}+00 & 1.195 \mathrm{E}+00 \\ \text { R16 } & 1.778 \mathrm{E}+00 & 1.298 \mathrm{E}+00 & 1.343 \mathrm{E}+00 & 1.067 \mathrm{E}+00 & 1.545 \mathrm{E}+00 & 1.172 \mathrm{E}+00 & 1.161 \mathrm{E}+00\end{array}$

$\begin{array}{cccccccc}\text { POINT } & \text { S15 } & \text { S16 } & \text { S17 } & \text { S18 } & \text { S19 } & \text { S20 } & \text { S21 } \\ \text { R01 } & 2.589 \mathrm{E}+00 & 2.486 \mathrm{E}+00 & 1.363 \mathrm{E}+00 & 2.374 \mathrm{E}+00 & 7.022 \mathrm{E}-01 & 1.133 \mathrm{E}+00 & 2.070 \mathrm{E}+00 \\ \mathbf{R} 02 & 2.644 \mathrm{E}+00 & 2.770 \mathrm{E}+00 & 1.414 \mathrm{E}+00 & 1.827 \mathrm{E}+00 & 7.183 \mathrm{E}-01 & 1.235 \mathrm{E}+00 & 1.910 \mathrm{E}+00 \\ \mathbf{R 0 3} & 2.340 \mathrm{E}+00 & 2.832 \mathrm{E}+00 & 1.518 \mathrm{E}+00 & 1.263 \mathrm{E}+00 & 7.655 \mathrm{E}-01 & 1.321 \mathrm{E}+00 & 1.776 \mathrm{E}+00 \\ \text { R04 } & 1.859 \mathrm{E}+00 & 2.637 \mathrm{E}+00 & 1.681 \mathrm{E}+00 & 1.240 \mathrm{E}+00 & 8.476 \mathrm{E}-01 & 1.364 \mathrm{E}+00 & 1.679 \mathrm{E}+00 \\ \text { R05 } & 1.391 \mathrm{E}+00 & 2.293 \mathrm{E}+00 & 1.909 \mathrm{E}+00 & 1.111 \mathrm{E}+00 & 9.722 \mathrm{E}-01 & 1.348 \mathrm{E}+00 & 1.621 \mathrm{E}+00 \\ \text { R06 } & 1.245 \mathrm{E}+00 & 1.947 \mathrm{E}+00 & 2.197 \mathrm{E}+00 & 1.018 \mathrm{E}+00 & 1.158 \mathrm{E}+00 & 1.279 \mathrm{E}+00 & 1.603 \mathrm{E}+00 \\ \text { R07 } & 1.109 \mathrm{E}+00 & 1.680 \mathrm{E}+00 & 2.489 \mathrm{E}+00 & 9.706 \mathrm{E}-01 & 1.402 \mathrm{E}+00 & 1.181 \mathrm{E}+00 & 1.626 \mathrm{E}+00 \\ \text { R08 } & 1.013 \mathrm{E}+00 & 1.494 \mathrm{E}+00 & 2.677 \mathrm{E}+00 & 9.683 \mathrm{E}-01 & 1.626 \mathrm{E}+00 & 1.081 \mathrm{E}+00 & 1.688 \mathrm{E}+00 \\ \text { R09 } & 9.627 \mathrm{E}-01 & 1.373 \mathrm{E}+00 & 2.670 \mathrm{E}+00 & 1.011 \mathrm{E}+00 & 1.715 \mathrm{E}+00 & 9.950 \mathrm{E}-01 & 1.790 \mathrm{E}+00\end{array}$




\section{REVISED METHODOLOGY FOR DETERMINING CESIUM-137 CONTENT OF HN-200 GROUT CONTAINERS.}

$\begin{array}{llllllll}\text { R10 } & 9.566 \mathrm{E}-01 & 1.309 \mathrm{E}+00 & 2.470 \mathrm{E}+00 & 1.099 \mathrm{E}+00 & 1.612 \mathrm{E}+00 & 9.309 \mathrm{E}-01 & 1.928 \mathrm{E}+00 \\ \text { R11 } & 9.940 \mathrm{E}-01 & 1.298 \mathrm{E}+00 & 2.175 \mathrm{E}+00 & 1.226 \mathrm{E}+00 & 1.383 \mathrm{E}+00 & 8.902 \mathrm{E}-01 & 2.090 \mathrm{E}+00 \\ \text { R12 } & 1.077 \mathrm{E}+00 & 1.337 \mathrm{E}+00 & 1.890 \mathrm{E}+00 & 1.260 \mathrm{E}+00 & 1.141 \mathrm{E}+00 & 8.731 \mathrm{E}-01 & 2.251 \mathrm{E}+00 \\ \text { R13 } & 1.204 \mathrm{E}+00 & 1.430 \mathrm{E}+00 & 1.667 \mathrm{E}+00 & 1.756 \mathrm{E}+00 & 9.606 \mathrm{E}-01 & 8.793 \mathrm{E}-01 & 2.371 \mathrm{E}+00 \\ \text { R14 } & 1.335 \mathrm{E}+00 & 1.585 \mathrm{E}+00 & 1.509 \mathrm{E}+00 & 2.324 \mathrm{E}+00 & 8.398 \mathrm{E}-01 & 9.090 \mathrm{E}-01 & 2.413 \mathrm{E}+00 \\ \text { R15 } & 1.713 \mathrm{E}+00 & 1.812 \mathrm{E}+00 & 1.409 \mathrm{E}+00 & 2.709 \mathrm{E}+00 & 7.607 \mathrm{E}-01 & 9.622 \mathrm{E}-01 & 2.360 \mathrm{E}+00 \\ \text { R16 } & 2.213 \mathrm{E}+00 & 2.126 \mathrm{E}+00 & 1.361 \mathrm{E}+00 & 2.730 \mathrm{E}+00 & 7.159 \mathrm{E}-01 & 1.038 \mathrm{E}+00 & 2.233 \mathrm{E}+00\end{array}$

$\begin{array}{ccc}\text { POIN } & \text { S22 } & \text { S23 } \\ \text { R01 } & 8.724 \mathrm{E}-01 & 8.679 \mathrm{E}-01 \\ \text { R02 } & 9.504 \mathrm{E}-01 & 9.987 \mathrm{E}-01 \\ \text { R03 } & 1.057 \mathrm{E}+00 & 1.118 \mathrm{E}+00 \\ \text { R04 } & 1.106 \mathrm{E}+00 & 1.177 \mathrm{E}+00 \\ \text { R05 } & 1.542 \mathrm{E}+00 & 1.144 \mathrm{E}+00 \\ \text { R06 } & 1.960 \mathrm{E}+00 & 1.037 \mathrm{E}+00 \\ \text { R07 } & 2.225 \mathrm{E}+00 & 9.045 \mathrm{E}-01 \\ \text { R08 } & 2.199 \mathrm{E}+00 & 7.881 \mathrm{E}-01 \\ \text { R09 } & 1.899 \mathrm{E}+00 & 7.012 \mathrm{E}-01 \\ \text { R10 } & 1.456 \mathrm{E}+00 & 6.415 \mathrm{E}-01 \\ \text { R11 } & 1.092 \mathrm{E}+00 & 6.057 \mathrm{E}-01 \\ \text { R12 } & 1.039 \mathrm{E}+00 & 5.920 \mathrm{E}-01 \\ \text { R13 } & 9.352 \mathrm{E}-01 & 5.995 \mathrm{E}-01 \\ \text { R14 } & 8.633 \mathrm{E}-01 & 6.288 \mathrm{E}-01 \\ \text { R15 } & 8.294 \mathrm{E}-01 & 6.812 \mathrm{E}-01 \\ \text { R16 } & 8.323 \mathrm{E}-01 & 5.798 \mathrm{E}-01\end{array}$

\begin{tabular}{|c|c|c|c|c|}
\hline \multicolumn{2}{|c|}{ SOURCE } & \multirow[b]{2}{*}{526} & \multirow[b]{2}{*}{$\mathbf{S 2 7}$} & \multirow[b]{2}{*}{$\mathbf{S 2 8}$} \\
\hline S24 & S25 & & & \\
\hline $9.156 \mathrm{E}-01$ & $1.587 \mathrm{E}+00$ & $1.748 \mathrm{E}+00$ & $6.209 \mathrm{E}-01$ & $1.578 \mathrm{E}+00$ \\
\hline $1.169 \mathrm{E}+00$ & $1.745 \mathrm{E}+00$ & $2.071 \mathrm{E}+00$ & $5.457 \mathrm{E}-01$ & $1.380 \mathrm{E}+00$ \\
\hline $1.411 \mathrm{E}+00$ & $1.821 E+00$ & $2.408 \mathrm{E}+00$ & $4.881 \mathrm{E}-01$ & $1.138 \mathrm{E}+00$ \\
\hline $1.515 \mathrm{E}+00$ & $1.782 \mathrm{E}+00$ & $2.619 \mathrm{E}+\infty 0$ & $4.519 \mathrm{E}-01$ & $9.483 \mathrm{E}-01$ \\
\hline $1.411 \mathrm{E}+00$ & $1.644 E+00$ & $2.584 \mathrm{E}+00$ & $4.366 \mathrm{E}-01$ & $8.260 \mathrm{E}-01$ \\
\hline $1.169 \mathrm{E}+00$ & $1.467 \mathrm{E}+00$ & $2.325 \mathrm{E}+00$ & $4.410 \mathrm{E}-01$ & $7.433 \mathrm{E}-01$ \\
\hline $9.145 \mathrm{E}-01$ & $1.300 \mathrm{E}+00$ & $1.980 \mathrm{E}+00$ & $4.657 \mathrm{E}-01$ & $6.928 \mathrm{E}-01$ \\
\hline $7.575 \mathrm{E}-01$ & $1.168 \mathrm{E}+00$ & $1.676 \mathrm{E}+00$ & $5.117 \mathrm{E}-01$ & $6.719 \mathrm{E}-01$ \\
\hline $6.626 \mathrm{E}-01$ & $1.075 E+00$ & $1.455 \mathrm{E}+00$ & $5.781 \mathrm{E}-01$ & $6.793 \mathrm{E}-01$ \\
\hline $5.966 \mathrm{E}-01$ & $1.019 \mathrm{E}+00$ & $1.306 \mathrm{E}+00$ & $6.711 \mathrm{E}-01$ & $7.153 \mathrm{E}-01$ \\
\hline $5.584 \mathrm{E}-01$ & $9.971 \mathrm{E}-01$ & $1.214 \mathrm{E}+00$ & $8.710 \mathrm{E}-01$ & $7.823 \mathrm{E}-01$ \\
\hline $5.459 \mathrm{E}-01$ & $1.008 \mathrm{E}+00$ & $1,173 \mathrm{E}+00$ & $1.080 \mathrm{E}+00$ & 8.839E-01 \\
\hline 5.584E-01 & $1.053 \mathrm{E}+00$ & $1.179 \mathrm{E}+00$ & $1.204 \mathrm{E}+00$ & $1.037 \mathrm{E}+00$ \\
\hline $5.966 \mathrm{E}-01$ & $1.134 \mathrm{E}+00$ & $1.233 \mathrm{E}+\infty 0$ & $1.165 \mathrm{E}+00$ & $1.263 \mathrm{E}+00$ \\
\hline $6.627 \mathrm{E}-01$ & $1.252 \mathrm{E}+00$ & $1.339 \mathrm{E}+\infty 0$ & $9.895 \mathrm{E}-01$ & $1.496 \mathrm{E}+00$ \\
\hline $7.576 \mathrm{E}-01$ & $1.409 E+00$ & $1.506 \mathrm{E}+00$ & $7.739 \mathrm{E}-01$ & $1.628 \mathrm{E}+00$ \\
\hline
\end{tabular}

$\begin{array}{cc}\text { POINT } & \text { S29 } \\ \text { R01 } & 1.206 \mathrm{E}+00 \\ \text { R02 } & 1.248 \mathrm{E}+00 \\ \text { R03 } & 1.286 \mathrm{E}+00 \\ \mathbf{R 0 4} & 1.313 \mathrm{E}+00 \\ \mathbf{R} 05 & 1.324 \mathrm{E}+00 \\ \text { R06 } & 1.315 \mathrm{E}+00 \\ \text { R07 } & 1.290 \mathrm{E}+00 \\ \text { R08 } & 1.253 \mathrm{E}+00 \\ \text { R09 } & 1.211 \mathrm{E}+00 \\ \text { R10 } & 1.173 \mathrm{E}+00\end{array}$

\begin{tabular}{ccc} 
& \multicolumn{2}{c}{ SOURCE } \\
S30 & S31 & S32 \\
$1.030 \mathrm{E}+00$ & $1.601 \mathrm{E}+00$ & $1.350 \mathrm{E}+00$ \\
$8.692 \mathrm{E}-01$ & $1.354 \mathrm{E}+00$ & $1.596 \mathrm{E}+00$ \\
$7.667 \mathrm{E}-01$ & $1.191 \mathrm{E}+00$ & $1.928 \mathrm{E}+00$ \\
$6.939 \mathrm{E}-01$ & $1.085 \mathrm{E}+00$ & $2.255 \mathrm{E}+00$ \\
$6.525 \mathrm{E}-01$ & $1.029 \mathrm{E}+00$ & $2.420 \mathrm{E}+00$ \\
$6.407 \mathrm{E}-01$ & $1.017 \mathrm{E}+00$ & $2.320 \mathrm{E}+00$ \\
$6.578 \mathrm{E}-01$ & $1.049 \mathrm{E}+00$ & $2.020 \mathrm{E}+00$ \\
$7.049 \mathrm{E}-01$ & $1.127 \mathrm{E}+00$ & $1.676 \mathrm{E}+00$ \\
$7.834 \mathrm{E}-01$ & $1.258 \mathrm{E}+00$ & $1.405 \mathrm{E}+00$ \\
$8.916 \mathrm{E}-01$ & $1.454 \mathrm{E}+00$ & $1.221 \mathrm{E}+00$
\end{tabular}

S33
$9.940 \mathrm{E}-01$
$8.871 \mathrm{E}-01$
$8.063 \mathrm{E}-01$
$7.635 \mathrm{E}-01$
$7.571 \mathrm{E}-01$
$7.865 \mathrm{E}-01$
$8.536 \mathrm{E}-01$
$9.553 \mathrm{E}-01$
$9.938 \mathrm{E}-01$
$1.331 \mathrm{E}+00$

$\mathbf{S 3 4}$

6.681E-01

$7.416 \mathrm{E}-01$

7.955E-01

9.931E-01

$1.338 \mathrm{E}+00$

$1.588 \mathrm{E}+00$

$1.653 \mathrm{E}+00$

$1.489 \mathrm{E}+00$

$1.193 E+00$

8.407E-01

\section{S35}

$2.912 \mathrm{E}+00$

$3.422 \mathrm{E}+00$

$3.479 E+00$

$3.039 E+00$

$2.375 E+00$

1.750E +00

$1.571 \mathrm{E}+00$

$1.395 \mathrm{E}+00$

$1.272 \mathrm{E}+00$

$1.209 E+00$ 


\section{REVISED METHODOLOGY FOR DETERMINING CESIUM-137 CONTENT OF HN-200 GROUT CONTAINERS.}

$\begin{array}{llllllll}\text { R11 } & 1.141 \mathrm{E}+00 & 1.081 \mathrm{E}+00 & 1.751 \mathrm{E}+00 & 1.100 \mathrm{E}+00 & 1.836 \mathrm{E}+00 & 7.832 \mathrm{E}-01 & 1.204 \mathrm{E}+00 \\ \text { R12 } & 1.121 \mathrm{E}+00 & 1.386 \mathrm{E}+00 & 2.126 \mathrm{E}+00 & 1.028 \mathrm{E}+00 & 2.197 \mathrm{E}+00 & 7.051 \mathrm{E}-01 & 1.254 \mathrm{E}+00 \\ \text { R13 } & 1.114 \mathrm{E}+00 & 1.657 \mathrm{E}+00 & 2.441 \mathrm{E}+00 & 1.002 \mathrm{E}+00 & 2.264 \mathrm{E}+00 & 6.427 \mathrm{E}-01 & 1.365 \mathrm{E}+00 \\ \text { R14 } & 1.120 \mathrm{E}+00 & 1.756 \mathrm{E}+00 & 2.522 \mathrm{E}+00 & 1.017 \mathrm{E}+00 & 1.988 \mathrm{E}+00 & 6.081 \mathrm{E}-01 & 1.532 \mathrm{E}+00 \\ \text { R15 } & 1.138 \mathrm{E}+00 & 1.616 \mathrm{E}+00 & 2.316 \mathrm{E}+00 & 1.076 \mathrm{E}+00 & 1.539 \mathrm{E}+00 & 6.008 \mathrm{E}-01 & 1.699 \mathrm{E}+00 \\ \text { R16 } & 1.168 \mathrm{E}+00 & 1.329 \mathrm{E}+00 & 1.952 \mathrm{E}+00 & 1.184 \mathrm{E}+00 & 1.001 \mathrm{E}+00 & 6.206 \mathrm{E}-01 & 2.232 \mathrm{E}+00\end{array}$

TABLE 9 - TOTAL RADIATION LEVEL AT EACH MEASURMENT POINT FROM AN UNGROUTED HN-200 GROUT CONTAINER FOR EACH SET OF SOURCES TO BE ANALYZED.

\begin{tabular}{cccccccc} 
& \multicolumn{7}{c}{ SOURCE SET } \\
POINT & S01-S35 & S01-S30 & S06-S35 & S01-S25 & S11-S35 & S01-S20 & S16-S35 \\
R01 & $5.108 E+01$ & $4.355 E+01$ & $4.333 E+01$ & $3.737 E+01$ & $3.617 E+01$ & $3.106 E+01$ & $2.808 E+01$ \\
R02 & $5.148 E+01$ & $4.348 E+01$ & $4.429 E+01$ & $3.737 E+01$ & $3.740 E+01$ & $3.059 E+01$ & $2.885 E+01$ \\
R03 & $5.078 E+01$ & $4.258 E+01$ & $4.431 E+01$ & $3.649 E+01$ & $3.750 E+01$ & $2.931 E+01$ & $2.917 E+01$ \\
R04 & $4.963 E+01$ & $4.149 E+01$ & $4.378 E+01$ & $3.547 E+01$ & $3.688 E+01$ & $2.821 E+01$ & $2.919 E+01$ \\
R05 & $4.841 E+01$ & $4.049 E+01$ & $4.293 E+01$ & $3.467 E+01$ & $3.576 E+01$ & $2.731 E+01$ & $2.874 E+01$ \\
R06 & $4.750 E+01$ & $4.004 E+01$ & $4.213 E+01$ & $3.458 E+01$ & $3.461 E+01$ & $2.734 E+01$ & $2.776 E+01$
\end{tabular}


HNF-4799, Rev 0

August 25, 1999

\section{REVISED METHODOLOGY FOR DETERMINING CESIUM-137 CONTENT OF HN-200 GROUT CONTAINERS.}

$\begin{array}{llllllll}\text { R07 } & 4.702 \mathrm{E}+01 & 3.987 \mathrm{E}+01 & 4.158 \mathrm{E}+01 & 3.479 \mathrm{E}+01 & 3.371 \mathrm{E}+01 & 2.782 \mathrm{E}+01 & 2.693 \mathrm{E}+01 \\ \mathbf{R 0 8} & 4.630 \mathrm{E}+01 & 3.965 \mathrm{E}+01 & 4.071 \mathrm{E}+01 & 3.484 \mathrm{E}+01 & 3.255 \mathrm{E}+01 & 2.824 \mathrm{E}+01 & 2.591 \mathrm{E}+01 \\ \mathbf{R} 09 & 4.541 \mathrm{E}+01 & 3.928 \mathrm{E}+01 & 3.942 \mathrm{E}+01 & 3.458 \mathrm{E}+01 & 3.101 \mathrm{E}+01 & 2.845 \mathrm{E}+01 & 2.472 \mathrm{E}+01 \\ \text { R10 } & 4.497 \mathrm{E}+01 & 3.891 \mathrm{E}+01 & 3.835 \mathrm{E}+01 & 3.416 \mathrm{E}+01 & 2.968 \mathrm{E}+01 & 2.851 \mathrm{E}+01 & 2.387 \mathrm{E}+01 \\ \text { R11 } & 4.571 \mathrm{E}+01 & 3.903 \mathrm{E}+01 & 3.840 \mathrm{E}+01 & 3.394 \mathrm{E}+01 & 2.946 \mathrm{E}+01 & 2.860 \mathrm{E}+01 & 2.408 \mathrm{E}+01 \\ \text { R12 } & 4.711 \mathrm{E}+01 & 3.980 \mathrm{E}+01 & 3.918 \mathrm{E}+01 & 3.415 \mathrm{E}+01 & 3.009 \mathrm{E}+01 & 2.872 \mathrm{E}+01 & 2.489 \mathrm{E}+01 \\ \text { R13 } & 4.864 \mathrm{E}+01 & 4.093 \mathrm{E}+01 & 4.037 \mathrm{E}+01 & 3.474 \mathrm{E}+01 & 3.138 \mathrm{E}+01 & 2.922 \mathrm{E}+01 & 2.612 \mathrm{E}+01 \\ \text { R14 } & 4.945 \mathrm{E}+01 & 4.178 \mathrm{E}+01 & 4.114 \mathrm{E}+01 & 3.524 \mathrm{E}+01 & 3.251 \mathrm{E}+01 & 2.961 \mathrm{E}+01 & 2.701 \mathrm{E}+01 \\ \text { R15 } & 4.962 \mathrm{E}+01 & 4.239 \mathrm{E}+01 & 4.152 \mathrm{E}+01 & 3.581 \mathrm{E}+01 & 3.342 \mathrm{E}+01 & 3.002 \mathrm{E}+01 & 2.725 \mathrm{E}+01 \\ \text { R16 } & 4.997 \mathrm{E}+01 & 4.298 \mathrm{E}+01 & 4.191 \mathrm{E}+01 & 3.658 \mathrm{E}+01 & 3.434 \mathrm{E}+01 & 3.077 \mathrm{E}+01 & 2.718 \mathrm{E}+01\end{array}$

$\begin{array}{cc}\text { POINT } & \text { S01-S18 } \\ \text { R01 } & 2.922 \mathrm{E}+01 \\ \text { R02 } & 2.864 \mathrm{E}+01 \\ \text { R03 } & 2.722 \mathrm{E}+01 \\ \text { R04 } & 2.600 \mathrm{E}+01 \\ \text { R05 } & 2.499 \mathrm{E}+01 \\ \text { R06 } & 2.490 \mathrm{E}+01 \\ \text { R07 } & 2.523 \mathrm{E}+01 \\ \text { R08 } & 2.553 \mathrm{E}+01 \\ \text { R09 } & 2.574 \mathrm{E}+01 \\ \text { R10 } & 2.597 \mathrm{E}+01 \\ \text { R11 } & 2.633 \mathrm{E}+01 \\ \text { R12 } & 2.670 \mathrm{E}+01 \\ \text { R13 } & 2.738 \mathrm{E}+01 \\ \text { R14 } & 2.786 \mathrm{E}+01 \\ \text { R15 } & 2.830 \mathrm{E}+01 \\ \text { R16 } & 2.901 \mathrm{E}+01\end{array}$

$\begin{array}{cc}\text { POINT } & \text { S23-S32 } \\ \text { R01 } & 1.250 \mathrm{E}+01 \\ \text { R02 } & 1.298 \mathrm{E}+01 \\ \text { R03 } & 1.356 \mathrm{E}+01 \\ \text { R04 } & 1.384 \mathrm{E}+01 \\ \text { R05 } & 1.347 \mathrm{E}+01 \\ \text { R06 } & 1.248 \mathrm{E}+01 \\ \text { R07 } & 1.127 \mathrm{E}+01 \\ \text { R08 } & 1.033 \mathrm{E}+01 \\ \text { R09 } & 9.809 \mathrm{E}+00 \\ \text { R10 } & 9.689 \mathrm{E}+00 \\ \text { R11 } & 1.010 \mathrm{E}+01\end{array}$

S19-S35
$2.186 \mathrm{E}+01$
$2.284 \mathrm{E}+01$
$2.356 \mathrm{E}+01$
$2.363 \mathrm{E}+01$
$2.342 \mathrm{E}+01$
$2.260 \mathrm{E}+01$
$2.179 \mathrm{E}+01$
$2.077 \mathrm{E}+01$
$1.967 \mathrm{E}+01$
$1.900 \mathrm{E}+01$
$1.938 \mathrm{E}+01$
$2.040 \mathrm{E}+01$
$2.126 \mathrm{E}+01$
$2.159 \mathrm{E}+01$
$2.132 \mathrm{E}+01$
$2.096 \mathrm{E}+01$

\section{SOURCE SET}

$\begin{array}{ccc}\text { S01-S12 } & \text { S13-S2A } & \text { S25-S35 } \\ 1.796 \mathrm{E}+01 & 1.783 \mathrm{E}+01 & 1.530 \mathrm{E}+01 \\ 1.736 \mathrm{E}+01 & 1.826 \mathrm{E}+01 & 1.586 \mathrm{E}+01 \\ 1.645 \mathrm{E}+01 & 1.823 \mathrm{E}+01 & 1.611 \mathrm{E}+01 \\ 1.554 \mathrm{E}+01 & 1.815 \mathrm{E}+01 & 1.594 \mathrm{E}+01 \\ 1.499 \mathrm{E}+01 & 1.804 \mathrm{E}+01 & 1.539 \mathrm{E}+01 \\ 1.486 \mathrm{E}+01 & 1.825 \mathrm{E}+01 & 1.439 \mathrm{E}+01 \\ 1.503 \mathrm{E}+01 & 1.846 \mathrm{E}+01 & 1.353 \mathrm{E}+01 \\ 1.530 \mathrm{E}+01 & 1.837 \mathrm{E}+01 & 1.263 \mathrm{E}+01 \\ 1.584 \mathrm{E}+01 & 1.766 \mathrm{E}+01 & 1.190 \mathrm{E}+01 \\ 1.670 \mathrm{E}+01 & 1.644 \mathrm{E}+01 & 1.183 \mathrm{E}+01 \\ 1.767 \mathrm{E}+01 & 1.527 \mathrm{E}+01 & 1.276 \mathrm{E}+01 \\ 1.853 \mathrm{E}+01 & 1.462 \mathrm{E}+01 & 1.396 \mathrm{E}+01 \\ 1.891 \mathrm{E}+01 & 1.477 \mathrm{E}+01 & 1.496 \mathrm{E}+01 \\ 1.880 \mathrm{E}+01 & 1.531 \mathrm{E}+01 & 1.534 \mathrm{E}+01 \\ 1.838 \mathrm{E}+01 & 1.618 \mathrm{E}+01 & 1.506 \mathrm{E}+01 \\ 1.825 \mathrm{E}+01 & 1.692 \mathrm{E}+01 & 1.480 \mathrm{E}+01\end{array}$

S01-S10

$1.491 \mathrm{E}+01$

$1.408 E+01$

$1.328 \mathrm{E}+01$

$1.275 \mathrm{E}+01$

$1.265 E+01$

$1.289 \mathrm{E}+01$

$1.331 \mathrm{E}+01$

$1.374 \mathrm{E}+01$

$1.439 \mathrm{E}+01$

$1.529 \mathrm{E}+01$

$1.624 \mathrm{E}+01$

$1.702 \mathrm{E}+01$

$1.727 \mathrm{E}+01$

$1.694 \mathrm{E}+01$

$1.620 \mathrm{E}+01$

$1.564 \mathrm{E}+01$
S13-S22

$1.604 \mathrm{E}+01$

$1.609 \mathrm{E}+01$

$1.570 \mathrm{E}+01$

$1.546 \mathrm{E}+01$

$1.548 \mathrm{E}+01$

$1.604 \mathrm{E}+01$

$1.664 \mathrm{E}+01$

$1.683 \mathrm{E}+01$

$1.630 \mathrm{E}+01$

$1.520 \mathrm{E}+01$

$1.411 \mathrm{E}+01$

$1.348 \mathrm{E}+01$

$1.361 \mathrm{E}+01$

$1.408 \mathrm{E}+01$

$1.484 \mathrm{E}+01$

$1.558 \mathrm{E}+01$

\section{SOURCE SET}

$\begin{array}{ccc}\text { S01-S07 } & \text { S08-S14 } & \text { S15-S21 } \\ 1.059 \mathrm{E}+01 & 9.817 \mathrm{E}+00 & 1.272 \mathrm{E}+01 \\ 9.743 \mathrm{E}+00 & 1.024 \mathrm{E}+01 & 1.252 \mathrm{E}+01 \\ 8.786 \mathrm{E}+00 & 1.049 \mathrm{E}+01 & 1.182 \mathrm{E}+01 \\ 7.985 \mathrm{E}+00 & 1.059 \mathrm{E}+01 & 1.131 \mathrm{E}+01 \\ 7.529 \mathrm{E}+00 & 1.075 \mathrm{E}+01 & 1.065 \mathrm{E}+01 \\ 7.404 \mathrm{E}+00 & 1.109 \mathrm{E}+01 & 1.045 \mathrm{E}+01 \\ 7.536 \mathrm{E}+00 & 1.145 \mathrm{E}+01 & 1.046 \mathrm{E}+01 \\ 7.822 \mathrm{E}+00 & 1.155 \mathrm{E}+01 & 1.055 \mathrm{E}+01 \\ 8.458 \mathrm{E}+00 & 1.127 \mathrm{E}+01 & 1.052 \mathrm{E}+01 \\ 9.451 \mathrm{E}+00 & 1.069 \mathrm{E}+01 & 1.031 \mathrm{E}+01 \\ 1.058 \mathrm{E}+01 & 1.006 \mathrm{E}+01 & 1.006 \mathrm{E}+01\end{array}$

$\begin{array}{cc}\text { S22-S28 } & \text { S29-S35 } \\ 8.190 \mathrm{E}+00 & 9.761 \mathrm{E}+00 \\ 8.860 \mathrm{E}+00 & 1.012 \mathrm{E}+01 \\ 9.441 \mathrm{E}+00 & 1.025 \mathrm{E}+01 \\ 9.599 \mathrm{E}+00 & 1.014 \mathrm{E}+01 \\ 9.588 \mathrm{E}+00 & 9.896 \mathrm{E}+00 \\ 9.142 \mathrm{E}+00 & 9.417 \mathrm{E}+00 \\ 8.483 \mathrm{E}+00 & 9.094 \mathrm{E}+00 \\ 7.772 \mathrm{E}+00 & 8.600 \mathrm{E}+00 \\ 7.050 \mathrm{E}+00 & 8.116 \mathrm{E}+00 \\ 6.406 \mathrm{E}+00 & 8.120 \mathrm{E}+00 \\ 6.121 \mathrm{E}+00 & 8.896 \mathrm{E}+00\end{array}$




\section{REVISED METHODOLOGY FOR DETERMINING CESIUM-137 CONTENT OF HN-200 GROUT CONTAINERS.}

$\begin{array}{lllllll}\text { R12 } & 1.094 \mathrm{E}+01 & 1.158 \mathrm{E}+01 & 9.559 \mathrm{E}+00 & 9.829 \mathrm{E}+00 & 6.322 \mathrm{E}+00 & 9.817 \mathrm{E}+00 \\ \mathbf{R 1 3} & 1.184 \mathrm{E}+01 & 1.210 \mathrm{E}+01 & 9.229 \mathrm{E}+00 & 1.027 \mathrm{E}+01 & 6.566 \mathrm{E}+00 & 1.049 \mathrm{E}+01 \\ \text { R14 } & 1.244 \mathrm{E}+01 & 1.205 \mathrm{E}+01 & 9.054 \mathrm{E}+00 & 1.091 \mathrm{E}+01 & 6.884 \mathrm{E}+00 & 1.054 \mathrm{E}+01 \\ \text { R15 } & 1.257 \mathrm{E}+01 & 1.158 \mathrm{E}+01 & 9.081 \mathrm{E}+00 & 1.173 \mathrm{E}+01 & 7.250 \mathrm{E}+00 & 9.985 \mathrm{E}+00 \\ \text { R16 } & 1.229 \mathrm{E}+01 & 1.122 \mathrm{E}+01 & 9.364 \mathrm{E}+00 & 1.242 \mathrm{E}+01 & 7.487 \mathrm{E}+00 & 9.487 \mathrm{E}+00\end{array}$

$\begin{array}{ccc}\text { POINT } & \text { S01-S05 } & \text { S06-S10 } \\ \text { R01 } & 7.747 \mathrm{E}+00 & 7.165 \mathrm{E}+00 \\ \text { R02 } & 7.189 \mathrm{E}+00 & 6.894 \mathrm{E}+00 \\ \text { R03 } & 6.475 \mathrm{E}+00 & 6.801 \mathrm{E}+00 \\ \text { R04 } & 5.844 \mathrm{E}+00 & 6.903 \mathrm{E}+00 \\ \text { R05 } & 5.480 \mathrm{E}+00 & 7.170 \mathrm{E}+00 \\ \text { R06 } & 5.372 \mathrm{E}+00 & 7.521 \mathrm{E}+00 \\ \text { R07 } & 5.443 \mathrm{E}+00 & 7.867 \mathrm{E}+00 \\ \text { R08 } & 5.589 \mathrm{E}+00 & 8.155 \mathrm{E}+00 \\ \text { R09 } & 5.990 \mathrm{E}+00 & 8.402 \mathrm{E}+00 \\ \text { R10 } & 6.621 \mathrm{E}+00 & 8.666 \mathrm{E}+00 \\ \text { R11 } & 7.303 \mathrm{E}+00 & 8.940 \mathrm{E}+00 \\ \text { R12 } & 7.924 \mathrm{E}+00 & 9.095 \mathrm{E}+00 \\ \text { R13 } & 8.269 \mathrm{E}+00 & 8.996 \mathrm{E}+00 \\ \text { R14 } & 8.310 \mathrm{E}+00 & 8.626 \mathrm{E}+00 \\ \text { R15 } & 8.096 \mathrm{E}+00 & 8.100 \mathrm{E}+00 \\ \text { R16 } & 8.061 \mathrm{E}+00 & 7.576 \mathrm{E}+00\end{array}$

\section{SOURCE SET}

\section{S11-S15 S16-S20}

$8.086 \mathrm{E}+00 \quad 8.058 \mathrm{E}+00$

$8.545 \mathrm{E}+00 \quad 7.964 \mathrm{E}+00$

$8.335 \mathrm{E}+00 \quad 7.700 \mathrm{E}+00$

$7.691 \mathrm{E}+00 \quad 7.770 \mathrm{E}+00$

$7.023 \mathrm{E}+00 \quad 7.633 \mathrm{E}+00$

$6.849 \mathrm{E}+00 \quad 7.599 \mathrm{E}+00$

$6.784 \mathrm{E}+00 \quad 7.723 \mathrm{E}+00$

$6.645 \mathrm{E}+00 \quad 7.846 \mathrm{E}+00$

$6.294 \mathrm{E}+00 \quad 7.764 \mathrm{E}+00$

$5.806 \mathrm{E}+00 \quad 7.421 \mathrm{E}+00$

$5.385 \mathrm{E}+00 \quad 6.972 \mathrm{E}+00$

$5.198 \mathrm{E}+00 \quad 6.501 \mathrm{E}+00$

$5.263 \mathrm{E}+00 \quad 6.693 \mathrm{E}+00$

$5.504 \mathrm{E}+00 \quad 7.167 \mathrm{E}+00$

$6.174 \mathrm{E}+00 \quad 7.653 \mathrm{E}+00$

$7.158 \mathrm{E}+00 \quad 7.971 \mathrm{E}+00$
S21-S25
$6.313 \mathrm{E}+00$
$6.773 \mathrm{E}+00$
$7.183 \mathrm{E}+00$
$7.259 \mathrm{E}+00$
$7.362 \mathrm{E}+00$
$7.236 \mathrm{E}+00$
$6.970 \mathrm{E}+00$
$6.601 \mathrm{E}+00$
$6.128 \mathrm{E}+00$
$5.641 \mathrm{E}+00$
$5.343 \mathrm{E}+00$
$5.436 \mathrm{E}+00$
$5.517 \mathrm{E}+00$
$5.636 \mathrm{E}+00$
$5.785 \mathrm{E}+00$
$5.812 \mathrm{E}+00$

S26-S30

6.183E+00

$6.114 \mathrm{E}+00$

$6.087 \mathrm{E}+00$

$6.026 \mathrm{E}+00$

$5.823 \mathrm{E}+00$

$5.465 \mathrm{E}+00$

$5.086 \mathrm{E}+00$

$4.818 \mathrm{E}+00$

$4.707 \mathrm{E}+00$

$4.757 \mathrm{E}+00$

$5.089 \mathrm{E}+00$

$5.644 \mathrm{E}+00$

$6.191 \mathrm{E}+00$

$6.537 \mathrm{E}+00$

$6.579 \mathrm{E}+00$

$6.405 \mathrm{E}+00$
S31-S35

$7.525 \mathrm{E}+00$

$8.001 \mathrm{E}+00$

$8.200 \mathrm{E}+00$

$8.136 \mathrm{E}+00$

$7.919 \mathrm{E}+00$

$7.462 \mathrm{E}+00$

$7.147 \mathrm{E}+00$

$6.642 \mathrm{E}+00$

$6.122 \mathrm{E}+00$

$6.056 \mathrm{E}+00$

$6.674 \mathrm{E}+00$

$7.310 \mathrm{E}+00$

$7.715 \mathrm{E}+00$

$7.667 \mathrm{E}+00$

$7.231 \mathrm{E}+00$

$6.990 \mathrm{E}+00$

$\begin{array}{ccc}\text { POINT } & \text { S01-S03 } & \text { S04-S06 } \\ \text { R01 } & 5.739 \mathrm{E}+00 & 3.439 \mathrm{E}+00 \\ \text { R02 } & 5.389 \mathrm{E}+00 & 3.091 \mathrm{E}+00 \\ \text { R03 } & 4.783 \mathrm{E}+00 & 2.837 \mathrm{E}+00 \\ \text { R04 } & 4.189 \mathrm{E}+00 & 2.679 \mathrm{E}+00 \\ \text { R05 } & 3.794 \mathrm{E}+00 & 2.622 \mathrm{E}+00 \\ \text { R06 } & 3.595 \mathrm{E}+00 & 2.655 \mathrm{E}+00 \\ \text { R07 } & 3.536 \mathrm{E}+00 & 2.757 \mathrm{E}+00 \\ \text { R08 } & 3.542 \mathrm{E}+00 & 2.897 \mathrm{E}+00 \\ \text { R09 } & 3.817 \mathrm{E}+00 & 3.052 \mathrm{E}+00 \\ \text { R10 } & 4.325 \mathrm{E}+00 & 3.232 \mathrm{E}+00 \\ \text { R11 } & 4.801 \mathrm{E}+00 & 3.528 \mathrm{E}+00 \\ \text { R12 } & 5.119 \mathrm{E}+00 & 3.952 \mathrm{E}+00\end{array}$

\section{SOURCE SET}

$\begin{array}{ccc}\text { SO7-S09 } & \text { S10-S12 } & \text { S13-S15 } \\ 4.309 E+00 & 4.470 E+00 & 5.041 E+00 \\ 4.049 E+00 & 4.834 E+00 & 5.266 \mathrm{E}+00 \\ 3.918 \mathrm{E}+00 & 4.908 \mathrm{E}+00 & 5.165 \mathrm{E}+00 \\ 3.913 \mathrm{E}+00 & 4.756 \mathrm{E}+00 & 4.901 \mathrm{E}+00 \\ 4.032 \mathrm{E}+00 & 4.537 \mathrm{E}+00 & 4.688 \mathrm{E}+00 \\ 4.271 \mathrm{E}+00 & 4.342 \mathrm{E}+00 & 4.879 \mathrm{E}+00 \\ 4.616 \mathrm{E}+00 & 4.121 \mathrm{E}+00 & 5.064 \mathrm{E}+00 \\ 5.031 \mathrm{E}+00 & 3.826 \mathrm{E}+00 & 5.093 \mathrm{E}+00 \\ 5.471 \mathrm{E}+00 & 3.503 \mathrm{E}+00 & 4.843 \mathrm{E}+00 \\ 5.916 \mathrm{E}+00 & 3.226 \mathrm{E}+00 & 4.395 \mathrm{E}+00 \\ 6.300 \mathrm{E}+00 & 3.044 \mathrm{E}+00 & 3.955 \mathrm{E}+00 \\ 6.483 \mathrm{E}+00 & 2.971 \mathrm{E}+00 & 3.691 \mathrm{E}+00\end{array}$

S16-S18

$6.223 \mathrm{E}+00$

$6.011 \mathrm{E}+00$

$5.613 E+00$

$5.558 \mathrm{E}+00$

$5.313 \mathrm{E}+00$

$5.162 \mathrm{E}+00$

$5.140 \mathrm{E}+00$

$5.139 E+00$

$5.054 \mathrm{E}+00$

$4.878 \mathrm{E}+00$

$4.699 \mathrm{E}+00$

$4.487 \mathrm{E}+00$
S19.S21

$3.905 \mathrm{E}+00$

$3.863 \mathrm{E}+00$

$3.863 E+00$

$3.891 E+00$

$3.941 \mathrm{E}+00$

$4.040 \mathrm{E}+00$

$4.209 \mathrm{E}+00$

$4.395 \mathrm{E}+00$

$4.500 \mathrm{E}+00$

$4.471 \mathrm{E}+00$

$4.363 \mathrm{E}+00$

$4.265 \mathrm{E}+00$ 


\section{REVISED METHODOLOGY FOR DETERMINING CESIUM-137 CONTENT} OF HN-200 GROUT CONTAINERS.

$\begin{array}{llllllll}\text { R13 } & 5.216 \mathrm{E}+00 & 4.345 \mathrm{E}+00 & 6.338 \mathrm{E}+00 & 3.014 \mathrm{E}+00 & 3.615 \mathrm{E}+00 & 4.853 \mathrm{E}+00 & 4.211 \mathrm{E}+00 \\ \mathbf{R 1 4} & 5.247 \mathrm{E}+00 & 4.496 \mathrm{E}+00 & 5.878 \mathrm{E}+00 & 3.179 \mathrm{E}+00 & 3.640 \mathrm{E}+00 & 5.418 \mathrm{E}+00 & 4.162 \mathrm{E}+00 \\ \mathbf{R 1 5} & 5.312 \mathrm{E}+00 & 4.307 \mathrm{E}+00 & 5.270 \mathrm{E}+00 & 3.487 \mathrm{E}+00 & 3.994 \mathrm{E}+00 & 5.930 \mathrm{E}+00 & 4.083 \mathrm{E}+00 \\ \mathbf{R 1 6} & 5.690 \mathrm{E}+00 & 3.892 \mathrm{E}+00 & 4.711 \mathrm{E}+00 & 3.955 \mathrm{E}+00 & 4.546 \mathrm{E}+00 & 6.217 \mathrm{E}+00 & 3.987 \mathrm{E}+00\end{array}$

\begin{tabular}{cccccc} 
& & \multicolumn{4}{c}{ SOURCE SET } \\
POINT & S22-S24 & S25-S27 & S28-S30 & S31-S33 & S34-S35 \\
R01 & $2.656 \mathrm{E}+00$ & $3.956 \mathrm{E}+00$ & $3.814 \mathrm{E}+00$ & $3.945 \mathrm{E}+00$ & $3.580 \mathrm{E}+00$ \\
R02 & $3.118 \mathrm{E}+00$ & $4.362 \mathrm{E}+00$ & $3.497 \mathrm{E}+00$ & $3.837 \mathrm{E}+00$ & $4.164 \mathrm{E}+00$ \\
R03 & $3.586 \mathrm{E}+00$ & $4.717 \mathrm{E}+00$ & $3.191 \mathrm{E}+00$ & $3.925 \mathrm{E}+00$ & $4.275 \mathrm{E}+00$ \\
R04 & $3.798 \mathrm{E}+00$ & $4.853 \mathrm{E}+00$ & $2.955 \mathrm{E}+00$ & $4.104 \mathrm{E}+00$ & $4.032 \mathrm{E}+00$ \\
R05 & $4.097 \mathrm{E}+00$ & $4.665 \mathrm{E}+00$ & $2.803 \mathrm{E}+00$ & $4.206 \mathrm{E}+00$ & $3.713 \mathrm{E}+00$ \\
R06 & $4.166 \mathrm{E}+00$ & $4.233 \mathrm{E}+00$ & $2.699 \mathrm{E}+00$ & $4.124 \mathrm{E}+00$ & $3.338 \mathrm{E}+00$ \\
R07 & $4.044 \mathrm{E}+00$ & $3.746 \mathrm{E}+00$ & $2.641 \mathrm{E}+00$ & $3.923 \mathrm{E}+00$ & $3.224 \mathrm{E}+00$ \\
R08 & $3.745 \mathrm{E}+00$ & $3.356 \mathrm{E}+00$ & $2.630 \mathrm{E}+00$ & $3.758 \mathrm{E}+00$ & $2.884 \mathrm{E}+00$ \\
R09 & $3.263 \mathrm{E}+00$ & $3.108 \mathrm{E}+00$ & $2.674 \mathrm{E}+00$ & $3.657 \mathrm{E}+00$ & $2.465 \mathrm{E}+00$ \\
R10 & $2.694 \mathrm{E}+00$ & $2.996 \mathrm{E}+00$ & $2.780 \mathrm{E}+00$ & $4.006 \mathrm{E}+00$ & $2.050 \mathrm{E}+00$ \\
R11 & $2.256 \mathrm{E}+00$ & $3.082 \mathrm{E}+00$ & $3.004 \mathrm{E}+00$ & $4.687 \mathrm{E}+00$ & $1.987 \mathrm{E}+00$ \\
R12 & $2.177 \mathrm{E}+00$ & $3.261 \mathrm{E}+00$ & $3.391 \mathrm{E}+00$ & $5.351 \mathrm{E}+00$ & $1.959 \mathrm{E}+00$ \\
R13 & $2.093 \mathrm{E}+00$ & $3.436 \mathrm{E}+00$ & $3.808 \mathrm{E}+00$ & $5.707 \mathrm{E}+00$ & $2.008 \mathrm{E}+00$ \\
R14 & $2.089 \mathrm{E}+00$ & $3.532 \mathrm{E}+00$ & $4.139 \mathrm{E}+00$ & $5.527 \mathrm{E}+00$ & $2.140 \mathrm{E}+00$ \\
R15 & $2.173 \mathrm{E}+00$ & $3.581 \mathrm{E}+00$ & $4.250 \mathrm{E}+00$ & $4.931 \mathrm{E}+00$ & $2.300 \mathrm{E}+00$ \\
R16 & $2.170 \mathrm{E}+00$ & $3.689 \mathrm{E}+00$ & $4.125 \mathrm{E}+00$ & $4.137 \mathrm{E}+00$ & $2.853 \mathrm{E}+00$
\end{tabular}

TABLE 10 - ESTIMATES OF CURIE CONTENT FOR VARIOUS SOURCE SETS COMPARED TO ACTUAL CURIE CONTENT FOR AN UNGROUTED CONTAINER. MULTIPLE SOURCE SET ESTIMATE AND ERROR - 5 FOOT MEASUREMENT

\begin{tabular}{|c|c|c|c|c|c|c|}
\hline & & 5 FOOT & & & & \\
\hline & AVG & CONV & EST & ACTUAL & & \\
\hline SOURCE & 5 FOOT & FACT & Cs-137 & Cs-137 & & $\%$ \\
\hline SET & $\mathbf{R} / \mathbf{h r}$ & $\mathbf{R} / \mathbf{h r} / \mathbf{C i}$ & CURIES & CURIES & ERROR & ERROR \\
\hline S01-S35 & $4.832 \mathrm{E}+01$ & $6.751 \mathrm{E}-02$ & 715.6945 & 708.6318 & 7.0627 & 0.9967 \\
\hline S01-S30 & $4.102 \mathrm{E}+01$ & $6.751 \mathrm{E}-02$ & 607.5673 & 604.0700 & 3.4973 & 0.5790 \\
\hline S06-S35 & $4.146 \mathrm{E}+01$ & $6.751 \mathrm{E}-02$ & 614.1219 & 610.0433 & 4.0786 & 0.6686 \\
\hline S01.S25 & $3.530 \mathrm{E}+01$ & $6.751 \mathrm{E}-02$ & 522.8484 & 520.3863 & 2.4621 & 0.4731 \\
\hline S11-S35 & $3.353 \mathrm{E}+01$ & $6.751 \mathrm{E}-02$ & 496.6602 & 490.8729 & 5.7873 & 1.1790 \\
\hline S01-S20 & $2.899 \mathrm{E}+01$ & $6.751 \mathrm{E}-02$ & 429.3489 & 426.0483 & 3.3006 & 0.7747 \\
\hline S16-S35 & $2.686 \mathrm{E}+01$ & $6.751 \mathrm{E}-02$ & 397.8424 & 393.8550 & 3.9874 & 1.0124 \\
\hline S01-S18 & $2.681 \mathrm{E}+01$ & $6.751 \mathrm{E}-02$ & 397.1875 & 394.2079 & 2.9795 & 0.7558 \\
\hline S19-S35 & $2.150 \mathrm{E}+01$ & $6.751 \mathrm{E}-02$ & 318.5071 & 314.4239 & 4.0832 & 1.2986 \\
\hline S01-S12 & $1.691 \mathrm{E}+01$ & $6.751 \mathrm{E}-02$ & 250.4767 & 247.8830 & 2.5937 & 1.0463 \\
\hline
\end{tabular}




\section{REVISED METHODOLOGY FOR DETERMINING CESIUM-137 CONTENT OF HN-200 GROUT CONTAINERS.}

\begin{tabular}{|c|c|c|c|c|c|c|}
\hline S13-S24 & $1.705 \mathrm{E}+01$ & $6.751 \mathrm{E}-02$ & 252.5032 & 252.7408 & -0.2376 & -0.094 \\
\hline S25-S35 & $1.436 \mathrm{E}+01$ & $6.751 \mathrm{E}-02$ & 212.7146 & 208.0080 & 4.7066 & 2.2627 \\
\hline S01-S10 & $1.479 \mathrm{E}+01$ & $6.751 \mathrm{E}-02$ & 219.0343 & 217.7589 & 1.2754 & 0.585 \\
\hline S13-S22 & $1.534 \mathrm{E}+01$ & $6.751 \mathrm{E}-02$ & 227.2604 & 227.1843 & 0.0761 & 0.0335 \\
\hline S23-S32 & $1.188 E+01$ & $6.751 \mathrm{E}-02$ & 176.0002 & 173.0444 & 2.9559 & 1.7082 \\
\hline S01-S07 & $9.650 \mathrm{E}+00$ & $6.751 \mathrm{E}-02$ & 142.9474 & 140.8425 & 2.1049 & 1.4945 \\
\hline S08-S14 & $1.027 \mathrm{E}+01$ & $6.751 \mathrm{E}-02$ & 152.0883 & 151.1873 & 0.9010 & 0.5959 \\
\hline S15-S21 & $1.103 E+01$ & $6.751 \mathrm{E}-02$ & 163.3913 & 162.6650 & 0.7263 & 0.4465 \\
\hline S22-S28 & $7.822 \mathrm{E}+00$ & $6.751 \mathrm{E}-02$ & 115.8705 & 116.2697 & -0.3992 & -0.343 \\
\hline S29-S35 & $9.546 \mathrm{E}+00$ & $6.751 \mathrm{E}-02$ & 141.3970 & 137.6673 & 3.7297 & 2.7092 \\
\hline S01-S05 & $6.857 \mathrm{E}+00$ & $6.751 \mathrm{E}-02$ & 101.5726 & 98.5885 & 2.9841 & 3.0268 \\
\hline S06-S10 & $7.930 \mathrm{E}+00$ & $6.751 \mathrm{E}-02$ & 117.4617 & 119.1704 & -1.7087 & -1.4339 \\
\hline S11.S15 & $6.671 E+00$ & 6.751E-02 & 98.8178 & 97.0179 & 1.7999 & 1.8552 \\
\hline S16-S20 & $7.527 \mathrm{E}+00$ & $6.751 \mathrm{E}-02$ & 111.4968 & 111.2715 & 0.2253 & 0.2025 \\
\hline S21-S25 & $6.312 E+00$ & $6.751 \mathrm{E}-02$ & 93.4995 & 94.3380 & -0.8385 & -0.888 \\
\hline S26-S30 & $5.719 E+00$ & $6.751 \mathrm{E}-02$ & 84.7189 & 83.6838 & 1.0352 & 1.2370 \\
\hline S31-S35 & $7.300 \mathrm{E}+00$ & $6.751 \mathrm{E}-02$ & 108.1272 & 104.5618 & 3.5654 & 3.4099 \\
\hline S01-S03 & $4.631 \mathrm{E}+00$ & $6.751 \mathrm{E}-02$ & 68.5954 & 66.9394 & 1.6560 & 2.4739 \\
\hline S04-S06 & $3.361 \mathrm{E}+00$ & $6.751 \mathrm{E}-02$ & 49.7918 & 47.9673 & 1.8244 & 3.8035 \\
\hline S07-S09 & $5.032 \mathrm{E}+00$ & $6.751 \mathrm{E}-02$ & 74.5316 & 76.0383 & -1.5068 & -1.9816 \\
\hline S10-S12 & $3.886 \mathrm{E}+00$ & $6.751 \mathrm{E}-02$ & 57.5580 & 56.9379 & 0.6200 & 1.0889 \\
\hline S13-S15 & $4.549 \mathrm{E}+00$ & $6.751 \mathrm{E}-02$ & 67.3754 & 66.8938 & 0.4816 & 0.7199 \\
\hline S16-S18 & $5.356 \mathrm{E}+00$ & $6.751 \mathrm{E}-02$ & 79.3354 & 79.4311 & -0.0958 & -0.1206 \\
\hline S19-S21 & $4.134 E+00$ & $6.751 \mathrm{E}-02$ & 61.2395 & 60.4869 & 0.7526 & 1.2442 \\
\hline S22-S24 & $3.008 \mathrm{E}+00$ & $6.751 \mathrm{E}-02$ & 44.5529 & 45.9289 & -1.3760 & -2.995 \\
\hline S25-S27 & $3.786 \mathrm{E}+00$ & $6.751 \mathrm{E}-02$ & 56.0762 & 54.8275 & 1.2488 & 2.2777 \\
\hline S28-S30 & $3.275 \mathrm{E}+00$ & $6.751 \mathrm{E}-02$ & 48.5111 & 48.6188 & -0.1076 & -0.2214 \\
\hline S31-S33 & $4.364 \mathrm{E}+00$ & $6.751 \mathrm{E}-02$ & 64.6425 & 61.8697 & 2.7728 & 4.4817 \\
\hline S34-S35 & $2.936 \mathrm{E}+00$ & $6.751 \mathrm{E}-02$ & 43.4848 & 42.6921 & 0.7926 & 1.8566 \\
\hline
\end{tabular}

Average \% Error $=\quad 0.9800$

INDIVIDUAL SOURCE ESTIMATE AND ERROR - 5 FOOT MEASUREMENT

\begin{tabular}{|c|c|c|c|c|c|c|}
\hline & & 5 FOOT & & & & \\
\hline & $\begin{array}{c}\text { AVG } \\
5 \text { FOOT }\end{array}$ & $\begin{array}{l}\text { CONV } \\
\text { FACT }\end{array}$ & $\begin{array}{c}\text { EST } \\
\text { Cs-137 }\end{array}$ & $\begin{array}{c}\text { ACTUAL } \\
\text { Cs-137 }\end{array}$ & & $\%$ \\
\hline SOURCE & $\mathbf{R} / \mathbf{h r}$ & $\mathrm{R} / \mathrm{hr} / \mathrm{Ci}$ & CURIES & CURIES & ERROR & ERROR \\
\hline S01 & $2.099 \mathrm{E}+00$ & $6.751 \mathrm{E}-02$ & 31.0871 & 29.4975 & 1.5895 & 5.3887 \\
\hline S02 & $9.619 \mathrm{E}-01$ & $6.751 \mathrm{E}-02$ & 14.2479 & 13.8502 & 0.3977 & 2.8712 \\
\hline S03 & $1.570 \mathrm{E}+00$ & $6.751 \mathrm{E}-02$ & 23.2604 & 23.5916 & -0.3312 & -1.4038 \\
\hline S04 & $1.474 \mathrm{E}+00$ & $6.751 \mathrm{E}-02$ & 21.8274 & 20.6982 & 1.1292 & 5.4558 \\
\hline S05 & $7.527 \mathrm{E}-01$ & $6.751 \mathrm{E}-02$ & 11.1498 & 10.9510 & 0.1988 & 1.8158 \\
\hline S06 & $1.135 \mathrm{E}+00$ & $6.751 \mathrm{E}-02$ & 16.8145 & 16.3182 & 0.4964 & 3.0417 \\
\hline S07 & $1.658 \mathrm{E}+00$ & $6.751 \mathrm{E}-02$ & 24.5603 & 25.9358 & -1.3756 & -5.3038 \\
\hline S08 & $1.732 \mathrm{E}+00$ & $6.751 \mathrm{E}-02$ & 25.6545 & 25.1678 & 0.4867 & 1.9339 \\
\hline S09 & $1.642 \mathrm{E}+00$ & $6.751 \mathrm{E}-02$ & 24.3168 & 24.9347 & -0.6179 & -2.4781 \\
\hline S10 & $1.763 E+00$ & $6.751 E-02$ & 26.1156 & 26.8139 & -0.6983 & -2.6043 \\
\hline S11 & $8.823 \mathrm{E}-01$ & $6.751 \mathrm{E}-02$ & 13.0692 & 12.5933 & 0.4758 & 3.7785 \\
\hline S12 & $1.240 \mathrm{E}+00$ & $6.751 \mathrm{E}-02$ & 18.3732 & 17.5307 & 0.8425 & 4.8058 \\
\hline S13 & $1.199 \mathrm{E}+00$ & $6.751 \mathrm{E}-02$ & 17.7612 & 17.9331 & -0.1719 & -0.9587 \\
\hline
\end{tabular}


HNF-4799, Rev 0

August 25, 1999

\section{REVISED METHODOLOGY FOR DETERMINING CESIUM-137 CONTENT OF HN-200 GROUT CONTAINERS.}

$\begin{array}{lllllll}\text { S14 } & 1.809 E+00 & 6.751 E-02 & 26.7979 & 26.2138 & 0.5841 & 2.2281 \\ \text { S15 } & 1.540 E+00 & 6.751 E-02 & 22.8163 & 22.7469 & 0.0694 & 0.3051 \\ \text { S16 } & 1.901 E+00 & 6.751 E-02 & 28.1523 & 27.6556 & 0.4967 & 1.7961 \\ \text { S17 } & 1.900 E+00 & 6.751 E-02 & 28.1431 & 28.5227 & -0.3797 & -1.3311 \\ \text { S18 } & 1.555 E+00 & 6.751 E-02 & 23.0400 & 23.2528 & -0.2128 & -0.9152 \\ \text { S19 } & 1.082 E+00 & 6.751 E-02 & 16.0345 & 15.3416 & 0.6929 & 4.5165 \\ \text { S20 } & 1.089 E+00 & 6.751 E-02 & 16.1270 & 16.4988 & -0.3718 & -2.2536 \\ \text { S21 } & 1.963 E+00 & 6.751 E-02 & 29.0781 & 28.6466 & 0.4315 & 1.5063 \\ \text { S22 } & 1.304 E+00 & 6.751 E-02 & 19.3101 & 20.3724 & -1.0623 & -5.2144 \\ \text { S23 } & 8.166 E-01 & 6.751 E-02 & 12.0953 & 13.0146 & -0.9193 & -7.0635 \\ \text { S24 } & 8.876 E-01 & 6.751 E-02 & 13.1475 & 12.5419 & 0.6056 & 4.8287 \\ \text { S25 } & 1.341 E+00 & 6.751 E-02 & 19.8684 & 19.7625 & 0.1060 & 0.5363 \\ \text { S26 } & 1.739 E+00 & 6.751 E-02 & 25.7517 & 24.8512 & 0.9005 & 3.6236 \\ \text { S27 } & 7.059 E-01 & 6.751 E-02 & 10.4560 & 10.2138 & 0.2423 & 2.3722 \\ \text { S28 } & 1.029 E+00 & 6.751 E-02 & 15.2414 & 15.5133 & -0.2720 & -1.7530 \\ \text { S29 } & 1.214 E+00 & 6.751 E-02 & 17.9797 & 18.0653 & -0.0856 & -0.4738 \\ \text { S30 } & 1.032 E+00 & 6.751 E-02 & 15.2900 & 15.0401 & 0.2499 & 1.6617 \\ \text { S31 } & 1.580 E+00 & 6.751 E-02 & 23.3975 & 22.3550 & 1.0425 & 4.6633 \\ \text { S32 } & 1.537 E+00 & 6.751 E-02 & 22.7726 & 21.6866 & 1.0859 & 5.0073 \\ \text { S33 } & 1.247 E+00 & 6.751 E-02 & 18.4724 & 17.8281 & 0.6444 & 3.6144 \\ \text { S34 } & 9.538 E-01 & 6.751 E-02 & 14.1280 & 14.6776 & -0.5496 & -3.7443 \\ \text { S35 } & 1.982 E+00 & 6.751 E-02 & 29.3568 & 28.0146 & 1.3422 & 4.7911 \\ & & & & \text { Average } \% & \text { Error }= & 1.0013\end{array}$




\section{REVISED METHODOLOGY FOR DETERMINING CESIUM-137 CONTENT OF HN-200 GROUT CONTAINERS.}

TABLE 1 - ESTIMATED VERSUS ACTUAL Cs-137 FOR EACH MULTIPLE SOURCE COMBINATION ANALYZED USING EACH METHOD OF DETERMINATION.

\begin{tabular}{|c|c|c|c|c|c|c|c|}
\hline \multicolumn{2}{|l|}{ Source } & \multicolumn{3}{|c|}{ Grouted } & \multicolumn{3}{|c|}{ Ungrouted } \\
\hline Set & Actual & Contact & 5 Feet & 10 Feet & Contact & 5 Feet & 10 Feet \\
\hline S01-S35 & 708.6318 & 193.7780 & 815.3966 & 833.9262 & 760.4690 & 715.6945 & 709.9558 \\
\hline S01-S30 & 604.0700 & 133.3947 & 602.0648 & 629.7304 & 621.7013 & 607.5673 & 605.4053 \\
\hline S06-S35 & 610.0433 & 155.6608 & 716.3037 & 709.7623 & 635.4184 & 614.1219 & 610.6073 \\
\hline S01-S25 & 520.3863 & 97.5422 & 501.6577 & 530.2328 & 517.3990 & 522.8484 & 521.3715 \\
\hline S11-S36 & 490.8729 & 142.8967 & 658.4350 & 647.2612 & 525.9006 & 496.6602 & 491.3635 \\
\hline S01-S20 & 426.0483 & 92.2078 & 408.2395 & 435.1891 & 442.1841 & 429.3489 & 427.2965 \\
\hline S16-S35 & 393.8550 & 119.1554 & 528.4853 & 519.7471 & 424.1038 & 397.8424 & 393.8557 \\
\hline S01-S18 & 394.2079 & 80.7628 & 385.6012 & 412.9800 & 402.3437 & 397.1875 & 395.2253 \\
\hline S19-S35 & 314.4239 & 113.0152 & 429.7954 & 420.9462 & 358.1253 & 318.5071 & 314.7306 \\
\hline S01-S12 & 247.8830 & 53.5439 & 191.6404 & 219.9637 & 263.7183 & 250.4767 & 249.2176 \\
\hline S13-S24 & 252.7408 & 43.5015 & 302.6889 & 302.6797 & 237.2968 & 252. & 252.2295 \\
\hline S25-S35 & 208.0080 & 96.7326 & 321.0673 & 311.2829 & 259.4539 & 212.7146 & 208.5087 \\
\hline S01-S10 & 217.7589 & 50.8813 & 156.9616 & 186.6650 & 234.5684 & 219.0343 & 218.5924 \\
\hline S13-S22 & 227.1843 & 39.9291 & 268.8898 & 269.1267 & 214.7626 & 227.2604 & 226.7596 \\
\hline S23-S32 & 173.0444 & 57.8887 & 199.1662 & 195.8188 & & & 174.1685 \\
\hline S01-S07 & 140.8425 & 48.03 & 135.22 & 163.1 & & & \\
\hline S08-S14 & 151.1873 & 12.9566 & 98.64 & 98.78 & 140.8031 & 152.0 & 152.1983 \\
\hline S15-S21 & 162.6650 & 31.8070 & 178.9 & 177.9648 & 159.4727 & 13 & 162.6982 \\
\hline S22-S28 & 116.2697 & 36.3937 & 156. & 157.1532 & 124. & 05 & 115.8784 \\
\hline S29-S35 & 137.6673 & 64.5819 & 246.5747 & 236.8935 & 168.4647 & 70 & 137.7559 \\
\hline S01-S05 & 98.5885 & 38.1173 & 99.09 & 124.1640 & 125.0506 & & 99.3485 \\
\hline S06-S10 & 119.1704 & 12.7641 & 57.8688 & 62.5010 & 109.5178 & 117. & 119.2439 \\
\hline S11-S15 & 97.0179 & 23.7412 & 129.9497 & 127.5142 & 101.7968 & & 97.5077 \\
\hline S16-S20 & 111.2715 & 17.5853 & 121.3282 & 121.0099 & 105.8189 & & 111.1964 \\
\hline S21.S25 & 94.3380 & 5.3344 & 93.4182 & 95.0437 & 75.2149 & 93.4995 & 94.0749 \\
\hline S26-S30 & 83.6838 & 35.8524 & 100.4071 & 99.4976 & 104.3023 & 4.7189 & 84.0338 \\
\hline S31-S35 & 104.5618 & 60.3834 & 213 & 204.1959 & 138.7677 & 72 & 104.5506 \\
\hline S01-S03 & 66.9394 & 29.2131 & 56.97 & 84.0272 & 86.9 & & 67.2825 \\
\hline S04-S06 & 47.9673 & 9.3949 & 47.9423 & 45.9077 & 52.9336 & 49.7918 & 48.6537 \\
\hline S07-S09 & 76.0383 & 11.5861 & 41.6691 & 45.3252 & 70.4577 & 74.5316 & 75.9157 \\
\hline S10-S12 & 56.9379 & 3.3499 & 45.0521 & 44.7035 & 53.4256 & & 57.3658 \\
\hline S13-S15 & 66.8938 & 21.0786 & 95.2710 & 94.2155 & 72.6469 & & 66.8825 \\
\hline S16-S18 & 79.43 & & 98.6 & 98.8 & & & 79.1252 \\
\hline S19-S21 & 60.4869 & 12.0397 & 27.20 & 26.8996 & 64.71 & 61.2395 & 61.0962 \\
\hline S22-S24 & 45.9289 & 4.2430 & 81.5183 & 82.7637 & 33.9519 & 44.5529 & 45.1256 \\
\hline S25-S27 & 54.8275 & 27.8024 & 52.7335 & 51.8058 & 74.8399 & 56.0762 & 55.3393 \\
\hline S28-S30 & 48.6188 & 8.5468 & 55.0020 & 55.2812 & 45.8462 & 48.5111 & 48.6189 \\
\hline S31-S33 & 61.8697 & 58.8015 & 107.1310 & 101.6609 & 108.7065 & 64.6425 & 62.3065 \\
\hline S34-S35 & 42.6921 & 1.5819 & 106.2008 & 102.5350 & 30.0612 & 43.4848 & 42.2441 \\
\hline A verage & 202.9501 & 52.6212 & 229.0406 & 234.0280 & 214.9797 & 204.7398 & 203.3117 \\
\hline
\end{tabular}




\section{REVISED METHODOLOGY FOR DETERMINING CESIUM-137 CONTENT OF HN-200 GROUT CONTAINERS.}

\section{TABLE 2 - ESTIMATED VERSUS ACTUAL Cs-137 FOR EACH INDIVIDUAL SOURCE ANALYZED USING EACH METHOD OF DETERMINATION.}

\begin{tabular}{|c|c|c|c|c|c|c|c|}
\hline \multirow[b]{2}{*}{ Source } & \multirow[b]{2}{*}{ Actual } & \multicolumn{3}{|c|}{ Grouted } & \multicolumn{3}{|c|}{ Ungrouted } \\
\hline & & Contact & 5 Feet & 10 Feet & Contact & 5 Feet & 10 Feet \\
\hline S01 & 29.4975 & 27.4247 & 46.0063 & 45.4521 & 57.5491 & 31.0871 & 29.9470 \\
\hline S02 & 13.8502 & 1.3879 & 9.4861 & 35.4894 & 10.7202 & 14.2479 & 13.6592 \\
\hline S03 & 23.5916 & 0.4005 & 1.4845 & 3.0858 & 18.6320 & 23.2604 & 23.6763 \\
\hline S04 & 20.6982 & 8.0173 & 37.8946 & 35.8599 & 27.3229 & 21.8274 & 20.9797 \\
\hline S05 & 10.9510 & 0.8869 & 4.2214 & 4.2769 & 10.8263 & 11.1498 & 11.0863 \\
\hline S06 & 16.3182 & 0.4907 & 5.8263 & 5.7709 & 14.7844 & 16.8145 & 16.5876 \\
\hline S07 & 25.9358 & 9.4308 & 30.3032 & 33.1948 & 26.9524 & 24.5603 & 25.4889 \\
\hline S08 & 25.1678 & 0.8206 & 4.9856 & 5.0626 & 22.8160 & 25.6545 & 25.5259 \\
\hline S09 & 24.9347 & 1.3347 & 6.3803 & 7.0678 & 20.6893 & 24.3168 & 24.9009 \\
\hline S10 & 26.8139 & 0.6873 & 10.3733 & 11.4049 & 24.2757 & 26.1156 & 26.7406 \\
\hline S11 & 12.5933 & 0.7885 & 7.4406 & 7.2655 & 12.2127 & 13.0692 & 12.8108 \\
\hline S12 & 17.5307 & 1.8741 & 27.2381 & 26.0331 & 16.9373 & 18.3732 & 17.8144 \\
\hline S13 & 17.9331 & 0.3894 & 2.9347 & 3.1761 & 14.5112 & 17.7612 & 18.0317 \\
\hline S14 & 26.2138 & 7.0621 & 39.2959 & 38.7750 & 29.3609 & 26.7979 & 26.3740 \\
\hline S15 & 22.7469 & 13.6272 & 53.0404 & 52.2644 & 28.7747 & 22.8163 & 22.4768 \\
\hline S16 & 27.6556 & 0.9474 & 23.1010 & 23.2134 & 22.9273 & 28.1523 & 27.9367 \\
\hline S17 & 28.5227 & 3.8457 & 15.7190 & 16.7470 & 27.4687 & 28.1431 & 28.5396 \\
\hline S18 & 23.2528 & 1.3471 & 59.8699 & 58.8404 & 15.5824 & 23.0400 & 22.6488 \\
\hline S19 & 15.3416 & 10.9540 & 19.6870 & 18.9295 & 26.8814 & 16.0345 & 15.5723 \\
\hline $\mathbf{S} 20$ & 16.4988 & 0.4911 & 2.9513 & 3.2795 & 12.9590 & 16.1270 & 16.4990 \\
\hline S21 & 28.6466 & 0.5946 & 4.5715 & 4.6906 & 24.8791 & 29.0781 & 29.0250 \\
\hline $\mathbf{S 2 2}$ & 20.3724 & 0.6706 & 47.7191 & 49.2107 & 11.4177 & 19.3101 & 19.6558 \\
\hline $\mathbf{S 2 3}$ & 13.0146 & 0.9994 & 7.4012 & 8.4840 & 9.5389 & 12.0953 & 12.8172 \\
\hline S24 & 12.5419 & 2.5730 & 26.3980 & 25.0690 & 12.9953 & 13.1475 & 12.6527 \\
\hline S25 & 19.7625 & 0.4968 & 7.3284 & 7.5894 & 16.3839 & 19.8684 & 19.9244 \\
\hline S26 & 24.8512 & 5.4794 & 22.9567 & 22.4148 & 29.6715 & 25.7517 & 25.2145 \\
\hline S27 & 10.2138 & 21.8262 & 22.4484 & 21.8015 & 28.7845 & 10.4560 & 10.2004 \\
\hline S28 & 15.5133 & 4.3483 & 21.7591 & 22.5837 & 16.1492 & 15.2414 & 15.4135 \\
\hline S29 & 18.0653 & 0.1467 & 1.1979 & 1.3031 & 14.3100 & 17.9797 & 18.2006 \\
\hline S30 & 15.0401 & 4.0519 & 32.0450 & 31.3945 & 15.3870 & 15.2900 & 15.0047 \\
\hline S31 & 22.3550 & 6.0887 & 31.0371 & 29.7613 & 27.3595 & 23.3975 & 22.6850 \\
\hline S32 & 21.6866 & 11.8783 & 26.5946 & 25.4175 & 34.7754 & 22.7726 & 22.0555 \\
\hline S33 & 17.8281 & 40.8344 & 49.4994 & 46.4821 & 46.5716 & 18.4724 & 17.5660 \\
\hline S34 & 14.6776 & 0.3584 & 35.0504 & 35.6104 & 8.4524 & 14.1280 & 14.2414 \\
\hline S35 & 28.0146 & 1.2235 & 71.1504 & 66.9246 & 21.6089 & 29.3568 & 28.0027 \\
\hline verage & 20.2466 & 5.5365 & 23.2970 & 23.8265 & 21.7277 & 20.4484 & 20.2845 \\
\hline
\end{tabular}

FIGURE 1 
REVISED METHODOLOGY FOR DETERMINING CESIUM-137 CONTENT OF HN-200 GROUT CONTAINERS.

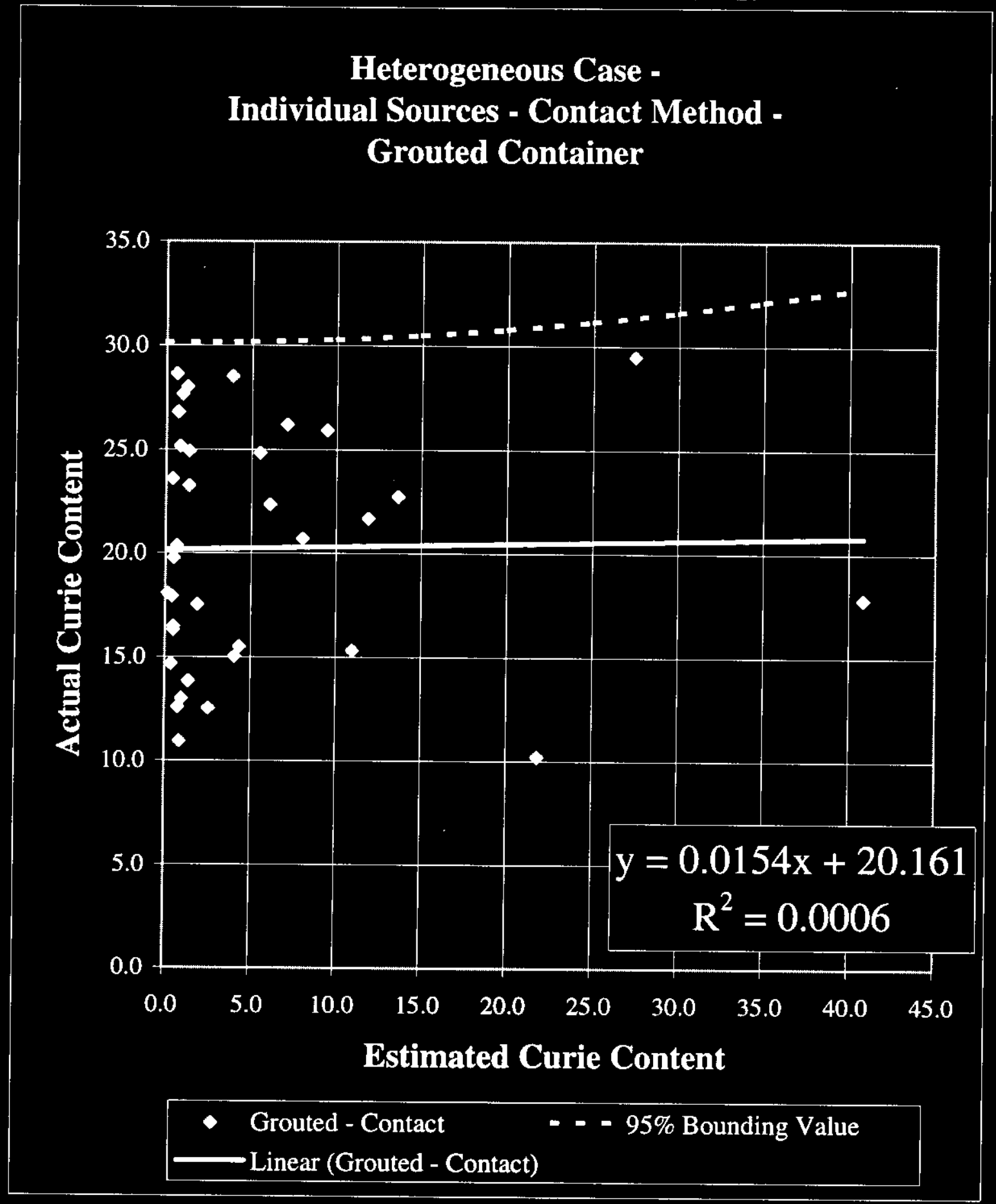

FIGURE 2 
REVISED METHODOLOGY FOR DETERMINING CESIUM-137 CONTENT OF HN-200 GROUT CONTAINERS.

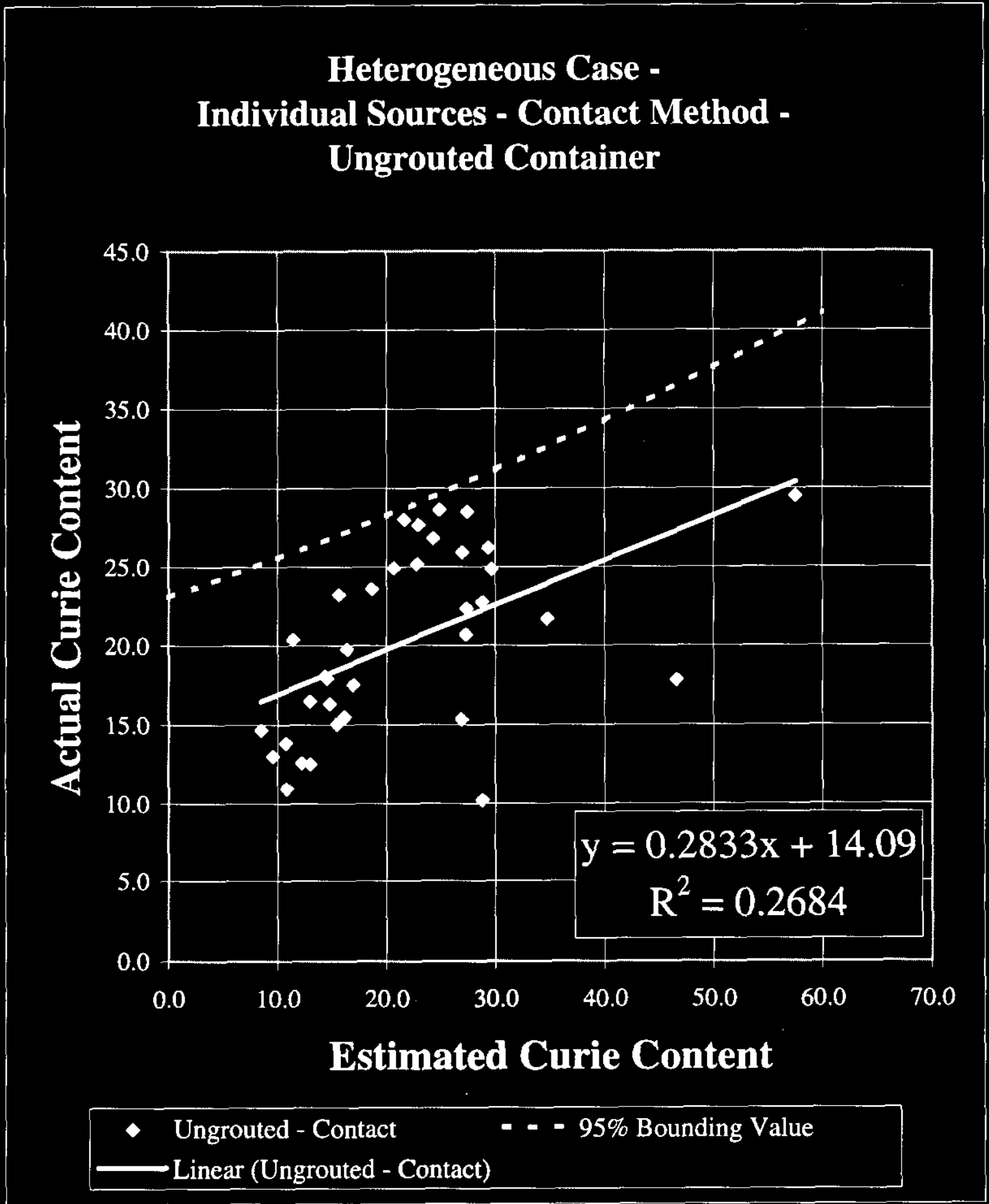


REVISED METHODOLOGY FOR DETERMINING CESIUM-137 CONTENT OF HN-200 GROUT CONTAINERS.

FIGURE 3

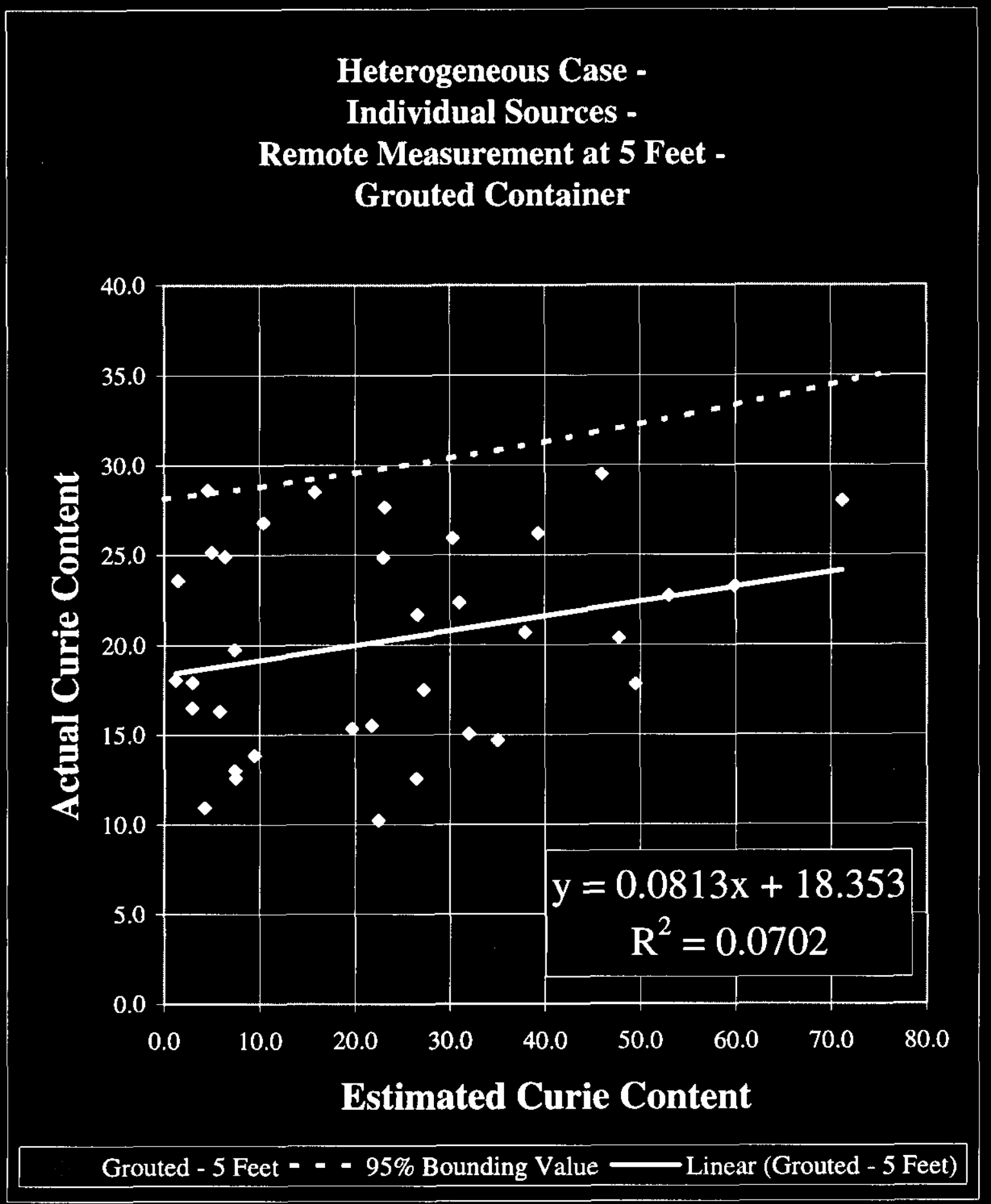

FIGURE 4 
REVISED METHODOLOGY FOR DETERMINING CESIUM-137 CONTENT OF HN-200 GROUT CONTAINERS.

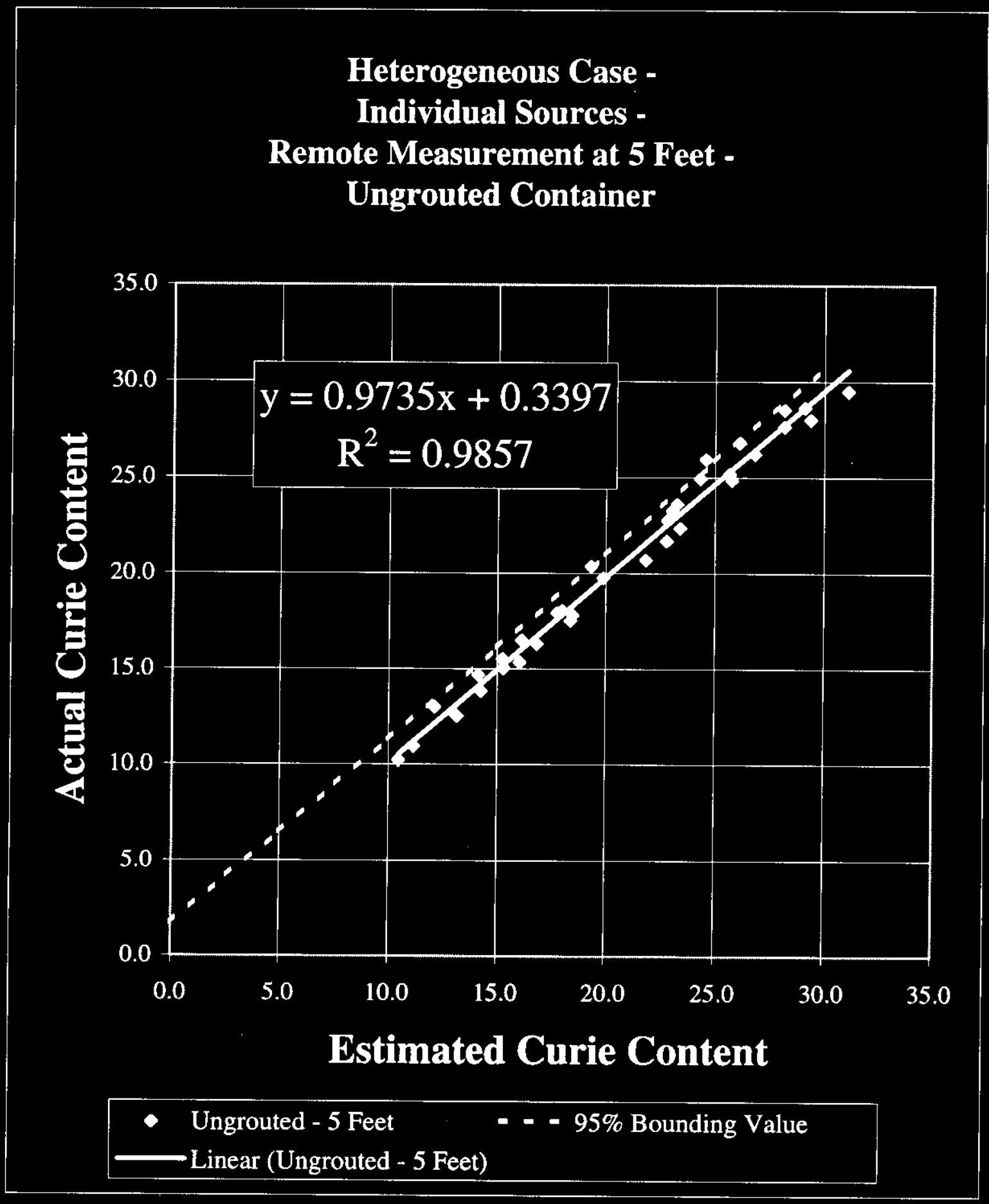

FIGURE 5 
HNF-4799, Rev 0 August 25, 1999

REVISED METHODOLOGY FOR DETERMINING CESIUM-137 CONTENT OF HN-200 GROUT CONTAINERS.

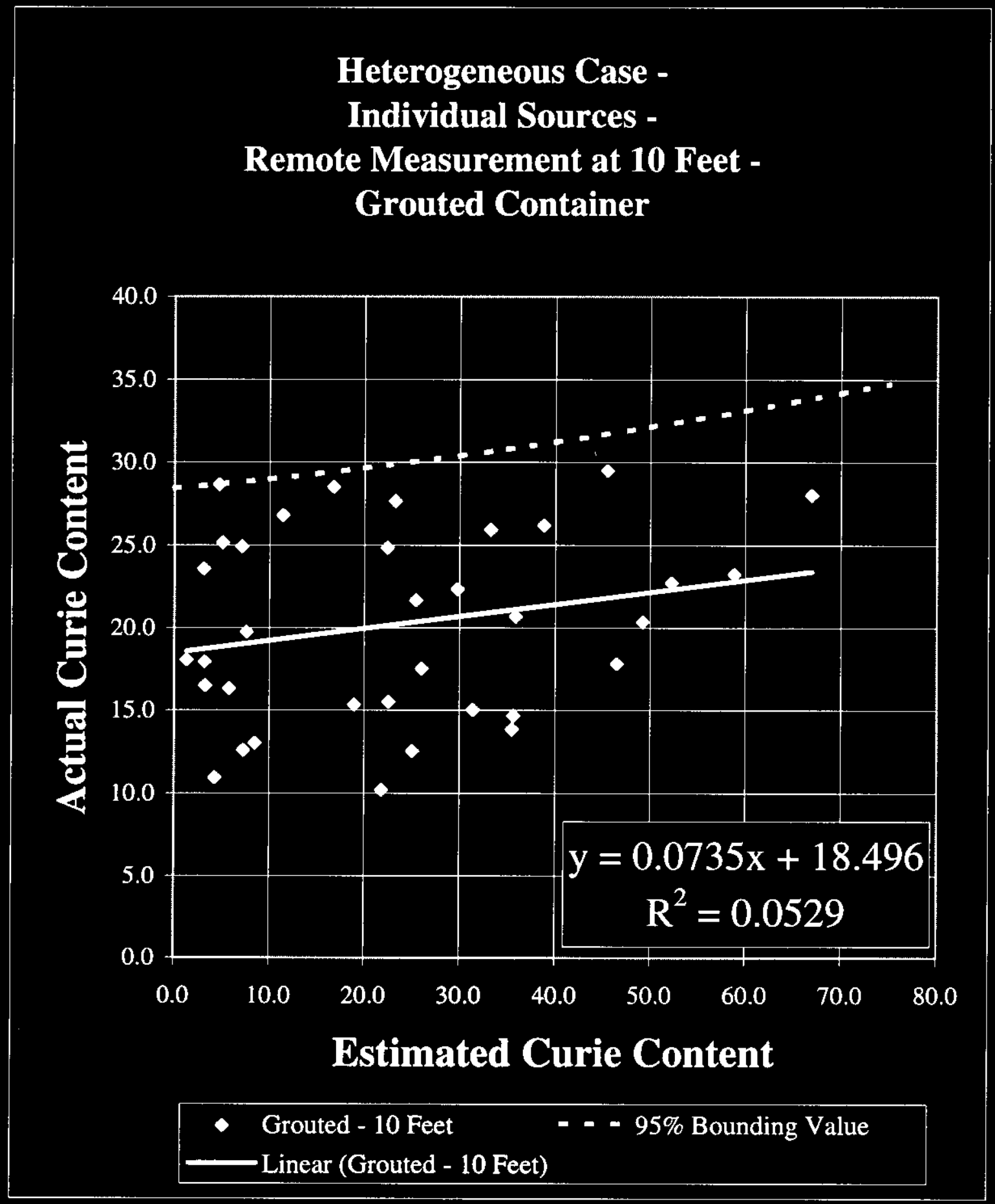

FIGURE 6 
HNF-4799, Rev 0

REVISED METHODOLOGY FOR DETERMINING CESIUM-137 CONTENT OF HN-200 GROUT CONTAINERS.

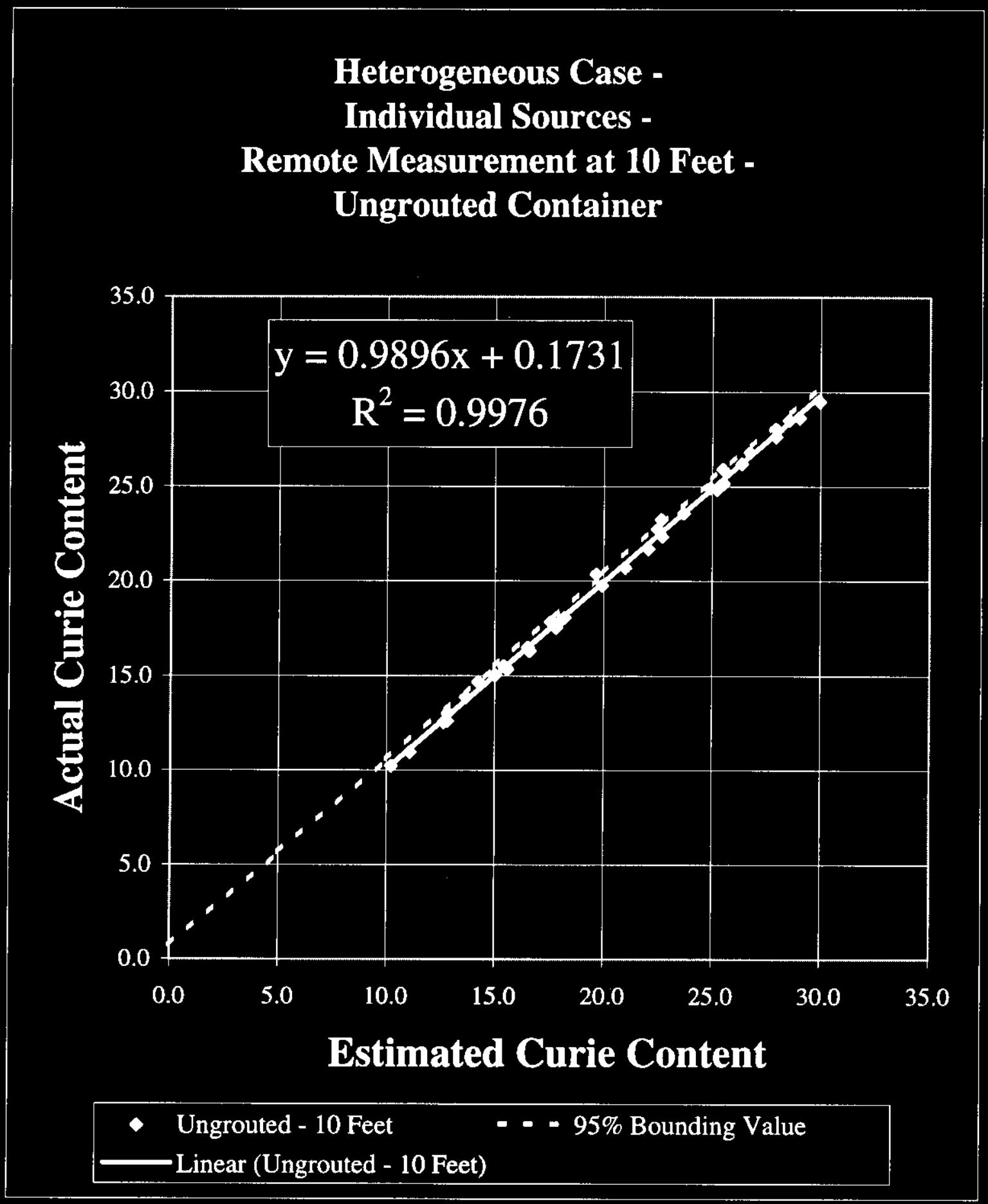




\section{REVISED METHODOLOGY FOR DETERMINING CESIUM-137 CONTENT OF HN-200 GROUT CONTAINERS.}

FIGURE 7

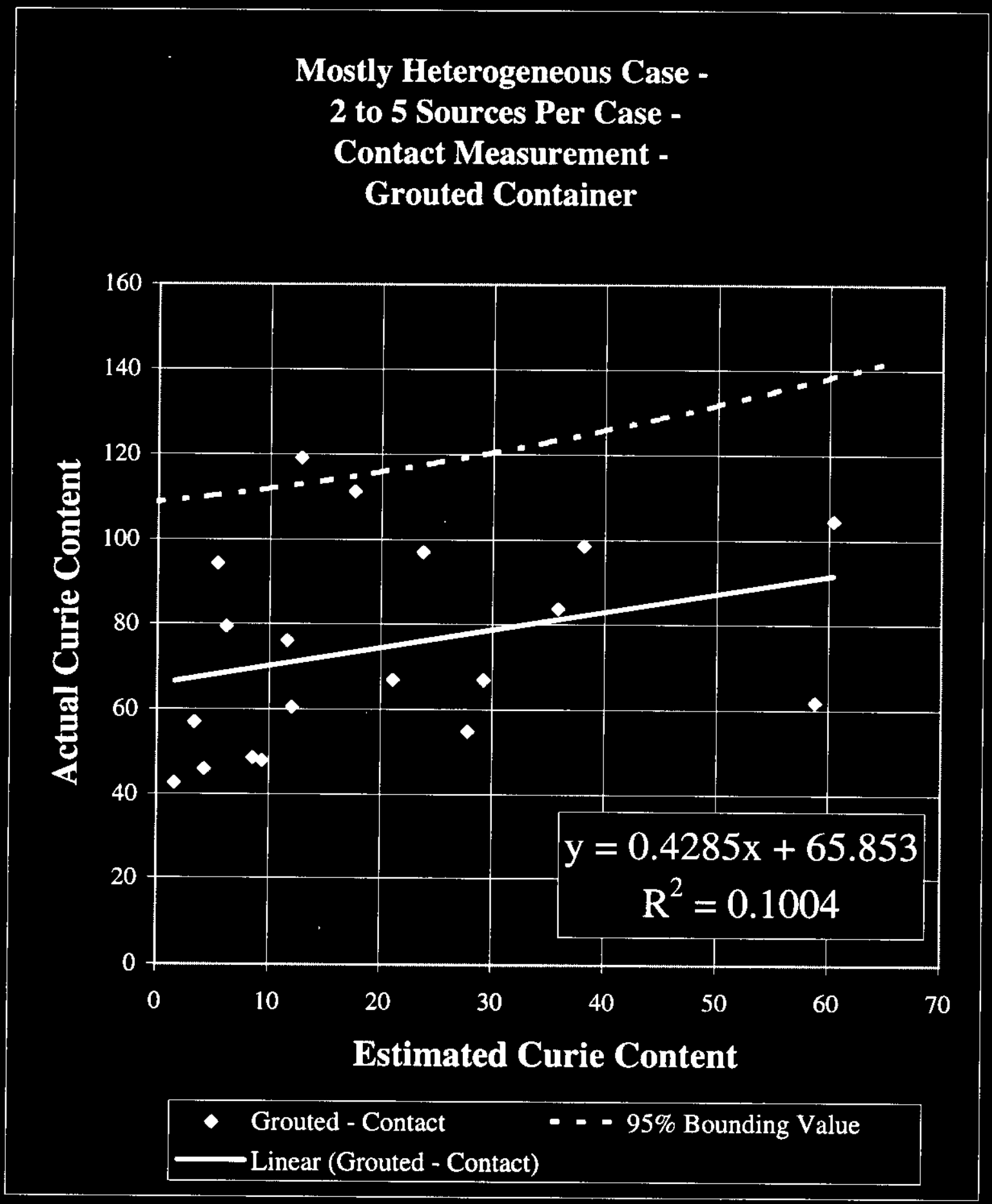

FIGURE 8 
HNF-4799, Rev 0

REVISED METHODOLOGY FOR DETERMINING CESIUM-137 CONTENT OF HN-200 GROUT CONTAINERS.

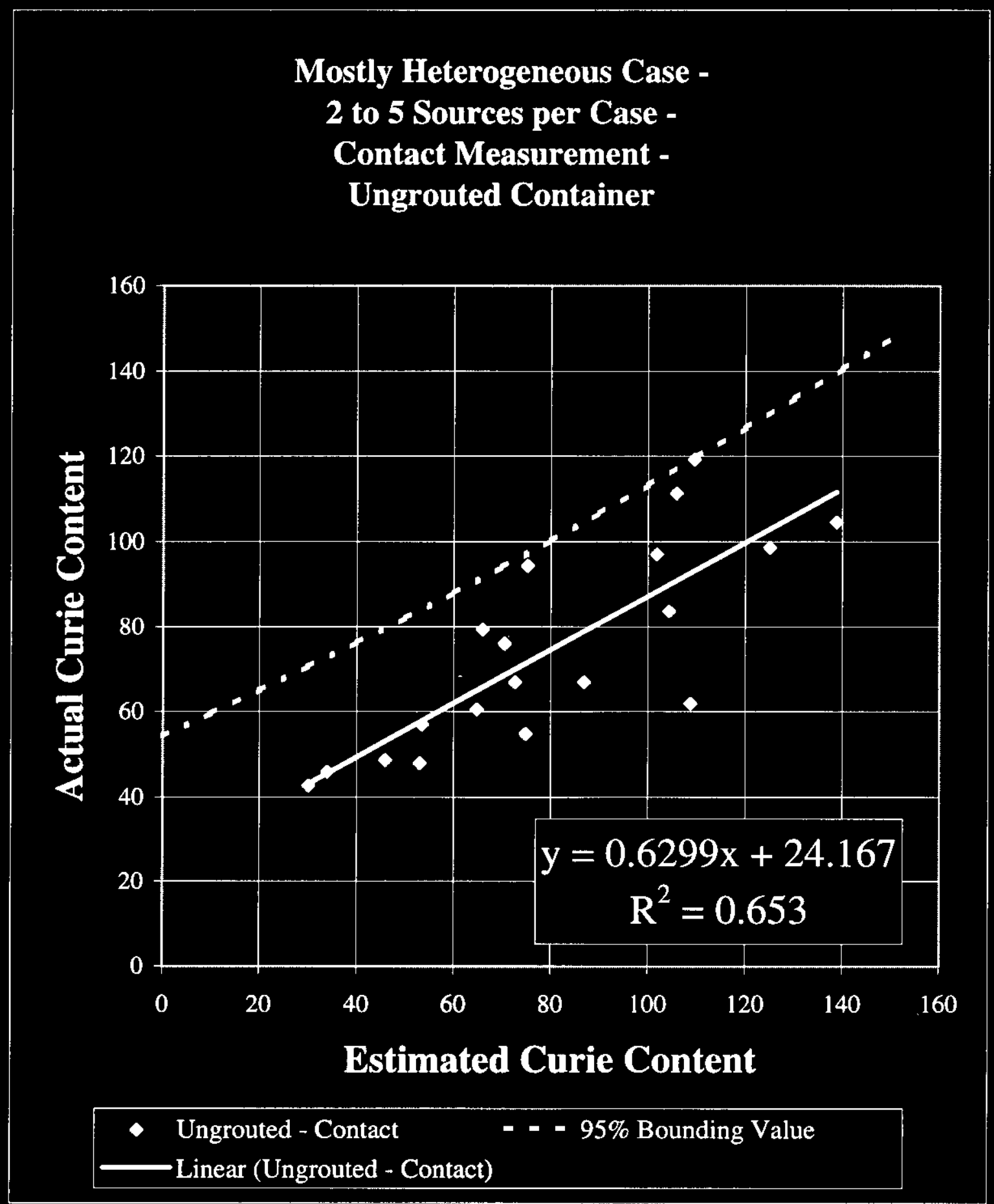

FIGURE 9 
REVISED METHODOLOGY FOR DETERMINING CESIUM-137 CONTENT OF HN-200 GROUT CONTAINERS.

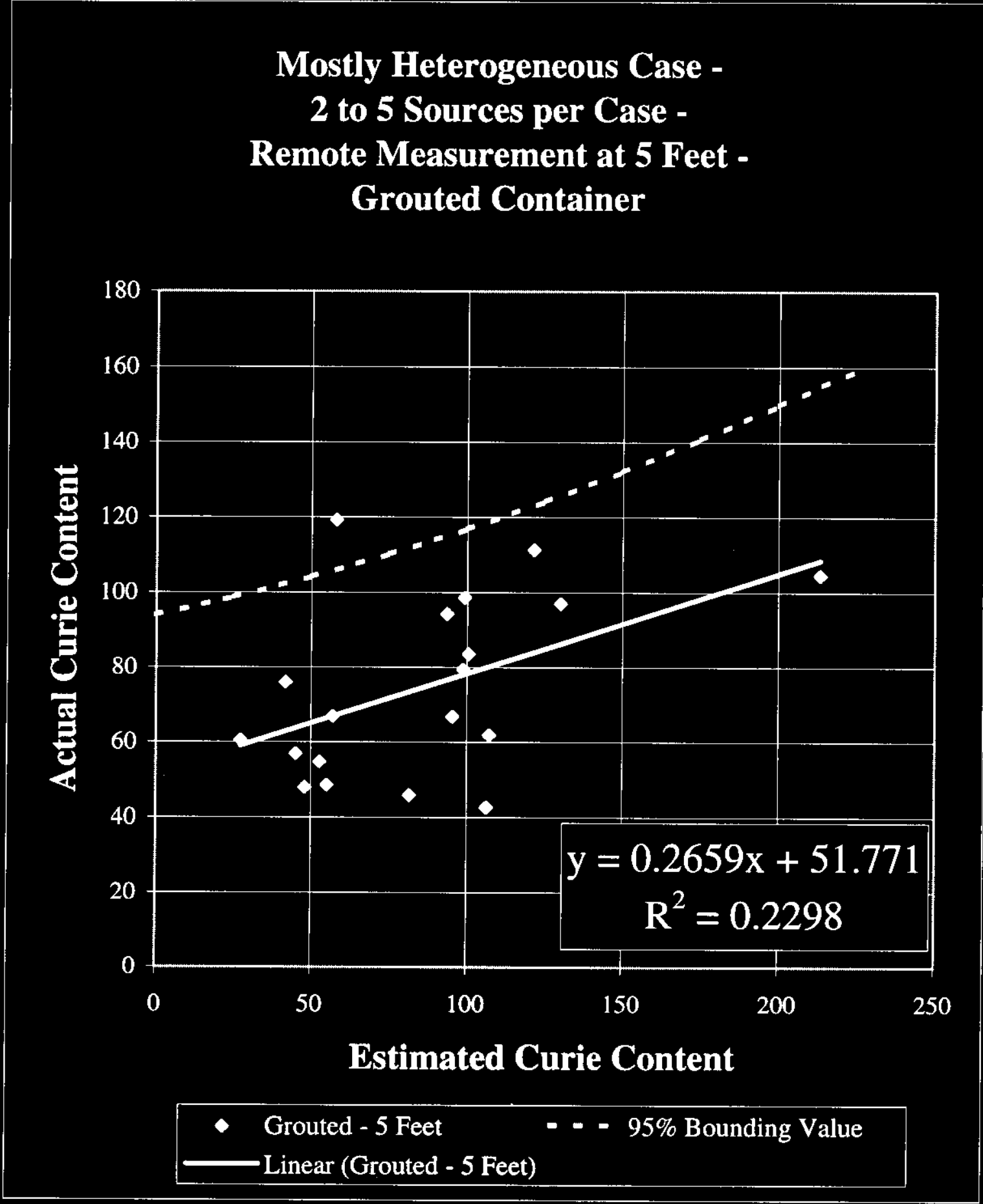

FIGURE 10 
HNF-4799, Rev 0

REVISED METHODOLOGY FOR DETERMINING CESIUM-137 CONTENT

OF HN-200 GROUT CONTAINERS.

\section{Mostly Heterogeneous Case -}

2 to 5 Sources per Case -

Remote Measurement at 5 Feet -

Ungrouted Container

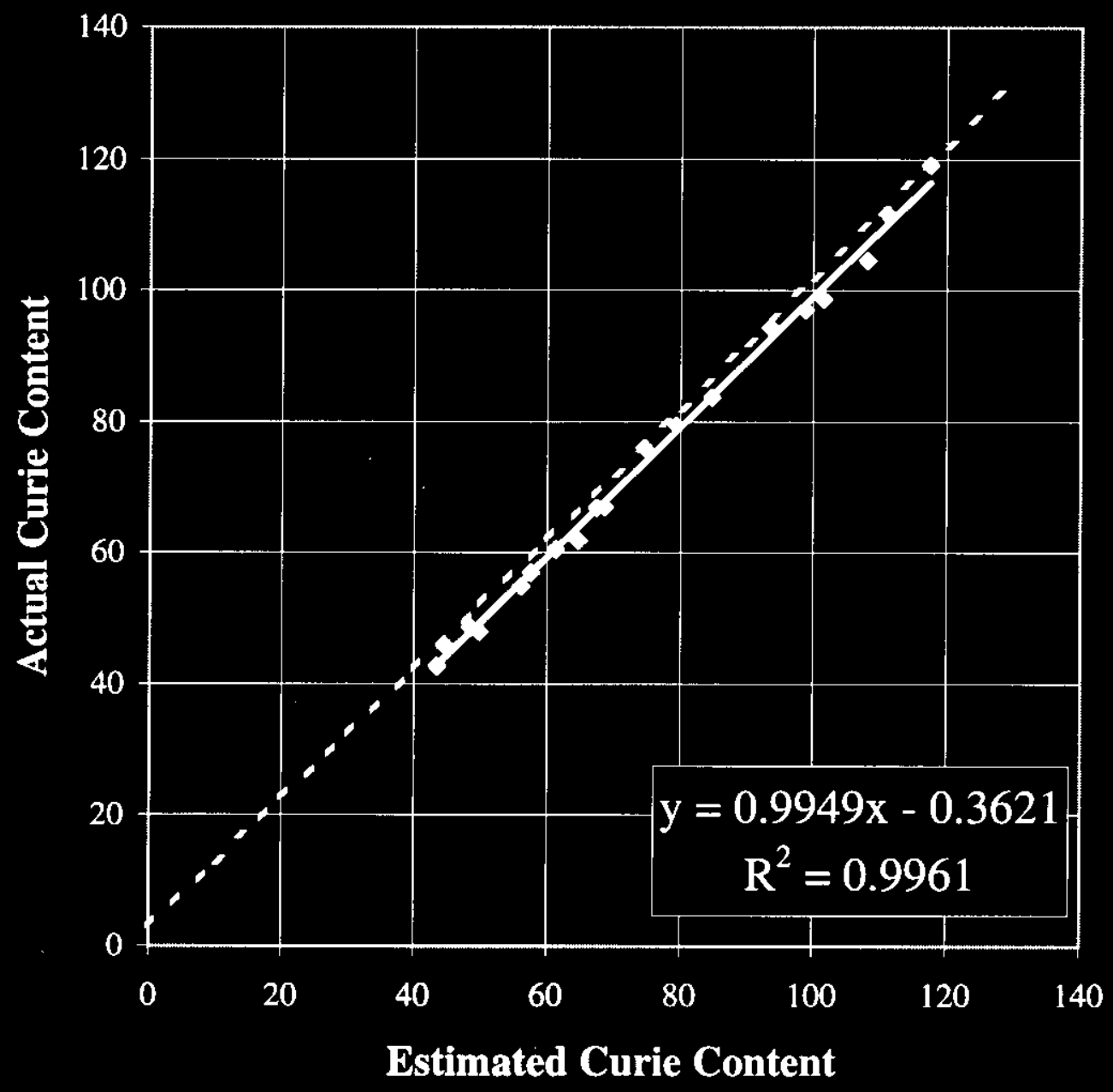

- Ungrouted - 5 Feet - - $95 \%$ Bounding Value Linear (Ungrouted - 5 Feet) 
HNF-4799, Rev 0

August 25, 1999

REVISED METHODOLOGY FOR DETERMINING CESIUM-137 CONTENT OF HN-200 GROUT CONTAINERS.

FIGURE 11

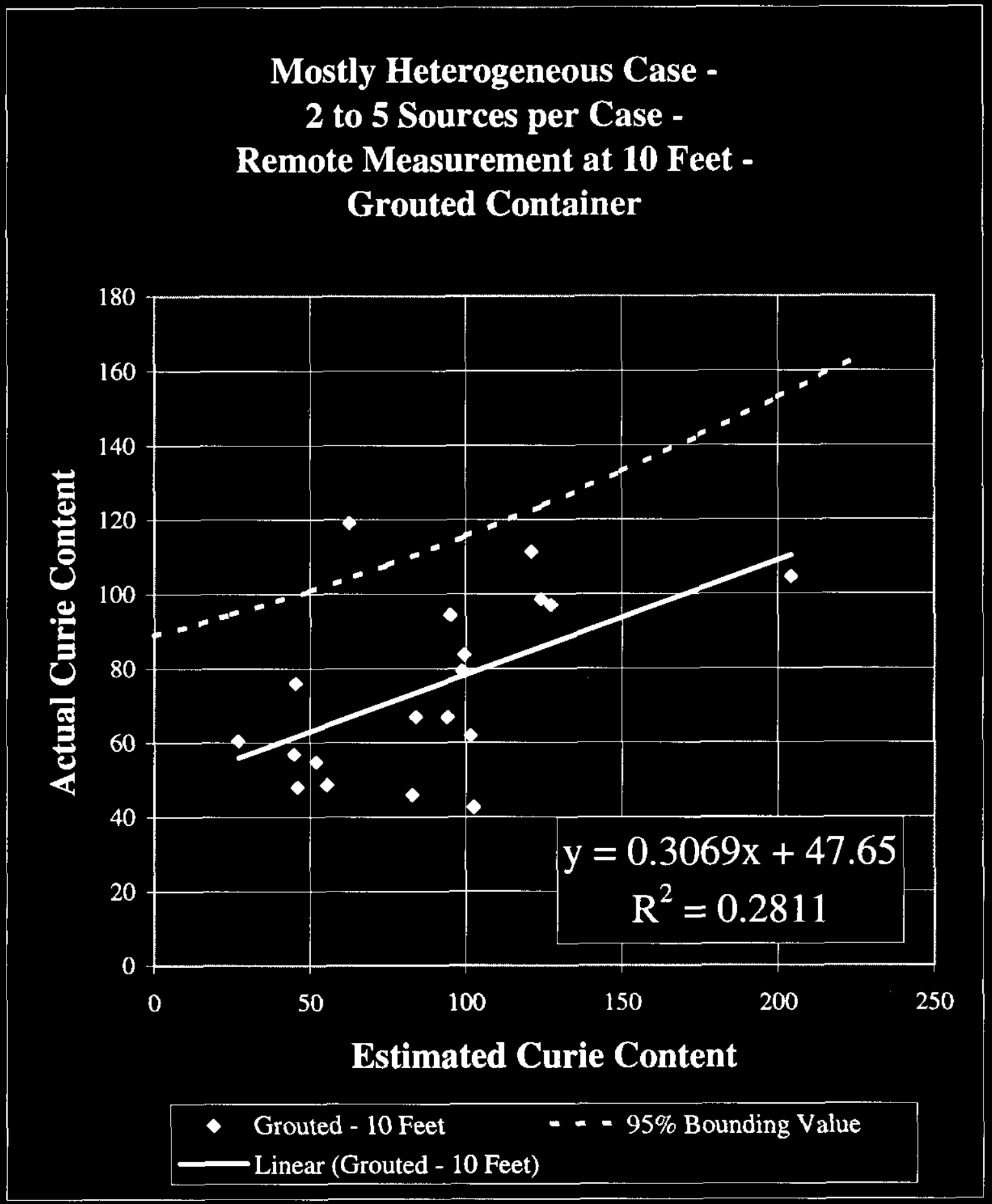

FIGURE 12 
REVISED METHODOLOGY FOR DETERMINING CESIUM-137 CONTENT OF HN-200 GROUT CONTAINERS.

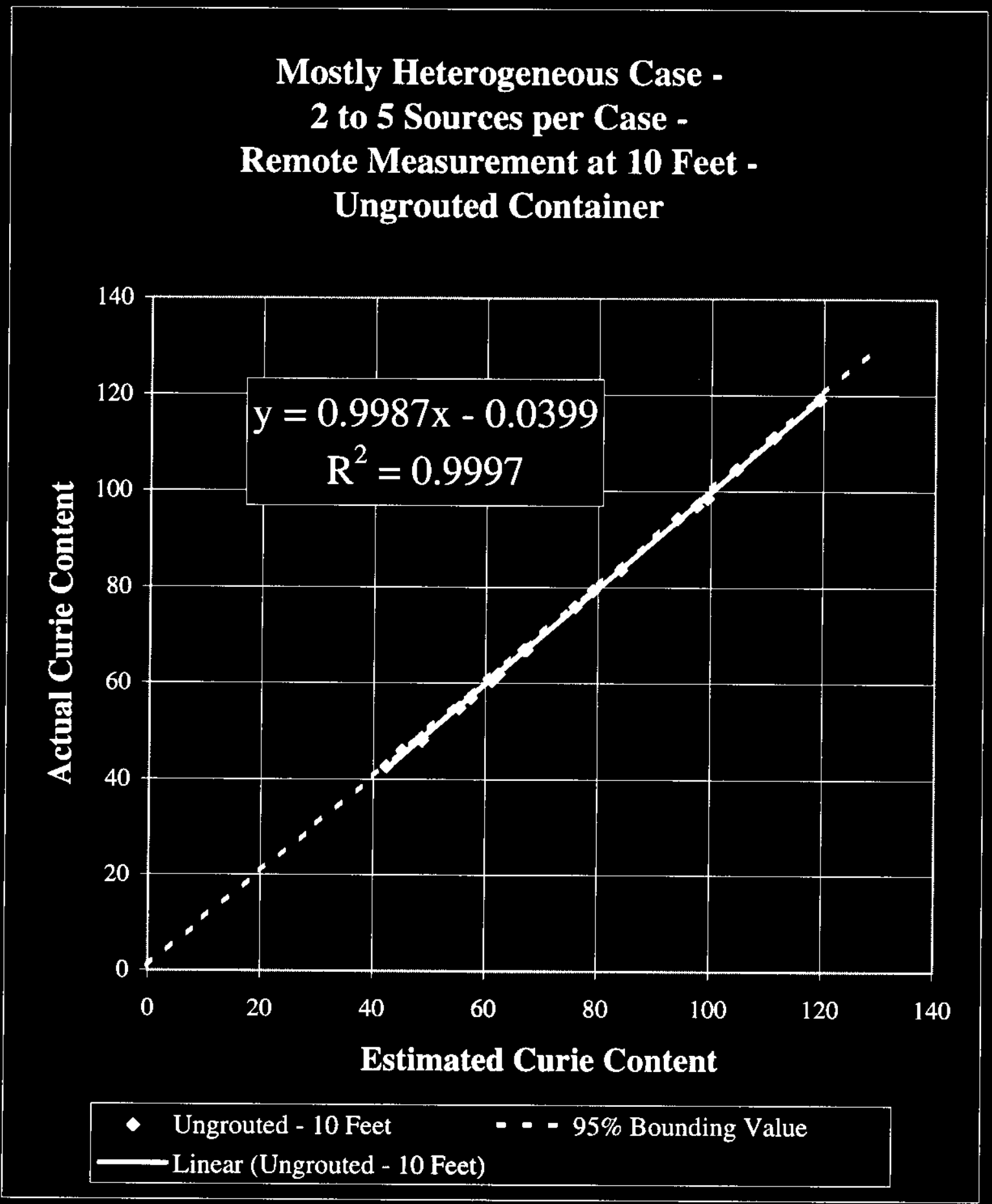

FIGURE 13 
REVISED METHODOLOGY FOR DETERMINING CESIUM-137 CONTENT OF HN-200 GROUT CONTAINERS.

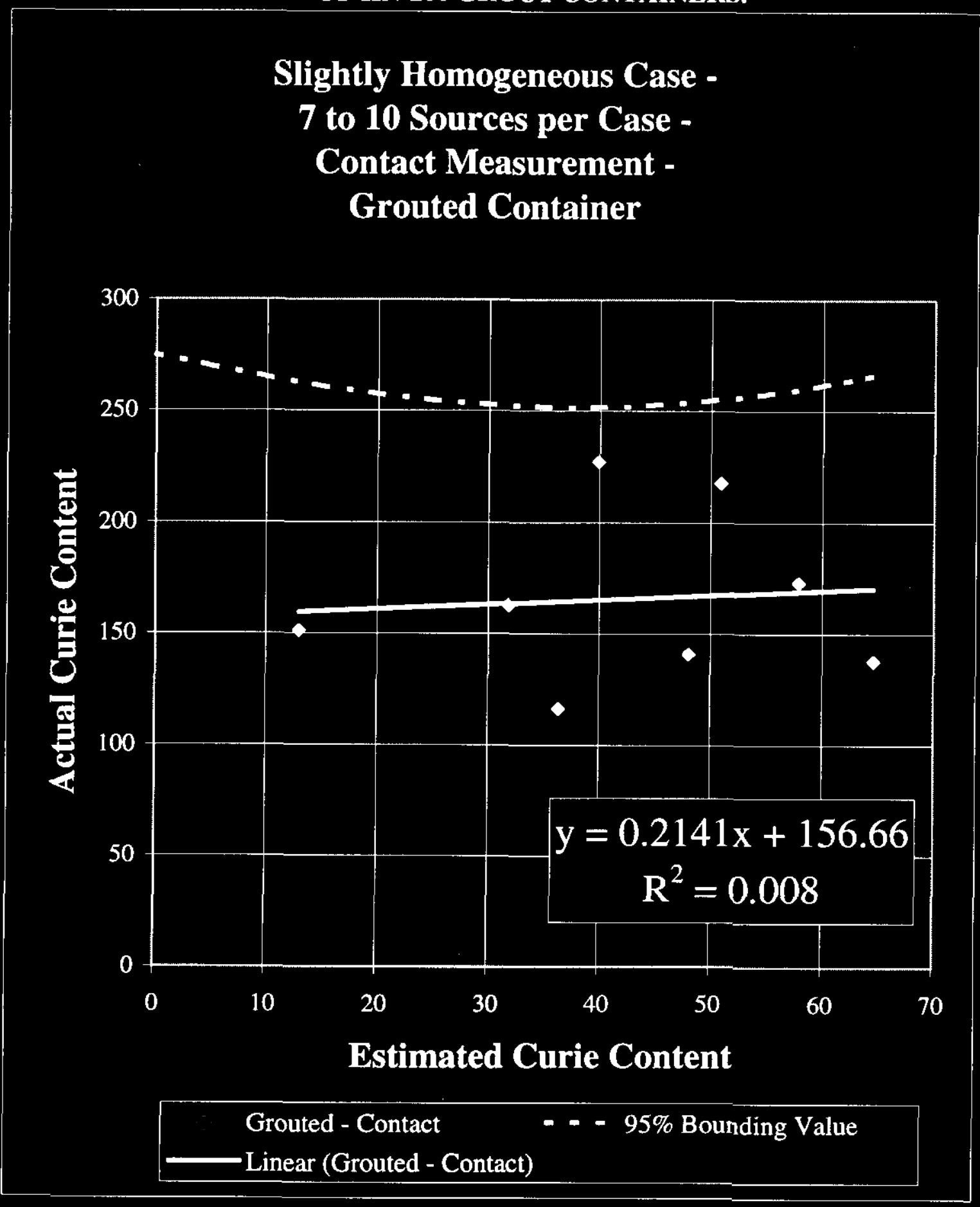

FIGURE 14 
REVISED METHODOLOGY FOR DETERMINING CESIUM-137 CONTENT OF HN-200 GROUT CONTAINERS.

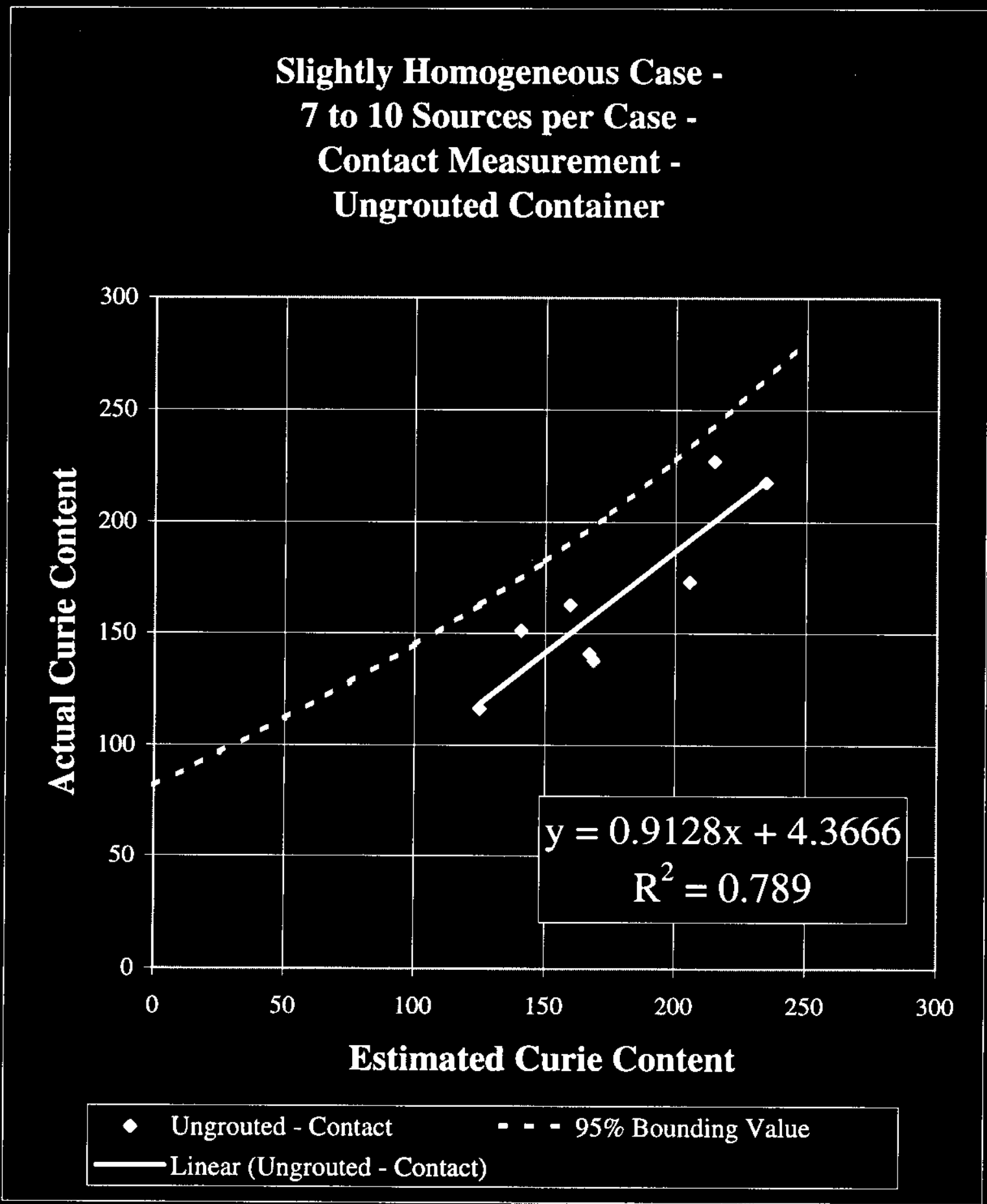




\section{REVISED METHODOLOGY FOR DETERMINING CESIUM-137 CONTENT OF HN-200 GROUT CONTAINERS.}

FIGURE 15

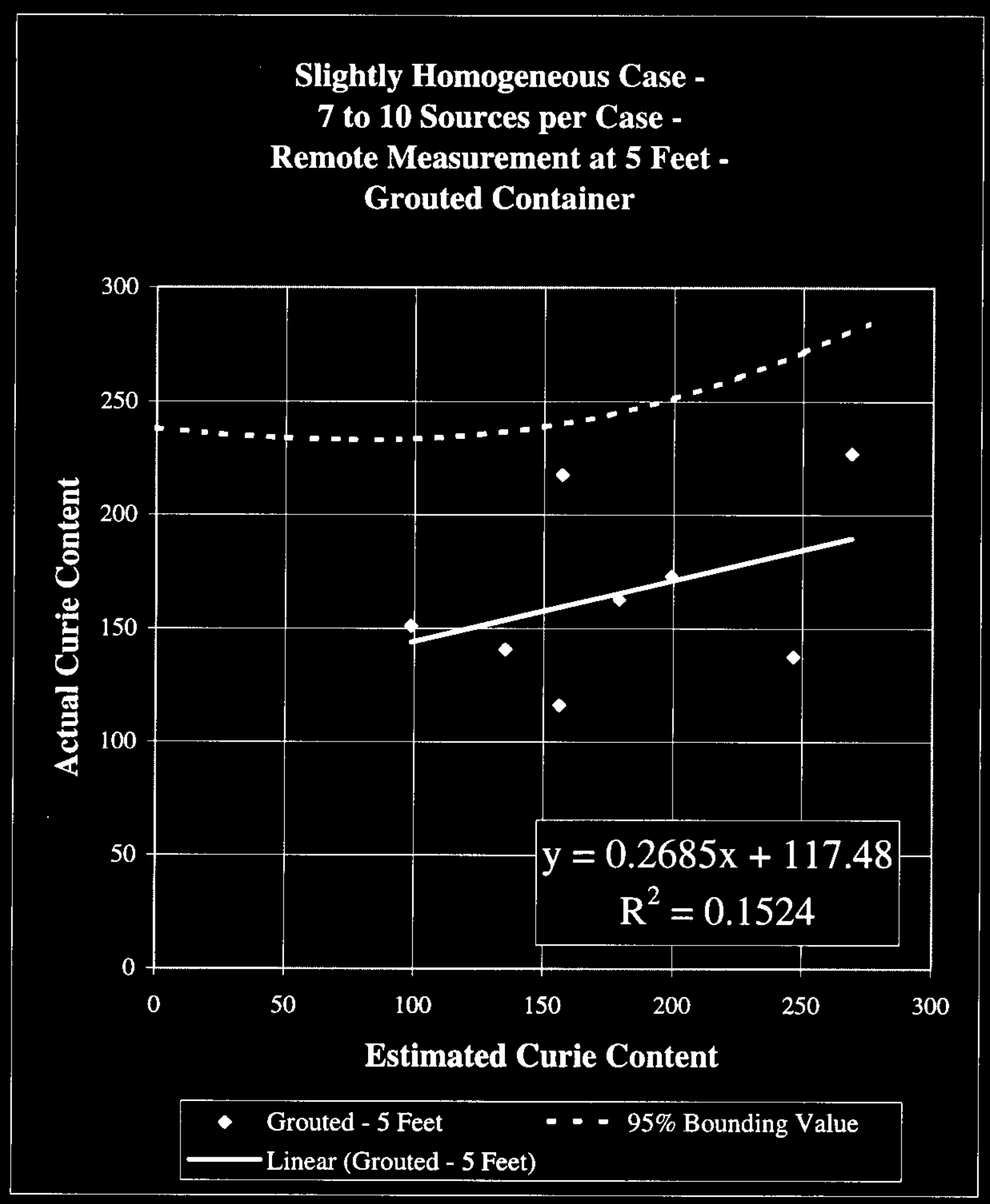

FIGURE 16 


\section{REVISED METHODOLOGY FOR DETERMINING CESIUM-137 CONTENT OF HN-200 GROUT CONTAINERS.}

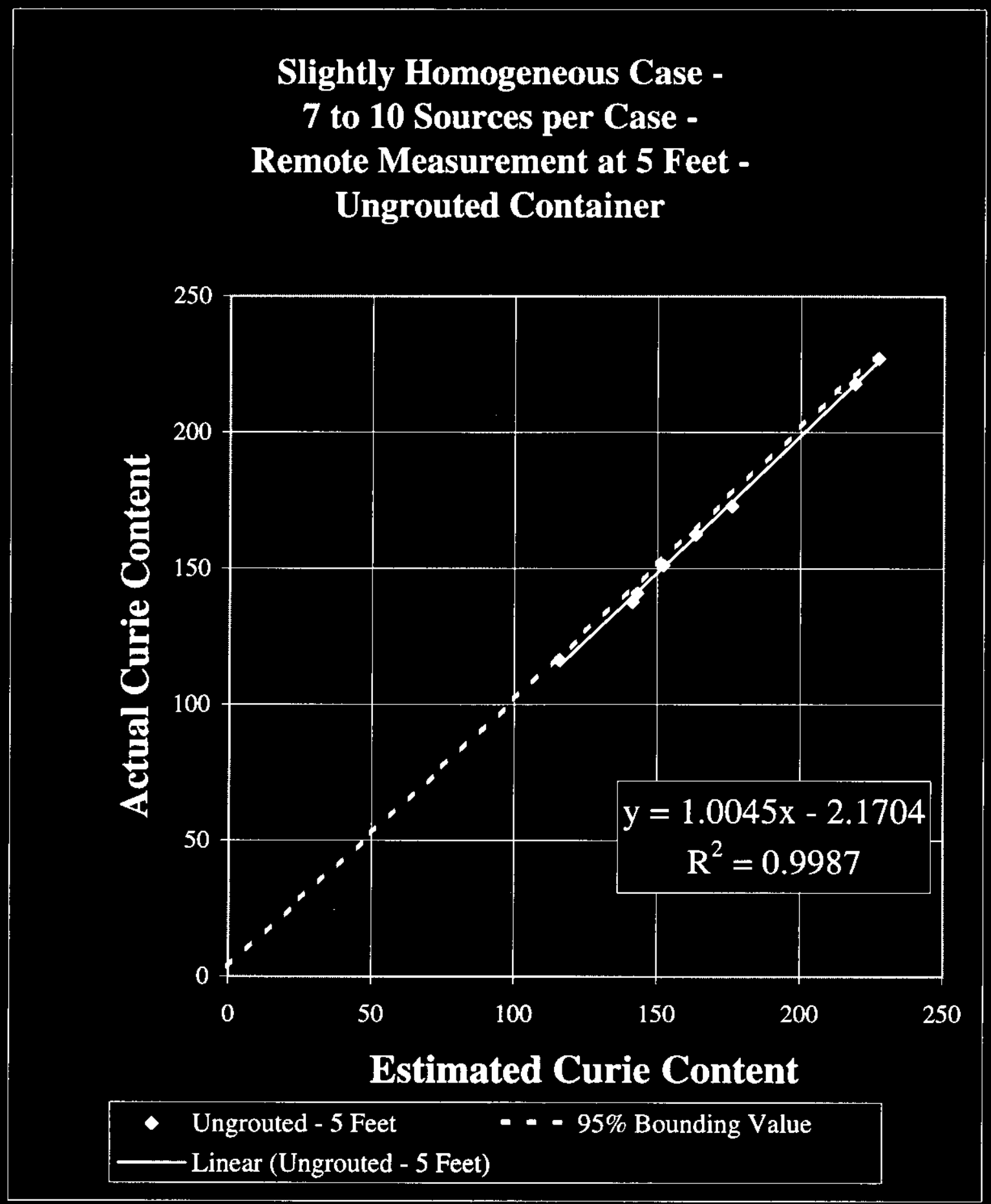

FIGURE 17 
REVISED METHODOLOGY FOR DETERMINING CESIUM-137 CONTENT OF HN-200 GROUT CONTAINERS.

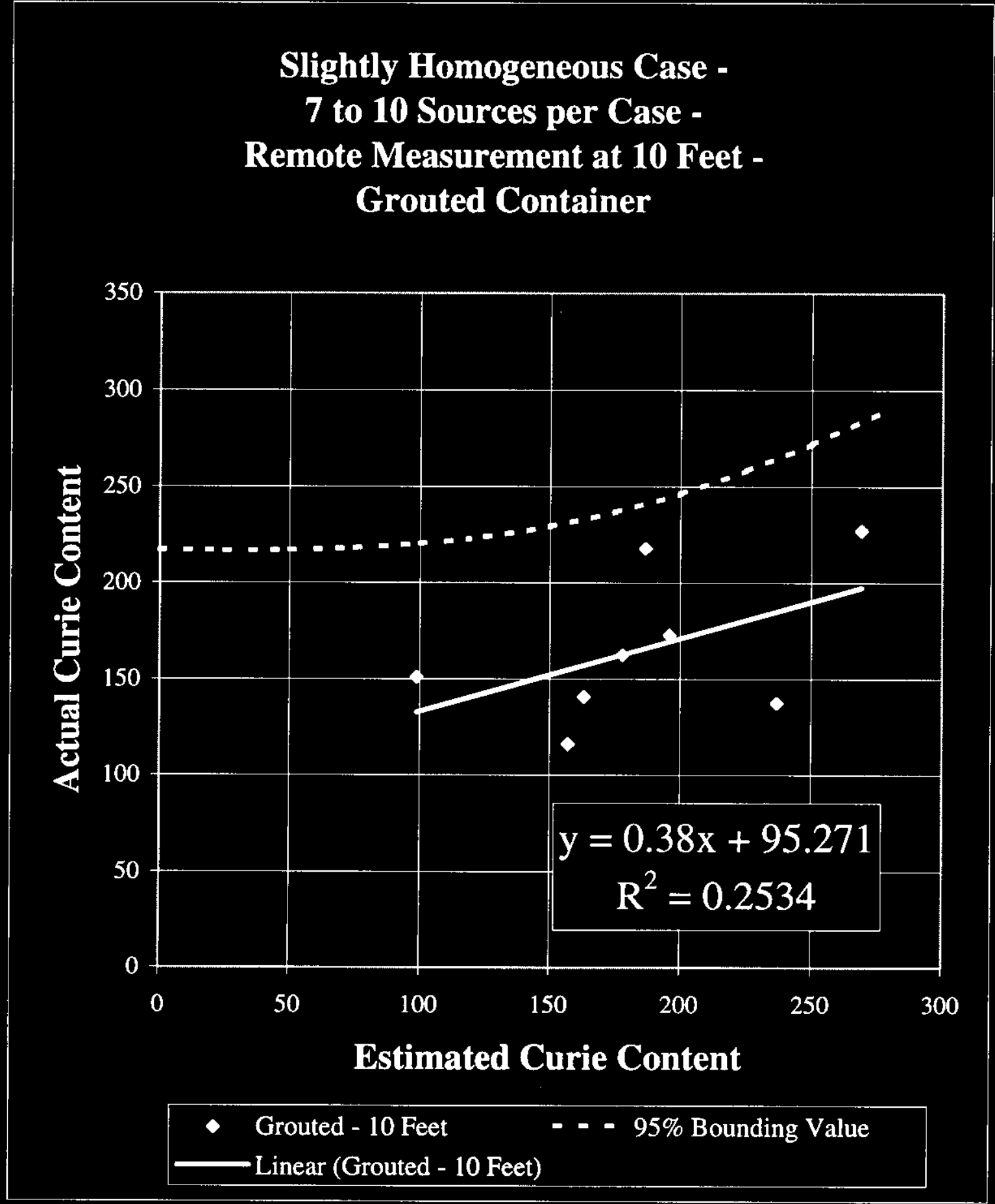

FIGURE 18 
REVISED METHODOLOGY FOR DETERMINING CESIUM-137 CONTENT OF HN-200 GROUT CONTAINERS.

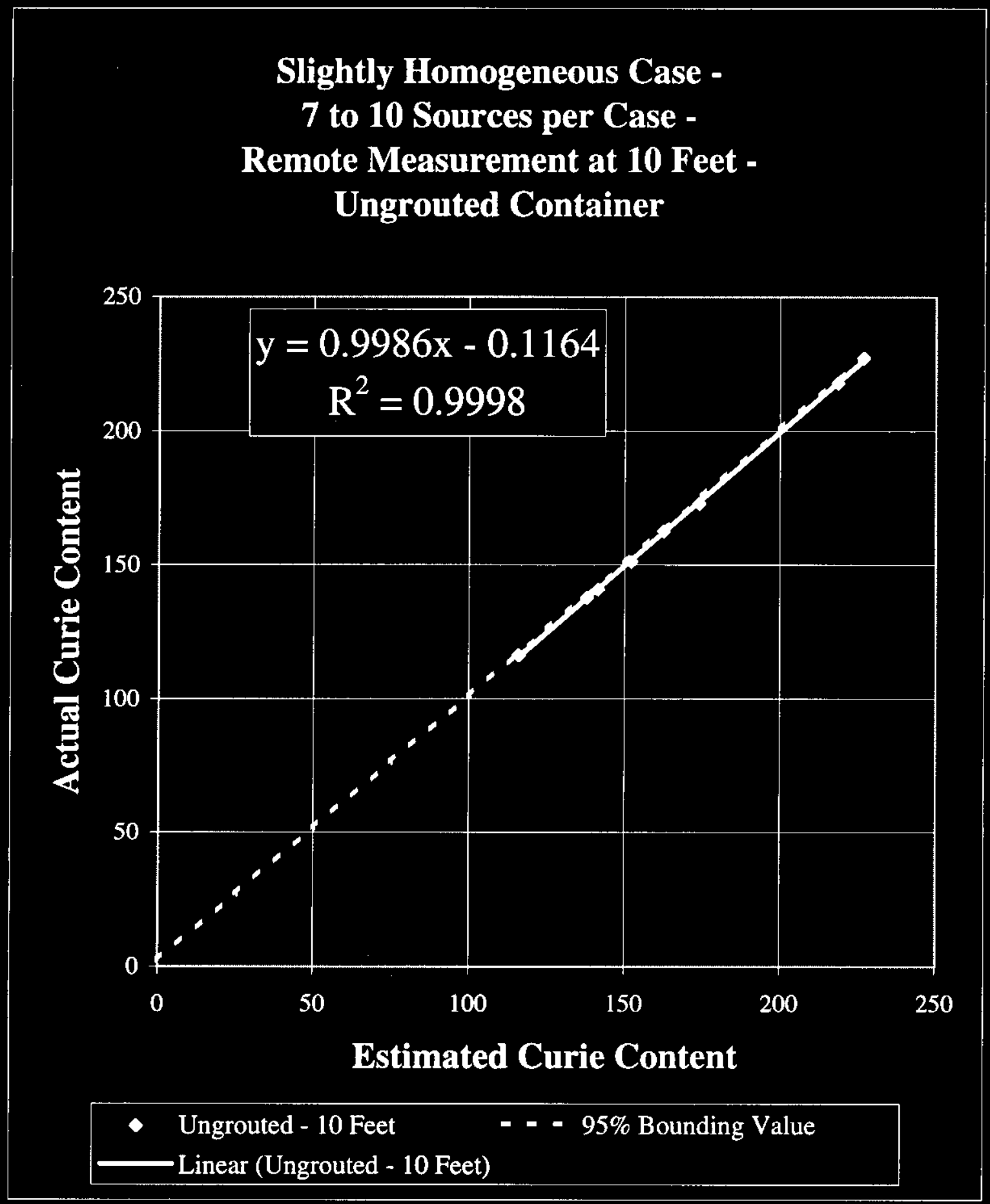


REVISED METHODOLOGY FOR DETERMINING CESIUM-137 CONTENT OF HN-200 GROUT CONTAINERS.

FIGURE 19

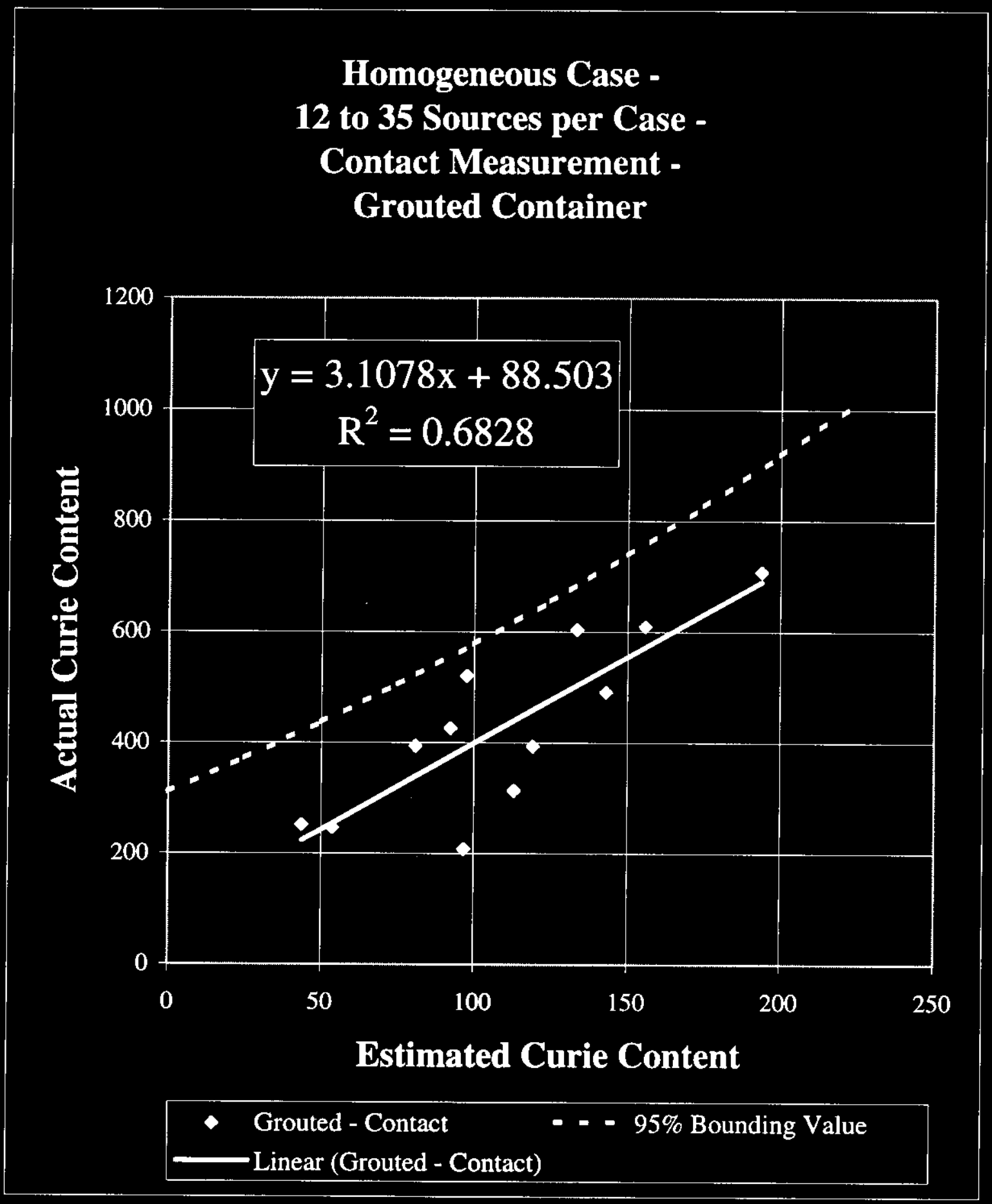

FIGURE 20 
REVISED METHODOLOGY FOR DETERMINING CESIUM-137 CONTENT OF HN-200 GROUT CONTAINERS.

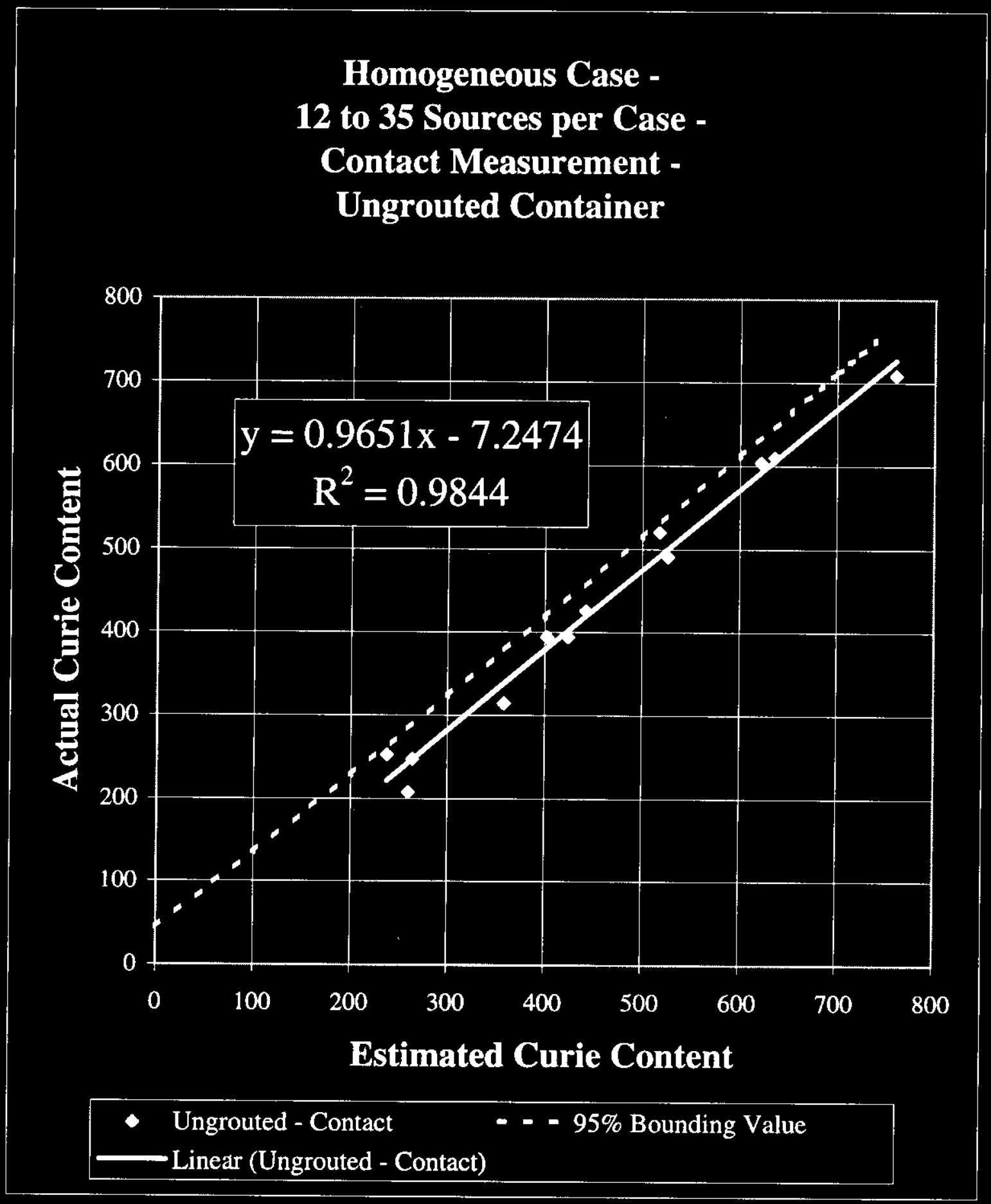

FIGURE 21 
REVISED METHODOLOGY FOR DETERMINING CESIUM-137 CONTENT OF HN-200 GROUT CONTAINERS.

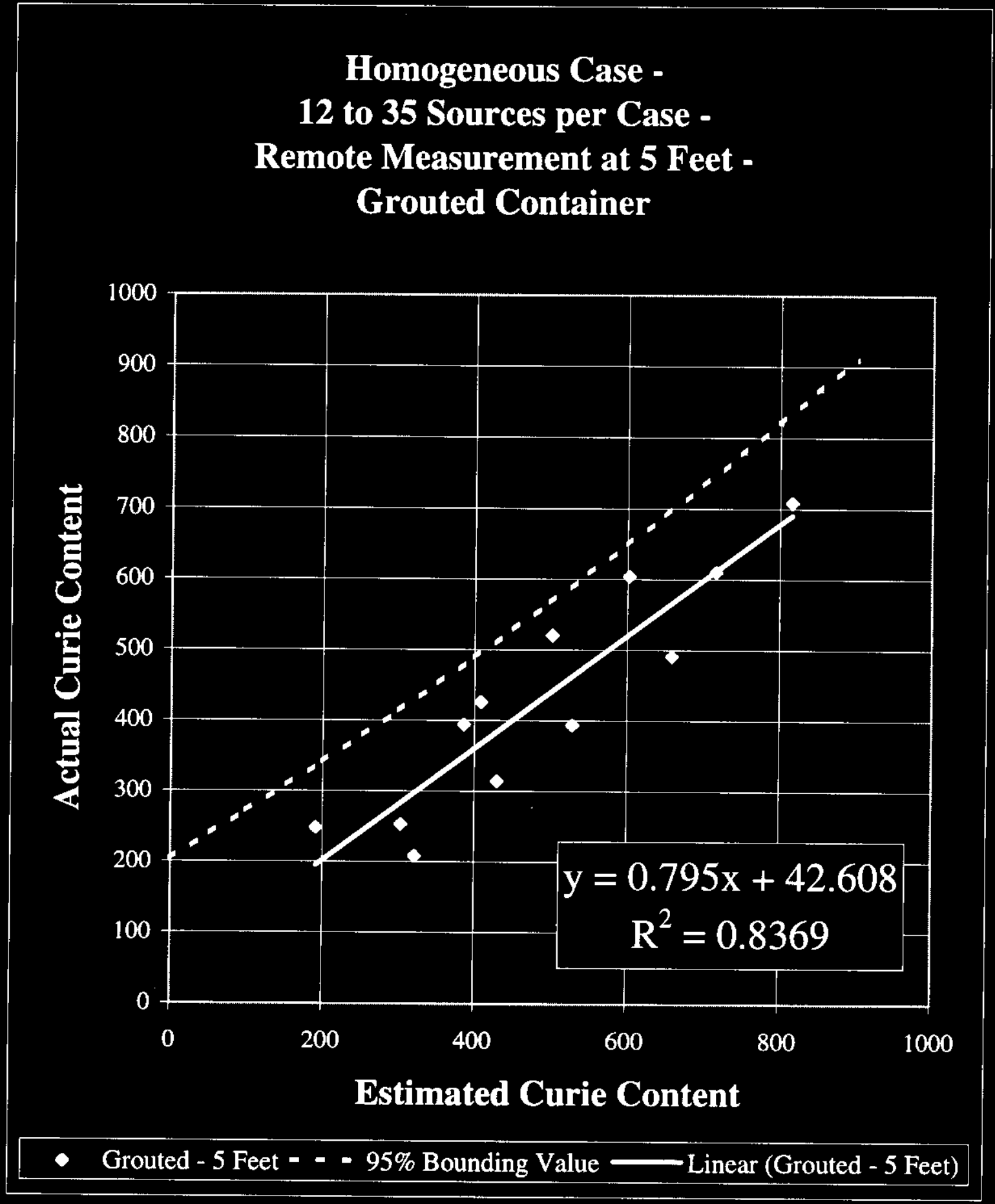

FIGURE 22 


\section{REVISED METHODOLOGY FOR DETERMINING CESIUM-137 CONTENT} OF HN-200 GROUT CONTAINERS.

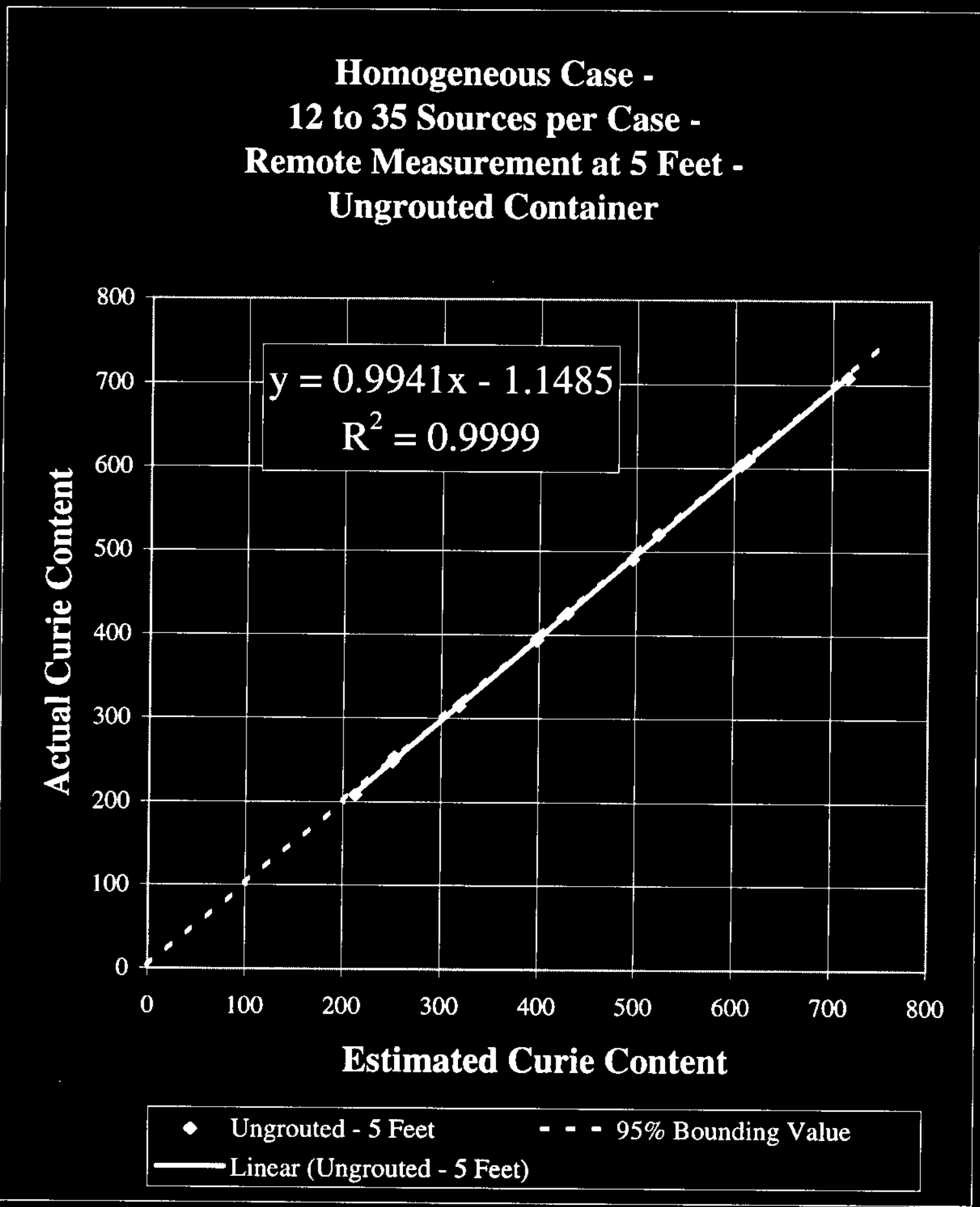


REVISED METHODOLOGY FOR DETERMINING CESIUM-137 CONTENT OF HN-200 GROUT CONTAINERS.

FIGURE 23

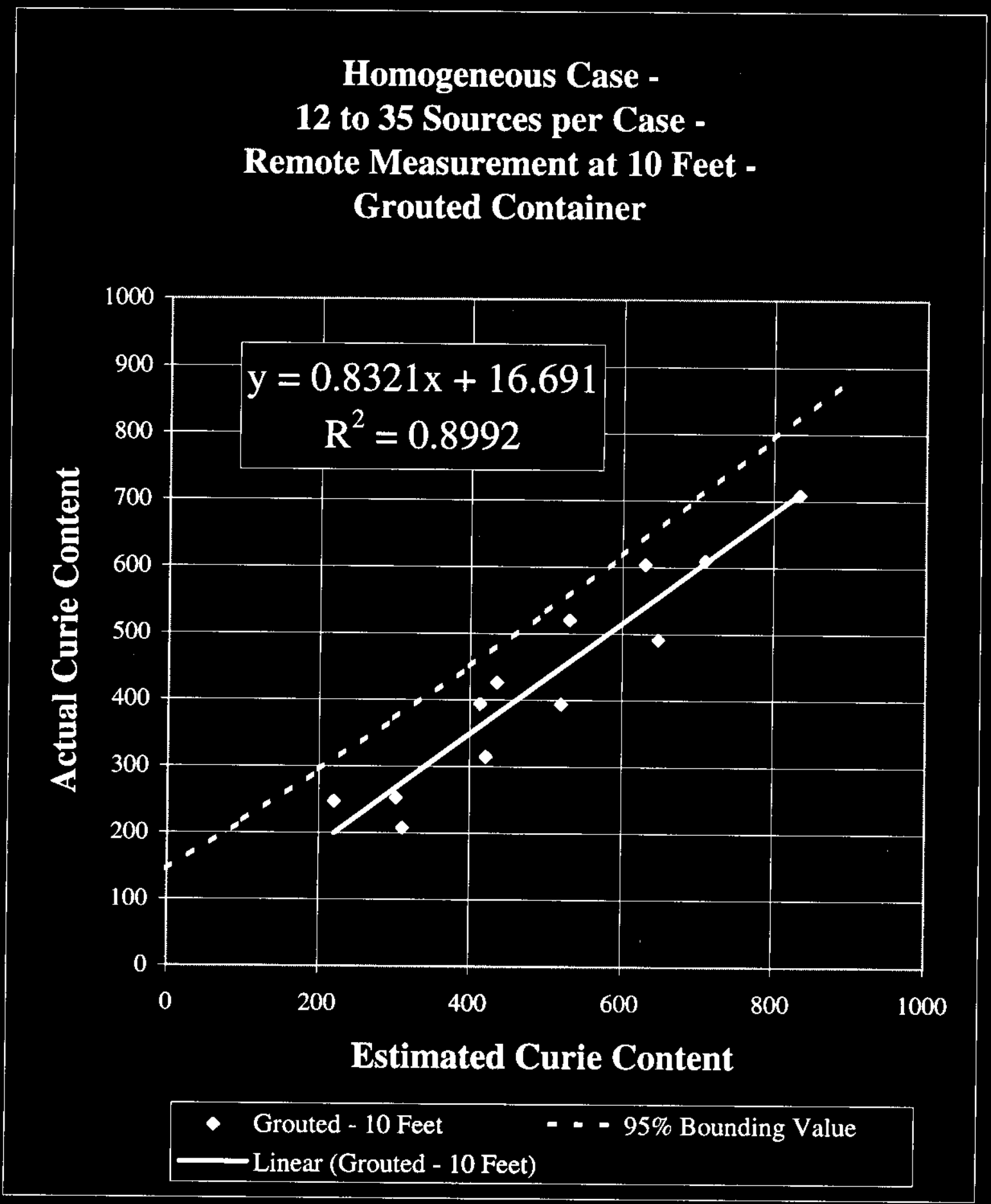

FIGURE 24 
HNF-4799, Rev 0

REVISED METHODOLOGY FOR DETERMINING CESIUM-137 CONTENT OF HN-200 GROUT CONTAINERS.

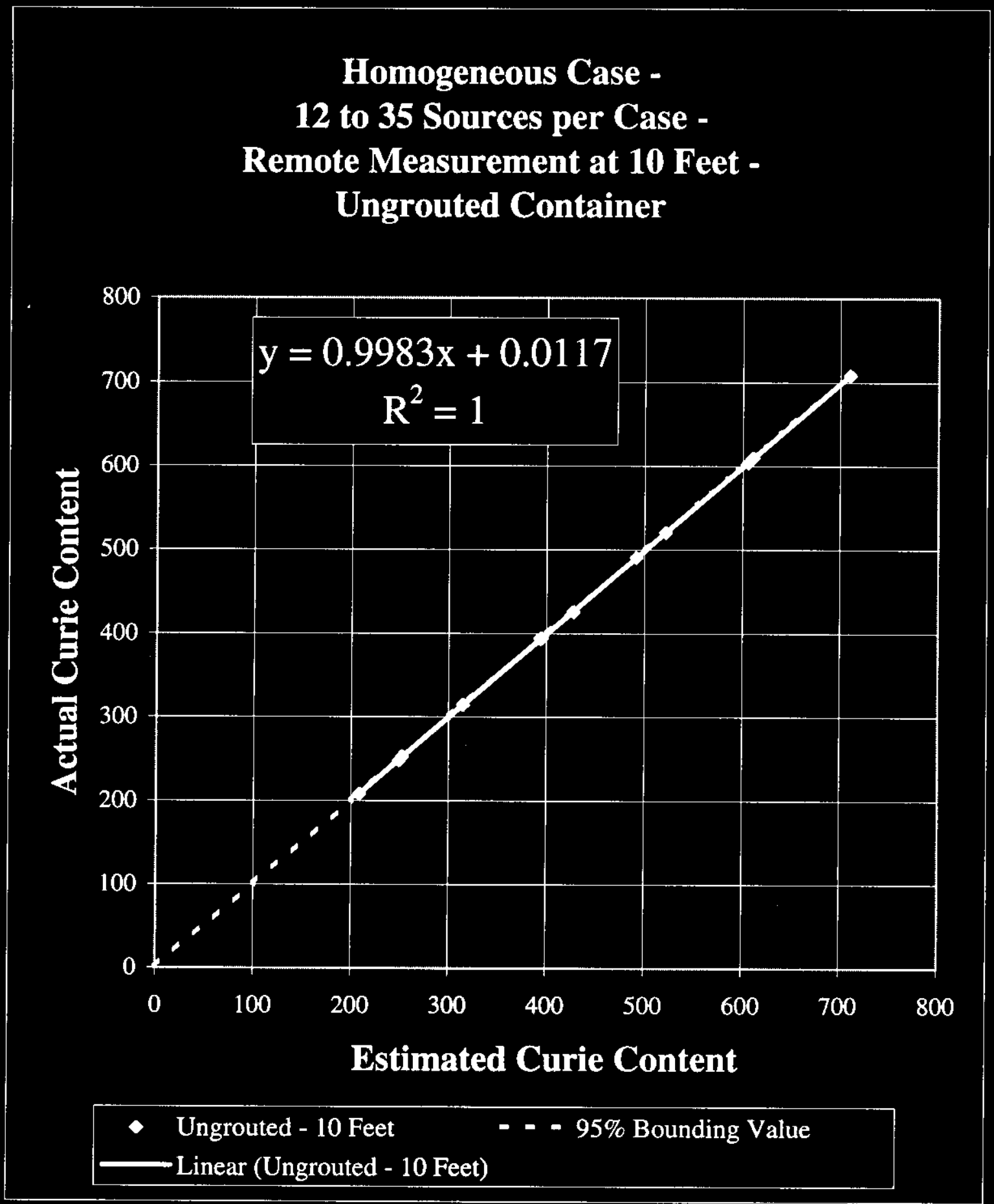

FIGURE 25 
HNF-4799, Rev 0 August 25, 1999

REVISED METHODOLOGY FOR DETERMINING CESIUM-137 CONTENT OF HN-200 GROUT CONTAINERS.

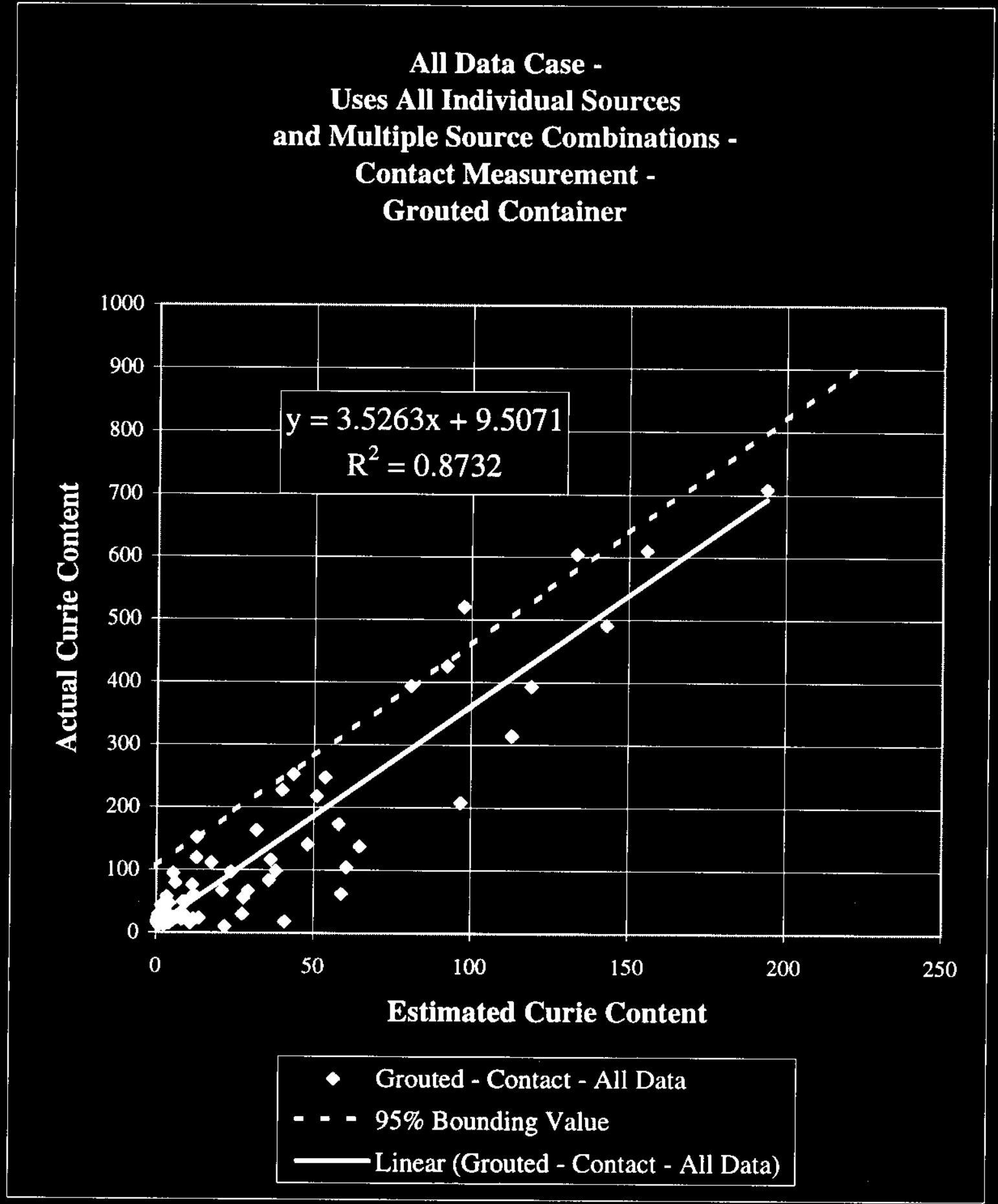

FIGURE 26 


\section{REVISED METHODOLOGY FOR DETERMINING CESIUM-137 CONTENT OF HN-200 GROUT CONTAINERS.}

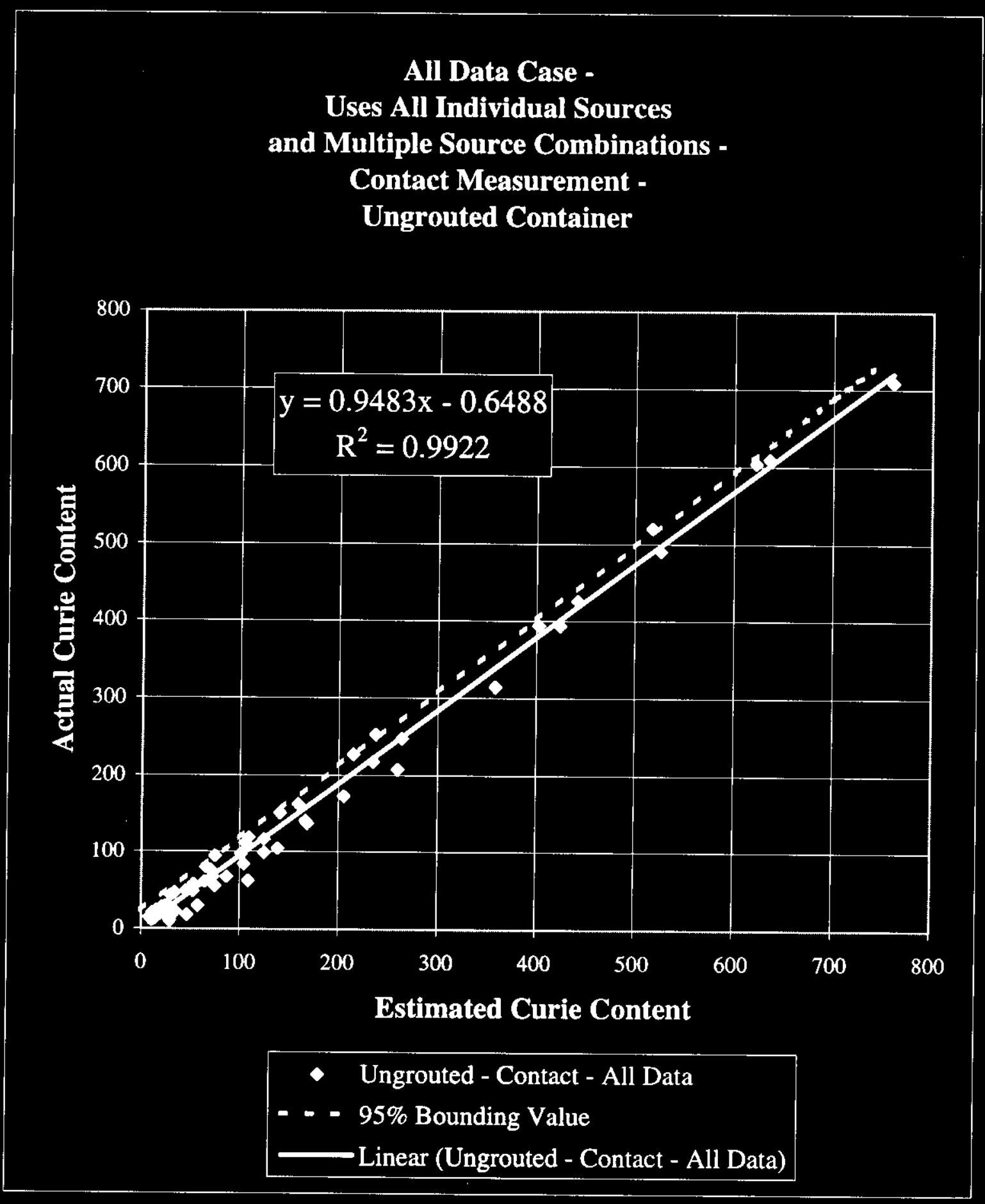




\section{REVISED METHODOLOGY FOR DETERMINING CESIUM-137 CONTENT OF HN-200 GROUT CONTAINERS. \\ TABLE 1 - MODEL FOR HN-200 GROUT CONTAINER FILLED WITH B CELL EQUIPMENT DUNNAGE AND SIFTED DEBRIS.}

\section{UNGROUTED CONTAINER WITH 2500 LBS NET WEIGHT IN WASTE}

\begin{tabular}{|c|c|c|c|c|c|c|}
\hline Shield & R-O (in) & R-I (in) & $\mathrm{A} \cdot \mathrm{O}(\mathrm{cm} 2)$ & A-I $(\mathrm{cm} 2)$ & Vol $(\mathrm{cm} 3)$ & Weight(lb) \\
\hline 1 & 22 & 21.75 & 47712.5838 & 47170.3954 & 30125.3459 & 519.56268 \\
\hline 2 & 19.5 & 19.25 & 42290.6993 & 41748.5109 & 26682.4492 & 460.18409 \\
\hline 3 & 17 & 16.75 & 36868.8148 & 36326.6263 & 23239.5526 & 400.8055 \\
\hline 4 & 14.5 & 14.25 & 31446.9303 & 30904.7418 & 19796.6559 & 341.42691 \\
\hline 5 & 12 & 11.75 & 26025.0457 & 25482.8573 & 16353.7592 & 282.04831 \\
\hline 6 & 9.5 & 9.25 & 20603.1612 & 20060.9728 & 12910.8625 & 222.66972 \\
\hline 7 & 7 & 6.75 & 15181.2767 & 14639.0882 & 9467.96586 & 163.29113 \\
\hline 8 & 4.75 & 4.5 & 10301.5806 & 9759.39215 & 6369.35885 & 109.8504 \\
\hline \multirow[t]{2}{*}{ Totals } & \multirow{2}{*}{\multicolumn{3}{|c|}{ Total Surface Area = }} & 226092.585 & 144945.95 & 2499.8387 \\
\hline & & & & 456522.677 & & \\
\hline
\end{tabular}

\begin{tabular}{|c|c|c|c|c|c|c|c|}
\hline & $\begin{array}{l}\text { Surface } \\
\text { Radius }\end{array}$ & $\begin{array}{l}\text { Source } \\
\text { Thickness }\end{array}$ & $\begin{array}{c}\text { Distance } \\
\text { to outer } \\
\text { wall }\end{array}$ & $\begin{array}{l}\text { Air Gap } \\
\text { to inner } \\
\text { wall }\end{array}$ & $\begin{array}{c}\text { Total } \\
\text { Steel } \\
\text { Thickness }\end{array}$ & Cesium & Barium \\
\hline 25-01 & 22 & 0.0001 & 0.9375 & 0.7992 & 0.1382 & 0.1045 & 0.0989 \\
\hline 25- 02 & 21.75 & 0.0001 & 1.1875 & 0.7992 & 0.3882 & 0.1033 & 0.0978 \\
\hline 25-03 & 19.5 & 0.0001 & 3.4375 & 3.0492 & 0.3882 & 0.0926 & 0.0876 \\
\hline 25-04 & 19.25 & 0.0001 & 3.6875 & 3.0492 & 0.6382 & 0.0914 & 0.0865 \\
\hline 25-05 & 17 & 0.0001 & 5.9375 & 5.2992 & 0.6382 & 0.0808 & 0.0764 \\
\hline 25-06 & 16.75 & 0.0001 & 6.1875 & 5.2992 & 0.8882 & 0.0796 & 0.0753 \\
\hline 25-07 & 14.5 & 0.0001 & 8.4375 & 7.5492 & 0.8882 & 0.0689 & 0.0652 \\
\hline 25-08 & 14.25 & 0.0001 & 8.6875 & 7.5492 & 1.1382 & 0.0677 & 0.0640 \\
\hline 25-09 & 12 & 0.0001 & 10.9375 & 9.7992 & 1.1382 & 0.0570 & 0.0539 \\
\hline 25-10 & 11.75 & 0.0001 & 11.1875 & 9.7992 & 1.3882 & 0.0558 & 0.0528 \\
\hline $25-11$ & 9.5 & 0.0001 & 13.4375 & 12.0492 & 1.3882 & 0.0451 & 0.0427 \\
\hline $25-12$ & 9.25 & 0.0001 & 13.6875 & 12.0492 & 1.6382 & 0.0439 & 0.0416 \\
\hline $25-13$ & 7 & 0.0001 & 15.9375 & 14.2992 & 1.6382 & 0.0333 & 0.0315 \\
\hline 25-14 & 6.75 & 0.0001 & 16.1875 & 14.2992 & 1.8882 & 0.0321 & 0.0303 \\
\hline 25-15 & 4.75 & 0.0001 & 18.1875 & 16.2992 & 1.8882 & 0.0226 & 0.0213 \\
\hline \multirow[t]{2}{*}{ 25-16 } & 4.5 & 0.0001 & 18.4375 & 16.2992 & 2.1382 & 0.0214 & 0.0202 \\
\hline & & & & & Total & 1.0000 & 0.9461 \\
\hline
\end{tabular}

Average Activity per 100 square $\mathrm{cm}=219.04717$ Cs-137 $207.23396 \quad \mathrm{Ba}-137 \mathrm{~m}$ 

GROUT CONTAINERS.

TABLE 2 - CALCULATE CONVERSION FACTORS FOR CONTACT, 5 FEET, AND 10 FEET FOR AN UNGROUTED HN-200 GROUT CONTAINER WITH 2500 LBS (NET) OF WASTE.

\begin{tabular}{|c|c|c|c|}
\hline Surface & Contact & 5 feet & 10 feet \\
\hline 25-01 & $1.130 \mathrm{E}-01$ & $7.080 \mathrm{E}-03$ & $2.328 \mathrm{E}-03$ \\
\hline 25-02 & $7.130 \mathrm{E}-02$ & $5.551 \mathrm{E}-03$ & $1.831 \mathrm{E}-03$ \\
\hline $25-03$ & $6.502 \mathrm{E}-02$ & $5.328 \mathrm{E}-03$ & $1.770 \mathrm{E}-03$ \\
\hline 25-04 & $4.570 \mathrm{E}-02$ & $4.185 E-03$ & $1.396 \mathrm{E}-03$ \\
\hline $25-05$ & 4.074E-02 & $3.876 \mathrm{E}-03$ & $1.300 \mathrm{E}-03$ \\
\hline $25-06$ & $2.947 \mathrm{E}-02$ & $3.017 \mathrm{E}-03$ & $1.016 \mathrm{E}-03$ \\
\hline $25-07$ & $2.591 \mathrm{E}-02$ & $2.724 \mathrm{E}-03$ & $9.223 E-04$ \\
\hline 25-08 & $1.896 \mathrm{E}-02$ & $2.103 \mathrm{E}-03$ & $7.146 \mathrm{E}-04$ \\
\hline 25-09 & $1.628 \mathrm{E}-02$ & $1.840 \mathrm{E}-03$ & $6.278 \mathrm{E}-04$ \\
\hline $25-10$ & $1.199 \mathrm{E}-02$ & $1.414 \mathrm{E}-03$ & $4.840 \mathrm{E}-04$ \\
\hline $25-11$ & 9.894E-03 & $1.182 E-03$ & $4.061 E-04$ \\
\hline 25-12 & $7.284 E-03$ & $9.031 \mathrm{E}-04$ & $3.112 \mathrm{E}-04$ \\
\hline $25-13$ & $5.626 \mathrm{E}-03$ & $7.036 \mathrm{E}-04$ & $2.430 \mathrm{E}-04$ \\
\hline $25-14$ & $4.106 \mathrm{E}-03$ & $5.309 \mathrm{E}-04$ & $1.840 \mathrm{E}-04$ \\
\hline $25-15$ & $2.936 \mathrm{E}-03$ & $3.815 \mathrm{E}-04$ & $1.324 \mathrm{E}-04$ \\
\hline $25-16$ & $2.100 \mathrm{E}-03$ & $2.813 \mathrm{E}-04$ & $9.794 \mathrm{E}-05$ \\
\hline ONVERSION FACTOR & $4.703 \mathrm{E}-01$ & $4.110 \mathrm{E}-02$ & $1.376 \mathrm{E}-02$ \\
\hline
\end{tabular}




\title{
REVISED METHODOLOGY FOR DETERMINING CESIUM-137 CONTENT OF HN-200 GROUT CONTAINERS.
}

\author{
TABLE 3 - MODEL FOR HN-200 GROUT CONTAINER FILLED WITH B CELL \\ EQUIPMENT DUNNAGE AND SIFTED DEBRIS.
}

\section{UNGROUTED CONTAINER WITH 2200 LBS NET WEIGHT IN WASTE}

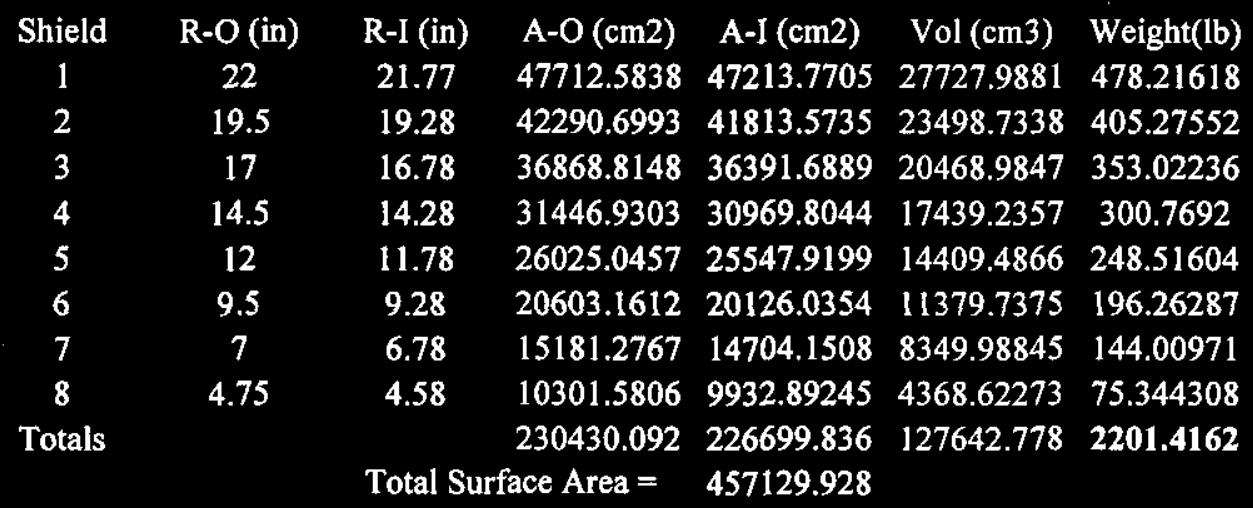

\begin{tabular}{|c|c|c|c|c|c|c|c|}
\hline & $\begin{array}{l}\text { Surface } \\
\text { Radius }\end{array}$ & $\begin{array}{l}\text { Source } \\
\text { Thickness }\end{array}$ & $\begin{array}{c}\text { Distance } \\
\text { to outer } \\
\text { wall }\end{array}$ & $\begin{array}{l}\text { Air Gap } \\
\text { to inner } \\
\text { wall }\end{array}$ & $\begin{array}{c}\text { Total } \\
\text { Steel } \\
\text { Thickness }\end{array}$ & Cesium & Barium \\
\hline 22-01 & 22 & 0.0001 & 0.9375 & 0.7992 & 0.1382 & 0.1044 & 0.0987 \\
\hline 22-02 & 21.77 & 0.0001 & 1.1675 & 0.7992 & 0.3682 & 0.1033 & 0.0977 \\
\hline $22-03$ & 19.5 & 0.0001 & 3.4375 & 3.0692 & 0.3682 & 0.0925 & 0.0875 \\
\hline 22-04 & 19.28 & 0.0001 & 3.6575 & 3.0692 & 0.5882 & 0.0915 & 0.0865 \\
\hline 22-05 & 17 & 0.0001 & 5.9375 & 5.3492 & 0.5882 & 0.0807 & 0.0763 \\
\hline $22-06$ & 16.78 & 0.0001 & 6.1575 & 5.3492 & 0.8082 & 0.0796 & 0.0753 \\
\hline 22-07 & 14.5 & 0.0001 & 8.4375 & 7.6292 & 0.8082 & 0.0688 & 0.0651 \\
\hline 22-08 & 14.28 & 0.0001 & 8.6575 & 7.6292 & 1.0282 & 0.0677 & 0.0641 \\
\hline 22-09 & 12 & 0.0001 & 10.9375 & 9.9092 & 1.0282 & 0.0569 & 0.0539 \\
\hline $22-10$ & 11.78 & 0.0001 & 11.1575 & 9.9092 & 1.2482 & 0.0559 & 0.0529 \\
\hline $22-11$ & 9.5 & 0.0001 & 13.4375 & 12.1892 & 1.2482 & 0.0451 & 0.0426 \\
\hline $22-12$ & 9.28 & 0.0001 & 13.6575 & 12.1892 & 1.4682 & 0.0440 & 0.0417 \\
\hline $22-13$ & 7 & 0.0001 & 15.9375 & 14.4692 & 1.4682 & 0.0332 & 0.0314 \\
\hline 22-14 & 6.78 & 0.0001 & 16.1575 & 14.4692 & 1.6882 & 0.0322 & 0.0304 \\
\hline $22-15$ & 4.75 & 0.0001 & 18.1875 & 16.4992 & 1.6882 & 0.0225 & 0.0213 \\
\hline \multirow[t]{2}{*}{$22-16$} & 4.58 & 0.0001 & 18.3575 & 16.4992 & 1.8582 & 0.0217 & 0.0206 \\
\hline & & & & & Total & 1.0000 & 0.9461 \\
\hline
\end{tabular}

Average Activity per 100 square $\mathrm{cm}=218.75619$ Cs-137 206.95867 Ba-137m 


\section{REVISED METHODOLOGY FOR DETERMINING CESIUM-137 CONTENT OF HN-200 GROUT CONTAINERS.}

TABLE 4 - CALCULATE CONVERSION FACTORS FOR CONTACT, 5 FEET, AND 10 FEET FOR AN UNGROUTED HN-200 GROUT CONTAINER WITH 2200 LBS (NET) OF WASTE.

\begin{tabular}{|c|c|c|c|}
\hline Surface & Contact & 5 feet & 10 feet \\
\hline 22-01 & $1.130 \mathrm{E}-01$ & $7.080 \mathrm{E}-03$ & $2.328 \mathrm{E}-03$ \\
\hline 22-02 & $7.388 \mathrm{E}-02$ & $5.669 \mathrm{E}-03$ & $1.870 \mathrm{E}-03$ \\
\hline $22-03$ & $6.672 \mathrm{E}-02$ & $5.420 \mathrm{E}-03$ & $1.800 \mathrm{E}-03$ \\
\hline 22-04 & 4.899E-02 & $4.396 \mathrm{E}-03$ & $1.465 \mathrm{E}-03$ \\
\hline $22-05$ & $4.322 \mathrm{E}-02$ & $4.049 \mathrm{E}-03$ & $1.358 \mathrm{E}-03$ \\
\hline $22-06$ & $3.260 \mathrm{E}-02$ & 3.267E-03 & $1.099 \mathrm{E}-03$ \\
\hline 22-07 & $2.839 \mathrm{E}-02$ & $2.934 \mathrm{E}-03$ & $9.923 \mathrm{E}-04$ \\
\hline 22-08 & $2.163 \mathrm{E}-02$ & $2.347 \mathrm{E}-03$ & $7.962 \mathrm{E}-04$ \\
\hline 22-09 & $1.842 \mathrm{E}-02$ & $2.043 \mathrm{E}-03$ & $6.962 \mathrm{E}-04$ \\
\hline $22-10$ & $1.408 \mathrm{E}-02$ & $1.624 \mathrm{E}-03$ & $5.549 \mathrm{E}-04$ \\
\hline 22-11 & $1.154 \mathrm{E}-02$ & $1.352 \mathrm{E}-03$ & 4.637E-04 \\
\hline 22-12 & $8.826 \mathrm{E}-03$ & $1.068 \mathrm{E}-03$ & 3.673E-04 \\
\hline 22-13 & $6.776 \mathrm{E}-03$ & 8.287E-04 & $2.857 \mathrm{E}-04$ \\
\hline 22-14 & $5.140 \mathrm{E}-03$ & $6.476 \mathrm{E}-04$ & $2.239 \mathrm{E}-04$ \\
\hline $22-15$ & $3.661 \mathrm{E}-03$ & $4.639 \mathrm{E}-04$ & $1.606 \mathrm{E}-04$ \\
\hline $22-16$ & $2.879 \mathrm{E}-03$ & $3.728 \mathrm{E}-04$ & $1.293 \mathrm{E}-04$ \\
\hline ONVERSION FACTOR & $4.998 \mathrm{E}-01$ & 4.356 -02 & $1.459 \mathrm{E}-02$ \\
\hline
\end{tabular}




\title{
REVISED METHODOLOGY FOR DETERMINING CESIUM-137 CONTENT OF HN-200 GROUT CONTAINERS.
}

\author{
TABLE 5 - MODEL FOR HN-200 GROUT CONTAINER FILLED WITH B CELL \\ EQUIPMENT DUNNAGE AND SIFTED DEBRIS.
}

\section{UNGROUTED CONTAINER WITH 1900 LBS NET WEIGHT IN WASTE}

\begin{tabular}{|c|c|c|c|c|c|c|}
\hline Shield & R-O (in) & R-I (in) & $\mathrm{A}-\mathrm{O}(\mathrm{cm} 2)$ & $A-I(\mathrm{~cm} 2)$ & $\mathrm{Vol}(\mathrm{cm} 3)$ & Weight(lb) \\
\hline 1 & 22 & 21.8 & 47712.5838 & 47278.8331 & 24127.8199 & 416.12518 \\
\hline 2 & 19.5 & 19.31 & 42290.6993 & 41878.6361 & 20310.0606 & 350.28144 \\
\hline 3 & 17 & 16.81 & 36868.8148 & 36456.7516 & 17693.4592 & 305.15371 \\
\hline 4 & 14.5 & 14.31 & 31446.9303 & 31034.867 & 15076.8577 & 260.02598 \\
\hline 5 & 12 & 11.81 & 26025.0457 & 25612.9825 & 12460.2562 & 214.89825 \\
\hline 6 & 9.5 & 9.31 & 20603.1612 & 20191.098 & 9843.65474 & 169.77052 \\
\hline 7 & 7 & 6.81 & 15181.2767 & 14769.2135 & 7227.05327 & 124.64279 \\
\hline 8 & 4.75 & 4.61 & 10301.5806 & 9997.95507 & 3609.25744 & 62.247766 \\
\hline \multirow[t]{2}{*}{ Totals } & & & 230430.092 & 227220.337 & 110348.419 & 1903.1456 \\
\hline & & \multicolumn{2}{|c|}{ Total Surface Area $=$} & 457650.429 & & \\
\hline
\end{tabular}

\begin{tabular}{|c|c|c|c|c|c|c|c|}
\hline & $\begin{array}{c}\text { Surface } \\
\text { Radius }\end{array}$ & $\begin{array}{l}\text { Source } \\
\text { Thickness }\end{array}$ & $\begin{array}{c}\text { Distance } \\
\text { to outer } \\
\text { wall }\end{array}$ & $\begin{array}{c}\text { Air Gap } \\
\text { to inner } \\
\text { wall }\end{array}$ & $\begin{array}{c}\text { Total } \\
\text { Steel } \\
\text { Thickness }\end{array}$ & Cesium & Barium \\
\hline 19-01 & 22 & 0.0001 & 0.9375 & 0.7992 & 0.1382 & 0.1043 & 0.0986 \\
\hline 19-02 & 21.8 & 0.0001 & 1.1375 & 0.7992 & 0.3382 & 0.1033 & 0.0977 \\
\hline $19-03$ & 19.5 & 0.0001 & 3.4375 & 3.0992 & 0.3382 & 0.0924 & 0.0874 \\
\hline 19-04 & 19.31 & 0.0001 & 3.6275 & 3.0992 & 0.5282 & 0.0915 & 0.0866 \\
\hline $19-05$ & 17 & 0.0001 & 5.9375 & 5.4092 & 0.5282 & 0.0806 & 0.0762 \\
\hline 19-06 & 16.81 & 0.0001 & 6.1275 & 5.4092 & 0.7182 & 0.0797 & 0.0754 \\
\hline 19.07 & 14.5 & 0.0001 & 8.4375 & 7.7192 & 0.7182 & 0.0687 & 0.0650 \\
\hline $19-08$ & 14.31 & 0.0001 & 8.6275 & 7.7192 & 0.9082 & 0.0678 & 0.0642 \\
\hline 19-09 & 12 & 0.0001 & 10.9375 & 10.0292 & 0.9082 & 0.0569 & 0.0538 \\
\hline $19-10$ & 11.81 & 0.0001 & 11.1275 & 10.0292 & 1.0982 & 0.0560 & 0.0529 \\
\hline $19-11$ & 9.5 & 0.0001 & 13.4375 & 12.3392 & 1.0982 & 0.0450 & 0.0426 \\
\hline 19-12 & 9.31 & 0.0001 & 13.6275 & 12.3392 & 1.2882 & 0.0441 & 0.0417 \\
\hline $19-13$ & 7 & 0.0001 & 15.9375 & 14.6492 & 1.2882 & 0.0332 & 0.0314 \\
\hline $19-14$ & 6.81 & 0.0001 & 16.1275 & 14.6492 & 1.4782 & 0.0323 & 0.0305 \\
\hline $19-15$ & 4.75 & 0.0001 & 18.1875 & 16.7092 & 1.4782 & 0.0225 & 0.0213 \\
\hline $19-16$ & 4.61 & 0.0001 & 18.3275 & 16.7092 & 1.6182 & 0.0218 & 0.0207 \\
\hline & & & & & Total & 1.0000 & 0.9461 \\
\hline
\end{tabular}

Average Activity per 100 square $\mathrm{cm}=218.50739 \quad$ Cs-137 206.72328 Ba-137m 


\section{REVISED METHODOLOGY FOR DETERMINING CESIUM-137 CONTENT OF HN-200 GROUT CONTAINERS.}

TABLE 6 - CALCULATE CONVERSION FACTORS FOR CONTACT, 5 FEET, AND 10 FEET FOR AN UNGROUTED HN-200 GROUT CONTAINER WITH 1900 LBS (NET) OF WASTE.

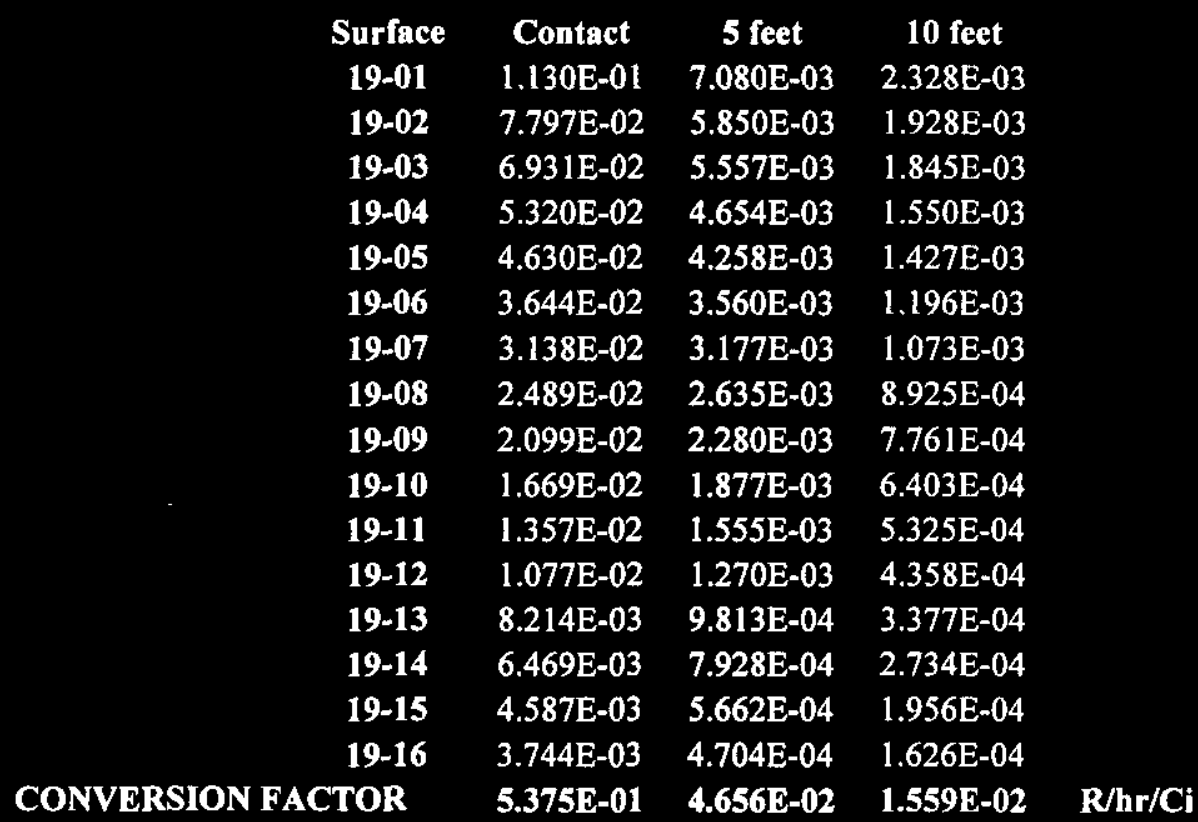

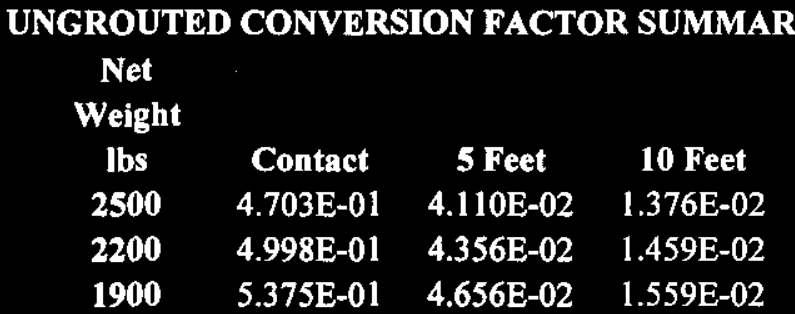


HNF-4799, Rev 0

August 25, 1999

REVISED METHODOLOGY FOR DETERMINING CESIUM-137 CONTENT OF HN-200 GROUT CONTAINERS.

FIGURE 1

\section{Conversion Factor \\ Variance with Net Weight of Waste \\ for Contact Measurements \\ Ungrouted Container}

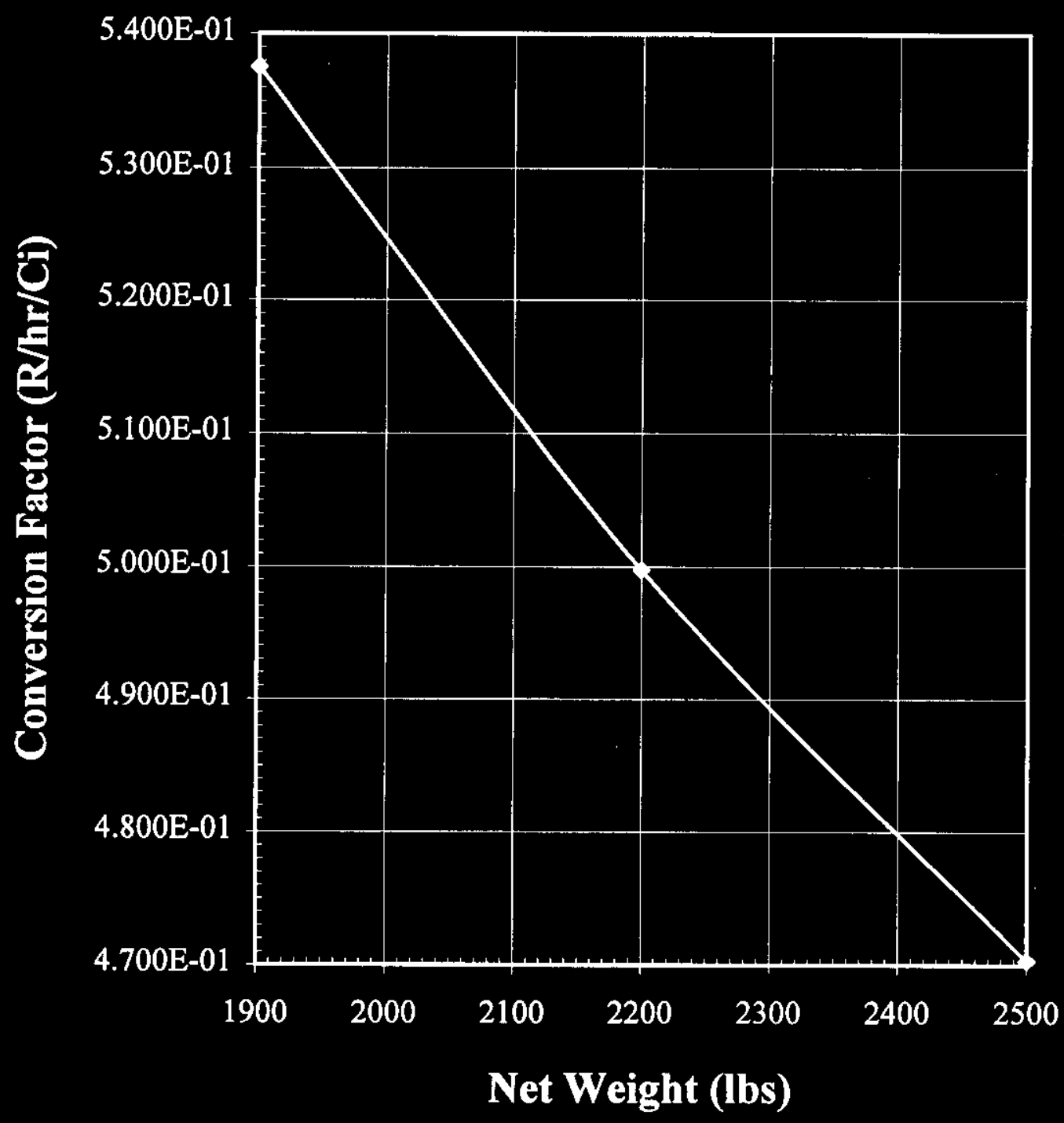


REVISED METHODOLOGY FOR DETERMINING CESIUM-137 CONTENT OF HN-200 GROUT CONTAINERS.

FIGURE 2

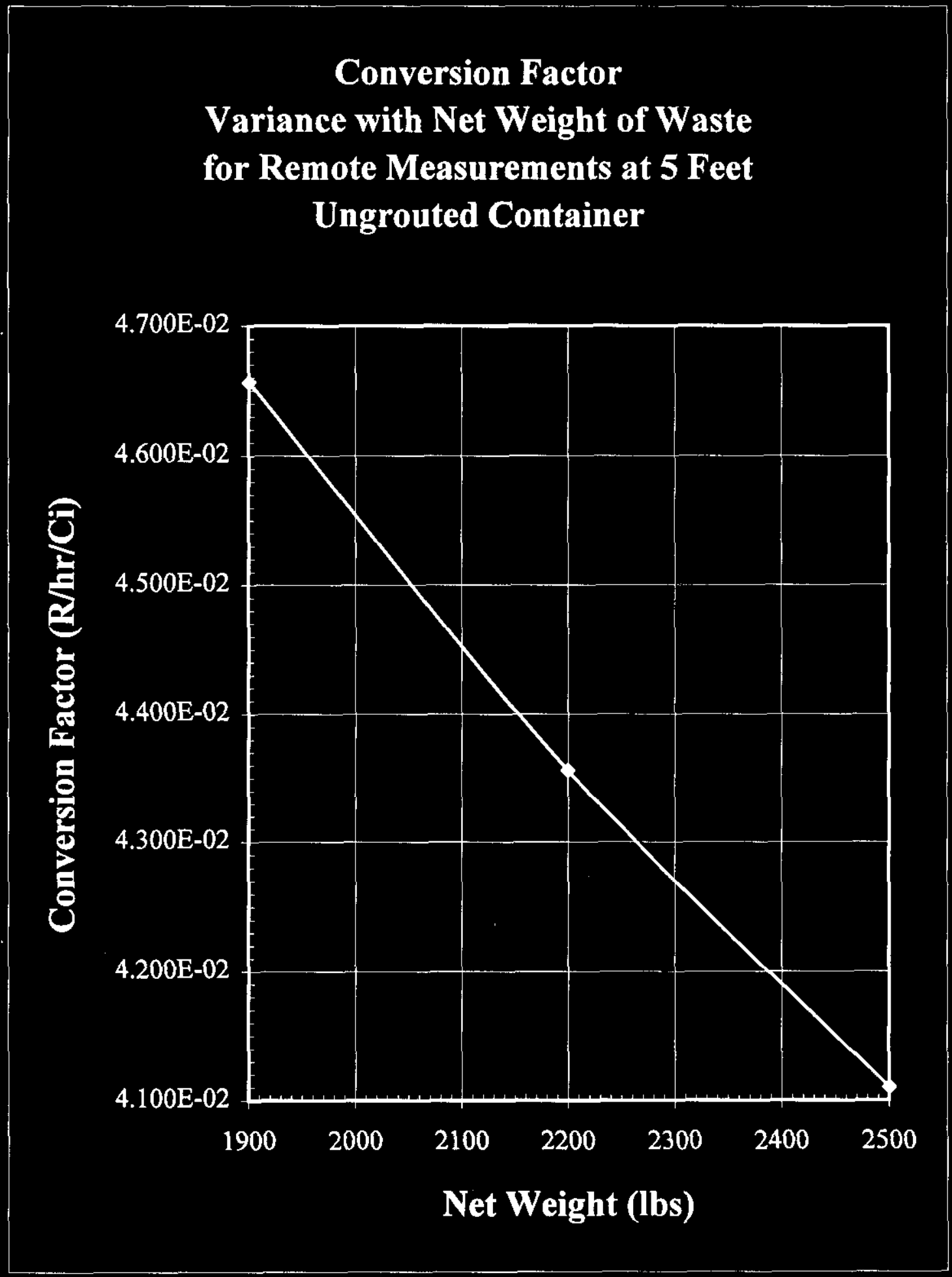


HNF-4799, Rev 0

August 25, 1999

REVISED METHODOLOGY FOR DETERMINING CESIUM-137 CONTENT OF HN-200 GROUT CONTAINERS.

FIGURE 3

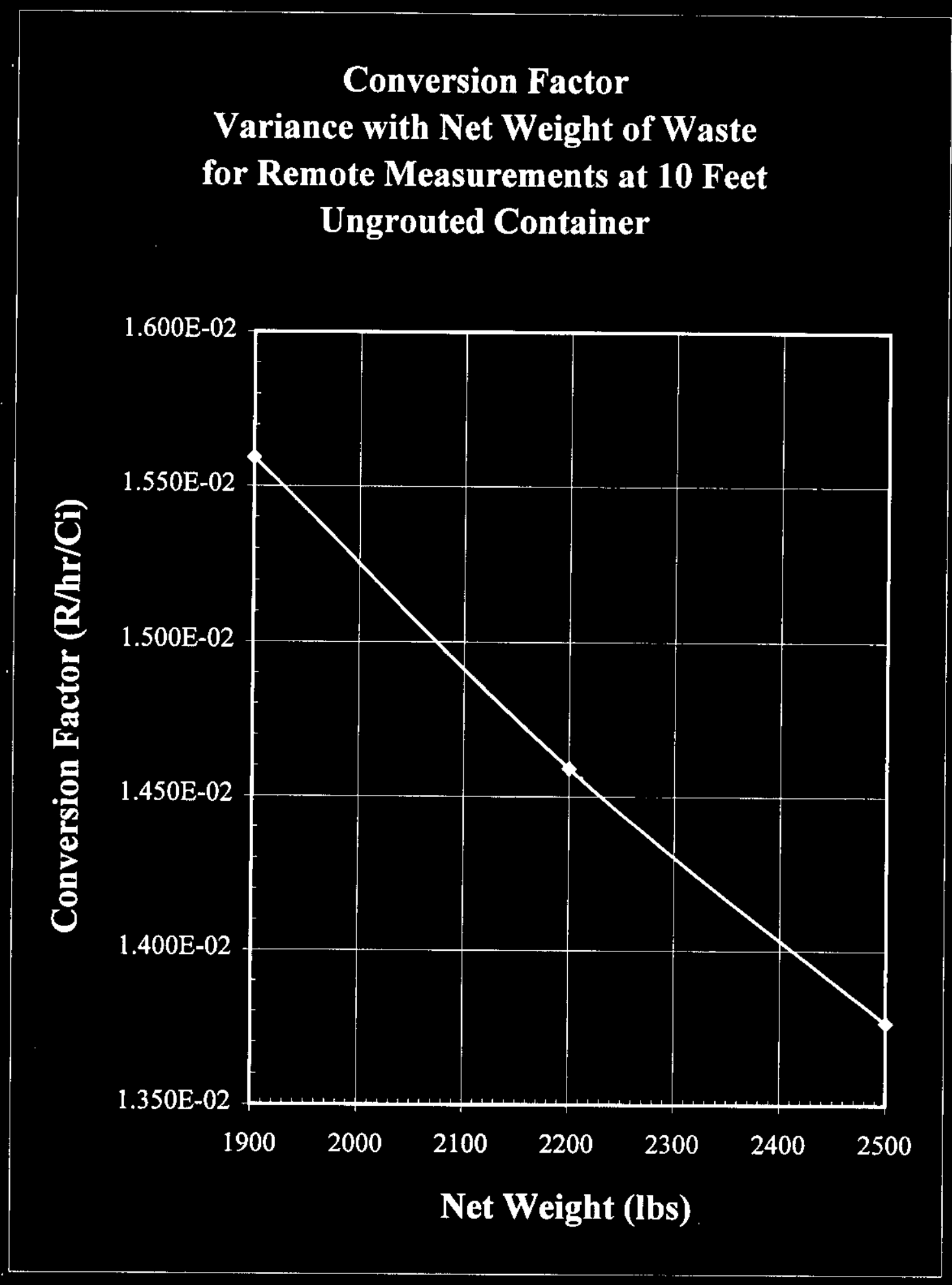




\title{
REVISED METHODOLOGY FOR DETERMINING CESIUM-137 CONTENT OF HN-200 GROUT CONTAINERS.
}

\author{
TABLE 7 - MODEL FOR HN-200 GROUT CONTAINER FILLED WITH B CELL \\ EQUIPMENT DUNNAGE AND SIFTED DEBRIS.
}

\section{GROUTED CONTAINER WITH 2500 LBS NET WEIGHT IN WASTE}

$\begin{array}{ccccccc}\text { Shield } & \text { R-O (in) } & \text { R-I (in) } & \text { A-O (cm2) } & \text { A-I (cm2) } & \text { Vol (cm3) } & \text { Weight(lb) } \\ 1 & 22 & 21.75 & 47712.5838 & 47170.3954 & 30125.3459 & 519.56268 \\ 2 & 19.5 & 19.25 & 42290.6993 & 41748.5109 & 26682.4492 & 460.18409 \\ 3 & 17 & 16.75 & 36868.8148 & 36326.6263 & 23239.5526 & 400.8055 \\ 4 & 14.5 & 14.25 & 31446.9303 & 30904.7418 & 19796.6559 & 341.42691 \\ 5 & 12 & 11.75 & 26025.0457 & 25482.8573 & 16353.7592 & 282.04831 \\ 6 & 9.5 & 9.25 & 20603.1612 & 20060.9728 & 12910.8625 & 222.66972 \\ 7 & 7 & 6.75 & 15181.2767 & 14639.0882 & 9467.96586 & 163.29113 \\ 8 & 4.75 & 4.5 & 10301.5806 & 9759.39215 & 6369.35885 & 109.8504 \\ \text { Totals } & & & 230430.092 & 226092.585 & 144945.95 & 2499.8387 \\ & & \text { Total Surface Area }= & 456522.677 & & \end{array}$

\begin{tabular}{|c|c|c|c|c|c|c|c|}
\hline & $\begin{array}{l}\text { Surface } \\
\text { Radius }\end{array}$ & $\begin{array}{l}\text { Source } \\
\text { Thickness }\end{array}$ & $\begin{array}{c}\text { Distance } \\
\text { to outer } \\
\text { wall }\end{array}$ & $\begin{array}{c}\text { Total } \\
\text { Grout } \\
\text { Thickness }\end{array}$ & $\begin{array}{c}\text { Total } \\
\text { Steel } \\
\text { Thickness }\end{array}$ & Cesium & Barium \\
\hline 25-01 & 22 & 0.0001 & 0.9375 & 0.7992 & 0.1382 & 0.1045 & 0.0989 \\
\hline 25-02 & 21.75 & 0.0001 & 1.1875 & 0.7992 & 0.3882 & 0.1033 & 0.0978 \\
\hline 25-03 & 19.5 & 0.0001 & 3.4375 & 3.0492 & 0.3882 & 0.0926 & 0.0876 \\
\hline 25-04 & 19.25 & 0.0001 & 3.6875 & 3.0492 & 0.6382 & 0.0914 & 0.0865 \\
\hline $25-05$ & 17 & 0.0001 & 5.9375 & 5.2992 & 0.6382 & 0.0808 & 0.0764 \\
\hline $25-06$ & 16.75 & 0.0001 & 6.1875 & 5.2992 & 0.8882 & 0.0796 & 0.0753 \\
\hline 25-07 & 14.5 & 0.0001 & 8.4375 & 7.5492 & 0.8882 & 0.0689 & 0.0652 \\
\hline 25-08 & 14.25 & 0.0001 & 8.6875 & 7.5492 & 1.1382 & 0.0677 & 0.0640 \\
\hline 25-09 & 12 & 0.0001 & 10.9375 & 9.7992 & 1.1382 & 0.0570 & 0.0539 \\
\hline $25-10$ & 11.75 & 0.0001 & 11.1875 & 9.7992 & 1.3882 & 0.0558 & 0.0528 \\
\hline 25-11 & 9.5 & 0.0001 & 13.4375 & 12.0492 & 1.3882 & 0.0451 & 0.0427 \\
\hline 25-12 & 9.25 & 0.0001 & 13.6875 & 12.0492 & 1.6382 & 0.0439 & 0.0416 \\
\hline $25-13$ & 7 & 0.0001 & 15.9375 & 14.2992 & 1.6382 & 0.0333 & 0.0315 \\
\hline $25-14$ & 6.75 & 0.0001 & 16.1875 & 14.2992 & 1.8882 & 0.0321 & 0.0303 \\
\hline 25-15 & 4.75 & 0.0001 & 18.1875 & 16.2992 & 1.8882 & 0.0226 & 0.0213 \\
\hline $25-16$ & 4.5 & 0.0001 & 18.4375 & 16.2992 & 2.1382 & 0.0214 & 0.0202 \\
\hline & & & & & Total & 1.0000 & 0.9461 \\
\hline
\end{tabular}

Average Activity per 100 square $\mathrm{cm}=219.04717$ Cs-137 $207.23396 \quad \mathrm{Ba}-137 \mathrm{~m}$ 


\section{REVISED METHODOLOGY FOR DETERMINING CESIUM-137 CONTENT OF HN-200 GROUT CONTAINERS.}

TABLE 8 - CALCULATE CONVERSION FACTORS FOR CONTACT, 5 FEET, AND 10 FEET FOR A GROUTED HN-200 GROUT CONTAINER WITH 2500 LBS (NET) OF WASTE.

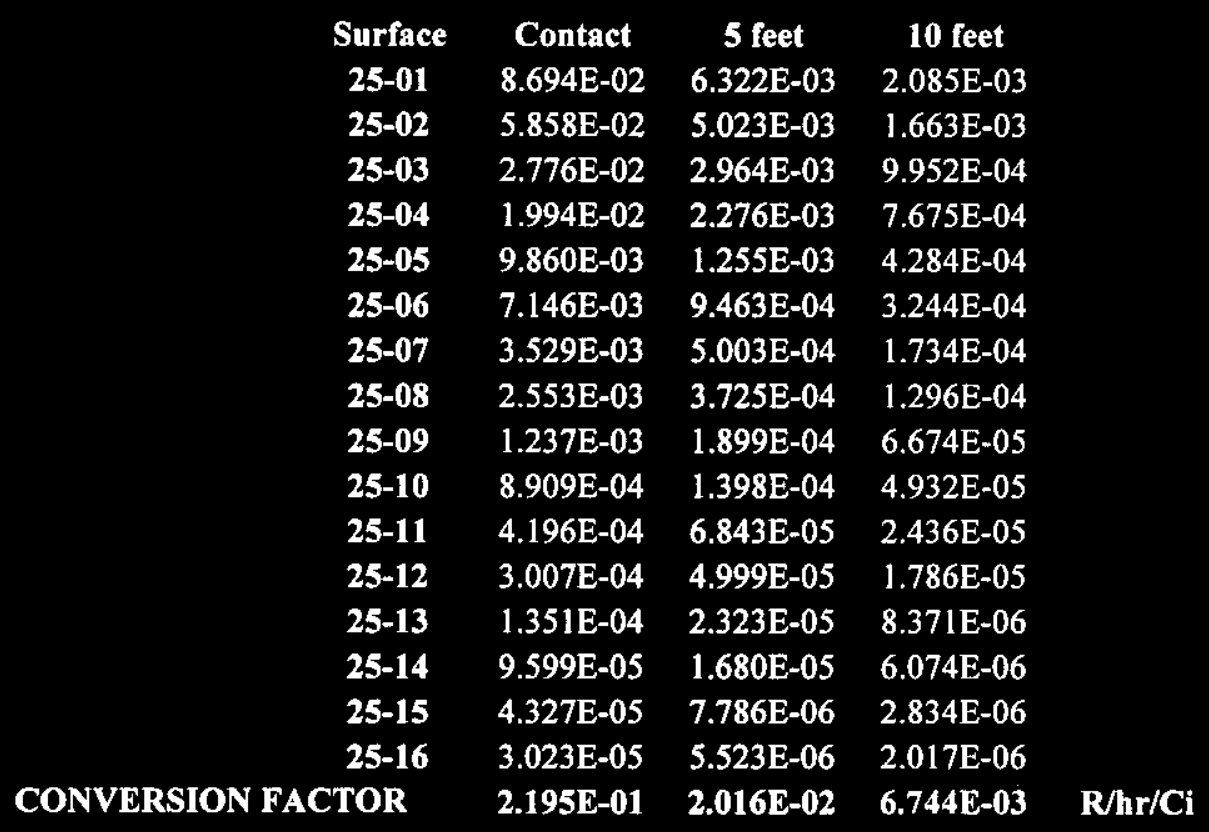




\section{REVISED METHODOLOGY FOR DETERMINING CESIUM-137 CONTENT OF HN-200 GROUT CONTAINERS.}

\section{TABLE 9 - MODEL FOR HN-200 GROUT CONTAINER FILLED WITH B CELL EQUIPMENT DUNNAGE AND SIFTED DEBRIS.}

\section{GROUTED CONTAINER WITH 2200 LBS NET WEIGHT IN WASTE}

\begin{tabular}{|c|c|c|c|c|c|c|}
\hline Shield & $\mathrm{R}-\mathrm{O}$ (in) & R-I (in) & $\mathrm{A}-\mathrm{O}(\mathrm{cm} 2)$ & $A-I(\mathrm{~cm} 2)$ & Vol (cm3) & Weight(lb) \\
\hline 1 & 22 & 21.77 & 47712.5838 & 47213.7705 & 27727.9881 & 478.21618 \\
\hline 2 & 19.5 & 19.28 & 42290.6993 & 41813.5735 & 23498.7338 & 405.27552 \\
\hline 3 & 17 & 16.78 & 36868.8148 & 36391.6889 & 20468.9847 & 353.02236 \\
\hline 4 & 14.5 & 14.28 & 31446.9303 & 30969.8044 & 17439.2357 & 300.7692 \\
\hline 5 & 12 & 11.78 & 26025.0457 & 25547.9199 & 14409.4866 & 248.51604 \\
\hline 6 & 9.5 & 9.28 & 20603.1612 & 20126.0354 & 11379.7375 & 196.26287 \\
\hline 7 & 7 & 6.78 & 15181.2767 & 14704.1508 & 8349.98845 & 144.00971 \\
\hline 8 & 4.75 & 4.58 & 10301.5806 & 9932.89245 & 4368.62273 & 75.344308 \\
\hline Totals & & & 230430.092 & 226699.836 & 127642.778 & 2201.4162 \\
\hline & & \multicolumn{2}{|c|}{ Total Surface Area = } & 457129.928 & & \\
\hline
\end{tabular}

\begin{tabular}{|c|c|c|c|c|c|c|c|}
\hline & $\begin{array}{l}\text { Surface } \\
\text { Radius }\end{array}$ & $\begin{array}{l}\text { Source } \\
\text { Thickness }\end{array}$ & $\begin{array}{c}\text { Distance } \\
\text { to outer } \\
\text { wall }\end{array}$ & $\begin{array}{c}\text { Total } \\
\text { Grout } \\
\text { Thickness }\end{array}$ & $\begin{array}{c}\text { Total } \\
\text { Steel } \\
\text { Thickness }\end{array}$ & Cesium & Barium \\
\hline 22-01 & 22 & 0.0001 & 0.9375 & 0.7992 & 0.1382 & 0.1044 & 0.0987 \\
\hline 22-02 & 21.77 & 0.0001 & 1.1675 & 0.7992 & 0.3682 & 0.1033 & 0.0977 \\
\hline 22-03 & 19.5 & 0.0001 & 3.4375 & 3.0692 & 0.3682 & 0.0925 & 0.0875 \\
\hline 22-04 & 19.28 & 0.0001 & 3.6575 & 3.0692 & 0.5882 & 0.0915 & 0.0865 \\
\hline 22-05 & 17 & 0.0001 & 5.9375 & 5.3492 & 0.5882 & 0.0807 & 0.0763 \\
\hline 22-06 & 16.78 & 0.0001 & 6.1575 & 5.3492 & 0.8082 & 0.0796 & 0.0753 \\
\hline 22-07 & 14.5 & 0.0001 & 8.4375 & 7.6292 & 0.8082 & 0.0688 & 0.0651 \\
\hline 22-08 & 14.28 & 0.0001 & 8.6575 & 7.6292 & 1.0282 & 0.0677 & 0.0641 \\
\hline $22-09$ & 12 & 0.0001 & 10.9375 & 9.9092 & 1.0282 & 0.0569 & 0.0539 \\
\hline $22-10$ & 11.78 & 0.0001 & 11.1575 & 9.9092 & 1.2482 & 0.0559 & 0.0529 \\
\hline $22-11$ & 9.5 & 0.0001 & 13.4375 & 12.1892 & 1.2482 & 0.0451 & 0.0426 \\
\hline $22-12$ & 9.28 & 0.0001 & 13.6575 & 12.1892 & 1.4682 & 0.0440 & 0.0417 \\
\hline $22-13$ & 7 & 0.0001 & 15.9375 & 14.4692 & 1.4682 & 0.0332 & 0.0314 \\
\hline $22-14$ & 6.78 & 0.0001 & 16.1575 & 14.4692 & 1.6882 & 0.0322 & 0.0304 \\
\hline 22-15 & 4.75 & 0.0001 & 18.1875 & 16.4992 & 1.6882 & 0.0225 & 0.0213 \\
\hline $22-16$ & 4.58 & 0.0001 & 18.3575 & 16.4992 & 1.8582 & 0.0217 & 0.0206 \\
\hline & & & & & Total & 1.0000 & 0.9461 \\
\hline
\end{tabular}

Average Activity per 100 square $\mathrm{cm}=218.75619 \quad$ Cs-137 $206.95867 \quad B a-137 m$ 


\section{REVISED METHODOLOGY FOR DETERMINING CESIUM-137 CONTENT OF HN-200 GROUT CONTAINERS.}

TABLE 10 - CALCULATE CONVERSION FACTORS FOR CONTACT, 5 FEET, AND 10 FEET FOR A GROUTED HN-200 GROUT CONTAINER WITH 2200 LBS (NET) OF WASTE.

\begin{tabular}{|c|c|c|c|}
\hline Surface & Contact & 5 feet & 10 feet \\
\hline 22-01 & 8.694E-02 & $6.322 \mathrm{E}-03$ & $2.085 \mathrm{E}-03$ \\
\hline 22-02 & $6.035 \mathrm{E}-02$ & $5.116 \mathrm{E}-03$ & $1.693 \mathrm{E}-03$ \\
\hline $22-03$ & $2.831 \mathrm{E}-02$ & $3.010 \mathrm{E}-03$ & $1.011 \mathrm{E}-03$ \\
\hline $22-04$ & $2.114 \mathrm{E}-02$ & $2.385 \mathrm{E}-03$ & $8.039 \mathrm{E}-04$ \\
\hline $22-05$ & $1.035 \mathrm{E}-02$ & $1.308 \mathrm{E}-03$ & $4.464 \mathrm{E}-04$ \\
\hline $22-06$ & $7.788 \mathrm{E}-03$ & $1.021 \mathrm{E}-03$ & $3.495 \mathrm{E}-04$ \\
\hline 22-07 & $3.814 \mathrm{E}-03$ & $5.367 \mathrm{E}-04$ & $1.859 \mathrm{E}-04$ \\
\hline $22-08$ & $2.865 \mathrm{E}-03$ & 4.137E-04 & $1.438 \mathrm{E}-04$ \\
\hline 22-09 & $1.378 \mathrm{E}-03$ & $2.098 \mathrm{E}-04$ & $7.364 \mathrm{E}-05$ \\
\hline 22-10 & $1.031 \mathrm{E}-03$ & $1.601 \mathrm{E}-05$ & $5.639 \mathrm{E}-05$ \\
\hline 22-11 & $4.816 \mathrm{E}-04$ & $7.788 \mathrm{E}-05$ & $2.768 \mathrm{E}-05$ \\
\hline 22-12 & $3.583 \mathrm{E}-04$ & $5.894 \mathrm{E}-05$ & $2.102 \mathrm{E}-05$ \\
\hline $22-13$ & $1.598 \mathrm{E}-04$ & $2.722 \mathrm{E}-05$ & $9.791 \mathrm{E}-06$ \\
\hline 22-14 & $1.179 \mathrm{E}-04$ & $2.040 \mathrm{E}-05$ & $7.360 \mathrm{E}-06$ \\
\hline 22-15 & $5.282 \mathrm{E}-05$ & $9.408 \mathrm{E}-06$ & $3.417 \mathrm{E}-06$ \\
\hline $22-16$ & $4.071 \mathrm{E}-05$ & $7.331 \mathrm{E}-06$ & $2.669 \mathrm{E}-06$ \\
\hline ONVERSION FACTOR & $2.252 \mathrm{E}-01$ & $2.054 \mathrm{E}-02$ & $6.920 \mathrm{E}-03$ \\
\hline
\end{tabular}


HNF-4799, Rev 0

August 25, 1999

\title{
REVISED METHODOLOGY FOR DETERMINING CESIUM-137 CONTENT OF HN-200 GROUT CONTAINERS.
}

\begin{abstract}
TABLE 11 - MODEL FOR HN-200 GROUT CONTAINER FILLED WITH B CELL EQUIPMINT DUNNAGE AND SIFTED DEBRIS.
\end{abstract}

\section{GROUTED CONTAINER WITH 1900 LBS NET WEIGHT IN WASTE}

\begin{tabular}{|c|c|c|c|c|c|c|}
\hline Shield & R-O (in) & R-I (in) & $\mathrm{A}-\mathrm{O}(\mathrm{cm} 2)$ & $A-I(\mathrm{~cm} 2)$ & $\mathrm{Vol}(\mathrm{cm} 3)$ & Weight(lb) \\
\hline 1 & 22 & 21.8 & 47712.5838 & 47278.8331 & 24127.8199 & 416.12518 \\
\hline 2 & 19.5 & 19.31 & 42290.6993 & 41878.6361 & 20310.0606 & 350.28144 \\
\hline 3 & 17 & 16.81 & 36868.8148 & 36456.7516 & 17693.4592 & 305.15371 \\
\hline 4 & 14.5 & 14.31 & 31446.9303 & 31034.867 & 15076.8577 & 260.02598 \\
\hline 5 & 12 & 11.81 & 26025.0457 & 25612.9825 & 12460.2562 & 214.89825 \\
\hline 6 & 9.5 & 9.31 & 20603.1612 & 20191.098 & 9843.65474 & 169.77052 \\
\hline 7 & 7 & 6.81 & 15181.2767 & 14769.2135 & 7227.05327 & 124.64279 \\
\hline 8 & 4.75 & 4.61 & 10301.5806 & 9997.95507 & 3609.25744 & 62.247766 \\
\hline \multirow[t]{2}{*}{ Totals } & & & 230430.092 & 227220.337 & 110348.419 & 1903.1456 \\
\hline & & \multicolumn{2}{|c|}{ Total Surface Area = } & 457650.429 & & \\
\hline
\end{tabular}

\begin{tabular}{|c|c|c|c|c|c|c|c|}
\hline & $\begin{array}{l}\text { Surface } \\
\text { Radius }\end{array}$ & $\begin{array}{l}\text { Source } \\
\text { Thickness }\end{array}$ & $\begin{array}{c}\text { Distance } \\
\text { to outer } \\
\text { wall }\end{array}$ & $\begin{array}{c}\text { Total } \\
\text { Grout } \\
\text { Thickness }\end{array}$ & $\begin{array}{c}\text { Total } \\
\text { Steel } \\
\text { Thickness }\end{array}$ & Cesium & Barium \\
\hline 19-01 & 22 & 0.0001 & 0.9375 & 0.7992 & 0.1382 & 0.1043 & 0.0986 \\
\hline 19-02 & 21.8 & 0.0001 & 1.1375 & 0.7992 & 0.3382 & 0.1033 & 0.0977 \\
\hline $19-03$ & 19.5 & 0.0001 & 3.4375 & 3.0992 & 0.3382 & 0.0924 & 0.0874 \\
\hline 19-04 & 19.31 & 0.0001 & 3.6275 & 3.0992 & 0.5282 & 0.0915 & 0.0866 \\
\hline 19-05 & 17 & 0.0001 & 5.9375 & 5.4092 & 0.5282 & 0.0806 & 0.0762 \\
\hline 19-06 & 16.81 & 0.0001 & 6.1275 & 5.4092 & 0.7182 & 0.0797 & 0.0754 \\
\hline 19-07 & 14.5 & 0.0001 & 8.4375 & 7.7192 & 0.7182 & 0.0687 & 0.0650 \\
\hline 19-08 & 14.31 & 0.0001 & 8.6275 & 7.7192 & 0.9082 & 0.0678 & 0.0642 \\
\hline $19-09$ & 12 & 0.0001 & 10.9375 & 10.0292 & 0.9082 & 0.0569 & 0.0538 \\
\hline $19-10$ & 11.81 & 0.0001 & 11.1275 & 10.0292 & 1.0982 & 0.0560 & 0.0529 \\
\hline $19-11$ & 9.5 & 0.0001 & 13.4375 & 12.3392 & 1.0982 & 0.0450 & 0.0426 \\
\hline 19-12 & 9.31 & 0.0001 & 13.6275 & 12.3392 & 1.2882 & 0.0441 & 0.0417 \\
\hline 19-13 & 7 & 0.0001 & 15.9375 & 14.6492 & 1.2882 & 0.0332 & 0.0314 \\
\hline 19-14 & 6.81 & 0.0001 & 16.1275 & 14.6492 & 1.4782 & 0.0323 & 0.0305 \\
\hline 19-15 & 4.75 & 0.0001 & 18.1875 & 16.7092 & 1.4782 & 0.0225 & 0.0213 \\
\hline 19-16 & 4.61 & 0.0001 & 18.3275 & 16.7092 & 1.6182 & 0.0218 & 0.0207 \\
\hline & & & & & Total & 1.0000 & 0.9461 \\
\hline
\end{tabular}

Average Activity per 100 square $\mathrm{cm}=218.50739 \quad$ Cs-137 $206.72328 \quad \mathrm{Ba}-137 \mathrm{~m}$ 


\section{REVISED METHODOLOGY FOR DETERMINING CESIUM-137 CONTENT OF HN-200 GROUT CONTAINERS.}

TABLE 12 - CALCULATE CONVERSION FACTORS FOR CONTACT, 5 FEET, AND 10 FEET FOR A GROUTED HN-200 GROUT CONTAINER WITH 1900 LBS (NET) OF WASTE.

\begin{tabular}{|c|c|c|c|}
\hline Surface & Contact & 5 feet & 10 feet \\
\hline 19-01 & $8.694 \mathrm{E}-02$ & 6.322E-03 & $2.085 \mathrm{E}-03$ \\
\hline 19-02 & 6.313E-02 & $5.256 \mathrm{E}-03$ & $1.738 \mathrm{E}-03$ \\
\hline $19-03$ & $2.917 \mathrm{E}-02$ & $3.080 \mathrm{E}-03$ & $1.034 \mathrm{E}-03$ \\
\hline 19-04 & $2.263 \mathrm{E}-02$ & $2.520 \mathrm{E}-03$ & $8.484 \mathrm{E}-04$ \\
\hline $19-05$ & $1.097 \mathrm{E}-02$ & $1.375 \mathrm{E}-03$ & $4.689 \mathrm{E}-04$ \\
\hline $19-06$ & $8.568 \mathrm{E}-03$ & 1.109E-03 & $3.794 \mathrm{E}-04$ \\
\hline $19-07$ & 4.161E-03 & 5.805E-04 & $2.008 \mathrm{E}-04$ \\
\hline $19-08$ & 3.245E-03 & 4.632E-04 & $1.608 \mathrm{E}-04$ \\
\hline 19-09 & $1.550 \mathrm{E}-03$ & 2.337E-04 & 8.193E-05 \\
\hline 19-10 & $1.203 \mathrm{E}-03$ & $1.848 \mathrm{E}-04$ & $6.497 \mathrm{E}-05$ \\
\hline 19-11 & 5.581E-04 & 8.939E-05 & 3.172E-05 \\
\hline 19-12 & 4.309E-04 & $7.008 \mathrm{E}-05$ & $2.494 \mathrm{E}-05$ \\
\hline $19-13$ & $1.908 \mathrm{E}-04$ & $3.216 \mathrm{E}-05$ & $1.155 \mathrm{E}-05$ \\
\hline 19-14 & $1.461 \mathrm{E}-04$ & $2.498 \mathrm{E}-05$ & $8.991 \mathrm{E}-06$ \\
\hline $19-15$ & $6.505 \mathrm{E}-05$ & $1.146 \mathrm{E}-05$ & $4.153 \mathrm{E}-06$ \\
\hline $19-16$ & 5.205E-05 & $9.254 \mathrm{E}-06$ & $3.360 \mathrm{E}-06$ \\
\hline CONVERSION FACTOR & $2.330 \mathrm{E}-01$ & $2.136 \mathrm{E}-02$ & $7.147 \mathrm{E}-03$ \\
\hline
\end{tabular}

GROUTED CONVERSION FACTOR SUMMARY
$\begin{gathered}\text { Net } \\ \text { Weight }\end{gathered}$
$\begin{array}{cccc}\text { lbs } & \text { Contact } & \text { 5 Feet } & \text { 10 Feet } \\ 2500 & 2.195 \mathrm{E}-01 & 2.016 \mathrm{E}-02 & 6.744 \mathrm{E}-03 \\ \mathbf{2 2 0 0} & 2.252 \mathrm{E}-01 & 2.054 \mathrm{E}-02 & 6.920 \mathrm{E}-03 \\ 1900 & 2.330 \mathrm{E}-01 & 2.136 \mathrm{E}-02 & 7.147 \mathrm{E}-03\end{array}$


HNF-4799, Rev 0 August 25, 1999

\section{REVISED METHODOLOGY FOR DETERMINING CESIUM-137 CONTENT OF HN-200 GROUT CONTAINERS.}

FIGURE 4

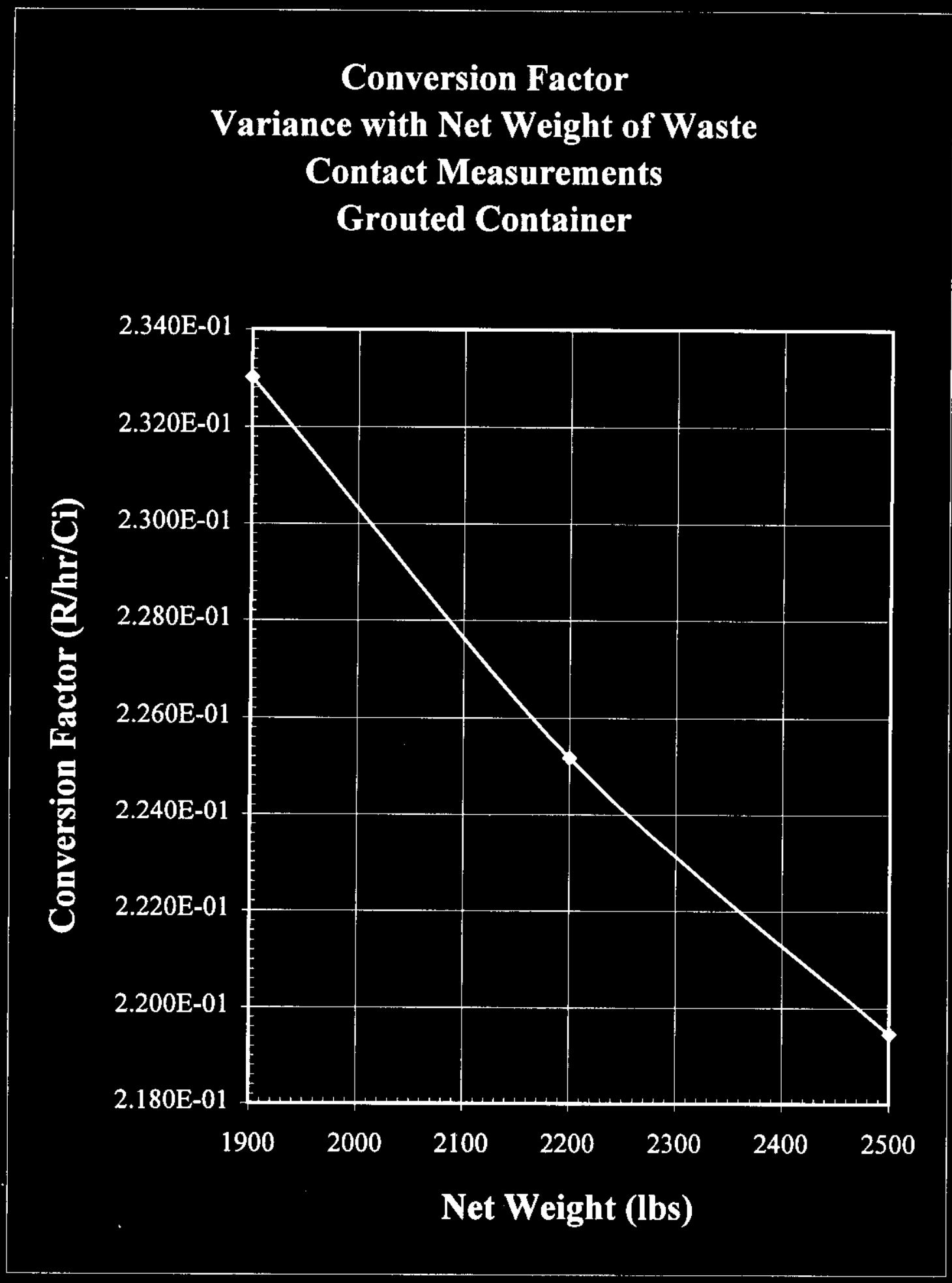


REVISED METHODOLOGY FOR DETERMINING CESIUM-137 CONTENT OF HN-200 GROUT CONTAINERS.

FIGURE 5

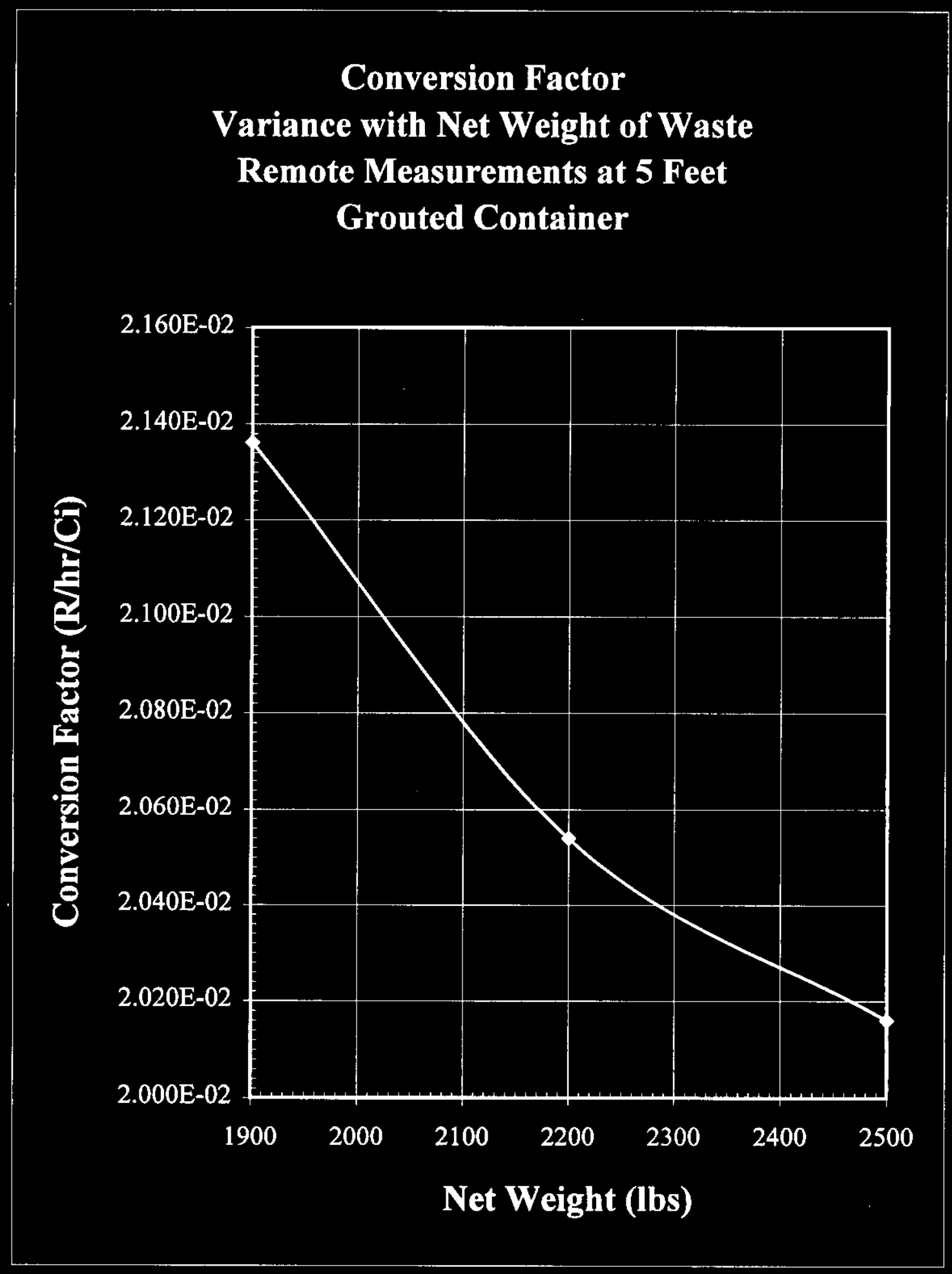


REVISED METHODOLOGY FOR DETERMINING CESIUM-137 CONTENT OF HN-200 GROUT CONTAINERS.

FIGURE 6

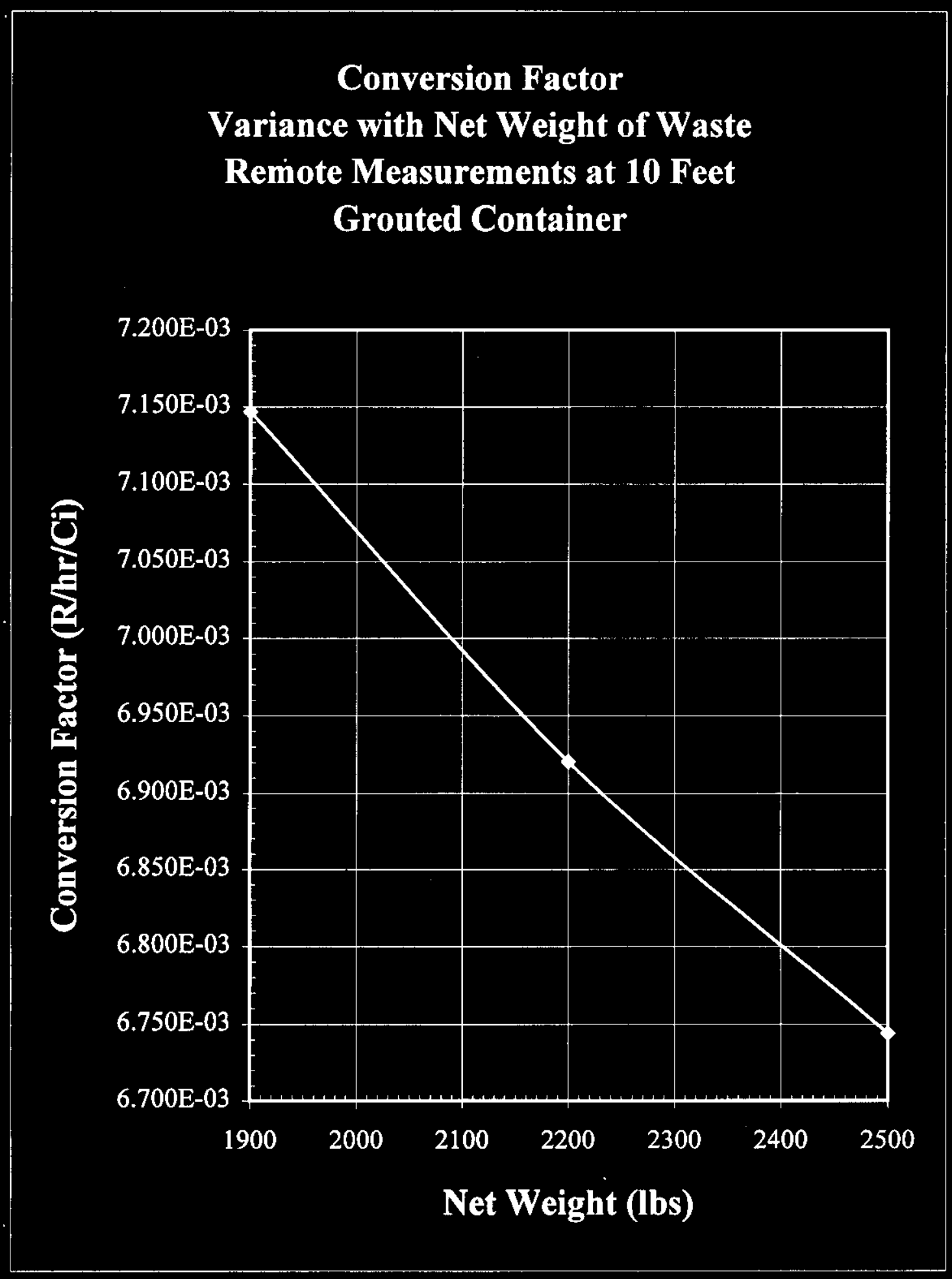




\title{
REVISED METHODOLOGY FOR DETERMINING CESIUM-137 CONTENT OF HN-200 GROUT CONTAINERS.
}

\author{
THIS ATTACHMENT PROVIDES DATA CORRELATION ANALYSIS TO SUPPORT \\ CONCLUSIONS DRAWN IN THIS TECHNICAL DOCUMENT AND PROVIDES \\ SPREADSHEETS FOR USE IN CALCULATING THE CURIE CONTENT OF HN-200 \\ GROUT CONTAINERS UNDER VARIOUS CONDITIONS.
}

TABLE 1 - DATA CORRELATION ANALYSIS

Data Correlation Analysis for Heterogeneous Case

\begin{tabular}{ccc} 
& & Actual \\
\cline { 2 - 3 } Grouted & Contact & $\mathbf{0 . 0 2 3 6 9 8 2 9}$ \\
Grouted & 5 Feet & 0.26495009 \\
Grouted & 10 Feet & 0.22996767 \\
Ungrouted & Contact & 0.51803993 \\
Ungrouted & 5 Feet & 0.99283017 \\
Ungrouted & 10 Feet & 0.99877489 \\
\hline
\end{tabular}

Data Correlation Analysis for Mostly Heterogeneous Case

\begin{tabular}{ccc} 
& & Actual \\
\cline { 2 - 3 } Grouted & Contact & 0.31679408 \\
Grouted & 5 Feet & 0.47940461 \\
Grouted & 10 Feet & 0.53018367 \\
Ungrouted & Contact & 0.80807004 \\
Ungrouted & 5 Feet & 0.99803202 \\
Ungrouted & 10 Feet & 0.99984409 \\
\hline
\end{tabular}

Data Correlation Analysis for Slightly Homogeneous Case

\begin{tabular}{ccc} 
& & Actual \\
\cline { 2 - 3 } Grouted & Contact & $\mathbf{0 . 0 8 9 5 3 6 2}$ \\
Grouted & 5 Feet & 0.3904012 \\
Grouted & 10 Feet & 0.50336033 \\
Ungrouted & Contact & 0.88823486 \\
Ungrouted & 5 Feet & 0.99934768 \\
Ungrouted & 10 Feet & 0.99987656 \\
\cline { 2 - 3 }
\end{tabular}

Data Correlation Analysis for Homogeneous Case

\begin{tabular}{ccc} 
& & Actual \\
\cline { 2 - 3 } Grouted & Contact & $\mathbf{0 . 8 2 6 2 9 2 2 7}$ \\
Grouted & 5 Feet & 0.91482892 \\
Grouted & 10 Feet & $\mathbf{0 . 9 4 8 2 5 6 0 6}$ \\
Ungrouted & Contact & $\mathbf{0 . 9 9 2 1 4 9 2 2}$ \\
Ungrouted & 5 Feet & 0.99995345 \\
Ungrouted & 10 Feet & $\mathbf{0 . 9 9 9 9 9 4 5 3}$ \\
\cline { 2 - 3 } & &
\end{tabular}

Data Correlation Analysis for All Data Case

\begin{tabular}{ccc} 
& & Actual \\
\cline { 2 - 3 } Grouted & Contact & 0.93447177 \\
Ungrouted & Contact & 0.99611592
\end{tabular}

TABLE 2 - CALCULA'TION DATA SHEET FOR AN UNGROUTED CONTAINER REMOTELY MEASURED AT 10 FEET FROM THE SURFACE OF THE CONTAINER. RO-7 Source Ck Correction =

lbs. 
HNF-4799, Rev 0

August 25, 1999

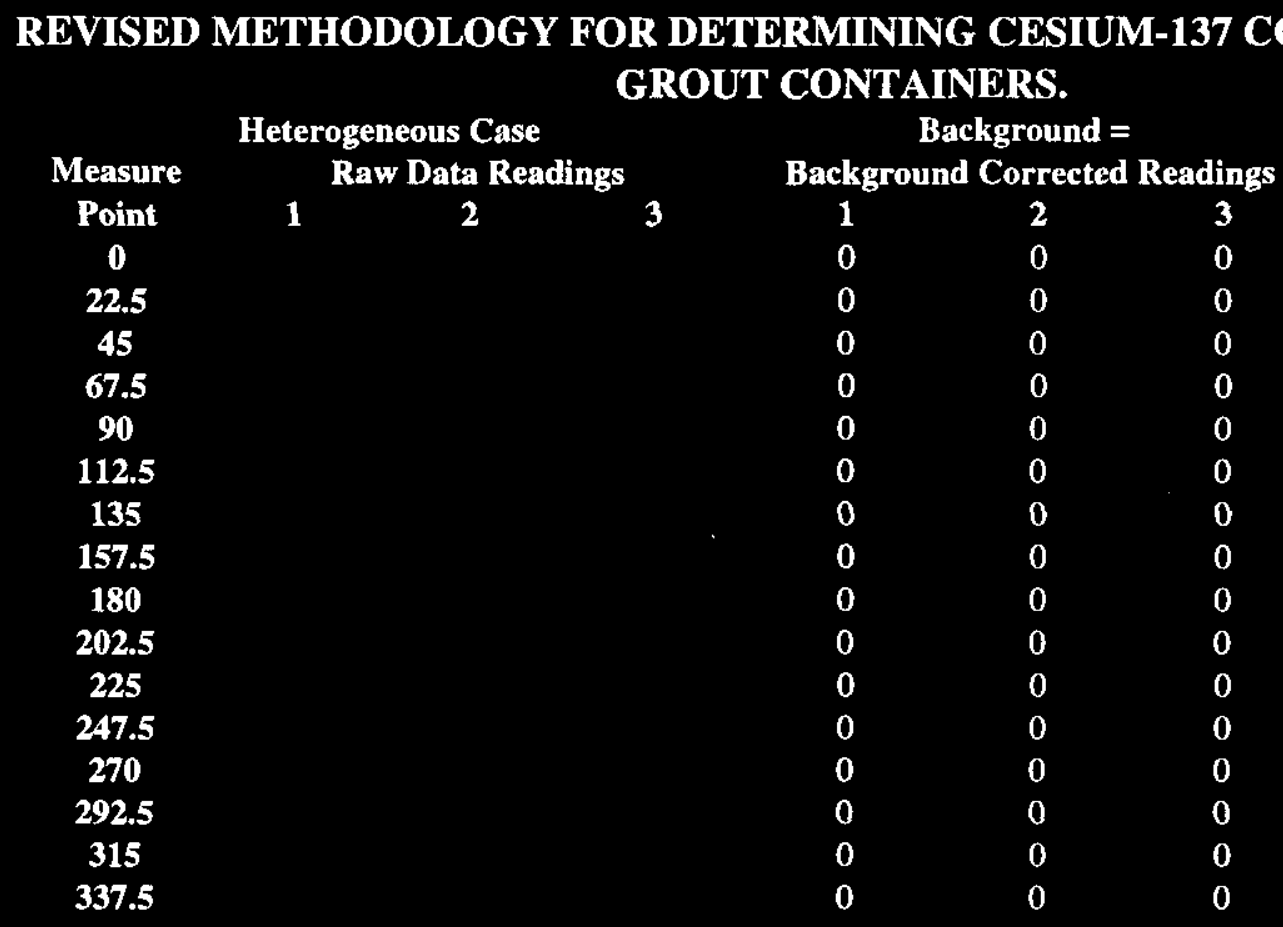

Average \#DIV/0! \#DIV/0! \#DIV/0! $\quad 0 \quad 000$

Error Correction $=1.1007$

Conversion Factor $=$
Overall Average $=0$

Corrected Overall A verage $=0$

Measured Cs-137 = \#DIV/0!

Predicted Cs-137 = \#DIV/0! \#DIV $/ 0$ !

95\% Bounding Value Cs-137 = \#DIV/0!

Regression Statistics from Attachment 5 Figure 6

$\begin{array}{ccc} & \text { Value } & \text { Std Error } \\ \text { Intercept } & 0.1731 & 0.1798 \\ \text { Slope } & 0.9896 & 0.0085 \\ \text { Regression } & \text { N/A } & 0.2867 \\ \text { Residual } & 33.0000 & \text { N/A } \\ \text { X Bar } & 20.2845 & \text { N/A }\end{array}$

TABLE 3 - CALCULATION DATA SHEET FOR AN UNGROUTED CONTAINER REMOTELY MEASURED AT 5 FEET FROM THE SURFACE OF THE CONTAINER.

RO-7 Source Ck Correction =

Heterogeneous Case

Measure

Point
Raw Data Readings

1
2
Net Weight $=$

Background $=$

Background Corrected Readings

R/hr

OF HN-200

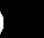




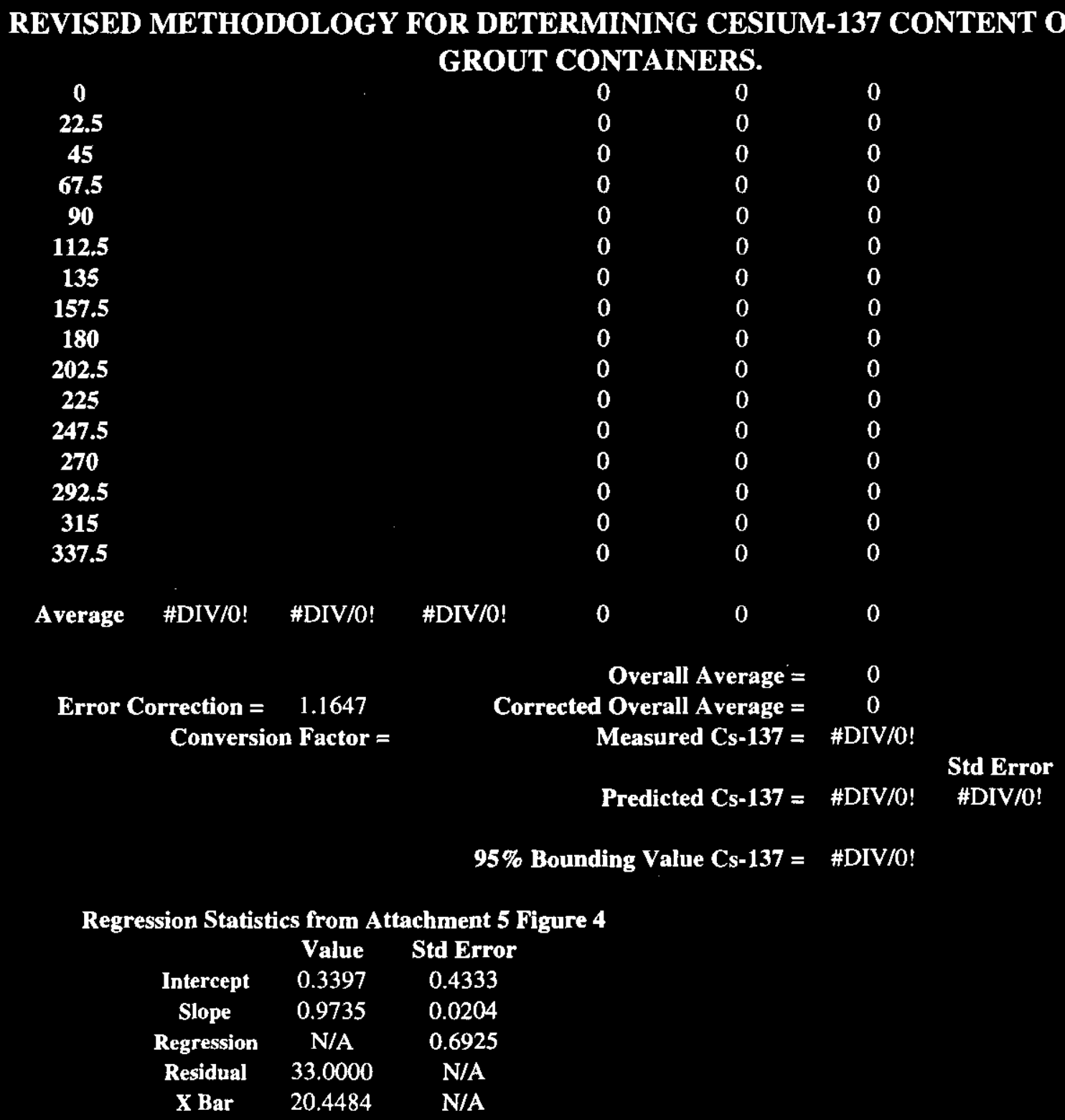

TABLE 4 - CALCULATION DATA SHEET FOR AN UNGROUTED CONTAINER MEASURED ON CONTACT WITH THE SURFACE OF THE CONTAINER. RO-7 Source Ck Correction $=$

All Data Case

Measure

Point

Raw Data Readings

0-1

0.2

$0-3$

\section{Net Weight $=$}

Background =

Background Corrected Readings

3

$\begin{array}{lll}\mathbf{1} & \mathbf{2} & \mathbf{3} \\ 0 & 0 & 0 \\ 0 & 0 & 0 \\ 0 & 0 & 0\end{array}$

lbs.

R/hr 
HNF-4799, Rev 0

August 25, 1999

\section{REVISED METHODOLOGY FOR DETERMINING CESIUM-137 CONTENT OF HN-200} GROUT CONTAINERS.

\section{$0-4$ \\ $0-5$ \\ 0-6 \\ 90-1 \\ 90-2 \\ 90.3 \\ 90-4 \\ $90-5$ \\ 90-6 \\ 180-1 \\ 180-2 \\ $180-3$ \\ 180-4 \\ 180-5 \\ 180-6 \\ 270-1 \\ 270-2 \\ 270-3 \\ 270-4 \\ 270-5 \\ 270-6}

Average \#DIV/0! \#DIV/0!

Error Correction $=\quad 1.21$

Conversion Factor $=$

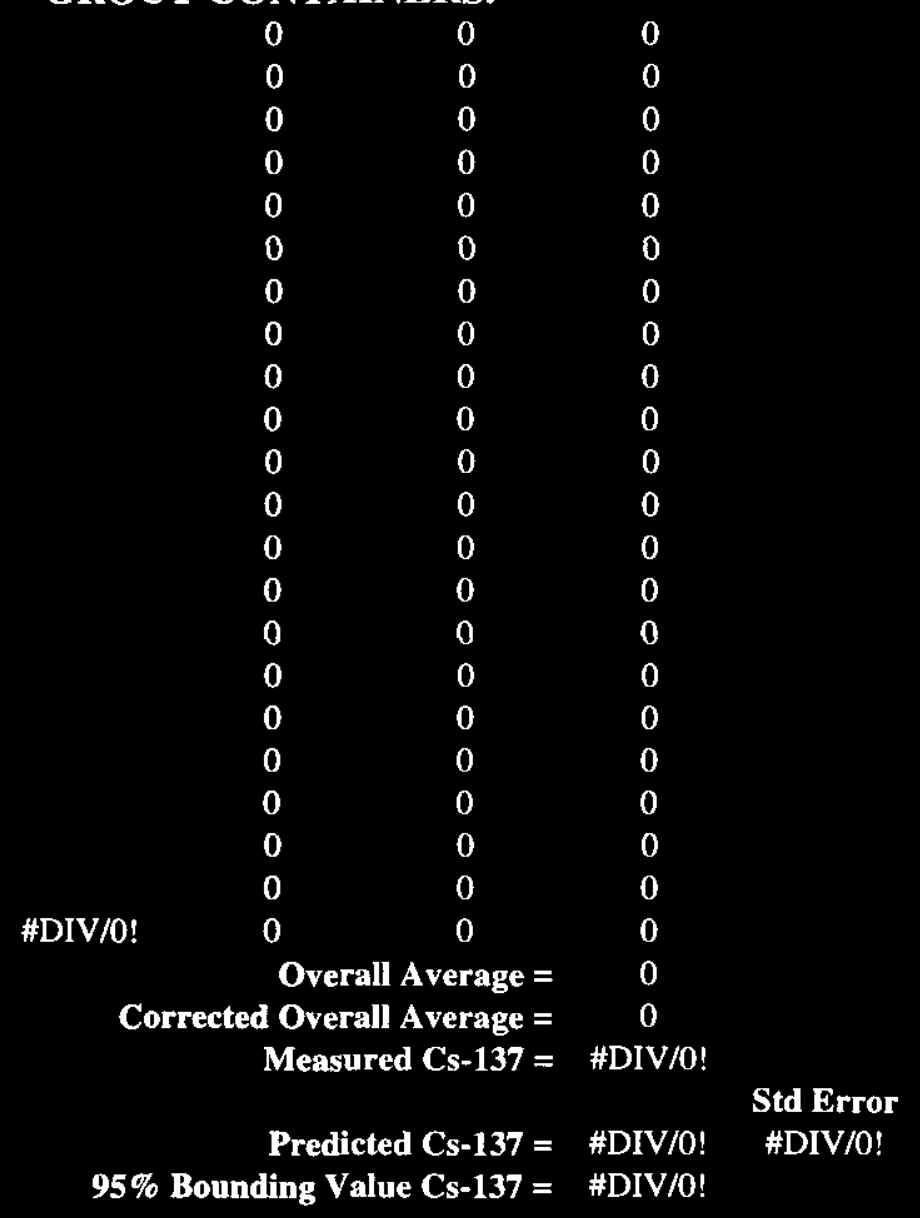

Regression Statistics from Attachment 5 Figure 26

$\begin{array}{ccc} & \text { Value } & \text { Std Error } \\ \text { Intercept } & -0.6488 & 2.0513 \\ \text { Slope } & 0.9483 & 0.0099 \\ \text { Regression } & \text { N/A } & 14.1807 \\ \text { Residual } & 72.0000 & \text { N/A } \\ \text { X Bar } & 123.5767 & \text { N/A }\end{array}$

TABLE 5 - CALCULATION DATA SHEET FOR A GROUTED CONTAINER REMOTELY MEASURED AT 10 FEET FROM THE SURFACE OF THE CONTAINER.

R0-7 Source Ck Correction = Homogeneous Case

Measure

Point

0

22.5

45

67.5

90

112.5

\begin{abstract}
Raw Data Readings
\end{abstract}
1

2

\section{3}

Net Weight $=$

Background =

Background Corrected Readings

$\begin{array}{lll}\mathbf{1} & \mathbf{2} & \mathbf{3} \\ \mathbf{0} & 0 & 0 \\ \mathbf{0} & 0 & 0 \\ 0 & 0 & 0 \\ \mathbf{0} & 0 & 0 \\ 0 & 0 & 0 \\ 0 & 0 & 0\end{array}$

lbs.

$\mathbf{R} / \mathbf{h r}$ 


\section{REVISED METHODOLOGY FOR DETERMINING CESIUM-137 CONTENT OF HN-200 GROUT CONTAINERS.}

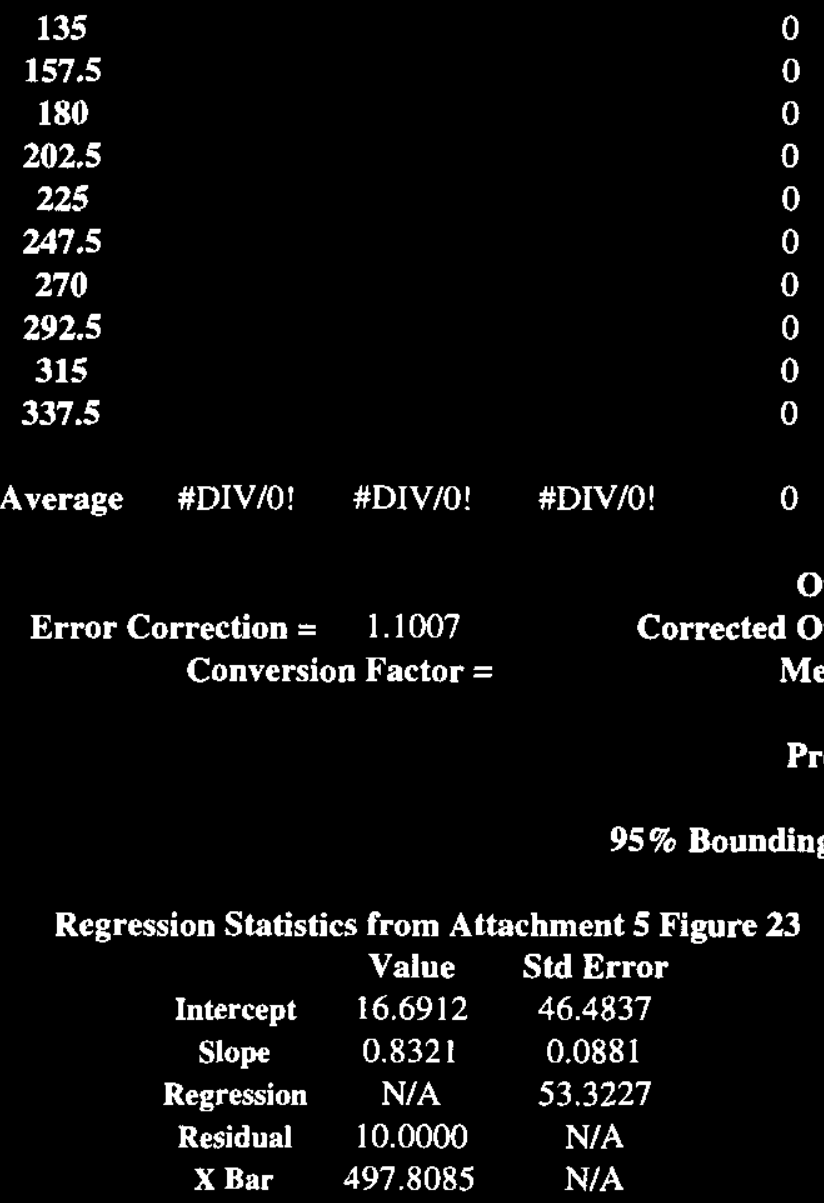

Overall Average $=\quad 0$

Measured Cs-137 = \#DIV/0!

Predicted Cs-137 = \#DIV/0! \#DIV/0!

Std Error 
HNF-4799, Rev 0

August 25, 1999

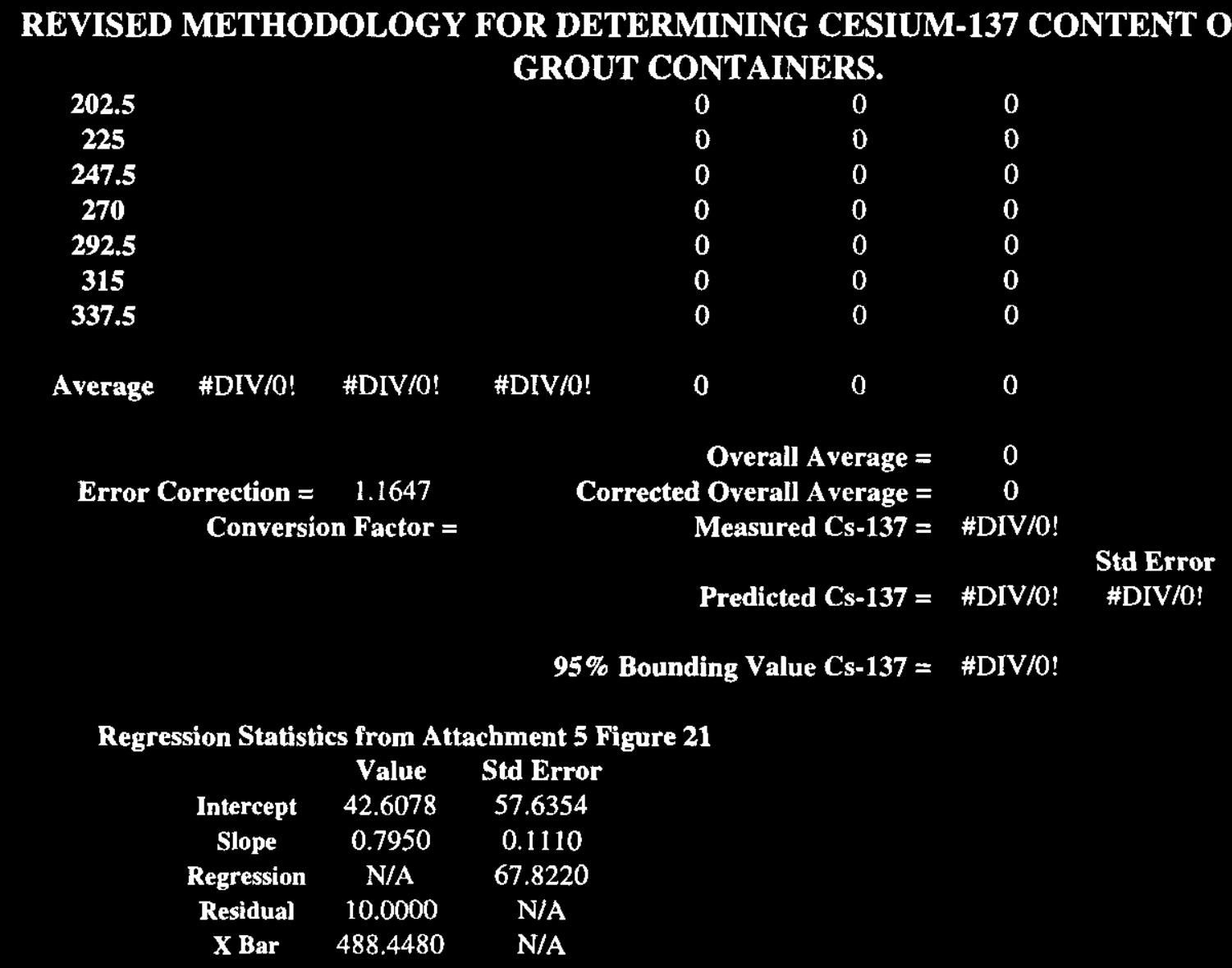

TABLE 7 - CALCULATION DATA SHEET FOR A GROUTED CONTAINER MEASURED ON CONTACT WITH THE SURFACE OF THE CONTAINER.

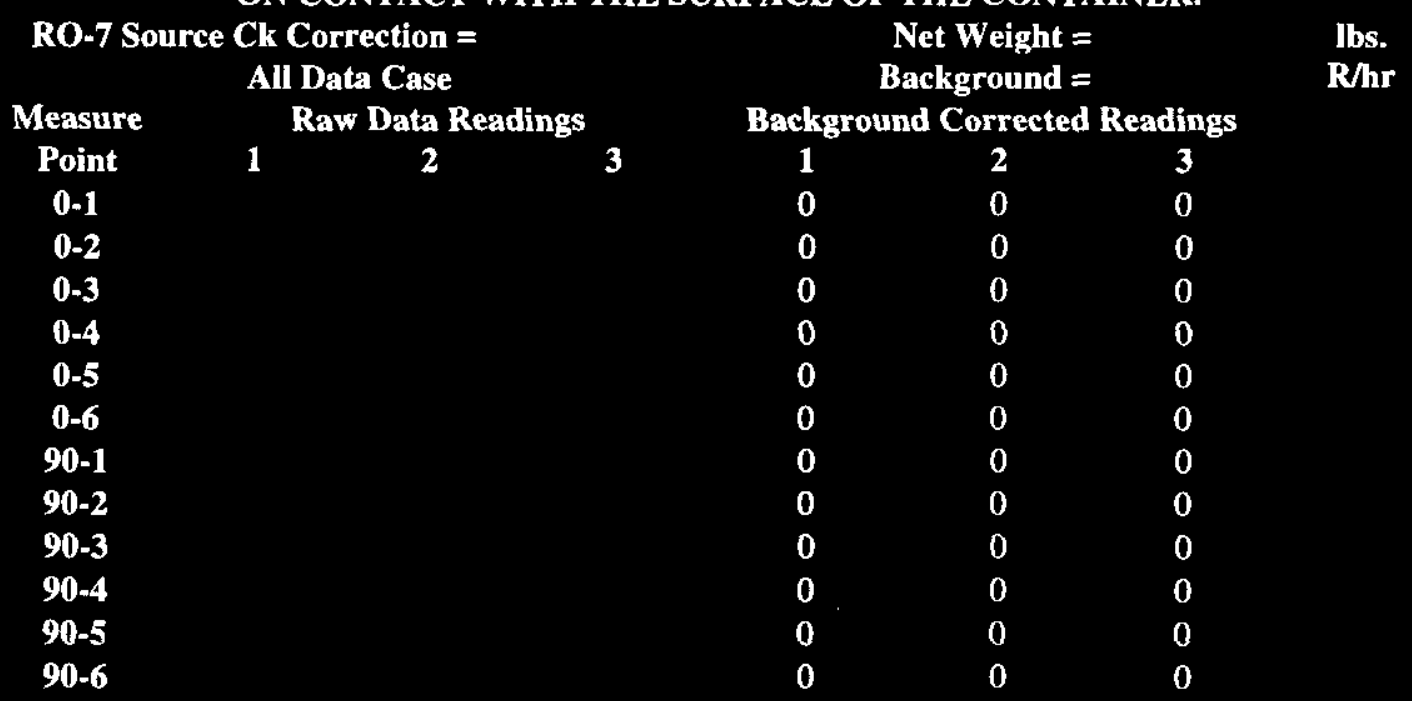




\section{REVISED METHODOLOGY FOR DETERMINING CESIUM-137 CONTENT OF HN-200 GROUT CONTAINERS.}

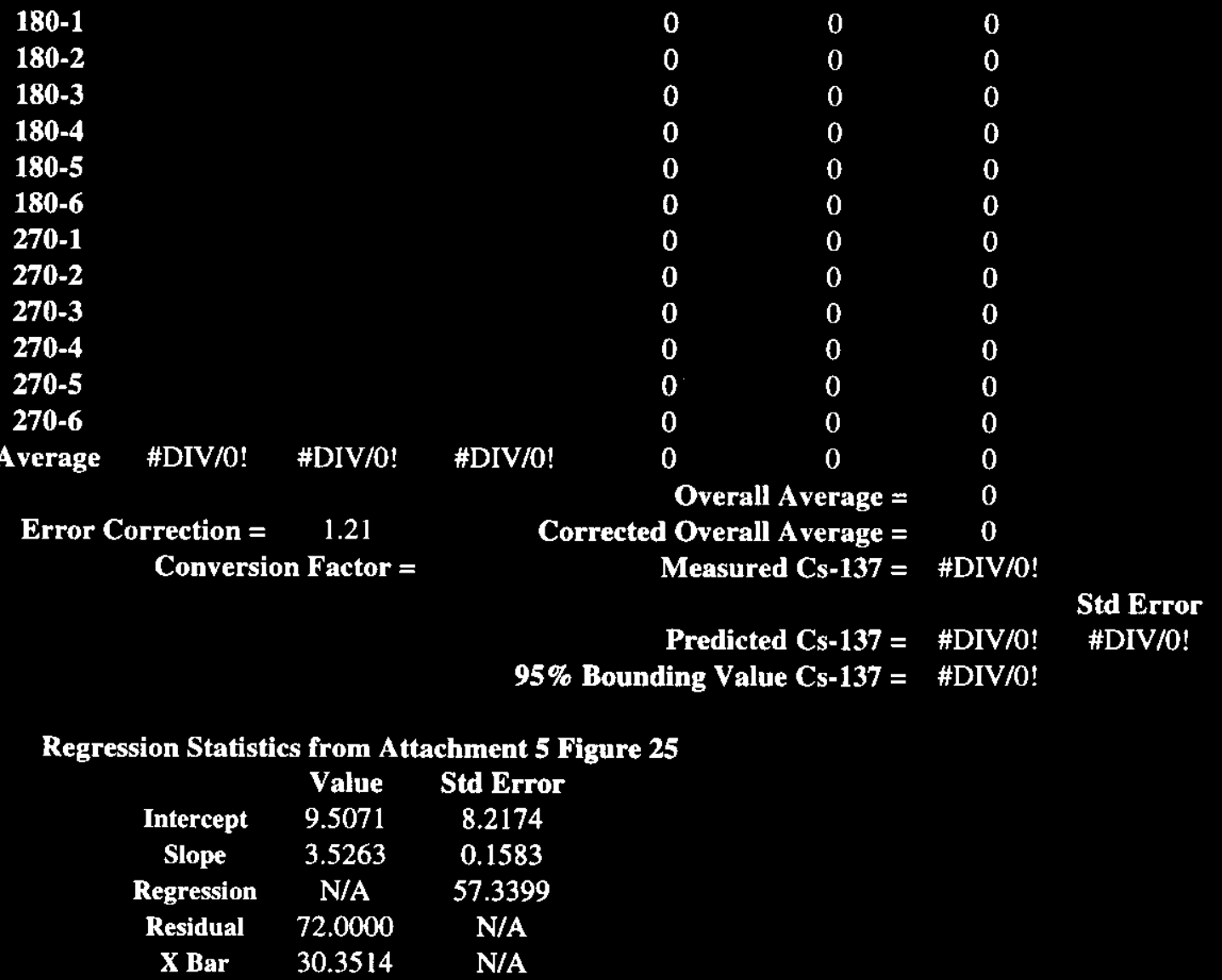

